The EIC Science case: a report on the joint BNL/INT/JLab program

\title{
Gluons and the quark sea at high energies: distributions, polarization, tomography
}

Institute for Nuclear Theory, University of Washington, USA

September 13 to November 19, 2010

\begin{abstract}
Editors:
D. Boer, Universiteit Groningen, The Netherlands

M. Diehl, Deutsches Elektronen-Synchroton DESY, Germany

R. Milner, Massachusetts Institute of Technology, USA

R. Venugopalan, Brookhaven National Laboratory, USA

W. Vogelsang, Universität Tübingen, Germany
\end{abstract}

Brookhaven National Laboratory, Upton, NY

Institute for Nuclear Theory, Seattle, WA

Thomas Jefferson National Accelerator Facility, Newport News, VA 
The EIC Science case: a report on the joint BNL/INT/JLab program

Gluons and the quark sea at high energies:

distributions, polarization, tomography

Seattle, Washington, September 13 to November 19, 2010

Program homepage:

http://www.int.washington.edu/PROGRAMS/10-3

Online proceedings at:

http://arxiv.org/abs/1108.1713

\section{DISCLAIMER:}

This report was prepared as an account of work sponsored by an agency of the United States Government. Neither the United States Government nor any agency thereof, nor any of their employees, nor any of their contractors, subcontractors, or their employees, makes any warranty, express or implied, or assumes any legal liability or responsibility for the accuracy, completeness, or any third party's use or the results of such use of any information, apparatus, product, or process disclosed, or represents that its use would not infringe privately owned rights. Reference herein to any specific commercial product, process, or service by trade name, trademark, manufacturer, or otherwise, does not necessarily constitute or imply its endorsement, recommendation, or favoring by the United States Government or any agency thereof or its contractors or subcontractors. The views and opinions of authors expressed herein do not necessarily state or reflect those of the United States Government or any agency thereof.

Published by:

Brookhaven National Laboratory, USA

Institute of Nuclear Theory, University of Washington, USA

Thomas Jefferson National Accelerator Facility, USA

August 2011

BNL-96164-2011

INT-PUB-11-034

JLAB-THY-11-1373

Printed at Brookhaven National Laboratory 


\section{Foreword}

The study of the fundamental structure of nuclear matter is a central thrust of physics research in the United States. As indicated in Frontiers of Nuclear Science, the $2007 \mathrm{Nu}-$ clear Science Advisory Committee long range plan, consideration of a future Electron-Ion Collider (EIC) is a priority and will likely be a significant focus of discussion at the next long range plan. We are therefore pleased to have supported the ten week program in fall 2010 at the Institute of Nuclear Theory which examined at length the science case for the EIC. This program was a major effort; it attracted the maximum allowable attendance over ten weeks.

This report summarizes the current understanding of the physics and articulates important open questions that can be addressed by an EIC. It converges towards a set of "golden" experiments that illustrate both the science reach and the technical demands on such a facility, and thereby establishes a firm ground from which to launch the next phase in preparation for the upcoming long range plan discussions. We thank all the participants in this productive program. In particular, we would like to acknowledge the leadership and dedication of the five co-organizers of the program who are also the co-editors of this report.

David Kaplan, Director, National Institute for Nuclear Theory

Hugh Montgomery, Director, Thomas Jefferson National Accelerator Facility

Steven Vigdor, Associate Lab Director, Brookhaven National Laboratory 


\section{Preface}

This volume is based on a ten-week program on "Gluons and the quark sea at high energies", which took place at the Institute for Nuclear Theory (INT) in Seattle from September 13 to November 19, 2010. The principal aim of the program was to develop and sharpen the science case for an Electron-Ion Collider (EIC), a facility that will be able to collide electrons and positrons with polarized protons and with light to heavy nuclei at high energies, offering unprecedented possibilities for in-depth studies of quantum chromodynamics. Guiding questions were

- What are the crucial science issues?

- How do they fit within the overall goals for nuclear physics?

- Why can't they be addressed adequately at existing facilities?

- Will they still be interesting in the 2020's, when a suitable facility might be realized?

The program started with a five-day workshop on "Perturbative and Non-Perturbative Aspects of QCD at Collider Energies", which was followed by eight weeks of regular program and a concluding four-day workshop on "The Science Case for an EIC".

More than 120 theorists and experimentalists took part in the program over ten weeks. It was only possible to smoothly accommodate such a large number of participants because of the extraordinary efforts of the INT staff, to whom we extend our warm thanks and appreciation. We thank the INT Director, David Kaplan, for his strong support of the program and for covering a significant portion of the costs for printing this volume. We gratefully acknowledge additional financial support provided by BNL and JLab.

The program was structured along several subtopics, which roughly correspond to the chapters in this report. For each topic, convenors were appointed, who played an important role in the scientific organization of the program weeks and in editing the corresponding chapters. We gratefully thank them for their work. Special thanks are due to Matt Lamont and Marco Stratmann, who took on the lion's share in the painstaking task of merging the different chapters and making final edits.

Last but not least, we thank all participants of the INT program and all authors of this report for the work and enthusiasm they put into their contributions. Thanks to their

efforts, much progress has been achieved, and we hope that the community will keep this momentum going in the continuing effort to build a compelling case for an Electron-Ion Collider.

August 2011

The program organizers

Daniël Boer

Markus Diehl

Richard Milner

Raju Venugopalan

Werner Vogelsang 


\section{Convenors and chapter editors}

A. Accardi

Hampton University, Hampton, VA 23668, USA

Thomas Jefferson National Accelerator Facility, Newport News, VA 23606, USA

E. C. Aschenauer

Brookhaven National Laboratory, Upton, NY 11973, USA

M. Burkardt

New Mexico State University, Las Cruces, NM 88003, USA

R. Ent

Thomas Jefferson National Accelerator Facility, Newport News, VA 23606, USA

V. Guzey

Thomas Jefferson National Accelerator Facility, Newport News, VA 23606, USA

D. Hasch

INFN, Laboratori Nazionali di Frascati, 00044 Frascati, Italy

K. Kumar

University of Massachusetts, Amherst, MA 01003, USA

M. A. C. Lamont

Brookhaven National Laboratory, Upton, NY 11973, USA

Y. Li

Brookhaven National Laboratory, Upton, NY 11973, USA

W. Marciano

Brookhaven National Laboratory, Upton, NY 11973, USA

C. Marquet

Physics Department, Theory Unit, CERN, CH-1211 Genève 23, Switzerland

F. Sabatié

CEA Saclay, Service de Physique Nucléaire, 91191 Gif-sur-Yvette, France

M. Stratmann

Brookhaven National Laboratory, Upton, NY 11973, USA

F. Yuan

Lawrence Berkeley National Laboratory, Berkeley, CA 94720, USA 


\section{List of Authors}

R. Sassot, P. Zurita

Instituto de Física de Buenos Aires and Departamento de Física, Facultad de Ciencias Exactas y Naturales, Universidad de Buenos Aires, Buenos Aires, Argentina

I. O. Cherednikov

Universiteit Antwerpen, 2020 Antwerpen, Belgium

V. P. Gonçalves

Instituto de Física e Matemática, Universidade Federal de Pelotas, Pelotas, RS, Brazil

R. Sandapen

Département de Physique et d'Astronomie, Université de Moncton, Canada

B. Z. Kopeliovich

Departamento de Física, Universidad Técnica Federico Santa María and Instituto de Estudios Avanzados en Ciencias e Ingeniería and Centro Científico-Tecnológico de Valparaíso, Valparaíso, Chile

J.-H. Gao

Department of Modern Physics, University of Science and Technology of China, Hefei, Anhui 230026, China

Z.-T. Liang

Department of Physics, Shandong University, Jinan, Shandong 250100, China

K. Passek-Kumerički

Theoretical Physics Division, Rudjer Bošković Institute, Zagreb, Croatia

K. Kumerički

Department of Physics, University of Zagreb, Zagreb, Croatia

T. Lappi

Department of Physics, 40014 University of Jyväskylä, Jyväskylä and Helsinki Institute of Physics, 00014 University of Helsinki, Helsinki, Finland

S. Wallon

LPT, Université d'Orsay, CNRS, 91404 Orsay and UPMC, Univ. Paris 06, Faculté de Physique, 75252 Paris, France

B. Pire

CPHT, École Polytechnique, CNRS, 91128 Palaiseau, France

R. Géraud, H. Moutarde, F. Sabatié

CEA, Centre de Saclay, Irfu/Service de Physique Nucléaire, 91191 Gif-sur-Yvette, France 
F. Gelis, G. Soyez

Institut de Physique Théorique, CEA/Saclay, 91191 Gif-sur-Yvette Cedex, France

M. Meškauskas, D. Müller, N. G. Stefanis

Institut für Theoretische Physik II, Ruhr-Universität Bochum, 44780 Bochum, Germany

K. Gallmeister, U. Mosel

Institute for Theoretical Physics, Giessen University, 35392 Giessen, Germany

M. Diehl

Deutsches Elektronen-Synchroton DESY, 22603 Hamburg, Germany

J. Bartels

Institute of Theoretical Physics, Universität Hamburg, 22761 Hamburg, Germany

H. J. Pirner

Institute of Theoretical Physics, Universität Heidelberg, 69120 Heidelberg, Germany

P. Hägler

Institut für Kernphysik, Johannes-Gutenberg-Universität, 55099 Mainz, Germany

B. Jäger, H. Spiesberger

Institut für Physik (THEP), Johannes-Gutenberg-Universität, 55099 Mainz, Germany

T. Lautenschlager, A. Schäfer

Institut für Theoretische Physik, Universität Regensburg, 93040 Regensburg, Germany

F. Ringer, W. Vogelsang

Institut für Theoretische Physik, Universität Tübingen, Auf der Morgenstelle 14, 72076

Tübingen, Germany

P. Kroll

Fachbereich Physik, Universität Wuppertal, 42097 Wuppertal, Germany

S. Alekhin, J Blümlein, S.-O. Moch

Deutsches Elektronen-Synchrotron DESY, Platanenallee 6, 15738 Zeuthen, Germany

C. Pisano

Dipartimento di Fisica, Università di Cagliari, and INFN, Sezione di Cagliari, Italy

D. Hasch

INFN Laboratori Nazionali di Frascati, 00044 Frascati, Italy

J. Rojo

Dipartimento di Fisica, Università di Milano and Instituto Nazionale di Fisica Nucleare, Sezione di Milano, Milano, Italy 
A. Bacchetta, B. Pasquini, M. Radici

Instituto Nazionale di Fisica Nucleare, Sezione di Pavia and Dipartimento di Fisica, Università di Pavia, Pavia, Italy

C. Ciofi degli Atti, C. B. Mezzetti, L. P. Kaptari

Instituto Nazionale di Fisica Nucleare, Sezione di Perugia and Department of Physics, University of Perugia, Perugia, 06123, Italy

M. Anselmino

Università di Torino and INFN, Sezione di Torino, 10125 Torino, Italy

K. Tanaka

Department of Physics, Juntendo University, Inzai, Chiba, Japan

Y. Koike

Department of Physics, Niigata University, Niigata, Japan

S. Kumano

KEK Theory Center, Institute of Particle and Nuclear Studies and Department of Particle and Nuclear Studies, Graduate University for Advanced Studies, Tsukuba, Japan

L. Motyka

Institute of Physics, Jagiellonian University, Cracow, Poland

K. Golec-Biernat, A. M. Staśto

Institute of Nuclear Physics, Polish Academy of Sciences, Cracow, Poland

K. Golec-Biernat

Institute of Physics, University of Rzeszów, Rzeszów, Poland

L. Szymanowski

Soltan Institute for Nuclear Studies, Warsaw, Poland

I. O. Cherednikov, L. P. Kaptari, A. Radyushkin

Bogoliubov Laboratory of Theoretical Physics, JINR, 141980 Dubna, Russia

S. Alekhin

Institute for High Energy Physics, 142281 Protvino, Moscow Region, Russia

A. Kondratenko

NTL Zaryad, Novosibirsk, Russia

W. A. Horowitz

Department of Physics, University of Cape Town, Rondebosch 7701, South Africa 
G. Schnell

Department of Theoretical Physics, University of the Basque Country UPV/EHU, 48080

Bilbao and IKERBASQUE, Basque Foundation for Science, 48011 Bilbao, Spain

C. Marquet

Physics Department, Theory Unit, CERN, 1211 Genève 23, Switzerland

P. Chevtsov

Paul Scherrer Institut, 5232 Villigen PSI, Switzerland

P. J. Mulders, T. C. Rogers

Department of Physics and Astronomy, Vrije Universiteit, Amsterdam, The Netherlands

D. Boer

Theory Group, KVI, University of Groningen, The Netherlands

J. R. Forshaw

Particle Physics Group, University of Manchester, Manchester, UK

A. Cooper-Sarkar

University of Oxford, Denys Wilkinson Bldg., Keble Road, Oxford OX1 3RH, UK

G. A. Chirilli, D. Müller, X.-N. Wang, F. Yuan

Nuclear Science Division, Lawrence Berkeley National Laboratory, Berkeley, CA 94720, USA

X. Qian

California Institute of Technology, Pasadena, CA 91125, USA

S. J. Brodsky

SLAC National Accelerator Laboratory, Stanford University, Stanford, CA 94309, USA

P. Schweitzer

Department of Physics, University of Connecticut, Storrs, CT 06269, USA

T. Horn

Catholic University of America, Washington, DC 20064, USA

K. Tuchin

Department of Physics and Astronomy, Iowa State University, Ames, IA 50011, USA

R. Dupré, B. Erdelyi, S. Manikonda, P. N. Ostrumov

Physics Division, Argonne National Laboratory, Argonne, IL 60439, USA

S. Abeyratne, B. Erdelyi

Northern Illinois University, De Kalb, IL 60115, USA 
A. Vossen

Department of Physics, Indiana University, Bloomington, IN 47408, USA

K. Kumar, S. Riordan

Department of Physics, University of Massachusetts, Amherst, MA 01002, USA

E. Tsentalovich

MIT Bates Linear Accelerator Center, Middleton, MA 01949, USA

G. R. Goldstein

Department of Physics and Astronomy, Tufts University, Medford, MA 02155, USA

E. Pozdeyev

FRIB, Michigan State University, East Lansing, MI 48824, USA

M. Huang

Department of Physics, Duke University, Durham, NC 27708, USA

M. Burkardt

New Mexico State University, Las Cruces, NM 88003, USA

C. Aidala

Physics Division, Los Alamos National Laboratory, Los Alamos, NM 87545, USA

A. Dumitru

Department of Natural Sciences, Baruch College, New York, NY 10010, USA

F. Dominguez

Department of Physics, Columbia University, New York, NY 10027, USA

I. Ben-Zvi, A. Deshpande, C. Faroughy, L. Hammons, Y. Hao, E. C. Johnson, V. N. Litvinenko, S. Taneja, N. Tsoupas, S. Webb

Department of Physics \& Astronomy, Stony Brook University, Stony Brook, NY 117943400, USA

J. Beebe-Wang, S. Belomestnykh, I. Ben-Zvi, M. M. Blaskiewicz, R. Calaga, X. Chang, A. Fedotov, D. Gassner, H. Hahn, L. Hammons, Y. Hao, P. He, W. Jackson, A. Jain, E. C. Johnson, D. Kayran, J. Kewisch, V. N. Litvinenko, Y. Luo, G. Mahler, G. McIntyre, W. Meng, M. Minty, B. Parker, A. Pikin, V. Ptitsyn, T. Rao, T. Roser, B. Sheehy, J. Skaritka, S. Tepikian, Y. Than, D. Trbojevic, N. Tsoupas, J. Tuozzolo, G. Wang, S. Webb, Q. Wu, W. Xu, A. Zelenski Collider-Accelerator Department, Brookhaven National Laboratory, Upton, NY 11973, USA

E. C. Aschenauer, G. Beuf, T. Burton, R. Debbe, S. Fazio, M. A. C. Lamont, Y. Li, W. J. Marciano, J.-W. Qiu, M. Stratmann, T. Toll, T. Ullrich

Physics Department, Brookhaven National Laboratory, Upton, NY 11973, USA 
A. Deshpande, A. Dumitru, Z.-B. Kang, A. M. Staśto, F. Yuan

RIKEN BNL Research Center, Brookhaven National Laboratory, Upton, NY 11973, USA

Y. V. Kovchegov, A Majumder

Department of Physics, The Ohio State University, Columbus, OH 43210, USA

A. Metz, J. Zhou

Department of Physics, Temple University, Philadelphia, PA 19122, USA

L. Gamberg

Penn State University-Berks, Reading, PA 19610, USA

A. M. Staśto, M. Strikman, B.-W. Xiao

Physics Department, Pennsylvania State University, State College, PA 16802, USA

M. Guzzi, P. Nadolsky, F. Olness

Southern Methodist University, Dallas, TX 75275, USA

H. BC

Department of Physics, University of Texas El Paso, El Paso, TX 79968, USA

S. Liuti

Department of Physics, University of Virginia, Charlottesville, VA 22904, USA

A. Accardi

Hampton University, Hampton, VA 23668, USA

S. Ahmed, A. Bogacz, Ya. Derbenev, A. Hutton, G. Krafft, R. Li, F. Marhauser, V. Morozov, F. Pilat, R. Rimmer, T. Satogata, M. Sullivan, M. Spata, B. Terzić, H. Wang, B. Yunn, Y. Zhang

Accelerator Division, Thomas Jefferson National Accelerator Facility, Newport News, VA 23606, USA

A. Accardi, H. Avakian, R. Ent, V. Guzey, B. Musch, P. Nadel-Turonski, A. Prokudin, A. Radyushkin, C. Weiss

Thomas Jefferson National Accelerator Facility, Newport News, VA 23606, USA

G. Krafft, A. Radyushkin, H. Sayed

Department of Physics, Old Dominion University, Norfolk, VA 23529, USA

G. P. Gilfoyle

Physics Department, University of Richmond, Richmond, VA 23173, USA

I. C. Cloët, G. Miller

Department of Physics, University of Washington, Seattle, WA 98195-1560, USA

M. Gonderinger

Department of Physics, University of Wisconsin-Madison, Madison, WI 53706, USA 


\section{Contents}

Executive summary 1

Tables of golden measurements . . . . . . . . . . . . . . 12

1 The spin and flavor structure of the proton 13

1.1 Introduction and chapter overview . . . . . . . . . . . . . 14

1.2 Status of perturbative QCD calculations . . . . . . . . . . . . 19

1.3 Unpolarized proton structure - HERA's legacy . . . . . . . . . . . 22

Unpolarized parton distribution functions:
questions to be addressed at an EIC . . . . . . . . . . . . . 27

1.5 Flavor separation from semi-inclusive DIS . . . . . . . . . . . . 31

1.6 The longitudinal structure function $F_{L}$ at an EIC . . . . . . . . 34

1.7 Theoretical status of inclusive heavy quark production in DIS . . . . . . . . . 38

$1.8 F_{2, L}(\mathrm{charm})$ at an EIC . . . . . . . . . . . . . . . . . 43

1.9 Probing intrinsic charm at the EIC . . . . . . . . . . . . 44

1.10 Status of helicity-dependent PDFs and

open questions to be addressed at an EIC . . . . . . . . . . . . . . 46

1.11 Opportunities in spin physics at an EIC . . . . . . . . . . . 53

1.12 Electroweak structure functions at the EIC . . . . . . . . . . . . 61

1.13 Charged current charm production and the strange sea . . . . . . . . . 75

1.14 Photoproduction processes at an EIC . . . . . . . . . . . . 77

1.15 Expectations for charm quark photoproduction . . . . . . . . . . 79

1.16 Polarized photoproduction at an EIC . . . . . . . . . . . . . . 81

2 Three-dimensional structure of the proton and nuclei: transverse momentum 83

2.1 Introduction and chapter summary . . . . . . . . . . . . . . . . . 84

2.2 Sivers function . . . . . . . . . . . . . . . . . . 95

2.3 Transverse polarization effects with gluons . . . . . . . . . . . . 120

2.4 Theory highlights . . . . . . . . . . . . . . . . 125

2.5 Chiral-odd partonic densities . . . . . . . . . . . . . . . 138

2.6 Overview on other TMDs . . . . . . . . . . . . . . . . . 149

3 Three-dimensional structure of the proton and nuclei: spatial imaging $\mathbf{1 5 3}$

3.1 Spatial imaging of sea quarks and gluons: summary . . . . . . . . . 154

3.2 Basics of generalized parton distributions . . . . . . . . . . . . 156

3.3 GPDs and transverse nucleon structure at collider energies . . . . . . . . . . . 166

3.4 How large can the distributions $E^{q}$ and $E^{g}$ be? . . . . . . . . . . . 170 
3.5 Imaging transverse distributions . . . . . . . . . . . . . . . . . . . 174

3.6 From transverse-momentum spectra to transverse images . . . . . . . . 177

3.7 GPDs from DVCS . . . . . . . . . . . . . . . . . . 183

3.8 Accessing GPDs from experiment: potential of a high-luminosity EIC . . . 185

3.9 Monte Carlo studies on DVCS with an EIC . . . . . . . . . . . . . . . 197

3.10 DVCS Beam Spin Asymmetries with an EIC . . . . . . . . . . . 205

3.11 Hard exclusive photoproduction of Quarkonia . . . . . . . . . . . . . . 208

3.12 Simulations of non-diffractive exclusive processes at an EIC . . . . . . . . 212

3.13 Partonic transverse spin in deep-inelastic exclusive experiments . . . . . . . 219

3.14 Ways to access transversity GPDs at the EIC . . . . . . . . . . . . . 222

4 Input from lattice QCD 226

4.1 Introduction . . . . . . . . . . . . . . . . . . . 227

4.2 Generalized form factors . . . . . . . . . . . . . . . . . . . . . . . . . . . . . . . . . .

4.3 TMDs on the lattice . . . . . . . . . . . . . . . . . . . 233

4.4 Spectroscopy and other physics topics . . . . . . . . . . . . . . 238

5 QCD matter under extreme conditions 239

5.1 Chapter summary: overview and golden measurements . . . . . . . . . . . 240

5.2 Review of linear and non-linear approaches in QCD at small- $x$. . . . . . 250

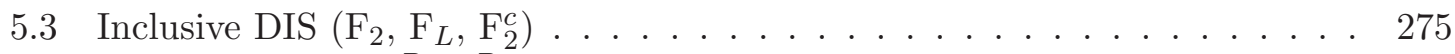

5.4 Diffractive DIS $\left(\mathrm{F}_{2}^{D}, \mathrm{~F}_{L}^{D}\right.$, charm contribution $) \ldots \ldots \ldots \ldots \ldots . \ldots \ldots$

$5.5 k_{T}$-dependent gluons: SIDIS and jets . . . . . . . . . . . . 304

$5.6 \quad b$-dependent gluons: exclusive VM, DVCS . . . . . . . . . . . . . . 310

5.7 Nuclear effects across the $x-Q^{2}$ plane: quarks and gluons . . . . . . . . . 332

5.8 Color transparency . . . . . . . . . . . . . . . . . . . . . 340

5.9 Nuclear GPDs and TMDs . . . . . . . . . . . . . . . . . . . . . . . . . . . . . . . . . . . . . . . . .

5.10 Current fragmentation . . . . . . . . . . . . . . . . . . 354

5.11 Jets . . . . . . . . . . . . . . . . . . . . 370

5.12 Target fragmentation . . . . . . . . . . . . . . . . . 376

5.13 Bose-Einstein correlations at an electron-ion collider . . . . . . . . . . . . 384

5.14 e+A Monte Carlo simulation tools . . . . . . . . . . . . . . . . . . 389

5.15 Connections to other fields . . . . . . . . . . . . . . . 397

6 Electroweak physics 409

6.1 Electroweak physics at the EIC . . . . . . . . . . . . . . 410

6.2 The weak mixing angle via polarized electron scattering asymmetries . . . . 411

6.3 Electron-to-Tau conversion . . . . . . . . . . . . . . . . . 418

7 Experimental aspects

7.1 High-energy high-luminosity electron-ion collider eRHIC . . . . . . . . . . 440

7.2 A polarized medium-energy electron-ion collider at JLab . . . . . . . . . . . 448

7.3 Kinematics and detector designs for the different EIC machine designs . . . 459

\begin{tabular}{ll} 
Acknowledgments & 498 \\
\hline
\end{tabular}

References 499

List of Authors 


\section{Executive summary}

Daniël Boer, Markus Diehl, Richard Milner, Raju Venugopalan, Werner Vogelsang

\section{Introduction}

Understanding the fundamental structure of matter in the physical universe is one of the central goals of scientific research. Strongly bound atomic nuclei predominantly constitute the matter from which humans and the observable physical world around us are formed. In the closing decades of the twentieth century, physicists developed a beautiful theory, Quantum Chromodynamics (QCD), which explains all strongly interacting matter in terms of point-like quarks interacting by the exchange of gauge bosons, known as gluons. Experiments have verified QCD quantitatively in processes involving a very large momentum exchange between the sub-atomic participants. Further confidence is obtained from significant progress in numerical computations of the static properties of the theory, in particular the excellent agreement of theory with the mass spectrum of low lying hadron resonances.

However, more than thirty years after QCD was first proposed as the fundamental theory of the strong force, and despite impressive theoretical and experimental progress made in the intervening decades, the understanding of how QCD works in detail remains an outstanding problem in physics. Very little is known about the dynamical basis of hadron structure in terms of the fundamental quark and gluon fields of the theory. How do these fundamental degrees of freedom dynamically generate the mass, spin, motion, and spatial distribution of color charges inside hadrons with varying momentum resolution and energy scales? Deep Inelastic Scattering (DIS) experiments at the HERA collider revealed clearly that at high momentum resolution and energy scales, the proton is a complex, many-body system of gluons and sea quarks, a picture very different from a more familiar view of the proton as a few point-like partons (a term that collectively refers to both quarks and gluons), each carrying a large fraction of its momentum. This picture, which is confirmed at hadron colliders, raises more questions than it answers about the dynamical structure of matter. For instance, how is the spin- $1 / 2$ of the proton distributed in this many-body system of sea quarks and gluons? In the early universe, how did the many-body plasma of quarks and gluons cool into hadrons with several simple structural properties? Recreating key features of this quark-hadron transition in heavy ion collisions has been a major activity in nuclear physics, with several surprising findings including the realization that this matter flows with very little resistance as a nearly perfect fluid. A deep understanding of the two cited examples, among many others, ultimately requires detailed knowledge of the quark-gluon structure of hadrons and nuclei.

This report on the science case for an Electron-Ion Collider (EIC) is the result of a ten-week program at the Institute for Nuclear Theory (INT) in Seattle (from September 
13-November 19, 2010), motivated by the need to develop a strong case for the continued study of the QCD description of hadron structure in the coming decades. Hadron structure in the valence quark region will be studied extensively with the Jefferson Lab $12 \mathrm{GeV}$ science program, the subject of an INT program the previous year. The focus of the INT program was on understanding the role of gluons and sea quarks, the important dynamical degrees of freedom describing hadron structure at high energies. Experimentally, the most direct and precise way to access the dynamical structure of hadrons and nuclei at high energies is with a high luminosity lepton probe in collider mode. An EIC with optimized detectors offers enormous potential as the next generation accelerator to address many of the most important, open questions about the fundamental structure of matter. The goal of the INT program, as captured in the writeups in this report, was to articulate these questions and to identify golden experiments that have the greatest potential to provide definitive answers to these questions.

At resolution scales where quarks and gluons become manifest as degrees of freedom, the structure of the nucleon and of nuclei is intimately connected with unique features of QCD dynamics, such as confinement and the self-coupling of gluons. Information on hadron sub-structure in DIS is obtained in the form of "snapshots" by the "lepton microscope" of the dynamical many-body hadron system, over different momentum resolutions and energy scales. These femtoscopic snapshots, at the simplest level, provide distribution functions which are extracted over the largest accessible kinematic range to assemble fundamental dynamical insight into hadron and nuclear sub-structure. For the proton, the EIC would be the brightest femtoscope scale lepton-collider ever, exceeding the intensity of the HERA collider a thousand fold. HERA, with its center-of-mass (CM) energy of $320 \mathrm{GeV}$, was built to search for quark substructure. An EIC, with its scientific focus on studying QCD in the regime where the sea quarks and gluons dominate, would have a lower CM energy. In a staged EIC design, the CM energy will range from 50-70 GeV in stage I to approximately twice that for the full design. In addition to being the first lepton collider exploring the structure of polarized protons, an EIC will also be the first electron-nucleus collider, probing the gluon and sea quark structure of nuclei for the first time.

Following the same structure as the scientific discussions at the INT, this report is organized around the following four major themes:

- The spin and flavor structure of the proton

- Three dimensional structure of nucleons and nuclei in momentum and configuration space

- QCD matter in nuclei

- Electroweak physics and the search for physics beyond the Standard Model

In this executive summary, we will briefly outline the outstanding physics questions in these areas and the suite of measurements that are available with an EIC to address these. The status of accelerator and detector designs is addressed at the end of the summary. Tables of golden measurements for each of the key science areas outlined are presented on page 12. In addition, each chapter in the report contains a comprehensive overview of the science topic addressed. Interested readers are encouraged to read these and the individual contributions for more details on the present status of EIC science. 


\section{The spin and flavor structure of the proton}

To understand how the constituents of the proton carry the proton's spin has been a defining question in hadron structure for several decades now. The proton spin problem presents the formidable challenge of understanding an essential feature of how a complex strongly-interacting many-body system organizes itself to produce a simple result. It goes directly to the heart of exploring and understanding the QCD dynamics of matter. From the surprising finding by the European Muon Collaboration that very little of the proton spin is provided by the spins of quarks and anti-quarks combined, the exploration of nucleon spin structure has by now developed into a world-wide quest central to nuclear and particle physics. To provide definitive answers in this area will be among the key tasks of an EIC.

Significant progress can be expected from the unique capability of an EIC to reach small momentum fractions $x$ and large momentum resolution scales $Q$, with high precision. A suite of measurements will be available. A golden measurement of nucleon spin structure at an EIC will be the precision study of the proton's spin structure function $g_{1}^{p}\left(x, Q^{2}\right)$ and its scaling violations, over wide ranges in $x$ and $Q^{2}$. As studies in this report will demonstrate, global analyses of spin-dependent parton distributions will determine the gluon helicity distribution $\Delta g$ and the quark singlet $\Delta \Sigma$ down to values of $x$ of about $10^{-4}$. This vastly extended reach should allow for the determination of the gluon and quark/anti-quark spin contributions to the proton spin to about $10 \%$ accuracy or better. The accuracy to which processes such as deeply-virtual Compton scattering can independently provide information on the remaining orbital angular momentum contributions will be addressed further in the section on spatial imaging.

An EIC will provide unprecedented insight into the flavor structure of the nucleon, a key element in mapping the "landscape" of hadron structure. There are two powerful golden measurements available at an EIC to achieve this. One of these methods, Semi-Inclusive Deep-Inelastic Scattering (SIDIS) has been used in previous fixed-target lepton scattering experiments HERMES and COMPASS. (Polarized proton-proton collisions at RHIC employ $W$-boson production for flavor identification.) At an EIC, semi-inclusive measurements would extend to much higher $Q^{2}$ than in fixed-target scattering, where the reaction becomes significantly cleaner, less contaminated with higher-twist effects (a technical term for contributions power suppressed in $1 / Q^{2}$ ), and therefore more tractable theoretically. The kinematic coverage for SIDIS in $x$ and $Q$ will be similar overall to what can be achieved in inclusive DIS. With the high luminosity of an EIC, extractions of the light-flavor helicity distributions $\Delta u, \Delta d$ and their anti-quark distributions from SIDIS will be possible with exquisite precision. With dedicated studies of kaon production, the strange and anti-strange distributions will also be accessible. All this will likely give insights into the question why it is that the combined quark and anti-quark spin contribution to the proton spin turns out to be so small.

The other independent method for accessing the quark and antiquark helicity distributions at an EIC is electroweak DIS. At high $Q^{2}$, the DIS process also proceeds significantly via the exchange of $Z$ and $W^{ \pm}$bosons. This gives rise to novel structure functions that are sensitive to various different combinations of the proton's helicity distributions. Studies show that both neutral current and charged current interactions would be observable at an EIC. To fully exploit the potential of an EIC for such measurements, positron beams are required, albeit not necessarily polarized. Besides the new insights into nucleon structure this would provide, studies of spin-dependent electroweak scattering at short distances with an EIC would be interesting physics in and of itself, much in the line of past and ongoing 
electroweak measurements at HERA, Jefferson Lab, and RHIC.

Polarized electron-proton physics can be expected to take center stage at an EIC because these would be the first such collider measurements. However, as studies in this report show, there is a large potential for unpolarized physics at an EIC. Thanks to its high luminosity and the feasibility for an energy scan, an EIC would vastly improve upon HERA data on measurements of the longitudinal structure function $F_{L}$. This quantity is a key observable for studies of gluon structure and the possible transition to a high parton density or saturation regime in the proton. At an EIC, several SIDIS measurements of flavor distributions and multi-particle correlations will be possible for the first time. In particular, pinning down the strange quark and antiquark content of the proton would close one of the last notable gaps in our knowledge of unpolarized parton densities. Extended rapidity coverage will also allow for detailed studies of the rapidity gap structure of hard diffractive final states. In addition, the very high luminosities will bring a vast improvement in the precision of measurements of the charm and beauty contributions to nucleon structure.

\section{Three dimensional structure of hadrons and nuclei: Trans- verse momentum distributions}

Partons can have a momentum component transverse to the direction of their parent nucleon and there exists experimental evidence to support an average transverse momentum of a few hundred $\mathrm{MeV} / \mathrm{c}$. However, much of our understanding of nucleon structure is in terms of integrated parton distributions that are only sensitive to the momentum resolution of the probe. A rigorous theoretical framework for parton transverse momentum distributions (TMDs) has been developed recently which allows for a description of specific scattering cross sections in terms of these distributions. TMDs are an essential step toward a more comprehensive understanding of the parton structure of the nucleon in QCD. An EIC will enable precise and detailed measurements of TMDs over a broad kinematic range.

For the scattering processes of interest, the large scale $Q^{2}$ justifies, in a leading twist approximation, the factorized description of the cross section in terms of several calculable or measurable factors, yielding a predictive framework. TMDs are examples of such measurable factors. In such descriptions not only does the magnitude of the parton transverse momentum enter, but also the transverse momentum direction, yielding strikingly asymmetric distributions. Several recently observed angular asymmetries are most naturally described by asymmetric, spin direction dependent TMDs.

A golden measurement at an EIC will be the Sivers asymmetry, a particular angular correlation between the target polarization and the direction of a produced final state hadron in polarized SIDIS. At the parton level, the Sivers effect is a spin-orbit coupling effect in QCD and is described by a TMD that quantifies how strongly the transverse momentum from orbital motion is coupled to spin. The Sivers effect is especially interesting because it is a consequence of phase interference peculiar to the gauge structure of QCD. The gauge invariant Sivers TMD is non-zero only if gluonic initial or final state interactions are taken into account. There is a calculable process dependence, most strikingly evident in SIDIS and Drell-Yan lepton pair production where the polarized Sivers function in the former is equal in magnitude but opposite in sign to the latter. Factorization breaking is also expected in more complicated processes, such as hadron-hadron collisions with hadronic final states. This process dependence has not yet been demonstrated but several such experiments, in particular at RHIC, will study the Sivers and other TMD effects. The comparison of these 
results with complementary information from an EIC will allow a detailed understanding of the nature and extent of factorization breaking for TMDs.

A goal at an EIC is to obtain a flavor-separated extraction of the Sivers TMD in an energy regime where its theoretical interpretation is unambiguous. Percent level azimuthal asymmetries measured by HERMES, COMPASS and at Jefferson Lab at rather modest $Q^{2}$ have enabled rough first estimates of the magnitude of the Sivers effect. With the $12 \mathrm{GeV}$ upgrade program at Jefferson Lab, the valence (large $x$ ) region will be explored in detail, whereas sea quark and gluon contributions at small $x$ (down to $10^{-4}$ ) will be mapped out with an EIC. The large $Q^{2}$ reach of an EIC will allow for extensive study of evolution effects in TMDs, and at large $x(x \sim 0.2)$ will have overlap with preceding experiments. High energies and high precision will enable a good understanding of the $x$ dependence of the Sivers functions for each quark flavor, including antiquarks and gluons. In addition, the larger transverse momentum range of final state particles at an EIC allows for studies of weighted asymmetries that are cleaner to interpret theoretically but are beyond the reach of fixed target experiments. The extensive transverse momentum range will for the first time in polarized SIDIS, allow studies of the transition region between the TMD description at low transverse momentum and the description in terms of collinear quark-gluon-quark correlation functions (known as the Qiu-Sterman mechanism) at high transverse momentum. Finally, with respect to previous SIDIS experiments and future Jefferson Lab experiments, a larger variety of final states can be considered at an EIC, such as (multiple) jets or Dmesons, all of great interest in isolating quark and gluon contributions to the various TMD effects.

Now that angular asymmetries consistent with the TMD framework have been observed, the road towards full-fledged experimental studies of TMDs can be mapped out and the essential role of an EIC identified. Besides the Sivers effect, essential information on the unpolarized TMD $f_{1}$ is obtained from unpolarized scattering cross sections. For reasons we shall outline, this extraction of $f_{1}$ can be classified as another golden measurement. This TMD determines the $Q^{2}$ dependence of the unpolarized cross section, which has been predicted but not yet verified. Predictions of the $x$, transverse momentum, scale and flavor dependence of $f_{1}$ allow for non-trivial checks of the fundamental TMD formalism corroborating and complementing what one learns from the Sivers and other spin TMD effects. The unpolarized SIDIS measurements at an EIC will give detailed information on the difference between sea and valence quark contributions, and on the role of gluons. Extracting unpolarized gluon TMDs at small $x$ is especially interesting because of the recently discovered agreement between predictions in the TMD framework and previous computations of the same in the Color Glass Condensate formalism as we shall discuss later.

The proposed silver experiments are 1) the distribution of transversely polarized quarks inside transversely polarized hadrons, 2) spin-orbit correlations inside unpolarized hadrons (the Boer-Mulders TMD), and 3) the Collins TMD fragmentation function, which describes a similar spin effect in the fragmentation of quarks into unpolarized hadrons. All three quantities involve transverse quark spin, which distinguishes them from the Sivers effect which deals with unpolarized partons inside a transversely polarized proton. An EIC will be able to provide multi-dimensional representations of all these quantities and the observables they give rise to. The TMD chapter illustrates by means of concrete examples and calculations how much further TMD studies can be pushed with an EIC compared to the present status. A prime example is shown in figure 2.11 on page 108. 


\section{Three dimensional structure of nucleons and nuclei: Spatial imaging}

The high luminosity and large kinematic reach of an EIC offers unique possibilities for exploring the spatial distribution of sea quarks and gluons in the nucleon and in nuclei. The "imaging" of partons is possible in suitable exclusive reactions. The transverse position of the quark or gluon on which the scattering took place is obtained by a Fourier transform from the transverse momentum of the scattered nucleon or nucleus. At the same time, the longitudinal momentum loss of the target is correlated with the longitudinal momentum fraction $x$ of the parton. By choosing particular final states, measurements at an EIC will be able to selectively probe the spatial distribution of sea quarks and gluons in a wide range of $x$. Such 'tomographic images' will provide essential insight into QCD dynamics inside hadrons, such as the interplay between sea quarks and gluons, the role of pion degrees of freedom at large transverse distances and, from a more general perspective, the mechanism for confinement in QCD.

The quantities that encode this tomographic information are generalized parton distributions (GPDs). The formalism of GPDs is applicable in the full range of $x$. An alternative description at small $x$ is the dipole formalism, which is expressed in terms of the amplitude for small color dipoles to scatter off gluons in the hadron target. GPDs allow direct comparison of tomographic images for sea quarks and gluons with their counterparts in the valence quark region, where the $12 \mathrm{GeV}$ program at Jefferson Lab will obtain information of unprecedented accuracy.

Potential golden measurements for parton imaging at an EIC are deeply virtual Compton scattering and photo- or electro-production of $J / \psi$ mesons. For Compton scattering, there are a large number of observables that can be calculated with high precision, whereas a unique advantage of $J / \psi$ production is its sensitivity to gluons. A suite of further reaction channels play the role of "silver measurements", which will provide complementary information and in particular help separate different quark flavors. Among those exclusive channels whose cross sections grow with energy, deeply virtual Compton scattering demands the highest luminosity. Simulations performed during the INT program indicate that precise and multi-differential measurements of this process can be envisaged with the projected EIC luminosity (see figures 3.34, 3.35 and 3.37 on pages 203, 204 and 207). Detailed studies including detector effects will be required to establish the achievable experimental accuracy.

The envisaged configuration of an EIC interaction region and detector will provide data in a wide enough range of transverse momentum transfer to permit a Fourier analysis of observables. With this, exclusive cross sections and angular or polarization asymmetries will give direct quantitative information about the spatial distribution of partons in a specified range of $x$. Estimates indicate that transverse distances ranging from about $0.1 \mathrm{fm}$ to 2 fm or higher will be accessible, provided that a good enough momentum resolution can be achieved experimentally. Such data will provide the basis for reconstructing generalized parton distributions and, ultimately, the joint distribution of partons in transverse position $b$ and longitudinal momentum fraction $x$. For this second step, an EIC's large lever arm in photon virtuality $Q^{2}$ at a given photon energy will be essential, since it is the scale evolution in $Q^{2}$ that carries the most detailed information about the longitudinal parton momentum.

Our current knowledge about the helicity distributions of quarks and gluons indeed suggests that the orbital angular momentum of partons plays a prominent role in the nucleon. Exclusive scattering on a transversely polarized target gives access to this degree of freedom 
in parton tomography and allows one to study spin-orbit correlations at the parton level. An especially interesting aspect is the relation between a polarization induced asymmetry in transverse parton position and the Sivers asymmetry in transverse parton momentum. Such a relation is profoundly dynamical, and its quantitative exploration in the sea quark and gluon domain will be a highlight of exploring hadron structure and dynamics at an EIC. Deeply virtual Compton scattering will again play an essential role in this context, along with vector meson production channels. Quantitative estimates of the achievable statistical and systematic accuracy were not made during the INT program, but the necessary tools are now in place and results should be available soon.

Ji's angular momentum sum rule condenses the connection between generalized parton distributions and parton angular momentum into a single number for each quark flavor and for the gluon. To evaluate this sum rule from exclusive measurements is truly challenging for several reasons. The most serious among them is that one needs to reconstruct the full $x$ dependence of GPDs from observed scaling violations in $Q^{2}$. As already mentioned, the large kinematic coverage of an EIC provides a good starting point for such a program, but it remains to be seen which accuracy can be attained for the angular momentum. We regard this as a long-term endeavor, which will profit from the progress one can expect in the coming years from the $12 \mathrm{GeV}$ program at Jefferson Lab.

\section{Physics opportunities in electron-nucleus collisions}

An EIC would be the world's first e+A collider. It will significantly extend parton studies of nuclear structure into the regime dominated by sea quarks and gluons. Prior fixed target DIS measurements on nuclei revealed that the ratio of nuclear to nucleon cross sections is significantly less than unity (normalized by the atomic mass number) both at large $x$ (the EMC effect) and at small $x$ (shadowing). These interesting nuclear phenomena were however only observed for valence and (to a lesser extent) sea quarks. The nuclear gluon distribution is very poorly constrained at all $x$ values, especially at $x<0.01$ where it is completely unknown. An EIC could reveal surprises in our fundamental understanding of the parton structure of nuclei in this terra incognita.

A fundamental feature of QCD is gluon saturation, which arises as a consequence of the fact that gluon distributions at a fixed $Q^{2}$ cannot grow rapidly indefinitely with decreasing $x$. The properties of matter in this novel saturation regime of strong color fields in QCD is described by a saturation scale which grows both with decreasing $x$ and with increasing nuclear size. Model estimates of this nuclear "oomph" give a saturation scale in a large nucleus at EIC energies to be of the same magnitude as the saturation scale in a proton at a $\mathrm{TeV}$ scale electron-proton collider; electron-nucleus collisions therefore provide an efficient method to explore saturation in QCD.

As a consequence of asymptotic freedom, the large saturation scale (relative to the intrinsic QCD scale $\Lambda_{Q C D}$ ) accessible at an electron-nucleus collider implies that the properties of saturated gluon matter at small $x$ can be computed systematically using weak coupling techniques and compared to experimental data. One such weak coupling approach is the Color Glass Condensate (CGC). Renormalization group (RG) methods in the CGC are used to compute observables in electron-nucleus collisions that are sensitive to the energy evolution of particular many-body gluon correlators. These correlators, classified as "dipole", "quadrupole" and "multipole" effective degrees of freedom from their color structure, are universal. Final states in proton-nucleus and nucleus-nucleus collisions can also 
be expressed in terms of these objects. Properties of multipole degrees of freedom can be inferred from measurements of cross-sections for specific final states in one of these reactions and used as input in computations of cross-sections for other final states, thereby providing an important test of the validity and limits of the CGC effective theory. A further interesting possibility is that multipole correlators at very high energies become independent of the initial conditions specific to a particular nucleus that are inputs at a given $x$ scale to the RG evolution equations. While it appears unlikely that an EIC would have sufficient energy to access this asymptotic regime, DIS off different nuclei can provide important constraints on pre-asymptotic trends in that direction.

At large $x$ in nuclei, DIS corresponds to the virtual photon scattering off quarks, with the nucleus acting as an extended colored medium that interacts with the hard colored probe. Because the energy and momentum resolution of the probe can be accurately controlled in DIS, one can quantitatively address, with a precision unmatched at hadron colliders, interesting questions about the nature of multiple scattering and $p_{\perp}$ broadening, energy loss and fragmentation, and the propagation of heavy quarks and jets in colored media. Perturbatively calculable short distance physics can be isolated from the hadronization mechanism by tuning the energy and momentum resolution of the virtual photon probe to shed new light on the latter both in medium and in the vacuum. While some such studies have been performed previously at fixed target DIS facilities and in proton-nucleus collisions, the extended kinematic reach, collider geometry and precision probes will vastly add to their scope, allowing for definitive answers to enduring questions about in-medium properties of QCD. For instance, the propagation of heavy charm and beauty quarks in medium will be quantitatively studied in DIS for the first time. In addition to being interesting in their own right, DIS studies of parton propagation in "cold" QCD media are an important benchmark for a quantitative understanding of their role in the hot QCD medium produced at RHIC and the LHC.

An important opportunity to understand the role of gluons in the structure of short range nuclear forces is made possible by exclusive measurements with an EIC of open heavy flavor and quarkonium in DIS off light nuclei. Other interesting studies at large $x$ where the kinematic reach of an EIC will complement the Jefferson Lab $12 \mathrm{GeV}$ program, including the EMC effect and generalized parton distributions for nuclei.

A number of experimental observables have been identified that can shed light on the compelling physics issues outlined. One set of golden measurements include the inclusive structure functions $F_{2}$ and $F_{L}$ for light and heavy nuclei. They will provide the first ever unambiguous measurements of nuclear gluon distributions. Studies of the evolution of quark singlet and gluon distributions with $x$ and $Q^{2}$ for light and heavy nuclei can systematically uncover the breakdown of leading twist evolution, the onset and development of non-linear saturation dynamics and enable the extraction of the corresponding saturation scale. Another set of golden measurements are provided by semi-inclusive DIS (SIDIS) off nuclei. Di-hadron correlations in particular, are very sensitive to non-linear QCD evolution, and allow for a clean extraction of the saturation scale. They will corroborate (or invalidate) claims of saturation seen in di-hadron correlations in deuteron-gold collisions; more generally, they enable the previously discussed tests of universality of multipole correlators at small $x$. Golden measurements at large $x$ are semi-inclusive production of light and heavy flavors and jets. These provide unique insight into energy loss and parton shower development in an extended colored medium, as well as into the dynamics of hadronization in this many-body environment. The heavy flavor and jet measurements will be the first of their kind in nuclear DIS; we note that feasibility studies for them are still in a preliminary stage. 
In addition to these golden measurements, there are several important measurements classified as "silver" instead of gold only in a relative sense. The most important among these are the diffractive structure functions $F_{2, D}$ and $F_{L, D}$ which will be extracted for nuclei for the very first time. At HERA, these structure functions for protons constituted more than $15 \%$ of the cross-section; the predictions of saturation models is that this fraction will be significantly larger in nuclei. Exclusive production of vector mesons and deeply virtual Compton scattering probe the spatial distribution of partons in nuclei; at small $x$, they can help clarify the interplay between saturation and the effects of chiral symmetry breaking and confinement.

Finally, a frequently posed question is whether proton/deuteron-nucleus scattering can provide the same information content as electron-nucleus collisions. In the former, the computation of final states, in leading twist kinematics, contains convolutions over parton distributions in the nucleon projectile as well as that in the target. In addition, for a number of final states, a large number of parton scattering reactions are likely to contribute. This significantly compromises the accuracy to which one determines the parton structure of the target. For fundamental questions regarding the spatial distribution of partons and color singlet structures exchanged in hard diffractive scattering, there are essential qualitative differences in hadron-hadron and lepton-hadron processes arising from the lack of universality in key aspects of the dynamical structure of nucleons and nuclei. Thus while proton/deuteron-nucleus scattering at high energies has the strong potential to be a discovery machine for new QCD physics, uncovering the origins of such physics and its implications for our fundamental understanding of the parton structure of nuclei, will require an EIC.

\section{Electroweak interactions and physics beyond the Standard Model}

While the physics of an EIC is primarily motivated by the study of strong interactions, its physics case is strengthened by its potential to contribute to electroweak studies as well. Experience has shown that a new accelerator that pushes the frontiers either in energy, and/or luminosity and intensity, is of interest for studies of electroweak physics. We have already mentioned that precision studies of (parity-violating) electroweak spin structure functions would be possible at an EIC, giving new insights into nucleon spin structure. However, the electroweak physics case for an EIC is broader as it would also allow measurements of parameters of electroweak theory. Studies presented in detail in the INT report suggest that for high energy and luminosity, there would be excellent prospects for extractions of the Weinberg angle, which should even be possible over a fairly wide range in $Q^{2}$ so that its running can be further studied in detail. In this way, an EIC would complement the precise LEP and SLD measurements on the $Z$-pole, atomic parity-violation measurements, the SLAC E158 Møller scattering data, and the NuTeV data whose final value is in fact around three standard deviations above the SM prediction. A comparison of EIC results for $\sin ^{2} \theta_{W}$ with those on the $Z$-pole in particular can be used to search for new physics effects. Some of the experimental systematics involved at an EIC are broadly understood, but may still need further work to clarify. A full "global survey" of electroweak parameters from EIC data - much in the spirit of the approach also taken at HERA - is still outstanding but planned. In addition, an EIC might possibly be able to open a direct window on beyond-Standard Model physics, assuming that conditions are favorable. Studies 
indicate that the EIC might be able to perform a sensitive search for a third generation leptoquark in electron-tau conversion $e p \rightarrow \tau X$, with potential reach well beyond that in previous studies at HERA.

\section{EIC Accelerator Design}

Two substantial, focused efforts at developing a design for an electron-ion collider in the U.S. based on existing accelerators are underway at Brookhaven National Laboratory and Thomas Jefferson National Accelerator Facility. At BNL, the eRHIC design utilizes a new linear electron accelerator to collide with the existing polarized proton and ion beams of the operating Relativistic Heavy Ion Collider (RHIC). At JLab, the ELIC design employs new electron and ion storage rings together with the $12 \mathrm{GeV}$ upgraded existing CEBAF. Although based on two different, existing accelerators, because they are driven by the same science objectives, the two U.S. EIC design efforts have similar characteristics. The most important include:

- highly polarized (> 70\%) electron and nucleon beams

- ion beams from deuterium to the heaviest nuclei - uranium or lead

- center of mass energies: from about $20 \mathrm{GeV}$ to about $150 \mathrm{GeV}$

- maximum collision luminosity $\sim 10^{34} \mathrm{~cm}^{-2} \mathrm{~s}^{-1}$

- non-zero crossing angle of colliding beams without loss of luminosity (so-called crab crossing)

- cooling of the proton and ion beams to obtain high luminosity

- staged designs where the first stage would reach $\mathrm{CM}$ energies of about $70 \mathrm{GeV}$

- the possibility to have multiple interaction regions

It is clear from the EIC physics studies that with a luminosity of $\sim 10^{33} \mathrm{~cm}^{-2} \mathrm{~s}^{-1}$, and operating for about a decade, ground breaking new experiments to probe our understanding of QCD will become feasible. This would require delivery of order $50 \mathrm{fb}^{-1}$ with polarized nucleon and heavy ion beams to experiments in about a decade. This would be 100 times more integrated luminosity than recorded over a decade at the only previous electron-proton

collider, HERA at DESY. With a luminosity of $\sim 10^{34} \mathrm{~cm}^{-2} \mathrm{~s}^{-1}$, precision imaging and electroweak experiments become feasible at an EIC.

The EIC accelerator designs being considered will require significant R\&D for realization. The cooling of the hadron beam is essential to attain the luminosities demanded by the science. The development of a new technique, coherent electron cooling, is underway at BNL while conventional electron cooling is being pushed to high RF power at JLab. Energy recovery linear accelerators at high energy and intensity are a key technology for an EIC. Further, the eRHIC design demands an increase in the intensity produced by polarized electron sources of over an order of magnitude beyond what is available at present. The ELIC design utilizes novel figure-8 storage rings for both electrons and ions.

In Europe, two electron-ion collider accelerators are under consideration. At the Large Hadron Collider at CERN, physicists are considering colliding an electron beam (either a 
linac or ring) with an energy of about $70 \mathrm{GeV}$ with the existing unpolarized proton and heavy-ion beams. The present LHeC design can reach a CM energy of about $1.4 \mathrm{TeV}$ with a luminosity of $10^{33} \mathrm{~cm}^{-2} \mathrm{~s}^{-1}$. At GSI in Germany, an Electron-Nucleon Collider (ENC) would be realized by colliding electrons in a $3 \mathrm{GeV}$ storage ring with $15 \mathrm{GeV}$ protons in the High Energy Storage Ring of the planned Facility for Antiproton and Ion Research (FAIR). The CM energy at an ENC is about $14 \mathrm{GeV}$ and the expected luminosity is about $10^{32}$ $\mathrm{cm}^{-2} \mathrm{~s}^{-1}$. Thus, the two European colliders differ in CM energy by about two orders of magnitude, in colliding luminosity by about one order of magnitude, and have very different scientific objectives.

\section{EIC Detectors}

Optimized detectors are essential to carry out the ground breaking experiments planned at an EIC. The design of EIC detectors is intimately connected to the design of the accelerator interaction regions (IR) through the location of magnets, configuration of crossing angles, and available space. A particular challenge is to detect forward-going scattered protons from exclusive reactions, as well as decay neutrons from the break-up of ions in incoherent diffraction. Past experience at colliders with lepton beams has shown that synchrotron radiation generated by bending the electron beam close to the IR can produce challenging backgrounds for detectors.

Detector concepts for an EIC are being developed and are guided both by the demands of the scientific program and by the experience with ZEUS and H1 at HERA. The EIC detector will certainly include a large central detector likely containing a solenoidal magnetic field (of order $4 \mathrm{~T}$ ); trackers for momentum and angular resolution; electromagnetic and hadronic calorimetry; particle identification involving Cerenkov detectors, and vertex detectors. Further, detectors in the forward and backward directions will be required to augment the large central detector. These are necessary to detect hadrons from low $x$ processes and will require particle identification, calorimetry (both electron and hadron) and possibly magnetic field. With multiple interaction regions, it may be more advantageous to consider different detectors (e.g. forward/backward vs. central, high luminosity vs. low luminosity) for different IRs.

Minimizing the effects of systematic uncertainties is an important aspect of detector design. Absolute and relative luminosity determination is a key to extracting important observables, for instance the longitudinal structure function or small polarization asymmetries. Measurement of the polarization of electron and hadron beams has a high priority. As with the accelerator, R\&D for EIC detectors will be essential. 


\section{Tables of golden measurements}

\begin{tabular}{|c|c|c|c|}
\hline \multicolumn{4}{|c|}{ Spin and flavor structure of the nucleon } \\
\hline Deliverables & Observables & What we learn & Requirements \\
\hline $\begin{array}{l}\text { polarized gluon } \\
\text { distribution } \Delta g\end{array}$ & $\begin{array}{l}\text { scaling violations } \\
\text { in inclusive DIS }\end{array}$ & $\begin{array}{l}\text { gluon contribution } \\
\text { to proton spin }\end{array}$ & $\begin{array}{c}\text { coverage down to } x \simeq 10^{-4} \\
\mathcal{L} \text { of about } 10 \mathrm{fb}^{-1}\end{array}$ \\
\hline $\begin{array}{l}\text { polarized quark and } \\
\text { antiquark densities }\end{array}$ & $\begin{array}{l}\text { semi-incl. DIS for } \\
\text { pions and kaons }\end{array}$ & $\begin{array}{l}\text { quark contr. to proton spin; } \\
\text { asym. like } \Delta \bar{u}-\Delta \bar{d} ; \Delta s\end{array}$ & $\begin{array}{l}\text { similar to DIS; } \\
\text { good particle ID }\end{array}$ \\
\hline $\begin{array}{c}\text { novel electroweak } \\
\text { spin structure functions }\end{array}$ & $\begin{array}{l}\text { inclusive DIS } \\
\text { at high } Q^{2}\end{array}$ & $\begin{array}{c}\text { flavor separation } \\
\text { at medium } x \text { and large } Q^{2}\end{array}$ & $\begin{array}{c}\sqrt{s} \geq 100 \mathrm{GeV} ; \mathcal{L} \geq 10 \mathrm{fb}^{-1} \\
\text { positrons; polarized }{ }^{3} \text { He beam }\end{array}$ \\
\hline
\end{tabular}

\begin{tabular}{|c|c|c|c|c|}
\hline \multicolumn{4}{|c|}{ Three-dimensional structure of the nucleon and nuclei: transverse momentum dependence } \\
\hline Deliverables & Observables & What we learn & Phase I & Phase II \\
\hline \hline Sivers and & SIDIS with transv. & quantum interference & valence+sea & 3D Imaging of \\
unpolarized & polarization/ions; & multi-parton and & quarks, overlap & quarks and gluon; \\
TMDs for & di-hadron (di-jet) & spin-orbit & with fixed target & $Q^{2}\left(P_{\perp}\right)$ range \\
quarks and gluon & heavy flavors & correlations & experiments & QCD dynamics \\
\hline
\end{tabular}

\begin{tabular}{|c|c|c|c|}
\hline \multicolumn{4}{|c|}{ Three-dimensional structure of the nucleon and nuclei: spatial imaging } \\
\hline Deliverables & Observables & What we learn & Requirements \\
\hline \hline sea quark and & DVCS and $J / \psi, \rho, \phi$ & transverse images of & $\mathcal{L} \geq 10^{34} \mathrm{~cm}^{-2} \mathrm{~s}^{-1}$ \\
gluon GPDs & production cross sect. & sea quarks and gluons \\
& and asymmetries & in nucleon and nuclei; & Roman Pots \\
& & total angular momentum; & polarized $e^{-}$and $p$ beams \\
& & onset of saturation & $e^{+}$beam for DVCS \\
\hline
\end{tabular}

\begin{tabular}{|c|c|c|c|c|}
\hline \multicolumn{5}{|c|}{ QCD matter in nuclei } \\
\hline Deliverables & Observables & What we learn & Phase I & Phase II \\
\hline $\begin{array}{c}\text { integrated gluon } \\
\text { distributions }\end{array}$ & $F_{2, L}$ & $\begin{array}{c}\text { nuclear wave function; } \\
\text { saturation, } Q_{s}\end{array}$ & $\begin{array}{c}\text { gluons at } \\
10^{-3} \leq x \leq 1\end{array}$ & $\begin{array}{c}\text { explore sat. } \\
\text { regime }\end{array}$ \\
\hline $\begin{array}{c}k_{T} \text {-dep. gluons; } \\
\text { gluon correlations }\end{array}$ & $\begin{array}{l}\text { di-hadron } \\
\text { correlations }\end{array}$ & $\begin{array}{c}\text { non-linear QCD } \\
\text { evolution/universality }\end{array}$ & $\begin{array}{c}\text { onset of } \\
\text { saturation; } Q_{s}\end{array}$ & RG evolution \\
\hline $\begin{array}{l}\text { transp. coefficients } \\
\text { in cold matter }\end{array}$ & $\begin{array}{c}\text { large- } x \text { SIDIS; } \\
\text { jets }\end{array}$ & $\begin{array}{l}\text { parton energy loss, } \\
\text { shower evolution; } \\
\text { energy loss mech. }\end{array}$ & $\begin{array}{l}\text { light flavors, charm } \\
\text { bottom; jets }\end{array}$ & $\begin{array}{l}\text { precision rare } \\
\text { probes; } \\
\text { large- } x \text { gluons }\end{array}$ \\
\hline
\end{tabular}

\begin{tabular}{|c|c|c|c|c|}
\hline \multicolumn{5}{|c|}{ Electroweak interactions and physics beyond the Standard Model } \\
\hline Deliverables & Observables & What we learn & Phase I & Phase II \\
\hline $\begin{array}{l}\text { Weak mixing } \\
\text { angle }\end{array}$ & $\begin{array}{l}\text { Parity violating } \\
\text { asymmetries in } \\
e p \text { - and } e d \text {-DIS }\end{array}$ & $\begin{array}{c}\text { physics behind electroweak } \\
\text { symmetry breaking } \\
\text { and BSM physics } \\
\end{array}$ & $\begin{array}{l}\text { good precision } \\
\text { over limited } \\
\text { range of scales }\end{array}$ & $\begin{array}{c}\text { high precision } \\
\text { over wide range } \\
\text { of scales }\end{array}$ \\
\hline$e-\tau$ conversion & $e p \rightarrow \tau, X$ & $\begin{array}{c}\text { flavour violation } \\
\text { induced by BSM physics }\end{array}$ & challenging & very promising \\
\hline
\end{tabular}


Chapter 1

\section{The spin and flavor structure of the proton}

Convenor and chapter editor:

M. Stratmann 


\subsection{Introduction and chapter overview}

\section{Marco Stratmann}

Two weeks of the INT program on "Gluons and the Quark Sea at High Energies" were devoted to the physics of unpolarized and polarized parton distribution functions. A compelling set of physics opportunities at an EIC has emerged from lively discussions among the participants and subsequent interactions with the hadron structure community. This Chapter outlines the identified open fundamental questions in hadronic physics and the "golden measurements" and experimental requirements to thoroughly address them at a future EIC. The anticipated results will have a profound influence on our understanding of the spin and flavor structure of nucleons.

Sixteen years of operations at DESY-HERA had a transformational impact on the way we view the internal partonic content of nucleons and have led to various new developments in the field of Quantum Chromodynamics. The experiments have left a rich legacy of results, the most prominent ones being the strong rise of the gluon density at small momentum fractions $x$, the large portion of diffractive events, and the transition from high to low momentum transfer $Q$ for various processes. Likewise, vigorous experimental programs with polarized beams and targets in the past twenty-five years at all major laboratories have brought us closer to pinpoint the various contributions to the proton's spin. They also revealed novel, often puzzling phenomena which initiated new directions of research in spin physics such as transverse-momentum dependent parton densities; see Chapter 2.

In each case, the experimental progress was matched by considerable theoretical efforts in Quantum Chromodynamics. Most notable in this context are the level of precision reached in higher-order calculations in perturbative QCD and the much refined global analysis tools to reliably extract information on parton densities from data and to determine their uncertainties. Yet, there is still a significant lack of understanding on quite a few outstanding issues. An EIC will prove crucial in addressing them by making use of the anticipated high luminosities and the variability of beam energies.

Of course, due to the lower center-of-mass system energies of an EIC as compared to HERA one cannot extend the kinematic reach towards smaller values of $x$ for unpolarized electron-proton collisions. Also, over the next couple of years the CERN-LHC will provide a great deal of information on helicity-averaged parton densities in a broad range of $x$ from various different hard scattering processes up to very large resolution scales $Q$. The 12 $\mathrm{GeV}$ upgrade of the CEBAF facility at Jefferson Laboratory is designed to map parton distributions up to very large values of $x$ at scales $Q$ of a few $\mathrm{GeV}$ to test how well, for instance, counting rules apply. Therefore, we expect that most aspects of unpolarized parton densities will be sufficiently well known by the time an EIC is expected to turn on, with some important exceptions to be discussed below.

The situation is rather different for spin physics where the bulk of experimental information stems from fixed-target lepton-nucleon scattering experiments at rather low energies. Ideas to turn HERA into a polarized electron-proton collider never materialized. Existing experiments studying the helicity structure of the nucleon, like PHENIX and STAR at RHIC, will continue to add data in the next couple of years. In particular, measurements of double-spin asymmetries for di-jets in $p p$ collisions at $500 \mathrm{GeV}$ should improve the current constraints on the polarized gluon density $\Delta g(x)$ and extend the covered $x$ range towards somewhat smaller values. Parity-violating, single-spin asymmetries for $W$ boson production should reach a level where they help to constrain the spin-dependent $u$ and $d$ 
quark and antiquark densities at medium-to-large $x$. At JLab-12 the focus is again on the large $x$ frontier at moderate values of $Q$ to address to what extent quarks obey helicity retention which predicts that in the limit $x \rightarrow 1$ quark and nucleon spins become fully aligned. Ultimately, all these efforts are limited by their kinematic coverage both in $x$ and in $Q$. Since the most fundamental open questions in spin physics concern the polarization of wee partons, see below, there are many opportunities for a high-energy polarized EIC to contribute significantly due to its unique capabilities to access values of $x$ down to about $10^{-4}$. This is central to finally determine and understand the role of quarks and gluons in the spin decomposition of the nucleon.

Factorization of experimental observables into non-perturbative parton densities and calculable hard scattering cross sections is the cornerstone for the theoretical application of QCD at high energies within perturbative methods. Available QCD calculations for inclusive and semi-inclusive deep-inelastic scattering processes will allow us to confront future high-statistics EIC data with theory at the necessary very high level of precision. A brief account of the status of perturbative QCD calculations for most of the key measurements at an EIC is given in Sec. 1.2.

Since the EIC is a natural extension of the physics program carried out at HERA both in terms of the anticipated significant increase in luminosity and the possibility to have polarized beams, we summarize the latest status of HERA data based on the recent combination of results from the H1 and ZEUS experiments in Sec. 1.3. This discussion also helps to expose the open questions about the structure of unpolarized nucleons an EIC can elucidate and which cannot be answered solely by measurements at the LHC. The most compelling ones comprise

- the longitudinal structure function $F_{L}$,

- the elusive strangeness and anti-strangeness densities,

- and heavy flavor contributions to deep-inelastic scattering.

A detailed account, including other second tier opportunities is given in Sec. 1.4.

An EIC could make the first precise measurement of $F_{L}$ in a kinematic range that overlaps both previous fixed-target and HERA data, none of which are very precise. $F_{L}$ is particularly sensitive to the gluon distribution and QCD dynamics at small $x$ which makes it a promising candidate to study the transition to the high parton density regime, i.e., the phenomenon of saturation, with an inclusive observable. While one does not expect non-linear effects to be of significant relevance in electron-proton collisions at an EIC, a measurement of $F_{L}$ provides the baseline for similar studies in electron-heavy ion collisions. Here, the onset of saturation effects is expected already at $x \simeq 10^{-3}$ which elevates $F_{L}$ to one of the golden measurements to be performed at the EIC; see Chapter 5 on QCD matter under extreme conditions for details. The determination of $F_{L}$ relies on an accurate measurement of the variation of the so-called reduced cross section for fixed values of $x$ and $Q$ at different c.m.s. energies $\sqrt{s}$. The large variability of beam energies at sustained large luminosities is a particular strength of an EIC and proves critical for this measurement. A first feasibility study for electron-proton collisions can be found in Sec. 1.6.

Semi-inclusive deep-inelastic production of identified pions and kaons is expected to be the most viable and promising way to determine differences among parton distribution functions for different quark flavors or between quarks and anti-quarks. Such measurements make use of the different probabilities for producing a certain hadron species from a given 
quark flavor or gluon and have been successfully performed at fixed-target experiments such as HERMES. The EIC offers unprecedented opportunities to extend the kinematic reach toward small $x$ or large $Q$. In particular, the elusive strangeness density and a possible asymmetry between strangeness and anti-strangeness distributions can be deduced from charged kaon production yields. Prerequisites are excellent particle identification in most of the phase space and a thorough theoretical understanding of the hadronization of quarks and gluons into the observed hadrons. In collinear factorization, the latter information is encoded in non-perturbative fragmentation functions which are constrained by a wealth of available experimental data on single-inclusive hadron yields. Further significant progress on the quality of such fits is expected once upcoming data from $B$ factories and the LHC are included. In Sec. 1.5 we present a first feasibility study for charged kaon production at the EIC.

Heavy flavors, in particular charm quarks, can give a sizable contribution to deepinelastic scattering structure functions. Within the foreseen EIC kinematics, charm yields up to $10 \div 15 \%$ of the inclusive cross section. The theoretical framework for heavy quark production is much more complex than for light (massless) quarks due to the presence of multiple scales. The mass of the heavy quarks prevents them from having a partonic interpretation, and they can be only produced externally, for instance, by photon-gluon fusion. This framework yields a very good description of all available HERA data within the present uncertainties and is expected to be relevant also in the entire kinematic regime of an EIC. Nonetheless, one may introduce heavy quark densities for asymptotically large scales, i.e., $Q \gg m$, and smooth interpolation schemes have been devised which incorporate the correct threshold and asymptotic behavior. The relevant theoretical framework and recent progress on higher order calculations is briefly reviewed in Sec. 1.7.

The charm contribution to the longitudinal structure function $F_{L}$ is expected to be particularly sensitive to mass effects and has never been measured before. A first feasibility study within the kinematics of an EIC can be found in Sec. 1.8. An EIC is also well suited to address the long-standing question of a possible relevance of a non-perturbative "intrinsic" charm contribution in the nucleon wave-function, mainly concentrated at large momentum fractions. Quantitative estimates based on models for an intrinsic charm contribution are promising and can be found in Sec. 1.9.

The physics opportunities with polarized lepton and proton beams are even more multifaceted and will address some of the most fundamental open questions in hadronic physics for which one has been seeking answers for more the two decades now. Thus, the anticipated results will have far-reaching impact on our understanding of the nucleon's spin structure. The unique capability of the EIC to reach small momentum fractions $x$ or large scales $Q$ in longitudinally polarized electron-proton collisions with high luminosity will enable us to explore in detail

- the polarized gluon distribution and its contribution to the proton's spin,

- the individual light quark helicity distributions in a broad kinematic range,

- novel electroweak structure functions,

- and the strangeness and anti-strangeness polarizations.

The latest status of global QCD fits to helicity dependent parton densities, which is not expected to improve much by the time the EIC would turn on, and the set of questions we want to address at the EIC are laid out in some detail in Sec. 1.10. 
Precise measurements of the polarized structure function $g_{1}$ in a wide kinematic range will be a flagship measurement for the EIC. The gluon helicity distribution $\Delta g$ is strongly correlated with QCD scaling violations, i.e., the $Q$ dependence of $g_{1}$ at a given $x$. This will allow for a determination of $\Delta g$ down to unprecedented small values of $x$ of about $10^{-4}$. This in turn will eventually pinpoint the elusive gluon contribution to the spin of the proton, given by the integral of $\Delta g$ over all momentum fractions $x$, to about $10 \%$ accuracy or better. The striking quantitative impact on extractions of $\Delta g$ based on projected EIC data is demonstrated in Sec. 1.11. The same set of inclusive measurements will also provide a significantly better determination of the total quark contribution $\Delta \Sigma$ both as function of $x$ and the integral relevant for the nucleon spin sum.

Like in the unpolarized case, see Sec. 1.5, the best strategy to achieve a full flavor and quark-antiquark separation of polarized helicity densities is based again on semi-inclusive deep-inelastic hadron production. The kinematic coverage in $x$ and $Q$ is similar to what can be achieved in inclusive DIS, with the extra theoretical complication of the need for fragmentation functions to model hadronization. At medium-to-large values of $x$ one can address with precision certain interesting asymmetries in the polarized quark sea like $\Delta \bar{u}-$ $\Delta \bar{d}$ (from charged pion yields) and perhaps even $\Delta s-\Delta \bar{s}$ (from charged kaon yields). The first quantity is predicted to be sizable in several model calculations of the nucleon but the precision of current experiments only gives a first hint of a possible non-zero asymmetry; the latter quantity may help to understand why the sum $\Delta s+\Delta \bar{s}$ appears to be much smaller in current experiments than expected. If $\Delta s$ and $\Delta \bar{s}$ have their spins anti-aligned, their sum could be small but the asymmetry would be sizable. Constraints from hyperon decay matrix elements and arguments based on $\mathrm{SU}(3)$ symmetry predict a significantly negative total ( $x$ integrated) strange quark polarization. To address the validity of this constraint and to access to what extent $\mathrm{SU}(3)$ symmetry is broken, one needs to determine $\Delta s$ down to small values of $x$ to obtain a reliable estimate of its $x$ integral. This is another unique measurement to be performed at the EIC.

First simulations of electroweak neutral and charged current deep-inelastic scattering at the EIC in Sec. 1.12 show that such measurements become feasible already with relatively modest integrated luminosities. The corresponding structure functions for polarized protons have never been measured before and probe combinations of quark flavors other than in one-photon-exchange dominating at low $Q$. To fully exploit the potential of the EIC for such measurements, positron beams are required, albeit not necessarily polarized. An effective source of polarized neutrons such as a Helium-3 beam would be highly desirable. When combined, these measurements will greatly aid the flavor decomposition of polarized parton densities at medium-to-large $x$, free of any hadronization ambiguities. At the highest c.m.s. energies and luminosities also photon- $Z$ boson interference contributions to structure functions should be accessible at the EIC. The production of charmed mesons in charged current DIS events is an alternative probe for the strange and anti-strange densities both unpolarized and polarized. This is discussed in Sec. 1.13.

Table 1.1 summarizes the identified golden measurements, science deliverables, and experimental requirements in spin-dependent lepton-proton collisions at an EIC. Other, second tier measurements with polarization involve the currently unknown charm contribution to the deep-inelastic structure function $g_{1}$ which offers sensitivity to $\Delta g$ through photon-gluon fusion. Some expectations can be found in Sec. 1.11. If an effective neutron beam is available one can also attempt to determine the fundamental Bjorken sum rule at a few percent level. The Bjorken sum is probably one of the most precisely calculated quantities in perturbative QCD and provides an interesting link to the Adler $D$ function in electron-positron 


\begin{tabular}{|c|c|c|c|}
\hline Deliverables & Observables & What we learn & Requirements \\
\hline $\begin{array}{l}\text { polarized gluon } \\
\text { distribution } \Delta g\end{array}$ & $\begin{array}{l}\text { scaling violations } \\
\text { in inclusive DIS }\end{array}$ & $\begin{array}{l}\text { gluon contribution } \\
\text { to proton spin }\end{array}$ & $\begin{array}{c}\text { coverage down to } x \simeq 10^{-4} ; \\
\mathcal{L} \text { of about } 10 \mathrm{fb}^{-1}\end{array}$ \\
\hline $\begin{array}{l}\text { polarized quark and } \\
\text { antiquark densities }\end{array}$ & $\begin{array}{l}\text { semi-incl. DIS for } \\
\text { pions and kaons }\end{array}$ & $\begin{array}{l}\text { quark contr. to proton spin; } \\
\text { asym. like } \Delta \bar{u}-\Delta \bar{d} ; \Delta s\end{array}$ & $\begin{array}{l}\text { similar to DIS; } \\
\text { good particle ID }\end{array}$ \\
\hline $\begin{array}{c}\text { novel electroweak } \\
\text { spin structure functions }\end{array}$ & $\begin{array}{l}\text { inclusive DIS } \\
\text { at high } Q^{2}\end{array}$ & $\begin{array}{c}\text { flavor separation } \\
\text { at medium } x \text { and large } Q^{2}\end{array}$ & $\begin{array}{l}\sqrt{s} \gtrsim 100 \mathrm{GeV} ; \mathcal{L} \gtrsim 10 \mathrm{fb}^{-1} \\
\text { positrons; polarized }{ }^{3} \mathrm{He} \text { beam }\end{array}$ \\
\hline
\end{tabular}

Table 1.1. Golden measurements in polarized ep collisions at an EIC.

annihilation through the Crewther relation.

Finally, the production of hadronic final states in electron-proton collisions is dominated by the exchange of photons of almost zero virtuality. Photoproduction measurements and, in particular, the exploration of kinematic regimes where "resolved photon" contributions dominate was one of the great successes of the HERA physics program. Resolved processes, where the photon interacts with the proton through its non-perturbative source of partons, offer a fresh look at these densities which are so far mainly determined from imprecise LEP data. Given the anticipated high luminosity, an EIC can elevate these studies to a level of unprecedented precision, and, thanks to the polarized beams, allows one to investigate for the first time also the non-perturbative structure of circularly polarized photons. A good knowledge of the partonic structure of photons is essential for part of the physics program of a possible future linear collider. The general framework for photoproduction and two examples of physics studies are presented in Secs. 1.14 1.16.

To summarize, the physics goals of the EIC should be ambitious and must offer detailed answers to all the open fundamental questions concerning the spin and flavor structure of nucleons laid out above. The following sections will outline the path to achieve these goals. The program bears significant experimental challenges which all need to be carefully addressed to reach the desired unprecedented level of precision. With the exception of some of the electroweak structure function measurements, most observables will be quickly limited by systematic uncertainties, intrinsic ambiguities of the extraction method like, for instance, the Rosenbluth separation for $F_{L}$, and the way how well we can control QED radiative corrections to unfold the information one is actually interested in. Experimental aspects are discussed in Chapter 7 . 


\title{
1.2 Status of perturbative QCD calculations
}

\author{
Sven-Olaf Moch
}

\subsubsection{Introduction}

Deep-inelastic scattering (DIS) and the observed scaling violations are at the very center of the formulation of QCD as the gauge theory of the strong interactions [1, 2].

Over the decades the experiments using lepton and neutrino scattering off fixed targets at CERN, FNAL, SLAC, and JLAB as well as electron-proton collisions at the HERA collider at DESY have provided unique insight into the nucleon structure with the available high precision experimental data spanning a large kinematical range. Dramatic further improvements can be expected from the planned electron-ion collider EIC.

The key observables are either inclusive structure functions or differential cross sections in the semi-inclusive case, which parametrize the hard hadronic interaction in the QCD improved parton model. The particle data group (PDG) 3] provides a very readable account of DIS, including the definitions of kinematic variables, etc.

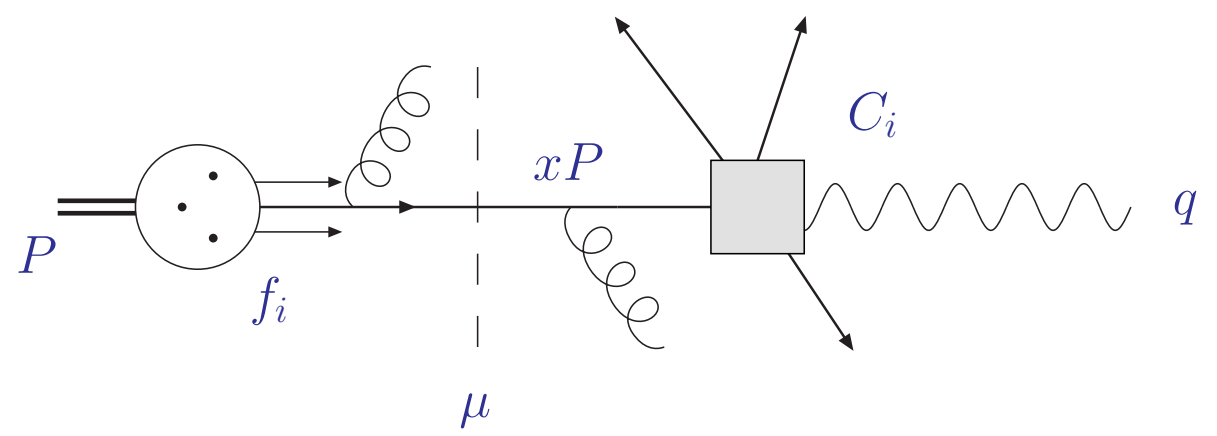

Figure 1.1. QCD factorization of the cross section for the scattering of a deeply virtual boson with (space-like) momentum $q\left(-q^{2}=Q^{2}>0\right)$ off a proton with momentum $P$ in their center-of-mass frame, see Eq. (1.1).

Precision predictions in perturbative QCD rest on the fact that we can separate the sensitivity to dynamics from different scales, i.e., the physics at scale of the proton mass from hard, high-energy scattering at a large scale $Q^{2}$. For lepton-proton DIS in the one-boson exchange approximation this is depicted in Fig. 1.1. For unpolarized DIS, this factorization at a scale $\mu$ allows to express the structure functions $F_{k}(k=2,3, L)$ as convolutions of parton distributions (PDFs) $f_{i}(i=q, \bar{q}, g)$ and short-distance Wilson coefficient functions $C_{k, i}$

$$
F_{k}\left(x, Q^{2}\right)=\sum_{i=q, \bar{q}, g} \int_{x}^{1} d z f_{i}\left(\frac{x}{z}, \mu^{2}\right) C_{k, i}\left(z, Q^{2}, \alpha_{s}(\mu), \mu^{2}\right)
$$

up to corrections of higher twist $\mathcal{O}\left(1 / Q^{2}\right)$. The coefficient functions $C_{k, i}$ are calculable perturbatively in QCD in powers of the strong coupling constant $\alpha_{s}$,

$$
C=C^{(0)}+\alpha_{s} C^{(1)}+\alpha_{s}^{2} C^{(2)}+\alpha_{s}^{3} C^{(3)}+\ldots,
$$


with the expansion coefficients $C^{(0)}$ denoted as the leading order (LO), $C^{(1)}$ the next-toleading order (NLO) and so on. The PDFs $f_{i}$ describe the fraction $x=Q^{2} /(2 P \cdot q)$ of the nucleon momentum carried by the quark or gluon. PDFs are non-perturbative objects and have to be obtained from global fits to experimental data or determined, e.g., by lattice computations. Perturbation theory, however, provides information about their scale dependence, i.e., the well-known evolution equations,

$$
\frac{d}{d \ln \mu^{2}}\left(\begin{array}{c}
f_{q_{i}}\left(x, \mu^{2}\right) \\
f_{g}\left(x, \mu^{2}\right)
\end{array}\right)=\sum_{j} \int_{x}^{1} \frac{d z}{z}\left(\begin{array}{cc}
P_{q_{i} q_{j}}(z) & P_{q_{i} g}(z) \\
P_{g q_{j}}(z) & P_{g g}(z)
\end{array}\right)\left(\begin{array}{c}
f_{q_{j}}\left(x / z, \mu^{2}\right) \\
f_{g}\left(x / z, \mu^{2}\right)
\end{array}\right) .
$$

The splitting functions $P_{i j}$ are universal quantities in QCD and describe the different possible parton splittings in the collinear limit. Like the $C_{k, i}$ also the $P_{i j}$ can be computed in a power series in $\alpha_{s}$,

$$
P=\alpha_{s} P^{(0)}+\alpha_{s}^{2} P^{(1)}+\alpha_{s}^{3} P^{(2)}+\ldots .
$$

Analogous formulae hold for the polarized DIS structure functions. In particular, for $g_{1}$ one may apply the obvious replacements $f_{i} \rightarrow \Delta f_{i}, C_{k, i} \rightarrow \Delta C_{g_{1}, i}$, and $P_{i j} \rightarrow \Delta P_{i j}$ in Eqs. (1.1)-(1.4). QCD factorization has also been established for (semi-)inclusive deepinelastic scattering (SIDIS), where the cross section $d^{2} \sigma / d x d Q^{2}$ is subject to a decomposition similar to Eq. (1.1). Although, in that case, the process dependent hard parton scattering cross sections need to be augmented by an additional prescription for the final state parton, e.g., a jet algorithm or fragmentation functions.

\subsubsection{Current status}

QCD predictions for DIS observables have reached over the years an unprecedented level of precision. All quantities in Eqs. (1.1)-(1.4) have been computed to higher orders in perturbation theory so that the effect of radiative corrections on those observables is well understood and largely under control. In the case of unpolarized DIS, the splitting functions $P_{i j}$ are known to NNLO [4, 5] and, likewise, the coefficient functions $C_{k, i}$ [6, 7, 8, 9]. For photon and charged current $W^{ \pm}$-boson exchange, even the hard corrections at order $\mathcal{O}\left(\alpha_{s}^{3}\right)$ are available [10, 11. In the case of polarized DIS, the spin dependent splitting functions $\Delta P_{i j}$ at two loop order have been obtained some time ago [12, 13]. At NNLO, the polarized splitting functions $\Delta P_{q q}$ and $\Delta P_{q g}$ have been reported [14], and the coefficient functions $\Delta C_{g_{1}, i}$ are available from [15]. For semi-inclusive observables, the QCD corrections are typically known to NLO. This corresponds to $\mathcal{O}\left(\alpha_{s}^{2}\right)$ since the underlying Born cross section behaves as $d^{2} \sigma^{(0)} / d x d Q^{2} \sim \mathcal{O}\left(\alpha_{s}\right)$ due to the additional final state parton. Processes considered include, for instance, the electro-production of hadrons with high transverse momentum [16, 17] or single inclusive DIS jet cross sections [18].

The currently available QCD predictions for inclusive DIS and SIDIS put us in comfortable position to confront experimental data with theory at a very high level of precision. In these comparisons, we no longer test QCD. Rather we use perturbative QCD as an essential and established part of our theory toolkit to deduce important information about PDFs or the value of the strong coupling constant $\alpha_{s}\left(M_{Z}\right)$. Of course, this is a situation that, generally, needs to be addressed also beyond DIS, since experimental data from the unpolarized (anti-)proton-proton colliders Tevatron at FNAL and the LHC at CERN as well as from the polarized proton-proton collider RHIC at BNL help to further constrain the non-perturbative input to QCD precision predictions. See, e.g., the analyses of 
unpolarized PDFs to NNLO in Refs. [19, 20, 21, 22] or recent studies of polarized PDFs in [23, 24, 25, 26, 27, 28].

Given the current status of perturbative QCD, experimental data from a future program of electron-ion collisions, EIC, can help to address and clarify a number of still open and yet very relevant questions; see also Secs. 1.4 and 1.11, For the case of unpolarized PDFs improvements can be made with respect to the flavor asymmetry of sea quarks at low $x$ and the valence quarks at large $x$, by studying, e.g., electron-deuteron collisions. Much of the physics case here had already been investigated in an assessment of the experimental prospects of electron-deuteron scattering at HERA some time ago [29]. More generally, the high luminosity of an EIC would further constrain PDFs, especially the gluon at low $x$ and $Q^{2}$. In this context, a precision measurements of the longitudinal structure function $F_{L}$, which is an observable predominantly driven by the gluon PDF is of high interest as it would complement and, eventually even supersede, existing experimental data, see, e.g., 30]. New high statistics DIS experiments can also improve the current precision of strong coupling constant $\alpha_{s}$ measurements in space-like kinematics.

For polarized DIS, a very fundamental question still remains the understanding of the proton spin, in particular, whether the polarized gluon PDF $\Delta f_{g}$ provides a significant contribution. To that end, an extension of the kinematical coverage in $x$ and $Q^{2}$, as it could be achieved by an electron-ion collider, is of paramount importance. This would help to access higher scales in $Q^{2}$ in order to test the perturbative evolution Eq. (1.3). Likewise, access to an extended $x$-range allows for a better determination of moments of the $\Delta f_{i}$. They also enter, e.g., in the Bjorken sum rule for polarized electro-production, which is again an observable very well-known in perturbative QCD [31, 32. Other issues of interest for polarized DIS in electron-ion collisions concern a reliable extraction of flavor structure as well as a study of strangeness PDFs, $\Delta f_{s}$.

\subsubsection{Summary}

We have briefly summarized the current status of perturbative QCD predictions for DIS experiments. To date, we can build on a very mature understanding of the theory, which could be confronted with experimental data from a future electron-ion collider in order to improve our knowledge about the fundamental structure of matter and the important dynamics of quarks and gluons in nucleons. 


\title{
1.3 Unpolarized proton structure - HERA's legacy
}

\author{
Amanda Cooper-Sarkar (for the H1 and ZEUS Collaborations)
}

\subsubsection{Introduction}

HERA data provide the most insight into the behaviour of unpolarized parton distribution functions (PDFs) at present and as such represent an integral part of all global QCD analyses. The $\mathrm{H} 1$ and ZEUS experiments are combining their various sub-sets of data so as to provide a legacy of HERA results. The combination of inclusive cross section data from HERA-I and the PDF fit based on these data are already published [20]. In 2010 further data have been combined and PDF fits to the augmented data sets have been made available in preliminary form. In Sec. 1.3 .2 results from the published combination are reviewed. In Sec. 1.3 .3 results from a combination of $F_{2}^{c \bar{c}}$ data are presented and their sensitivity to the mass of the charm quark and the choice of the heavy flavor scheme adopted in the global PDF fit is discussed. In Sec. 1.3.4 results from the combination of inclusive cross section data taken at lower proton beam energies are discussed. Finally, in Sec. 1.3.5 an updated combination of all inclusive data from HERA-I and HERA-II running is shown and a PDF fit to these data is presented.

\subsubsection{Inclusive data from HERA-I running (1992-2001)}

The inclusive cross section data, from the HERA-I running period, for Neutral Current (NC) and Charged Current (NC), $e^{+} p$ and $e^{-} p$ scattering have been combined 20]. The combination procedure pays particular attention to the correlated systematic uncertainties of the data sets such that resulting combined data benefits from the best features of each detector. The combined data set has systematic uncertainties which are smaller than its statistical errors and the total uncertainties are small $(1-2 \%)$ over a large part of the kinematic plane. The combined data is compared to the separate input data sets of ZEUS and $\mathrm{H} 1$ in Fig. 1.2.

These data are used as the sole input to a PDF fit called the HERAPDF1.0 [20]. The motivations for performing a HERA-only fit are firstly, that the combination of the HERA data yields a very accurate and consistent data set such that the experimental uncertainties on the PDFs may be estimated from the conventional $\chi^{2}$ criterion $\Delta \chi^{2}=1$. Global fits which include dats sets from many different experiments often use inflated $\chi^{2}$ tolerances in order to account for marginal consistency of the input data sets. Secondly, the HERA data are proton target data so that there is no uncertainty from heavy target corrections or deuterium corrections and there is no need to assume that $d$ in the proton is the same as $u$ in the neutron since the $d$-quark PDF may be extracted from $e^{+} p$ CC data. Thirdly, the HERA inclusive data give information on the gluon, the Sea and the $u$ - and $d$-valence PDFs over a wide kinematic region: the low- $Q^{2} \mathrm{NC} e^{+} p$ cross-section data are closely related to the low- $x$ Sea PDF and the low- $x$ gluon PDF is derived from its scaling violations; the high$x u$ - and $d$-valence PDFs are closely related to the high- $Q^{2} \mathrm{NC} e^{ \pm} p, \mathrm{CC} e^{-} p$, and $\mathrm{CC} e^{+} p$ cross sections, respectively; the difference between the high- $Q^{2} e^{-} p$ and $e^{+} p$ cross-sections gives the valence shapes down to low $x, x \sim 10^{-2}$.

HERAPDF provides model and parametrisation uncertainties on the PDFs as well as experimental uncertainties; for details, see Ref. [20]. A major contribution to the total uncertainties in the HERAPDF1.0 set comes from the model uncertainty on the charm mass value. This can be improved using information from data on $F_{2}^{c \bar{c}}$. 
H1 and ZEUS

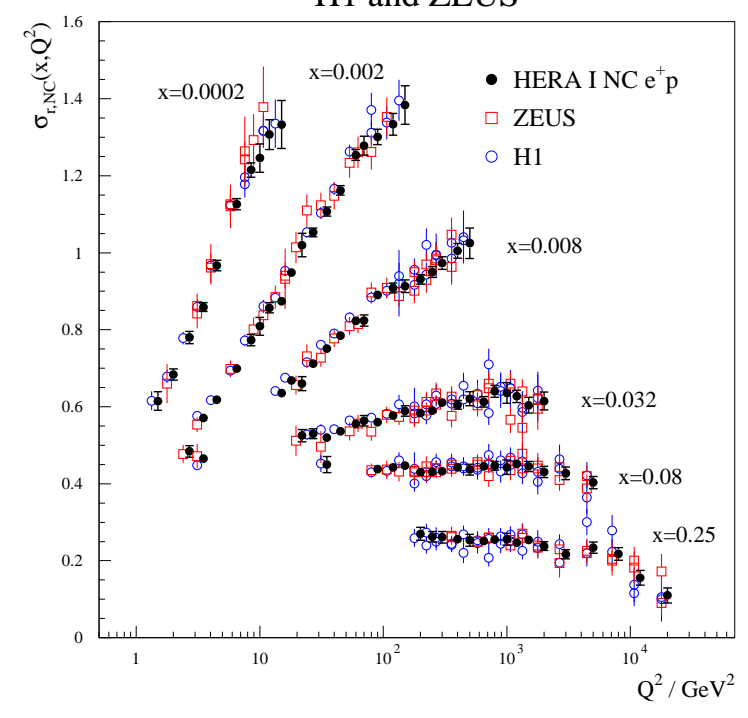

Figure 1.2. HERA combined data points for the $\mathrm{NC} e^{+} p$ cross section as a function of $Q^{2}$ in selected bins of $x$, compared to the separate ZEUS and H1 data sets input to the combination.

\subsubsection{Charm data from HERA-I and II running}

H1 and ZEUS have also combined their data on $F_{2}^{c \bar{c}}[20$. In Fig. 1.3 the combined data are compared to the separate data sets which go into the combination. These data are input to the HERAPDF fit together with the inclusive data which were used for HERAPDF1.0. The $\chi^{2}$ of this fit is sensitive to the value of the charm quark mass. Fig. 1.4 compares the $\chi^{2}$, as a function of this mass, for a fit which includes these data (left) to that for the HERAPDF1.0 fit (middle). However, it would be premature to conclude that the data can be used to determine the charm pole-mass. The HERAPDF formalism uses the ThorneRoberts (RT) variable-flavour-number (VFN) scheme for heavy quarks. This scheme is not unique, specific choices are made for threshold behaviour. In Fig. 1.4 (right) the $\chi^{2}$ profiles for the standard and the optimized versions of this scheme are compared to two alternative ACOT VFN schemes and the Zero-Mass VFN scheme. Each of these schemes favours a different value for the charm quark mass, and the fit to the data is equally good for all the heavy quark mass schemes; see Fig. 1.3 (right). However, the Zero-Mass scheme is $\chi^{2}$ disfavoured; see Ref. [20] for further details.

\subsubsection{Low energy proton beam data from 2007}

In $2007 \mathrm{NC}^{+} p$ data were taken at two lower values of the proton beam energy in order to determine the longitudinal strucure function $F_{L}$. Some of the H1 and ZEUS data sets from these runs have now been combined [20] and the results for the $\mathrm{NC} e^{+} p$ cross section are shown in Fig. 1.5. These data have been input to the HERAPDF fit together with the inclusive data from HERA-I. The resulting PDFs are compared with those of HERAPDF1.0 in Fig. 1.5. The low energy data are sensitive to the choice of minimum $Q^{2}$ (standard cut $Q^{2}>3.5 \mathrm{GeV}^{2}$ ) for data entering the fit. If a somewhat harder cut, $Q^{2}>5 \mathrm{GeV}^{2}$, is made, a steeper gluon distribution results, see Fig. 1.5, whereas for the HERAPDF1.0 this variation of cuts results in PDFs which lie within the PDF uncertainty bands. This sensitivity is also present if an $x$ cut, $x>5 \times 10^{-4}$, or a "saturation inspired" cut, $Q^{2}>0.5 x^{-0.3}$, is made. This sensitivity may indicate the breakdown of the DGLAP formalism at low $x$ [33]. 

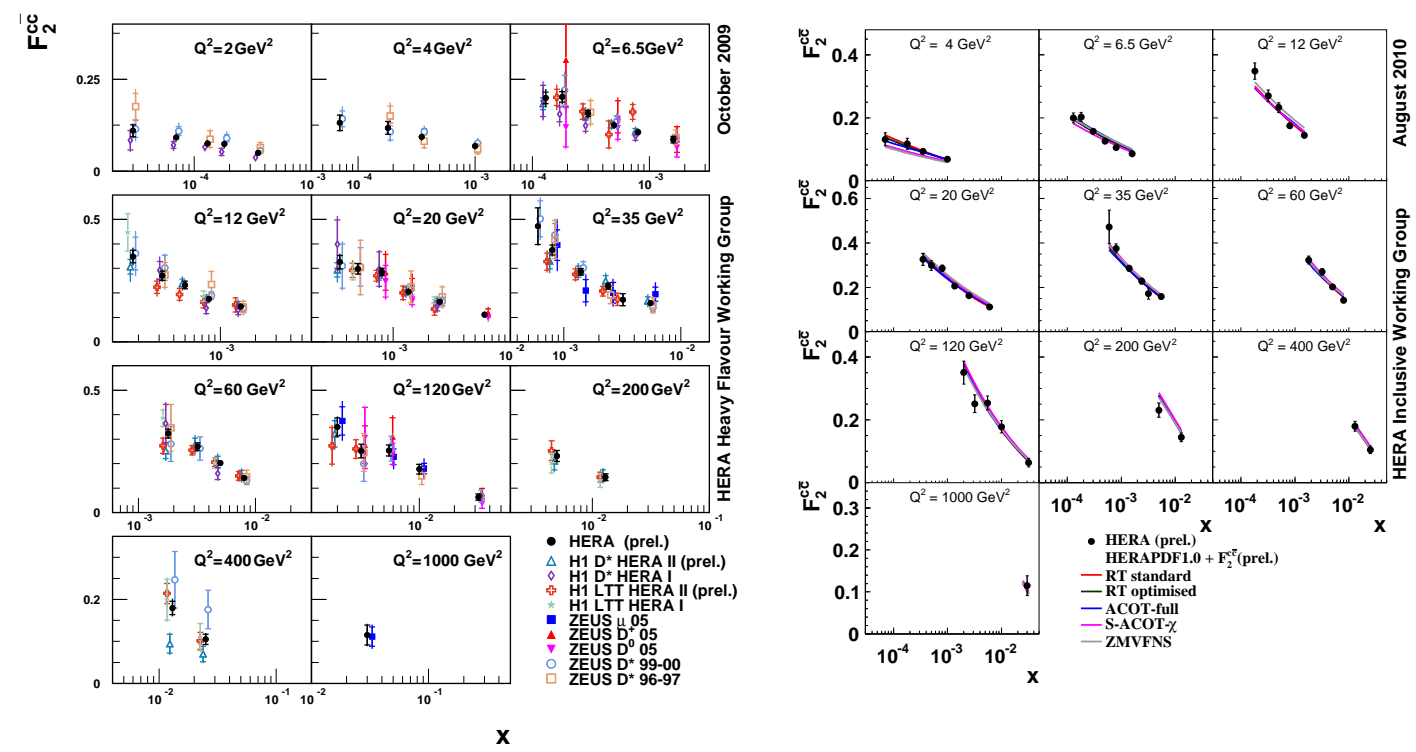

Figure 1.3. Left: HERA combined data points for $F_{2}^{c \bar{c}}$ compared to the separate ZEUS and H1 data sets. Right: HERA combined data points for $F_{2}^{c \bar{c}}$ compared to HERAPDF fits to these plus the inclusive DIS data, for various different heavy-quark-mass schemes.
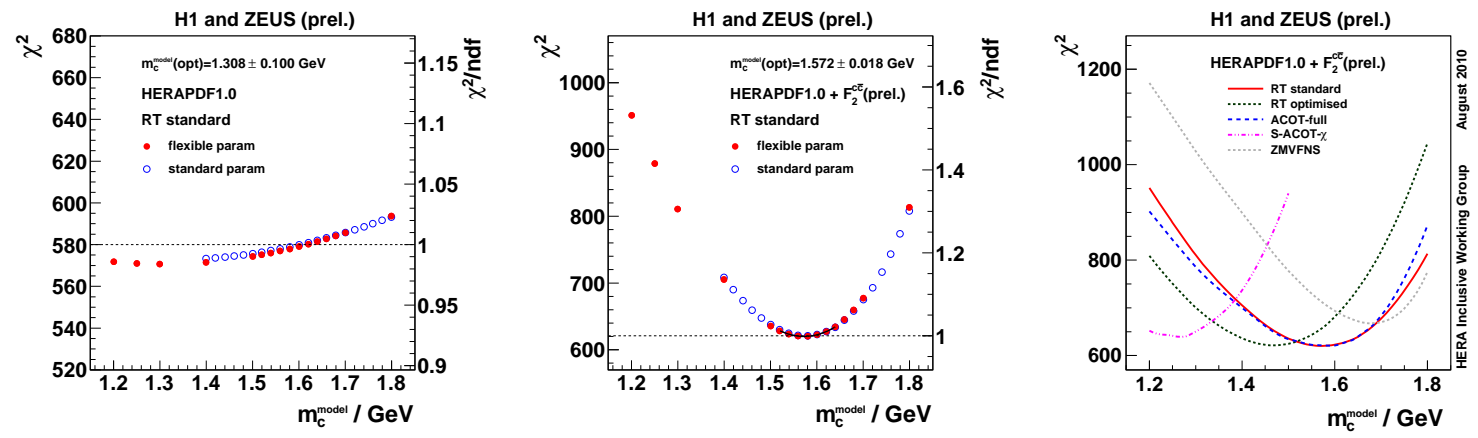

Figure 1.4. The $\chi^{2}$ of the HERAPDF fit as a function of the charm mass $m_{c}^{\text {model }}$. Left and Middle: using the RT-standard scheme, when $F_{2}^{c \bar{c}}$ data are not included and included in the fit, respectively. Right: results for using various mass schemes in the fit to $F_{2}^{c \bar{c}}$ data. 

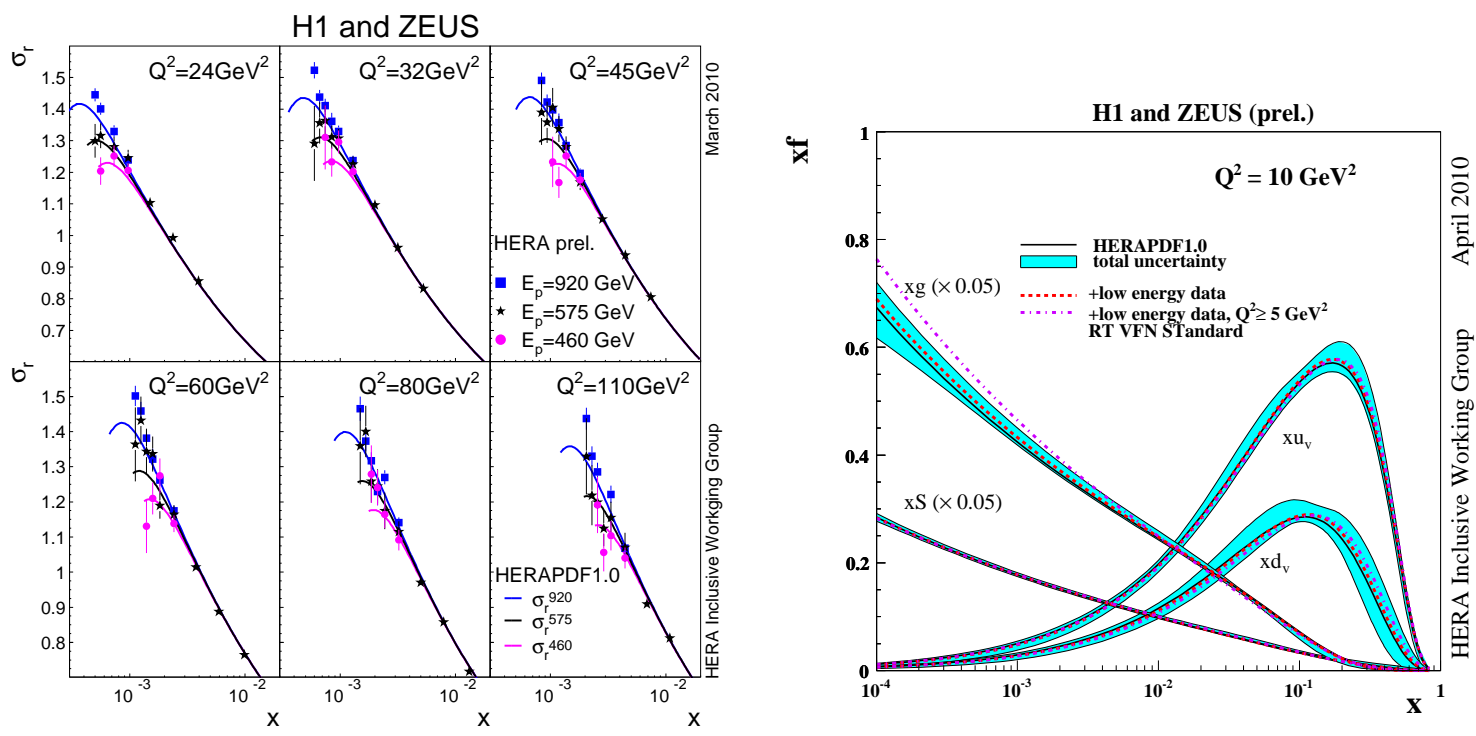

Figure 1.5. Left: HERA combined data points for the $\mathrm{NC} e^{+} p$ cross-section for three different proton beam energies. Right: PDFs, $x u_{v}, x d_{v}, x S=2 x(\bar{U}+\bar{D})$, and $x g$ at $Q^{2}=10 \mathrm{GeV}^{2}$, for HERAPDF1.0 and for a HERAPDF fit which also includes the low-energy proton beam data, with the standard $Q^{2}$ cut, $Q^{2}>3.5 \mathrm{GeV}^{2}$, and for $Q^{2}>5.0 \mathrm{GeV}^{2}$.

\subsubsection{High- $Q^{2}$ data from HERA-II running}

Preliminary $\mathrm{H} 1$ data on $\mathrm{NC}$ and $\mathrm{CC} e^{+} p$ and $e^{-} p$ inclusive cross-sections and published ZEUS data on NC and $\mathrm{CC} e^{-} p$ and $\mathrm{CC} e^{+} p$ data, from HERA-II running, have been combined with the HERA-I data to yield an inclusive data set with improved accuracy at high $Q^{2}$ and high $x$ [34]. The HERA-I data set and the new HERA I+II data sets are compared for $\mathrm{CC} e^{-} p$ data in Fig. 1.6. This new data set is used as the sole input to a new PDF fit called the HERAPDF1.5 which uses the same formalism and assumptions as the HERAPDF1.0 fit [35. These fits are superimposed on the corresponding data sets in the figure. Fig. 1.7 (left) shows the combined data for $\mathrm{NC} e^{ \pm} p$ cross-sections with the HERAPDF1.5 fit superimposed. The PDFs from HERAPDF1.0 and HERAPDF1.5 are compared in Fig. 1.7 (right). The improvement in precision at high $x$ is clearly visible.

\subsubsection{Summary}

The status of the combinations of $\mathrm{H} 1$ and ZEUS data has been discussed. HERA leaves rich legacy of results which are the basis for all present QCD analyses of unpolarized PDFs and define the goals for any future DIS experiment. 

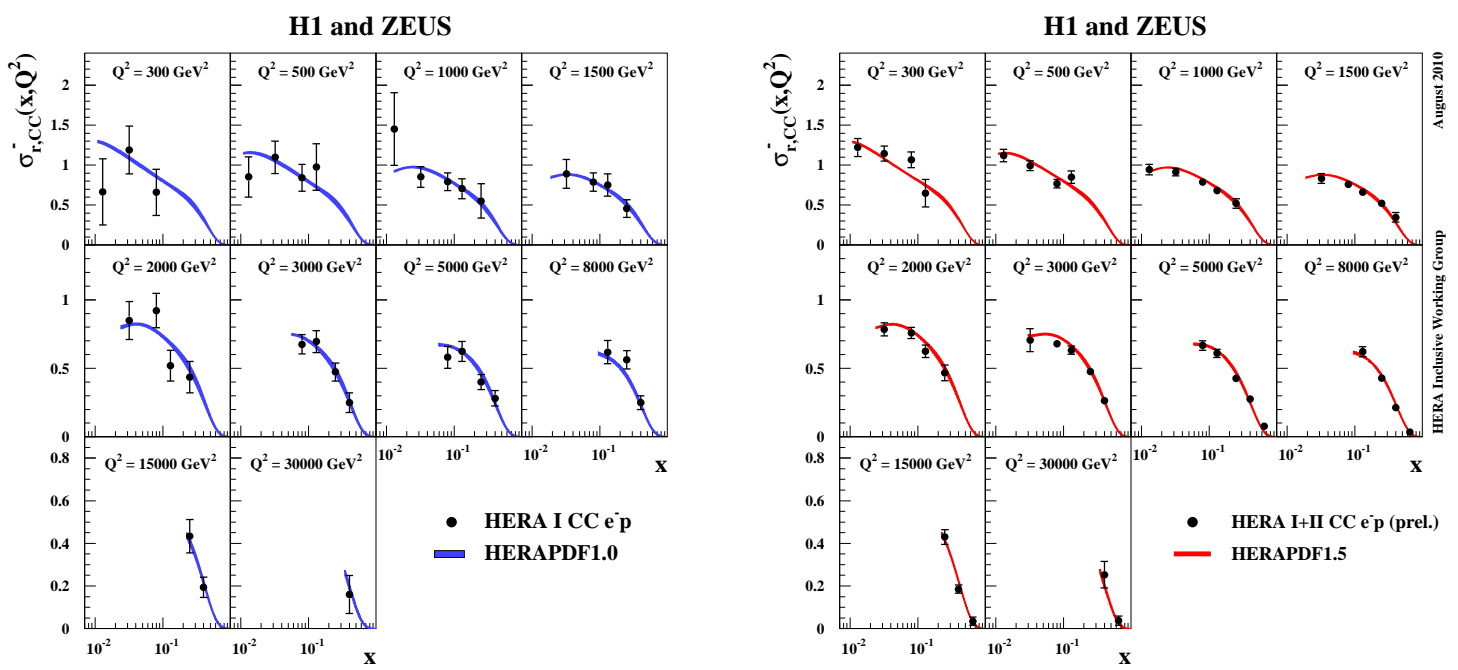

Figure 1.6. HERA combined data points for the $\mathrm{CC} e^{-} p$ cross-section. Left: from the HERA-I run period. Right: from the HERA-I and II run periods. On each plot the HERAPDF fit which includes the corresponding data is illustrated: the HERAPDF1.0 fit on the left hand plot and the HERAPDF1.5 on the right hand plot.
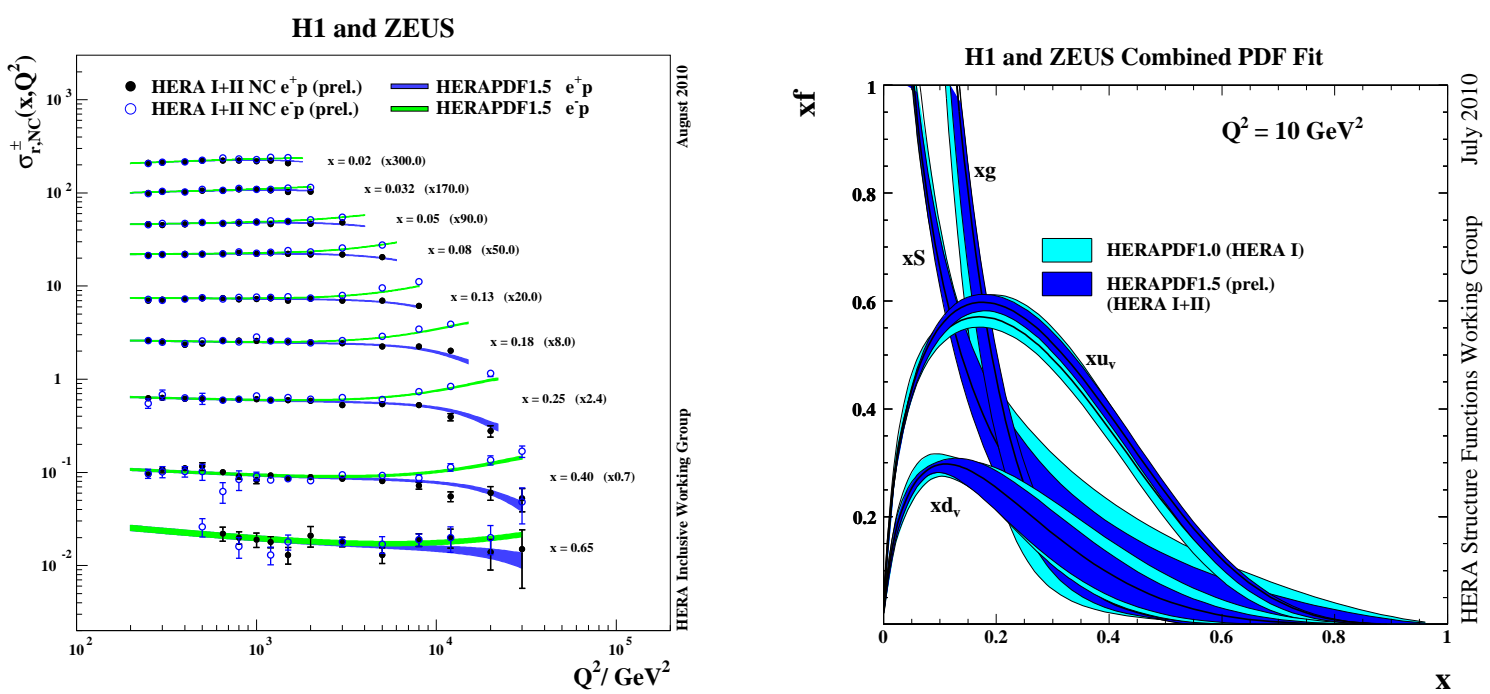

Figure 1.7. Left: HERA combined data points for the $\mathrm{NC} e^{ \pm} p$ cross-sections for data from the HERA-I and II run periods. The HERAPDF1.5 fit to these data is also shown on the plot. Right: Parton distribution functions from HERAPDF1.0 and HERAPDF1.5; $x u_{v}, x d_{v}, x S=2 x(\bar{U}+\bar{D})$ and $x g$ at $Q^{2}=10 \mathrm{GeV}^{2}$. 


\title{
1.4 Unpolarized parton distribution functions: questions to be addressed at an EIC
}

\author{
Marco Guzzi, Pavel Nadolsky, Fredrick Olness
}

\subsubsection{Introduction}

The Electron-Ion Collider (EIC) will operate at a time when the Large Hadron Collider (LHC) has established a new "gold standard" for perturbative QCD by measuring a variety of hard-scattering processes. High-luminosity EIC measurements will be very complementary to those at the LHC, as they will accurately probe various aspects of hadronic structure using independent experimental techniques. In the next few years, when next-tonext-to-leading order (NNLO) accuracy of QCD calculations becomes the norm, a variety of perturbative and nonperturbative effects need to be taken into account to match the precision of multi-loop radiative contributions. Some of these effects can be constrained solely by the LHC data; others need independent measurements, not affected by systematical uncertainties present at the LHC. With an integrated luminosity of $10 \mathrm{fb}^{-1}$ or more, the EIC will disentangle many such effects, including modifications of the nucleon structure within heavy-nuclei targets, flavor dependence of parton distribution functions (PDFs), and QCD dynamics at very large or small $x$.

As compared with previous lepton-nucleus experiments, the EIC will probe to smaller $x$ values with high precision. In contrast to the HERA ep collider, which explores the same $\left\{x, Q^{2}\right\}$ region, heavy-ion scattering will achieve much higher partonic densities that are a prerequisite for the onset of saturation. It will help delineate the kinematical boundary between the DGLAP factorization and saturated dynamics in the nuclear medium.

The $Q^{2}$ range of the EIC will cover the transition region from the perturbative to the non-perturbative regime. Here, we wish to learn how the perturbative parton-scattering picture valid at large momentum transfers matches on nonperturbative models describing the strongly-coupled resonance region. Understanding of this region is important for hadronic experiments at the intensity frontier.

\subsubsection{Open Questions}

Several questions about PDFs will likely remain open at the time of the EIC operation. Figure 1.8 shows the kinematic domains in $x$ and $Q^{2}$ probed by current experiment and the PDFs that are most strongly constrained in these reqions.

Nuclear PDFs. Several groups extract nuclear PDFs and their uncertainties by analyzing the global data on nuclear targets [36, 37, 38, 39, 40]. In their studies, they find that the nuclear corrections depend on the type of the nucleus (its atomic number $A$ ), flavor of the probed parton, and even the type of the probing boson. For example, it was found recently [41, 37] that the nuclear correction factors preferred by the $\nu$ Fe DIS data by NuTeV [42] are surprisingly different from predictions based on the $\ell^{ \pm} \mathrm{Fe}$ charged-lepton results.

By performing deep inelastic scattering (DIS) both on proton and heavy-nuclei targets, the EIC can distinguish between intrinsic properties of the proton and those of the extended nuclear medium. A high-intensity EIC could use a variety of nuclear beams to precisely map the $A$-dependent nuclear correction factors in the $\left\{x, Q^{2}\right\}$ kinematic plane and clarify the behavior of nuclear corrections to NC DIS. Such information is of importance for determining the proton PDFs, in particular, the strange quark PDF that is constrained largely 


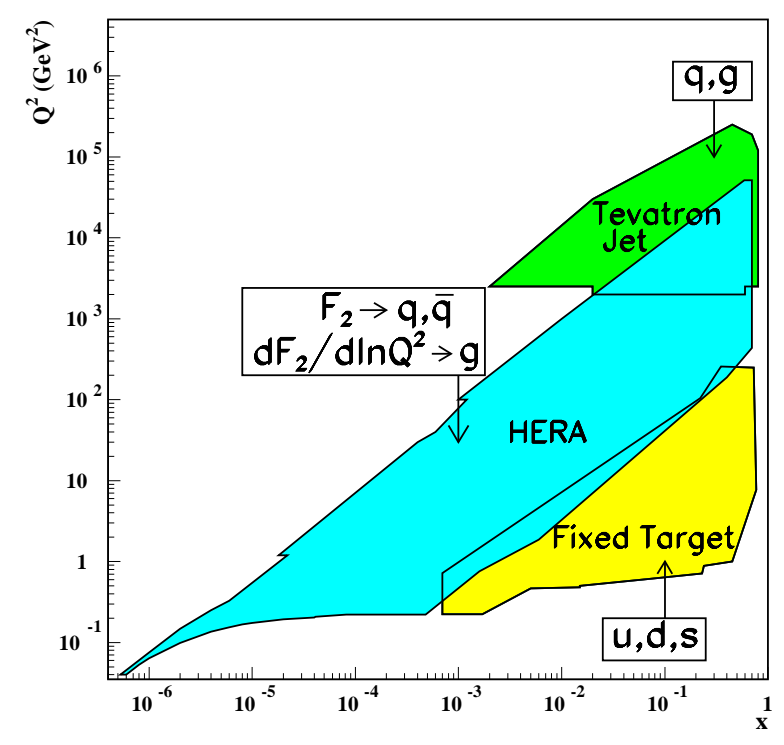

Figure 1.8. Kinematic domains in $x$ and $Q^{2}$ probed by fixed-target and collider experiments, shown together with the PDFs that are most strongly constrained by the indicated regions [3]. DIS data off nuclear targets exist only in the fixed-target region.

from the $\mathrm{NuTeV}$ data. The nuclear correction affects the uncertainty in $s(x, Q)$, which is large at present and may limit the precision of electroweak studies in $W$ and $Z$ boson production at the LHC [43].

The topics of nuclear PDFs and saturation will be extensively discussed in the Chapter 5 devoted to $e A$ physics at an EIC.

Better constraints on the strangeness PDF. Despite extensive investigation, there remain large uncertainties in flavor differentiation of sea-quark PDFs both in the proton and nuclei. In particular, the strange quark+antiquark distribution in the proton, $s_{+}(x)=$ $s(x)+\bar{s}(x)$, and its asymmetry, $s_{-}(x)=s(x)-\bar{s}(x)$, are still poorly known [44, 45, 46, 22, 47, despite their significance for understanding of the nucleon structure. Existing constraints on the strangeness come predominantly from neutrino (semi-)inclusive DIS [48, 42]. At the EIC, both $s_{+}(x)$ and $s_{-}(x)$ can be probed in semi-inclusive DIS production of kaons; see Sec. 1.5 for some quantitative studies. This measurement will rely on a good understanding of fragmentation functions, which will be known much better by the time an EIC turns on.

The $\mathrm{d} / \mathrm{u}$ ratio at large $\mathrm{x}$. Because of its intermediate energy and high beam intensity, the EIC is ideal for studying parton distributions at large Bjorken $x(x>0.1)$, where separation of parton flavors is not fully understood despite many years of experiments. For example, even the ratio $d(x, Q) / u(x, Q)$ of the dominant up and down quark proton PDFs at $x>0.3$ has been recently put in doubt by contradicting constraints from DIS on deuteron targets [49, 50] and charged lepton asymmetry at the Tevatron [51, 52]. While the PDF analysis groups labor to understand these differences [22, 46, 53, (and new clean LHC measurements of the $d / u$ ratio in proton scattering are in the queue), the EIC will help to resolve this controversy by extracting the ratio $F_{2}^{n}(x, Q) / F_{2}^{p}(x, Q)$ from DIS data on various nuclear targets. Such measurement will help to separate several types of kinematical and nuclear corrections ([54], and references therein) that influence the $F_{2}^{n} / F_{2}^{p}$ ratio derived 
from nuclear-target DIS.

Gluon PDF in the proton and charm production at large x. Even more uncertainty exists in the gluon PDF $g(x, Q)$ at large $x$, where it can be larger than the down-quark $d(x, Q)$ at $x>0.5$ in some recent parametrizations for proton PDFs [55]. This ambiguity will be reduced by upcoming high- $p_{T}$ jet production at the LHC, but significant systematic limitations of both experimental and theoretical nature may persistent at the largest $x$, where the EIC could independently contribute. Production of heavy-quark $(c, b)$ pairs or heavy mesons $(J / \psi, \Upsilon)$ in deep-inelastic scattering could accurately probe the large- $x$ gluon PDF. The EIC detectors will have excellent charm tagging efficiency, in a relatively clean scattering environment as compared to the LHC.

Inclusive charm production is interesting in its own right, given that large radiative contributions are known to exist near the heavy-quark production threshold, i.e., at $Q$ comparable to the charm quark mass; see Sec. 1.7 for a detailed account of heavy quark contributions to DIS structure functions. The rate for charm production at large $x, x \gtrsim 0.1$, can be increased by up to an order of magnitude by nonperturbative intrinsic charm production suggested by light-cone models [56, 57]. An EIC will be a unique opportunity to cleanly test for the presence of intrinsic charm contributions; see Sec. 1.9 for some quantitative studies.

Transition to the high-density regime. There is a long-standing question of partonic saturation and recombination in the small- $x$ region. As a related phenomenon, BFKL [58, 59, 60] effects from large $\ln [1 / x]$ contributions may supersede the usual DGLAP evolution in the small- $x$ regime. The EIC should be capable of probing the transition from DGLAP factorization to BFKL/saturation dynamics, particularly using heavy nuclei beams in order to produce large partonic densities; see Chapter 5 for details on $e A$ physics.

Perturbative-nonperturbative QCD boundary. The general kinematic parameters of an EIC would span across both the perturbative (large $Q^{2}$ ) region and the nonperturbative (small $Q^{2}$ ) region. The theoretical description of the physics in these two regions is very different, and precise EIC data might enable us to better connect these two disparate theoretical descriptions.

The longitudinal structure function. The longitudinal structure function $F_{L}=$ $F_{2}-2 x F_{1}$ is of special interest, in view that its leading $\mathcal{O}(1)$ term vanishes according to the Callan-Gross relation. The first non-vanishing, leading order contribution is of $\mathcal{O}\left(\alpha_{s}\right)$ and dominated by photon-gluon fusion. Hence, $F_{L}$ is particularly sensitive to the gluon distribution $g\left(x, Q^{2}\right)$. Corrections up to $\mathcal{O}\left(\alpha_{s}^{3}\right)$ are known [10, allowing for a consistent analysis of $F_{L}$ at NNLO accuracy. An EIC could make the first precise measurements of $F_{L}$ in a kinematic range that overlaps both the fixed-target and HERA collider data [30] which have large statistical uncertainties; see Sec. 1.6 for more details on such a measurement at an EIC.

Electroweak contributions to proton PDFs. Some, if not all, NLO electroweak effects will be included in future PDF analyses, as their magnitude is comparable to the size of NNLO QCD radiative contributions that will be routinely included. The QCD+EW PDFs require additional experimental input to constrain nonperturbative parametrizations for photon PDFs, as well as charge asymmetry effects (isospin violation) between PDFs for up-type quarks and down-type quarks at the initial scale $Q \approx 1 \mathrm{GeV}$. An EIC has the potential to contribute toward improving limits on electroweak PDF terms either directly or in combination with neutrino DIS measurements.

When extracting information about the proton PDFs from scattering on nuclear targets, we generally make use of isospin symmetry to relate the proton and neutron PDFs via a 
$u \leftrightarrow d$ interchange. While the isospin symmetry is elegant, it is nonetheless approximate and can be violated at the level of a few percent [61, 62, 63, 64, 65, 66, 67, 41, 68]. Violation of the exact $p \leftrightarrow n$ isospin symmetry, or charge symmetry violation (CSV), invalidates the parton model relations that reduce the number of independent nonperturbative distributions; e.g., $u^{n}(x) \not \equiv d^{p}(x)$ and $u^{p}(x) \not \equiv d^{n}(x)$. It is important to be aware of the potential magnitude of isospin symmetry violation and its consequences for flavor separation of proton PDFs.

It is noteworthy that isospin symmetry is automatically violated both perturbatively and nonperturbatively. This is because the photon couples to the up quark distribution $u^{p}(x)$ differently than to the down quark distribution $d^{n}(x)$. These terms can be comparable to the NNLO DGLAP evolution effects [69, 70, 71].

Some combinations of structure functions, such as $\Delta F_{2} \equiv \frac{5}{18} F_{2}^{C C}\left(x, Q^{2}\right)-F_{2}^{N C}\left(x, Q^{2}\right)$ and $\Delta x F_{3}=x F_{3}^{W^{+}}-x F_{3}^{W^{-}}$, can be particularly sensitive to isospin violations, and an EIC can contribute to their measurement. For example, the EIC is capable of measuring precisely the structure function $F_{2}^{N C}$ mediated by the neutral-current $\gamma / Z$ exchange processes. Measurement of $F_{2}^{C C}$, mediated by the charged-current $W^{ \pm}$exchange, would rely on compensating the $M_{W}^{2} / Q^{2}$ suppression of the $W$ boson propagator with high intensity of the beams; see Sec. 1.12 for more details on electroweak structure function measurements at an EIC.

In separate experiments, $\Delta x F_{3}$ can be measured precisely via the neutrino-nucleon DIS process; as these measurements are performed with heavy nuclear targets, the nuclear correction factors can be the limiting factor as to the derived CSV constraint. Since an EIC will use a variety of nuclear targets, it can obtain very precise nuclear correction factors; this information could, in principle, be used together with the neutrino-nucleon DIS data to extract improved CSV limits.

The structure functions $\Delta F_{2}$ and $\Delta x F_{3}$ receive contributions from both heavy flavors as well as CSV contributions; improved understanding of the heavy-quark components (discussed previously) can indirectly contribute to better CSV limits [68].

The combination of high-statistics EIC measurements and constraints could thus yield important information on the fundamental charge symmetry.

\subsubsection{Acknowledgments}

FIO thanks I. Schienbein, J.Y. Yu, K. Kovarik, C. Keppel, J.G. Morfin, J.F. Owens, K. Park, and T. Stavreva for valuable discussions. 


\title{
1.5 Flavor separation from semi-inclusive DIS
}

\author{
Elke-Caroline Aschenauer, Marco Stratmann
}

\subsubsection{Motivation and Method}

The strangeness distribution and a possible asymmetry between strangeness and antistrangeness densities have been identified as two of the most compelling open questions in hadronic physics which are difficult to address without an EIC; see Sec. 1.4.

Existing constraints in global fits come predominantly from neutrino (semi-)inclusive DIS [48, 42] but both $s_{+}(x) \equiv s(x)+\bar{s}(x)$ and $s_{-}(x) \equiv s(x)-\bar{s}(x)$ are still only poorly known [46, 22, 72]. Figure 1.9 summarizes recent uncertainty estimates for $s_{ \pm}$from three global QCD fits.
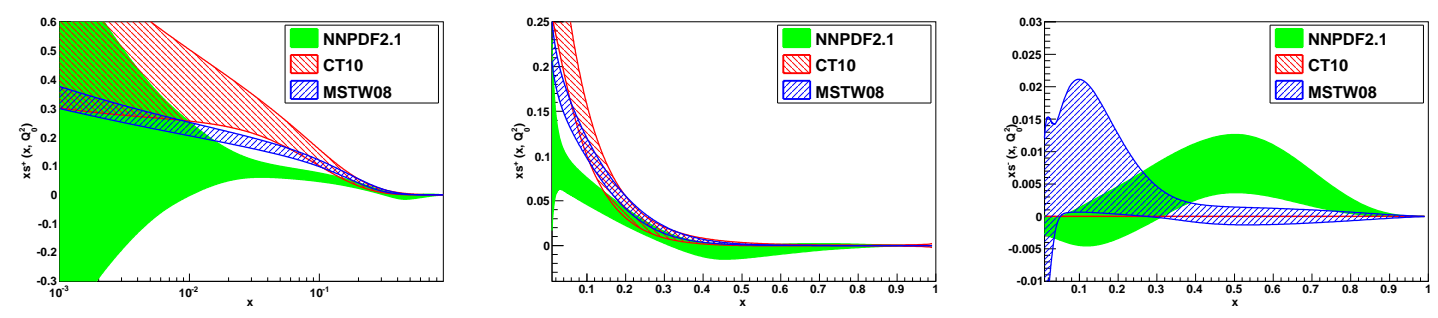

Figure 1.9. Uncertainty bands for $s_{ \pm}$at $Q^{2}=2 \mathrm{GeV}^{2}$ for recent fits. Figure taken from [72].

Semi-inclusive DIS with identified charged kaons is expected to be a viable method to determine the elusive strange quark density and perhaps a possible asymmetry $s_{-}$experimentally. One can access basically the same a broad kinematic range in $x$ and $Q^{2}$ as in inclusive DIS. The HERMES collaboration has successfully performed such a measurement in the range $0.02<x<0.6$ at an average $Q^{2}$ of about $2.5 \mathrm{GeV}$ [3]. Compared to $s(x)$ from most global PDF fits, they find a softer strangeness distribution in their LO analysis. Clearly, more data in a larger range of $x$ and $Q^{2}$ are necessary to clarify this issue.

The SIDIS measurement relies, however, on a good understanding of the hadronization mechanism which is encoded in non-perturbative, collinear parton-to-hadron fragmentation functions (FFs) $D_{i}^{H}$ if factorization is assumed in a pQCD calculation. Like PDFs, FFs are extracted from global QCD analyses. One can resort to a wealth of single-inclusive hadron production data obtained at different c.m.s. energies in $e^{+} e^{-}$annihilation and in $e p$ and $p p(p \bar{p})$ scattering. Pion FFs are currently known best with uncertainties of about $5 \div 10 \%$ depending on the flavor of the fragmenting parton [74. Ambiguities for kaon FFs are about twice as large [74]. Significant progress on the quality of fits to FFs is expected once data from $B$ factories and the LHC become available. Also, NNLO evolution kernels are expected to become available in the near future [75], which will help to reduce theoretical scale ambiguities further.

All relevant SIDIS cross sections are known at least to NLO accuracy [76, 77, 78, 79], and the analytical expressions are relatively simple and easy to implement into global fits of PDFs, see, e.g., [80]. Schematically the unpolarized SIDIS cross section for the production of a hadron $H$ in the current fragmentation region reads

$$
\frac{d \sigma^{H}}{d x d y d z}=\frac{2 \pi \alpha^{2}}{Q^{2}}\left[\frac{1+(1-y)^{2}}{y} 2 F_{1}^{H}\left(x, z, Q^{2}\right)+\frac{2(1-y)}{y} F_{L}^{H}\left(x, z, Q^{2}\right)\right]
$$


with $x$ and $y$ denoting the usual DIS variables, $-q^{2}=Q^{2}=S x y$, and $z=p_{H} \cdot p / p \cdot q$ the momentum fraction taken by the hadron $H$. Assuming factorization, the structure functions $F_{1, L}^{H}$ at a factorization scale $\mu \sim Q$ can be expressed as convolutions of non-perturbative PDFs $f_{j}(x, \mu)$ and FFs $D_{i}^{H}(z, \mu)$ with short-distance Wilson coefficients $C_{i j}^{1, L}(x, z, \mu)$.

\subsubsection{Expectations for Charged Kaon Production at an EIC}

Figures 1.10 and 1.11 show expectations for the $K^{+}$and $K^{-}$production cross section (1.5) at NLO accuracy, respectively, as a function of $x$ in bins of $Q^{2}$, using $0.01 \leq y \leq 0.95$ and $\sqrt{S}=70.7 \mathrm{GeV}$ (i.e., $5 \times 250 \mathrm{GeV}$ collisions at an EIC). To reduce uncertainties from kaon FFs, $z$ is integrated in the range $0.2 \leq z \leq 0.8$. The DSS set 74 is used. The solid lines are the statistical average over 100 replicas in the NNPDF2.0 neural network analysis [47] and the dashed lines reflect the corresponding PDF uncertainties.

Also shown in Figs. 1.10 and 1.11 are simulations based on the PYTHIA 81 event generator in the same kinematic range. Here, the CTEQ6L set of PDFs [82] has been used. The hadronic final state was simulated using JETSET based on LEP fragmentation settings and a suppression of $s \bar{s}$ pair production from the vacuum of 0.3 [PARJ (2)] compared to $u \bar{u}$ or $d \bar{d}$ creation. The results turn out to be remarkably similar to the NLO calculations based on collinear factorization despite the very different way hadronization is implemented in PYTHIA and the fact that only LO matrix elements are used, albeit matched with a parton shower. This gives us quite some confidence that the PYTHIA generator can be used to provide very reasonable estimates of yields for DIS-type processes at an EIC. In addition, it also tells us that the current DSS kaon FFs are doing a good job and include a realistic amount of "strangeness suppression". Already after one month of operation, corresponding to an integrated luminosity of about $20 \mathrm{fb}^{-1}$ the measurement will be limited by systematic uncertainties which need to be carefully studied. The statistical accuracy is significantly better than indicated by size of the points shown in the figures.

If one compares the results for $K^{+}$and $K^{-}$in Figs. 1.10 and 1.11 one finds hardly any difference at the smallest $x$ values in each $Q^{2}$ bin. At larger $x$ values, where $s_{-}$is largest, see Fig. 1.9, the yields for $K^{-}$are significantly lower than the ones for $K^{+}$. An EIC should be able to provide accurate measurements of both $s_{+}$and $s_{-}$in a broad kinematic range up to $Q^{2}$ values of a few hundred $\mathrm{GeV}$.

Within the neural network approach it is in principle fairly straightforward to quantify by how much a new data set will reduce present PDF uncertainties. The original ensemble of replicas is constructed in such a way that all have the same weight. Information contained in new data sets can be incorporated without the need for refitting by reweighting each PDF in the ensemble by the probability that it agrees with the new data 47, 53. Sets with small weights will become largely irrelevant in statistical averages. If too many sets receive small weights the accuracy of results from the new PDF ensemble will deteriorate, and the reweighting procedure becomes unreliable, necessitating a full refit. One reason for this to happen is, that the new data set contains significant new information which leads to much smaller uncertainties in certain kinematic regions. This is exactly what happens when one applies the reweighting method to the SIDIS data shown in Figs. 1.10 and 1.11 even if one assigns a fictitious $\mathcal{O}(5 \%)$ systematic uncertainty to each data point.

There are many other things which can be studied in SIDIS at an EIC. For instance, one can also bin in $z$ which makes the measurement more sensitive to the shape of the kaon FFs. This will provide a more stringent check whether FFs are universal functions in $e^{+} e^{-}, e p$, and $p p$ scattering. Pion yields will allow one to study other interesting and 
relevant PDF combinations such as $\bar{u}(x)-\bar{d}(x)$. Similar measurements can be also done with longitudinally polarized beams which will give access to the helicity-dependent quark and antiquark densities, see Sec. 1.11, Detailed quantitative studies including more timeconsuming global QCD analyses with simulated SIDIS data for various c.m.s. energies are planned to quantify the impact of such measurements on our understanding of the spin and flavor structure of the nucleon. These studies should include also some estimates of the various sources of systematic uncertainties, like detector resolution, uncertainties in the particle identification, luminosity, and polarization measurements, details on these can be found in Sec. 7.3 .
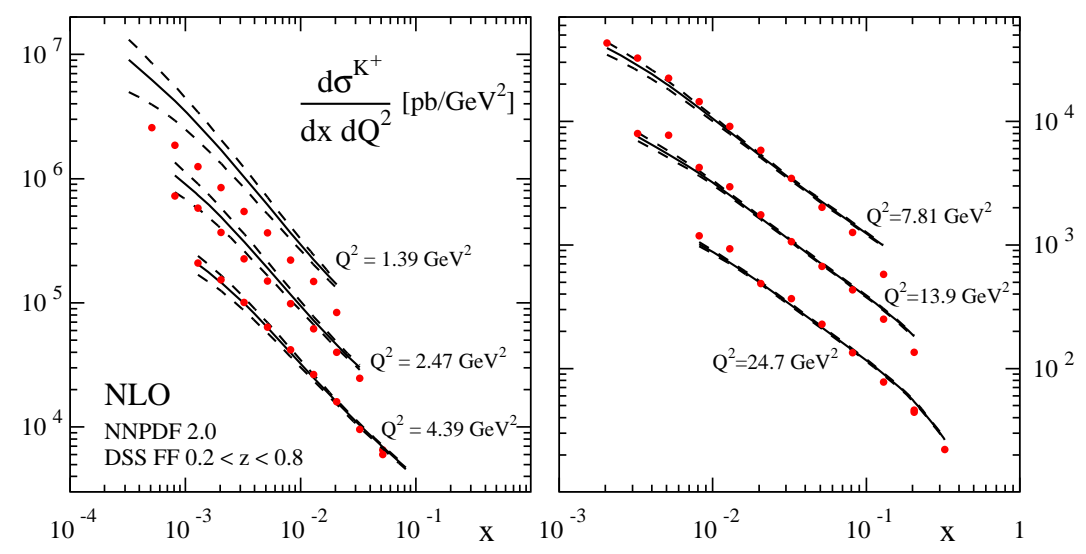

Figure 1.10. SIDIS cross section for $K^{+}$production at NLO accuracy using NNPDF2.0 PDFs [47. The dashed lines denote the PDF uncertainties. Also shown (points) are the results from a PYTHIA simulation (see text).

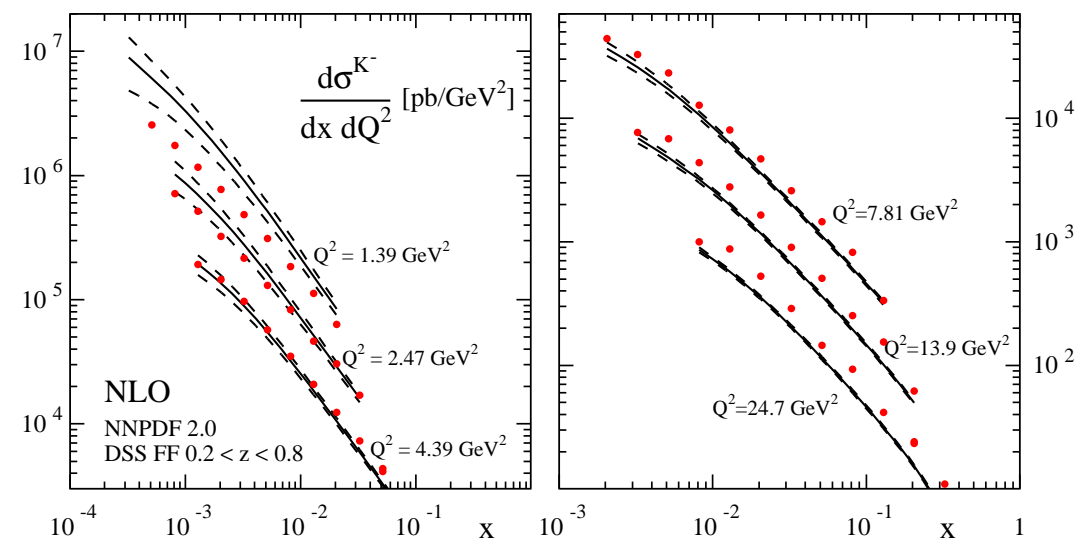

Figure 1.11. Same as in Fig. 1.10 but now for $K^{-}$production. 


\title{
1.6 The longitudinal structure function $F_{L}$ at an EIC
}

\author{
Elke C. Aschenauer, Ramiro Debbe, Marco Stratmann
}

\subsubsection{Motivation and Current Status of $F_{L}$ Results}

The DIS reduced cross section $\sigma_{r}$ for one-photon-exchange can be represented as the sum of two independent structure functions $F_{2}$ and $F_{L}$ as follows

$$
\sigma_{r} \equiv \frac{Q^{4} x}{2 \pi \alpha_{e m}^{2} Y_{+}} \frac{d^{2} \sigma}{d x d Q^{2}}=F_{2}\left(x, Q^{2}\right)-\frac{y^{2}}{Y_{+}} F_{L}\left(x, Q^{2}\right)
$$

where $Y_{+} \equiv 1+(1-y)^{2}$ depends on the inelasticity $y=Q^{2} /(s x)$ of the process.

$F_{L}$ is proportional to the cross section for probing the proton with a longitudinally polarized virtual photon and vanishes in the naive Quark Parton Model due to helicity conservation. Starting from $\mathcal{O}\left(\alpha_{s}\right)$, the longitudinal structure function differs from zero, receiving contributions from both quarks and gluons.

At low $x$, the gluon contribution due to photon-gluon fusion greatly exceeds the quark contribution. Therefore, measuring $F_{L}$ provides a rather direct way of studying the gluon density and QCD dynamics at small $x$, i.e., the transition to the high parton density regime. Measurements can be used to test several phenomenological and QCD models describing the low $x$ behavior of the DIS cross section, including color dipole models [83, 84, 85] and expectations from DGLAP fits performed at NLO and NNLO accuracy of QCD. Possible deviations from the DGLAP behavior in the small $x$, low $Q^{2}$ region can be studied by varying kinematic cuts to the data used in the fits.

The longitudinal structure function, or the equivalent cross section ratio $R=\sigma_{L} / \sigma_{T}=$ $F_{L} /\left(F_{2}-F_{L}\right)$, was first measured in fixed target experiments and found to be small at large $x, x \geq 0.01$, see, e.g., Ref. [86]. H1 [30] and ZEUS [87] have recently combined their measurements of $\sigma_{r}$ for three different proton beam energies [20], $E_{p}=920,575$, and 460 GeV, see Fig 1.5 in Sec. 1.3, The extracted $F_{L}$, shown in Fig. 1.12, covers a wide kinematic range, spanning $2.5<Q^{2}<800 \mathrm{GeV}^{2}$ and $0.0006<x<0.0036$. As can be seen, $F_{L}$ is clearly non-zero, and there is some mild tension with the HERAPDF1.0 fit based on DGLAP evolution [20] at the lowest values of $x$ and $Q^{2}$ where one expects non-linear effects to be relevant; see Chapter 5 on $e A$ physics. In this regime, predictions from the dipole model provide a better description of the data. However, the achieved statistical precision of the combined H1 and ZEUS measurement is too limited to be conclusive.

\subsubsection{Measurement Strategy and Experimental Challenges}

The measurement of $F_{L}$ relies on an accurate determination of the variation of the reduced cross section (1.6) for common values of the $\left(x, Q^{2}\right)$ bin centers at different beam energies, i.e., c.m.s. energies $\sqrt{s}$. Relative normalizations and systematic uncertainties of the different data sets for $\sigma_{r}$ have to be well under control.

$F_{L}$ and $F_{2}$ can be extracted simultaneously from $\sigma_{r}$ by plotting $\sigma_{r}$ for fixed values of $\left(x, Q^{2}\right)$ as a function of $y^{2} / Y_{+} . F_{L}$ is then determined as the slope of the line fitted to the measurements of $\sigma_{r}$ for different values of $\sqrt{s}: F_{L}\left(x, Q^{2}\right)=-\partial \sigma_{r}\left(x, Q^{2}, y\right) / \partial\left(y^{2} / Y_{+}\right)$. Likewise, $F_{2}$ is the intercept of the fitted line with the $y$ axis: $F_{2}\left(x, Q^{2}\right)=\sigma_{r}\left(x, Q^{2}, y=0\right)$. All measurements at HERA are observed to be consistent with the expected linear dependence [30, 87, 20. At any given value of $Q^{2}$, the lowest possible $x$ values are only accessed by 
$\mathrm{H} 1$ and ZEUS

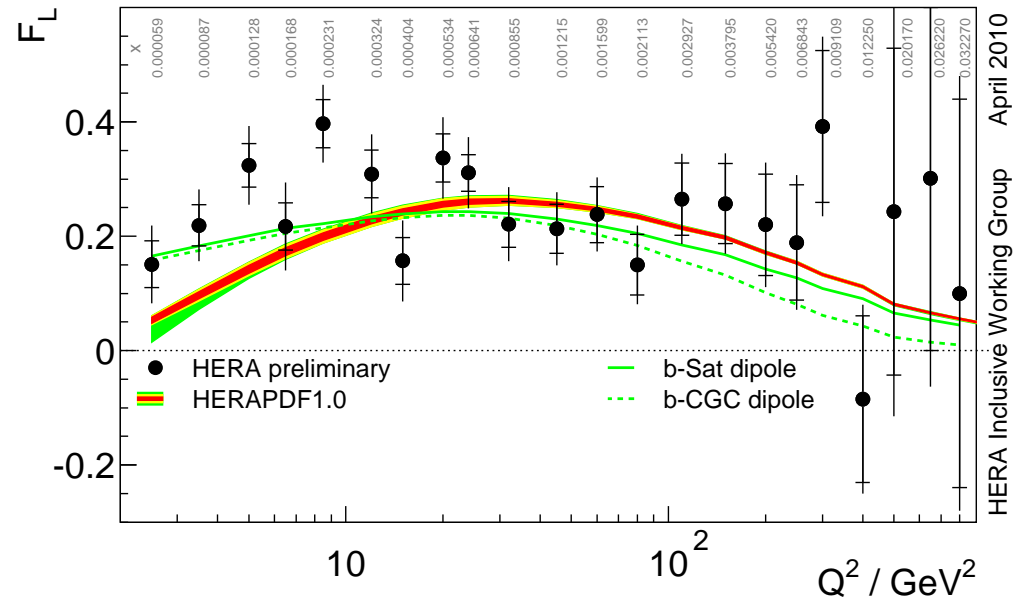

Figure 1.12. Combined H1 and ZEUS extraction of $F_{L}$ [20] as a function of $Q^{2}$ averaged over $x$ compared to the HERAPDF1.0 fit and predictions from dipole models.

the highest $\sqrt{s}$, and the slope related to $F_{L}$ cannot be determined. Hence, the Rosenbluth separation limits the kinematic coverage of $F_{L}$ at small $x$. At larger values of $x$, measurements of $\sigma_{r}$ for various different $\sqrt{s}$ are available and the slopes can be straightforwardly extracted.

The contribution of $F_{L}$ to the reduced cross section (1.6) can be sizable only at large values of $y$. For low values of $y, \sigma_{r}$ is very well approximated by the structure function $F_{2}$ [30, 87, 20]. Low $y$ data can be used to normalize data sets taken at different c.m.s. energies relative to each other. For measurements at high $y$ the reconstruction of the DIS kinematics using the scattered lepton, the so called "electron method", has the best resolution and was used at HERA.

In the large $y$ region, $y \gtrsim 0.5$, and low $x$ the electron method is prone to large QED radiative corrections which can reach a level of more than $50 \%$ of the Born cross section. Studies based on the DJANGO [88] and HECTOR [89] programs for HERA kinematics show that the largest radiative contributions arise because of hard initial-state radiation (ISR) from the incoming lepton [30]. The radiated photon usually escapes in the beam pipe and the $E-P_{z}$ of the event is reduced. Therefore, hard ISR can be efficiently suppressed to a level of about $10 \%$ at HERA with only a slight residual dependence on $y$ by requiring $E-P_{z}$ close to the nominal value of twice the electron beam energy implied by energymomentum conservation [30]. $E-P_{z}$ can be reconstructed from the measured final-state particles. At the highest $y, y \gtrsim 0.7$, corrections increase due to QED Compton events which can be rejected by certain topological cuts. All cross section measurements at HERA are corrected for QED radiation up to $\mathcal{O}\left(\alpha_{e m}\right)$ using HERACLES [90] which is included in the DJANGOH package; further details can be found in Sec. 7.3.

Kinematically, for low $Q^{2}$, large values of $y$ correspond to low energies of the scattered lepton. Selecting high $y$ events is thus further complicated due to a possibly large background from energy deposits of hadronic final state particles leading to fake electron signals. However, the cut on $E-P_{z}$ also suppresses such type of backgrounds. In addition, electron tracking, which is foreseen for an EIC detector, will largely eliminate fake electron signals as an additional cut on $E / p \simeq 1$ can be placed to identify the lepton.

Extractions of $F_{L}$ are certainly the most demanding inclusive structure function measurements but an EIC will have many advantages compared to HERA, in particular, the possibility to vary $\sqrt{s}$ in a wide range for high luminosity collisions. Also, much better detector capabilities, for instance, concerning the electron, are foreseen. One can also take 


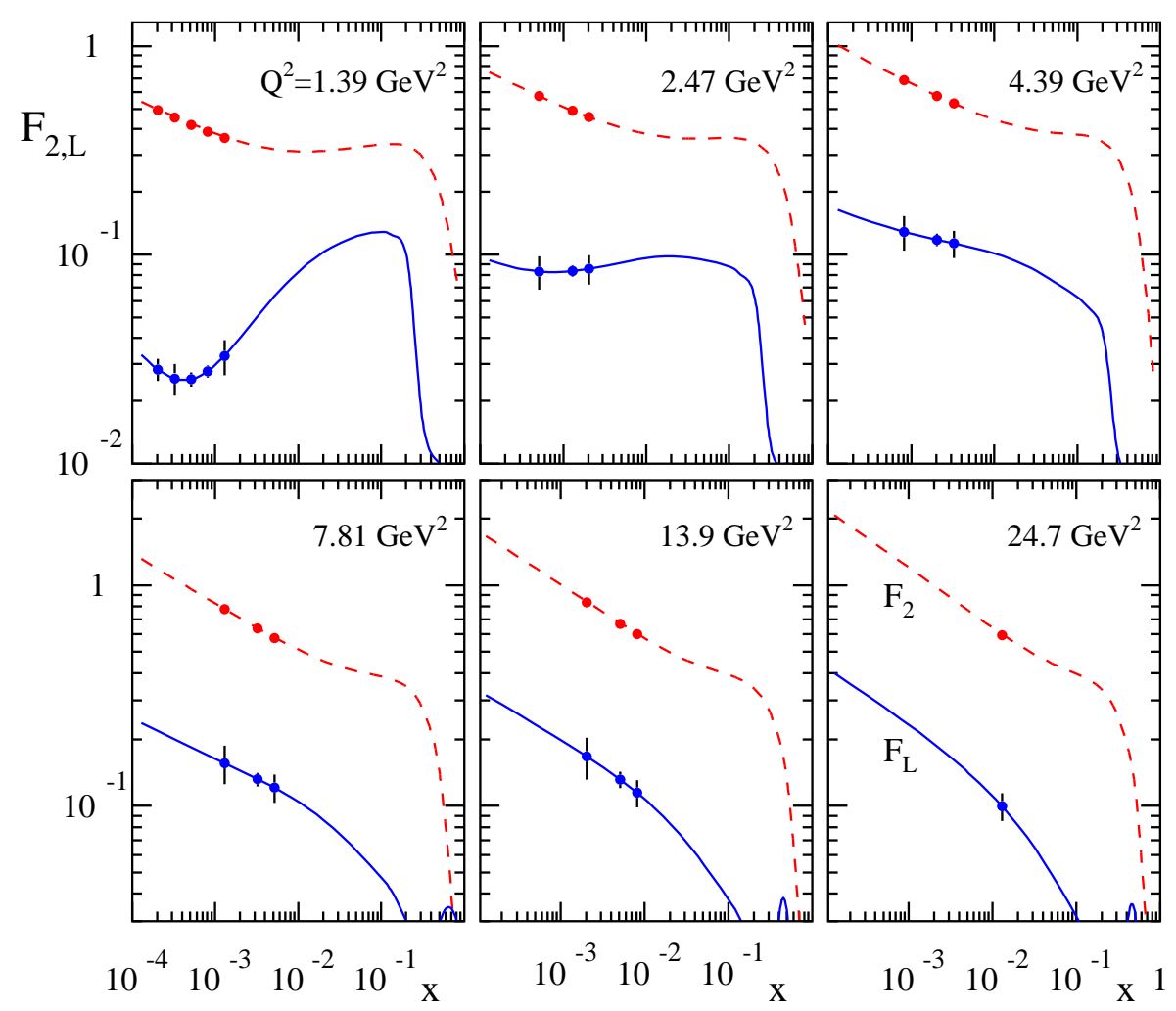

Figure 1.13. Projected uncertainties for an extraction of $F_{2}$ and $F_{L}$ from a Rosenbluth separation for data taken at three different c.m.s. energies. Also shown are theoretical expectations at NNLO based on the ABKM09 set of PDFs [19] (see text).

advantage of all the analysis techniques and Monte Carlo codes developed for HERA to deal with QED radiative corrections.

\subsubsection{Expectations for the EIC}

Pseudo-data for the reduced cross section (1.6) have been generated using the Monte Carlo generator LEPTO [91] for the first stage of an EIC (5 GeV electrons on 100, 250, and $325 \mathrm{GeV}$ protons). The CTEQ6L set of PDFs [82 has been used in the simulations. The hadronic final state was simulated using JETSET [81]. We note that the current pseudo-data do not include any simulations of QED radiative effects and reflect statistical uncertainties which could be achieved by running one month at each of the beam energy settings with the projected luminosities for eRHIC. In addition, a 1\% systematic uncertainty is added.

Figure 1.13 shows the structure functions $F_{L}$ and $F_{2}$ extracted from the pseudo-data of the reduced cross section by means of a Rosenbluth separation, requiring a minimum scattered lepton momentum of $0.5 \mathrm{GeV}, Q^{2}>1 \mathrm{GeV}^{2}, 0.01<y<0.90$, and $0.5^{\circ}<\theta<$ $179.5^{\circ}$. To guide the eye, the expected uncertainties are placed on theoretical expectations for $F_{2, L}$ at NNLO accuracy using the ABKM09 set of PDFs [19]. One should note that these PDFs use only data with $Q^{2}>2.5 \mathrm{GeV}^{2}$ in their fit and, hence, the behavior of $F_{2, L}$ in the lowest $Q^{2}$ bin must be taken with a grain of salt and are only for illustration. The extracted uncertainties take detector smearing of the scattered electron momentum into account. The momentum resolution was taken from ZEUS, i.e., $\delta p / p=0.85 \%+0.25 \% \times p$. 


\subsubsection{Summary and To-Do Items}

Like for most inclusive and semi-inclusive measurements at the EIC, an extraction of $F_{L}$ will be dominated by systematic uncertainties which need to be thoroughly addressed. This is work in progress. It is planned to study the unfolding of $F_{L}$ in great detail both in $e p$ and $e A$ scattering, including QED radiative corrections and a full simulation of the detector. This will elucidate to what extent the methods developed and used at HERA [30, 87, 20] are suited for high precision measurements of $F_{L}$ aimed at the EIC. In any case, it will be crucial to design the relevant detector components very carefully to optimize

- the luminosity measurement and its relative calibration for running at different c.m.s. energies,

- the lowest lepton momentum we can detect ( $0.5 \mathrm{GeV}$ would be desirable),

- the identification of the scattered lepton to suppress potential background from misidentified hadrons,

- the resolution in momentum and scattering angle of the scattered lepton, and

- the acceptance for the hadronic final state to suppress events which have a photon radiated from the incoming or outgoing lepton as well as quasi real photo-production events.

Details on the design of the detector are given in Sec. 7.3. Also, it will be possible to extract $\mathrm{F}_{L}$ from the EIC data alone, but the combination of the EIC reduced cross section measurements with the ones from HERA may provide an even better lever arm in a larger $x, Q^{2}$ range. This needs to be investigated.

Finally, we note that even for statistically very precise measurements of $\sigma_{r}$, the Rosenbluth separation of $F_{L}$, i.e., the determination of the slope with respect to $y^{2} / Y_{+}$, can lead to significantly larger uncertainties if the measured values of $\sigma_{r}$ have very similar $y^{2} / Y_{+}$. This source of uncertainties needs be minimized by optimizing the binning in $y$ and the set of different c.m.s. energies $\sqrt{s}$. Studies is this direction are ongoing as well. 


\subsection{Theoretical status of inclusive heavy quark production in DIS}

Sergey Alekhin, Johannes Blümlein, Sven-Olaf Moch

\subsubsection{Introduction}

Heavy quark production gives a sizable contribution to the unpolarized DIS structure functions at small $x$, see, e.g., [92, 93, 94, 95]. For the foreseen EIC kinematics of DIS it yields up to $10 \%$ of the inclusive cross section. Therefore in order to employ the full potential of the small- $x$ EIC data for phenomenology one has to provide an accurate theoretical description of heavy-quark electro-production within perturbative QCD.

For the light-parton contributions to DIS structure functions a theoretical accuracy of $O($ few $\%)$ is achieved, with the complete QCD corrections up to 3-loops being available, see also Sec. 1.2, In the case of the fixed-flavor-number scheme (FFNS) the heavy flavor corrections are available only to $O\left(\alpha_{s}^{2}\right)$. This can be a bottleneck for the analysis of high-precision data. Therefore, progress in the higher-order calculations of heavy-quarkproduction coefficient functions is quite important for the EIC phenomenology. For the variable-flavor-number scheme (VFNS) the massive quarks are considered on the same footing as the massless ones. Furthermore, the heavy-quark PDFs appearing in the VFNS are derived from the light-parton PDFs and the appropriate massive operator-matrix elements (OMEs). The VFNS coefficient functions are known up to 3-loop accuracy due but the massive OMEs are only available to the NLO corrections. This limits the theoretical accuracy of the VFNS as well.

In the following we summarize the state-of-art in calculations of the NNLO corrections to the unpolarized heavy-quark coefficient functions and to the massive OMEs. The FFNS and VFNS are compared to the available HERA data and to each other. We also discuss the implementation of the running-mass scheme for the NLO and NNLO heavy-quark coefficient functions and the resulting improvement in the perturbative stability related to this definition.

\subsubsection{General framework}

The heavy flavor corrections to deep-inelastic structure functions emerge in the Wilson coefficients for the respective processes, i.e., they contribute in terms of virtual and final state effects. Heavy quarks have no strict partonic interpretation since partons are massless, and by virtue of this, infinitely long lived, with the possibility to move collinear to each other. Adopting this picture, heavy quarks can be singly or pair produced from massless partons and the gauge bosons of the Standard Model as final states. This description is called FFNS, which is the genuine scheme in any quantum-field theoretic calculation. The DIS structure functions $F_{i}\left(x, Q^{2}\right)$ obey the representation

$$
F_{i}\left(x, Q^{2}\right)=\left[\sum_{k=q_{l}, g}\left[C_{i, \text { light }}^{k}\left(x, Q^{2} / \mu^{2}\right)+C_{i, \text { heavy }}^{k}\left(x, Q^{2} / \mu^{2}, m_{h}^{2} / \mu^{2}\right)\right] \otimes f^{k}\left(\mu^{2}\right)\right](x),
$$

where $q_{l}$ and $g$ label the massless quarks and gluons, $f^{k}\left(\mu^{2}\right)$ are the PDFs, $C_{i \text {,ight(heavy) }}^{k}$ the massless (massive) Wilson coefficients, $h=c, b$ the charm and bottom quarks, and $\otimes$ denotes the Mellin convolution. Other approaches derive from this description. 
In case of unpolarized DIS the LO contributions were given in [96, 97, 98, 99] and the NLO corrections were calculated in semi-analytic form in [100, 101]. For asymptotic values $Q^{2} \gg m_{h}^{2}$ one may obtain the massive Wilson coefficients in analytic form. This is due to a factorization theorem [102] relating the massive Wilson coefficients $C_{i \text {,heavy }}^{k}$ to universal massive OMEs and the massless Wilson coefficients [77, 6, 103, 10. As comparisons up to NLO showed [102, these representations are valid for the structure function $F_{2}\left(x, Q^{2}\right)$ if $Q^{2} / m_{h}^{2} \gtrsim 10$. To $O\left(\alpha_{s}^{2}\right)$ the Wilson coefficients were obtained in [102, 104, 105] at general values of the Mellin variable $N$. A first contribution to the 3-loop corrections was given in [106] by the $O\left(\alpha_{s}^{2} \varepsilon\right)$ terms which contribute to the logarithmic terms $O\left(\ln ^{k}\left(Q^{2} / m_{h}^{2}\right)\right), k=$ $1,2,3$, in $O\left(\alpha_{s}^{3}\right)$. A large number of even Mellin-moments for all unpolarized 3-loop massive OMEs have been calculated in [107] up to $N=10 \ldots 14$ depending on the respective channel. For the structure function $F_{L}\left(x, Q^{2}\right)$ the asymptotic 3-loop corrections were given in [108] for general values of $N$. However, they are valid at $1 \%$ accuracy at much higher scales of $Q^{2} / m_{h}^{2} \gtrsim 800$ only. All logarithmic terms at $O\left(\alpha_{s}^{3}\right)$ for the heavy flavor Wilson coefficients contributing to the structure function $F_{2}\left(x, Q^{2}\right)$ are known [109, 110]. More than this, all the contributions to the constant terms emerging from lower order contributions by renormalization have been calculated, cf. [107] for details. Due to the size of the constant contributions phenomenological applications for the kinematic range available at HERA and the EIC cannot be based on only the logarithmic contributions. QCD corrections to charged current heavy flavor production have been considered in [111, 112, 113].

\subsubsection{FFNS and VFNS}

The logarithmic contributions in the heavy flavor Wilson coefficients $\propto \ln ^{k}\left(Q^{2} / m_{h}^{2}\right)$ never become large enough in the kinematic region of HERA or the EIC that their resummation would be required [114. Nonetheless one may introduce a description changing the number of light flavors effectively, which refers to the universal contributions to the heavy flavor Wilson coefficients, consisting of the twist-2 parton densities and the massive OMEs [115, 107, 116, 117]. This requires the knowledge of also the gluonic OMEs to 3-loop order [107.

By matching at typical scales $\mu_{f}$ one performs the transition from $n_{f}$ to $n_{f}+1$ massless flavors using the asymptotic relations. In this way one may introduce a heavy quark density. The corresponding representation, which is obtained in terms of a reformulation of the FFNS, is called zero mass variable flavor number scheme (ZMVFNS). It is unique up to the choice of the matching point(s). An important issue is the choice of the scale $\mu_{f}$, for which very often $\mu_{f} \simeq m_{h}$ is used. In Ref. [118] it was shown, however, comparing exact and flavor number matched calculations that this scale is process dependent and often very different scales have to be chosen. In this context various problems arise. Because of the value of the charm to bottom mass ratio, $m_{c}^{2} / m_{b}^{2} \sim 1 / 9$, power corrections due to $m_{c}^{2}$ usually cannot be neglected at scales $\mu^{2} \simeq m_{b}^{2}$. Therefore, sequential decoupling of both charm and bottom quarks is problematic. Furthermore, starting at $O\left(\alpha_{s}^{3}\right)$, Feynman diagrams with both bottom and charm quarks contribute, which cannot be attributed to either the charm or the bottom quark PDF [119]. The description of the FFNS, on the other hand, is still possible. Therefore, representations based on the ZMVFNS remain approximations to which one may refer for specific applications. Furthermore, it applies only for the asymptotic case $Q^{2} \gg m_{h}^{2}$

For the description of data one would like to have a smooth description of the structure 

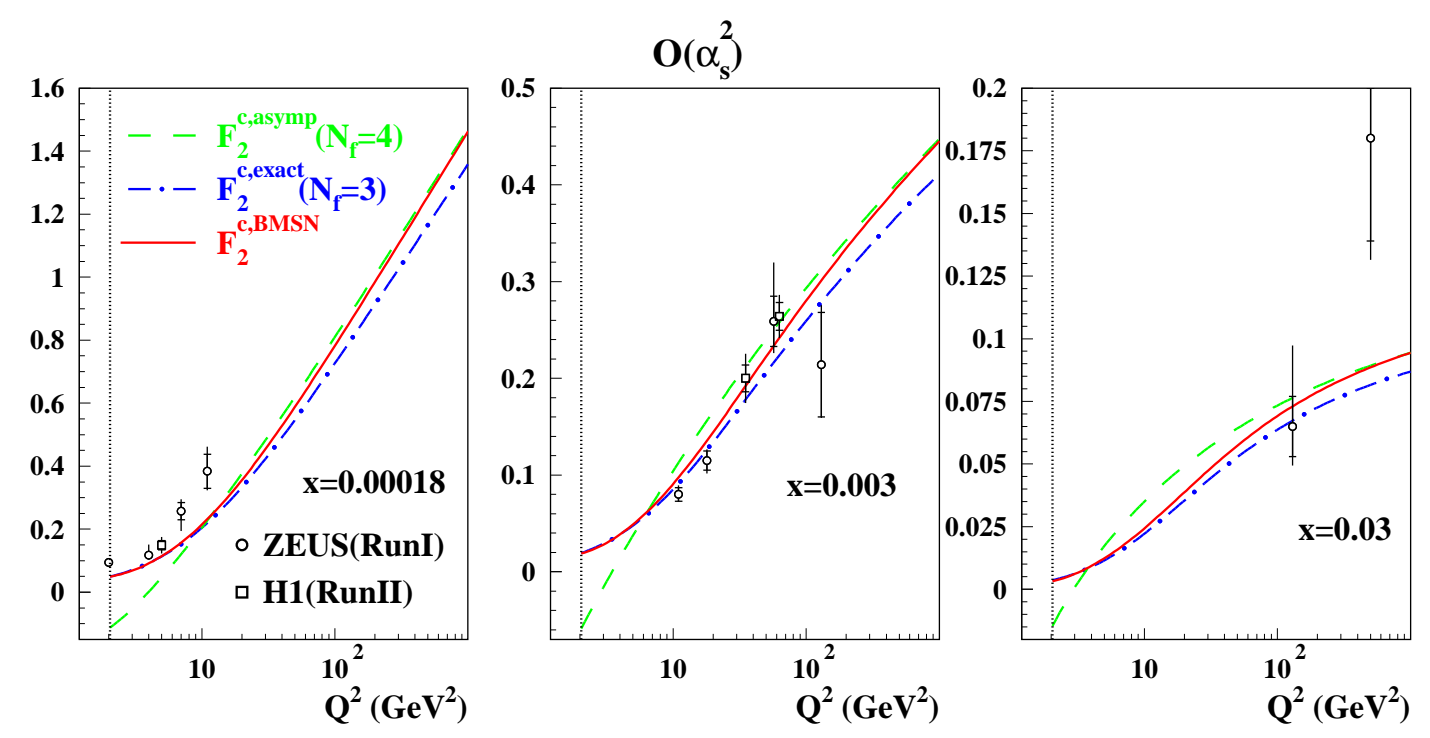

Figure 1.14. Comparison of $F_{2}^{c}$ computed in different schemes to H1 and ZEUS data: GMVFNS in the BMSN prescription (solid lines), 3-flavor scheme (dot-dashed lines), and 4-flavor scheme (dashed lines). The vertical dotted line denotes the position of $m_{c}=1.43 \mathrm{GeV}$. Taken from Ref. [19.

functions at both large and low values of $Q^{2}$, which is called the general mass variable flavor number scheme (GMVFNS). Here, a smooth interpolation is provided by the BMSN scheme [116, 19] given by

$$
F_{2}^{h, \text { BMSN }}\left(n_{f}+1\right)=F_{2}^{h, \text { exact }}\left(n_{f}\right)+F_{2}^{h, \text { ZMVFNS }}\left(n_{f}+1\right)-F_{2}^{h, \text { asymp }}\left(n_{f}\right),
$$

where exact corresponds to [100, 101], asymp to its asymptotic form for $Q^{2} \gg m_{h}^{2}$, and ZMVFNS to the value in the zero mass variable flavor scheme. In Fig. 1.14 the transition is shown for values of $x$ between 0.00018 and 0.03 for the kinematics at HERA according to (1.8) (see Ref. [120] for phenomenological variants of the GMVFNS).

\subsubsection{The massive NNLO corrections and the running mass}

The radiative corrections to the massive Wilson coefficients are known to be sizable. In particular, near the production threshold $s \simeq 4 m_{h}^{2}$, where large Sudakov double logarithms $\alpha_{s}^{k} \ln ^{2 k}\left(1-4 m_{h}^{2} / s\right)$ dominate at each order, one may wish to apply resummations; see Refs. [121, 122, 123] for details.

Another aspect at higher orders concerns the definition of the heavy quark mass, since it is a scheme dependent quantity. It is of particular interest to investigate which choice of scheme leads to the best convergence of the perturbative series. Upon conversion of the conventionally used on-shell (pole) mass for heavy quark DIS to the running mass $m_{h}(\mu)$ in the $\overline{\mathrm{MS}}$-scheme, one observes a considerable improvement of scale stability and convergence of the perturbative expansion. The latter aspect is demonstrated in Fig. 1.15. Here one uses the Wilson coefficients to NLO and refers to the approximate result valid in the threshold region [121, 122, 123, to give an estimate for the NNLO value, see Ref. [124. 

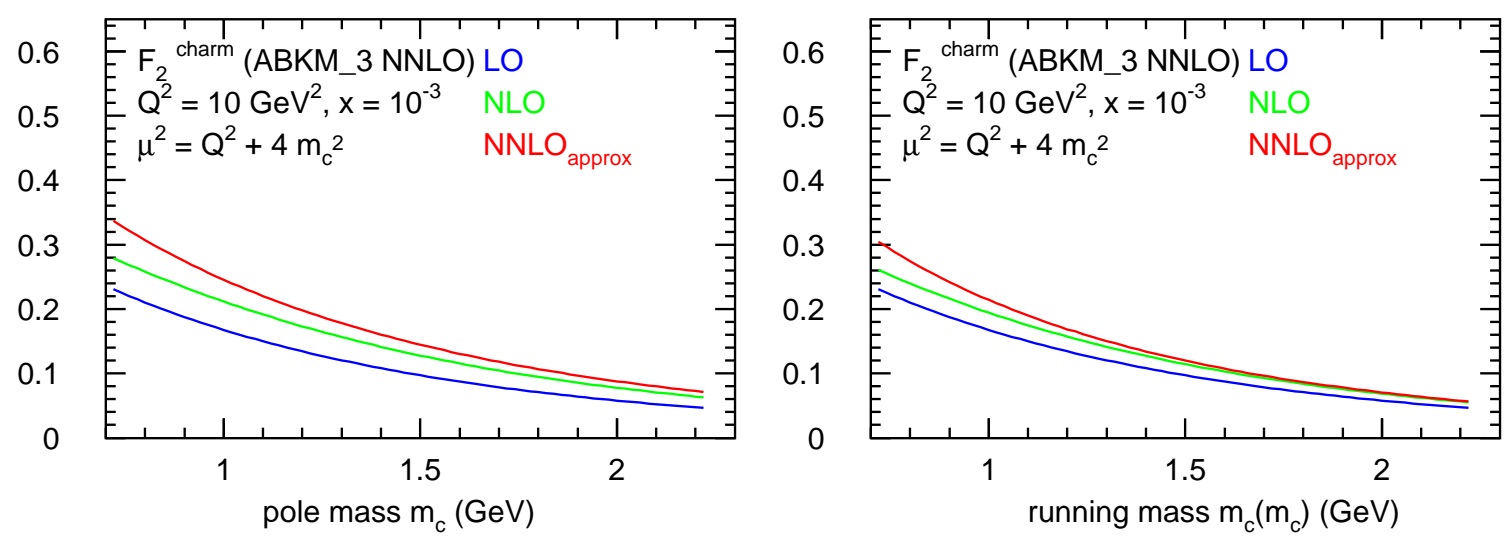

Figure 1.15. The mass dependence of $F_{2}^{c}$ for $Q^{2}=10 \mathrm{GeV}^{2}, x=10^{-3}$, and $\mu_{r}=\mu_{f}=\sqrt{Q^{2}+4 m_{c}^{2}}$ using the PDFs of [19]. $m_{c}$ is taken in the on-shell scheme (left) and in the $\overline{\mathrm{MS}}$ scheme (right) at LO (blue), NLO (green), and $\mathrm{NNLO}_{\text {approx }}$ (red); from Ref. [124].

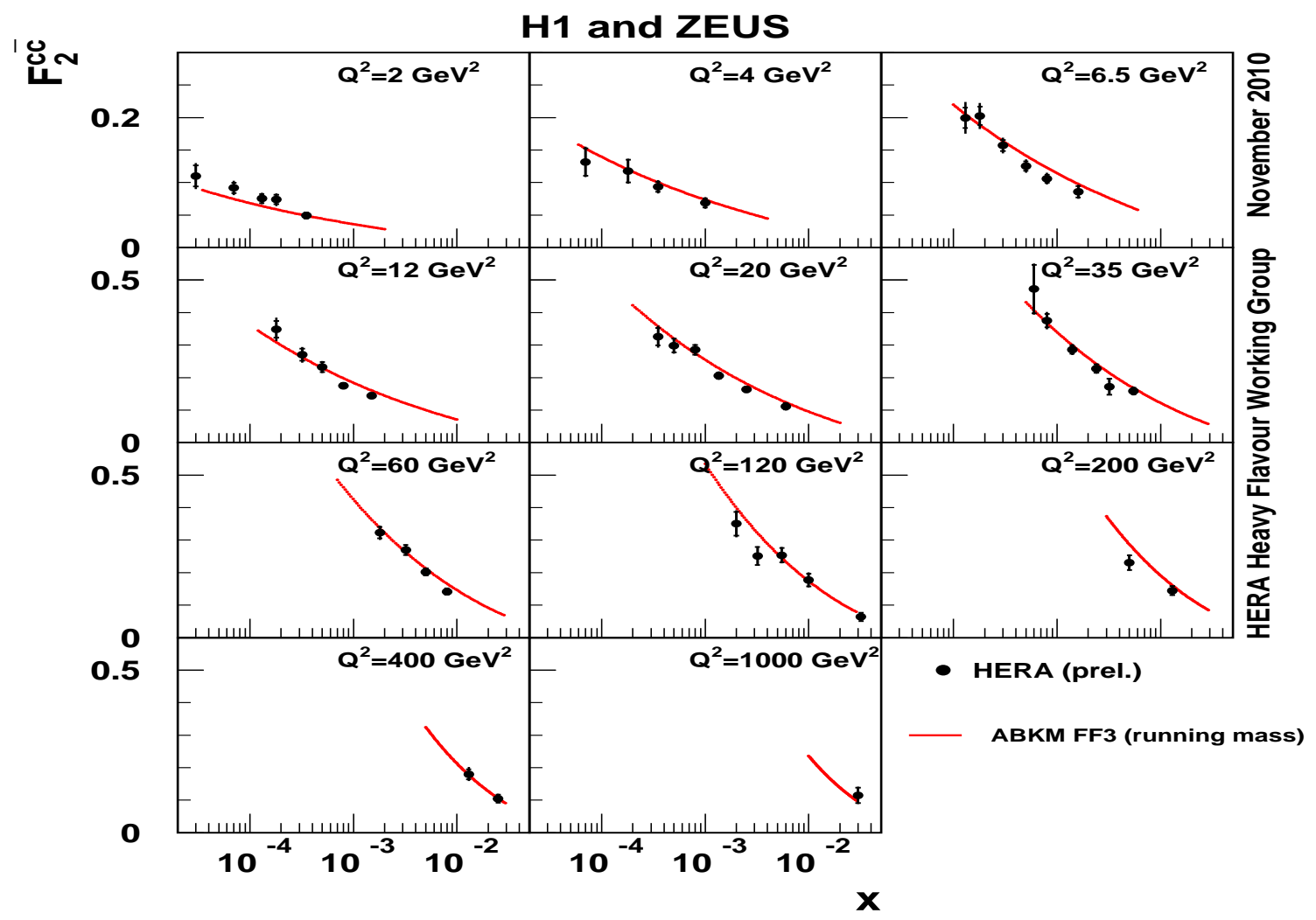

Figure 1.16. The combined HERA data on $F_{2}^{c}$ in comparison with the prediction of a fit [124] performed in the running-mass scheme [125]. 

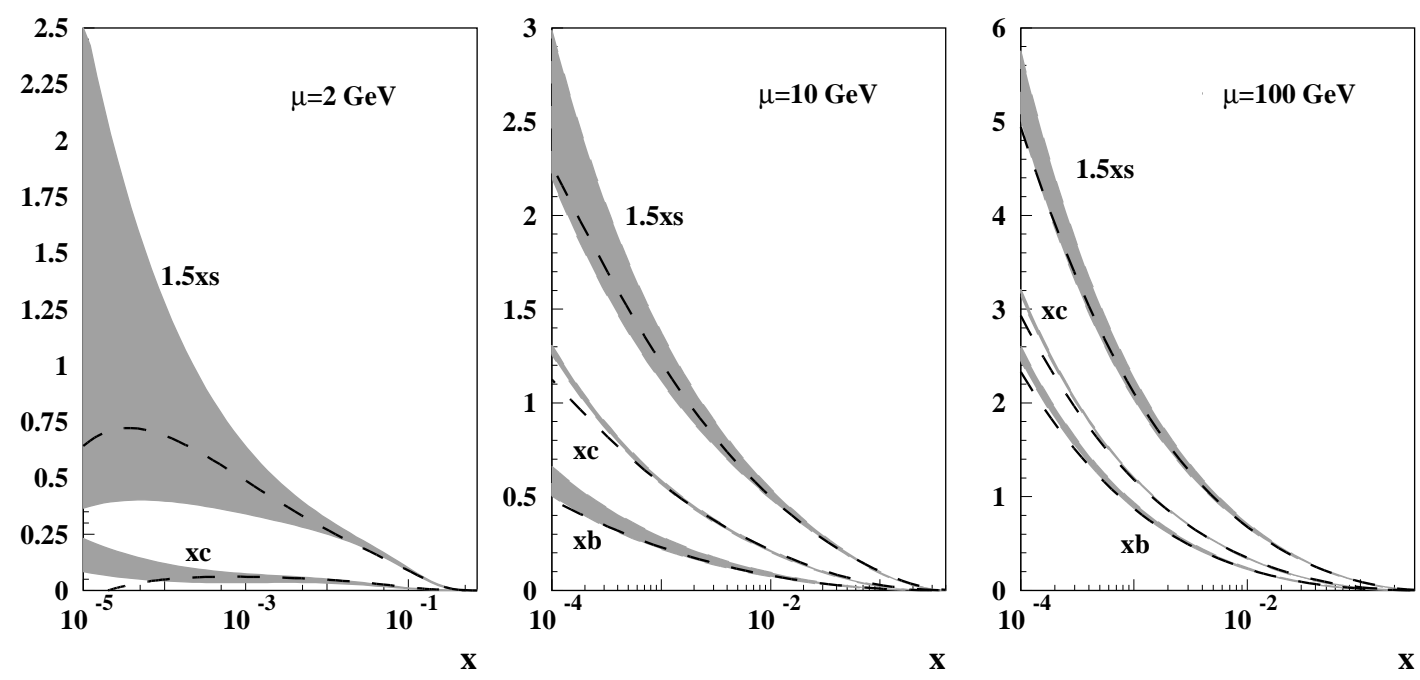

Figure 1.17. The 1- $\sigma$ error bands (shaded area) for our NNLO 4-flavor (left panel) and 5-flavor (central and right panels) $s, c$, and $b$ quark distributions in comparison to the corresponding MSTW2008 NNLO PDFs 22] (dashed lines); from Ref. [19].

The phenomenological impact of the mass scheme re-definition was checked for the ABKM fit of Ref. [19]. In a variant of this fit [124] the heavy-quark electro-production was considered in the running mass scheme and with the approximate NNLO corrections taken into account. $m_{c}$ was fitted to the DIS data simultaneously with the PDG world average [3] added to the fit as an additional constraint. In this way the value of $m_{c}\left(m_{c}\right)=$ $1.18 \pm 0.06 \mathrm{GeV}$ was obtained. The corresponding predictions for the semi-inclusive structure function $F_{2}^{c \bar{c}}$ are in good agreement with the preliminary HERA data in a wide kinematical region, cf. Fig. 1.16. This result gives an additional justification of the validity of the FFNS up to $Q^{2} \sim 1000 \mathrm{GeV}^{2}$, i.e., in the entire kinematic range relevant for an EIC.

\subsubsection{Heavy-flavor PDFs}

For applications at high-energy hadron colliders, schemes with 4- and 5-light flavors need to be considered. The necessary charm- and bottom PDFs are generated perturbatively. In Fig. 1.17 the results for the $s, c$, and $b$ quark flavors are shown at NNLO accuracy as determined in two global fits to the world data [19, 22. The 1- $\sigma$ error bands correspond to the analysis of [19]. The central values of the MSTW08 distributions turn out to lie below those found in the ABKM09 analysis for the $c$ and $b$ quark distributions in the whole kinematic range of HERA due to the smaller gluon density [22]. The strange quark distribution still exhibits large errors; see also Sec. 1.5. Measurements at the EIC are expected to considerably improve both the strange and charm quark densities thanks to the much higher luminosities than at HERA. 


\title{
$1.8 \quad F_{2, L}($ charm $)$ at an EIC
}

\author{
Elke C. Aschenauer, Marco Stratmann
}

Section 1.7 gave an outline of the theoretical status of heavy flavor contributions to DIS structure function and a comparison to HERA data. The mass $m_{h}$ of the heavy quark introduces extra theoretical complications including the need for a smooth prescription to cover both the threshold $\left(Q \simeq m_{h}\right)$ and the asymptotic $\left(Q \gg m_{h}\right)$ region, the scheme used for $m_{h}$ (on-shell or $\overline{\mathrm{MS}}$ ), and the actual value of $m_{h}$ used in the calculations.

Detailed experimental results from the EIC, in particular, for the so far unmeasured charm contribution to $F_{L}$, will help to refine the current theoretical understanding. In the entire kinematic domain of the EIC one expects the FFNS to be applicable for $F_{2}^{c}$; see Sec. 1.7. Differences between the exact, massive FFNS results, and the ZMVFNS are expected to be much more pronounced for $F_{L}^{c}$, see, e.g., Fig. 7 in [114], than for $F_{2}^{c}$ shown in Fig. 1.14,

The extraction of $F_{L}^{c}$ requires a Rosenbluth separation and should proceed along very similar lines as discussed already in Sec. 1.6. The extra experimental complication is the requirement to detect a charm quark in the final state. A quantitative feasibility study is still ongoing. We note that the detection of charmed mesons is important also for other physics topics. Therefore the design of the detector foresees to have particle identification for pions and kaons to fully reconstruct charmed mesons via their $K \pi$ decay channel. In addition, a micro-vertex detector is expected to provide a vertex resolution of $5 \mu \mathrm{m}$ to separate charmed mesons from B- and other mesons by measuring a displaced decay vertex. Using such techniques for a measurement of $F_{L}$ requires to detect a second decay lepton with a displaced vertex in addition to the scattered lepton. This, together with good lepton identification, should provide a high charmed meson detection efficiency. The required luminosities for a precise measurement of $F_{2, L}^{c}$ will scale with the achieved charm detection efficiency of the EIC detectors and the smaller reduced cross section for charm as compared to the fully inclusive $\sigma_{r}$ studied in Sec. 1.6. To illustrate the relative size of $F_{L}^{c}$ and $F_{2}^{c}$ we present in Fig. 1.18 some theoretical expectations at NLO accuracy based on the ABKM set of PDFs [19]; see Sec. 1.7] for details.

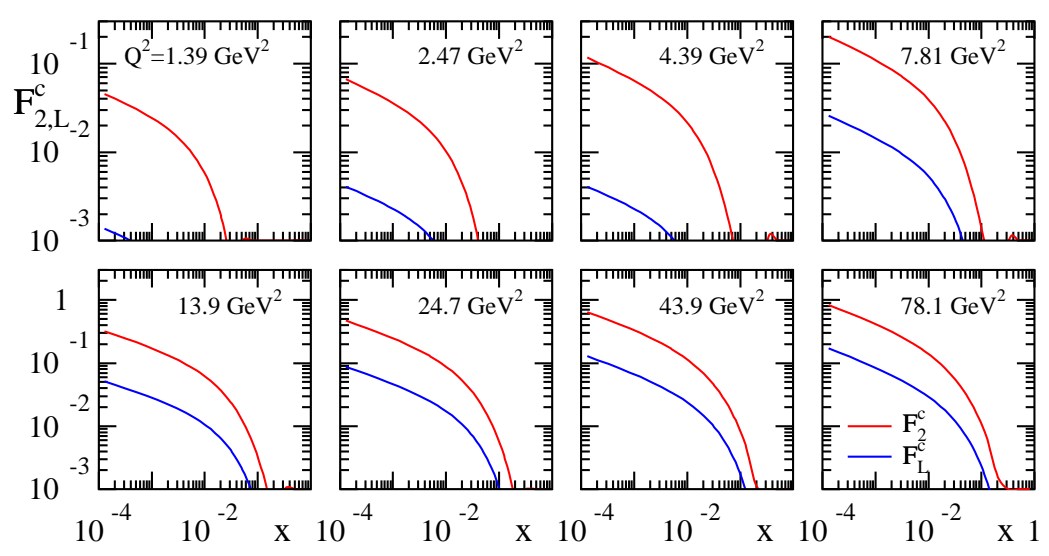

Figure 1.18. Expectations for $F_{2, L}^{c}\left(x, Q^{2}\right)$ in bins of $Q^{2}$ using the ABKM set of PDFs [19]. 


\title{
1.9 Probing intrinsic charm at the EIC
}

\author{
Marco Guzzi, Pavel Nadolsky, Fredrick Olness
}

In the variable flavor number (VFN) factorization scheme [126, 127, 68, heavy quark flavors are actively included in the PDF evolution via gluon splitting to a heavy quark pair $g \rightarrow Q \bar{Q}$. While the heavy quark PDF $f_{Q}(x, \mu)$ is often taken to vanish below the mass threshold $\left(\mu<m_{Q}\right)$, there is the possibility that the proton contains non-vanishing heavy quark constituents even for scales below $m_{Q}$; this component of the heavy quark PDF is identified as the intrinsic parton distribution [56, 57, 128, 129], in contrast to the extrinsic distribution generated by gluon splitting $g \rightarrow Q \bar{Q}$.

While we can introduce intrinsic parton distributions for both charm and bottom quarks, we will focus here on the intrinsic charm (IC). Operationally, the total charm PDF is then composed as $f_{c}(x, \mu)=f_{c}^{e x t}(x, \mu)+f_{c}^{\text {int }}(x, \mu)$. For the extrinsic component, we generally take the boundary condition $f_{c}^{e x t}(x, \mu)=0$ for $\mu<m_{c}$, i.e., we do not need to assume an initial functional form for $f_{c}^{e x t}$, as it is determined purely by the gluon evolution.

Conversely, for the IC component $f_{c}^{i n t}$ we do need to assume a functional form. Here, we consider two typical shapes of $f_{c}^{\text {int }}$ at the initial scale $\mu=m_{c}$, assuming $m_{c}=1.3 \mathrm{GeV}$.

- In the BHPS model [56, 57, 130], the intrinsic charm is concentrated at large $x$.

- In sea-like models [129], the intrinsic charm is spread over all $x$ values.

Sample distributions of IC PDFs were obtained in a global QCD fit of hadronic data [129]. We display them in Fig. 1.19. In these models, the momentum fraction carried by the charm can be varied in some range. Roughly, an intrinsic momentum fraction of $2 \%$ or $3 \%$ is at the outer limit of what is allowed in the context of a global fit.
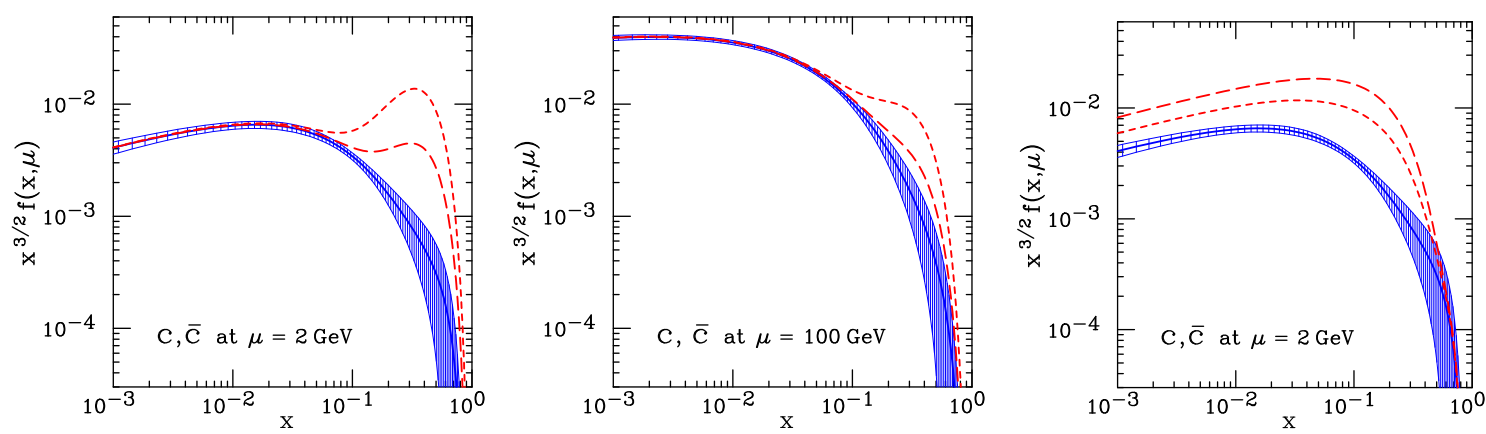

Figure 1.19. Left, middle: charm PDFs for the BHPS model, at $\mu=2$ and $100 \mathrm{GeV}$. The upper dashed curve is for a momentum fraction of $2 \%$, and the lower for $0.57 \%$. The shaded band is the CTEQ6.5 PDF uncertainty. Right: charm PDFs for the sea-like model. The upper curve is for a momentum fraction of $2.4 \%$, and the lower for $1.1 \%$. Figs. are taken from [129.

For heavy quark production in the threshold region $\left(\mu \sim m_{Q}\right)$, the magnitude of the intrinsic component will be large on the relative scale compared to the extrinsic contribution. At higher $\mu$ scales, the DGLAP evolution will increase the extrinsic component via $g \rightarrow Q \bar{Q}$ splitting. However, the distinctive shape of the BHPS distribution, with its characteristic large- $x$ enhancement, remains clearly evident even at much higher scales $\mu \gg m_{c}$.

We now consider two different c.m.s. energies for an EIC [131] and investigate the degree to which one can distinguish the IC component based on measurements of the charm 

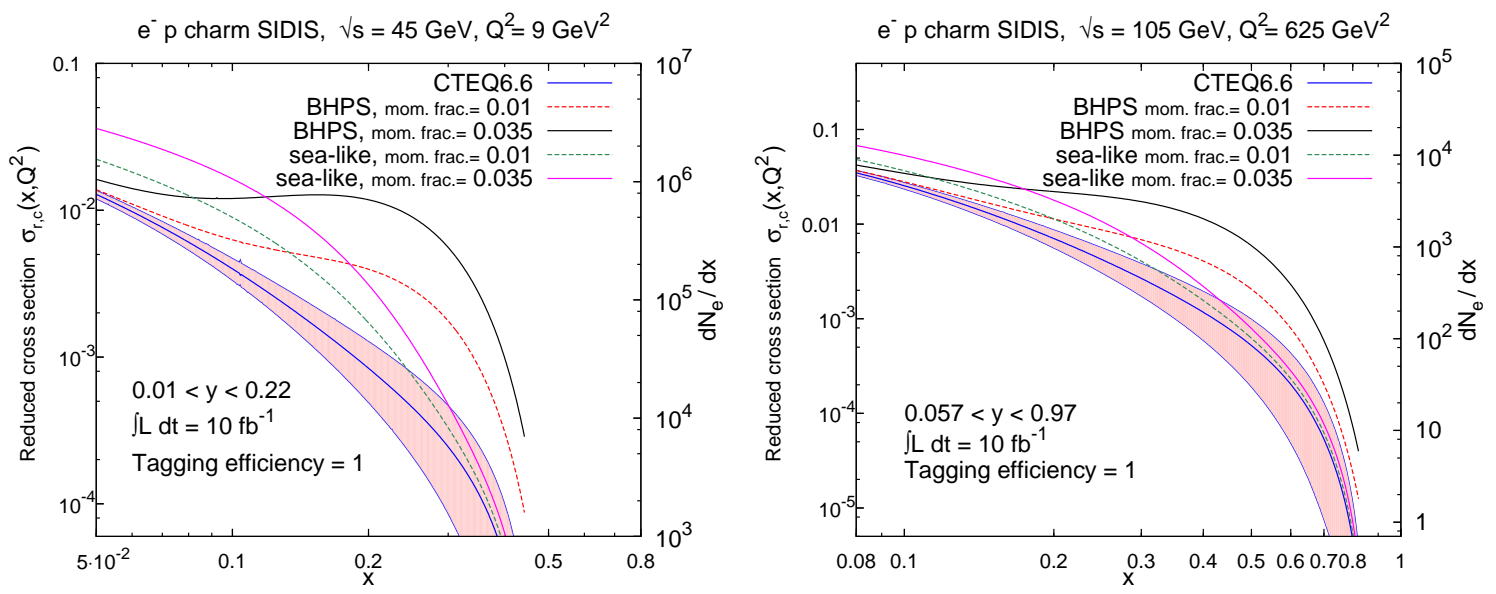

Figure 1.20. Charm contribution to the reduced NC $e^{-} p$ DIS cross section at $\sqrt{s}=45$ and $105 \mathrm{GeV}$. For each IC model, curves for charm momentum fractions of $1 \%$ and $3.5 \%$ are shown. For comparison we display the number of events $d N_{e} / d x$ for $10 \mathrm{fb}^{-1}$, assuming perfect charm tagging efficiency.

contribution to the DIS cross section. Alternatively, the IC can be searched for by measuring the longitudinal structure function $F_{L}$ or angular distributions [132]. In Fig. 1.20 we display the reduced cross section $\sigma_{r, c}$ for semi-inclusive DIS charm production at an EIC. The reduced charm cross section is defined as in Eq. (1.6). The probed ranges of $y$ are displayed in the figures.

The number of events for a conservative integrated luminosity $\mathcal{L}=10 \mathrm{fb}^{-1}$ has been computed as $d N_{e} / d x=\mathcal{L}\left\langle d \sigma_{c} / d x\right\rangle$ where $\left\langle d \sigma_{c} / d x\right\rangle$ is the average cross section in a $Q$ bin of size $0.15 \mathrm{GeV}$, evaluated at NLO accuracy. The shaded band represents the error on the cross section induced by the CTEQ6.6 PDF uncertainty [43].

For both BHPS and sea-like IC, we observe that the cross sections significantly exceed the nominal CTEQ6.6 values. While a momentum fraction of $3.5 \%$ is easily distinguished, even the intrinsic charm models with $1 \%$ can be resolved with moderate integrated luminosities.

\section{Acknowledgments}

We appreciate a discussion with J. Pumplin. 


\title{
1.10 Status of helicity-dependent PDFs and open questions to be addressed at an EIC
}

\author{
Rodolfo Sassot, Marco Stratmann
}

\subsubsection{Introduction}

Helicity-dependent or polarized PDFs (pPDFs) tell us precisely how much quarks and gluons with a given momentum fraction $x$ tend to have their spins aligned with the spin direction of a nucleon in a helicity eigenstate. Their knowledge is essential in the quest to answer one of the most basic and fundamental questions in hadronic physics, namely how the spin of a nucleon is composed of the spins and orbital angular momenta of its constituents.

The nucleon spin structure can be best understood in high-energy scattering experiments where quarks and gluons behave as almost free particles at scales $\mu \gg \Lambda_{Q C D}$. The relevance of pPDFs or research in spin physics in general is reflected in more than a dozen vigorous experimental programs in the wake of the unexpected finding that only very little of the proton spin is actually carried by its three valence quarks almost twenty-five years ago. The experiments have measured with increasing precision various observables sensitive to different combinations of quark and gluon polarizations in the nucleon. This progress was matched by advancements in corresponding theoretical higher order calculations in the framework of pQCD and phenomenological analyses of available data. Potentially large sea quark and/or gluon polarizations were initially thought to be ways to account for the "missing" proton spin, but at the same time, both turned out to be challenging to access experimentally.

The most comprehensive global fits include all available data taken in spin-dependent DIS, semi-inclusive DIS (SIDIS) with identified pions and kaons, and proton-proton collisions. They allow for extracting sets of pPDFs consistently at NLO accuracy along with estimates of their uncertainties [25, 26]. Contributions from the orbital angular momenta of quarks and gluons completely decouple from such type of experimental probes and need to be quantified by other means. Here, transverse momentum-dependent PDFs or generalized PDFs appear to be the most promising approaches which will be discussed elsewhere in Chapters 2 and 3, respectively.

Despite the impressive progress made in the past couple of years both experimentally and theoretically many fundamental questions related to the proton's helicity structure still remain unanswered and shall be summarized below; addressing them and providing answers is a prime target for an EIC.

Present fixed-target experiments suffer from their very limited kinematic coverage in $x$ and $Q^{2}$, which is insufficient to precisely study, for instance, QCD scaling violations for the polarized DIS structure function $g_{1}\left(x, Q^{2}\right)$ which in turn can be linked to the $x$ dependence of the polarized gluon density $\Delta g(x)$. There are numerous other opportunities for an EIC to further our understanding of the nucleon spin structure which will be listed below and discussed in some details in Secs. 1.11, 1.12, and 1.13.

\subsubsection{Current status of global pPDF fits - baseline for EIC projections}

Unlike unpolarized PDF fits, where a separation of different quark flavors is obtained from inclusive DIS data taken with neutrino beams, differences in polarized quark and 

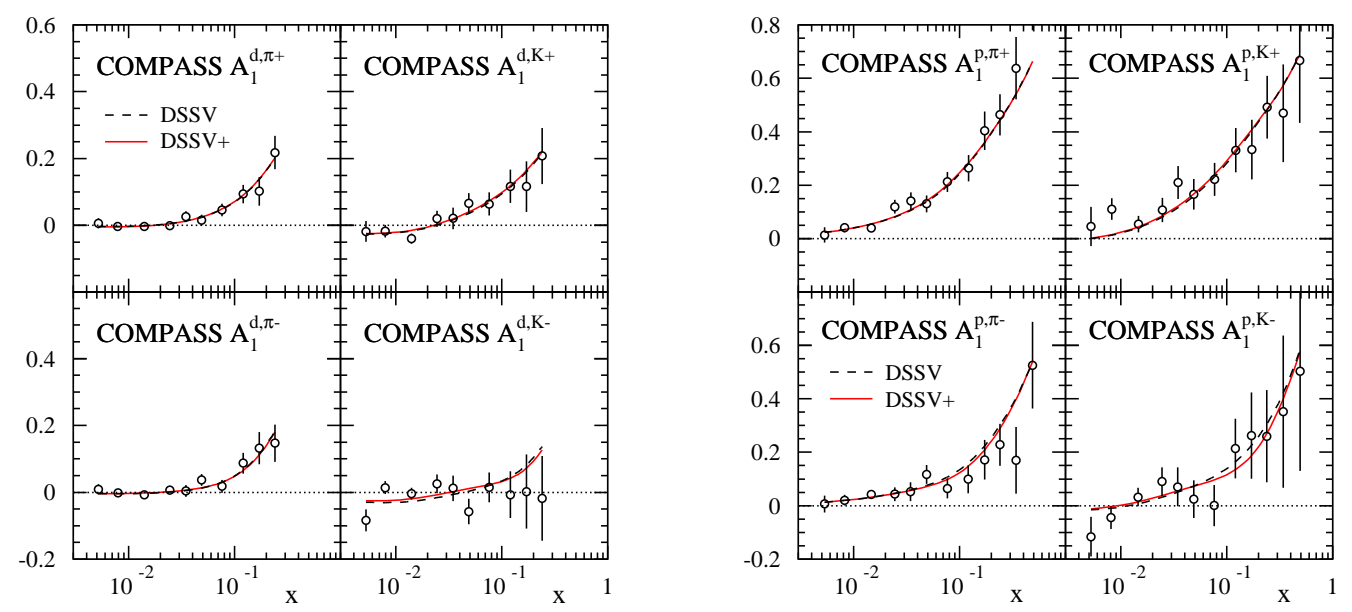

Figure 1.21. COMPASS results 133, 134 for SIDIS spin asymmetries on a deuteron (left) and proton target (right) compared to DSSV and DSSV+ fits (see text).

antiquark densities are at present determined exclusively from SIDIS data and hence require knowledge of fragmentation functions. Recently published SIDIS data from the COMPASS collaboration [133, 134] extend the coverage in $x$ down to about $x \simeq 5 \times 10^{-3}$, almost an order of magnitude lower than the kinematic reach of the HERMES data used in the DSSV global analysis of 2008 [25, 26]. For the first time, the new results comprise measurements of identified pions and kaons in the final state taken with a longitudinally polarized proton target. Clearly, these data can have a significant impact on fits of pPDFs and estimates of their uncertainties.

In particular, the COMPASS kaon data will serve as an important check of the validity of the strangeness density obtained in the DSSV analysis, which instead of favoring a negative polarization as in most fits based exclusively on DIS data, prefers a vanishing or perhaps even slightly positive $\Delta s$ in the measured range of $x$. One reason for concern is the dependence on fragmentation functions. Even though pion fragmentation functions are rather well constrained [74] by data, kaon fragmentation functions suffer from much larger uncertainties, and this could explain the unexpected result for $\Delta s$ obtained in the DSSV analysis.

Figure 1.21 shows a comparison between the new SIDIS spin asymmetries from COMPASS [133, 134] and the DSSV fit of 2008 [25, 26]. Also shown is the result of re-analysis at NLO accuracy based on the updated data set. This fit, henceforth called "DSSV+", will serve as baseline pPDFs when quantifying the potential impact of projected EIC data on our knowledge of the nucleon spin structure in Sec. 1.11. The differences between the original and the updated fit are hard to notice for both identified pions and kaons. In terms of $\chi^{2}$ values, the original DSSV analysis amounts to 392 units for the original set of 467 data points used in the fit [74. Adding both deuteron and proton data from COMPASS (88 points) it goes up to 456 and drops by about 4 units upon refitting (DSSV+), which is not really a significant improvement for a PDF analysis in view of non-Gaussian theoretical uncertainties. Recall that in the DSSV analysis a $\Delta \chi^{2} \simeq 9$ (corresponding to $\Delta \chi^{2} / \chi^{2}=2 \%$ ) was tolerated as a faithful, albeit conservative estimate of PDF uncertainties.

In Fig. 1.22 we compare the individual sea quark densities obtained in the original and 


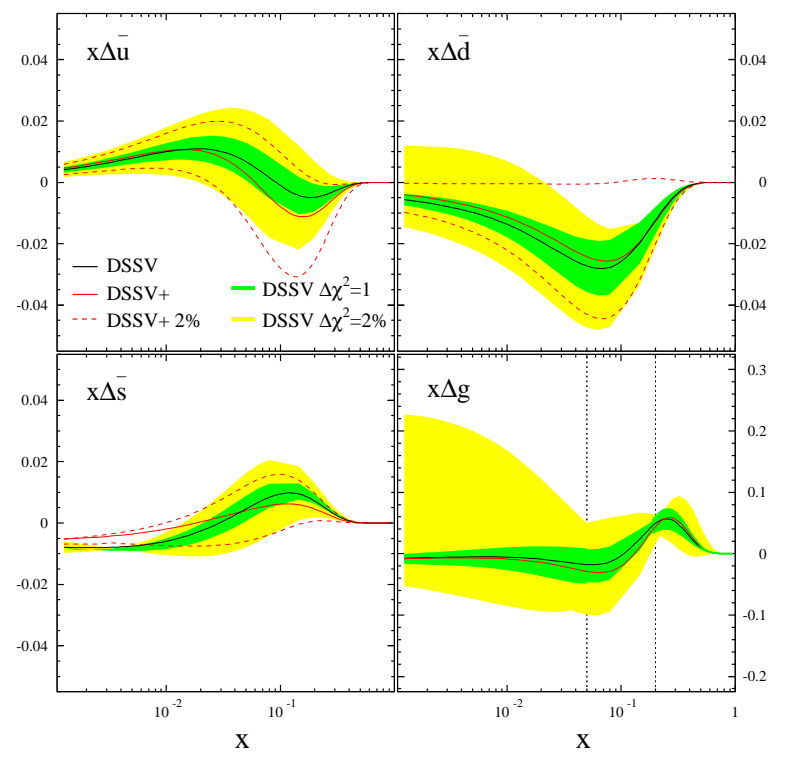

Figure 1.22. DSSV and DSSV+ sea quark pPDFs and uncertainty bands at $Q^{2}=10 \mathrm{GeV}^{2}$. Also shown is $\Delta g$. The vertical lines indicate the $x$ region constrained by RHIC $p p$ data.

updated DSSV analyses. As can be seen, except for $\Delta s$, the new central fits fall well within the $\Delta \chi^{2}=1$ uncertainty bands of DSSV. The gluon distribution is hardly affected by the new SIDIS data. For DSSV+ we only give the new uncertainty bands (dashed lines) referring to the $\Delta \chi^{2} / \chi^{2}=2 \%$ tolerance criterion.

Although it may seem that the new SIDIS data have little impact on the fit, this is not the case if one studies individual $\chi^{2}$ profiles in more detail. Figure 1.23 shows the contributions to $\Delta \chi^{2}$ from various data sets against variations of the truncated first moments for $\Delta \bar{u}$ and $\Delta \bar{d}$ in the range $0.001 \leq x \leq 1$. Compared to the original DSSV fit one notices a trend towards smaller net polarization as the best fit values shift towards zero. This is induced by the new COMPASS SIDIS data. Both pions and kaons pull in the same direction and to a common smaller best fit value. There is, however, some mild tension with older SIDIS sets, but this is well within the tolerance of the fit and most likely caused by the different $x$ ranges covered by the different data sets. In addition, one finds a significant reduction in the uncertainties, as determined by the width of the $\chi^{2}$ profiles at a given $\Delta \chi^{2}$.

A much debated feature of the strangeness pPDF obtained in the DSSV fit is its unexpected small value at medium-to-large $x$ which, when combined with a node at intermediate $x$, still allows for acquiring a significant negative first moment at small $x$, in accordance with expectations from $\mathrm{SU}(3)$ symmetry (hyperon decay constants $F$ and $D$ ) and fits to DIS data only (see, e.g., Ref. [23]). To investigate the possibility of a node in $\Delta s(x)$ further we present in Fig. 1.24 the $\chi^{2}$ profiles for two different intervals in $x$ : $0.001 \leq x \leq 0.02$ and $0.02 \leq x \leq 1$. Again, the new COMPASS SIDIS data have quite some impact on the profiles but the central value for the combined range, $0.001 \leq x \leq 1$, does not shift from its original DSSV value.

The profiles in Fig. 1.24 clearly show that for $0.001 \leq x \leq 0.02$ the result for $\Delta s$ is a compromise between DIS and SIDIS data, the latter favoring much less negative values. 


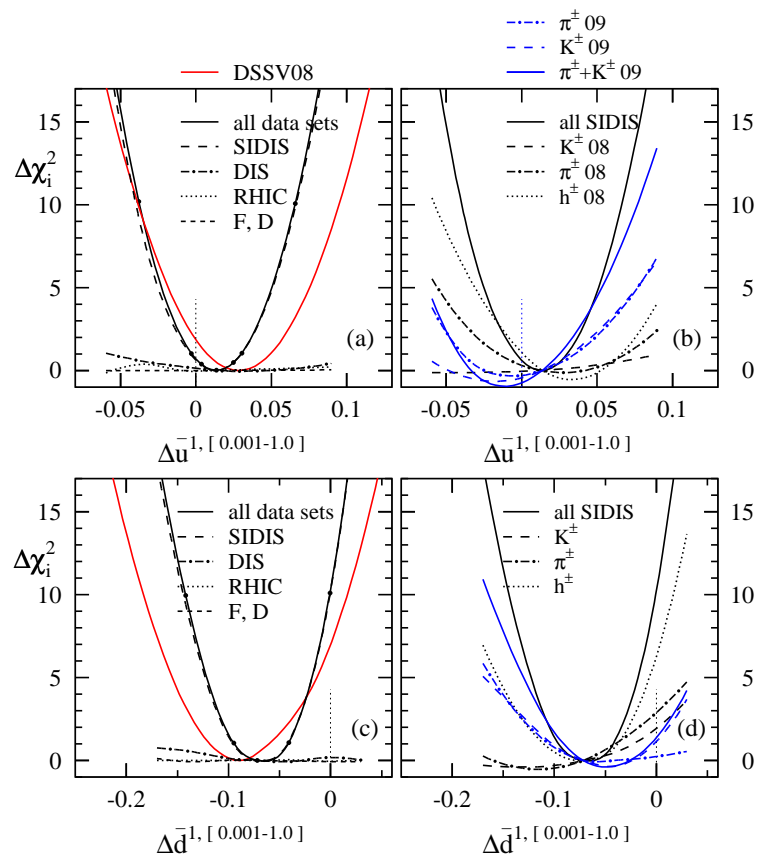

Figure 1.23. $\chi^{2}$ profiles for the first moments of $\Delta \bar{u}$ and $\Delta \bar{d}$ truncated to $0.001 \leq x \leq 1$.
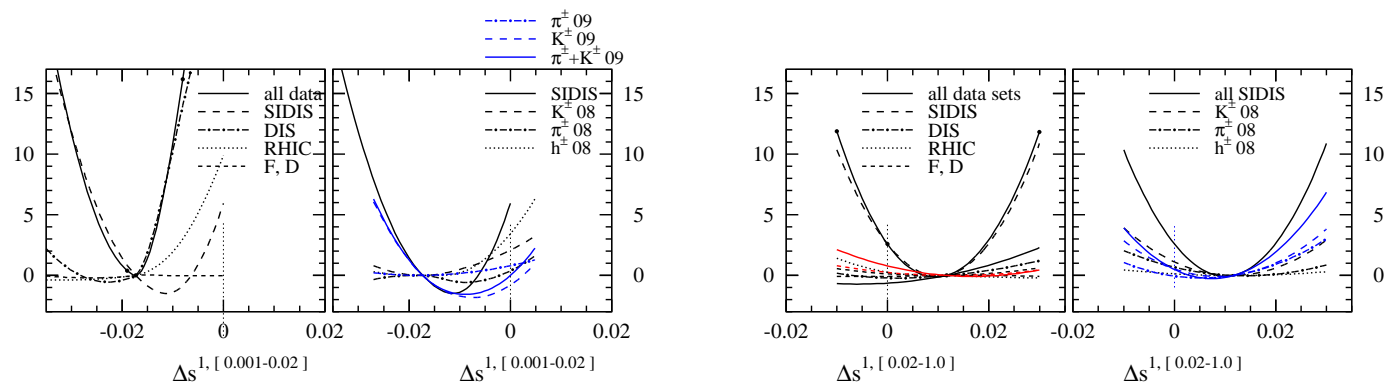

Figure 1.24. $\chi^{2}$ profiles for the truncated first moment of $\Delta s$ in two different $x$ intervals.

For $0.02 \leq x \leq 1$ everything is determined by SIDIS data and all sets consistently ask for a small, slightly positive strange quark polarization. There is no hint of a tension with DIS data as they do not provide a useful constraint at medium-to-large $x$. We note that at low $x$, most SIDIS sets give indifferent results except the new COMPASS data which extend towards the smallest $x$ values so far and actually do show some preference for a slightly negative value for $\Delta s$. This exemplifies the need for measurements at small $x$. Clearly, all current extractions of $\Delta s$ from SIDIS data show a significant dependence on kaon FFs, see, e.g., Ref. [133, 134]. Better determinations of $D^{K}(z)$ are highly desirable, but should be possible with forthcoming data from $B$-factories, DIS multiplicities, and LHC data. We also notice that in the range $x \gtrsim 0.001$ the hyperon decay constants, the so-called $F$ and $D$ values, do not play a significant role in constraining $\Delta s$ as can be deduced from their relative contribution to $\Delta \chi^{2}$ in Fig. 1.24. Computations of SU(3) breaking effects in axial current matrix elements [135, 136], and, more recently, also first lattice results for the first moment of $\Delta s+\Delta \bar{s}$ [137] point towards a sizable breaking of $\mathrm{SU}(3)$ symmetry. To study its validity of one needs to probe $\Delta s(x)$ at smaller values of $x$ at an EIC. 
An interesting recent development is that the LSS group produced an update of their pPDF fit using for the first time DIS and SIDIS data simultaneously [28]. As in the DSSV analysis they also utilize DSS fragmentation functions [74]. Their functional form is also very similar to the one used in DSSV and DSSV+. As in their previous analyses they carefully include target mass corrections and phenomenological higher twist corrections for inclusive DIS data. Nevertheless, their obtained pPDFs are very similar to the best fit of DSSV shown in Fig. 1.22. Their strange quark polarization also changes sign as in DSSV but is overall slightly smaller in magnitude. LSS finds non negligible higher twist corrections to inclusive DIS data, however, these conclusions are not fully shared by another recent analysis of polarized DIS data [23. Ref. [23] also provides an extraction of $\alpha_{s}$ from polarized DIS data. There are also interesting first attempts to perform a pPDF analysis based on neural networks [138, 139] similar to successful global fits of unpolarized data [47]. This would provide independent estimates of pPDF uncertainties not biased by the choice of a particular functional form.

\subsubsection{Open Questions}

The status of pPDFs outlined above will likely not change much until the time of EIC operations. Most of the remaining, compelling open questions in spin physics related to pPDFs will be still with us and can be only addressed by extending the kinematic coverage to smaller values of $x$; see the items listed below.

Existing experiments, like PHENIX and STAR at RHIC, will continue to add data in the next couple of years. Parity-violating, single-spin asymmetries for $W$ boson production should reach a level where they help to constrain $\Delta u, \Delta \bar{u}, \Delta d$, and $\Delta \bar{d}$ at large $x$, $0.07 \leq x \leq 0.4$ at scales $Q \simeq M_{W}$ much larger than typically probed in SIDIS [140]. Measurements of double spin asymmetries for di-jets in $p p$ collisions at $500 \mathrm{GeV}$ should improve the current constraints on $\Delta g(x)$ and extend them towards somewhat smaller values of $x$. The strangeness polarization is, however, very hard to access in polarized $p p$ collisions. In the future, JLab12 will add very precise DIS data at large $x$. They will allow us to challenge ideas like helicity retention [141, 142] which predict that $\Delta f(x) / f(x) \rightarrow 1$ as $x \rightarrow 1$. Currently, only $\Delta u / u$ exhibits this trend, while $\Delta d / d$ remains negative up to $x \simeq 0.6$.

We expect an EIC to make significant contributions on the following topics:

Polarized gluon density $\Delta \mathbf{g}(\mathbf{x})$ : precise data for the DIS structure function $F_{2}$ in a broad kinematic range in $x$ and $Q^{2}$ from HERA provide the world's best and theoretically cleanest constraint on the unpolarized gluon density; see Sec. 1.3. One of the most important results of HERA was to establish the strong rise of the gluon density at small $x$ which could not be anticipated from previous fixed-target results.

Figure 1.25 summarizes the current situation for polarized DIS. The kinematic coverage is limited to the fixed-target regime. There are no data below $x \simeq 0.005$, and the lever-arm in $Q^{2}$ is very limited, in particular, for the smallest $x$ values. As a consequence, $\Delta g(x)$ is basically unconstrained at small $x$ as is reflected in the large uncertainty band shown in Fig. 1.22. There are theoretical arguments that $\Delta g(x) \simeq x g(x)$ at small $x$ [141] but they cannot be verified experimentally due to the lack of data

The fact that current RHIC data favor a very small gluon density in $0.05 \lesssim x \lesssim 0.2$ [25], perhaps with a node, also greatly complicates the determination of the first moment, $\int_{0}^{1} \Delta g\left(x, Q^{2}\right) d x$, which enters in the fundamental proton spin sum rule in its light-cone gauge formulation [143, 144]. Since contributions to the moment largely cancel in the measured $x$ range, the unmeasured small $x$ region may contribute significantly even up to one unit of 


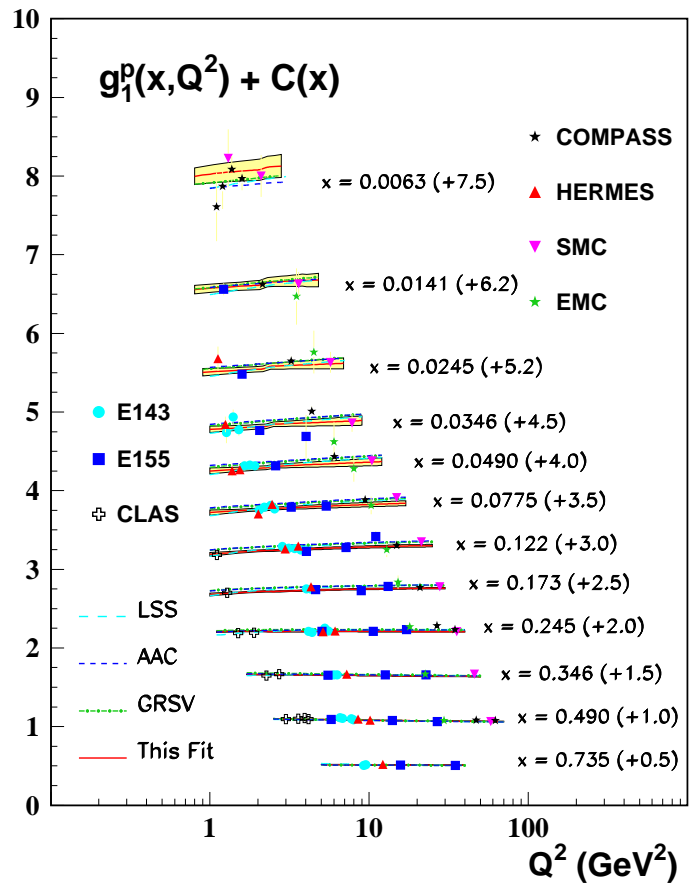

Figure 1.25. Scaling violations for the structure function $x g_{1}^{p}$ in bins of $x$. Experimental data are compared to various fits at NLO accuracy. Figure taken from [23].

$\hbar$.

Precise measurements of the structure function $g_{1}\left(x, Q^{2}\right)$ in a wide kinematic range will be a flagship measurement for an EIC. The polarized gluon density is strongly correlated with QCD scaling violations, $d g_{1}\left(x, Q^{2}\right) / d \ln Q^{2} \simeq-\Delta g\left(x, Q^{2}\right)$, i.e., a large positive $\Delta g$ at small $x$ is expected to drive $g_{1}$ towards large negative values for $x \simeq 10^{-(3 \div 4)}$. A precise DIS measurement will also constrain the quark singlet density $\Delta \Sigma\left(x, Q^{2}\right)$ and its first moment, i.e., the total quark spin contribution to the proton spin, much better.

Complete flavor separation: given the significant impact present SIDIS data already have in global analyses of pPDFs, it is easy to imagine that an EIC with its extended kinematic coverage can turn SIDIS measurements into a precision tool for detailed studies of $\Delta u, \Delta \bar{u}, \Delta d, \Delta \bar{d}, \Delta s$, and $\Delta \bar{s}$. For instance, a precise determination of a possible asymmetry in the light quark sea, $\Delta \bar{u}(x)-\Delta \bar{d}(x)$ will challenge expectations from model calculations. Again, current QCD fits have revealed rather complicated functional forms with possible nodes for the quark densities which need to be studied more precisely.

Prerequisites are a detector with excellent particle ID in an as large as possible portion of phase space and an improved theoretical knowledge of FFs, in particular, for kaons. For the latter, significant progress will be made by the time the EIC turns on. In any case, there will be also plenty of opportunities to further constrain them at an EIC if necessary.

Novel electroweak probes in DIS: At large enough $Q^{2}$ and with the envisioned luminosities of up to $10^{34} \mathrm{~cm}^{-2} \mathrm{~s}^{-1}$ an EIC has the unique opportunity to access polarized electroweak structure functions via charged and neutral current DIS measurements. These novel probes depend on various combinations of polarized quark PDFs and provide an alternative way of separating different quark flavors for $x \gtrsim 10^{-2}$. Prerequisites are both electron and positron beams to fully exploit charged current (CC) DIS, i.e., the pPDF combinations probed in the exchange of $W^{-}$and $W^{+}$bosons. Also, one needs to be able 
to reconstruct $x$ and $Q^{2}$ from the final state hadrons in the absence of a scattered lepton in CC DIS.

Strangeness polarization, $\Delta \mathbf{s}-\Delta \overline{\mathbf{s}}$, and SU(3) symmetry: As mentioned already, the surprisingly small strangeness density determined from SIDIS data has triggered a lot of discussions recently. It is certainly of outmost importance to precisely map $\Delta s(x)$ and $\Delta \bar{s}(x)$ down to sufficiently small values of $x$ to reliably determine their first moments. If $\mathrm{SU}(3)$ symmetry is approximately valid, one expects a significantly negative first moment for strangeness; if, on the other hand, SU(3) symmetry is badly broken at a $20 \div 30 \%$ level, $\Delta s(x)$ can remain small and perhaps even slightly positive down to small $x$. Ideas have been put forward that $\Delta s(x)$ and $\Delta \bar{s}(x)$ may have opposite polarizations which could explain the smallness of $\Delta s+\Delta \bar{s}$ in DIS but would result in a potentially sizable $\Delta s-\Delta \bar{s}$.

At an EIC there are different strategies to determine $\Delta s$ and $\Delta \bar{s}$. The most promising one is through SIDIS production of charged kaons. Once $K^{+}$and $K^{-}$yields are known with high precision and uncertainties for kaon FFs are well understood one can attempt an extraction of $\Delta s(x)$ and $\Delta \bar{s}(x)$ in a large range of $x$. Alternatively, one can study charm production in CC DIS with a polarized proton target. If one has electron and positron beams available, the yields of $D$ and $\bar{D}$ mesons should be related to $\Delta s(x)$ and $\Delta \bar{s}(x)$, respectively.

Heavy flavor contributions to $\mathrm{g}_{1}$ : for presently available data, any contribution from heavy quarks, i.e., charm and bottom, can be safely ignored. From HERA we know, however, that at sufficiently small values of $x$ and large enough $Q^{2}$, charm quarks can contribute as much as $20 \div 25 \%$ to a measurement of $F_{2}$. It is important to determine the charm contribution to $g_{1}$ at small $x$ experimentally and to properly include it in future global analyses. Since $g_{1}^{c}$ is mainly driven by photon-gluon-fusion it can be also a viable probe of $\Delta g$ in the small $x$ region.

Bjorken sum rule: the Bjorken sum rule is certainly one of the best known quantities in perturbative QCD. Corrections up to $\mathcal{O}\left(\alpha_{s}^{4}\right)$ have been calculated [32]. There is also a nontrivial connection to Adler's $D\left(Q^{2}\right)$ function defined in $e^{+} e^{-}$annihilation through the generalized Crewther relation [145, 32] involving the QCD $\beta$ function which incorporates the deviation from the limit of exact conformal invariance. It is certainly important and legitimate to ask to what level of precision an EIC can verify this fundamental sum rule.

Since the Bjorken sum rule relates the moments of the $g_{1}$ structure functions for protons and neutrons, it first of all requires an "effective neutron target" such as Helium-3. Perhaps the biggest challenge is then to develop a polarimeter to control its polarization with high accuracy. Most likely this will be the limited factor for a measurement of the Bjorken sum.

In addition, the sum rule involves the first moments of $g_{1}$, i.e., one has to worry about possible extrapolation uncertainties for $x \rightarrow 0$. However, since the Bjorken sum is a nonsinglet quantity, contributions from the small $x$ region should be under control up to a $1 \div 2 \%$ once a measurement down to $x \simeq 10^{-4}$ can be performed. At this level of accuracy one may also expect contributions to matter which break isospin symmetry. 


\title{
1.11 Opportunities in spin physics at an EIC
}

\author{
Elke C. Aschenauer, Rodolfo Sassot, Marco Stratmann
}

Here, we demonstrate how an EIC can address the fundamental open questions concerning the proton's helicity structure raised in the previous Section. A detailed, quantitative discussion of novel electroweak effects in polarized DIS can be found in Secs. 1.12 and 1.13

\subsubsection{Scaling violations in inclusive DIS and their impact on $\Delta \mathrm{g}(\mathrm{x})$}

A precise determination of the polarized gluon distribution $\Delta g\left(x, Q^{2}\right)$ in a broad kinematic regime is a primary goal for the EIC. Current determinations of $\Delta g$ suffer from both a limited $x$ coverage and fairly large theoretical scale ambiguities in polarized $p p$ collisions for inclusive (di)jet [146, 147] and pion production [148, 149]. Several channels are sensitive to $\Delta g$ in $e p$ scattering at collider energies such as DIS jet [150, 151] or charm [152, 153, 154] production but QCD scaling violations in inclusive polarized DIS have been identified as the golden measurement.

The inclusive structure function $g_{1}\left(x, Q^{2}\right)$ is the most straightforward probe in spin physics and has been determined in various fixed-target experiments at medium-to-large values of $x$ in the last two decades. It is also the best understood quantity from a theoretical point of view. Unlike for most other processes, full NNLO corrections of the relevant hard scattering coefficient functions are available [15], and partial results for the polarized splitting functions at NNLO have been reported in [14] recently. A consistent framework up to NNLO accuracy will be in place by the time of first EIC operations and is required in order to limit the size of residual theoretical scale uncertainties to the anticipated unprecedented level of precision for a polarized DIS experiment. To achieve the latter, systematic uncertainties need to be controlled extremely well which imposes stringent requirements on the detector performance, acceptance, and the design of the interaction region. Necessary, on-going studies comprise the detection of scattered electrons down to small momenta of $\mathcal{O}(0.5 \mathrm{GeV})$ to access small $x$, the required resolution in momentum and angle of the scattered lepton, and the unfolding of QED radiative corrections, see Sec. 7.3 .

For studying scaling violations $d g_{1}\left(x, Q^{2}\right) / d \log Q^{2}$ efficiently, it is not only essential to have good precision but also to cover the largest possible range in $Q^{2}$ for any given fixed value of $x$. The accessible range in $Q^{2}$ is again linked (via the inelasticity $y$ ) to the capabilities of detecting electrons in an as wide as possible range of momenta and scattering angles. For a detailed discussion of the kinematic coverage at the EIC see Sec. 7.3 .

Figure 1.26 highlights the main motivation for a measurement of $g_{1}$ at the EIC. The significant uncertainty in $\Delta g\left(x, Q^{2}\right)$ at $x \lesssim 0.01$ shown in Fig. 1.22 translates into a large spread of predictions for the behavior of $g_{1}$ at small $x$. The spin-dependent scale evolution is such that $d g_{1}\left(x, Q^{2}\right) / d \log Q^{2}$ at low $x$ is strongly correlated with the negative of $\Delta g\left(x, Q^{2}\right)$, i.e., a positive gluon distribution drives $g_{1}$ at small $x$ to more and more negative values as $Q^{2}$ increases, and vice versa. Hence, a precision measurement of $g_{1}$ and its logarithmic scale dependence will determine $\Delta g\left(x, Q^{2}\right)$ at small $x$, hereby dramatically reducing the extrapolation uncertainties of the integral $\int_{0}^{1} \Delta g\left(x, Q^{2}\right) d x$ entering the proton spin sum rule. Depending on the shape of $\Delta g\left(x, Q^{2}\right)$ in the unmeasured region, it is currently still possible to accommodate up to one unit of $\pm \hbar$ at small $x$ [25, 26], i.e., twice the proton spin! Having determined the functional form of $\Delta g\left(x, Q^{2}\right)$ down to about $10^{-4}$, even extreme extrapolations to $x \rightarrow 0$ are not expected to contribute anymore significantly to the integral 


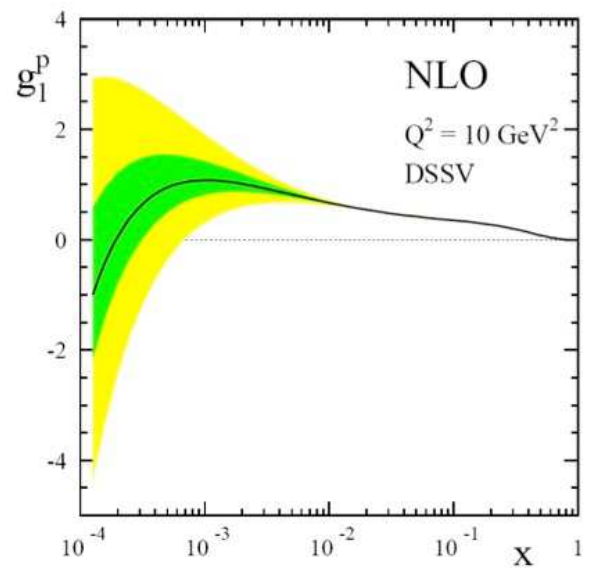

Figure 1.26. Spread of predictions for $g_{1}(x)$ induced by the current uncertainty in $\Delta g(x)$.

$\int_{0}^{1} \Delta g\left(x, Q^{2}\right) d x$

To quantify the impact of polarized DIS measurements on our knowledge of the gluon density we have performed a series of global QCD analyses based on realistic pseudo-data for various c.m.s. energies at a first stage of eRHIC: $5 \mathrm{GeV}$ electrons on 50, 100, 250, and 325 $\mathrm{GeV}$ protons. The simulations are based on the PEPSI Monte Carlo code [155] using the GRSV "std" set of polarised PDFs 24]. The statistical precision of the data sets for 100-325 $\mathrm{GeV}$ protons corresponds to about two months of running at the anticipated luminosities for eRHIC with an assumed operations efficiency of $50 \%$. For $5 \times 50 \mathrm{GeV}$ an integrated luminosity of $5 \mathrm{fb}^{-1}$ was assumed. Demanding a minimum $Q^{2}$ of $1 \mathrm{GeV}^{2}, W^{2}>10 \mathrm{GeV}^{2}$, the depolarization factor of the virtual photon to be $D(y)>0.1$, and $0.1 \leq y \leq 0.95$, the highest $\sqrt{s} \simeq 70 \div 80 \mathrm{GeV}$ allows one to access $x$ values down to about $2 \times 10^{-4}$. As can be seen from the kinematic plots in Sec. 7.3, the lever-arm in $Q^{2}$ more and more diminishes if smaller $x$ values are probed. For instance, choosing $Q_{\min }^{2}=2 \mathrm{GeV}^{2}$ would limit the $x$ range to $x \gtrsim 4 \times 10^{-4}$ at the first stage of eRHIC. Clearly, one wants to utilize $Q^{2}$ values as low as possible in a QCD analysis but once actual EIC data become available one needs to systematically study how far down $Q_{\text {min }}^{2}$ can be pushed before the pQCD framework breaks down. We plan to investigate the impact of the $Q_{\min }^{2}$ cut on constraining $\Delta g$ based on analyses with the pseudo-data. At small enough $x$ one may observe also deviations from standard DGLAP evolution as we will discuss briefly below. A full eRHIC with energies of up to $30 \mathrm{GeV}$ electrons on $325 \mathrm{GeV}$ protons is certainly desirable as it would cover the most interesting kinematic region around $x=10^{-4}$ at larger values of $Q^{2}$.

The l.h.s. of Fig. 1.27 shows the $x$ and $Q^{2}$ coverage for one of the simulated data sets for the spin asymmetry $A_{1}$. The statistical uncertainties are in general way too small to be visible. For the smallest $x$ and $Q^{2}$ values, the expected size of the asymmetries is of the order of a few times $10^{-3}$, which sets the scale for the required experimental precision. On the r.h.s. of Fig. 1.27 we show the $Q^{2}$ dependence of the structure function $g_{1}$ for various bins in $x$. As can be seen, combining the data sets for the different c.m.s. energies extends the coverage in $x$ and $Q^{2}$. We note that present fixed-target data, cf. Fig. 1.25, all fall in the lower right corner of the plot but have some overlap with the projected EIC data.

The pseudo-data for the spin asymmetry $A_{1}$ have been added to a global QCD fit of helicity-dependent PDFs based on the DSSV framework [25, 26]. We have used the projected uncertainties to randomize the pseudo-data by one sigma around their central 

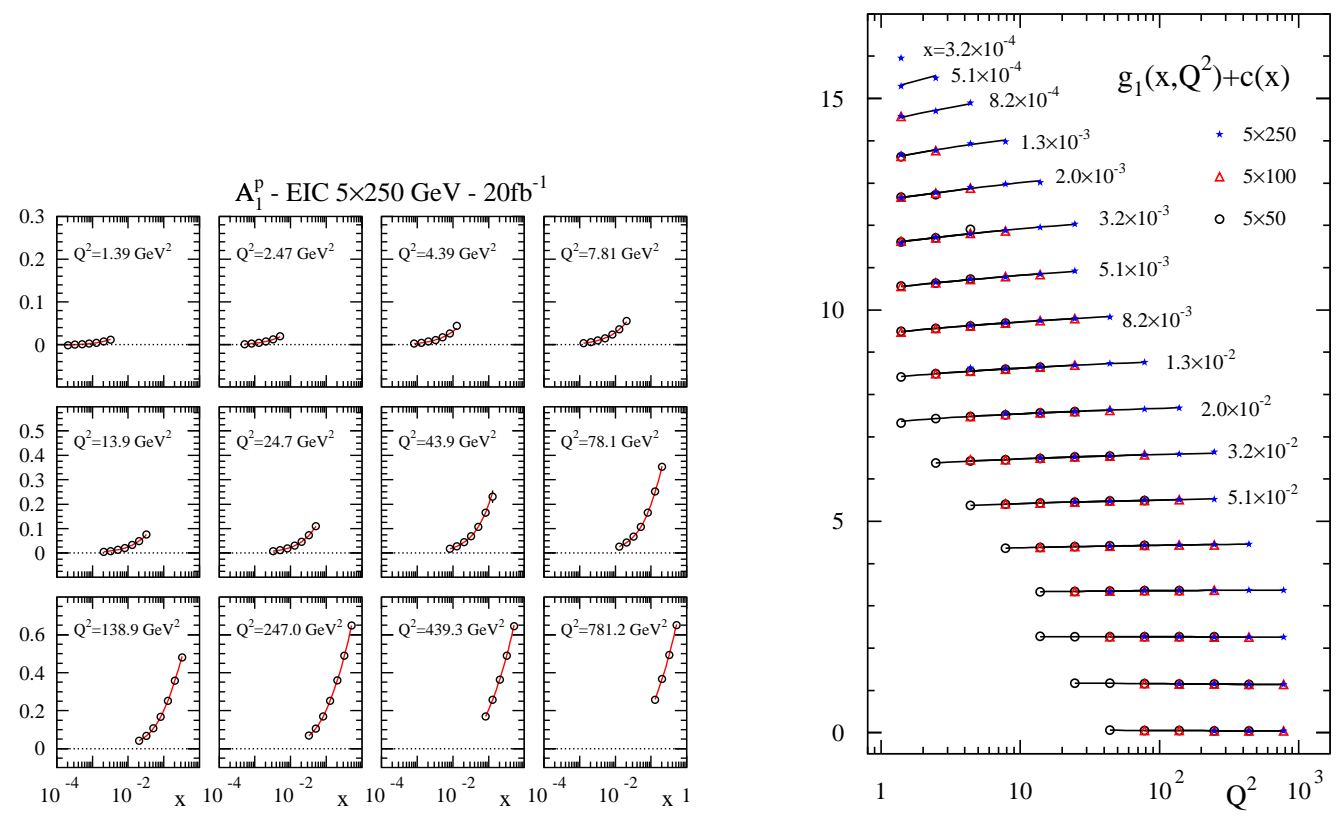

Figure 1.27. left: generated pseudo-data for $A_{1}$ in bins of $Q^{2}$ for $5 \times 250 \mathrm{GeV}$ collisions; right: $g_{1}$ as a function of $Q^{2}$ for fixed $x$ for $5 \mathrm{GeV}$ electrons on three different proton energies.
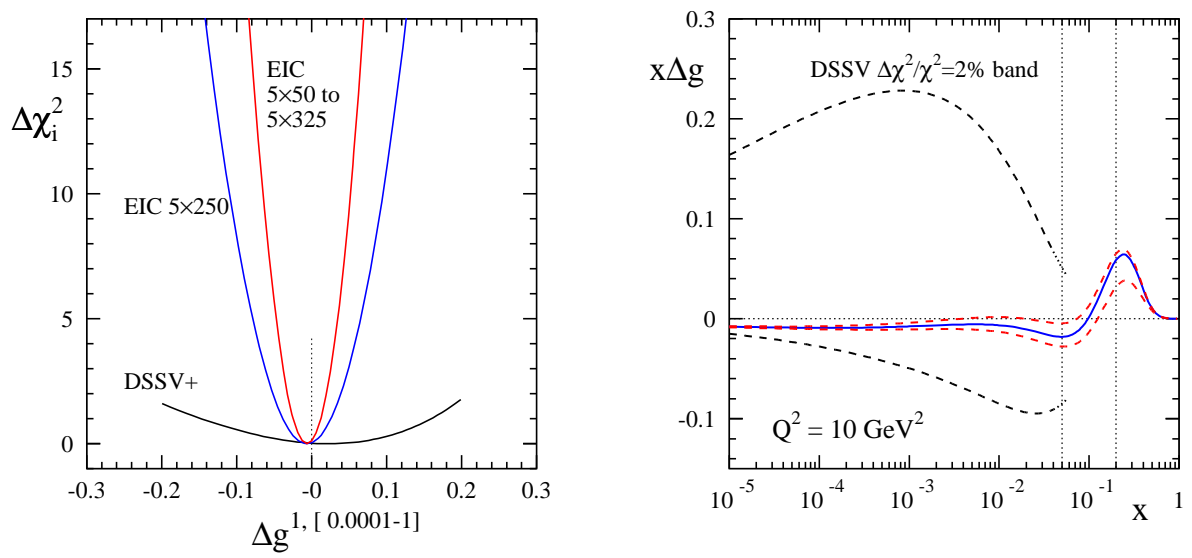

Figure 1.28. $\chi^{2}$ profiles for the truncated $x$ integral of $\Delta g$ (l.h.s.) and uncertainty bands for $x \Delta g$ referring to $\Delta \chi^{2} / \chi^{2}=2 \%$ (r.h.s.) with and without including the generated EIC pseudo-data in the fit.

values determined by the DSSV set of PDFs. To demonstrate the impact of the generated EIC data on $\Delta g$, we show on the 1.h.s. of Fig. 1.28 the $\chi^{2}$ profile for the first moment of $\Delta g$ truncated to the range $10^{-4} \leq x<1$ where EIC DIS data with $Q^{2}>1 \mathrm{GeV}^{2}$ can potentially constrain its value. As can be inferred from the plot, the fit based on all presently available DIS, SIDIS, and RHIC $p p$ data set (labeled as "DSSV+" and described in the previous Section) only very marginally constrains the integral. Adding in the projected data for 
$5 \times 250 \mathrm{GeV}$, shown in Fig. 1.27, already greatly improves the $\chi^{2}$ profile. Including all four EIC data sets determines the integral very well; recall that the width of the profile determines the uncertainty for a given, tolerated increase $\Delta \chi^{2}$. To achieve such a level of accuracy, the data sets with the highest $\sqrt{s} \simeq 70 \div 80 \mathrm{GeV}$ are most critical in the fit as they probe the smallest $x$ values.

Even more impressive is the reduction of the ambiguities on the $x$ shape of $\Delta g\left(x, Q^{2}\right)$ shown on the r.h.s. of Fig. 1.28. The currently completely undetermined shape for $x \lesssim 0.01$ can be mapped precisely to an accuracy of about $\pm 10 \%$ (or better) for $\gtrsim 10^{-4}$. Below $\approx$ $2 \times 10^{-4}$ the shown $\Delta g\left(x, Q^{2}\right)$ and its uncertainties are not constrained by the projected EIC data and merely result from an extrapolation of the used functional form. We note that since one needs to control all sources of uncertainties extremely well it might be advantageous to measure and analyze polarized cross sections instead of spin asymmetries traditionally used so far. This should greatly simplify the theoretical analysis as one does not need any information on unpolarized PDFs or the ratio of $\sigma_{L} / \sigma_{T}$ anymore. There are also first, very interesting attempts to analyze polarized DIS data within the methodology of neural networks [138, 139], which provides a less biased way to estimate PDF uncertainties than standard approaches based on pre-defined functional forms.

As was mentioned above, one expects to find deviations from DGLAP evolution at sufficiently small values of $x$. In contrast to the unpolarized case, the dominant contribution of gluons mixes with quarks also at $x \ll 1$. From DGLAP evolution one expects for the small $x$ behavior of gluons and quarks

$$
\Delta q\left(x, Q^{2}\right), \Delta g\left(x, Q^{2}\right) \simeq \exp \left[\text { const } \times \alpha_{s} \ln \left(Q^{2} / \mu^{2}\right) \ln (1 / x)\right]^{1 / 2}
$$

assuming for simplicity a fixed coupling $\alpha_{s}$. In [156, 157] it was demonstrated that this simple behavior can strongly underestimate the rise at small $x$ due to other potentially large double logarithmic contributions of the type $\alpha_{s} \ln ^{2}(1 / x)^{n}$ in the $n$-th order of $\alpha_{s}$ which are beyond the standard DGLAP framework. This gives rise to a power-like behavior of $g_{1}$ at small $x$ of the form $g_{1}\left(x, Q^{2}\right) \sim(1 / x)^{\mathcal{O}\left(\alpha_{s}\right)}$. There are qualitative arguments that in the polarized case the relevance of these logarithms in $1 / x$ is larger than the difference between DGLAP and BFKL evolution in the unpolarized case [156, 157]. However, more detailed quantitative studies are still lacking, and it remains to be seen if the kinematic reach of an EIC is large enough to actually observe deviations from DGLAP in polarized DIS. Clearly, any such estimate will strongly depend upon the initial input distributions, and eventually one needs data to clarify the relevance of small $x$ enhancements. Finally, we note that in Ref. [158] the leading small $x$ logarithms were combined with DGLAP evolution, and some effects of running coupling were addressed in [159].

\subsubsection{Charm Contribution to $\mathrm{g}_{1}$}

As discussed in Sec. 1.7 in the context of unpolarized DIS structure functions, the contributions from heavy flavors require a special theoretical framework. For the kinematic regime covered at the EIC it is expected that effects of the finite heavy quark mass play an important role and should not be neglected. This is, of course, particularly relevant not too far from threshold, i.e., for $Q^{2}$ less than a few times $m_{h}^{2}$.

For all presently available DIS data, the charm contribution to $g_{1}$ can be safely neglected and, hence, is usually not included in any of the QCD analyses except for the fit presented in Ref. [23]. The relevant coefficient functions for $\gamma^{*} g \rightarrow c \bar{c} X$ have been calculated only to LO accuracy [152] so far which is not sufficient for the anticipated experimental precision. 

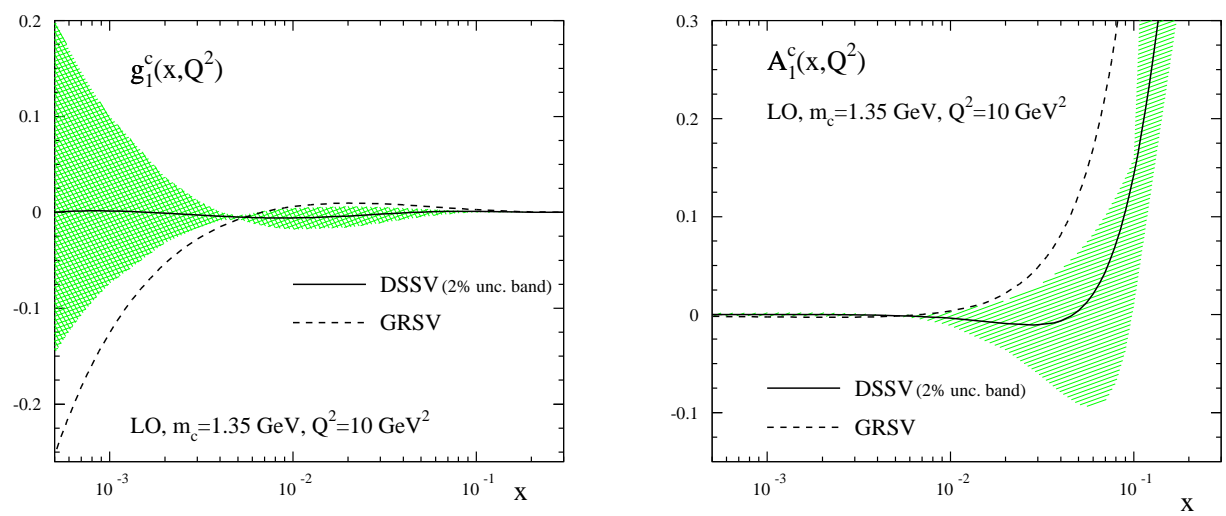

Figure 1.29. LO expectations for $g_{1}^{c}$ (l.h.s.) and $A_{1}^{c}$ (r.h.s) for the $Q^{2}=10 \mathrm{GeV}^{2}, m_{c}=1.35 \mathrm{GeV}$, and using the DSSV and GRSV "std" sets of PDFs. The shaded band corresponds to the $\Delta \chi^{2} / \chi^{2}=2 \%$ uncertainty estimate of DSSV.

The computation of the NLO corrections is, however, work in progress and results should become available for more detailed quantitative studies soon.

For spin dependent DIS the heavy quark contributions are expected to be smaller than in the helicity-averaged case but, of course, will very much depend on the currently unknown size of $\Delta g\left(x, Q^{2}\right)$ at small $x$. There is also an interesting constraint on the gluonic Wilson coefficient for heavy quark production, demanding a vanishing first moment when regulated dimensionally or with a quark mass [160, 161]. This leads to a non-trivial oscillating pattern for $g_{1}^{c}$ depending on the sign of $\Delta g$ which will look rather different in the case that $\Delta g$ itself changes sign within the $x$ range probed.

Figure 1.29 shows some expectations for the spin asymmetry $A_{1}^{c}$ for DIS charm production (r.h.s.) and the charm contribution to the structure function $g_{1}$ (l.h.s.) both computed at LO accuracy with two different polarized gluon distributions. For a small $\Delta g$ with a node, as in the best fit of DSSV, the charm contribution turns out to be at most at the percent level even at collider kinematics, and the corresponding spin asymmetry is most likely too small, $\mathcal{O}\left(\right.$ few $\left.\times 10^{-5}\right)$, to be measured directly. For a larger gluon distribution at small $x$, as in the GRSV fit, or for a gluon within the current uncertainty band of DSSV, asymmetries can be significantly larger, reaching $\mathcal{O}$ (few $\times 10^{-3}$ ), and at $x=10^{-3}$ and $Q^{2} \simeq 10 \mathrm{GeV}^{2}$ charm quarks can contribute about $10 \div 15 \%$ to the inclusive $g_{1}$. The experimental aspects for detecting charmed mesons have beed discussed already in Sec. 1.8 and apply also here.

\subsubsection{Remark on the Bjorken sum rule}

The Bjorken sum rule

$$
\int_{0}^{1} d x\left[g_{1}^{p}\left(x, Q^{2}\right)-g_{1}^{n}\left(x, Q^{2}\right)\right]=\frac{1}{6} C_{B j}\left[\alpha_{s}\left(Q^{2}\right)\right] g_{A}
$$

is not only one of the most fundamental relations in QCD but presumably also one of the best known quantities in pQCD. Corrections up to $\mathcal{O}\left(\alpha_{s}^{4}\right)$ have been calculated [162, 31, 32]. Given the anticipated precision of DIS measurements at the EIC, it is natural to ask what can be achieved concerning the Bjorken sum. The major obstacle is, of course, the need for an effective, longitudinally polarized neutron beam. One conceivable option would be to run 
with ${ }^{3} \mathrm{He}$ but developing a method to measure its polarization to the required percent level is certainly an extremely challenging $R \& D$ task requiring novel ideas. From the theoretical side it might be advantageous to analyze the data not in terms of PDFs but directly on the structure function level with the help of so called "physical anomalous dimensions" [163]. This reduces not only the number of parameters but also theoretical scale uncertainties.

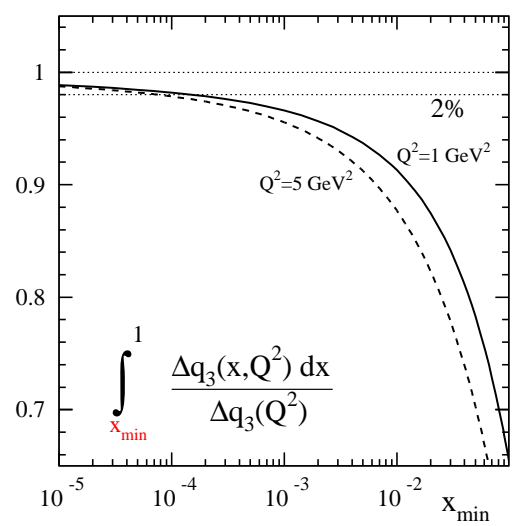

Figure 1.30. The truncated ("running") $x$ integral for the non-singlet combination $\Delta q_{3}$ related to the Bjorken sum normalized to the full first moment for two values of $Q^{2}$.

From present fixed target experiments the sum rule is currently verified to about $10 \%$, which sets the target for any future measurement to the $1 \div 2$ percent level. One of the current limitations is the extrapolation uncertainty from the unmeasured small $x$ region. Since the Bjorken sum probes a non-singlet (NS) quark combination, the small $x$ uncertainties are considerably less severe than for $\Delta g\left(x, Q^{2}\right)$, but to reduce them to a level of about $2 \%$, measurements of $g_{1}^{p, n}$ down to $x \simeq 10^{-4}$ are required. This is illustrated in Fig. 1.30 where we show the "running" $x$ integral for the relevant NS quark combination $\Delta q_{3}$ normalized to its full first moment, assuming the functional form from the DSSV analysis. At the required $1 \div 2 \%$ level of accuracy one might start to see deviations from (1.10) due to isospin and charge symmetry violations. Very little is known about these effects, and, if experimentally feasible, measurements could reveal genuine new insights into the hadronic structure.

The fundamental relation (1.10) between a high-energy measurement of DIS structure functions and a low-energy quantity like the axial charge $g_{A}$ by itself warrants an experimental exploration at the EIC. From a more theoretical perspective one might argue that since $\mathcal{O}\left(\alpha_{s}^{4}\right)$ corrections are available, a precision measurement of the Bjorken sum can be turned into one of the most accurate determinations of $\alpha_{s}$. One can easily convince oneself, however, that this does not work out. Changing $\alpha_{s}$ by about one percent, translates only in a $0.1 \%$ change of the Bjorken sum, which is impossible to resolve experimentally. Perhaps more interesting is the non-trivial connection of the Bjorken sum rule to the Adler $D\left(Q^{2}\right)$ function which naturally appears, for instance, in the $e^{+} e^{-}$annihilation into hadrons [164. These two, seemingly unrelated quantities are connected through the generalized Crewther relation [145, 32. For large enough $Q^{2}$, the Adler function can be expanded as a power series in $\alpha_{s}$ like $C_{B j}\left[\alpha_{s}\left(Q^{2}\right)\right]$ in (1.10), and results are available up to $\mathcal{O}\left(\alpha_{s}^{4}\right)$ as well [165]. 


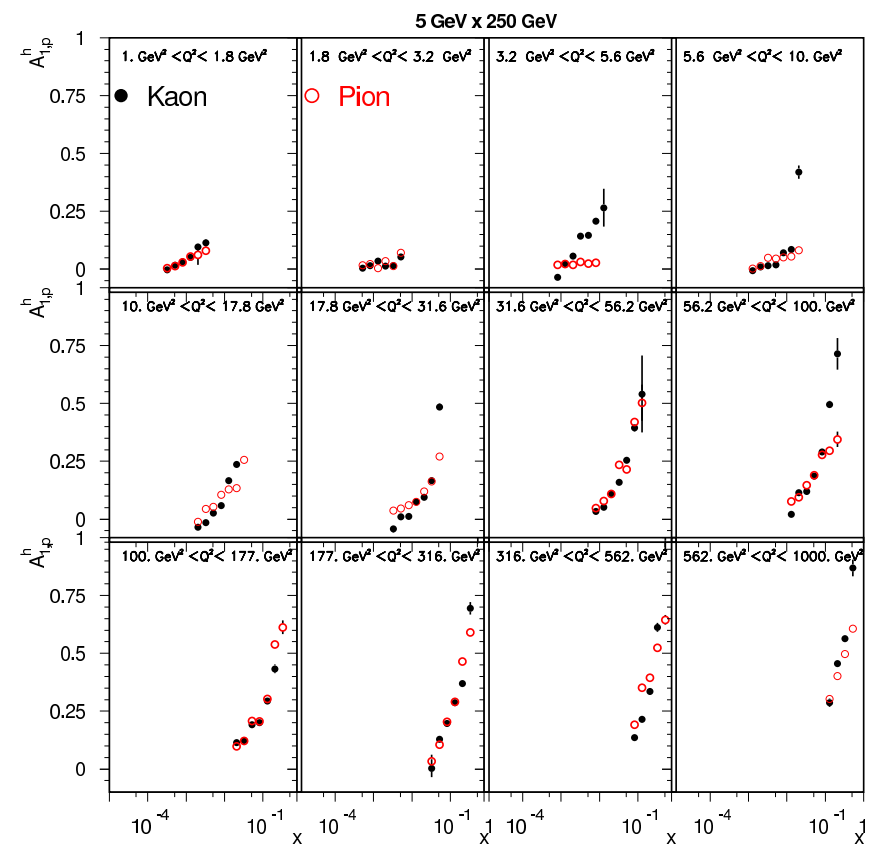

Figure 1.31. Projected spin asymmetries for pion and kaon production in SIDIS for beam energies of $5 \times 250 \mathrm{GeV}$ and various bins in $Q^{2}$.

The Crewther relation then states for the NS part of the $D$ function that

$$
D\left[\alpha_{s}\left(Q^{2}\right)\right] C_{B j}\left[\alpha_{s}\left(Q^{2}\right)\right]=3\left[1+\frac{\pi \beta\left(\alpha_{s}\right)}{\alpha_{s}} K\left[\alpha_{s}\left(Q^{2}\right)\right]\right]
$$

where $\beta$ denotes the QCD beta function, and the first four terms in the expansion of $K\left[\alpha_{s}\left(Q^{2}\right)\right]$ are known. The term proportional to $\beta$ in (1.11) describes the deviation from the limit of exact conformal invariance of QCD [145, 166]. We also note that since the Bjorken sum rule can be measured down to small values of $Q^{2}$ it provides a way to define an effective strong coupling constant [167, 168] which is by construction gauge and scheme invariant and approaches the standard running of $\alpha_{s}$ in the perturbative domain.

\subsubsection{Opportunities in semi-inclusive DIS}

As has been mentioned in Sec. 1.10, the flavor separation of polarized PDFs in current fits is largely based on pion and kaon yields in SIDIS. An EIC can easily extend the existing kinematic coverage in the same way as for inclusive DIS. Prerequisites for exploiting SIDIS as a precision tool at the EIC, such as good particle identification and well constrained fragmentation functions, have been already discussed in Sec. 1.5 for the unpolarized case.

Figure 1.31 shows projected data for the longitudinal spin asymmetry in SIDIS with identified pions and kaons in the same $Q^{2}$ bins as used for inclusive DIS studies in Fig. 1.27, The simulation is based on the PEPSI Monte Carlo [155] using the GRSV "std" set of polarised PDFs [24]. The following cuts have been applied to model some detector and acceptance effects: $Q^{2}>1 \mathrm{GeV}^{2}, 0.1<y<0.95$, photon depolarization factor $D(y)>0.1$, $W^{2}>10 \mathrm{GeV}^{2}, 0.2<z<0.8, p_{H}>1.5 \mathrm{GeV}$, and $1^{\circ}<\theta_{H}<179^{\circ}$. The momentum cut on the detected hadron $H$ is placed to ensure to be above the PID Cherenkov threshold. The statistical precision reflects one month of running at the luminosities anticipated for 
the first stage of eRHIC. Again, these measurements will be limited by systematic uncertainties, which have to be addressed in detail. In addition to the sources of systematic uncertainties present for inclusive DIS, the detector performance for the identification of different produced hadron species is most critical for SIDIS. Additional sets of data have been generated for other combinations of electron and proton beam energies. They are currently being implemented into the same global QCD analysis framework used to analyze the projected inclusive DIS data above. Plots similar to those for the $\chi^{2}$ profile of the truncated $x$ integral and the $x$ dependent uncertainty bands for $\Delta g\left(x, Q^{2}\right)$ in Fig. 1.28 will be prepared to quantify the impact of SIDIS data on our knowledge of helicity-dependent quark densities. We expect that all light quark and anti-quark flavors, i.e., $\Delta u, \Delta \bar{u}, \Delta d$, $\Delta \bar{d}, \Delta s$, and $\Delta \bar{s}$, can be determined with a precision close to the one obtained for $\Delta g\left(x, Q^{2}\right)$ in Fig. 1.28,

Although knowledge of individual quark and anti-quark flavors is in principle not required for an understanding of the proton spin sum rule, where only the total quark singlet $\Delta \Sigma$ enters, it would provide deeper insight into the question why the observed total quark polarization is considerably smaller than in naive quark models. Here, it is essential to understand in detail how sea quarks are polarized, i.e., whether they have a preference for spinning "against" the direction of the proton spin thereby diluting the total quark polarization. Current QCD fits [25, 26] start to reveal rather complicated patters of polarization at medium-to-large $x$ with possible sign changes but the statistical precision and kinematic reach of the fixed-target data is not sufficient for any definitive conclusions.

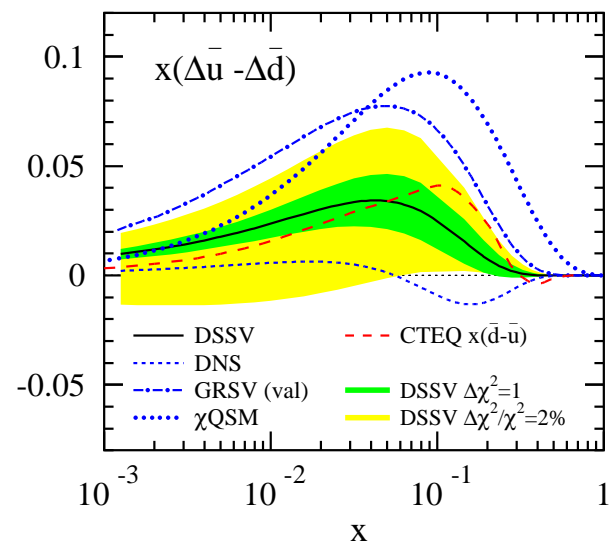

Figure 1.32. $x(\Delta \bar{u}-\Delta \bar{d})$ at $Q^{2}=10 \mathrm{GeV}^{2}$ along with the uncertainty bands from DSSV, results from earlier global fits, and predictions from the chiral quark soliton model [169, 170].

To give an example, Fig. 1.32 shows the current significance of a possible asymmetry in the light quark sea, $\Delta \bar{u}(x)-\Delta \bar{d}(x)$. Given the well-established pronounced difference between $\bar{u}$ and $\bar{d}$ in the spin-averaged case, a precise determination of $\Delta \bar{u}(x)-\Delta \bar{d}(x)$ is of of particular interest. Different patterns of symmetry breaking in the light anti-quark sea polarizations have been predicted qualitatively by a number of models of nucleon structure. For instance, within the large- $N_{c}$ limit of QCD as incorporated in the chiral quark soliton model [169, 170, 171, 172] one expects $|\Delta \bar{u}-\Delta \bar{d}|>|\bar{u}-\bar{d}|$. In addition, charged kaon data should help to clarify issues related to $\mathrm{SU}(3)$ symmetry and the polarized strangeness density $\Delta s\left(x, Q^{2}\right)$ by providing sufficient input to determine its first moment reliably. 


\title{
1.12 Electroweak structure functions at the EIC
}

\author{
Abhay Deshpande, Krishna Kumar, Felix Ringer, Seamus Riordan, Swadhin Taneja, \\ Werner Vogelsang
}

\subsubsection{Motivation and Introduction}

The use of charged leptons to probe the structure of nucleons through electroweak interactions has proven to be an invaluable tool in our exploration of the strong force. Experiments on deep inelastic scattering (DIS) $e p \rightarrow e X$, which dominantly proceeds via the exchange of a virtual photon between the electron and the nucleon, have established the existence of quarks and provided detailed studies of the short range aspects of the strong coupling.

It is well known that neutral current (NC) interactions can also be mediated by the $Z$-bosons of the weak interactions, and their interference with the photon. This gives rise to parity violating effects, which offer complementary access to nucleon structure. This has been a theme at parity violating electron scattering experiments, both at fixed target facilities [173, 3] and at HERA [174, 175]. For an unpolarized target, the NC parity violating asymmetry is given by

$$
A_{\text {beam }} \equiv \frac{\sigma_{R}-\sigma_{L}}{\sigma_{R}+\sigma_{L}}
$$

where $\sigma_{R}\left(\sigma_{L}\right)$ denotes the cross section for right- (left-) handed electrons. For fixed-target experiments, where the virtuality $Q$ of the exchanged boson is typically much smaller than the $Z$-boson mass $M_{Z}$, only $\gamma Z$-interference is relevant, and one obtains

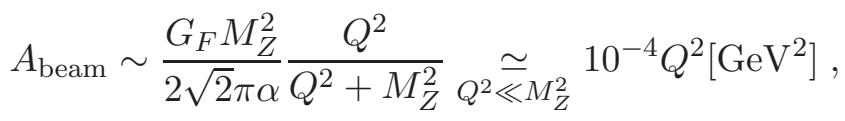

with the Fermi constant $G_{F}$ and the fine structure constant $\alpha$. At modern fixed target facilities, measured asymmetries were typically of the order of $10^{-4}$ or less [173]. At HERA, on the other hand, with its enormous kinematic reach in $Q^{2}$, also contributions by pure $Z$-exchange play a role [174].

Charged current (CC) interactions in DIS lepton scattering measurements have been performed at HERA in $e^{ \pm} p$ collisions [174] and at various neutrino scattering experiments [176]. They are inaccessible at fixed target charged lepton beam facilities where $Q^{2} \ll M_{W}^{2}$.

An EIC provides a number of advantages in the study of structure functions through electroweak interactions over previous and existing facilities. As the asymmetries and relative likelihood of $Z^{0}$ and $W^{ \pm}$exchange monotonically increase with $Q^{2}$, larger c.m.s. energies are more favorable for such measurements. Additionally, advances in accelerator and source technologies should provide luminosities on the order of $\sim 10^{34} \mathrm{~cm}^{-2} \mathrm{~s}^{-1}$, two orders of magnitude higher than what was available at HERA. A new feature will be the ability for bunch-by-bunch variation of the sign of the longitudinal polarization of both the electron and hadron beams. A broader $Q^{2}$ and $y$ acceptance than at fixed target facilities, and variable beam energy, also allow for separation of the various structure functions. High precision is possible over a broad range in Bjorken- $x, 0.01 \lesssim x \lesssim 0.4$, whereas fixed target facilities typically are sensitive only to $x>0.1$.

\section{Polarized Hadrons}

Arguably the most important feature at the EIC is the availability of polarized ${ }^{1} \mathrm{H}$, and potentially ${ }^{2} \mathrm{H}$ and ${ }^{3} \mathrm{He}$, beams with rapid polarization flips, which offers access to 
electroweak spin structure functions that may provide additional constraints on polarized PDFs. The counterpart of $A_{\text {beam }}$ in (1.12) with polarized protons has never been measured before, and neither have spin asymmetries in CC interactions. Both would in principle be accessible at the EIC.

The theoretical study of electroweak spin-dependent structure functions dates back to the seventies 177, 178, 179, 180, 181, 182, 183, 184, 185. Renewed interest arose in the nineties in the context of a possible polarized ep program at HERA [186, 187, 188, 189, 190, 191, 192, 193, 194, 195, 196, 197, 198, and later in terms of studies for a neutrino factory [199]. Parity-violating spin structure functions were shown to contain rich information on polarized PDFs. For example, as we shall discuss in more detail in the next section, for CC interactions via $W^{-}$exchange in the parton model, two structure functions $g_{1}^{W^{-}}$and $g_{5}^{W^{-}}$contribute to the spin asymmetry [196, 197]:

$$
A^{W^{-}}=\frac{2 b g_{1}^{W^{-}}+a g_{5}^{W^{-}}}{a F_{1}^{W^{-}}+b F_{3}^{W^{-}}},
$$

where $a=2\left(y^{2}-2 y+2\right), b=y(2-y)$, and

$$
g_{1}^{W^{-}}(x)=\Delta u(x)+\Delta \bar{d}(x)+\Delta c+\Delta \bar{s}(x), \quad g_{5}^{W^{-}}(x)=-\Delta u(x)+\Delta \bar{d}(x)-\Delta c+\Delta \bar{s}(x) .
$$

In Eq. (1.14), $F_{1}^{W^{-}}$and $F_{3}^{W^{-}}$are the corresponding unpolarized CC structure functions. Extraction of $g_{1}^{W^{-}}$and $g_{5}^{W^{-}}$hence offers new and independent constraints on the quark and anti-quark helicity distributions, with $g_{1}^{W^{-}}$measuring singlet contributions, while $g_{5}^{W^{-}}$is a flavor non-singlet. If additionally positrons and polarized neutrons are available, which is possible at the EIC, one could obtain a full flavor decomposition of the nucleon polarized quark and anti-quark sector. For instance, for proton scattering $g_{1}^{W^{-}}+g_{1}^{W^{+}}$provides the full quark singlet distribution $\Delta \Sigma$, whose first moment gives the quark and anti-quark spin contribution to the proton's spin. Likewise, $g_{5}^{W^{-}}+g_{5}^{W^{+}}$determines the "valence" distributions $\Delta q-\Delta \bar{q}$. Adding neutrons, one has, for example, $g_{5, p}^{W^{+}, p}-g_{5, n}^{W^{+}, n}=\Delta u+$ $\Delta \bar{u}-(\Delta d+\Delta \bar{d})$, which satisfies a sum rule equally fundamental as the Bjorken sum rule:

$$
\int_{0}^{1} d x\left[g_{5}^{W^{+}, p}-g_{5}^{W^{+}, n}\right]=\left(1-\frac{2 \alpha_{s}}{3 \pi}\right) g_{A},
$$

where we have included the first-order QCD correction [193]. NC structure functions offer independent insights into nucleon structure. For example, for the $\gamma-Z$ interference contribution, the structure function $g_{1}$ becomes to good approximation $g_{1}^{\gamma Z} \propto \Delta u+\Delta \bar{u}+\Delta d+$ $\Delta \bar{d}+\Delta s+\Delta \bar{s}$ and thus again probes the full quark and anti-quark singlet. The structure function $g_{5}$, on the other hand, probes the valence densities: $g_{5}^{\gamma Z} \propto 2 \Delta u_{v}+\Delta d_{v}$.

We present a few first studies of the prospects for measurements of electroweak spin structure functions in CC and NC scattering at an EIC. These are not meant to present an exhaustive assessment of all the opportunities the EIC would provide in this area.

\subsubsection{Electroweak Deep Inelastic Scattering}

\section{Structure Functions and Parton Model Expressions}

In the determination of cross sections and asymmetries, we follow closely the PDG review [3]. The spin-averaged DIS cross section for $Q^{2} \gg M^{2}$, where $M$ is the mass of the nucleon, is 
given by

$$
\frac{d^{2} \sigma^{i}}{d x d y}=\frac{2 \pi \alpha^{2}}{x y Q^{2}} \eta^{i}\left[Y_{+} F_{2}^{i} \mp Y_{-} x F_{3}^{i}-y^{2} F_{L}^{i}\right],
$$

where $i$ is for $\mathrm{NC}$ or $\mathrm{CC}$ and $Y_{ \pm}=1 \pm(1-y)^{2}$. We have introduced the longitudinal structure function $F_{L}^{i}=F_{2}^{i}-2 x F_{1}^{i}$, which vanishes to lowest order according to the CallanGross relation. The NC structure functions for $e^{ \pm} N$ scattering can be represented as the sums of the photon, $Z^{0}$, and interference contributions:

$$
F_{2}^{\mathrm{NC}}=F_{2}^{\gamma}-\left(g_{V}^{e} \pm \lambda g_{A}^{e}\right) \eta_{\gamma Z} F_{2}^{\gamma Z}+\left(g_{V}^{e}{ }^{2}+g_{A}^{e}{ }^{2} \pm 2 \lambda g_{V}^{e} g_{A}^{e}\right) \eta_{Z} F_{2}^{Z}
$$

and

$$
x F_{3}^{\mathrm{NC}}=-\left(g_{A}^{e} \pm \lambda g_{V}^{e}\right) \eta_{\gamma Z} x F_{3}^{\gamma Z}+\left[2 g_{V}^{e} g_{A}^{e} \pm \lambda\left(g_{V}^{e}{ }^{2}+g_{A}^{e} 2\right)\right] \eta_{Z} x F_{3}^{Z} .
$$

Here and above, the sign \pm is commensurate to the lepton charge. We have

$$
\eta_{\gamma}=1 ; \quad \eta_{\gamma Z}=\left(\frac{G_{F} M_{Z}^{2}}{2 \sqrt{2} \pi \alpha}\right)\left(\frac{Q^{2}}{Q^{2}+M_{Z}^{2}}\right) ; \quad \eta_{Z}=\eta_{\gamma Z}^{2}
$$

and $g_{V}^{e}=-\frac{1}{2}+2 \sin ^{2} \theta_{W}, g_{A}^{e}=-\frac{1}{2} . \lambda= \pm 1$ is the electron/positron helicity.

The spin-averaged structure functions can be written as

$$
\begin{aligned}
& {\left[F_{2}^{\gamma}, F_{2}^{\gamma Z}, F_{2}^{Z}\right]=x \sum_{q}\left[e_{q}^{2}, 2 e_{q} g_{V}^{q}, g_{V}^{q}{ }^{2}+g_{A}^{q}{ }^{2}\right](q+\bar{q}),} \\
& {\left[F_{3}^{\gamma}, F_{3}^{\gamma Z}, F_{3}^{Z}\right]=\sum_{q}\left[0,2 e_{q} g_{A}^{q}, 2 g_{V}^{q} g_{A}^{q}\right](q-\bar{q}),}
\end{aligned}
$$

where $e_{q}$ is the fractional electric charge of the quark, $g_{V}^{q}= \pm \frac{1}{2}-2 e_{q} \sin ^{2} \theta_{W}$, and $g_{A}^{q}= \pm \frac{1}{2}$, with the + sign for up-type quarks and the - sign for down-type quarks.

For $Q^{2} \ll M_{Z}^{2}$, the pure $Z$ contribution can be neglected, and one finds in this limit

$$
A_{\text {beam }}=\frac{G_{F} Q^{2}}{2 \sqrt{2} \pi \alpha}\left[g_{A}^{e} \frac{F_{1}^{\gamma Z}}{F_{1}^{\gamma}}+g_{V}^{e} \frac{Y_{-}}{2 Y_{+}} \frac{F_{3}^{\gamma Z}}{F_{1}^{\gamma}}\right] .
$$

For the case of a polarized target, there are similar spin dependent structure functions. The difference $\Delta \sigma$ of cross sections for the two nucleon helicity states is

$$
\frac{d^{2} \Delta \sigma^{i}}{d x d y}=\frac{8 \pi \alpha^{2}}{x y Q^{2}} \eta^{i}\left[Y_{+} x g_{5}^{i} \pm Y_{-} x g_{1}^{i}-y^{2} g_{L}^{i}\right],
$$

where again $i$ is for NC or CC and where $g_{L}^{i}=g_{4}^{i}-2 x g_{5}^{i}$. We note that, like $F_{L}$, the latter quantity vanishes to $\mathcal{O}\left(\alpha_{s}^{0}\right)$ [178. The NC spin dependent structure functions are

$$
\begin{aligned}
& g_{5}^{\mathrm{NC}}=-\left(g_{V}^{e} \pm \lambda g_{A}^{e}\right) \eta_{\gamma Z} g_{5}^{\gamma Z}+\left(g_{V}^{e}{ }^{2}+g_{A}^{e}{ }^{2} \pm 2 \lambda g_{V}^{e} g_{A}^{e}\right) \eta_{Z} g_{5}^{Z}, \\
& g_{1}^{\mathrm{NC}}=\lambda g_{1}^{\gamma}-\left(g_{A}^{e} \pm \lambda g_{V}^{e}\right) \eta_{\gamma Z} g_{1}^{\gamma Z}+\left(2 g_{V}^{e} g_{A}^{e} \pm \lambda\left(g_{V}^{e}+g_{A}^{e}\right)\right) \eta_{Z} g_{1}^{Z} .
\end{aligned}
$$

Their components can be written as

$$
\begin{aligned}
& {\left[g_{1}^{\gamma}, g_{1}^{\gamma Z}, g_{1}^{Z}\right]=\frac{1}{2} \sum_{q}\left[e_{q}^{2}, 2 e_{q} g_{V}^{q}, g_{V}^{q 2}+g_{A}^{q 2}\right](\Delta q+\Delta \bar{q})} \\
& {\left[g_{5}^{\gamma}, g_{5}^{\gamma Z}, g_{5}^{Z}\right]=\sum_{q}\left[0, e_{q} g_{A}^{q}, g_{V}^{q} g_{A}^{q}\right](\Delta q-\Delta \bar{q})}
\end{aligned}
$$


The spin asymmetry for scattering an unpolarized lepton off a polarized nucleon is then given by

$$
A_{\mathrm{L}}=\eta^{\gamma Z}\left[g_{V}^{e} \frac{g_{5}^{\gamma Z}}{F_{1}^{\gamma}} \mp \frac{Y_{-}}{Y_{+}} g_{A}^{e} \frac{g_{1}^{\gamma Z}}{F_{1}^{\gamma}}\right]
$$

In the CC case, we have

$$
\eta_{\mathrm{CC}}=(1 \pm \lambda)^{2} \eta_{W}=\frac{(1 \pm \lambda)^{2}}{2}\left(\frac{G_{F} M_{W}}{4 \pi \alpha} \frac{Q^{2}}{Q^{2}+M_{W}^{2}}\right)^{2}
$$

For $W^{-}$exchange (electron scattering), the structure functions (assuming four active flavors) are in the parton model:

$$
\begin{aligned}
F_{2}^{W-}=2 x(u+\bar{d}+\bar{s}+c), & F_{3}^{W-}=2(u+\bar{d}+\bar{s}+c), \\
g_{1}^{W-}=\Delta u+\Delta \bar{d}+\Delta \bar{s}+\Delta c, \quad & g_{5}^{W-}=-\Delta u+\Delta \bar{d}+\Delta \bar{s}-\Delta c .
\end{aligned}
$$

For $W^{+}$exchange, one replaces $u \leftrightarrow d$ and $s \leftrightarrow c$. The spin asymmetries for electron and positron scattering then take the simple parton model forms

$$
A_{W^{-}}=\frac{\Delta u+\Delta c-(1-y)^{2}(\Delta \bar{d}+\Delta \bar{s})}{u+c+(1-y)^{2}(\bar{d}+\bar{s})}, \quad A_{W^{+}}=\frac{(1-y)^{2}(\Delta d+\Delta s)-\Delta \bar{u}-\Delta \bar{c}}{(1-y)^{2}(d+s)+\bar{u}+\bar{c}} .
$$

By measuring over a range in $y$, one can perform a separation of the $\Delta u+\Delta c, \Delta d+\Delta s$ quark or anti-quark combinations.

\section{Next-to-leading Order QCD Corrections}

The NLO QCD corrections to the spin-dependent structure functions have been computed in Refs. 192, 193]. To NLO, the expression for a given structure function can be cast into the generic form [199]

$$
\begin{aligned}
g_{1}^{\mathrm{NLO}}\left(x, Q^{2}\right) & =\Delta C_{q, 1} \otimes g_{1}^{\mathrm{LO}}+f_{\Sigma} \Delta C_{g} \otimes \Delta g \\
\frac{g_{4}^{\mathrm{NLO}}\left(x, Q^{2}\right)}{2 x} & =\Delta C_{q, 4} \otimes\left[\frac{g_{4}^{\mathrm{LO}}}{2 x}\right], \\
g_{5}^{\mathrm{NLO}}\left(x, Q^{2}\right) & =\Delta C_{q, 5} \otimes g_{5}^{\mathrm{LO}}
\end{aligned}
$$

where the symbol $\otimes$ denotes a convolution, and $g_{i}^{\mathrm{LO}}$ is the LO (parton model) expression for the respective structure function. The coefficient functions to NLO in the $\overline{\mathrm{MS}}$ scheme can be found in [192, 193]. The factor $f_{\Sigma}$ in Eq. (1.30) is the sum over the coefficient of each quark or anti-quark distribution in the LO expression for $g_{1}$. For example, for the electromagnetic $g_{1}^{\gamma}$ with four flavors, $f_{\Sigma}=10 / 9$, while for $g_{1}^{W^{-}}$one has $f_{\Sigma}=4$. Needless to say that when including the NLO corrections in the calculation of the structure functions, one also has to perform the evolution of the polarized PDFs to NLO [12, 200, 13. For the most part of our study, we will only use the LO expressions for the structure functions, which are expected to be entirely sufficient for estimating the sensitivities at an EIC. We will, however, briefly investigate the typical size of the NLO corrections in Figs. 1.36 and 1.40 below. 


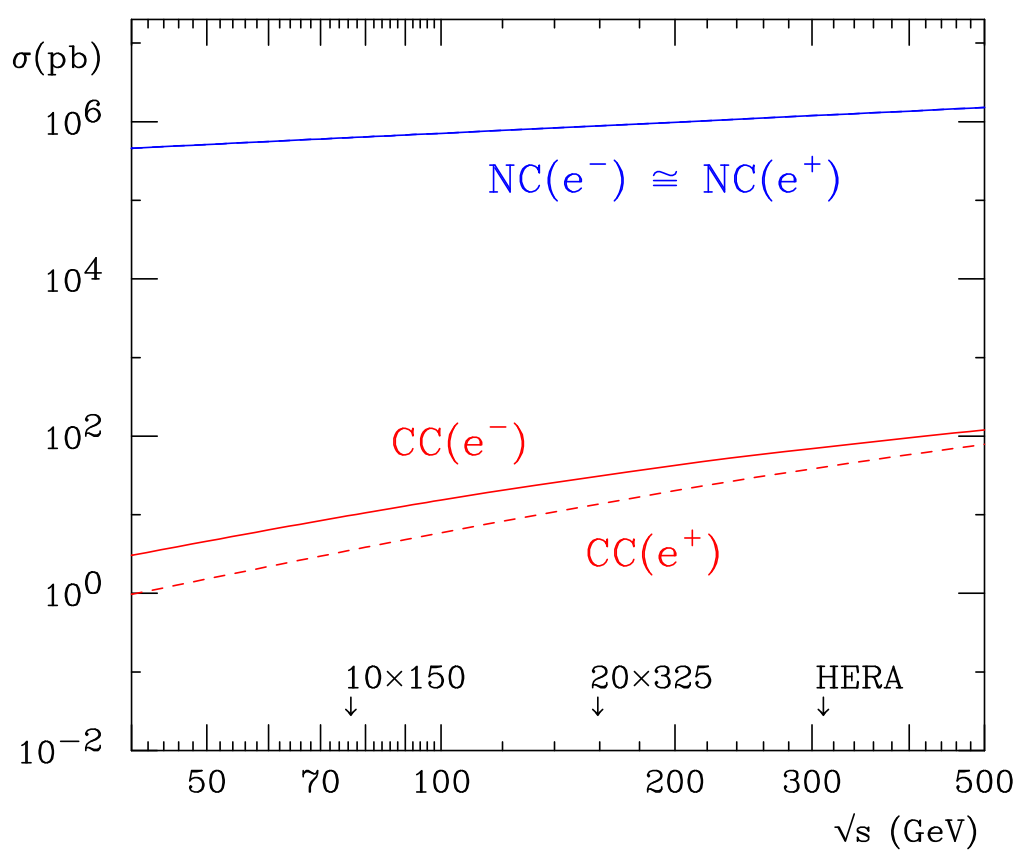

Figure 1.33. Total NC and CC cross sections for $Q^{2}>1 \mathrm{GeV}^{2}$ as functions of the ep $\sqrt{s}$.

\subsubsection{Measurements of Parton Distribution Functions}

In the following, we will present estimates for rates and spin asymmetries for electroweak DIS at an EIC. For the spin-averaged case, we use the CTEQ6.5 [201] unpolarized PDFs. For the helicity PDFs we use the ones of [25]. We note that the latter do not contain a charm quark distribution.

\section{Basic kinematics and machine considerations}

Proposed EIC parameters allow for electron energies of $5-30 \mathrm{GeV}$ and ion energies of $50-325 \mathrm{GeV}$. Figure 1.33 shows the spin-averaged NC and CC total cross sections for electron and positron scattering, as functions of the ep c.m.s. energy $\sqrt{s}$. We have integrated over all $Q^{2}>1 \mathrm{GeV}^{2}$, based on a simple theoretical LO calculation. One can see that the cross section of course rises with energy, but relatively mildly so. Therefore, measurements of electroweak structure functions may well be feasible in collisions at energies significantly lower than those at HERA.

The upper two plots in Figure 1.34 show distributions of the CC cross section in $\log \left(Q^{2}\right)$ and $\log (x)$, respectively, at three different c.m.s. energies. One can see that the largest statistical weight would be at $x \sim 0.1$ and $Q^{2} \sim 1000 \mathrm{GeV}^{2}$, which is a consequence of the $W$-propagator factor in Eq. (1.27). Binning in $x$ and $Q^{2}$ of course allows to investigate more detailed distributions, see below. For NC interactions, the $\gamma$-exchange contribution dominates the spin-averaged cross section and strongly pushes the $Q^{2}$ distribution towards $Q^{2} \rightarrow 0$ (see center row of the figure). Taking the parity-violating electron beam-helicity difference of cross sections, however, essentially singles out the $\gamma Z$-interference contribution. For this piece, which of course is much smaller than the full spin-averaged cross section, the $Q^{2}$ distribution levels off towards $Q^{2} \rightarrow 0$, as follows from the expressions in Sec. 1.12.2 

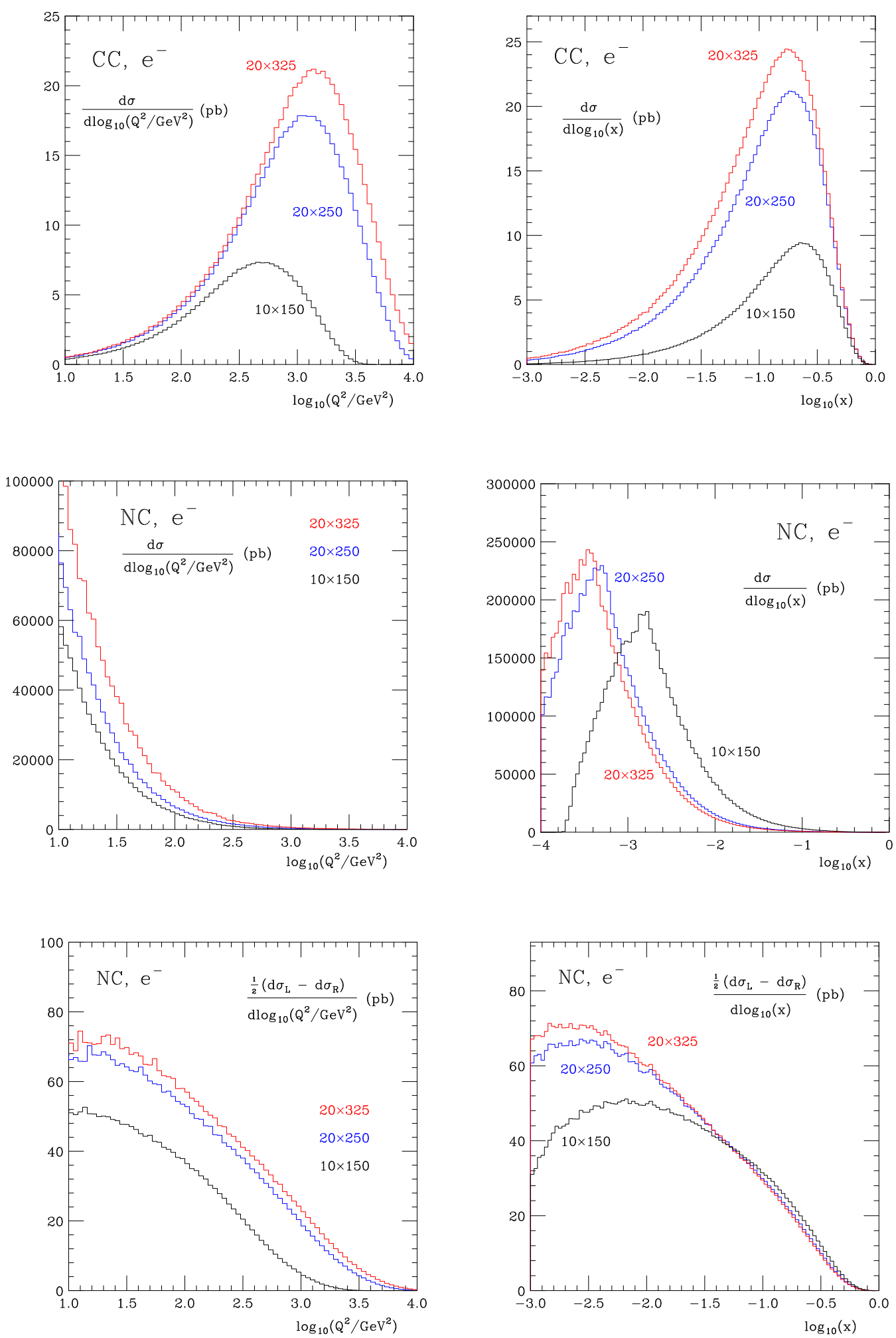

Figure 1.34. Top row: Distributions of the CC spin-averaged cross section in $Q^{2}$ (left) and $x$ (right). We have applied the cuts $Q^{2} \geq 1 \mathrm{GeV}^{2}$ and $0.1 \leq y \leq 0.9$. Center row: same for the NC case. Bottom row: Same for the NC parity-violating electron beam-helicity difference of cross sections. 

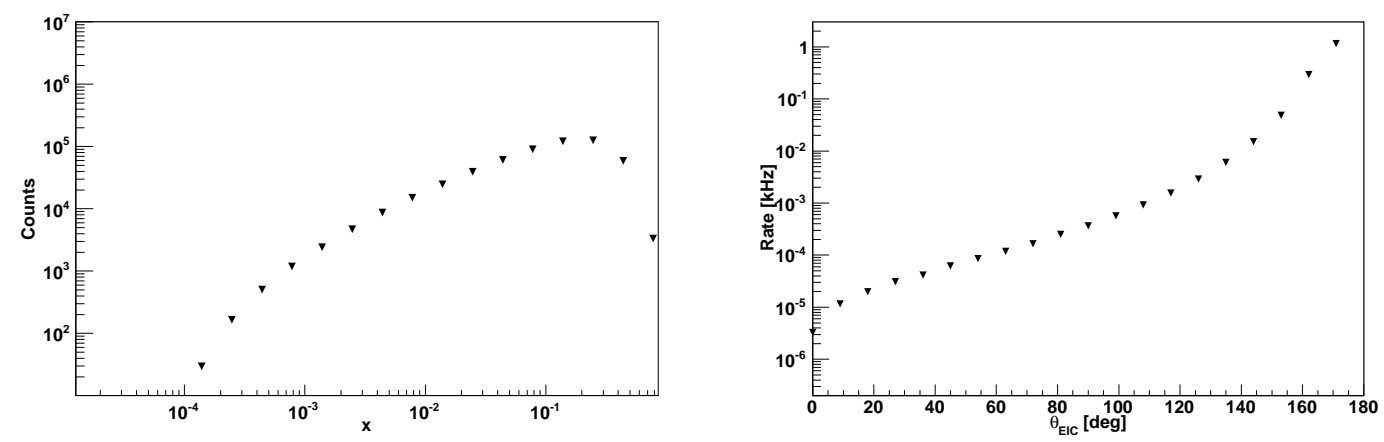

Figure 1.35. Left: Total number of CC events for $20 \times 250 e^{-} p$ scattering for an integrated luminosity of $10 \mathrm{fb}^{-1}$. Right: Binned NC event rate as function of the electron scattering angle, for $20 \times 325$ $e^{-} p$ collisions at $\mathcal{L}=1 \times 10^{33} / \mathrm{s} / \mathrm{cm}^{2}$.

and as shown in the bottom row of Fig. 1.34,

In $\mathrm{CC}$ electron scattering, $e^{-} p \rightarrow \nu_{e} X$, the neutrino remains undetected. To identify a $\mathrm{CC}$ event and to reconstruct $x$ and $Q^{2}$, the final-state hadrons must then be reconstructed instead. The detectors must hence be optimized to detect resulting hadronic jet formation. There will likely be some additional detection and reconstruction efficiency associated with this type of analysis. The discussion of the specific requirements is beyond the scope of this study, and we will assume that this reconstruction is possible. In practice, CC measurements could be performed simultaneously with the NC ones, though at a reduced duty factor if the electron helicity is flipped, as the interaction is purely $V-A$. We also assume that polarized positron beams would be available at an EIC.

For the following analysis, we will consider configurations of $E_{e}[\mathrm{GeV}] \times E_{\text {ion }}[\mathrm{GeV}]$ with $20 \times 325$ and $20 \times 250$. For each of these, a luminosity of about $\sim 1 \times 10^{34} / \mathrm{s} / \mathrm{cm}^{2}$ was considered, with estimates for machine availabilities, detector acceptance and efficiency, and beam polarization. Based on an expected five year run time, we consider a realistic effective integrated luminosity of $100 \mathrm{fb}^{-1}$ for $\mathrm{NC}$ processes and $10 \mathrm{fb}^{-1}$ for CC. For the studies below, a Monte Carlo simulation framework was developed to evaluate rates and asymmetries of both the $\mathrm{NC}$ and $\mathrm{CC}$ processes. No detector responses have yet been included, and a full azimuthal acceptance was assumed. In all analyses we consider a minimum scattered electron energy of $2 \mathrm{GeV}$ within $3^{\circ}<\theta<177^{\circ}$ scattering angle. The smaller integrated luminosity for $\mathrm{CC}$ studies is because of a factor of 2 loss due to helicity flips and also because efficiency of hadron jet and kinematic reconstruction has not yet been studied.

Of practical importance is to evaluate how well a separation of the structure functions can be done at individual points in $x$, though it remains for a future Monte Carlo study to evaluate the $x$ resolution after reconstruction. We bin all data in $20 x$ bins logarithmically spaced from $10^{-5}$ to 1 . When binned in $Q^{2}$, we use 20 bins from 2 to $5 \times 10^{4} \mathrm{GeV}^{2}$. These $Q^{2}$ bins were also used in determining any $y$ dependence. Figure 1.35 (left) shows the total number of events expected for CC interactions in $e^{-} p$ scattering at $\sqrt{s}=141 \mathrm{GeV}$ and $\mathcal{L}=10 \mathrm{fb}^{-1}$, binned in $x$.

Typical rates in NC scattering are up to $1 \mathrm{kHz}$, as shown in the right part of Fig. 1.35 for $\sqrt{s}=161 \mathrm{GeV}$. The highest rate occurs in the forward direction of the electron beam. Here, pipeline electronics will likely be necessary in order to avoid significant deadtime effects. 


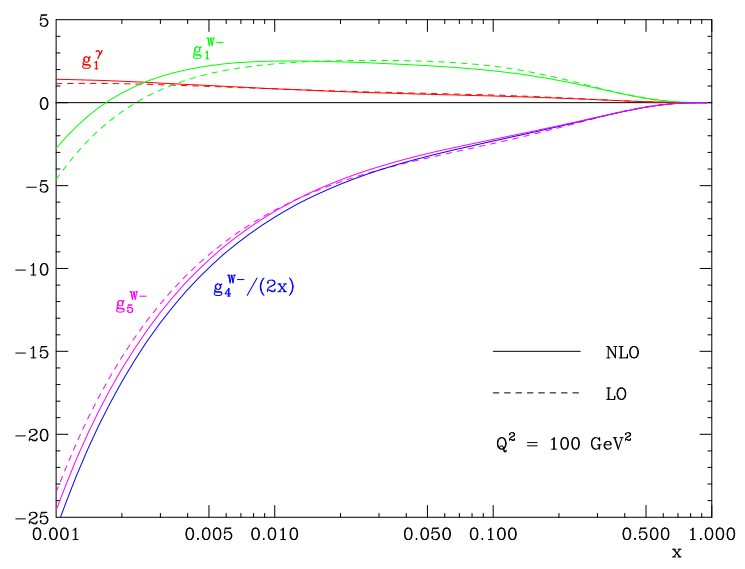

Figure 1.36. CC spin dependent structure functions $g_{1}^{W^{-}}, g_{5}^{W^{-}}$, and $g_{4}^{W^{-}} / 2 x$, at $Q^{2}=100 \mathrm{GeV}^{2}$. The dashed lines show the LO results (the one for $g_{4}^{W^{-}} / 2 x$ is not shown in this case, since it coincides with that for $g_{5}^{W^{-}}$), while the solid curves are NLO. For comparison, we also show the electromagnetic $g_{1}^{\gamma}$.

\section{Polarized Parton Distributions from CC Interactions}

As follows from Eq. (1.29), CC processes in electron scattering off polarized targets offer a unique method to extract combinations of $\Delta u+\Delta c$ and $\Delta \bar{d}+\Delta \bar{s}$. With positron beams, one could also extract $\Delta d+\Delta s$ and $\Delta \bar{u}+\Delta \bar{c}$. For the present analysis, we have assumed a $100 \%$ polarized electron/positron source. As mentioned before, we have assumed only $10 \mathrm{fb}^{-1}$ integrated luminosity, making our estimates somewhat conservative.

In Fig. [1.36 we show the spin structure functions $g_{1}^{W^{-}}, g_{5}^{W^{-}}$, and $g_{4}^{W^{-}} / 2 x$, at $Q^{2}=$ $100 \mathrm{GeV}^{2}$, using the PDFs of [25]. Results are shown both at LO (dashed) and at NLO (solid). One observes that the NLO corrections are well under control. To guide the eye, also the ordinary electromagnetic structure function $g_{1}^{\gamma}$ is shown. Figure 1.37(left) displays the asymmetry $A_{W^{-}}$for $\mathrm{CC} e^{-} \vec{p}$ scattering, as function of $x$. Different data points at same $x$ correspond to different bins in $Q^{2}$. As mentioned above, we have chosen here 20 bins in $Q^{2}$, spaced logarithmically from $2 \mathrm{GeV}^{2}$ to $5000 \mathrm{GeV}^{2}$. The lower asymmetries correspond to the lower bins in $Q^{2}$. Thanks to the simple structure of the LO expressions for the cross sections, the asymmetries in CC interactions become very large in the valence region, much larger than those in the $\mathrm{NC}$ case to be discussed below. On the other hand, as we saw in Figs. 1.34 and 1.35, event rates are much more suppressed at lower $Q^{2}$ and therefore $x$. The right part of Fig. 1.37 gives the resulting values for the relative uncertainty $\delta A_{W^{-}} / A_{W^{-}}$ of the asymmetry. Here we have summed over all $Q^{2}$ bins. The results shown look very promising, with better than $10 \%$ measurements appearing feasible all the way down to $x \sim 10^{-2}$. It is worth keeping in mind that relative polarimetry uncertainties at an EIC are also expected to be at the $0.5-1 \%$ level for electrons and $2-3 \%$ level for hadrons, so that these might become the dominant sources of uncertainty in the regions where the statistical $\delta A_{W^{-}} / A_{W^{-}}$is very small, especially at high $x$.

Using Eq. (1.29), the asymmetries give direct access to the polarized quark and antiquark distributions. As we discussed, higher-order QCD corrections (and also Cabibbosuppressed contributions) will somewhat modify the expressions in Eq. (1.29). However, for a first estimate use of Eq. (1.29) as a means to gauge the sensitivity to the distributions 

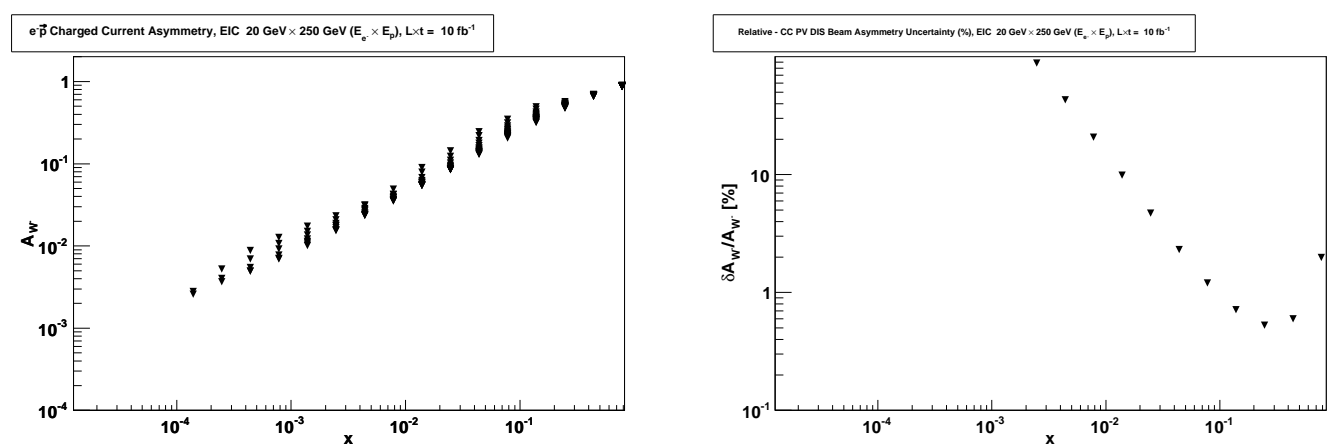

Figure 1.37. Left: spin asymmetry for $\mathrm{CC} e^{-} \vec{p}$ scattering, as function of $x$ for various bins in $Q^{2}$. Right: resulting relative uncertainties of the asymmetry.

is justified. The additional contributions will not make a qualitative difference and can be systematically included in future studies. If furthermore full knowledge of the unpolarized parton distributions is assumed, then extraction of the sums of the two up-type quarks and down-type anti-quarks can be performed by a linear fit in $(1-y)^{2}$. The results of such fits are shown for electron and positron running in Figs. 1.38 and 1.39, respectively. We note that if a polarized deuterium or ${ }^{3} \mathrm{He}$ beam were available, additional opportunities would arise; $e^{-} \vec{n}$ scattering would probe the combinations $\Delta u+\Delta d+2 \Delta c$ and $\Delta \bar{d}+\Delta \bar{u}+2 \Delta \bar{s}$. At larger $x$ where the sea quarks are suppressed relative to the valence quarks, $e^{-} \vec{p}$ and $e^{-} \vec{n}$ scattering could be used to separate the valence polarizations.

\section{Structure Functions and Polarized PDFs from NC Interactions}

Again we first show the spin-dependent structure functions; see Figure 1.40. As the contributions from pure $Z$-exchange are small, we only consider the electromagnetic $g_{1}^{\gamma}$, and the $\gamma-Z$ interference contributions $g_{1}^{\gamma Z}$ and $g_{4,5}^{\gamma Z}$, whose expressions were given in Eq. (1.25).

The left part of Fig. 1.41 shows the parity-violating spin asymmetry in Eq. (1.22), obtained for a polarized lepton beam scattering off an unpolarized proton beam, as function of $x$ in various different $Q^{2}$ bins. The lower (upper) asymmetries correspond to $Q^{2} \sim 2 \mathrm{GeV}^{2}$ $\left(Q^{2} \sim 4000 \mathrm{GeV}^{2}\right)$. As one can see, typical asymmetries range from $10^{-4}$ to 0.1 . The right part of the figure gives the resulting values for the relative uncertainty $\delta A_{\text {beam }} / A_{\text {beam }}$ of the asymmetry. Here we have summed over all $Q^{2}$ bins and assumed an integrated luminosity of $\mathcal{L}=100 \mathrm{fb}^{-1}$. The relative uncertainty is found to be near $2 \%$ over a relatively wide range in $x$; the relative electron polarization uncertainty achievable with modern polarimetry techniques should be better than this.

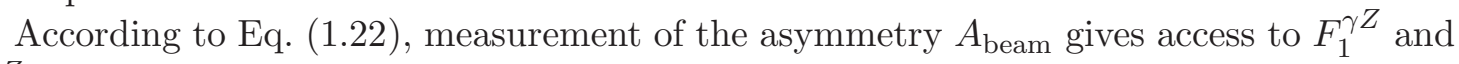
$F_{3}^{\gamma Z}$. Figure 1.42 presents the expected relative uncertainties for these structure functions, corresponding to the results shown in Fig. 1.41. Figure 1.43 shows the corresponding result for the case of $\vec{e}^{-} D$ scattering, for the structure function $F_{1}^{\gamma Z}$. Due to the suppression by the electron vector coupling, the uncertainty of $F_{3}^{\gamma Z}$ is about an order of magnitude worse than that of $F_{1}^{\gamma Z}$. The sensitivity is maximized in the region of $x \sim 0.01-0.4$. The approved PVDIS experiment using the SoLID spectrometer in Hall A at Jefferson Lab [202] anticipates achieving an extraction of $A_{\text {beam }}$ with relative accuracy $\approx 0.5-1 \%$ over several bins in $x$ in the range of $0.2 \leq x \leq 0.7$, both from proton and deuterium targets. The 

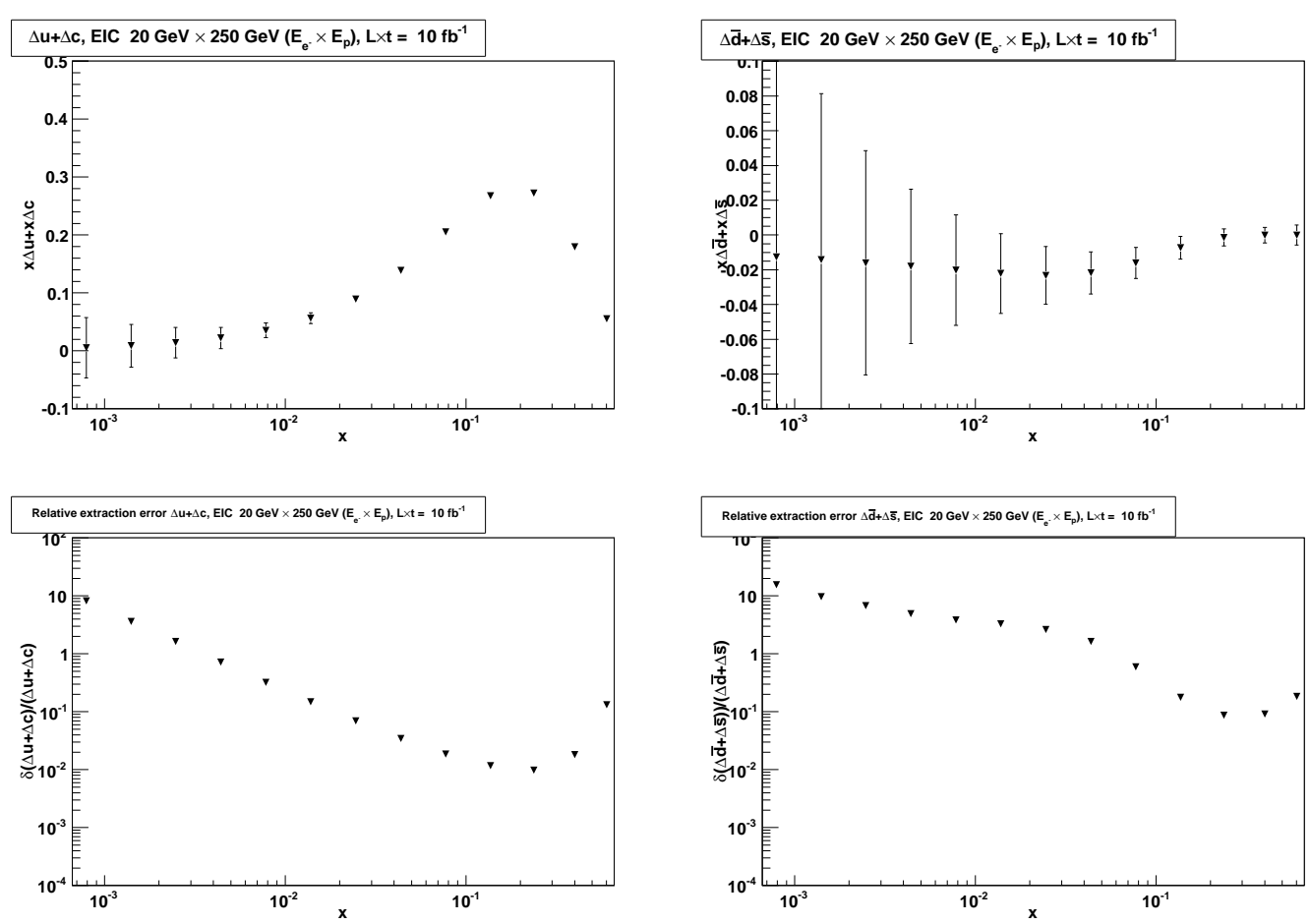

Figure 1.38. Top: LO extraction of polarized quark and anti-quark distributions from the spin asymmetry for $\mathrm{CC} e^{-} \vec{p}$ scattering. Bottom: Corresponding relative uncertainties of the extracted distributions.
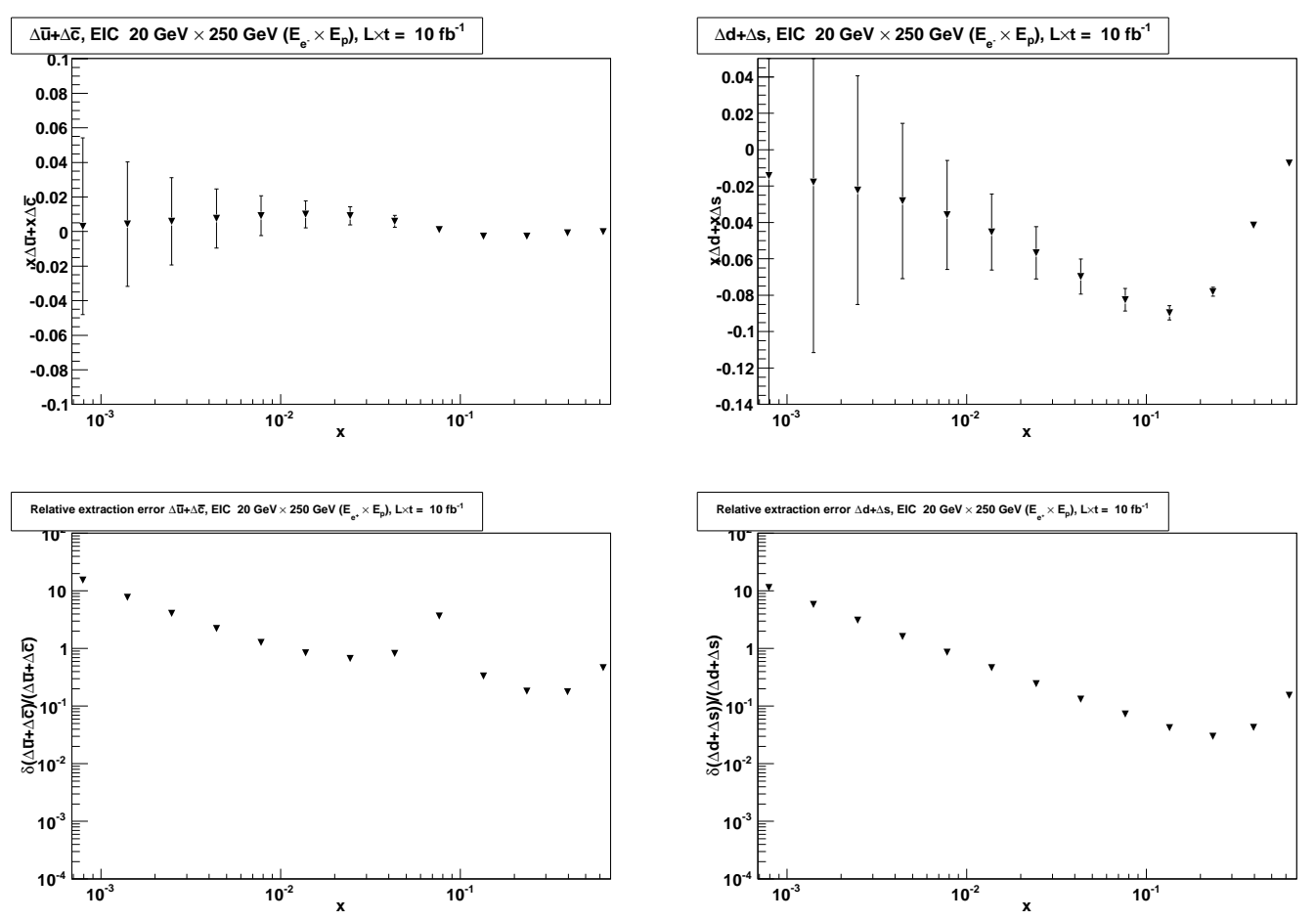

Figure 1.39. Same as Fig. 1.38 but for $e^{+} \vec{p}$ scattering. 


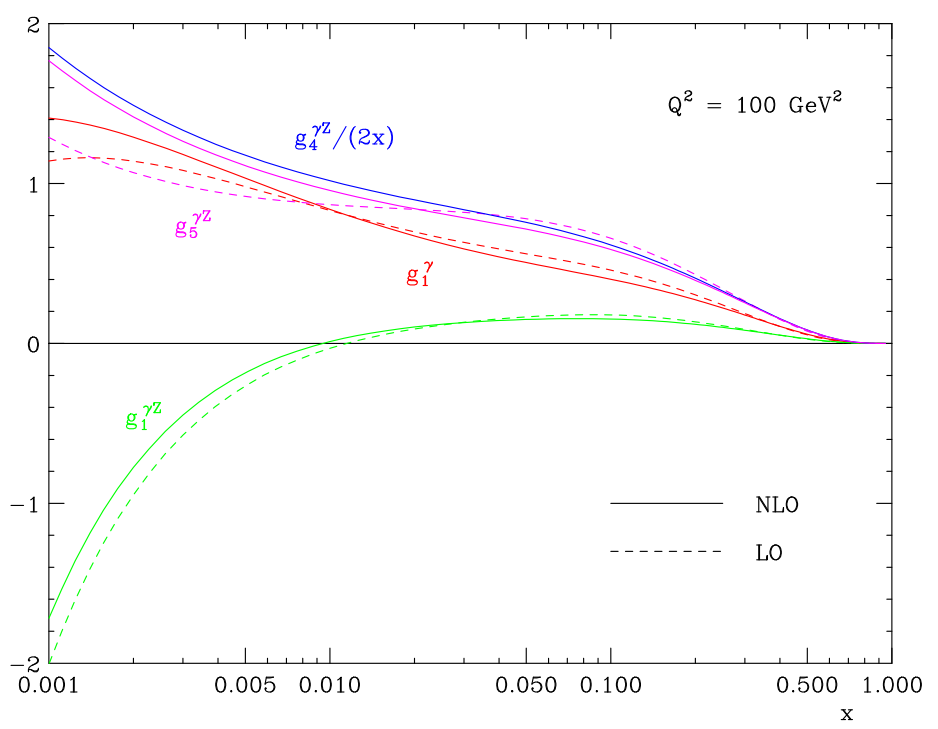

Figure 1.40. NC spin-dependent structure functions for $\gamma-Z$ interference, at $Q^{2}=100 \mathrm{GeV}^{2}$, calculated at LO (dashed) and NLO (solid), using the polarized PDFs of [25.
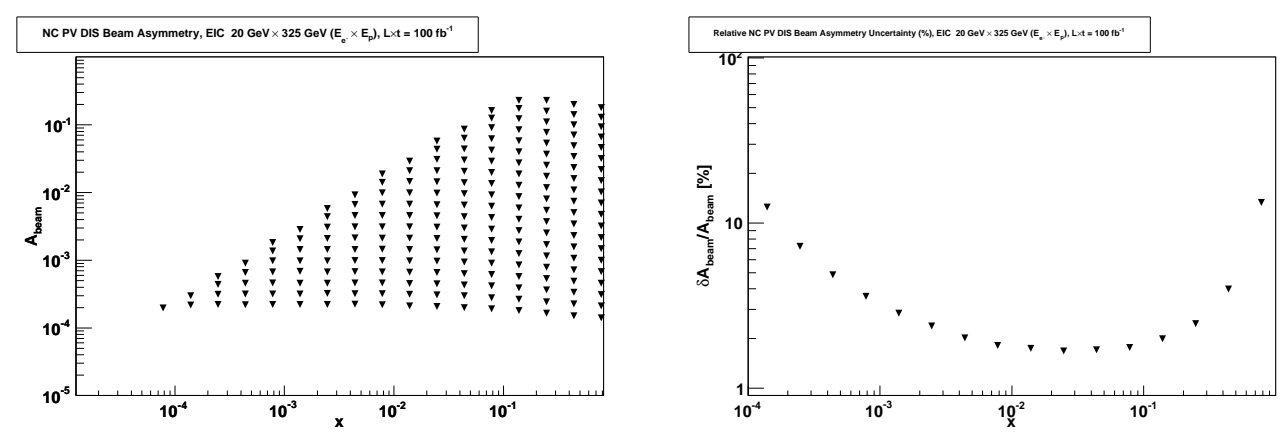

Figure 1.41. Left: Parity violating NC spin asymmetries for polarized electrons on unpolarized protons, binned logarithmically in $x$ and $Q^{2}$. Right: Resulting relative uncertainties of the asymmetry.
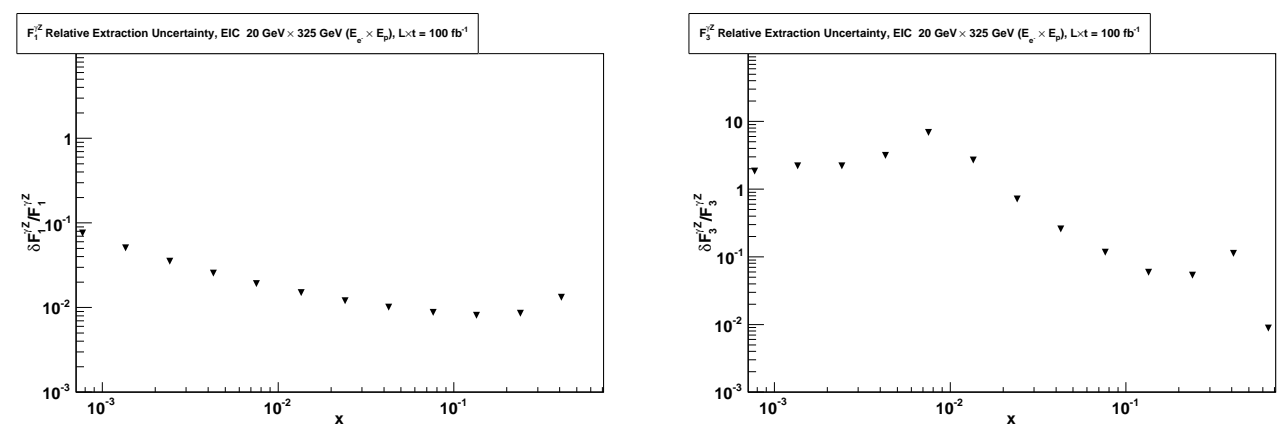

Figure 1.42. Relative uncertainties of $F_{1}^{\gamma Z}$ (left) and $F_{3}^{\gamma Z}$ (right) extracted from NC $\vec{e}^{-} p$ scattering. 


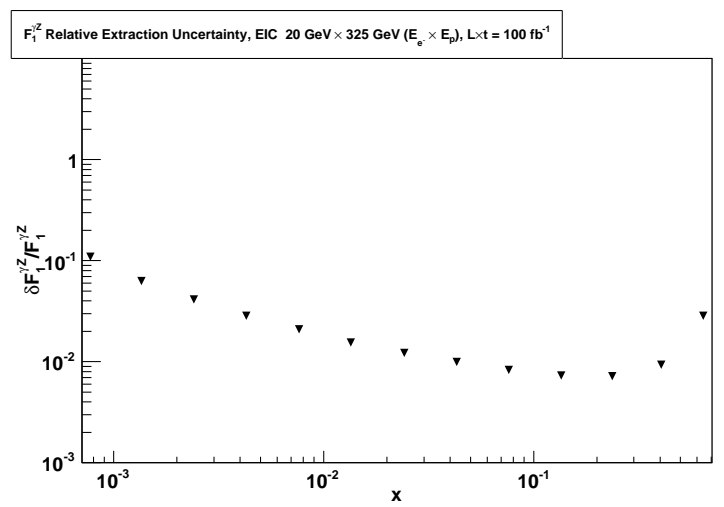

Figure 1.43. Same as left part of Fig. 1.42, but for $\vec{e}^{-} \mathrm{D}$ scattering.
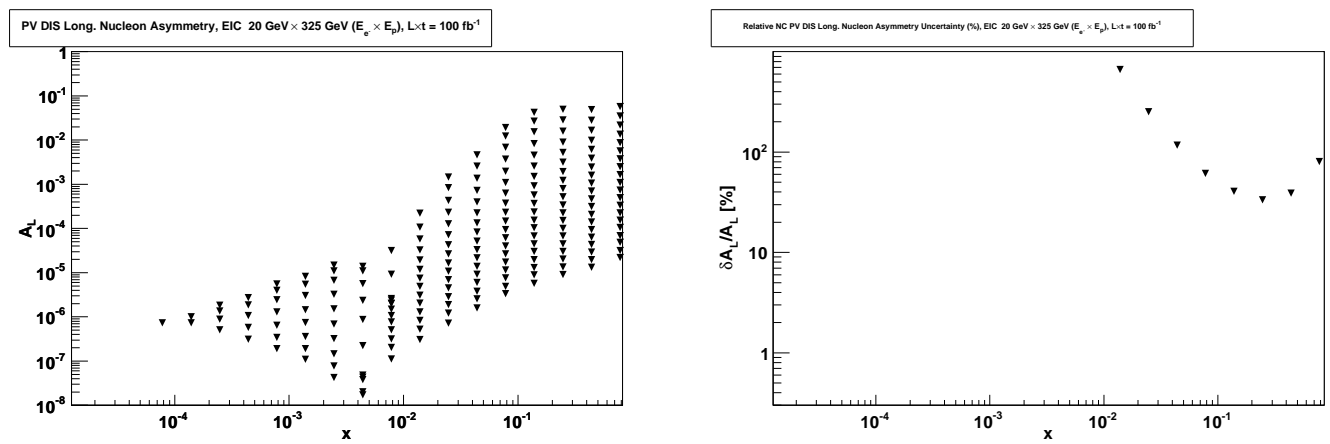

Figure 1.44. Same as Fig. 1.41, but for unpolarized electrons on polarized protons.

products of the quarks' electric charges and their vector charges are approximately equal for up-type and down-type quarks, $e_{u} g_{V}^{u} \approx e_{d} g_{V}^{d} \approx 0.1$. Therefore, one has from Eq. (1.21) that $F_{1}^{\gamma Z} \propto u+\bar{u}+d+\bar{d}+s+\bar{s}$, both for proton and deuterium. On the other hand, for the corresponding products of the charges and axial charges one finds $e_{u} g_{A}^{u} \approx 2 e_{d} g_{A}^{d}$, and hence in the valence region $F_{3}^{\gamma Z} \propto 2 u_{v}+d_{v}$ for protons and $\propto u_{v}+d_{v}$ for deuterium. While $F_{3}^{\gamma Z}$ could thus give a clean separation of the $u$ and $d$ valence distributions, its contribution to the beam asymmetry is unfortunately suppressed.

Of significant interest are measurements of $g_{1}^{\gamma Z}$ and $g_{5}^{\gamma Z}$, which contain complementary information on the polarized PDFs. Similarly to what we discussed for the case of $F_{1}^{\gamma Z}$, one finds that to a good approximation $g_{1}^{\gamma Z} \propto \Delta u+\Delta \bar{u}+\Delta d+\Delta \bar{d}+\Delta s+\Delta \bar{s}$, which would in principle make this structure function an complementary probe of the quark and anti-quark singlet and spin contribution to the proton spin. Furthermore, $g_{5}^{\gamma Z}$ offers probes of the valence regime. According to Eq. (1.23), $g_{1}^{\gamma Z}$ and $g_{5}^{\gamma Z}$ may be accessed by flipping the proton helicity while leaving the electron polarization unchanged. The corresponding spin asymmetries, obtained after summing over the electron helicities, are unfortunately overall much smaller than their counterparts with polarized electron and unpolarized proton. They are shown in Fig. 1.44, along with the their expected relative uncertainties, computed again for $\mathcal{L}=100 \mathrm{fb}^{-1}$. The best sensitivity is in the valence quark region, $x>0.1$. Even here, it remains at the $10 \%$ level. This directly translates into similar uncertainties for the structure functions $g_{1}^{\gamma Z}$ and $g_{5}^{\gamma Z}$, which are shown in Fig. 1.45, In the valence region, where 

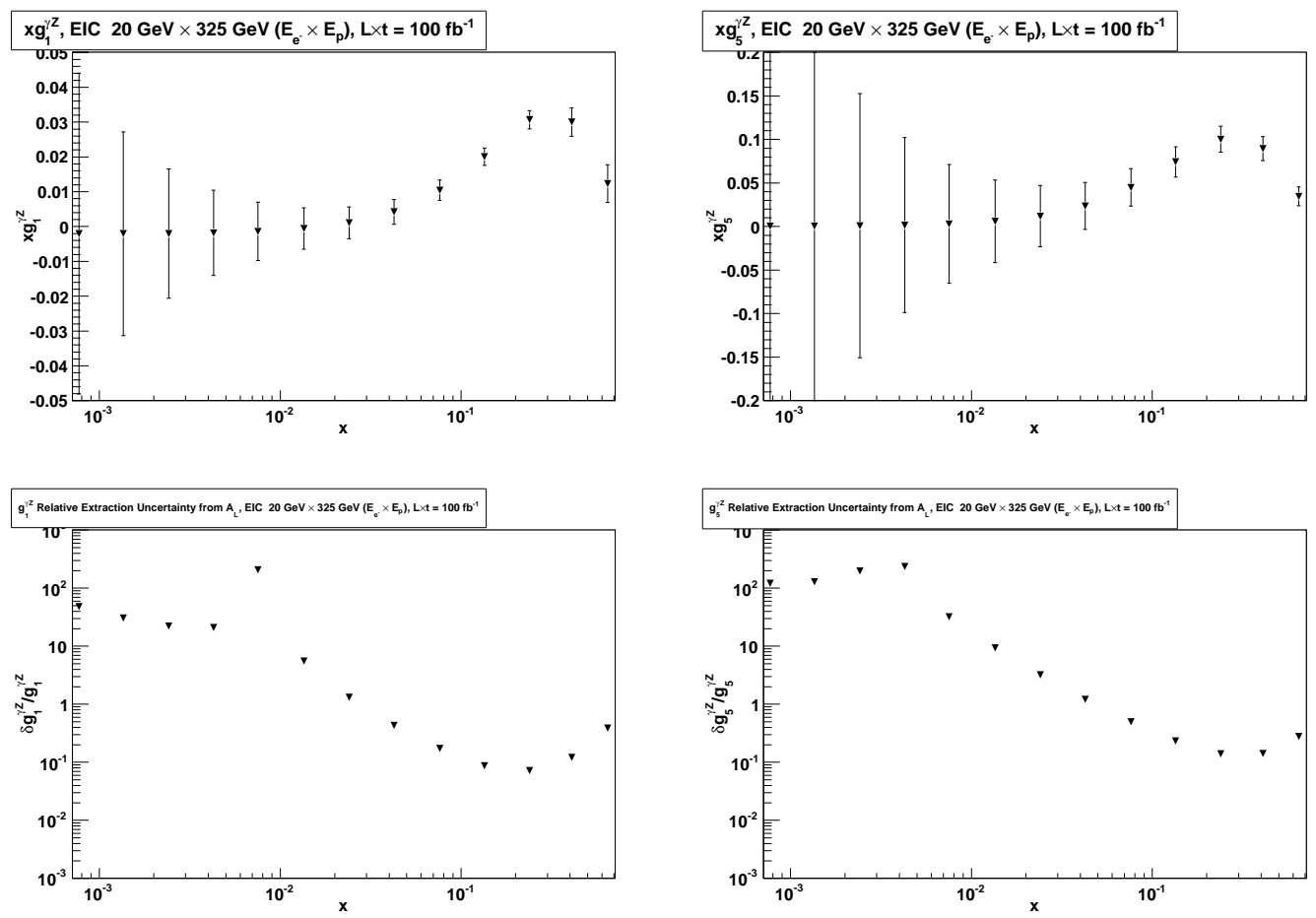

Figure 1.45. Structure functions $g_{1}^{\gamma Z}$ and $g_{5}^{\gamma Z}$ (top) and their relative uncertainties resulting from Fig. 1.44 (bottom).

sea quarks are irrelevant, we have $g_{1}^{\gamma Z} \propto \Delta u_{v}+\Delta d_{v}$ and $g_{5}^{\gamma Z} \propto 2 \Delta u_{v}+\Delta d_{v}$, which may provide a separation of $\Delta u$ and $\Delta d$.

Finally, assuming perfect knowledge of $\Delta u$ and $\Delta d$ and their anti-quark distributions from other sources, one might ask if an extraction of $\Delta s+\Delta \bar{s}$ from $g_{1}^{\gamma Z}$ and $g_{5}^{\gamma Z}$ could be possible. This quantity, and in particular its integral, is a key ingredient to nucleon spin structure and for understanding why quarks and anti-quarks combined appear to carry little of the proton spin. Constraints on $\Delta s+\Delta \bar{s}$ are presently available from an $\mathrm{SU}(3)$ symmetry analysis of hyperon $\beta$-decays, and from kaon production in semi-inclusive DIS, which are both inflicted with sizable uncertainties and in fact show some tension (for discussion, see [26]). The result for the extraction of $\Delta s+\Delta \bar{s}$ from electroweak DIS at the EIC is shown in Fig. 1.46. As can be seen, a non-zero measurement would be challenging for the assumed $100 \mathrm{fb}^{-1}$ integrated luminosity. Nevertheless, this measurement might become interesting if independent methods of extracting $\Delta s+\Delta \bar{s}$ were to provide surprising results. If this measurement is deemed sufficiently interesting and important, larger integrated luminosities will indeed help, since the measurement will continue to remain statistics limited, provided relative hadron polarization errors can be kept at the $3 \%$ level or better.

\subsubsection{Summary}

We have performed a basic analysis of the potential of an EIC in terms of measurements of structure functions in electroweak NC and CC scattering. Precise measurements of the CC functions $F_{1}^{W}, F_{3}^{W}, g_{1}^{W}$, and $g_{5}^{W}$ become feasible with a relatively modest integrated luminosity. These measurements will greatly aid the flavor decomposition of polarized and 


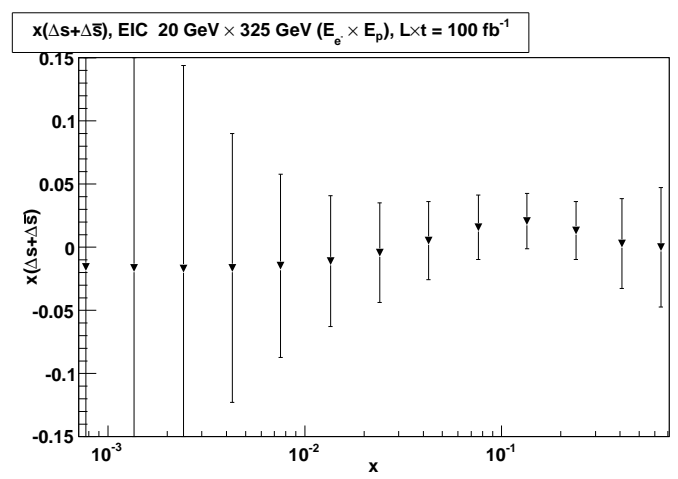

Figure 1.46. Results for the $x(\Delta s+\Delta \bar{s})$ distribution extracted from the $A_{\mathrm{L}}$ spin asymmetry under the assumption that all other helicity distributions are known.

unpolarized PDFs in the region $x \gtrsim 0.01$. NC structure functions become accessible with good precision at high integrated luminosities. Measurements of $F_{1}^{\gamma Z}$ and $F_{3}^{\gamma Z}$ seem to be of limited use in improving present or approved measurements. At the highest luminosities and center of mass energies, $g_{1}^{\gamma Z}$ and $g_{5}^{\gamma Z}$ become accessible; these structure functions have never before been measured. The combined analysis of the new $\mathrm{CC}$ and NC structure functions with electrons and positrons as well as with polarized protons and neutrons at these highest luminosities could potentially open a new window into precision QCD tests of the spin structure of the nucleon; this will be the focus of future experimental and theoretical investigations. 


\subsection{Charged current charm production and the strange sea}

Marco Stratmann

\subsubsection{Basic idea}

The leading order contribution to $\mathrm{CC}$ charm production in $e^{+} p$ DIS is given by the $\mathcal{O}\left(\alpha_{s}^{0}\right)$ parton model process $W^{+} s^{\prime} \rightarrow c$, where $s^{\prime}$ denotes the Cabibbo-Kobayashi-Maskawa (CKM) "rotated" combination $s^{\prime} \equiv\left|V_{c s}\right|^{2} s+\left|V_{c d}\right|^{2} d$. Due to the smallness of $\left|V_{c d}\right|^{2}[3$, the process is expected to be essentially sensitive to the strange sea content. Only at large $x$, where quark sea contributions are less relevant, the $\left|V_{c d}\right|^{2}$ suppression is balanced by the valence enhancement of the well-known $d(x)$ density. Likewise, in $e^{-} p$ DIS, the process $W^{-} \bar{s}^{\prime} \rightarrow \bar{c}$ predominantly probes the anti-strange density $\bar{s}(x)$. With a polarized proton beam one can access also $\Delta s(x)$ and $\Delta \bar{s}(x)$.

Current determinations of $s(x)$ rely mainly on fixed-target neutrino scattering off nuclear targets with potentially large uncertainties, see Fig. 1.9 in Sec. 1.5. Much less is known about the longitudinally polarized $\Delta s(x)$ so far, see Sec. 1.10. Due to the limited luminosity and charm detection efficiency, charm production in CC DIS could not be studied at HERA. CC DIS would provide an independent way to extract the unpolarized and polarized strange sea distributions at much larger scales, typically $Q \sim M_{W}$, than probed in semi-inclusive kaon production, cf. Sec. 1.5. On the downside, such a measurement requires also a positron beam, though not polarized.

Next-to-leading order QCD corrections also complicate the simple picture for CC charm production and may deteriorate the sensitivity to strangeness. Apart from the $\mathcal{O}\left(\alpha_{s}\right)$ corrections to the LO process $W^{+} s^{\prime} \rightarrow c$, the genuine NLO, gluon induced subprocess $W^{+} g \rightarrow c \bar{s}^{\prime}$ has to be taken into account as well. It contributes significantly to the charm production cross section in certain regions of phase space and hence dilutes the sensitivity to the strange sea. In addition, a proper theoretical calculation also needs to take into account the mass of the produced heavy (charm) quark, as was also discussed in the context of $F_{2, L}^{c}$ in Sec. 1.7. In order to make contact with experiment, a fully inclusive calculation [203, 111] is not entirely sufficient, and one should compute also the momentum $z$ spectrum of the detected charmed $D$ mesons. In the unpolarized case this was achieved in [204]. The corresponding polarized results can be found in Ref. 205]. Imposing a lower cut $z_{\min }$ on the $D$ meson momentum fraction was shown to considerably reduce gluon-initiated NLO contributions and enhance the sensitivity to the strange sea.

Concerning the mass $m_{c}$ of the charm quark, it turns out that the naive "rescaling prescription" [206], i.e., $s(x) \rightarrow s(\xi)$ where $\xi \equiv x\left(1+m_{c}^{2} / Q^{2}\right)$, applies also at NLO accuracy as it allows for a consistent factorization of all initial-state collinear singularities.

\subsubsection{Sensitivity to the Strange Sea}

So far, detailed phenomenological studies have been provided only for HERA kinematics [205], and they still need to be updated for EIC kinematics. However, these projections are sufficient to demonstrate the idea of the measurement and give a rough estimate of the size of cross sections and spin asymmetries. From the studies of inclusive CC electroweak DIS structure functions in Sec. 1.12 we already know that such measurements appear to be feasible at an EIC despite its lower c.m.s. energy than HERA even with moderate integrated luminosities of about $10 \mathrm{fb}^{-1}$. 

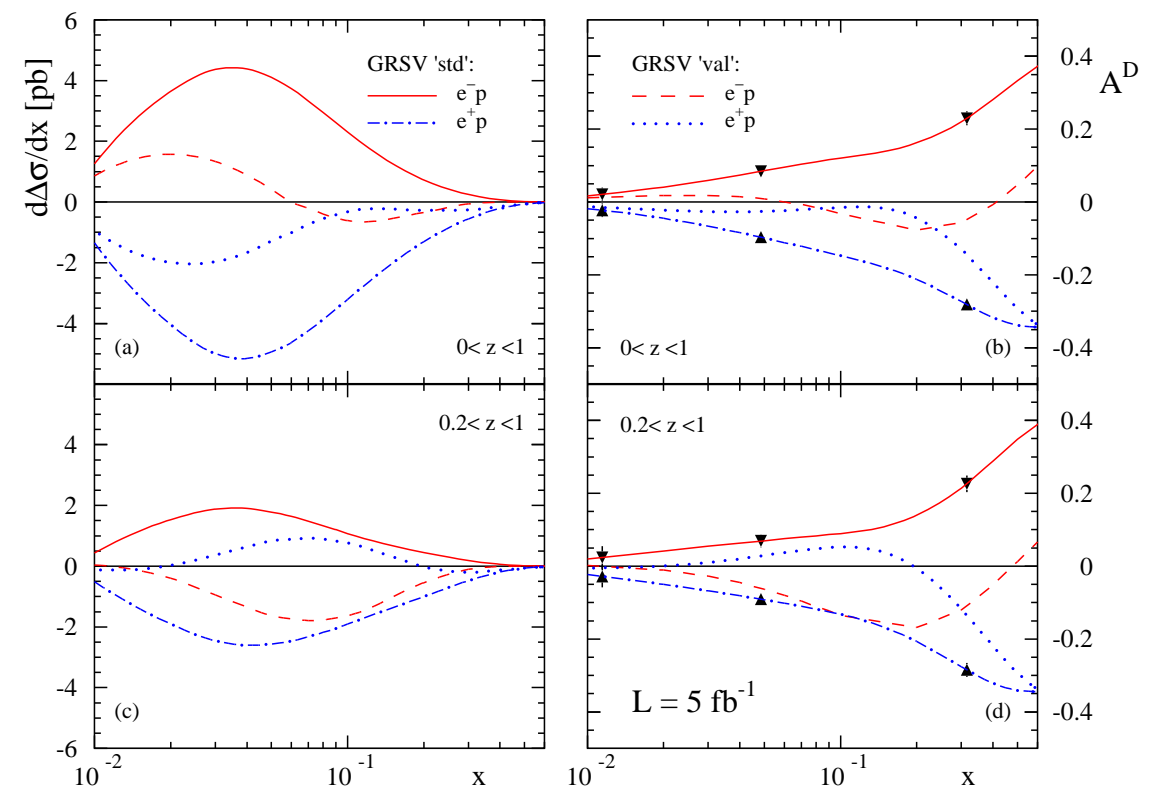

Figure 1.47. The $z$ integrated polarized cross section for CC charm production in $e^{-} p$ and $e^{+} p$ collisions and the corresponding spin asymmetry $A^{D}$ for $(\mathbf{a}, \mathbf{b})$ : $0<z<1$, (c,d): $0.2<z<1$, using the GRSV "std" and "val" sets of PDFs. Projected uncertainties are for $70 \%$ polarization, $100 \%$ charm detection efficiency, and an integrated luminosity of $5 \mathrm{fb}^{-1}$.

As an example, Fig. 1.47 shows the sensitivity of $\mathrm{CC}$ charm ( $D$ meson) production in $e^{-} p$ and $e^{+} p$ collisions at $\sqrt{S}=300 \mathrm{GeV}, Q^{2}>500 \mathrm{GeV}^{2}$, and $0.01 \leq y \leq 0.9$, to the choice of $\Delta s$. The momentum fraction of the detected $D$ meson has been integrated using $z_{\text {min }}=0$ (upper row) and 0.2 (lower row). The GRSV valence set [24] has a very small positive $\Delta s(x)$ in the relevant region $x \gtrsim 0.01$, roughly comparable to what is nowadays obtained from fixed target SIDIS data, e.g., in the DSSV analysis [25, 26]; see Sec. 1.10, On the contrary, the GRSV standard set has a sizable negative strangeness polarization as favored by fits including only inclusive DIS data [23]. Other PDFs, in particular the gluon density, are very similar in both GRSV sets. Note that $\Delta s(x)=\Delta \bar{s}(x)$ is assumed in all current polarized PDF analyses due to the lack of data constraining them separately.

The solid and dashed lines in Fig. 1.47 show the results for $e^{-} p$ scattering for GRSV standard and valence PDFs, respectively. Within the projected statistical uncertainties, obtained for $70 \%$ proton polarization, $100 \%$ charm detection efficiency, and an integrated luminosity of $5 \mathrm{fb}^{-1}$, differences in $\Delta \bar{s}(x)$ can be easily resolved. The dot-dashed and dotted lines show the results for a corresponding measurement with positron beams. Having results for both $W^{-}$and $W^{+}$exchange, one should be able to study a possible asymmetry in $\Delta s(x)-$ $\Delta \bar{s}(x)$. The results presented here need to be backed up with more detailed simulations of $\mathrm{CC}$ charm production for EIC kinematics. 


\title{
1.14 Photoproduction processes at an EIC
}

\author{
Hubert Spiesberger, Marco Stratmann
}

The production of hadronic final states in $e p$ collisions is dominated by photoproduction where the electron is scattered by a small angle producing photons of almost zero virtuality $\left(Q^{2} \simeq 0\right)$. At $\mathrm{LO}$ of $\mathrm{pQCD}$, the dominant process for the production of high- $p_{T}$ hadrons, jets, or heavy quarks is often photon-gluon fusion, $\gamma g \rightarrow q \bar{q}$. Here, the photon interacts directly with a gluon from the nucleon. Besides this so-called "direct" photoproduction channel, the scattering can proceed also via "resolved" processes. In this case, the photon acts as a source of partons which interact with the partons in the nucleon through any of the standard $2 \rightarrow 2$ LO QCD hard scattering processes such as $g g \rightarrow g g$ or $q \bar{q} \rightarrow q \bar{q}$. The large number of possible subprocesses can make the resolved contribution sizable in certain regions of phase space. Examples for a direct and a resolved process are shown in Fig. 1.48,

At LO, the two interaction mechanisms in Fig. 1.48 both contribute at $\mathcal{O}\left(\alpha_{e m} \alpha_{s}\right)$ but otherwise appear to be independent. Starting from NLO, however, the separation into direct and resolved contributions becomes factorization scheme dependent. This is due to soft and collinear singularities appearing in a perturbative approach. These singularities have to be identified and consistently factorized into non-perturbative PDFs of the nucleon and the photon. This procedure is not unique, and it is therefore important that the direct and resolved parts are treated together consistently. Only their sum is an experimentally meaningful and measurable cross section. For a theoretical review on photoproduction, see, e.g., Ref. 208].

The differential cross section for electron-nucleon scattering, $d \sigma_{e N}$, at a c.m.s. energy $\sqrt{s}$ is related to the photoproduction cross section $d \sigma_{\gamma N}$ through

$$
d \sigma_{e N}(\sqrt{s})=\int_{y_{\min }}^{y_{\max }} d y f_{e \gamma}(y) d \sigma_{\gamma N}(y \sqrt{s}) .
$$

Here, $f_{e \gamma}$ is the energy spectrum of the exchanged photon which in the Weizsäcker-Williams approximation is given by

$$
f_{e \gamma}(y)=\frac{\alpha_{e m}}{2 \pi}\left[\frac{1+(1-y)^{2}}{y} \ln \frac{(1-y) Q_{\max }^{2}}{y^{2} m_{e}^{2}}+2(1-y)\left(\frac{y m_{e}^{2}}{(1-y) Q_{\max }^{2}}-\frac{1}{y}\right)\right] .
$$

The photon flux $f_{e \gamma}$ depends $y=E_{\gamma} / E_{e} . Q_{\max }$ and the range $y_{\min } \leq y \leq y_{\max }$ are determined by cuts in the experimental analysis. Typically, a lower cut $y_{\min }=\mathcal{O}(0.1)$ is

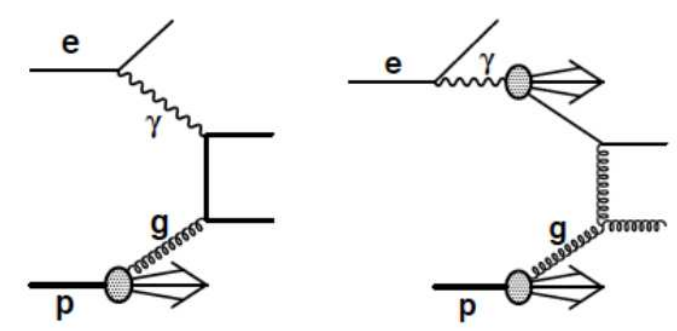

Figure 1.48. Example of a gluon-initiated direct and resolved contributions to photoproduction at LO (taken from Ref. 207]). 
applied in order to exclude low-mass hadronic final states, and an upper limit on $y$, e.g., $y_{\max }=0.7 \div 0.9$, is used to reduce the kinematic range where radiative corrections are expected to be large.

The photoproduction cross section is then obtained as the sum of its direct and resolved parts, $d \sigma_{\gamma N}=d \sigma_{\gamma N}^{\text {dir }}+d \sigma_{\gamma N}^{\text {res }}$, as convolutions $\otimes$ of the appropriate partonic hard scattering cross sections $d \sigma_{a b}$ with the PDFs $f_{a / \gamma}\left(x_{\gamma}\right)$ and $f_{b / N}\left(x_{N}\right)$ of the photon and nucleon, respectively, at a factorization scale $\mu_{f}$, i.e.,

$$
d \sigma_{\gamma N}^{\mathrm{res}}=\sum_{a, b} f_{a / \gamma}\left(x_{\gamma}, \mu_{f}\right) \otimes f_{b / N}\left(x_{N}, \mu_{f}\right) \otimes d \sigma_{a b}\left(x_{\gamma}, x_{N}, \mu_{f}\right)
$$

$d \sigma_{\gamma N}^{\operatorname{dir}}$ can be obtained from (1.33) by replacing the photon PDFs by a $\delta$-function and considering only photon-parton scattering processes $d \sigma_{\gamma b}$ in the sum.

The resolved process is accompanied by a hadronic remnant of the photon which carries the fraction $1-x_{\gamma}$ of the photon energy. At LO, the presence of a hadronic remnant could be used to distinguish different event topologies for the two mechanisms. In addition, for two-jet final states $x_{\gamma}$ can be reconstructed experimentally from the measured transverse momenta and rapidities of the jets. It is customary to define

$$
x_{\gamma}^{\text {obs }} \equiv\left(E_{\mathrm{T}}^{\mathrm{jet}_{1}} e^{-\eta^{\mathrm{jet}_{1}}}+E_{\mathrm{T}}^{\mathrm{jet}_{2}} e^{-\eta^{\mathrm{jet}_{2}}}\right) /\left(2 y E_{e}\right) .
$$

However, at higher orders of pQCD, initial- and final-state radiation of additional partons will also give rise to hadrons emitted in the direction of the incoming photon. Moreover, non-perturbative hadronization may contribute to the appearance of hadrons in the same kinematic region. Both effects lead to a reduction of the experimentally determined value of $x_{\gamma}$. Therefore a unique separation of the direct and resolved parts is not possible anymore. Nevertheless, the variable $x_{\gamma}$ can still be used to define kinematic regimes where direct (large $x_{\gamma}$ ) or resolved (small $x_{\gamma}$ ) contributions dominate.

At HERA, photoproduction has been used to test pQCD and the presence of both direct and resolved photon processes for final-states comprising hadrons, jets, prompt photons, and heavy quarks. Generally, the data are well described by NLO calculations in regimes expected to be dominated by the direct process. Kinematic regions where resolved processes are sizable are somewhat less well described; for a review see, e.g., 209]. This is mainly due to the fact that the photon PDFs needed for the calculation of the resolved contribution are significantly less well constrained by data than the partonic structure of protons. Only data for inclusive DIS off a quasi-real photon target, i.e., $\gamma^{*}\left(Q^{2}\right) \gamma$ scattering in $e^{+} e^{-}[210$, have been used in fits of photon PDFs so far, see, e.g., [211. No attempts have been made to perform global analyses or to quantify uncertainties at a level similar to current fits of proton PDFs. Any additional, more precise data are therefore of vital importance for an improved understanding of the theoretical description of photoproduction processes and a reliable determination of photon PDFs. The latter are of great phenomenological relevance at a possible future linear $e^{+} e^{-}$collider to describe processes involving quasi-real photons.

The next two sections show some examples how an EIC can contribute to further our knowledge of photoproduction processes both in unpolarized and in polarized electronproton scattering. 


\title{
1.15 Expectations for charm quark photoproduction
}

\author{
Hubert Spiesberger
}

The description of heavy quark production in the framework of perturbative QCD is complicated due to the presence of several large scales, like the transverse momentum $p_{T}$ of the produced charmed meson, the momentum transfer $Q$ in DIS, or the mass of the produced heavy hadron. Depending on the kinematic range considered, the mass $m_{c}$ of the charm quark may have to be taken into account. Different calculational schemes (see, e.g. [212, 213], and references therein) have been developed to obtain predictions from pQCD, depending on the specific kinematical region and the relative importance of the different scales.

In the case of relatively small transverse momentum, $p_{T} \lesssim m_{c}$, the fixed-flavor number scheme (FFNS) is usually applied. Here one assumes that the light quarks and the gluon are the only active flavors and the charm quark appears only in the final state. The charm quark mass can explicitly be taken into account together with the $p_{T}$ of the produced heavy meson; this approach is therefore expected to be reliable when $p_{T}$ and $m$ are of the same order of magnitude.

In the complementary kinematical region where $p_{T} \gg m_{c}$, calculations are usually based on the zero-mass variable-flavor-number scheme (ZM-VFNS) where $m_{c}=0$ and the charm quark acts as an active parton with its own PDF; see also Sec. 1.7. The charmed meson is produced not only by fragmentation from the charm quark but also from the light quarks and the gluon. The fragmentation process is described with the help of scale-dependent fragmentation functions (FFs), $D(z, \mu)$, which determine the probability that the produced heavy meson carries the fraction $z$ of the momentum of the parton it is produced from. The predictions obtained in this scheme are expected to be reliable only in the region of large $p_{T}$ since all terms of the order $m_{c}^{2} / p_{T}^{2}$ are neglected in the hard scattering cross section.

A unified scheme that combines the virtues of the FFNS and the ZM-VFNS is the so-called general-mass variable-flavour-number scheme (GM-VFNS) [212, 213. In this approach the large logarithms $\ln \left(p_{T}^{2} / m_{c}^{2}\right)$ are factorized into the PDFs and FFs and summed to all orders by the well-known DGLAP evolution equations. At the same time, massdependent power corrections are retained in the hard-scattering cross sections, as in the FFNS. In order to conform with standard $\overline{\mathrm{MS}}$ factorization, finite subtraction terms must be supplemented to the results of the FFNS. As in the ZM-VFNS, one has to take into account processes with incoming charm quarks, as well as light quarks and gluons in the final state which fragment into the heavy meson. It is expected that this scheme is valid not only in the region $p_{T}^{2} \gg m_{c}^{2}$, but also in the kinematic region where $p_{T}$ is only a few times larger than $m_{c}$. The basic features of the GM-VFNS are described in Ref. [214]. Analytic results for the required hard scattering cross sections can be found in Refs. [213, 215, 216, 217].

Next, we present theoretical predictions [214] for the photoproduction of $D^{*}$-mesons in $e p$ scattering at the EIC. We assume an experimental analysis with $Q_{\max }=1 \mathrm{GeV}$ in Eq. (1.32). Since the cross section is dominated by low $Q^{2}$, our results should not depend too strongly on the precise value of $Q_{\max }$. The relevant direct and resolved hard scattering cross sections are calculated at NLO accuracy. For the photon PDFs we use the parametrization of Ref. 218] with the standard set of parameter values, and for the proton PDF we have chosen the CTEQ6.5 set [201]. For the FFs we use the Global-GM set of Ref. [219] based on a fit to the combined Belle [220], CLEO [221], ALEPH [222], and OPAL [223, 224] data. We choose the renormalization and factorization scales to be equal and use $\mu_{r}=\mu_{f}=m_{T}$, 

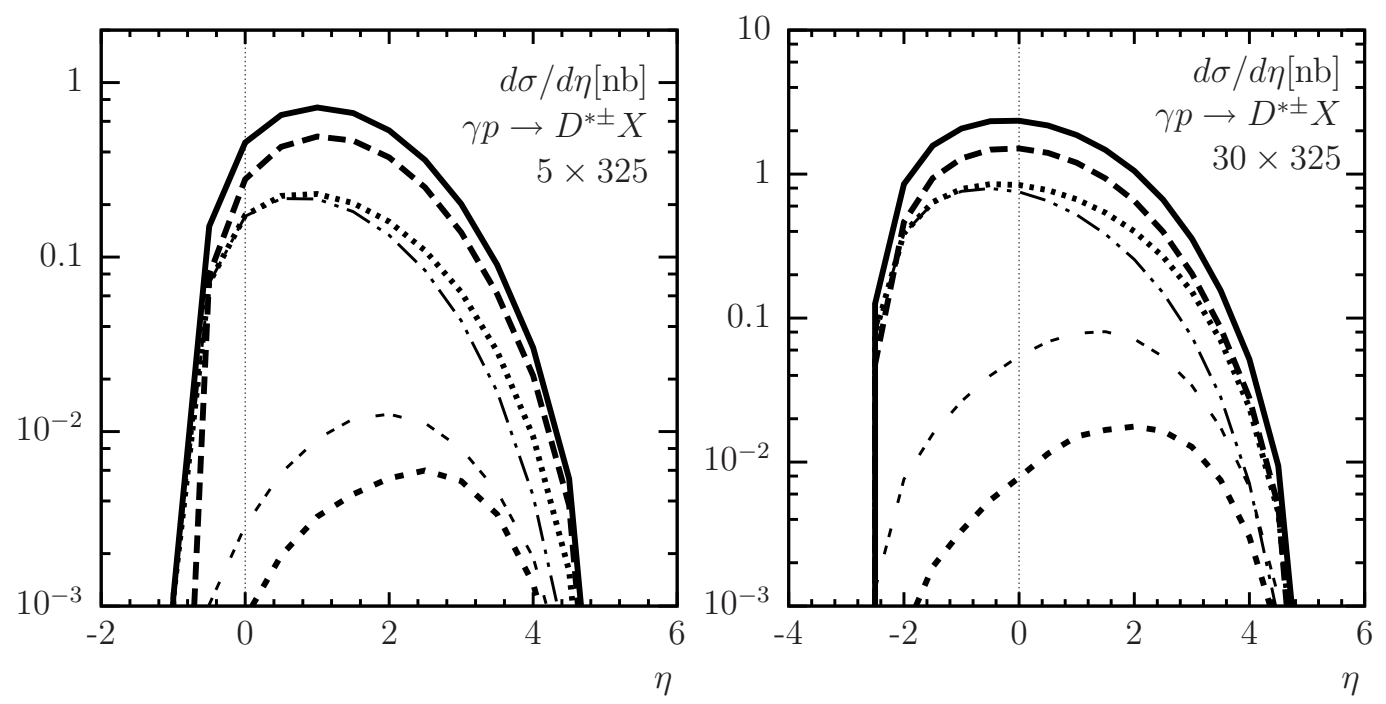

Figure 1.49. $d \sigma / d \eta$ for the production of $D^{*}$ mesons at the EIC for two settings of beam energies integrated over transverse momenta $3 \mathrm{GeV} \leq p_{T} \leq 5 \mathrm{GeV}$. The different curves are explained in the text.

where $m_{T}=\sqrt{m_{c}^{2}+p_{T}^{2}}$ is the transverse mass and $m_{c}=1.5 \mathrm{GeV}$. In Ref. [214] we studied scale uncertainties for photoproduction at HERA, as well as ambiguities due to various possible choices for input variables, such as the proton and photon PDFs, the $D^{*} \mathrm{FFs}$, and the dependence on $m_{c}$.

In our calculation of the differential cross section $d \sigma / d \eta$ (where $\eta$ is the rapidity of the observed heavy meson, $D^{* \pm}$ ) we use $E_{p}=325 \mathrm{GeV}$ and consider two choices for the energy of the electron beam: $E_{e}=5 \mathrm{GeV}$ (left panel of Fig. 1.49) and $E_{e}=30 \mathrm{GeV}$ (right panel). The transverse momentum $p_{T}$ is integrated over the range $3<p_{T}<5 \mathrm{GeV}$. The results show that the higher electron beam energy would lead to an increase of the cross section by roughly a factor of three and the rapidity distribution is shifted towards the backwards region, as expected.

The figure shows a split-up of the total cross section into contributions from different subprocesses. From top to bottom, the curves correspond to the total cross section (full line), the direct contribution (long dashed), the total resolved part (dotted), the contribution due to charm in the photon (dash-dot-dotted) and charm in the proton (long double-dashed), and, finally, the part due to resolved subprocesses with light partons in the initial state. The direct contribution, which is sensitive mainly to the gluon distribution in the proton, is dominating throughout the shown range of $p_{T}$ and $\eta$. The resolved part is mainly due to the charm content of the photon, in particular, at negative rapidities. Here one may hope that measurements at an EIC, in particular, for the option with the highest $\sqrt{s}$, will contribute to a better determination of the photon PDFs.

The total cross sections for charm production at an EIC are not very different from those measured at HERA; however, an increase in the precision of corresponding measurements can be expected due to the higher luminosity. Apart from providing a better testing-ground for pQCD, one may expect that the experimental information will contribute to an improved determination of the charm content of the proton and, perhaps, the charm FFs. 


\title{
1.16 Polarized photoproduction at an EIC
}

\author{
Barbara Jäger, Marco Stratmann
}

The framework for photoproduction outlined in Sec.1.14 can be readily extended to longitudinally polarized $e p$ collisions by replacing all unpolarized hard scattering cross sections and PDFs with their helicity-dependent counterparts. The energy spectrum of circularly polarized photons is given by [225]

$$
\Delta f_{e \gamma}(y)=\frac{\alpha_{e m}}{2 \pi}\left[\frac{1-(1-y)^{2}}{y} \ln \frac{Q_{\max }^{2}(1-y)}{m_{e}^{2} y^{2}}+2 m_{e}^{2} y^{2}\left(\frac{1}{Q_{\max }^{2}}-\frac{1-y}{m_{e}^{2} y^{2}}\right)\right] .
$$

The polarized beams available at an EIC offer unique opportunities for studying the spin structure of circularly polarized photons in photoproduction processes. Such measurements could yield also valuable, complementary information on the gluon helicity density of the proton as we shall demonstrate below.

To study the sensitivity of an EIC to the parton content of polarized photons, which is completely unmeasured so far, we consider two extreme models [226] based on the current knowledge of the unpolarized $f^{\gamma}\left(x, \mu_{0}\right)$ [211] and the positivity constraint $\left|\Delta f^{\gamma}\left(x, \mu_{0}\right)\right| \leq$ $f^{\gamma}\left(x, \mu_{0}\right)$. In the "minimal" scenario we assume $\Delta f^{\gamma}\left(x, \mu_{0}\right)=0$ at a scale $\mu_{0} \simeq 1 \mathrm{GeV}$ and we saturate the bound in the "maximal" scenario, i.e., $\Delta f^{\gamma}\left(x, \mu_{0}\right)=f^{\gamma}\left(x, \mu_{0}\right)$.

We present results of NLO calculations for single-inclusive jet photoproduction at a c.m.s. energy of $\sqrt{s}=100 \mathrm{GeV}$. In order to compute the cross section for jet production, an algorithm has to be specified describing the formation of jets by the final-state partons produced in the hard scattering. A frequently adopted choice is to define a jet as the deposition of the total transverse energy of all final-state partons that fulfill $\left(\eta-\eta^{i}\right)^{2}+$ $\left(\phi-\phi^{i}\right)^{2} \leq R^{2}$, where $\eta^{i}$ and $\phi^{i}$ denote the pseudo-rapidities and azimuthal angles of the particles and $R$ the jet cone aperture. We work in the so-called "small-cone approximation" [227, 228, 229, 230, 231] which can be considered as an expansion of the jet cross section in terms of $R$ of the form $A \log R+B+\mathcal{O}\left(R^{2}\right)$. Neglecting $\mathcal{O}\left(R^{2}\right)$ pieces, the evaluation and phase-space integration of the partonic cross sections can be performed analytically. This approximation has been shown [231, 232, 233, 147] to account extremely well for jet observables up to cone sizes of about $R \approx 0.7$ in related $p p$-scattering reactions by explicit comparison to calculations that take $R$ fully into account.

Figure 1.50 presents our results 234] for the expected NLO double-spin asymmetry $A_{L L}^{\text {jet }}$ for single-inclusive jet photoproduction at $\sqrt{S}=100 \mathrm{GeV}$ for two different choices of proton helicity densities [24, 25] and the two extreme sets of polarized photon densities introduced above. In (1.35) we chose $Q_{\max }^{2}=1 \mathrm{GeV}^{2}$ and the range of photon energies is limited to $0.2 \leq y \leq 0.85$; see also Sec. 1.14. The jet transverse momentum is integrated over for $p_{T}>4 \mathrm{GeV}$, and the factorization and renormalization scales are chosen to be $p_{T}$.

For single-inclusive observables, the rapidity-differential cross sections and the spin asymmetry are particularly interesting, since the relevant ranges of momentum fractions of the partons in the photon and the proton are related to the rapidity of the observed jet. As explained, e.g., in Ref. [235], if counting positive rapidity in the forward direction of the proton, large momentum fractions $x_{\gamma} \simeq 1$ are probed at large negative values of $\eta$. In this region, the direct contribution is expected to be largest and the photon structure is dominated by the purely perturbative "pointlike" QED part 226] which does not depend on the unknown non-perturbative input. As can be seen in Fig. 1.50, measurements of $A_{L L}^{\text {jet }}$ 


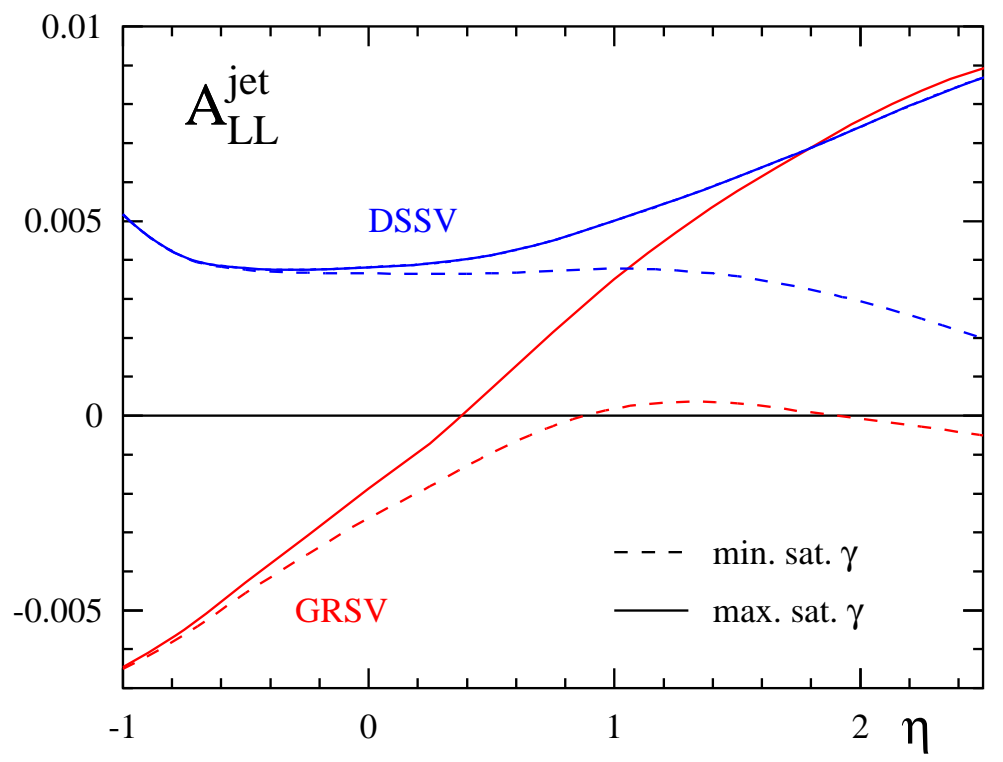

Figure 1.50. Pseudo-rapidity dependence of the NLO QCD spin asymmetry for single-inclusive jet photoproduction at $\sqrt{S}=100 \mathrm{GeV}$ integrated over $p_{T}>4 \mathrm{GeV}$ for two different choices of proton helicity PDFs and two extreme sets of polarized photon densities. Taken from Ref. [234].

for negative $\eta$ can provide valuable information on the proton's spin structure, in particular, the gluon helicity density due to the dominance of gluon-induced processes. On the other hand, at large positive rapidities, $A_{L L}^{j e t}$ is particularly sensitive to the parton content of the resolved photon, $x_{\gamma} \ll 1$, as is also exemplified in the figure. The size of $A_{L L}^{j e t}$ increases if the lower cut for the jet transverse momentum is raised to larger values. The range in $p_{T}$ where jets can be reliably reconstructed at an EIC still needs to be investigated in detail.

If one has determined the proton helicity PDFs from elsewhere, see Sec. 1.11, the prospects for learning about the parton content of polarized photons are excellent. We note that the latter may become relevant in estimates of photon induced cross sections at a future linear collider if the lepton beams will be longitudinally polarized. Resolved photon contributions also complicate current extractions of $\Delta g(x, \mu)$ in polarized-lepton nucleon scattering experiments at fixed-target energies [236, 237]. We have estimated the expected size of statistical uncertainties in case of the related single-inclusive pion photoproduction at an EIC in Ref. 238. Measurements appear to be feasible already with very moderate integrated luminosities of a few $\mathrm{fb}^{-1}$ thanks to the sizable cross sections for small $Q^{2}$.

We note that other promising observables, like di-jet production where one has a better control of the range of $x_{\gamma}$ probed, see Sec. 1.14, or heavy quark production still need to be studied. Some theoretical results and simulations, mainly for HERA energies, can be found in Refs. 235, 239, 225. 


\title{
Chapter 2
}

\section{Three-dimensional structure of the proton and nuclei: transverse momentum}

\author{
Convenors and chapter editors: \\ D. Hasch, F. Yuan
}




\title{
2.1 Introduction and chapter summary
}

\author{
Mauro Anselmino, Andreas Metz, Peter Schweitzer
}

The exploration of the internal structure of the nucleon in terms of quarks and gluons, the fundamental degrees of freedom of Quantum Chromodynamics (QCD), has been and still is at the frontier of hadronic high energy physics research. After four decades of Deep Inelastic Scattering (DIS) experiments of high energy leptons off nucleons, our knowledge of the nucleon structure has made impressive progress. To leading order in the electromagnetic coupling constant $\alpha_{\mathrm{QED}} \sim \frac{1}{137}$ the lepton with initial momentum $l$ interacts via one photon exchange with the quarks inside the nucleon. By observing the momentum $l^{\prime}$ of the lepton in the final state one obtains information about the quark and gluon content of the nucleon.

This information is encoded in the Parton Distribution Function (PDF) $f_{1}^{a}\left(x, Q^{2}\right)$ where $x=Q^{2} /(2 P \cdot q)$ is the fraction of the nucleon momentum $P$ which is carried by the parton with $Q^{2}=-q^{2}$ and $q=l-l^{\prime}$. This PDF can be interpreted as the number density of partons of type $q$ inside the nucleon, carrying a momentum fraction $x$. Similar information has been obtained about the number density of longitudinally polarized partons inside longitudinally polarized nucleons, the helicity distribution $g_{1}^{a}\left(x, Q^{2}\right)$. The successful prediction of the scale $\left(Q^{2}\right)$ dependence of the PDFs is one of the great triumphs of QCD.

However consolidated our understanding of the nucleon structure from DIS experiments is, it is basically one-dimensional. From DIS we 'only' learn about the longitudinal motion of partons in a fast moving nucleon or, which is equivalent, about their momentum distributions along the light-cone direction singled out by the hard momentum flow in the process (i.e., in DIS, of the virtual photon). In DIS the nucleon is seen as a bunch of fast-moving quarks, antiquarks and gluons, whose transverse momenta are not resolved. A fast moving nucleon is Lorentz-contracted but its transverse size is still about $1 \mathrm{fm}$, which is a large distance on the strong interaction scale.

It makes therefore sense to ask questions like: how are quarks spatially distributed inside the nucleon? How do they move in the transverse plane? Do they orbit, and carry orbital angular momentum? Is there a correlation between orbital motion of quarks, their spin and the spin of the nucleon? How can we access information on such spin-orbit correlations, and what will this tell us about the nucleon? Recent theoretical progress has put many of these questions on a firm field-theoretical basis. We do not know all answers, yet, but we have now a much better idea on how to get them. The past decade has also witnessed tremendous experimental achievements which lead to fascinating new phenomenological insights into the structure of the nucleon.

The above questions address two complementary aspects of the nucleon structure: the description of quarks in the transverse plane in momentum space and in coordinate space. The field-theoretical tools adequate to describe the former are the Transverse Momentum Dependent Parton Distribution Functions (TMD PDFs, or, shortly, TMDs). The fieldtheoretical objects tailored to describe the spatial distributions of quarks in the transverse plane are the Generalized Parton Distributions (GPDs), which are discussed in chapter 3.1 . The focus of this chapter is on the TMDs, their theoretical properties and phenomenological implications.

Several fascinating topics are related to the study of TMDs:

- 3D-imaging. The TMDs depend on the intrinsic motion of partons inside the nucleon and allow the reconstruction of the nucleon structure in momentum space. Such an 
information, when combined with the analogous information on the parton spatial distribution from GPDs, leads to a complete 3-dimensional imaging of the nucleon.

- Orbital motion. Most TMDs would vanish in the absence of parton orbital angular momentum. The possibility of learning about the orbital motion of quarks inside a nucleon emerges from the study of TMDs.

- Spin-orbit correlations. Most TMDs and related, observable, azimuthal asymmetries, are due to couplings of the transverse momentum of quarks with the nucleon (or the quark) spin. Spin-orbit correlations, similar to those in hydrogen atoms, can therefore be studied.

- QCD gauge invariance and universality. The origin of some TMDs and the related spin asymmetries, when considered at partonic level, reveal fundamental properties of QCD, mainly its color gauge invariance. This interpretation leads to expect some clear differences, between TMDs, in different processes (universality breaking). A test of such ideas is crucial for our understanding of QCD at work.

\subsubsection{What are TMDs?}

The 'simplest' TMD is the unpolarized function $f_{1}^{q}\left(x, k_{\perp}\right)$ which describes, in a fast moving nucleon, the probability to find a quark carrying the longitudinal momentum fraction $x$ of the nucleon momentum, and a transverse momentum $k_{\perp}=\left|\boldsymbol{k}_{\perp}\right|$. It is formally related to the collinear ('integrated') $\mathrm{PDF}$ by $\int \mathrm{d}^{2} \boldsymbol{k}_{\perp} f_{1}^{q}\left(x, k_{\perp}\right)=f_{1}^{q}(x)$ (notice that, for brevity, the dependence of TMDs and PDFs on auxiliary scales is often not indicated).

This and other quark TMDs are defined in terms of the unintegrated quark-quark correlator [240, 241]

$$
\Phi_{i j}^{q}\left(x, \boldsymbol{k}_{\perp}, \boldsymbol{S}\right)_{\eta}=\left.\int \frac{d z^{-} d^{2} z_{\perp}}{(2 \pi)^{3}} \mathrm{e}^{i k \cdot z}\left\langle\boldsymbol{P}, \boldsymbol{S}\left|\bar{\psi}_{j}^{q}(0) \mathcal{W}_{\eta}(0, z) \psi_{i}^{q}(z)\right| \boldsymbol{P}, \boldsymbol{S}\right\rangle\right|_{z^{+}=0},
$$

in which the gauge link operator $\mathcal{W}_{\eta}(0, z)$ ensures the color gauge invariance of the matrix element. $\mathcal{W}_{\eta}(0, z)$ depends on a path. Factorization theorems give the prescription along which path the positions 0 and $z$ of the quark fields have to be connected, and the index $\eta$ indicates that strictly speaking $\mathcal{W}_{\eta}(0, z)$ depends on the process, as it will be further discussed. The light-cone coordinates are defined as $a^{\mu}=\left(a^{-}, a^{+}, \boldsymbol{a}_{\perp}\right)$ with $a^{ \pm}=\frac{1}{\sqrt{2}}\left(a^{0} \pm\right.$ $\left.a^{3}\right)$ and $\boldsymbol{a}_{\perp}=\left(a^{1}, a^{2}\right)$.

The power and rich possibilities of the TMD approach arise from the simple fact that $\boldsymbol{k}_{\perp}$ is a vector, which allows various correlations with the other vectors involved: the nucleon momentum $\boldsymbol{P}$ and the nucleon spin $\boldsymbol{S}$. A systematic description of the information content of the correlator was initiated in $[242,243,244]$. Of particular importance are 'leading-twist' TMDs, i.e. TMDs which enter in observables without power suppression. In this context, a TMD or observable is said to be twist-t if its contribution to a cross section is suppressed by the factor $(M / Q)^{t-2}$ [245] in addition to kinematic overall factors ( $M$ represents a generic hadronic scale including the transverse momentum.).

The leading-twist TMDs are associated with the large + component of the nucleon momentum (in a frame where the nucleon moves fast). For a spin $\frac{1}{2}$ particle like the nucleon there are 8 leading-twist TMDs, namely (we suppress the $\eta$ process dependence 
label)

$$
\begin{aligned}
\frac{1}{2} \operatorname{tr}\left[\gamma^{+} \Phi^{q}\left(x, \boldsymbol{k}_{\perp}, \boldsymbol{S}\right)\right] & =f_{1}^{q}\left(x, k_{\perp}\right)-\frac{\varepsilon^{j k} k_{\perp}^{j} S_{T}^{k}}{M} f_{1 T}^{\perp q}\left(x, k_{\perp}\right), \\
\frac{1}{2} \operatorname{tr}\left[\gamma^{+} \gamma_{5} \Phi^{q}\left(x, \boldsymbol{k}_{\perp}, \boldsymbol{S}\right)\right] & =S_{L} g_{1 L}^{q}\left(x, k_{\perp}\right)+\frac{\boldsymbol{k}_{\perp} \cdot \boldsymbol{S}_{T}}{M} g_{1 T}^{q}\left(x, k_{\perp}\right), \\
\frac{1}{2} \operatorname{tr}\left[i \sigma^{j+} \gamma_{5} \Phi^{q}\left(x, \boldsymbol{k}_{\perp}, \boldsymbol{S}\right)\right] & =S_{T}^{j} h_{1}^{q}\left(x, k_{\perp}\right)+S_{L} \frac{k_{\perp}^{j}}{M} h_{1 L}^{\perp q}\left(x, k_{\perp}\right) \\
& +\frac{\left(k_{\perp}^{j} k_{\perp}^{k}-\frac{1}{2} \boldsymbol{k}_{\perp}^{2} \delta^{j k}\right) S_{T}^{k}}{M^{2}} h_{1 T}^{\perp q}\left(x, k_{\perp}\right)+\frac{\varepsilon^{j k} k_{\perp}^{k}}{M} h_{1}^{\perp q}\left(x, k_{\perp}\right)(.2 .4)
\end{aligned}
$$

Dirac structures other than those above yield higher twist TMDs [246, 247]. TMDs of antiquarks and gluons are defined similarly in terms of correlators analogous to (2.1). The notation used in Eqs. (2.2)-(2.4) follows [243, 244, 245], where the common subscript 1 is used to indicate twist-2 TMDs. (Notice that in the TMD literature also a different notation is often used, in which, for instance, $\Delta^{N} f_{q / p^{\uparrow}}\left(x, k_{\perp}\right)=-\left(2 k_{\perp} / M\right) f_{1 T}^{\perp q}\left(x, k_{\perp}\right)$. We refer to 248, for an overview.)

The leading twist TMDs (2.2 2.4) have partonic interpretations. The gamma-structures signal the quark polarizations. $\gamma^{+}$describes unpolarized quarks, thus Eq. (2.2) gives the number density of unpolarized quarks inside an unpolarized (first term) or transversely polarized (second term) proton. $\gamma^{+} \gamma_{5}$, which appears in Eq. (2.3), singles out longitudinally polarized quarks, either in a longitudinally (first term) or transversely polarized (second term) proton. Finally, in Eq. (2.4), the gamma-factor $i \sigma^{+j} \gamma_{5}$ selects transversely polarized quarks inside transversely polarized (first and third terms), longitudinally polarized (second term) or unpolarized (fourth term) protons.

\subsubsection{Partonic interpretation and properties of the TMDs}

As they are the central focus of interest in this Chapter, let us further elaborate on the leading order TMDs and their partonic interpretation. We also introduce the Transverse Momentum Dependent Fragmentation Functions (TMD FFs). The TMDs contain information on the longitudinal and transverse (or intrinsic) motion of quarks and gluons inside a fast moving nucleon. When adding the spin degree of freedom they link the parton spin (say a quark, $\boldsymbol{s}_{q}$ ) to the parent proton spin $(\boldsymbol{S})$ and to the intrinsic motion $\left(\boldsymbol{k}_{\perp}\right)$. The correlator (2.1) restricted to leading twist defines the most general spin dependent TMD, which we denote by $f_{1}^{q}\left(x, \boldsymbol{k}_{\perp} ; \boldsymbol{s}_{q}, \boldsymbol{S}\right)$, and may depend on all possible combinations of the pseudo-vectors $\boldsymbol{s}_{q}, \boldsymbol{S}$ and the vectors $\boldsymbol{k}_{\perp}, \boldsymbol{P}$ which are allowed by parity invariance. At leading order in $1 / Q$, there are eight such combinations, leading to the eight independent TMDs in Eqs. (2.2 2.4).

A similar correlation between spin and transverse motion can occur in the fragmentation process of a transversely polarized quark, with spin vector $\boldsymbol{s}_{q}$ and three-momentum $\boldsymbol{k}_{q}$, into a hadron with longitudinal momentum fraction $z$ and transverse momentum $\boldsymbol{P}_{\perp}$ (with respect to the quark direction); such a mechanism is called the Collins effect [249] and appears in the fragmentation function via a $\boldsymbol{s}_{q} \cdot\left(\boldsymbol{k}_{q} \times \boldsymbol{P}_{\perp}\right)$ term. For a quark fragmentation into a spinless hadron there are two independent leading-twist transverse momentum dependent fragmentation functions.

We briefly list here the eight leading-twist Transverse Momentum Dependent Partonic Distributions of a proton and the two Fragmentation Functions (for a final spinless hadron), 
which are the main objects in our investigation of the nucleon momentum structure.

- $f_{1}^{a}\left(x, k_{\perp}\right)$ is the unpolarized, $k_{\perp}$ dependent distribution of parton $a$ inside a proton. Its integrated version is the usual PDF measured in DIS. Common notations are $q(x)=\int d^{2} \boldsymbol{k}_{\perp} f_{1}^{q}\left(x, k_{\perp}\right)$, and $g(x)=\int d^{2} \boldsymbol{k}_{\perp} f_{1}^{g}\left(x, k_{\perp}\right)$ for quarks of flavor $q$ and gluons respectively.

Most experimental and theoretical efforts have so far been dedicated to $q\left(x, Q^{2}\right)$ and $g\left(x, Q^{2}\right)$; these are by now the best known partonic distributions, and the comparison of the predicted $Q^{2}$ dependence with data has been a great success for perturbative QCD.

- $g_{1 L}^{a}\left(x, k_{\perp}\right.$ ) (or simply $g_{1}^{a}$ ) is the unintegrated helicity distribution: the difference between the number density of partons $a$ with the same and opposite helicity of the parent proton. Common notations for the integrated helicity distributions are $\Delta q(x)=\int d^{2} \boldsymbol{k}_{\perp} g_{1 L}^{q}\left(x, k_{\perp}\right)$ for quarks and similarly $\Delta g(x)$ for gluons. See the relevant discussions in section 1.10.

The $\Delta q(x)$ 's are not so well known as the corresponding $q(x)$, as they require polarized DIS, but have been measured by several experiments. The least known of the helicity distributions is the gluon one, $\Delta g(x)$, despite some attempts to measure it.

- $h_{1}^{q}\left(x, k_{\perp}\right)$ is the analogue of the helicity distribution, for transverse nucleon spin, i.e. the transversity distribution. The integrated version has several notations in the literature $\Delta_{\perp} q(x)=h_{1}^{q}(x)=\int d^{2} \boldsymbol{k}_{\perp} h_{1}^{q}\left(x, k_{\perp}\right)$ for quarks of flavor $q$. There is no transversity distribution for gluons in a spin $\frac{1}{2}$ hadron.

The unpolarized, the helicity and the transversity distributions are the only three independent PDFs which survive in the collinear limit, $\boldsymbol{k}_{\perp}=0$. The transversity distribution is chiral-odd and needs to be coupled to another chiral-odd quantity to be observed. So far only one extraction of the $u$ and $d$ quark transversities is available in the literature [250], obtained by a combined fit of SIDIS and $e^{+} e^{-}$data.

A good knowledge of the transversity distributions for quarks and antiquarks would allow computation of the tensor charge, given by $\int_{0}^{1} d x\left[h_{1}^{q}(x)-h_{1}^{\bar{q}}(x)\right]$, a non perturbative quantity for which lattice and model computations exist.

- $f_{1 T}^{\perp a}\left(x, k_{\perp}\right)$ is the Sivers function [251], appearing in the distribution of unpolarized partons $a$ inside a polarized proton. It links the parton intrinsic motion to the proton spin:

$$
f_{1}^{a}\left(x, \boldsymbol{k}_{\perp} ; \boldsymbol{S}\right)=f_{1}^{a}\left(x, k_{\perp}\right)-\frac{k_{\perp}}{M} f_{1 T}^{\perp a}\left(x, k_{\perp}\right) \boldsymbol{S} \cdot\left(\hat{\boldsymbol{P}} \times \hat{\boldsymbol{k}}_{\perp}\right) .
$$

The Sivers function offers new information and plays a crucial role in our understanding of the nucleon structure. Its observation, already confirmed, is a clear indication of parton orbital motion; the opposite values for $u$ and $d$ quarks is argued to be linked to the nucleons' anomalous magnetic moments; its very origin and expected process dependence are related to fundamental QCD effects. Due to its importance the Sivers TMD for quarks will be discussed at length in Sec. 2.2 and for gluons in Sec. 2.3. Theoretical issues concerning $f_{1 T}^{\perp a}$, its origin and relation with basic QCD properties like the color gauge links and color gauge invariance will be treated in Sec. 2.4. 
- $h_{1}^{\perp q}\left(x, k_{\perp}\right)$ is the Boer-Mulders function [244], appearing in the distribution of polarized quarks $q$ inside an unpolarized proton:

$$
f_{1}^{q}\left(x, \boldsymbol{k}_{\perp} ; \boldsymbol{s}_{q}\right)=\frac{1}{2} f_{1}^{q}\left(x, k_{\perp}\right)-\frac{k_{\perp}}{2 M} h_{1}^{\perp q}\left(x, k_{\perp}\right) \boldsymbol{s}_{q} \cdot\left(\hat{\boldsymbol{P}} \times \hat{\boldsymbol{k}}_{\perp}\right) .
$$

This function has the striking peculiarity that it might give unexpected spin effects even in unpolarized processes, as it singles out polarized quarks from unpolarized protons and neutrons. It will be discussed in Sec. 2.5.

- The remaining three TMDs, $g_{1 T}^{a}\left(x, k_{\perp}\right), h_{1 L}^{\perp q}\left(x, k_{\perp}\right)$ and $h_{1 T}^{\perp q}\left(x, k_{\perp}\right)$ are related to double spin correlations in the PDFs; respectively, the amount of longitudinally polarized partons in a transversely polarized proton, of transversely polarized quarks in a longitudinally polarized proton, and of transversely polarized quarks in a transversely (but in a different direction) polarized proton. Neglecting higher-twist terms, some approximate relationships with the other TMDs can be obtained [252]. They will briefly be discussed in Sec. 2.6.

- $D_{1}^{a}\left(z, P_{\perp}\right)$ (also denoted as $D_{h / a}$ ) is the unpolarized, $P_{\perp}$ dependent, parton $a$ fragmentation function (into a hadron $h$ ). Its integrated version $D_{1 h}^{a}(z)=\int d^{2} \boldsymbol{P}_{\perp} D_{1}^{a}\left(z, P_{\perp}\right)$ is the usual FF.

- $H_{1}^{\perp q}\left(z, P_{\perp}\right)$ is the Collins function [249], describing the fragmentation of a polarized quark into a spinless (or unpolarized) hadron:

$$
D_{1}^{q}\left(z, \boldsymbol{P}_{\perp} ; \boldsymbol{s}_{q}\right)=D_{1}^{q}\left(z, P_{\perp}\right)+\frac{P_{\perp}}{z M_{h}} H_{1}^{\perp q}\left(z, P_{\perp}\right) \boldsymbol{s}_{q} \cdot\left(\hat{\boldsymbol{p}}_{q} \times \hat{\boldsymbol{P}}_{\perp}\right) .
$$

The Collins effect has been observed by several experiments and is well established. It is considered as a universal property of the quark hadronization process and it plays a crucial role in many spin effects. Its chiral-odd nature makes it the ideal partner to access chiral-odd TMDs like the transversity distribution and the Boer-Mulders function. All these will be discussed in Sec. 2.5

\subsubsection{How do we obtain information on TMDs?}

Our guiding experiments involve again lepton-nucleon scattering at high energy, with the difference, with respect to the usual DIS, that one observes in the final state a hadron in addition to the scattered lepton, $\ell(l)+N(P) \rightarrow \ell\left(l^{\prime}\right)+h\left(P_{h}\right)+X$, the so-called SemiInclusive Deep-Inelastic Scattering (SIDIS). In this case the hadron, which results from the fragmentation of a scattered quark, 'remembers' the original motion of the quark, including the transverse one, and offers new information.

In general, SIDIS depends on six kinematic variables. In addition to the variables for inclusive DIS, $x, y=(P \cdot q) /(P \cdot l)$, and the azimuthal angle $\phi_{S}$ describing the orientation of the target spin vector for transverse polarization, one has three variables for the final state hadron, which we denote by $z=\left(P \cdot P_{h}\right) /(P \cdot q)$ (longitudinal hadron momentum), $P_{h T}$ (magnitude of transverse hadron momentum), and the angle $\phi_{h}$ for the orientation of $\boldsymbol{P}_{h T}$ (see also Fig. 2.1). In the one-photon exchange approximation, the SIDIS cross section can be decomposed in terms of structure functions [242, 247, 254, 255] where, largely following 


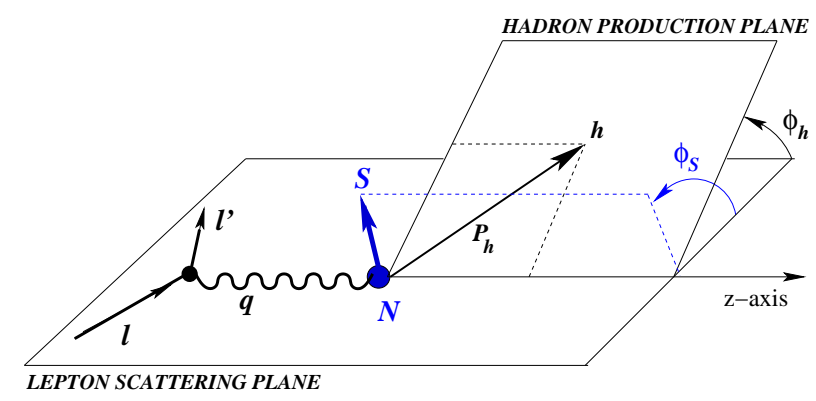

Figure 2.1. Illustration of the kinematics, especially the azimuthal angles, for SIDIS in the target rest frame 253. $\boldsymbol{P}_{h T}$ and $\boldsymbol{S}_{T}$ are the transverse parts of $\boldsymbol{P}_{h}$ and $\boldsymbol{S}$ with respect to the virtual photon momentum $\boldsymbol{q}=\boldsymbol{l}-\boldsymbol{l}^{\prime}$.

the notation of [247], one has

$$
\begin{aligned}
\frac{d \sigma}{d x_{B} d y d \phi_{S} d z_{h} d \phi_{h} d P_{h T}^{2} \propto} & \left\{F_{U U, T}+\varepsilon \cos \left(2 \phi_{h}\right) F_{U U}^{\cos 2 \phi_{h}}\right. \\
& +S_{\|} \varepsilon \sin \left(2 \phi_{h}\right) F_{U L}^{\sin 2 \phi_{h}}+S_{\|} \lambda_{\ell} \sqrt{1-\varepsilon^{2}} F_{L L} \\
& +\left|S_{\perp}\right|\left[\sin \left(\phi_{h}-\phi_{S}\right) F_{U T, T}^{\sin \left(\phi_{h}-\phi_{S}\right)}+\varepsilon \sin \left(\phi_{h}+\phi_{S}\right) F_{U T}^{\sin \left(\phi_{h}+\phi_{S}\right)}\right. \\
& \left.+\varepsilon \sin \left(3 \phi_{h}-\phi_{S}\right) F_{U T}^{\sin \left(3 \phi_{h}-\phi_{S}\right)}\right] \\
& \left.+\left|S_{\perp}\right| \lambda_{e} \sqrt{1-\varepsilon^{2}} \cos \left(\phi_{h}-\phi_{S}\right) F_{L T}^{\cos \left(\phi_{h}-\phi_{S}\right)}+\ldots\right\}
\end{aligned}
$$

In Eq. (2.8), $\varepsilon$ is the degree of longitudinal polarization of the virtual photon which can be expressed through $y$ [247], $S_{\|}$denotes longitudinal target polarization, and $\lambda_{e}$ is the lepton helicity. The structure functions $F_{X Y}(X$ and $Y$ refer to the lepton and the nucleon, respectively: $U=$ unpolarized; $L, T=$ longitudinally, transversely polarized) merely depend on $x, z$, and $P_{h T}$. The third subscript $F_{X Y, T}$ specifies the polarization of the virtual photon. By choosing specific polarization states and weighting with the appropriate azimuthal dependence, one can extract each structure function in (2.8) as pioneering experiments have already unambiguously shown.

For TMD studies one is interested in the kinematic region defined by

$$
P_{h T} \simeq \Lambda_{\mathrm{QCD}} \ll Q,
$$

for which the structure functions can be written as certain convolutions of TMDs. In this region, the components in Eq. (2.8) appear at leading order when expanding the cross section in powers of $1 / Q$, while additional ones show up at sub-leading order [242, 247, 254, 255]. Measuring the structure functions in Eq. (2.8) allows one to obtain information on all eight leading quark TMDs. To be specific, one has (for a spinless final state hadron) [247, 255],

$$
\begin{array}{ll}
F_{U U} \sim \sum_{q} e_{q}^{2} f_{1}^{q} \otimes D_{1}^{q} & F_{L T}^{\cos \left(\phi-\phi_{S}\right)} \sim \sum_{q} e_{q}^{2} g_{1 T}^{q} \otimes D_{1}^{q} \\
F_{L L} \sim \sum_{q} e_{q}^{2} g_{1 L}^{q} \otimes D_{1}^{q} & F_{U T}^{\sin \left(\phi-\phi_{S}\right)} \sim \sum_{q} e_{q}^{2} f_{1 T}^{\perp q} \otimes D_{1}^{q} \\
F_{U U}^{\cos (2 \phi)} \sim \sum_{q} e_{q}^{2} h_{1}^{\perp q} \otimes H_{1}^{\perp q} & F_{U T}^{\sin \left(\phi+\phi_{S}\right)} \sim \sum_{q} e_{q}^{2} h_{1 T}^{q} \otimes H_{1}^{\perp q}
\end{array}
$$




$$
F_{U L}^{\sin (2 \phi)} \sim \sum_{q} e_{q}^{2} h_{1 L}^{\perp q} \otimes H_{1}^{\perp q} \quad F_{U T}^{\sin \left(3 \phi-\phi_{S}\right)} \sim \sum_{q} e_{q}^{2} h_{1 T}^{\perp q} \otimes H_{1}^{\perp q},
$$

where $e_{q}$ is the charge of the struck quark in units of the elementary charge. Notice that the four chiral-even TMDs couple to the well known unpolarized fragmentation function $D_{1}$, while the chiral-odd TMDs couple to the (chiral-odd) Collins function $H_{1}^{\perp}$. In the subsequent sections the major focus will be on $F_{U T}^{\sin \left(\phi-\phi_{S}\right)}$ containing the Sivers function.

The factorized expressions for the structure functions in Eqs. (2.10)-(2.13) hold in this form in the parton model approximation. If loop corrections are included, one not only obtains a nontrivial higher order term describing the hard scattering part of the process but also a leading-twist contribution arising from soft gluon emission (soft factor) [240, 256, 257, 258, 259, 260]. In the case of inclusive DIS such soft gluon effects cancel between real and virtual radiative corrections, but they survive in the SIDIS cross section for $P_{h T} \simeq \Lambda_{\mathrm{QCD}}$. While the hard coefficient enters the structure functions in a simple multiplicative way, the soft factor gets convoluted with the parton distributions and the fragmentation functions. The presence of uncanceled soft gluon emission also requires to somewhat generalize the field-theoretical definition of TMDs given above. More details about this point will be presented in Sec. 2.4.

Almost all existing analyses of TMD-observables are based on the parton model approximation. This is sufficient for getting a good first idea about the general features of the TMDs and also at the present stage of the data, which often are plagued by considerable uncertainties. However, precision studies will be necessary to reveal features of QCD dynamics. The parton model approach will then be no longer appropriate, and one will have to deal with soft gluon effects, especially when high quality data from the EIC become available that will cover a large kinematic range.

\subsubsection{Gauge invariance, universality, and beyond}

Local gauge invariance is the underlying principle of the Standard Model of Particle Physics. In the case of QCD it is the SU(3) gauge invariance associated with the color degree of freedom of the quarks which matters. This color gauge invariance plays a particularly crucial role for TMDs. Here a brief introduction to this topic is given, while especially in Sec. 2.4 more details about this very active and fascinating field can be found.

As discussed in Sec. 2.1.1, in order to have a gauge invariant definition of TMDs a gauge link (Wilson line) has to be inserted between the two quark fields showing up in the correlator in Eq. (2.1). This is not specific for TMDs but applies also to, e.g., ordinary PDFs. However, two features are unique in the case of TMDs: first, certain TMDs are non-zero only if the Wilson line is taken into account [261, 262, 263, 264]. Second, the Wilson line depends on the process, which leads to a nontrivial universality behavior of TMDs 262.

The mere existence of two TMDs depends on the presence of the Wilson line - the Sivers function $f_{1 T}^{\perp}$ and the Boer-Mulders function $h_{1}^{\perp}$. They are also denoted as naive timereversal odd (T-odd) functions. (This term is not related to real violation of T-invariance but, roughly speaking, is associated with a nontrivial phase at the amplitude level of a process.)

The Wilson line is automatically generated when carrying out factorization. In the case of SIDIS, it arises due to the exchange of (infinitely many) gluons between the active struck quark and the remnants of the target. Since in DIS these exchanges happen after the 
virtual photon strikes the quark one also talks about final state interactions (FSI). On the other hand, for the Drell-Yan process, there exist corresponding gluon exchanges before the photon-quark interaction, which we call initial state interactions (ISI). As a consequence, the Wilson-lines for the two processes are running along different paths. This in turn endangers the universality (process-independence) of TMDs, which is a crucial prerequisite for factorization being of any practical use.

Although the paths of the Wilson lines are different, the TMDs for both processes can be related by using the parity and time-reversal transformation [262]. One finds that the six T-even TMDs are actually universal, while the T-odd TMDs are non-universal. However, this non-universality is well under control and 'merely' consists of a sign change [262],

$$
\left.f_{1 T}^{\perp}\right|_{\mathrm{DY}}=-\left.f_{1 T}^{\perp}\right|_{\mathrm{DIS}},\left.\quad h_{1}^{\perp}\right|_{\mathrm{DY}}=-\left.h_{1}^{\perp}\right|_{\mathrm{DIS}} .
$$

In other words, the predictive power of factorization is maintained. The experimental check of this sign change is currently one of the outstanding topics in hadronic physics.

We are now in a position to further motivate why the study of the Sivers effect should play a central role in the EIC science case. First, the Sivers function not only tells us something about the three-dimensional structure of the nucleon, a particular spin-orbit correlation, etc. Its physics is also intimately related to the gauge invariance of QCD. Second, existing data for non-zero transverse single-spin asymmetries in SIDIS and in proton-proton collisions can be explained on the basis of the Sivers effect. In other words, the physics of FSI/ISI is the key to describing these asymmetries (which can be as large as 40\%) in QCD. Third, according to our present knowledge, in SIDIS the Sivers function is easier to measure than the Boer-Mulders function. Fourth, the check of the predicted sign reversal in (2.14), strictly speaking, is more direct for $f_{1 T}^{\perp}$ than for the chiral-odd $h_{1}^{\perp}$. In the latter case input from models is required.

Quite some progress was made in recent years to further elucidate this physics associated with the underlying gauge structure of QCD. In particular, for hadron-hadron collisions with hadronic final states the presence of both ISI and FSI may unable any kind of (standard) TMD-factorization [265, 266, 267, 268, 269, 270, 271, 272]. The consequences of a breakdown of TMD-factorization are far-reaching. For instance, in such a case also the so-called QCD resummation technique [273], which is widely used whenever there is more than one physical momentum scale in a process, becomes questionable. Moreover, if the sign reversal of the Sivers function in Eq. (2.14) is not confirmed by experiment, the general procedure of applying QCD to hard scattering processes may have to be revisited. Further striking developments in this rather new field can be expected, and only the close interplay between lepton-nucleon scattering and hadronic collisions will allow us to fully explore this physics, as is also obvious from the relations (2.14).

\subsubsection{TMDs and orbital angular momentum}

The helicity PDFs $g_{1}^{a}(x)$ are still not well known, especially in the sea quark and gluon sector, but by now one fact seems clear: the spin of quarks and gluons accounts only for a part of the nucleon spin. A substantial fraction of the nucleon spin must be due to orbital angular momentum (OAM). It is important to keep in mind that in gauge theories there is no unique decomposition of the nucleon spin into contributions due to the spin and OAM of quarks and gluons [143, 274]. Nevertheless it is possible [274, 275] to learn about OAM from GPDs which describe the dynamics of partons in the transverse plane in position space. 
TMDs provide complementary information on the dynamics of partons in the transverse plane in momentum space, and one naturally expects TMDs to teach us about parton OAM. That the OAM of partons plays an important role is well known: in the light-cone wave function of the nucleon components with OAM $L_{z} \neq 0$ must be present in order to have a non-zero anomalous magnetic moment [276, 277, and the situation is similar for several other quantities 278]. Model calculations have also shown that the leading twist TMDs

$f_{1 T}^{\perp q}, g_{1 T}^{q}, h_{1}^{\perp q}, h_{1 L}^{\perp q}, h_{1 T}^{\perp q}$ and many sub-leading twist TMDs would vanish without different components in the nucleon wave function with $\Delta L_{z} \neq 0$. But although OAM seems to play a crucial role also for many TMDs, so far no rigorous connection between the OAM contribution of partons and the nucleon spin could be established.

\subsubsection{Further important topics}

In this subsection some further important aspects about TMDs are briefly discussed; more details will be presented in the other Sections of this TMD Chapter.

\section{Models and lattice QCD}

Model calculations have had a particularly strong impact on the TMD field. It suffices to recall the calculations in the quark-diquark model [261] which helped to establish the existence of the Sivers effect within QCD and the TMD factorization framework [262. Models may allow to see more clearly the relevant aspects of TMDs which are obscured in the much more complicated QCD dynamics. We encountered one promising instance of that above, in Sec. 2.1.5. Model results have, however, also very practical applications. Nearly nothing is known about most of the TMDs. Models provide information on the sign and magnitude of TMDs, or possible (model) relations among different TMDs. This information can be applied to make predictions for the planned experiments, and in this way help to better explore the opportunities of the available and planned facilities. The importance of model studies is discussed in Sec. 2.4.

Lattice QCD is in principle a powerful approach. What can be handled presently in lattice studies are calculations of the matrix element in the integrand of the correlator in Eq. (2.1), i.e., TMDs in Fourier-space. Most readily accessible is information on $x$-integrated TMDs such as $\int d x f_{1}^{q}\left(x, k_{\perp}\right)[279,280$. The caveat is that lattice results presently available have been obtained with a simplified gauge-link in the correlator (2.1). This simplified gauge-link differs from the link-geometry dictated by factorization in a particular scattering process. Investigations with more realistic gauge-links are ongoing.

\section{Gluon TMDs}

In addition to the eight TMDs for quarks, there also exist eight TMDs for gluons [248, 281, 282. The most prominent one is the unpolarized gluon TMD, which is a widely used ingredient of many calculations in high-energy processes. Because of the initial and final state interactions, the universality of this object is nontrivial and has attracted renewed interest lately [283. Moreover, linearly polarized gluons for an unpolarized nucleon can, in principle, be explored through, e.g., heavy quark pair production in $\ell p$-collisions [284]. A particularly important role is played by the Sivers function for gluons, which will be discussed in quite some detail in Sec. 2.3. Experimentally, the sector of gluon TMDs is largely unexplored so far, and the EIC could provide extremely valuable information in this respect. 


\section{Moments of TMDs}

Momentum moments of some of the TMDs are of particular interest because of their relation to certain collinear 3-parton correlators, which appear in the QCD-description of, e.g., SIDIS structure functions at large $P_{h T} \simeq Q$ or weighted asymmetries (see Sec. 2.2). For instance, in the case of the Sivers function one can consider the moment [264, 285]

$$
f_{1 T}^{\perp(1)}(x) \equiv \int d^{2} \boldsymbol{k}_{\perp} \frac{\boldsymbol{k}_{\perp}^{2}}{2 M^{2}} f_{1 T}^{\perp}\left(x, \boldsymbol{k}_{\perp}^{2}\right)=\pi T_{F}(x, x),
$$

where $T_{F}$ represents a quark-gluon-quark correlator. These correlation functions were also introduced in the literature to describe the single-spin asymmetries in hard scattering processes in the collinear factorization framework [286, 287, 288, 289]. Equation (2.15) is a model-independent result which allows one to relate different observables. A corresponding

relation holds for the Boer-Mulders function [264, 285]. Also the moments $g_{1 T}^{(1)}$ and $h_{1 L}^{\perp(1)}$ can be expressed through collinear 3-parton correlators [290].

\section{Integrated/weighted observables}

In Sec. 2.1.3 leading-twist soft gluon effects were mentioned. Such effects can cancel if the components in Eqs. (2.8) are integrated upon the transverse momentum $P_{h T}$ of the hadron. For instance, a cancellation occurs for the unpolarized structure function $F_{U U}$, and also for the term associated with $F_{U T}^{\sin \left(\phi_{h}-\phi_{S}\right)}$ which is related to the Sivers effect [291]. In the latter case the integration needs to be done with a proper weight factor (a more elaborate account on this topic will be given in Sec. 2.2). Such weighted observables are therefore rather attractive from a theoretical point of view. They depend on moments of the TMDs just discussed above and as such provide additional complementary information. The EIC would be ideal for seriously studying these interesting observables.

\section{Structure functions from low to high transverse momenta}

While at low $P_{h T}$ the SIDIS structure functions can be described by means of TMDfactorization, for $P_{h T} \simeq Q$ collinear factorization is the appropriate framework. Recently, a lot of progress has been made to understand the quantitative relation between TMDfactorization on the one hand and collinear factorization on the other in the region $\Lambda_{\mathrm{QCD}} \ll$ $P_{h T} \ll Q$ where both approaches apply [292, 293, 294, 295, 296, 297]. An extended discussion of these aspects, with a focus on the EIC, will also be given in Sec. 2.2.

\section{Higher twist TMDs}

The focus of present research is on the leading-twist TMDs. However, there is also a lot of important information encoded in twist-3 TMDs, which contain detailed information on the quark-gluon correlators. Experimentally, such twist-3 effects can be explored by measuring sub-leading structure functions appearing in the general decomposition of the SIDIS cross section (2.8) [242, 247, 254, 255]. In fact, the first clear single-spin phenomena in SIDIS, which crucially vitalized the field, were sub-leading twist observables. Although studied in numerous works, these first data on single-spin asymmetries in SIDIS remain basically unexplained. Some aspects of the interesting topic of higher twist TMDs will be discussed in more detail in Sec. 2.6. 


\begin{tabular}{|c|c|c|c|c|}
\hline Deliverables & Observables & What we learn & Phase I & Phase II \\
\hline \hline Sivers + unp. & SIDIS with Tran. & Quant. Interf. & valence+sea & 3D Imaging of \\
TMD quarks & polarization/ion; & Multi-parton \& & quarks, overlap & quarks \& gluon; \\
and gluon & di-hadron (di-jet) & Spin-Orbit & with the fixed & $Q^{2}\left(P_{\perp}\right)$ range \\
& heavy flavor & correlations & target exp. & QCD dynamics \\
\hline \hline Chiral-odd & SIDIS with Tran. & $3^{\text {rd }}$ basic quark & valence+sea & $Q^{2}\left(P_{\perp}\right)$ range \\
functions: & polarization/ion; & PDF; novel & quarks, overlap & for detailed \\
Transversity; & di-hadron & hadronization & with the fixed & QCD dynamics \\
Boer-Mulders & production & effects & target exp. & \\
\hline
\end{tabular}

Table 2.1. Science Matrix for TMD physics: 3D structure in transverse momentum space: golden measurements (upper part) and silver measurements (lower part).

\subsubsection{TMDs and the EIC}

Despite the tremendous progress in understanding TMDs and the related physics, without a new lepton-hadron collider many aspects of this fascinating field will remain untouched or at least on a qualitative level. Existing facilities either suffer from a much too restricted kinematic coverage or from low luminosity or from both. Based on the present status of research we see the following potential in an EIC:

- clean quantitative measurements of TMDs in the valence region due to high luminosity, and ability to go to sufficiently large $Q^{2}$ in order to suppress potential higher twist contaminations. Primordial orbital motion is expected for valence quarks.

- related to the wide kinematic coverage and the high luminosity, ability to provide multi-dimensional representations of the observables, which is basically impossible on the basis of current experiments.

- production and possible observation of jets with significantly larger particle multiplicities, allowing for the study a larger variety of hadronic final states.

- first access to TMDs for antiquarks.

- (first) access to TMDs for gluons, for instance through dihadron correlations, dijet correlations, or semi-inclusive production of quarkonium.

- systematic study of perturbative QCD techniques (for polarization observables). Tests and studies of QCD evolution properties of TMDs.

We strongly believe that the EIC will bring our knowledge of the partonic structure of the nucleon to an entirely new level. Keeping in mind deeply QCD rooted effects, like the (potential) sign-change of the Sivers function, the EIC can be expected to stimulate further developments in the application of perturbative QCD to other hard scattering processes. A series of "golden" and "silver" measurements are outlined in table 2.1. The significance of these points is further enhanced by newly planned (polarized) Drell-Yan experiments, which will study complementary physics aspects. 


\subsection{Sivers function}

Christine Aidala, Elke Aschenauer, Alessandro Bacchetta, Thomas Burton, Leonard Gamberg, Delia Hasch, Min Huang, Zhong-Bo Kang, Yuji Koike, Bernhard Musch, Alexei Prokudin, Xin Qian, Gunar Schnell, Kazuhiro Tanaka, Anselm Vossen, Feng Yuan

We choose the example of the Sivers function to illustrate the physics case for TMD distributions at the EIC. This function incorporates all new facets and intriguing physical aspects of TMD distributions outlined in the introduction and discussed in more detail in the following sections. We start this discussion with a brief review of the peculiarities of the Sivers function thereby illustrating the crucial role TMDs play in our understanding of the nucleon structure.

The Sivers function $f_{1 T}^{\perp a}\left(x, k_{\perp}\right)$, appearing in the distribution of unpolarized partons $a$ inside a polarized nucleon:

$$
f_{1}^{a}\left(x, \boldsymbol{k}_{\perp} ; \boldsymbol{S}\right)=f_{1}^{a}\left(x, k_{\perp}\right)-\frac{k_{\perp}}{M} f_{1 T}^{\perp a}\left(x, k_{\perp}\right) \boldsymbol{S} \cdot\left(\hat{\boldsymbol{P}} \times \hat{\boldsymbol{k}}_{\perp}\right)
$$

describes the correlation between the momentum direction of the struck parton and the spin of its parent nucleon and is hence related to the orbital motion of partons inside the nucleon. This correlation generates a dipole pattern in the transverse $k_{\perp}$-plane. We illustrate this fascinating aspect of certain TMDs in providing a three-dimensional imaging of the nucleon in momentum space by choosing a specific configuration for the vectors involved in Eq. (2.16). Taking for example $\hat{\boldsymbol{P}} \equiv \frac{\mathbf{P}}{\mid \mathbf{P}}=(0,0,-1)$ and the spin of the proton along the $y$ direction, so that $S=(0,1,0)$ and the transverse momentum of the parton $k_{\perp}=\left(k_{\perp x}, k_{\perp y}, 0\right)$, yields a typical "dipole" modulation of the distribution:

$$
f_{1}^{a}\left(x, \boldsymbol{k}_{\perp} ; \boldsymbol{S}\right)=f_{1}^{a}\left(x, k_{\perp}\right)+\frac{k_{\perp x}}{M} f_{1 T}^{\perp a}\left(x, k_{\perp}\right) .
$$

The $f_{1}$ term provides an axially symmetric contribution, while the second term containing

$f_{1 T}^{\perp}$ gives rise to the dipole pattern. A superposition of both effects results in a distribution that is shifted away from the center (distorted) in the $k_{\perp}$-plane as shown in fig. 2.2. This distortion turns out to be of opposite sign for up and down quarks.

The Sivers function manifests the importance of initial and final state interaction effects in hard scattering processes as the presence of these effects is required for the existence of a non-zero Sivers function. Their inclusion in the TMD factorization approach yields a peculiar breaking of the universality of the Sivers function. As introduced in sec. 2.1.4 and detailed in sec. 2.4.1, this non-universality is well under control and 'merely' consists of a sign change of the Sivers function when appearing in the Drell-Yan process as compared to DIS. The experimental verification of this sign change is currently one of the outstanding topics in hadronic physics and presents a crucial test for our understanding of hadron production in high-energy reactions. We will therefore briefly review the prospects for measurements of the Sivers effect in Drell-Yan in sec. 2.2.3.

A further intriguing aspects of the Sivers function is its connection to the orbital angular momentum in the nucleon. A non-zero quark Sivers function involves a transition between initial and final nucleon states that differ by one unit of orbital angular momentum. This property together with the potential for a three-dimensional imaging, puts the Sivers function in close relation to the GPD $E$ discussed in chapter 3. In particular, it was proposed that there is a dynamical relation called "chromodynamic lensing", where 

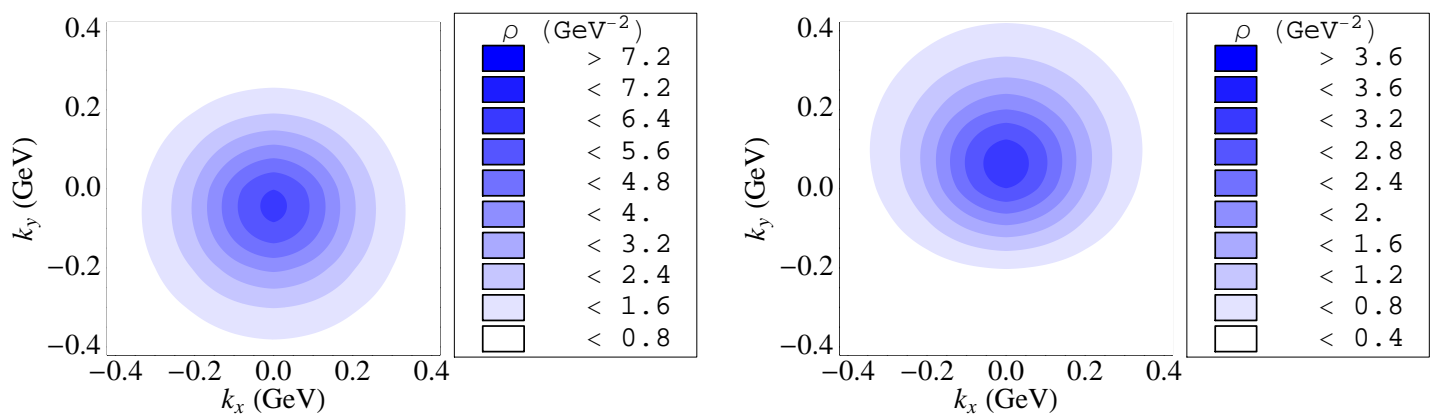

Figure 2.2. Spin density in the transverse-momentum plane for unpolarized quarks in a transversely polarized nucleon, as described by the Sivers function. The left panel is for up quarks and the right one for down quarks. The model calculation of Ref. 298 was used.

the spatial distortion of the transverse quark distribution (in a transversely polarized proton) leads to a distortion in transverse momentum distribution described by the Sivers function [299, 300, 301].

\subsubsection{What do we know so far from experiments?}

Though the Sivers function was first suggested to explain the surprisingly large singlespin asymmetries measured in $p p$ collisions, our guiding experiments for obtaining unambiguous information about this function, and most of the other TMDs, involve high-energy lepton-nucleon scattering with the observation of one or more hadrons in coincidence with the scattered lepton (semi-inclusive DIS). In addition, model calculations of TMDs, discussed in sec. 2.4. guide Ansätze for global fits of TMD parameterizations and provide an interpretation of the various aspects of TMDs.

In this section, after a brief review of the results from $p p$ collisions, we will summarize available semi-inclusive DIS measurements of observables related to the Sivers effect and present phenomenological extractions of the Sivers function from data. The following section will then highlight the potential of an EIC for a detailed and systematic exploration of the various aspects of the quark Sivers functions illustrative of TMDs in general.

\section{Transverse-spin effects in proton-proton collisions}

Historically, the surprisingly large left-right asymmetries observed in hadronic reactions with transversely polarized protons initiated the idea about a transverse momentum dependence of quark distributions in polarized protons. The pioneering measurements [302, 303] of these large (up to 0.3-0.4 in magnitude) transverse-spin asymmetries in inclusive forward production of pions in $p p$ collisions $p^{\uparrow} p \rightarrow \pi+X$, have been extensively confirmed by experiments at FermiLab 304, 305, 306, 307, 308, and at RHIC (BNL) at much higher center-of-mass energies of up to $\sqrt{s}=200 \mathrm{GeV}$ [309]. The observation of such asymmetries was frequently quoted as a puzzle or challenge for theory. In fact, for a long time, transverse single-spin asymmetries were assumed to be negligible in hard scattering processes [310]. 
The work of 251] introduced a transverse momentum dependent quark distribution, now termed the Sivers function, which provides a mechanism for the observed asymmetries that does not vanish at high energies.

A rich variety of single-spin asymmetries for identified hadrons $\left(\pi^{ \pm}, \pi^{0}, K^{ \pm}, p, \bar{p}\right)$ measured over a wide kinematic range is now available from the BRAHMS, PHENIX and STAR experiments at RHIC (BNL) [311, 312, 313, 314, 315, 316, 317, 318. The results exhibit a general pattern: sizable asymmetries are measured at forward-rapidity and for positive Feynman $x_{F}>0.3$ which increase in magnitude with increasing $x_{F}$ and $P_{h T}$. In contrast, for negative $x_{F}$ and at mid-rapidity all asymmetries are found to be consistent with zero.

Several mechanisms have been suggested to explain these asymmetries. At large values of $P_{h T}$ collinear factorization involving twist-3 distributions can be applied. However, the intrinsic prediction of a $1 / P_{h T}$ fall-off has yet to be confirmed. An alternative approach using a generalized parton model that takes intrinsic transverse momentum dependences into account has been used to describe existing data, achieving a fairly successful description of the observed asymmetries for pion production in $p p$ collisions [319]. If less inclusive measurements are performed, with an observed soft momentum scale in addition to a hard scale, one can attempt to describe the data using a TMD approach in pQCD. However, as discussed in sec. 2.4.1, the presence of both initial and final state interactions in hadronhadron collisions may prevent any kind of (standard) TMD-factorization. More insight might be gained regarding the intricate color structure of $p p$ reactions for example by measuring di-jet production. In di-jet production both large scales (e.g., jet $p_{T}$ ) and small scales (e.g., $\Delta p_{T}$ of nearly back-to-back jets) can be observed. To assess factorization breaking due to color interactions in $p p$ collisions, the experimental measurements can be compared to calculations using TMDs extracted from DIS and Drell-Yan, for which TMDfactorization has been demonstrated. Little experimental information currently exists on these processes, but they are part of the physics program at RHIC.

Many questions still need to be answered, but it is clear that for a strict assessment of whether the TMD Ansatz is indeed possible and appropriate to describe results from hadronic collisions, more precise parameterizations of the Sivers function and, hence, more precise data on the Sivers effect in a well-understood process like DIS is needed.

\section{Semi-inclusive Deep-Inelastic Scattering}

In semi-inclusive DIS, the Sivers function leads to single-spin asymmetries in the distribution of hadrons in the azimuthal angles illustrated in fig. 2.1. The azimuthal modulations of the SIDIS cross section are given in Eq. (2.8). The Sivers effect manifests itself as a $\sin \left(\phi_{h}-\phi_{S}\right)$ modulation and requires transverse polarization of the target nucleon. The additional information provided by the azimuthal angle $\phi_{S}$ of the transverse component of the target-proton spin about the virtual photon direction allows for an unambiguous extraction of the Sivers effect. Experimentally, the so-called Sivers amplitude $2\left\langle\sin \left(\phi_{h}-\phi_{S}\right)\right\rangle_{U T}^{h}$ [253], which projects out the structure function $F_{U T, T}^{\sin \left(\phi_{h}-\phi_{S}\right)}$ in Eq. (2.8) for a specific hadron $h$, is extracted from the asymmetry

$$
A_{U T}^{h}\left(\phi_{h}, \phi_{S}\right) \equiv \frac{1}{\left|\mathbf{S}_{T}\right|} \frac{d \sigma^{h}\left(\phi_{h}, \phi_{S}\right)-d \sigma^{h}\left(\phi_{h}, \phi_{S}+\pi\right)}{d \sigma^{h}\left(\phi_{h}, \phi_{S}\right)+d \sigma^{h}\left(\phi_{h}, \phi_{S}+\pi\right)},
$$

where the subscript $U$ indicates an unpolarized lepton beam and $T$ a transversely polarized target nucleon. This amplitude has so far been extracted by three polarized fixed-target experiments as summarized in Tab. 2.2. From these measurements, fig. 2.3] shows a selection 


\begin{tabular}{lcccc}
\hline experiment (laboratory) & $\sqrt{s}$ in GeV & target type & hadron types & references \\
\hline COMPASS (CERN) & 18 & deuteron & $h^{ \pm}, \pi^{ \pm}, K^{ \pm}, K^{0}$ & {$[320$, 321] } \\
& & $\begin{array}{c}\text { proton } \\
\text { proton }\end{array}$ & $h^{ \pm}, K^{ \pm}$ & prelim. [323] \\
\hline HERMES (DESY) & 7.4 & proton & $\pi^{ \pm}$ & {$[324$} \\
& & proton & $\pi^{ \pm},\left(\pi^{+}-\pi^{-}\right), \pi^{0}, K^{ \pm}$ & {$[325]$} \\
\hline HallA (JLab) & 3.5 & neutron & $\pi^{ \pm}$ & prelim. [326] \\
\hline
\end{tabular}

Table 2.2. Summary of currently available measurements of Sivers asymmetry amplitudes from lepton-nucleon DIS experiments, their center-of-mass energy, transversely polarized target type, and analyzed hadron types.

of results that are significantly non-zero and help in determining the shape of the Sivers function. All other asymmetry amplitudes listed in Tab. 2.2 are small or consistent with zero.

The results have so far been interpreted in the parton model as a convolution of distribution and fragmentation functions, where the Sivers amplitude can be approximated by

$$
2\left\langle\sin \left(\phi_{h}-\phi_{S}\right)\right\rangle_{U T}^{h}\left(x_{B}, y, z_{h}, P_{h T}\right)=-\frac{\sum_{q} e_{q}^{2} f_{1 T}^{\perp q}\left(x, k_{\perp}^{2}\right) \otimes_{\mathcal{W}} D_{1}^{q}\left(z, P_{\perp}^{2}\right)}{\sum_{q} e_{q}^{2} f_{1}^{q}\left(x, k_{\perp}^{2}\right) \otimes D_{1}^{q}\left(z, P_{\perp}^{2}\right)} .
$$

Here the sums run over the quark flavors, the $e_{q}$ are the quark charges, and $f_{1}\left(x, k_{\perp}^{2}\right)$ and $D_{1}\left(z, P_{\perp}^{2}\right)$ are the spin-independent quark distribution and fragmentation functions, respectively. The symbol $\otimes(\otimes \mathcal{W})$ represents a (weighted) convolution integral over intrinsic and fragmentation transverse momenta, $\boldsymbol{k}_{\perp}$ and $\boldsymbol{P}_{\perp}$ respectively, as explicitely given in (2.21).

A qualitative picture of the Sivers function can already be derived from the measured asymmetry amplitudes. The non-zero results shown in fig. 2.3 are obtained with a proton target. As scattering off $u$ quarks dominates these data due to the charge factor, the positive Sivers amplitudes for $\pi^{+}$and $K^{+}$suggest a large and negative Sivers function for up quarks. This is supported by the positive amplitudes of the pion difference asymmetry, which originates mainly from the difference $\left(f_{1 T}^{\perp d_{v}}-4 f_{1 T}^{\perp u_{v}}\right)$ in the Sivers functions for valence down and up quarks and is dominated by the contribution from valence $u$ quarks. The vanishing amplitudes for $\pi^{-}$require cancellation effects, e.g. from a $d$ quark Sivers function opposite in sign to the $u$ quark Sivers function. Such cancellation effects between Sivers functions for up and down quarks are supported by the vanishing asymmetry amplitudes extracted from deuteron data by the COMPASS collaboration. An interesting facet of the data shown in fig. 2.3 is the magnitude of the $K^{+}$amplitudes, which are nearly twice as large as those of the $\pi^{+}$. Again, on the basis of $u$ quark dominance, one might naively expect that the $\pi^{+}$and $K^{+}$amplitudes should be similar. Their difference in size may thus point to a significant role of other quark flavors, e.g. sea quarks.

Phenomenological analyses of HERMES and COMPASS data [327, 328, 329, 330, 331, 332 , confirm the picture drawn above as discussed in the following. So far, only the analysis of Ref. [327] makes use of a subset of the most recent data listed in Tab. 2.2 and all fits have yet to be updated for the results from proton data from COMPASS and the first neutron data from HallA. 


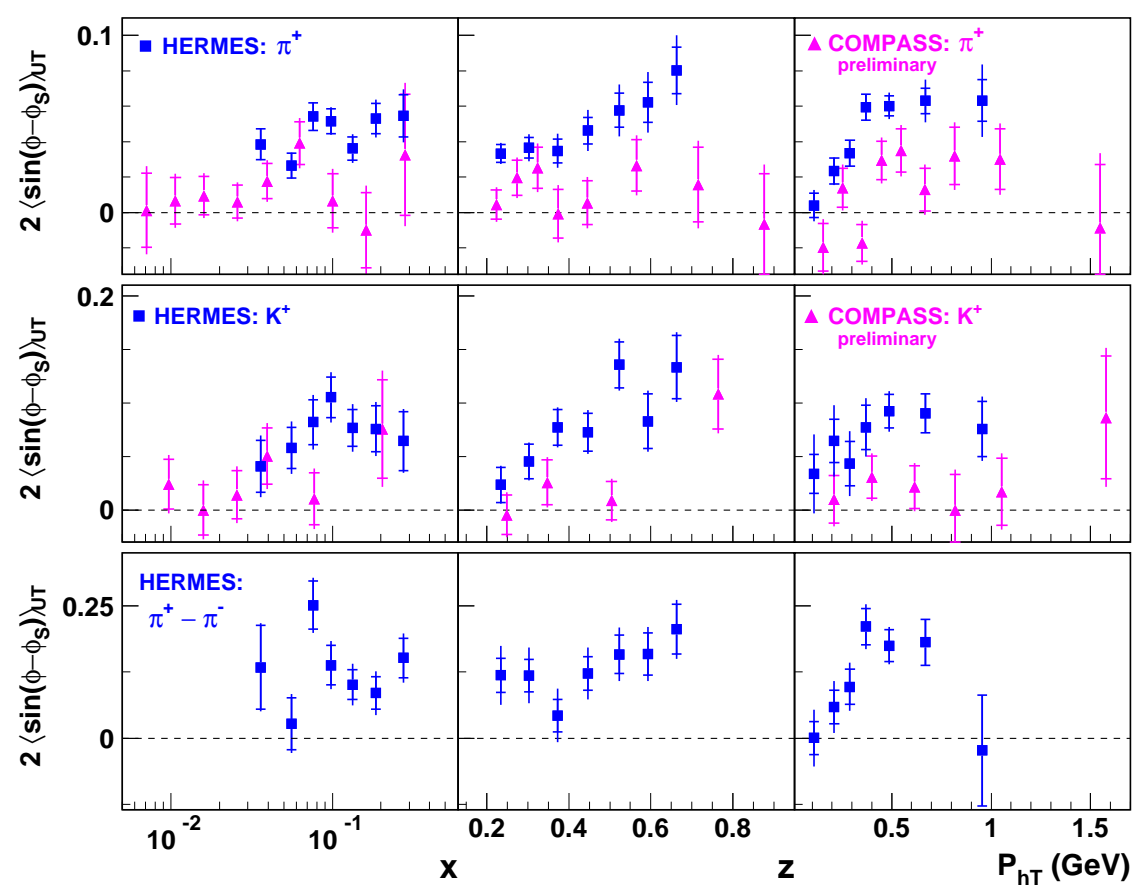

Figure 2.3. Sivers amplitudes for $\pi^{+}, K^{+}$and the pion-difference (as denoted in the panels) from HERMES [325] and for $\pi^{+}$and $K^{+}$from COMPASS 323 . measured with a proton target. Inner error bars present statistical uncertainties and full error bars the quadratic sum of statistical and systematic uncertainties. Note that the average kinematics in each bin differs for HERMES and COMPASS.

\section{Phenomenological extractions and models of the Sivers function}

The strong impact and success of model calculations and lattice QCD on the TMD field is discussed in detail in sec. 2.4 and sec. 4.1, respectively. Models provide information on the magnitudes and signs of TMDs and guide Ansätze for global fits of TMD parameterizations. For example, from chiral models [333] and the QCD limit of a large number of colours (large $N_{c}$ limit) 334 a Sivers function for up and down quarks of equal size but with opposite $\operatorname{sign}\left(f_{1 T}^{\perp u}=-f_{1 T}^{\perp d}\right)$ is predicted.

Phenomenological analyses provide extractions of TMDs from data. As discussed in sec. 2.1.3, existing analyses of TMD observables are so far based on the parton model approximation, where the measured amplitudes of the SIDIS cross section in Eq. (2.8), are expressed as convolutions of distribution $f^{q}$ and fragmentation functions $D^{q}$. For the Sivers amplitude it reads

$$
F_{U T, T}^{\sin \left(\phi_{h}-\phi_{S}\right)} \propto \sum_{q} e_{q}^{2} f_{1 T}^{\perp q}\left(x, k_{\perp}^{2}\right) \otimes_{\mathcal{W}} D_{1}^{q}\left(z, P_{\perp}^{2}\right)
$$

where $\otimes_{\mathcal{W}}$ is defined as

$$
\otimes_{\mathcal{W}} \equiv \int d^{2} \boldsymbol{k}_{\perp} d^{2} \boldsymbol{P}_{\perp} \delta^{(2)}\left(z \boldsymbol{k}_{\perp}+\boldsymbol{P}_{\perp}-\boldsymbol{P}_{h T}\right) \mathcal{W}
$$

with the kinematic factor $\mathcal{W}$ depending on the involved transverse momenta. This convolution can be resolved by either employing a particular model for the transverse momentum 

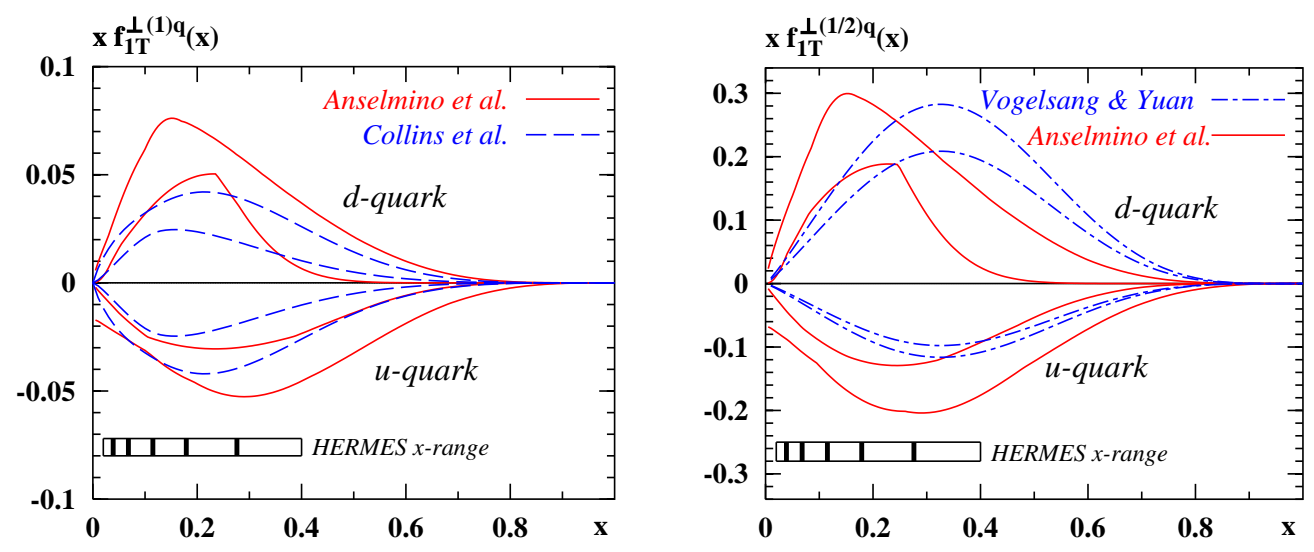

Figure 2.4. Up and down quark Sivers distributions extracted from HERMES (and for the full line also from COMPASS) data using three different parameterizations [329, 330, 331] (see text). The left and right panels show, respectively, the first and the $1 / 2$ moment. The curves indicate the 1 -sigma regions of the various parameterizations. None of three parameterizations makes use of the latest experimental results listed in Tab. 2.2 .

dependence or by integrating over the transverse momentum $P_{h T}$ using a proper weight factor in the extraction of the asymmetry amplitudes which involves $P_{h T}$, building for example $2\left\langle\frac{P_{h T}}{M_{p}} \sin \left(\phi_{h}-\phi_{S}\right)\right\rangle_{U T}^{h}$. The latter approach is very attractive but experimentally challenging for measurements at current fixed target facilities as it requires full $P_{h T}$ coverage, which cannot be obtained at any of the existing experiments. An EIC would be the ideal facility to study such weighted asymmetries and to seriously explore the advantages of these observables, as further discussed in sec. 2.2.7

An intuitive and common Ansatz for the transverse momentum dependence of distribution and fragmentation functions, which provides an analytic solution of (2.21), is a Gaussian distribution like

$$
f_{1 T}^{\perp q}\left(x, k_{\perp}^{2}\right)=f_{1 T}^{\perp q}(x) \frac{1}{\pi\left\langle k_{\perp}^{2}\right\rangle} \exp \left(-\frac{\boldsymbol{k}_{\perp}^{2}}{\left\langle k_{\perp}^{2}\right\rangle}\right), D_{1}^{q}\left(z, P_{\perp}^{2}\right)=D_{1}^{q}(z) \frac{1}{\pi\left\langle P_{\perp}^{2}\right\rangle} \exp \left(-\frac{\boldsymbol{P}_{\perp}^{2}}{\left\langle P_{\perp}^{2}\right\rangle}\right)
$$

with typical values for $\left\langle k_{\perp}^{2}\right\rangle$ and $\left\langle P_{\perp}^{2}\right\rangle$ of 0.2 to $0.3 \mathrm{GeV}^{2}$.

The Sivers function was among the first to be extracted from data, as it couples to the usual unpolarized fragmentation function $D_{1}^{q}$. This fragmentation function is reasonably well parameterized [335, 336] using precise data from electron-positron annihilation into charged hadrons and, most recently, also from single-hadron production in $p p$ collisions and semi-inclusive DIS [74], which provide complementary information on the flavour dependence of the fragmentation process.

Figure 2.4 shows the extraction of the up and down quark Sivers distributions using three different parameterizations for the Sivers function [329, 330, 331], presenting $k_{\perp}$-moments defined as

$$
f^{(1)}(x) \equiv \int d^{2} \boldsymbol{k}_{\perp} \frac{\boldsymbol{k}_{\perp}^{2}}{2 M^{2}} f\left(x, k_{\perp}^{2}\right) \quad \text { and } \quad f^{(1 / 2)}(x) \equiv \int d^{2} \boldsymbol{k}_{\perp} \frac{\left|\boldsymbol{k}_{\perp}\right|}{2 M} f\left(x, k_{\perp}^{2}\right) .
$$

The parameterization from Ref. [329] (full line) is based on a combined fit to previous HERMES and COMPASS data, while the other two fit HERMES data only but describe 


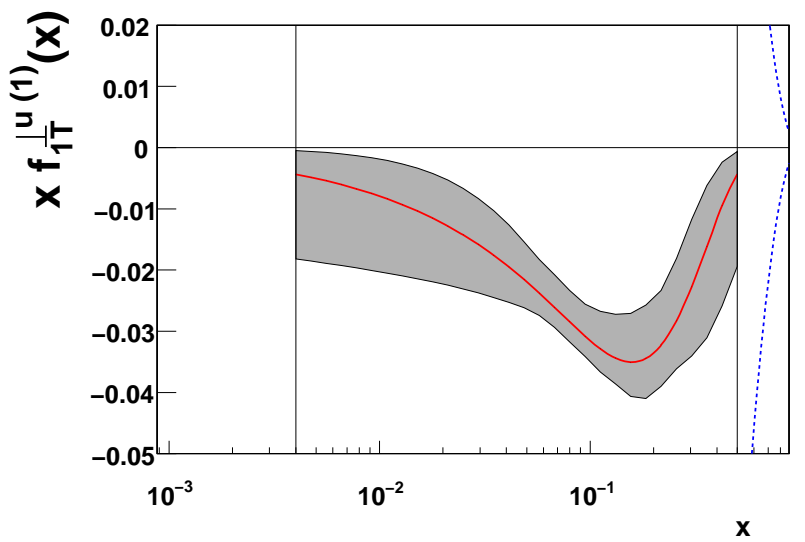

Figure 2.5. The Sivers function for $u$ quarks extracted from recent experimental data 327. Vertical lines indicate the region where experimental data are available. The band represents the 2-sigma range for the chosen parameterization. The dashed blue lines indicate the positivity bound.

the COMPASS data well when using the obtained parameters to calculate the asymmetries for COMPASS kinematics. All three extractions use the parameterization from Ref. 335] for the unpolarized fragmentation function. The two curves of each set indicate the 1-sigma regions of the various parameterizations, taking into account solely statistical uncertainties of the data sets employed in the fit. The three approaches describe the HERMES Sivers asymmetries equally well. The differences in size and shape of the extracted Sivers up and down quark distributions hence reflect the model dependence of the fit results. The parameterization of [331] imposes the constraint from the large $N_{c}$ limit, which results in the symmetric parametrization of up and down Sivers distributions, shown in the left panel of fig. 2.4 with dashed lines. None of the extractions involve parameterizations for sea quarks as they could not be constrained by the data used in the fits.

However, the recent, surprisingly large, Sivers asymmetry amplitudes for $K^{+}$measured by HERMES, which were found to be nearly twice as large as those of the $\pi^{+}$, might hint at a possibly important role of sea quarks. In Ref. [327, the sensitivity of these data to sea quark contributions was tested. A fit including Sivers functions for only up and down quarks was compared with a second fit that allowed also for sea quark contributions $(\bar{u}, \bar{d}, s, \bar{s})$ to the Sivers amplitude. Both fits describe the data with equally good $\chi^{2}$, demonstrating that their precision is not yet good enough to independently constrain the Sivers function for six quark flavours. In this analysis, the usage of new parameterizations of the fragmentation functions from Ref. [74] was essential for obtaining a good description of the kaon data.

The available parameterizations of the Sivers function for up and down quarks 327, 328, 331, 332 agree, within their large uncertainties, with calculations based on a lightcone model [298] and on a diquark spectator model [337, 338, while predictions based on the bag model [339] appear to be too small in magnitude for both the up and down quark Sivers function (see also sec. 2.4).

\section{Open issues in extractions of the Sivers function}

Figure 2.5 illustrates our current knowledge of the Sivers function. So far, only the up and down quark Sivers functions can be constrained with relatively large uncertainties within the range $0.004<x<0.5$ using basic parameterizations for their shapes.

The precision of current data permits neither constraints of the Sivers functions for 
sea quarks nor an employment of more flexible functional forms, which would also allow for a sign change as suggested by a spectator model (see fig. 2.22 in sec. 2.4). The band in fig. 2.5 represents the 2-sigma range for the chosen parameterization and reflects the precision of the data, but does not account for model uncertainties or for variations of the functional form of the parameterizations. Also not estimated so far, is any uncertainty stemming from the Gaussian Ansatz used to resolve the convolution in (2.21). For example, the average value, $\left\langle k_{\perp}^{2}\right\rangle$, of the quark intrinsic transverse momentum used in this Ansatz might be flavour dependent, and both $\left\langle k_{\perp}^{2}\right\rangle$ and $\left\langle P_{\perp}^{2}\right\rangle$ dependent on the energy scale. The latter is particularly relevant for the fragmentation functions, which are extracted from data collected at much higher energy than the available SIDIS asymmetry data used in the fits. The EIC would provide both TMD observables at substantially higher scale than any fixed target DIS experiment and unique data sets of hadron production for a flavour tagging in the fragmentation process and a study of its transverse momentum dependence.

At this stage of analysis, also specific known issues of experimental data are ignored. For example, the limited precision of currently available SIDIS data usually allows only for presenting the results as a function of one kinematic variable while integrating over the others within the experimental acceptance. Hence, the asymmetry amplitudes from a specific experiment, presented for different kinematic variables are correlated. Moreover, the experimental acceptance usually does not provide a full coverage in $P_{h T}$. Thus, the 'unweighthed' asymmetry amplitudes extracted as function of $x$ or $z$ present only partial $P_{h T}$ moments in contrast to theoretical considerations. A fully differential analysis of SIDIS data, which requires high statistic datasets, would resolve these issues.

Turning our essentially qualitative picture of the Sivers function and the related physics into a quantitative description, which goes beyond the tree-level approximation, requires new facilities providing high precision polarized data over a wide kinematic range as discussed in the following section.

\subsubsection{The Sivers function at the EIC}

A systematic and detailed study of the Sivers function, and TMDs in general, can only be performed on the basis of precise spin- and azimuthal-asymmetry amplitude measurements in semi-inclusive DIS over a wide kinematic range. The availability of experimental results that are fully differential in the kinematic variables $x, Q^{2}, z$ and $P_{h T}$ would be a great asset for phenomenological analyses, as they permit testing the underlying perturbative QCD techniques and assumptions. Particle identification over the full momentum range and measurements with both proton and (effective) neutron targets would allow for a full flavour separation of the distribution functions under study.

Planned experiments at the upcoming JLab12 facility aim at providing high precision semi-inclusive DIS data in the valence quark region at relatively low $Q^{2}$, taken with transversely polarized neutrons (HallA) [340, protons and deuterons (CLAS12) 341. The expected high luminosities should allow for fully differential extractions of the relevant azimuthal and transverse-spin asymmetries. The kinematic range of JLab12 experiments will be complementary to COMPASS measurements [342, partially overlap with those of HERMES, and provide data in the so-far unexplored high- $x$ region.

The kinematic coverage of these experiments is compared in fig. 2.6 with the coverage of an EIC for an energy setting of $\sqrt{s}=50 \mathrm{GeV}$. As discussed in sec.7.1 and sec.7.2, the ability to vary the energy of both the electron and proton (ion) beams at the EIC provides variable energy in the range $\sqrt{s}=15-65 \mathrm{GeV}$ or $\sqrt{s}=45-200 \mathrm{GeV}$ depending on the realization 


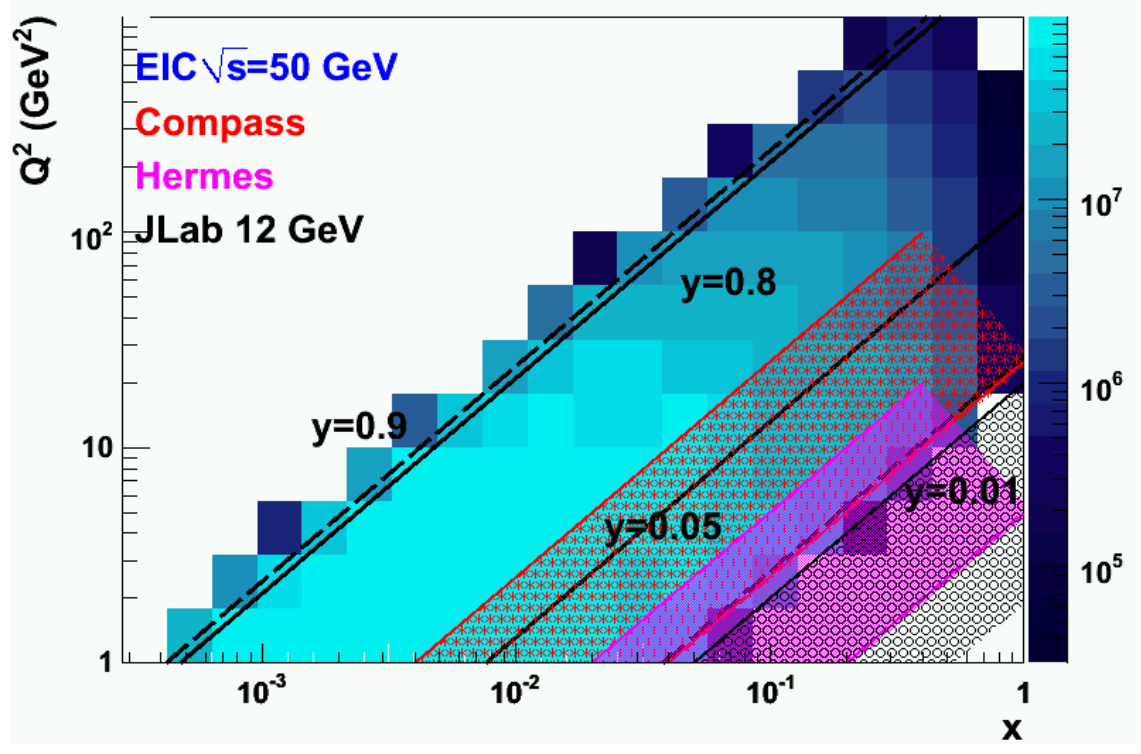

Figure 2.6. [color online] Kinematic coverage in $x$ and $Q^{2}$ for the EIC for an energy setting of $\sqrt{s}=50 \mathrm{GeV}$ compared to the coverage of COMPASS, HERMES and future JLab12 experiments represented by the red, purple and black hatched areas, respectively.

options under discussion. This ability puts the EIC in the unique position of accessing the valence region at much larger $Q^{2}$ than current and near-future experiments (thereby suppressing potential higher twist contaminations) while also accessing low $x$ down to values of about $10^{-5}$, where sea quarks and gluons could be studied in detail. The expected high luminosity will allow for a fully differential analysis over almost the whole wide kinematic range. In this section we will illustrate this potential for fully differential analyses of TMD observables and test the sensitivity to sea quark distributions. The unique features of the EIC for access to TMDs for gluons, a study of the evolution properties of TMDs, and of the transition from low to high transverse momenta will be discussed, using the Sivers function as an example, in secs. 2.3, 2.4.2 and 2.2.5, respectively.

\section{Generation of pseudo-data}

The projections presented in the following for the Sivers asymmetry where estimated using either modified existing Monte Carlo generators or standard parameterizations of the unpolarized parton distribution and fragmentation functions. Events were generated for $Q^{2}>1 \mathrm{GeV}^{2}, 0.01<y<0.9$ and $0.1<z<0.9$, over the full kinematically allowed range in $x$. At this stage no cuts were applied on the scattered electron or produced hadron. Events were divided into four-dimensional $\left(x, Q^{2}, z, P_{h T}\right)$ bins and the mean asymmetry in each bin was evaluated. Full acceptance in azimuth was assumed and statistical uncertainties of $\sqrt{2 / N}$ were assigned in each bin. More details about the simulations can be found in 343. For all projections shown in the following, no losses due to detector acceptance were applied, but an overall operational efficiency of $50 \%$ was assumed. The transverse proton beam polarization is set to $70 \%$. No estimate of systematic uncertainties is applied.

Most of the projections will be given for an integrated luminosity of $4 \mathrm{fb}^{-1}$ or $30 \mathrm{fb}^{-1}$. These statistics would be achieved in approximately one week to one month $\left(4 \mathrm{fb}^{-1}\right)$ or 


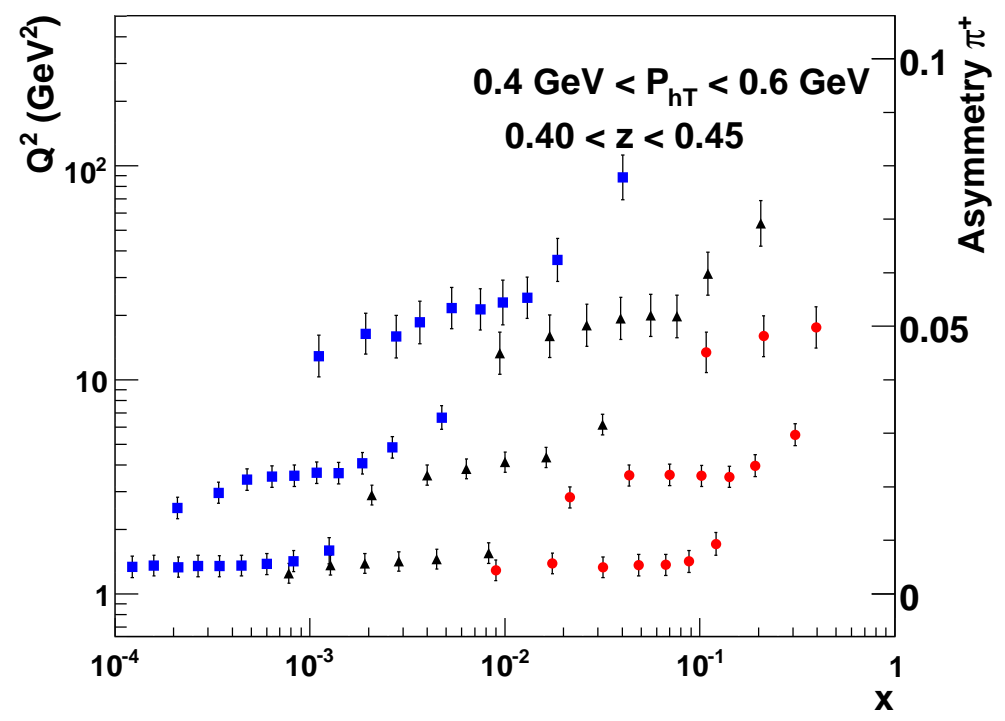

Figure 2.7. Projected accuracy for $\pi^{+}$production in semi-inclusive DIS off the proton for a particular $P_{h T}$ and $z$ range as indicated in the figure. The position of each point is according to its $Q^{2}$ and $x$ value, within the range $0.05<y<0.9$. The projected event rate, represented by the error bar, is scaled to the (arbitrarily chosen) asymmetry value at the right axis. The blue squares, black triangles and red dots represent the $\sqrt{s}=140 \mathrm{GeV}, \sqrt{s}=50 \mathrm{GeV}$ and $\sqrt{s}=15 \mathrm{GeV}$ EIC configurations, respectively. Event counts correspond to an integrated luminosity of $30 \mathrm{fb}^{-1}$ for each of the three configurations.

one month to six month $\left(30 \mathrm{fb}^{-1}\right)$ for luminosities ranging from $1 \times 10^{34} \mathrm{~cm}^{-2} \mathrm{~s}^{-1}$ to $3 \times$ $10^{33} \mathrm{~cm}^{-2} \mathrm{~s}^{-1}$. Therefore the statistical precision in the figures presented here should be understood as that achievable in a relatively brief period of operation for an EIC.

\section{Four-dimensional mapping of the phase space}

The great potential of the EIC for obtaining a fully differential mapping of almost the entire phase space relevant for TMD studies is illustrated in figs. 2.7 and 2.8. A wide $x$ and $Q^{2}$ range can be mapped using different beam energies. The projected accuracy for single $\pi^{+}$production is given for a four-dimensional binning in the kinematic variables $x$, $Q^{2}, z$ and $P_{h T}$, using three different energy configurations for the EIC $(\sqrt{s}=15,50$ and 140 $\mathrm{GeV}$ ) and an integrated luminosity of $30 \mathrm{fb}^{-1}$ for each configuration. Events are selected for $0.05<y<0.9$ and $W^{2}>5 \mathrm{GeV}^{2}$. For a clearer view and explanation of the presented projections, we show in fig. 2.7 one of the panels from fig. 2.8 corresponding to a specific $z$ and $P_{h T}$ range. In both figures, the position of each point is according to its $x$ and $Q^{2}$ value (abscissa and left ordinate, respectively) and each panel is for a specific $z$ and $P_{h T}$ bin as indicated in the figure. The projected event rate is represented by the error bar scaled with respect to the (arbitrarily chosen) asymmetry value given at the right ordinate.

The simulations demonstrate that a four-dimensional mapping of TMD observables for pions over the whole phase space of main interest, meaning $P_{h T}$ values of up to about $1 \mathrm{GeV}$, could be achieved in about 3-5 month of running for each energy configuration. Kaon rates 


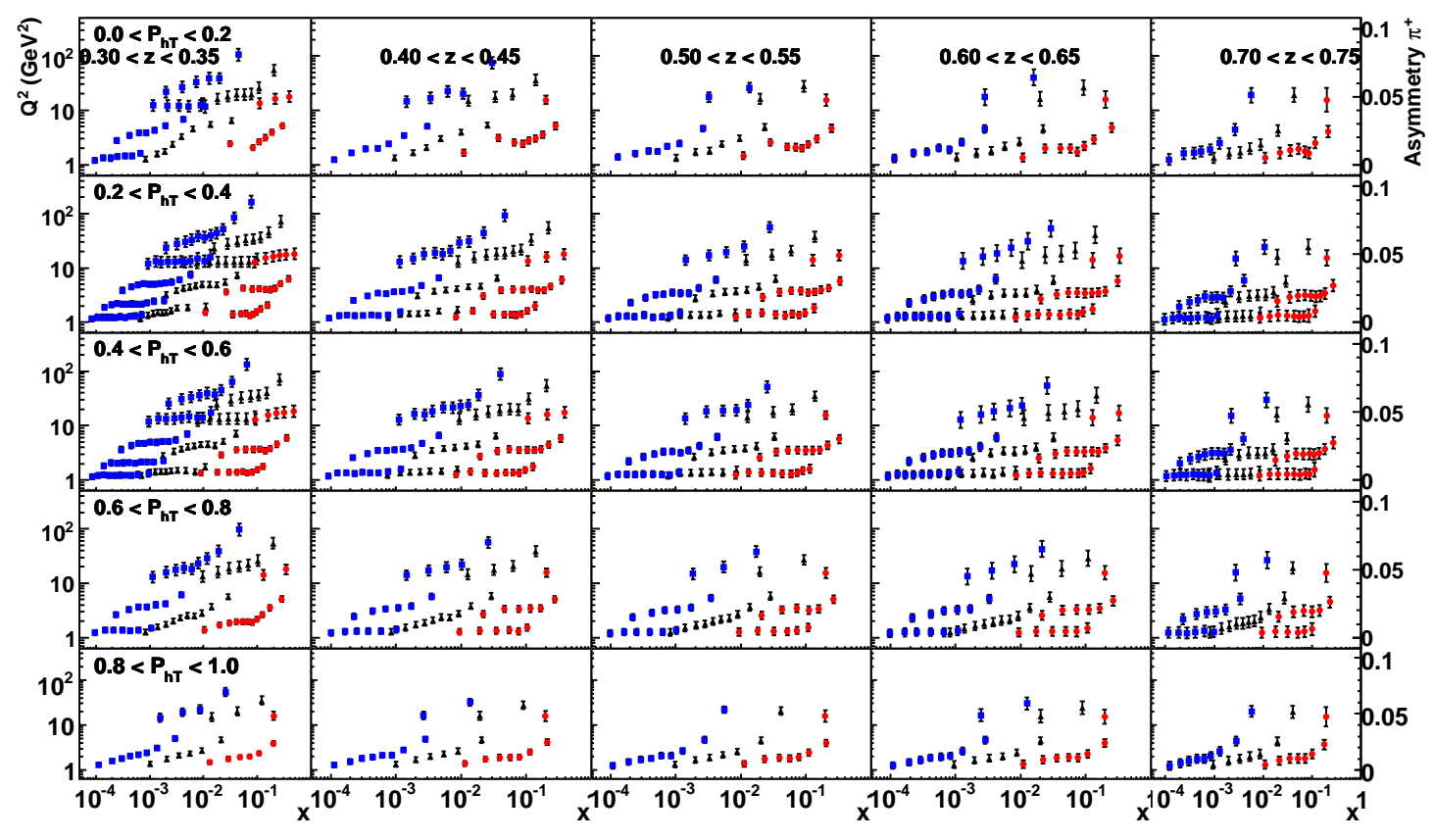

Figure 2.8. Four-dimensional representation of the projected accuracy for $\pi^{+}$production in semiinclusive DIS off the proton. Each panel corresponds to a specific $z$ bin with increasing value from left to right and a specific $P_{h T}$ bin with increasing value from top to bottom, with values given in the figure. The position of each point is according to its $Q^{2}$ and $x$ value, within the range $0.05<y<0.9$. The projected event rate, represented by the error bar, is scaled to the (arbitrarily chosen) asymmetry value at the right axis. Blue squares, black triangles and red dots represent the $\sqrt{s}=140 \mathrm{GeV}, \sqrt{s}=50 \mathrm{GeV}$ and $\sqrt{s}=15 \mathrm{GeV}$ EIC configurations, respectively. Event counts correspond to an integrated luminosity of $30 \mathrm{fb}^{-1}$ for each of the three configurations.

are typically a factor 4-5 lower than those for pions and a similar quality of data can be achieved within a correspondingly longer running time.

The strategy for a full flavour separation of the Sivers distribution, and TMD distributions in general, involves both pion and kaon identification over almost the whole momentum range and measurements with proton and effective neutron targets. For the latter, the usage of polarized ${ }^{3} \mathrm{He}$ ions is foreseen for both EIC concepts. Compared to the projections shown in fig. 2.8 the dilution factor of $1 / 3$ has to be compensated with higher luminosities (respectively longer running times). The resulting different phase space for the neutron measurements compared with the proton case due to the $Z / A$ factor entering the momentum distribution and the expected lower center-of-mass energy (by about $2 / 3$ ) because of the different rigidities of the beams can be compensated to a large extent by using the different beam energy settings.

In addition, valuable and necessary information about the transverse momentum dependence of the fragmentation process will be obtained from the same data using a fully differential extraction of the individual hadron multiplicities.

\section{Sensitivity to sea quarks}

Among the unique features of the EIC is its sensitivity for an exploration of the Sivers function for sea quarks, which are expected to play an important role in the lower $x$ region. 


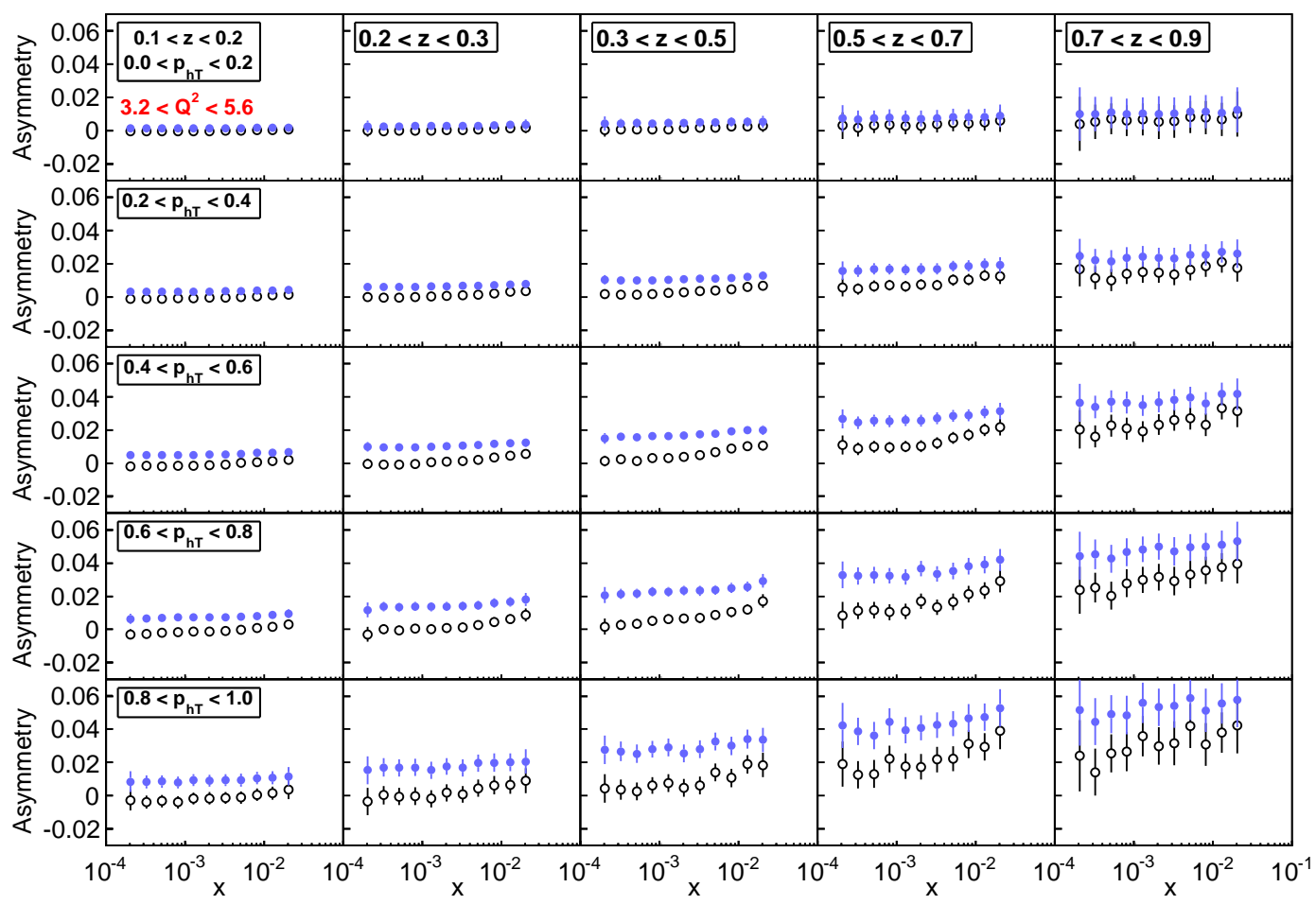

Figure 2.9. Simulated Sivers asymmetry amplitudes for $\pi^{+}$, obtained with an energy of $\sqrt{s}=140$ $\mathrm{GeV}$, as a function of $x$ in bins in $z, P_{h T}$ and for a single bin in $Q^{2}$ as given in the panels. Closed blue (open black) dots correspond to (non-)zero Sivers functions for sea quarks. Error bars represent the projected accuracy corresponding to an integrated luminosity of $4 \mathrm{fb}^{-1}$.

We investigate this sensitivity by generating two sets of events, one with and one without contributions from sea quarks. As the Sivers distribution is essentially unknown, it was parameterized via a constant multiplied by the unpolarized PDF with independent constants for $u, d$ and sea quarks. The Sivers asymmetry is returned by the generator on an eventby-event basis. The unpolarized PDFs of [82] and the fragmentation functions of [74] were used.

In both cases, the same parameterization for up and down quark Sivers functions was used, which were set equal to $25 \%$ of the unpolarized distribution, but with opposite sign, i.e. $f_{1 T}^{\perp u}(x)=-0.25 f_{1}^{u}(x)$ and $f_{1 T}^{\perp d}(x)=0.25 f_{1}^{d}(x)$. In the first data set, the Sivers functions for sea quarks were also set to $25 \%$ of the corresponding unpolarized distribution. In the second data set the sea quark Sivers distributions were fixed to zero. This allowed for a comparison of the case in which the sea quark Sivers function was significant compared to that of the valence quarks with the case of a vanishing sea quark contribution.

Figure 2.9 shows the asymmetry amplitudes for $\pi^{+}$, obtained with an energy of $\sqrt{s}=140$ $\mathrm{GeV}$, for a single bin in $Q^{2}$ as a function of $x$, binned in $z$ and $P_{h T}$ as indicated in the panels. Open black dots represent the case of non-zero Sivers functions for sea quarks and closed blue dots the case of vanishing contributions. Error bars correspond to an integrated luminosity of $4 \mathrm{fb}^{-1}$, already yielding sufficient precision to resolve small resulting differences in the asymmetry. Because of their different quark content, kaon production is expected to have a higher sensitivity to sea quark contributions. Figure 2.10 shows the asymmetry amplitudes 


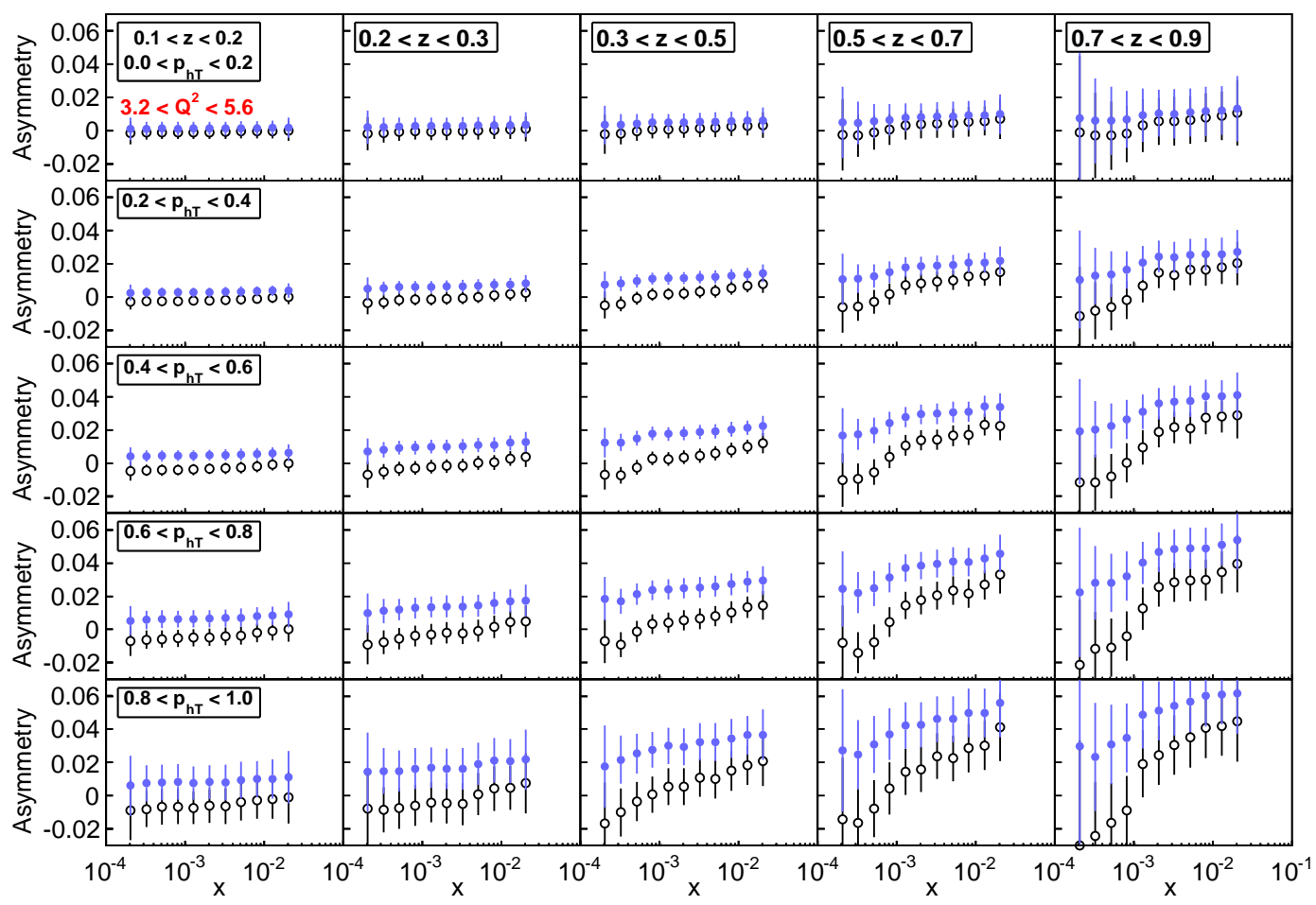

Figure 2.10. Simulated Sivers asymmetry amplitudes for $K^{+}$, obtained with an energy of $\sqrt{s}=140$ $\mathrm{GeV}$, as a function of $x$ in bins in $z, P_{h T}$ and for a single bin in $Q^{2}$ as given in the panels. Closed blue (open black) dots correspond to (non-)zero Sivers functions for sea quarks. Error bars represent the projected accuracy corresponding to an integrated luminosity of $4 \mathrm{fb}^{-1}$.

for $K^{+}$where indeed both scenarios are more distinct. As for $\pi^{+}$, the estimate is based on an integrated luminosity of $4 \mathrm{fb}^{-1}$ and obtained with an energy of $\sqrt{s}=140 \mathrm{GeV}$.

The study demonstrates that even a relatively brief running of the EIC provides the potential to distinguish zero and non-zero Sivers functions for sea quarks. Note that these parameterizations are intended not as a prediction of what asymmetries will actually be seen at an EIC, but as an indicator of sensitivity given the expected statistical precision.

\section{Impact of the EIC}

The EIC will be the unique facility for exploring the Sivers function (and TMDs in general) for sea quarks and the gluon, to study the evolution properties of TMD distributions and to investigate experimentally the transition from low to high transverse momenta. As discussed in sec. 2.2.1, our current knowledge is restricted to an essentially qualitative picture of the Sivers function. Available data permit to constrain parameterizations for up and down quarks only, employing relatively simple functional forms.

We illustrate the expected impact of data from the EIC using the parameterization from Ref. 327] as an arbitrarily chosen model of the Sivers function. This parameterization,

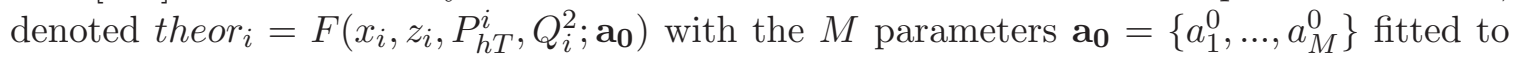



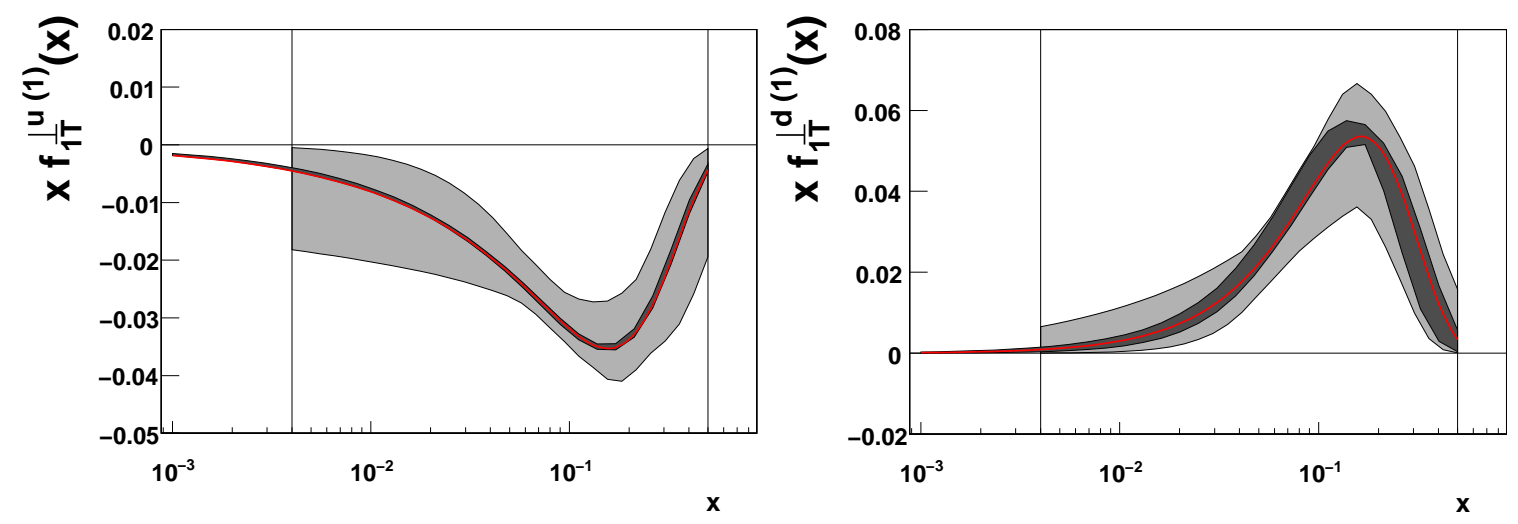

Figure 2.11. [color online] Comparison of the precision (2- $\sigma$ uncertainty) of extractions of the Sivers function for $u$ quarks (left) and $d$ quarks (right) from currently available data 327] (grey band) and from pseudo-data generated for the EIC with energy setting of $\sqrt{s}=45 \mathrm{GeV}$ and an integrated luminosity of $4 \mathrm{fb}^{-1}$ (dark grey band around the red line). The uncertainty estimates are for the specifically chosen underlying functional form (see text for details).

existing data, serves to generate a set of pseudo-data in each kinematic bin $i$

$$
f\left(\text { value }_{i} ; \text { theor }_{i}, \sigma_{i}^{2}\right)=\frac{1}{\sqrt{2 \pi \sigma_{i}^{2}}} e^{-\left(\text {value }_{i}-\text { theor }_{i}\right)^{2} / 2 \sigma_{i}^{2}} .
$$

In each $x_{i}, Q_{i}^{2}, z_{i}$ and $P_{h T}^{i}$ bin, the obtained values, value $i$, for the Sivers function are distributed using a Gaussian smearing with a width $\sigma_{i}$ corresponding to the simulated event rate at an energy of $\sqrt{s}=45 \mathrm{GeV}$ obtained with an integrated luminosity of $4 \mathrm{fb}^{-1}$. For illustration of the obtainable statistical precision the event rate for the production of $\pi^{+}$in semi-inclusive DIS was used.

This new set of pseudo-data was then analysed like the real data in Ref. [327]. Figure 2.11 shows the result for the extraction of the Sivers function for $u$ and $d$ quarks. The central value of $f_{1 T}^{\perp u}$, represented by the red line, follows by construction the underlying model. The 2-sigma uncertainty of this extraction, valid for the specifically chosen functional form, is indicated by the dark grey area, which is hardly seen around the red line. This precision, obtainable with an integrated luminosity of $4 \mathrm{fb}^{-1}$, is compared with the uncertainty of the extraction from existing data, represented by the light grey band and shown before in fig. 2.5.

Remembering that the event rate of the generated pseudo-data is achievable in a brief period of operation for an EIC, the impressive impact of the EIC on studies of TMDs is greatly illustrated.

\subsubsection{TMDs in Drell-Yan processes}

One of the intriguing facets of the Sivers effect is its peculiar breaking of universality, as discussed in secs. 2.1.4 and 2.4. The symmetry properties of QCD require a reversal of sign of the Sivers function when appearing in the Drell-Yan process, the production of dilepton pairs in the collision of two hadrons, as compared to DIS. The important test of this fundamental QCD prediction remains outstanding, its invalidation would have profound consequences for our understanding of high-energy reactions involving hadrons. It is thus 

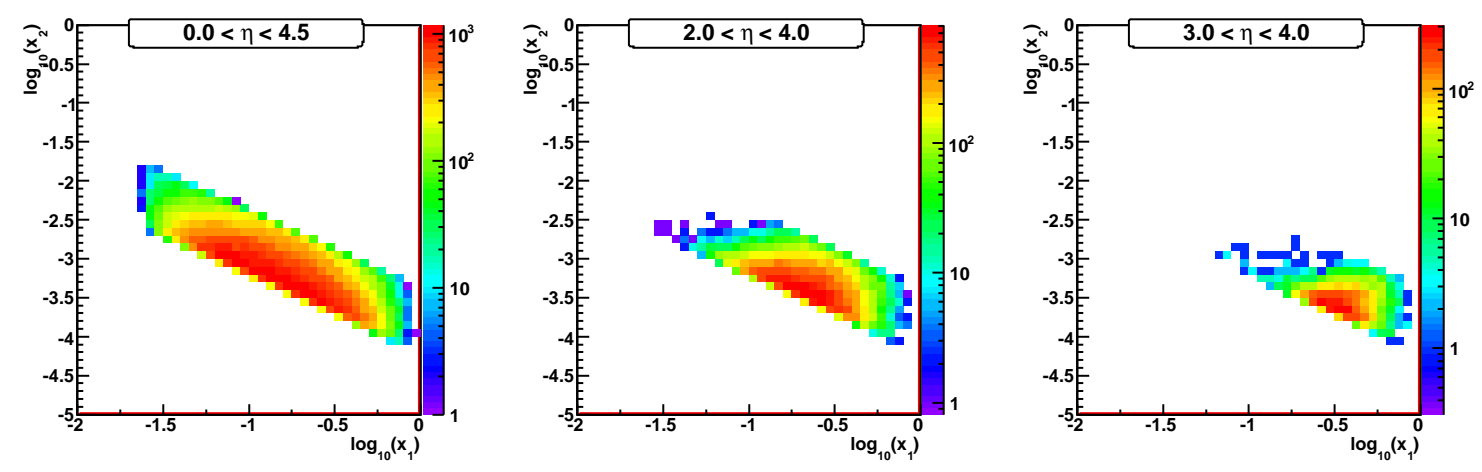

Figure 2.12. The correlation of the quark and antiquark momentum fractions, $x_{1}$ and $x_{2}$, in DrellYan for different rapidity bins in proton-proton collisions at $\sqrt{s}=500 \mathrm{GeV}$.

not surprising to see the Drell-Yan process appear as a milestone measurement in the update for the future spin program at RHIC [344].

The Drell-Yan process with unpolarized hadrons has been studied at numerous fixedtarget experiments [345, 346, 347, 348, 349]. There are several proposals for future polarized Drell-Yan measurements, either at fixed-target experiments (CERN, FermiLab, GSI, and J-Parc), but also at colliders (BNL, GSI). So far no measurement exists for Drell-Yan with transverse hadron polarization to isolate the Sivers effect, unlike the case for the related mechanism of the Boer-Mulders function. Being a naive-T-odd distribution the latter also involves a reversal of sign when going from DIS to Drell-Yan. For the BoerMulders function data from the Drell-Yan process exist. In particular the violation of the Lam-Tung relation [350] is a substantial hint of the Boer-Mulders effect, as discussed in sec. 2.5.2. However, being also a chiral-odd distribution, presents an additional challenge for experimental measurements and their interpretation, given that a second, presently poorly constrained, chiral-odd function is needed. In the case of Drell-Yan the other chiralodd function is a second Boer-Mulders function, making it especially tricky to look for the sign change between Drell-Yan and DIS.

Among the proposed measurements of the Sivers effect in Drell-Yan two have timescales of a few years from now. One is an experiment set at IP2 of RHIC (BNL) where transversely polarized "beam" protons will interact with effectively unpolarized "target" protons [344. At the COMPASS experiment at CERN it is not the beam - in this case consisting of pions - that will be polarized but the target [342]. This configuration is the theoretically more challenging one of the two as the partonic structure of the pion enters besides the structure of the proton.

The choice of measuring Drell-Yan single-spin asymmetries at a collider like RHIC has various advantages. Among others, the asymmetries depend only weakly on the partonic momentum $x_{2}$ of the (anti)quark in the unpolarized nucleon. When integrated over $x_{2}$ the cross section increases with the center-of-mass energy $\sqrt{s}$ as one can reach lower values of $x_{2}$ where anti-quarks are more abundant. Furthermore, it is easier to differentiate between "forward" and "backward" production at a collider allowing easy access to the valence region of the (transversely polarized) beam nucleon. In fig. 2.12, we show the correlation of the quark and antiquark momentum fractions, $x_{1}$ and $x_{2}$, in the Drell-Yan (DY) process

\footnotetext{
${ }^{1}$ The proton beams at IP2 are transversely polarized but due to rapid spin flips they can be spin balanced to get unpolarized protons.
} 


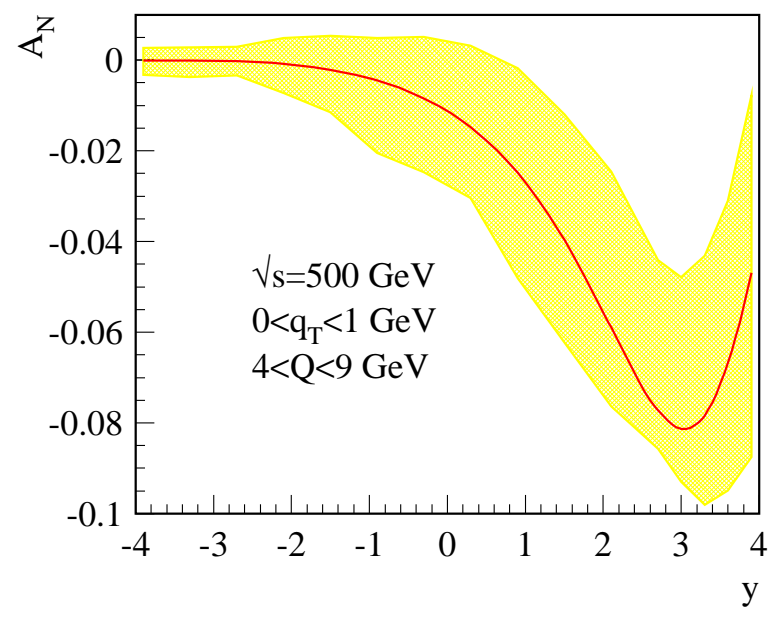

Figure 2.13. Sivers asymmetries for the Drell-Yan process at RHIC, as a function rapidity for $\sqrt{s}=500 \mathrm{GeV}$ 351.

for different rapidity bins in proton-proton collisions at $\sqrt{s}=500 \mathrm{GeV}$. The plot assumes an invariant mass range of the DY lepton pair between the $J / \psi$ and the Upsilon. To select DY at masses below the $J / \psi$ and/or at rapidities below 2.0 will be experimentally extremely challenging due to the dominance of the QCD $2 \rightarrow 2$ processes $\left(>10^{8}\right)$. The expected single-spin asymmetry, $A_{N}$, is presented in fig. 2.13 as a function of rapidity, $y$, for $\sqrt{s}=500 \mathrm{GeV}$ and integrated over the range $4 \div 9 \mathrm{GeV}$ in the invariant mass of the di-lepton pair [351]. The estimate makes use of a recent "DIS" Sivers function parameterization from fits to COMPASS and HERMES data 332. Asymmetries of this size should be readily measurable with a limited data set. Nevertheless, one should keep in mind that the change of sign applies to the flavor-dependent Sivers function. For a stringent test of this sign change it is therefore of utter importance not only to measure the Sivers effect in DIS and Drell-Yan, but to perform a flavor-decomposition of the Sivers effect as well. In $p p$ collisions one will be mainly sensitive to the $u$-quark Sivers function due to the charge factor. Using pion beams one can vary the sensitivity to the various quark flavors via the choice of the pion charge as the valence anti-quark flavor in the pion will either be an anti- $u$ or an anti- $d$. This will help in a subsequent flavor decomposition of the Sivers effect in Drell-Yan.

\subsubsection{Single-spin asymmetry in the collinear factorization: Twist-three mechanism}

The quark Sivers function discussed in the last subsection is also closely related to the twist-3 quark-gluon-quark correlation functions in the collinear factorization approach which can generate large single spin asymmetries in hard scattering process, in particular, in inclusive hadron production in $p p$ collisions. The single-transverse spin asymmetry in the process like $p p \rightarrow \pi X$ is among the simplest spin observables in hadronic scattering. One scatters a beam of transversely polarized protons off unpolarized protons and measures the numbers of pions produced to either the left or the right of the plane spanned by the momentum and spin directions of the initial polarized protons. Measurements of single-spin asymmetries in hadronic scattering experiments over the past three decades have shown spectacular results. Large asymmetries of up to several tens of percents were observed at forward (with respect to the polarized initial beam) angles of the produced pion. Despite the 
conceptual simplicity of $A_{N}$, the theoretical analysis of single-spin asymmetries in hadronic scattering is remarkably complex. The reason for this is that the asymmetry for a singleinclusive reaction like $p p \rightarrow \pi X$ is power-suppressed as $1 / \ell_{\perp}$ in the hard scale set by the observed large pion transverse momentum. Power-suppressed contributions to hardscattering processes are generally much harder to describe in QCD than leading-twist ones. In the case of the single-spin asymmetry, a complete and consistent framework could be developed [286, 288, 289, 352]. It is based on a collinear factorization theorem at nonleading twist that relates the single-spin cross section to convolutions of twist-three quarkgluon correlation functions for the polarized proton with the usual parton distributions for the unpolarized proton and the pion fragmentation functions, and with hard-scattering functions calculated from an interference of two partonic scattering amplitudes: one with a two-parton initial state and the other with a three-parton initial state.

In the following, we briefly describe the collinear factorization formalism for the twist3 single-spin-dependent cross section in the semi-inclusive deep inelastic scattering, $e p \rightarrow$ $e h X$. This factorization applies when the transverse momentum of the final state hadron is large compared to the non-perturbative scale $\Lambda_{\mathrm{QCD}}$. The usual leading twist spin-average cross section for this process can be schematically written as

$$
d \sigma \sim \sum_{a, b} f_{a}(x, \mu) \otimes D_{h / b}(z, \mu) \otimes \hat{\sigma}^{a b}(x, z, Q, \mu)
$$

where $f_{a}(x, \mu)$ and $D_{h / b}(z, \mu)(a, b=q, \bar{q}, g)$ are, respectively, the parton density in the nucleon and the fragmentation function for $b \rightarrow h$, convoluted with the hart part $\hat{\sigma}^{a b}$. The twist-3 cross section relevant for SSA in $e p^{\uparrow} \rightarrow e h X$ takes the factorized form,

$$
\begin{aligned}
d \sigma^{\mathrm{tw} 3} & \sim \sum_{a, b} G_{a}^{(3)}\left(x_{1}, x_{2}, \mu\right) \otimes D_{h / b}(z, \mu) \otimes \hat{\sigma}_{1}^{a b}\left(x_{1}, x_{2}, z, Q, \mu\right) \\
& +\sum_{a, b} \delta f_{a}(x, \mu) \otimes D_{h / b}^{(3)}\left(z_{1}, z_{2}, \mu\right) \otimes \hat{\sigma}_{2}^{a b}\left(x, z_{1}, z_{2}, Q, \mu\right)
\end{aligned}
$$

where $\otimes$ represents the appropriate convolution, similarly as the twist- 2 factorization formula (2.25) , with the relevant momentum fractions $x_{1,2}, z, x, z_{1,2}$ integrated over. $G_{a}^{(3)}\left(x_{1}, x_{2}, \mu\right)$ is the twist-3 distribution function in the transversely-polarized nucleon $p^{\uparrow}$, and $D_{h / b}^{(3)}\left(z_{1}, z_{2}, \mu\right)$ is the twist- 3 fragmentation function for the hadron $h$; the latter function is chiral-odd, combined with the chiral-odd transversity distribution $\delta f_{a}(x, \mu)$ for $p^{\uparrow}$. (In the TMD approach, the first term in (2.26) is described in terms of the Sivers function, and the second term is described using the Collins function.) These twist-3 distribution and fragmentation functions describe the multi-parton correlations in the nucleon and in the fragmentation process, respectively, and thus provides us with an opportunity to reveal the more detailed internal structure of hadrons beyond the parton-model picture. Each twist-3 function has its own logarithmic scale dependence, which differs from that of the twist-2 functions; for the corresponding $\mu$-dependence, see section (2.4.3).

For $G_{a}^{(3)}\left(x_{1}, x_{2}, \mu\right)$ with $a=q$ in (2.26) , two independent quark-gluon correlation functions, $G_{F}\left(x_{1}, x_{2}\right)$ and $\widetilde{G}_{F}\left(x_{1}, x_{2}\right)$, participate. They are defined as dimensionless, real, Lorentz-scalar functions in terms of nucleon matrix element associated with the gluon field strength tensor $F^{\alpha \beta}$ as well as the quark field $\psi$ on the light-cone [352, 353. Similarly, the twist-3 purely gluonic correlation functions $O\left(x_{1}, x_{2}\right)$ and $N\left(x_{1}, x_{2}\right)$ as $G_{g}^{(3)}\left(x_{1}, x_{2}, \mu\right)$ in (2.26), are defined through the gauge-invariant lightcone correlation of three field-strength 
tensors 354. Thus, a complete set of the twist-3 correlation functions in the transverselypolarized nucleon is now provided by $G_{F}\left(x_{1}, x_{2}\right), \widetilde{G}_{F}\left(x_{1}, x_{2}\right), O\left(x_{1}, x_{2}\right)$ and $N\left(x_{1}, x_{2}\right)$, taking into account all symmetry constraints in QCD. We note that the twist-3 correlation functions, $T_{F}\left(x_{1}, x_{2}\right), T_{G}^{(f, d)}(x, x)$, etc., used in the literature [355, 356, 357] can be expressed by the above correlation functions.

Another origin of SSA is in the fragmentation process for the final hadron, as represented in terms of the twist-3 fragmentation function $D_{h / b}^{(3)}\left(z_{1}, z_{2}, \mu\right)$ of (2.26) , which is also defined as a multi-parton light-cone correlation function (see [297]).

For SIDIS, $e p \rightarrow e h X$, the large transverse-momentum $P_{h T}$ of the hadron $h$ should come from a perturbative mechanism, i.e. from the recoil from the hard (unobserved) final-state partons. Then, the factorization formula (2.26) is derived in the LO perturbative QCD, manifesting their gauge invariance at the twist-3 level, and a practical procedure to calculate the relevant partonic hard part $\hat{\sigma}_{i}^{a b}$ is provided in [352, 354, 297]: an extra gluon, which emanates from nonperturbative multi-parton correlation and carries the momentum fraction $x_{2}-x_{1}$, participates in the partonic hard scattering. The coupling of this gluon allows an internal propagator in the partonic subprocess to be on-shell, and this produces the required imaginary phase. The results for those partonic subprocesses imply [352, 358, 359],

$$
\begin{aligned}
& \frac{d^{5} \sigma^{\mathrm{tw} 3}}{d x_{B} d Q^{2} d z_{h} d P_{h T}^{2} d \phi_{h}}=\sin \left(\phi_{h}-\phi_{S}\right) F^{\sin \left(\phi_{h}-\phi_{S}\right)}+\sin \left(2 \phi_{h}-\phi_{S}\right) F^{\sin \left(2 \phi_{h}-\phi_{S}\right)} \\
& \quad+\sin \phi_{S} F^{\sin \phi_{S}}+\sin \left(3 \phi_{h}-\phi_{S}\right) F^{\sin \left(3 \phi_{h}-\phi_{S}\right)}+\sin \left(\phi_{h}+\phi_{S}\right) F^{\sin \left(\phi_{h}+\phi_{\phi} \phi_{2} .27\right)}
\end{aligned}
$$

with the azimuthal angles $\phi_{h}$ and $\phi_{S}$ of $P_{h T}$ and $S_{\perp}$, respectively, measured from the lepton plane; the five azimuthal dependences in (2.26) are similar as those in the TMD approach. Here, each structure function $F^{\sin (\cdots)}$ is expressed in a factorized form, convoluted with $G_{F}(x, x)$ and $d G_{F}(x, x) / d x$. The similar twist-3 effects from $G_{F}$ and $\widetilde{G}_{F}$ have been investigated for SSA in Drell-Yan and direct photon productions, and hadron production in $p p$ collisions.

Charm production in SIDIS and $p p$ collisions is useful to study the twist-2 gluon distributions in the nucleon, since the $c \bar{c}$-pair creation through the photon-gluon or gluon-gluon fusion is their driving subprocess. Likewise, the three-gluon correlation functions can be probed by SSA in these processes. From this point of view, the three-gluon contribution to SSA in $D$-meson production processes, $e p^{\uparrow} \rightarrow e D X$ and $p^{\uparrow} p \rightarrow D X$, have been studied in [356, 357, 354, 360]. For both processes, the twist-3 cross sections for SSA can be derived entirely as the gluonic pole contribution leading to $x_{1}=x_{2}$, and thus receive the contributions $O(x, x), O(x, 0), N(x, x)$ and $N(x, 0)$ (and their derivatives) [354, 360]. The result for $e p^{\uparrow} \rightarrow e D X$ has five azimuthal dependences like in (2.27) 354].

So far, RHIC at BNL reported a significant amount of data of $A_{N}$ for $p^{\uparrow} p \rightarrow h X$ $(h=\pi, K, \eta, D, J / \Psi)$. Given that the NLO QCD in collinear factorization can provide a reasonable description of the corresponding unpolarized cross section, we expect that one can apply the above twist-3 formalism to analyze the $A_{N}$ data [289, 355, 361]. The complete LO QCD formula for $A_{N}$ from the twist-3 quark-gluon correlation functions to $p^{\uparrow} p \rightarrow h X$ has been derived: It consists of the contribution associated with $G_{F}(x, x)$ and $d G_{F}(x, x) / d x\left(\widetilde{G}_{F}(x, x)=0\right)$ [355], and the contribution 362] associated with $G_{F}(x, 0)$ and $\widetilde{G}_{F}(x, 0)$. Phenomenological analysis of RHIC data shows that both contributions are important, although the main contribution comes from the $G_{F}(x, x)$ contribution [289, 355], the $G_{F}(x, 0)\left(\widetilde{G}_{F}(x, 0)\right)$ contribution also plays an important role, and the combination of both contributions provides a reasonable description of the RHIC data, shedding light on 

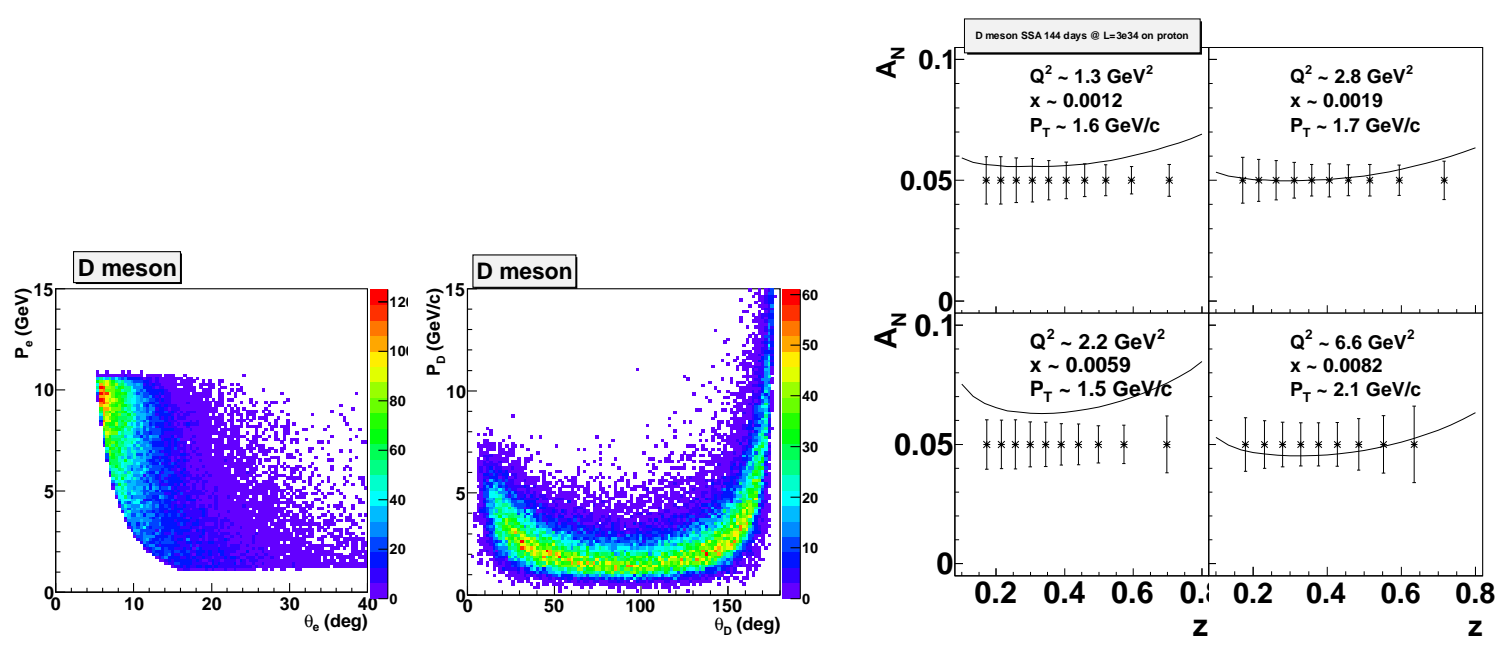

Figure 2.14. Left: kinematics for $D$-meson events showing the momentum vs. polar angle distribution for the electron and $D$ (or equivalently $\bar{D}$ ) meson in the laboratory frame. Right: Projected accuracy for transverse single-spin asymmetries from single $D$ meson production using an energy of $\sqrt{s}=50 \mathrm{GeV}$ and an integrated luminosity of $370 \mathrm{fb}^{-1}$.

the behavior of $G_{F}$ and $\widetilde{G}_{F}$ 361. There are also some initial efforts to calculate the twist-3 fragmentation contribution to $A_{N}$ 363. Global analysis of RHIC and future EIC data is expected to reveal more details on the role of the multi-parton correlations, including the three-gluon correlation functions.

The potential of the EIC for measuring transverse single-spin asymmetries in charm production is illustrated in fig. 2.14. In the simulation, based on the PYTHIA event generator, the main decay channel for $D$ mesons, $D \rightarrow \pi^{+} K^{-}$, with a branching ratio of $3.8 \pm 0.1 \%$ is investigated. Events are selected for $P_{h T}>1 \mathrm{GeV}$ and $Q^{2}>1 \mathrm{GeV}$ within $0.05<y<0.9$ and $1.86<M_{D}<1.87$. The signal-to-background ratio for the reconstructed $D$ mesons strongly depends on the detector resolution. In this study, we assume a momentum resolution of $0.8 \% \cdot \frac{p}{10 \mathrm{GeV}}$ and a resolution of the polar and azimuthal angles of $0.3 \mathrm{mrad}$ and $1 \mathrm{mrad}$, respectively. The resulting resolution of the reconstructed invariant mass of the $D$ meson is $1.8 \mathrm{MeV}$ yielding an overall signal-to-background ratio of about 1.6 to 1 . The overall detection efficiency for this triple coincidence process is assumed to be $60 \%$. The polarization of the proton beam is set to $80 \%$.

The projected accuracy for measuring transverse single-spin asymmetries in single $D$ meson production is shown in fig. 2.14 (right) as a function of $z$ for different regions in $Q^{2}$, $x$ and $P_{h T}$, as indicated in the figure, together with model calculations of the asymmetry from Ref. [356]. An energy of $\sqrt{s}=50 \mathrm{GeV}$ and an integrated luminosity of $370 \mathrm{fb}^{-1}$ were used. The study demonstrates a very promising feasibility of extracting observables involving charm production. It will significantly benefit from higher energies up to $\sqrt{s}=200$ $\mathrm{GeV}$.

In summary, the twist-3 collinear factorization framework provides us with a systematic way for describing SSA in the region of large transverse-momentum $P_{h T}$ of the final hadron, and is thus complementary to the TMD description of SSA which is valid in the low $P_{h T}$ region. For the twist-3 distribution functions in the transversely-polarized nucleon, relevant to SSA, there are two independent quark-gluon correlation functions and the two indepen- 
dent three-gluon correlation functions, all of which are process-independent. Twist-3 cross section formulae for SSA are available for many important processes, which can be used for confronting with the RHIC and EIC data and may serve to reveal multi-parton correlation effects in QCD hard processes.

\subsubsection{Unifying the Mechanisms for the Sivers effect}

Recent developments have shown that the TMD approach and the collinear factorization approach can be unified to describe the Sivers effect for the single transverse-spin asymmetries in semi-inclusive DIS. The TMD approach covers the kinematic region $P_{h T} \ll Q$ where $Q \gg \Lambda_{\mathrm{QCD}}$, while the twist-3 approach covers the large $P_{h T}$ region, $P_{h T} \gg \Lambda_{\mathrm{QCD}}$. A natural question here is whether the two mechanisms give rise to equivalent (or consistent) SSA in the overlapping region, $\Lambda_{\mathrm{QCD}} \ll P_{h T} \ll Q$. To address this issue, we first recall the relation between the Sivers function $f_{1 T}^{\perp}\left(x, k_{\perp}\right)$ and the quark-gluon correlation function $G_{F}(x, x)$ [364]: $\int d \boldsymbol{k}_{\perp}^{2} \boldsymbol{k}_{\perp}^{2} f_{1 T}^{\perp}\left(x, k_{\perp}\right)=\pi M_{N}^{2} G_{F}(x, x)$, which indicates that the two mechanisms are closely related.

A more explicit relation for the SSA in the two approaches has also been derived for the Sivers cross section, $F^{\sin \left(\phi_{h}-\phi_{S}\right)}$ in (2.27) 292, 294, 295]: In the TMD approach, $F^{\sin \left(\phi_{h}-\phi_{S}\right)}$ is expressed in terms of the Sivers function $f_{1 T}^{\perp}\left(x, k_{\perp}\right)$. In the large $k_{\perp}$-region, relevant to $\Lambda_{\mathrm{QCD}} \ll P_{h T} \ll Q$, the $k_{\perp}$-dependence of $f_{1 T}^{\perp}\left(x, k_{\perp}\right)$ can be generated perturbatively, such that $f_{1 T}^{\perp}\left(x, k_{\perp}\right)$ is expressed as the convolution of the corresponding perturbative coefficient functions with the nonperturbative correlation functions $G_{F}$ and $\widetilde{G}_{F}$. By inserting this form of $f_{1 T}^{\perp}\left(x, k_{\perp}\right)$ into the TMD factorization formula for $F^{\sin \left(\phi_{h}-\phi_{S}\right)}$, one obtains the cross section written in terms of $G_{F}$ and $\widetilde{G}_{F}$, and this expression turns out to be identical to the leading $P_{h T}$ behavior of the twist-3 mechanism for $F^{\sin \left(\phi_{h}-\phi_{S}\right)}$ in the overlap region $\Lambda_{\mathrm{QCD}} \ll P_{h T} \ll Q$. From these studies, the two mechanisms for single-spin asymmetries represent a unique QCD effect over the entire $P_{h T}$ region. The same equivalence was also shown for the SSA in the Drell-Yan process. It should be noted that the sign of the Sivers function changes from SIDIS to the Drell-Yan case, while the twist-3 quark-gluon correlation functions are process-independent. The connection between the two mechanisms is also consistent with such process-(in)dependence [293.

The contribution from the twist-3 fragmentation function in (2.26) gives rise to the structure function $F^{\sin \left(\phi_{h}+\phi_{S}\right)}$ in (2.27), and dominates the leading $P_{h T}$ behavior of $F^{\sin \left(\phi_{h}+\phi_{S}\right)}$ compared to that from the quark-gluon correlation functions. This leading $P_{h T}$ behavior in $F^{\sin \left(\phi_{h}+\phi_{S}\right)}$ turns out to be identical to the corresponding contribution from the Collins function in the TMD approach, similarly as the above equivalence for $F^{\sin \left(\phi_{h}-\phi_{S}\right)}$ [297.

These are nontrivial and important results, which demonstrate that we indeed have a unique picture for single transverse-spin asymmetries in DIS and hadronic collisions. The discussion can be further generalized to other structure functions in SIDIS as well.

To analyze the general power behavior of the structure functions, it is important to realize that the power expansions are done in two different ways in the above two descriptions. At low $q_{T}$, first we expand in $\left(q_{T} / Q\right)^{n-2}$ and neglect terms with $n$ bigger than a certain value (so far, analyses have been carried out only up to $n=3$, i.e., twist-3). To study the behavior at intermediate $q_{T}$ we further expand in $\left(M / q_{T}\right)^{k}$. Vice versa, at high $q_{T}$ we first expand in $\left(M / q_{T}\right)^{n}$ (also in this case, analyses are available up to $n=3$, i.e., twist-3). To study the intermediate- $q_{T}$ region, we further expand in $\left(q_{T} / Q\right)^{k-2}$. We can encounter two different situations: 
- Type-I observables, where the leading terms at high and low transverse momentum have the same behavior. For instance,

$$
F\left(q_{T}, Q\right)=A\left[\frac{q_{T}}{Q}\right]^{0}\left[\frac{M}{q_{T}}\right]^{2}+B\left[\frac{q_{T}}{Q}\right]^{2}\left[\frac{M}{q_{T}}\right]^{2}+\ldots,
$$

where the term $A$ is leading in both the low- and high- $q_{T}$ calculations. In this case, the calculations at high and low transverse momentum must yield exactly the same result at intermediate transverse momentum [273, 292]. If a mismatch occurs, it means that one of the calculations is incorrect or incomplete.

- Type-II observables, where the leading terms at high and low transverse momentum have different behavior. For instance,

$$
F\left(q_{T}, Q\right)=A^{\prime}\left[\frac{q_{T}}{Q}\right]^{0}\left[\frac{M}{q_{T}}\right]^{4}+B^{\prime}\left[\frac{q_{T}}{Q}\right]^{2}\left[\frac{M}{q_{T}}\right]^{2}+\ldots .
$$

where the first term is leading and the second term sub-leading in the low- $q_{T}$ calculation, whereas the reverse holds in the high- $q_{T}$ calculation. In this case, if the calculations at high and low transverse momentum are performed at their respective leading order, they describe two different mechanisms and will not lead to the same result at intermediate transverse momentum. In order to "match", the calculations should be carried out in both regimes up to the sub-subleading order. We could call this situation an "expected mismatch", since it is simply due to the difference between the two expansions.

In Tab. 2.3 we list the power behavior of the structure functions at intermediate transverse momentum, as obtained from the limits of the low- $q_{T}$ and high- $q_{T}$ calculation. For details of the calculation, we refer to [296]. The structure functions with a "yes" or "no" in the last column of Tab. 2.3 are type-I observables, where on the basis of power counting we know that two calculations describe the same physics and should therefore exactly match. In these cases, the high- $q_{T}$ calculation describes the perturbative tail of the low- $q_{T}$ effect. The two mechanisms need not be distinguished. Using resummation it should be possible to construct expressions for these observables that are valid at any $q_{T}$. Six of these structure functions have been calculated explicitly.

For the functions identified as type-II in the last column of Tab. 2.3. the low- $q_{T}$ and high- $q_{T}$ calculations at leading order pick up two different components of the full structure function. They therefore describe two different mechanisms and do not match. For such type-II observables, if one aims at studying the leading-twist contribution from transverse momentum distributions, some considerations have to be kept in mind:

- the leading contribution from the high- $q_{T}$ calculation (often referred to as a pQCD or radiative correction) is a competing effect that has to be taken into account 365 , 366, 367];

- $q_{T}$-weighted asymmetries enhance the high- $q_{T}$ mechanism and thus are not appropriate to extract type-II TMDs;

- it is at present impossible to construct an expression that extends the high- $q_{T}$ calculation to $q_{T} \approx M$, since this requires a smooth merging into unknown twist-4 contributions, which most probably cannot be factorized (see also Ref. [368]); 


\begin{tabular}{|l|c|c|c|}
\hline $\begin{array}{l}\text { structure } \\
\text { function }\end{array}$ & $\begin{array}{c}\text { low- } q_{T} \\
\text { power }\end{array}$ & $\begin{array}{c}\text { high- } q_{T} \\
\text { power }\end{array}$ & $\begin{array}{c}\text { exact } \\
\text { match }\end{array}$ \\
\hline$F_{U U, T}$ & $1 / q_{T}^{2}$ & $1 / q_{T}^{2}$ & yes \\
$F_{U U}^{\cos 2 \phi_{h}}$ & $1 / q_{T}^{4}$ & $1 / Q^{2}$ & type II \\
$F_{U L}^{\sin 2 \phi_{h}}$ & $1 / q_{T}^{4}$ & & (type II) \\
$F_{L L}$ & $1 / q_{T}^{2}$ & $1 / q_{T}^{2}$ & yes \\
\hline
\end{tabular}

\begin{tabular}{|l|c|c|c|}
\hline $\begin{array}{l}\text { structure } \\
\text { function }\end{array}$ & $\begin{array}{c}\text { low- } q_{T} \\
\text { power }\end{array}$ & $\begin{array}{c}\text { high- } q_{T} \\
\text { power }\end{array}$ & $\begin{array}{c}\text { exact } \\
\text { match }\end{array}$ \\
\hline$F_{U T, T}^{\sin \left(\phi_{h}-\phi_{S}\right)}$ & $1 / q_{T}^{3}$ & $1 / q_{T}^{3}$ & yes \\
$F_{U T}^{\sin \left(\phi_{h}+\phi_{S}\right)}$ & $1 / q_{T}^{3}$ & $1 / q_{T}^{3}$ & yes \\
$F_{U T}^{\sin \left(3 \phi_{h}-\phi_{S}\right)}$ & $1 / q_{T}^{3}$ & $1 /\left(Q^{2} q_{T}\right)$ & type II \\
$F_{L T}^{\cos \left(\phi_{h}-\phi_{S}\right)}$ & $1 / q_{T}^{3}$ & & (yes) \\
\hline
\end{tabular}

Table 2.3. Behavior of SIDIS structure functions in the region $M \ll q_{T} \ll Q$, as deduced from the low- $q_{T}$ calculation based on TMD factorization and the high- $q_{T}$ calculation based on collinear factorization. Empty fields indicate that no calculation is available. The last column indicates whether the expressions match exactly, do not match exactly, or should not be expected to match. In parentheses: expected answers based on analogy, rather than actual calculation.

- it is desirable from the experimental point of view to build observables that are least sensitive to the effect of radiative corrections.

We stress that the above considerations apply not only to semi-inclusive DIS, but also to Drell-Yan and $e^{+} e^{-}$annihilation [369], which have been already used to extract the BoerMulders and Collins functions [367, 370].

In summary, at the moment there is the hope to build descriptions of the structure functions that go from low to high transverse momentum for the five structure functions with a "yes" in the last column of Tab. 2.3.

\subsubsection{From low to high transverse momentum}

Based on the above results, we can write down a unique formula for the transverse momentum dependence. Following the procedure of [273], the differential cross section for the spin dependent SIDIS process can be written as,

$$
\frac{d \Delta \sigma\left(S_{\perp}\right)}{d y d x_{B} d z_{h} d^{2} P_{h T}}=\frac{d \Delta \sigma^{\mathrm{TMD}}}{d y d x_{B} d z_{h} d^{2} P_{h T}}+\left(\frac{d \Delta \sigma^{\mathrm{CO}}}{d y d x_{B} d z_{h} d^{2} P_{h T}}-\left.\frac{d \Delta \sigma^{\mathrm{CO}}}{d y d x_{B} d z_{h} d^{2} P_{h T}}\right|_{P_{h T} \ll Q}\right)(2
$$

which is valid in the whole transverse momentum region at leading power of $1 / Q^{2}$. In the above equation, the first term comes from the TMD factorization formalism, and the second term from the collinear factorization, $\mathrm{CO}$, with the twist-three quark-gluon correlations contributions. The second term will dominate the SSA at large transverse momentum, and its $q_{T}$-dependence can be calculated from perturbative QCD. On the other hand, at low transverse momentum $P_{h T} \ll Q$, the second term vanishes, because the two contributions are exactly the same in this limit, and cancel each other out. Experimentally, if we can study the transverse momentum dependence of the SSA for a wide range, we shall explore the transition from the perturbative region to the nonperturbative region.

The potential of the EIC for a study of this transition is illustrated in fig. 2.15, which shows the projected accuracy for single $\pi^{+}$production for a four-dimensional binning in the kinematic variables $x, Q^{2}, z$ and $P_{h T}$, using three different energy configurations for the $\operatorname{EIC}(\sqrt{s}=15,50$ and $140 \mathrm{GeV})$ and an integrated luminosity of $120 \mathrm{fb}^{-1}$ for each configuration. Events are selected for $0.05<y<0.9$ and $W^{2}>5 \mathrm{GeV}^{2}$ and for the $z$ range of $0.30<z<0.35$, as example. An overall detection efficiency of $50 \%$ and a beam polarization of $70 \%$ are assumed. The position of each point is according to its $x$ and $Q^{2}$ 


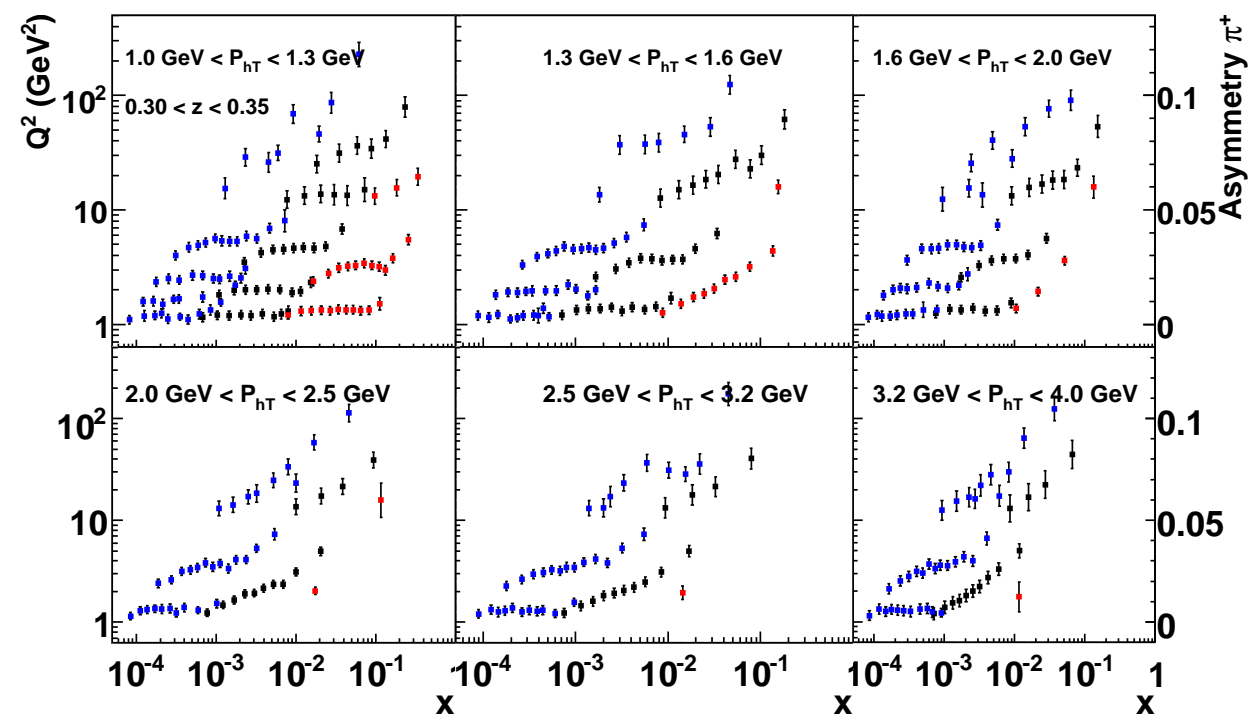

Figure 2.15. Four-dimensional representation of the projected accuracy for single $\pi^{+}$production in semi-inclusive DIS off the proton focussing on the transition region from low to high $P_{h T}\left(q_{T} \approx\right.$ $\left.P_{h T} / z_{h}\right)$ as indicated in the panels. The position of each point is according to its $Q^{2}$ and $x$ value for a specific bin in $z$ of $0.30<z<0.35$ and within the range $0.05<y<0.9$. The projected event rate, represented by the error bar, is scaled to the (arbitrarily chosen) asymmetry value at the right axis. Blue squares, black triangles and red dots represent the $\sqrt{s}=140 \mathrm{GeV}, \sqrt{s}=50 \mathrm{GeV}$ and $\sqrt{s}=15$ $\mathrm{GeV}$ EIC configurations, respectively. Event counts correspond to an integrated luminosity of 120 $\mathrm{fb}^{-1}$ for each of the three configurations.

value (abscissa and left ordinate, respectively) and each panel is for a $P_{h T}$ bin as indicated in the figure. The projected event rate is represented by the error bar scaled with respect to the (arbitrarily chosen) asymmetry value given at the right ordinate. The parameterization of Ref. 371] was used to simulate the cross section in the transition region. The simulation demonstrates that the transition region $q_{T} \approx P_{h T} / z_{h} \sim 4 \div 8 \mathrm{GeV}$ can be explored in great detail. Energies up to $\sqrt{s}=200 \mathrm{GeV}$ and longer running times will allow for exploring even higher values of $P_{h T}$.

The most important example to study the transition between low and high transverse momentum and the role of resummation is the structure function $F_{U U, T}$. The doublelongitudinal structure function $F_{L L}$ is the only other example where the theoretical framework has been developed at the same level [372].

Fig. 2.16 shows an example of resummation results for DIS at a high-energy EIC option. These results give us an idea of the extension of the region of intermediate transverse momentum (and therefore also of the regions of high and low transverse momentum). This extension obviously depends on experimental kinematics, in particular on $Q^{2}$. As a lower boundary of this region we can consider the values of $q_{T}$ where the nonperturbative component of the Sudakov factor becomes relevant. As an upper boundary we can consider the values of $q_{T}$ for which the fixed-order cross section becomes comparable to the resummed cross section. From fig. 2.16 we can estimate that the intermediate-transverse-momentum region corresponds to $4 \mathrm{GeV} \lesssim q_{T} \lesssim 8 \mathrm{GeV}$.

A lot remains to be done to better pin down the nonperturbative Sudakov factors, their functional form, their flavor dependence, and their errors. This should be a high-priority 


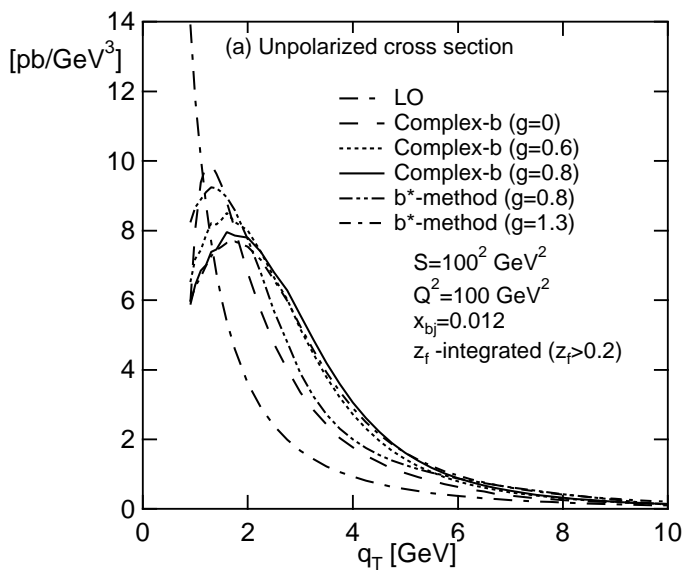

Figure 2.16. Unpolarized SIDIS cross section for EIC kinematics from Ref. 372. Shown are: the fixed-order result, the resummation results with different high- $b$ regularizations and different values of the nonperturbative Sudakov factor.

task for the EIC. The same is true for the doubly-longitudinally polarized case, where the nonperturbative components are unknown.

To conclude this section, we mention that the same program of resumming radiative contributions should be pursued also for the Sivers, Collins, and $F_{L T}^{\cos \left(\phi_{h}-\phi_{S}\right)}$ structure functions. At the moment the only discussion of similar topics is done in sec. 9 of Ref. [369]. However, we can expect developments in this direction in the near future and hope to obtain an expression of the above-mentioned structure functions that includes transverse-momentum resummation and describes the physics in the whole transverse-momentum spectrum.

\subsubsection{Weighted Asymmetries}

Currently, experimental studies in semi-inclusive DIS have limited access to single-spin asymmetries at large transverse momentum, and most of the data are in the low transverse momentum region, where the TMD formalism dominates. In phenomenological studies, in order to compare with the experimental data, one has to make model assumptions for the transverse momentum dependence of the distribution and fragmentation functions. However, there is a class of observables that does not require detailed model assumptions about transverse momentum dependence. These are transverse momentum weighted single-spin asymmetries, which transform the convolutions in the factorized cross section into simple products [244, 373].

Staying for the moment in the framework of collinear factorization, an example for a weighted differential cross section at leading order in $\alpha_{s}$ is

$$
\int d^{2} P_{h T} \frac{P_{h T}}{z_{h} M_{P}} \sin \left(\phi_{h}-\phi_{S}\right) \frac{d \Delta \sigma^{\mathrm{TMD}}\left(S_{\perp}\right)}{d x_{B} d y d z_{h} d^{2} \vec{P}_{h T}}=\sigma_{0} \sum_{q} e_{q}^{2} \frac{g_{s}}{2 M_{P}} T_{F}^{q}(x) D(z),
$$

where $e_{q}$ is the electric charge for a quark of flavor $q$, and where $T_{F}(x)$ is the Qiu-Sterman matrix element of the quark-gluon correlation function, and has been defined above. With the standard choice of $P_{h T}$-weights $w_{1}=P_{h T} / z_{h} M_{P}$ for the numerator and $w_{0}=1$ for the 
denominator, the $P_{h T}$-weighted Sivers-asymmetry thus becomes

$$
\frac{\left\langle\frac{P_{h T}}{z_{h} M_{P}} \sin \left(\phi_{h}-\phi_{S}\right)\right\rangle_{\mathrm{UT}}}{\langle 1\rangle_{\mathrm{UU}}}=\frac{\frac{1}{Q^{4}}\left(1-y+\frac{y^{2}}{2}\right) \frac{x_{B}}{2 M_{P}} \sum_{q} e_{q}^{2} g_{s} T_{F}^{q}(x) D(z)}{\frac{1}{Q^{4}}\left(1-y+\frac{y^{2}}{2}\right) x_{B} \sum_{q} e_{q}^{2} f_{1}(x) D(z)} .
$$

We can go beyond the above leading order results and establish a collinear factorization formalism for the weighted single transverse spin dependent cross section. A similar study has been performed for the Drell-Yan lepton pair production process, where a next-to-leading order perturbative corrections have been obtained [291]. We expect similar calculations for SIDIS shall appear soon.

Recently, a generalization to employ Bessel functions as weights $w_{n} \propto J_{n}\left(\left|\mathbf{P}_{h T}\right| \mathcal{B}_{T}\right)$ has been suggested [374]. The Sivers asymmetry with generalized weights reads

$$
\begin{aligned}
\left.\frac{\left\langle\frac{2 J_{1}\left(\left|\mathbf{P}_{h T}\right| \mathcal{B}_{T}\right)}{z M \mathcal{B}_{T}}\right.}{\left\langle J_{0}\left(P_{h T} \mathcal{B}_{T}\right)\right\rangle} \sin \left(\phi_{h}-\phi_{s}\right)\right\rangle_{U T} \\
\quad-2 \frac{\frac{1}{Q^{4}}\left(1-y+\frac{y^{2}}{2}\right) \sum_{q} e_{q}^{2} \tilde{f}_{1 T}^{\perp(1) q}\left(x, z^{2} \mathcal{B}_{T}^{2}\right) \tilde{D}\left(z, \mathcal{B}_{T}^{2}\right)}{\frac{1}{Q^{4}}\left(1-y+\frac{y^{2}}{2}\right) \sum_{q} e_{q}^{2} \tilde{f}_{1}\left(x, z^{2} \mathcal{B}_{T}^{2}\right) \tilde{D}_{1}\left(z, \mathcal{B}_{T}^{2}\right)}
\end{aligned}
$$

where now $\tilde{f}_{1 T}^{\perp(1) q}, \tilde{f}_{1}^{q}$ and $\tilde{D}$ are TMDs and TMD FFs Fourier transformed with respect to transverse momentum. In the asymptotic limit $\mathcal{B}_{T} \rightarrow 0$, we recover the conventional weighted asymmetry Eq. (2.32), and the Fourier transformed TMDs and FFs can be identified with the moments in that equation.

An important advantage of the generalized weights is that a non-zero choice of the parameter $\mathcal{B}_{T}$ can reduce the sensitivity to large transverse momenta. This property also applies to the Fourier transformed TMDs and TMD FFs entering the asymmetries. The new approach thus avoids the problem of divergent $k_{\perp}$-integrals that affects moments of TMDs and TMD FFs. Additionally, the analysis in Ref. 374 shows that soft factors appearing beyond tree level cancel out of the weighted asymmetry.

We conclude that an EIC presents a unique opportunity to obtain the necessary coverage and resolution in $P_{h T}$ to explore the nucleon spin structure in the language of weighted asymmetries. 


\subsection{Transverse polarization effects with gluons}

Daniël Boer, Stanley J. Brodsky, Piet J. Mulders, Cristian Pisano, Markus Diehl, Bo-Wen Xiao, Feng Yuan

The gluon Sivers function shares the same characteristic features as its counterpart in the quark sector, the quark Sivers function, as discussed in the last section. Among the important information we can obtain from this distribution is the spin-orbit correlation of gluons inside the nucleon, which will help us to understand the gluon spin contribution to the proton spin. The EIC is the unique machine to map out in much detail the gluon distribution, including the spin-dependent and spin-averaged transverse momentum dependent distributions. In this section, we will focus on the gluon Sivers function. The study of this distribution is strongly related to other measurements such as the gluon GPDs and the unintegrated gluon distributions of nucleon/nucleus at small- $x$.

Various processes in DIS can be used to probe the transverse momentum dependent gluon distributions, such as heavy quark and quarkonium production. Also the dijet/dihadron correlation has been proposed as a promising probe for the gluon Sivers function and other TMD gluon distributions.

In Ref. [375], it was suggested to use the dijet-correlation to study the gluon Sivers function in $p p$ collisions. However, because of both initial and final state interaction effects involved in $p p$ scattering, the factorization of this process is shown to be broken (see detailed discussions in next section). On the other hand, for the DIS processes, because only one hadron is involved in the initial state, the dijet-correlation process could be factorized in the same spirit as the semi-inclusive hadron production discussed in the previous sections.

We consider here the dijet/quark-antiquark production in DIS

$$
\gamma^{*} N^{\uparrow} \rightarrow H_{1}\left(k_{1}\right)+H_{2}\left(k_{2}\right)+X
$$

where $N$ represents the transversely polarized nucleon, $H_{1}$ and $H_{2}$ are the two final state particles with momenta $k_{1}$ and $k_{2}$, respectively. We are interested in the kinematic region where the transverse momentum imbalance between them is much smaller than the individual transverse momenta: $\boldsymbol{k}_{\perp}=\left|\boldsymbol{k}_{1 T}+\boldsymbol{k}_{2 T}\right| \ll P_{J T}$ where $\boldsymbol{P}_{J T}$ is defined as $\left(\boldsymbol{k}_{1 T}-\boldsymbol{k}_{2 T}\right) / 2$. This is referred to as the (back-to-back) correlation limit. An important advantage of taking this correlation limit is that we can apply the power counting method to obtain the leading order contribution of $k_{\perp} / P_{J T}$ where the differential cross section directly depends on the TMD gluon distribution. As illustrated in Fig. 2.17, with transverse spin in the dijet plane, the correlation between the two jets will lead to a preferred direction in the transverse plane. This will signal the gluon Sivers effect if the process is dominated by the gluonic subprocesses.

As demonstrated in Ref. 283, the TMD gluon distribution in the quark-antiquark jet correlation in the DIS process of (2.34) follows the original gluon distribution definition of Ref. [241],

$$
x f_{1}^{g}\left(x, k_{\perp}\right)=\int \frac{d \xi^{-} d^{2} \xi_{\perp}}{(2 \pi)^{3} P^{+}} e^{i x P^{+} \xi^{-}-i k_{\perp} \cdot \xi_{\perp}}\left\langle P\left|F^{+i}\left(\xi^{-}, \xi_{\perp}\right) \mathcal{L}_{\xi}^{\dagger} \mathcal{L}_{0} F^{+i}(0)\right| P\right\rangle,
$$

where $F^{\mu \nu}$ is the gauge field strength tensor $F_{a}^{\mu \nu}=\partial^{\mu} A_{a}^{\nu}-\partial^{\nu} A_{a}^{\mu}-g f_{a b c} A_{b}^{\mu} A_{c}^{\nu}$ with $f_{a b c}$ the antisymmetric structure constants for $S U(3)$, and the gauge-link follows the similar definition as that for the quark distribution but in the adjoint representation. The physics behind 


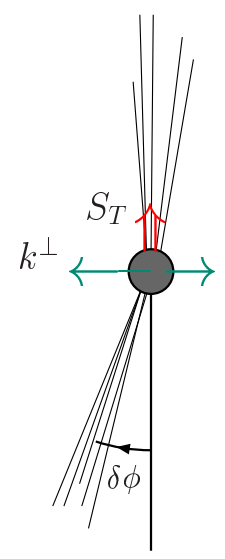

Figure 2.17. Back-to-back dijet correlation can be used to probe the TMD gluon distributions.

this factorization is the following. The virtual photon scatters on the nucleon target and produces a quark-antiquark pair through the partonic process $\gamma^{*} g \rightarrow q \bar{q}$. In the correlation limit, the quark-antiquark pair stays close in the coordinate space, and act as a color-octet object, which effectively behaves like a single gluon. In particular, the net effect of the final state interactions between the nucleon target and the quark-antiquark pair is exactly the same antisymmetric structure $f_{a b c}$ as in the TMD gluon definition of Eq. (2.35). This is totally different from the analogous QED process where the final state interactions cancel out completely with the fermion-antifermion pair.

In the following, we will present some recent phenomenological studies on the gluon TMDs from the quark-antiquark correlation in DIS processes. We expect more interesting results shall be obtained in the near future.

\subsubsection{The gluonic Sivers effect in dihadron production}

The production of a pair of hadrons with high transverse momenta in DIS is sensitive to the transverse-momentum dependent gluon distribution. In particular, it has a transverse target spin asymmetry due to the gluon Sivers function. The relevant parton-level subprocess is $\gamma^{*} g \rightarrow q \bar{q}$, and to eliminate contributions from $\gamma^{*} q \rightarrow q g$ and $\gamma^{*} \bar{q} \rightarrow \bar{q} g$ we focus on charm production.

As a straight forward generalization of the unpolarized case recently studied in [283], the cross section for the dijet/c $\bar{c}$ production from a nucleon with transverse polarization $\boldsymbol{S}_{\perp}$ can be written as

$$
\frac{d \sigma^{\gamma_{T, L}^{*} p \rightarrow c \bar{c}+X}}{d z d^{2} k_{1 T} d^{2} k_{2 T}}=\frac{H^{\gamma_{T, L}^{*} g \rightarrow c \bar{c}}}{z \bar{z}}\left[f_{1}^{g}\left(x, k_{\perp}\right)+\frac{\left(\boldsymbol{S}_{\perp} \times \boldsymbol{k}_{\perp}\right)^{3}}{M} f_{1 T}^{g \perp}\left(x, k_{\perp}\right)\right] .
$$

Here, $f_{1}^{g}$ is the usual gluon TMD, $f_{1 T}^{g \perp}$ the gluon Sivers distribution and $\bar{z}=1-z$. Again, we are interested in the back-to-back correlation limit. The gluon momentum fraction is then given by $x / x_{B} \approx 1+\left(P_{J T}^{2}+m_{c}^{2}\right) /\left(z \bar{z} Q^{2}\right)$, where $m_{c}$ is the charm quark mass. The hard-scattering cross sections $H^{\gamma_{T, L}^{*} g \rightarrow c \bar{c}}$ for transverse and longitudinal photons depend on $P_{h T}^{2}, Q^{2}, z$ and $m_{c}$ and can be found in [283].

It may be possible to study the cross section (2.36) experimentally through the production of two heavy-quark jets, but the interpretation of this process requires a quantitative 
understanding of the relative transverse momentum between a reconstructed jet and the heavy quark it originates from. As an alternative, we consider here the production of two heavy hadrons, e.g. $D$ mesons. Its cross section reads

$$
\begin{gathered}
\frac{d \sigma^{\gamma_{T, L}^{*} p \rightarrow h_{1} h_{2}+X}}{d z_{1} d z_{2} d^{2} P_{h_{1} T} d^{2} P_{h_{2} T}}=\int_{z_{1}}^{1-z_{2}} d z \frac{H^{\gamma_{T, L}^{*} g \rightarrow c \bar{c}}}{z^{2} \bar{z}^{2}} \int d^{2} \lambda_{1 T} d^{2} \lambda_{2 T}\left[f_{1}^{g}\left(x, k_{\perp}\right)\right. \\
\left.+\frac{\left(\boldsymbol{S}_{\perp} \times \boldsymbol{k}_{\perp}\right)^{3}}{M} f_{1 T}^{g \perp}\left(x, k_{\perp}\right)\right] D^{h_{1} / c}\left(\frac{z_{1}}{z}, \frac{z_{1}}{z} \lambda_{1 T}\right) D^{h_{2} / \bar{c}}\left(\frac{z_{2}}{\bar{z}}, \frac{z_{2}}{\bar{z}} \lambda_{2 T}\right)
\end{gathered}
$$

where

$$
\boldsymbol{k}_{1 T}=\boldsymbol{\lambda}_{1 T}+\frac{z}{z_{1}} \boldsymbol{P}_{h_{1} T}, \quad \quad \boldsymbol{k}_{2 T}=\boldsymbol{\lambda}_{2 T}+\frac{\bar{z}}{z_{2}} \boldsymbol{P}_{h_{2} T} .
$$

Here $h_{1}$ is the hadron containing a $c$ quark and $h_{2}$ the one containing a $\bar{c}$, with $\boldsymbol{P}_{h_{1} T}, \boldsymbol{P}_{h_{2} T}$ denoting their transverse momenta and $z_{1}, z_{2}$ their momentum fractions w.r.t. the virtual photon. The fragmentation functions $D\left(z, P_{\perp}\right)$ depend on the momentum fraction $z$ and the relative transverse momentum $P_{\perp}$ of the hadron with respect to the quark or antiquark.

The parton-level variables $\boldsymbol{k}_{1 T}, \boldsymbol{k}_{2 T}$ and $z$ are not directly measurable, but a detailed analysis of the kinematics [376] reveals that they can be partly determined from the hadronic final state. In particular, one can define variables $\boldsymbol{k}_{\perp}^{\prime}, \boldsymbol{P}_{T}^{\prime}$ and $z^{\prime}$ that are measurable and closely related to $\boldsymbol{k}_{\perp}=\boldsymbol{k}_{1 T}+\boldsymbol{k}_{2 T}, \boldsymbol{P}_{T}=\left(\boldsymbol{k}_{1 T}-\boldsymbol{k}_{2 T}\right) / 2$ and $z$, respectively. The cross product $\left(\boldsymbol{S}_{\perp} \times \boldsymbol{k}_{\perp}\right)$ in (2.37) gives rise to an angular modulation

$$
\frac{d \sigma^{\gamma^{*} p \rightarrow h_{1} h_{2}+X}}{d k^{\prime} d \phi_{S, k^{\prime}}} \approx A\left(k_{\perp}^{\prime}\right)+B\left(k_{\perp}^{\prime}\right) \sin \left(\phi_{S k^{\prime}}+\gamma\right),
$$

where $\phi_{S k^{\prime}}$ is the azimuthal angle between $\boldsymbol{S}_{\perp}$ and $\boldsymbol{k}_{\perp}^{\prime}$. The coefficient $B\left(k^{\prime}\right)$ depends on the gluon Sivers function, as well as the phase $\gamma$.

To estimate the possible size of the Sivers asymmetry, we follow [377] and assume

$$
f_{1 T}^{g \perp}\left(x, k_{\perp}\right)=\frac{2 \sigma M}{k_{\perp}^{2}+\sigma^{2}} f_{1}^{g}\left(x, k_{\perp}\right), \quad f_{1}^{g}\left(x, k_{\perp}\right)=\frac{e^{-k_{\perp}^{2} / \sigma^{2}}}{\pi \sigma^{2}} f_{1}^{g}(x)
$$

with $\sigma=800 \mathrm{MeV}$ and the integrated gluon distribution $f_{1}^{g}(x)$ from MSTW 2008 [22]. This Ansatz saturates the positivity bound $\frac{k_{\perp}}{M}\left|f_{1 T}^{g \perp}\left(x, k_{\perp}\right)\right| \leq f_{1}^{g}\left(x, k_{\perp}\right)$ at $k_{\perp}=\sigma$ and undershoots it for all other values of $k_{\perp}$. We consider the production of $D$ meson pairs and take a fragmentation function $D\left(z, P_{\perp}\right)=D(z) e^{-P_{\perp}^{2} / \sigma^{2}} /\left(\pi \sigma^{2}\right)$ with the same Gaussian width as in (2.40). We take $D(z) \propto z^{\alpha}(1-z)^{\beta} e^{\gamma z(1-z)}$ with $\alpha=2.86, \beta=1.57, \gamma=5.66$, which gives a fair description of the $D^{0}$ spectrum observed in $e^{+} e^{-}$annihilation [221, 220]. In Fig. 2.18 we show the transverse target spin asymmetry

$$
A\left(k_{\perp}^{\prime}, \phi_{S k^{\prime}}\right)=\frac{d \sigma\left(k_{\perp}^{\prime}, \phi_{S k^{\prime}}\right)-d \sigma\left(k_{\perp}^{\prime}, \phi_{S k^{\prime}}+\pi\right)}{d \sigma\left(k_{\perp}^{\prime}, \phi_{S k^{\prime}}\right)+d \sigma\left(k_{\perp}^{\prime}, \phi_{S k^{\prime}}+\pi\right)}
$$

for the process $\gamma^{*} p \rightarrow D^{0} \bar{D}^{0}+X$ summed over transverse and longitudinal photon polarization. We find that the phase shift $\gamma$ in (2.39) is tiny. The asymmetry is found to be sizable with our Ansatz, which suggests that DIS production of heavy meson pairs at EIC has good sensitivity to the gluon Sivers function. 


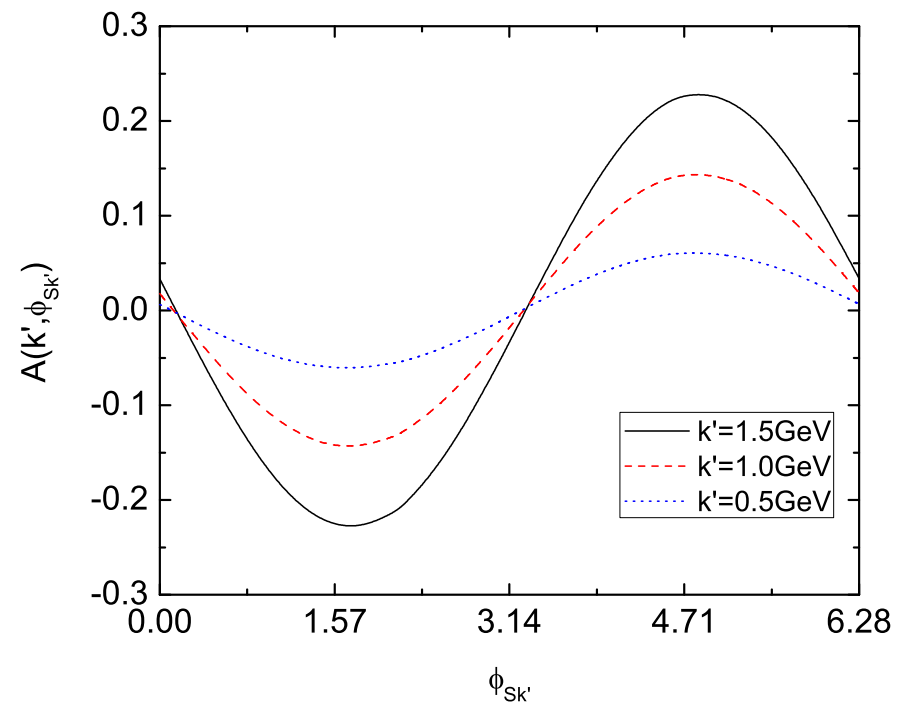

Figure 2.18. The transverse target asymmetry (2.41) for $\gamma^{*} p \rightarrow D^{0} \bar{D}^{0}+X$. The kinematics is specified by $W=100 \mathrm{GeV}, Q^{2}=16 \mathrm{GeV}^{2}, z_{1}=z_{2}=0.3,0.25<z^{\prime}<0.75$ and $5 \mathrm{GeV}<P_{T}^{\prime}<$ $40 \mathrm{GeV}$.

\subsubsection{Probing the linear polarization of gluons in unpolarized hadrons}

Gluons inside unpolarized hadrons can be linearly polarized provided they have a nonzero transverse momentum. The simplest and theoretically safest way to probe this TMD distribution of linearly polarized gluons is through $\cos 2 \phi$ asymmetries in heavy quark pair or dijet production in electron-hadron collisions. Future EIC or LHeC experiments are ideally suited for this purpose. Here we estimate the maximum asymmetries for EIC kinematics.

Linearly polarized gluons in an unpolarized hadron, carrying a light-cone momentum fraction $x$ and transverse momentum $\boldsymbol{k}_{\perp}$ w.r.t. to the parent's momentum, are described by the TMD $h_{1}^{\perp g}\left(x, k_{\perp}\right)$ [281, 284, 378]. Unlike the quark TMD $h_{1}^{\perp q}$ of transversely polarized quarks inside an unpolarized hadron (also frequently referred to as Boer-Mulders function) [244], $h_{1}^{\perp g}$ is chiral-even and $T$-even. This means it does not require initial or final state interactions (ISI/FSI) to be non-zero. Nevertheless, as any TMD, $h_{1}^{\perp g}$ can receive contributions from ISI or FSI and therefore can be process dependent, in other words, non-universal, and its extraction can be hampered in non-factorizing cases.

Thus far no experimental studies of $h_{1}^{\perp g}$ have been performed. As recently pointed out, it is possible to obtain an extraction of $h_{1}^{\perp g}$ in a simple and theoretically safe manner, since unlike $h_{1}^{\perp q}$ it does not need to appear in pairs [284. Here we will discuss observables that involve only a single $h_{1}^{\perp g}$ in semi-inclusive DIS to two heavy quarks or to two jets, which allow for TMD factorization and hence a safe extraction. The corresponding hadroproduction processes run into the problem of factorization breaking [272, 284].

Again, we consider heavy quark production, $e(\ell)+h(P) \rightarrow e\left(\ell^{\prime}\right)+Q\left(k_{1}\right)+\bar{Q}\left(k_{2}\right)+X$, where the four-momenta of the particles are given within brackets, and the heavy quark-antiquark pair in the final state is almost back-to-back in the plane perpendicular to the direction of the exchanged photon and hadron. The calculation proceeds along the lines explained in Refs. [378, 379]. We obtain for the cross section integrated over the angular distribution of 

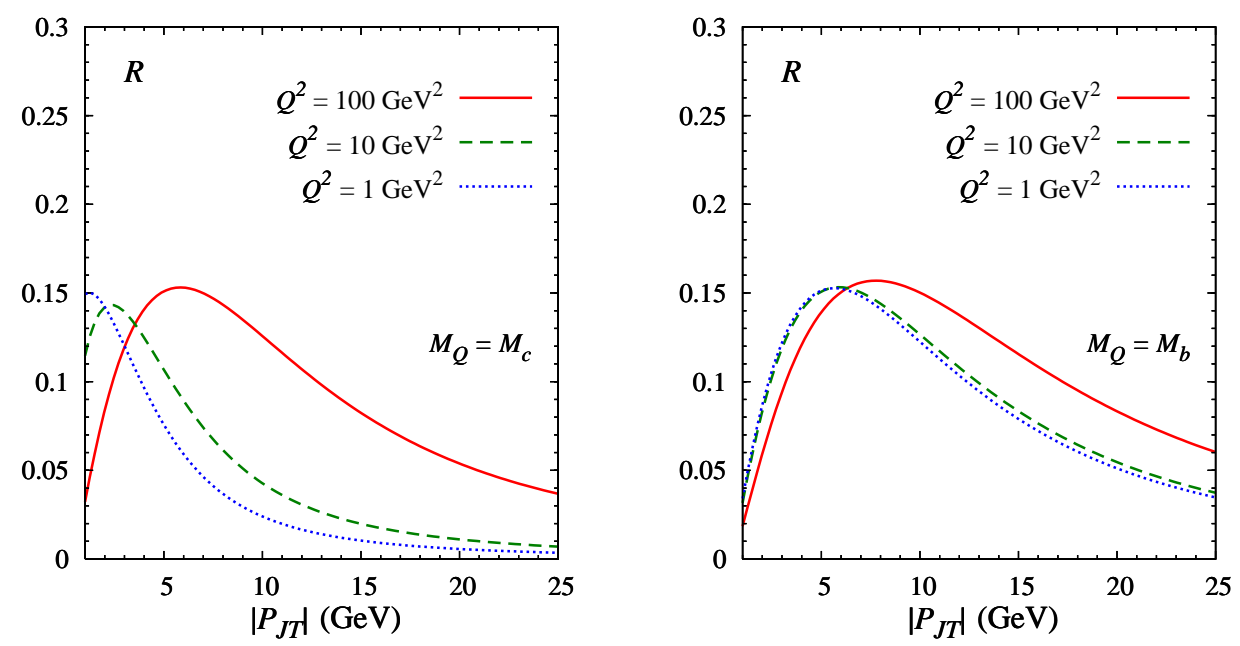

Figure 2.19. Upper bounds of the asymmetry ratio $R$ in equation (2.44) as a function of $\left|\boldsymbol{P}_{J T}\right|$ at different values of $Q^{2}$, with $y=0.01$ and $z=0.5$.

the back-scattered electron $e\left(\ell^{\prime}\right)$ :

$$
\frac{d \sigma}{d y_{1} d y_{2} d y d x_{B} d^{2} \boldsymbol{k}_{\perp} d^{2} \boldsymbol{P}_{J T}}=\frac{\alpha^{2} \alpha_{s}}{\pi s M_{T}^{2}} \frac{\left(1+y x_{B}\right)}{y^{5} x_{B}}\left(A+\frac{\boldsymbol{k}_{\perp}^{2}}{M^{2}} B \cos 2 \phi\right) \delta\left(1-z_{1}-z_{2}\right)
$$

The kinematics are the same as in the last subsection with the heavy quark mass $M_{Q}$, $M_{i T}^{2} \approx M_{T}^{2}=M_{Q}^{2}+\boldsymbol{P}_{J T}^{2}$ and the rapidities $y_{i}$ for the quark momenta along photon-target direction. The azimuthal angles of $\boldsymbol{k}_{\perp}$ and $\boldsymbol{P}_{J T}$ are denoted by $\phi_{\perp}$ and $\phi_{T}$, respectively, and $\phi \equiv \phi_{\perp}-\phi_{T}$. The functions $A$ and $B$ depend on $y, z\left(\equiv z_{2}\right), Q^{2} / M_{T}^{2}, M_{Q}^{2} / M_{T}^{2}$, and $\boldsymbol{k}_{\perp}^{2}$. The angular independent part $A$ involves only the unpolarized TMD gluon distribution $f_{1}^{g}$, while the magnitude $B$ of the $\cos 2 \phi$ asymmetry is determined by $h_{1}^{\perp g}\left(x, k_{\perp}\right)$. Since $h_{1}^{\perp g}$ is completely unknown, we estimate the maximum asymmetry that is allowed by the bound 284

$$
\left|h_{1}^{\perp g(2)}(x)\right| \leq \frac{\left\langle k_{\perp}^{2}\right\rangle}{2 M^{2}} f_{1}^{g}(x),
$$

where the superscript (2) denotes the $n=2$ transverse moment (defined as $f^{(n)}(x) \equiv$ $\left.\int d^{2} \boldsymbol{k}_{\perp}\left(\boldsymbol{k}_{\perp}^{2} / 2 M^{2}\right)^{n} f\left(x, \boldsymbol{k}_{\perp}^{2}\right)\right)$. The maximal (absolute) value of the asymmetry ratio

$$
R=\left|\frac{\int d^{2} \boldsymbol{k}_{\perp} \boldsymbol{k}_{\perp}^{2} \cos 2\left(\phi_{\perp}-\phi_{T}\right) d \sigma}{\int d^{2} \boldsymbol{k}_{\perp} \boldsymbol{k}_{\perp}^{2} d \sigma}\right|=\frac{\int d \boldsymbol{k}_{\perp}^{2} \boldsymbol{k}_{\perp}^{4}|B|}{2 M^{2} \int d \boldsymbol{k}_{\perp}^{2} \boldsymbol{k}_{\perp}^{2} A}
$$

is depicted in Fig. 2.19 as a function of $\left|\boldsymbol{P}_{J T}\right|$ at different values of $Q^{2}$ for charm (left panel) and bottom (right panel) production, where we have selected $y=0.01, z=0.5$, and taken $M_{c}^{2}=2 \mathrm{GeV}^{2}, M_{b}^{2}=25 \mathrm{GeV}^{2}$. Such large asymmetries, together with the relative simplicity of the suggested measurement (polarized beams are not required), would probably allow an extraction of $h_{1}^{\perp g}\left(x, k_{\perp}\right)$ at the EIC (or LHeC). 


\subsection{Theory highlights}

Igor O. Cherednikov, Zhong-Bo Kang, Piet J. Mulders, Barbara Pasquini, Ted Rogers, Peter Schweitzer, Nicolaos G. Stefanis, Jian-Wei Qiu

The candidates for the golden measurement at the EIC are the spin-dependent Sivers function $f_{1 T}^{\perp}$, as well as the unpolarized quark distribution $f_{1}$. The proposed silver candidates are the transversity, the Boer-Mulders, and the Collins functions. All these objects are transverse-momentum dependent parton densities that describe the inner structure of hadrons by taking into account the longitudinal and the transversal partonic degrees of freedom.

In the last few years, there has been tremendous progress on the theory developments for the transverse momentum dependent parton distributions. In particular, there have been intensive investigations on the QCD factorization and the associated universality of the TMD parton distributions in various hard processes; the energy scale dependence for the TMD distributions and related quark-gluon correlation functions. In this section, we will highlight these developments.

\subsubsection{Gauge-links, TMD-factorization, and TMD-factorization breaking}

In this section, we discuss some basic features of transverse momentum dependent parton distribution functions. In hard processes, parton distribution functions and fragmentation functions are expressed as matrix elements of nonlocal combinations of quark or gluon fields. In the collinear situation that all transverse momenta of partons are integrated over in the definitions, the nonlocality is in essence light-like. These correlation functions are convoluted with the squared amplitude for the partonic subprocess (in essence the partonic cross section) of a hard process. When the transverse momenta of partons are involved, the non-locality in the matrix elements includes a transverse separation, and a transverse momentum dependent (TMD) factorization theorem is needed. In all cases the definitions of the non-perturbative functions include gluon contributions resummed into gauge-links (or Wilson lines) that bridge the nonlocality.

It is important to realize that the appearance of the gauge-links is a consequence of the systematic resummation of extra gluon contributions in the derivations of factorization, so their structure is dictated by the requirements of factorization.

In processes like $\ell+H \longrightarrow \ell^{\prime}+h+X$ (semi-inclusive DIS), $\ell+\bar{\ell} \longrightarrow h_{1}+h_{2}+X$ (annihilation process) or $H_{1}+H_{2} \longrightarrow \ell+\bar{\ell}+X$ (Drell-Yan process) one has, at leading power in the hard scale, a simple underlying hard process, which is a virtual photon (or weak boson) coupling to a parton line. The color flow from the hard part to collinear or soft parts is simple. Additional gluons with polarizations collinear to the parton momenta are resummed into gauge-links, which exhibit the interesting behavior that for transverse momentum dependent functions they bridge the transverse separation between the nonlocal field combinations at lightcone past or future infinity. Which gauge-link is relevant in a particular non-perturbative function depends on the color flow in the full process. For a quark distribution function one has a link via (future) lightcone $+\infty$ if the color flows into the final state, and a link via (past) lightcone $-\infty$ if the color is annihilated by another incoming parton.

QCD factorization theorems are central to understanding high energy hadronic scattering cross sections in terms of the fundamentals of perturbative QCD. In addition to 
providing a practical prescription for order-by-order calculations, derivations of factorization provide a solid theoretical underpinning for concepts like PDFs and FFs which are crucial in the quest to expand the basic understanding of hadronic structure. The most natural first attempt at a TMD-factorization formula is simply to extend the classic parton model intuition familiar from collinear factorization. For the semi-inclusive deep inelastic scattering (SIDIS) cross section, for example, the cross section might be written schematically as

$$
d \sigma \sim|\mathcal{H}|^{2} \otimes \Phi\left(x, \boldsymbol{k}_{\perp}\right) \otimes D\left(z, \boldsymbol{P}_{\perp}\right) \delta^{(2)}\left(\boldsymbol{q}_{T}+\boldsymbol{k}_{\perp}-\boldsymbol{P}_{\perp}\right) .
$$

Here $\Phi\left(x, \boldsymbol{k}_{\perp}\right)$ is the TMD PDF while $D\left(z, \boldsymbol{P}_{\perp}\right)$ is the TMD FF, with the usual probability interpretations, and $|\mathcal{H}|^{2}$ represents the hard part. The momentum $\boldsymbol{q}_{T}$ is the small momentum sensitive to intrinsic transverse momenta, $\boldsymbol{k}_{\perp}$ and $\boldsymbol{P}_{\perp}$, carried by the colliding proton and the produced hadron. The $\otimes$ symbol denotes all relevant convolution integrals, and the $x$ and $z$ arguments are the usual longitudinal momentum fractions.

In a perturbative derivation of factorization, a small-coupling perturbative expansion of the cross section is analyzed in terms of "leading regions", and the sum is shown orderby-order to separate into the factors of Eq. (2.45). The precise field theoretic definitions of the correlation functions, $\Phi\left(x, \boldsymbol{k}_{\perp}\right)$ and $D\left(z, \boldsymbol{P}_{\perp}\right)$, should emerge naturally from the requirements of factorization. In the hard part $|\mathcal{H}|^{2}$, all propagators must be off-shell by order the hard scale $Q$ so that asymptotic freedom applies, and small-coupling perturbation theory is valid, with non-factorizing higher-twist contributions suppressed by powers of $Q$. Such factorization theorems are well-established for inclusive processes that utilize the standard integrated correlation functions (see [380] and references therein), but TMDfactorization theorems involve other subtleties, particularly with regard to the definitions of the TMD PDFs and FFs and their associated gauge-links.

In cases where there is a more complex color flow such as is often the case when the underlying hard process involves multiple color flows and/or if the incoming partons are gluons, this can potentially lead to a more complex gauge-link structure including traced closed loops or looping gauge-links. For situations in which only one TMD correlation function is studied, these structures have been examined in [265, 267, 381, 382] for two-totwo partonic subprocesses. In situations that involve several TMD functions, factorization using separate TMD functions fails completely.

To understand the issues that arise in defining TMDs, it is instructive to start with a review of the definition of the standard integrated quark PDF. It is

$$
f(x ; \mu)=\text { F.T. }\left\langle p\left|\bar{\psi}\left(0, w^{-}, \mathbf{0}_{t}\right) \gamma^{+} V_{[0, w]}\left(u_{\mathrm{J}}\right) \psi(0)\right| p\right\rangle,
$$

where "F.T." stands for the Fourier transform from coordinate space to momentum space. The above definition contains UV divergences which must be renormalized. This gives dependence on an extra scale $\mu$, and ultimately results in the well-known DGLAP evolution equations for the integrated PDF. For a gauge invariant definition, the PDF must contain a path ordered exponential of the gauge field that connects the points 0 and $\left(0, w^{-}, \mathbf{0}_{t}\right)$. This is the gauge-link and its formal definition is

$$
V_{[0, w]}\left(u_{J}\right)=P \exp \left(-i g t^{a} \int_{0}^{w^{-}} d \lambda u_{J} \cdot A^{a}\left(\lambda u_{J}\right)\right) .
$$

The path of the gauge-link is determined by the light-like vector $u_{\mathrm{J}}=\left(0,1, \mathbf{0}_{t}\right)$. That is, the gauge-link follows a straight path connecting 0 and $\left(0, w^{-}, \mathbf{0}_{t}\right)$ along the exactly 


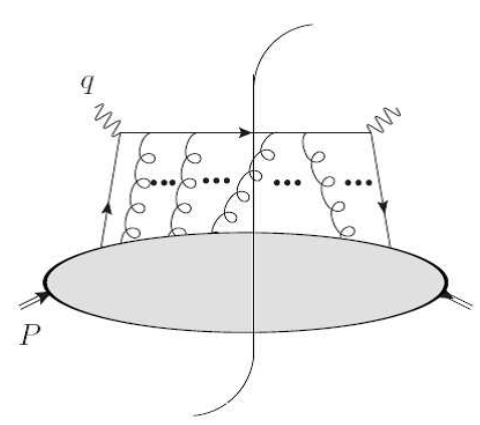

(a)

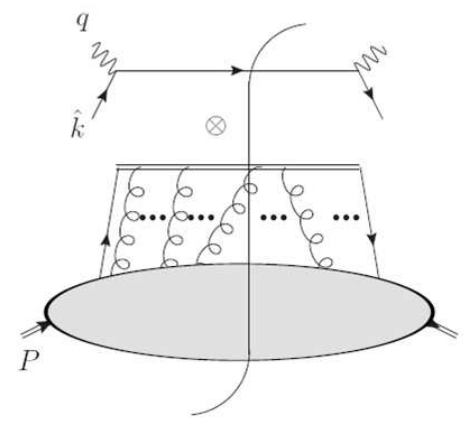

(b)

Figure 2.20. (a) Target-collinear gluons in a graph for SIDIS. (b) Factorization of extra gluons into gauge-link contributions.

light-like minus direction. In Feynman graph calculations, the contribution from the gaugelink corresponds to the so-called "eikonal factors," which have definite Feynman rules that follow naturally from factorization proofs. After a sum over graphs, and the application of appropriate approximations and Ward identity arguments, extra collinear gluons like those shown in Fig. 2.20(a) for SIDIS factor into gauge-link contributions. In Fig. 2.20(b), the eikonal factors are shown as gluon attachments from the target-collinear bubble to a double line.

The most natural first try at extending the PDF definition in Eq. (2.46) to the TMD case is to simply leave the integration over transverse momentum in the TMD PDF definition undone. That is, instead of Eq. (2.46) one may try

$$
\Phi\left(x, \boldsymbol{k}_{t}\right)=\text { F.T. }\left\langle p\left|\bar{\psi}\left(0, w^{-}, \boldsymbol{w}_{t}\right) \gamma^{+} U_{[0, w]}\left(u_{J}\right) \psi(0)\right| p\right\rangle .
$$

The separation is now 0 and $\left(0, w^{-}, \boldsymbol{w}_{t}\right)$ - it has acquired a transverse component and the Fourier transform is now in both $w^{-}$and $\boldsymbol{w}_{t}$. As a result, the structure of the gauge-link $U_{[0, w]}\left(u_{J}\right)$ must also be modified from the simple straight light-like $V_{[0, w]}\left(u_{\mathrm{J}}\right)$ gauge-link of Eq. (2.46). The eikonal attachments on either side of the cut in Fig. 2.20 still give minusdirection Wilson lines, but now in order to have a closed link there must also be a small transverse detour at light-cone infinity. This detour arises naturally from boundary terms that are needed as subtractions to make higher twist contributions gauge invariant [263, 264].

The gauge-link structure in Eq. (2.48), with its two exactly light-like legs and a transverse link at infinity is commonly cited as the gauge-link that is necessary for the definition of the TMD PDFs. However, there are a number of further subtleties, and we will find that the definition needs to be modified. One complication is that rapidity divergences, which in collinear factorization would cancel in the sum of graphs, remain uncanceled in the definition of the TMD correlation functions. Rapidity divergences correspond to gluons moving with infinite rapidity in the direction opposite the containing hadron, and remain even when infrared gluon mass regulators are included. (For a more complete review of these and related issues, see for example [259, 383.) The most common way to regularize the lightcone divergences is to make the gauge links slightly non-light-like. In the coordinate space picture, the gauge-link therefore becomes more like the tilted hook shape. This introduces a new arbitrary rapidity parameter - the "tilt" of the gauge-link. A generalization of renormalization group techniques is needed to recover predictability in the factorization formula. A system of evolution equations for the TMD case was developed by Collins, Soper 
and Sterman (CSS) and has been successfully applied to specific processes [240, 241, 273].

A complete treatment of TMD-factorization involves soft gluons, which give rise to an extra "soft factor" $S(\boldsymbol{q})$ in the factorization formula of Eq. (2.45). The TMD-factorization formula then becomes

$$
d \sigma \sim|\mathcal{H}|^{2} \otimes \Phi\left(x, \boldsymbol{k}_{\perp}\right) \otimes D\left(z, \boldsymbol{P}_{\perp}\right) \otimes S\left(\boldsymbol{h}_{T}\right) \delta^{(2)}\left(\boldsymbol{q}_{T}+\boldsymbol{k}_{\perp}-\boldsymbol{P}_{\perp}-\boldsymbol{h}_{T}\right) .
$$

The soft factor describes the role of gluons with nearly zero center-of-mass rapidity. One difficulty with the usual presentation of the CSS formulation is that the explicit appearance of a soft factor seems somewhat counter to the basic parton model intuition wherein all nonperturbative effects are associated with functions for each external hadron with simple and specific probabilistic interpretations. A natural hope is that, with an appropriate sequence of redefinitions, the role of the soft gluons can be absorbed into the definitions of the PDFs and FFs. The recent work of Collins [384 has shown how this is possible. Indeed, this treatment of the soft factor is necessary for a completely correct factorization derivation with fully consistent definitions for the correlation functions.

While the CSS formalism has been implemented for specific spin independent processes (see, for example, [385]), much work remains to be done in tabulating and classifying the TMDs. This is especially true for cases that involve spin. Work in this direction has been started in [260].

\section{TMD-factorization breaking}

The discussion has focussed on situations where factorization is known to hold. There are also, however, situations where TMD-factorization is now known to break down [265, 267, 268, 270, 271, 272, 381, 382. The key issue is the failure of the usual Ward identity arguments that ordinarily allow eikonalized gluons to be factorized and identified with a particular gauge-link structure in the definitions of the TMDs. A hint of what leads to TMD-factorization breaking is already suggested by the well-known overall relative sign flip in the Sivers function for SIDIS as compared to the Drell-Yan (DY) process [261, 262. The difference comes because in the SIDIS TMD-factorization formula, the gauge link in the Sivers function is future pointing, whereas it is past pointing in the DY case. At the level of Feynman graphs, the difference can be seen in the fact that the "extra" gluons which contribute to the gauge-link attach before the hard scattering in one case, and after the hard scattering in the other. This illustrates that the direction of the flow of color through the eikonal lines is a critical factor in the definition of the correlation functions.

In the more complicated hadro-production processes, $H_{1}+H_{2} \rightarrow H_{3}+H_{4}+X$, where $H_{3}$ and $H_{4}$ may be either jets or hadrons, a reasonable first approach would be to trace the flow of color through the eikonal factors and use analogous arguments to what we used for SIDIS and DY in the previous section. One finds that the resulting structures are not simply the future or past pointing gauge-links familiar from SIDIS or DY, but rather are complicated and highly process dependent objects [265, 267, 381, 382. That this corresponds (at least) to a breakdown of universality is most directly seen in an explicit spectator model calculation. For example, one may consider an Abelian scalar-quark / Dirac spectator model with multiple flavors as in [270]. Then, in addition to the standard gauge-link attachments, there are extra gluon attachments that do not cancel in a simple Ward identity argument, and which give contributions that are not consistent with having a simple gauge-link like what is found SIDIS or DY (opposite pointing). 
Therefore, it is clear that there is at least a violation of universality in the hadroproduction of hadrons. The natural next approach to try is to maintain a basic factorization structure, but to loosen the requirement that the TMDs be universal, resulting in a kind of "generalized" TMD-factorization formalism. That is, the cross section might still be expected to factorize order-by-order into a hard part and well-defined, albeit non-universal, matrix elements for each separate external hadron [268]. However, a careful order-by-order consideration of multiple gluons in the derivation of TMD-factorization shows that even this is not possible [272]. If, for example, one extends the model of [270] to allow the gluons to carry color (while still considering a hard part that involves only the exchange of a colorless boson) then it is straightforward to see that the flow of color spoils the possibility of factorizing the graph into TMD PDFs with separate gauge-links for each TMD, regardless of what kind of gauge-link geometries are allowed. Therefore, the problem with factorization in the hadro-production of hadrons is more than just a problem with universality - separate correlation functions cannot even be defined in a way that is consistent with factorization.

The root of the problem is a failure of Ward identity arguments, which normally allow "extra" gluons to be factorized after a sum over graphs. The Ward identity arguments are only valid after an appropriate sequence of contour deformations on the momentum integrals. In the case of hadro-production of hadrons the necessary deformations are prohibited. In other cases where the direction of color flow may at first appear to pose a problem for factorization (such as in $e+p \rightarrow h_{1}+X$ and $e+p \rightarrow h_{1}+h_{2}+X$ ), the necessary contour deformations are possible and factorization holds. (See the explanation in chapter 12 of [384].)

To summarize, we list the status of TMD-factorization for various well-known processes with a check mark for processes where factorization appears to be valid and !! where it has been shown to fail:

$\checkmark$ Semi-inclusive DIS $\left(e+p \rightarrow e^{\prime}+h_{1}+X\right)$.

$\checkmark$ Drell-Yan (up to overall minus signs for some spin-dependent TMDs).

$\checkmark$ Back-to-Back hadron or jet production in $e^{+} e^{-}$annihilation.

$\checkmark$ Back-to-back hadron or jet production in DIS $\left(e+p \rightarrow e^{\prime}+h_{1}+h_{2}+X\right)$.

!! Hadro-production of back-to-back jets or hadrons $\left(H_{1}+H_{2} \rightarrow H_{3}+H_{4}+X\right)$.

In cases where TMD-factorization is valid, there is still much work left to be done (and much potential insight to be gained) in terms of implementing the evolution of precisely defined TMDs [260]. Much already exists for the case of unpolarized scattering, but even here the most complete and formal identification of evolution effects with separate TMDs has only recently been clarified in [384]. For polarization dependent functions, it is also important to include evolution, but to date there has been very little work that accounts for evolution in actual fits to data.

Finally, the experimental search for TMD-factorization breaking effects opens the possibility of new and exciting insights into the transverse dynamics of hadronic collisions. The breakdown of TMD-factorization in the hadro-production of hadrons implies that unexpected and exotic correlations between partons in different hadrons can exist. Calculations that allow for experiments to distinguish between factorization and factorization-breaking scenarios are therefore very important, and a quantitative understanding of factorization (via the methods of [283], for example) are part of the next step toward understanding hadronic structure in high energy collisions. 


\subsubsection{Evolution of transverse-momentum-dependent densities}

Much of the success of QCD collinear factorization relies on our ability to calculate the short-distance partonic dynamics in QCD perturbation theory order-by-order in powers of $\alpha_{s}$ and the universality as well as the scale evolution of the non-perturbative collinear parton distribution and correlation functions. With its dependence on the parton's transverse momentum, TMDs carry much richer information on the partonic structure of a hadron than what collinear PDFs could provide. Like the case of collinear factorization, the predictive power of the TMD factorization formalism also requires our ability to calculate the short-distance dynamics and the evolution of TMDs. However, the theoretical framework for calculating the evolution of TMDs and radiative corrections to short-distance dynamics has not been fully established. All existing parameterizations of TMDs are extracted from SIDIS data at relatively low $Q^{2}$. The available hard scale $Q^{2}$ at a future EIC is expected to be much larger. The TMDs, like PDFs, depend on the momentum scale $Q^{2}$ where they are probed. Understanding the $Q^{2}$ dependence of the TMDs is crucial for testing the TMD factorization formalism and for extracting correct information on the partonic structure of hadrons at the EIC. However, the $Q^{2}$-dependence of TMDs in the existing TMD factorization formalism is very different from the factorization scale $\mu_{F}^{2}$ dependence of the PDFs. The factorization scale is not a physical scale. Any factorized physical cross section should not be sensitive to the choice of the factorization scale. The perturbatively calculated factorization scale dependence of PDFs is necessarily compensated by the same scale dependence in the high order short-distance partonic dynamics. On the other hand, the TMDs in the existing proved TMD factorization formalism are effectively physical quantities. They are connected to a physical observable by a partonic scattering cross section without strong interaction and a soft factor which can be absorbed into the redefinition of TMDs 384. Unlike the DGLAP evolution equation of PDFs, the $Q^{2}$-dependence of TMDs cannot be derived by a simple renormalization group equation. The $Q^{2}$-dependence of TMDs was systematically studied in the context of the transverse momentum $\left(q_{T}\right)$ distribution of the Drell-Yan process and the two-jet momentum imbalance in $e^{+} e^{-}$collisions [273]. The $Q^{2}$-dependence was derived by resumming $\ln ^{2}\left(Q^{2} / q_{T}^{2}\right)$-type large logarithms perturbatively in the impact parameter $b_{T}$-space (a Fourier transform of the parton's transverse momentum space). The CSS formalism was extended to SIDIS [386, 387] as well as spin observables [257, 388]. With the proof that the soft factor of the TMD factorization formalism could be absorbed into the redefinition of TMDs [384, the CSS resummation formalism was recently applied to the TMDs directly [260]. Within the CSS formalism, it is not the $Q^{2}$-dependence of TMDs that is derived but rather the $Q^{2}$-dependence of the Fourier transformed TMDs at small $b_{\perp}$. In order to obtain the $Q^{2}$-dependence of TMDs, one has to perform the Fourier transform from the impact parameter $b_{\perp}$-space to the parton's transverse momentum $k_{T}$-space. The procedure of Fourier transform requires necessarily input from the nonperturbative large $b_{\perp}$ region, which could significantly reduce the predictive power of the TMDs [389. Various treatments/models for the extrapolation into the large $b_{\perp}$ region have been proposed to fit the existing data [385. For the precision study of TMDs at the EIC, it is very important to examine the universality of the nonperturbative extrapolation to the large $b_{\perp}$ region and its dependence on the observed kinematic variables; and most important, the predictive power of the formalism [389]. In order to understand the $Q^{2}$-dependence of spin-dependent TMDs, a careful generalization of the CSS resummation formalism to $\boldsymbol{k}_{\perp}$-dependent TMDs is needed [388], which is necessary for the study of asymmetries generated by the TMDs at the EIC. 


\subsubsection{QCD Evolution for the Correlation Functions}

As introduced in Sec. 2.2, a collinear factorization formalism at twist-3 is relevant for describing the SSAs of high $P_{h T}$ particle production. Even though the phenomenological applications of this approach have been successful, the theoretical calculations so far have been mainly limited to the "bare" parton model, that is, to the zeroth order of perturbation theory without any QCD corrections. These leading order (LO) calculations have some disadvantages: they strongly depend on the choice of the renormalization as well as the factorization scale, while the physically observed SSAs should not depend on the choice of these scales. The strong dependence on the choice of these scales is an artifact of the LO perturbative calculation, and a significant cancellation of the scale dependence between the leading and the next-to-leading (NLO) contribution is expected from the QCD factorization theorem. As demonstrated by many examples, NLO contributions are typically very important in hadronic processes, and often offer a more comprehensive test of the relevant QCD factorization formalism.

To move forward to the NLO QCD dynamics, it is necessary to study the evolution (or the scale dependence) of the universal long distance distributions and to evaluate the perturbative short-distance contribution beyond the LO. The evolution equation of the twist-3 distribution functions have been derived by different groups [290, 291, 390, 391]. Recently the evolution equations for the twist-3 fragmentation functions have also become available [392]. A first NLO calculation for the short-distance hard part function has been presented in [291].

As emphasized in Sec. 2.3, there are close connections between the twist-3 collinear factorization formalism and the TMD factorization formalism. The twist- 3 correlation functions are closely related to the relevant TMD functions. Even though the Collins-Soper evolution equations have been derived for all the leading-twist TMD functions [393], these evolution equations are available in $b$-space ( $b$ is conjugate to the transverse momentum $k_{\perp}$ ). How these evolution equations are transformed into the scale (or energy) dependence of the SSAs (thus leading to a similar Collins-Soper-Sterman transverse momentum resummation) is not yet fully understood.

The evolution equations of twist-3 distribution functions, particularly for the so-called soft-gluonic-pole correlation functions have been derived in [290, 291, 390, 391]. Among them, $T_{F}\left(x_{1}, x_{2}\right)$ and $T_{F}^{(\sigma)}\left(x_{1}, x_{2}\right)$ are the most discussed ones and they are related to the Sivers and Boer-Mulders functions [264]:

$$
\begin{aligned}
T_{F}(x, x) & =-\left.\int d^{2} k_{\perp} \frac{\left|\boldsymbol{k}_{\perp}\right|^{2}}{M_{p}} f_{1 T}^{\perp}\left(x, k_{\perp}^{2}\right)\right|_{\mathrm{DIS}}, \\
T_{F}^{(\sigma)}(x, x) & =-\left.\int d^{2} k_{\perp} \frac{\left|\boldsymbol{k}_{\perp}\right|^{2}}{M_{p}} h_{1}^{\perp}\left(x, k_{\perp}^{2}\right)\right|_{\mathrm{DIS}},
\end{aligned}
$$

where $M_{p}$ is the nucleon mass. The evolution equations for both $T_{F}(x, x)$ and $T_{F}^{(\sigma)}(x, x)$ have the following generic form:

$$
\frac{\partial T\left(x, x, \mu^{2}\right)}{\partial \ln \mu^{2}}=\frac{\alpha_{s}}{2 \pi} \int \frac{d x^{\prime}}{x^{\prime}}\left[A(\hat{\xi}) T\left(x^{\prime}, x^{\prime}, \mu^{2}\right)+B\left(x, x^{\prime}\right) T\left(x, x^{\prime}, \mu^{2}\right)\right],
$$

where $T$ represents either $T_{F}$ or $T_{F}^{(\sigma)}$, and $\hat{\xi}=x / x^{\prime}$. As can be seen in (2.51), the evolution equation for the diagonal correlation function $\left(x_{1}=x_{2}=x\right)$ is not a closed equation since it also depends on the off-diagonal piece (the $B\left(x, x^{\prime}\right)$ term). The diagonal $A(\hat{\xi})$ terms are typically similar to the relevant twist- 2 splitting kernel: for $T_{F}$, it is the same as the $q \rightarrow q$ 
splitting kernel for the unpolarized distribution functions; for $T_{F}^{(\sigma)}$, it is the same as the splitting kernel for the transversity distribution. It might be worth pointing out that there are some discrepancies for the evolution equation of $T_{F}$ in the literature: Ref. [391] contains additional contributions compared to [290, 291, 390]. One additional piece corresponds to a contribution from the mixing between a gluon state and quark-antiquark state, which are missing in [290, 291, 390] and could be easily reproduced. Another term $\left[-N_{c} T_{F}(x, x)\right]$ seems difficult to reconcile at the moment, and further study is needed to resolve this discrepancy.

Similarly, one could study the evolution of the three-gluon correlation functions. For an initial effort, see [390]. They receive contributions from themselves, as well as from the quark-gluon correlation functions $T_{F}$. Even though our information on three-gluon correlation functions is very scarce, one can not rule out the possibility that they might be large since they could be generated through the QCD radiation from the quark-gluon correlation. It is also worth pointing out that we now have data from PHENIX on the SSA of $J / \Psi$ [313], which turns out to be non-zero and gives some indication that three-gluon correlation functions might be sizable. It has been suggested that open charm production in a future Electron Ion Collider (EIC) with broader kinematics could be used to unravel the three-gluon correlation functions.

Within the same method, one could study the evolution equations for the twist-3 fragmentation functions. The two most important ones are related to the first transversemomentum-moment of the Collins function $H_{1}^{\perp}\left(z, z^{2} k_{\perp}^{2}\right)$ and the polarizing fragmentation function $D_{1 T}^{\perp}\left(z, z^{2} k_{\perp}^{2}\right)$ [297, 394]:

$$
\hat{H}(z)=-z^{3} \int d^{2} k_{\perp} \frac{\left|\boldsymbol{k}_{\perp}\right|^{2}}{M_{h}} H_{1}^{\perp}\left(z, z^{2} k_{\perp}^{2}\right), \quad \hat{T}(z)=-z^{3} \int d^{2} k_{\perp} \frac{\left|\boldsymbol{k}_{\perp}\right|^{2}}{M_{h}} D_{1 T}^{\perp}\left(z, z^{2} k_{\perp}^{2}\right),
$$

with both $H_{1}^{\perp}$ and $D_{1 T}^{\perp}$ from the convention in [243]. These twist-3 fragmentation functions belong to the more general two-argument fragmentation functions denoted as $\hat{H}_{F}\left(z, z_{1}\right)$ and $\hat{T}_{F}\left(z, z_{1}\right)$, for details on the operator definitions, see [392]. The evolution equation for $\hat{H}(z)$ takes the following generic form (same form for $\hat{T}(z)$ ):

$$
\frac{\partial \hat{H}\left(z_{h}, \mu^{2}\right)}{\partial \ln \mu^{2}}=\frac{\alpha_{s}}{2 \pi} \int \frac{d z}{z}\left[A(\hat{z}) \hat{H}\left(z, \mu^{2}\right)+\int \frac{d z_{1}}{z_{1}^{2}} \mathrm{PV}\left(\frac{1}{\frac{1}{z}-\frac{1}{z_{1}}}\right) B\left(z_{h}, z, z_{1}\right) \hat{H}_{F}\left(z, z_{1}, \mu^{2}\right)\right],
$$

where $\hat{z}=z / z_{h}$, and in the case of $\hat{H}\left(z_{h}, \mu^{2}\right), A(\hat{z})$ is the same as the evolution kernel for the transversity distribution; while for $\hat{T}\left(z_{h}, \mu^{2}\right), A(\hat{z})$ is the same as the $q \rightarrow q$ splitting kernel for the unpolarized fragmentation function.

We have reviewed the evolution equations for the twist-3 distribution and fragmentation functions. Particularly for those related to the first transverse-momentum-moment of the Sivers and Boer-Mulders function, and Collins and polarizing fragmentation function. These evolution equations are generally not a closed set of equations. However, the diagonal pieces are very similar to those appearing in the evolution of leading-twist distribution and fragmentation functions. For the Sivers function and polarizing fragmentation function, this piece is the same as for the unpolarized distribution functions. For the Boer-Mulders function and Collins function, this piece is the same as for transversity. The evolution equations of these functions will transform into the scale dependence of the spin observables, which could be studied at EIC. With a wide coverage in $x$ and $Q^{2}$, EIC offers a great opportunity to study these scale dependences - a direct test of QCD dynamics. 


\subsubsection{Non-perturbative studies of TMDs in effective approaches}

TMDs are matrix elements of certain non-local QCD light-front operators in hadron states and can only be calculated using non-perturbative frameworks. Several low-energy QCD-inspired models have been employed. Although they all have in common that they strongly oversimplify the complexity of the QCD dynamics in hadrons, studies in different models based on often complementary assumptions, help to unravel non-perturbative aspects of TMDs. Insights into non-perturbative properties are of particular interest when confirmed in various models. The practical value of model results is that they can be used to predict new observables, or to guide educated Ansätze for fits of TMD parameterizations. Especially in the context of TMDs one should not underestimate the conceptual importance of model calculations. Model calculations demonstrated the existence of effects [261], paved the way towards an understanding of universality in the fragmentation process [395], established new TMDs [396, 397, see [398] for a review. The distinction of T-even and T-odd TMDs is important also from the point of view of modeling. In order to model the former it is sufficient to use a model with explicit quark degrees of freedom. In contrast, the modeling of T-odd TMDs requires the explicit presence of gauge-field degrees of freedom.

In the following we will briefly review TMD models, though a detailed classification of all models in which TMDs have been studied would go far beyond the scope of this section.

\section{Models of TMDs}

An interesting model is QCD in the multicolor limit, i.e. one works with $N_{c} \rightarrow \infty$ instead of $N_{c}=3$ colors. In the large- $N_{c}$ limit the nucleon can be described as a classical soliton of the chiral field [399]. Also for $N_{c} \rightarrow \infty$ QCD cannot be solved (in $3+1$ dimensions). But certain symmetry properties of the soliton field are known [399] and can be used to derive relations which compare the relative magnitudes of different flavor combinations [334,

$$
\begin{array}{rlrl}
\left(f_{1}^{u}+f_{1}^{d}\right) & \gg\left|f_{1}^{u}-f_{1}^{d}\right|, & & \left|f_{1 T}^{\perp u}-f_{1 T}^{\perp d}\right| \gg\left|f_{1 T}^{\perp u}+f_{1 T}^{\perp d}\right|, \\
\left|g_{1}^{u}-g_{1}^{d}\right| \gg\left|g_{1}^{u}+g_{1}^{d}\right|, & & \left|g_{1 T}^{\perp u}-g_{1 T}^{\perp d}\right| \gg\left|g_{1 T}^{\perp u}+g_{1 T}^{\perp d}\right|, \\
\left|h_{1}^{u}-h_{1}^{d}\right| \gg\left|h_{1}^{u}+h_{1}^{d}\right|, & & \left|h_{1 L}^{\perp u}-h_{1 L}^{\perp d}\right| \gg\left|h_{1 L}^{\perp u}+h_{1 L}^{\perp d}\right|, \\
\left|h_{1}^{\perp u}+h_{1}^{\perp d}\right| \gg\left|h_{1}^{\perp u}-h_{1}^{\perp d}\right|, & \left|h_{1 T}^{\perp u}-h_{1 T}^{\perp d}\right| \gg\left|h_{1 T}^{\perp u}+h_{1 T}^{\perp d}\right|,
\end{array}
$$

where the not indicated arguments of the TMDs scale with $N_{c}$ as $x \sim 1 / N_{c}$ and $k_{\perp} \sim N_{c}^{0}$. Analogous relations hold for antiquarks [334]. In (2.54) the respectively 'large' flavor combinations are one order in $N_{c}$ enhanced compared to the 'small' ones. For known distribution functions the hierarchies in (2.54) are roughly supported in nature [400]. The large- $N_{c}$ prediction [334] also proved useful as a guideline for a first extraction of the Sivers function from SIDIS [328]. Conclusions about gluon TMDs can also be drawn. For instance, $f_{1 T}^{\perp g}$ is predicted to be one order in $N_{c}$ suppressed with respect to the quark Sivers distributions [328], which seems supported by phenomenology [401, 402].

The first quark model to give practical results on T-even TMDs was the quark-diquark spectator model [403]. The basic idea of this model is to make a spectral decomposition of the correlation function which defines the TMDs, and to evaluate it in the spectator approximation, i.e. by truncating the sum over intermediate states to a single on-shell spectator with definite mass. The spectator can have the quantum numbers of a scalar (spin 0) isoscalar or axial-vector (spin 1) iso-vector diquark, and it plays the role of an effective particle which effectively takes into account non-perturbative effects related to the 
sea and gluon content of the nucleon. The nucleon-quark-diquark coupling is described by an effective vertex which may contain a model-dependent form factor. This class of models with various vertex functions and different choices for the axial-vector diquark polarization states have been used extensively in literature [337, 404, 405, 406]. These results for TMDs can also be interpreted in terms of overlap of light-cone wave functions (LCWFs) for the diquark [407. The advantage of the spectator model is that the complicated many-particle system can be effectively treated by a simple two-particle technique. However, the price to pay is that basic properties like the momentum and quark-number sum rules cannot be satisfied simultaneously, since the number of quarks "seen" in the spectator model is only one. This fundamental limitation can be resolved only by considering the diquark not as an elementary particle, but as formed by two quarks which play the role of active particles (see, e.g., ref. [408]).

A different approach consists in exploiting LCWFs to model the three-quark structure of the nucleon. The three-quark LCWFs encode the bound state quark properties of hadrons, including their momentum, spin and flavor correlations, in the form of universal processand frame-independent amplitudes. Such amplitudes have also the important property to be eigenstates of the total quark orbital-angular momentum $L_{z}^{q}[278$, 407] and therefore, allow for mapping in a transparent way the multipole pattern in $\boldsymbol{k}_{\perp}$ associated with each TMD [409, 410]. In particular, $f_{1}^{q}, g_{1 L}^{q}$ and $h_{1}^{q}$ describe monopole distributions with $\Delta L_{z}^{q}=0$ between the initial and final nucleon states, with $f_{1}^{q}, g_{1 L}^{q}$ containing $S, P$ and $D$ wave contributions, and $h_{1}^{q}$ only $S$ and $P$ waves. The other twist-2 T-even TMDs are non-diagonal in the orbital angular momentum, with $g_{1 T}^{q}$ and $h_{1 L}^{q}$ describing dipole distributions due to the interference of $S-P$ and $P-D$ waves, and $h_{1 T}^{\perp q}$ being related to a quadrupole shape due to a transfer of two units of orbital angular momentum [411, 301]. Two phenomenologically successful models were used to compute the quark LCWFs: the light-cone constituent quark model (LCCQM) 409] and the chiral quark-soliton model ( $\chi \mathrm{QSM})$ [412, 413, 414, 415]. In the LCCQM one describes the baryon state in terms of three free on-shell valence quarks. The three-quark state is however not on-shell, i.e. $M \neq \sum_{i} \omega_{i}$, where $\omega_{i}$ is the energy of free quark $i$ and $M$ is the physical mass of the bound state. The motion of the quarks inside the nucleon is described by a momentum-dependent function which is assumed to have a simple analytical expression, with free parameters fitted, e.g., to the anomalous magnetic moments and the axial charge of the nucleon. In the $\chi$ QSM quarks are not free but bound by a relativistic chiral mean field (semi-classical approximation). This field creates a discrete level in the one-quark spectrum and distorts at the same time the Dirac sea. Despite the different model assumptions in LCCQM and $\chi$ QSM, it turns out that the corresponding LCWFs are very similar in structure. It should be noticed that the $\chi$ QSM naturally incorporates higher Fock states and it has been applied to describe the unpolarized TMD for both quark and antiquarks [416].

A different model used to compute TMDs is the bag model. In its simplest version it describes the nucleon as three non-interacting massless quarks confined inside a sphere. This is therefore the only quark model discussed so far which incorporates confinement, which is modeled by the bag boundary condition, i.e. in some sense the boundary condition mimics gluons [418. All twist-2 and twist-3 T-even TMDs were studied in this model in [419], and a complete set of linear and non-linear relations among them was derived. Another remarkable insight was that the bag model strongly supports the Gaussian $k_{\perp}$-dependence of TMDs observed in phenomenology [420].

A physical picture nearly "opposite" to the bag model is provided by the covariant 

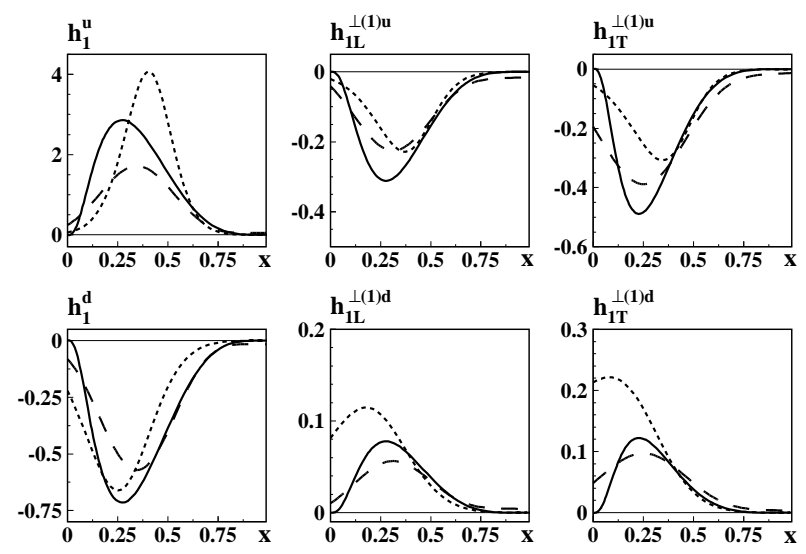

Figure 2.21. Results for $h_{1}^{q}(x)$ (left panels), $h_{1 L}^{\perp(1) q}$ (middle panels) and $h_{1 T}^{\perp(1) q}$ (right panels) as functions of $x$ within different models at low scales for up (upper panels) and down quarks (lower panels). Dashed curves: spectator model of ref. 403]. Dotted curves: bag model of ref. [417]. Solid curves: light-cone constituent quark model of ref. [298.

parton model 421, 422, 423]. In this approach the partons are free, and assumed to be described in terms of 3D spherically symmetric momentum distributions in the nucleon rest frame. Compliance of the model with relations derived from QCD equations of motion allows the existence of only two such covariant momentum distributions: one describes unpolarized and the other polarized quarks. All twist-2 TMDs are described in terms of these two covariant distributions. This also implies relations among TMDs discussed in [421. The most interesting aspect of the model is that the symmetry of the covariant momentum distributions tightly connects longitudinal and transverse parton momenta. As a consequence, it is possible to predict the $x$ - and $k_{\perp}$-dependence of TMDs from the $x$ dependence of known PDFs [423]. Interestingly, also this model supports the Gaussian $k_{\perp}$-dependence. An important feature is that the covariant parton model yields results which refer to a large scale. Other parton model approaches in the context of TMDs were discussed in [424, 425, 426].

TMDs in the non-relativistic limit were studied for an arbitrary number of colors $N_{c}$ in [421. In this context we recall the popular non-relativistic model prediction $h_{1}^{q}(x)=g_{1}^{q}(x)$. The non-relativistic model makes similar predictions for other TMDs. In particular, it naturally explains why in many models the integrated pretzelosity function, $h_{1 T}^{\perp q}(x)$, is so large compared to other TMDs.

Results for selected T-even TMDs computed within different models are shown in Fig. 2.21, In order to model T-odd TMDs one needs to invoke also gauge-boson degrees of freedom. We shall devote a separate section to that. But before that we discuss relations among TMDs.

In QCD all TMDs are independent functions. However, in a large class of quark models [409, 403, 405, 413, 414, 415, 419, 421, 422, 423] there appear relations among different TMDs. In fact, certain relations, the so-called 'LIRs' ('Lorentz-invariance relations') must hold in any consistent quark model framework without gauge-field degrees of freedom. The 14 T-even leading- and subleading-twist TMDs can be expressed in terms of 9 independent 'quark-nucleon scattering amplitudes' which implies the relations [243, 427] (see [428] for a review). 


\section{T-odd TMDs}

T-odd TMDs emerge from the gauge-link structure of the parton correlation functions which describe initial/final-state interactions (ISI/FSI) via soft-gluon exchanges between the struck parton and the target remnant. Here we will summarize the status of model calculations for the two leading-twist T-odd TMDs, namely the Sivers function $f_{1 T}^{\perp}$ and the Boer-Mulders function $h_{1}^{\perp}$. Both these functions require orbital angular momentum in the nucleon, since they involve a transition between initial and final nucleon states whose orbital angular momentum differ by $\Delta L_{z}^{q}= \pm 1$. Following the first calculation which explicitly predicted a non-zero Sivers function within a scalar-diquark model [261], more refined calculation of the T-odd TMDs were performed in the spectator models with both scalar and axial-vector diquark [337, 404, 429, 430, 431, 432, 433, 434. Other model calculations include the bag model [339, 435, 436], the non-relativistic constituent quark model [437] and a light-cone constituent quark model [298]. Within all these models, the FSI/ISI are approximated by taking into account only the leading contribution due to the one-gluon exchange mechanism. As a result, the final expressions for the T-odd functions are proportional to the strong coupling constant, which plays the role of a global normalization factor with different values depending on the intrinsic hadronic scale of the model. Meanwhile, we also notice that it may be not appropriate to use a perturbative coupling for these non-perturbative calculations. A non-perturbative approach was studied in refs. [438, 439], where T-odd distributions were obtained from the non-perturbative chromomagnetic quarkgluon interaction induced by instantons. A complementary approach is also to take into account the physics of the FSI/ISI by constructing augmented LCWFs which incorporate the rescattering effects by acquiring an imaginary (process-dependent) phase [440]. Finally we remark that an interesting way to circumvent the no-go theorem concerning the modeling of T-odd TMDs in chiral quark models [441] was discussed in 333] where the role of gluons is played by a 'hidden vector-meson gauge symmetry'.

Recently, interesting studies were presented, which go beyond the one-gluon exchange approximation by resumming all order contributions [338, 442, 443]. This is achieved using approximate relations between TMDs and GPDs. In particular, the T-odd TMDs are described via factorization of the effects of FSIs, incorporated in a so-called "chromodynamics lensing function", and a spatial distortion of impact parameter space parton distributions [444, 299, 300]. While such relations are fulfilled from lowest order contributions in spectator models [282, 300], they are not expected to hold in general [445, 446]. However, the interesting novelty in the approach of refs. [338, 442, 443] is the calculation of the lensing function using non-perturbative eikonal methods which permit to take into account higher order gluonic contributions from the gauge-link.

A non trivial constraint in modeling or fitting the Sivers function is given by the Burkardt sum rule [447. This sum rule is related to momentum conservation, which requires that the first transverse-momentum moment of the Sivers function, i.e. the net transverse momentum due to final state interactions, should vanish. In the bag model this sum rule is violated by a few percent [339, 435], since the bag states are not good momentum eigenstates. Analogously, the non-relativistic calculation in constituent quark models leads to a small violation of the sum rule. In spectator models, the sum rule is expected to be fulfilled only when taking into account both the quark and the diquark as explicit degrees of freedom [432]. On the other side, it was proven to hold in light-cone constituent quark models [298].

In fig. 2.22 the results from different models for the first transverse-momentum moment of the Sivers and Boer-Mulders functions are compared with phenomenological parametriza- 

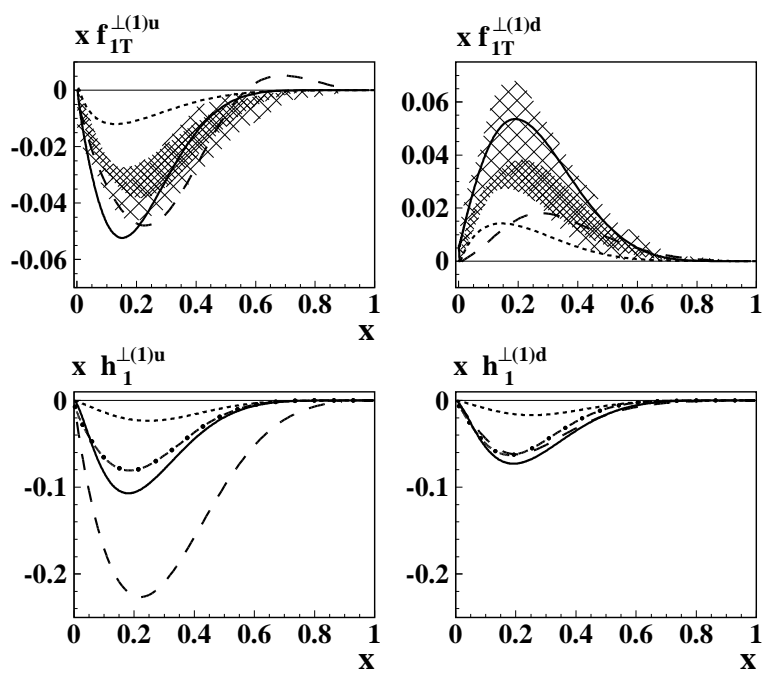

Figure 2.22. Results for the (1)-moments of the quark Sivers (upper panels) and Boer-Mulders (lower panels) functions as function of $x$. The different curves correspond to the results after (approximate) evolution from the model scale to $Q^{2}=2.5 \mathrm{GeV}^{2}$. Solid curves: light-cone constituent quark model of ref. [298]. Dashed curves: spectator model of ref. [337]. Dotted curves: bag model of ref. [339, 436]. In the case of the Sivers function, the lighter and darker shaded areas indicate statistical uncertainties of the parameterizations of ref. [332] and [328, 331]. For the Boer-Mulders function the dashed-dotted curves are the results of the phenomenological parametrization of refs. [366, 367.

tions [328, 332, 331, valid at an average scale of $Q^{2}=2.5 \mathrm{GeV}^{2}$, extracted by a fit to available experimental data for pion and kaon production in semi-inclusive deep inelastic scattering. The model results are evolved from the corresponding hadronic scale to $Q^{2}=2.5$ $\mathrm{GeV}^{2}$, by employing those evolution equations which seem most promising to be able to simulate the correct evolution, which is presently not available. In particular, we evolved the (1)-moment of the Sivers function by means of the evolution pattern of the unpolarized parton distribution, while for the (1)-moment Boer-Mulders function we used the evolution pattern of the chiral-odd transversity. Within the large error bar, the results of both the LCCQM and spectator model for the Sivers function are compatible with the parameterizations for both up and down quark, although the shapes of the distributions and the magnitude of the up- and down-quark contributions are quite different. On the other hand, the bag model predicts much smaller results, for both the Sivers and Boer-Mulders functions. In all the models the Boer-Mulders function has the same sign for both the up and down contributions, confirming theoretical expectations [334, 448]. Furthermore, the up and down contributions to the Boer-Mulders function are expected to have the same order of magnitude within the available parametrizations [366, 367, 370, 449]. This is confirmed from the predictions of the LCCQM and bag model, while it is at variance with the spectator model where the up distribution is more than twice bigger than the down distribution. However, we note that the available data do not allow yet a full fit of $h_{1}^{\perp}$ with its $x$ and $k_{\perp}^{2}$ dependence and the available phenomenological parameterizations are only first attempts to extract information on this distribution. New experimental data will play a crucial role to better constrain these analyses. 


\title{
2.5 Chiral-odd partonic densities
}

\author{
Harut Avakian, Alessandro Bacchetta, Andreas Metz, Marco Radici
}

Half of the leading-twist TMDs are denoted by the letter $h$, which means that they describe the distribution of transversely polarized partons. In the helicity basis for a spin

$\frac{1}{2}$ nucleon, where the unpolarized distribution $f_{1}$ and the helicity distribution $g_{1}$ have their well known probabilistic interpretation, transverse polarization states are given by linear combinations of positive and negative helicity states. Since helicity and chirality are the same at leading twist [245, they are called chiral-odd distributions.

One of the four leading-twist chiral-odd TMDs, the transversity distribution $h_{1}$, survives the integration upon transverse momentum. From the experimental point of view, transversity is quite an elusive object. In any observable the chiral-odd transversity needs to be coupled to a chiral-odd nonperturbative partner. In SIDIS, as discussed in Sec. 2.1. $h_{1}$ can appear in the leading-twist part of the cross section together with the chiral-odd Collins fragmentation function $H_{1}^{\perp}$, which can be determined separately, e.g., by measuring azimuthal asymmetries of the distribution of back-to-back pions in two-jet events in electronpositron annihilations, i.e. $e^{+} e^{-} \rightarrow \pi^{+} \pi^{-} X$ [250, 369]. Another promising approach to access transversity is semi-inclusive production of pion pairs, $e p^{\uparrow} \rightarrow e^{\prime}\left(\pi^{+} \pi^{-}\right) X$ [450], where the chiral-odd partner of $h_{1}$ is represented by the chiral-odd Dihadron Fragmentation Function (DiFF) $H_{1}^{\Varangle}$ [451].

Among the remaining chiral-odd quark distributions, the so-called Boer-Mulders function attracted great interest from both experiment and theory. It shares some common features as the quark Sivers function discussed in Sec. 2.2. In this section, we will dedicate one subsection to briefly describe this function, including the unique opportunity of exploring it using unpolarized hadrons.

\subsubsection{The quark transversity distribution}

At leading twist, three collinear distribution functions are needed to describe the quark distribution in the nucleon. Transversity is a leading-twist collinear PDF and enjoys the same status as $f_{1}$ and $g_{1}$ [452, 418]. An important difference between $h_{1}$ and $g_{1}$ is that in spin- $\frac{1}{2}$ hadrons there is no gluonic function analogous to transversity. The most important consequence is that $h_{1}^{q}$ for a quark with flavor $q$ does not mix with gluons in its evolution and it behaves as a non-singlet quantity; this has been verified up to NLO, where chiral-odd evolution kernels have been studied so far [453, 454, 455.

The tensor charge of the nucleon is defined as the sum of the Mellin moments $\delta q\left(Q^{2}\right)=$ $\int d x\left[h_{1}^{q}\left(x, Q^{2}\right)-h_{1}^{\bar{q}}\left(x, Q^{2}\right)\right]$. Contrary to the axial charge - which is related to $g_{1}^{q}\left(x, Q^{2}\right)$ - it has a nonvanishing anomalous dimension: it evolves with the hard scale $Q^{2}$ [418]. It has been calculated on the lattice [456] and in various models [408, 457, 458, 459, 460], and was found to be sizable. For a more comprehensive review, we refer to Ref. [461].

The extraction of transversity is of fundamental interest for obtaining a complete description of the nucleon structure even for the case when internal transverse momenta are integrated over. To achieve this goal, it is crucial to cover the widest possible range in $\left(x, Q^{2}\right)$, to measure the related asymmetries differential in the relevant kinematic variables and to be able to perform a flavor separation. 


\begin{tabular}{lcccc}
\hline experiment (laboratory) & $\sqrt{s}$ in $\mathrm{GeV}$ & target type & hadron types & references \\
\hline COMPASS (CERN) & 18 & deuteron & $h^{ \pm}, \pi^{ \pm}, K^{ \pm}, K^{0}$ & {$[320$, 321] } \\
& & proton & $h^{ \pm}$ & {$[322]$} \\
& & proton & $\pi^{ \pm}, K^{ \pm}$ & prelim. [323] \\
\hline HERMES (DESY) & 7.4 & proton & $\pi^{ \pm}$ & {$[324]$} \\
& & proton & $\pi^{ \pm}, \pi^{0}, K^{ \pm}$ & {$[462]$} \\
\hline HallA (JLab) & 3.5 & neutron & $\pi^{ \pm}$ & prelim. [326] \\
\hline
\end{tabular}

Table 2.4. Summary of currently available measurements of Collins asymmetry amplitudes from lepton-nucleon DIS experiments, their center-of-mass energy, transversely polarized target type, and analyzed hadron types.

\section{The Collins effect}

As discussed in Sec. 1, at tree-level and leading-twist, the SIDIS $F_{U T}^{\sin \left(\phi_{h}+\phi_{S}\right)}$ structure function of Eq. (2.8) can be described as a convolution between the transversity $h_{1 T}^{q}$ and the Collins fragmentation function $H_{1}^{\perp q}$, i.e.,

$$
F_{U T}^{\sin \left(\phi_{h}+\phi_{S}\right)} \sim \sum_{q} e_{q}^{2} h_{1 T}^{q} \otimes H_{1}^{\perp q}
$$

In order to project out the structure function $F_{U T, T}^{\sin \left(\phi_{h}+\phi_{S}\right)}$ in Eq. (2.8), the so-called Collins amplitude $2\left\langle\sin \left(\phi_{h}+\phi_{S}\right)\right\rangle_{U T}^{h}$ for a specific hadron $h$ is extracted from the asymmetry

$$
A_{U T}^{h}\left(\phi_{h}, \phi_{S}\right) \equiv \frac{1}{\left|\mathbf{S}_{T}\right|} \frac{d \sigma^{h}\left(\phi_{h}, \phi_{S}\right)+d \sigma^{h}\left(\phi_{h}, \phi_{S}+\pi\right)}{d \sigma^{h}\left(\phi_{h}, \phi_{S}\right)+d \sigma^{h}\left(\phi_{h}, \phi_{S}+\pi\right)},
$$

where the subscript $U$ indicates an unpolarized lepton beam and $T$ a transversely polarized target nucleon. The azimuthal angles are illustrated in Fig. 2.1. This amplitude has so far been extracted by three polarized fixed-target experiments as summarized in Table 2.4. From these measurements, Fig. 2.23 shows a selection of results that are significantly nonzero and help in determining both the shape of transversity and the relative size and sign of the Collins fragmentation function. All other asymmetry amplitudes listed in Table 2.4 are small or consistent with zero.

For the second unknown in Eq. (2.55), the Collins fragmentation function, model calculations are available [382, 463, 464, 465, 466, 467, 468, 469]. However, for a modelindependent extraction of transversity from the SIDIS asymmetry amplitudes we need to determine the Collins function from an independent source. This is represented by the measurement of azimuthal asymmetries in the distribution of back-to-back pions in two-jet events in electron-positron annihilations, i.e. $e^{+} e^{-} \rightarrow \pi^{+} \pi^{-} X$ [470].

The relevant vectors and angles involved in $e^{+} e^{-}$annihilations leading to back-to-back jets are depicted in Fig. 2.24 (left panel). The following asymmetry can be measured [250, 369 ]

$$
A_{12}\left(z_{1}, z_{2}, \theta_{2}, \phi_{1}+\phi_{2}\right)=1+\frac{\sin ^{2} \theta}{1+\cos ^{2} \theta} \cos \left(\phi_{1}+\phi_{2}\right) \frac{\sum_{q} e_{q}^{2} H_{1}^{\perp(1) q}\left(z_{1}\right) H_{1}^{\perp(1) \bar{q}}\left(z_{2}\right)}{\sum_{q} e_{q}^{2} D_{1}^{q}\left(z_{1}\right) D_{1}^{\bar{q}}\left(z_{2}\right)} .
$$




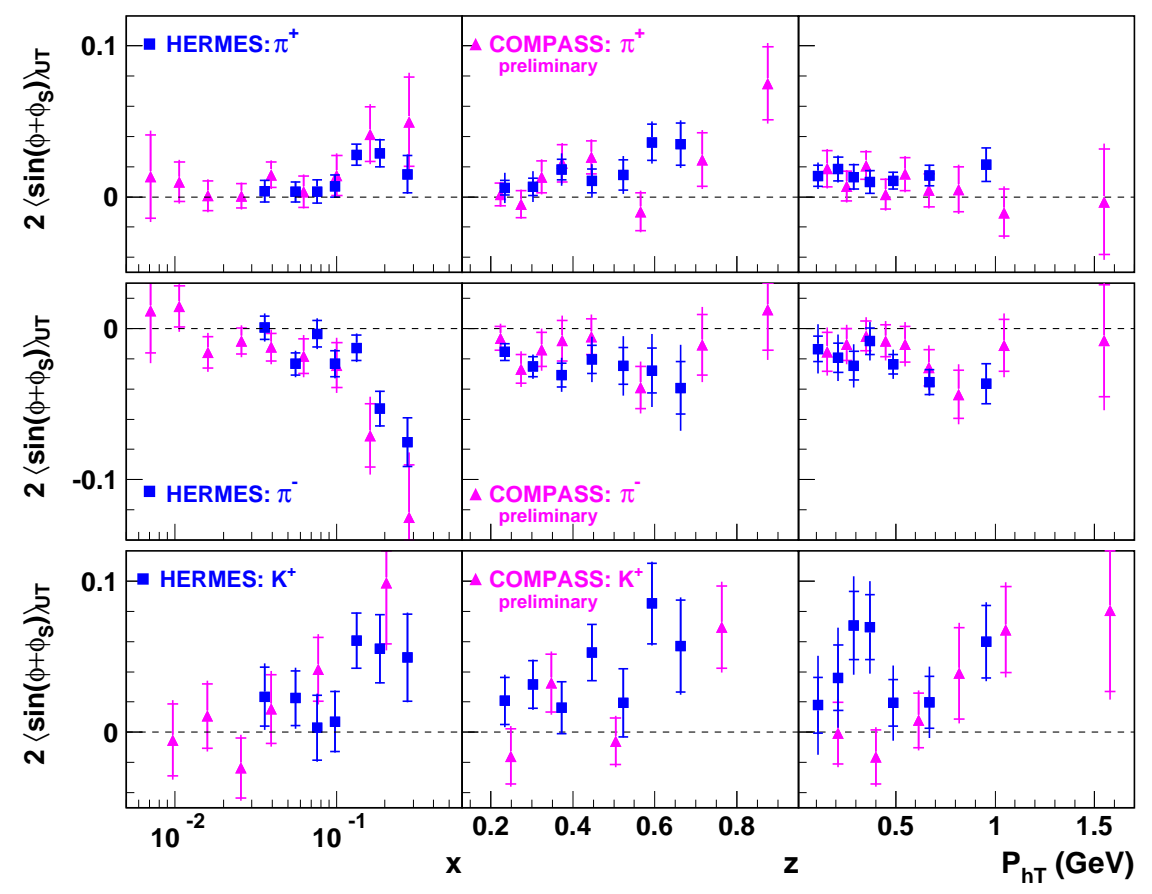

Figure 2.23. Collins amplitudes for $\pi^{+}, \pi^{-}$and $K^{+}$(as denoted in the panels) from HERMES [462] and COMPASS 323 measured with a proton target. Inner error bars present statistical uncertainties and full error bars the quadratic sum of statistical and systematic uncertainties. Note that the average kinematics in each bin differs for HERMES and COMPASS and the sign of the COMPASS asymmetries have been reversed.

Pioneering measurements of this spin-dependent fragmentation function have been performed by the BELLE Collaboration (KEK) [472, 471]. Experimentally, double ratios of asymmetries for like-sign (L), unlike-sign (U) and any charged (C) pion pairs are built in order to cancel (to a large extent) contributions from the experimental acceptance and radiative effects. The resulting asymmetries, $A^{U L}$ and $A^{U C}$, are then sensitive to different combinations of the favored and unfavored Collins fragmentation functions as given in [471. These asymmetries are presented in Fig. 2.24 as function of $z_{2}$ for four bins of $z_{1}$ for the light quarks $(u, d, s)$, where $z_{1}$ and $z_{2}$ are for a hadron in each of the back-to-back jets.

The experimental results shown in Figs. 2.23 and 2.24 are striking. First, they clearly demonstrate that the Collins effect as a manifestation of chiral-odd and naïve T-odd mechanisms is different from zero and not suppressed, both in SIDIS and in $e^{+} e^{-}$annihilations. Second, the results for oppositely charged pions (hadrons) in Fig. 2.23 suggest a very peculiar feature for the Collins fragmentation function. As scattering off $u$ quarks dominates these data due to the charge factor, the large magnitude of $\pi^{-}$amplitudes being of similar size than the $\pi^{+}$ones but having opposite sign, can only be understood if the disfavored Collins function $H_{1}^{\perp}$ unfav is large and of opposite sign to the favored one. Opposite signs for the favored and unfavored Collins functions are also supported by the different size of $A^{U L}$ and $A^{U C}$ asymmetries from BELLE in Fig. 2.24, They can be understood in light of the string model of fragmentation [463] (and also of the Schäfer-Teryaev sum rule [473]). If a favored pion is created at the string end by the first break, an unfavored pion from the 

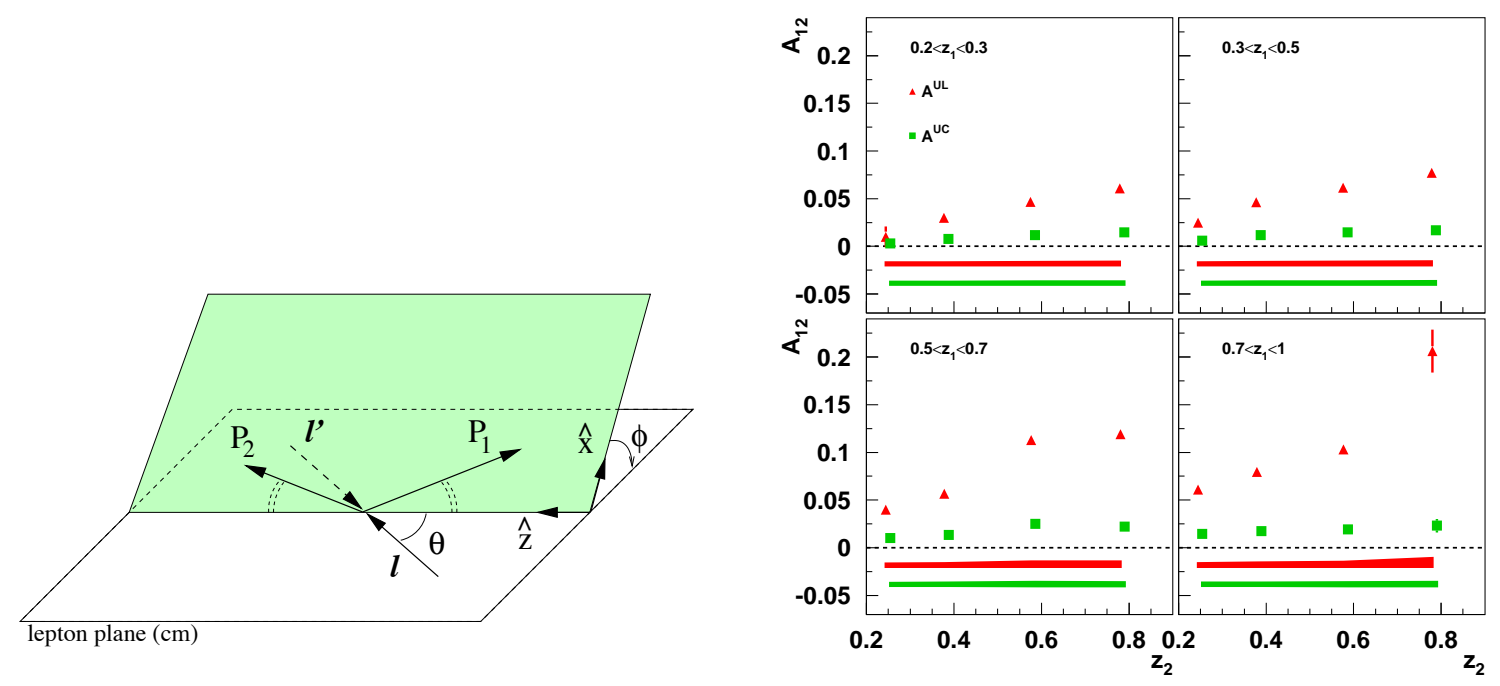

Figure 2.24. Left: the kinematics of $e^{+} e^{-}$annihilation leading to back-to-back jets along the $\hat{\boldsymbol{z}}$ axis (jet frame), $P_{1}$ is the momentum of a hadron in one jet, $P_{2}$ is the momentum of a hadron in the other jet. Right: Collins asymmetry $A_{12}$ for the double ratios for like-sign (L), unlike-sign (U) and any charged (C) pion pairs as function of $z_{2}$ in bins of $z_{1}$ from BELLE [471. $A^{U L}$ and $A^{U C}$ are sensitive to different combinations of the favored and unfavored Collins fragmentation functions.

next break is likely to inherit transverse momentum in the opposite direction.

The extraction of transversity and Collins functions from available data faces the same issues as discussed for the Sivers function in Sec. 2.2.1 for resolving the convolution in Eq. (2.55) and the same strategies are applied here. Employing the Gaussian Ansatz in Eq. (2.22) both transversity and Collins function have been extracted [250, 474] from (part of) the experimental data discussed before. The new COMPASS proton or Hall-A neutron data are not yet included in this fit. The results of this global analysis are presented in Fig. 2.25 for $u$ and $d$ transversity distributions (left panel) and favored and unfavored Collins fragmenation functions (right panel). The decrease in the presented uncertainties for the specifically chosen parametrization, which is the same as in [250, 474], is due to the new BELLE and HERMES data. The extracted favored and unfavored Collins functions confirm the features discussed before.

\section{Dihadron Fragmentation Functions}

A complementary approach to transversity is provided by semi-inclusive two-hadron production, $e p^{\uparrow} \rightarrow e^{\prime}\left(h_{1} h_{2}\right) X$, where the two unpolarized hadrons with momenta $P_{1}$ and $P_{2}$ emerge from the fragmentation of the struck quark. The underlying mechanism differs from the Collins mechanism in that the transverse spin of the fragmenting quark is transferred to the relative orbital angular momentum of the hadron pair. Consequently, this mechanism does not require transverse momentum of the hadron pair and collinear factorization applies.

Dihadron fragmentation functions were introduced in Ref. 475] and studied for the polarized case in Refs. [450, 476, 477]. The decomposition of the SIDIS cross section in terms of quark distributions and dihadron fragmentation functions was carried out to leading twist in Ref. [451] and to sub-leading twist in Ref. 478.

The kinematics is similar to the one in single-hadron SIDIS except for the final hadronic 

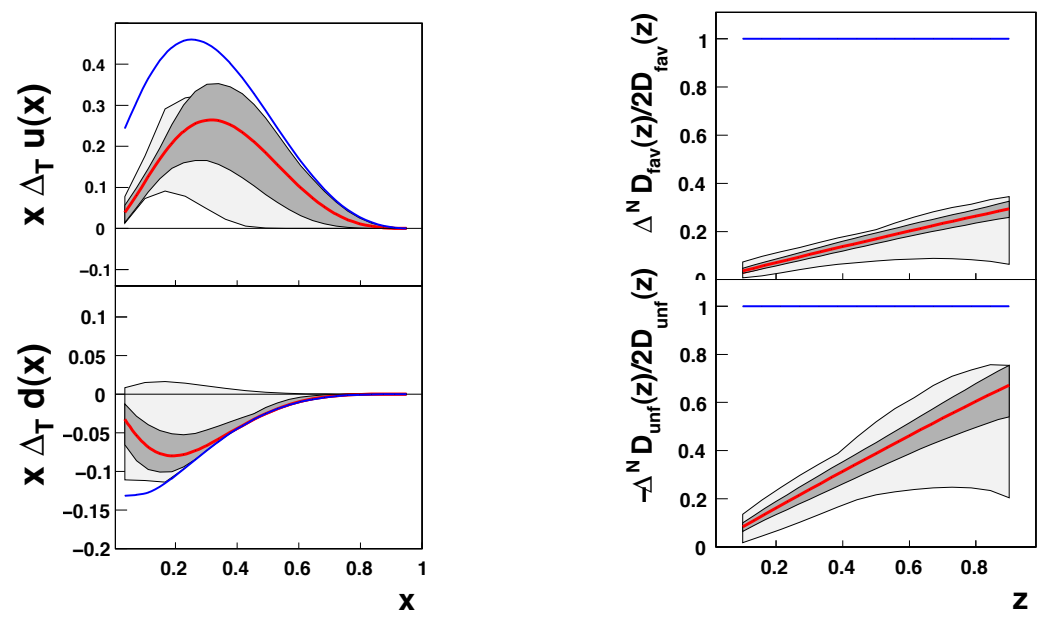

Figure 2.25. Left: transversity $x h_{1}^{q}(x)$ for $u$ (upper panel) and $d$ (lower panel) quarks. Right: the normalized Collins functions $\sqrt{2} H_{1}^{\perp(1 / 2)}(z) / D_{1}(z)$ for favored (upper panel) and unfavored (lower panel) fragmentation. The light grey band represents the uncertainty for the extraction in Ref. [250] and the dark grey band from the updated analysis 474 . Blue lines indicate the Soffer and positivity bound for transversity and Collins function, respectively.

state, where now $z=z_{1}+z_{2}$ is the fractional energy carried by the hadron pair and we introduce the vectors $P_{h}=P_{1}+P_{2}$ and $R=\left(P_{1}-P_{2}\right) / 2$ (see Fig. 2.26), together with the pair invariant mass $M_{h}$, which must be considered much smaller than the hard scale (e.g., $P_{h}^{2}=M_{h}^{2} \ll Q^{2}$ ). We shall often use the quantity [479],

$$
|\boldsymbol{R}|=\frac{1}{2} \sqrt{M_{h}^{2}-2\left(M_{1}^{2}+M_{2}^{2}\right)+\left(M_{1}^{2}-M_{2}^{2}\right)^{2}},
$$

where $P_{1}^{2}=M_{1}^{2}, P_{2}^{2}=M_{2}^{2}$ and $R_{T}^{2}$ is related to $M_{h}^{2}$ [479].

In analogy with the Collins function, the expression for unpolarized hadrons $\left(h_{1}, h_{2}\right)$ produced by a transversely polarized quark reads

$$
D_{h_{1} h_{2} / q^{\uparrow}}\left(z, M_{h}^{2}, \boldsymbol{R}_{T}\right)=D_{1}^{q}\left(z, M_{h}^{2}\right)-H_{1 s p}^{\Varangle q}\left(z, M_{h}^{2}\right) \frac{\boldsymbol{S}_{\perp q} \cdot\left(\hat{\boldsymbol{p}} \times \boldsymbol{R}_{T}\right)}{M_{h}} .
$$

Choosing $\hat{\boldsymbol{p}} \| \hat{z}$ and $\boldsymbol{S}_{\perp q} \| \hat{y}$, a positive $H_{1 s p}^{\Varangle q}$ means that hadron $h_{1}$ is preferentially emitted along $-\hat{x}$ and hadron $h_{2}$ along $\hat{x}$.

Since $\boldsymbol{R}_{T}=\boldsymbol{R} \sin \theta$, where in the c.m. frame of the hadron pair $\theta$ is the angle between $P_{1}$ and the direction of $P_{h}$ in the laboratory frame (for more details, see refs. 479, 480, 481, 482]), the relevant asymmetry that should be measured in SIDIS is

$$
\begin{aligned}
A_{U T}^{\sin \left(\phi_{R}+\phi_{S}\right) \sin \theta} & \equiv 2 \frac{\int d \cos \theta d \phi_{R} d \phi_{S} \sin \left(\phi_{R}+\phi_{S}\right)\left[d \sigma\left(\phi_{R}, \phi_{S}\right)-d \sigma\left(\phi_{R}, \phi_{S}+\pi\right)\right] / \sin \theta}{\int d \cos \theta d \phi_{R} d \phi_{S}\left[d \sigma\left(\phi_{R}, \phi_{S}\right)+d \sigma\left(\phi_{R}, \phi_{S}+\pi\right)\right]} \\
& \sim \frac{|\boldsymbol{R}|}{M_{h}} \frac{\sum_{q} e_{q}^{2} h_{1}^{q}(x) H_{1 s p}^{\Varangle q}\left(z, M_{h}^{2}\right)}{\sum_{q} e_{q}^{2} f_{1}^{q}(x) D_{1}^{q}\left(z, M_{h}^{2}\right)}
\end{aligned}
$$

As in the single-hadron production case, transversity can be extracted from the asymmetry (2.60) only if the unknown $H_{1 s p}^{\Varangle}$ is independently determined from the $e^{+} e^{-}$annihilation producing, in this case, two hadron pairs: $e^{+} e^{-} \rightarrow\left(\pi^{+} \pi^{-}\right)_{\text {jet1 }}\left(\pi^{+} \pi^{-}\right)_{\text {jet} 2} X$ with kinematics 

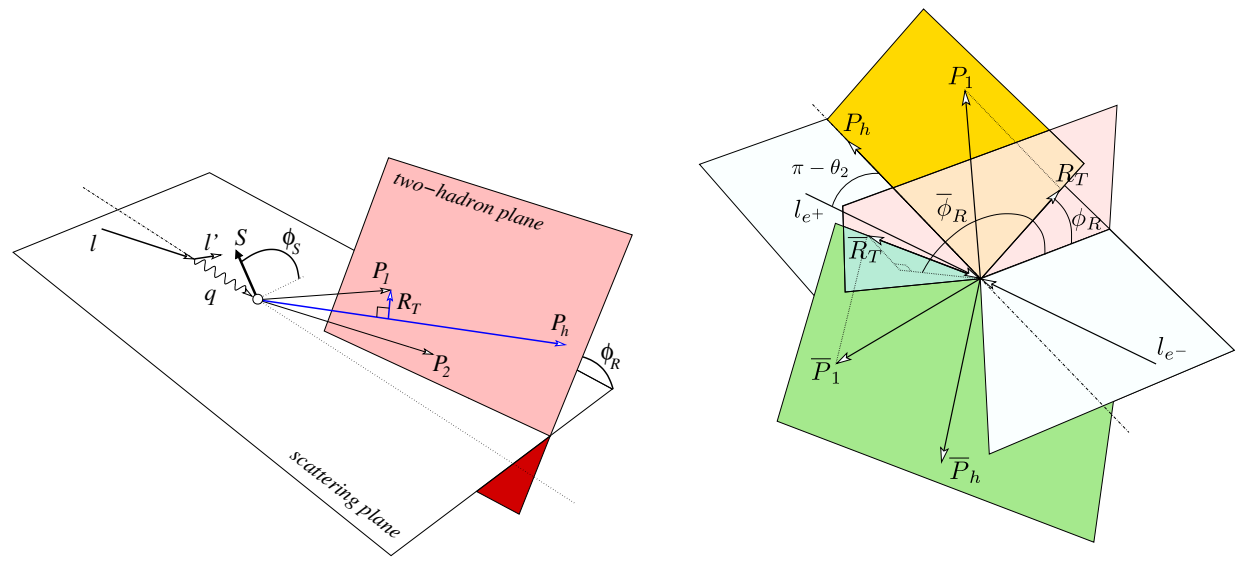

Figure 2.26. Kinematics for the production of two hadrons (left) and for the $e^{+} e^{-} \rightarrow$ $\left(\pi^{+} \pi^{-}\right)_{\text {jet1 }}\left(\pi^{+} \pi^{-}\right)_{\text {jet } 2} X$ process (right).

depicted in Fig. 2.26 (right). The relevant signal is similar to that of the Collins function, except that each transverse polarization of the quark-antiquark pair is now correlated to the azimuthal orientation of the plane formed by the momenta of the corresponding hadron pairs, suggesting that $H_{1}^{\Varangle}$ is related to the concept of handedness of the jet containing a specific pair 483, 484, 485.

The leading-twist cross section of this process contains many terms [485], among which there is one involving the product of $H_{1 s p}^{\Varangle q}$ for the quark $q$ and of $\bar{H}_{1 s p}^{\Varangle q}$ for the $\bar{q}$ partner, weighted by $\cos \left(\phi_{R}+\bar{\phi}_{R}\right)$. Thus, we can properly weight the cross section and extract this contribution by defining the so-called Artru-Collins azimuthal asymmetry [485, 482]

$A^{\cos \left(\phi_{R}+\bar{\phi}_{R}\right)}\left(\cos \theta_{2}, z, M_{h}^{2}, \bar{z}, \bar{M}_{h}^{2}\right)=\frac{\sin ^{2} \theta_{2}}{1+\cos ^{2} \theta_{2}} \frac{\pi^{2}}{32} \frac{|\boldsymbol{R}||\overline{\boldsymbol{R}}|}{M_{h} \bar{M}_{h}} \frac{\sum_{q} e_{q}^{2} H_{1 s p}^{\Varangle q}\left(z, M_{h}^{2}\right) \bar{H}_{1 s p}^{\Varangle q}\left(\bar{z}_{\bar{z}}, \bar{M}_{h}^{2}\right)}{\sum_{q} e_{q}^{2} D_{1}^{q}\left(z, M_{h}^{2}\right) \bar{D}_{1}^{q}\left(\bar{z}, \bar{M}_{h}^{2}\right)}$,

where the dihadron fragmentation functions $D_{1}^{q}$ and $H_{1 s p}^{\Varangle q}$ are the same universal functions appearing in the SIDIS asymmetry of equation (2.60).

Pioneering measurements of $A_{U T}^{\sin \left(\phi_{R}+\phi_{S}\right) \sin \theta}$ from HERMES [481] gave evidence for a non-zero dihadron fragmentation function $H_{1 s p}^{\Varangle q}$ as shown in Fig. 2.27. The $M_{h}$ dependence does not exhibit any sign change and rules out the model of Ref. 476]: interference patterns in semi-inclusive $\pi^{+} \pi^{-}$production are different from those in $\pi^{+} \pi^{-}$elastic scattering. Calculations based on the spectator model [480, 486] are compatible with data. They, however, overestimate the asymmetries if $h_{1}^{q}$ is taken from the parametrization 474] discussed in Fig. 2.25. This estimate is presented in Fig. 2.27 by the grey band where the model $H_{1 s p}^{\Varangle q}$ is reduced by a factor $\alpha=0.32 \pm 0.06$ in order to reproduce the magnitude of the asymmetry.

Preliminary SIDIS data are also available from the COMPASS Collaboration using transversely polarized deuteron and hydrogen [487] targets. While $A_{U T}^{\sin \left(\phi_{R}+\phi_{S}\right) \sin \theta}$ is basically vanishing on the deuteron, the proton data show a signal larger than the HERMES results in Fig. 2.27, which might be due to different kinematics.

Last but not least, results from pioneering measurements of the $A^{\cos \left(\phi_{R}+\bar{\phi}_{R}\right)}$ asymmetry related to the dihadron fragmentation function became recently available from the BELLE 


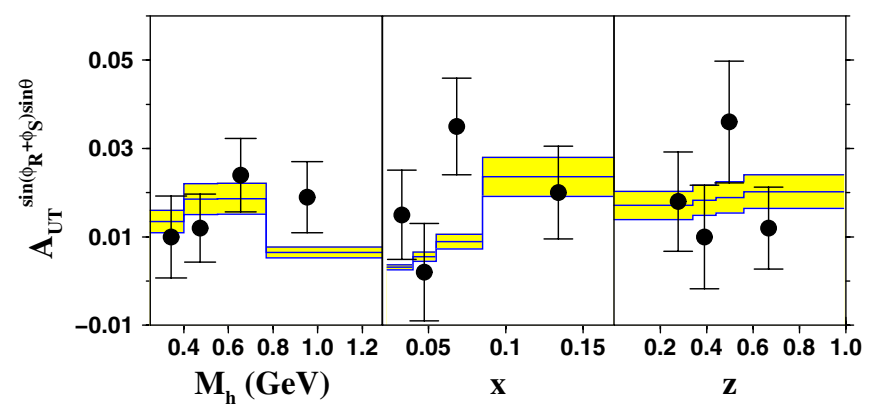

Figure 2.27. The spin asymmetry for the semi-inclusive production of a pion pair in deep-inelastic scattering on a transversely polarized proton 481 . The grey band presents a fit to the data involving the dihadron FF calculated in the spectator model of Ref. [480] and on the parametrization for $h_{1}$ from Ref. 474.

Collaboration in Ref. [488.

For a real breakthrough of this promising approach to transversity, much more data over wide kinematic range are needed. We only mention that the SIDIS cross section does now depend on nine kinematic variables compared to six for the single-hadron case, which calls even more for a multi-dimensional analysis for a bias-free extraction of the asymmetries.

\section{Collins effect at EIC}

The exploration of chiral-odd structures using the Collins effect is far from being complete. Several aspects need to be significantly improved. The $x$ dependence is largely unconstrained due to the lack of SIDIS asymmetries outside the range $0.005 \lesssim x \lesssim 0.3$. The antiquark and sea-quark content of transversity in the proton is completely unknown. Together with the loose constraints on the $x$ dependence, this missing piece of information makes the calculation of the tensor charge still unsatisfactory. Also the transverse momentum dependence of both the transversity and the Collins function has a significant degree of arbitrariness. Lastly, the $Q^{2}$ range of HERMES and COMPASS measurements is approximately the same: it would be desirable to study the $A_{U T}^{\sin \left(\phi_{h}+\phi_{S}\right)}\left(Q^{2}\right)$ dependence in a wide range of $Q^{2}$.

All these remarks call for more data in order to enlarge the phase space and perform a multi-dimensional analysis in all relevant kinematic variables simultaneously. An ambitious

program is planned at JLab12, that would aim for exploring $A_{U T}^{\sin \left(\phi_{h}+\phi_{S}\right)}$ in the valence region with high luminosity [340, 341]. The EIC would be the ideal facility to carry out this program over a uniquely wide range in $x$ and $Q^{2}$. This potential for a mapping of the multi-dimensional phase-space in an unprecedented kinematic range is illustrated by the studies presented in Sec. 2.2.2 and are equally valid for transversity.

The promising and complementary approach of extracting transversity with help of the dihadron fragmentation function will even more profit from the high energy option of an EIC. Fig. 2.28 shows the projected accuracy for semi-inclusive kaon pair production at an energy $\sqrt{s}=140 \mathrm{GeV}$ and for an integrated luminosity of $30 \mathrm{fb}^{-1}$. The PYTHIA event generator has been used to obtain the SIDIS event rate, and an overall detection efficiency of $50 \%$ and beam polarization of $70 \%$ were assumed. Data are shown as function of $x$ for the various different $z$ and $M_{K K}$ bins indicated in the panels. The invariant mass range of the kaon pair, $M_{K K}$, is chosen for the vicinity of the $\phi$ meson, which provides unique access 


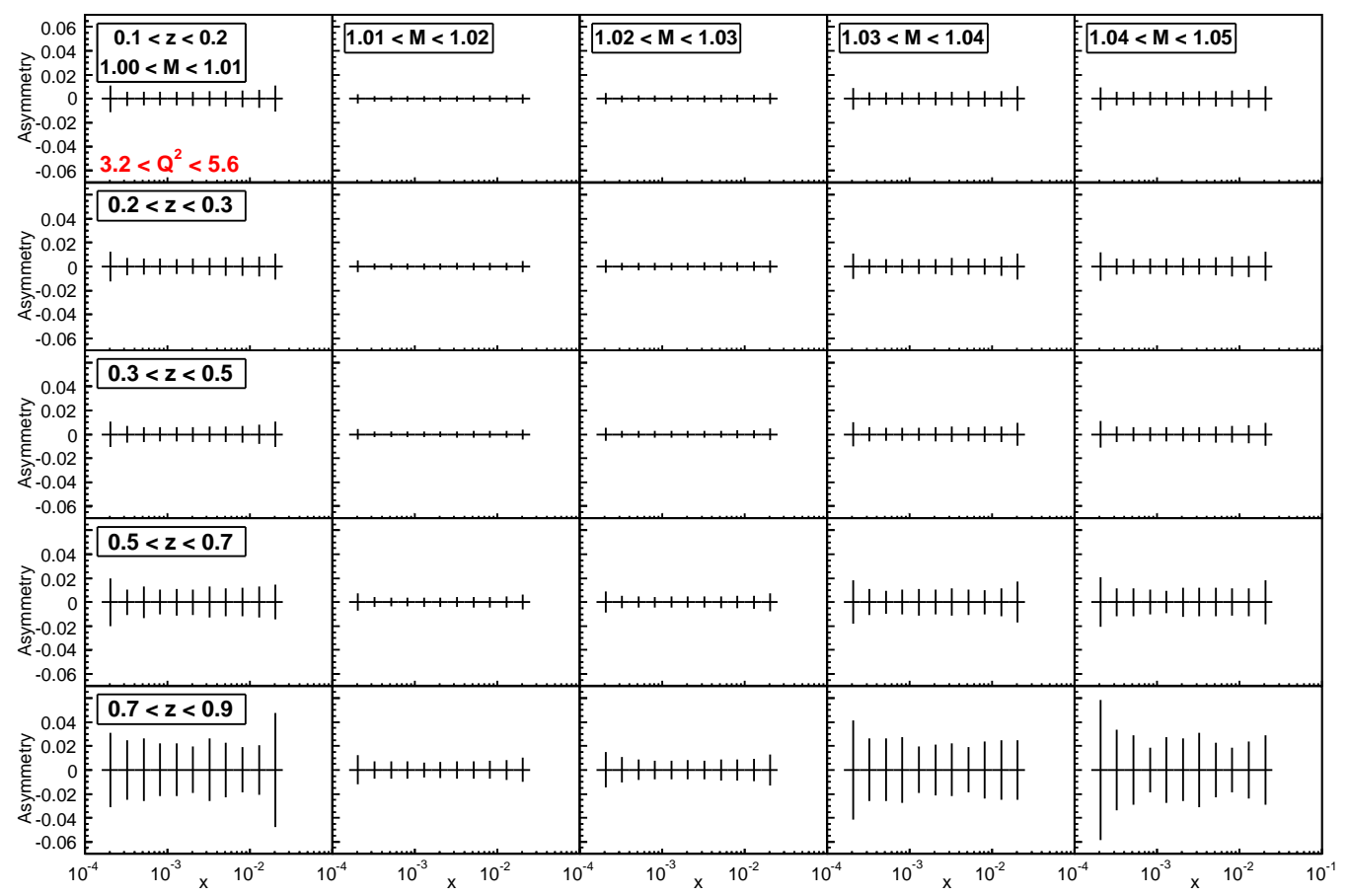

Figure 2.28. Projected accuracy, represented by the error bars, for semi-inclusive kaon pair production obtained with an energy of $\sqrt{s}=140 \mathrm{GeV}$ for an integrated luminosity of $30 \mathrm{fb}^{-1}$, as a function of $x$ in bins in $z, M_{K K}$ and for a single bin in $Q^{2}$ as indicated in the panels.

to strange quark distributions.

Furthermore, the general picture obtained so far would significantly profit from data available over a wide $Q^{2}$ range which can only be provided by the EIC. This picture obtained so far, is based on a tree-level analysis of transverse-momentum dependent azimuthal (spin) asymmetries occurring at very different energies: while the average scale of SIDIS experiments is approximately $2.5 \mathrm{GeV}^{2}$, the BELLE measurement was performed at the typical bottonium mass, i.e. $Q^{2} \sim 100 \mathrm{GeV}^{2}$. Beyond tree level, the evolution effects with running scale were included (at LO) only in the modification of the $x$ and $z$ dependence of the various functions. At low $\boldsymbol{P}_{h T}^{2} / Q^{2}\left(Q_{T}^{2} / Q^{2}\right.$ for $e^{+} e^{-}$annihilation, where $Q_{T}=\left|\boldsymbol{q}_{T}\right|$ is the transverse momentum of the virtual photon), the correct $Q^{2}$ dependence beyond tree level of transverse-momentum dependent structure functions should be studied extending the Collins-Soper-Sterman formalism mentioned in Sec. 2.2.6 273. A quantitative attempt to go in this direction was presented in Ref. [369], where it was estimated that transversemomentum resummation produces a suppression of the tree level result by almost a factor 5 at BELLE energies. Therefore, the extraction of the Collins function using the tree level formula could significantly underestimate its actual magnitude. In order to fit the available SIDIS asymmetries, a larger $H_{1}^{\perp}$ would automatically imply a transversity smaller than that one illustrated in Fig. 2.25 


\subsubsection{Boer-Mulders function}

The Boer-Mulders function $h_{1}^{\perp}$ 244] can be considered as the counterpart of the Sivers function $f_{1 T}^{\perp}$ : while $f_{1 T}^{\perp}$ describes the distribution of unpolarized quarks in a transversely polarized target, $h_{1}^{\perp}$ describes the distribution of transversely polarized quarks in an unpolarized target. Both functions are T-odd, and therefore vanish if the gauge-link is not taken into account in their operator definition, which makes them somewhat unique among the TMDs. Put it differently, their existence depends on the presence of initial and/or final state interactions between the active partons of a process and the target remnants (see the corresponding discussion in Sec. 2.4.1). It is expected that both TMDs change their sign when going from SIDIS to the Drell-Yan process [262]. There is, however, one important difference between them. The Sivers function is chiral-even, whereas the Boer-Mulders function is chiral-odd. Since the elementary interactions of the Standard Model do not change the chirality (helicity) of fermions, one has to couple the Boer-Mulders function - like any other chiral-odd object too - to another nonperturbative chiral-odd correlator in order to generate a non-zero observable. This implies that $h_{1}^{\perp}$, in general, is harder to measure than $f_{1 T}^{\perp}$.

On the other hand, in the case of the Boer-Mulders function no polarized target is required, which makes this distribution rather attractive. In fact, it is believed that the Boer-Mulders effect is essential for understanding data on the angular distribution of the unpolarized Drell-Yan process [489]. To be more specific, the general structure of the DrellYan cross section reads (see [490] and references therein)

$$
\frac{1}{\sigma_{D Y}} \frac{d \sigma_{D Y}}{d \Omega}=\frac{3}{4 \pi} \frac{1}{\lambda+3}\left(1+\lambda \cos ^{2} \theta+\mu \sin 2 \theta \cos \phi+\frac{\nu}{2} \sin ^{2} \theta \cos 2 \phi\right)
$$

where the angles $\theta$ and $\phi$ characterize the orientation of the lepton pair in a dilepton rest frame like the Collins-Soper frame [491. What attracted particular attention is the so-called Lam-Tung relation between the coefficients $\lambda$ and $\nu$ [350, 492],

$$
\lambda+2 \nu=1 .
$$

This relation is exact if one computes the Drell-Yan process to $\mathcal{O}\left(\alpha_{s}\right)$ in the standard collinear perturbative QCD framework. Even at $\mathcal{O}\left(\alpha_{s}^{2}\right)$ the numerical violation of (2.63) is small [493]. However, data for $\pi^{-} N \rightarrow \mu^{-} \mu^{+} X$ taken at CERN [345, 346] and at Fermilab [494] were found to clearly violate the Lam-Tung relation. In particular, an unexpectedly large $\cos 2 \phi$ modulation of the cross section was observed. Various explanations of this experimental result have been put forward, with the most favorable one being based on intrinsic transverse motion of partons leading to the Boer-Mulders effect [489]. The product of two Boer-Mulders functions — one for each initial state hadron - contributes to the $\cos 2 \phi$ term in the cross section in (2.62) [489]. An ultimate understanding of the angular distribution in (2.62), and thus also of the role played by the Boer-Mulders function, is of crucial importance if one keeps in mind that, from a theoretical point of view, the Drell-Yan process is the cleanest hard hadron-hadron reaction.

Several model calculations have been carried out for the Boer-Mulders function of both the nucleon [282, 298, 337, 338, 404, 429, 435, 436] and the pion [443, 446, 495, where the treatments for the nucleon comprise spectator models, the MIT bag model, and constituent quark models. In the case of the nucleon two general features emerge: first, the BoerMulders function comes out to be as large as the Sivers function or even larger. Second, it has the same sign for up-quarks and down-quarks. This finding nicely agrees with a 
model-independent analysis according to which $h_{1}^{\perp u}=h_{1}^{\perp d}$ to leading order of an expansion in powers of $1 / N_{c}$, with $N_{c}$ being the number of colors [334].

A lot of attention has been paid to an intuitive relation between the Boer-Mulders function and (a specific linear combination of) chiral-odd Generalized Parton Distributions in impact parameter space [496, 497]. (This connection between two types of parton distributions is the analogue of a corresponding relation involving the Sivers function which was proposed earlier [444, 299].) The intuitive picture is compatible with the two general results from model calculations discussed above. In particular, it also suggests a significant size for the Boer-Mulders function in the valence region. In Quantum Field Theory one can make such a relation quantitative in the framework of simple spectator models [282, 433, 300]. However, according to current knowledge, a general model-independent relation cannot exist [45, 446].

The Boer-Mulders function describes the strength of a correlation between the transverse momentum and the transverse spin of the active quark. This correlation generates a dipole pattern in the transverse $k_{\perp}$-plane - like the correlations associated with $f_{1 T}, g_{1 T}$, and $h_{1 L}^{\perp}$ do. One way of visualizing the Boer-Mulders effect is by looking at the density

$$
\rho_{h_{1}^{\perp}}^{q}\left(\boldsymbol{k}_{\perp}, \boldsymbol{s}_{\perp}\right)=\int d x \frac{1}{2}\left[f_{1}^{q}\left(x, \boldsymbol{k}_{\perp}^{2}\right)+\frac{\epsilon_{\perp}^{i j} s_{\perp}^{i} k_{\perp}^{j}}{M} h_{1}^{\perp q}\left(x, \boldsymbol{k}_{\perp}^{2}\right)\right]
$$

describing the distribution of transversely polarized quarks in an unpolarized nucleon [298]. The quark polarization is specified by the spin vector $s_{\perp}$. Note that the longitudinal momentum fraction has been integrated over. In Eq. (2.64), the $f_{1}$ term provides an axially symmetric contribution, while the second term containing $h_{1}^{\perp}$ gives rise to the mentioned dipole pattern. If both effects are superimposed, the resulting distribution is shifted away from the center (distorted) in the $k_{\perp}$-plane.

The Boer-Mulders function can also be studied in SIDIS and therefore at the EIC. In this process it couples to the chiral-odd Collins fragmentation function $H_{1}^{\perp}$ [249] and gives rise to a $\cos 2 \phi_{h}$-modulation of the cross section. The pertinent structure function takes the generic form

$$
F_{U U}^{\cos 2 \phi_{h}} \sim \sum_{q} e_{q}^{2}\left(h_{1}^{\perp q} \otimes H_{1}^{\perp q}+\frac{C}{Q^{2}} f_{1}^{q} \otimes D_{1}^{q}+\ldots\right)
$$

where $C$ is a kinematic factor. The second term on the right hand side of (2.65) is the so-called Cahn effect [498, 499], which is also caused by intrinsic transverse parton motion. It is a kinematic twist- 4 contribution, i.e., it is suppressed by a factor $1 / Q^{2}$ relative to the first term. Theoretical estimates of this effect are still plagued by large uncertainties, mainly related to the insufficient knowledge of the transverse momentum dependence of $f_{1}^{q}$ and $D_{1}^{q}$. The explicit form of all potential additional (dynamical) twist-4 effects in this structure function is presently not known. These considerations show that a reliable extraction of the Boer-Mulders function from SIDIS requires data in a kinematic region for which the (largely unknown) higher-twist contributions can be neglected. Since $f_{1}^{q} \gg h_{1}^{\perp q}$ and $D_{1}^{q} \gg H_{1}^{\perp q}$, the suppression of the Cahn effect requires very large $Q^{2}$.

The SIDIS structure function $F_{U U}^{\cos 2 \phi_{h}}$ has already been measured by the CLAS Collaboration at JLab [500, the HERMES Collaboration at DESY [501], and the COMPASS Collaboration at CERN [502]. More precisely, typically data are shown for the relevant azimuthal asymmetry given by $F_{U U}^{\cos 2 \phi_{h}} / F_{U U}$. However, due to the limited range in $Q^{2}$ the present SIDIS data allow at most a qualitative extraction of $h_{1}^{\perp}$, as is also obvious from 
a first exploratory study [367]. Moreover, the Boer-Mulders function for antiquarks is not at all constrained by the available data from SIDIS. Some information about antiquarks is available from recent Fermilab data on proton-deuteron [348] and proton-proton [349] DrellYan, though the uncertainties are again significant and not the least due to the presently large uncertainties for the Boer-Mulders function of quarks [370, 503, 504].

Even without further detailed reasoning it is clear that a quantitative knowledge about the Boer-Mulders function can only be obtained with data from new facilities. Measurements of the structure function $F_{U U}^{\cos 2 \phi_{h}}$ in the valence region in electroproduction of pions and kaons compose an important part of the upgraded JLab program on TMD studies. However, the $Q^{2}$ range obtainable with JLab12 will not be sufficient to suppress the contribution from the Cahn effect.

Only the unprecedented wide kinematic range of the EIC would provide clean measurements of the Boer-Mulders function for valence and sea quarks, and will allow for studying both, its $Q^{2}$ evolution and transition behavior from low to high $P_{h T}$.

Finally, there also exists a Boer-Mulders function for gluons, $h_{1}^{\perp g}$, describing the distribution of linearly polarized gluons in an unpolarized hadron [248, 281, 282. In contrast to the Boer-Mulders function for quarks, $h_{1}^{\perp g}$ is T-even. See the relevant discussions in Sec. 3 . 


\title{
2.6 Overview on other TMDs
}

\author{
Harut Avakian, Alessandro Bacchetta, Andreas Metz, Peter Schweitzer
}

In previous Sections, we discussed the unpolarized TMD $f_{1}$, the Sivers distribution $f_{1 T}^{\perp}$, the transversity distribution $h_{1}$, and the Boer-Mulders distribution $h_{1}^{\perp}$. They have been given more emphasis because at the present state of our knowledge they seem to be the most attractive and promising for EIC studies.

Nevertheless, interesting physics is embodied also in all other TMDs. Only the combination of information from all TMDs will fully explore the information contained in the unintegrated quark correlator, and provide a complete picture of the parton structure of the nucleon in transverse momentum space. This wealth of information may become one of the biggest legacies of the EIC.

In this Section, we briefly discuss the leading-twist TMDs that have not been analyzed in previous Sections and some of the sub-leading twist TMDs.

quark pol.

\begin{tabular}{|c|c|c|c|c|}
\hline \multirow{4}{*}{ 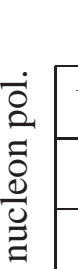 } & $\mathrm{U}$ & $\mathrm{L}$ & \multicolumn{2}{|c|}{$\mathrm{T}$} \\
\hline & $f_{1}$ & & \multicolumn{2}{|c|}{$h_{1}^{\perp}$} \\
\hline & & $g_{1}$ & \multicolumn{2}{|c|}{$h_{1 L}^{\perp}$} \\
\hline & $f_{1 T}^{\perp}$ & $g_{1 T}$ & $h_{1}$ & $h_{1 T}^{\perp}$ \\
\hline
\end{tabular}

(a)

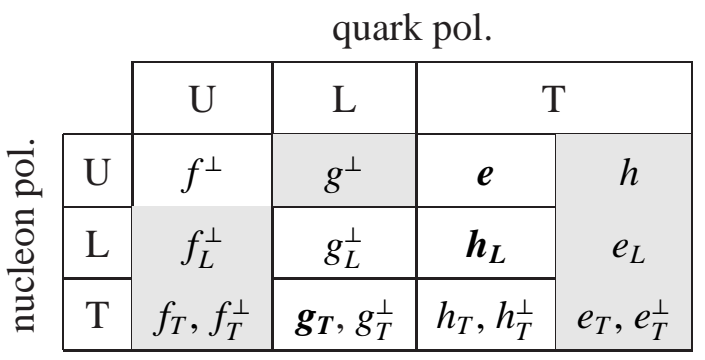

(b)

Table 2.5. Transverse momentum dependent (a) twist-2, (b) twist-3 distribution functions. The U,L,T correspond to unpolarized, longitudinally polarized and transversely polarized nucleons (rows) and quarks (columns). Functions in boldface survive transverse momentum integration. Functions in gray cells are T-odd.

\subsubsection{Other leading-twist TMDs}

Table 2.5 a summarizes the full list of leading-twist TMDs. The helicity distribution $g_{1}$, together with $f_{1}$ and $h_{1}$, survives integration over transverse momentum and has been already discussed extensively. Here we mention the importance of also studying its transverse momentum dependence. It may be possible that the transverse momentum distribution of quarks with spin antiparallel to the nucleon is different from that of quarks with spin parallel to the nucleon as suggested by lattice calculations [280] shown in Fig. 2.29] The structure function $F_{L L}$, involving the transverse-momentum dependence of $g_{1}$, is the only one where transverse-momentum resummation studies have been carried out to a level similar to $F_{U U, T}$ [372], but no extraction of the nonperturbative component has ever been attempted. The EIC will be an ideal machine to address this question.

The chiral-odd T-even TMD $h_{1 T}^{\perp}$ appears in the SIDIS structure function $F_{U T}^{\sin \left(3 \phi-\phi_{S}\right)}$. This function may be interpreted as the distribution of quarks with a polarization transverse but orthogonal to that of a transversely polarized nucleon. The popular name "pretzelosity" is due to the fact that this distribution has a quadrupole shape, vaguely reminiscent of a 


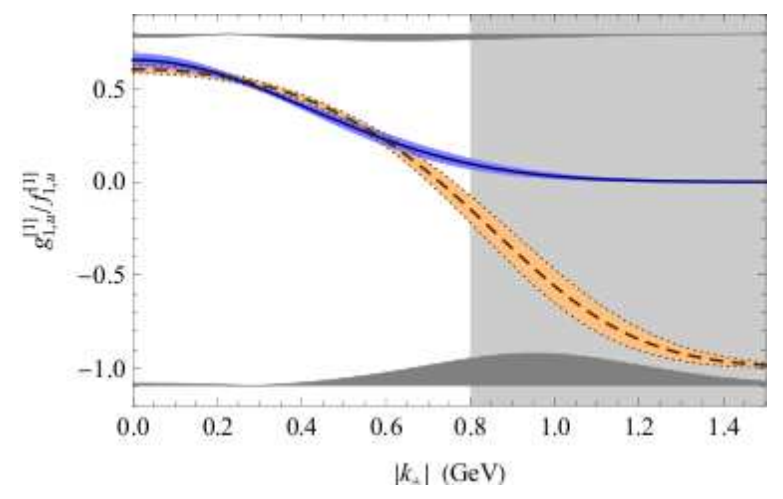

Figure 2.29. Ratio between the helicity distribution and the unpolarized distribution for up quarks based on lattice QCD computations [280]: the significant $k_{\perp}$ dependence of the two curves (corresponding to two different parameterizations) suggests that quarks with different spin orientation have different transverse momentum distributions.

pretzel [411, 301]. This TMD has attracted a lot of interest in the literature recently because of its possible connection with orbital angular momentum (see detailed discussion in Sec. 2.4.4). It is also interesting that, in a number of nonperturbative models, $h_{1 T}^{\perp}$ is just the difference between the quark helicity and the transversity distribution [419]. Moreover, in simple spectator models of the nucleon it can be related to a particular linear combination of chiral-odd generalized parton distributions [282]. In general, $h_{1 T}^{\perp}$ involves an interference between light-cone wave function components that differ by two units of orbital angular momentum. Preliminary data from COMPASS [505] and from HERMES [506] taken with transversely polarized deuterons or protons, respectively, showed an effect compatible with zero, however, within large experimental uncertainties.

The TMDs $g_{1 T}$ and $h_{1 L}^{\perp}$ appear in the structure functions $F_{L T}^{\cos \left(\phi-\phi_{S}\right)}$ and $F_{U L}^{\sin 2 \phi}$, respectively. The chiral-even (chiral-odd) $g_{1 T}\left(h_{1 L}^{\perp}\right)$ describes longitudinally (transversely) polarized quarks in a transversely (longitudinally) polarized nucleon. Since both functions link two perpendicular spin directions, they are sometimes named "worm-gear" functions. Both functions are related to quark orbital motion inside nucleons. They represent the real part of an interference between nucleon wave functions that differ by one unit of orbital angular momentum, while the imaginary parts are related to the Sivers and Boer-Mulders functions [278, 507]. Because of this, they appear in positivity bounds together with the Sivers and Boer-Mulders function [507]. They do not depend on final-state interactions and may offer cleaner insights into orbital angular momentum compared to the Sivers and Boer-Mulders functions. Interestingly, these functions are the first TMDs that have been computed on the lattice [279, 280]. The results (with the due caveats) indicate that they are sizable, $g_{1 T}^{u_{v}}>0, g_{1 T}^{d_{v}}<0$, and $g_{1 T} \approx-h_{1 L}^{\perp}$. These general findings also agree with some model calculations, see Sec. 2.4.4.

Notice that due to their chirality properties, $g_{1 T}$ couples through evolution to its analogous function for gluons (named $\Delta G_{T}$ in Ref. [281] and $g_{1 T}^{g}$ in Ref. [282]), while this is not true for $h_{1 L}^{\perp}$. This difference will be particularly relevant at the EIC, where gluons will play an important role.

By exploring QCD equations of motion, and neglecting "pure twist-3" quark-gluon correlators and current quark mass terms, one can express $g_{1 T}\left(h_{1 L}^{\perp}\right)$ in terms of $g_{1}\left(h_{1}\right)$ (see, e.g., [243, 252, 427, 508, and references therein). This is similar in spirit to the clas- 
sic Wandzura-Wilczek approximation [509] for the twist-3 distribution function $g_{T}^{q}(x) \approx$ $\int_{x}^{1} \mathrm{~d} y g_{1}^{q}(y) / y$, (which is supported by the instanton QCD vacuum model [510, 511] and lattice QCD [512, 513]). At an initial stage, it may be convenient to exploit such WandzuraWilczek-type approximations, in order to make estimates for planned experiments [252, 508, 514. In fact, existing data suggest that they are reasonable [508, even though at present there are no compelling grounds for supporting their validity [515, 516, 517]. In the end, these approximations should be tested and twist-3 effects should be extracted from the data, as we will also argue in the next subsection.

\subsubsection{Subleading-twist TMDs}

Eight out of the 18 structure functions providing the complete description of the SIDIS cross section are leading twist and were discussed in detail in previous sections. However, 10 structure functions are higher twist, where the underlying twist-classification follows 245]: "an observable is twist- $t$ if its effect is effectively suppressed by $(M / Q)^{t-2}$."

Higher twist functions, see Table 2.5b for a full list of twist-3 TMDs, are of interest for several reasons. Their understanding is required not only to complete the description of the SIDIS process. Besides being indispensable to correctly extract twist-2 parts from data, the knowledge of higher twists will also offer important tools to access the physics of the largely unexplored quark-gluon correlations which provide direct and unique insights into the dynamics inside hadrons, see, e.g., [518]. The EIC, which will span a large $Q$-range, will be an ideal tool to identify higher-twist effects, which fall off as powers of $1 / Q$.

Although suppressed with respect to twist- 2 observables by $1 / Q$, twist-3 observables are not small in the kinematics of fixed target experiments. Indeed, the first unambiguously measured single spin phenomena in SIDIS which triggered important theoretical developments, were the sizable longitudinal target $\left(A_{U L}^{\sin \phi}\right)$ and beam $\left(A_{L U}^{\sin \phi}\right)$ spin asymmetries observed at HERMES and JLab [519, 520, 521, 522, 523, 524]. Further data on twist-3 spin asymmetries are underway [505, 525, 526]. In unpolarized SIDIS, the sizable twist-3 effects $\left(A_{U U}^{\cos \phi}\right)$ are known since EMC [527, 528], see also recent results from JLab, HERMES and COMPASS [501, 529, 500, 530. At high energies $A_{U U}^{\cos \phi}$ can be described in perturbative QCD, and the unique possibilities of EIC could bridge 371 the gap to high energy data [531, 532, 533, 534. The understanding of the "matching" of the TMD formalism and the large- $p_{T}$ collinear description is of fundamental importance, see Sec. 2.2.5 and references therein.

The theoretical description of twist-3 observables is challenging. A good illustration of this point is that in spite of the enormous dedicated theoretical and phenomenological effort [396, 397, 465, 535, 536, 537, 538, 539, 540, 541, 542, 543, 544, 545, 546, 547, 548, 549, 550, 551, 552, 553, 554, 555] to explain the first single spin phenomena in SIDIS,

$A_{U L}^{\sin \phi}$ and $A_{L U}^{\sin \phi}$, these observables are still not understood. The theoretical challenge is that presently it is not understood how to control light-cone divergences in SIDIS at $1 / Q$ order [555]. This does not necessarily mean there is no factorization, but it indicates that possibly new techniques are needed to pave the way towards a factorization proof in SIDIS at twist-3. If one assumes twist-3 TMD factorization, the phenomenological challenge is that each twist-3 observable receives contributions from several unknown twist-3 TMDs or fragmentation functions [247]. The situation simplifies in semi-inclusive jet production, a promising process to study at EIC energies, which could provide valuable complementary information on twist-3 TMDs [556]. 
An important process which can provide independent information on twist-3 (and, of course, also twist-2) TMDs are interference functions [451, 478, 479, 480, 476, 557, 558]. The advantage of this approach is that here collinear factorization applies, i.e. one cannot access TMDs. However, those functions which "survive" the $k_{\perp}$-integration of the quark correlator can be studied, and this includes at the twist-3 $e^{a}(x), g_{T}^{a}(x), h_{L}^{a}(x)$. These functions contribute to observables in convolution with specific interference fragmentation functions, which can be inferred from azimuthal asymmetries in $e^{+} e^{-}$annihilations [485.

There is no doubt that experimental, phenomenological and theoretical efforts to go beyond twist-2 are worth. Twist-3 functions describe multiparton distributions corresponding to the interference of higher Fock components in the hadron wave functions, and as such have no probabilistic partonic interpretations. Yet they offer fascinating insights into the nucleon structure [559. The Mellin moment $\int \mathrm{d} x x^{2} \tilde{g}_{T}^{a}(x)$ of the pure twist-3 piece in $g_{T}^{a}$ describes the transverse impulse the active quark acquires after being struck by the virtual photon due to the color Lorentz force. The Mellin moment $\int \mathrm{d} x x^{2} \tilde{e}^{a}(x)$ of the pure twist-3 piece in $e^{a}(x)$ describes the average transverse force acting on a transversely polarized quark in an unpolarized target after interaction with the virtual photon.

Twist-3 TMDs are closely related to projections of different combinations of the collinear twist-3 correlation functions $G_{F}(x, x \prime)$ and $\tilde{G}_{F}(x, x \prime)$ discussed in Sec. 2.2.4, which are involved in the evolution equations of twist-3 collinear PDFs [560, 561, 562, 563, 564, 565, 566], and play important roles also in derivations of the evolution equations for transverse moments of TMDs [290, 291, 390, 391, 392, calculations of processes at high transverse momentum [352, or calculations of the high transverse momentum tails of TMDs [292, 295]. Ultimately, through a global study of all of these observables, one could simultaneously obtain better knowledge of twist-3 collinear functions and twist-2 TMDs, and at the same time test the validity of the formalism. Gathering as much information as one can on the quark-gluon-quark correlator is essential to reach this goal. 


\section{Chapter 3}

\section{Three-dimensional structure of the proton and nuclei: spatial imaging}

Convenors and chapter editors:

M. Burkardt, V. Guzey, F. Sabatié 


\title{
3.1 Spatial imaging of sea quarks and gluons: summary
}

\author{
V. Guzey, F. Sabatié, M. Burkardt
}

The internal landscape of the nucleon and nuclei in terms of the fundamental quarks and gluons can be studied in different hard processes and can be characterized by different quantities (distributions). Hard exclusive reactions such as deeply virtual Compton scattering (DVCS) and exclusive production of mesons give an access to the aspects of the hadron structure that are encoded in generalized parton distributions (GPDs) and dipole amplitudes.

GPDs generalize the well-known form factors, distribution amplitudes and parton distributions and quantify various correlations/distributions of quarks and gluons in terms of their momentum fractions and positions in the transverse plane. Thus, GPDs provide a rigorous framework for studies of the three-dimensional parton structure of hadrons as well as many additional important aspects of the hadron structure such as the parton angular momentum and the related "spin puzzle", spin and flavor content, the role of chiral symmetry, and many more.

At the moment, our knowledge about GPDs is mostly limited to valence quark GPDs (Hermes, Compass, Jefferson Lab $6 \mathrm{GeV}$ and also Jefferson Lab $12 \mathrm{GeV}$ in the near future) and rather low precision data from HERA. A high-energy high-luminosity Electron-Ion Collider (EIC) will be an ideal machine for the studies of hard exclusive reactions and sea quark and gluon GPDs as summarised in table 3.1 .

\begin{tabular}{|c|c|c|c|}
\hline Deliverables & Observables & What we learn & Requirements \\
\hline $\begin{array}{l}\text { sea quark and } \\
\text { gluon GPDs }\end{array}$ & $\begin{array}{l}\text { DVCS and } J / \psi, \rho, \phi \\
\text { production cross sect. } \\
\text { and asymmetries }\end{array}$ & $\begin{array}{l}\text { transverse images of } \\
\text { sea quarks and gluons } \\
\text { in nucleon and nuclei; } \\
\text { total angular momentum; } \\
\text { onset of saturation }\end{array}$ & $\begin{array}{c}\mathcal{L} \geq 10^{34} \mathrm{~cm}^{-2} \mathrm{~s}^{-1}, \\
\quad \text { Roman Pots } \\
\text { wide range of } x_{B} \text { and } Q^{2} \\
\text { polarized } e^{-} \text {and } p \text { beams } \\
e^{+} \text {beam for DVCS }\end{array}$ \\
\hline $\begin{array}{c}\text { sea and valence } \\
\text { quark GPDs }\end{array}$ & $\begin{array}{l}\text { cross sections for } \\
\pi^{+}, K, K^{*}, \rho^{+} \\
\text {electroproduction }\end{array}$ & $\begin{array}{l}\text { flavor decomposition and } \\
\text { polarization of quarks } \\
\text { in the transverse plane }\end{array}$ & $\begin{array}{c}\mathcal{L} \geq 10^{34} \mathrm{~cm}^{-2} \mathrm{~s}^{-1} \\
\text { Roman Pots } \\
\text { high } Q^{2} \\
\text { range of beam energies } \\
\text { for } \sigma_{L} / \sigma_{T} \text { separation } \\
\end{array}$ \\
\hline
\end{tabular}

Table 3.1. Science Matrix for Exclusive Processes at EIC.

(i) One essential aspect of the GPD program is obtaining the transverse image of quarks and gluons in the nucleon/nucleus through the measurement of the $t$ dependence of cross sections of various exclusive processes (DVCS, production of $J / \psi, \phi, \pi, K$, etc. mesons) in a wide range of $t$. In the nucleon case, covering the interval $0 \approx|t| \leq 2 \mathrm{GeV}^{2}$ will enable one to map out the parton distributions in the transverse plane of the impact parameter $b$ down to as low as $b \approx 0.1 \mathrm{fm}$.

(ii) One area where an EIC shines is the large range in $Q^{2}$ available in the full $x_{B}$ interval. QCD evolution equations of GPDs, similarly to the PDF case, allow one to globally fit the data using flexible parameterizations of GPDs and to extract accurate and model- 
independent information on GPDs. One also will use the large lever arm in $Q^{2}$ to establish the reaction mechanisms (scaling properties, higher twist effects).

(iii) Another clear advantage of an EIC is the availability of different polarizations for the lepton and proton beams that allows one to fully disentangle the various GPDs from the experimental observables. While DVCS is sensitive to singlet quark and gluon GPDs, other exclusive diffractive processes (electroproduction of $\rho, J / \psi, \phi$, etc.) and non-diffractive processes (electroproduction of $\pi^{+}, K^{+}$, etc.) will allow one to access the spin and flavor dependences of GPDs. Note that the non-diffractive processes push the requirements for high luminosity much further than DVCS or other diffractive processes.

(iv) Exclusive processes with nuclei in a collider and, subsequently, the spatial image of sea quarks and gluons in nuclei will be studied for the first time. All the processes mentioned above will benefit from the high luminosity of an EIC (of the order of $10^{34} \mathrm{~cm}^{-2} \mathrm{~s}^{-1}$ ) as well as excellent detection capabilities and particle identification guaranteeing exclusivity.

The contributions below describe in detail various aspects of the rich program of spatial imaging of sea quarks and gluons at an EIC. In conclusion, a high-energy high-luminosity EIC, studying various deep exclusive processes through cross sections and polarization observables, would uniquely extend and complement our knowledge of the 3D partonic structure of the nucleon/nucleus to the sea of quarks and gluons. 


\title{
3.2 Basics of generalized parton distributions
}

\author{
Anatoly Radyushkin
}

\subsubsection{Introduction}

The fundamental physics to be accessed via the generalized parton distributions (GPDs) [274, 567, 568, 569, 570, 571, 572] is the structure of hadrons. This is a rather general statement, and we may want to have a more specific one. A classic example of such a specific case is the search for the Higgs boson (HB) performed currently at the Large Hadron Collider (LHC). The motivation for the search is that HB is supposed to be responsible for generation of masses, in particular, quark masses. However, by far, the largest part of visible mass is due to the nucleons, and out of $940 \mathrm{MeV}$ of the nucleon mass, less than 30 $\mathrm{MeV}$ (current quark masses) may be related to HB. The remaining $97 \%$ of the nucleon mass is due to gluons - which are massless! This is a characteristic illustration of the situation in hadron physics:

i) All the relevant particles are already established, i.e., no "higgses" to find.

ii) The QCD Lagrangian is known.

iii) However, we still need to understand how QCD works, i.e., to understand hadronic structure in terms of quark and gluon fields.

Projecting quark and gluon fields $q\left(z_{1}\right), q\left(z_{2}\right), \ldots$ onto hadronic states $|p, s\rangle$ gives matrix elements:

$$
\left\langle 0\left|\bar{q}_{\alpha}\left(z_{1}\right) q_{\beta}\left(z_{2}\right)\right| M(p), s\right\rangle \quad, \quad\left\langle 0\left|q_{\alpha}\left(z_{1}\right) q_{\beta}\left(z_{2}\right) q_{\gamma}\left(z_{3}\right)\right| B(p), s\right\rangle
$$

that can be interpreted as hadronic wave functions. In particular, in the light-cone (LC) formalism [573, a hadron is described by its Fock components in the infinite-momentum frame. For the nucleon, one can schematically write:

$$
|P\rangle=\Psi_{q q q}\left|q\left(x_{1} P, k_{1 \perp}\right) q\left(x_{2} P, k_{2 \perp}\right) q\left(x_{3} P, k_{3 \perp}\right)\right\rangle+\Psi_{q q q G}|q q q G\rangle+\Psi_{q q q \bar{q} q}|q q q \bar{q} q\rangle+\ldots,
$$

where $x_{i}$ are momentum fractions satisfying $\sum_{i} x_{i}=1 ; k_{i \perp}$ are transverse momenta, $\sum_{i} k_{i \perp}=0 ; \Psi$ are light-cone wave functions. In principle, solving the bound-state equation $H|P\rangle=E|P\rangle$ one should get the wave function $|P\rangle$ that contains complete information about the hadron structure. In practice, however, the equation (involving an infinite number of Fock components) has not been solved yet in the realistic 4-dimensional case. Moreover, the LC wave functions are not directly accessible experimentally.

The way out of this situation is the description of hadron structure in terms of phenomenological functions. Among the "old" functions used for a long time we can list form factors, usual parton densities, and distribution amplitudes. The "new" functions, generalized parton distributions (for reviews, see [574, 575, 576, 577]), are hybrids of form factors, parton densities and distribution amplitudes. Furthermore, the "old" functions are limiting cases of the "new" ones.

\subsubsection{Form factors}

The form factors are defined through matrix elements of electromagnetic (EM) and weak currents between hadronic states. In particular, the nucleon electromagnetic form factors are given by

$$
\left\langle p^{\prime}, s^{\prime}\left|J^{\mu}(0)\right| p, s\right\rangle=\bar{u}\left(p^{\prime}, s^{\prime}\right)\left[\gamma^{\mu} F_{1}(t)+\frac{r^{\nu} \sigma^{\mu \nu}}{2 m_{N}} F_{2}(t)\right] u(p, s),
$$


where $r=p-p^{\prime}$ is the momentum transfer and $t=r^{2}$. The electromagnetic current is given by the sum of its flavor components:

$$
J^{\mu}(z)=\sum_{f} e_{f} \bar{\psi}_{f}(z) \gamma^{\mu} \psi_{f}(z)
$$

The nucleon helicity non-flip form factor $F_{1}(t)$ can also be written as a sum $\sum_{f} e_{f} F_{1 f}(t)$. A similar decomposition holds for the helicity flip form factor $F_{2}(t)=\sum_{f} e_{f} F_{2 f}(t)$. At $t=0$, these functions have well known limiting values. In particular, $F_{1}(t=0)=e_{N}=\sum_{f} N_{f} e_{f}$ gives total electric charge of the nucleon $\left(N_{f}\right.$ is the number of valence quarks of flavor $f$ ) and $F_{2}(t=0)=\kappa_{N}$ gives its anomalous magnetic moment. The form factors are measurable through elastic $e N$ scattering.

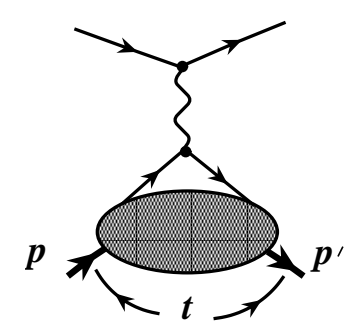

Figure 3.1. Elastic $e N$ scattering in the one-photon exchange approximation.

\subsubsection{Usual parton densities}

The parton densities are defined through forward matrix elements of quark/gluon fields separated by light-like distances. In particular, in the unpolarized case we have

$$
\left.\left\langle p\left|\bar{\psi}_{a}(-z / 2) \gamma^{\mu} \psi_{a}(z / 2)\right| p\right\rangle\right|_{z^{2}=0}=2 p^{\mu} \int_{0}^{1}\left[e^{-i x(p z)} f_{a}(x)-e^{i x(p z)} f_{\bar{a}}(x)\right] d x .
$$

In the local limit $z=0$, the operators in this definition coincide with the operators contributing into the non-flip form factor $F_{1}$. Since $t=0$ for the forward matrix element, we obtain the sum rule for the numbers of valence quarks:

$$
\int_{0}^{1}\left[f_{a}(x)-f_{\bar{a}}(x)\right] d x=N_{a}
$$

The definition of parton densities has the form of the plane wave decomposition. This observation allows one to give the momentum space interpretation: $f_{a(\bar{a})}(x)$ is the probability to find $a(\bar{a})$-quark with momentum $x p$ inside a nucleon with momentum $p$. The classic process to access the usual parton densities is deep inelastic scattering (DIS) $\gamma^{*} N \rightarrow X$.

Using the optical theorem, the $\gamma^{*} N \rightarrow X$ cross section is given by the imaginary part of the forward virtual Compton scattering amplitude. The momentum transfer $q$ is spacelike $q^{2} \equiv-Q^{2}$, and when it is sufficiently large, perturbative QCD factorization works. At the leading order, one deals with the so-called handbag diagram, see figure 3.2 .

Through simple algebra, $\frac{1}{\pi} \operatorname{Im} 1 /(q+x p)^{2} \approx \delta\left(x-x_{B}\right) / 2(p q)$, one finds that DIS measures parton densities at the point $x=x_{B}$, where the parton momentum fraction equals the Bjorken variable $x_{B}=Q^{2} / 2(p q)$. Comparing parton densities to form factors, we note that the latter have a point vertex instead of a light-like separation and $p \neq p^{\prime}$. 


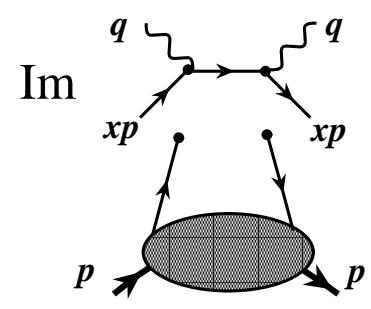

Figure 3.2. Lowest order pQCD factorization for DIS.

\subsubsection{Nonforward parton densities}

"Hybridization" of different parton distributions is the key idea of the GPD approach. As the first step, we can combine form factors with parton densities [578] and write the flavor components $F_{1 a}(t)$ of form factors as integrals over the momentum fraction variable $x$ :

$$
F_{1 a}(t)=\int_{0}^{1}\left[\mathcal{F}_{a}(x, t)-\mathcal{F}_{\bar{a}}(x, t)\right] d x .
$$

In the forward limit $t=0$, the new objects-nonforward parton densities $\mathcal{F}_{a(\bar{a})}(x, t)$ (NPDs) - coincide with the usual ("forward") densities:

$$
\mathcal{F}_{a(\bar{a})}(x, t=0)=f_{a(\bar{a})}(x) .
$$

NPDs can be also treated as Fourier transforms of the impact parameter $b_{\perp}$ distributions $f\left(x, b_{\perp}\right)$ describing the variation of parton densities in the transverse plane [579, 580].

A nontrivial question is the interplay between $x$ and $t$ dependencies of $\mathcal{F}_{a(\bar{a})}(x, t)$. The simplest factorized ansatz $\mathcal{F}_{a}(x, t)=f_{a}(x) F_{1}(t)$ satisfies both the forward constraint, $\mathcal{F}_{a}(x, t=0)=f_{a}(x)$, and also the local constraint (3.7). The reality may be more complicated: light-cone wave functions with Gaussian $k_{\perp}$ dependence

$$
\Psi\left(x_{i}, k_{i \perp}\right) \sim \exp \left[-\frac{1}{\lambda^{2}} \sum_{i} k_{i \perp}^{2} / x_{i}\right]
$$

suggest that

$$
\mathcal{F}^{a}(x, t)=f_{a}(x) e^{\bar{x} t / 2 x \lambda^{2}},
$$

where $\bar{x} \equiv 1-x$. Taking $f_{a}(x)$ from existing parametrizations and adjusting $\lambda^{2}$ to provide the standard value of the quark intrinsic transverse momentum $\left\langle k_{\perp}^{2}\right\rangle \approx(300 \mathrm{MeV})^{2}$ gives a rather reasonable description of the proton form factor $F_{1}(t)$ in a wide range of momentum transfers $-t \sim 1-10 \mathrm{GeV}^{2}$ [578. To comply with the Regge behavior, one may wish to change $e^{\bar{x} t / 2 x \lambda^{2}} \rightarrow x^{-\alpha^{\prime} t}$, where $\alpha^{\prime}$ is the Regge trajectory slope. The modified Regge ansatz,

$$
\mathcal{F}^{a}(x, t)=f_{a}(x) x^{-\alpha^{\prime}(1-x) t},
$$

allows one to easily fit electromagnetic form factors for the proton and neutron [581]. A similar model was proposed in Ref. [582.

The same nonforward parton densities appear in the handbag diagrams for the wideangle real Compton scattering, see figure 3.3 . 


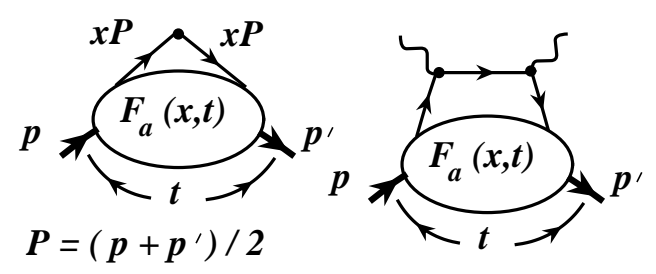

Figure 3.3. Form factor and wide-angle Compton scattering amplitude in terms of nonforward parton densities.

The handbag contribution is approximately given by the product of a new form factor, $R_{V}^{a}(t)$, and the cross section of the Compton scattering off an elementary fermion (given by Klein-Nishina expression):

$$
\frac{d \sigma}{d t}=\left.\left[\sum_{a} e_{a}^{2} R_{V}^{a}(t)\right]^{2} \frac{d \sigma}{d t}\right|_{K N} \quad \text { with } \quad R_{V}^{a}(t)=\int_{0}^{1} \frac{\mathcal{F}^{a}(x, t)}{x} d x .
$$

The predictions based on handbag dominance and NPDs [578, 583] are in much better agreement with the existing data [584] than the predictions based on two-gluon hard exchange mechanism of asymptotic perturbative QCD: the predicted cross section is too small in the latter case. The absolute normalization for predictions is settled by the form of the nonperturbative functions (NPDs in the handbag approach and nucleon distribution amplitudes in the pQCD approach) which were fixed by fitting the $F_{1}$ form factor data. Still, when there is an uncertain overall factor, it is risky to make strong statements. Remarkably, the perturbative QCD hard scattering mechanism and soft handbag mechanism give drastically different predictions for the polarization asymmetry $A_{L L}$ [583. Experiment E-99-114 performed at Jefferson Lab [584] strongly favors handbag mechanism that predicts the value close to the asymmetry for the scattering on a single quark.

\subsubsection{Distribution amplitudes}

Another example of nonperturbative functions describing the hadron structure are the distribution amplitudes (DAs). They can be interpreted as light cone wave functions integrated over transverse momentum, or as $\langle 0|\ldots| p\rangle$ matrix elements of light cone operators. In the case of the pion, we have

$$
\left.\left\langle 0\left|\bar{\psi}_{d}(-z / 2) \gamma_{5} \gamma^{\mu} \psi_{u}(z / 2)\right| \pi^{+}(p)\right\rangle\right|_{z^{2}=0}=i p^{\mu} f_{\pi} \int_{-1}^{1} e^{-i \alpha(p z) / 2} \varphi_{\pi}(\alpha) d \alpha,
$$

with $x_{1}=(1+\alpha) / 2, x_{2}=(1-\alpha) / 2$ being the fractions of the pion momentum carried by the quarks. The distribution amplitudes describe the hadrons in situations when the pQCD hard scattering approach is applicable to exclusive processes. The classic example is the $\gamma^{*} \gamma \rightarrow \pi^{0}$ transition; its amplitude is proportional to the $1 /\left(1-\alpha^{2}\right)$ moment of $\varphi_{\pi}(\alpha)$, see figure 3.4, left. The predictions for the $\gamma^{*} \gamma \rightarrow \pi^{0}$ form factor based on two competing models for the pion DA, the asymptotic $\varphi_{\pi}^{\text {as }}(\alpha)=\frac{3}{4}\left(1-\alpha^{2}\right)$ and Chernyak-Zhitnitsky DA $\varphi_{\pi}^{\mathrm{CZ}}(\alpha)=\frac{15}{4} \alpha^{2}\left(1-\alpha^{2}\right)$ differ by factor of $5 / 3$, and the hope was that this difference would allow for an experimental discrimination between them. Indeed, the comparison with CLEO and CELLO data for $Q^{2} F_{\gamma^{*} \gamma \pi^{0}}\left(Q^{2}\right)$ that extend to $Q^{2} \lesssim 10 \mathrm{GeV}^{2}$ favors DAs that are closer 
to $\varphi^{\text {as }}(\alpha)$. However, recent BABAR data covering the range up to $Q^{2} \sim 40 \mathrm{GeV}^{2}$ show the increase of $Q^{2} F_{\gamma^{*} \gamma \pi^{0}}\left(Q^{2}\right)$ for $Q^{2} \gtrsim 10 \mathrm{GeV}^{2}$. To explain this increase, the scenarios were proposed in which the pion DA does not vanish at the end-points, e.g., $\varphi_{\pi}^{\text {flat }}(\alpha)=1$.

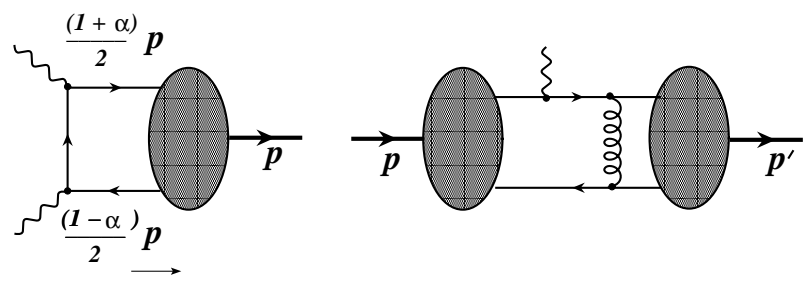

Figure 3.4. Lowest-order pQCD factorization for $\gamma^{*} \gamma \rightarrow \pi^{0}$ transition amplitude and for the pion electromagnetic form factor.

Another classic application of pQCD to exclusive processes is the pion electromagnetic form factor, see figure 3.4, right. With the asymptotic pion DA $\varphi_{\pi}^{\text {as }}(\alpha)$, the hard pQCD contribution to $F_{\pi}\left(Q^{2}\right)$ is $\left(2 \alpha_{s} / \pi\right)\left(0.7 \mathrm{GeV}^{2}\right) / Q^{2}$, which is less than $1 / 3$ of the experimental value. Taking wider DAs formally increases the size of the one-gluon-exchange contribution, but it is dominated then by the regions where the gluon virtuality is too small to be treated perturbatively. So, in this case we deal with the dominance of the competing soft mechanism which is described by nonforward parton densities, exactly in the same way as the proton form factor $F_{1}^{p}(t)$ discussed in the previous section.

\subsubsection{Hard electroproduction processes}

An attempt to use perturbative QCD to extract new information about hadronic structure is the study of deep exclusive photon [274] or meson [569, 572] electroproduction reactions. In the hard kinematics when both $Q^{2}$ and $s \equiv(p+q)^{2}$ are large while the momentum transfer $t \equiv\left(p-p^{\prime}\right)^{2}$ is small, one can use pQCD factorization which represents the amplitudes as a convolution of a perturbatively calculable short-distance amplitude and nonperturbative parton functions describing the hadron structure. The hard pQCD subprocesses in these two cases have different structure, see figure 3.5. Since the photon is a pointlike particle, the deeply virtual Compton scattering (DVCS) amplitude has the structure similar to that of the $\gamma^{*} \gamma \pi^{0}$ form factor: the pQCD hard term is of zero order in $\alpha_{s}$ (the handbag mechanism), and there is no competing soft contribution. Thus, we can expect that pQCD works from $Q^{2} \sim 2 \mathrm{GeV}^{2}$. On the other hand, the deeply virtual meson production process is similar to the pion EM form factor: the hard term has a $O\left(\alpha_{s} / \pi\right) \sim 0.1$ suppression factor. As a result, the dominance of the hard pQCD term may be postponed to $Q^{2} \sim 5-10 \mathrm{GeV}^{2}$.
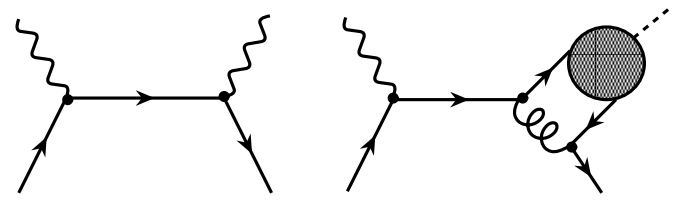

Figure 3.5. Lowest-order factorization for deeply virtual photon and meson production. 
One should also have in mind that the competing soft mechanism can mimic the same power-law $Q^{2}$-behavior (just like in case of pion and nucleon EM form factors). Hence, a mere observation of a "right" power-law behavior of the cross section may be insufficient to claim that pQCD is already working. One should look at other characteristics of the reaction, especially its spin properties, to make strong statements about the reaction mechanism.

\subsubsection{Deeply virtual Compton scattering and generalized parton distri- butions}

It is convenient to visualize DVCS in the $\gamma^{*} N$ center-of-mass frame, with the initial hadron and the virtual photon moving in opposite directions along the $z$-axis. Since the momentum transfer $t$ is small, the hadron and the real photon in the final state also move close to the $z$-axis. This means that the virtual photon momentum $q=q^{\prime}-x_{B} p$ has the component $-x_{B} p$ canceled by the momentum transfer $r$. In other words, the momentum transfer $r$ has the longitudinal component $r^{+}=x_{B} p^{+}$, where $x_{B}=Q^{2} / 2(p q)$ is the DIS Bjorken variable. One can say that DVCS has a skewed kinematics in which the final hadron has the "plus" momentum $(1-\zeta) p^{+}$that is smaller than that of the initial hadron. In the particular case of DVCS, we have $\zeta=x_{B}$.

The parton picture for DVCS has some similarity to that of DIS, with the main difference that the plus-momenta of the incoming and outgoing quarks in DVCS are not equal; they are $X p^{+}$and $(X-\zeta) p^{+}$, see figure 3.6. Another difference is that the invariant momentum transfer $t$ in DVCS is nonzero: the matrix element of partonic fields is essentially nonforward.

Thus, the nonforward parton distributions (NFPDs) $\mathcal{F}_{\zeta}(X, t)$ describing the hadronic structure in DVCS depend on $X$ (the fraction of $p^{+}$carried by the outgoing quark), $\zeta$ (the skewness parameter characterizing the difference between initial and final hadron momenta), and $t$ (the invariant momentum transfer). In the forward $r=0$ limit, we have a reduction formula

$$
\mathcal{F}_{\zeta=0}^{a}(X, t=0)=f_{a}(X)
$$

relating NFPDs with the usual parton densities. The nontriviality of this relation is that $\mathcal{F}_{\zeta}(X, t)$ appear in the amplitude of the exclusive DVCS process, while the usual parton densities are measured from the cross section of the inclusive DIS reaction.

Another limit for NFPDs is zero skewness $\zeta=0$, where they correspond to nonforward parton densities: $\mathcal{F}_{\zeta=0}^{a}(X, t)=\mathcal{F}^{a}(X, t)$. The local limit relates NFPDs to form factors:

$$
\int_{0}^{1} \mathcal{F}_{\zeta}^{a}(X, t) \frac{d X}{1-\zeta / 2}=F_{1}^{a}(t)
$$

The description in terms of NFPDs has the advantage of using the variables most close to those of the usual parton densities. However, the initial and final hadron momenta are not treated symmetrically in this scheme. Ji [274] proposed to use symmetric variables in which the plus-momenta of the hadrons are $(1+\xi) P^{+}$and $(1-\xi) P^{+}$, and those of the active partons are $(x+\xi) P^{+}$and $(x-\xi) P^{+}, P$ being the average momentum $P=\left(p+p^{\prime}\right) / 2$, see figure 3.6. In the simplified case of scalar fields, the GPD parametrization of the nonforward matrix element is

$$
\langle P+r / 2|\psi(-z / 2) \psi(z / 2)| P-r / 2\rangle=\int_{-1}^{1} e^{-i x(P z)} H(x, \xi) d x+\mathcal{O}\left(z^{2}\right) .
$$



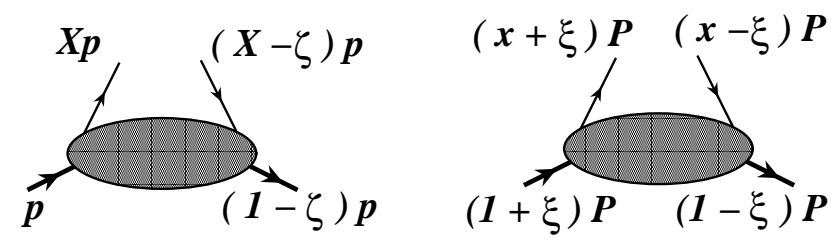

Figure 3.6. Comparison of NFPDs and OFPDs.

To take into account the spin properties of hadrons and quarks, one needs four offforward parton distributions $H, E, \tilde{H}, \widetilde{E}$, each of which is a function of $x, \xi$, and $t$. The skewness parameter $\xi \equiv r^{+} / 2 P^{+}$can be expressed in terms of the Bjorken variable, $\xi=$ $x_{B} /\left(2-x_{B}\right)$, but it does not coincide with it.

Depending on the value of $x$, each GPD has 3 distinct regions. When $\xi<x<1$, GPDs are analogous to usual quark distributions; when $-1<x<-\xi$, they are similar to antiquark distributions. In the region $-\xi<x<\xi$, the "returning" quark has a negative momentum and should be treated as an outgoing antiquark with momentum $(\xi-x) P$. The total $q \bar{q}$ pair momentum $r=2 \xi P$ is shared by the quarks in fractions $r(1+x / \xi) / 2$ and $r(1-x / \xi) / 2$. Hence, a GPD in the region $-\xi<x<\xi$ is similar to a distribution amplitude $\Phi(\alpha)$ with $\alpha=x / \xi$.

In the local limit, GPDs reduce to elastic form factors:

$$
\sum_{a} e_{a} \int_{-1}^{1} H^{a}(x, \xi ; t) d x=F_{1}(t) \quad, \quad \sum_{a} e_{a} \int_{-1}^{1} E^{a}(x, \xi ; t) d x=F_{2}(t) .
$$

The $E$ function, like $F_{2}(t)$, comes with the $r_{\mu}$ factor. Hence, it is invisible in DIS described by the forward $r=0$ Compton amplitude. However, the $t=0, \xi=0$ limit of $E$ exists:

$$
E^{a, \bar{a}}(x, \xi=0 ; t=0) \equiv \kappa^{a, \bar{a}}(x) .
$$

In particular, its integral gives the proton anomalous magnetic moment $\kappa_{p}$,

$$
\sum_{a} e_{a} \int_{-0}^{1}\left(\kappa^{a}(x)-\kappa^{\bar{a}}(x)\right) d x=\kappa_{p}
$$

while its first moment enters Ji's sum rule for the total quark contribution $J_{q}$ to the proton spin:

$$
J_{q}=\frac{1}{2} \sum_{a} \int_{-0}^{1} x\left[f^{a}(x)+f^{\bar{a}}(x)+\kappa^{a}(x)+\kappa^{\bar{a}}(x)\right] d x .
$$

Note that only valence quarks contribute to $\kappa_{p}$, while $J_{q}$ involves also sea quarks. Furthermore, the values of $\kappa_{p, n}$ (unlike $e_{p, n} \equiv F_{1}^{p, n}(0)$ ) strongly depend on dynamics, e.g., $\kappa_{N} \sim 1 / m_{q}$ in constituent quark models. 


\subsubsection{Double distributions}

To model GPDs, two approaches are used: a direct calculation in specific dynamical models: bag model, chiral soliton model, light-cone formalism, etc., and a phenomenological construction based on the relation of GPDs to usual parton densities $f_{a}(x), \Delta f_{a}(x)$ and form factors $F_{1}(t), F_{2}(t), G_{A}(t), G_{P}(t)$. The key question in the second approach is the interplay between $x, \xi$ and $t$ dependencies of GPDs. There are not so many cases in which the pattern of the interplay is evident. One example is the function $\widetilde{E}(x, \xi, t)$ which is related to the $G_{P}(t)$ form factor and is dominated for small $t$ by the pion pole term $1 /\left(t-m_{\pi}^{2}\right)$. It is also proportional to the pion distribution amplitude $\varphi_{\pi}(\alpha)$ taken at $\alpha=x / \xi$. The construction of self-consistent models for other GPDs can be performed using an ansatz based on the formalism of double distributions (DD) [585].

The main idea behind the double distributions is a "superposition" of $\mathrm{P}^{+}$and $r^{+}$momentum flows, i.e., the representation of the parton momentum $k^{+}=\beta P^{+}+(1+\alpha) r^{+} / 2$ as the sum of a component $\beta P^{+}$due to the average hadron momentum $P$ (flowing in the $s$-channel) and a component $(1+\alpha) r^{+} / 2$ due to the $t$-channel momentum $r$, see figure 3.7 In the simplified case of scalar fields, the DD parametrization reads

$$
\langle P-r / 2|\psi(-z / 2) \psi(z / 2)| P+r / 2\rangle=\int_{\Omega} F(\beta, \alpha) e^{-i \beta(P z)-i \alpha(r z) / 2} d \beta d \alpha+\mathcal{O}\left(z^{2}\right) .
$$

Thus, the double distribution $f(\beta, \alpha)$ (we consider here for simplicity the $t=0$ limit) looks like a usual parton density with respect to $\beta$ and like a distribution amplitude with respect to $\alpha$. The connection between the DD variables $\beta, \alpha$ and the GPD variables $x, \xi$ is obtained from $r^{+}=2 \xi P^{+}$, which results in the basic relation $x=\beta+\xi \alpha$. The formal connection between DDs and GPDs is

$$
H(x, \xi)=\int_{\Omega} F(\beta, \alpha) \delta(x-\beta-\xi \alpha) d \beta d \alpha .
$$

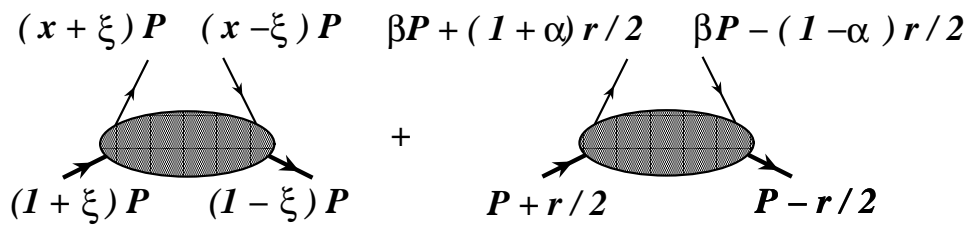

Figure 3.7. Comparison of GPD and DD descriptions.

The forward limit $\xi=0, t=0$ corresponds to $x=\beta$, and gives the relation between DDs and the usual parton densities:

$$
\int_{-1+|\beta|}^{1-|\beta|} F_{a}(\beta, \alpha ; t=0) d \alpha=f_{a}(\beta)
$$

The DDs live on the rhombus $|\alpha|+|\beta| \leq 1$ [denoted by $\Omega$ in (3.21) and (3.22)] and are symmetric functions of the "DA" variable $\alpha: f_{a}(\beta, \alpha ; t)=f_{a}(\beta,-\alpha ; t)$ ("Munich" symmetry [586]). These restrictions suggest a factorized representation for a DD in the form of 
a product of a usual parton density in the $\beta$-direction and a distribution amplitude in the $\alpha$-direction:

$$
F(\beta, \alpha)=f(\beta) h(\beta, \alpha), h_{N}(\beta, \alpha) \sim \frac{\left[(1-|\beta|)^{2}-\alpha^{2}\right]^{N}}{(1-|\beta|)^{2 N+1}}, \int_{-1+|\beta|}^{1-|\beta|} h(\beta, \alpha) d \alpha=1 .
$$

To obtain usual parton densities from DDs, one should integrate (scan) them over the vertical lines $\beta=x=$ const. To obtain the GPD $H(x, \xi)$ with nonzero $\xi$ from DDs $f(\beta, \alpha)$, one should integrate (scan) DDs along the parallel lines $\alpha=(x-\beta) / \xi$ with a $\xi$-dependent slope. One can call this process the DD-tomography. The basic feature of GPDs $H(x, \xi)$ resulting from DDs is that for $\xi=0$ they reduce to usual parton densities, and for $\xi=1$ they have a shape like a meson distribution amplitude. A more complete truth is that such a DD modeling misses terms invisible in the forward limit: meson-exchange contributions and so-called D-term, which can be interpreted as $\sigma$-exchange. The inclusion of the D-term induces nontrivial behavior in the central $|x|<\xi$ region (for details, see [587]).

\subsubsection{GPDs and the structure of hadrons}

Hadronic structure is a complicated subject, and it requires a study from many sides and in many different types of experiments. The description of specific aspects of hadronic structure is provided by several different functions: form factors, usual parton densities, distribution amplitudes. Generalized parton distributions provide a unified description: all these functions can be treated as particular or limiting cases of GPDs $H(x, \xi, t)$.

Usual parton densities $f(x)$ correspond to the case $\xi=0, t=0$. They describe a hadron in terms of probabilities $\sim|\Psi|^{2}$. However, QCD is a quantum theory: GPDs with $\xi \neq 0$ describe correlations $\sim \Psi_{1}^{*} \Psi_{2}$. Taking only the point $t=0$ corresponds to integration over impact parameters $b_{\perp}$ - information about the transverse structure is lost.

Form factors $F(t)$ contain information about the distribution of partons in the transverse plane, but $F(t)$ involve integration over momentum fraction $x$ - information about longitudinal structure is lost.

A simple "hybridization" of usual densities and form factors in terms of NPDs $\mathcal{F}(x, t)$ (GPDs with $\xi=0$ ) shows that the behavior of $F(t)$ is governed both by transverse and longitudinal distributions. GPDs provide adequate description of nonperturbative soft mechanism. They also allow to study transition from soft to hard mechanism.

Distribution amplitudes $\varphi(x)$ provide quantum-level information about the longitudinal structure of hadrons. In principle, they are accessible in exclusive processes at large momentum transfer, when hard scattering mechanism dominates. GPDs have DA-type structure in the central region $|x|<\xi$.

Generalized parton distributions $H(x, \xi, t)$ provide a 3-dimensional picture of hadrons. GPDs also provide some novel possibilities, such as "magnetic distributions" related to the spin-flip GPD $E(x, \xi, t)$. In particular, the structure of nonforward density $E(x, \xi=0, t)$ determines the $t$-dependence of $F_{2}(t)$. Recent JLab data give $F_{2}(t) / F_{1}(t) \sim 1 / \sqrt{-t}$ rather than $1 / t$ expected in hard pQCD and many models - a puzzle waiting to be resolved. The forward reductions $\kappa^{a}(x)$ of $E(x, \xi, t)$ look as fundamental as $f^{a}(x)$ and $\Delta f^{a}(x)$ : Ji's sum rule involves $\kappa^{a}(x)$ on equal footing with $f(x)$. Magnetic properties of hadrons are strongly sensitive to dynamics providing a testing ground for models. Another novel possibility is the study of flavor-nondiagonal distributions, e.g., proton-to-neutron GPDs accessible through processes like exclusive charged pion electroproduction, proton-to- $\Lambda$ GPDs (they appear in kaon electroproduction), and proton-to- $\Delta$ GPDs - these can be related to form factors of 
proton-to- $\Delta$ transition (another puzzle for hard pQCD). The GPDs for $N \rightarrow N+$ soft $\pi$ processes can be used for testing the soft pion theorems and physics of chiral symmetry breaking.

An interesting problem is the separation and flavor decomposition of GPDs. The DVCS amplitude involves all four types of GPDs, $H, E, \widetilde{H}, \widetilde{E}$, so we need to study other processes involving different combinations of GPDs. An important observation is that, in hard electroproduction of mesons, the spin nature of produced meson dictates the type of GPDs involved, e.g., for pion electroproduction, only $\widetilde{H}, \widetilde{E}$ appear, with $\widetilde{E}$ dominated by the pion pole at small $t$. This gives an access to (generalization of) polarized parton densities without polarizing the target.

In summary, the structure of hadrons is the fundamental physics to be accessed via GPDs. GPDs describe hadronic structure on the quark-gluon level and provide a threedimensional picture ("tomography") of the hadronic structure. GPDs adequately reflect the quantum-field nature of QCD (correlations, interference). They also provide new insights into spin structure of hadrons (spin-flip distributions, orbital angular momentum). GPDs are sensitive to chiral symmetry breaking effects, a fundamental property of QCD. Furthermore, GPDs unify existing ways of describing hadronic structure. The GPD formalism provides nontrivial relations between different exclusive reactions and also between exclusive and inclusive processes. 


\title{
3.3 GPDs and transverse nucleon structure at collider ener- gies
}

\author{
C. Weiss
}

Generalized parton distributions (GPDs) have emerged as a key concept in nucleon structure and the theory of high momentum-transfer processes in QCD. They unify the traditional notions of parton densities and elastic form factors and describe the transverse spatial distribution of quarks and gluons in a fast-moving hadron. A general introduction to GPDs and hard exclusive processes is given in section 3.2. Here we summarize the properties of GPDs at collider energies, where the parton picture can be combined with methods specific to high-energy scattering ("small- $x$ physics"). This includes the transverse spatial structure of the nucleon at small $x$; gluon and quark imaging with hard exclusive processes at ep colliders (HERA, EIC); the correspondence with the QCD dipole model and the role of transverse nucleon structure in saturation at small $x$; and the application of GPDs to high-energy $p p$ collisions with hard processes (Tevatron, LHC).

GPDs are defined as the transition matrix elements of the QCD twist-2 operators between nucleon states of different momenta. They are functions of the longitudinal momentum fractions of the partons, $x$ and $x^{\prime}$, and the invariant momentum transfer $t$, as well as the resolution scale $Q^{2}$ (see figure 3.8 a). Of particular interest is the "diagonal" limit $x=x^{\prime}$, where the momentum transfer is in the transverse direction only, $t=-|\boldsymbol{\Delta}|^{2}$, and the GPD can be regarded as the form factor of partons carrying longitudinal momentum fraction $x$. Its two-dimensional Fourier transform

$$
f\left(x, b, Q^{2}\right) \equiv \int \frac{d^{2} \Delta}{(2 \pi)^{2}} e^{-i(\boldsymbol{\Delta} \boldsymbol{b})} \operatorname{GPD}\left(x, t=-\boldsymbol{\Delta}^{2}, Q^{2}\right)
$$

describes the transverse spatial distribution of partons with momentum fraction $x$ and thus provides a "tomographic" image of the structure of the fast-moving nucleon (see figure 3.8b) [580. The coordinate $b$ measures the distance from the transverse center-of-mass (CM), defined as the average of the transverse positions of all constituents weighted with their longitudinal momentum fractions. In general, the removal of a parton with momentum fraction $x$ changes the position of the CM, and this effect must be taken into account in interpreting the coordinate distributions at $x \sim 1$. At $x \ll 1$ however, the contribution of the removed parton to the CM is negligible and one can think of the $b$-distributions of (3.25) as referring to a fixed transverse center of the nucleon. This considerably simplifies the spatial interpretation of GPDs at small $x$.

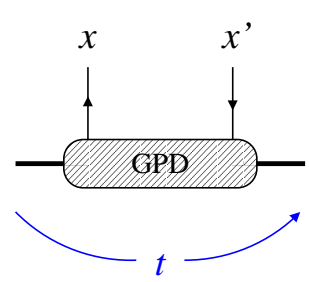

(a)

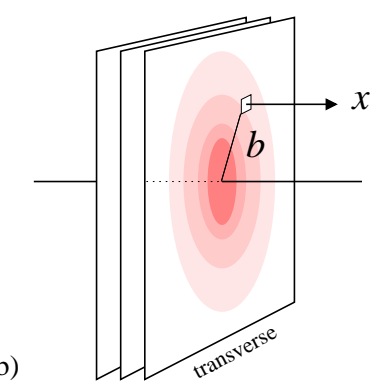

(b)

(c)

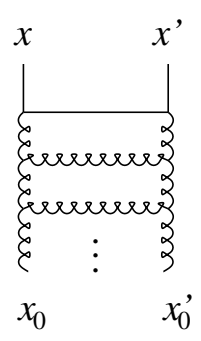

Figure 3.8. (a) GPD and partonic variables. (b) Transverse spatial distribution of partons. (c) QCD evolution generates small $x, x^{\prime}$ from the quasi-diagonal GPD at lower scale. 

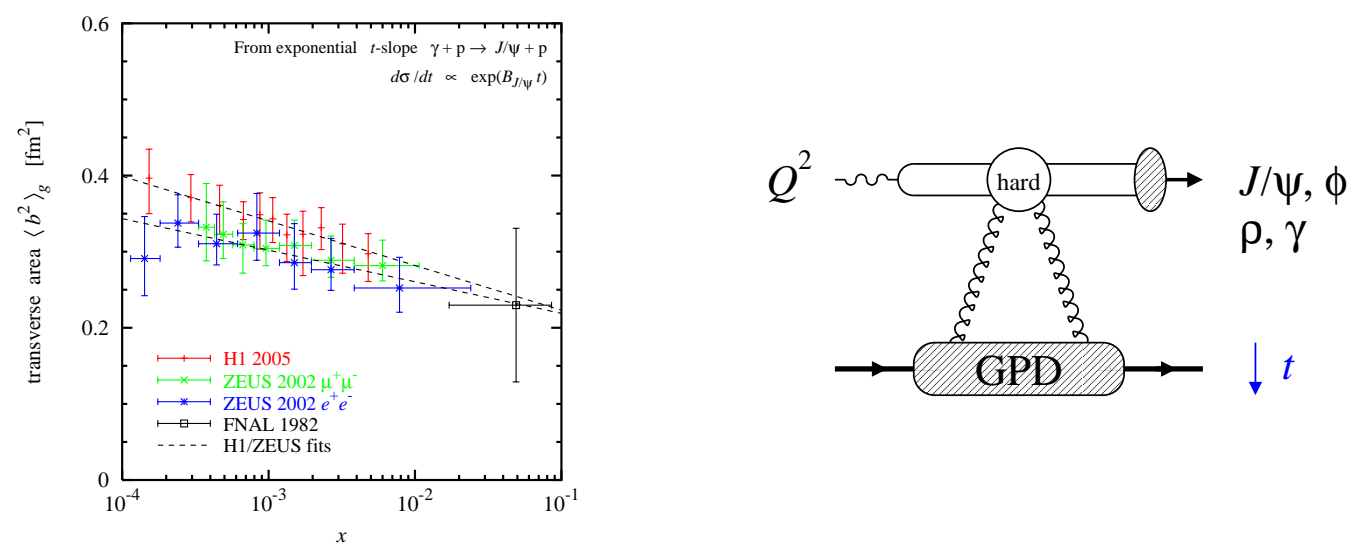

Figure 3.9. (left) Exclusive $J / \psi$ production as a probe of the gluon GPD. (right) Average transverse gluonic size of the nucleon $\left\langle b^{2}\right\rangle_{g}$ extracted from $J / \psi$ photoproduction at HERA [588, 589] and FNAL [590] (adapted from [591). The effective scale at which the GPD is probed is $Q_{\text {eff }}^{2} \approx 3 \mathrm{GeV}^{2}$.

Hard exclusive processes require a nonzero longitudinal momentum transfer to the nucleon and probe the GPDs at $x-x^{\prime} \equiv$ $2 \xi \neq 0$, where the "skewness" is related to the Bjorken variable by $\xi=x_{B} /\left(2-x_{B}\right)$. Models or additional assumptions are generally needed to extract the diagonal GPD from the data. However, at $x_{B} \ll 1$ and sufficiently large $Q^{2}$ the "skewed" GPD can approximately be reconstructed from the diagonal limit [592, 593. In this case QCD evolution generates the GPD with $x$ and $x^{\prime}$ from configurations at a lower scale with momentum fractions $x_{0}, x_{0}^{\prime} \gg x, x^{\prime}$; because the difference of the parton momentum fractions is preserved under evolution, the lower-scale GPD is effectively evaluated in the diagonal limit $x_{0}-x_{0}^{\prime} \ll x_{0}, x_{0}^{\prime}$ (see figure 3.8k). This approximation allows one to relate the measured $t$-dependence of the differential cross sections directly to the transverse structure of the nucleon at fixed $x$.

The transverse spatial distribution of partons changes with the momentum fraction $x$ and the scale $Q^{2}$. The valence quarks and gluons at $x>0.1$ are concentrated at small transverse distances $b \ll 1 \mathrm{fm}$, as can be inferred from the nucleon axial form factor and exclusive processes at large $x$. Be-

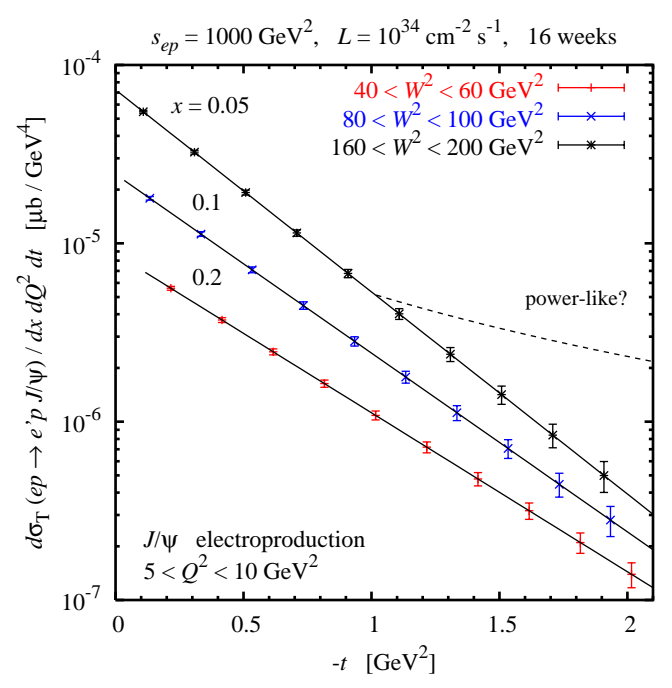

Figure 3.10. A simulated measurement of exclusive $J / \psi$ electro-production with a mediumenergy EIC for an integrated luminosity of 100 $\mathrm{fb}^{-1}$. The expected statistical errors in the $t$ dependence of the $J / \psi$ dilepton cross section in a fully differential measurement in $W, Q^{2}$ and $t$ are shown. The values of $x \equiv M_{J / \psi}^{2} / W^{2}$ in the bins are indicated above the curves, corresponding approximately to the $x$-values where the gluon GPD is probed. Such measurements can image the transverse distribution of gluons at $x>0.1$ and explore the unknown $t$-dependence at $|t|>1 \mathrm{GeV}^{2}$. 
low $x<M_{\pi} / M_{N}$ chiral dynamics gives rise to a distinct large-distance contribution to the parton density at $b \sim 2 / M_{\pi}$ [594]. At even smaller values of $x$ the nucleon's transverse size is expected to grow as a result of Gribov diffusion in the successive parton branchings building up the small- $x$ parton density. The transverse distribution also shrinks with increasing $Q^{2}$ as a result of DGLAP evolution [595]. Overall, much interesting information on nucleon structure and non-perturbative dynamics can be obtained from the study of the transverse spatial distributions of quarks and gluons.

The transverse spatial distribution of gluons can be measured cleanly through exclusive $J / \psi$ photo- or electroproduction $\gamma^{(*)} N \rightarrow J / \psi+N$, or electroproduction of $\phi$ mesons at $Q^{2} \gtrsim 10 \mathrm{GeV}^{2}$ (see figure 3.9a). Measurements at HERA have confirmed the applicability of QCD factorization, with corrections for the finite size of the produced meson, and tested the universality of the gluon GPD; see [596] for a review. The data show that the nucleon's transverse gluonic radius at $x<0.01$ is substantially smaller than the transverse charge radius (see figure $3.9 \mathrm{~b}$ ). It increases only moderately with decreasing $x$, with a logarithmic slope much smaller than that of the Pomeron trajectory, $\alpha_{P}^{\prime}=0.25 \mathrm{GeV}^{-2}$, showing that Gribov diffusion is suppressed for partons with virtualities $\sim$ few $\mathrm{GeV}^{2}$. Both observations are of central importance for nucleon structure and small- $x$ physics.

While the HERA experiments have provided basic information on the nucleon's transverse gluonic size at small $x$, many important questions remain unanswered:

- How are the gluons at $x>10^{-2}$ distributed in transverse space? Global PDF fits indicate a substantial momentum density of gluons in that $x$-range at low scales $Q^{2} \sim$ few $\mathrm{GeV}^{2}$. Knowledge of their spatial distribution would help to explain their dynamical origin, one of the key issues of nucleon structure in QCD.

- Do singlet quarks and gluons have the same transverse distribution? This can be studied by comparing the $t$-dependence of $J / \psi$ and $\phi$ with $\rho^{0}$ and $\gamma$ electroproduction. A larger radius for quarks than gluons is expected from non-perturbative effects [597].

- How are non-singlet sea quarks distributed in transverse space? The non-singlet sea at $x<0.1$ reveals non-perturbative QCD interactions (vacuum fluctuations, mesonic degrees of freedom) in the nucleon. This component is probed in exclusive $\pi, K, \rho^{+}$ or $K^{*}$ production - non-diffractive processes involving quantum number exchange.

- How does the nucleon's gluon GPD behave at $|t| \sim$ few $\mathrm{GeV}^{2}$ ? The large- $|t|$ behavior of GPDs is important not only to obtain accurate images at small $b$, but also to understand how soft Regge-like dynamics is connected to QCD at short distances.

- What is the probability for a nucleon to break up into a low-mass hadronic state $\left(M_{H} \sim\right.$ few $\left.\mathrm{GeV}\right)$ in an exclusive process at small $x_{B}$ ? Such "diffractive dissociation" reveals the quantum fluctuations of the nucleon's gluon density - new information going beyond the average densities described by the GPDs [598.

An EIC would enable a comprehensive program of transverse imaging of gluons and sea quarks in the nucleon. Measurements of $J / \psi$ photo- and electroproduction, as well as $\phi$ meson electroproduction at $Q^{2}>10 \mathrm{GeV}^{2}$, would cleanly map the transverse distribution of gluons, including the gluons at $x>0.1$ (see the example in figure 3.10). They could also explore the unknown $t$-dependence of the GPD at $|t|>1 \mathrm{GeV}^{2}$. Measurements of $\rho^{0}$ and $\gamma$ production (DVCS) would provide additional information on the singlet quarks. With a high-luminosity EIC, even the non-diffractive channels $\left(\pi, K, \rho^{+}, K^{*}\right)$ could be measured 

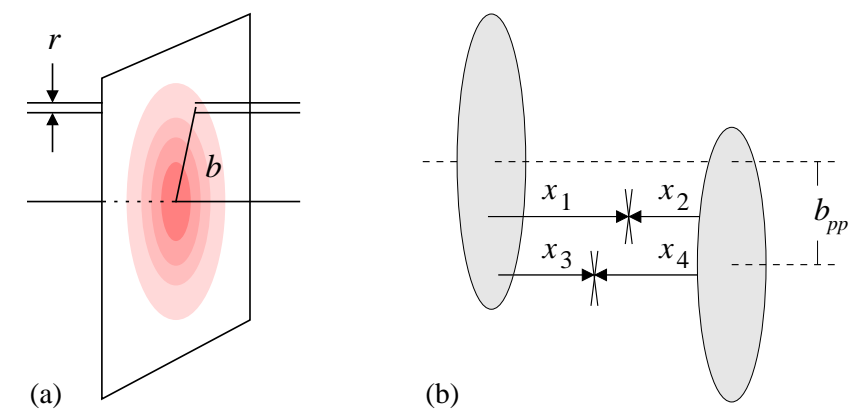

Figure 3.11. (a) Dipole picture of high-energy scattering in the target rest frame. (b) Multiparton processes in high-energy $p p$ collisions.

for the first time down to $x \sim 0.01$, providing detailed information on the spatial distribution of the non-singlet sea, including its spin and flavor composition (see section 3.12).

The QCD factorization theorem for hard exclusive processes at small $x$ (figure $3.9 \mathrm{a}$ ) is equivalent to the dipole picture of exclusive processes in the nucleon rest frame in the leading $\alpha_{s} \log Q^{2}$ approximation [599. The scattering amplitude for a dipole of size $r$ with impact parameter $b$ is proportional to the $b$-dependent gluon density of (3.25) at a scale $Q^{2} \approx \pi^{2} / r^{2}$ (see figure 3.3 $\mathrm{a}$ ). This correspondence relates GPDs to the dipole model phenomenology of small- $x$ physics [596]. In particular, the transverse spatial distribution of gluons is an essential input to studies of the unitarity limit in hard processes at small $x$ ("black-disk regime"). It defines the spatial profile of the initial conditions of non-linear QCD evolution equations leading to gluon saturation at small $x$. Detailed studies of saturation in the dipole model have used the transverse gluonic size extracted from the HERA data (see figure 3.9 b) 600, 601]; better knowledge of the transverse profile would help to accurately predict the $x$ and $b$-dependence of the saturation scale.

The transverse distribution of partons also plays an important role in high-energy $p p$ collisions with hard processes. It determines the probability of hard parton-parton processes as a function of the $p p$ impact parameter. Using knowledge of the transverse distribution of partons from $e p$ scattering one can explain many features of the underlying event in $p p$ collisions with hard processes [591]. In particular, one can predict the rate of multiparton processes (see figure $3.3 \mathrm{~b}$ ), which form a potentially large background to new physics events at the LHC. The enhancement of such processes beyond their geometric probability signals dynamical correlations between partons, the study of which represents a new frontier of nucleon structure. 


\title{
3.4 How large can the distributions $E^{q}$ and $E^{g}$ be?
}

\author{
Markus Diehl
}

\subsubsection{Positivity bounds}

The generalized parton distributions $E$ for quarks and gluons play a key role in the study of nucleon structure through exclusive processes. In the following I focus on the case of zero skewness, $\xi=0$, where the physics interpretation is most intuitive and where constraints on these distributions are most easily obtained. The density of unpolarized quarks in a proton polarized along the $x$-axis is given by

$$
q^{X}(x, \vec{b})=q\left(x, b^{2}\right)-\frac{b^{y}}{m} \frac{\partial}{\partial b^{2}} e_{q}\left(x, b^{2}\right),
$$

where $m$ is the proton mass. The quarks have impact parameter $\vec{b}$ and move in the $z$ direction with momentum fraction $x$. The term with

$$
e_{q}\left(x, b^{2}\right)=\int \frac{d^{2} \Delta}{(2 \pi)^{2}} e^{-i \vec{b} \vec{\Delta}} E^{q}\left(x, \xi=0, t=-\vec{\Delta}^{2}\right)
$$

quantifies the transverse shift of the density due to the proton polarization. The density interpretation of (3.26) (together with its analog for longitudinal quark and proton polarization) entails a positivity bound [602]:

$$
\frac{b^{2}}{m^{2}}\left[\frac{\partial}{\partial b^{2}} e_{q}\left(x, b^{2}\right)\right]^{2} \leq\left[q\left(x, b^{2}\right)+\Delta q\left(x, b^{2}\right)\right]\left[q\left(x, b^{2}\right)-\Delta q\left(x, b^{2}\right)\right] .
$$

The theoretical status of this bound is the same as for the positivity of unpolarized parton densities and for the Soffer inequality: they hold in the parton model and are preserved by leading-order DGLAP evolution to higher scales, but they can be violated by higher-order evolution effects or at very low scales. Since so little is known about $E$, I suggest to use (3.28) as a guide, with proper caution. A consequence of (3.28) is that $\left(\partial / \partial b^{2}\right) e_{q}$ must decrease faster with $b$ than $\sqrt{q^{2}-\Delta q^{2}}$. This has immediate consequences for parameterizations: using Gaussian forms $E^{q} \propto e^{B_{e} t}$ and $\sqrt{q^{2}-\Delta q^{2}} \propto e^{B_{q} t}$ for the momentum-space distributions at $\xi=0$, one must have $B_{e}<B_{q}$, and with power laws $E^{q} \propto\left(1-t / M_{e}^{2}\right)^{-3}$ and $\sqrt{q^{2}-\Delta q^{2}} \propto\left(1-t / M_{q}^{2}\right)^{-2}$, one must have $1 / M_{e}<1 / M_{q}$, with equality of the parameters not being allowed in either case. Starting from (3.28) one can also derive a bound 602 for the integrated distribution:

$$
e_{q}(x)=\int d^{2} b e_{q}\left(x, b^{2}\right)=E^{q}(x, \xi=0, t=0) .
$$

That bound constrains the large $x$ behavior of $e_{q}(x)$, but numerically turns out to be rather weak for $x$ below 0.5 , see e.g. 603.

Analogous definitions and bounds apply to antiquark and gluon distributions $e_{\bar{q}}$ and $e_{g}$.

\subsubsection{Sum rules}

An important constraint follows from the sum rule

$$
\kappa_{q}=\int_{0}^{1} d x\left[e_{q}(x)-e_{\bar{q}}(x)\right]
$$



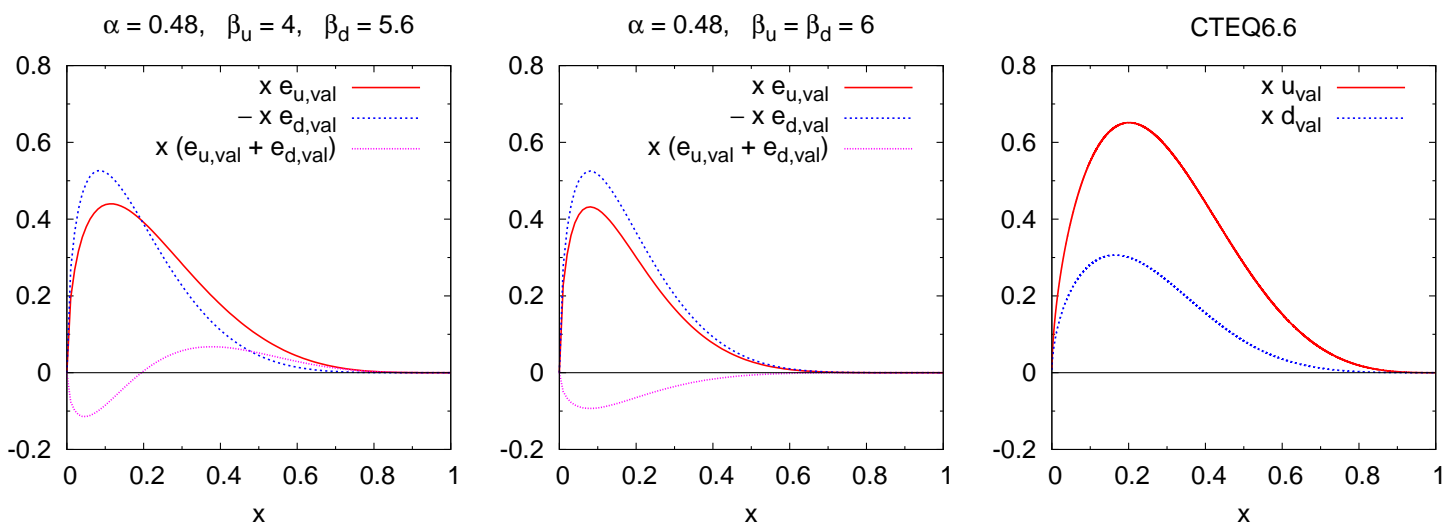

Figure 3.12. GPDs in the forward limit obtained in a phenomenological fit $[582$ to the nucleon form factors. The first two panels correspond to two parameter sets giving a good fit. The valence quark distributions $u_{\mathrm{val}}=u-\bar{u}$ and $d_{\mathrm{val}}=d-\bar{d}$ in the third panel are shown for comparison. All distributions are shown at the scale $\mu=2 \mathrm{GeV}$.

where $\kappa_{q}$ is the contribution of quark flavor $q$ to the anomalous magnetic moment of the proton. From the magnetic moments of proton and neutron one obtains $\kappa_{u}-\kappa_{d}=3.71$ and $\kappa_{u}+\kappa_{d}+\kappa_{s}=-0.36$. Under the reasonable assumption that $\kappa_{s}$ is small compared with $\kappa_{u}$ and $\kappa_{d}$, these numbers imply that $\kappa_{u}$ and $\kappa_{d}$ are both large but have opposite signs and largely cancel in the flavor sum. As a consequence, the functions $e_{u, \text { val }}(x)=e_{u}(x)-e_{\bar{u}}(x)$ and $e_{d, \mathrm{val}}(x)=e_{d}(x)-e_{\bar{d}}(x)$ must be large at least in some region of $x$. This is illustrated in figure 3.12, which shows distributions obtained by fitting a model ansatz for $u$ and $d$ quark GPDs to the electromagnetic nucleon form factors [582] (neglecting strange-quark contributions). The fit suggests that $e_{u, \text { val }}$ and $e_{d, \text { val }}$ are of similar size as the unpolarized valence distributions, whereas $e_{u, \mathrm{val}}+e_{d \text {,val }}$ is small and poorly known, to the point that we do not know whether it has zero crossings.

The second moments of $e(x)$ appear in Ji's angular momentum sum rules,

$$
2 J^{q}=\int_{0}^{1} d x x[q(x)+\bar{q}(x)]+\int_{0}^{1} d x x\left[e_{q}(x)+e_{\bar{q}}(x)\right], \quad 2 J^{g}=\int_{0}^{1} d x x g(x)+\int_{0}^{1} d x x e_{g}(x),
$$

where they give "nontrivial" contributions in addition to the "trivial" ones from the momentum integrals of quarks and gluons (whose values are well known). Summed over all partons, the momentum integrals add up to 1 and the angular momenta to $\frac{1}{2}$, so that

$$
\int_{0}^{1} d x x e_{\operatorname{sing}}(x)+\int_{0}^{1} d x x e_{g}(x)=0,
$$

where $e_{\text {sing }}(x)=\sum_{q}\left[e_{q}(x)+e_{\bar{q}}(x)\right]$. Note that both (3.30) and (3.32) are exact relations in QCD, in contrast to the positivity bound (3.28). The scale dependence of $e_{g}(x)$ and $e_{\text {sing }}(x)$ is governed by coupled DGLAP equations, with the same kernels as for the unpolarized gluon and quark singlet distributions. With (3.32) one finds that to leading order in $\alpha_{s}$

$$
\int_{0}^{1} d x x e_{g}(x, \mu)=\left(\frac{\alpha_{s}(\mu)}{\alpha_{s}\left(\mu_{0}\right)}\right)^{\gamma} \int_{0}^{1} d x x e_{g}\left(x, \mu_{0}\right)
$$


where $\gamma=50 / 81$ for $n_{f}=3$ and $56 / 75$ for $n_{f}=4$ active flavors. All numbers in the following refer to $\mu=2 \mathrm{GeV}$; the evolution of (3.33) to higher scales is rather slow.

With the distributions in [582] one finds that $\int d x x e_{\text {sing }}$ has a very small valence part $\int d x x\left[e_{u}-e_{\bar{u}}+e_{d}-e_{\bar{d}}\right]$ between -0.042 and 0.068. A similar situation is found in lattice calculations, which obtain a small contribution to $\int d x x\left[e_{u}+e_{\bar{u}}+e_{d}+e_{\bar{d}}\right]$ from connected graphs, with values between $-0.077(16)$ and 0.015(11) for different extrapolations to the physical quark masses 604.

Assuming that $\int d x x\left[e_{u}-e_{\bar{u}}+e_{d}-e_{\bar{d}}\right]$ is indeed small (and barring the possibility of an implausibly large $\left.e_{s}-e_{\bar{s}}\right)$ we find that the sum $\int d x x e_{g}+\int d x x e_{\text {sea }}$ of second moments must be small, where $e_{\text {sea }}=2 \sum_{q} e_{\bar{q}}$. This still leaves us with a number of possible scenarios:

1. both $e_{g}(x)$ and $e_{\text {sea }}(x)$ are small (note that this does not exclude large $e_{\bar{q}}(x)$ for individual quark flavors: only the flavor sum must be small),

2. $e_{g}(x)$ and $e_{\text {sea }}(x)$ are both large but have opposite signs,

3. both distributions are large but have nodes such that their second moments are small.

Scenario 2 is illustrated in figure 3.13, which shows two variants of model distributions proposed in 605]. The absolute size of the distributions is limited by the bound (3.28) and its analogs for $e_{\bar{q}}$ and $e_{g}$, and the opposite signs of $e_{g}$ and $e_{\text {sea }}$ ensure that (3.32) can be fulfilled. We see that scenarios where both $e_{g}$ and $e_{\text {sea }}$ are large cannot be ruled out with our present knowledge. If the above model distributions are evolved to higher scales, $e_{g}$ becomes even larger and steeper at small $x$ [603].
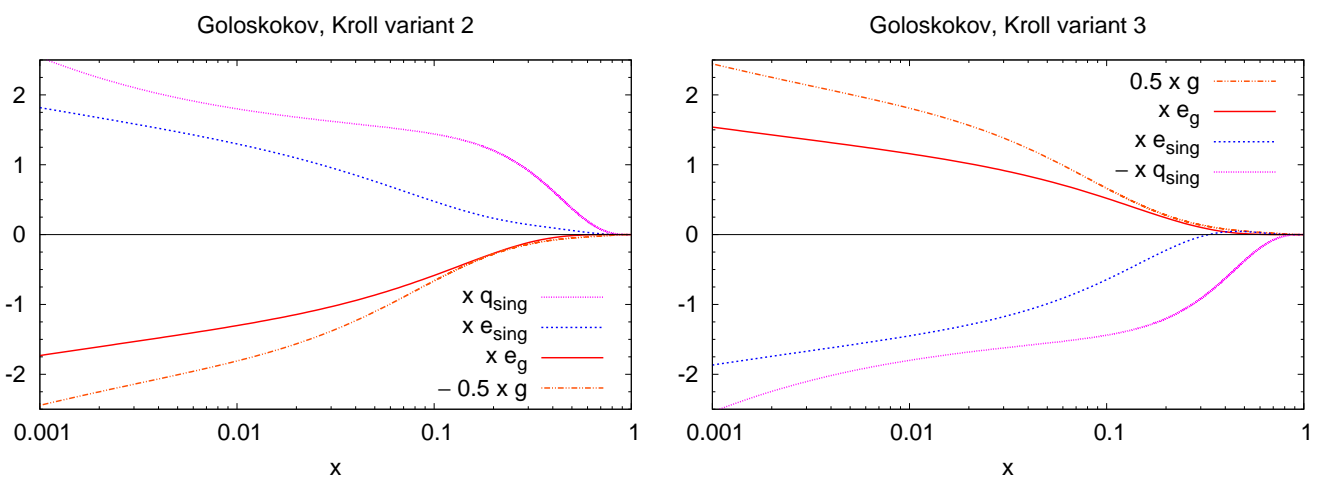

Figure 3.13. Two variants of model distributions $e_{g}$ and $e_{\text {sing }}$ at $\mu=2 \mathrm{GeV}$ from [605]. The distributions of the quark singlet $q_{\text {sing }}=\sum_{q}(q+\bar{q})$ and the gluon are shown for comparison.

\subsubsection{Exclusive processes}

Up to now I discussed $E^{q}, E^{\bar{q}}$ and $E^{g}$ at zero skewness $\xi=0$, but in exclusive processes like DVCS and meson production $\xi$ is always nonzero. Nevertheless, experience from phenomenology and models suggests that GPDs at $\xi=0$ are closely enough related to those at $\xi \neq 0$ to serve as a guide for their overall size, see e.g. [577, 606].

Note that even the large model distributions $e_{g}$ and $e_{\text {sea }}$ in figure 3.13 result in small values for the transverse target spin asymmetry $A_{U T}$ in exclusive $\rho$ electroproduction [605]. This is in part due to cancellations in the sum over $u$ and $d$ quarks in this process (the 
same distributions give a larger asymmetry for $\omega$ production). Moreover, $A_{U T}$ in exclusive meson production is proportional to $\operatorname{Im}\left(\mathcal{H} \mathcal{E}^{*}\right)$, where $\mathcal{H}$ and $\mathcal{E}$ are the scattering amplitudes associated with $H$ and $E$ distributions, respectively. Hence $A_{U T}$ is also small when both amplitudes are large but have a small relative phase. The transverse target asymmetry in DVCS is therefore of special importance, because the interference between Compton scattering and the Bethe-Heitler process is linear in $\operatorname{Im} \mathcal{E}$. 


\title{
3.5 Imaging transverse distributions
}

\author{
Gerald A. Miller
}

\subsubsection{Introduction}

Much effort has gone into measuring electromagnetic form factors, which are related to the charge and magnetization densities within the nucleons. The influence of relativistic motion of the quarks within the nucleon causes the standard textbook interpretation of form factors as three-dimensional Fourier transforms to be wrong [607]. The use of transverse densities [608, 609] avoids various difficulties by working in the infinite momentum frame and taking the spacelike momentum transfer to be in the direction transverse to that of the infinite momentum. In this case, the different momenta of the initial and final nucleon states are accommodated by using two-dimensional Fourier transforms and transverse charge and magnetization densities are constructed from density operators that are the absolute square of quark-field operators.

The transverse charge density is given by [608, 610]

$$
\rho(b)=\int d x^{-} \rho\left(x^{-}, b\right)=\frac{1}{2 \pi} \int Q d Q J_{0}(Q b) F_{1}\left(Q^{2}\right),
$$

where $\rho\left(x^{-}, b\right)$ is the three dimensional spatial density.

The transverse charge densities are shown in [608, 609]. The interesting feature is that the central neutron charge density is negative. An interpretation of this finding based on the impact parameter distribution [580, 611 was presented in 612. All models of these quantities are based on the Drell-Yan-West relation, which connects large values of $x$ with large values of $Q^{2}$. These models tell us that the $d$ quarks that dominate deep inelastic scattering from the neutron at large values of $x$ dominate the neutron center. It is also possible that the negatively charge pionic cloud may penetrate the center [613].

The transverse anomalous magnetization density is obtained from the matrix element of the magnetization density operator $\frac{1}{2} \vec{b} \times \vec{j}$, where $\vec{j}$ is taken in the $z$-direction:

$$
\rho_{M}(b)=\frac{\sin ^{2} \phi}{2 M} b \int \frac{Q^{2} d Q}{2 \pi} F_{2}\left(Q^{2}\right) J_{1}(Q b) .
$$

The integral $\int d^{2} b \rho_{M}(b)$ gives the anomalous magnetic moment.

\subsubsection{Realistic transverse images of the proton charge and magnetic den- sities}

The word "realistic" refers to the ability to know the uncertainty in the transverse densities derived from experiment. The previously obtained transverse densities are derived from various parameterizations of the form factors. A more detailed treatment is needed to be able to extract uncertainties. The following discussion is based on the analysis [614].

The basic idea behind our approach is to use the observation that $\rho(b) \approx 0$ for $b \geq R$, where $R$ is a finite distance. Since the functions $\rho$ and $F$ are Fourier transforms, $F$ is bandlimited. We proceed in the spirit of the Nyquist-Shannon sampling theorem and expand the function $\rho$ as

$$
\rho(b)=\sum_{n=1}^{\infty} \frac{1}{2 \pi} \frac{2}{R^{2} J_{1}\left(X_{n}\right)^{2}} F\left(Q_{n}^{2}\right) J_{0}\left(X_{n} \frac{b}{R}\right),
$$




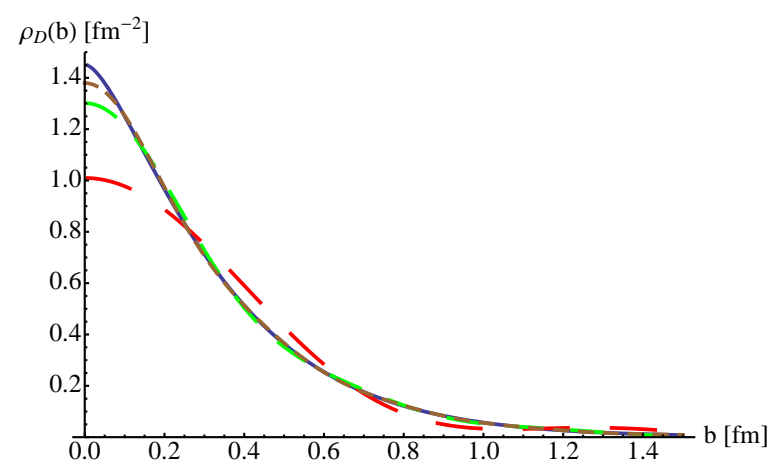

Figure 3.14. Plot of $\rho_{D}$ (solid), 5 term approximation (red, long dash), 10 term approximation (green, medium dash) and 15 term approximation (brown, short dash). From Ref. 614].

where $X_{n}$ is the $n$-th zero of the regular cylindrical Bessel function of order $0, J_{0} ; Q_{n} \equiv$ $X_{n} / R$ and $X_{n} \approx(n+3 / 4) \pi$. Equation (3.36) defines the so-called finite radius approximation (FRA). Using, for example, $R=3 \mathrm{fm}$ and $n=10, Q_{n}^{2} \approx 4 \mathrm{GeV}^{2}$. Thus, the measurement up to $Q^{2}=4 \mathrm{GeV}^{2}$ determines the first ten terms of the expansion. As an example, let us consider the expansion (3.36) for the dipole form factor: $F_{D}\left(Q^{2}\right)=1 /\left(1+Q^{2} / \Lambda^{2}\right)^{2}$ with $\Lambda^{2}=0.71 \mathrm{GeV}^{2}$. The results shown in figure 3.14 indicate that relatively few terms suffice to give an accurate representation.

The relationship between the FRA and the usual expansion into a complete set of functions is examined in 614 where it is shown that the FRA is very accurate. The available data set consists of ep scattering up to $31 \mathrm{GeV}^{2}$ and $G_{E, M}$ are separately extracted for up to $10 \mathrm{GeV}^{2}$. The form factors $G_{E}$ and $G_{M}$ have been extracted from a global analysis of the world's cross section and polarization data, including corrections for two-photon exchange corrections [615]. The analysis is largely identical to that of [616], although additional high $Q^{2}$ form factor results 617 have been included. In addition, the slopes of $G_{E}$ and $G_{M}$ at $Q^{2}=0$ were constrained in the global fit based on a dedicated analysis of the low $Q^{2}$ data. In writing $G_{E}\left(Q^{2}\right)=1-Q^{2} R_{E}^{2} / 6$, the value of $R_{E}$ was constrained to be $0.878 \mathrm{fm}$ and $R_{M}$ was constrained to be $0.860 \mathrm{fm}$. This is important in the extraction of the large scale structure of the density. The fit is given in 614.

We then use the fit and uncertainties for $G_{E}$ and $G_{M}$ to extract $F_{1}$ and $F_{2}$, treating the uncertainties in $G_{E}$ and $G_{M}$ as uncorrelated, yielding:

$$
\begin{aligned}
& \left(d F_{1}\right)^{2}=\left(\frac{1}{1+\tau}\right)^{2}\left(d G_{E}\right)^{2}+\left(\frac{\tau}{1+\tau}\right)^{2}\left(d G_{M}\right)^{2}, \\
& \left(d F_{2}\right)^{2}=\left(\frac{1}{1+\tau}\right)^{2}\left(d G_{E}\right)^{2}+\left(\frac{1}{1+\tau}\right)^{2}\left(d G_{M}\right)^{2} .
\end{aligned}
$$

For $Q^{2}<30 \mathrm{GeV}^{2}$, we use $d F_{1}$ above in the FRA to get $d \rho(b)$. For $Q^{2}>30 \mathrm{GeV}^{2}$, we use the FRA and take $d F_{1}= \pm \mid F_{1}$ (fit)|. This corresponds to a maximum value of $n=30$. The resulting transverse charge density is shown in figure 3.15. The proton transverse charge density is now very well known.

Our FRA technique can be exploited to image other quantities that depend on the transverse position. Suppose there is a transverse quantity $\rho^{(\lambda)}(b)$ that is a two-dimensional 


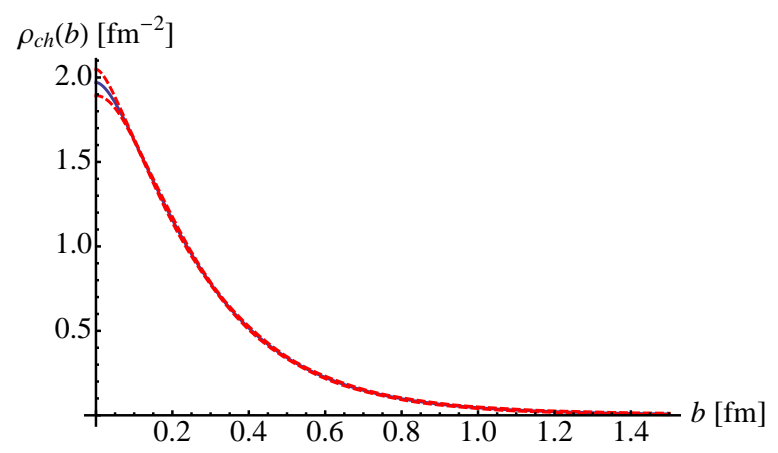

Figure 3.15. (Color online) $\rho_{c h}$ (solid, blue) with error bands (short dashed, red). From Ref. 614].

Fourier transform of an experimental observable $F^{(\lambda)}\left(Q^{2}\right)$ such that

$$
\rho^{(\lambda)}(b)=\frac{1}{2 \pi} \int Q d Q J_{\lambda}(Q b) F^{(\lambda)}\left(Q^{2}\right) .
$$

An example, discussed in detail in [614], is the magnetization density $\rho_{M}$. The index $(\lambda)$ is associated with a given number of units of the orbital angular momentum. The extraction of $\rho^{(\lambda)}(b)$ is facilitated by using the expansion

$$
\rho^{(\lambda)}(b)=\sum_{n=1}^{\infty} \frac{2}{R^{2} J_{\lambda+1}\left(X_{\lambda, n}\right)^{2}} F^{(\lambda)}\left(Q_{\lambda, n}^{2}\right), J_{\lambda}\left(X_{\lambda, n} \frac{b}{R}\right),
$$

where $X_{\lambda, n}$ is the $n$-th zero of the Bessel function of order $\lambda ; Q_{\lambda, n}=X_{\lambda, n} / R$. The result (3.39) can be used to relate accessible kinematic ranges with transverse regions.

\subsubsection{Summary}

Much data for form factors exist and JLab12 will further improve the data set. The charge density is not a three-dimensional Fourier transform of $G_{E}$. One can interpret form factors as determining transverse charge and magnetization densities. The nucleon transverse densities are known now to high precision. The new FRA technique can be used for other quantities that depend on transverse position, in particular, for the exclusive scattering amplitudes and generalized parton distributions discussed in this chapter.

Acknowledgments. I thank S. Venkat, J. Arrington, and X. Zhan for their extensive efforts in producing the paper 614] on which this presentation is based. I also wish to thank Jefferson Laboratory for its hospitality during a visit while this work was being completed. 


\title{
3.6 From transverse-momentum spectra to transverse im- ages
}

\author{
Elke-Caroline Aschenauer, Markus Diehl, Salvatore Fazio
}

\subsubsection{Imaging partons in the transverse plane}

The principle of "parton imaging" using exclusive processes such as DVCS or hard exclusive meson production is rather simple. The key variable to measure is the transverse momentum transfer $\vec{\Delta}_{T}$ to the target proton or nucleus in the $\gamma^{*}$-target c.m. The invariant momentum transfer is then given by

$$
t=-\frac{x^{2} m^{2}+\vec{\Delta}_{T}^{2}}{1-x} \quad \text { with } \quad x=\frac{Q^{2}+M_{V}^{2}}{Q^{2}+W^{2}}
$$

where $m$ is the target mass and $M_{V}$ the mass of the produced meson. For DVCS one should omit $M_{V}$, so that $x$ coincides with the Bjorken variable. In the limit of large $Q^{2}+M_{V}^{2}$, the $\gamma^{*} p$ scattering amplitude is a linear combination of generalized parton distributions convoluted with hard-scattering kernels. The distribution of partons in the transverse plane is obtained by a Fourier transform w.r.t. $\vec{\Delta}_{T}$ [580, 611]. In the simple case where the unpolarized quark or gluon GPDs $H^{i}$ dominate the $\gamma^{*} p$ cross section $d \sigma / d t$, the impact parameter profile is

$$
F\left(b, x, Q^{2}\right) \propto \frac{1}{(2 \pi)^{2}} \int d^{2} \vec{\Delta}_{T} e^{-i \vec{b} \vec{\Delta}_{T}} \sqrt{\frac{d \sigma}{d t}}=\frac{1}{2 \pi} \int_{0}^{\infty} d \Delta_{T} \Delta_{T} J_{0}\left(b \Delta_{T}\right) \sqrt{\frac{d \sigma}{d t}},
$$

where $\Delta_{T}=\left|\vec{\Delta}_{T}\right|$ and $b=|\vec{b}|$. For simplicity we drop the information from the absolute size of the cross section in this contribution and focus our attention on the normalized $b$-space profile, which satisfies $\int d^{2} b F\left(b, x, Q^{2}\right)=1$. For polarization asymmetries and for the interference term between DVCS and the Bethe-Heitler process, the extraction of the relevant $\gamma^{*} p$ amplitudes is more involved, but the principle of Fourier transforming these amplitudes w.r.t. $\vec{\Delta}_{T}$ remains the same.

In the present contribution, we estimate how accurately one can hope to determine $F\left(b, x, Q^{2}\right)$ from cross section measurements for DVCS on the proton. Firstly, $d \sigma / d t$ will have statistical and systematic errors. Secondly, the range of $\Delta_{T}$ in a measurement will be restricted both from above and from below, so that an extrapolation is required in order to perform the Fourier integral in (3.41).

\subsubsection{Acceptance in transverse momentum}

To achieve the precision discussed below for imaging partons in the impact parameter space, it is critical to integrate from the beginning the detection of the scattered proton into the detector and interaction region design. The scattered proton in exclusive reactions is characterized by carrying almost the full beam momentum and a transverse momentum $\Delta_{T}$ between several $\mathrm{MeV}$ and a few $\mathrm{GeV}$, corresponding to very small scattering angles. Figure 3.16 shows the relation between the longitudinal momentum of the protons and their scattering angle for two different $e p$ center-of-mass energies.

The commonly used method to detect these protons is to integrate "Roman pots" in the machine lattice. The standard technologies for such detectors are silicon strip detectors or 

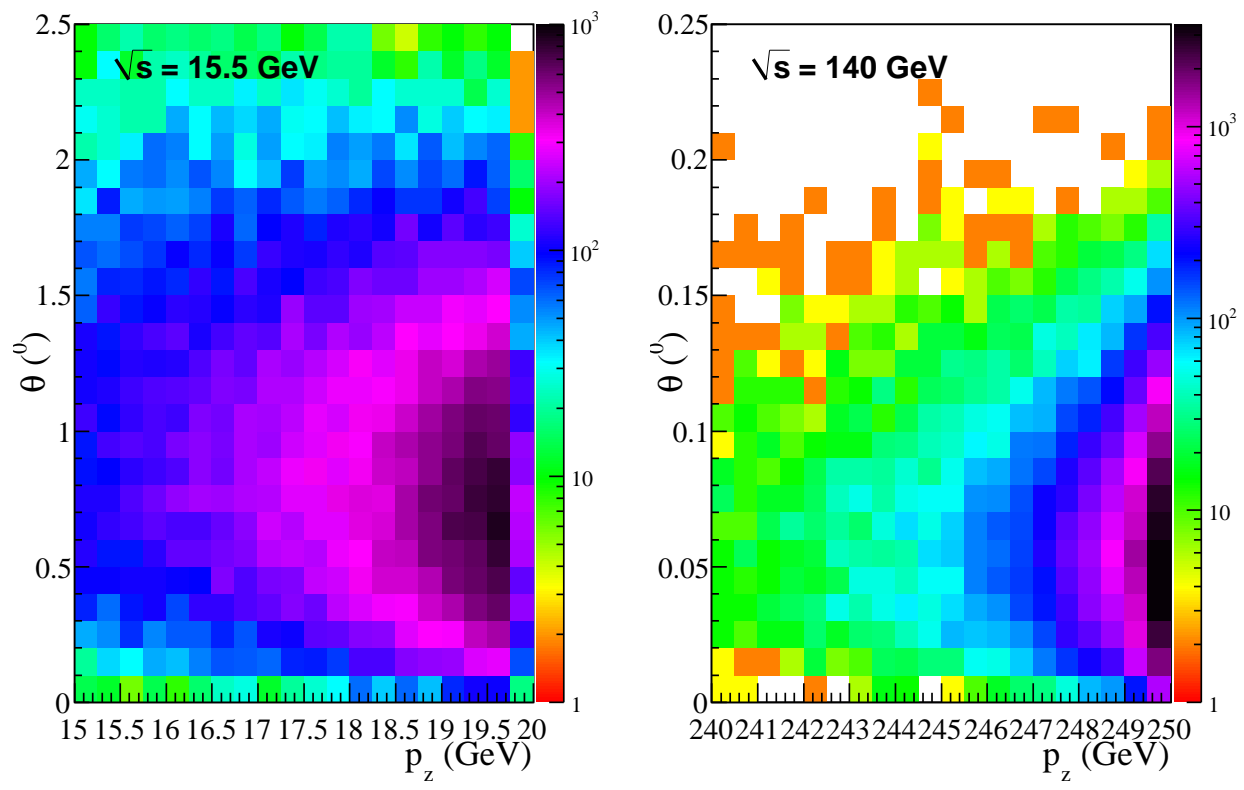

Figure 3.16. (Color online) The longitudinal momentum $p_{z}$ of the scattered proton in exclusive reactions vs. its scattering angle $\theta$ for an $e p$ center-of-mass energy of $15.5 \mathrm{GeV}$ (left) and $145 \mathrm{GeV}$ (right).

scintillating fiber detectors. The acceptance for protons with the transverse momentum in the $\mathrm{MeV}$ region is limited by the requirement that Roman pots must have a beam clearance distance of 10 times the beam emittance. The upper transverse acceptance is given by the apertures of the magnets that the protons have to transverse. For transverse momenta above $1 \mathrm{GeV}$, the proton can be detected in the main solenoidal detector. Details on the solutions for the eRHIC and ELIC interaction region designs are given in section 7.3 .

\subsubsection{Precision of the measurement}

A detailed simulation of DVCS events is described in section 3.9. To illustrate the expected statistical accuracy of a measurement, we show $d \sigma / d t$ for a selected bin of $x$ and $Q^{2}$ in figure 3.17. The value of $y$ in this bin ranges from 0.05 to 0.14 . For bins with lower $x$ or lower $Q^{2}$, the statistical errors are smaller, except for kinematics where the $y>0.01$ cut applied in the simulation becomes relevant.

The $t$ spectrum shown in the figure 3.17 was generated with an exponential dependence $d \sigma / d t \propto \exp (B t)$ with $B=5 \mathrm{GeV}^{-2}$. An exponential fit to the generated spectrum gives $B=5.02 \mathrm{GeV}^{-2}$ with an error below $1 \%$. Data of this quality also allows one to explore possible deviations from an exponential spectrum. To this end, we have also fitted to $d \sigma / d t \propto \exp \left(B t-C t^{2}\right)$. This fit and its $1 \sigma$ error band is shown in the figure and gives $B=(4.92 \pm 0.10) \mathrm{GeV}^{-2}$ and $C=(0.079 \pm 0.076) \mathrm{GeV}^{-4}$. Although the relative uncertainty on the extra parameter $C$ is large, the term $C t^{2}$ in the exponential is small compared with $B t$ in the fitted $t$ range (as it should be for a spectrum generated with a pure exponential law). The logarithmic $t$ slope at $|t|=1.75 \mathrm{GeV}^{2}$ in this fit is $(5.20 \pm 0.18) \mathrm{GeV}^{-2}$.

We conclude at this point that with the projected luminosity available at an EIC, the $t$ spectrum for the DVCS cross section will be dominated by systematic uncertainties and not 


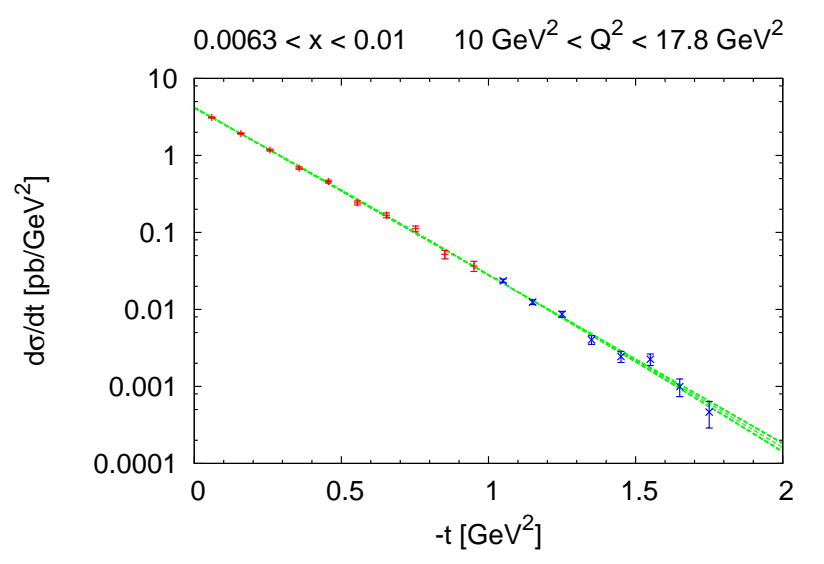

Figure 3.17. (Color online) Generated $t$ spectrum for the DVCS cross section in a selected bin of $x$ and $Q^{2}$. The errors are statistical only and correspond to the integrated luminosity of $11.9 \mathrm{fb}^{-1}$ for $|t|<1 \mathrm{GeV}^{2}$ and to $151 \mathrm{fb}^{-1}$ for $|t|>1 \mathrm{GeV}^{2}$. The curve represents a fit explained in the text.

by statistics, even if one measures differentially in $x$ and $Q^{2}$. Systematic uncertainties, for instance due to momentum resolution, strongly depend on details of the experimental setup and have not been studied yet. We note that the normalized $b$ space profile $F\left(b, x, Q^{2}\right)$ is not affected by errors on the overall luminosity and acceptance.

\subsubsection{Uncertainty from the extrapolation in $t$}

We now estimate the uncertainty in the impact parameter profile $F(b)$ due to the lack of knowledge of the scattering amplitude for all $t$. Since the projected statistical errors are so small, we do not include them in this exercise.

For the extrapolation to large $|t|$, we assume a measured $t$-spectrum $d \sigma / d t \propto \exp (B t)$ with $B=4 \mathrm{GeV}^{-2}$ up to $|t|_{\max }=1$ or $2 \mathrm{GeV}^{2}$. Larger values of $B$ give a smaller cross section at high $|t|$ and thus a smaller extrapolation uncertainty in the Fourier integral (3.41). In turn, the statistical errors on the cross section at high $|t|$ are then larger, so that in $F(b)$ there is a tradeoff between the uncertainties from the measured $t$ spectrum and those from its extrapolation.

To estimate the extrapolation uncertainty, we adopt a strategy similar to that in 618 and assume different forms for the scattering amplitude (i.e., for $\sqrt{d \sigma / d t}$ ) at $|t|>|t|_{\max }$ :

1. an exponential $\propto \exp (B t / 2)$, labeled "exp" in figure 3.18,

2. a dipole form $\propto\left(1+|t| / M^{2}\right)^{-2}$, labeled "dip",

3. a modified dipole form $\propto\left(1+0.05|t| / M^{2}\right)^{-1}\left(1+0.45|t| / M^{2}\right)^{-1}$, labeled "mod dip",

4. a modified exponential $\propto \exp \left(-D t^{2}\right)$, labeled "mod exp".

In each case we require the amplitude and its first derivative to be continuous at $|t|=|t|_{\max }$. Note that in the measured $t$ region, forms 2 to 4 would give unacceptable fits to the simulated spectrum in figure 3.17. Forms 3 and 4 should be regarded as examples for functions falling off especially slowly or especially fast and do not claim to be particularly realistic. When performing the Fourier transform (3.41), we neglect the term $x^{2} m^{2}$ in (3.40), which is justified in a large region of phase space. 
In figure 3.18 we show the resulting scattering amplitude (normalized to unity at $\vec{\Delta}_{T}=$ $\overrightarrow{0})$ and its Fourier transform $F(b)$. We observe that the curves in $b$ space are close together in a wide region and rather quickly start to differ below a certain critical value $b_{\mathrm{cr}}$. For $|t|_{\max }=$ $1 \mathrm{GeV}^{2}$, we find $b_{\mathrm{cr}} \sim 0.25 \mathrm{fm}$ and an appreciable spread of $F(b)$ at lower $b$. This would be a serious limitation for studying the central region of the proton. Interesting physical effects like the variation of $F(b)$ with $x$ or $Q^{2}$ are typically expected to be only logarithmic (see, e.g., the estimates in [619]) and hence require sufficiently precise measurements. Clearly, there is a very significant gain of accuracy in impact parameter space if $|t|_{\text {max }}$ can be raised from 1 to $2 \mathrm{GeV}^{2}$, i.e., if a scattered proton in the corresponding kinematics can be seen in the main detector. We then find $b_{\mathrm{cr}} \sim 0.1 \mathrm{fm}$ and a small uncertainty even at $b=0$.

As an alternative scenario we assume a dipole form instead of an exponential $t$ dependence in the measured region 1 with a dipole mass $M=770 \mathrm{MeV}$ that gives the same scattering amplitude at $|t|=1 \mathrm{GeV}^{2}$ as the exponential with $B=4 \mathrm{GeV}^{-2}$. The extrapolation error is larger in the dipole scenario, but since the cross section decreases much more slowly, it can be measured out to higher values of $|t|$ before statistics becomes an issue. We recall however that a description in terms of generalized parton distributions requires $|t| \ll Q^{2}+M_{V}^{2}$. As seen in figure 3.19, a measurement up to $|t|_{\max }=3.3 \mathrm{GeV}^{2}$ in the dipole scenario gives a very precise $F(b)$ down to $b_{\text {cr }} \sim 0.1 \mathrm{fm}$. The extrapolation uncertainty at lower $b$ is larger than for $|t|_{\max }=2 \mathrm{GeV}^{2}$ in the exponential scenario.

Let us now investigate the extrapolation to small $|t|$. We assume again an exponential cross section $d \sigma / d t \propto \exp (B t)$, but now with a larger slope $B=6.6 \mathrm{GeV}^{-2}$ in order to maximize the importance of low $|t|$ in the Fourier integral. We consider either $300 \mathrm{MeV}$ or $200 \mathrm{MeV}$ as minimum measured values of $\Delta_{T}$, and take the following extrapolations for $\Delta_{T}$ down to zero:

1. an exponential in $t$, labeled "exp" in figure 3.20 ,

2. a dipole form $\propto\left(1+|t| / M^{2}\right)^{-2}$, labeled "dip",

3. a linear function in $t$, labeled "lin",

4. a monopole form $\propto\left(1+|t| / M^{2}\right)^{-1}$, labeled "mono",

5. an inverse square root $\propto\left(1+|t| / M^{2}\right)^{-1 / 2}$, labeled "sqrt".

We see in figure 3.20 that with a measurement down to $\Delta_{T}=300 \mathrm{MeV}$, one has a rapidly growing extrapolation uncertainty for $b$ above about $1.25 \mathrm{fm}$. The situation dramatically improves if one has to extrapolate only below $\Delta_{T}=200 \mathrm{MeV}$. Repeating this study with a dipole form in the measured region yields the same conclusion [621. Whether a measurement down to even lower $\Delta_{T}$ can still improve the accuracy of $b$ space images can only be decided after an estimate of experimental uncertainties.

Let us recall the specific physics interest of the impact parameter profile of the proton at very large $b$. This is the region where the dynamics of chiral symmetry breaking should manifest itself. A description in terms of virtual pion fluctuations yields definite predictions, such as a behavior $F(b) \propto b^{-1} e^{-\kappa b}$ with $\kappa \approx 2 m_{\pi} \approx(0.7 \mathrm{fm})^{-1}$ at large $b$ [594]. This translates into a small $|t|$ behavior given by the inverse square root law in point 5 (with $M^{2}=\kappa^{2}$ ). These predictions should be tested quantitatively.

\footnotetext{
${ }^{1}$ We note that the measurement of $J / \Psi$ photoproduction at HERA [589, 620] strongly favors an exponential $t$ dependence at $|t|$ below $1 \mathrm{GeV}^{2}$, but the behavior of exclusive hard scattering cross sections at larger $t$ is poorly known. For a conservative error estimate, we do not want to rule out a dipole behavior at $|t|>1 \mathrm{GeV}^{2}$.
} 

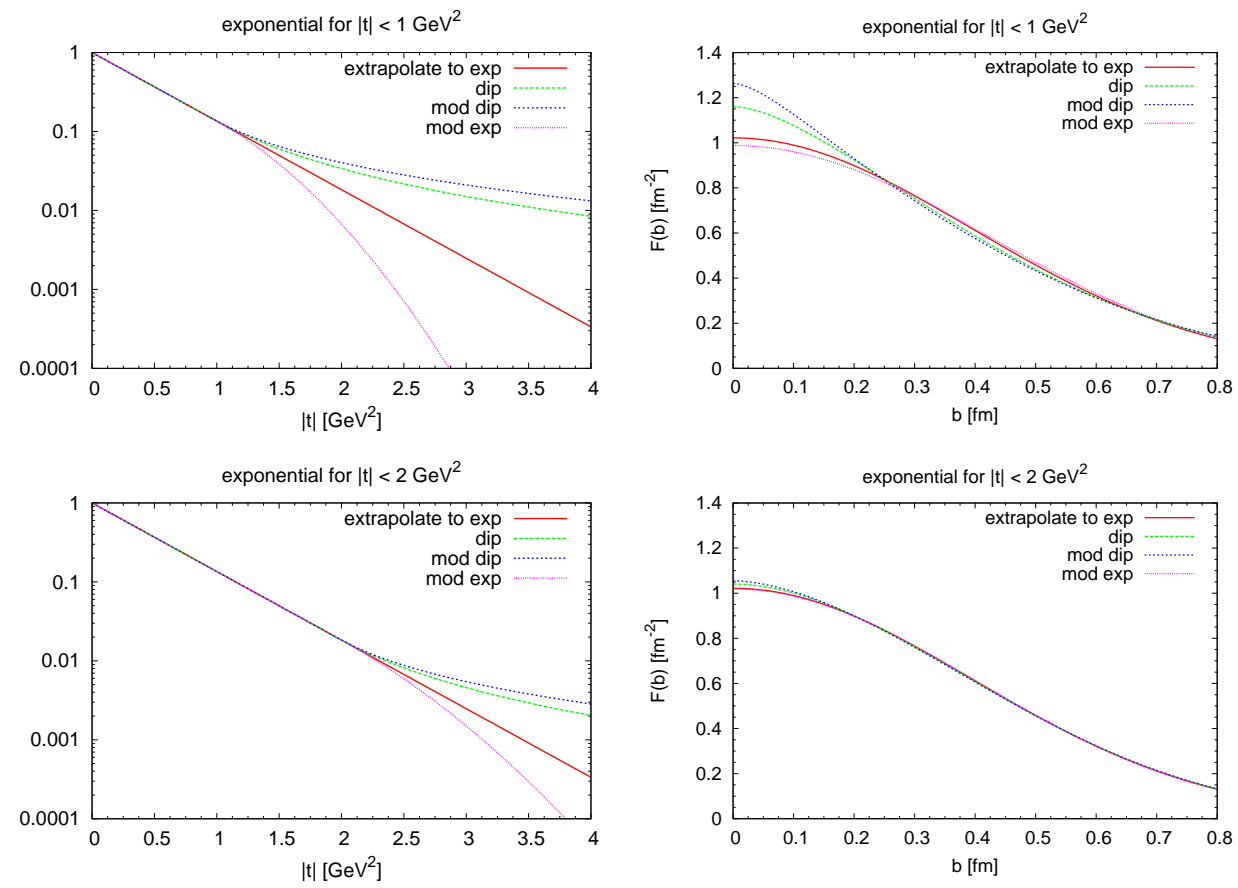

Figure 3.18. (Color online) Examples for normalized amplitudes (left) with different extrapolations to large $|t|$, together with their Fourier transforms to impact parameter space (right).
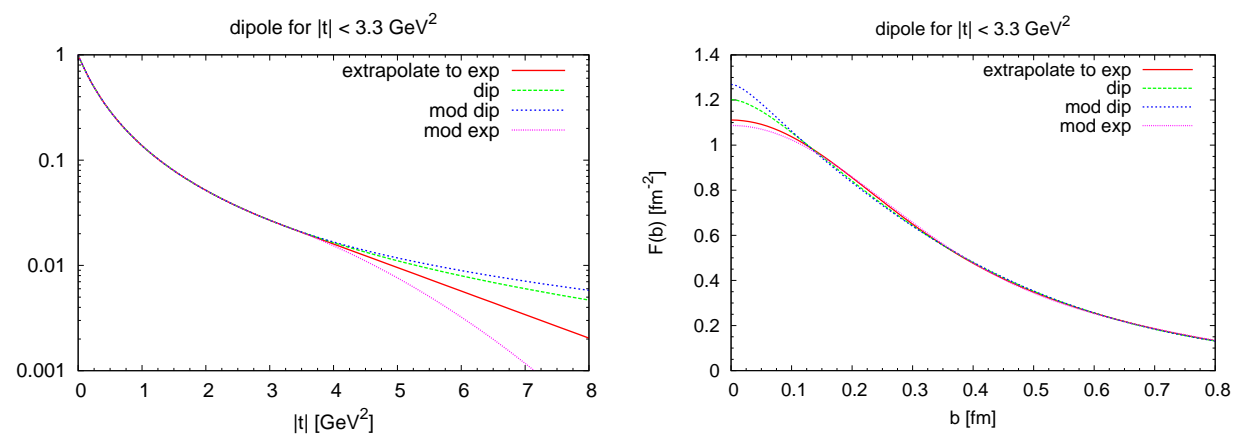

Figure 3.19. (Color online) As in figure 3.18 but with a dipole form of the amplitude up to $|t|=$ $3.3 \mathrm{GeV}^{2}$. 

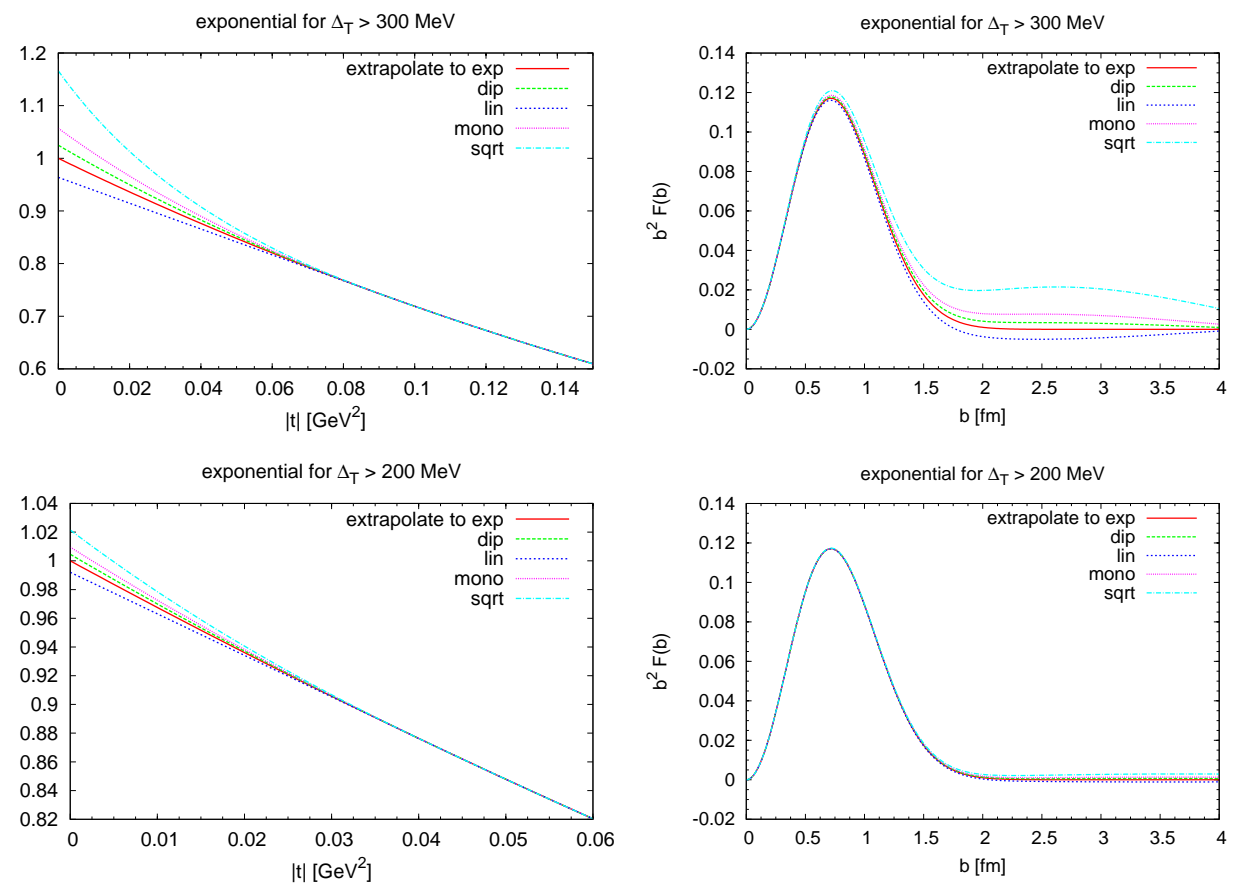

Figure 3.20. (Color online) As in figure 3.18, but with extrapolation to small $|t|$, i.e. small $\Delta_{T}$. The impact parameter profile $F(b)$ is multiplied with $b^{2}$ in order to make the large $b$ behavior visible.

In summary, we find that with the parameters we have assumed, neither statistics nor acceptance in $t$ will seriously limit $b$ space imaging at an EIC, with an accessible $b$ range from $0.1 \mathrm{fm}$ up to $1.5 \mathrm{fm}$ or larger. Detailed estimates of experimental uncertainties will be necessary to assess the limiting factors of accuracy in this endeavor. 


\title{
3.7 GPDs from DVCS
}

\author{
Matthias Burkardt, Hikmat BC
}

\subsubsection{Introduction}

GPDs are linked to many processes and observables involving hadrons 622, but their most intuitive application is in the context of Ji's angular momentum decomposition (see section 3.4) and in three-dimensional imaging (see section 3.6). Both involve GPDs in the $\xi=0$ limit (the $\xi$-dependence drops out in the Ji sum rule). At the same time, the DVCS amplitude $\mathcal{A}_{\text {DVCS }}$ provides direct access only to GPDs along the "diagonal" $x=\xi$ (through the imaginary part of the DVCS amplitude) as well as to a convolution integral involving GPDs (through the real part of $\mathcal{A}_{\mathrm{DVCS}}$ ). In the leading order (LO) factorization, one finds

$$
\begin{aligned}
\Im m \mathcal{A}_{\mathrm{DVCS}} & \longrightarrow G P D^{(+)}(\xi, \xi, t), \\
\Re e \mathcal{A}_{\mathrm{DVCS}} & \longrightarrow \int_{-1}^{1} d x \frac{G P D^{(+)}(x, \xi, t)}{x-\xi} .
\end{aligned}
$$

The '(+)' superscript in (3.42) emphasizes that DVCS is sensitive only charge-even (i.e., quark+antiquark) combinations of GPDs. Moreover, the accessible range in $\xi$ is limited, $\xi_{\min }<\xi<\xi_{\max }$. The lower limit $\xi_{\min }$ is defined by the DIS kinematics. The upper limit $\xi_{\max }$ follows from the relation

$$
-t=\frac{4 \xi^{2} M^{2}+\Delta_{\perp}^{2}}{1-\xi^{2}}
$$

and the positivity of $\boldsymbol{\Delta}_{\perp}^{2}$. Thus, even in an idealized DVCS experiment (fixed $Q^{2}$ ), where angular dependencies as well as spin asymmetries have been used to disentangle different GPDs and the proton and 'neutron' targets have been used to accomplish the flavor decomposition, one can at best expect a determination of the observables in (3.42) for $\xi_{\min }<\xi<\xi_{\max }$. One of the key question in the context of DVCS is whether this information will allow an unambiguous and model-independent extraction of GPDs.

\subsubsection{Constraints on GPDs: polynomiality, dispersion relations and QCD evolution}

GPDs are not only constrained by DVCS, but also by DIS and form factor data. However, the form factor data constrains only charge-odd distributions and helps only in kinematical regimes where antiquark contributions are negligible. While DIS data is sensitive to charge-even distributions, there is no DIS data that would constrain the forward $(\xi=0$, $t=0)$ limit of $E^{q}(x, \xi, t)$.

Fortunately, multiple theoretical constraints exist that will be helpful in determining GPDs from DVCS data. For example, Lorentz invariance implies the polynomiality conditions on GPDs [274, 574]:

$$
\int_{-1}^{1} d x x^{n} G P D(x, \xi, t)=A_{n, 0}(t)+A_{n, 2}(t) \xi^{2}+\ldots+A_{n, n+1} \xi^{n+1}
$$

where the highest power $\xi^{n+1}$ is only present when $n$ is odd. These polynomiality conditions imply that the dependence of GPDs on the variables $x$ and $\xi$ cannot be independent. This imposes significant and rigorous constraints on any GPD extraction from DVCS data. 
Rigorous dispersion relations exist for the DVCS amplitude $\mathcal{A}\left(\nu, t, Q^{2}\right)$ :

$$
\Re e \mathcal{A}\left(\nu, t, Q^{2}\right)=\frac{\nu^{2}}{\pi} \int_{0}^{\infty} \frac{d \nu^{\prime 2}}{\nu^{\prime 2}} \frac{\Im m \mathcal{A}\left(\nu^{\prime}, t, Q^{2}\right)}{\nu^{\prime 2}-\nu^{2}}+\Delta\left(t, Q^{2}\right)
$$

where $\Delta\left(t, Q^{2}\right)$ is a possible subtraction that can be identified with the $D$-form factor [587]. In combination with the leading order (LO) factorization (3.42), this implies for GPDs

$$
\Re e \mathcal{A}\left(\xi, t, Q^{2}\right) \sim \int_{-1}^{1} d x \frac{G P D^{(+)}\left(x, \xi, t, Q^{2}\right)}{x-\xi}=\int_{-1}^{1} d x \frac{G P D^{(+)}\left(x, x, t, Q^{2}\right)}{x-\xi}+\Delta\left(t, Q^{2}\right) .
$$

Although its derivation from dispersion relations is more physical, (3.46) was first derived from polynomiality [574].

One of the consequences of (3.46) is that it allows to "condense" the information from the DVCS amplitude (including the real part) into GPDs along the diagonal $x=\xi$ plus the D-form factor. However, it should be emphasized that this does not render measurements of the real part of the DVCS amplitude redundant. Indeed, measurements of $\Im m \mathcal{A}_{\text {DVCS }}$ for a given beam energy do not cover the whole region $0<\xi<1$ that enters (3.46). This implies that one can, for example, use $\Re e \mathcal{A}\left(\xi, t, Q^{2}\right)$ at fixed $Q^{2}$ to constrain $G P D\left(\xi, \xi, t, Q^{2}\right)$ for the values of $\xi$ that are not accessible directly through the measurement of $\Im m \mathcal{A}\left(\xi, t, Q^{2}\right)$. In summary, a DVCS experiment at fixed $Q^{2}$ (large enough for GPD factorization to hold) should in principle allow for the determination of GPDs along the diagonal $x=\xi$ as well as the D-form factor, which (through the polynomiality condition) impose some constraints on GPDs for $x \neq \xi$.

Additional important constraints on GPDs come from their QCD evolution. The $Q^{2}$ evolution equations can be "diagonalized" by expanding GPDs in terms of Gegenbauer polynomials $C_{n}^{3 / 2}(x)$ :

$$
G P D\left(x, \xi, t, Q^{2}\right)=\left(1-x^{2}\right) \sum_{n=0}^{\infty} C_{n}^{3 / 2}(x) \sum_{m=0(\text { even })}^{n} a_{n m}(\xi) \mathcal{C}_{n-m}\left(\xi, t, Q^{2}\right)
$$

where $a_{n m}(\xi)$ are known polynomials. The coefficients $\mathcal{C}_{k}\left(\xi, t, Q^{2}\right)$ are a priori unknown, but their $Q^{2}$ evolution is known. This allows one (in principle) to determine $\mathcal{C}_{k}\left(\xi, t, Q^{2}\right)$ model independently. For this purpose, let us consider $x=\xi$, where GPDs can be measured directly. Upon relabeling $k=n-m$, (3.47) reads

$$
G P D\left(\xi, \xi, t, Q^{2}\right)=\left(1-\xi^{2}\right) \sum_{k=0}^{\infty} \mathcal{C}_{k}\left(\xi, t, Q^{2}\right) f_{k}(\xi)
$$

where $f_{k}(\xi)=\sum_{m=0(\text { even })}^{\infty} a_{m+k, m}(\xi) C_{m+k}^{3 / 2}(\xi)$ are known functions. For any fixed $\xi$, each term in (3.48) evolves differently and, thus, a measurement over a wide range of $Q^{2}$ should allow for the determination of $\mathcal{C}_{k}\left(\xi, t, Q^{2}\right)$ as well as the GPDs for $x \neq \xi$ [via (3.47)]. At an EIC with its wide $Q^{2}$ range and high luminosity, it may be possible for the first time to carry out a model-independent extraction of GPDs. More detailed numerical studies will be required to quantify this expectation. 


\subsection{Accessing GPDs from experiment: potential of a high- luminosity EIC}

K. Kumerički, T. Lautenschlager, D. Müller, K. Passek-Kumerički, A. Schäfer, M. Meškauskas

\subsubsection{Introduction}

Generalized parton distributions (GPDs) [567, 568, 569] have received much attention from both the theoretical and experimental sides. This was triggered by the hope to solve the "spin puzzle" that refers to the mismatch between the quark contribution to the proton spin extracted from polarized DIS and the one given by the constituent quark model. We view the "spin puzzle" first and foremost as a quest to quantify the partonic structure of the nucleon in terms of quark and gluon angular momenta [274]. Furthermore, it has been realized that GPDs allow for a three-dimensional imaging of nucleons and nuclei [623], providing, in the zero-skewness case $(\xi=0)$, a probabilistic interpretation in terms of partonic degrees of freedom [579]. In fact, GPDs build up a whole framework for description of hadron structure [575, 576], with the "spin puzzle" being just one interesting aspect.

In phenomenology, GPDs are used for modeling elastic form factors and the description of hard exclusive leptoproduction and even photoproduction. For hard exclusive processes, factorization theorems have been proven in the collinear framework at twist-two level [572, 624. In the last decade, various hard exclusive processes have been measured by the H1 and ZEUS collaborations (DESY) in the small $x_{B}$ region and by HERMES (DESY), CLAS (JLAB), and Hall A (JLAB) in the moderate $x_{B}$ region in the fixed-target experiments.

Deeply virtual Compton scattering (DVCS) off nucleon is considered as the theoretically cleanest process offering access to GPDs. Its amplitude can be parameterized by twelve Compton form factors (CFFs) [625], which are given in terms of twist-two (including gluon transversity) and twist-three GPDs. For instance, at leading order (LO), parity-even twisttwo CFFs, $\mathcal{H}$ and $\mathcal{E}$, can be expressed through quark GPDs $H$ and $E$ :

$$
\left\{\begin{array}{l}
\mathcal{H} \\
\mathcal{E}
\end{array}\right\}\left(x_{B}, t, \mathcal{Q}^{2}\right) \stackrel{\text { LO }}{=} \int_{-1}^{1} d x \frac{2 x}{\xi^{2}-x^{2}-i \epsilon}\left\{\begin{array}{l}
H \\
E
\end{array}\right\}\left(x, \eta=\xi, t, \mathcal{Q}^{2}\right),
$$

where both quark and anti-quark GPDs are defined in the region $x \in[-\xi, 1] ; x_{B}=$ $2 \xi /(1+\xi)$. Similar expressions can be written for twist-two parity-odd CFFs $\widetilde{\mathcal{H}}$ and $\widetilde{\mathcal{E}}$, while for other CFFs they are a bit more intricate 625. Analogous formulae hold for the LO description of $\gamma^{*} N \rightarrow M N$ transition form factors (TFFs), measurable in deeply virtual electroproduction of mesons (DVEM). Here, in addition to GPDs, the non-perturbative meson distribution amplitude enters, which describes the transition of a quark-antiquark state into the final meson. This induces an additional uncertainty in the GPD phenomenology.

Let us briefly clarify which GPD information can be extracted from experimental measurements. Neglecting radiative and higher twist-contributions, one might view the GPD on the $\eta=x$ cross-over line as a "spectral function", which provides also the real part of the CFF via the "dispersion relation" [626, 627, 628, 629]:

$$
\begin{aligned}
\Im \mathrm{m} \mathcal{F}\left(x_{B}, t, \mathcal{Q}^{2}\right) & \stackrel{\mathrm{LO}}{=} \pi F\left(\xi, \xi, t, \mathcal{Q}^{2}\right), \quad F=\{H, E, \widetilde{H}, \widetilde{E}\}, \\
\Re \mathrm{e}\left\{\begin{array}{c}
\mathcal{H} \\
\mathcal{E}
\end{array}\right\}\left(x_{B}, t, \mathcal{Q}^{2}\right) & \stackrel{\mathrm{LO}}{=} \operatorname{PV} \int_{0}^{1} d x \frac{2 x}{\xi^{2}-x^{2}}\left\{\begin{array}{c}
H \\
E
\end{array}\right\}\left(x, x, t, \mathcal{Q}^{2}\right) \pm \mathcal{D}\left(t, \mathcal{Q}^{2}\right) .
\end{aligned}
$$


The GPD support properties ensure that (3.50) and (3.51) are in one-to-one correspondence to the perturbative formula (3.49), where the subtraction constant $\mathcal{D}$, which is related in a specific GPD representation to the so-called $D$-term [587], can be calculated from either $H$ or E. However, we note that the "dispersion relation" (3.51) is given in terms of partonic variables and compared to the dispersion relation formulated in physical variables it differs by power suppressed contributions. To pin down the GPD in the outer region $y \geq \eta=x$, one might employ evolution. For instance, in the non-singlet case, the change of the GPD on the cross-over line is governed by (the equation in the whole outer region is needed)

$$
\mu^{2} \frac{d}{d \mu^{2}} F\left(x, x, t, \mu^{2}\right)=\int_{x}^{1} \frac{d y}{x} V\left(1, y / x, \alpha_{s}(\mu)\right) F\left(y, x, t, \mu^{2}\right),
$$

where $V$ is the evolution kernel [567]. Unfortunately, a large enough $\mathcal{Q}^{2}$ range is not available in fixed target experiments. Hence, we must conclude that in such measurements, essentially only the GPD on the cross-over line [thanks to (3.51), also outside of the experimentally accessible part of this line [629]] and the subtraction constant $\mathcal{D}$ can be accessed. Moments, such as those entering the spin sum rule, can only be obtained from a GPD model, fitted to data, or more generally with help of some "holographic" mapping [629]:

$$
\left\{F\left(x, \eta=0, t, Q^{2}\right), F\left(x, \eta=x, t, Q^{2}\right)\right\} \quad \Longrightarrow \quad F\left(x, \eta, t, Q^{2}\right) .
$$

Here, $F\left(x, \eta=0, t, \mathcal{Q}^{2}\right)$ are constrained from form factor measurements and, additionally, GPDs $\widetilde{H}(H)$ by (un)polarized phenomenological PDFs. Of course, a given 'holographic' mapping holds only for a specific class of GPD models.

\subsubsection{GPD modeling}

The implementation of radiative corrections, even including LO evolution (3.52), requires to model CFFs or TFFs in terms of GPDs. This can be done in different representations, which should be finally considered as equivalent. However, for a specific purpose a particular representation may be more suitable than the others.

Neglecting positivity constraints, we model GPDs by means of a conformal $\mathrm{SL}(2, \mathbb{R})$ partial wave expansion, which can be written as a Mellin-Barnes integral [630]:

$$
F\left(x, \eta, t, \mu^{2}\right)=\frac{i}{2} \int_{c-i \infty}^{c+i \infty} d j \frac{p_{j}(x, \eta)}{\sin (\pi j)} F_{j}\left(\eta, t, \mu^{2}\right) .
$$

Here, $p_{j}(x, \eta)$ are the partial waves given in terms of associated Legendre functions of the first and second kind, and the integral conformal GPD moments $F_{j}\left(\eta, t, \mu^{2}\right)$ are even polynomials in $\eta$ of order $j$ or $j+1$. Other representations of GPDs based on the $\mathrm{SL}(2, \mathbb{R})$ partial wave expansion include the so-called "dual" parameterization 6631, 632, 633, 634.

In the Mellin-Barnes representation, the CFFs possess a rather convenient form, e.g., (3.49) can be rewritten in the following form [627, 635]:

$$
\begin{aligned}
\left\{\begin{array}{c}
\mathcal{H} \\
\mathcal{E}
\end{array}\right\}\left(x_{B}, t, Q^{2}\right) \stackrel{\mathrm{LO}}{=} & \frac{1}{2 i} \int_{c-i \infty}^{c+i \infty} d j \xi^{-j-1}\left[i+\tan \left(\frac{\pi j}{2}\right)\right] \\
& \times\left.\frac{2^{j+1} \Gamma(j+5 / 2)}{\Gamma(3 / 2) \Gamma(j+3)}\left\{\begin{array}{c}
H_{j} \\
E_{j}
\end{array}\right\}\left(\eta=\xi, t, Q^{2}\right)\right|_{\xi=\frac{x_{B}}{2-x_{B}}} .
\end{aligned}
$$

This integral is numerically implemented in an efficient routine in two different factorization schemes, including the standard minimal subtraction $(\overline{\mathrm{MS}})$ one at next-to-leading order (NLO) accuracy. Further advantages of this representation are: 
(i) The conformal moments evolve autonomously at LO.

(ii) One can employ conformal symmetry to obtain next-to-next-to-leading order (NNLO) corrections to the DVCS amplitude [635, 636].

(iii) PDF and form factor constraints can be straightforwardly implemented. Namely, $F_{j}\left(\eta=0, t=0, \mu^{2}\right)$ are the Mellin moments of PDFs, $F_{j=0}$ are partonic contributions to elastic form factors, $H_{j=1}$ and $E_{j=1}$ are the energy-momentum tensor form factors, and for general $j$ one immediately makes contact to lattice measurements.

To parameterize the degrees of freedom that can be accessed in hard exclusive reactions, one can expand the conformal moments in terms of $t$-channel $\mathrm{SO}(3)$ partial waves expressed

in terms of the Wigner rotation matrices $\hat{d}_{j}(\eta)\left(\hat{d}_{j}(\eta=0)=1\right)$ [637]. An effective GPD model at given input scale $Q_{0}^{2}$ is provided by taking into account three partial waves,

$$
F_{j}(\eta, t)=\hat{d}_{j}(\eta) f_{j}^{j+1}(t)+\eta^{2} \hat{d}_{j-2}(\eta) f_{j}^{j-1}(t)+\eta^{4} \hat{d}_{j-4}(\eta) f_{j}^{j-3}(t),
$$

which is valid for integral $j \geq 4$. In the simplest version of such a model, one might introduce just two additional parameters by setting the non-leading partial wave amplitudes to:

$$
f_{j}^{j-k}(\eta, t)=s_{k} f_{j}^{j+1}(\eta, t), \quad k=2,4, \ldots
$$

Such a model allows us to control the size of the GPD on the cross-over line and its $Q^{2}$ evolution, see fig. 3.28. A flexible parameterization of the skewness effect in the large $x$ region requires to decorate the skewness parameters $s_{k}$ with some $j$ dependence and for more convenience one might replace Wigner's rotation matrices by some effective $\mathrm{SO}(3)$ partial waves.

\subsubsection{GPDs from hard exclusive measurements}

Based on the experimental data set from the collider experiments $\mathrm{H} 1$ and ZEUS at DESY, the fixed target experiment HERMES at DESY, and the Hall A, CLAS, and Hall C experiments at JLAB, GPDs have been accessed from hard exclusive meson and photon electroproduction in the last few years. Favorably, DVCS enters as a subprocess into the hard photon electroproduction where its interference with the Bethe-Heitler (BH) bremsstrahlung process provides variety of handles on the real and imaginary part of twist-two and twistthree CFFs 625, 638. However, switching from a proton to a neutron target allows only for a partial flavor separation, which is much more intricate than in DIS. On the other hand, DVEM can be used as a flavor filter, however, here one expects that both radiative 639, 640, 641] and (non-factorizable) higher-twist contributions might be rather important. The onset of the collinear description remains here an issue which should be explored.

For the DVCS process, the collinear factorization approach has been employed in a specific scheme up to NNLO in the small $x_{B}$ region [627, 635, 636]. It turns out that NLO corrections are moderate, while NNLO ones are becoming much smaller 627. Experimentally, the unpolarized DVCS cross section has been provided by the H1 and ZEUS collaborations 642, 643, 644, 645]. In the collider kinematics, the DVCS cross section is primarily given in terms of two CFFs, $\mathcal{H}$ and $\mathcal{E}$ :

$$
\left.\frac{d \sigma^{\text {DVCS }}}{d t}\left(W, t, Q^{2}\right) \approx \frac{\pi \alpha^{2}}{\mathcal{Q}^{4}} \frac{W^{2} x_{B}^{2}}{W^{2}+Q^{2}}\left[|\mathcal{H}|^{2}-\frac{t}{4 M_{p}^{2}}|\mathcal{E}|^{2}\right]\left(x_{B}, t, \mathcal{Q}^{2}\right)\right|_{x_{B} \approx \frac{Q^{2}}{W^{2}+Q^{2}}} .
$$




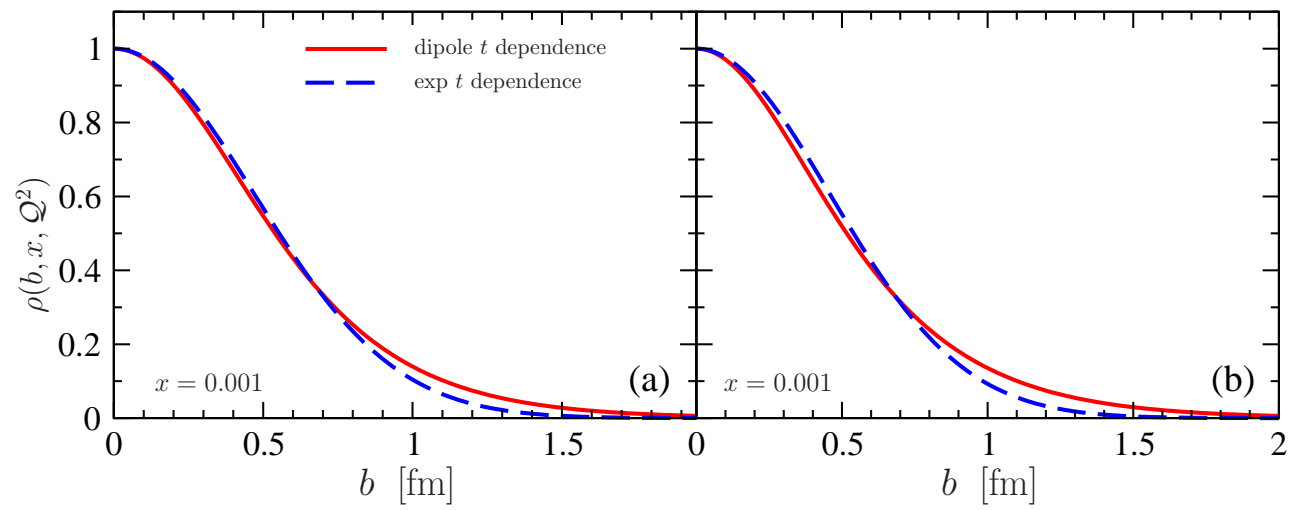

Figure 3.21. Quark (a) and gluon (b) transverse profile function (3.59) for $Q^{2}=4 \mathrm{GeV}^{2}$ and $x=10^{-3}$ from a six parameter DVCS fit 646.

Although at a fixed scale and to LO accuracy the CFFs are given by (dominant sea) quark GPDs, evolution will induce a gluonic contribution, too. Indeed, the experimental lever arm $3 \mathrm{GeV}^{2} \lesssim Q^{2} \lesssim 80 \mathrm{GeV}^{2}$ is sufficiently large to access the gluonic GPD. In our fitting procedure, the Mellin-Barnes integral was utilized within a $\mathrm{SO}(3)$ partial wave ansatz for the conformal moments and good fits $\left(\chi^{2} /\right.$ d.o.f. $\left.\approx 1\right)$ could be obtained at LO to NNLO accuracy, exemplifying that flexible GPD models were at hand. From such fits, one can then obtain the image of quark and gluon distributions. It is illustrated in figure 3.21 that in impact space, the (normalized) transverse profiles,

$$
\rho\left(b, x, Q^{2}\right)=\frac{\int_{-\infty}^{\infty} d^{2} \vec{\Delta} e^{i \vec{\Delta} \vec{b}} H\left(x, \eta=0, t=-\vec{\Delta}^{2}, Q^{2}\right)}{\int_{-\infty}^{\infty} d^{2} \vec{\Delta} H\left(x, \eta=0, t=-\vec{\Delta}^{2}, Q^{2}\right)},
$$

determined for dipole and exponential $t$-dependence of $H$, mainly differ for distances larger than the disc radius of the proton, i.e., for $b>0.6 \mathrm{fm}$. Hence, the larger values of the transverse widths, $\sqrt{\left\langle\vec{b}^{2}\right\rangle} \approx 0.9 \mathrm{fm}$ and $\sqrt{\left\langle\vec{b}^{2}\right\rangle} \approx 0.8 \mathrm{fm}$ for the dipole ansatz, arise from the long-range tail of the profile function, see the solid curves in figure 3.21, For an exponential ansatz, we find slightly smaller values $\sqrt{\left\langle\vec{b}^{2}\right\rangle_{\text {sea }}} \approx 0.7 \mathrm{fm}$ and $\sqrt{\left\langle\vec{b}^{2}\right\rangle_{\mathrm{G}}} \approx 0.6 \mathrm{fm}$, where the gluonic one is compatible with the analysis of $J / \psi$ production [594]. Note that the model uncertainty in the extrapolation of the GPD to $t=0$ corresponds to the uncertainty in the long-range tail. Moreover, the model uncertainty of the extrapolation into the region $-t>1 \mathrm{GeV}^{2}$ is essentially canceled in the profile (3.59) normalized at $b=0$.

We also note that at LO the gluonic GPD (as the gluonic PDF) is rather steep and radiative corrections might provide a large GPD/PDF reparameterization effect, which will be studied in more detail in the future. Our first successful LO description of DVCS within a flexible GPD model [646] is in agreement with aligned-jet model considerations [647]. We also mention that an attempt has been undertaken to access the $\mathcal{E}$ CFF from the beam charge asymmetry measurement [648, proportional to the combination $\Re$ e $\left[F_{1}(t) \mathcal{H}-\frac{t}{4 M^{2}} F_{2}(t) \mathcal{E}\right]$. Unfortunately, the size of the experimental uncertainties does not allow one to separate the $\mathcal{H}$ and $\mathcal{E}$ contributions.

An approach analogous to the one employed for DVCS [627] is also suitable for LO and NLO analysis of DVEM. Hence, one can simultaneously make use of DVCS and DVEM measurements in a global fitting procedure, which is in progress.

GPD studies were also performed for the DVCS process in the fixed target kinematics 
to LO accuracy. In this region, relying on the scaling hypothesis, one might directly ask for the value of the GPDs on their cross-over line. For instance, for valence quarks we use the following generically motivated ansatz:

$$
H^{\mathrm{val}}(x, x, t)=\frac{1.35 r}{1+x}\left(\frac{2 x}{1+x}\right)^{-\alpha(t)}\left(\frac{1-x}{1+x}\right)^{b}\left(1-\frac{1-x}{1+x} \frac{t}{M^{\mathrm{val}}}\right)^{-1},
$$

where $r=\lim _{x \rightarrow 0} H(x, x) / H(x, 0)$ is the skewness ratio; $\alpha(t)=0.43+0.85 t / \mathrm{GeV}^{2} ; b$ controls the $x \rightarrow 1$ limit and $M^{\text {val }}$ controls the residual $t$-dependence, which we set to $M^{\mathrm{val}}=0.8 \mathrm{GeV}$. For the forward limit $q(x)=H(x, 0)$, we used the LO parameterization of Alekhin [649]. The generic $(-t)^{-2}$ fall-off at large $-t$ for generalized form factors is indirectly encoded in the Regge-trajectory and the residual $t$ dependence is modeled by a monopole form with an $x$-dependent cut-off mass. The subtraction constant (3.51) is taken an a dipole form:

$$
\mathcal{D}(t)=d\left(1-\frac{t}{M_{d}^{2}}\right)^{-2}
$$

In a first global fit [646] to hard exclusive photon electroproduction off unpolarized proton, we took sea quark and gluon GPD models with two $\mathrm{SO}(3)$ partial waves at small $x$, reparameterized the outcome from $\mathrm{H} 1$ and ZEUS DVCS fits at $Q^{2}=2 \mathrm{GeV}^{2}$, and employed it in fits of fixed target data within the scaling hypothesis. To relate the CFFs with the observables, we employed the BKM formulas 625] within the 'hot-fix' convention [650] and used the Sachs parameterization for the electromagnetic form factors. Thereby, we utilized the "dispersion relation" (3.5013.51), where the ansatz (3.60) specifies a valence-like GPD on the cross-over line. Besides the subtraction constant (3.61), we also included the parameterfree pion-pole model for the $\tilde{E}$ GPD 651 and parameterized the $\widetilde{H}$ GPD rather analogously to (3.60) with $b=3 / 2$. For the fixed target fits, we chose two data sets resulting in two fits (KM09a and KM09b). Out fit gives:

$$
\begin{aligned}
& \text { KM09a: } \quad b^{\text {sea }}=3.09, \quad r^{\mathrm{val}}=0.95, \quad b^{\mathrm{val}}=0.45, \quad d=-0.24, \quad M_{d}=0.5 \mathrm{GeV}, \\
& \text { KM09b: } \quad b^{\text {sea }}=4.60, \quad r^{\text {val }}=1.11, \quad b^{\text {val }}=2.40, \quad d=-6.00, \quad M_{d}=1.5 \mathrm{GeV}(.3 .62)
\end{aligned}
$$

These values of the fit parameters are compatible with our generic expectations: the skewness effect at small $x$ should be small, i.e., $r \sim 1$, the subtraction constant should be negative [574, 652], and, according to counting rules [653], $b$ should be smaller than the corresponding $\beta$ value of the relevant PDF [646, 654.

To improve the models that we just described, we now use a hybrid technique where the sea quark and gluon GPDs are represented in terms of conformal moments, while, for convenience, the valence quarks are still modeled in momentum fraction space and within the "dispersion integral" approach. Also, the residue of the pion-pole contribution is now considered as a parameter, and the Hall A data forces a roughly three times larger value than expected from the model 651. Optionally, we might also use the improved formulae from 655 applicable for a longitudinally polarized target. The new parameters read:

$$
\begin{array}{ll}
\text { KM10a: } & r^{\mathrm{val}}=0.88, \quad M^{\mathrm{val}}=1.5 \mathrm{GeV}, \quad b^{\mathrm{val}}=0.40, d=-1.72, \quad M_{d}=2.0 \mathrm{GeV}, \\
\text { KM10b: } & r^{\mathrm{val}}=0.81, \quad M^{\mathrm{val}}=0.8 \mathrm{GeV}, \quad b^{\mathrm{val}}=0.77, d=-5.43, \quad M_{d}=1.33 \mathrm{Ge} \text { (3.63) }
\end{array}
$$

Note that for the valence part of the $H$ GPD, these results are qualitatively compatible with those from the pure KM09 "dispersion relation" fits. 


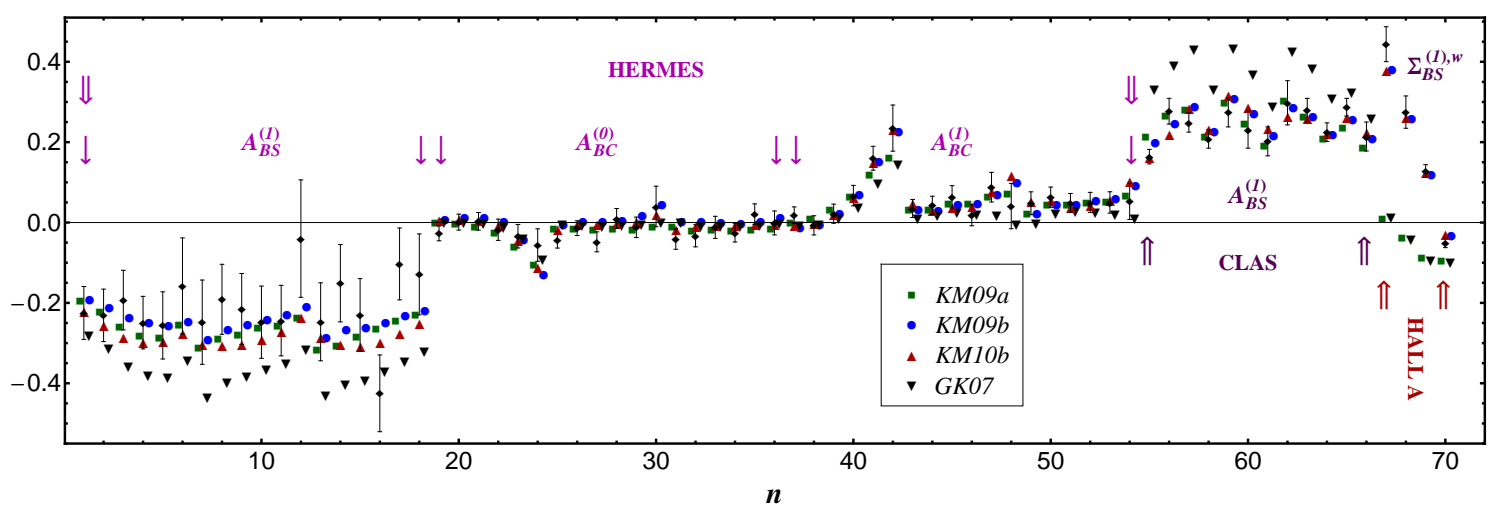

Figure 3.22. Experimental measurements for fixed target kinematics (circles) labeled by data point number $n$ : $A_{\mathrm{BS}}^{(1)}(1-18), A_{\mathrm{BC}}^{(0)}(19-36), A_{\mathrm{BC}}^{(1)}(37-54)$ from 656 ; $A_{\mathrm{BS}}^{(1)}(55-66)$ and $\Sigma_{\mathrm{BS}}^{(1), w}(67-70)$ are derived from [657] and 658]. Model results are from the "dispersion-relation" fits KMO9a without Hall A data 646] (squares, slightly shifted to the left) and KMO9b with the Hall A data (circles, slightly shifted to the right), hybrid model fit KM10b (triangles-up), and a hand-bag prediction GK07 from hard vector meson production (triangles-down, slightly shifted to the r.h.s.) 659].

We also performed an additional fit where we directly used the harmonics of beam spin sums and differences measured by Hall A (fit KM10). The results of our two "dispersionrelation" fits and three hybrid model fits are available as a computer program providing the four-fold cross section of polarized lepton scattering on unpolarized proton for a given kinematics, see http://calculon.phy.hr/gpd/. Unlike "dispersion-relation" fits, the hybrid model fits, where LO evolution of sea quark and gluon GPDs has been taken into account, are suitable for estimates in the small $x_{B}$ region.

In figure 3.22 we confront our fit results $\left(\chi^{2} /\right.$ d.o.f. $\approx 1$ w.r.t. the employed data sets) to experimental data: KM09a (squares), KM09b (circles), and the hybrid model fit KM10b (triangles-up) in which we now utilized the improved formulae set 655] and the Kelly form factor parameterization [660. We also include the predictions from the GK07 model [659] (triangles-down), where we adopt the hypothesis of $H$ dominance. Qualitatively, these predictions are consistent with a VGG2 code estimate, which tends to over-estimate the BSAs [657, 656] and describes the BCAs from HERMES rather well without the $D$-term [661]. This is perhaps not astonishing, since the employed $H$ GPD model relies on Radyushkin's DD ansatz, too. We would like to emphasize that at LO, the GKO7 model is in reasonable agreement with the H1 and ZEUS DVCS data $\left(\chi^{2} /\right.$ d.o.f. $\left.\approx 2\right)$, essentially thanks to the rather small and stable skewness ratio $r^{\text {sea }}$ of sea quarks.

Longitudinally polarized target data from CLAS [662] and HERMES 663] provide a handle on $\widetilde{H}$ 625], where the mean values of CFF fits 664] in the JLAB kinematics give two to three times bigger $\widetilde{H}$ contribution compared to our expectations $\left(r_{\widetilde{H}} \simeq 1, b_{\widetilde{H}} \simeq 2\right)$. These findings are one to two standard deviations away from our big $\widetilde{H}$ ad hoc scenario of the $K M 09 \mathrm{~b}$ fit, which is indeed disfavored by the longitudinally polarized proton data. We like to add that with our present hybrid model a reasonable global fit, such as KM10 above, is possible. In such a fit, the Hall A data require a rather large pion pole contribution, inducing a large DVCS cross section contribution. Still, we have not included the transversal

\footnotetext{
${ }^{2}$ VGG refers to a computer code originally written by M. Vanderhaeghen, P. Guichon, and M. Guidal. To our best knowledge, the code for DVCS presently used by experimentalists employs a model that adopts Radyushkin's DD ansatz [574].
} 

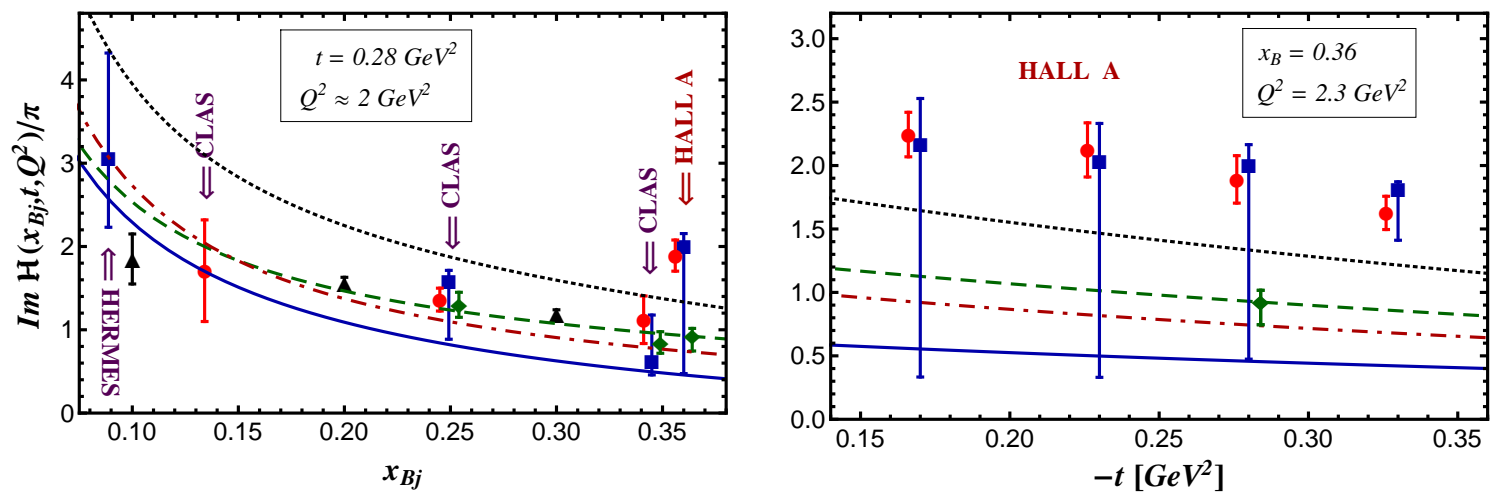

Figure 3.23. $\Im \mathrm{m} \mathcal{H} / \pi$ from different strategies: our DVCS fits [dashed (solid) curve excludes (includes) Hall A data from "dispersion relation" KM09a (KM09b) 646] and hybrid KM10b (dashdotted) models], GK07 model from DVEM (dotted), seven-fold CFF fit 666, 667] with boundary conditions (squares), $\mathcal{H}, \widetilde{\mathcal{H}} \mathrm{CFF}$ fit 664 (diamonds), smeared conformal partial wave model fit 668 within $H$ GPD (circles). The triangles result from our neural network fit, cf. figure 3.24

target data from the HERMES collaboration [661] or the neutron data from Hall A 665].

So far we did not study model uncertainties or experimental error propagation, since both tasks might be rather intricate. To illuminate this, in figure 3.23 we compare our results for $\Im m \mathcal{H}\left(x_{B}, t\right) / \pi$ with the results that do provide error estimates. The squares arise from constrained least squares fits [666, 667] at given kinematic means of HERMES and JLAB measurements on unpolarized proton, where the imaginary and real parts of twist-two CFFs are taken as parameters. The huge size of the error bars shows the limited accuracy with which $H$ can be extracted from unpolarized proton data alone [625]. A pure $H$ GPD model fit 668 (circles) to JLAB data provides much smaller errors, arising from error propagation and some estimated model uncertainties. All three of our curves are compatible with the findings [666, 667] and the $H$ GPD model analysis [668] of CLAS data. However, for Hall A kinematics, the deviation of the two predictions that are based on the $H$ dominance hypothesis (the dashed curve and circles in the right panel) are obvious and are explained by our underestimation of the cross section normalization by about $50 \%$. Moreover, the quality of fit $668, \chi^{2} /$ d.o.f. $\sim 1.7$, might provide another indication that CLAS and Hall A data are not compatible, when this hypothesis is assumed, see, e.g., the two rightmost circles in the left panel for CLAS $\left(x_{B}=0.34, t=-0.3 \mathrm{GeV}^{2}, Q^{2}=2.3\right.$ $\left.\mathrm{GeV}^{2}\right)$ and Hall $\mathrm{A}\left(x_{\mathrm{Bj}}=0.36, t=-0.28 \mathrm{GeV}^{2}, Q^{2}=2.3 \mathrm{GeV}^{2}\right)$. While the pure $\mathcal{H}$ and $\widetilde{\mathcal{H}} \mathrm{CFF}$ fit 664 (diamonds), including longitudinally polarized target data, is within error bars inconsistent with the $H$ dominated scenario [668] (circles), it (accidentally) reproduces our dashed curve.

Another source of uncertainties are twist-three contributions and perhaps also gluon transversity related contributions, which might be strongly affected by twist-four effects [669].

All this exemplifies that within (strong) assumptions and the present set of measurements, the propagated experimental errors cannot be taken as an estimate of GPD uncertainties. An error estimation in model fits might be based on twist-two sector projection technique 625, boundaries for the unconstrained model degrees of freedom, and error propagation in the twist-two sector. Alternatively, neural networks, already successfully used for PDF fits [47, may be an ideal tool to extract CFFs or GPDs. In figure 3.24, we present a first example in which, within the $H$-dominance hypothesis, $\mathcal{H}$ is extracted using a pro- 


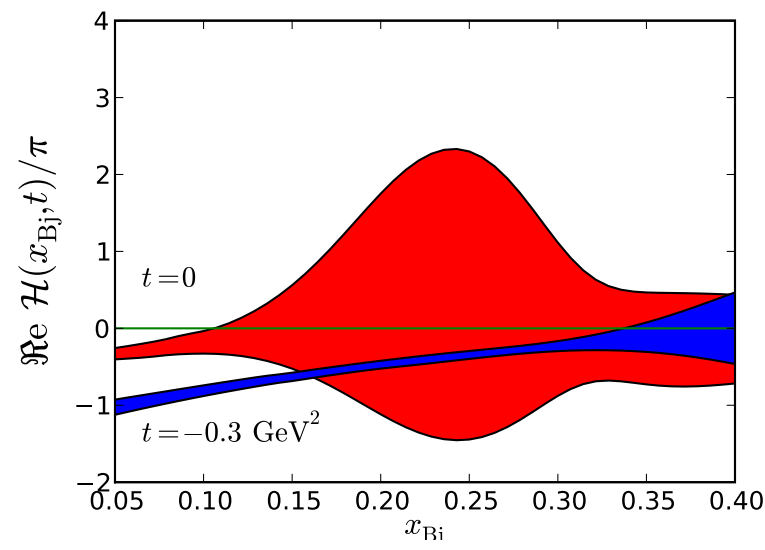

Figure 3.24. Neural network extraction of $\Re \mathrm{e} \mathcal{H}\left(x_{B}, t\right) / \pi$ from BCA [656] and BSA [657] data.

cedure similar to the one of [670]. Here, 50 feed-forward neural nets with two hidden layers were trained using HERMES BCA [656] and CLAS BSA [657] data. Hence, only the experimental errors were propagated, which, in the absence of a model hypothesis, become large for the $t \rightarrow 0$ extrapolation.

\subsubsection{Potential of an electron-ion collider}

A high luminosity machine in the collider mode with polarized electron and proton or ion beams would be an ideal instrument to quantify QCD phenomena. It is expected that such a machine, combined with designated detectors, would allow for precise measurements of exclusive channels. Besides hard exclusive vector meson and photon electroproduction, one might address the behavior of parity-odd GPDs $\widetilde{\mathcal{H}}$ (related to polarized PDFs) and $\tilde{\mathcal{E}}$ via the exclusive production of pions even in the small $x$ region. It is obvious from what was said above that an access of GPDs requires a large data set with small errors. In the following we would like to illustrate the potential of such a machine for DVCS studies, where we also address the GPD deconvolution problem.

Let us remind that already the isolation of CFFs is rather intricate. For a spin- $1 / 2$ target, we have four twist-two, four twist-three, and four gluon transversity-related complex valued CFFs. The photon helicity non-flip amplitudes are dominated by twist-two CFFs, the transverse-longitudinal flip amplitudes by twist-three effects, and the transverse-transverse flip ones by gluon transversity. Hence, the first, second, and third harmonics w.r.t. the azimuthal angle of the interference term are twist-two, twist-three, and gluon transversity dominated, respectively. In an ideal experiment, assuming that transverse photon helicity flip effects are negligible, cross section measurements would allow to separate the sixteen quantities that are then given in terms of twist-two and twist-three CFFs. The reader might find a more detailed discussion, based on a $1 / Q$ expansion, in [625]. We also note that the definition of CFFs is convention-dependent.

In a twist-two analyzes on unpolarized, longitudinally and transversally polarized protons, one might be able to disentangle the four different twist-two CFFs via the measurement of single beam and target spin asymmetries. In figure 3.25, we illustrate that the beam spin asymmetry for a proton target (solid curves),

$$
A_{\mathrm{BS}}^{(1)} \propto \frac{\sqrt{t_{\min }-t}}{2 M} y\left[F_{1}(t) H\left(\xi, \xi, t, Q^{2}\right)-\frac{t}{4 M^{2}} F_{2}(t) E\left(\xi, \xi, t, Q^{2}\right)+\cdots\right],
$$




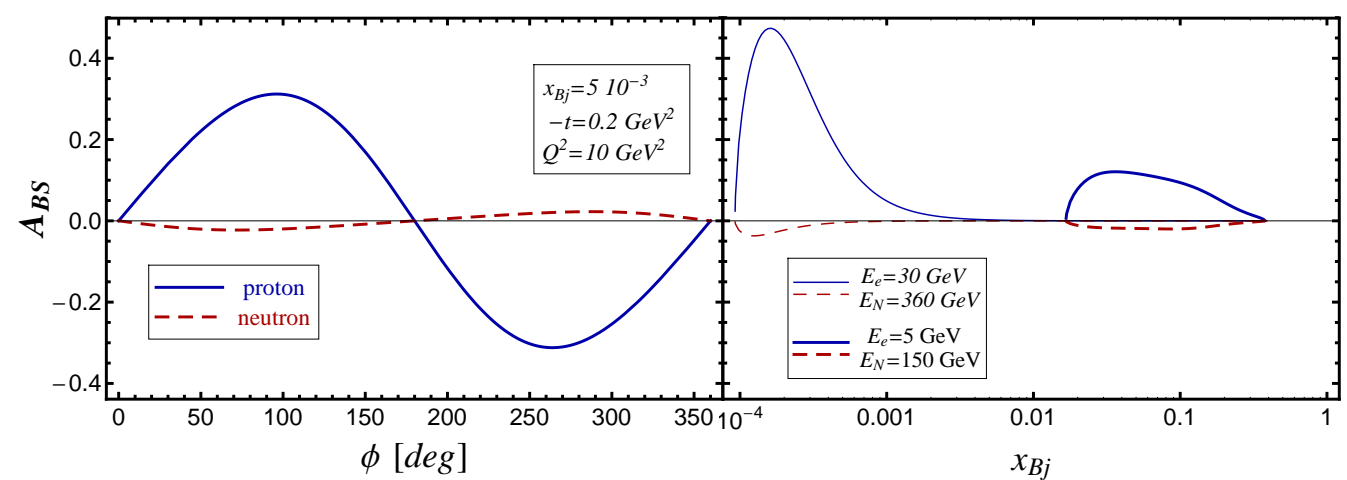

Figure 3.25. KM10b model estimate for the DVCS beam spin asymmetry with a proton (solid) and neutron (dashed) target. Left panel: $A_{\mathrm{BS}} v s$. $\phi$ for $E_{N}=250 \mathrm{GeV}, E_{e}=5 \mathrm{GeV}, x_{B}=5 \times 10^{-3}$, $Q^{2}=10 \mathrm{GeV}^{2}$, and $t=-0.2 \mathrm{GeV}^{2}$. Right panel: Amplitude $A_{\mathrm{BS}}^{(1)}$ of the first harmonic vs. $x_{\mathrm{Bj}}$ at $t=-0.2 \mathrm{GeV}^{2}$ for small $x_{B}$ (thin) $\left[E_{e}=30 \mathrm{GeV}, E_{p}=360 \mathrm{GeV}, Q^{2}=4 \mathrm{GeV}^{2}\right]$ and large $x_{B}$ (thick) $\left[E_{e}=5 \mathrm{GeV}, E_{p}=150 \mathrm{GeV}, Q^{2}=50 \mathrm{GeV}^{2}\right]$ kinematics.

might be rather sizeable over a large kinematical region in which the lepton energy loss $y$ is not too small. Here the helicity conserved $\mathrm{CFF} \mathcal{H}$ is the dominant contribution, while $\mathcal{E}$ appears with a kinematic suppression factor $t / 4 M^{2}$, induced by the helicity flip. For a neutron target, the $\mathcal{H}$ contribution is suppressed by the accompanying Dirac form factor $F_{1}^{n}$ $\left(F_{1}^{n}(t=0)=0\right)$ and, hence, one becomes sensitive to the CFF $\mathcal{E}$. Unfortunately, one also has to worry about other non-dominant CFF contributions, indicated by the ellipsis. Note that the asymmetry for the neutron (dashed curves in figure 3.25) might be underestimated since we set in our model $E\left(x, x, t, Q^{2}\right)$ to zero.

For a longitudinally polarized target, the asymmetry

$$
A_{\mathrm{TS}}^{\Rightarrow(1)} \propto \frac{\sqrt{t_{\mathrm{min}}-t}}{2 M}\left[F_{1}(t) \widetilde{H}\left(\xi, \xi, t, \mathcal{Q}^{2}\right)-\frac{t}{4 M^{2}} F_{2}(t) \xi \widetilde{E}\left(\xi, \xi, t, \mathcal{Q}^{2}\right)+\cdots\right]
$$

is sensitive to the GPD $\widetilde{H}$, while $\xi \widetilde{E}$ and other GPDs might contribute to some extent. Naively, one would expect that this asymmetry vanishes in the small $x_{B}$ region and might be sizeable at $x_{B} \sim 0.1$, see the left panel of figure 3.26. Not much is known about the small $x$ behavior of $\widetilde{H}$ and it might be even accessible at smaller values of $x_{B}$, as illustrated by the KM09b model with its big $\widetilde{H}$ contribution (solid curve, the right panel of figure 3.26). For a neutron target, the asymmetry becomes sensitive to the $\xi \widetilde{E}$ GPD. Note that here the factor $\xi$ is annulled by a conventional $1 / \xi$ factor in the definition of the $\widetilde{E}$ GPD.

Finally, we emphasize that a single spin asymmetry measurement with a transversally polarized target provides another handle on the helicity-flip GPDs $E$ and $\widetilde{E}$. If the target spin is perpendicular to the reaction plane, the asymmetry

$$
A_{\mathrm{TS}}^{\Uparrow(1)} \propto \frac{t}{4 M^{2}}\left[F_{2}(t) H\left(\xi, \xi, t, \mathcal{Q}^{2}\right)-F_{1}(t) E\left(\xi, \xi, t, \mathcal{Q}^{2}\right)+\cdots\right],
$$

is dominated by a linear combination of the GPDs $H$ and $E$. In the case when the target spin is aligned with the reaction plane, the asymmetry

$$
A_{\mathrm{TS}}^{\Downarrow(1)} \propto \frac{t}{4 M^{2}}\left[F_{2}(t) \widetilde{H}\left(\xi, \xi, t, \mathcal{Q}^{2}\right)-F_{1}(t) \xi \widetilde{E}\left(\xi, \xi, t, \mathcal{Q}^{2}\right)+\cdots\right]
$$




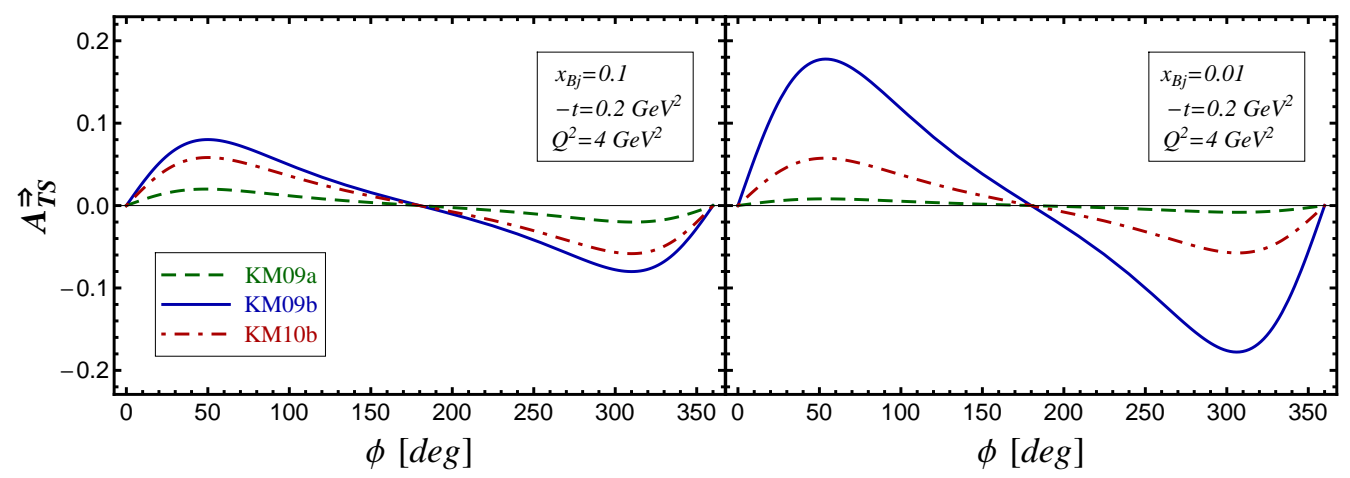

Figure 3.26. DVCS longitudinal target spin asymmetry vs. $\phi$ for $K M 09 a$ (dashed), KM09b (solid), and $K M 10 b$ hybrid (dash-dotted) models at $E_{e}=5 \mathrm{GeV}, t=-0.2 \mathrm{GeV}^{2}, \mathcal{Q}^{2}=4 \mathrm{GeV}^{2}$ within $E_{p}=150 \mathrm{GeV}, x_{\mathrm{Bj}}=0.1$ (left) and $E_{p}=350 \mathrm{GeV}, x_{\mathrm{Bj}}=0.01$ (right).

Unfortunately, compared to the single beam spin (3.64) and longitudinal target (3.65) asymmetries, the transversally ones are kinematically suppressed by an additional factor $\sim \sqrt{-t} /(2 M)$ and, for a neutron target, in addition by the Dirac form factor $F_{1}(t)$.

Although the given formulae (3.64 3.67) are rather crude, they illustrate that a measurement of single spin asymmetries would allow to access the imaginary part of the four twist-two related CFFs. However, the normalization of these asymmetries depends to some extent also on the real part of the twist-two related CFFs and the remaining eight ones. Measurements of cross section differences would allow one to eliminate the normalization uncertainty, and in combination with the harmonic analysis, one can separate to some extent twist-two, twist-three, and gluon transversity contributions. However, the extracted harmonics might also be contaminated by DVCS cross section contributions which are bilinear in the CFFs. To get rid of these admixtures, one needs cross section measurements with a positron beam. Forming differences and sums of cross section measurements with both kinds of leptons, allows one to extract the pure interference and DVCS squared terms and, thus, might allow one to quantify twist-three effects. Existing data indicate that these effects are small as expected based on kinematic factors. However, even obtaining only an upper limit is important for the determination of the systematic uncertainties of twist-two CFFs.

We also emphasize that having both kinds of lepton beams available allows one to measure the real part of CFFs. In figure 3.27, we show the beam charge asymmetry,

$$
A_{\mathrm{BC}}^{(1)} \propto \Re \mathrm{e}\left[F_{1}(t) \mathcal{H}\left(x_{\mathrm{Bj}}, t, \mathcal{Q}^{2}\right)-\frac{t}{4 M^{2}} F_{2}(t) \mathcal{E}\left(x_{\mathrm{Bj}}, t, \mathcal{Q}^{2}\right)+\cdots\right]
$$

for an unpolarized target, which is expected to be sizeable. For a proton target, this asymmetry should possess a node in the transition from the valence to sea region(thick solid curve, right panel). In our parameterization, the real part of the $\mathcal{E}$ CFF is determined by the $\mathcal{D}$ subtraction term, which induces a sizeable asymmetry (thick dashed curve, right panel), even for a neutron target.

The large kinematical coverage of the proposed high-luminosity EIC raises the question: Can one utilize evolution, even at moderate $x_{B}$ values, to access GPDs away from their crossover line? Similarly to what has been done for the small $x_{B}$ region, we use the Mellin-Barnes integral technique to address the problem. Taking different non-leading $\mathrm{SO}(3)$ partial waves in the ansatz for the conformal moments (3.56 33.57), we build three different GPD models 


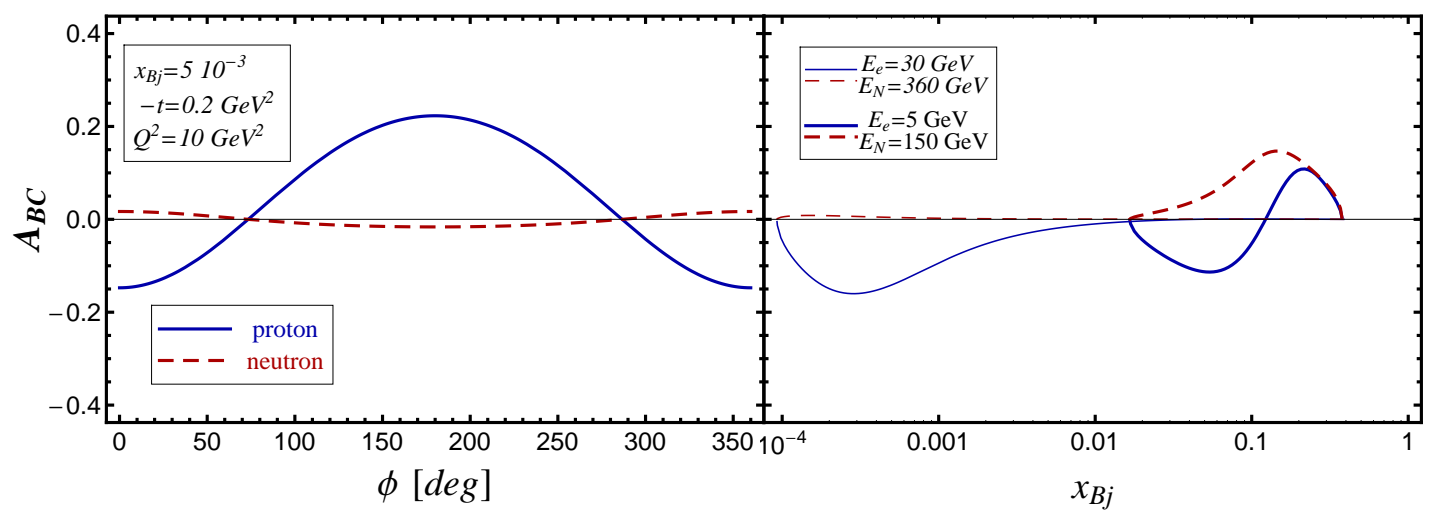

Figure 3.27. KM10b model estimate for the DVCS beam charge asymmetry with a proton (solid) and neutron (dashed) target. Left panel: $A_{\mathrm{BC}}$ vs. $\phi$ for $E_{N}=250 \mathrm{GeV}, E_{e}=5 \mathrm{GeV}, x_{B}=5 \times 10^{-3}$, $Q^{2}=10 \mathrm{GeV}^{2}$, and $t=-0.2 \mathrm{GeV}^{2}$. Right panel: Amplitude $A_{\mathrm{BC}}^{(1)}$ of the first harmonic vs. $x_{B}$ at $t=-0.2 \mathrm{GeV}^{2}$ for small $x_{B}$ (thin) $\left[E_{e}=30 \mathrm{GeV}, E_{p}=360 \mathrm{GeV}, Q^{2}=4 \mathrm{GeV}^{2}\right]$ and large $x_{B}$ (thick) $\left[E_{e}=5 \mathrm{GeV}, E_{p}=150 \mathrm{GeV}, Q^{2}=50 \mathrm{GeV}^{2}\right]$ kinematics.

for valence quarks that provide almost identical CFFs, see the upper left panel in figure 3.28 , They are compatible with (3.60) from the "dispersion-relation" fit KM09a (dotted curves). We note that the different model behavior at large $x_{B}$ results only in a small discrepancy for the real part of the CFF in the kinematics of interest. In the lower left panel of figure 3.28 , we illustrate that for fixed $\eta$, the $x$-shape of the three GPD models looks quite differently. Compared to the minimalist model (dotted curve), a model with a negative next-to-leading partial wave (solid) decreases the size of the GPD on the cross-over line $\eta=x$ and generates an oscillating behavior in the central region. The model with an alternating-sign $\mathrm{SO}(3)$ partial wave expansion (dash-dotted) possesses more pronounced oscillation effects in the central region or even nodes. In the third model (dashed curve), the reduction on the cross-over line is reached within a next-to-next leading $\mathrm{SO}(3)$ partial wave. Note that the GPDs in the region $\eta \ll x$ are governed by the $x$-behavior of the PDF analogues. In the right panels, we demonstrate that for a large lever arm in $Q^{2}$ (e.g., $Q^{2}=50 \mathrm{GeV}^{2}$ ), the evolution effects are important in the valence quark region. However, for CFFs (the upper right panel), the discriminating power of evolution effects remains moderate even if the GPD shapes look rather different.

\subsubsection{Conclusions and summary}

With all the theoretical tools sketched above plus those which are presently under development, it is clear that our understanding of hadron structure will be revolutionized once most of the diverse asymmetries are measured with percent or permille precision (depending on the observable). At present, first steps have been undertaken to access GPDs from experimental data in the small $x_{B}$ region and in the fixed target kinematics providing us with some insight into the GPD $H$. In particular, for DVCS in the fixed target kinematics, LO model fits are compatible with least-square CFF fits and first results from neural networks (assuming $H$ dominance). The large uncertainties in extracting CFFs are mainly related to the lack of experimental data. Thus, not only the extraction of the very desired $\mathcal{E}$ playing an important role in the "spin-puzzle", but also of other CFFs, requires a comprehensive measurement of all possible observables in dedicated experiments. A further comparison 

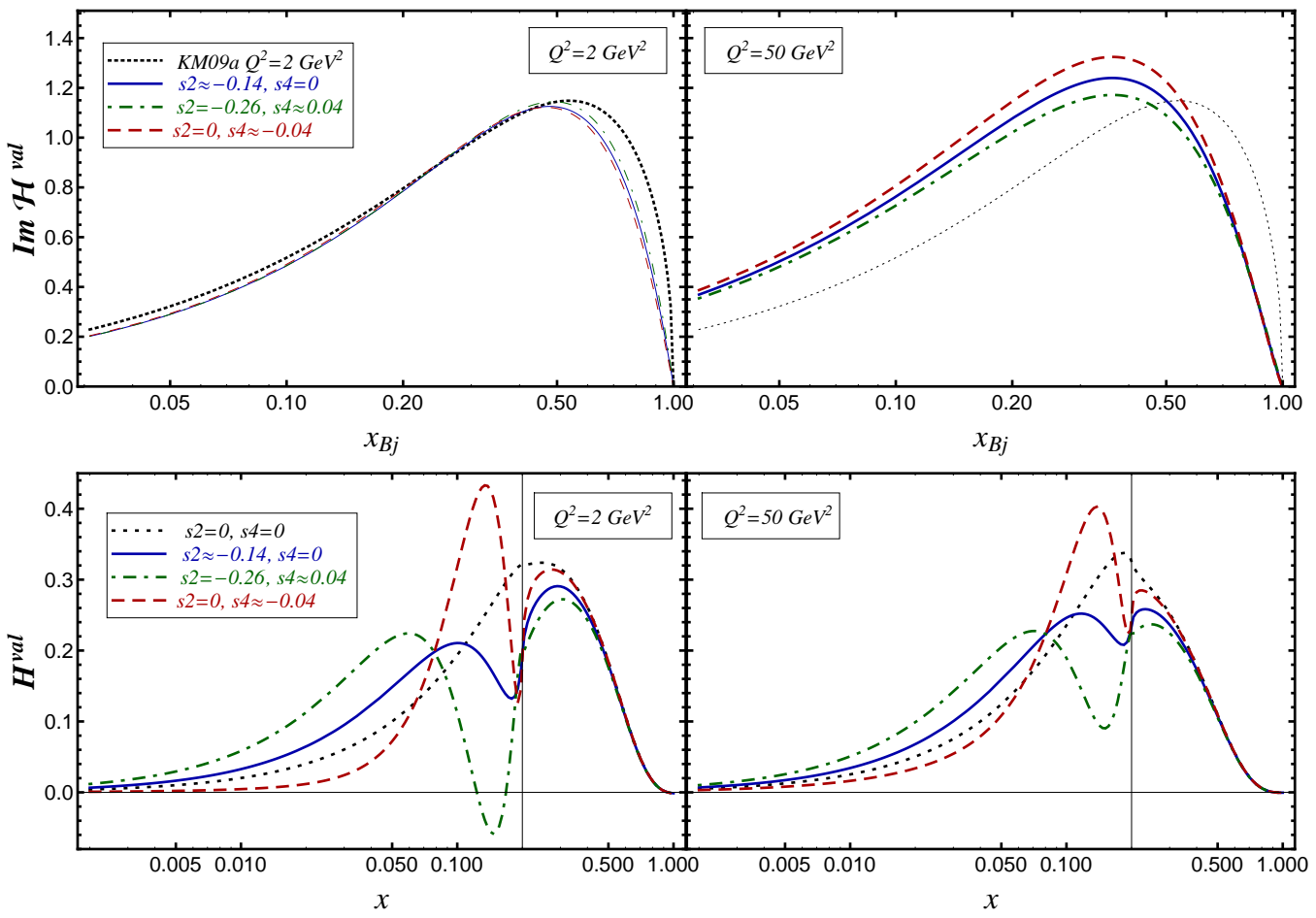

Figure 3.28. Upper left panel: The valence-like contribution (3.60) to the CFF $\mathcal{H}$ extracted with a "dispersion-relation" fit KM09a from fixed target measurements (dotted) at $t=-0.2 \mathrm{GeV}^{2}$ and $Q^{2}=2 \mathrm{GeV}^{2}$ vs. $x_{B}$ together with various models. Lower left panel: The corresponding models of the GPD $x H\left(x, \eta, t, \mathcal{Q}^{2}\right)$ together with a minimalist GPD parameterization (dotted curve) vs. $x$ at $\eta=0.2, t=-0.2 \mathrm{GeV}^{2}$, and $Q^{2}=2 \mathrm{GeV}^{2}$. The same quantities at $Q^{2}=50 \mathrm{GeV}^{2}$ are displayed in the right panels.

shows that while in the valence region the extracted quark GPDs are somewhat different, they become compatible for small $x$. The main difference lies in the gluonic sector; a more appropriate analysis requires the inclusion of radiative corrections in a global fitting procedure, which is in progress. We should also mention here that hard exclusive processes with nuclei, which at present are not extensively studied, open a new window for the partonic view of nuclei.

Imaging the partonic content of the nucleon and the phenomenological access to the proton spin sum rule from hard exclusive processes can only be reached through proper understanding of GPD models. We also point out that GPDs can also be formulated in terms of an effective nucleon (light-cone) wave function, which links GPDs to transverse momentum dependent parton distributions. The whole framework consisting of perturbative QCD, lattice simulations, and dynamical modeling is available to reveal GPDs and access the nucleon wave function. Such a unifying description can be considered as the primary goal in quantifying the partonic picture. While such a task looks rather straightforward, much effort is needed on the theoretical, phenomenological, and experimental sides, with experimental data with small uncertainties playing the key role. A high-luminosity EIC is an ideal machine that would cover a wide kinematical range and complement the planned fixed target experiments at JLab@12 GeV. Thus, besides new measurements, an EIC has a great potential to significantly improve existing data sets.

Acknowledgments. We are grateful to P. Kroll for many fruitful discussions. 


\title{
3.9 Monte Carlo studies on DVCS with an EIC
}

\author{
Salvatore Fazio
}

\subsubsection{Exclusive processes with a dedicated EIC detector}

Our current knowledge of the role of gluons in hadronic matter comes mainly from DIS experiments of electrons off protons most notably from HERA at DESY. Although electrons only interact with electrically charged particles, and gluons carry only color charge, a highenergy electron beam can still be used as an excellent gluon microscope.

The HERA physics program of ep collisions surprisingly showed a large fraction of diffractive events contributing $10-15 \%$ to the total DIS cross-section. One of the key signatures of these "diffractive" events is an intact proton traveling at nearly beam energies, together with a gap in rapidity before some final-state particles are produced at mid-rapidity. However, the detectors (H1 and ZEUS) were not optimized for this important physics and were unable to measure the scattered proton; this was only achievable after a program of upgrades. In fact, to measure diffractive physics events, it is desirable to have very forward detectors at small angles with respect to the beam line, referred to as "Roman Pots". Other requirements are that the detector should be able to measure all processes: inclusive $\left(e p \rightarrow e^{\prime} X\right)$, semi-inclusive ( $e p \rightarrow e^{\prime} X+$ hadrons), and exclusive (e.g., $e p \rightarrow e^{\prime} p+J / \psi$ ) reactions. The requirements for $e p$ and $e A$ collisions are very similar, the only additional complication in $e A$ collisions arises from the need to tag the struck nucleus, or to veto events with the nucleus break-up by detecting neutrons and other breakup products with high efficiencies.

Briefly, a possible EIC detector consists (in the barrel region at mid-rapidity) of a solenoidal field with Si tracking with full rapidity coverage around the interaction point itself, followed by Cherenkov detectors for particle identification and then by both electromagnetic and hadronic calorimeters. There are further trackers and calorimeters at forward rapidities, but this time, the magnetic field is a dipole. A Roman Pots spectrometer can be installed along the beam-pipe in the direction of the outgoing proton. This is just a starting point and other technologies are under active investigation.

\subsubsection{DVCS and GPDs: from HERA to an EIC}

Measurements of observables associated with hard exclusive processes at an $e p / e A$ collider requires substantially higher luminosities than traditional inclusive DIS because of the small cross sections and the need for differential measurements. The detectors and the interaction region have to be designed to permit full reconstruction of the final state.

In assessing the prospects for measurements of exclusive processes in $e p$ scattering at collider energies, $W^{2} \gg 10 \mathrm{GeV}^{2}$, one needs to distinguish between "diffractive" (no exchange of quantum numbers between the target and the projectile/produced system) and "non-diffractive" processes (exchange of quantum numbers). In diffractive channels, such as $J / \psi, \rho, \phi$ production and DVCS (production of a real photon), the cross sections rapidly rise with the collision energy, $W$. At large $Q^{2}$, these processes probe the gluon GPD and/or the singlet quark GPD. In non-diffractive channels, such as $\pi^{ \pm}, \pi^{0}, \rho^{+}, K$ production, the cross sections decrease with energy. These processes at high $Q^{2}$ probe the flavor/charge/spin non-singlet quark GPDs describing the quark structure of the target.

The final state of a DVCS event, shown in figure $3.29 \mathrm{a}$, contains one track and two 


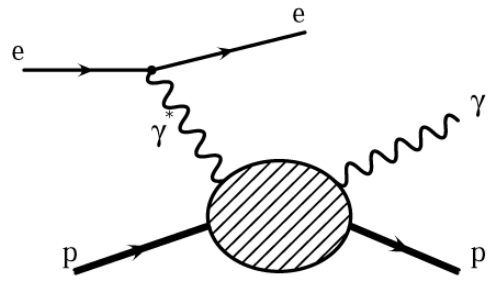

a)

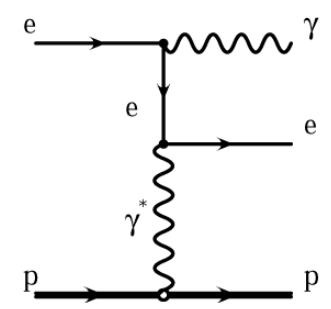

b)

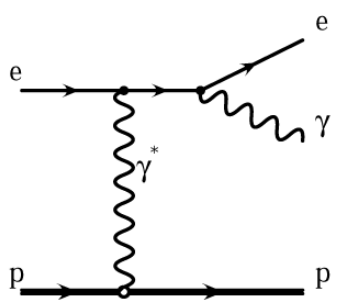

c)

Figure 3.29. Diagram of the DVCS (a) and BH processes for a photon emitted from the initial (b) and final (c) lepton line.

electromagnetic clusters together with a proton scattered at a very small angle. The technique for measuring DVCS consists of first extracting a data sample of events characterized by the DVCS topology. Apart from the DVCS process, the data selection comprises also Bethe-Heitler (BH) events, a well known QED process, because DVCS and BH share the same final state. The selected sample will contain a mixture of DVCS and BH contributions. For not too large $y$ (see figure 3.31), the $\mathrm{BH}$ contribution is not much larger than DVCS and thus can be subtracted from the data using the MC predictions and control data samples containing $\mathrm{BH}$ only since at high enough $Q^{2}$ the interference contribution drops out to a good accuracy when averaging over the angle $\phi$ between the production and scattering planes.

The differential cross section as a function of $|t|$ can be parameterized by an exponential: $d \sigma / d t \propto e^{b|t|}$. The H1 Collaboration [648] measured $|t|$ from the transverse momentum distribution of the photon and studied the $b$-slope in a few bins in $Q^{2}$ and $W$. The slope $b$ seems to decrease with $Q^{2}$ up to the value expected for a hard process but it does not depend on $W$. The ZEUS Collaboration 645] performed a direct measurement of the proton final state using a Roman Pots spectrometer: the resulting $b=4.5 \pm 1.3 \pm 0.4 \mathrm{GeV}^{-2}$ at $Q^{2}=3.2 \mathrm{GeV}^{2}$ and $W=104 \mathrm{GeV}$ is consistent, within the large uncertainties due to the low acceptance of the spectrometer, with the $\mathrm{H} 1$ result of $b=5.45 \pm 0.19 \pm 0.34 \mathrm{GeV}^{-2}$ at $Q^{2}=8 \mathrm{GeV}^{2}$ and $W=82 \mathrm{GeV}[648]$.

A comprehensive program of parton imaging in the nucleon would need precise measurements of $b$ for wide range of $x_{B}$ values, $10^{-4}<x_{B}<10^{-1}$; this is currently beyond the possibilities of any experiment. Building an EIC with a properly designed detector could finally make it possible - a preliminary feasibility study is reported in section 3.6.

The beam-charge asymmetry (BCA) provides an access to the real part of the DVCS amplitude through the interference between the DVCS and BH amplitudes (for illustration, we keep only the dominant $\cos (\phi)$ harmonic):

$$
A_{C}=\frac{\frac{d \sigma^{+}}{d|t|}-\frac{d \sigma^{-}}{d|t|}}{\frac{d \sigma^{+}}{d|t|}+\frac{d \sigma^{-}}{d|t|}}=p_{1} \cos (\phi) \propto 2 \mathcal{A}_{\mathrm{BH}} \frac{\Re e\left(\mathcal{A}_{\mathrm{DVCS}}\right)}{\left|\mathcal{A}_{\mathrm{DVCS}}\right|^{2}+\left|\mathcal{A}_{\mathrm{BH}}\right|^{2}} \cos (\phi) .
$$

The measurement of $A_{C}$ is complementary to the measurement of the $|t|$ distribution. The DVCS beam-charge asymmetry has been measured by the H1 [648] and HERMES [671, 672] experiments.

The large rapidity acceptance and high precision tracker of the EIC detector together with its very accurate electromagnetic calorimeter and the high luminosity of the machine, 
make it an ideal tool for the measurement of both the DVCS cross section differential in $|t|$ and the DVCS $+\mathrm{BH}$ cross section asymmetries. Specifically for the BCA, a positron beam would be required.

\subsubsection{Monte Carlo simulations of DVCS at an EIC}

The Monte Carlo generator used for our studies is MILOU [673], which simulates both the DVCS and the BH processes together with their interference term. DVCS is simulated using the framework of GPDs at next-to-leading order (NLO) accuracy, including the NLO evolution of GPDs 625. The $t$ dependence is introduced as an exponential $d \sigma / d t \propto e^{B\left(Q^{2}\right)|t|}$, where $B\left(Q^{2}\right)$ is either a constant or can have a weak logarithmic dependence on $Q^{2}, B\left(Q^{2}\right) \sim \ln \left(Q^{2}\right)$. In the present simulation, we used the former option, $B\left(Q^{2}\right)=5 \mathrm{GeV}^{-2}$. In addition, the proton dissociation background, $e p \rightarrow e \gamma Y$, has not been included.

The DVCS and BH processes have been simulated in the following kinematic range:

- $Q^{2} \geq 1 \mathrm{GeV}^{2}$;

- $10^{-4}<x_{B}<10^{-1}$;

- $0.01<y<0.85$;

- $0.01<|t|<1 \mathrm{GeV}^{2}$.

The $Q^{2}$ and $x_{B}$ ranges correspond to the phase space achievable with an EIC; the lower $y$ limit is chosen according to the acceptance of the detector; the interval in $|t|$ relates to the acceptance of a forward proton spectrometer. The energy configuration considered for the present study is a $5-20 \mathrm{GeV}$ electron beam colliding with a $250 \mathrm{GeV}$ proton beam.

Figure 3.30 shows the correlation between the scattering angle of the real photon produced in the interaction and its energy for different EIC energy configurations. For the $20 \times 250$ configuration, the photons with an energy greater than $\sim 5 \mathrm{GeV}$ are produced backward at an angle larger than $2.7 \mathrm{rad}$, corresponding to the rear end-cap calorimeter in the detector. Since for the DVCS process the electron is always scattered backward, this can lead to problems in discriminating the photon and electron clusters and makes it crucial to have an electromagnetic calorimeter with high spatial resolution and a good tracker coverage at backward rapidity to measure the electron track. Indeed, this is extremely important for the $t$ resolution in the case of a measurement performed without a Roman Pots because the four-momentum $t$ must be reconstructed, using momentum conservation, from the transverse momenta of the electron and the photon.

The fraction of $\mathrm{BH}$ events has been estimated using an MC sample containing both DVCS and $\mathrm{BH}$ processes. The samples have been normalized to the luminosity. The fraction of $\mathrm{BH}$ events is calculated as follows:

$$
F_{B H}=\frac{B H_{e v t}}{B H_{e v t}+D V C S_{e v t}} .
$$

Figure 3.31 shows the fraction of $\mathrm{BH}$ events as a function of $y, Q^{2}$, and $|t|$. As expected, DVCS is dominant at low $y$ whereas BH dominates at higher $y$ with its fraction increasing up to $100 \%$ for $y>0.85$.

In the present study, the kinematic domain has been binned logarithmically in $1<Q^{2}<$ $100 \mathrm{GeV}^{2}$ and $10^{-4}<x_{B}<10^{-1}$. Figure 3.32 shows the distribution of the statistics per bin for the $20 \times 250$ configuration (left panel) and $5 \times 50$ configuration (right panel). 

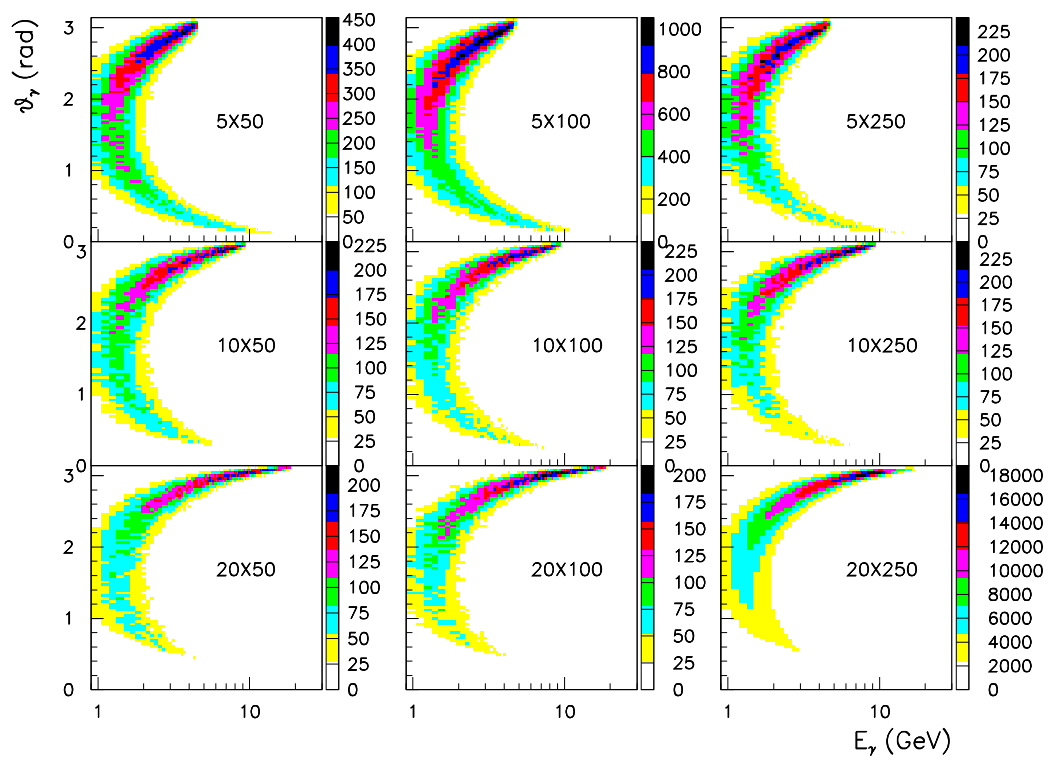

Figure 3.30. The angle of the produced real photon in a DVCS event as a function of the photon energy for different EIC energy configurations. Each plot shows also the distribution of the photon and scattered electron energies.
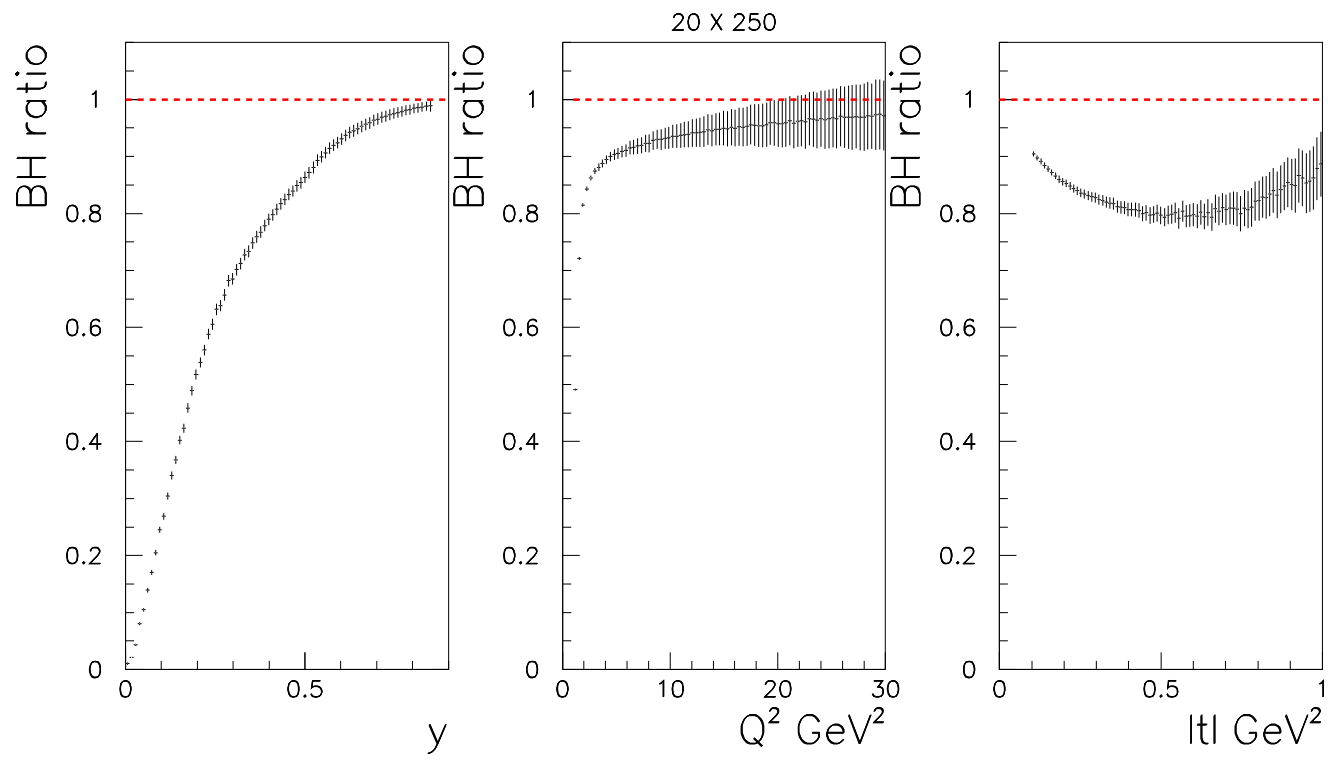

Figure 3.31. The fraction of $\mathrm{BH}$ events in the $e p \rightarrow e p \gamma$ sample as a function of $y$ (left), $Q^{2}$ (middle), and $|t|$ (right). 


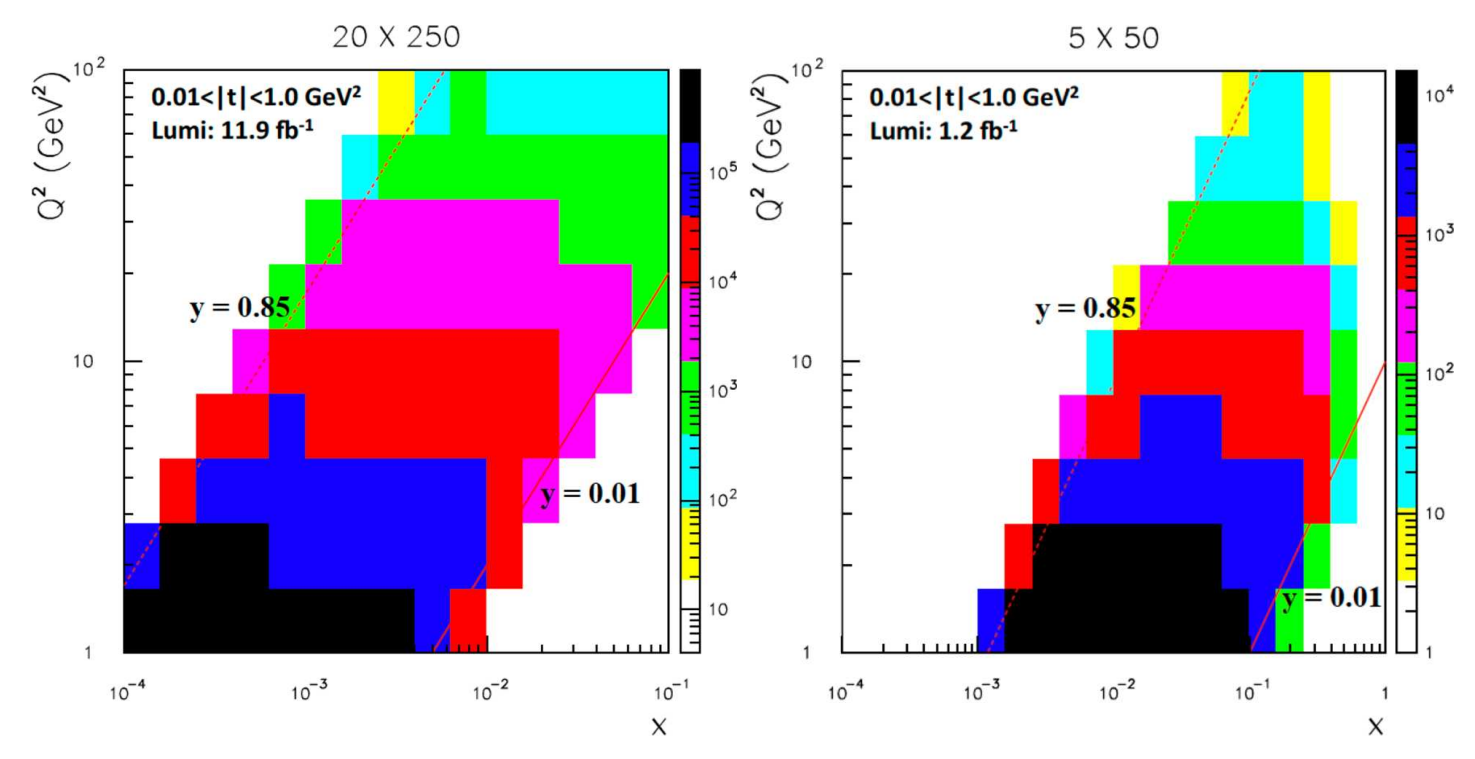

Figure 3.32. The distribution of the DVCS events in a logarithmically binned phase space for the $20 \times 250$ (left) and $5 \times 50$ (right) EIC beam energy configurations.

As an example of the precision that could be achieved at an EIC, figure 3.34 shows the expectations for a measurement of the DVCS ep cross section differential in $|t|, \mathrm{d} \sigma_{e p \rightarrow e p \gamma} / \mathrm{d} t$, for several bins of $x_{B}$ and $Q^{2}$. The estimated luminosity for the $20 \times 250$ configuration is $10^{34} \mathrm{~cm}^{-2} \mathrm{~s}^{-1}$. The integrated luminosity of the simulated events is $11.9 \mathrm{fb}^{-1}$, corresponding to approximately one month of running at $20 \times 250$ and assuming $50 \%$ operational efficiency. The $b$ slope parameter with its uncertainty is extracted for each data set via a an exponential fit $\sim e^{-b|t|}$, and its value is reported in figure 3.34 together with uncertainties.

One can see that an excellent measurement (binning over a wide range in $Q^{2}$ and $x_{B}$ ) can already be obtained with a relatively modest beam time, allowing for numerous detailed studies of the reaction mechanism $\left(Q^{2}\right.$-scaling behavior, QCD evolution) and extraction of information about the nucleon GPDs and its change with $x_{B}$. The statistical uncertainty for the differential cross section can be, at small $|t|$ values, significantly below $1 \%$, as well as the uncertainty on the extracted slope parameter, $b$. This implies that the measurement is actually limited by systematics. Thus the utilization of a high resolution spectrometer based on the Roman Pots technique becomes important for an EIC. For example, the leading proton spectrometer based on 6 Roman Pots stations equipped with silicon microstrips detectors used at ZEUS for the DVCS $d \sigma / d|t|$ measurement, allowed to measure $P_{t}$ with a resolution of $5 \mathrm{MeV}$ [645] under test beam conditions which corresponded to $\Delta\left(P_{t}^{2}\right)=|\Delta t|=10^{-2} P_{t}$ for a $|t|$ measurement. A new properly designed Roman Pots spectrometer, potentially based on a radiation-hard silicon pixel technology, could reach a geometrical acceptance of about $60 \%$, with a better $P_{t}$ resolution. Since for an EIC systematics are the challenge, it is worth sacrificing the acceptance and therefore increasing the beam time for a more accurate measurement.

Figure 3.35 shows the expectations for a DVCS measurement for large- $|t|$. The data have been simulated for $1<|t|<2 \mathrm{GeV}^{2}$ in several bins of $x_{B}$ and $Q^{2}$. The luminosity of the simulated sample is $151 \mathrm{fb}^{-1}$ corresponding to approximately 52 weeks of data taking in the $20 \times 250$ configuration. One can see that even if the cross section drops drastically for large $|t|$ values, the EIC still allows for good binned measurements, but this requires 
years of data taking. (For a relevant discussion, see section 3.6.) In this regime, the main detector offers a much better acceptance then Roman Pots and can be used for measuring $|t|$.

A data sample containing DVCS, BH and their interference term has been simulated considering separately an electron beam (luminosity is $44 \mathrm{pb}^{-1}$ ) and a positron beam (luminosity is $47 \mathrm{pb}^{-1}$ ) and used to calculate the beam-charge asymmetry, $A_{C}$. The result is shown in figure 3.33 together with a fit in the form $A_{C}=p_{1} \cos (\phi)$ (3.69), where $p_{1}$ is a free parameter. One can see that a fair accuracy for the BCA can be obtained at an EIC for a modest integrated luminosity.

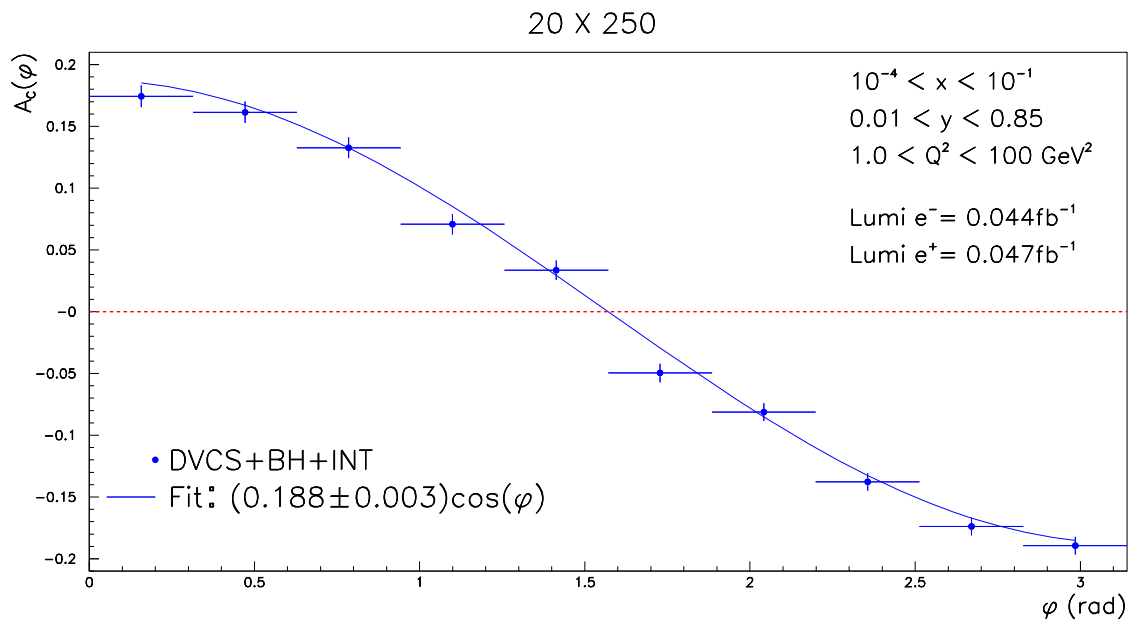

Figure 3.33. The beam-charge asymmetry $A_{C}$ as a function of the azimuthal angle $\phi$ between the production and scattering planes. 


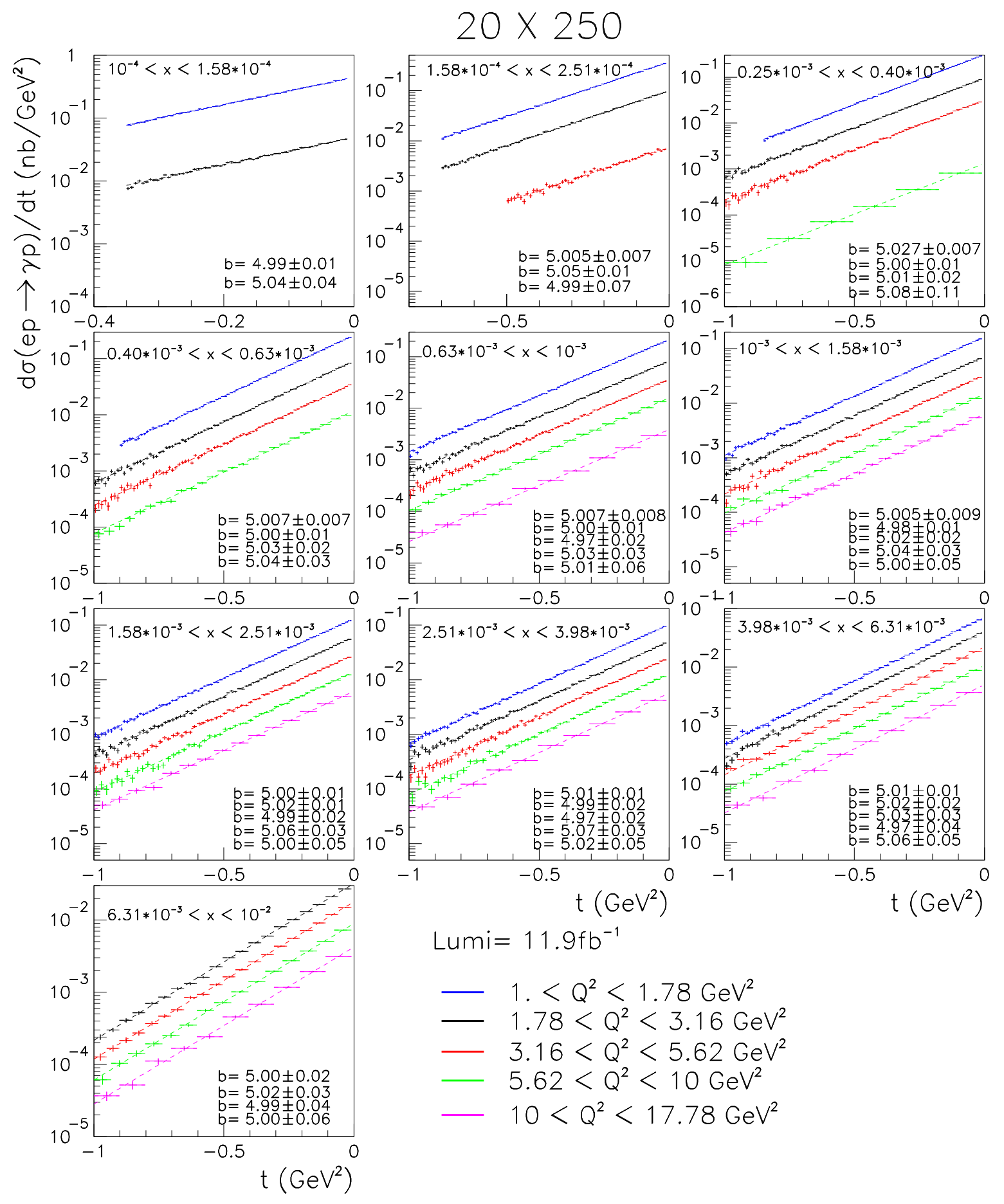

Figure 3.34. The DVCS cross section has been simulated in the range $1.0<Q^{2}<100 \mathrm{GeV}^{2}$, $10^{-4}<x_{B}<0.1$ for the $20 \times 250 \mathrm{GeV}$ energy configuration. The DVCS cross section is simulated in several bins of $x_{B}$ and $Q^{2}$ and is shown for small $|t|$ values. 


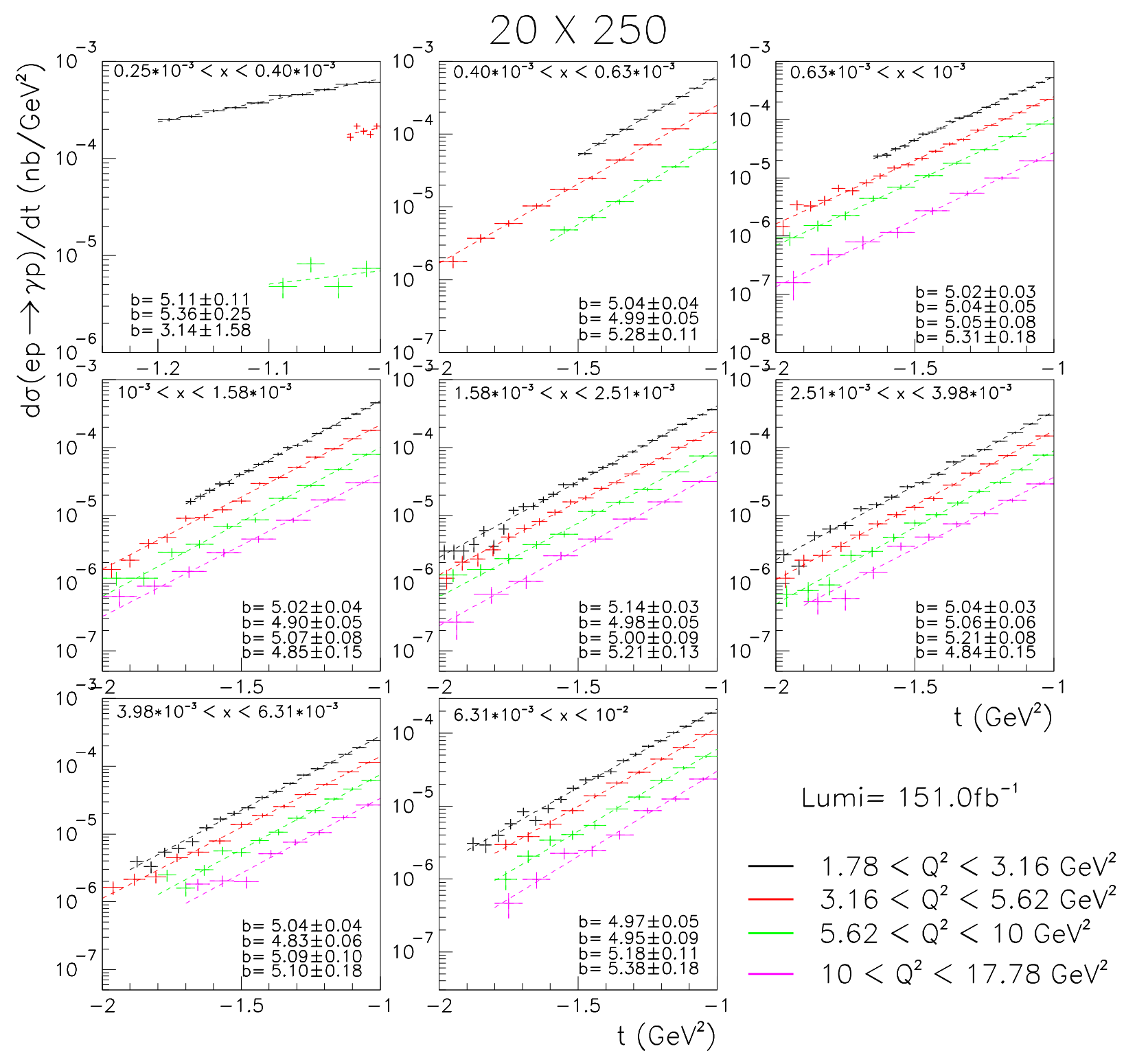

Figure 3.35. The DVCS cross section has been simulated in the range $1.0<Q^{2}<100 \mathrm{GeV}^{2}$, $10^{-4}<x_{B}<0.1$ for the $20 \times 250 \mathrm{GeV}$ energy configuration. The DVCS cross is section simulated in several bins of $x_{B}$ and $Q^{2}$ and is shown for large $|t|$ values. 


\title{
3.10 DVCS Beam Spin Asymmetries with an EIC
}

\author{
R. Géraud, H. Moutarde, F. Sabatié
}

\subsubsection{Deeply Virtual Compton Scattering polarization observables}

The photon electroproduction $e p \rightarrow e p \gamma$ can either occur by radiation along one of the electron lines (Bethe-Heitler or BH) or by emission of a real photon by the nucleon (Deeply Virtual Compton Scattering or DVCS). The total cross section as given by [625] reads:

$$
\frac{\mathrm{d} \sigma^{e p \rightarrow e p \gamma}}{\mathrm{d} x_{B} d y \mathrm{~d} \Delta^{2} \mathrm{~d} \phi \mathrm{d} \varphi}=\frac{\alpha^{3} x_{B} y}{16 \pi^{2} Q^{2} \sqrt{1+\epsilon^{2}}}\left|\frac{\mathcal{T}}{e^{3}}\right|^{2},
$$

where $\Delta$ is the 4-momentum transfer between the initial and final proton; $Q^{2}$ the virtuality of the exchanged photon; $x_{B}$ the usual Bjorken variable; $\epsilon=2 x_{B} M / Q$ ( $M$ is the proton mass); $y$ is the fraction of the electron energy lost in the nucleon rest frame; $\phi$ is the angle between the leptonic plane $\left(e, e^{\prime}\right)$ and the photonic plane $\left(\gamma^{*}, \gamma\right)$ as shown in figure 3.36, The angle $\varphi$ is defined as the difference between $\phi$ and $\phi_{S}$, the orientation of the target spin in the case of a polarized target, shown also in figure 3.36 .

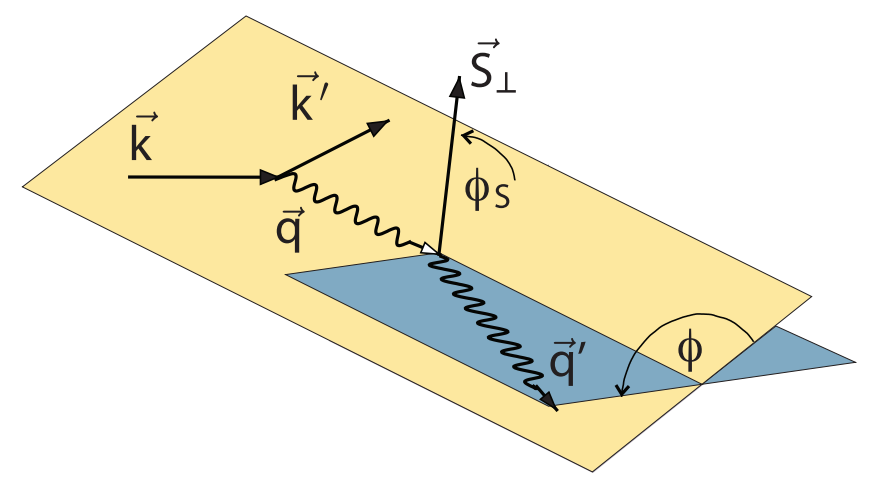

Figure 3.36. Kinematics of the photon leptoproduction in the target rest frame (the Trento notations). The incoming and outgoing leptons define the scattering plane, and the outgoing photon and recoil protons define the hadronic plane. In this reference system, the azimuthal angle between the lepton and recoil proton planes is $\phi$. The angle $\phi_{S}$ defines the orientation of the target spin in the case of a polarized target (it will not be used in the present contribution).

The total amplitude $\mathcal{T}$ is the superposition of the BH and DVCS amplitudes:

$$
\begin{aligned}
|\mathcal{T}|^{2} & =\left|\mathcal{T}_{\mathrm{BH}}\right|^{2}+\left|\mathcal{T}_{\mathrm{DVCS}}\right|^{2}+\mathcal{I}, \\
\mathcal{I} & =\mathcal{T}_{\mathrm{DVCS}}^{*} \mathcal{T}_{\mathrm{BH}}+\mathcal{T}_{\mathrm{DVCS}} \mathcal{T}_{\mathrm{BH}}^{*},
\end{aligned}
$$

where $\mathcal{T}_{\mathrm{DVCS}}$ and $\mathcal{T}_{\mathrm{BH}}$ are the amplitudes for the DVCS and Bethe-Heitler processes, and $\mathcal{I}$ denotes the interference between these amplitudes. The individual contributions to the total $e p \rightarrow e p \gamma$ cross section can be written as (up to twist-3 contributions and corrections 
in $1 / Q)$ 625]:

$$
\begin{aligned}
\left|\mathcal{T}_{\mathrm{BH}}\right|^{2} & =\frac{\Gamma_{\mathrm{BH}}\left(x_{B}, Q^{2}, t\right)}{\mathcal{P}_{1}(\phi) P_{2}(\phi)}\left\{c_{0}^{\mathrm{BH}}+\sum_{n=1}^{2} c_{n}^{\mathrm{BH}} \cos (n \phi)+s_{1}^{\mathrm{BH}} \sin \phi\right\} \\
\left|\mathcal{T}_{\mathrm{DVCS}}\right|^{2} & =\Gamma_{\mathrm{DVCS}}\left(x_{B}, Q^{2}, t\right)\left\{c_{0}^{\mathrm{DVCS}}+\sum_{n=1}^{2}\left[c_{n}^{\mathrm{DVCS}} \cos (n \phi)+s_{n}^{\mathrm{DVCS}} \sin (n \phi)\right]\right\} \\
\mathcal{I} & \left.=\frac{\Gamma_{I}\left(x_{B}, Q^{2}, t\right)}{\mathcal{P}_{1}(\phi) P_{2}(\phi)}\right]\left\{c_{0}^{I}+\sum_{n=1}^{3}\left[c_{n}^{I} \cos (n \phi)+s_{n}^{I} \sin (n \phi)\right]\right\}
\end{aligned}
$$

where $\Gamma_{\mathrm{BH}}, \Gamma_{\mathrm{DVCS}}$ and $\Gamma_{I}$ are known kinematical prefactors. $\mathcal{P}_{1}(\phi)$ and $\mathcal{P}_{2}(\phi)$ come from the $\mathrm{BH}$ electron propagators and can be written as:

$$
Q^{2} \mathcal{P}_{1}=Q^{2}+2 k \cdot \Delta, \quad Q^{2} \mathcal{P}_{2}=-2 k \cdot \Delta+\Delta^{2},
$$

where $k$ is the 4-momentum of the incoming lepton.

In the case of scattering on unpolarized or longitudinally polarized targets, all $\sin (n \phi)$ coefficients in (3.73 3.75) depend either on the beam helicity $\lambda$ or on the target longitudinal polarization $\Lambda$; they disappear in the unpolarized cross section.

Using a polarized beam, two separate quantities can be extracted: the difference of cross section with opposite beam helicities and the total cross section, which at leading twist can be written respectively as:

$$
\begin{aligned}
d \sigma^{\rightarrow}-d \sigma^{\leftarrow} & =2 \cdot \mathcal{T}_{\mathrm{BH}} \cdot \Im m\left(\mathcal{T}_{\mathrm{DVCS}}\right) \\
d \sigma^{\rightarrow}+d \sigma^{\leftarrow} & =\left|T_{\mathrm{BH}}\right|^{2}+2 \cdot \mathcal{T}_{\mathrm{BH}} \cdot \Re e\left(\mathcal{T}_{\mathrm{DVCS}}\right)+\left|T_{\mathrm{DVCS}}\right|^{2}
\end{aligned}
$$

where the arrows correspond to the beam helicity. At low $y$, the interference term entering the total cross section is small compared to the DVCS and BH contributions, which contrasts with the case of intermediate or large $y$ where the DVCS contribution is small with respect to the interference, which itself is in general significantly smaller than the BH term. Note that the DVCS contribution to the difference of cross section only appears at higher twist.

From these two natural observables, one can write asymmetries which are experimentally easier to determine than cross sections:

$$
A_{L U}=\frac{d \sigma^{\leftarrow}-d \sigma^{\rightarrow}}{d \sigma^{\leftarrow}+d \sigma^{\rightarrow}}
$$

Beam spin asymmetries are mostly sensitive to the GPD $H$ and are complementary to unpolarized cross sections and beam charge asymmetry measurements presented in section 3.9 .

\subsubsection{Monte Carlo}

The PROPHET package [674] was used in its Monte Carlo configuration to generate photon electroproduction pseudo-data in the EIC kinematics. We relied on the GoloskokovKroll model for GPDs [605] evaluated at NLO, integrated over the LO hard kernel to obtain Compton Form Factors $\mathcal{H}, \widetilde{\mathcal{H}}, \mathcal{E}$ and $\widetilde{\mathcal{E}}$ which are the complex counterparts of GPDs and directly relate to the DVCS amplitude at the leading order of $\alpha_{s}$ [625]. Note that $\widetilde{\mathcal{E}}$ only enters the unpolarized cross section for DVCS, but was neglected in this evaluation. 
The photon electroproduction observables were evaluated using the GV package [675], which in contrast with the usual BMK formalism [625], does not make approximations of the order of $1 / Q$ in the treatment of the interference term. It was checked that the unpolarized cross sections generated by our Monte Carlo give the results simular to those shown in section [3.9, but with more realistic $b$-slopes since they were not taken as a constant but form a part of the Goloskokov-Kroll model.

\subsubsection{Projected results}

The Beam Spin Asymmetry evaluated in a typical $\left(x_{B}, Q^{2}\right)$ bin is shown in figure 3.37 . The $\sin \phi$ coefficient turns out 3 to 5 times lower than that at typical lower energy and higher $x_{B}$ kinematics. Therefore, this asks for a rather large integrated luminosity. For the considered $x_{B}$ range (and lower), about three months of EIC at $10^{34} \mathrm{~cm}^{-2} \mathrm{~s}^{-1}$ luminosity assuming $50 \%$ operational efficiency is necessary to achieve a 10 to $15 \%$ accuracy on the extracted $\sin \phi$ coefficient, mostly linked to the imaginary part of CFF $\mathcal{H}$. For higher $x_{B}$ and $Q^{2}$ values, much larger integrated luminosities will be necessary to achieve similar statistical accuracy.

Using longitudinal and transverse target asymmetries, one will be able to obtain information on $\widetilde{H}$ and even the elusive $E$, essential for the evaluation of Ji's sum rule. Studies of these observables for the EIC are in progress using the same formalism.
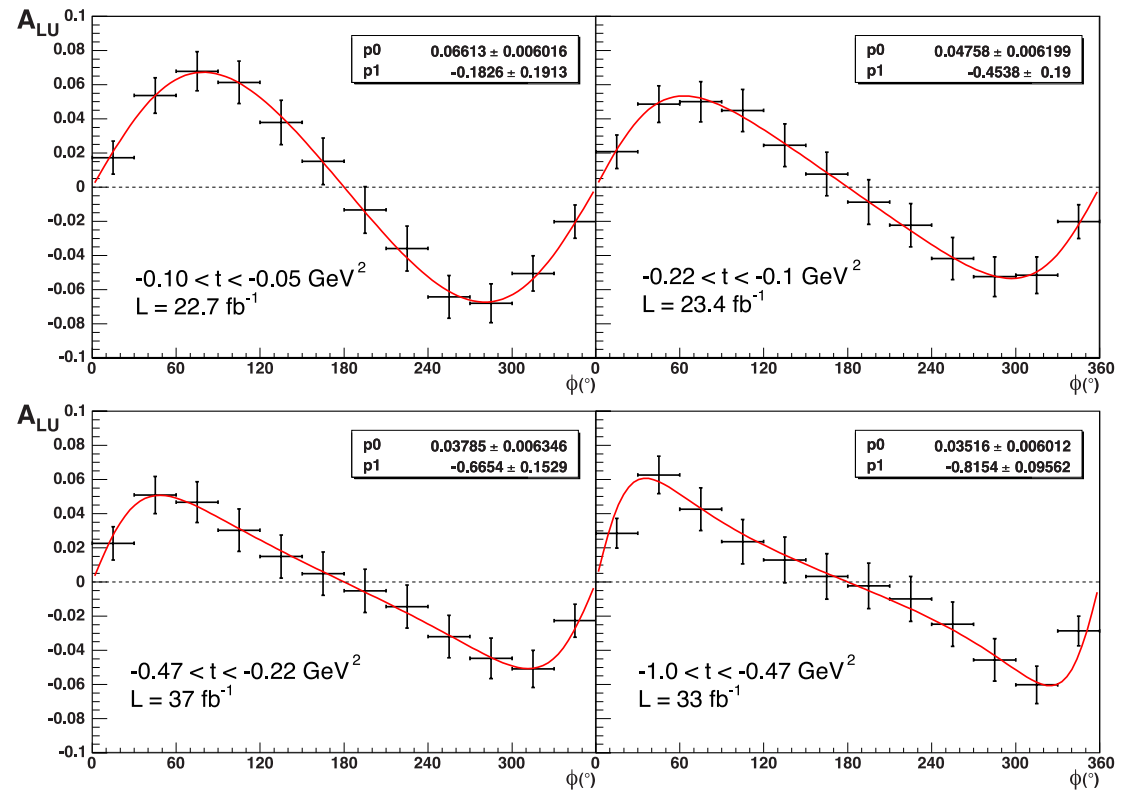

Figure 3.37. Photon electroproduction Beam Spin Asymmetries for the $20 \times 250$ EIC configuration, in the typical kinematic bin: $1.58 \cdot 10^{-3}<x_{B}<2.51 \cdot 10^{-3}, 3.16<Q^{2}<5.61 \mathrm{GeV}^{2}$ for four different $t$-bins as shown on each plot. The Monte Carlo was set up as to generate $90 \mathrm{k}$ events for each $t$-bin and the corresponding integrated luminosity is shown on each plot. Up to about 3 months of beam time with $50 \%$ efficiency is necessary to achieve 10 to $15 \%$ accuracy on the extracted $\sin \phi$ coefficient p0, sensitive to the imaginary part of CFF $\mathcal{H}$. 


\subsection{Hard exclusive photoproduction of Quarkonia}

\section{Peter Kroll}

Photoproduction of quarkonia (e.g., $J / \Psi$ and $\Upsilon$ ) forms another class of hard exclusive processes which allow one to scrutinize the handbag approach and extract information on generalized parton distributions (GPDs). Neglecting intrinsic heavy quarks in the proton, only the gluonic subprocess $\gamma^{*} g \rightarrow M g$ (accompanied by the gluon GPDs) contributes to the scattering amplitude. A particular feature of quarkonium production is the appearance of the large mass, $m_{Q}$, of the heavy quark which provides a hard scale and allows one to treat photoproduction within a QCD factorization approach. A first leading-order calculation of quarkonium production within the handbag approach has been carried out in 676]. Recently this analysis has been improved by the inclusion of NLO corrections [677. In these studies, a non-relativistic scenario for the description of the quarkonium state has been adopted in which the quark and antiquark share the meson's momentum equally. Hence, the quarkonium wave function is proportional to $\delta(x-1 / 2)$ and the quarkonium mass is given by $M_{Q} \simeq 2 m_{Q}$. There are other theoretical approaches to the process of interest, e.g., the dipole approach, the leading $\ln (1 / x)$ approximation or the BFKL Pomeron. Due to limitation of space these approaches will not be discussed here.

In the kinematical range of large photon-proton center-of-mass energy, $\sqrt{s} \gg M_{Q}$, and small momentum transfer, $t$, the skewness is given by

$$
\xi=M_{Q}^{2} /(2 s)
$$

Neglecting terms of order of $\sqrt{-t} / m_{Q}, t / 4 m^{2}$ and $\xi$, one finds the following expressions for the helicity amplitudes of the quarkonium photoproduction:

$$
\begin{aligned}
\mathcal{M}_{\mu+, \mu+} & =\frac{e_{0}}{2} e_{Q} \int_{0}^{1} \frac{d x}{(x+\xi)(x-\xi+i \varepsilon)} \sum_{\lambda} \mathcal{H}_{\mu \lambda, \mu \lambda}\left[H^{g}+\lambda \widetilde{H}^{g}\right] \\
\mathcal{M}_{\mu-, \mu+} & =-e_{0} e_{Q} \frac{\sqrt{-t}}{4 m} \int_{0}^{1} \frac{d x}{(x+\xi)(x-\xi+i \varepsilon)} \sum_{\lambda} \mathcal{H}_{\mu \lambda, \mu \lambda} E^{g} \\
\mathcal{M}_{--,++} & =e_{0} e_{Q} \frac{\sqrt{-t}}{2 m} \int_{0}^{1} \frac{d x}{(x+\xi)(x-\xi+i \varepsilon)} \mathcal{H}_{--,++} H_{T}^{g} .
\end{aligned}
$$

Other helicity amplitudes are zero except for those related by parity conservation to the above ones. The helicity labels $\mu$ and $\mu^{\prime}$ refer to the initial photon and final meson, respectively; the labels $\lambda$ and $\lambda^{\prime}$ refer to the initial and final gluon, respectively. The explicit helicity labels of $\mathcal{M}$ refer to the proton. In the non-relativistic scenario, the LO subprocess amplitudes read

$$
\mathcal{H}_{\mu^{\prime} \lambda^{\prime}, \mu \lambda}=\frac{8 \pi \alpha_{s}\left(\mu_{R}\right) f_{Q}}{3 m_{Q}} \delta_{\mu^{\prime} \mu} \delta_{\lambda^{\prime} \lambda}
$$

where $f_{Q}$ is the decay constant of the quarkonium; $\mu_{R}$ is an appropriate renormalization scale. Thus, at this level of accuracy, only the process amplitudes,

$$
\mathcal{M}_{\mu+, \mu+}=e_{0} e_{Q} \frac{8 \pi \alpha_{s} f_{Q}}{3 m_{Q}}\left\langle H^{g}\right\rangle, \quad \mathcal{M}_{\mu-, \mu+}=-e_{0} e_{Q} \frac{8 \pi \alpha_{s} f_{Q}}{3 m_{Q}} \frac{\sqrt{-t}}{2 m}\left\langle E^{g}\right\rangle,
$$

are non-zero. The terms $\langle F\rangle$ denote the convolutions of the subprocess amplitudes and GPDs. One sees that the unpolarized cross section for quarkonium production at small $t$ 
is only fed by $H^{g}$, while the asymmetry measured with a transversally polarized target is given by an interference term of $E^{g}$ and $H^{g}$. Other GPDs, like the chiral-odd $H_{T}^{g}$, do not contribute at this level of accuracy.

For the EIC kinematics for which $\xi<0.01$, quarkonium production is a diffractive process, i.e., the amplitudes are dominantly imaginary. Thus, essentially the GPDs are only needed at the cross-over line $x=\xi$, while the small real part may be estimated with the help of analyticity. Many methods for the construction of GPDs, e.g., the double distribution ansatz [678] or the Shuvaev transform [593], lead to GPDs which at $x=\xi$ are proportional to the usual parton distributions. In particular, for the reggeized double distribution ansatz used in 659, 679, one has

$$
H^{g}(\xi, \xi, t)=c_{h}[2 \xi g(2 \xi)](2 \xi)^{-\alpha_{h}^{\prime} t} e^{b_{h} t} .
$$

Assuming that $x g(x)=c x^{-\delta_{h}}$ at low $x$, the constant $c_{h}$ reads (with $b=2[678$ ):

$$
c_{h}=c\left[\left(1-\delta_{h} / 5\right)\left(1-\delta_{h} / 4\right)\left(1-\delta_{h} / 3\right)\right]^{-1} .
$$

Since $\delta_{h}$ is positive, $c_{h}>c$, which implies that $H^{g}(\xi, \xi, t=0)>2 \xi g(2 \xi)$ (this is termed the skewness effect). In (3.83), a linear gluonic ('Pomeron-like') Regge trajectory is assumed:

$$
\alpha_{h}=1+\delta_{h}+\alpha_{h}^{\prime} t
$$

Its slope is taken from the $J / \Psi$ photoproduction data 588 ] $\left(\alpha_{h}^{\prime}=0.15 \mathrm{GeV}^{-2}\right)$, while $\delta_{h}$, the intercept minus 1, can be fixed from the data on the cross section for electroproduction of $\rho^{0}$ and $\phi$ mesons [680] that behaves as $\sigma \propto s^{2 \delta_{h}}$. The data provide a scale-dependent intercept (see figure 3.38):

$$
\delta_{h}(\mu)=0.1+0.06 \ln \left(\mu^{2} / \mu_{0}^{2}\right),
$$

where $\mu_{0}=2 \mathrm{GeV}$ and $\mu=Q$ for electroproduction. The scale dependence of $\delta_{h}$ is in agreement with evolution.
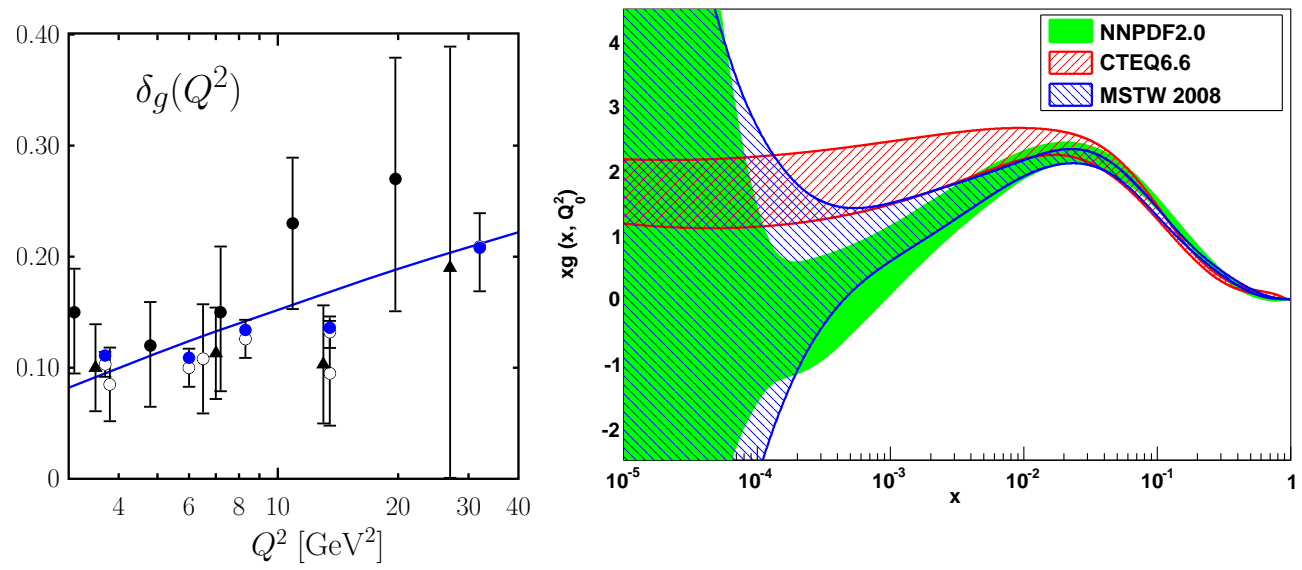

Figure 3.38. Left: The intercept of the gluonic trajectory shifted by one unit; the data points are from [680]. Right: Various NLO gluon PDFs. The figure is from [681.

The construction of the GPD along the lines described above requires the knowledge of the gluon PDF; in figure 3.38, some recent results for it are shown. For $x$ smaller than 
about $10^{-3}$, the errors of most PDFs become very large. The only exception is the CTEQ6 results 82 ] for which the error stays constant at the level of about $25 \%$ for $x<10^{-2}$. Since the uncertainties of the PDF are conveyed to the GPDs and, hence, to the quarkonium cross section, any prediction of the latter will suffer from huge uncertainties rendering any comparison with experiment meaningless. In order to arrive at reasonable predictions, the following remedial measure has been proposed in [659, 679]: The gluon PDF is expanded as the following series,

$$
x g(x, \mu)=x^{-\delta_{h}(\mu)}(1-x)^{5} \sum_{i=0}^{2} c_{i}(\mu) x^{i / 2},
$$

and the expansion parameters are fitted to a given PDF for intermediate values of $x$, say, $0.003<x<0.3$, and relevant scales. The power $\delta_{h}$ is fixed at the experimental value (3.86). Applying this prescription to the NLO CTEQ6 PDF [82, one can reproduce the CTEQ6 result for $x<0.003$. Therefore, the CTEQ6 gluon PDF with its errors may be used for numerical predictions. (The same method applied to other current gluon PDFs leads, in most cases and within uncertainties, to the results for cross sections that are in a reasonable agreement with those evaluated using the CTEQ6 PDF 659.)

The calculation of the quarkonium cross section is further complicated by large NLO corrections 677, see figure 3.39. The results shown in figure 3.39 are evaluated from a GPD that is also generated from the NLO CTEQ6 PDF but under the assumption that, at the initial scale, $H^{g}$ is given by the PDF multiplied by an appropriate function of $t$. Evidently, the large NLO corrections necessitate a resummation of higher orders for a reliable prediction of the quarkonium cross section.
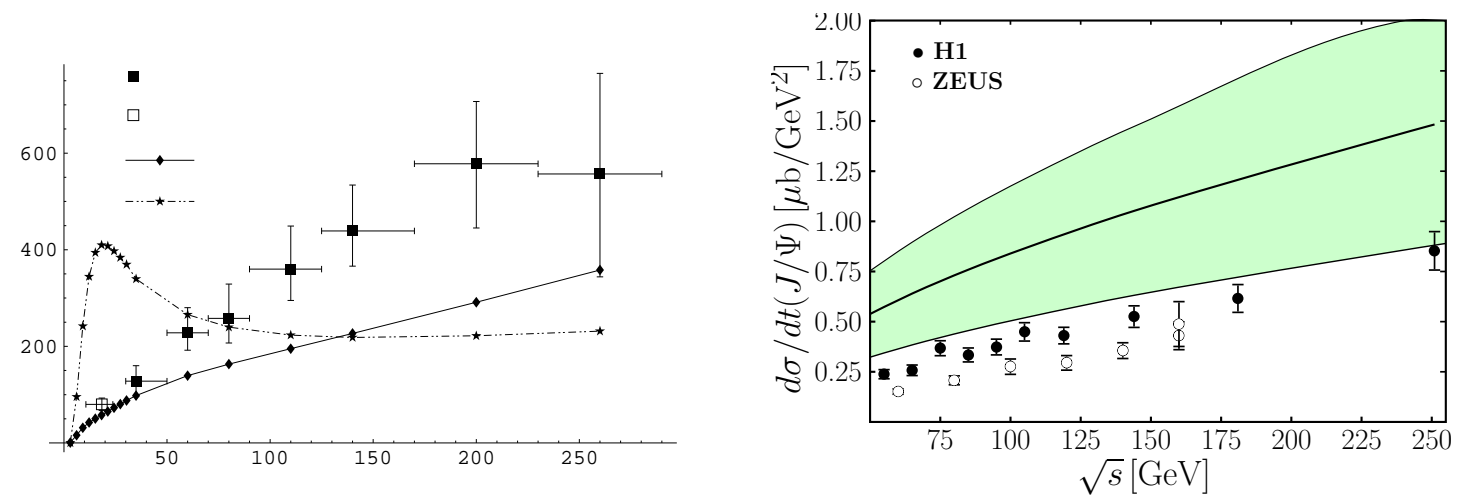

Figure 3.39. Left: LO and NLO predictions for the forward $J / \Psi$ photoproduction $v s . \sqrt{s}$ (the figure is from [677). Right: LO prediction using the GPD given in [679, 659, see the text. The green band indicates the uncertainty of the prediction; the data points are from [589, [588.

In order to examine the dependence of the predictions on the GPD used in the calculation, I have repeated the LO order calculation exploiting the GPD proposed in [659, 679] and whose construction is briefly described above. The result, obtained for a scale of $2 m_{Q}$ which is chosen in concord with the construction of the GPD [see (3.86)], overestimates the data but is in agreement with the experimental energy dependence. It however differs strongly from the LO result presented in [677]. Similar observations can be made for photoproduction of the $\Upsilon$. 
The target asymmetry for quarkonium production may give access to the GPD $E^{g}$, of which not much is known, see the discusion in section 3.4. Indeed,

$$
A_{U T}=-\frac{\sqrt{-t}}{m} \frac{\left|\left\langle E^{g}\right\rangle\right|}{\left|\left\langle H^{g}\right\rangle\right|} \sin \phi
$$

where $\phi$ is the relative phase between $\left\langle H^{g}\right\rangle$ and $\left\langle E^{g}\right\rangle$. Since $E^{g}$ is expected to behave similarly to $H^{g}$ at small $\xi$ but with a Regge trajectory $\alpha_{e}=1+\delta_{e}+\alpha_{e}^{\prime} t$, analyticity tells us that the relative phase is approximately given by

$$
\phi(t)=\pi / 2\left(\alpha_{e}(t)-\alpha_{h}(t)\right) .
$$

In a soft Pomeron scenario, one would have $\alpha_{e}=\alpha_{h}$ and, hence, $A_{U T}=0$. However, the QCD evolution of the GPDs may generate differences in the trajectories and, therefore, $A_{U T}$ may be non-zero. To work this out, one needs a detailed study of the evolution of $E^{g}$, which is lacking at present. There are examples of $\alpha_{e}$ in the literature that lead to tiny asymmetries of the order of $1-2 \%$. In section 3.4 it has been proposed a parameterization of $E^{g}$ with a node at some intermediate value of $x$. In this case one may obtain a larger $A_{U T}$. However, this possibility has not yet been explored in detail.

Summary: Comparing precise data of the cross section for photoproduction of quarkonia with theoretical calculations within the handbag approach may allow for an extraction of $H^{g}$ at the cross-over line and small values of $\xi$ since the real part of the amplitude provides only small corrections to the cross section of the order of $10 \%$. This may lead to a useful constraint on $H^{g}$ and $g(x)$ at low $x$. However, in order to arrive at reliable results for $H^{g}$, the theoretical calculation should include resummed higher orders of perturbative QCD. Also, deviations from the non-relativistic scenario should be investigated and the strength of contributions from intrinsic heavy quarks estimated. Furthermore, a detailed comparison of various gluon GPDs should be made and their errors taken into account. For photoproduction of charmonium production in particular, one should be aware of possible substantial power corrections since the charm quark mass although being large enough to allow for a perturbative treatment of the subprocess, is not large enough to suppress power corrections decisively. On the other hand, for electroproduction of charmonium power corrections are likely to be smaller. In principle the target asymmetry gives an access to $E^{g}$. However, $A_{U T}$ will likely be very small except for the case when $E^{g}$ markedly differs from common parameterizations. 


\subsection{Simulations of non-diffractive exclusive processes at an EIC}

\section{T. Horn}

\subsubsection{Introduction}

Exclusive processes in ep scattering at collider energies can be either "diffractive" (no exchange of quantum numbers between the target and the projectile/produced system) or "non-diffractive" (there is an exchange of quantum numbers). By measuring diffractive channels $\left(J / \Psi, \rho^{0}\right.$, or $\phi$ production) at sufficiently high $Q^{2}$, one probes the gluon GPDs and/or the singlet quark GPDs. In particular, $J / \Psi$ production probes the gluon GPD in the nucleon, and its $t$-dependence reveals the transverse spatial distribution of the gluons. Measurements of DVCS and exclusive $\rho^{0}$ production at high $Q^{2}$ provide access to the singlet quark and gluon GPDs.

Non-diffractive channels like $\pi^{+}, \pi^{0}$, or $K^{+}$production are sensitive to the flavor and spin structure of the nucleon at small $x_{B}$, which complements the information obtained from DVCS and meson production experiments in the valence region, e.g., HERMES and $6 \mathrm{GeV}$ and $12 \mathrm{GeV}$ JLab.

For moderate values of $x_{B}$, the proposed electron-ion collider (EIC) could reach $Q^{2}>10$ $\mathrm{GeV}^{2}$, where higher-twist contributions, which complicate the extraction of GPDs from the data, are expected to be small. Indeed, the comparison of different meson channels alone provides model-independent information about the ratio of quark spin and spatial distributions, and a comparison between, for instance, $\pi^{+}$and $K^{+}$production may allow for the studies of $\mathrm{SU}(3)$ symmetry in parton distributions.

\subsubsection{Rate predictions}

Rate predictions were made for several exclusive reaction channels using a new exclusive Monte Carlo generator. Here, we will focus on the $\pi^{+}$and $K^{+}$channels. These are the simplest systems also allowing for comparisons of non-strange and strange distributions similarly to the comparative studies of singlet quarks and gluons with diffractive exclusive channels.

Figure 3.40 shows the simulated cross section for exclusive pion and kaon production in the $5 \times 50 \mathrm{GeV}$ configuration in $e p$ collisions $(\sqrt{s}=31.6 \mathrm{GeV})$ at a luminosity of $10^{34} \mathrm{~cm}^{-2}$ $\mathrm{s}^{-1}$ 3, and data taking for 100 days. The simulated data shown here are divided into four $Q^{2}$ bins between 10 and $45 \mathrm{GeV}^{2}$ for a bin in $x_{B}$ between 0.02 and 0.05 . Each $Q^{2}$ bin was divided into nine $-t$ bins. The simulated pion data cover a range in $-t$ up to $1 \mathrm{GeV}^{2}$ with acceptable rates for the assumed run time and luminosity for all $Q^{2}$ bins. The rates in each $Q^{2}$ bin are highest at small values of $-t$ and smallest at high values of $-t$. This makes sense as one of the features of pion production is the dominance of the "pole term" at low $-t$. Furthermore, the pion rate decreases rapidly with higher $Q^{2}$ bins as the cross sections decrease, which is a characteristic behavior of exclusive reactions. However, one should keep in mind that reaching high $Q^{2}$ is needed due to the factorization requirement for studying the transverse spatial structure of sea quarks. The kaon simulated data are presented in the same kinematic bins as the pion data. The kaon cross section is smaller than the pion

\footnotetext{
${ }^{3}$ These energies and luminosities correspond to those given as medium-energy collider design prior to the INT 10-3 program, e.g., $\sqrt{s}=31.0 \mathrm{GeV}$.
} 
one, although it does not fall off as rapidly with increasing $-t$ for each $Q^{2}$ bin, because the kaon pole is not as dominant as the pion pole. The kaon rates are generally lower than the pion rates, but the effect is most visible in the largest $Q^{2}$ bins. For a fully differential kaon measurement, it is thus essential to have luminosities of at least $10^{34} \mathrm{~cm}^{-2} \mathrm{~s}^{-1}$. Given the design parameters of the medium-energy collider, this would correspond to a range in $\sqrt{s}$ between 31 and about $45 \mathrm{GeV}$.
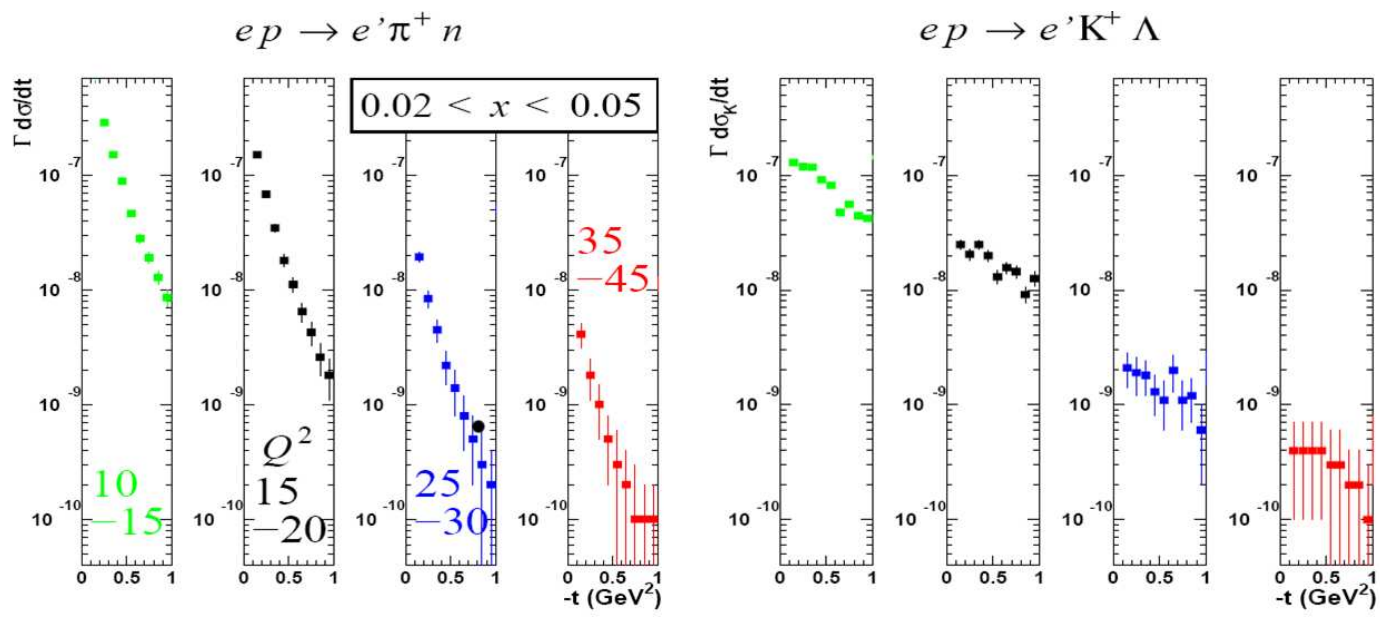

Figure 3.40. EIC simulations for the exclusive pion and kaon electroproduction cross sections at a electron beam energy of $5 \mathrm{GeV}$ and a proton beam energy of $50 \mathrm{GeV}(\sqrt{s}=31.6 \mathrm{GeV}), 100$ days running, and a luminosity of $10^{34} \mathrm{~cm}^{-2} \mathrm{~s}^{-1}$. The points are shown for a bin in $x_{B}$ of 0.02 to 0.05 . The four panels denote bins in $Q^{2}$ (from left to right) of $10-15 \mathrm{GeV}^{2}, 15-20 \mathrm{GeV}^{2}, 25-30 \mathrm{GeV}^{2}$, and $35-40 \mathrm{GeV}^{2}$.

The rate prediction depends to some extent on the cross section models included in the simulation. For pions, a Regge-based cross section model that describes existing data well was used 682. The model dependence of the rate prediction was estimated using a different cross section model based on an empirical parameterization of charged pion data 683]. For kaons, the rate estimate was based on the empirical fits to world kaon production data. The resulting uncertainty in the simulated rates was about a factor of two.

\subsubsection{Kinematic considerations}

Measurements in exclusive reactions require, besides knowledge of the beam quantities, information of all particles in the exclusive reaction, i.e., the scattered electron, the scattered meson, and the recoil baryon. Below we will illustrate the kinematic features of exclusive reactions using the $\mathrm{H}\left(e, e^{\prime} \pi^{+}\right) \mathrm{n}$ reaction. However, the kinematic distributions shown are independent of the exclusive channel, and are thus generally applicable to all exclusive reactions (diffractive and non-diffractive).

Figure 3.41 shows the accessible phase space for exclusive reactions in $e p$ collisions for five center of mass energies 4 . A cut of $Q^{2}>10 \mathrm{GeV}^{2}$ was applied to focus on the region of interest for transverse spatial structure studies. At a value of $\sqrt{s}=13.8 \mathrm{GeV}$, the meson distribution covers the angular range of about 30-40 degrees at relatively small momentum.

\footnotetext{
${ }^{4}$ The energies have been chosen to correspond to the preliminary values of the medium and high energy collider designs as given prior to the INT 10-3 program.
} 
Up to values of $\sqrt{s}=44.7 \mathrm{GeV}$, which correspond to nearly symmetric collisions, the exclusive meson distribution spreads over a wide angular range, still at a moderate momentum. At even higher values of $\sqrt{s}$, the angular spread is reduced significantly. Indeed, the meson distribution is pushed into a relatively narrow forward cone. Furthermore, the events of interest in this narrow angular range also have very high momentum approaching the beam energy. Exclusive measurements at these large values of $\sqrt{s}$ would thus require the detection of a high energy meson over a very small angular range.

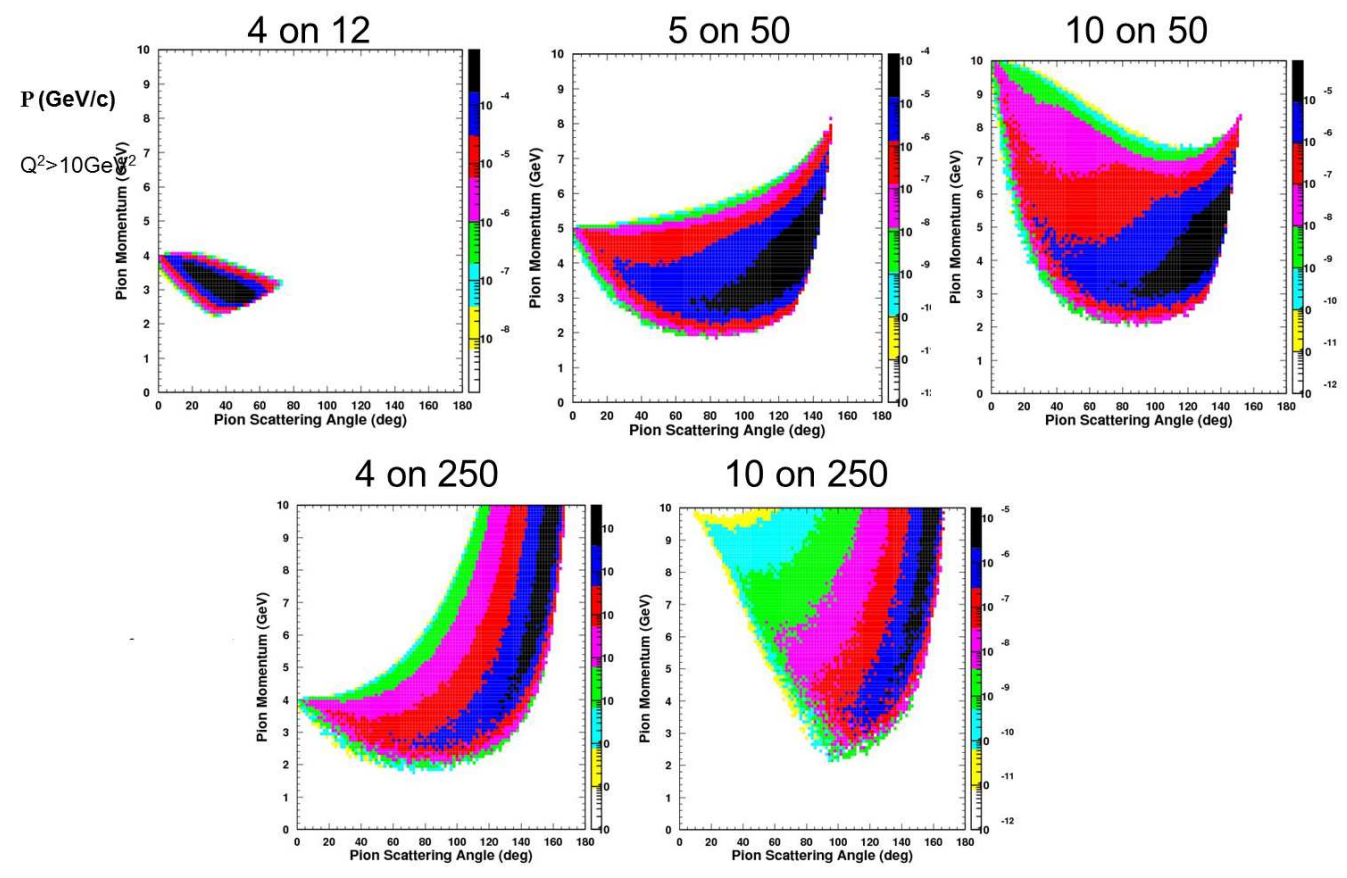

Figure 3.41. The kinematic phase space for light mesons in deep exclusive reactions for different $e p$ collisions. A cut of $Q^{2}>10 \mathrm{GeV}^{2}$ is applied to focus on events needed for studies of transverse spatial distributions. The darker regions in the figures denote regions of the highest intensity. The center of mass energies for the medium energies are $\sqrt{s}=13.8 \mathrm{GeV}, 31.6 \mathrm{GeV}$, and $44.7 \mathrm{GeV}$, and for the high energies $\sqrt{s}=63.2 \mathrm{GeV}$ and $100 \mathrm{GeV}$. In this simulation, the direction of the electron beam is toward increasing angles.

The momentum resolution $(d p / p)$ to first order scales linearly with the momentum. The best resolution is thus achieved by keeping the laboratory momenta as low as possible for a given $\sqrt{s}$. This is achieved in symmetric, or nearly symmetric collisions. As illustrated in figures 3.41 and 3.42 such kinematics also offer the advantage that the angular distribution of the outgoing electrons and mesons covers nearly $4 \pi$, providing the best angular resolution.

Figure 3.42 shows the scattered electron distribution in deep exclusive reactions. At modest electron energies (up to about $6 \mathrm{GeV}$ ) electrons predominantly scatter into the central and forward direction. Kinematically these correspond to high- $Q^{2}$ events, which are also the events of interest in studies of the transverse spatial structure of sea quarks. On the other hand, electrons at larger energies (up to the electron beam energy) scatter into the forward-electron direction. These events correspond to low- $Q^{2}$ events, which are of interest in photoproduction or heavy meson measurements.

The meson momentum distribution has a strong $Q^{2}$ dependence with the high momentum region dominated by low- $Q^{2}$ (photoproduction) events. This is illustrated in figure 3.43 


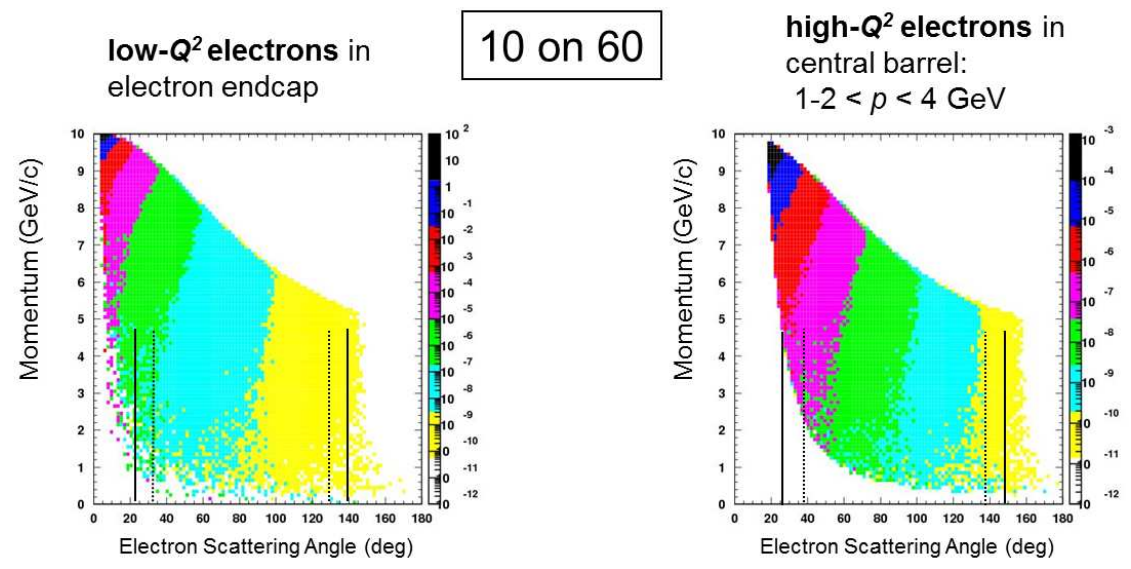

Figure 3.42. The kinematic phase space for electrons in exclusive reactions at low and high $Q^{2}$ at a fixed value of $\sqrt{s}=49.0 \mathrm{GeV}$.

with a comparison of photo- and electroproduction at fixed $\sqrt{s}=22 \mathrm{GeV}$ using a cut of $Q^{2}>$ $10 \mathrm{GeV}^{2}$ to select the electroproduced light mesons. The forward scattered photoproduced mesons dominate the low $Q^{2}$ region populating a narrow angle cone with high momentum while the light mesons with $Q^{2}>10 \mathrm{GeV}^{2}$ are centered around central angles at momenta between 2 and $4 \mathrm{GeV}$. The scattered electron distribution shows the same general features as discussed above. The $t$ distribution of the recoil baryons does not change with selecting the low or high $Q^{2}$ region.
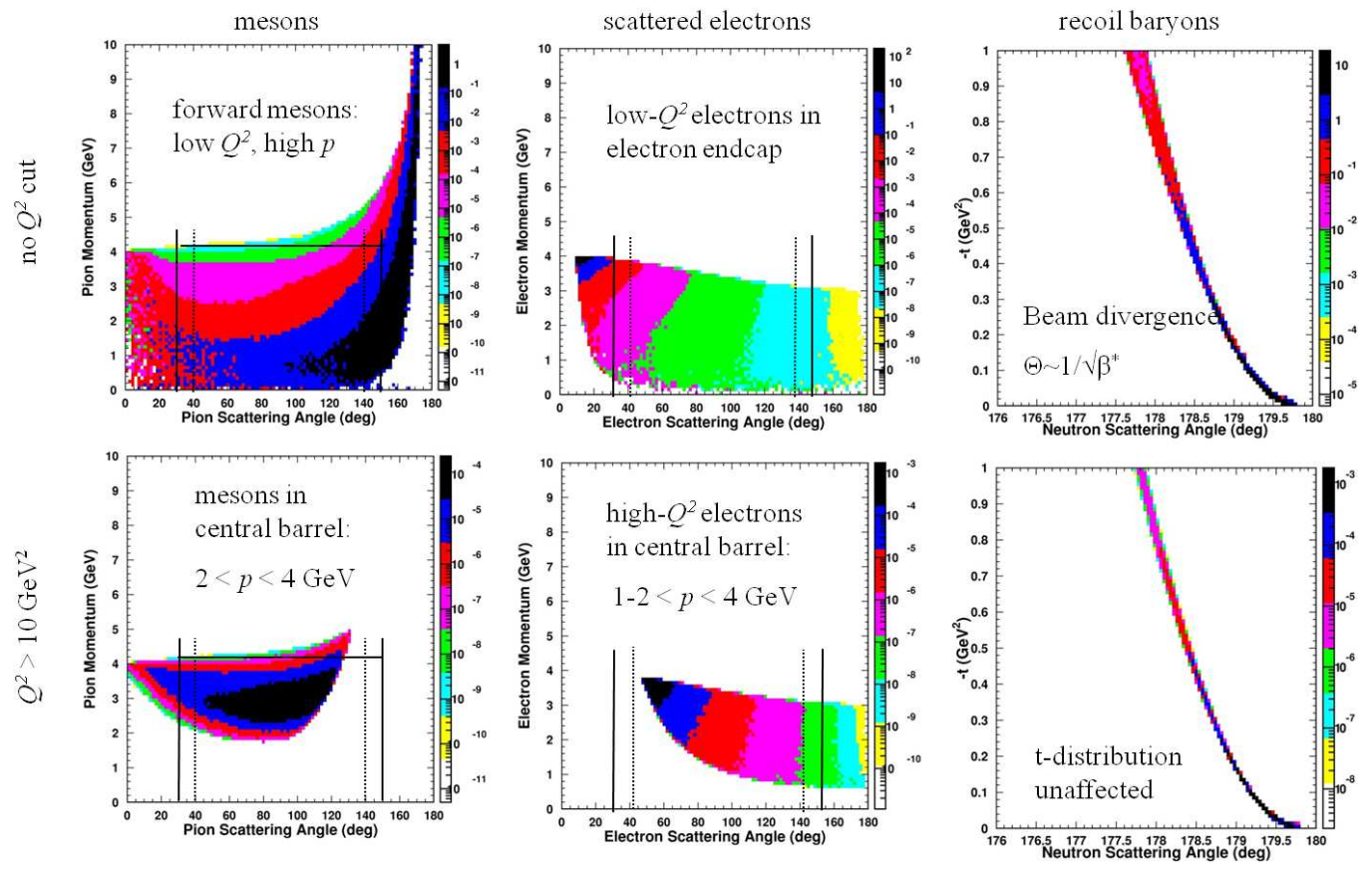

Figure 3.43. A comparison of the kinematic phase space for the scattered meson, electron, and the recoiling baryon in exclusive photo- and meson electroproduction at $\sqrt{s}=21.9 \mathrm{GeV}$. The three upper panels are dominated by photoproduction; the three lower panels focus on light meson events for studies of transverse spatial distributions. 
The EIC includes the option of using higher electron energies up to $11 \mathrm{GeV}$. Figure 3.44 shows a comparison of the meson distribution at fixed values of the ion beam energy for three values of $\sqrt{s}=21.9,31.6,44.7 \mathrm{GeV}$. Here, we will focus on the distribution in the central region, which is indicated by the vertical lines. As mentioned above, the meson distribution is pushed into a narrow angular cone with an increasingly higher momentum as $\sqrt{s}$ increases. Furthermore, the average meson momentum in the central region between $\pm 30 \mathrm{deg}$ increases from $4 \mathrm{GeV} / \mathrm{c}$ to about $8 \mathrm{GeV} / \mathrm{c}$ as the electron beam energy doubles from about 5 to $10 \mathrm{GeV}$ at a fixed ion beam energy. Measurements of exclusive reactions at electron beam energies of about $10 \mathrm{GeV} / \mathrm{c}$ and fixed ion beam energies would thus require the detection of high momentum mesons in the central angle region $\left( \pm 30^{\circ}\right)$.
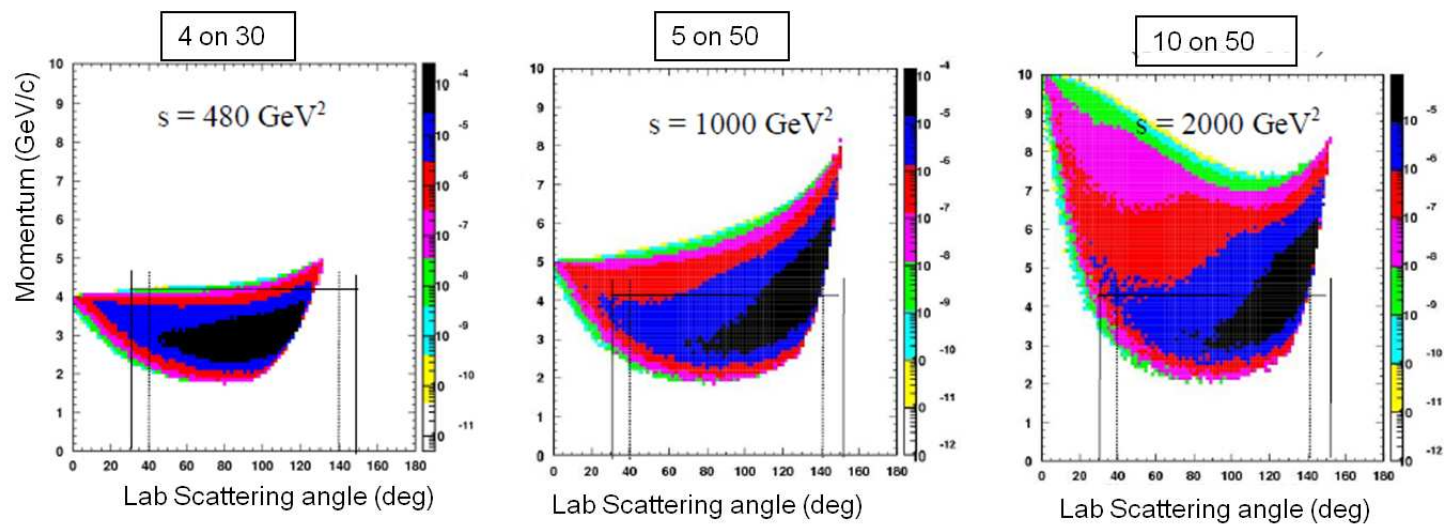

Figure 3.44. The meson kinematic phase space for higher energies for deep exclusive reactions for different combinations of the beam electron and proton energies. The first value in the labels denotes the electron beam energy. A cut of $Q^{2}>10 \mathrm{GeV}^{2}$ is applied to focus on events needed for studies of transverse spatial distributions.

To access the physics of interest in exclusive reactions and extract information about the GPDs, one needs data binned over a sufficiently large range in $-t$ : a range of at least $0<|t|<1 \mathrm{GeV}^{2}$ is needed. In ep collisions, the main challenge is that the outgoing baryons are scattered at relatively small angles, especially at low $-t$, as the resolution goes roughly as the inverse of the proton beam energy,

$$
\frac{\delta t}{t} \sim \frac{t}{E_{p}}
$$

Figure 3.45 illustrates the deep exclusive recoil baryon $-t$ angular resolutions for values of $\sqrt{s}=(13.8,31.6,44.7,63.2,100) \mathrm{GeV}$. The nearly symmetric collisions at lower proton beam energy provide the largest recoil baryon angular distributions of values of at least $1^{\circ}$. For asymmetric collisions, the distribution rapidly decreases to the angular distributions of less than $0.3^{\circ}$. To access the physics of interest, a better $-t$ resolution would thus be achieved with lower-energy and more symmetric kinematics.

\subsubsection{L/T separation}

Beyond studies of transverse spatial structure of sea quarks, non-diffractive processes provide the opportunity for additional studies, for instance, the tests of hard-soft QCD factorization and measurements of the pion form factor. These measurements require isolating the longitudinal part of the electroproduction cross section using the L/T separations. This 


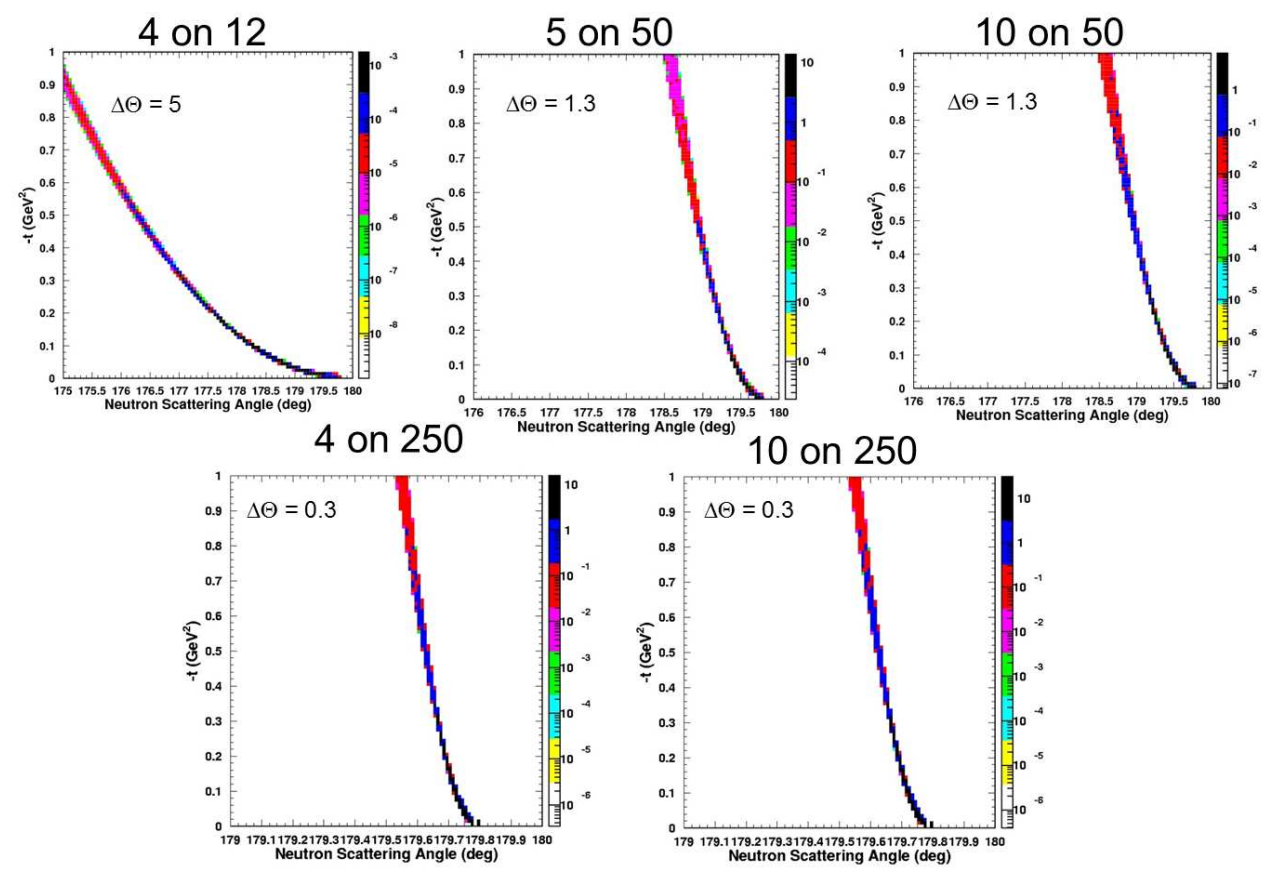

Figure 3.45. The kinematic phase space as $-t v s$. the scattering angle of the recoil baryon in exclusive reactions for five values of $\sqrt{s}$. The first value in the labels denotes the electron beam energy. A cut of $Q^{2}>10 \mathrm{GeV}^{2}$ is applied to focus on events needed for studies of transverse spatial distributions.

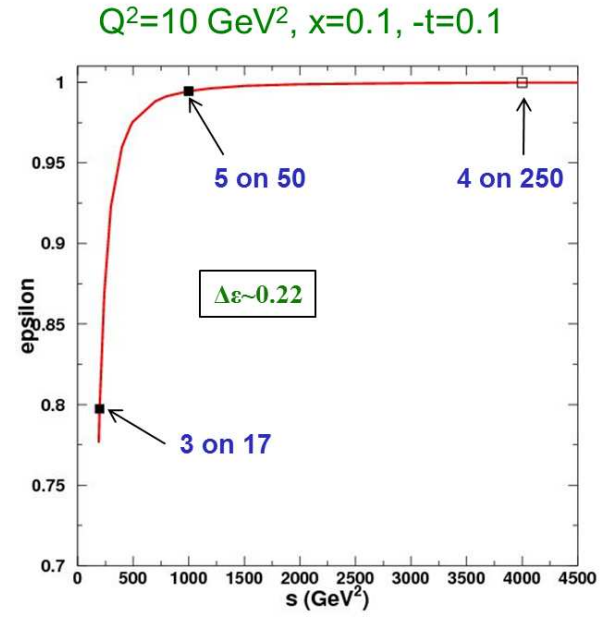

Figure 3.46. The virtual photon polarization, $\epsilon$ as a function of $s$ for different combinations of the electron and proton beam energies, at fixed $Q^{2}=10 \mathrm{GeV}^{2}, x_{B}=0.1$, and $-t=0.1 \mathrm{GeV}^{2}$. At high values of $s, \epsilon \rightarrow 1$ complicating the $\mathrm{L} / \mathrm{T}$ separation. 
technique requires comparing data taken at two different beam energies with sufficiently large separation of the virtual photon polarization, $\Delta \epsilon$, to control systematic uncertainties. Based on previous L/T separations, a minimum acceptable value of $\Delta \epsilon$ is 0.1 .

Figure 3.46 shows the accessible values of $\epsilon$ as a function of $s$ at fixed values of $Q^{2}$, $x_{B}$, and $-t$. The lowest value of $\epsilon$ of about 0.8 is reached at $\sqrt{s}=14.3 \mathrm{GeV}$, increasing to near unity as $s$ increases. Beyond $\sqrt{s}=31.6 \mathrm{GeV}, \epsilon$ is effectively unity making the $\mathrm{L} / \mathrm{T}$ distributions impossible.

\subsubsection{Summary of basic requirements for exclusive reactions}

Studies of exclusive non-diffractive processes provide important information on the transverse spatial distribution of non-perturbative sea quarks. These measurements require high luminosity for fully differential measurements in $x_{B},-t$, and $Q^{2}$ as well as recoil detection for exclusivity. They require a kinematic reach in $t$ of at least up to $1 \mathrm{GeV}^{2}$ with good resolution. Our studies suggest that exclusive processes for values of $x_{B}>0.01$ have better prospects with lower-energy and more symmetric kinematics.

The following list summarizes the basic experimental requirements for studies of the transverse spatial structure of sea quarks through non-diffractive exclusive processes.

Energies

- More symmetric energies favorable in exclusive non-diffractive reactions;

- Lower energies essential for a range in $\epsilon$ for the $\mathrm{L} / \mathrm{T}$ separation.

$\underline{\text { Kinematic Reach }}$

- Need $Q^{2}>10 \mathrm{GeV}^{2}$ (pointlike configurations);

$-x_{B}$ range between 0.001 and 0.1 overlapping with HERA and JLab $12 \mathrm{GeV}$;

$-s$ range between 200 and $1000 \mathrm{GeV}^{2}$.

Luminosity

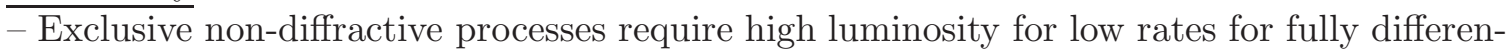
tial measurements; - Kaons push luminosity to $>10^{34} \mathrm{~cm}^{-2} \mathrm{~s}^{-1}$.

Detection

- Need recoil detection for exclusivity;

- Range in $-t$ and resolution. 


\title{
3.13 Partonic transverse spin in deep-inelastic exclusive ex- periments
}

\author{
Gary R. Goldstein, Simonetta Liuti
}

\subsubsection{Introduction}

In this contribution, we suggest a class of deeply virtual exclusive reactions, namely pseudoscalar meson electroproduction, as a means to access chiral-odd distributions. These are described by a set of four chiral-odd GPDs which enter the matrix elements for the various terms of the cross section. We conducted an analysis using a parameterization of the GPDs that is inspired by a physically motivated picture of the nucleon as a quark-diquark system with a Regge behavior. In the chiral-even sector a quantitative parameterization can be obtained from a global fit to PDFs, nucleon form factors, and DVCS data where the masses, couplings and Regge power behavior that set the scale for the dependence on the kinematic variables, $X, \zeta, t, Q^{2}$, are determined via a recursive procedure [684].

The extension of this parameterization scheme to the chiral-odd GPDs is critical for the phenomenology of deeply virtual meson electroproduction, which was begun particularly for the $\pi^{0}$ in [685]. In the diquark spectator model, chiral-even helicity amplitudes are simply related to their chiral-odd counterparts via parity transformations. For the $d$-quark case it is only the axial diquark relations that are involved, while the $u$-quark involves the scalar contribution, as well. We thereby obtain the full set of four chiral-odd GPDs, each being linearly related to helicity amplitudes. This allows us to predict the behavior of pseudoscalar electroproduction [686].

It has now become particularly pressing to study the heavy quark components of the nucleon because of the advent of the LHC. For the types of precision measurements in the unprecedented multi-TeV CM energy regimes envisaged at the LHC it will be necessary to provide accurately determined QCD inputs. The analyses in [129] have shown how the inclusion of non perturbative charm quarks could modify the outcome of global PDF analyses. However, the situation is not clear-cut. We therefore extended our analysis to strange and charm pseudoscalar meson production [687. We proposed that in order to refine analyses such as the one in [129], new observables need to be identified from deeply virtual meson production and spin correlation measurements. We presented preliminary results involving the following electroproduction exclusive processes: (1) $\gamma^{*} p \rightarrow J / \psi p^{\prime}$; (2) $\gamma^{*} p \rightarrow D \bar{D} p^{\prime} ;(3) \gamma^{*} p \rightarrow \bar{D} \Lambda_{c}$; (4) $\gamma^{*} p \rightarrow \eta_{C} p^{\prime}$. These processes necessitate: i) high luminosity because they are exclusive; ii) high enough $Q^{2}$ to produce the various charmed mesons, and iii) a wide kinematical range in Bjorken $x$.

Finally, a few questions have emerged concerning on one side the applicability of dispersion relations to deeply virtual exclusive processes [688], and on the other, the commonly assumed partonic picture of the ERBL region [689. Newer deeply virtual exclusive cross section and asymmetry measurements in extended kinematical regimes will provide essential tests of the theory.

\subsubsection{Transverse spin from pseudoscalar meson production}

The basic definition of the quark-nucleon GPDs is through off-forward matrix elements of quark field correlators. Contracting with the Dirac matrices, $\gamma^{\mu}$ or $\gamma^{\mu} \gamma^{5}\left(\sigma^{\mu \nu} \gamma^{5}\right)$, and integrating over the internal quark momenta gives rise to the four chiral-even GPDs, $H, E$ 
or $\widetilde{H}, \widetilde{E}$, and four chiral-even GPDs, $H_{T}, E_{T}, \widetilde{H}_{T}, \widetilde{E}_{T}$ 575. The crucial connection of the eight GPDs to spin dependent observables in DVCS and DVMP is through the helicity decomposition [575]. For example,

$$
\begin{gathered}
A_{++,++}(X, \xi, t)=\frac{\sqrt{1-\xi^{2}}}{2}\left(H^{q}+\tilde{H}^{q}-\frac{\xi^{2}}{1-\xi^{2}}\left(E^{q}+\tilde{E}^{q}\right)\right), \\
A_{++,--}(X, \xi, t)=\sqrt{1-\xi^{2}}\left(H_{T}^{q}+\frac{t_{0}-t}{4 M^{2}} \tilde{H}_{T}^{q}-\frac{\xi}{1-\xi^{2}}\left(\xi E_{T}^{q}+\tilde{E}_{T}^{q}\right)\right) .
\end{gathered}
$$

We have constructed a robust model for the GPDs, extending previous work [690] that is based on the parameterization of diquark spectators and Regge behavior at small $X$. The GPD model parameters are constrained by their relations to PDFs (at $\zeta=0, t=0$ ) and to nucleon form factors $F_{1}(t), F_{2}(t), g_{A}(t)$, and $g_{P}(t)$ through the first $x$ moments. For the chiral-odd GPDs, there are fewer constraints. In particular, $H_{T}(X, 0,0)=h_{1}(X)$ can be fit using the loose constraints in [250] since the first moment of $H_{T}(X, \xi, t)$ is the "tensor form factor", called $g_{T}(t)$. It is conjectured that the first moment of $2 \tilde{H}_{T}^{q}(X, 0,0)+E_{T}^{q}(X, 0,0)$ is a "transverse anomalous moment", $\kappa_{T}^{q}$, defined in [497].

With our ansatz, many observables can be determined in parallel with the corresponding Regge predictions. Since the initial work [685, we have undertaken a more extensive parameterization and presented several new predictions [684]. In figure 3.47, we show an example corresponding to the transversely polarized proton target.
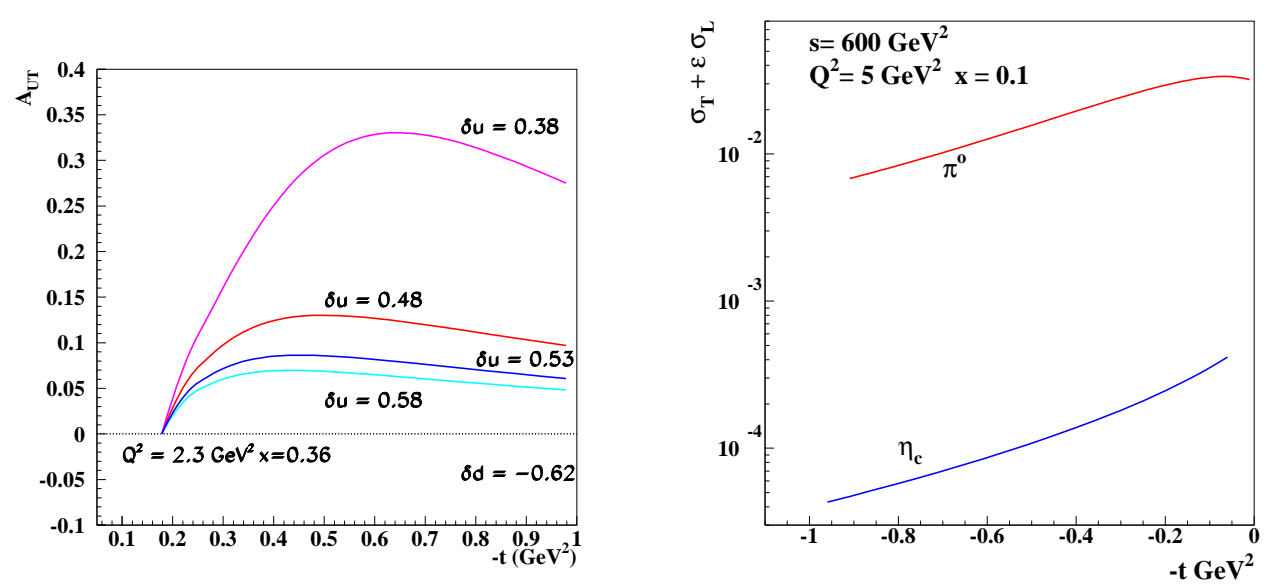

Figure 3.47. Left: Transverse spin asymmetry, $A_{U T}$, vs. $-t$ at $Q^{2}=2.3 \mathrm{GeV}^{2}$ and $x_{B}=0.36$ for different values of the tensor charge, $\delta u$, with fixed $\delta d=-0.62$. Right: Comparison of $\pi^{0}$ and $\eta_{c}$ cross sections. The range between the two lines gives an estimate of where the cross sections for the other processes will lie.

The measured cross section for $\pi^{0}$ is sizable and has large transverse $\gamma^{*}$ contributions. This indicates that the main contributions should come from chiral-odd GPDs, for which the $t$-channel decomposition is richer. In particular, because these GPDs arise from the Dirac matrices $\sigma^{\mu \nu}$, there are two series of $J^{P C}$ values for each GPD [691] corresponding to spacespace or time-space combinations $1^{--}$and $1^{+-}$. These series occur for three of the four chiral-odd GPDs, with exception of $\widetilde{E}_{T}$. We are thus led to the conclusion that chiral-odd GPDs will dominate the neutral pseudoscalar leptoproduction cross sections. This result 
has interesting consequences. First, in a factorized handbag picture, these GPDs will couple to the hard part, the $\gamma^{*}+$ quark $\rightarrow \pi^{0}+$ quark, provided that $\pi^{0}$ couples through $\gamma^{5}$, which is naively twist-three, rather than the twist-two coupling $\gamma^{+} \gamma^{5}$. Second, the vector $1^{--}$and axial-vector $1^{+-}$in the $t$-channel, viewed as particles $\left(\rho^{0}, \omega\right.$ and $\left.b_{1}^{0}, \mathrm{~h}\right)$, couple primarily to the transverse virtual photon. For Reggeons, the $1^{--}$does not couple at all to the longitudinal photon, while the axial-vector $1^{+-}$does so through helicity flip 692. Guided by these observations [685], we assume that the hard part depends on whether the exchange quantum numbers are in the vector or axial-vector series, thereby introducing orbital angular momentum into the model. We use $Q^{2}$ dependent electromagnetic "transition" form factors for vector or axial-vector quantum numbers going to a pion. We calculate these using pQCD for $q+\bar{q}+\gamma^{*}\left(Q^{2}\right) \rightarrow q+\bar{q}$ and a standard $z$-dependent pion wave function, convoluted in the impact parameter representation that allows orbital excitations to be easily implemented.

With our model for the chiral-odd spin-dependent GPDs and these transition form factors, we can obtain the full range of cross sections and asymmetries in kinematic regimes that coincide with ongoing JLab experiments. (A similar emphasis on chiral-odd contributions for $\pi$ electroproduction has recently been proposed [693], although the details of that model are quite different from ours.) We are able to predict the important transverse photon contributions to the observables [685]. In figure 3.47 (left), we show one striking example of the predictions that depend on the values of the tensor charges, thereby providing a means to narrow down those important quantities. This program has been presented 684 and further details will soon appear, as the refinements of the chiral-odd parameterization are completed [686]. In figure 3.47 (right), we show the cross section, $\sigma_{T}+\epsilon \sigma_{L}$, for charmed meson production and compare it to the one for $\pi^{0}$ production [687.

In summary, through the use of physically motivated models and the new horizons provided by the EIC, a far reaching interpretation of the separate spin-dependent GPDs and thereby, a picture of the transverse structure of the nucleons will emerge. The connection of chiral-odd GPDs to the transversity structure of the nucleon is of great interest as a manifestation of quark and gluon orbital angular momentum. 


\title{
3.14 Ways to access transversity GPDs at the EIC
}

\author{
B. Pire, L. Szymanowski, S. Wallon
}

\subsubsection{Introduction}

Transversity quark distributions in the nucleon remain among the most unknown leading twist hadronic observables. This is mostly due to their chiral-odd character which enforces their decoupling in most hard amplitudes. Generalized parton distributions (GPDs) offer a new way to access the transversity dependent quark content of the nucleon. The factorization properties of exclusive amplitudes allow in principle to extract the four chiral-odd transversity GPDs [694], $H_{T}, E_{T}, \tilde{H}_{T}, \tilde{E}_{T}$. However, one-photon or one-meson electroproduction leading twist amplitudes are insensitive to them [695, 696]. The strategy which we followed in 697, 698] is to study the leading twist contribution to exclusive processes where more mesons are present in the final state. Note that, contrarily to transversity PDFs, transversity GPDs enter the formulae for exclusive cross sections even when considering unpolarized proton target, provided one selects the polarization state of an outgoing meson.

\subsubsection{Diffractive photoproduction of two $\rho$ mesons}

We consider [697, 698], in analogy with the virtual photon exchange occurring in the deep inelastic electroproduction of a meson, the subprocess:

$$
\mathbb{P}\left(q_{P}\right)+p\left(p_{2}\right) \rightarrow \rho_{T}\left(p_{\rho}\right)+N^{\prime}\left(p_{2^{\prime}}\right)
$$

of almost forward scattering of a virtual Pomeron (the hard scale is the virtuality $-q_{P}^{2}$ of this Pomeron) on a nucleon. This subprocess is at work in the process

$$
\gamma_{L / T}^{(*)}(q)+p\left(p_{2}\right) \rightarrow \rho_{L, T}^{0}\left(q_{\rho}\right)+\rho_{T}\left(p_{\rho}\right)+N^{\prime}\left(p_{2^{\prime}}\right),
$$

where a real or virtual photon scatters on a proton $p$, which leads via a two-gluon exchange to the production of two vector mesons separated by a large rapidity gap and the scattered nucleon $N^{\prime}$, as shown on figure 3.48. The final state may be either $\rho^{0} \rho^{0} p$ or $\rho^{0} \rho^{+} n$. In both cases, the two-gluon exchange with the nucleon line is forbidden by charge conjugation or charge conservation and the process is thus sensitive only to quark GPDs. We consider the kinematical region where the rapidity gap between $\rho\left(p_{\rho}\right)$ and $N^{\prime}$ is much smaller than the one between $\rho\left(q_{\rho}\right)$ and $\rho\left(p_{\rho}\right)$, i.e., the energy of the $\left(\rho\left(p_{\rho}\right)+N^{\prime}\right)$ system is smaller than that of the $(\rho+\rho)$ system but is still large enough to justify our approach (in particular, it is much larger than baryonic resonance masses). Since quasi-real transverse photons are more abundant in electron-ion collisions and charged pions are most easily detected, one may specialize to the reaction:

$$
\gamma_{T}(q)+p\left(p_{2}\right) \rightarrow \rho_{L, T}^{0}\left(q_{\rho}\right)+\rho_{T}^{0}\left(p_{\rho}\right)+p\left(p_{2^{\prime}}\right),
$$

where the initial quasi-real photon is treated as if it were real.

In this kinematical regime, the amplitude for this process is calculable consistently within the collinear factorization method, as an integral (over the longitudinal momentum fractions of the quarks) of the product of two amplitudes: the first one (the impact factor) describes the transition $\gamma^{(*)} \rightarrow \rho_{L, T}^{0}$ via a two-gluon exchange and the second one describes the subprocess $\mathbb{P}+p \rightarrow \rho_{T}^{0}+p$. The fact that the latter process is closely related to 


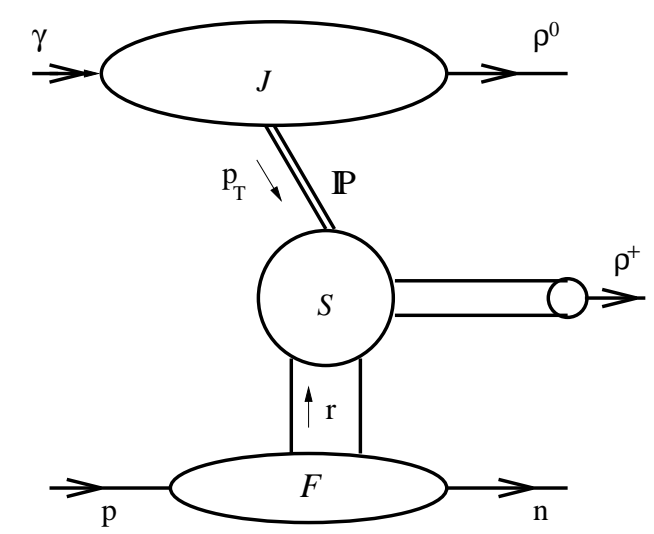

Figure 3.48. Factorization of the process $\gamma_{T}(q)+p\left(p_{2}\right) \rightarrow \rho_{L, T}^{0}\left(q_{\rho}\right)+\rho_{T}^{+}\left(p_{\rho}\right)+n\left(p_{2^{\prime}}\right)$ in the asymmetric kinematics discussed in the text. $\mathbb{P}$ is the hard Pomeron modeled by a two-gluon exchange.

the electroproduction process $\gamma^{*} p \rightarrow \rho^{0} p$ allows us to separate its long distance dynamics expressed through the GPDs from a perturbatively calculable coefficient function. The skewness parameter $\xi$ is related in the usual way $\left(\xi \approx x_{B} /\left(2-x_{B}\right)\right)$ to the Bjorken variable defined by the Pomeron momentum $x_{B}=-q_{P}^{2} /\left(2 q_{P} \cdot p_{2}\right)$. The choice of a transversely polarized vector meson $\rho_{T}^{0}$ involves a chiral-odd distribution amplitude, which in turn selects the chiral-odd GPDs.

The resulting scattering amplitude $\mathcal{M}^{\gamma^{*}} p \rightarrow \rho_{L}^{0} \rho_{T}^{0} p$ then receives contributions from the four chiral-odd GPDs $H_{T}, \tilde{H}_{T}, E_{T}$ and $\tilde{E}_{T}$, but only the first one does not vanish kinematically in the forward direction. Thus, assuming that the Mandelstam variable $-t=$ $-\left(p_{2}-p_{2^{\prime}}\right)^{2}$ is sufficiently small, the transversity GPD $H_{T}$ contribution dominates the amplitude of process (3.95) which reads :

$$
\begin{aligned}
& \mathcal{M}^{\gamma p \rightarrow \rho_{L}^{0} \rho_{T}^{0} p}=\sin \theta 16 \pi^{2} s \alpha_{s} f_{\rho}^{T} \xi \sqrt{\frac{1-\xi}{1+\xi}} \frac{C_{F}}{N\left(p_{T}^{2}\right)^{2}} \\
& \times \int_{0}^{1} \frac{d u \phi_{\perp}(u)}{u^{2} \bar{u}^{2}} J^{\gamma \rightarrow \rho_{L}^{0}}\left(u p_{T}, \bar{u} p_{T}\right) \frac{H_{T}^{u} d(\xi(2 u-1), \xi, t)}{\sqrt{2}},
\end{aligned}
$$

where $H_{T}^{u d}=H_{T}^{u}-H_{T}^{d} ; \phi_{\perp}(u)$ is the distribution amplitude (DA) of the $\rho_{T}$ meson; $\theta$ is the angle between the transverse polarization vector of the target $\vec{n}$ and the polarization vector $\vec{\epsilon}_{T}$ of the produced $\rho_{T}^{0}$ meson; $\vec{\varepsilon}$ is the polarization vector of the initial photon. The impact factor reads

$$
J^{\gamma \rightarrow \rho_{L}}\left(k_{T 1}, k_{T 2}=p_{T}-k_{T 1}\right)=-\frac{e \alpha_{s} \pi f_{\rho}^{0}}{\sqrt{2} N} \int_{0}^{1} d z(2 z-1) \phi_{\|}(z)\left(\vec{\varepsilon} \cdot \vec{Q}_{P}\right),
$$

with

$$
\begin{gathered}
\vec{Q}_{P}\left(k_{T 1}, k_{T 2}=p_{T}-k_{T 1}\right)=\frac{z \vec{p}_{T}}{z^{2} p_{T}^{2}+Q^{2} z \bar{z}+m_{q}^{2}}-\frac{\bar{z} \vec{p}_{T}}{\bar{z}^{2} p_{T}^{2}+Q^{2} z \bar{z}+m_{q}^{2}} \\
+\frac{\vec{k}_{T 1}-z \vec{p}_{T}}{\left(k_{T 1}-z p_{T}\right)^{2}+Q^{2} z \bar{z}+m_{q}^{2}}-\frac{\vec{k}_{T 1}-\bar{z} \vec{p}_{T}}{\left(k_{T 1}-\bar{z} p_{T}\right)^{2}+Q^{2} z \bar{z}+m_{q}^{2}}
\end{gathered}
$$

The scattering amplitude (3.96) receives a contribution only from the ERBL region. 


\subsubsection{Cross section estimates}

To obtain an estimate of the differential cross section of this process, we need a model for the transversity GPD $H_{T}^{q}(x, \xi, t)(q=u, d)$. We proposed a simple meson-pole approach starting with the effective interaction Lagrangian,

$$
\mathcal{L}_{\mathcal{A N N}}=\frac{g_{A N N}}{2 M} \bar{N} \sigma_{\mu \nu} \gamma_{5} \partial^{\nu} A^{\mu} N
$$

in which $g_{A N N}$ is the coupling constant determining the strength of the interaction of the axial meson $A$ with the nucleon $N$. This yields

$$
H_{T}^{a}(x, \xi)=\frac{g_{A N N} f_{A}^{a \perp}\left(\Delta \cdot S_{T}\right)^{2}}{2 M_{N} m_{A}^{2}} \frac{\phi_{\perp}\left(\frac{x+\xi}{2 \xi}\right)}{2 \xi},
$$

where $\Delta$ is the transverse part of the momentum transfer vector $r ; f_{A}^{a \perp}$ is related to the $A$ meson decay constant. Identifying the scalar product $\left(\Delta \cdot S_{T}\right)^{2}$ with the average of the intrinsic transverse momentum of the quarks, $\left(\Delta \cdot S_{T}\right)^{2} \rightarrow 1 / 2\left\langle k_{\perp}^{2}\right\rangle$, and the axial meson $A$ with the $b_{1}$ meson, $A=b_{1}(1235)$, we obtain our final expression for $H_{T}^{u d}$ :

$$
H_{T}^{u d}(x, \xi, 0)=\frac{g_{b_{1} N N} f_{b_{1}}^{T}\left\langle k_{\perp}^{2}\right\rangle}{2 \sqrt{2} M_{N} m_{b_{1}}^{2}} \frac{\phi_{\perp}^{b_{1}}\left(\frac{x+\xi}{2 \xi}\right)}{2 \xi},
$$

where $f_{b_{1}}^{T}=\sqrt{2} f_{a_{1}} / m_{b_{1}}$ with $f_{a_{1}}=(0.19 \pm 0.03) \mathrm{GeV}^{2} ; g_{b_{1} N N}=5 /(3 \sqrt{2}) g_{a_{1} N N}$ with $g_{a_{1} N N}=7.49 \pm 1.0 ;\left\langle k_{\perp}^{2}\right\rangle=(0.58-1.0) \mathrm{GeV}^{2}$. The $t$ dependence of the chiral-odd GPDs may be parameterized in the following simple way:

$$
H_{T}^{q}(x, \xi, t)=H_{T}^{q}(x, \xi, t=0) \times \frac{C^{2}}{(t-C)^{2}},
$$

with the standard dipole form factor with $C=0.71 \mathrm{GeV}^{2}$.

In figure 3.49, we show our model estimates for the differential cross sections of photoproduction of two vector mesons (3.94), $\rho^{0}$ and transversely polarized $\rho^{+}$, with the unpolarized beam and target. Note that these cross sections depend on the $\gamma$-nucleon energy only through the variable $\xi$. The cross sections for the processes with two neutral $\rho^{0}$ mesons in the final state are two times smaller than those with $\rho^{0} \rho^{+}$.

\subsubsection{Photoproduction at lower photon energies}

Diffractive physics requires high photon energies. If low energy photon tagging may be performed at the EIC, a QCD study based solely on the collinear factorization (i.e., without any Pomeron exchange) approach may be followed, opening other interesting channels. In [699, 700], we considered the process:

$$
\gamma(q)+p\left(p_{1}, \lambda\right) \rightarrow \pi^{+}\left(p_{\pi}\right)+\rho_{T}^{0}\left(p_{\rho}\right)+n\left(p_{2}, \lambda^{\prime}\right),
$$

on a polarized or unpolarized proton target, in the kinematical regime of large invariant mass $M_{\pi \rho}$ of the final meson pair (the hard factorization scale is now this invariant mass) and small momentum transfer $t=\left(p_{1}-p_{2}\right)^{2}$ between the initial and the final nucleons. Roughly speaking, this kinematics means a moderate-to-large, and approximately opposite, transverse momentum of each meson. The cross sections obtained are sizeable at values of 

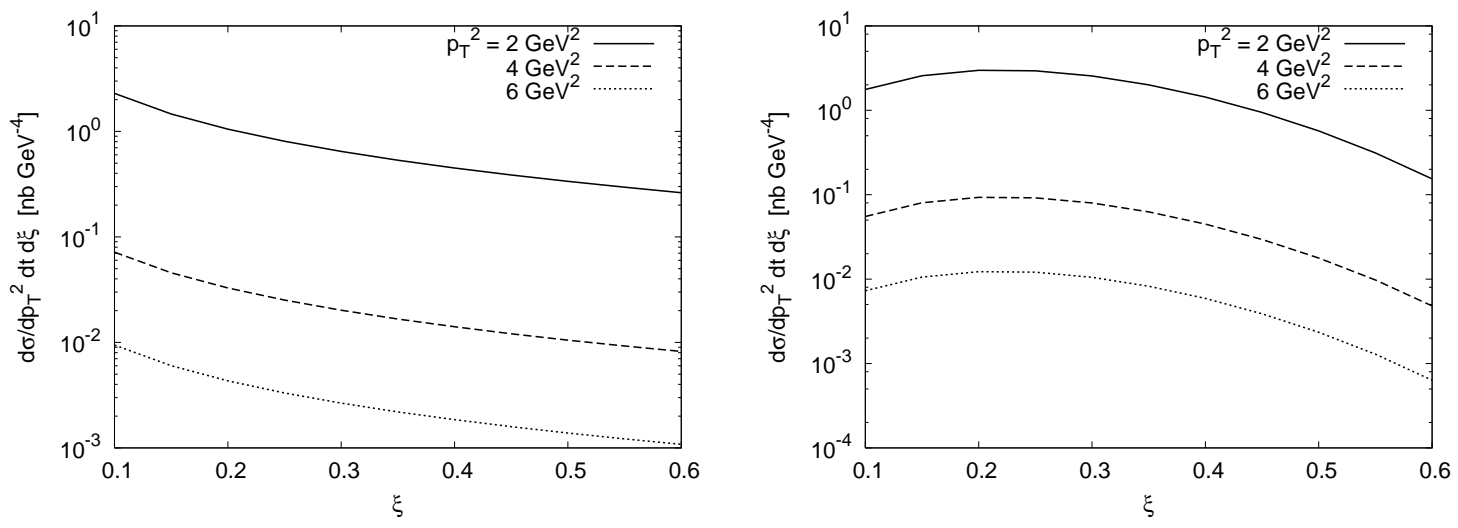

Figure 3.49. The differential cross section for the photoproduction of $\rho_{T}^{0}$ and $\rho_{T}^{+}$(left panel) and $\rho_{L}^{0}$ and $\rho_{T}^{+}$(right panel) as a function of $\xi$ for $p_{T}^{2}=2,4$, and $6 \mathrm{GeV}^{2}$. The cross sections for the processes with two neutral $\rho^{0}$ mesons in the final state are two times smaller than those with $\rho^{0} \rho^{+}$.

$s_{\gamma N}$ of the order $10-20 \mathrm{GeV}^{2}$ but decrease quickly with the photon energies. This regime is more in the range of the JLab 12 program than of EIC.

In conclusion, we stress that this approach only assumes leading twist factorization of non-perturbative quantities, such as meson DAs and chiral-odd GPDs.

Acknowledgments. We are grateful to D.Yu. Ivanov, R. Enberg and O.V. Teryaev for their contributions to the results presented here. 


\section{Chapter 4}

\section{Input from lattice QCD}

Chapter editors:

D. Hasch, F. Yuan 


\subsection{Introduction}

\section{Philipp Hägler, Bernhard Musch, Andreas Schäfer}

The focus of research at an EIC is a precise and comprehensive understanding of the quark-gluon structure and dynamics of hadrons and nuclei within the scope of traditional QCD, as well as beyond it, e.g., beyond the formalism based on collinear parton distributions. This requires the combination of input from many different fields, including lattice QCD (LQCD). In this chapter, the status of LQCD as well as the prospects for the next decade are sketched. The main tasks of LQCD is to increase precision and to extend the scope of LQCD calculations. [Both depends also significantly on progress in the understanding of perturbative QCD (pQCD).] Although working out solutions for all technical details is a formidable task, recent developments suggest that LQCD should have settled most of the open theory issues by the time the EIC starts operating.

LQCD results are by now routinely used as input for phenomenology if direct experimental information is not available. This trend will intensify when in the future ever more subtle aspects are investigated. Therefore, the EIC and a dedicated effort in LQCD have to form a strong union. If direct comparison with experiment has proven certain types of LQCD calculations to be reliable, LQCD can provide easily information which is hard to obtain experimentally, for example on moments of PDFs and GPDs and the flavour decomposition of structure functions. In this context it is, unfortunately, quite often not sufficiently appreciated that most quantities of interest calculated on the lattice can only be linked to experiment by highly non-trivial input from pQCD. Thus all three elements, experiment, LQCD and pQCD have to be combined to reach optimal results.

The two main sources of difficulty are:

- The basis of LQCD is the observation that the analytic continuation to imaginary times $x^{0} \rightarrow \mathrm{i} x^{4}$ relates quantum field theory to statistics/thermodynamics. The latter allows for a purely numerical treatment by means of Monte Carlo techniques. This analytic continuation is only simple for time-independent quantities. The quantities of this type usually studied are matrix elements of local operators (which can be evaluated at $x^{0}=0=x^{4}$ ).

$$
\left\langle h^{\prime}\left(p^{\prime}\right)|\mathcal{O}(x=0)| h(p)\right\rangle .
$$

Here $h, h^{\prime}$ can be any hadronic state, including the QCD vacuum. One typically needs the continuum operator product expansion (OPE) to link such quantities to observables.

- Most QCD quantities of interest are scheme and scale dependent. Only in leading order (LO) this dependence can be neglected, but LO calculations are in most cases insufficient for a high precision machine like the EIC. Thus LQCD results for matrix elements of the type Eq. (4.1) have to be matched to a specific pQCD setting, typically the $\overline{M S}$ scheme at a certain scale $\mu$. This requires also a matching of renormalization effects, which are quite different in the continuum and on the lattice due to the loss of continuum symmetries (as discussed below). The lattice discretization leads to different Feynman rules, in particular the appearance of tadpole diagrams. Another concrete, simple example is the modification of the fermion propagator on the lattice, 


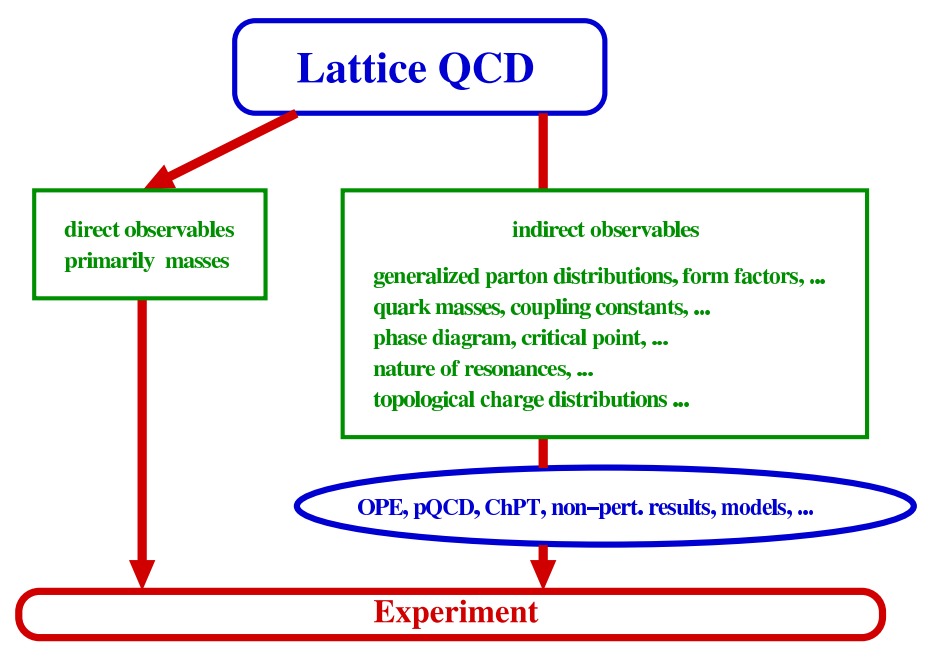

Figure 4.1. Sketch of the different types of lattice observables. For nearly all quantities of interest for an EIC, a combination of pQCD and LQCD is needed to make contact to experiment.

which typically might read (depending on the specific lattice action)

$$
D_{\text {Lattice }}(p)=\frac{m-\mathrm{i} a^{-1} \sum_{\mu} \gamma_{\mu} \sin \left(p_{\mu} a\right)}{m^{2}+a^{-2} \sum_{\mu} \sin ^{2}\left(p_{\mu} a\right)}
$$

Thus, renormalization factors on the lattice and in the continuum differ by finite amounts, typically of the order of a few up to 30 percent. If one aims at an overall precision of order percent, the matching of the renormalization factors between nonperturbative (i.e. all order) lattice calculations and fixed order continuum calculations has to be achieved with high precision. To achieve this for all quantities of interest is clearly one of the major challenges for theory, both LQCD and pQCD, in the next decade.

The points just discussed apply to 'indirect observables', as illustrated in the right column of Fig. 4.1. There do also exist some observables which can be compared directly, without the need for renormalization, especially hadron masses. However, these are well known experimentally, while the aim of LQCD is clearly to provide information on hitherto unknown correlators. The observables of interest at the EIC, require nearly always nonperturbative renormalization in the corresponding lattice studies.

The calculation of matrix elements like (4.1) proceeds as follows:

1. One generates a number of ensembles of gauge field configurations with the correct statistical weights. The parameters for these ensembles are chosen such that one has best control (for given computer resources) of the combined limit: lattice spacing $a \rightarrow 0$; physical lattice size $L \gg 1 / \Lambda_{Q C D}$; quark masses $m_{q} \rightarrow m_{q}$ (physical); large number of independent field configurations, typically $N \gg 100$.

2. One generates hadronic states using products of quark fields with the correct quantum numbers (sources), e.g., one can use for a proton $\left(C=i \gamma^{2} \gamma^{4}\right.$ is the charge conjugation matrix, $i, j, k$ run over the three color states):

$$
\hat{B}_{\alpha}(t, \vec{p})=\sum_{\vec{x}} e^{\mathrm{i} \vec{p} \cdot \vec{x}} \epsilon_{i j k} \hat{u}_{\alpha}^{i}(x) \hat{u}_{\beta}^{j}(x)\left(C^{-1} \gamma_{5}\right)_{\beta \gamma} \hat{d}_{\gamma}^{k}(x)
$$


Propagation in Euclidean time generates real exponentials rather than phases. Consequently, when expanding into a series in the correct physical multi-particle hadronic states, propagation in Euclidean time filters out the lowest mass state for large enough times,

$$
\begin{aligned}
\hat{B}(0, \vec{p})|0\rangle & =c_{0}|N\rangle+c_{1}\left|N^{\prime}\right\rangle+c_{2}|N \pi\rangle+\ldots \\
\hat{B}(t, \vec{p})|0\rangle & =c_{0} e^{-E_{N} t}|N\rangle+c_{1} e^{-E_{N^{\prime}} t}\left|N^{\prime}\right\rangle+c_{2} e^{-E_{N \pi} t}|N \pi\rangle+\ldots \\
& \sim c_{0} e^{-E_{N} t}|N\rangle
\end{aligned}
$$

To improve signals and to investigate higher lying states one uses a set of sources and calculates a full correlation matrix.

3. One constructs ratios for quantities of interest in which the exponential factors cancel, e.g.,

$$
\frac{\tilde{\Gamma}_{\alpha \beta}\left\langle B_{\beta}(t, \vec{p}) \mathcal{O} \bar{B}_{\alpha}(0, \vec{p})\right\rangle}{\Gamma_{\alpha \beta}\left\langle B_{\beta}(t, \vec{p}) \bar{B}_{\alpha}(0, \vec{p})\right\rangle}
$$

4. Finally, one determines the relevant renormalization factors for the operator $\mathcal{O}$ nonperturbatively on the lattice and relates the lattice results to a specific pQCD scheme.

One has to appreciate that the efficient combination of experimental and LQCD results requires a good and efficient parametrization for the quantities of interest. If there exists e.g. an efficient parametrization of a specific GPD etc. in terms of just a few parameters, each result will constrain the acceptable parameter range. Thus also high quality model building is necessary.

Over the years it became clear that it is very non-trivial to derive realistic estimates, in particular of the systematic uncertainties, from the highly correlated quantities extracted from Lattice Monte Carlo data. The ultimate test revealing potentially underestimated systematic uncertainties is the comparison of certain benchmark observables with experimental measurements, in this case, EIC data. The next best option is to compare results obtained with substantially different lattice formulations. In principle, each analysis should be repeated at least once with a different action. The latter is typically done in such a way that different collaborations specialise on one specific action each.

The most critical extrapolation is the continuum limit $a \rightarrow 0$. Lattice actions violate basic symmetries of QCD (isotropy and homogeneity of space-time, chiral symmetry, isospin symmetry in the case of twisted-mass fermions ...) for finite lattice spacing. Thus the $a \rightarrow 0$ limit, which restores all symmetries, could be non-trivial. Unfortunately, $a$ can only be varied in very limited ranges because the needed CPU time is always proportional to a large power of $1 / a$. Therefore, a variety of improved lattice actions was proposed in which lattice artifacts are not proportional to $a$ but e.g. $a^{2}$. Many variants exist, all of which are well motivated in one way or the other. Substantial effort is invested to further improve such actions, and it would be very surprising if by the time an EIC starts operating also the systematic uncertainties due to the multiple extrapolation $a \rightarrow 0, L \rightarrow \infty$, $m_{q} \rightarrow m_{q}$ (physical). were not much better under control.

The purely statistical uncertainty will for sure become much smaller due to increased computer power. While the most powerful present day computers are of the Petaflop class, various initiatives aim already at Exaflop computing. In the next sections we will discuss in detail some of the physics quantities calculated on the lattice, which are especially important for the EIC. 


\subsection{Generalized form factors}

Most correlators relevant for hadron structure which were determined on the lattice are related to Generalized Parton Distributions (GPDs) or Distribution Amplitudes (DAs). For GPDs the hadronic states in Eq. (4.1) are equal, $h=h^{\prime}$ but the momenta are usually different $\left(p \neq p^{\prime}\right)$. For the best known GPDs $H_{q}$ and $E_{q}$,

$$
\begin{aligned}
& \left.\int \frac{d z^{-}}{2 \pi} e^{i x \bar{P}^{+} z^{-}}\left\langle P_{2}\left|\bar{q}\left(-\frac{1}{2} z\right) \gamma^{+} q\left(\frac{1}{2} z\right)\right| P_{1}\right\rangle\right|_{z^{+}=0, z_{\perp}=0} \\
= & \frac{1}{P^{+}}\left[H_{q}(x, \xi, t) \bar{N}\left(P_{2}\right) \gamma^{+} N\left(P_{1}\right)+E_{q}(x, \xi, t) \bar{N}\left(P_{2}\right) \frac{i \sigma^{+\alpha} \Delta_{\alpha}}{2 M} N\left(P_{1}\right)\right] .
\end{aligned}
$$

The OPE gives moments in terms of generalized form factors $A_{n, k}(t), B_{n, k}(t), C_{n}(t)$,

$$
\begin{aligned}
\int_{-1}^{1} d x x^{n-1} H(x, \xi, t) & =\sum_{\substack{k=0 \\
\text { even }}}^{n-1}(2 \xi)^{k} A_{n, k}(t)+(\mathrm{n}+1 \bmod 2)(2 \xi)^{n} C_{n}(t) \\
\int_{-1}^{1} d x x^{n-1} E(x, \xi, t) & =\sum_{\substack{k=0 \\
\text { even }}}^{n-1}(2 \xi)^{k} B_{n, k}(t)-(\mathrm{n}+1 \bmod 2)(2 \xi)^{n} C_{n}(t)
\end{aligned}
$$

which can be expressed in terms of local correlators by equations like

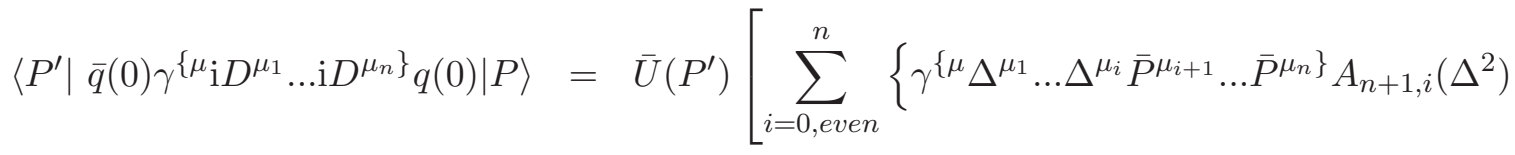

$$
\begin{aligned}
& -\mathrm{i} \frac{\Delta_{\alpha} \sigma^{\alpha\{\mu} \Delta^{\mu_{1}} \ldots \Delta^{\mu_{i}} \bar{P}^{\mu_{i+1}} \ldots \bar{P}^{\left.\mu_{n}\right\}}}{2 m} B_{n+1, i}\left(\Delta^{2}\right) \\
& \left.+\left.\frac{\Delta^{\mu} \ldots \Delta^{\mu_{n}}}{m} C_{n+1,0}\left(\Delta^{2}\right)\right|_{n \text { odd }}\right] U(P),
\end{aligned}
$$

where $\{\cdots\}$ denotes symmetrization and subtraction of trace terms. The fact that the sum in Eq. (4.7) extends only up to $n-1$ is called polynomiality. The proton alone has eight independent quark GPDs for each quark flavour and typically one can calculate the leading three moments with satisfactory accuracy on the lattice. Adding the gluon GPDs and repeating the analysis for all octet and decuplet baryons and octet mesons one is already speaking about several hundred quantities. In future one will also increasingly analyse hadron resonances and transition form factors, such that the lattice data base will become even richer. For each of these observables one has to analyse the renormalization properties, the quark/pion mass dependence and the finite volume dependence (within suitable versions of effective field theory/chiral perturbation theory (ChPT)). Finally one has to compare results for different lattice actions and analyse the origin of discrepancies. Obviously it is impossible to review all of this here. Rather, we refer to the comprehensive paper [604] for an example of a state of the art analysis. Fig. 4.2, taken from this paper, gives a typical example. This figure shows a number of common aspects:

1. Fluctuations are strongly suppressed for heavy quark/pion masses. This is why the statistical errors (dark blue bars) increase drastically for smalles pion masses. 


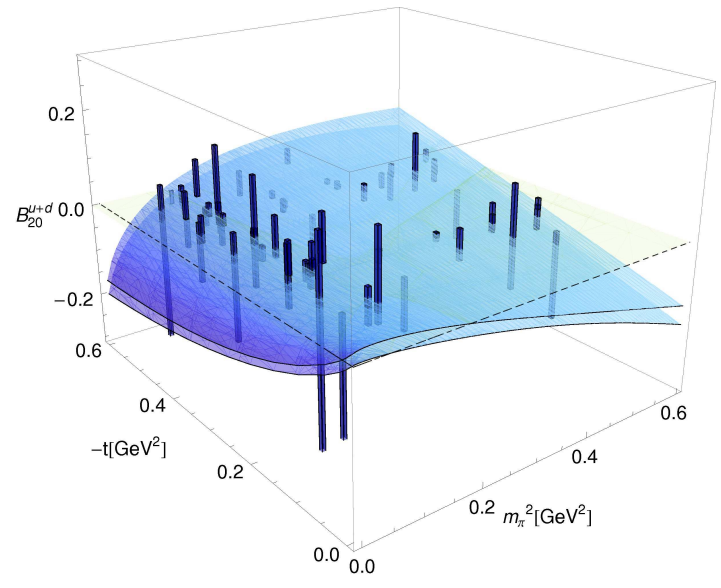

Figure 4.2. The isosinglet moment $B_{20}^{u+d}(t)$ as a function of simulated pion mass and $t$ 604].

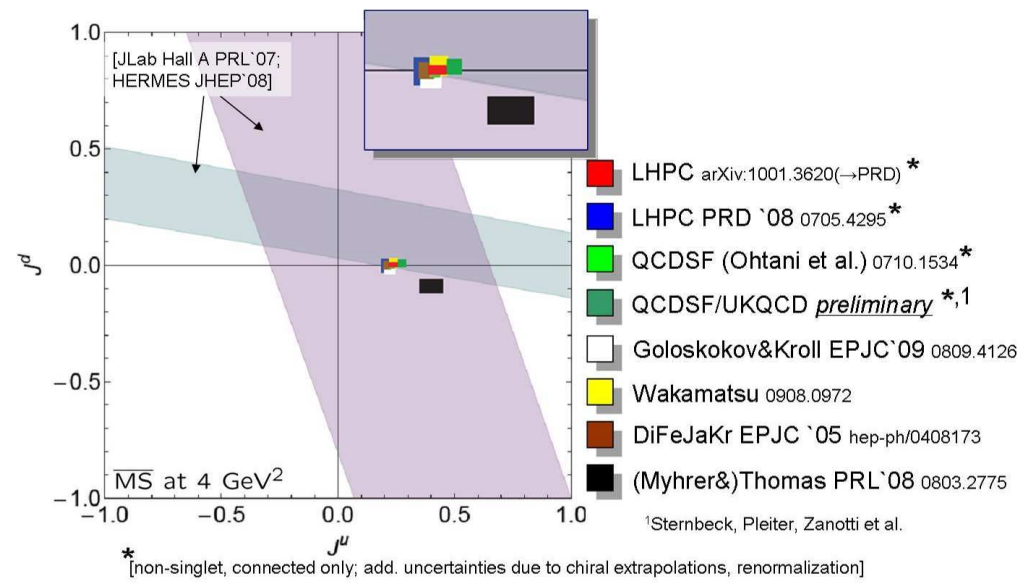

Figure 4.3. Lattice results for $J_{u}$ and $J_{d}$ compared with various models [582, 701, 605, 702] and constraints derived from experiment (colored bands)

2. The difference between the two sheets gives the variation of HBChPT fits. However, it would be safer to only use ensembles with squared pion masses below $m_{\pi}^{2} \leq 0.25 \mathrm{GeV}^{2}$, where ChPT is rather well under control, which was obviously not possible with the ensembles available for this analysis.

3. One is especially interested in the $t=0$ limit of $B_{20}$ in view of Ji's sum rule,

$$
\left\langle J_{q}^{3}\right\rangle=\frac{1}{2}\left[A_{2,0}^{q}(0)+B_{2,0}^{q}(0)\right] .
$$

Already today lattice simulations give rather precise results for the total angular momentum carried by the different quark species in a nucleon, see Fig. 4.3. In future these results will further improve, e.g. due to the use of twisted boundary conditions to realize proton momenta different from the natural ones on a lattice, i.e. different from $p_{j}=\frac{2 \pi}{L} n_{j}$.

Thus, much has been done already, and much more will be done in future. Extrapolating the progress of recent years to the time an EIC will start operation it seems realistic to expect 


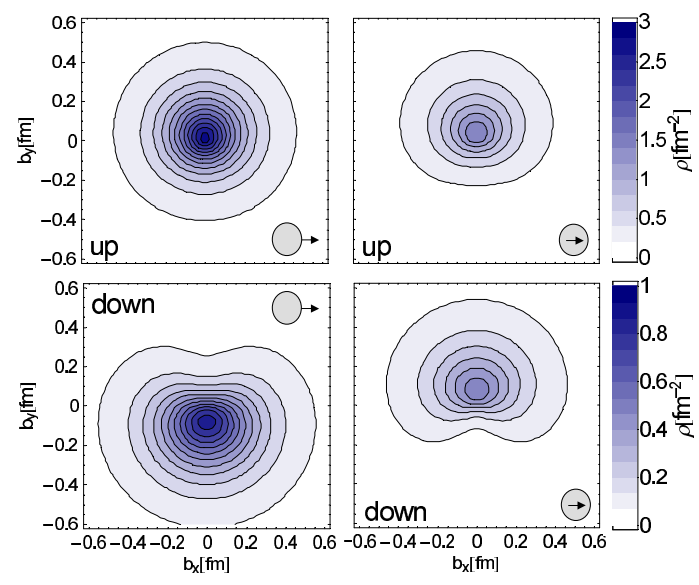

Figure 4.4. An illustration for the transverse probability distribution of the nucleon quark distributions as a function of the transverse quark and nucleon spin direction. Figure taken from [456].

that by then pictures like Fig. 4.2 will be numerically precise and will include reliable error bands.

Another important example are the quark density distributions in the transverse plane plotted in Fig. 4.4. Their form is mainly determined by the Fourier transformations of (moments of) the GPDs.

$$
\begin{aligned}
B_{n 0}^{q}\left(x, 0, b_{\perp}^{2}\right) & =\frac{1}{(2 \pi)^{2}} \int_{-1}^{1} d x x^{n-1} \int d^{2} \Delta_{\perp} e^{\mathrm{i} b_{\perp} \cdot \Delta_{\perp}} E\left(x, 0, \Delta_{\perp}^{2}\right) \\
\bar{B}_{T n 0}^{q}\left(x, 0, b_{\perp}^{2}\right) & =\frac{1}{(2 \pi)^{2}} \int_{-1}^{1} d x x^{n-1} \int d^{2} \Delta_{\perp} e^{\mathrm{i} b_{\perp} \cdot \Delta_{\perp}} \bar{E}_{T}\left(x, 0, \Delta_{\perp}^{2}\right)
\end{aligned}
$$

The information on transverse structure contained in GPDs is, e.g., relevant in the following context: First LHC data show strong disagreement between observed interaction rates and predictions from event generators, see e.g. [703] for the so-called "underlying event" which denotes the whole of all medium hard reaction channels, which are completely dominated by QCD. Part of the explanation might be related to multiple-hard interactions, a class of reactions which was shown to be already relevant at the Tevatron, see [704. In these reactions multiple hard quark-gluon interactions occur in the same proton-proton collision, which are not described by the usual inclusive factorization theorems. The correction terms have a complicated structure, see e.g. [705] and references cited there, but can be partially related to GPD profiles in the transverse coordinate plane. By combining experimental results from an EIC with improved lattice calculations it should be possible to describe these effects much more precisely than currently. In this context, as always, experimental results are crucial, because it is very difficult to judge the reliability of lattice results without being able to compare with at least some experimental facts.

A multitude of angular asymmetries and hadronic correlations, many of which include spin degrees of freedom, can be measured with a high luminosity EIC. For many of these, the microscopic reaction mechanism is not yet understood. Some of the proposals made depend crucially on the transverse hadronic structure encoded in GPDs, see e.g. [299]. One of the main missions of an EIC is to clarify both the transverse structure and the reaction mechanisms. This is a demanding task which can only be mastered with input from LQCD. 


\subsection{TMDs on the lattice}

The availability of methods to study GPDs on the lattice motivates us to develop similar techniques for the calculation of TMDs [279, 280]. In contrast to other, more mature areas of lattice QCD, the present focus of TMD calculations on the lattice is on the development of methodology and on qualitative observations rather than precision. The ultimate goal is to obtain results from first principles only that can potentially be compared to experimental observations. The first step to reach this goal is to describe precisely which matrix elements need to be calculated, and how they can be regularized in the context of TMD factorization. Already at this step, the situation is much more challenging for TMDs than for moments of GPDs, where the matrix elements needed are well-known. These issues are not specific to lattice QCD, but they play a central role in the development of methods to calculate TMDs non-perturbatively.

In its basic form, the correlator that needs to be calculated is that of eq. (2.1). For our purposes, we write the trace projections $\Phi^{[\Gamma]}=\frac{1}{2} \operatorname{Tr}(\Gamma \Phi)$ of this correlator as

$$
\Phi^{[\Gamma]}\left(x, \mathbf{k}_{\perp}\right)=\left.\frac{1}{P^{+}} \underbrace{\int \frac{d(l \cdot P)}{2 \pi} e^{-i(l \cdot P) x}}_{\mathcal{F}_{x}} \underbrace{\int \frac{d^{2} \mathbf{l}_{\perp}}{(2 \pi)^{2}} e^{i \mathbf{l}_{\perp} \cdot \mathbf{k}_{\perp}}}_{\mathcal{F}_{\perp}} \underbrace{\frac{1}{2}\left\langle P, S\left|\bar{q}(l) \Gamma \mathcal{W}_{\eta} q(0)\right| P, S\right\rangle}_{\widetilde{\Phi}^{[\Gamma]}(l, P, S)}\right|_{l^{+}=0}
$$

where $\Gamma$ is a Dirac matrix. The gauge link $\mathcal{W}_{\eta}$ is discussed in sec. 2.4.1, and its geometry is depicted for the SIDIS process in figure 4.5 a). With the generalization of eq. (2.47) it can be written as a concatenation of straight Wilson lines $\mathcal{W}_{\eta}=V_{[l, l+\eta v]} V_{[l+\eta v, \eta v]} V_{[\eta v, 0]}$. Here $v$ is a time-like vector normalized to $v^{2}=1$. A staple shaped gauge link $\mathcal{W}_{\infty}$ extending to $\eta \rightarrow \infty$ corresponds to SIDIS, while a staple $\mathcal{W}_{-\infty}$ directed in the opposite direction corresponds to the Drell-Yan process. Beyond tree level, eq. (4.10) needs to be modified in order to take the collective effect of soft momentum gluons into account and to subtract divergences. This can be achieved, e.g., by dividing $\tilde{\Phi}^{[\Gamma]}$ by appropriate vacuum expectation values (soft factors), see, e.g., [706, 256, 257, 260, 384.

First studies of transverse momentum dependence on the lattice follow the strategy to determine matrix elements of the form $\tilde{\Phi}^{[\Gamma]}$ in eq. (4.10) directly from three-point functions. The idea of using a discrete representation of the non-local operator $\bar{q}(l) \Gamma \mathcal{W}_{\eta} q(0)$ is a novel technique and requires investigations about the properties of such extended operators on the lattice. Considering this and the ambiguities about the precise operator geometry suitable for TMD extraction, it seems reasonable to begin with a simplified setup. The following two operator geometries are under investigation:

- straight gauge link connecting the two quark fields directly, $\mathcal{W}_{0}=V_{[l, 0]}$. This simple setup yields high statistics results, but does not correspond to the situation in SIDIS or Drell-Yan. For example, non-zero time-reversal odd TMDs such as the Sivers function $f_{1 T}^{\perp}$ are forbidden by symmetry with this link geometry. However, the qualitative features of the results are interesting, especially the spin-dependence. A brief outline of findings obtained with straight links is given in sec. 4.3.1.

- staple shaped gauge link of finite extent $\mathcal{W}_{\eta}$ for a spacelike choice of the direction $v$ as depicted in figure $4.5 \mathrm{a}$ ). Results for the SIDIS link $\mathcal{W}_{+\infty}$ and the Drell-Yan link $\mathcal{W}_{-\infty}$ can be read off if the lattice results converge to a constant for longer and longer staple extents $\eta$. Ongoing studies with this operator geometry are discussed in sec. 4.3.2. 
a)

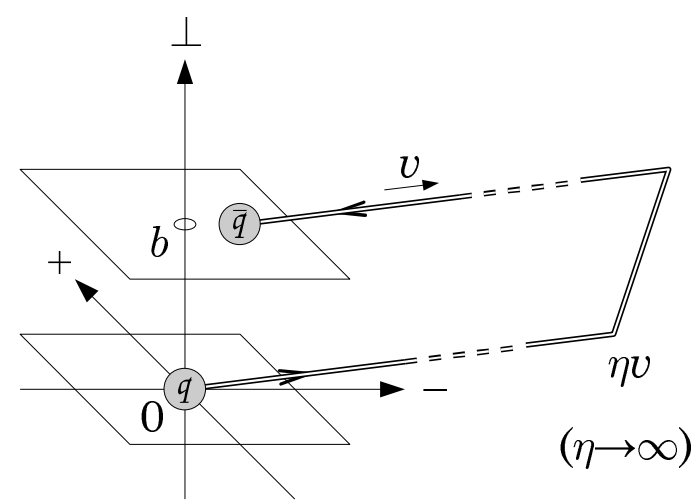

b)

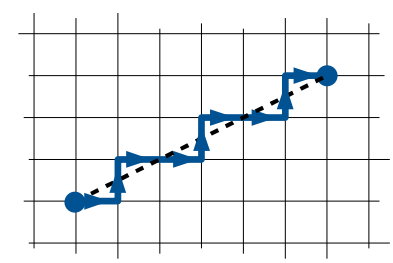

Figure 4.5. a) Staple shaped Wilson line. b) Representation of a straight Wilson line (dashed line) as a step-like product of link variables.

\subsubsection{Straight gauge links}

In lattice QCD, it is possible to determine matrix elements $\widetilde{\Phi}^{[\Gamma]}$ appearing in eq. (4.10) directly from a ratio of three- and two-point functions, provided the operator has no extent in Minkowski-time. To do this, we employ the standard methods described in sec. 4.1. Only the operator we insert is specific to our method. Operators with straight gauge links can be approximated on the lattice by a step-like product of link variables, as depicted in figure 4.5. b). At present, disconnected diagrams are neglected. Disconnected diagrams cancel in the isovector channel, i.e., in $u-d$ quark distributions.

The key element to relate the matrix elements $\widetilde{\Phi}^{[\Gamma]}$ determined on the lattice to the TMDs is a parametrization in terms of Lorentz-invariant amplitudes $\widetilde{A}_{i}\left(l^{2}, l \cdot P\right)$, similar to the parametrization in terms of amplitudes $A_{i}\left(k^{2}, k \cdot P\right)$ in ref. [243]. For straight gauge links one obtains

$$
\begin{aligned}
\widetilde{\Phi}^{\left[\gamma^{\mu}\right]} & =2 P^{\mu} \tilde{A}_{2}+2 i M^{2} l^{\mu} \widetilde{A}_{3}, \\
\widetilde{\Phi}^{\left[\gamma^{\mu} \gamma^{5}\right]} & =-2 M S^{\mu} \widetilde{A}_{6}-2 i M P^{\mu}(l \cdot S) \widetilde{A}_{7}+2 M^{3} l^{\mu}(l \cdot S) \widetilde{A}_{8},
\end{aligned}
$$

To translate the amplitudes into TMDs, the Fourier transform in eq. (4.10) must be carried out. For example

$$
\begin{aligned}
f_{1}\left(x, \mathbf{k}_{\perp}^{2}\right) & =2 \mathcal{F}_{\perp} \mathcal{F}_{x} \widetilde{A}_{2}\left(l^{2}, l \cdot P\right), \\
g_{1 T}\left(x, \mathbf{k}_{\perp}^{2}\right) & =4 M^{2} \partial_{\mathbf{k}_{\perp}^{2}} \mathcal{F}_{\perp} \mathcal{F}_{x} \widetilde{A}_{7}\left(l^{2}, l \cdot P\right),
\end{aligned}
$$

where the two independent Fourier transforms $\mathcal{F}_{x}$ and $\mathcal{F}_{\perp}$ are defined in eq. (4.10). On the Euclidean lattice, the matrix elements can only be evaluated in the range

$$
l^{2} \leq 0 \quad|l \cdot P| \leq\left|\mathbf{P}_{\text {lat }}\right| \sqrt{-l^{2}}
$$

where $\mathbf{P}_{\text {lat }}$ is the three-momentum of the nucleon chosen on the lattice. As a result, data points are only available in a wedge shaped area. The opening angle of the wedge can be potentially increased by using larger lattice nucleon momenta $\mathbf{P}_{\text {lat }}$, but full coverage of the $|l|, l \cdot P$-plane can never be achieved with this method. Thus the information required to reconstruct the $x$-dependence of the distributions is not fully available; the Fourier transform $\mathcal{F}_{x}$ in eqs. (4.13)-(4.14) cannot be carried out. However, model assumptions about the 


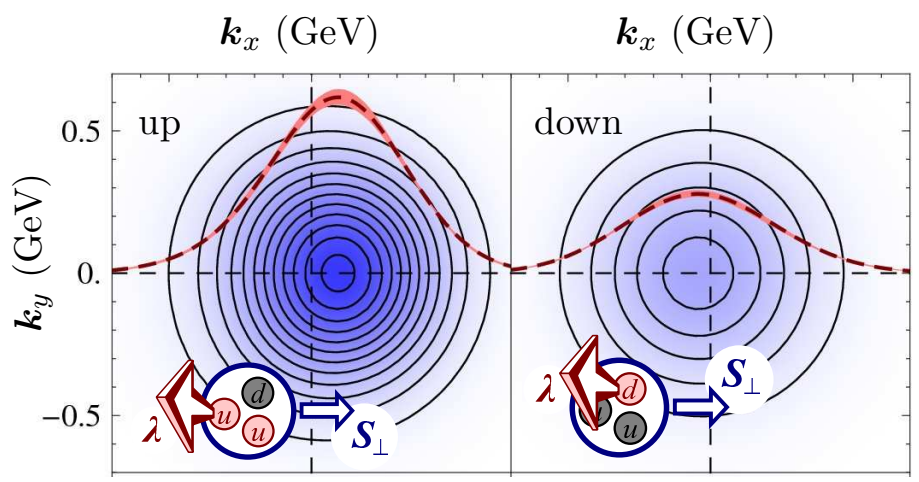

Figure 4.6. $x$-integrated density of longitudinally polarized quarks inside a nucleon polarized in the transverse $x$-direction. These results have been obtained with straight gauge links at a pion mass $m_{\pi} \approx 500 \mathrm{MeV}[279$. The insets display the spin polarization of the quarks and of the nucleon.

correlation of $x$ - and $\mathbf{k}_{\perp}$-dependence can be compared to the lattice data, see sec. VI of ref. [280. Moreover, the lowest $x$-moment of TMDs can be calculated, since the required information is encoded in the data at $l \cdot P=0$. For example,

$$
\begin{aligned}
f_{1}^{[1]}\left(\mathbf{k}_{\perp}^{2}\right) & \equiv \int d x f_{1}\left(x, \mathbf{k}_{\perp}^{2}\right)=\int_{0}^{1} d x\left(f_{1}\left(x, \mathbf{k}_{\perp}^{2}\right)-\bar{f}_{1}\left(x, \mathbf{k}_{\perp}^{2}\right)\right) \\
& =2 \mathcal{F}_{\perp} \widetilde{A}_{2}\left(l^{2}, 0\right)
\end{aligned}
$$

where $\bar{f}_{1}$ is the unpolarized anti-quark distribution function. First results for the lowest $x$-moments $f_{1}^{[1]}, g_{1 T}^{[1]}$ and $h_{1 L}^{\perp[1]}$ using straight gauge links have been presented in Ref. [279, 280]. The calculations were carried out at a pion mass of about $500 \mathrm{MeV}$, taking advantage of existing gauge configurations from the MILC collaboration [707] and propagators from the LHP collaboration [708]. The worm gear distribution $g_{1 T}$ gives rise to dipole deformations in the $x$-integrated, $\mathbf{k}_{\perp}$-dependent density of longitudinally polarized quarks inside a transversely polarized nucleon, as shown in figure 4.6. Due to the dipole deformation, this density is not axially symmetric. The peak is clearly shifted away from the center along the axis defined by the transverse spin vector. This shift is associated with a non-zero average transverse quark momentum $\left\langle\mathbf{k}_{x}\right\rangle_{T L}$, which can be expressed in terms of a ratio of amplitudes $\widetilde{A}_{7}(0,0) / \widetilde{A}_{2}(0,0)$. The lattice computations yield $\left\langle\mathbf{k}_{x}\right\rangle_{T L}=67(5) \mathrm{MeV}$ for down quarks and $\left\langle\mathbf{k}_{x}\right\rangle_{T L}=-30(5) \mathrm{MeV}$ for up quarks (errors statistical only). Reference [709] reveals that these results are of the same sign and of quite similar magnitudes as those obtained with a light-cone constituent quark model [409, despite the unphysically large quark masses employed in the lattice calculation.

We note that the straight link results discussed above depend on two additional important ingredients: a non-perturbative renormalization condition and a Gaussian parametrization to perform the Fourier transform, see ref. 280] for details. The renormalization condition is necessary to fix the length-dependent renormalization factor $\exp (-\delta m|l|)$ due to the self-energy of the spacelike Wilson line $\mathcal{V}_{[l, 0]}$ [710, 711, 712]. At the present level of statistical precision, the parametrization of the renormalized data as Gaussian functions is very successful and acts as a provisional regulator of contributions from large $\mathbf{k}_{\perp}$. A better understanding of the operator in the transition from the short range (small $\sqrt{-l^{2}}$, corresponding to large $\mathbf{k}_{\perp}$ ) to the long range behavior may lead to a an improved parametrization of the lattice data, beyond the Gaussian assumption, and may open the possibility to make 


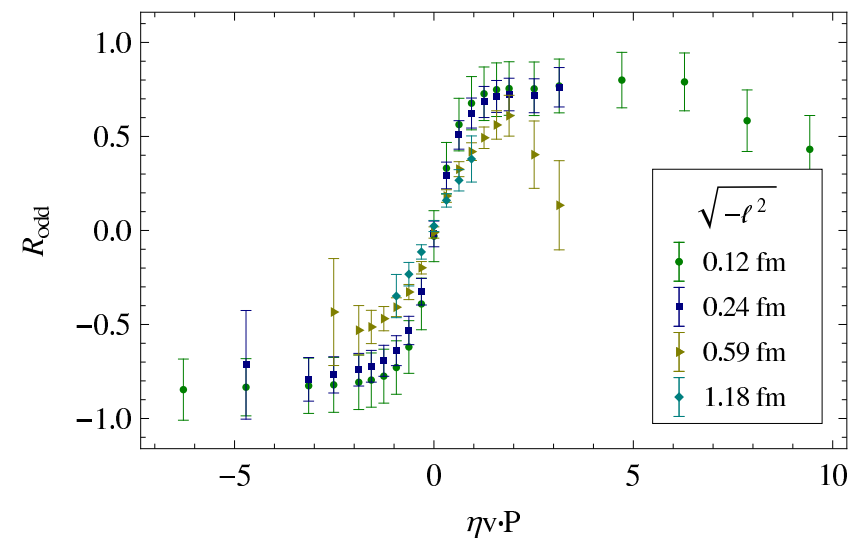

Figure 4.7. Test calculation of a T-odd ratio of amplitudes using staple shaped links at $m_{\pi} \approx$ $800 \mathrm{MeV}$ 713.

contact with a perturbatively defined renormalization scheme.

\subsubsection{Staple-shaped links and the Sivers function}

TMDs obtained with a straight gauge link as discussed in the previous section are not strictly identical to those relevant in, e.g., SIDIS or the Drell-Yan process. Instead, a link geometry as depicted in figure $4.5 \mathrm{a}$ ) is required. In particular, naively time-reversal odd TMD such as the Sivers function can only be non-vanishing once the operator structure involves another direction $v$ related to final or initial state interactions. Lattice QCD can profit from frameworks that avoid rapidity divergences by considering directions $v$ slightly off the lightlike $n^{-}$-direction [240, 241, 256, 257, 260, 384]. TMDs introduced this way follow an evolution equation in the rapidity cutoff parameter $\zeta \equiv(2 P \cdot v)^{2} /\left|v^{2}\right|$ [240, 393]. The restriction to operators $\bar{q}(l) \Gamma \mathcal{W}_{\eta} q(0)$ that have no extent in Euclidean time permits only the implementation of spacelike directions $v$ on the lattice, and furthermore limits the rapidity cutoff parameter to the range $0 \leq \zeta \leq 4\left|\mathbf{P}_{\text {lat }}\right|^{2}$, where $\mathbf{P}_{\text {lat }}$ is the selected nucleon three-momentum on the lattice. The dependence on $v$ leads to additional amplitudes $\tilde{A}_{i}$, $\tilde{B}_{i}$ in the decomposition of the correlator eq. (4.11), compare also [246]:

$$
\begin{aligned}
& \widetilde{\Phi}^{\left[\gamma^{\mu}\right]}=\frac{2}{\widetilde{S}}\left\{P^{\mu} \widetilde{A}_{2}+i M^{2} l^{\mu} \widetilde{A}_{3}+i M \epsilon^{\mu \nu \alpha \beta} P_{\nu} l_{\alpha} S_{\beta} \widetilde{A}_{12}+\frac{M^{2}}{(v \cdot P)} v^{\mu} \widetilde{B}_{1}\right. \\
&+ \frac{M}{v \cdot P} \epsilon^{\mu \nu \alpha \beta} P_{\nu} v_{\alpha} S_{\beta} \widetilde{B}_{7}+\frac{i M^{3}}{v \cdot P} \epsilon^{\mu \nu \alpha \beta} l_{\nu} v_{\alpha} S_{\beta} \widetilde{B}_{8} \\
&\left.-\frac{M^{3}}{v \cdot P}(l \cdot S) \epsilon^{\mu \nu \alpha \beta} P_{\nu} l_{\alpha} v_{\beta} \widetilde{B}_{9}+\frac{i M^{3}}{(v \cdot P)^{2}}(v \cdot S) \epsilon^{\mu \nu \alpha \beta} P_{\nu} l_{\alpha} v_{\beta} \widetilde{B}_{10}\right\} .
\end{aligned}
$$

Here $\widetilde{S}$ generically represents a soft factor modification, as needed, e.g., in the formalism of refs. [256, 257, 260, 384. First lattice studies are ongoing for ratios of amplitudes, in which renormalization factors and potential soft factors cancel [713, 280], similar as in the asymmetries discussed in sec. 2.2.7. Figure 4.7 shows results from a test calculation 713. of a ratio of time-reversal odd over time-reversal even amplitudes $R_{\text {odd }} \equiv\left(\widetilde{A}_{12}-\right.$ $\left.\left(M /\left|\mathbf{P}_{\text {lat }}\right|\right)^{2} \widetilde{B}_{8}\right) / \widetilde{A}_{2}$, evaluated at $l \cdot P=0,\left|\mathbf{P}_{\text {lat }}\right| \approx 0.5 \mathrm{GeV}$, for selected values of $l^{2}$. Note that $\widetilde{A}_{12}$ would correspond to the Sivers function $f_{1 T}^{\perp}$ for lightlike $v$. In the test calculation, the operator has been evaluated with staple shaped links $\mathcal{W}_{\eta}$ for a large range of extents $\eta$. 
The result of the test calculation is, within statistics, an odd function of $\eta v \cdot P$, as expected for a time-reversal odd function. Moreover, we see the onset of a plateau at $|\eta v \cdot P| \gtrsim 2$. The plateau at large positive $\eta$ correspond to the SIDIS result with a $\mathcal{W}_{\infty}$ link, while the plateau at large negative $\eta$ correspond to the Drell-Yan result with the $\mathcal{W}_{-\infty}$ link. This is a promising indication that lattice estimates could be feasible for, e.g., the average transverse momentum shift due to the Sivers function given by

$$
\left.\left\langle\mathbf{k}_{y}\right\rangle_{T U} \equiv \frac{\int d^{2} \mathbf{k}_{\perp} \mathbf{k}_{y} \Phi^{\left[\gamma^{+}\right]}}{\int d^{2} \mathbf{k}_{\perp} \Phi^{\left[\gamma^{+}\right]}}\right|_{\mathbf{S}_{\perp}=(1,0)}=M \frac{\int d x f_{1 T}^{\perp(1)}(x)}{\int d x f_{1}^{(0)}(x)}
$$

see also [264, 444. Here $\Phi^{\left[\gamma^{+}\right]}$intuitively has an interpretation as the density of unpolarized quarks in a transversely polarized proton, and $f_{1 T}^{\perp(1)}$ and $f_{1}^{(0)}(x)$ are $\mathbf{k}_{\perp}$-moments defined as $f^{(n)}(x) \equiv \int d^{2} \mathbf{k}_{\perp}\left(\mathbf{k}_{\perp}^{2} / 2 M^{2}\right)^{n} f\left(x, \mathbf{k}_{\perp}^{2}\right)$. A generalized version of the above quantity can be formed directly from the amplitudes determined on the lattice, namely

$$
\left\langle\mathbf{k}_{y}\right\rangle_{T U}\left(\mathcal{B}_{\perp}\right) \equiv M \frac{\int d x \tilde{f}_{1 T}^{\perp(1)}\left(x, \mathcal{B}_{\perp}^{2}\right)}{\int d x \tilde{f}_{1}\left(x, \mathcal{B}_{\perp}^{2}\right)}=-\left.M \frac{\widetilde{A}_{12}-R(\zeta) \widetilde{B}_{8}}{\widetilde{A}_{2}+R(\zeta) \widetilde{B}_{1}}\right|_{l=-\mathcal{B}_{\perp}^{2}}
$$

with $R(\zeta)=1-\sqrt{1+4 M^{2} / \zeta}$ and where $\tilde{f}_{1}$ and $\tilde{f}_{1 T}^{\perp(1)}$ are now $\mathbf{k}_{\perp}$-Fourier-transformed TMDs as they appear in eq. (2.33). Keeping the length $\mathcal{B}_{\perp}$ sufficiently large compared to the lattice spacing and correspondingly assuming renormalization properties as in continuum field theory, one finds that multiplicative renormalization factors, including Wilson line self-energies, as well as potential soft factors cancel in the ratio of amplitudes above. The extrapolation to $\mathcal{B}_{\perp}=0$, where $\left\langle\mathbf{k}_{y}\right\rangle_{T U}\left(\mathcal{B}_{\perp}\right)$ is equal to $\left\langle\mathbf{k}_{y}\right\rangle_{T U}$, will require special attention to UV divergences and cutoff effects. However, already the generalized object $\left\langle\mathbf{k}_{y}\right\rangle_{T U}\left(\mathcal{B}_{\perp}\right)$ at nonzero $\mathcal{B}_{\perp}$ may offer opportunities to compare with phenomenology, by means of an $x$-integrated version of the Bessel-weighted quantities introduced in eq. (2.33) A possible difficulty for lattice computations will be to reach large enough values $\zeta$, in the regime where evolution equations [240, 393] can be applied. 


\subsection{Spectroscopy and other physics topics}

Still another approach to elucidate hadron structure is offered by lattice spectroscopy. Spectroscopy has the great advantage that it allows to avoid the subtle renormalization issues mentioned above, but the disadvantage that the deduction of information on hadron structure is less direct. A typical recent example is found in [714, see Fig. 4.8, The basic idea is that one uses a set of interpolating currents (sources) $\mathcal{O}_{i}$ with the same quantum numbers to calculate and analyse the correlation matrix as a function of separation of the time-hyper planes

$$
C_{i j}(t)=\left\langle 0\left|\mathcal{O}_{i}(t) \mathcal{O}_{j}(0)\right| 0\right\rangle
$$

and solves the generalized eigenvalue problem. One thus obtains not only the eigenvalues (masses) but also the eigenvectors in terms of the different sources. If done with care the relative overlap of the physical mass states with the different sources allows to draw conclusions about their structure. This provides information, which is often complementary to that obtained with the methods sketched above. Again for practical purposes this information is most sensitive to leading Fock-state components. While this is a very powerful method, its results must be interpreted with care. The eigenvectors of different mass states give the amplitudes with which each source contributes. All of these are forced to be orthonormal by construction. This constraint can lead to substantial artifacts if the chosen source functions span too small a function space. To avoid premature conclusions one, therefore, has to compare results obtained for different lattice actions and many different choices of sources. Presently the lattice community is still in the process of optimizing this method, but it seems already clear by now that in a few years this approach will be a standard source of many detailed information about hadron structure.

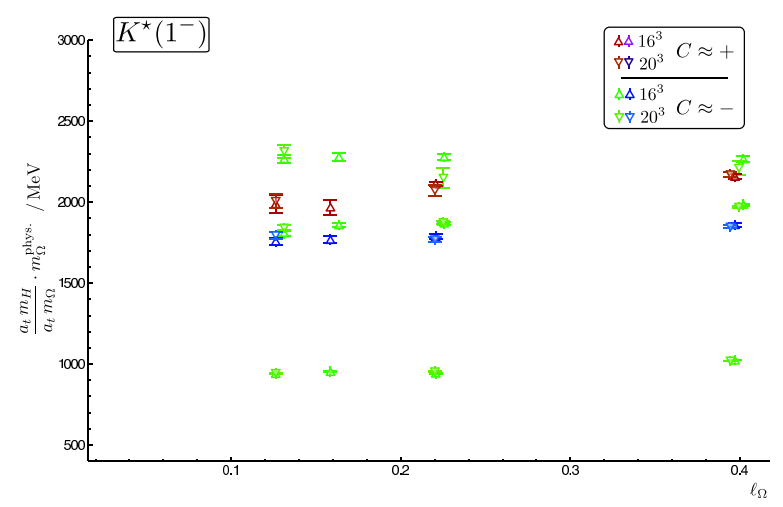

Figure 4.8. A typical example from [714]. The $1^{-}$kaon states are shown. Color coding indicates dominance of a particular charge-conjugation eigenstate

\section{Acknowledgments}

Thanks are due to our collaborator John Negele. We are grateful to the LHP and MILC collaborations, for providing gauge configurations and propagators. We also thank Gunnar Bali, Alexei Bazavov, Vladimir Braun, Markus Diehl, Robert Edwards, Meinulf Göckeler, Alexei Prokudin, Dru Renner and David Richards for helpful discussions. Our software uses the Chroma-library [715, and we use USQCD computing resources at Jefferson Lab. 


\section{Chapter 5}

\section{QCD matter under extreme conditions}

Convenors and chapter editors:

A. Accardi, M. Lamont, C. Marquet 


\title{
5.1 Chapter summary: overview and golden measurements
}

\author{
Alberto Accardi, Matthew Lamont and Cyrille Marquet
}

A basic quest of nuclear physics is the understanding of the structure of hadrons and nuclei (nucleon number $A>1$ ) in terms of QCD Lagrangian degrees of freedom, the quarks and gluons. Deviations of the nuclear quark and gluon densities from the sum of the free nucleon densities directly attest to binding effects and elucidate the QCD origin of the inter-nucleon interactions. Such deviations can arise through different mechanisms, such as a modification of the free nucleon structure, the presence of non-nucleonic degrees of freedom, and quantum-mechanical interference of the quark/gluon fields of different nucleons at small parton fractional momentum $x$ ("shadowing"), creating a fascinating landscape of many-body QCD. At even smaller $x$, the gluon density increases to the point where gluons become closely packed, leading to a strong field regime of non-linear QCD evolution called saturation. This regime is argued to have universal properties for any hadronic system, ranging from pions, to protons and nuclei, but its onset is enhanced in nuclear targets due to the superposition of the gluon field of many nucleons.

A peculiar pattern of nuclear modifications was observed in fixed-target experiments and caused much excitement; it shows suppression for $0.2<x<0.8$ ("EMC effect"), some signs of enhancement for $0.05<x<0.2$, and significant suppression (shadowing) at smaller $x$. However, such experiments were unable to reach deep into the shadowing region or probe gluons. The EIC will overcome these limitations, extend measurements to very high scales of $Q^{2}$, and determine with high precision the nuclear effects on gluon distributions. Full reconstruction of the hadronic final state also opens up for the first time the possibility of measuring charged current interactions on nuclei, and to perform a full quark flavour separation based on nuclear DIS data only. Crucially, an EIC will access much lower values of $x<0.01$ and study the onset of the saturation regime, which has never been directly probed experimentally, although tantalising (but not unequivocal) signatures have been found at the Relativistic Heavy-Ion Collider (RHIC).

Another possibility offered by nuclear targets is the study of the propagation of colour charges in nuclear matter and the space-time evolution of hadronization. The unique feature of an EIC, compared to previous fixed target experiments, is its large energy span. This allows one to experimentally boost hadronization effects completely out of the nucleus, in order to focus attention on the propagation of fast quarks and gluons, and their accompanying parton showers, through the nucleus. Thus one can use the partons as coloured probes of the soft components of the target nuclear wave function, and conversely experimentally test QCD mechanisms of parton energy loss in a known nuclear medium. At lower energies, hadronization happens partially inside the nucleus, which can then be used as a femtometer scale detector of the process. A good control of energy loss mechanisms in the partonic phase will yield unambiguous insights into the dynamics of colour confinement whereby hadrons emerge from coloured quarks and gluons.

Novel observables will be available thanks to the high energy reach, namely heavy flavours, charmonium and bottomonium, and jets, greatly expanding the experimental toolbox and sensitivity to nuclear effects, and thereby allowing a close connection to first principles calculations in QCD. The collider mode will also make it feasible to study in detail target fragmentation and its correlation to current fragmentation through multi-particle correlations, thereby expanding considerably the study of shower development and hadronization mechanisms. 


\subsubsection{Gold and silver measurements}

One of the goals of the program at the INT was to identify a small number of measurements whose ability to extract novel physics is beyond question and which are feasible at an EIC. Such measurements are referred to as "golden" measurements. These are complemented by other "silver" measurements/observables, to form a broad, robust, and compelling physics program. The gold and silver measurements are summarised in Tables 5.1 and 5.2. where also their feasibility in phase-I (medium energy) and phase-II (full energy) is indicated, and further discussed below. Many more observables than can fit in this section will be available at an EIC, contributing to a very rich physics program exploring the QCD basis of nuclear physics. Many of these will be reviewed in detail in the rest of this chapter.

\begin{tabular}{|c|c|c|c|c|}
\hline Deliverables & Observables & What we learn & Phase-I & Phase-II \\
\hline $\begin{array}{c}\text { integrated gluon } \\
\text { distributions }\end{array}$ & $F_{2, L}$ & $\begin{array}{c}\text { nuclear wave.fn.; } \\
\text { saturation, } Q_{s}\end{array}$ & $\begin{array}{c}\text { gluons at } \\
10^{-3} \lesssim x \lesssim 1\end{array}$ & $\begin{array}{l}\text { explore sat. } \\
\text { regime }\end{array}$ \\
\hline $\begin{array}{c}k_{T} \text {-dep. gluons; } \\
\text { gluon correlations }\end{array}$ & $\begin{array}{l}\text { di-hadron } \\
\text { correlations }\end{array}$ & $\begin{array}{c}\text { non-linear QCD } \\
\text { evolution/universality }\end{array}$ & $\begin{array}{c}\text { onset of } \\
\text { saturation; } Q_{s}\end{array}$ & RG evolution \\
\hline $\begin{array}{l}\text { transp. coefficients } \\
\text { in cold matter }\end{array}$ & $\begin{array}{c}\text { large- } x \text { SIDIS; } \\
\text { jets }\end{array}$ & $\begin{array}{l}\text { parton energy loss, } \\
\text { shower evolution; } \\
\text { energy loss mech. }\end{array}$ & $\begin{array}{c}\text { light flavours, charm } \\
\text { bottom; jets }\end{array}$ & $\begin{array}{l}\text { precision rare } \\
\text { probes; } \\
\text { large- } x \text { gluons }\end{array}$ \\
\hline
\end{tabular}

Table 5.1. Golden measurements in $e+A$ collisions at an EIC

\begin{tabular}{|c|c|c|c|c|}
\hline Deliverables & Observables & What we learn & Phase-I & Phase-II \\
\hline \hline $\begin{array}{c}\text { integrated gluon } \\
\text { distributions }\end{array}$ & $F_{2, L}^{c}, F_{2, L}^{D}$ & $\begin{array}{c}\text { nuclear w.fn.; } \\
\text { saturation, } Q_{s}\end{array}$ & $\begin{array}{c}\text { early sat. onset } \\
\text { challenge to measure }\end{array}$ & $\begin{array}{c}\text { saturation } \\
\text { regime }\end{array}$ \\
\hline $\begin{array}{c}\text { flavour separated } \\
\text { nuclear PDFs }\end{array}$ & $\begin{array}{c}\text { charged current } \\
\& \gamma Z \text { str. fns. }\end{array}$ & EMC effect origin & $\begin{array}{c}\text { full } q_{i} \text { separation } \\
\text { at } 0.01 \lesssim x \lesssim 1\end{array}$ & $\begin{array}{c}\text { larger } Q^{2}, \\
\text { smaller } x\end{array}$ \\
\hline$k_{T}$-dep. gluons & SIDIS at & non-linear QCD & extract $Q_{s} ;$ & RG evol.; \\
& small- $x$ & multipole corr. & flavour sep. \\
\hline $\begin{array}{c}b \text {-dep. gluons; } \\
\text { gluon correlations }\end{array}$ & $\begin{array}{c}\text { DVCS; } \\
\text { diffractive } J / \Psi, \\
\& \text { vector mesons }\end{array}$ & $\begin{array}{c}\text { interplay between } \\
\text { small- } x \text { evolution } \\
\text { and confinement }\end{array}$ & $\begin{array}{c}\text { moderate } x \text { with } \\
\text { light, heavy nuclei }\end{array}$ & $\begin{array}{c}\text { smaller } x, \\
\text { saturation }\end{array}$ \\
\hline
\end{tabular}

Table 5.2. Silver measurements in $e+A$ collisions at an EIC

\subsubsection{QCD at high gluon density}

The fact that we do not know the dynamics of gluons in nuclei over basically any $x$ range seems a compelling enough reason to build an EIC. The non-Abelian nature of QCD is its most distinguishing feature and controls emergent phenomena such as colour confinement, chiral symmetry breaking and the generation of the vast bulk of the visible mass in the Universe. These are, however, non-perturbative phenomena which are difficult to attack from first principles; where this is possible, such as in the case of the hadron spectrum from lattice QCD, only static aspects of the strong interactions are addressed. An EIC would allow one for the first time to experimentally probe at small $x$ dynamical non-Abelian aspects of a fundamental force of nature in a controlled setting where weak coupling methods apply. The physics in this regime is the non-perturbative physics of strong 


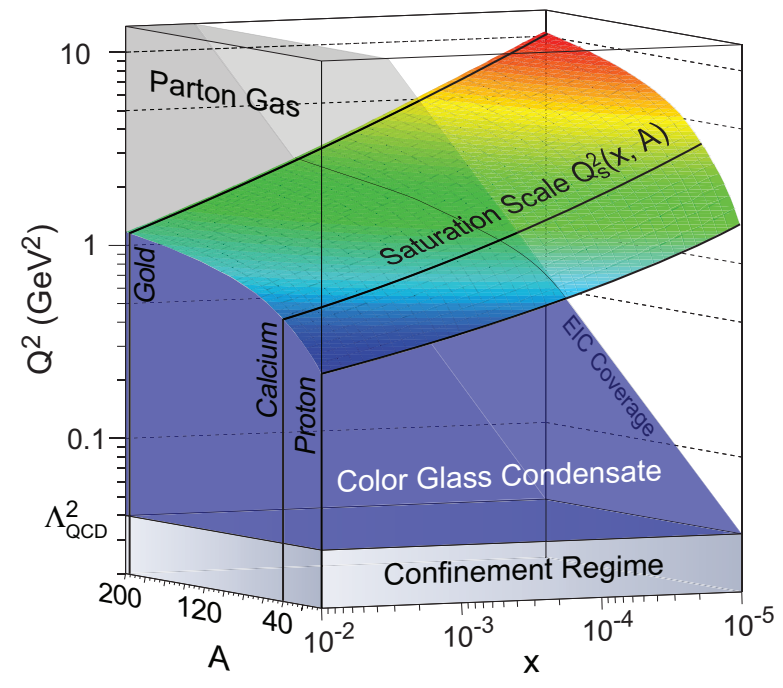

Figure 5.1. The saturation scale, $Q \equiv Q_{s}$, and how it scales with $x$, and $A$.

colour fields; the important new feature is that the applicability of weak coupling methods allow for systematic comparisons of theory to experiment.

In addition to the intrinsic interest in this novel many-body regime of QCD, experimentally establishing and refining an effective field theory for the saturation regime - such as the Colour Glass Condensate (CGC) - as well as precisely imaging the distribution and correlations of small- $x$ partons in nuclei, besides being of intrinsic interest, would have wide-ranging applications. The universality of the saturation regime implies that such a theory would provide a microscopic basis for understanding and calculating total hadronic cross sections, with important applications to, for example, ultra-high energy cosmic ray physics, where extrapolations in energy of several orders of magnitude are required to compute their spectrum and detect possible new physics effects. In high-energy relativistic heavy-ion collisions, the release of saturated low- $x$ partons represents the starting point of the subsequent space-time evolution of the Quark-Gluon Plasma (QGP). Testing and benchmarking the underlying theory opens the prospect of a controlled, and precise, first principles calculation of such an initial state. Thereby reducing one of the largest sources of uncertainty in the interpretation of experimental observables, and the measurements of the QGP properties: an EIC would offer to the RHIC and LHC heavy-ion programs an important asset, as valuable as the one HERA provided to the LHC $\mathrm{p}+\mathrm{p}$ program.

The onset of the saturation regime, when the gluon density becomes so large that further growth with energy is tamed, is characterised by the saturation scale $Q_{s}(x)$; partons with momenta below this scale overlap in transverse space, so that parton recombination and screening stops further growth in their number density. Given that parton distributions grow as $x$ decreases, and dramatically so as discovered at HERA, the saturation scale is clearly expected to grow as $x$ decreases. It is further enhanced in nuclear targets because of the overlap of the gluon fields originating from different nucleons. This is illustrated in figure 5.1. In the saturated, dense regime at small $x$, non-linear QCD dynamics becomes dominant but at the scale being set by a semi-hard $Q_{s}$ (of order $1 \mathrm{GeV}$ ), calculations can be carried out by weak coupling techniques and suitable effective field theories, of which the CGC is a prime example, can be derived from first principles.

The dilute-dense separation of scales is more subtle than just described. The larger 
the gluon's transverse momentum $k_{T}$, the smaller its longitudinal energy fraction $x$ needs to be to enter the saturation regime. In a scattering process, dilute partons (with $k_{T} \gg$ $\left.Q_{s}(x)\right)$ behave incoherently, whilst when the parton density is large $\left(k_{T} \lesssim Q_{s}(x)\right)$, gluons scatter coherently. Therefore, transverse momentum dependent observables will be able to uncover more details than inclusive observables, which can only access averaged saturation effects. The interplay of saturation and the transverse spatial distribution of gluons is also important; as $x$ decreases, gluon densities saturate first in the centre of the nucleus. To accommodate further growth, gluons will be pushed more and more to the periphery, so that the average gluon radius is expected to increase with decreasing $x$.

For all these reasons regarding the small- $x$ physics program in e+A collisions, the physics deliverables of an EIC have been classified in three main categories giving access to the integrated, transverse-momentum-dependent, and impact-parameter-dependent gluons. Here, by "gluons" we mean not only the conventional single-gluon distributions but also multigluon correlations. These have often been of secondary interest, but are now recognised as essential to a full understanding of the low- $x$ regime. Indeed, except for the most inclusive observables which are subject to cancellations, consistent QCD calculations in the nonlinear regime require the knowledge of multi-gluon distributions. Integrated, transversemomentum-dependent, and impact-parameter-dependent gluon distributions and correlations in nuclei are all unknown, and the processes we discuss below have never been measured at small $x$.

\section{Integrated gluons and sea-quarks}

As the most basic observables from both the theory and experimental sides, the inclusive $(e+\mathrm{A} \rightarrow e+\mathrm{X})$ structure functions $F_{2}$ and $F_{L}$ stood out among other measurements, already well before the INT programme. They were the first potential golden measurements discussed; the pros and cons of these candidates to pin down the gluon and sea-quark distributions in nuclei were further reviewed during the program.

$F_{2}$ is the most inclusive observable in deep inelastic scattering. Its measurement presents no particular experimental challenge. On the theory side, it is the simplest process to calculate, along with $F_{L}$, with the fewest input assumptions. For instance, $F_{2}$ and $F_{L}$ will be the first observables for which a full NLO calculation in QCD including non-linear effects will be available. (The existing phenomenology is still based on leading-order "impact factor" computations.)

Although it is harder to extract experimentally, $F_{L}$ is a golden measurement for high density QCD because it is more directly related to the gluon distribution. Furthermore, it is more sensitive to non-linear effects than $F_{2}$. In the latter, higher-twist contributions cancel each other out delaying the onset of non-linear effects. The necessity of performing an energy scan to measure $F_{L}$ implies that the accessible $x$ range is a bit smaller than accessible with the $F_{2}$ measurement. However, the increased sensitivity to non-linear effects more than compensates for this shortcoming. Deviations of DGLAP fits of the simultaneous "singlet" dominated (at small $x$ ) evolution of $F_{2}$ and $F_{L}$ determined from EIC data should be able to quantitatively determine the onset of the saturation regime. The case for the low-energy EIC needs to be investigated more; in particular, the implementation of non-linear effects must be made more accurate, and more detailed DGLAP fits of EIC pseudo-data should be performed before establishing its sensitivity to saturation physics in the inclusive channel. One caveat discussed is that QED radiative corrections for nuclear targets can be large, and it remains to be proven that they can be controlled to the required precision. Computations 
addressing the role of these radiative corrections are underway and are discussed further in the detector studies section of this report.

The charm structure functions $F_{2, L}^{c}$ were considered as silver measurements for the nonlinear regime. As in the case of $F_{L}$, these observables give more direct access to the gluon distribution relative to $F_{2}$; however due to the mass of the charm quark, they also probe higher values of $x$ and are therefore less sensitive to non-linear effects. In addition, QCD calculations with non-zero charm mass are scheme dependent, which can absorb signals of non-linear effects if not appropriately handled. However, since they can be measured precisely with a properly-designed vertex detector, charm structure functions will be a very important complementary measurement to pin down the nuclear gluon distribution throughout the $\left(x, Q^{2}\right)$ plane.

Last but not least, silver measurements in the inclusive category are those of the diffractive structure functions $F_{2}^{D}$ and $F_{L, D}$. These are sensitive to the square of the gluon distribution. As may therefore be anticipated, the strongest hints for manifestations of non-linear effects in $\mathrm{e}+\mathrm{p}$ collisions at HERA come from diffractive measurements. A striking example is the fact that the ratio of the diffractive to inclusive structure function is constant with energy, an observation not easily reconciled in a leading twist scenario. Furthermore, the leading-twist approximation does not explain the geometric scaling of the diffractive cross section. Finally, as a sign of enhanced sensitivity to non-linear effects, the DGLAP analysis of diffractive structure functions from HERA is problematic at larger values of $Q^{2}$ relative to the same for $F_{2}\left(\sim 8 \mathrm{GeV}^{2}\right.$ in the former compared to $2 \mathrm{GeV}^{2}$ in the latter). However, measurements of diffractive structure functions are relatively more difficult and the additional kinematic variables make the analyses more involved than for $F_{2, L}$.

\section{Transverse momentum dependent gluons and sea-quarks}

The golden measurement here is that of di-hadron azimuthal correlations in $e+\mathrm{A} \rightarrow$ $e+\mathrm{h}_{1}+\mathrm{h}_{2}+\mathrm{X}$ processes. Di-hadron correlations are not only sensitive to the $k_{T}$ dependence of the gluon distribution but also to the $k_{T}$ dependence of gluon correlations. These correlations are sensitive to multi-gluon distributions for which first principles computations are only now becoming available. Precise measurements of these di-hadron correlations at an EIC would allow one to extract these multi-gluon correlations and study their non-linear evolution. Saturation effects in this channel correspond to a progressive disappearance with decreasing $x$ of the peak in the di-hadron azimuthal angle difference around $\Delta \phi=\pi$. In a leading twist picture, where there is only one hard scattering, one expects, from momentum conservation, that the peak will persist. A comparison of the heights and widths of the dihadron azimuthal distributions in $e+\mathrm{A}$ and $e+\mathrm{p}$ collisions respectively would clearly mark out such an effect experimentally. An analogous phenomenon has already been observed for di-hadrons produced at forward rapidity in $\mathrm{d}+\mathrm{Au}$ and $\mathrm{p}+\mathrm{p}$ collisions at RHIC. In that case, di-hadron production proceeds from valence quarks in the deuteron (proton) scattering on small- $x$ gluons in the target $\mathrm{Au}$ nucleons (proton), $q_{V}+\mathrm{Au}(\mathrm{p}) \rightarrow \mathrm{h}_{1}+\mathrm{h}_{2}+\mathrm{X}$. Lacking direct experimental control over $x$, the onset of the saturation regime is controlled by changing the centrality of the collision, the di-hadron rapidity and the transverse momenta of the produced particles. Experimentally, a striking flattening of the $\Delta \phi$ peak in $\mathrm{d}+\mathrm{Au}$ collisions is observed in central collisions, but the peak reappears in peripheral collisions or for mid-rapidity di-hadrons. Directly using a point-like electron probe, as opposed to a quark bound in a proton or deuteron, is extremely beneficial. It is experimentally much cleaner as there is no "spectator" background to subtract and the access to the exact kinematics of 
the process allows for more accurate extraction of the physics than is possible at RHIC or in the future with $\mathrm{p}+\mathrm{A}$ collisions at the LHC. Because there is such a clear correspondence between the physics of this particular final state in $\mathrm{e}+\mathrm{A}$ collisions to the same in $\mathrm{p}+\mathrm{A}$ collisions, this measurement is an excellent testing ground for quantitative studies of the universality of multi-gluon correlations in $\mathrm{p}+\mathrm{A}$ and $\mathrm{e}+\mathrm{A}$ collisions.

The simplest process to extract the transverse momentum dependence of the gluon distribution is single inclusive DIS (SIDIS), $e+\mathrm{A} \rightarrow e+\mathrm{h}+\mathrm{X}$. One reason why these processes are especially interesting is that by having two momentum scales at one's disposal, it is possible to keep $Q^{2}$ large and access the saturation regime at transverse momenta $p_{T} \lesssim Q_{s}$. This way, non-perturbative effects and higher-twist contributions are suppressed, but one can nonetheless access non-linear QCD dynamics. Considering that $Q_{s}$ will not exceed a few $\mathrm{GeV}$ at an EIC, this helps one disentangle strong coupling effects, characterized by a fixed scale $\left(\Lambda_{Q C D}\right)$, from weak coupling non-linear effects more cleanly relative to inclusive observables. Furthermore, in the large $Q^{2}$ and small $x$ limits, the relation between the transverse momentum of the produced hadron $p_{T}$ and that of the small- $x$ glue $k_{T}$ is quite direct, enabling a rather straightforward experimental probe of the gluon transverse momentum distribution. From the theoretical point of view, important connections have been established between the framework of Transverse Momentum Dependent (TMD) distributions discussed previously in this report and the CGC effective theory at small $x$. Thus SIDIS has all the pre-requisites to be considered a golden observable. It is nonetheless classified as silver because di-hadron correlations are more directly sensitive to non-linear QCD evolution.

\section{Transverse position dependence of gluons and sea-quarks}

To pin down the transverse distribution and correlations of small- $x$ gluons, exclusive measurements are needed. The prototypical observables discussed are diffractive vector meson production (DVMP) and deeply virtual Compton scattering (DVCS). Coherent diffraction, where the nuclear target is intact, gives access to the transverse spatial distribution of the gluon density in a nucleus. Incoherent diffraction, where the nuclear target breaks up, but is separated by a rapidity gap from the projectile fragmentation region, allows one to extract, in addition, transverse plane correlations. These shed important light on the spatial picture of the partonic sub-structure of nuclei. In addition, both contribute crucial information necessary to understand the spatial gluon distributions that form the initial conditions for heavy ion collisions. $J / \Psi$ meson production off nuclei is the most widely considered exclusive channel; those of other vector mesons $\rho, \phi)$ provide important complementary information. DVCS, though luminosity hungry, is free of the uncertainty from incomplete knowledge of vector meson wave-functions.

Coherent diffractive $J / \Psi$ production has been extensively discussed as potentially the golden measurement in this category. However, while the physics goals are golden, the technical challenges are formidable. Coherent diffraction dominates over incoherent diffraction only at rather low values of $t$. It was determined that a rejection of the target-dissociation background with at least $95 \%$ efficiency is required in order to measure the coherent cross section up to large enough momentum transfers, and a $20 \mathrm{MeV}$ resolution on the momentum transfer is also needed in order to extract precise enough information in impact parameter space. While this measurement is more feasible in light nuclei, it becomes more challenging in heavier nuclei. For light nuclei, coherent diffraction could shed important light on short range nuclear forces. For larger nuclei, it is unclear at present whether what one learns is 
distinguishable from the distribution of gluons obtained from the Woods-Saxon distribution of nucleons in the nucleus; the ability for coherent diffraction to distinguish between different dynamical models for large nuclei is disputed. For these reasons, it is classed as a silver measurement.

Studies of the incoherent regime of diffractive vector meson production are slowly but surely emerging. This process is a priori more sensitive to high parton densities than is coherent diffraction. This is because it is much easier to measure at large $t$, corresponding to small values of $b$, nearer the center of the nucleus where the gluon density is the largest. However, the amount of information that can be extracted from nuclear fragments is not clear, since the theoretical description of the nuclear break-up remains a challenge. The minimum requirement is to be able to identify if the nucleus breaks up into its constituent nucleons or if the nucleons themselves break-up, as the corresponding calculations require different theoretical tools. Neither experimental or theoretical works on this process are mature enough to classify it as a golden measurement. However, the effects predicted by saturation models are large and unique enough that an observation of these will be convincing evidence of this physics. Therefore, both theoretical and experimental studies of this channel should be pursued vigorously.

\subsubsection{Parton substructure of nuclei}

Nuclear deep-inelastic scattering with an EIC will provide a unique measurement of gluon and sea quark densities in the "dilute" regime at $x \gtrsim 0.01$ in a range of nuclei. While the quark densities in the region $0.05 \lesssim x \lesssim 0.6$ were studied in fixed target experiments and will be further explored at JLab with $12 \mathrm{GeV}$ electron beams, the behaviour of the gluon and sea quark densities in this region is essentially unknown. An EIC will have sufficient coverage in $Q^{2}$ to extract the nuclear gluon density through the $Q^{2}$ dependence of the nuclear structure function $F_{2}^{A}$. Furthermore, direct access to gluons can be gained from the longitudinal structure function $F_{L}^{A}$ through measurements at different beam energies, or additionally, by tagging charm production.

A reliable determination of the nuclear gluon density in the dilute regime is essential for a quantitative assessment of the onset of the new QCD regime of high parton densities and non-linear gluon interactions, which will be more widely accessible at a full-energy EIC. At $x \gtrsim 0.1$, an EIC will also explore gluon anti-shadowing and EMC effects - a step that might prove as revolutionary for our understanding of nuclei as the discovery of the quark EMC effect 30 years ago. For these reasons, inclusive $F_{2, L}$ structure function measurements at larger $x$ complement those discussed for the small- $x$ regime.

As it turns out, the high luminosity envisaged for an EIC enables measurements of nuclear electromagnetic structure functions up to $x \approx 1$ competitively with, or even surpassing, what has been achieved to date in fixed target experiments. (Since the maximum $x$ in a nucleus is $A$, collider high luminosity measurements could uncover interesting physics in the Fermi regime where partons carry more more momenta than a bound nucleon.) Furthermore, the large $Q^{2}$ range and hadronic event reconstruction capabilities will also likely allow measurement of charged current structure functions, and possibly of $\gamma-Z$ interference structure functions. See the chapter on electroweak physics for further discussion. This will enable full quark flavour separation utilising only nuclear DIS data, and offer, for example, new handles on the origin of the EMC effect such as its flavour dependence. In this context, one should also mention the possibility of extracting information on particular twist four operators, which play an important role in parity violating DIS in the EMC region. 
These measurements are highly interesting, important, and in some cases unique to an EIC compared to previous facilities. However, more work is needed to establish to what extent full flavour separation can be effectively carried out at an EIC; we therefore classify them as silver measurements.

Much more information on the nuclear modification of the quark/gluon structure of the proton and neutron can be gained from deep-inelastic measurements with detection of the spectator system of $(A-1)$ nucleons in the final state. In particular, measurements on deuterium with a spectator proton can measure structure functions of the bound neutron ranging from nearly on-shell to far off-shell, facilitating the extrapolation to an on-shell neutron. Measurements with a spectator neutron, which are extremely difficult with a fixed target but feasible at a collider using a zero degree calorimeter, provide completely new information on the off-shell proton structure functions, and constrain theoretical models by comparison to the well known free proton wave function. With heavier nuclear targets, one could explore the effects of parton/nucleon embedding in a complex nuclear environment. While no technical difficulty is foreseen, detailed studies of the required detectors are needed to determine the feasibility and precision of these measurements.

\subsubsection{Parton propagation and hadronization in nuclear matter}

The transition from coloured partons (quarks and gluons) to colourless hadrons - the so-called hadronization or fragmentation process - exemplifies a fundamental process in QCD which still lacks a quantitative understanding from first principles calculations. Fragmentation functions, which encode the probability that a parton fragments into a hadron, have been obtained by fitting experimental data covering large kinematic ranges and numerous hadron species. However, knowledge about the dynamics of the process remains limited and model dependent. A particular model, see figure 5.2. posits a separation of scales between a short time scale for colour neutralization due to confinement generating a colourless "pre-hadron" and a longer time scale (presumably controlled by chiral symmetry breaking), which governs the formation of hadrons. The dynamical consequences of such a model are distinguishable from other models where the separation of scales is reversed or is non-existent. Extracting these time scales would be an important step towards understanding how hadrons emerge dynamically from partons, complementing the information on properties of colour confinement extracted from lattice measurements of ground state "static" correlators.

Nuclear deep inelastic scattering (nDIS) provides a known and stable nuclear medium ("cold QCD matter") and a final state with strong experimental control on the kinematics of the hard scattering. This permits one to use nuclei as femtometer-scale detectors of the hadronization process, see figure 5.2. In fact, both the energy loss due to medium-induced gluon bremsstrahlung off a quasi-free parton and the pre-hadron re-interaction with the surrounding nucleons lead to attenuation and transverse momentum broadening of hadron yields compared to proton targets, and allow experimental access to the space-time evolution of hadronization. Theoretical models of this process can be calibrated in nDIS and then applied, for example, to the study of the Quark-Gluon Plasma ("hot QCD matter") created in high-energy nucleus-nucleus collisions.

The combination of high energy and luminosity offered by an EIC promises a truly qualitative advance in this field, compared with current and planned fixed target experiments. The large $Q^{2}$ range permits measurements in the fully calculable perturbative regime with enough leverage to determine nuclear modifications in the QCD evolution of 


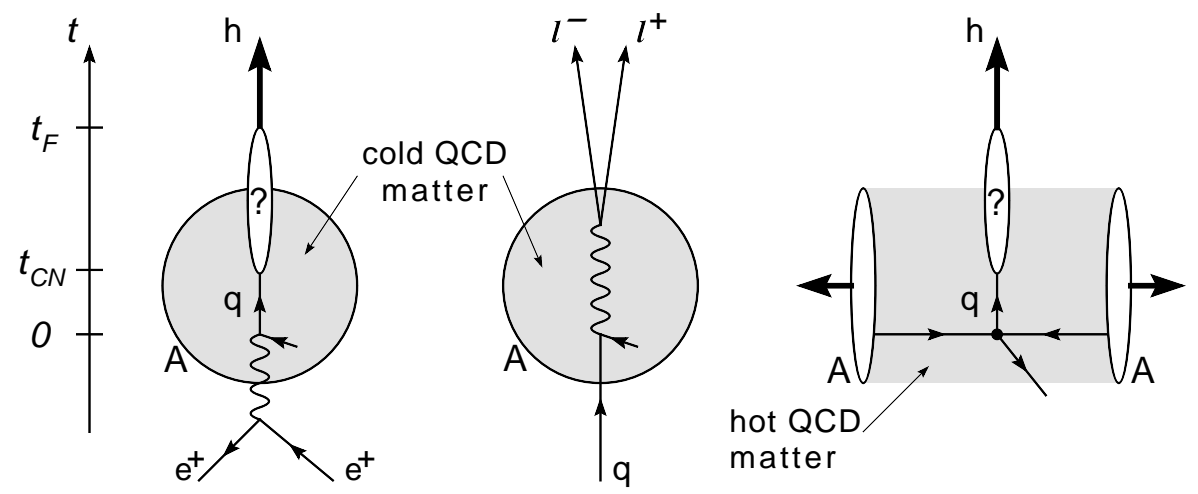

Figure 5.2. Parton propagation and hadronization in cold and hot nuclear matter. A scenario of possibly distinct colour neutralization $\left(t_{C N}\right)$ and hadron formation $\left(t_{F}\right)$ time scales is illustrated on the vertical time axis.

fragmentation functions; the high-luminosity permits the multidimensional binning necessary for separating the many competing effects and for detecting rare hadrons. The large $\nu$ range $(\approx 10-1000 \mathrm{GeV})$ allows one to experimentally boost the hadronization process in and out of the nuclear medium, in order to isolate in-medium parton propagation effects (large $\nu$ ) and cleanly extract colour neutralization and hadron formation times (small $\nu$ ); furthermore, using the quark flavour separated nuclear PDFs expected from an EIC, one could analyze nuclear Drell-Yan data, which are free from hadronization effects, and isolate initial state parton energy loss from nuclear wave function effects, enabling a complete experimental study of colour charge interactions in cold nuclear matter. For the first time, one will be able to study hadronization of open charm and open bottom meson production in $e+A$ collisions, as well as the in-medium propagation of the associated heavy quarks: these allow one to fundamentally test high-energy QCD predictions for energy loss, and confront puzzling measurements of heavy flavour suppression in the Quark-Gluon Plasma at RHIC. Within a collider environment, one would also be able to separate target from current hadronization and cross-correlate these two, adding a new dimension to hadronization studies.

The scattered quarks and gluons, from which the final-state hadrons emerge, couple to other nuclear gluons. Good control over the colour neutralization time scale will allow one to use this internally created colour radiation to explore the structure of nuclear matter in close analogy with the well-known exploration of matter with electromagnetic radiation or electrically charged particles. Furthermore, an EIC with $\sqrt{ } s \gtrsim 30 \mathrm{GeV}$ will permit for the first time the measurement of jets and their substructure in $e+\mathrm{A}$ collisions, furnishing a novel and extensive set of observables which directly access quark energy loss and the as yet untested parton shower mechanism, fundamentally described in QCD and pervasive in applications to particle physics simulations. Jet nuclear modifications can also be directly related to the propagation of the coloured partons shower in the nuclear medium, and used to measure the cold nuclear matter transport coefficients which encode basic information on the non-perturbative soft gluon structure of the nuclei. These measurements are complementary to direct inclusive and diffractive structure functions measurements at small $x$ in accessing the high-density non-linear QCD regime, but are entirely feasible with a low-energy EIC.

The outlined parton propagation and hadronization program can for the most part be carried out in phase-I. In phase-II, we do not anticipate any qualitative new lesson will be 
learned, while the increased energy and $Q^{2}$ range may prove useful, for instance, for more refined studies in the jet and heavy flavour sectors, and offering an increased reach towards small $x$ for nuclear gluon measurements via $2+1$ jet production.

In conclusion, due to the physics interest, theoretical interpretability and feasibility in phase-I, this jet and heavy quark study program as a whole was classified as a golden measurement for $e+\mathrm{A}$ collisions at an EIC, with light quark SIDIS classified as a silver measurement. 


\title{
5.2 Review of linear and non-linear approaches in QCD at small- $x$
}

\section{Collinear factorization and DGLAP evolution}

\author{
Anna M. Staśto
}

The evaluation of strong interaction cross-sections which involve hard scales is possible thanks to QCD factorization theorems. The latter are derived from first principles in QCD [716, 717] and allow the factorization of cross sections into hard scattering coefficients (computed in a perturbative expansion in the strong coupling constant) and parton densities which contain information about nonperturbative dynamics. Parton densities, due to their intrinsically non-perturbative nature, cannot be directly evaluated from first-principles lattice computations except perhaps in very limited kinematic windows. Nevertheless, their evolution with hard scale can be calculated. This is done usually using the renormalization group DGLAP equations,

$$
\mu \frac{d}{d \mu} f_{j / h}(x, \mu)=\sum_{k} \int_{x}^{1} \frac{d z}{z} P_{j k}\left(z, \alpha_{s}(\mu)\right) f_{k / h}(x / z, \mu),
$$

with the splitting functions which have perturbative expansion in powers of the strong coupling constant $\alpha_{s}$,

$$
P_{j k}\left(z, \alpha_{s}(\mu)\right)=\sum_{i}\left(\alpha_{s}(\mu)\right)^{i} P_{j k}^{(i)}(z) .
$$

Coefficient functions and splitting functions are known up to NNLO accuracy [4, 718, 5]. It has been found that at this order large corrections appear which are enhanced by the logarithmic terms in $1 / x$. The collinear approach suffers also from other limitations. The kinematical approximations mostly suitable for the evaluation of the inclusive observables are not sufficient for exclusive processes and can lead to large discrepancies [719].

There are also other direct indications of the breakdown of the fixed order approach. From the global fits [22, 43], it is known that the gluon density suffers from large uncertainties at the NLO level in the region of small values of $x$, and the gluon density even turns negative. Even though the gluon density is not a directly observable quantity, the aforementioned uncertainties propagate into the observable longitudinal structure function $F_{L}$. The problem is concentrated in the low $Q$ and low- $x$ region, though the uncertainties remain even at larger values of $Q$ when $x$ is decreased. A systematic study of the compatibility of the HERA deep inelastic data with DGLAP evolution has been performed in [720]. This analysis, originally based on the NNPDF1.2 analysis [681, 721], was then extended to the global NNPDF2.0 set, which includes the very precise combined HERA-I dataset as well as all the relevant hadronic data. A 'safe' region was defined as the one in which the non-DGLAP effects are expected to be negligible, and it was defined by the cut on low- $x$ and $Q$ data. A fit was then performed to the data that pass the cut and only belong to the safe region and the structure functions evaluated at different scales. It turned out that the prediction for the structure functions at low $Q^{2}$ obtained from the backward-evolution of the data above the cut exhibits a systematic downward trend. Thus the precise HERA measurements indicate that the fixed order DGLAP evolution is incompatible with the data in the low $Q^{2}$ and low $x$ region. 


\section{Small- $x$ re-summations}

\section{Anna M. Staśto}

Since the seminal works [59, 60, it is well known that observables at small $x$ receive substantial corrections due to the large logarithms $\alpha_{s} \ln 1 / x$ which need to be re-summed in this regime. The BFKL approach [59, 60, provides a framework for this summation and it is known up to next-to-leading logarithmic accuracy. The resulting evolution of the gluon Green's function provided by this framework is with respect to the $\ln 1 / x$ or rapidity variable, with the transverse momenta of the gluons being summed over all possible configurations. The evolution has the following form

$$
G\left(Y ; \mathbf{k}, \mathbf{k}_{0}\right)=\delta^{(2)}\left(\mathbf{k}-\mathbf{k}_{0}\right)+\int d^{2} \mathbf{k}^{\prime} K\left(\mathbf{k}, \mathbf{k}^{\prime}\right) G\left(Y ; \mathbf{k}^{\prime}, \mathbf{k}_{0}\right),
$$

with the branching kernel having also the perturbative expansion

$$
K\left(\mathbf{k}, \mathbf{k}^{\prime}\right)=\sum_{i}\left(\alpha_{s}(\mu)\right)^{i} K^{(i)}\left(\mathbf{k}, \mathbf{k}^{\prime}\right) .
$$

A solution for the gluon Green's function and therefore the resulting cross sections exhibit strong growth with the energy, the hard Pomeron, with the intercept being significantly larger than unity in the LO approximation, $\omega_{P}=1+\alpha_{s} N_{c} 4 \ln 2 / \pi$. This growth turns out to be incompatible with both the hadronic data and the data on structure functions from deep-inelastic scattering. NLL (next-to-leading-log) corrections [722, 723] turned out to be rather large numerically and point to the need for the resummation of subsequent powers of higher order corrections $\alpha_{s}^{k} \ln 1 / x$. The sizes of the various NLL corrections can be understood on physical grounds. Firstly, unlike in the DGLAP limit, the strong coupling constant is not naturally a small parameter. On top of that, the BFKL approach does not satisfy the momentum sum rule for the longi-

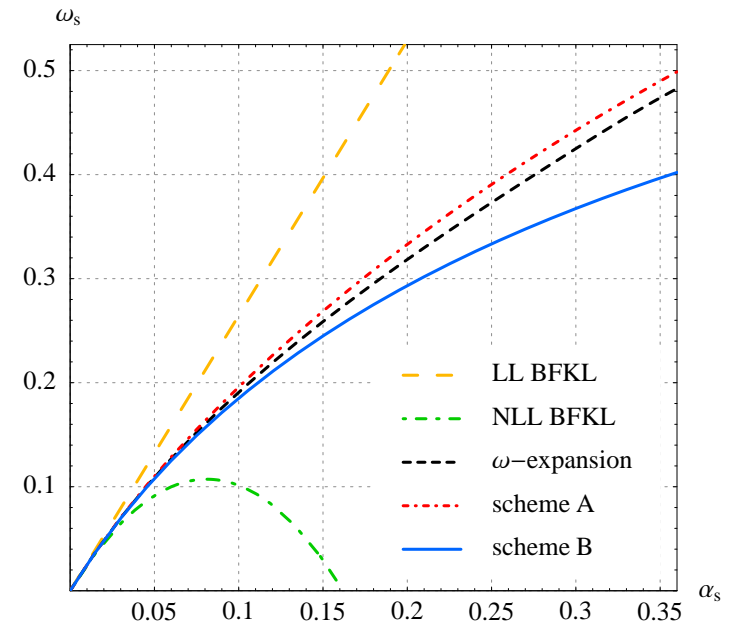

Figure 5.3. The intercept of the hard Pomeron extracted from the BFKL equation with fixed strong coupling in LL, NLL and re-summed cases. tudinal momentum fractions (the transverse momenta are however conserved, unlike in the collinear approach). The kinematical approximations made in the BFKL limit cannot be efficiently recovered by the truncated higher orders of the perturbative expansion.

The strategy of re-summation at small $x$ has been developed in a series of works [724, 725, 726, 727, 728. It involves the construction of the appropriate re-summed kernel of the form given by Eq. 5.4, which includes at the same time known terms in the expansion of the splitting function, Eq. 5.2. Although the details of the various approaches differ, there are common fundamental ingredients. The evolution in rapidity is subjected to kinematical constraints which originate from the requirement of the consistency of the 

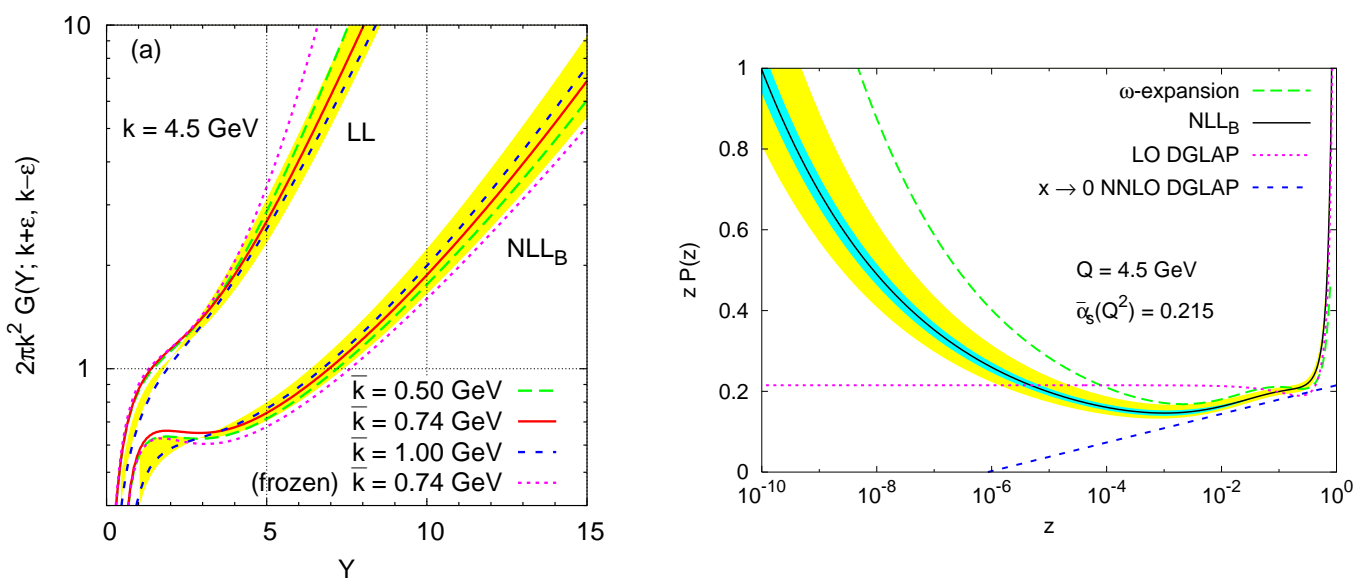

Figure 5.4. Left: The gluon Green's function extracted from the BFKL equation in LL and resummed cases. The coupling is running in all computations, and $\bar{k}$ denotes the scale at which the strong coupling is regularized. Right: The extracted effective splitting function from the re-summed approach: solid line. The scale was taken to be $Q=4.5 \mathrm{GeV}$. Dotted pink line indicates the LO DGLAP splitting function and the blue dashed indicates small $x$ part of the NNLO DGLAP. The dashed green line corresponds to the re-summed spliitting function from the $\omega$ expansion. The band correspond to the scale variation.

assumption about the Regge kinematics. The evolution is matched with the DGLAP evolution by including the splitting function at LO and NLO. The momentum sum rule is imposed onto the resulting re-summed splitting function. The running of the coupling is included into the evolution. Finally, matching to the NLL BFKL is performed with the suitable subtractions in order to avoid double counting. The resulting Green's function and splitting function turn out to be very stable with minimal variations across the different re-summation schemes.

c Gluon Green's function and the splitting function: In Fig. 5.3, we show the results on the intercept of the gluon Green's function in the case of the fixed strong coupling constant, obtained within the re-summation framework of [724]. The linear growth is given by the LO approximation. The NLO value of the intercept is significantly below the lowest order, and turns negative even for the intermediate values of $\alpha_{s}$. The re-summed result is between the NLO and $\mathrm{LO}$, it exhibits clear growth with increasing values of the coupling constant, albeit much reduced with respect to the $\mathrm{LO}$ value and much closer to the phenomenology.

The rapidity dependence of the gluon Green's function is shown in Fig. 5.4 (left). The scale was chosen to be equal to $k=4.5 \mathrm{GeV}$. The reduction of the speed of growth is clear in the re-summed case. Also the scale variations are relatively small in this case.

By using the deconvolution of the integral equation, one can calculate the integrated gluon density. As a result, it is possible to solve the re-summed splitting function numerically. In this way, the perturbative and non-perturbative contributions are factorized in $Q^{2}$. In Fig. 5.4 (right), we show the results for the splitting function as a function of the momentum fraction for the re-summed case. It is compared with the results on the LO and NNLO (only small $x$ part) splitting functions. The results on the splitting function demonstrate that the small $\mathrm{x}$ growth is delayed to much smaller values of $\mathrm{x}$ (beyond HERA). The splitting function also has an interesting feature, namely that of the dip. It turns out that 
this is a universal feature, present also in other schemes of re-summation. In general, it was found that the dip comes from the interplay between NNLO order and the re-summation.

Thus far, re-summation was demonstrated to give stable results for the gluon channel only. For the complete description, however, one needs to include quarks in the evolution. A matrix approach was developed which was shown to be consistent with the collinear matrix factorization of the parton densities in the singlet evolution [726]. This approached enables the calculation of the anomalous dimensions matrix, which can be directly compared with the standard DGLAP matrix. It was shown that it is possible to incorporate NLLx BFKL + NLO DGLAP in this framework [726].

Conclusions and outlook: The small- $x$ regime requires a formalism which incorporates re-summation of the large terms $\alpha_{s} \ln 1 / x$. The BFKL formalism was extended to include re-summation to higher orders. This formalism includes both DGLAP NLO and BFKL NLL and higher order terms. Stability of the results was demonstrated for scale changes and model changes. There are certain universal and characteristic features which come from the solutions to the evolution equations: the rapid growth with $x$ is delayed to smaller values of $x$, and the splitting function has a minimum. A matrix model was developed which gives consistent results on the gluon Green's function and the splitting functions. For the complete framework, one needs to include the re-summed coefficient functions. Detailed fits to the data need to be performed. In this regard, the EIC will generate very important information on parton densities at small $x$, and in distinguishing small $x$ re-summation effects from higher twist saturation effects.

\section{Parton Saturation}

\section{Yuri V. Kovchegov and Cyrille Marquet}

The QCD description of hadrons in terms of quarks and gluons depends on the processes considered and on what part of the hadron wave function they are sensitive to. Consider a hadron moving at nearly the speed of light along the light cone direction $x^{+}$, with momentum $P^{+}$. Depending on their transverse momentum $k_{T}$ and longitudinal momentum $x P^{+}$, the virtual partons inside the hadron behave differently, reflecting the different regimes of the hadron wave function. Soft hadronic processes are mostly sensitive to the non-perturbative part of the wave function, they involve quantum fluctuations with transverse momenta of the order of $\Lambda_{Q C D} \sim 200 \mathrm{MeV}$. A hadron can then be thought of as a bound state of stronglyinteracting partons, but a QCD description of the associated dynamics is still lacking. By contrast, hard processes in hadronic collisions are sensitive to the weakly-coupled part of the wave function and resolve the partonic structure of hadrons. They probe partons with $k_{T} \gg \Lambda_{Q C D}$ whose QCD dynamics is better understood.

One can distinguish two weakly-coupling regimes in the wave function: a linear one called the hard regime, involving a small density of partons, typically with $x \lesssim 1$, in which the hadron looks like a dilute system of independent partons, and a non-linear one called the saturation regime, involving a large density of partons with $x \ll 1$, in which the hadron looks like a dense system of nevertheless weakly-interacting partons, mainly gluons (called small- $x$ gluons). The dilute-dense separation is a bit subtler than that: the larger $k_{T}$ is, the smaller $x$ needs to be to enter the saturation regime. Indeed the separation between the two regimes is characterized by a momentum scale $Q_{s}(x)$, called the saturation scale, which increases as $x$ decreases. In a scattering process, dilute partons (with $k_{T} \gg Q_{s}(x)$ ) behave 
incoherently, while when the parton density is large $\left(k_{T} \lesssim Q_{s}(x)\right)$, gluons scatter coherently. The dynamics of the dilute regime is well described by the leading-twist approximation of QCD, whose hallmark is collinear factorization. As explained in the previous section, when $x$ becomes small while not yet reaching the non-linear regime, so-called small- $x$ re-summations are also needed to improve the approximation.

To describe the small- $x$ non-linear part of hadronic/nuclear wave functions in QCD, the Color Glass Condensate (CGC) effective theory was proposed. Rather than using the standard Fock-state expansion which is ineffective in dealing with numerous small $-x$ gluons, the CGC approach employs collective degrees of freedom, static color sources at large $x$ and dynamical classical color fields at small $x$. The traditional approach to saturation physics consists of two stages, corresponding to two different levels of approximations. The first level corresponds to the classical gluon field description of nuclear wave functions and scattering cross sections. It re-sums all multiple re-scatterings in the nucleus, but lacks energy dependence. The latter is included through quantum corrections, which are re-summed by non-linear evolution equations. This constitutes the second level of approximation. We will present both stages below.

\section{Classical gluon fields}

McLerran-Venugopalan model: Imagine a single large nucleus, which was boosted to some ultrarelativistic velocity, as shown in Fig. 5.5 (left). We are interested in the dynamics of small- $x$ gluons in the wave function of this relativistic nucleus. The small- $x$ gluons interact with the whole nucleus coherently in the longitudinal direction: therefore, only the transverse plane distribution of nucleons is important for the small- $x$ wave function. As one can see from Fig. 5.5, after the boost, the nucleons, as "seen" by the small- $x$ gluons, appear to overlap with each other in the transverse plane, leading to high parton density. Large occupation numbers of color charges (partons) lead to classical gluon fields dominating the small- $x$ wave function of the nucleus. This is the essence of the McLerran-Venugopalan (MV) model [729, 730, 731]. According to the MV model, the dominant gluon field is given by the solution of the classical Yang-Mills equations $\mathcal{D}_{\mu} F^{\mu \nu}=J^{\nu}$ where the classical color current $J^{\nu}$ is generated by the valence quarks in the nucleons of the nucleus from Fig. 5.5.
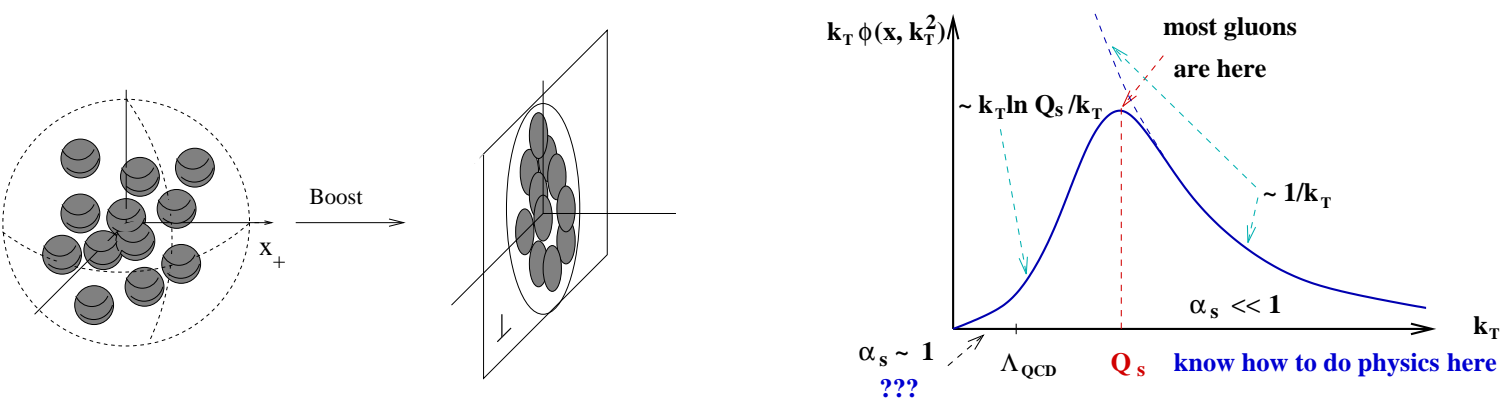

Figure 5.5. Left: Large nucleus before and after an ultrarelativistic boost. Right: Unintegrated gluon distribution $\phi\left(x, k_{T}^{2}\right)$ of a large nucleus due to classical gluon fields (solid line). Dashed curve denotes the lowest-order perturbative result.

The Yang-Mills equations were solved for a single nucleus exactly [732, 733, resulting in the unintegrated gluon distribution $\phi\left(x, k_{T}^{2}\right)$ (multiplied by the phase space factor of the gluon's transverse momentum $k_{T}$ ) shown in Fig. 5.5 right as a function of $k_{T}$. (Note that in the MV model, $\phi\left(x, k_{T}^{2}\right)$ is independent of Bjorken- $x$.) Fig. 5.5 demonstrates the emergence 
of the saturation scale $Q_{s}$. As one can see from Fig. 5.5, the majority of gluons in this classical distribution have transverse momentum $k_{T} \approx Q_{s}$. Since in this classical approximation $Q_{s}^{2} \sim A^{1 / 3}$, for a large enough nucleus, all of its small- $x$ gluons would have large transverse momenta $k_{T} \approx Q_{s} \gg \Lambda_{Q C D}$, justifying the applicability of the perturbative approach to the problem. Note that the gluon distribution slows down its growth with decreasing $k_{T}$ for $k_{T}<Q_{s}$ (from power-law of $k_{T}$ to a logarithm) and the distribution saturates.

DIS at high energy: Glauber-Mueller formula: Let us consider deep inelastic scattering (DIS) on a large nucleus. In DIS, the incoming electron emits a virtual photon, which in turn interacts with the proton or nucleus. In the rest frame of the nucleus, the interaction can be thought of as the virtual photon splitting into a quark-antiquark pair, which then interacts with the nucleus (see Fig. 5.6, left panel). Since the light cone lifetime of the $q \bar{q}$ pair is much longer than the size of the target nucleus, the total cross section for the virtual photon-nucleus scattering can be written as a convolution of the virtual photon's light cone wave function (the probability for it to split into a $q \bar{q}$ pair) with the forward scattering amplitude of a $q \bar{q}$ pair interacting with the nucleus

$$
\sigma_{t o t}^{\gamma * A}\left(Q^{2}, x_{B j}\right)=\int \frac{d^{2} x d z}{2 \pi}\left[\Phi_{T}(\underline{x}, z)+\Phi_{L}(\underline{x}, z)\right] d^{2} b N(\underline{x}, \underline{b}, Y)
$$

with the help of the light-cone perturbation theory [734. Here the incoming photon with virtuality $Q$ splits into a quark-antiquark pair with the transverse separation $\underline{x}$ and the impact parameter (transverse position of the center of mass of the $q \bar{q}$ pair) $\underline{b}$. $Y$ is the rapidity variable given by $Y=\ln \left(s x_{T}^{2}\right) \approx \ln 1 / x_{B j}$. The square of the light cone wave function of $q \bar{q}$ fluctuations of a virtual photon is denoted by $\Phi_{T}(\underline{x}, z)$ and $\Phi_{L}(\underline{x}, z)$ for transverse and longitudinal photons correspondingly, with $z$ being the fraction of the photon's longitudinal momentum carried by the quark. At the lowest order in electromagnetic coupling $\left(\alpha_{E M}\right)$ $\Phi_{T}(\underline{x}, z)$ and $\Phi_{L}(\underline{x}, z)$ are given by [735, 736]

$$
\begin{gathered}
\Phi_{T}(\underline{x}, z)=\frac{2 N_{c}}{\pi} \sum_{f} \alpha_{E M}^{f}\left\{a_{f}^{2} K_{1}^{2}\left(x_{\perp} a_{f}\right)\left[z^{2}+(1-z)^{2}\right]+m_{f}^{2} K_{0}\left(x_{\perp} a_{f}\right)^{2}\right\}, \\
\Phi_{L}(\underline{x}, z)=\frac{2 N_{c}}{\pi} \sum_{f} \alpha_{E M}^{f} 4 Q^{2} z^{2}(1-z)^{2} K_{0}^{2}\left(x_{\perp} a_{f}\right),
\end{gathered}
$$

with $a_{f}^{2}=Q^{2} z(1-z)+m_{f}^{2}, x_{\perp}=|\mathbf{x}|$ and $\sum_{f}$ denoting the sum over all relevant quark flavors with quark masses denoted by $m_{f} . \alpha_{E M}^{f}=e_{f}^{2} / 4 \pi$ with $e_{f}$ the electric charge of a quark with flavor $f$.

Our first goal is to calculate the forward scattering amplitude of a quark-anti-quark dipole interacting with the nucleus, which is denoted by $N(\underline{x}, \underline{b}, Y)$ in Eq. (5.5)), including all multiple re-scatterings of the dipole on the nucleons in the nucleus. To do this we need to construct a model of the target nucleus. We assume that the nucleons are dilutely distributed in the nucleus [737]. There we can represent the dipole-nucleus interaction as a sequence of successive dipole-nucleon interactions, as shown in Fig. 5.6, right panel. Since each nucleon is a color singlet, the lowest order dipole-nucleon interaction in the forward amplitude from Fig. 5.6 is a two-gluon exchange. The exchanged gluon lines in Fig. 5.6 are disconnected at the top: this denotes a summation over all possible connections of these gluon lines either to the quark or to the anti-quark lines in the incoming dipole. 

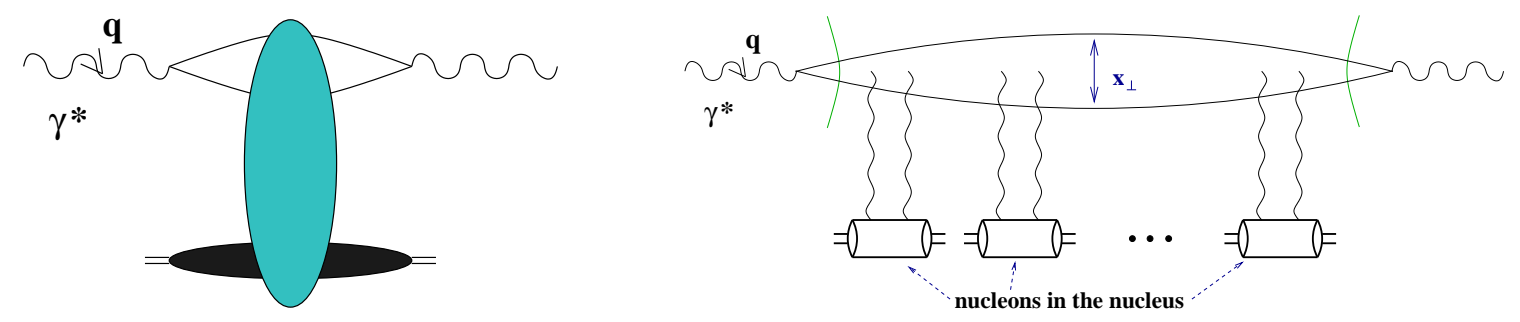

Figure 5.6. Left: Deep inelastic scattering in the rest frame of the target. Right: Deep inelastic scattering in the quasi-classical Glauber-Mueller approximation in $\partial_{\mu} A^{\mu}=0$ gauge.

Re-summation of the diagrams like the one in Fig. 5.6 yields [737]

$$
N(\underline{x}, \underline{b}, Y=0)=1-\exp \left\{-\frac{x_{\perp}^{2} Q_{s}^{2}(\underline{b}) \ln \left(1 / x_{\perp} \Lambda\right)}{4}\right\}
$$

with the saturation scale defined by

$$
Q_{s}^{2}(\underline{b}) \equiv \frac{4 \pi \alpha_{s}^{2} C_{F}}{N_{c}} \rho T(\underline{b}) .
$$

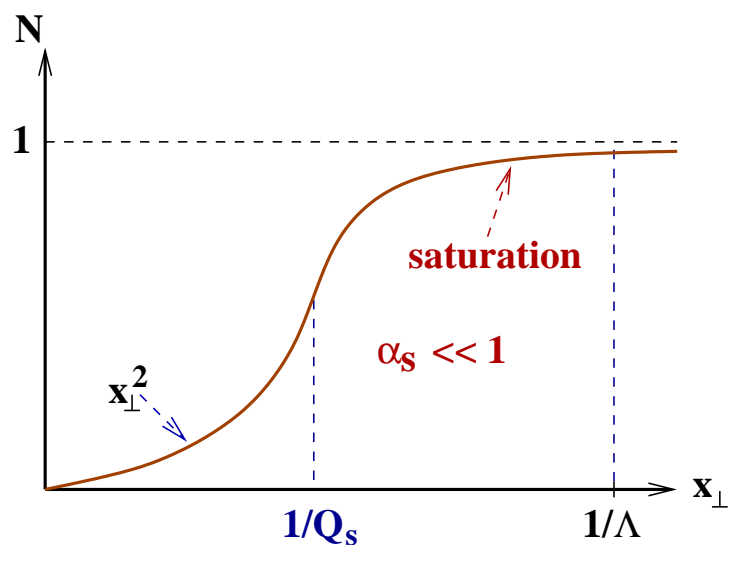

Figure 5.7. The forward amplitude of the dipolenucleus scattering $N$,plotted as a function of the transverse separation between the quark and the anti-quark in a dipole $\left(x_{\perp}\right)$ using Eq. (5.8). $\alpha_{s}^{2} A^{1 / 3}$. The physical meaning of the parameter $\alpha_{s}^{2} A^{1 / 3}$ is rather straightforward: at a given impact parameter the dipole interacts with $\sim A^{1 / 3}$ nucleons exchanging two gluons with each. Since the two-gluon exchange is parametrically of the order $\alpha_{s}^{2}$ we obtain $\alpha_{s}^{2} A^{1 / 3}$ as the re-summation parameter for the quasi-classical approximation.

The dipole amplitude $N$, from Eq. (5.8), is plotted (schematically) in Fig. 5.7 as a function of $x_{\perp}$. One can see that, at small $x_{\perp}, x_{\perp} \ll 1 / Q_{s}$, we have $N \sim x_{\perp}^{2}$ and the amplitude is a rising function of $x_{\perp}$. However, at large dipole sizes $x_{\perp} \gtrsim 1 / Q_{s}$, the growth stops and the amplitude levels off (saturates) at $N=1$. This regime corresponds to the black disk limit for the dipole-nucleus scattering where, for large dipoles, the nucleus appears 
as a black disk. To understand that the $N=1$ regime corresponds to the black disk limit, let us note that the total dipole-nucleus scattering cross section is given by:

$$
\sigma_{\text {tot }}^{q \bar{q} A}=2 \int d^{2} b N(\underline{x}, \underline{b}, Y)
$$

where the integration goes over the cross sectional area of the nucleus. If $N=1$ at all impact parameters $\underline{b}$ inside the nucleus, for a spherical nucleus of radius $R$, Eq. (5.10) becomes $\sigma_{\text {tot }}^{q \bar{q} A}=2 \pi R^{2}$, which is a well-known formula for the cross section of a particle scattering on a black sphere [739].

The transition between the $N \sim x_{\perp}^{2}$ to $N=1$ behavior in Fig. 5.7 happens at around $x_{\perp} \sim 1 / Q_{s}$. For dipole sizes $x_{\perp} \gtrsim 1 / Q_{s}$, the amplitude $N$ saturates to a constant. This translates into the saturation of quark distribution functions in the nucleus, as was shown in [737] (as $x q+x \bar{q} \sim F_{2} \sim \sigma_{\text {tot }}^{\gamma * A}$ ), and thus can be identified with parton saturation, justifying the name of the saturation scale.

Before we proceed, let us finally note that since $T(\underline{b}) \sim A^{1 / 3}$, the saturation scale in Eq. (5.9) scales as $Q_{s}^{2} \sim A^{1 / 3}$ with the nuclear atomic number [730, 731, 729, 737]. This implies that for a very large nucleus, the saturation scale would become very large, much larger than $\Lambda_{Q C D}$. If $Q_{s} \gg \Lambda_{Q C D}$, the transition to the black disk limit in Fig. 5.7 happens at momentum scales (corresponding to inverse dipole sizes) where the physics is perturbative and gluons are the correct degrees of freedom.

\section{Nonlinear evolution equations}

General picture: While the classical gluon fields of the MV model exhibit many correct qualitative features of saturation physics, and give predictions about the $A$-dependence of observables which may be compared to the data, they do not lead to any rapidity/Bjorken$x$ dependence of the corresponding observables, which is essential in the data on nuclear and hadronic collisions. To include rapidity dependence, one has to calculate quantum corrections to the classical fields described above.

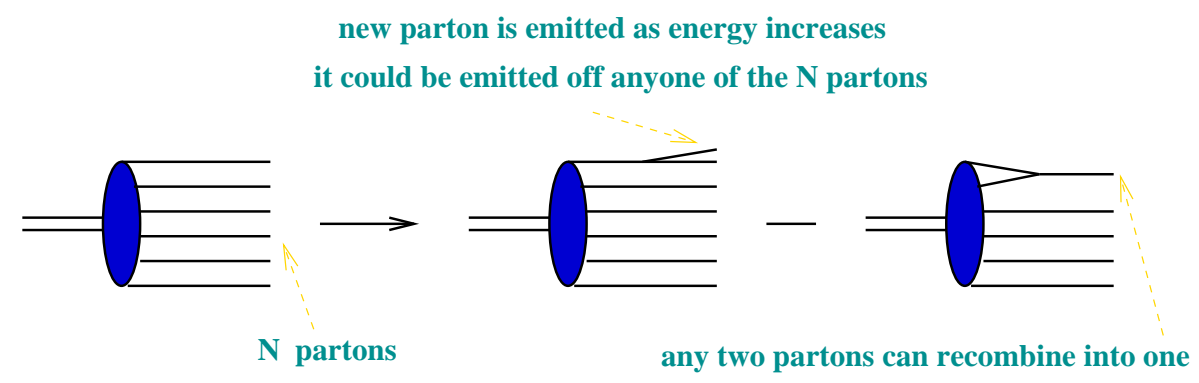

Figure 5.8. Nonlinear small- $x$ evolution of a hadronic or nuclear wave functions. All partons (quarks and gluons) are denoted by straight solid lines for simplicity.

The inclusion of quantum corrections is accomplished by the small- $x$ evolution equations. The first small- $x$ evolution equation was constructed before the birth of saturation physics. This is the Balitsky-Fadin-Kuraev-Lipatov (BFKL) evolution equation [60, 59]. This is a linear evolution equation, which is illustrated by the first term on the right hand side of Fig. 5.8. Consider a wave function of a high-energy nucleus or hadrons: it contains many partons, as shown on the left of Fig. 5.8. As we make one step of evolution by boosting the nucleus/hadron to higher energy, either one of the partons can split into two partons, 
leading to an increase in the number of partons proportional to the number of partons $N$ at the previous step,

$$
\frac{\partial N\left(x, k_{T}^{2}\right)}{\partial \ln (1 / x)}=\alpha_{s} K_{B F K L} \otimes N\left(x, k_{T}^{2}\right),
$$

with $K_{B F K L}$ an integral kernel. Clearly the BFKL equation (5.11) introduces a Bjorken$x /$ rapidity dependence in the observables it describes.

The main problem with the BFKL evolution is that it leads to the power-law growth of the total cross sections with energy, $\sigma_{t o t} \sim s^{\alpha_{P}-1}$, with the BFKL pomeron intercept $\alpha_{P}-1=\left(4 \alpha_{s} N_{c} \ln 2\right) / \pi>0$. Such a power-law cross section increase violates the Froissart bound, which states that the total hadronic cross section can not grow faster than $\ln ^{2} s$ at very high energies. Moreover, the power-law growth of cross sections with energy violate the black disk limit known from quantum mechanics: the high-energy total scattering cross section $\sigma_{\text {tot }}$ of a particle on a sphere of radius $R$ is bounded by $2 \pi R^{2}$ (note the factor of 2 which is due to quantum mechanics, this is not simply a hard sphere from classical mechanics!).

We see that something has to modify Eq. (5.11) at high energy. The modification is illustrated on the far right of Fig. 5.8, at very high energies, partons may start to recombine with each other on top of the splitting. The recombination of two partons into one is proportional to the number of pairs of partons, which, in turn, scales as $N^{2}$. We end up with the following non-linear evolution equation:

$$
\frac{\partial N\left(x, k_{T}^{2}\right)}{\partial \ln (1 / x)}=\alpha_{s} K_{B F K L} \otimes N\left(x, k_{T}^{2}\right)-\alpha_{s}\left[N\left(x, k_{T}^{2}\right)\right]^{2} .
$$

This is the Balitsky-Kovchegov (BK) evolution equation [740, 741], which is valid for QCD in the limit of large number of colors $N_{c}$. An equation of this type was originally suggested by Gribov, Levin and Ryskin [742 and by Mueller and Qiu [743, though at the time it was assumed that the quadratic term is only the first non-linear correction with higher order terms possibly appearing as well: in [740, 741] the exact form of the equation was found, and it was shown that in the large- $N_{c}$ limit, Eq. (5.12) does not have any higherorder terms in $N$. Generalization of Eq. (5.12) beyond the large- $N_{c}$ limit is accomplished by the Jalilian-Marian-Iancu-McLerran-Weigert-Leonidov-Kovner (JIMWLK) [744, 745] evolution equation, which is a functional differential equation. Both the BK and JIMWLK evolution equations will be discussed in more details later.

The physical impact of the quadratic term on the right of Eq. (5.12) is clear: it slows down the small- $x$ evolution, leading to parton saturation and to total cross sections adhering to the black disk limit. The effect of gluon mergers becomes important when the quadratic term in Eq. (5.12) becomes comparable to the linear term on the right-hand-side. This gives rise to the saturation scale $Q_{s}$, which now grows with energy (on top of its increase with $A$ ).

The Balitsky-Kovchegov equation: Let us now include the energy dependence in the dipole amplitude $N$ from Eq. (5.8). Similar to the BFKL evolution equation [59, 60, we are interested in quantum evolution in the leading longitudinal logarithmic approximation re-summing the powers of $\alpha_{s} \ln \frac{1}{x_{B j}} \sim \alpha_{s} Y$, with $Y$ the rapidity variable. Again we will be working in the rest frame of the nucleus, but this time we choose to work in the light cone gauge of the projectile $A^{+}=0$ if the dipole is moving in the light cone + direction.

Leading logs in $x$ corrections appear in the diagrams through emissions of long-lived $s$-channel gluons, as shown in Fig. [5.9. These $s$-channel gluons interact with the target 

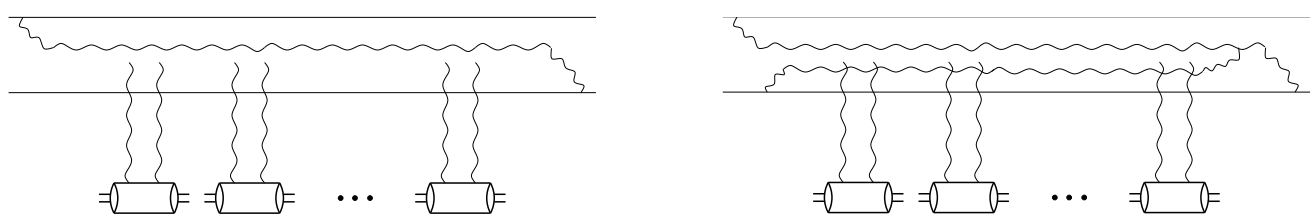

Figure 5.9. Quantum corrections to dipole-nucleus scattering.

nucleus through multiple re-scatterings. In the large- $N_{c}$ limit of QCD such diagrams can be re-summed by the BK evolution equation [740, 746, 741, 747]:

$$
\begin{array}{r}
\frac{\partial N\left(\underline{x}_{0}, \underline{x}_{1}, Y\right)}{\partial Y}=\frac{\alpha_{s} C_{F}}{\pi^{2}} \int d^{2} x_{2} \frac{x_{01}^{2}}{x_{20}^{2} x_{21}^{2}}\left[N\left(\underline{x}_{0}, \underline{x}_{2}, Y\right)+N\left(\underline{x}_{2}, \underline{x}_{1}, Y\right)-N\left(\underline{x}_{0}, \underline{x}_{1}, Y\right)\right. \\
\left.-N\left(\underline{x}_{0}, \underline{x}_{2}, Y\right) N\left(\underline{x}_{2}, \underline{x}_{1}, Y\right)\right],
\end{array}
$$

where we have redefined the arguments of $N$ to depend on the transverse coordinates of the quark and antiquark (instead of dipole size and the impact parameter as was done in Eq. (5.8)). Here $x_{i j}=\left|\underline{x}_{i j}\right|$ and $\underline{x}_{i j}=\underline{x}_{i}-\underline{x}_{j}$.

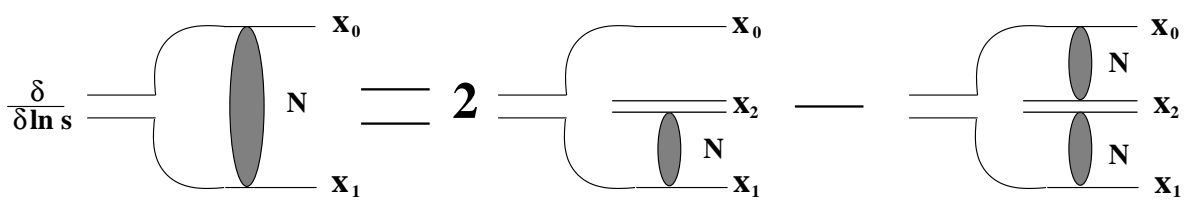

Figure 5.10. Diagrammatic representation of the nonlinear evolution equation (5.13).

In the large- $N_{c}$ limit, gluon cascades reduce to a cascade of color dipoles. Summation of the dipole cascade is illustrated in Fig. 5.10 where the dipole cascade and its interaction with the target are denoted by a shaded oval. In one step of the evolution in energy (or rapidity) a soft gluon is emitted in the dipole. If the gluon is real, than the original dipole would be split into two dipoles, as shown in Fig. 5.10. Either one of these dipoles can interact with the nucleus with the other one not interacting, which is shown by the first term on the right hand side of Fig. 5.10 with the factor of 2 accounting for the fact that there are two dipoles in the wave function now. Alternatively, both dipoles may interact simultaneously, which is shown by the second term on the right hand side of Fig. 5.10. This term comes in with the minus sign. The emitted gluon in one step of evolution may be a virtual correction, which is not shown in Fig. 5.10, in that case, the original dipole would not split into two, it would remain the same and would interact with the target. In the end, the evolved system of dipoles interacts with the nucleus. In the large- $N_{c}$ limit, each dipole does not interact with other dipoles during the evolution which generates all the dipoles. For a large nucleus, the dipole-nucleus interaction was given above in Eq. (5.8). That result re-sums powers of $\alpha_{s}^{2} A^{1 / 3}$ : hence the BK equation re-sums powers of $\alpha_{s} Y$ and powers of $\alpha_{s}^{2} A^{1 / 3}$.

\section{Map of high-energy QCD}

Solutions of the BK and JIMWLK evolution equations have been calculated numerically [748, 749, 750], with asymptotic limits studied analytically. The numerical solution 

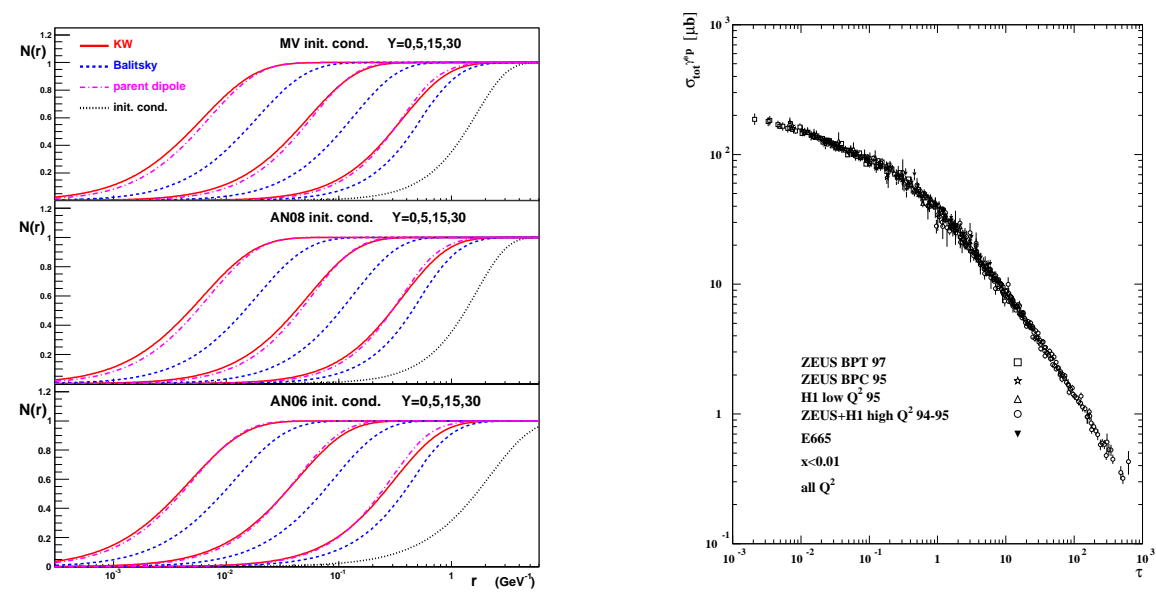

Figure 5.11. Left: Solutions of the BK equation at rapidities $\mathrm{Y}=0,5,15$ and 30 (curves are labeled from right to left) for the three different running coupling schemes considered in [751] Right: HERA data on the total DIS $\gamma^{*} p$ cross section plotted in 752 as a function of the scaling variable $\tau=$ $Q^{2} / Q_{s}^{2}\left(x_{B j}\right)$.

(for the BK equation with running coupling, which will be described later) is presented in Fig. 5.11 751. These plots are the same dipole amplitude $N$ plotted as a function of the dipole size labeled $r$ as was done in Fig. 5.7. In Fig. 5.11, different curves correspond to different energies/rapidities $Y$. One can clearly see that the curves tend to drift to the left with increasing energies, corresponding to increasing saturation scale with the energy/rapidity. Therefore we see that the saturation scale increases with rapidity, making the corresponding physics more perturbative.

We summarize our knowledge of high energy QCD in Fig. 5.12, in which different regimes are plotted in the $\left(Q^{2}, Y=\ln 1 / x\right)$ plane, by analogy with DIS. For hadronic and nuclear collisions one can think of typical transverse momentum $p_{T}^{2}$ of the produced particles instead of $Q^{2}$. Also rapidity $Y$ and Bjorken- $x$ variable are interchangeable. On the left of Fig. 5.12 we see the region with $Q^{2} \leq \Lambda_{Q C D}^{2}$ in which the coupling is large, $\alpha_{s} \sim 1$, and small-coupling approaches do not work. In the perturbative region, $Q^{2} \gg \Lambda_{Q C D}^{2}$, we see the standard DGLAP evolution and the linear BFKL evolution. The BFKL equation evolves gluon distributions toward small- $x$, where parton densities becomes large and parton saturation sets in. The transition to saturation is described by the non-linear BK and JIMWLK evolution equations. Most importantly, this transition happens at $Q_{s}^{2} \gg \Lambda_{Q C D}^{2}$ where the small-coupling approach is valid.

One of the most important predictions of nonlinear small- $x$ evolution is that, at high enough rapidity, the scattering amplitude $N$ (and, consequently, DIS structure functions) would be a function of a single variable $x_{\perp} Q_{s}(Y)$, such that $N\left(x_{\perp}, Y\right)=N\left(x_{\perp} Q_{s}(Y)\right)$. This prediction is spectacularly confirmed by HERA data. Geometric scaling has been demonstrated in an analysis of the HERA DIS data [752, presenting one of the strongest arguments for the observation of saturation phenomena at HERA. These results are shown here in Fig. 5.11 from [752, where the authors combined HERA data on the total DIS $\gamma^{*} p$ cross section $\sigma_{\text {tot }}^{\gamma^{*} p}$ for $x_{B j}<0.01$ as a function of the scaling variable $\tau=Q^{2} / Q_{s}^{2}\left(x_{B j}\right)$. One can see that, amazingly enough, all the data falls on the same curve, indicating that $\sigma_{\text {tot }}^{\gamma^{*} p}$ is a function of a single variable $Q^{2} / Q_{s}^{2}\left(x_{B j}\right)$ ! This gives us the best to date experimental proof of geometric scaling. (For a similar analysis of DIS data on nuclear targets see [753].) 


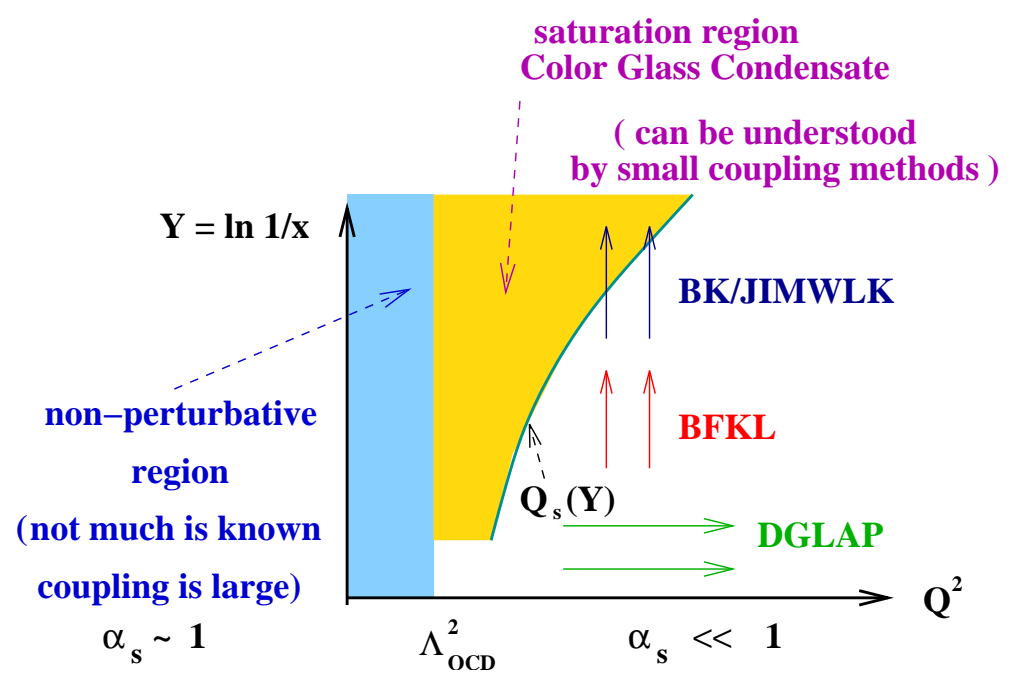

Figure 5.12. Map of high energy QCD in the $\left(Q^{2}, Y=\ln 1 / x\right)$ plane.

The fact that geometric scaling is a property of the solution of the BK equation was later demonstrated in [754, 755].

\section{Universality aspects of the Color Glass Condensate}

François Gelis

The Color Glass Condensate (CGC) is an effective field theory (EFT) based on the separation of the degrees of freedom into fast frozen color sources and slow dynamical color fields [729, 731, 730]. A renormalization group equation -the JIMWLK equation 756, 757, 758, 759, 760, 761, 745, 762, 763 - ensures the independence of physical quantities with respect to the cutoff that separates the two kinds of degrees of freedom.

The fast gluons with longitudinal momentum $k^{+}>\Lambda^{+}$are frozen by Lorentz time dilation in configurations specified by a color current $J_{a}^{\mu} \equiv \delta^{\mu+} \rho^{a}$, where $\rho^{a}\left(x^{-}, x_{\perp}\right)$ is the corresponding color charge density. On the other hand, slow gluons with $k^{+}<\Lambda^{+}$are described by the usual gauge fields $A^{\mu}$ of QCD. Because of the hierarchy in $k^{+}$between these two types of degrees of freedom, they are coupled eikonaly by a term $J_{\mu} A^{\mu}$. The fast gluons thus act as sources for the fields that represent the slow gluons. Although it is frozen for the duration of a given collision, the color source density $\rho^{a}$ varies randomly event by event. The CGC provides a gauge invariant distribution $W_{\Lambda^{+}}[\rho]$, which gives the probability of a configuration $\rho$. This encodes all the correlations of the color charge density at the cutoff scale $\Lambda^{+}$, separating the fast and slow degrees of freedom. Given this statistical distribution, the expectation value of an operator at the scale $\Lambda^{+}$is given by

$$
\langle\mathcal{O}\rangle_{\Lambda^{+}} \equiv \int[D \rho] W_{\Lambda^{+}}[\rho] \mathcal{O}[\rho]
$$

where $\mathcal{O}[\rho]$ is the expectation value of the operator for a particular configuration $\rho$ of the color sources.

The power counting of the CGC EFT is such that in the saturated regime, the sources $\rho$ are of order $g^{-1}$. Attaching an additional source to a given Feynman graph does not alter its order in $g$; the vertex where this new source attaches to the graph is compensated by the 
$g^{-1}$ of the source. Thus, computing an observable at a certain order in $g^{2}$ requires the resummation of all the contributions obtained by adding extra sources to the relevant graphs. The leading order in $g^{2}$ is given by a sum of tree diagrams, which can be expressed in terms of classical solutions of the Yang-Mills equations. Moreover, for inclusive observables [764, 765], these classical fields obey a simple boundary condition: they vanish when $t \rightarrow-\infty$.

Next-to-leading order (NLO) computations in the CGC EFT involve a sum of one-loop diagrams embedded in the above classical field. To prevent double counting, momenta in loops are required to be below the cutoff $\Lambda^{+}$. This leads to a logarithmic dependence in $\Lambda^{+}$of these loop corrections. These logarithms are large if $\Lambda^{+}$is well above the typical longitudinal momentum scale of the observable considered, and must be re-summed.

For inclusive observables, the leading logarithms are universal and can be absorbed into a redefinition of the distribution $W_{\Lambda^{+}}[\rho]$ of the hard sources. The evolution of $W_{\Lambda^{+}}[\rho]$ with $\Lambda^{+}$is governed by the functional JIMWLK equation

$$
\frac{\partial W_{\Lambda^{+}}[\rho]}{\partial \ln \left(\Lambda^{+}\right)}=-\mathcal{H}\left[\rho, \frac{\delta}{\delta \rho}\right] W_{\Lambda^{+}}[\rho]
$$

where $\mathcal{H}$ is known as the JIMWLK Hamiltonian. This operator contains up to two derivatives $\partial / \partial \rho$, and arbitrary powers in $\rho$. Its explicit expression can be found in refs. [756, 757, 758, 759, 760, 761, 745, 762, 763, 766, 767]. The derivation of the JIMWLK equation will be sketched below.

Numerical studies of JIMWLK evolution were performed in 750, 768. An analytic, albeit formal, solution to the JIMWLK equation was constructed in [769] in the form of a path integral. Alternatively, the evolution can can be expressed as an infinite hierarchy of coupled non-linear equations for $n$-point Wilson line correlators-often called the Balitsky hierarchy [770]. In this framework, the BK equation is a mean field approximation of the JIMWLK evolution, valid in the limit of a large number of colors $N_{c} \rightarrow \infty$. Numerical studies of the JIMWLK equation [750, 768] have found only small differences with the BK equation.

Let us finally comment on the initial condition for the JIMWLK equation which is also important in understanding its derivation. The evolution should start at some cutoff value in the longitudinal momentum scale $\Lambda_{0}^{+}$at which the saturation scale is already a (semi)hard scale, say $Q_{s 0} \gtrsim 1 \mathrm{GeV}$, for perturbation theory to be applicable. The gluon distribution at the starting scale is in general non-perturbative and requires a model. A physically motivated model for the gluon distribution in a large nucleus is the McLerran-Venugopalan model [729, 731, 730]. In a large nucleus, there is a window in rapidity where evolution effects are not large but $x$ is still sufficiently small for a probe not to resolve the longitudinal extent of the nucleus. In this case, the probe "sees" a large number of color charges, proportional to $A^{1 / 3}$. These charges add up to form a higher dimensional representation of the gauge group, and can therefore be treated as classical color distributions 729, 731, 730, 771, Further, the color charge distribution $W_{\Lambda_{0}^{+}}[\rho]$ is a Gaussian distribution 11 in $\rho$. The variance of this distribution -the color charge squared per unit area- is proportional to $A^{1 / 3}$ and provides a semi-hard scale that makes weak coupling computations feasible. In addition to its role in motivating the EFT and serving as the initial condition in JIMWLK evolution, the MV model allows for direct phenomenological studies in $\mathrm{p}+\mathrm{A}$ and $\mathrm{A}+\mathrm{A}$ collisions in

\footnotetext{
${ }^{1}$ There is a additional term, corresponding to the cubic Casimir; which is parametrically suppressed for large nuclei [772. This term generates Odderon excitations in the JIMWLK/BK evolution 773, 774].
} 
regimes where the values of $x$ are not so small as to require evolution.

The CGC in DIS at small $x$ : We denote $\sigma_{\text {dipole }}\left(x, \boldsymbol{r}_{\perp}\right)$ the QCD "dipole" cross-section for the quark-antiquark pair to scatter off the target. This process is shown in fig. 5.13 left, where we have assumed that the target moves in the $-z$ direction. In the leading order (LO) CGC description of DIS, the target is described, as illustrated in fig. 5.13 right, as static sources with $k^{-}>\Lambda_{0}^{-}$. The field modes do not contribute at this order.
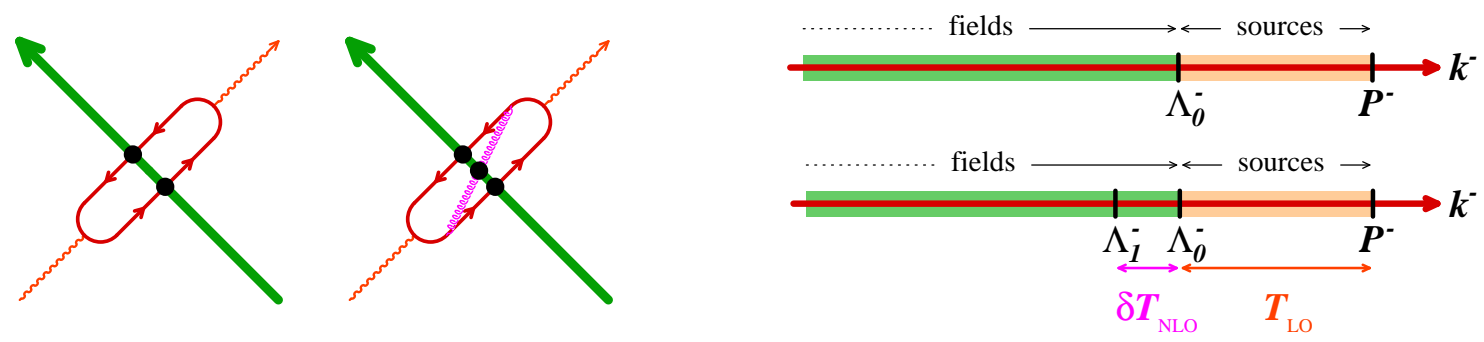

Figure 5.13. Left: LO and NLO contributions to DIS off the CGC. Top right: sources and fields in the CGC effective theory. Bottom right: NLO correction from a layer of field modes just below the cutoff.

Employing the optical theorem, $\sigma_{\text {dipole }}\left(x, \boldsymbol{r}_{\perp}\right)$ can be expressed in terms of the forward scattering amplitude $\boldsymbol{T}\left(\boldsymbol{x}_{\perp}, \boldsymbol{y}_{\perp}\right)$ of the $q \bar{q}$ pair at LO as

$$
\sigma_{\text {dipole }}^{\mathrm{LO}}\left(x, \boldsymbol{r}_{\perp}\right)=2 \int d^{2} \mathbf{b} \int[D \rho] W_{\Lambda_{0}^{-}}[\rho] \boldsymbol{T}_{\mathrm{LO}}\left(\boldsymbol{b}+\frac{\boldsymbol{r}_{\perp}}{2}, \boldsymbol{b}-\frac{\boldsymbol{r}_{\perp}}{2}\right),
$$

where, for a fixed configuration of the target color sources [775, 776]

$$
\boldsymbol{T}_{\mathrm{LO}}\left(\boldsymbol{x}_{\perp}, \boldsymbol{y}_{\perp}\right)=1-\frac{1}{N_{c}} \operatorname{tr}\left(U\left(\boldsymbol{x}_{\perp}\right) U^{\dagger}\left(\boldsymbol{y}_{\perp}\right)\right)
$$

with $U\left(\boldsymbol{x}_{\perp}\right)$ a Wilson line representing the interaction between a quark and the color fields of the target, defined to be

$$
U\left(\boldsymbol{x}_{\perp}\right)=\mathrm{T} \exp i g \int^{1 / x P^{-}} d z^{+} \mathcal{A}^{-}\left(z^{+}, \boldsymbol{x}_{\perp}\right) .
$$

In this formula, $\mathcal{A}^{-}$is the minus component of the gauge field generated (in Lorentz gauge) by the sources of the target; it is obtained by solving classical Yang-Mills equations with these sources. The upper bound $x P^{-}$(where $P^{-}$is the target longitudinal momentum) indicates that source modes with $k^{-}<x P^{-}$do not contribute to this scattering amplitude. Thus if the cutoff $\Lambda_{0}^{-}$of the CGC EFT is lower than $x P^{-}, \boldsymbol{T}_{\mathrm{LO}}$ is independent of $\Lambda_{0}^{-}$.

However, when $\Lambda_{0}^{-}$is larger than $x P^{-}$, the dipole cross-section is in fact independent of $x$ (since the CGC EFT does not have source modes near the upper bound $x P^{-}$) and depends on the unphysical parameter $\Lambda_{0}^{-}$. As we shall see now, this is related to the fact that eq. (5.16) is incomplete and receives large corrections from higher order diagrams. Consider now the NLO contributions (one of them is shown in the right panel in figure 5.13 left with gauge field modes in the slice $\Lambda_{1}^{-} \leq k^{-} \leq \Lambda_{0}^{-}$(see fig. 5.13 right). An explicit computation of the contribution of field modes in this slice gives

$$
\delta \boldsymbol{T}_{\mathrm{NLO}}\left(\boldsymbol{x}_{\perp}, \boldsymbol{y}_{\perp}\right)=\ln \left(\frac{\Lambda_{0}^{-}}{\Lambda_{1}^{-}}\right) \mathcal{H} \boldsymbol{T}_{\mathrm{LO}}\left(\boldsymbol{x}_{\perp}, \boldsymbol{y}_{\perp}\right)
$$


where $\mathcal{H}$ is the JIMWLK Hamiltonian. All dependence on the cutoff scales is in the logarithmic prefactor alone. This Hamiltonian has two derivatives with respect to the classical field $\mathcal{A} \sim \mathcal{O}(1 / g) ; \mathcal{H} \boldsymbol{T}_{\mathrm{LO}}$ is of order $\alpha_{s} \boldsymbol{T}_{\mathrm{LO}}$ and therefore clearly an NLO contribution. However, if the new scale $\Lambda_{1}^{-}$is such that $\alpha_{s} \ln \left(\Lambda_{0}^{-} / \Lambda_{1}^{-}\right) \sim 1$, this NLO term becomes comparable in magnitude to the LO contribution. Averaging the sum of the LO and NLO contributions over the distribution of sources at the scale $\Lambda_{0}^{-}$, one obtains

$$
\int[D \rho] W_{\Lambda_{0}^{-}}[\rho]\left(\boldsymbol{T}_{\mathrm{LO}}+\delta \boldsymbol{T}_{\mathrm{NLO}}\right)=\int[D \rho] W_{\Lambda_{1}^{-}}[\rho] \boldsymbol{T}_{\mathrm{LO}},
$$

where $W_{\Lambda_{1}^{-}} \equiv\left(1+\ln \left(\Lambda_{0}^{-} / \Lambda_{1}^{-}\right) \mathcal{H}\right) W_{\Lambda_{0}^{-}}$. We have shown here that the NLO correction from quantum modes in the slice $\Lambda_{1}^{-} \leq k^{-} \leq \Lambda_{0}^{-}$can be absorbed in the LO term, provided we now use a CGC effective theory at $\Lambda_{1}^{-}$with the modified distribution of sources shown in eq. (5.20). In differential form, the evolution equation of the source distribution is the JIMWLK equation stated previously.

Repeating this elementary step, one progressively re-sums quantum fluctuations down to the scale $k^{-} \sim x P^{-}$. Thanks to eq. (5.20), the result of this re-summation for the dipole cross-section is formally identical to eq. (5.16), except that the source distribution is $W_{x P^{-}}$ instead of $W_{\Lambda_{0}^{-}}$. Note that if one further lowers the cutoff below $x P^{-}$, the dipole crosssection remains unchanged.

The CGC in A+A collisions: Collisions between two nuclei ("dense-dense" scattering) are complicated to handle on the surface. However, in the CGC framework, because the wave functions of the two nuclei are saturated, the collision can be treated as the collision of classical fields coupled to fast partons of each nucleus respectively described by the external current $J^{\mu}=\delta^{\mu+} \rho_{1}+\delta^{\mu-} \rho_{2}$. The source densities of fast partons $\rho_{1,2}$ are both parametrically of order $1 / \mathrm{g}$, which implies that graphs involving multiple sources from both projectiles must be re-summed.

At leading order, inclusive observable 22 depends on the retarded classical color field $\mathcal{A}^{\mu}$, which solves the Yang-Mills equations $\left[\mathcal{D}_{\mu}, \mathcal{F}^{\mu \nu}\right]=J^{\nu}$ with the boundary condition $\lim _{x^{0} \rightarrow-\infty} \mathcal{A}^{\mu}=0$. Among the observables to which this result applies is the expectation value of the energy-momentum tensor at early times after the collision. At leading order,

$$
T_{\mathrm{LO}}^{\mu \nu}=\frac{1}{4} g^{\mu \nu} \mathcal{F}^{\lambda \sigma} \mathcal{F}_{\lambda \sigma}-\mathcal{F}^{\mu \lambda} \mathcal{F}_{\lambda}^{\nu}
$$

where $\mathcal{F}^{\mu \nu}$ is the field strength of the classical field $\mathcal{A}^{\mu}$.

Although $\mathrm{A}+\mathrm{A}$ collisions are more complicated than $\mathrm{e}+\mathrm{A}$ or $\mathrm{p}+\mathrm{A}$ collisions, one can still factorize the leading higher order corrections into the evolved distributions $W_{\Lambda^{-}}\left[\rho_{1}\right]$ and $W_{\Lambda^{+}}\left[\rho_{2}\right]$. At the heart of this factorization is a generalization of eq. (5.19) to the case where the two projectiles are described in the CGC framework [777, 778, 779]. When one integrates out the field modes in the slices $\Lambda_{1}^{ \pm} \leq k^{ \pm} \leq \Lambda_{0}^{ \pm}$, the correction to the energy momentum tensor is

$$
\delta T_{\mathrm{NLO}}^{\mu \nu}=\left[\ln \left(\frac{\Lambda_{0}^{-}}{\Lambda_{1}^{-}}\right) \mathcal{H}_{1}+\ln \left(\frac{\Lambda_{0}^{+}}{\Lambda_{1}^{+}}\right) \mathcal{H}_{2}\right] T_{\mathrm{LO}}^{\mu \nu},
$$

where $\mathcal{H}_{1,2}$ are the JIMWLK Hamiltonians of the two nuclei respectively. What is crucial here is the absence of mixing between the coefficients $\mathcal{H}_{1,2}$ of the logarithms of the two

\footnotetext{
${ }^{2}$ Exclusive observables may also be expressed in terms of solutions of the same Yang-Mills equations, but with more complicated boundary conditions than for inclusive observables.
} 
projectiles; they depend only on $\rho_{1,2}$ respectively and not on the sources of the other projectile. Although the proof of this expression is somewhat involved, the absence of mixing is deeply rooted in causality. The central point is that because the duration of the collision (which scales as the inverse of the energy) is so brief, soft radiation must occur before the two nuclei are in causal contact. Thus logarithms associated with this radiation must have coefficients that do not mix the sources of the two projectiles.

Following the same procedure for eq. (5.22), as for the $\mathrm{e}+\mathrm{A}$ and $\mathrm{p}+\mathrm{A}$ cases, one obtains for the energy-momentum tensor in an $\mathrm{A}+\mathrm{A}$ collision the expression

$$
\left\langle T^{\mu \nu}\right\rangle_{\mathrm{LLog}}=\int\left[D \rho_{1} D \rho_{2}\right] W_{1}\left[\rho_{1}\right] W_{2}\left[\rho_{2}\right] T_{\mathrm{LO}}^{\mu \nu} .
$$

This result can be generalized to multi-point correlations of the energy-momentum tensor,

$$
\left\langle T^{\mu_{1} \nu_{1}}\left(x_{1}\right) \cdots T^{\mu_{n} \nu_{n}}\left(x_{n}\right)\right\rangle_{\mathrm{LLog}}=\int\left[D \rho_{1} D \rho_{2}\right] W_{1}\left[\rho_{1}\right] W_{2}\left[\rho_{2}\right] T_{\mathrm{LO}}^{\mu_{1} \nu_{1}}\left(x_{1}\right) \cdots T_{\mathrm{LO}}^{\mu_{n} \nu_{n}}\left(x_{n}\right) .
$$

In this expression, all the correlations between the energy-momentum tensor at different points are from the distributions $W_{1,2}\left[\rho_{1,2}\right]$. Thus, the leading correlations are already built into the wavefunctions of the projectiles prior to the collision.

Note that the expressions in eqs. (5.23) and (5.24) are valid for proper times $\tau \sim 1 / Q_{s}$ after the heavy ion collision. Complicated final state effects, possibly driven by instabilities, are expected to bring this non-equilibrium gluonic matter into a quark-gluon plasma. Although this aspect of $\mathrm{A}+\mathrm{A}$ collisions is very different from what happens in DIS reactions, the Color Glass Condensate provides a universal description of the hadronic and nuclear wavefunctions prior to the collision in both cases, and a powerful framework to show that the logarithms of the collision energy are universal for inclusive enough observables. Thanks to this universality, measurements at small $x$ in e+A collisions can provide valuable constraints on the distributions $W[\rho]$ for a nucleus, that can then be used in order to compute the state of the system formed at early times in $\mathrm{A}+\mathrm{A}$ collisions.

\section{Shadowing}

\section{Boris Z. Kopeliovich}

In terms of the dipole formalism, nuclear shadowing is related to the interaction of different Fock components of the projectile particle with the nuclear target. The lowest Fock states (i.e. $\gamma^{*} \rightarrow \bar{q} q$ ) are responsible for higher twist shadowing, while higher Fock components (i.e. $\gamma^{*} \rightarrow \bar{q} q g$ ) give rise to leading twist gluon shadowing.

Quark shadowing: The magnitude of higher twist shadowing is controlled by the interplay between two fundamental quantities.

(i) The lifetime of photon fluctuations, or coherence time.

$$
l_{c}=\frac{2 \nu}{Q^{2}+M^{2}}=\frac{P}{x_{B j} m_{N}}=P l_{c}^{\max },
$$

where $x_{B j}=Q^{2} / 2 m_{N} \nu, M$ is the effective mass of the fluctuation, $P=\left(1+M^{2} / Q^{2}\right)^{-1}$, and $l_{c}^{\max }=1 / m_{N} x_{B j}$. The usual approximation is to assume that $M^{2} \approx Q^{2}$ since $Q^{2}$ is the only large dimensional scale available. In this case, $P=1 / 2$ and the corresponding value of $l_{c}$ is called Ioffe length of time. 
Shadowing is possible only if the coherence time exceeds the mean nucleon spacing in nuclei, and shadowing saturates (for a given Fock component) if the coherence time substantially exceeds the nuclear radius.

(ii) Equally important for shadowing is the transverse separation of the $\bar{q} q$. This controls the dipole-nucleon cross section $\sigma_{\bar{q} q}^{N}(r)$, and correspondingly the total nuclear cross section [780, 781],

$$
\left(\sigma_{\text {tot }}^{\gamma^{*} A}\right)_{l_{c} \gg R_{A}}^{T, L}=2 \int d \alpha \int d^{2} r\left|\Psi_{\bar{q} q}^{T, L}(\varepsilon r)\right|^{2} \int d^{2} b\left[1-\exp \left(-\frac{1}{2} \sigma_{q \bar{q}}^{N}(r) T_{A}(b)\right)\right]
$$

where the perturbative light-cone distribution function for the $\bar{q} q$ has the form [782, 783],

$$
\Psi_{\bar{q} q}^{T, L}\left(\vec{r}_{T}, \alpha\right)=\frac{\sqrt{\alpha_{e m}}}{2 \pi} \bar{\chi} \widehat{O}^{T, L} \chi K_{0}\left(\epsilon r_{T}\right)
$$

$\chi$ and $\bar{\chi}$ are the spinors of the quark and antiquark respectively; $K_{0}\left(\epsilon r_{T}\right)$ is the modified Bessel function; $\epsilon^{2}=\alpha(1-\alpha) Q^{2}+m_{q}^{2}$; and the operators $\widehat{O}^{T, L}$ for transversely and longitudinally polarized photons have the form,

$$
\begin{gathered}
\widehat{O}^{T}=m_{q} \vec{\sigma} \cdot \vec{e}+i(1-2 \alpha)(\vec{\sigma} \cdot \vec{n})\left(\vec{e} \cdot \vec{\nabla}_{r}\right)+(\vec{\sigma} \times \vec{e}) \cdot \vec{\nabla}_{r}, \\
\widehat{O}^{L}=2 Q \alpha(1-\alpha) \vec{\sigma} \cdot \vec{n} .
\end{gathered}
$$

Here $\vec{n}=\vec{p} / p$ is a unit vector parallel to the photon momentum; $\vec{e}$ is the polarization vector of the photon; $m_{q}$ and and $\alpha$ are the mass, and fractional light-cone momentum carried by the quark. See also eqs. (5.6) and (5.7) discussed previously.

In order to be shadowed, a $\bar{q} q$-fluctuation of the photon has to interact with a large cross section. As a result of color transparency [780, 784, small size dipoles with $r^{2} \sim$ $1 / Q^{2}$ interact only weakly and are therefore less shadowed. The dominant contribution to shadowing comes from the aligned jet configurations $(\alpha \rightarrow 0,1)$ [785] of $\bar{q} q$ pairs, which have large transverse separation, $\left\langle r^{2}\right\rangle \sim 1 /\left[Q^{2} \alpha(1-\alpha)\right]$ according to (5.27). Although the weight of such configurations is small, $1 / Q^{2}$, this is compensated by the large interaction cross section [786.

The coherence length (Eq. (5.25) ) averaged over interacting $|\bar{q} q\rangle$ and $|\bar{q} q g\rangle$ fluctuations calculated in [787] is presented in Fig. 5.14. The mean values of the factor $P=l_{c} / l_{c}^{\max }$ in (5.25) are plotted for $\bar{q} q$ fluctuations of transverse and longitudinal photons, as well as for $\bar{q} q g$ fluctuations as a function of $Q^{2}$ at fixed $x_{B j}$ (left panel). We see that $\bar{q} q$ fluctuations in a longitudinal photon live about twice as long as in a transverse one. Both are different from $P=1 / 2$ corresponding to the Ioffe time. The lifetime of the higher order Fock states containing gluons is about order of magnitude shorter.

Onset of shadowing: Eq. (5.26) describing quark shadowing is valid only in the limit of $l_{c} \gg R_{A}$, i.e. at very small $x_{B j}$ where the magnitude of shadowing nearly saturates. However, all available data for DIS on nuclei are in the region of shorter coherence length, and one needs theoretical tools to describe the onset of shadowing.

The Gribov theory of inelastic shadowing [791] relates nuclear shadowing to the cross section of diffractive dissociation. In the case of a deuteron target, this approach provides a full and model independent description of shadowing. The onset of shadowing can be accurately calculated, since the phase shift $\Delta z / l_{c}$ between the impulse approximation term and the inelastic shadowing term is under control. However, a description of shadowing 

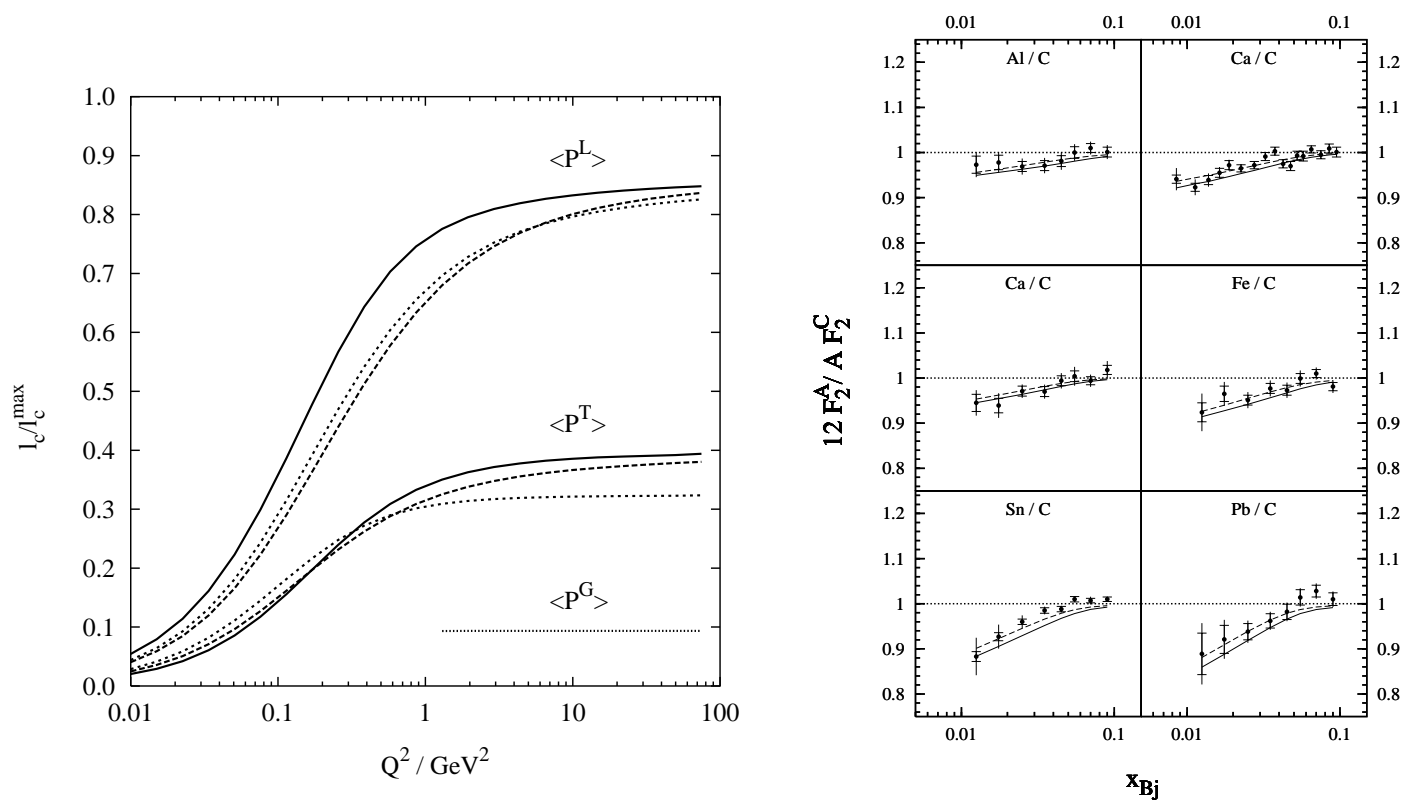

Figure 5.14. Left panel: Factor $\left\langle P^{T, L}\right\rangle$ and $\left\langle P^{g}\right\rangle$ defined in (5.25) for $\bar{q} q$ fluctuations of transverse and longitudinal photons, and for $\bar{q} q g$ fluctuations, from the top to bottom. Calculations are done as a function of $Q^{2}$ at $x_{B j}=0.01$. Dotted curves correspond to perturbative wave functions and an approximate dipole cross section $\propto r_{T}^{2}$. Dashed curves rely on the realistic parameterization for the dipole cross section [788. The solid curves show the most realistic case based on the nonperturbative wave functions. Right panel: Comparison between calculations for quark shadowing and experimental data from NMC [789, 790, for the structure functions of different nuclei relative to carbon as function of $x_{B j}$. The $Q^{2}$ range covered by the data is approximately $3 \mathrm{GeV}^{2} \leq Q^{2} \leq 17 \mathrm{GeV}^{2}$ from the lowest to the highest $x_{B j}$ bin. Solid and dashed curves are calculated with and without the real part of the light-cone potential in (5.31).

for heavy nuclei is a challenge in this approach. Indeed, only the lowest order of Gribov corrections can be calculated using data on diffraction. The higher order corrections, illustrated in Fig. 5.15 a , need information unavailable from data, like the diffractive amplitudes between different excited states, $X^{*}, X^{* *}$, the attenuation of these states in the nuclear medium, etc.
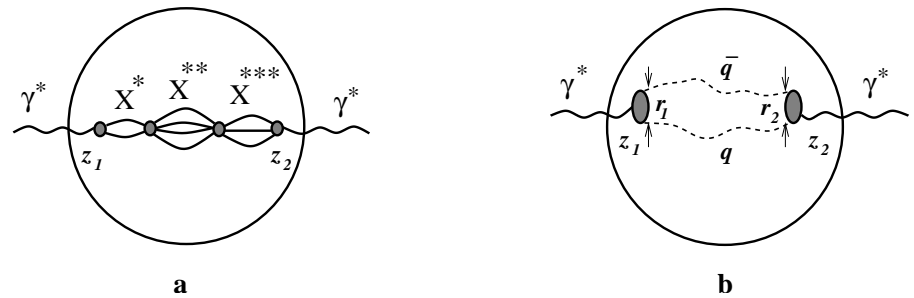

Figure 5.15. a: A high order term in Gribov inelastic shadowing corrections to $F_{2}^{A}\left(x, Q^{2}\right)$; b: Dipole description based on the path integral technique, which sums up the Gribov corrections in all orders.

An alternative description with the path integral technique was proposed in [787]. One should sum up over all possible trajectories of the quark and antiquark propagating through the nucleus, as is illustrated in Fig. $5.15 \mathrm{~b}$. This leads to the 2-dimensional Schrödinger equation for the Green function describing propagation of a dipole with initial (final) transverse 
separation $\vec{r}_{1}\left(\vec{r}_{2}\right)$ at longitudinal coordinate $z_{1}\left(z_{2}\right)$,

$$
\left[i \frac{\partial}{\partial z_{2}}+\frac{\Delta_{\perp}\left(r_{2}\right)-\varepsilon^{2}}{2 \nu \alpha(1-\alpha)}+\frac{i}{2} \rho_{A}\left(b, z_{2}\right) \sigma_{q \bar{q}}^{N}\left(r_{2}\right)-\frac{a^{4}(\alpha) r_{2}^{2}}{2 \nu \alpha(1-\alpha)}\right] G\left(r_{2}, z_{2} \mid r_{1}, z_{1}\right)=0
$$

The last two terms represent the imaginary and real parts of the light cone potential. The former describes the attenuation of the dipole in the nuclear medium, while the latter models the non-perturbative interactions inside the dipole. Solving this equation, one can calculate the shadowing corrections as

$$
\begin{gathered}
\left(\sigma_{\text {tot }}^{\gamma^{*} A}\right)^{T, L}=A\left(\sigma_{\text {tot }}^{\gamma^{*} N}\right)^{T, L}-\frac{1}{2} \operatorname{Re} \int d^{2} b \int_{0}^{1} d \alpha \int_{-\infty}^{\infty} d z_{1} \int_{z_{1}}^{\infty} d z_{2} \int d^{2} r_{1} \int d^{2} r_{2} \\
\times \quad\left[\Psi_{\bar{q} q}^{T, L}\left(\varepsilon, \lambda, r_{2}\right)\right]^{*} \rho_{A}\left(b, z_{2}\right) \sigma_{q \bar{q}}^{N}\left(s, r_{2}\right) G\left(r_{2}, z_{2} \mid r_{1}, z_{1}\right) \rho_{A}\left(b, z_{1}\right) \sigma_{q \bar{q}}^{N}\left(s, r_{1}\right) \Psi_{\bar{q} q}^{T, L}\left(\varepsilon, \lambda, r_{1}\right)
\end{gathered}
$$

At $l_{c} \ll 1 / \rho \sigma$, the second term vanishes. For $l_{c} \gg R_{A}$, it saturates at the value given by Eq. (5.26). The numerical results are compared with data from the NMC experiment [789, 790] in the right panel of Fig. 5.14. The solid and dashed curves are calculated with and without the real part of the light-cone potential in (5.31). It worth emphasizing that this is a parameter-free calculation, no adjustment to nuclear data has been done. The dipole cross section was fitted to DIS data on a proton.

Note that these calculations were performed for the lowest Fock component $|\bar{q} q\rangle$ of the photon; they miss gluon shadowing related to the higher Fock states containing gluons.

Gluon shadowing: Gluon shadowing is related to specific channels of diffractive gluon radiation. In terms of Regge phenomenology, these processes correspond to the triplePomeron contribution, and can be seen in data as the large mass tail of the invariant mass distribution, $d \sigma_{d i f f} / d M_{X}^{2} \propto 1 / M_{X}^{2}$. Such an $M_{X}^{2}$-dependence is the undebatable evidence of radiation of a vector particle, i.e. a gluon.

Data show that the magnitude of diffractive gluon radiation is amazingly small. The way to see that is to express the single diffraction cross section in terms of the Pomeronproton cross section as is illustrated in the left panel of Fig. 5.16. The Pomeron can be
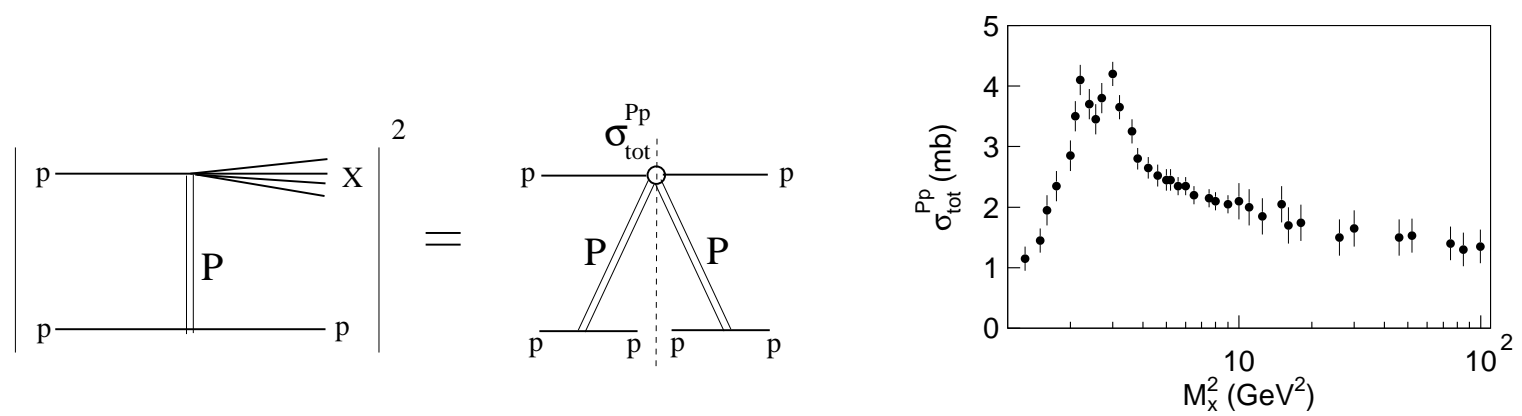

Figure 5.16. Left panel: The amplitude squared of diffractive excitation of the projectile proton, summed over all the excitations with invariant mass $M_{X}$, is related via the optical theorem with the total Pomeron-proton cross section at c.m. energy $M_{X}$. Right panel: The Pomeron-proton cross section extracted 792 from data on single diffraction $p p \rightarrow p X$ as function of $\mathbb{P}-p$ c.m. squared.

treated as a gluonic dipole and its cross section is expected to be about twice as big as for a $\bar{q} q$ dipole, i.e. $\sigma_{\text {tot }}^{\mathbb{P} p} \sim 50 \mathrm{mb}$. However, data depicted in the right panel of Fig. [5.16] show 
that $\sigma_{\text {tot }}^{\mathbb{P p} p}<2 \mathrm{mb}$. Such a dramatic disagreement gives a clue that diffractive gluon radiation is strongly suppressed compared with the expectation based on pQCD. This problem has been known in the Regge phenomenology as smallness of the triple-Pomeron coupling [793].

Gluon radiation can be described within the dipole approach via the propagation of a $\bar{q} q g$ dipole through the nuclear medium [794]. As the mean fractional momentum of the radiated gluon is very small, $\left\langle\alpha_{g}\right\rangle \sim 1 / \ln (s)$, one can rely on eq. (5.26) for very small $x_{B j}$, or eqs. (5.30)- 5.31) for the onset of gluon shadowing, by replacing $\sigma_{\bar{q} q}(r) \Rightarrow \sigma_{g g}(r)$. The only way to explain the observed suppression of gluon radiation is to reduce the mean size of the glue-glue dipole. This can be achieved by introducing a specifically strong nonperturbative interaction within the glue-glue dipole, which comes as the real part of the light-cone potential in eq. (5.30). Adjusting the strength of this interaction to data on diffractive gluon radiation (triple-Pomeron term) one arrives at the light-cone distribution functions in (5.26) and (5.31) with the mean glue-glue separation $r_{0} \approx 0.3 \mathrm{fm}$ [795]. This distance is smaller than the confinement radius $\sim 1 / \Lambda_{Q C D}=1 \mathrm{fm}$ and is in accord with the lattice evaluations of the $g g$ correlation radius [796], and the instanton radius [797]. There is more experimental evidence supporting the existence of a semi-hard scale in hadrons [798].

Thus, the magnitude of gluon shadowing evaluated in [795, 799] is expected to be rather small, as is depicted in Fig. 5.17. The nuclear ratio $R_{g}=G_{A}\left(x, Q^{2}\right) / A G_{N}\left(x, Q^{2}\right)$ is plotted as a function of $x_{B j}$ at $Q^{2}=4$ and $40 \mathrm{GeV}^{2}$ (left panel); and as a function of the path length in nuclear matter at $Q^{2}=4 \mathrm{GeV}^{2}$ and different values of $x_{B j}$.
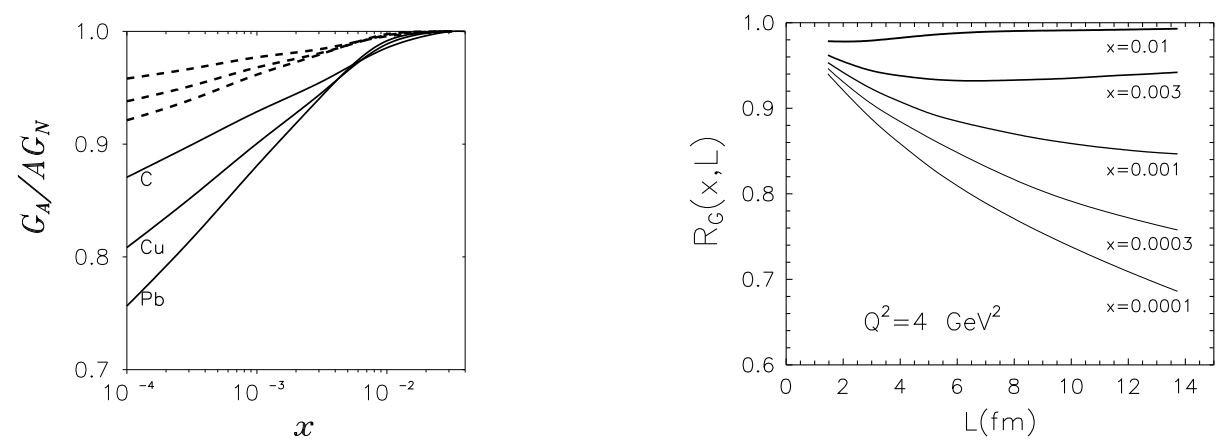

Figure 5.17. Left panel: Ratio of the gluon distribution functions in nuclei (carbon, copper and lead) and nucleons versus Bjorken $x$ at $Q^{2}=4 \mathrm{GeV}^{2}$ (solid curves) and $40 \mathrm{GeV}^{2}$ (dashed curves) [795. Right panel: Nuclear ratio $R_{g}=G_{A}\left(x, Q^{2}\right) / A G_{N}\left(x, Q^{2}\right)$ for gluons as function of path length in nuclear matter, calculated in [799] at $Q^{2}=4 G e V^{2}$ for several fixed values of $x$.

The path-integral approach is the most accurate method, which is valid in all regimes of gluon radiation, from incoherent to fully coherent. Nevertheless, this is still the lowest order calculation, which might be a reasonable approximation only for light nuclei, or for the onset of shadowing. The contribution of higher Fock components is still a challenge. This problem has been solved so far only in the unrealistic limit of long coherence lengths for all radiated gluons, described by the Balitsky-Kovchegov (BK) equation [740, 741]. A numerical solution of this equation is quite complicated and includes lots of modelling [800]. A much simpler bootstrap equation, which only requires modelling the shape of the saturated gluon distribution, was derived in [801]. It includes the self-quenching effect for gluon shadowing, and leads to a gluon distribution in nuclei which satisfies the unitarity bound [802] The results are quite similar to the numerical solutions of the BK equation [800]. The magnitude of the self-quenched gluon shadowing found in [801] is similar to the above results obtained in the leading order. 


\title{
Leading-twist nuclear shadowing
}

\author{
Vadim Guzey and Mark Strikman
}

Nuclear shadowing in hadron (photon)-nucleus scattering is the firmly established experimental phenomenon that at high energies the scattering cross section on a nuclear target is smaller than the sum of the scattering cross sections on the individual nucleons. In the nucleus rest frame, the theory of nuclear shadowing is based on the connection between nuclear shadowing and diffraction which has been established long time ago by Gribov [791]. In the derivation, the key assumption is that nuclei can be described as dilute systems of nucleons. The accuracy of the resulting theory for hadron-nucleus cross sections is very high, with the corrections at the level of a few \% which reflect the small admixture of nonnucleonic degrees of freedom in nuclei and the small off-shellness of the nucleons in nuclei as compared to the soft strong interaction scale. Gribov's result can be understood [803] as a manifestation of unitarity as reflected in the Abramovsky-Gribov-Kancheli (AGK) cutting rules 804 .

The connection between shadowing and diffraction is also valid in deep inelastic scattering (DIS) with nuclei; the approach based on this connection is called the leading twist theory of nuclear shadowing [803, 805, 806, 807]. In this theory, parton distribution functions (PDFs) in nuclei at small $x$ are calculated combining the unitarity relations for different cuts of the shadowing diagrams corresponding to the diffractive and inelastic final states (AGK cutting rules) with the QCD factorization theorem for hard diffraction [808] (which provides a good description of the totality of the HERA hard diffractive data). The resulting multiple scattering series for the quark nuclear PDFs is presented in fig. 5.18, where graphs $a, b$, and $c$ correspond to the interaction with one, two, and three nucleons of the target, respectively. Graph $a$ gives the impulse approximation; graphs $b$ and $c$ contribute to the shadowing correction. The interaction with $N>3$ nucleons, though not shown, is taken into account in the final expression for nuclear PDFs.

At the level of the interaction with two nucleons of a nucleus with the atomic mass number $A$, one can derive the model-independent expression for the shadowing correction to the nuclear PDF of flavor $j$ [803] (corresponding to graph $b$ of fig. [5.18):

$$
\begin{aligned}
x f_{j / A}^{(b)}\left(x, Q^{2}\right) & =-8 \pi A(A-1) \Re e \frac{(1-i \eta)^{2}}{1+\eta^{2}} \int_{x}^{0.1} d x_{\mathbb{P}} \beta f_{j}^{D(4)}\left(\beta, Q^{2}, x_{\mathbb{P}}, t_{\mathrm{min}}\right) \\
& \times \int d^{2} \vec{b} \int_{-\infty}^{\infty} d z_{1} \int_{z_{1}}^{\infty} d z_{2} \rho_{A}\left(\vec{b}, z_{1}\right) \rho_{A}\left(\vec{b}, z_{2}\right) e^{i\left(z_{1}-z_{2}\right) x_{\mathbb{P}} m_{N}},
\end{aligned}
$$

where $f_{j}^{D(4)}$ is the diffractive parton distribution of the nucleon; $\rho_{A}$ is the nuclear matter density; $\eta$ is the ratio of the real to imaginary parts of the elementary diffractive amplitude, $\eta=\Re e A^{\text {diff }} / \Im m A^{\text {diff }} \approx 0.17$. The diffractive $\mathrm{PDF} f_{j}^{D(4)}$ depends on two light-cone fractions $x_{\mathbb{P}}=\left(M_{X}^{2}+Q^{2}\right) /\left(W^{2}+Q^{2}\right)$ and $\beta=x / x_{\mathbb{P}}$ and the invariant momentum transfer $t$, where $W$ is the invariant virtual photon-nucleon energy, $W^{2}=(q+p)^{2}$, and $M_{X}^{2}$ is the invariant mass squared of the produced intermediate diffractive state denoted as " $\mathrm{X}$ " in fig. 5.18. The longitudinal (collinear with the direction of the photon momentum) coordinates $z_{1}$ and $z_{2}$ and the transverse coordinate (impact parameter) $\vec{b}$ refer to the two interacting nucleons; $m_{N}$ is the nucleon mass. The $t$ dependence of $f_{j}^{D(4)}$ can be safely neglected as compared to the strong fall-off of the nuclear form-factor for $A>4$ and, as a result, $f_{j}^{D(4)}$ enters eq. (5.32) at $t_{\min } \approx-x^{2} m_{N}^{2}\left(1+M_{X}^{2} / Q^{2}\right)^{2}$ and all nucleons enter with the same impact 


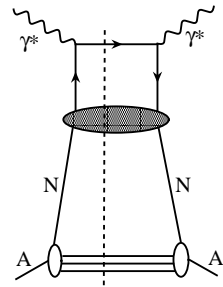

a)

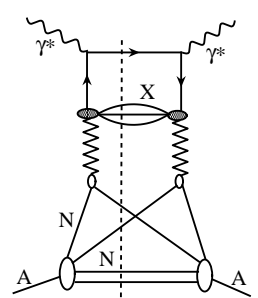

b)

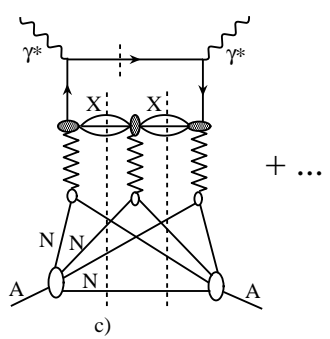

Figure 5.18. Multiple scattering series for nuclear quark PDFs. Graphs $a, b$, and $c$ correspond to the interaction with one, two, and three nucleons, respectively. Graph $a$ gives the impulse approximation; graphs $b$ and $c$ contribute to the shadowing correction.

parameter $\vec{b}$. Equation (5.32) satisfies the QCD evolution equations at all orders in the strong coupling constant $\alpha_{s}$.

To evaluate the contribution to nuclear shadowing of the interactions with $N \geq 3$ nucleons in fig. 5.18, one needs to invoke additional model-dependent considerations, since the interaction of a hard probe (virtual photon) with $N \geq 3$ nucleons is sensitive to fine details of the diffractive dynamics. In particular, the hard probe can be viewed as a coherent superposition of configurations which interact with the target nucleons with very different strengths. This effect of color (cross section) fluctuations is analogous to the inelastic shadowing in hadron-nucleus scattering with the important difference that the dispersion of the interaction strengths is much smaller in the hadron case than in DIS. However, the observation that $\alpha_{\mathbb{P}}(0)=1.11$ found in the analysis of hard diffraction at HERA [809] is very close to $\alpha_{\mathbb{P}}^{\text {soft }}(0)=1.08$ in soft hadronic interactions [810] indicates that hard diffraction in DIS is dominated by large-size hadron-like (aligned jet) configurations which evolve to large $Q^{2}$ via the DGLAP evolution. (As to the point-like configurations, they give an important and increasing with $Q^{2}$ contribution to graph $a$ in fig. 5.18.)

This important observation reduces theoretical uncertainties in the treatment of the interactions with $N \geq 3$ nucleons and allows one to reliably parameterize the strength of the interaction with $N \geq 3$ nucleons by a single effective hadron-like cross section $\sigma_{\text {soft }}^{j}$. The final expression for the nuclear PDFs at a certain initial scale $Q_{0}^{2}$ reads [806, 807]:

$$
\begin{aligned}
x f_{j / A}\left(x, Q_{0}^{2}\right) & =A x f_{j / N}\left(x, Q_{0}^{2}\right) \\
& -8 \pi A(A-1) \Re e \frac{(1-i \eta)^{2}}{1+\eta^{2}} \int_{x}^{0.1} d x_{\mathbb{P}} \beta f_{j}^{D(4)}\left(\beta, Q_{0}^{2}, x_{\mathbb{P}}, t_{\min }\right) \int d^{2} b \int_{-\infty}^{\infty} d z_{1} \\
& \int_{z_{1}}^{\infty} d z_{2} \rho_{A}\left(\vec{b}, z_{1}\right) \rho_{A}\left(\vec{b}, z_{2}\right) e^{i\left(z_{1}-z_{2}\right) x_{\mathbb{P}} m_{N}} e^{-\frac{A}{2}(1-i \eta) \sigma_{\text {soft }}^{j}\left(x, Q_{0}^{2}\right) \int_{z_{1}}^{z_{2}} d z^{\prime} \rho_{A}\left(\vec{b}, z^{\prime}\right)} .
\end{aligned}
$$

Due to the QCD factorization theorem, these nuclear PDFs $f_{j / A}\left(x, Q^{2}\right)$ can be used to calculate many different observables at small $x$ including the nuclear structure function $F_{2 A}$ and the longitudinal structure function $F_{L}^{A}$, the charmed contributions to these structure functions $F_{2 A}^{c}$ and $F_{L}^{A(c)}$, etc.; $f_{j / A}\left(x, Q^{2}\right)$ can also be applied to the calculations of hard processes in heavy-ion collisions.

Removing the integration over $d^{2} b$ in right-hand side of eq. (5.33), one obtains the impact parameter dependent nuclear PDFs (nuclear GPDs in the $\xi=0$ limit in the impact parameter representation) [807, see Section 5.9.1. 

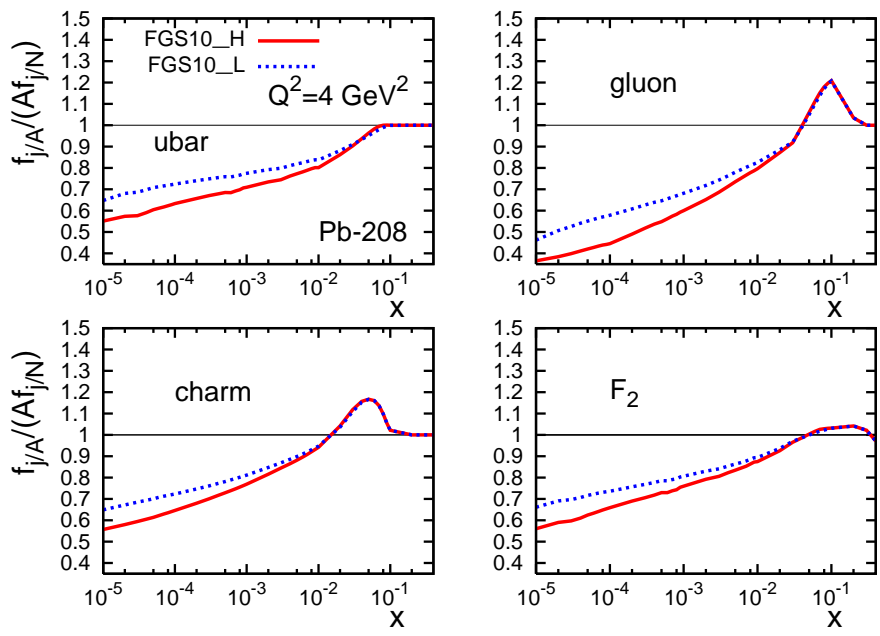

Figure 5.19. The leading twist theory of nuclear shadowing predictions for $f_{j / A} /\left(A f_{j / N}\right)$ ( $\bar{u}$ and $c$ quarks and gluons) and $F_{2 A} /\left(A F_{2 N}\right)$ as functions of $x$ at $Q_{0}^{2}=4 \mathrm{GeV}^{2}$. The two sets of curves correspond to the two extreme scenarios of nuclear shadowing (see the text).
In our analysis 807, we used two models for the color fluctuations in the virtual photon which correspond to two models for $\sigma_{\text {soft }}^{j}$ which cover essentially all reasonable possibilities for the resulting nuclear shadowing. An example of our predictions for the gluon, $\bar{u}$ quark, $c$-quarks, and $F_{2 A}$ structure functions is presented in fig. 5.19 for the two scenarios for $\sigma_{\text {soft }}^{j}$ that we have mentioned above (labeled FGS10_H and FGS10_L). As one can see from fig. 5.19, we predict large nuclear shadowing for each singlet parton flavor with the characteristic feature that nuclear shadowing in the gluon channel is larger than that in the quark channel. The difference between the two extreme scenarios of color fluctuations (the solid and dotted

curves in fig. (5.19) is less than $20 \%$ for $A \sim 200$ and much smaller for light nuclei. The spread between the solid and dotted curves is the theoretical uncertainty of our predictions. Note also that these results weakly depend on the choice of nucleon PDFs.

Accounting for the color fluctuations as done in eq. (5.33) tends to reduce the amount of nuclear shadowing as compared to the quasi-eikonal approximation used in the literature [803, 811. Also, the AGK technique allows one to calculate other quantities such as nuclear diffractive PDFs and fluctuations of multiplicity in non-diffractive DIS [803, 807, 812] both of which turn out to be sensitive to the pattern of the color fluctuations, see Section 5.4.

Our approach to nuclear shadowing assumes the applicability of the linear (in parton densities) leading-twist DGLAP approximation. Numerical studies indicate that the dominant contribution to nuclear shadowing in eq. (5.33) comes from the region of relatively large $\beta=Q^{2} /\left(M^{2}+Q^{2}\right)$ corresponding to the rapidity intervals $\leq 3$ for which the small$x$ approximation used in the BFKL-type approaches is not applicable. These approaches predict $\alpha_{\mathbb{P}}(0) \sim 1.25$, while the HERA experiments find $\alpha_{\mathbb{P}}(0) \sim 1.11 \approx \alpha_{\mathbb{P}}^{\text {soft }}(0)$ consistent with the expectations of the QCD aligned jet approximation [813] that we effectively implemented in the derivation of eq. (5.33).

\section{Non-perturbative approaches}

\section{Hans J. Pirner}

One of the challenges in QCD is the description and understanding of high-energy scattering on protons and nuclei. Even for high energies and large $Q^{2}$ in deep inelastic electron scattering a non-perturbative framework may be necessary. For the transverse structure function, the $q \bar{q}$ dipole in the photon can be large and the saturation scale $Q_{s}$ is small for the energies we discuss. In the following I will present the main features of such an 
approach, which of course will also include the perturbative aspects.

The most important phenomenon observed in high-energy scattering is the rise of the total cross sections with increasing c.m. energy. While the rise is slow in hadronic reactions of large particles such as protons, pions, kaons, or real photons, it is steep if one small particle is involved such as an incoming virtual photon or an outgoing charmonium. This energy behavior is best seen in the proton structure function $F_{2}\left(x, Q^{2}\right)$. With increasing photon virtuality $Q^{2}$, the increase of $F_{2}\left(x, Q^{2}\right)$ towards small Bjorken $x$ becomes significantly stronger. It is tempting to test the growth of the structure function with nuclei. In the following I will summarize my work with Shoshi, Steffen and Dosch which is published in two main papers $[814,815$. For references to other work please see these two papers.

In the two-pomeron model of Donnachie and Landshoff, the energy dependence of the cross sections at high energies results from the exchange of a soft and a hard pomeron. The first dominates in hadron-hadron and $\gamma^{*} p$ reactions at low $Q^{2}$ and the second in $\gamma^{*} p$ reactions at high $Q^{2}$. The two pomerons may be related to a glueball trajectory, which is inherently non-perturbative, and a gluon ladder à la BFKL, which includes the perturbative aspects. The two-pomeron model, however, does not contain parton saturation nor unitarity effects. A model motivated by the concept of parton saturation is the one of Golec-Biernat and Wüsthoff which allows very successful fits to $\gamma^{*} p$ data, but cannot be applied to hadronhadron reactions. A successful description of dipole nucleon scattering which can be used for hadron-nucleon scattering and DIS with moderate $Q^{2}$ has been found 795 .

We have combined perturbative and non-perturbative QCD to compute high-energy reactions of hadrons and photons with special emphasis on saturation effects that manifest $S$-matrix unitarity 814. We follow the functional integral approach to high-energy scattering of Nachtmann, in which the $S$-matrix element factorizes into the universal correlation of two light-like Wegner-Wilson loops $S_{D D}$. The light-like Wegner-Wilson loops describe color dipoles given by the quark and antiquark in the meson or photon projectile and the quark and di-quark in the baryon target. This approach treats projectile and target symmetrically. S-matrix unitarity is respected as a consequence of a matrix cumulant expansion and the Gaussian approximation of the functional integrals. The resulting dipole cross sections do not show Glauber-like behavior with the dipole size as in the Golec-Biernat model. The loop-loop correlation function $S_{D D}$ is expressed in terms of the gauge invariant bi-local gluon field strength correlator integrated over two connected minimal surfaces. Due to the symmetric treatment of the two dipoles this formalism can explicitly investigate the dependence on the impact parameter of the two scattering partners.

The gluon field strength correlator has a non-perturbative and a perturbative component. The stochastic vacuum model of Dosch and Simonov is used for the non-perturbative low frequency background field and perturbative BFKL gluon exchange for the high frequency contributions. This combination allows us to describe long and short distance correlations in agreement with Euclidean lattice calculations of the static quark-antiquark potential with color-Coulomb behavior at short distances and confining linear rise at long distances. We have tried to model both components in AdS/QCD, but the long range looploop correlation cannot be established on a classical level, since the connecting surface in 5 dimensions breaks off at large distances [816.

Energy dependence in the loop-loop correlation function, $S_{D D}$, is introduced by hand in order to describe simultaneously the energy behavior in hadron-hadron, photon-hadron, and photon-photon reactions involving real and virtual photons as well. Motivated by the twoPomeron picture of Donnachie and Landshoff, we ascribe to the soft and hard component a weak and strong energy dependence, respectively. The parameter describing the energy 
dependence of the perturbative correlation function is very large because we include multiple gluonic interactions. In ref. [814] we have considered not only the dependence of the dipole cross section on dipole size with increasing energy and the resulting $k_{t}$-saturation, but also the scattering amplitudes in impact parameter space, where the $S$-matrix unitarity imposes rigid limits on the impact parameter profiles such as the black disc limit. We present profile functions for longitudinal photon-proton scattering that provide an intuitive geometrical picture for the energy dependence of the cross sections. The profile function first becomes greyer, turns black and then increases in transverse size. Using a leading-twist NLO DGLAP relation, we estimated the impact parameter dependent gluon distribution of the proton $x G\left(x, Q^{2},\left|\vec{b}_{\perp}\right|\right)$ from the profile function for longitudinal photon-proton scattering. We have not found saturation of the profile function at HERA energies, but at higher energies, $x G\left(x, Q^{2},\left|\vec{b}_{\perp}\right|\right)$ does saturate as a manifestation of the $S$-matrix unitarity.

In the same framework, we have studied the unintegrated gluon distribution $x G\left(x, k_{t}\right)$ as a function of transverse momentum $k_{t}$ for increasing energies [815]. To obtain the unintegrated gluon distribution, one uses the possibility to rewrite the non-perturbative scattering of an artificial external dipole as a superposition of perturbative contributions. In other words the string of the projectile dipole can be decomposed mathematically in a superposition of dipoles of smaller sizes, from which $x G\left(x, k_{t}\right)$ can be extracted.

The long range confining character of the non-perturbative field strength correlators determines the low $k_{t}$ behavior of the gluon structure function of the hadron as $x G\left(x, k_{t}\right) \propto$ $1 / k_{t}$. In the low momentum limit, $x G\left(x, k_{t}\right) \cdot k_{t}$ converges towards a constant independent of $x$, related to the size of the hadron. The cross-over from the non-perturbative region to the perturbative region occurs at around $k_{t}=1 \mathrm{GeV}$ at $x$-values $10^{-4}<x<10^{-2}$.

On a more fundamental level, we have analysed correlations of Wilson lines in vacuum as one approaches the light cone from space-like distances [817]. The dominant terms of the near light cone Hamiltonian for the Wilson lines define a field theory in $2+1$ dimensions. In the limit of small $\mathrm{x}$, the $\mathrm{SU}(3) \mathrm{QCD}$ for Wilson lines reduces to a critical $\mathrm{Z}(3)$ theory with a diverging correlation length $\xi(x) \propto x^{-1 /\left(2 \lambda_{2}\right)}$ where the exponent $\lambda_{2}=2.52$ is obtained from the center group $\mathrm{Z}(3)$ of $\mathrm{SU}(3)$. We conjecture that the dipole wave function of the virtual photon behaves as the correlation function of Wilson lines in the vacuum. For transverse sizes smaller than the correlation size it scales like $\Psi \propto 1 /\left(x_{t}\right)^{1+n}$ with $n=0.04$ and for distances larger than the correlation length it decays exponentially which makes this region negligible. For $F_{2}$ we integrate the square of the photon wave function weighted with a dipole proton cross section of fixed size $R_{0}$ independent of x. All the energy dependence is absorbed into the photon. Because of the approximate conformality of the dipole wave function $(n \approx 0)$, the result depends only on $R_{0}^{2} / \xi(x)^{2} \propto R_{0}^{2} x^{1 / \lambda_{2}}$, i.e. the saturation scale varies as as $Q_{s}^{2}=Q\left(x_{o}\right)^{2}(x 0 / x)^{1 / \lambda_{2}}$. The critical index in this theory is a characteristic feature of $\mathrm{Z}(3)$ theory i.e. the center group of $\mathrm{SU}(3)$ in an external field given by the light quarks. This is very different from the perturbative color glass condensate where $Q_{s}$ depends on the running coupling similarly to the power behaviour of BFKL. 


\title{
5.3 Inclusive DIS $\left(\mathbf{F}_{2}, \mathbf{F}_{L}, \mathbf{F}_{2}^{c}\right)$
}

\section{Estimates of higher twist in deep inelastic nucleon and nucleus scattering}

\author{
Joachim Bartels, Krzysztof Golec-Biernat and Leszek Motyka
}

A deeper understanding of the transition region at low $Q^{2}$ and small $x$ in deep inelastic electron proton scattering has been one of the central tasks of HERA physics. It will be one of the key questions to be addressed by a future Electron Ion Collider. Approaching this transition region from the perturbative side, one expects to see the onset of corrections to the successful DGLAP description, based upon leading twist operators in QCD. The twist expansion defines a systematic approach to the short distance limit probed in deep inelastic scattering. The study of higher-twist corrections therefore provides an attractive route for investigating the region of validity of the leading twist DGLAP evolution equations.

The validity of the leading-twist QCD evolution equations is based upon the fact that, for sufficiently large $Q^{2}$ and not too small $x$, the gluons inside the proton are dilute. The DGLAP evolution equations, however, predict that, at small $x$ and low $Q^{2}$, the gluon density grows. As a result, the gluons start to interact and the gluon density eventually saturates. The onset of saturation is encoded in the saturation scale, $Q_{\text {sat }}^{2}(x)$.

The investigation of saturation is of highest importance for our understanding of QCD. Saturation can be viewed as a first step of entering the strong interaction region. While the QCD coupling constant is still small, saturation phenomena probe nonlinear dynamics of the gluon sector which plays a crucial role in many areas of strong interactions. It is expected that saturation effects in deep inelastic scattering on a nucleus are enhanced in comparison with deep inelastic scattering on a proton. In the former case, the incoming photon 'sees' the gluons of many nucleons, whereas in the case of a single nucleon, one has to go to smaller $x$ values (higher energies) in order to reach the same gluon density.

A brief discussion of the connection between saturation and the twist expansion has been given in [818. Whereas in the GBW model [83, 819] there is a rather direct classification of eikonal-type exchanges of gluon ladders in terms of twist quantum numbers, in saturation models based upon the nonlinear BK-equation [85, 820] a twist decomposition is much less obvious. In the following we present some numerical estimates of higher-twist contributions, using the improved version of the GBW model [821].

The method: The theory of higher-twist operators and their evolution equations has been outlined in 822]: in leading order, the higher-twist evolution equations are described by the nonforward DGLAP splitting functions, and there is a particular pattern of mixing between different operators of the same twist. In the same way as for leading twist, a numerical analysis of higher twists requires initial conditions for the set of evolution equations, which have to be adjusted to data. In [818] the magnitude of higher-twist corrections was evaluated in a slightly different way. Starting from the observation that within the GBW saturation model the multiple exchanges of leading-twist gluon ladders can be put into a one-toone correspondence with contributions of definite twist quantum numbers, it is possible to arrive at quantitative estimates of the leading-twist contributions and corrections due to twist $\tau=4,6, \ldots$. Details have been described in [818] and will not be repeated here.

While the analysis in [818] was performed for the case of $e+p$ scattering, it is straightforward to extend it to electron-nucleus scattering. Assuming a cylindrical nucleus with a

characteristic size $R_{A} \approx A^{1 / 3} R_{p}$ (with $R_{p}$ being the proton radius), we simply replace the 

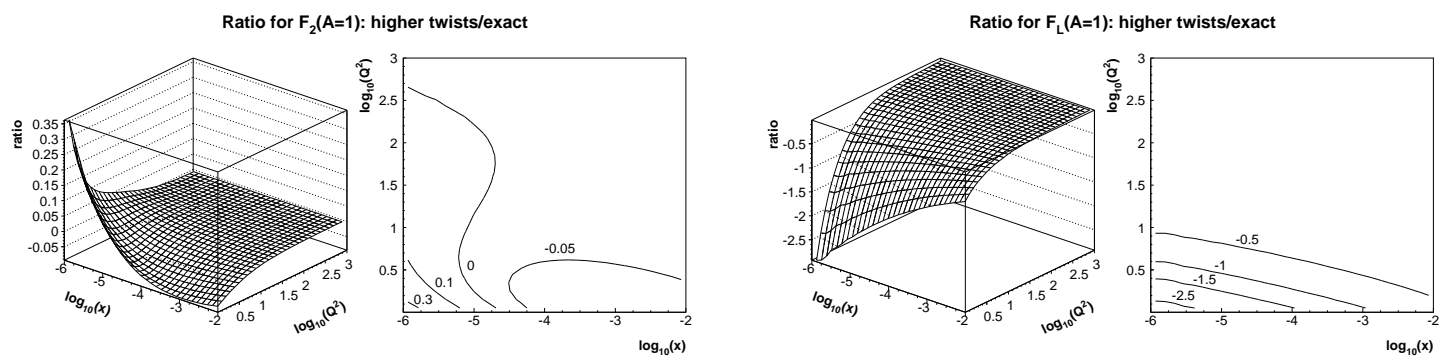

Figure 5.20. Higher-twist contribution estimate for $F_{2}$ (left) and $F_{L}$ (right) of the proton.

dipole-proton cross section (eq.(42) in [818])

$$
\sigma_{\text {dipole- }} \text { proton }=\sigma_{0}\left(1-\exp \left(-\Omega\left(x, r^{2}\right)\right)\right)
$$

by the dipole-nucleus cross section

$$
\sigma_{\text {dipole-nucleus }}=A^{2 / 3} \sigma_{0}\left(1-\exp \left(-A^{1 / 3} \Omega\left(x, r^{2}\right)\right)\right),
$$

where $\Omega\left(x, r^{2}\right)$ is the eikonal function given in [818. With the parameters from [818] we simply repeat the electron proton calculations for electron gold scattering, using the modified dipole cross section formula in (2).
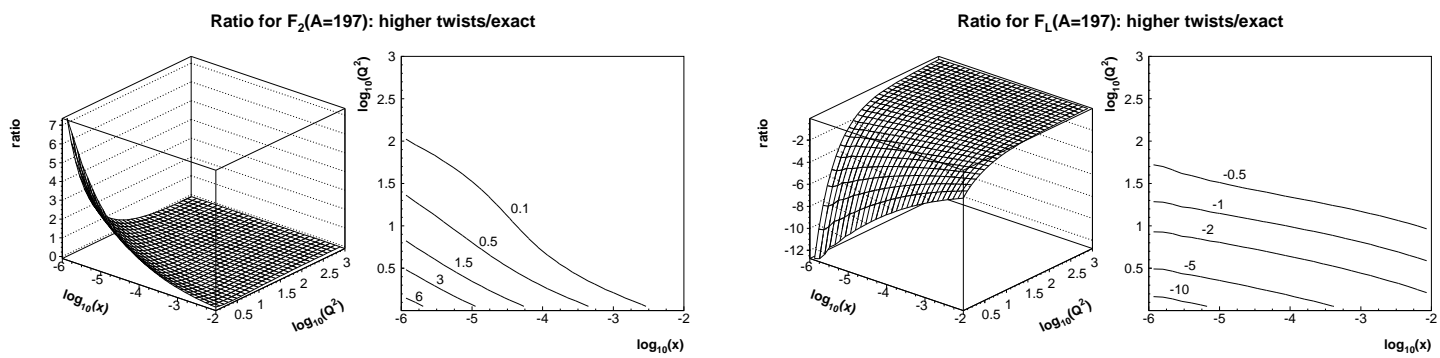

Figure 5.21. Higher-twist contribution estimate for $F_{2}$ (left) and $F_{L}$ (right) of the gold nucleus.

Numerical results: The numerical results for $F_{2}$ and $F_{L}$ are shown in Fig. 5.20 for the proton, and Fig. 5.21 for the gold nucleus. In each figure we show, on the l.h.s in a 3dimensional view, the ratio of the higher-twist corrections and the full structure function as a fucntion of $x$ and $Q^{2}$,

$$
\text { ratio }=\frac{F_{2, L}^{(\text {total })}-F_{2, L}^{(\tau=2)}}{F_{2, L}^{(\text {total })}} .
$$

The r.h.s. shows the projection onto the $\left(\log x, \log Q^{2}\right)$ plane: the lines belong to fixed values of the ratio (5.36). One recognizes the general trend: the corrections are getting larger when $x$ and $Q^{2}$ decrease (moving towards the lower left corner). For given $x$ and $Q^{2}$, the corrections for the longitudinal structure functions are larger than for $F_{2}$. This is a consequence of the sign structure of the corrections in $F_{L}$ and $F_{T}$ : twist four corrections to $F_{L}$ and $F_{T}$ have opposite signs, and in the analysis [818] of $F_{2}=F_{T}+F_{L}$ a strong cancellation has been found. This explains the small higher twist contribution for $F_{2}$. 
One also recognizes the general trend that for gold, all corrections are larger than for the proton. Finally, the corrections to $F_{L}$ are negative and those to $F_{2}$ are mostly positive. In the case of the proton $F_{2}$ there is a change in sign in the region of very small values of $Q^{2}$ : this again is a consequence of the sign structure of the twist corrections to $F_{T}$ and $F_{L}$.

Conclusions: Our numerical analysis confirms that, in general, the structure functions $F_{L}$ are more sensitive to higher-twist corrections than $F_{2}$ which, because of the sign structure in the twist 4 corrections, seems to much better "protected" against higher twist. Also, nuclear targets are more sensitive to higher twist corrections relative to the proton. In view of these results, it seems clear that in a future electron-ion collider, the measurement of $F_{L}$ is of vital importance in the search for saturation.

\section{Strength of nonlinear effects in nucleons and nuclei}

\section{Tuomas Lappi}

The effects of nonlinearity and unitarity in small $x$ DIS are most clearly visible in the dipole framework. We denote $\mathcal{N}\left(x, \mathbf{b}_{T}, \mathbf{r}_{T}\right)$ the imaginary part of the scattering amplitude for a dipole of size $\mathbf{r}_{T}$ and rapidity $y=\ln (1 / x)$ to scatter off the target at impact parameter $\mathbf{b}_{T}$. The total dipole cross section is given by twice the integral of $\mathcal{N}\left(x, \mathbf{b}_{T}, \mathbf{r}_{T}\right)$ over the impact parameter. While the formal unitarity limit would be for $\mathcal{N}$ to lie between 0 and 2 , in practice the reasonable physical area is between 0 (no scattering) and 1 (complete absorption or the black disk limit). The typical value of the dipole scattering amplitude therefore serves as a good measure of the degree of nonlinearity of the scattering process.

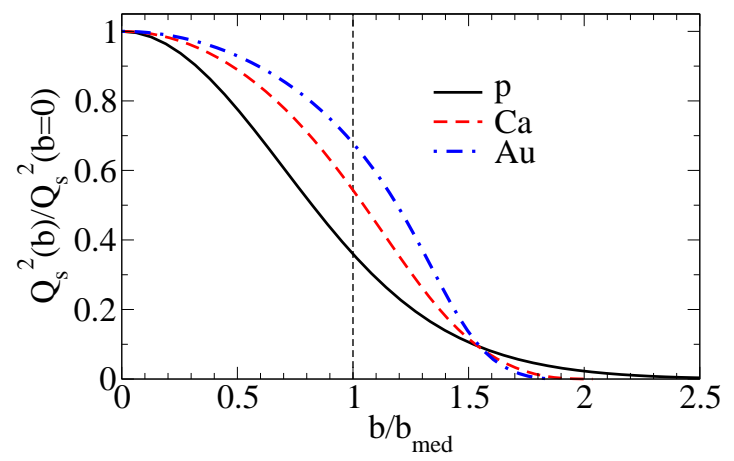

Figure 5.22. The saturation scale in a proton and $\mathrm{Ca}$ and $\mathrm{Au}$ nuclei as a function of $b / b_{\text {med }}$, where $b_{\text {med }}$ is the median impact parameter probed in inclusive DIS at $x=0.001$ and $Q^{2}=1 \mathrm{GeV}^{2}$.

As the total cross section depends on the integral of the scattering amplitude over the impact parameter, statements about the magnitude of the scattering amplitude depend on the profile of the target in $\mathbf{b}_{T}$. The $\mathbf{b}_{T^{-}}$ depdendence for the scattering amplitude on a nucleon is, however, very much constrained by the $t$-dependence of exclusive vector meson production. Using this information, in addition to the total cross section, results in the two commonly used $\mathbf{b}_{T}$-dependent dipole amplitude parametrizations that we will use here, the IPsat and bCGC models [600, 823, 824]. They have successfully been used to describe HERA data on the inclusive cross section, exclusive vector meson production and diffractive structure functions [825].

The saturation scale: To a first approximation the impact parameter dependence of the nuclear scattering amplitude can then be obtained by combining the nucleon one with basic knowledge of nuclear geometry in a Glauber-like treatment (see e.g. Refs. 826, 827] for details). This yields a characteristic pattern of nuclear suppression (shadowing) of the inclusive cross section, a nuclear enhancement of diffraction to small mass states and a 
suppression in diffraction to large masses (small $\beta$ ) 825 .

One way of quantifying the importance of nonlinear effects is to compare the value of the $\left(\mathbf{b}_{T}\right.$ and $x$-dependent) saturation scale $Q_{\mathrm{s}}^{2}$ to the virtuality $Q^{2}$ of the process. The saturation scale is defined as the inverse of the dipole size at which the scattering amplitude $\mathcal{N}$ reaches some specific value defined by convention. For $Q^{2} \gg Q_{\mathrm{s}}^{2}$ one is in the dilute limit and for $Q^{2} \sim Q_{\mathrm{s}}^{2}$ nonlinear effects become important. A naive argument of the $A$ dependence of the saturation scale for nuclei would give $Q_{\mathrm{s} A}^{2} \sim A^{1 / 3}$. The importance of a realistic impact parameter dependence for nuclei was discussed in more detail in Ref. [826], where it was found that this dependence is indeed true to a very good approximation, but the picture is more intricate than that. For the center of a nucleus vs. the center of a proton the saturation scale is suppressed by a geometrical factor $\sim 0.3 \approx R_{p}^{2} A^{2 / 3} / R_{A}^{2}$. Both a nucleon and a nucleus have a dilute edge at large impact parameters. The thickness of this edge is determined by confinement scale physics and is thus of the same order for both. The proton is, however, a much smaller object and therefore the dilute edge region is responsible for a much larger fraction of the total cross section than in a nucleus. One way to see this is to look at the saturation scale at the median impact parameter contributing to the inclusive cross DIS cross section. The value of $Q_{\mathrm{s}}^{2}\left(b_{\text {med }}\right)$ is $\sim 35 \%$ of the value at $b=0$ for a proton, but $\sim 70 \%$ for a gold nucleus (see Fig. [5.22, [826]).
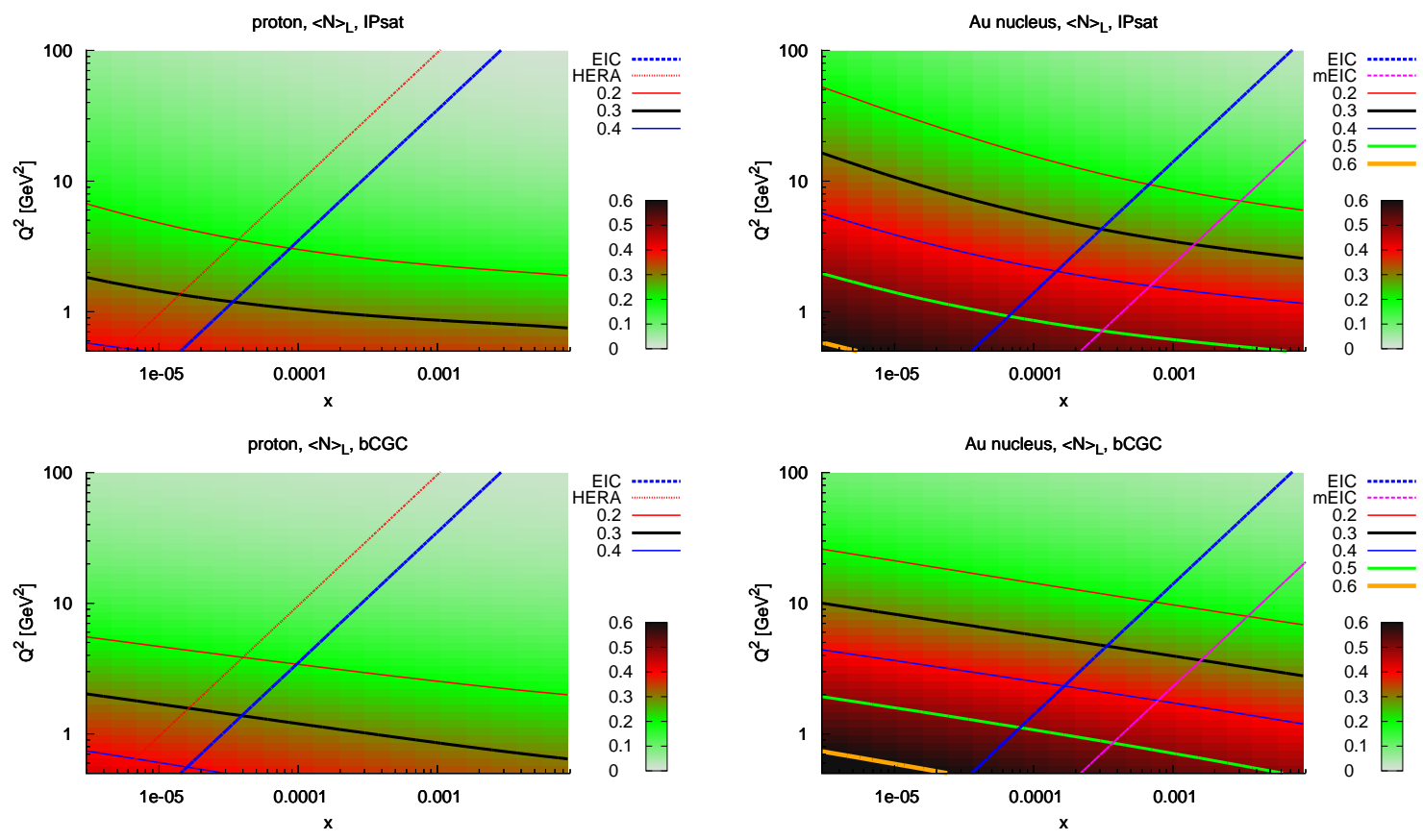

Figure 5.23. Longitudinal mean scattering amplitude $\langle\mathcal{N}\rangle_{L}$ for a proton (left) and a a gold nucleus (right) with the IPsat parametrization (first row) and bCGC parametrization (second row).

The mean scattering amplitude: An alternative way of assessing the typical values of the scattering amplitude is to calculate its expectation value weighted by the cross section 
of a particular process. We thus define the mean scattering amplitude as

$$
\langle\mathcal{N}\rangle_{T, L}=\frac{\int \mathrm{d}^{2} \mathbf{r}_{T} \int_{0}^{1} \mathrm{~d} z\left|\Psi_{L, T}^{\gamma^{*}}\right|^{2} \int \mathrm{d}^{2} \mathbf{b}_{T} \mathcal{N}^{2}\left(x, \mathbf{b}_{T}, \mathbf{r}_{T}\right)}{\int \mathrm{d}^{2} \mathbf{r}_{T} \int_{0}^{1} \mathrm{~d} z\left|\Psi_{L, T}^{\gamma^{*}}\right|^{2} \int \mathrm{d}^{2} \mathbf{b}_{T} \mathcal{N}\left(x, \mathbf{b}_{T}, \mathbf{r}_{T}\right)}
$$

This will yield a value between 0 and 1 for all points in the $Q^{2}, x$-plane. Note that although in principle $\langle\mathcal{N}\rangle$ varies between 0 and 1 , the maximal value for a Gaussian $\mathbf{b}_{T}$-distribution, which describes the proton very well, is only $1 / 2$. The longitudinal and transverse structure functions probe a slightly different distribution of dipole sizes $r$, with the longitudinal structure function showing a stronger $Q^{2}$-dependence. The same quantities can easily be computed also for charm quarks only.
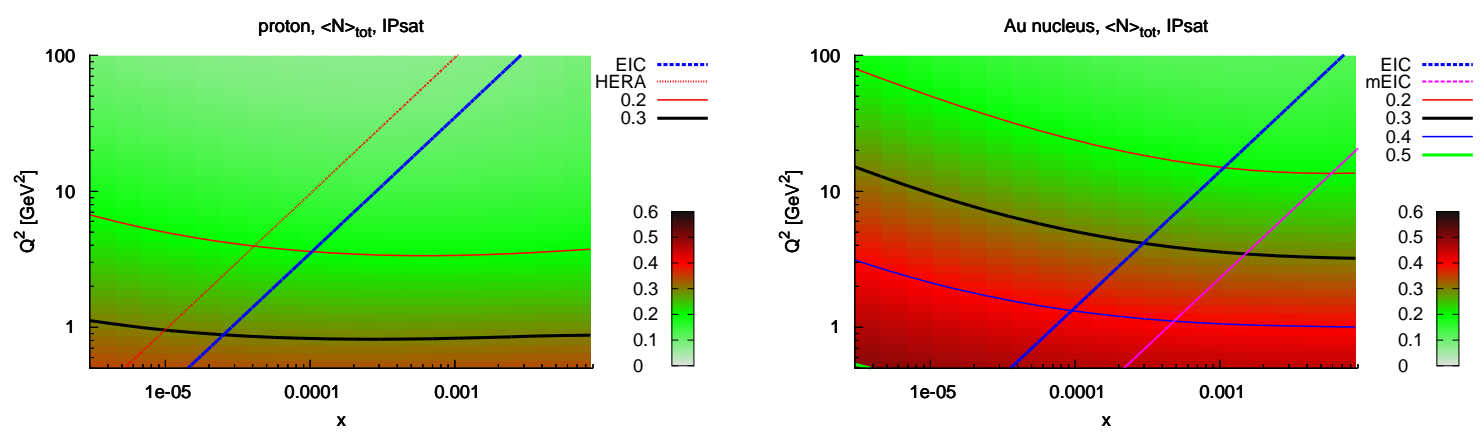

Figure 5.24. The mean scattering amplitude $\langle\mathcal{N}\rangle_{\text {tot }}$ for the total cross section for a proton (left) and a a gold nucleus (right) with the bCGC parametrization.

Figure 5.23 shows the mean scattering amplitude probed in the longitudinal total cross section in a proton and a gold nucleus in the IPsat model. The characteristic feature of the eikonalized DGLAP-evolved gluon distribution in this parametrization is the fact that the $x$-dependence becomes faster at higher energies. The same quantity for the bCGC cross section is plotted in fig. 5.23. Here one sees the characteristic constant energy dependence $Q_{\mathrm{s}}^{2} \sim x^{-\lambda}$ in the bCGC parametrization leading to straight lines of constant $\mathcal{N}$ in a log-log plot. The amplitude weighted by the total cross section is shown in Fig. 5.24 for the IPsat parametrization. It shows a slower $Q^{2}$-dependence than the longitudinal one, connected with the well-known fact that the longitudinal structure function is more sensitive to higher-twist effects than the total one.

In all plots for protons we have shown the kinematical limits for HERA and the EIC $(325 \mathrm{GeV}$ proton on $30 \mathrm{GeV}$ electron with $y<0.9)$ and in the nucleus plots for the EIC $(130 \mathrm{~A} \mathrm{GeV}$ nucleus on $30 \mathrm{GeV}$ electron with $y<0.9)$ and lower energy mEIC option $(130 \mathrm{~A} \mathrm{GeV}$ nucleus on $5 \mathrm{GeV}$ electron with $y<0.9)$. The comparison between nuclei and protons is striking. In the IPsat parametrization, as is typical of DGLAP evolution, the energy dependence at the initial small $Q^{2}$-scale is very slow. Thus the lower energy of the EIC compared to HERA would be insignificant in face of the effect of using nuclei. A value of $\langle\mathcal{N}\rangle_{\text {tot }}$ of 0.3 could, for example, be reached at $Q^{2}=4 \mathrm{GeV}^{2}$ at the EIC vs. $Q^{2}=1 \mathrm{GeV}^{2}$ at HERA; much more safely in the weak coupling regime. With nuclei the EIC could, at $Q^{2}=1 \mathrm{GeV}$, reach values of $\langle\mathcal{N}\rangle_{L} \approx 0.5$ that are simply inaccessible in an ep collider at practically any energy for an approximately Gaussian proton profile. 
Acknowledgments: M. Diehl came up with the idea of visualizing the strength of the nonlinear effects in the way presented here.

\title{
Nuclear PDFs and deviations from DGLAP evolution
}

\author{
Alberto Accardi, Vadim Guzey and Juan Rojo
}

In this contribution we present a preliminary analysis which aims at determining the potential of the EIC to measure gluon shadowing and anti-shadowing and its sensitivity to saturation dynamics.

The input for this analysis is the EIC pseudo data for the inclusive DIS cross section in two scenarios, a medium energy $\operatorname{EIC}(\sqrt{s}=$ $12,17,24,32,44 \mathrm{GeV}$, denoted by stage I) and a full energy $\operatorname{EIC}(\sqrt{s}=63,88,124 \mathrm{GeV}$, stage II), with $0.004<y<0.8$ in either case. The kinematic coverage is summarized in Fig. 5.25. The pseudo-data was generated starting from $e+p$ and $e+n$ cross sections computed using the central values of the NNPDF2.0 parton distributions [47. An integrated luminosity of $4 \mathrm{fb}^{-1}$ was assumed for all energies, and the pseudodata has been corrected for the expected statistical fluctuations. For most of the $x$ range the resulting statistical errors are negligible compared to the assumed $2 \%$ systematic error. Nuclear effects have been included in a $K$-factor approx-

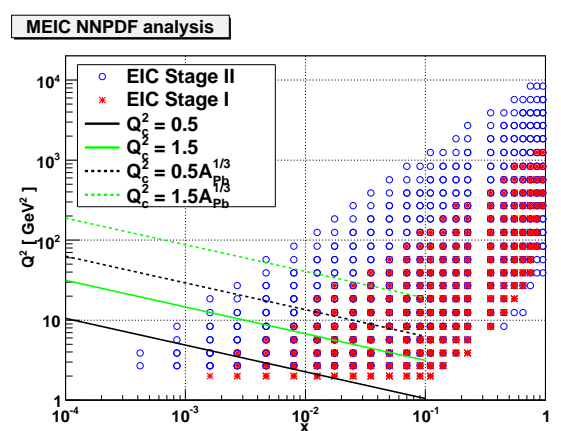

Figure 5.25. Kinematical coverage of the pseudo-data included in the NNPDF analysis of the EIC e+Pb cross-sections, both for stage I and for stage II. Possible kinematical cuts relevant to the study of the onset of non-linear phenomena are also shown. imation, so that the longitudinal and transverse cross sections in ${ }^{208} \mathrm{~Pb}$ can be expressed in terms of the proton cross sections as

$$
\sigma_{T, L}^{\mathrm{Pb}}\left(x, Q^{2}, y\right)=K_{T, L}^{\lambda}\left(x, Q^{2}, y\right) \sigma_{T, L}^{\mathrm{p}}\left(x, Q^{2}, y\right),
$$

where the label $\lambda$ sets the intensity of the assumed saturation effects, and $\lambda=1$ corresponds to the nominal saturation in the IP Non-sat model 600. In particular, the $K$-factor in Eq. (5.38) is given by the following piece-wise expression. For small $x, x \leq 0.01$,

$$
K_{T, L}^{\lambda}=\frac{2}{\left\langle\sigma_{q \bar{q}}\right\rangle_{T, L}} \int d^{2} b\left\langle\left(1-e^{-\lambda \frac{1}{2} A \sigma_{q \bar{q}} T_{A}(b)}\right)\right\rangle_{T, L},
$$

where $\sigma_{q \bar{q}}$ is the dipole cross section in the IP Non-sat model (we assume for simplicity that in the EIC kinematic range there is no saturation at the proton level, and search for the nuclear medium-induced saturation); $T_{A}(b)=\int d z \rho_{A}(b, z)$, where $\rho_{A}(b, z)$ is the nuclear density normalized to unity; the brackets $\langle\ldots\rangle_{T, L}$ stand for the integration with the wave function squared of a virtual photon with transverse or longitudinal polarization, respectively. In the $0.01 \leq x \leq 0.1$ interval, we assume that $K_{T, L}^{\lambda}$ increases linearly from the value given by Eq. (5.39) at $x=0.01$ up to $K_{T, L}^{\lambda}=1$ at $x=0.1$. For $x>0.1$, we assumed that $K_{T, L}^{\lambda}$ is equal to the ratio of the nuclear to free nucleon structure functions, $F_{2 A}\left(x, Q^{2}\right) /\left[A F_{2 N}\left(x, Q^{2}\right)\right]$, which is given by the leading-order parameterization of Ref. [828] 
Nuclear parton distributions are then determined by a Next-to-Leading Order QCD fit of the pseudo-data within the NNPDF framework [681, 47]. The kinematic cuts used to ensure the validity of DGLAP evolution are $Q^{2} \geq 2 \mathrm{GeV}^{2}$ and $W^{2} \geq 12.5 \mathrm{GeV}^{2}$. In this preliminary study, we consider pseudo-data for $\mathrm{Pb}$ targets only, and postpone discussion of the dependence of the nuclear PDFs on $A$ to a future investigation. In the collinear factorization approximation, $\mathrm{Pb}$ structure functions are related to $\mathrm{Pb}$ parton distributions in the same way as in the proton case (see Section 5.7 and Ref. 829]). We also assumed for simplicity the $\mathrm{Pb}$ nucleus to be isoscalar, so that the structure functions depend only on three independent nuclear PDFs: the singlet quark PDF, $\Sigma^{\mathrm{Pb}}\left(x, Q^{2}\right)$, the gluon PDF $g^{\mathrm{Pb}}\left(x, Q^{2}\right)$, and the strange PDF; the latter was furthermore set to be a fixed fraction of the singlet PDF.

Now we discuss some preliminary results of the nuclear PDF fits. We show in Fig. 5.26 the singlet and the gluon PDFs at the initial scale $Q^{2}=2 \mathrm{GeV}^{2}$ obtained using only stage I data for e $+\mathrm{Pb}$ collisions, and then adding the stage II data. To illustrate the accuracy that the EIC can reach in the determination of nuclear PDFs we show in Fig. 5.27 their relative uncertainties alongside those of the proton's NNPDF2.0 47] combined with those of the EPS09 nuclear modifications [40] for ${ }^{208} \mathrm{~Pb}$, which allows a comparison of the relative error bands. Since the restrictive EPS09 parametrization may underestimate the nuclear uncertainties outside the region where data is presently available, notably at $x \lesssim 0.01$, we added the relative NNPDF2.0 and EPS09 relative uncertainties linearly for a conservative estimate of the total uncertainty.
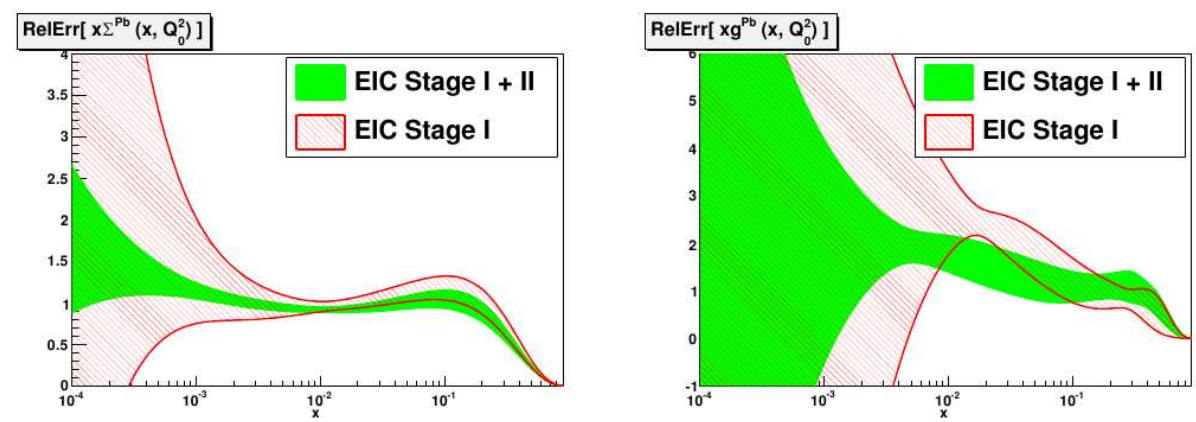

Figure 5.26. The quark singlet (left plot) and the gluon PDFs (right plot) in $\mathrm{Pb}$ at the initial evolution scale $Q_{0}^{2}=2 \mathrm{GeV}^{2}$, for stage I and stage I+II.

The measurement of the nuclear modifications of the gluon are one of the most important measurements at the EIC, as this quantity is essentially unknown from present data. Inclusive cross sections are sensitive to the gluon distribution both via scaling violations and, to a lesser extent, through the longitudinal structure function accessed through the proposed $\sqrt{s}=12-124 \mathrm{GeV}$ energy scan. From Fig. 5.26 we see that one can determine, with reasonable accuracy, the gluon shadowing down to $x \sim 10^{-3}$ in stage II and down to $x \sim 10^{-2}$ in stage I. The better capabilities of stage II stem both from its greater lever arm in $Q^{2}$ and its coverage of smaller values of $x$, see Fig. 5.25, In particular, the precision of the $\mathrm{Pb}$ gluon in Stage II at small $x$ is comparable to estimates from global proton fits. On top of this, at the EIC it will be possible to study gluon anti-shadowing, EMC and Fermi motion effects with much better accuracy than afforded by current global nuclear fits (see Sections 5.7 and 5.7. We can also see that EIC will measure accurately the sea quark shadowing, and that nuclear modifications of light quarks at large $x$ could be measured a precision similar or even better than for the proton case. 

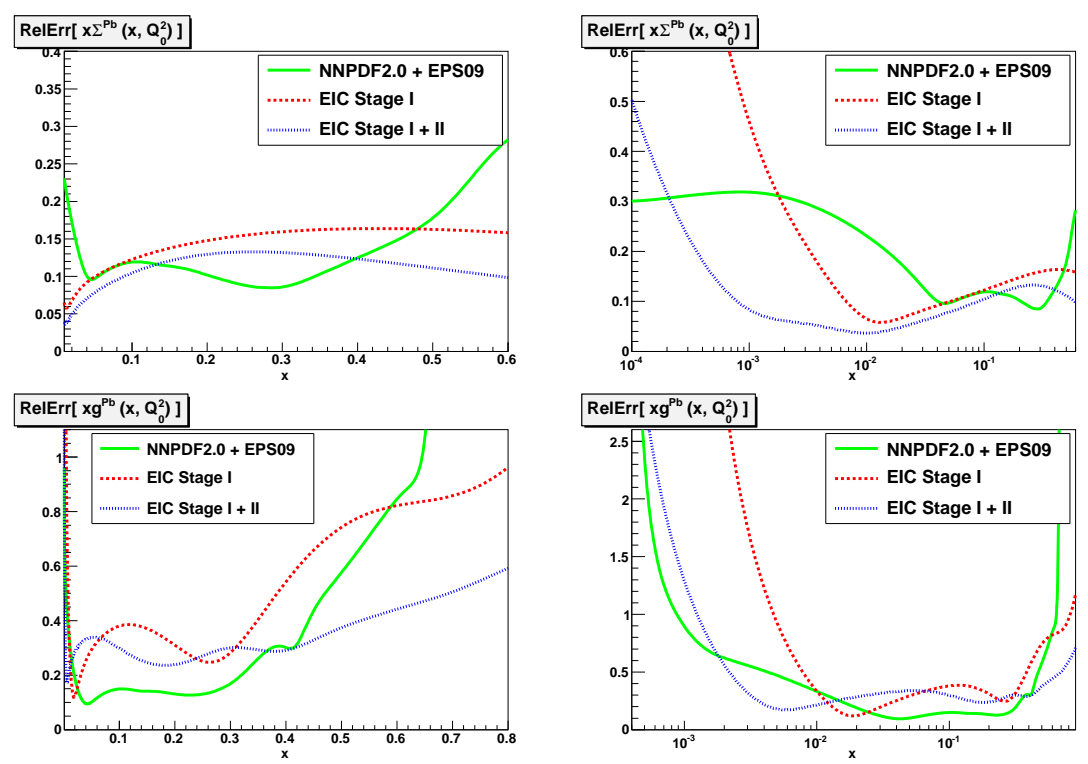

Figure 5.27. The relative uncertainty in the quark singlet (two upper panels) and the gluon PDFs in $\mathrm{Pb}$ (two lower panels) at the initial evolution scale $Q_{0}^{2}=2 \mathrm{GeV}^{2}$, with stage I and stage I+II data. Results are shown on linear (left plot) and logarithmic (right plot) scales. For reference, the analogous results for the Pb PDFs using NNPDF2.0+EPS09 parametrizations are also shown.

This analysis was based on the validity of collinear factorization for nuclei, and the validity of linear DGLAP evolution in $Q^{2}$. However, at small enough $x$ and $Q^{2}$, deviations from linear fixed order DGLAP evolution are expected to appear, e.g., due to small- $x$ resummation effects 830, or gluon saturation, see Section 5.2. In heavy nuclei, the effects due to gluon saturation are boosted to higher $Q^{2}$ and $x$ by the atomic number; one then has the possibility of experimentally separating small- $x$ and saturation effects, which is not be possible with HERA $e+p$ data.

In Refs. [720, 831] a general strategy was presented to quantify potential deviations from NLO DGLAP evolution, which was then applied to proton HERA data. In a global PDF fit, deviations from DGLAP in the data can be hidden in a distortion of parton distributions; however, these can be singled out by determining undistorted PDFs from data in regions where such effects are expected to be small. In more detail, one can fit PDFs using data at large $x$ and $Q^{2}$, where DGLAP is likely to hold with high accuracy, and then evolving them down in the $Q^{2}$ region where deviations are expected to arise. DGLAP deviations can then be quantitatively determined by comparing calculations to data in this region, which were not used in the PDF determination.

This approach can be applied as well to the nuclear case. From simple theoretical arguments about the energy and $A$ dependence of the saturation scale (see Section [5.2), we expect deviations from linear evolution to appear when $Q^{2} \lesssim \bar{Q}^{2}(A \bar{x} / x)^{\frac{1}{3}}$, where $\bar{x}$ is a reference value, say $\bar{x}=10^{-3}$, and $\bar{Q}^{2}$ is the scale where DGLAP evolution at $\bar{x}$ would be broken in the proton. Note however that the $A$-dependence of the saturation scale may in fact be tamed by the leading twist nuclear shadowing, see Section 5.2 . While saturation models may give an indication of the value of $\bar{Q}^{2}$, we wish to determine this scale in a model independent way as the scale at which deviations from DGLAP evolution can be detected from EIC nuclear target (pseudo-)data. The unsafe region for DGLAP evolution can also be 
written as $Q^{2} \lesssim Q_{c}^{2} x^{-\frac{1}{3}}$ with $Q_{c}^{2}$ some constant setting the strength of the deviations from DGLAP. In Refs. [720, 831] the range $Q_{c}^{2} \in[0.5,1.5] \mathrm{GeV}^{2}$ was considered for the proton case; in the nuclear case this range should be rescaled by a factor $A_{\mathrm{Pb}}^{1 / 3} \approx 6$. Typical values of these kinematical cuts for the $\mathrm{Pb}$ nucleus are shown in Fig. 5.25.
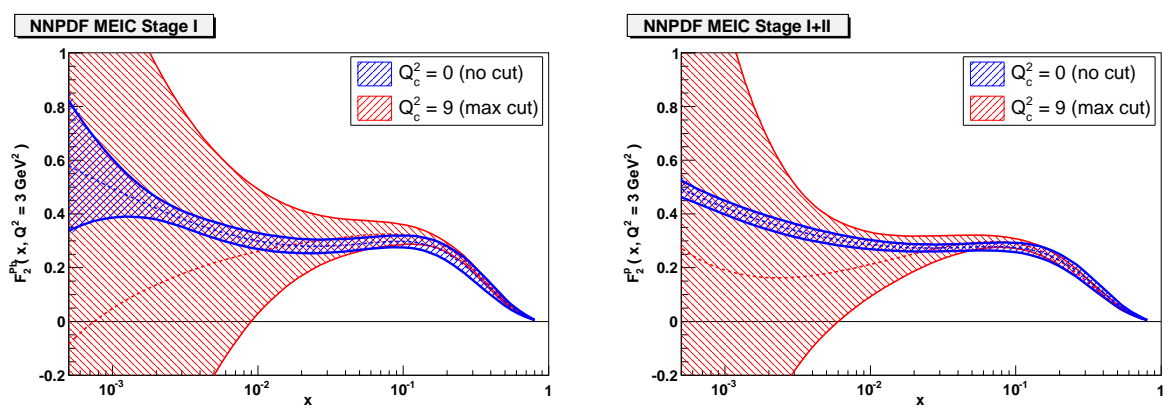

Figure 5.28. The $\mathrm{Pb}$ structure function $F_{2}^{\mathrm{Pb}}\left(x, Q^{2}\right)$ at $Q^{2}=3 \mathrm{GeV}^{2}$ from the analysis of the EIC stage I (left plot) and stage I+II (right plot) simulated data with $\lambda=1$, without kinematical cuts and with cuts using $Q_{c}^{2}=1.5 A_{\mathrm{Pb}}^{1 / 3} \sim 9$.

We show in Fig. 5.28 a representative result of the fits to the EIC pseudo-data after applying the cut with $\bar{Q}^{2}=1.5 A_{\mathrm{Pb}}^{1 / 3} \sim 9$, compared to the reference uncut fits to stages I and I+II pseudo-data with $\lambda=1$. As expected when data is removed the uncertainties in the physical observables become much larger, but one can still see a systematic downwards shift in the central value, which is the signature of the departure from linear evolution [720, 831]. Note that this signal is already apparent with stage I data only, although its statistical significance might be marginal.

We plan to systematically explore the sensitivity of the EIC to non-linear dynamics using this technique, by optimizing the kinematical cuts for different values of the saturation scale used to generate the pseudo-data, exploit the interplay between the $F_{2}^{\mathrm{Pb}}$ and $F_{L}^{\mathrm{Pb}}$ structure functions, and quantitatively measuring the statistical significance of the signal. This will determine in a fairly model-independent way the smallest saturation scale that can be detected at the EIC in either stage I or stage II.

Acknowledgments: We thank F. Caola, R. Ent, S. Forte and L. Zhu for discussions and collaboration.

\section{Constraining the nuclear gluon distribution using inclusive observables}

\section{Victor P. Gonçalves}

Data from HERA allow for a good determination of the gluon density of the proton. A much harder task has been to determine the gluon distribution of nucleons bound in a nucleus, the nuclear gluon distribution $\left(x g^{A}\left(x, Q^{2}\right)\right)$. Existing data, taken over a wide kinematic range $10^{-5} \leq x \leq 0.1$ and $0.05 \mathrm{GeV}^{2} \leq Q^{2} \leq 100 \mathrm{GeV}^{2}$, show a systematic reduction of the nuclear structure function $F_{2}^{A}\left(x, Q^{2}\right) / A$ with respect to the free nucleon structure function $F_{2}^{N}\left(x, Q^{2}\right)$. This phenomenon is known as the nuclear shadowing effect and is associated to the modification of the target parton distributions so that $x q^{A}\left(x, Q^{2}\right)<$ $A x q^{N}\left(x, Q^{2}\right)$, as expected from a superposition of ep interactions. The modifications depend on the parton momentum fraction: for momentum fractions $x<0.1$ (shadowing region) 
and $0.3<x<0.7$ (EMC region), a depletion is observed in the nuclear structure functions. These two regions are bridged by an enhancement known as antishadowing for $0.1<x<$ 0.3. The experimental data for the nuclear structure function determine the behaviour of the nuclear quark distributions, while the behaviour of the nuclear gluon distribution is indirectly determined using the momentum sum rule as a constraint and/or studying the $\log Q^{2}$ slope of the ratio $F_{2}^{S n} / F_{2}^{C}$. Currently, the behaviour of $x g^{A}\left(x, Q^{2}\right)$ at small $x$ (high energy) is completely uncertain as shown in Fig. 5.29. where we present the ratio $R_{g}=x g^{A} /\left(A . x g^{N}\right)$, for $A=208$, predicted by four different groups which realize a global analysis of the nuclear experimental data using the DGLAP evolution equations in order to determine the parton densities in nuclei. In particular, the magnitude of shadowing and the presence or not of the antishadowing effe

In this contribution we study the behaviour of the nuclear longitudinal structure function $F_{L}^{A}$ and the charm structure function $F_{2}^{c, A}$ and analyse the possibility to constrain the nuclear effects present in $x g^{A}$ using these inclusive observables (For more details and references see Ref. [832]).

$F_{L}^{A}$ and $F_{2}^{c, A}$ in the collinear formalism: The longitudinal structure function in deep inelastic scattering is one of the observables from which the gluon distribution can be unfolded. In the collinear formalism, $F_{L}$ is described in terms of the AltarelliMartinelli equation

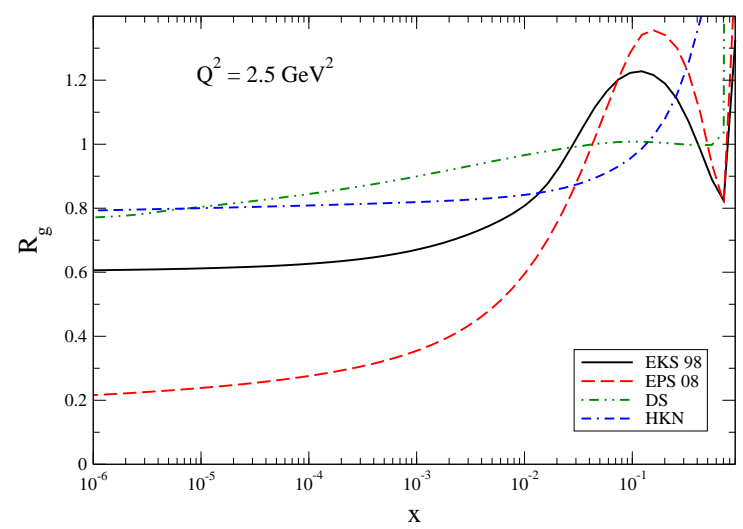

Figure 5.29. The ratio $R_{g}=x g^{A} / A \cdot x g^{N}$ predicted by the EKS, DS, HKN and EPS parametrizations for $A=208$ and $Q^{2}=2.5 \mathrm{GeV}^{2}$.

$$
F_{L}\left(x, Q^{2}\right)=\frac{\alpha_{s}\left(Q^{2}\right)}{2 \pi} x^{2} \int_{x}^{1} \frac{d y}{y^{3}}\left[\frac{8}{3} F_{2}\left(y, Q^{2}\right)+4 \sum_{q} e_{q}^{2}\left(1-\frac{x}{y}\right) y g\left(y, Q^{2}\right)\right] .
$$

At small $x$, the second term with the gluon distribution is the dominant one. This expression can be reasonably approximated by $F_{L}\left(x, Q^{2}\right) \approx 0.3 \frac{4 \alpha_{s}}{3 \pi} x g\left(2.5 x, Q^{2}\right)$, which demonstrates the close relation between the longitudinal structure function and the gluon distribution. Therefore, we expect the longitudinal structure function to be sensitive to nuclear effects.

In order to estimate the charm contribution to the structure function we treat the charm quark as a heavy quark and estimate its contribution by fixed-order perturbation theory. This involves the computation of the boson-gluon fusion process. A $c \bar{c}$ pair can be created by boson-gluon fusion when the squared invariant mass of the hadronic final state is $W^{2} \geq 4 m_{c}^{2}$. Since $W^{2}=\frac{Q^{2}(1-x)}{x}+M_{N}^{2}$, where $M_{N}$ is the nucleon mass, the charm production can occur well below the $Q^{2}$ threshold, $Q^{2} \approx 4 m_{c}^{2}$, at small $x$. The charm contribution to the proton/nucleus structure function, in leading order (LO), is given by

$$
\frac{1}{x} F_{2}^{c}\left(x, Q^{2}, m_{c}^{2}\right)=2 e_{c}^{2} \frac{\alpha_{s}\left(\mu^{\prime 2}\right)}{2 \pi} \int_{a x}^{1} \frac{d y}{y} C_{g, 2}^{c}\left(\frac{x}{y}, \frac{m_{c}^{2}}{Q^{2}}\right) g\left(y, \mu^{\prime 2}\right),
$$

where $a=1+\frac{4 m_{c}^{2}}{Q^{2}}$ and the factorization scale $\mu^{\prime}$ is assumed $\mu^{\prime 2}=4 m_{c}^{2} . C_{g, 2}^{c}$ is the coefficient 


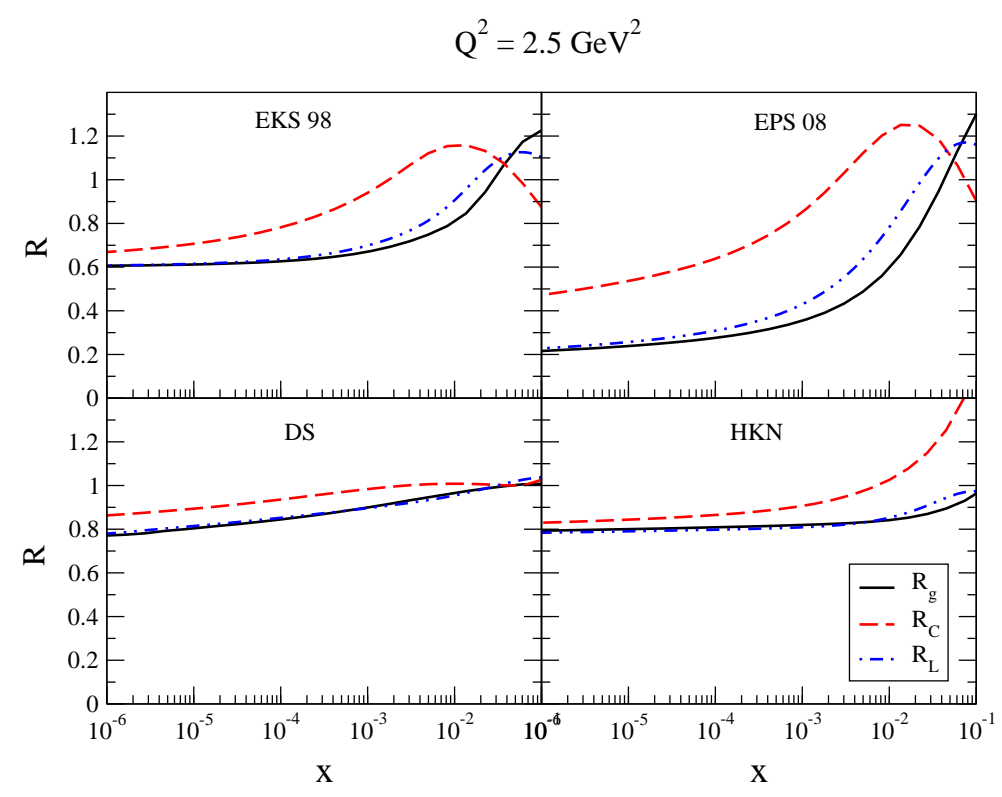

Figure 5.30. Ratios $R_{g}, R_{C}$ and $R_{L}$ for the four considered nuclear parametrizations, $Q^{2}=2.5$ $\mathrm{GeV}^{2}$ and $A=208$.

function given by

$$
\begin{aligned}
C_{g, 2}^{c}\left(z, \frac{m_{c}^{2}}{Q^{2}}\right) & =\frac{1}{2}\left\{\left[z^{2}+(1-z)^{2}+z(1-3 z) \frac{4 m_{c}^{2}}{Q^{2}}-z^{2} \frac{8 m_{c}^{4}}{Q^{4}}\right] \ln \frac{1+\beta}{1-\beta}\right. \\
& \left.+\beta\left[-1+8 z(1-z)-z(1-z) \frac{4 m_{c}^{2}}{Q^{2}}\right]\right\},
\end{aligned}
$$

where $\beta=1-\frac{4 m_{c}^{2} z}{Q^{2}(1-z)}$ is the velocity of one of the charm quarks in the boson-gluon centerof-mass frame. Therefore, in leading order, $\mathcal{O}\left(\alpha_{s}\right), F_{2}^{c}$ is directly sensitive only to the gluon density via the well-known Bethe-Heitler process $\gamma^{*} g \rightarrow c \bar{c}$. The dominant uncertainty in the QCD calculations arises from the uncertainty in the charm quark mass. In this contribution we assume $m_{c}=1.5 \mathrm{GeV}$.

The nuclear ratios: Let us now study the behaviour of the nuclear longitudinal structure function $F_{L}^{A}$ and the charm structure function $F_{2}^{c, A}$ and analyze the possibility to constrain the nuclear effects present in $x g^{A}$ using these inclusive observables. We estimate the normalized ratios

$$
R_{L}\left(x, Q^{2}\right)=\frac{F_{L}^{A}\left(x, Q^{2}\right)}{A F_{L}^{p}\left(x, Q^{2}\right)} \text { and } R_{C}\left(x, Q^{2}\right)=\frac{F_{2}^{c, A}\left(x, Q^{2}\right)}{A F_{2}^{c, p}\left(x, Q^{2}\right)}
$$

considering four distinct parametrizations for the nuclear gluon distributions and compare their behaviour with those predicted for the ratio $R_{g}=x g^{A} / A x g^{N}$.

In Fig. 5.30 we present our results. Firstly, let us discuss the small- $x$ region, $x \leq 10^{-3}$, determined by shadowing effects. We observe that $R_{L}$ practically coincides with $R_{g}$ for all parametrizations and for the two values of $Q^{2}$ considered. This suggests that shadowing effects can be easily constrained in an $e A$ collider by measuring $F_{L}$. This conclusion is, to a good extent, model independent. On the other hand, the ratio $R_{C}$ gives us an upper 
bound for the magnitude of the shadowing effects. For example, if it is found that $R_{C}$ is equal to $\approx 0.6$ at $x=10^{-4}$ and $Q^{2}=2.5 \mathrm{GeV}^{2}$ the nuclear gluon distributions from DS and HKN parametrizations are very large and should be modified. Considering now the kinematical range of $x>10^{-3}$ we can analyse the correlation between the behaviour of $R_{L}$ and $R_{C}$ and the antishadowing present or not in the nuclear gluon distribution. Similarly to what is observed at small values of $x$, the behaviour of $R_{L}$ is very close to the $R_{g}$ one in the large- $x$ range. In particular, the presence of antishadowing in $x g^{A}$ directly implies an enhancement in $F_{L}^{A}$. It is almost $10 \%$ smaller in magnitude that the enhancement predicted for $x g^{A}$ by the EKS and EPS parametrizations. Inversely, if we assume the non-existence of the antishadowing in the nuclear gluon distribution at $x<10^{-1}$, as in the DS and HKN parametrizations, no enhancement will be present in $F_{L}^{A}$ in this kinematical region. Therefore, it suggests that also the antishadowing effects can be easily constrained in an $e A$ collider measuring $F_{L}$. On the other hand, in this kinematical range the behavior of $R_{C}$ is distinct of $R_{g}$ at a same $x$. However, we observe that the behavior of $R_{C}$ at $x=10^{-2}$ is directly associated to $R_{g}$ at $x=10^{-1}$. In other words, the antishadowing is shifted in $R_{C}$ by approximately one order of magnitude in $x$. For example, the large growth of $R_{g}$ predicted by the HKN parametrization at $x \geq 10^{-1}$ shown in Fig. 5.29 implies the steep behavior of $R_{C}$ at $x \geq 10^{-2}$ observed in Fig. 5.30. Consequently, by measuring $F_{2}^{c}$ it is also possible to constrain the existence and magnitude of the antishadowing effects.

Acknowledgments: The author thanks E.R. Cazaroto, F. Carvalho, and F.S. Navarra for collaboration.

\section{DIS in the high-energy limit at next-to-leading order}

\section{Giovanni A. Chirilli}

Nowadays, it is widely accepted that non-linear dynamics effects dominate deep inelastic lepton hadrons scattering processes (DIS) at very high-energy (Regge limit), and non-linear equations have been derived in order to describe the evolution of the structure of hadronic matter at this regime. One of these equations is the Balitsky-Kovchegov equation (BK) derived by Balitsky [770] in the Wilson lines formalism, and by Kovchegov [741, 747] in the dipole frame. The Wilson line formalism is an operator language based on the concept of factorization of the scattering amplitude in rapidity space and on the extension of the application of the Operator Product Expansion (OPE) formalism to high-energy (Regge limit). So far, the OPE formalism was known only in the Bjorken limit as an expansion in terms of local operators or in terms of light ray operators.

The relevance of the BK equation for future experiments at an Electron Ion Collider (EIC) or Large electron Hadron Collider (LeHC) can be determined by the running of the coupling constant and the evolution kernel at the next-to-leading-order (NLO) approximation (NLO corrections in power of the strong coupling constant $\alpha_{s}$ ). The argument of the coupling constant has been obtained by the authors of ref. [833, 834] where only the quark contribution has been calculated explicitly, while the gluonic part was obtained conjecturing that its contribution would follow the same pattern of the quark contribution. However, this result did not fully solve the problem of the argument of the running coupling constant due to an ambiguity of one term which is not proportional to $b=\frac{11}{3} N_{c}-\frac{2}{3} n_{f}$. The com-

plete results of the NLO-BK kernel including the gluon contribution to the argument of the coupling constant has been obtained in [835] where it was shown that the result agrees with 
the NLO Balitsky-Fadin-Kuraev-Lipatov (BFKL) kernel. The BFKL equation [836, 60] can be obtained from the BK equation by dropping out the non linear terms. Indeed, a caveat of such a linear evolution equation is the violation at very high energy of the unitarity condition which is instead preserved by the BK equation.

Conformal symmetry is a symmetry violated in QCD by the running of the coupling constant. What one would then expect from the calculation of the NLO BK-kernel is that the only source of violation of such symmetry come from the running of coupling while the rest of the kernel preserves conformal (Möbius) symmetry. However, although Wilson lines are formally conformal invariant, at one loop correction they are rapidity-divergent, and since it is not known how to regulate them in a conformally invariant way, the NLOBK kernel contains non-conformal terms (besides to the running coupling constant) as a remnant of the prescription used to cure such divergences. In order to study the source of the loss of conformal invariance, it is convenient to consider a conformally invariant theory like the $\mathcal{N}=4$ super-symmetric Yang-Mills (SYM) theory. The NLO evolution kernel obtained in this framework is also not conformally invariant [837, contrary to what one would expect from a conformal field theory. It was then shown in [837, that suitable operators for the description of processes at high-energy (Regge) theory are composite conformal (Wilson line) operators constructed order by order in perturbation theory. These operators absorb the undesired non conformal terms in the same way as counterterms are added to renormalize local composite operators in order to restore the symmetry that the bare operator lost at the level of NLO (and higher) corrections. Indeed, the NLO evolution of such composite conformal operators in QCD resolve in a running coupling part and in a conformally invariant part. In ref. [837, 838, the conformal expression for the NLO BFKL has been obtained for the first time.

In order to obtain the full NLO amplitude for DIS at high energy, one needs to calculate the coefficient function (photon impact factor) at NLO and convolute it with the NLO evolution kernel of the relative operator (the NLO BK kernel). The NLO impact factor has been calculated in ref. [839] where an analytic expression (in coordinate space) has been obtained for the first time.

High-energy operator product expansion: In the usual OPE, due to the presence of two different scales of the transverse momentum $k_{\perp}$, one introduces a factorization scale, usually denoted by $\mu$, which factorizes the amplitude of DIS processes in pertubatively calculable contributions (hard part) and in a non-pertubatively calculable ones (soft part) represented by matrix elements made of light-ray operators. The evolution of such matrix elements with respect to the renormalization point $\mu$ is the DGLAP evolution equation.

At high-energy (Regge limit), all the transverse momenta are of the same order of magnitude. Therefore, a suitable factorization scale would be the rapidity scale: one introduces rapidity $(\eta)$ which separates "fast" fields from "slow" fields. Thus, the amplitude of the process can be represented as a convolution of contributions coming from fields with rapidity $\eta<Y$ (fast fields) and contributions coming from fields with rapidity $\eta>Y$ (slow fields). As in the case of the usual OPE, the integration over the fields with rapidity $\eta<Y$ gives us the coefficient functions while the integrations over fields with rapidity $\eta>Y$ are the matrix elements of the operators. A general feature of high-energy scattering is that a fast particle moves along its straight-line classical trajectory and the only quantum effect is the eikonal phase factor acquired along this propagation path. In QCD, for the fast quark or gluon scattering off some target, this eikonal phase factor is a Wilson line - an infinite 
gauge link ordered along the straight line collinear to the particle's velocity $n^{\mu}$ :

$$
U^{\eta}\left(x_{\perp}\right)=\operatorname{Pexp}\left\{i g \int_{-\infty}^{\infty} d u n_{\mu} A^{\mu}\left(u n+x_{\perp}\right)\right\}
$$

Here, $A_{\mu}$ is the gluon field of the target, $x_{\perp}$ is the transverse position of the particle which remains unchanged throughout the collision, and the index $\eta$ labels the rapidity of the particle. Repeating the above argument for the target (moving fast in the spectator's frame) we see that particles with very different rapidities perceive each other as Wilson lines and therefore Wilson-line operators are the convenient effective degrees of freedom in high-energy QCD (for a review, see Ref. [840]). The expansion of the T product of two electromagnetic currents at high-energy (Regge limit) is then in terms of Wilson lines

$$
\begin{aligned}
& T\left\{\hat{j}_{\mu}(x) \hat{j}_{\nu}(y)\right\}=\int d^{2} z_{1} d^{2} z_{2} I_{\mu \nu}^{\mathrm{LO}}\left(x, y ; z_{1}, z_{2}\right) \hat{\mathcal{U}}\left(z_{1}, z_{2}\right) \\
& +\int d^{2} z_{1} d^{2} z_{2} d^{2} z_{3} I_{\mu \nu}^{\mathrm{NLO}}\left(x, y ; z_{1}, z_{2}, z_{3}\right)\left[\hat{\mathcal{U}}\left(z_{1}, z_{3}\right)+\hat{\mathcal{U}}\left(z_{2}, z_{3}\right)-\hat{\mathcal{U}}\left(z_{1}, z_{2}\right)-\hat{\mathcal{U}}\left(z_{1}, z_{3}\right) \hat{\mathcal{U}}\left(z_{3}, z_{2}\right)\right]
\end{aligned}
$$

where

$$
\hat{\mathcal{U}}^{\eta}\left(x_{\perp}, y_{\perp}\right)=1-\frac{1}{N_{c}} \operatorname{Tr}\left\{\hat{U}^{\eta}\left(x_{\perp}\right) \hat{U}^{\dagger \eta}\left(y_{\perp}\right)\right\}
$$

The evolution of the Wilson line operator in eq. (5.45) is given by the BK equation [770, 741, 747.

$$
\begin{array}{r}
\frac{d}{d \eta} \hat{\mathcal{U}}(x, y)=\frac{\alpha_{s} N_{c}}{2 \pi^{2}} \int d^{2} z \frac{(x-y)^{2}}{(x-z)^{2}(z-y)^{2}}[\hat{\mathcal{U}}(x, z)+\hat{\mathcal{U}}(y, z) \\
-\hat{\mathcal{U}}(x, y)-\hat{\mathcal{U}}(x, z) \hat{\mathcal{U}}(z, y)]
\end{array}
$$

The first three terms correspond to the linear BFKL evolution equation [836, 60] and describe parton emission while the last term is responsible for parton annihilation. For sufficiently low $x_{B}$, parton emission balances parton annihilation so the partons reach the state of saturation [742, 841, 842, with the characteristic transverse momentum $Q_{s}$ growing with energy $1 / x_{B}$. The NLO evolution equation for composite Wilson line operator (preserving conformal invariance as explained in the introduction) has been calculated in [835], where one can find the full analytic expression.

In order to obtain the DIS amplitude at high-energy at the NLO, we now need the coefficient function ("impact factor") at next to leading order. Here, we present the NLO impact factor for the study of DIS in the linearized case (two gluon approximation) where the NLO BK equation reduces to the NLO BFKL equation. In this case the OPE at high energy for DIS reduces to

$$
\begin{aligned}
& \frac{1}{N_{c}}(x-y)^{4} T\left\{\overline{\hat{\psi}}(x) \gamma^{\mu} \hat{\psi}(x) \overline{\hat{\psi}}(y) \gamma^{\nu} \hat{\psi}(y)\right\} \\
& =\frac{\partial \kappa^{\alpha}}{\partial x^{\mu}} \frac{\partial \kappa^{\beta}}{\partial y^{\nu}} \int \frac{d z_{1} d z_{2}}{z_{12}^{4}} \hat{\mathcal{U}}_{a_{0}}\left(z_{1}, z_{2}\right)\left[\mathcal{I}_{\alpha \beta}^{\mathrm{LO}}\left(1+\frac{\alpha_{s}}{\pi}\right)+\mathcal{I}_{\alpha \beta}^{\mathrm{NLO}}\right]
\end{aligned}
$$

where

$$
\mathcal{I}_{\mathrm{LO}}^{\alpha \beta}\left(x, y ; z_{1}, z_{2}\right)=\mathcal{R}^{2} \frac{g^{\alpha \beta}\left(\zeta_{1} \cdot \zeta_{2}\right)-\zeta_{1}^{\alpha} \zeta_{2}^{\beta}-\zeta_{2}^{\alpha} \zeta_{1}^{\beta}}{\pi^{6}\left(\kappa \cdot \zeta_{1}\right)\left(\kappa \cdot \zeta_{2}\right)}
$$


is the LO impact factor and where we used the notation $\mathcal{R} \equiv \frac{\kappa^{2}\left(\zeta_{1} \cdot \zeta_{2}\right)}{2\left(\kappa \cdot \zeta_{1}\right)\left(\kappa \cdot \zeta_{2}\right)}$, and the conformal vectors $\kappa=\frac{\sqrt{s}}{2 x_{*}}\left(\frac{p_{1}}{s}-x^{2} p_{2}+x_{\perp}\right)-\frac{\sqrt{s}}{2 y_{*}}\left(\frac{p_{1}}{s}-y^{2} p_{2}+y_{\perp}\right), \zeta_{i}=\left(\frac{p_{1}}{s}+z_{i \perp}^{2} p_{2}+z_{i \perp}\right)$ with $x_{*}=p_{2}^{\mu} x_{\mu}=\frac{\sqrt{2}}{s} x^{+}$(s is the Mandelstam variable). The analytic expression of the NLO impact factor for DIS at high energies can be found in Ref. 839]. Note that the NLO impact factor is conformally (Möbius) invariant and is given by a linear combination of five conformal tensor structures as predicted in [843]. The next natural step would be the Fourier transformation of the result in ref. [839] (the NLO impact factor), which gives the momentum-space impact factor convenient for phenomenological applications (and available at present only as a combination of numerical and analytical expressions [844, 845, 846]).

Conclusions: We have briefly summarized the status of the NLO calculation of the structure function for DIS at high energy. The main ingredients for the full amplitude, namely the NLO BK kernel and the NLO IF, have been calculated. The main result of this analysis is that the OPE for high energy (Regge limit) is at the same status as the usual OPE in the Bjorken limit. This means that the factorization in rapidity did not break down at NLO accuracy. As an application of the factorization in rapidity, the full NLO analytic amplitude in $\mathcal{N}=4 \mathrm{SYM}$ was calculated, the NLO result for the Pomeron intercept at small $\alpha_{s}$ was confirmed, and for the first time the NLO Pomeron residue was obtained [847].

The Wilson line formalism proved to be very successful, not only in obtaining in a more efficient way many results that in the usual pertubative QCD mechanism (pQCD), were obtained after many years of calculations by several groups, but also to obtain some results that have not been obtained (not for lack of efforts) in the usual pQCD mechanism, like the NLO impact factor, the NLO conformal BFKL kernel and the NLO pomeron residue, and in addition to generalize these results to include the non linear effects dominant at high energies. Another example which proves the efficiency of this formalism is the calculation, in a very easy way, of the triple pomeron vertex for diffractive and non-diffractive ("fan diagrams") processes, including the subleading $N_{c}$ contributions 848.

Acknowledgments: The author is grateful to the organizer of the workshop, in particular to Markus Diehl and Raju Venugopalan, and to the INT institute for the warm hospitality.

\section{Running Coupling in Small-x Physics}

\section{Yuri V. Kovchegov}

Running coupling corrections have been included into BFKL/BK/JIMWLK evolution following the Brodsky-Lepage-Mackenzie (BLM) scale-setting procedure [849] in [850, 834, 833, 851, 751. The BLM prescription requires one to first re-sum the contribution of all quark bubble corrections giving powers of $\alpha_{\mu} N_{f}$, with $N_{f}$ the number of quark flavors and $\alpha_{\mu}$ the physical coupling at some arbitrary renormalization scale $\mu$. One then has to complete $N_{f}$ to the full beta-function by replacing $N_{f} \rightarrow-6 \pi \beta_{2}$ in the obtained expression. Here, $\beta_{2}=\left(11 N_{c}-2 N_{f}\right) /(12 \pi)$ is the one-loop QCD beta-function. After this, the powers of $\alpha_{\mu} \beta_{2}$ should combine into physical running couplings $\alpha_{s}\left(Q^{2}\right)=\alpha_{\mu} /\left(1+\alpha_{\mu} \beta_{2} \ln \left(Q^{2} / \mu^{2}\right)\right)$ at various momentum scales $Q$ which would follow from this calculation. The running coupling below will be written in the $\overline{\mathrm{M} S}$ renormalization scheme.

Below we will concentrate on the case of running coupling corrections to the BFKL and BK evolution equations. Running-coupling corrections to the JIMWLK equation can 
be found in [834, 751]. At the moment the running coupling corrections to BK have been better explored numerically than those for JIMWLK.

Analytic result: Let us briefly summarize the results of [834, 833, 751]. The BalitskyKovchegov evolution equation with the running coupling corrections included (rcBK) reads

$$
\frac{\partial S\left(\underline{x}_{0}, \underline{x}_{1} ; Y\right)}{\partial Y}=\mathcal{R}[S]-\mathcal{S}[S] .
$$

Here we use the $S$-matrix notation, related to the forward dipole amplitude by $S\left(\underline{x}_{0}, \underline{x}_{1} ; Y\right)=$ $1-N\left(\underline{x}_{0}, \underline{x}_{1} ; Y\right)$. The first term on the right hand side of Eq. (5.49) is referred to as the running coupling contribution, while the second term on the right hand side of Eq. (5.49) is referred to as the subtraction contribution. Separation into the two parts is arbitrary, and was done differently in 833] and 834, with the net sum being the same [751].

The running coupling part was calculated independently in [833] and in [834]: the results of those calculations are

$$
\begin{aligned}
\mathcal{R}^{\mathrm{Bal}}[S] & =\int d^{2} z \tilde{K}^{\mathrm{Bal}}\left(\underline{x}_{0}, \underline{x}_{1}, \underline{z}\right)\left[S\left(\underline{x}_{0}, \underline{z} ; Y\right) S\left(\underline{z}, \underline{x}_{1} ; Y\right)-S\left(\underline{x}_{0}, \underline{x}_{1} ; Y\right)\right] \\
\mathcal{R}^{\mathrm{KW}}[S] & =\int d^{2} z \tilde{K}^{\mathrm{KW}}\left(\underline{x}_{0}, \underline{x}_{1}, \underline{z}\right)\left[S\left(\underline{x}_{0}, \underline{z} ; Y\right) S\left(\underline{z}, \underline{x}_{1} ; Y\right)-S\left(\underline{x}_{0}, \underline{x}_{1} ; Y\right)\right] .
\end{aligned}
$$

The integral kernels in the two cases are given by

$$
\tilde{K}^{\mathrm{Bal}}\left(\underline{r}, \underline{r}_{1}, \underline{r}_{2}\right)=\frac{N_{c} \alpha_{s}\left(r^{2}\right)}{2 \pi^{2}}\left[\frac{r^{2}}{r_{1}^{2} r_{2}^{2}}+\frac{1}{r_{1}^{2}}\left(\frac{\alpha_{s}\left(r_{1}^{2}\right)}{\alpha_{s}\left(r_{2}^{2}\right)}-1\right)+\frac{1}{r_{2}^{2}}\left(\frac{\alpha_{s}\left(r_{2}^{2}\right)}{\alpha_{s}\left(r_{1}^{2}\right)}-1\right)\right]
$$

as found in 833 , and by

$$
\tilde{K}^{\mathrm{KW}}\left(\underline{r}, \underline{r}_{1}, \underline{r}_{2}\right)=\frac{N_{c}}{2 \pi^{2}}\left[\alpha_{s}\left(r_{1}^{2}\right) \frac{1}{r_{1}^{2}}-2 \frac{\alpha_{s}\left(r_{1}^{2}\right) \alpha_{s}\left(r_{2}^{2}\right)}{\alpha_{s}\left(R^{2}\right)} \frac{\underline{r}_{1} \cdot \underline{r}_{2}}{r_{1}^{2} r_{2}^{2}}+\alpha_{s}\left(r_{2}^{2}\right) \frac{1}{r_{2}^{2}}\right],
$$

as found in 834, where

$$
R^{2}\left(\underline{r}, \underline{r}_{1}, \underline{r}_{2}\right)=r_{1} r_{2}\left(\frac{r_{2}}{r_{1}}\right)^{\frac{r_{1}^{2}+r_{2}^{2}}{r_{1}^{2}-r_{2}^{2}}-2 \frac{r_{1}^{2} r_{2}^{2}}{\underline{r}_{1} \cdot \underline{r}_{2}} \frac{1}{r_{1}^{2}-r_{2}^{2}}}
$$

One notices immediately that $\mathcal{R}^{\mathrm{Bal}}[S]$ calculated in 833 is different from $\mathcal{R}^{\mathrm{KW}}[S]$ calculated in 834] due to the difference in the kernels $\tilde{K}^{\mathrm{Bal}}$ and $\tilde{K}^{\mathrm{KW}}$ in Eqs. (5.52) and (5.53). However that does not imply disagreement between the calculations of [833] and [834]: after all, it is the full kernel on the right of Eq. (5.49), $\mathcal{R}[S]-\mathcal{S}[S]$, that needs to be compared. To do that, one has to calculate the second term on the right hand side of Eq. (5.49) (the subtraction contribution). This was done in [751], yielding

$$
\begin{aligned}
\mathcal{S}[S]= & \alpha_{\mu}^{2} \int d^{2} z_{1} d^{2} z_{2} K_{(1)}\left(\underline{x}_{0}, \underline{x}_{1} ; \underline{z}_{1}, \underline{z}_{2}\right) \\
& \times\left[S\left(\underline{x}_{0}, \underline{w}, Y\right) S\left(\underline{w}, \underline{x}_{1}, Y\right)-S\left(\underline{x}_{0}, \underline{z}_{1}, Y\right) S\left(\underline{z}_{2}, \underline{x}_{1}, Y\right)\right]
\end{aligned}
$$

and the re-summed BK kernel $K_{(1)}$ can be found in the original reference. Substituting $\underline{w}=\underline{z}_{1}$ (or, equivalently, $\underline{w}=\underline{z}_{2}$ ) in Eq. (5.55) yields the subtraction term $\mathcal{S}^{\mathrm{Bal}}[S]$, which has to be subtracted from $\mathcal{R}^{\mathrm{Bal}}[S]$ calculated in [833] and given by Eq. (5.50) to obtain 


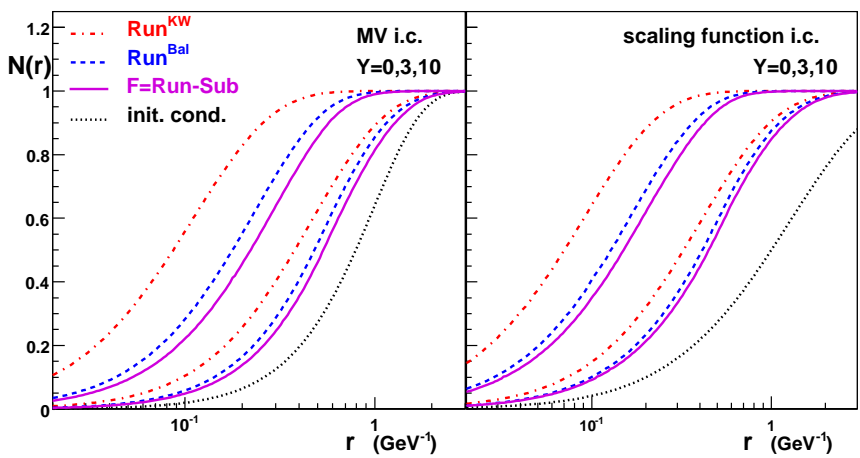

Figure 5.31. Solutions of the complete (all orders in $\alpha_{s} \beta_{2}$ ) evolution equation given in Eq. (5.49) (solid lines), and of the equation with Balitsky's (dashed lines) and KW's (dashed-dotted) running coupling schemes at rapidities $Y=0,3$ and 10. Left plot uses quasi-classical McLerran-Venugopalan (MV) initial condition. The right plot employs the initial condition given by the dipole amplitude at rapidity $Y=35$ evolved using Balitsky's running coupling scheme and with $r$-dependence rescaled down such that $Q_{s}=Q_{s}^{\prime}=1 \mathrm{GeV}$.

the complete evolution equation re-summing all orders of $\alpha_{s} N_{f}$ in the kernel. Substituting $\underline{w}=\underline{z}=\alpha \underline{z}_{1}+(1-\alpha) \underline{z}_{2}$ in Eq. (5.55) yields the term $\mathcal{S}^{\mathrm{KW}}[S]$, which has to be subtracted from $\mathcal{R}^{\mathrm{KW}}[S]$ calculated in 834 and given in Eq. (5.51) again to obtain the complete evolution equation re-summing all orders of $\alpha_{s} N_{f}$ in the kernel.

Numerical Solution: The numerical solution of the running-coupling BK (rcBK) evolution just presented was performed in [751] and plotted in Fig. [5.31, One plots the runningcoupling parts from Eqs. (5.50) and (5.51) [834, 833] (dashed and dash-dotted lines correspondingly), along with the full solution (solid line). As one can see the full solution is best approximated by the Balitsky's running coupling scheme from Eq. (5.50) [833]. Hence in most phenomenological applications one simply solves rcBK with Balitsky's prescription [85, 852. Note that the rcBK solution also exhibits the property of geometric scaling [752], as was shown in [751.

Running-coupling BFKL evolution: The running-coupling BFKL equation (rcBFKL) was constructed in 851] and reads

$$
\begin{aligned}
\frac{\partial \phi(k, Y)}{\partial Y}=\frac{N_{c}}{2 \pi^{2}} \int d^{2} q\{ & \frac{2}{(\boldsymbol{k}-\boldsymbol{q})^{2}} \alpha_{s}\left((\boldsymbol{k}-\boldsymbol{q})^{2} e^{-5 / 3}\right) \phi(q, Y) \\
& \left.-\frac{\boldsymbol{k}^{2}}{\boldsymbol{q}^{2}(\boldsymbol{k}-\boldsymbol{q})^{2}} \frac{\alpha_{s}\left(\boldsymbol{q}^{2} e^{-5 / 3}\right) \alpha_{s}\left((\boldsymbol{k}-\boldsymbol{q})^{2} e^{-5 / 3}\right)}{\alpha_{s}\left(\boldsymbol{k}^{2} e^{-5 / 3}\right)} \phi(k, Y)\right\},
\end{aligned}
$$

where the unintegrated gluon distribution $\phi(k, Y)$ is defined by

$$
N\left(x_{01}, Y\right)=\int \frac{d^{2} k}{(2 \pi)^{2}}\left(1-e^{i \boldsymbol{k} \cdot \boldsymbol{x}_{01}}\right) \tilde{N}(k, Y)
$$

with

$$
\alpha_{s}\left(k^{2}\right) \phi(k, Y)=\frac{N_{c} S_{\perp}}{(2 \pi)^{3}} k^{2} \tilde{N}(k, Y) .
$$


Here $S_{\perp}$ is the transverse area of the target. The running-coupling BFKL equation (5.56) was originally conjectured in [853, 854] by postulating the validity of the bootstrap equation for running-coupling corrections.

\section{Running-coupling and higher-order effects on the saturation scale}

\section{Guillaume Beuf}

The DGLAP [855, 856, 857] and BFKL [858, 59, 60] equations give the evolution with kinematics of the partonic content of hadrons and nuclei in the regime where these are dilute. As these equations are linear, they can be solved analytically by using a Mellin tranform. By contrast, when the phenomenon of gluon saturation [742] is taken taken into account, the relevant evolution equations - B-JIMWLK [770, 756, 757, 759, 760, 761, 745, 762, 763] or BK [770, 741, 747] - are nonlinear, and thus cannot be solved analytically.

Nevertheless, the solutions of these nonlinear equations in the leading order (LO) approximation (where the coupling $\alpha_{s}$ is kept fixed) are well understood, by combining results from numerical simulations [859, 750, 860] and analytical asymptotic expansions [755, 861, 862, 863, 864. Indeed, the BK equation belongs to a well-studied class of nonlinear equations, whose solutions develop asymptotically a universal traveling wave-front structure [865, 866], which is independent of the initial condition 3 . In the context of QCD, that traveling wavefront structure of the solution implies the geometric scaling [752] property found in the DIS data at HERA: the total virtual photon - target cross sections $\sigma_{T, L}^{\gamma^{*}}\left(Y, Q^{2}\right)$ depend on $Y$ and $Q^{2}$ essentially only through the combination $Q^{2} / Q_{s}^{2}(Y)$, because the dipole-target amplitude solution of the BK equation depends only on $r^{2} Q_{s}^{2}(Y)$ at large $Y, r$ being the dipole size. The evolution of the saturation scale $Q_{s}^{2}(Y)$ is obtained from the propagation of the wave-front. For the LO BK equation, one gets a large $Y$ expansion of the form

$$
\log Q_{s}^{2}(Y)=a_{1} Y+a_{0} \log Y+\text { Const. }+a_{-1 / 2} Y^{-1 / 2}+\mathcal{O}\left(Y^{-1}\right),
$$

where $a_{1}, a_{0}$ and $a_{-1 / 2}$ are three known universal coefficients [864], whereas the constant term and all the ones of order $Y^{-1}$ or less do depend on the initial conditions, i.e. on the nature of the target used for the DIS. From geometric considerations, the initial $Q_{s}^{2}$ of a nucleus $A$ is enhanced by a factor $A^{1 / 3}$ with respect to that of a proton. That nuclear enhancement of $Q_{s}^{2}(Y)$ is preserved by the LO high-energy evolution, in the constant term of the expansion (5.59). Both from numerical simulations and from the expansion (5.59), one learns that the evolution of $Q_{s}^{2}(Y)$ implied by the LO BK equation is too fast to be compatible with the data for DIS and other observables, which favor $\log Q_{s}^{2}(Y) \sim \lambda Y$, with $\lambda \simeq 0.2$ or 0.3 . We are thus forced to consider higher order corrections to the BK equation.

Running vs. fixed coupling: As discussed in this section by Chirilli, the BK equation is now known at next-to-leading order (NLO) [835, 837]. However, its solutions are much less understood than the ones of the LO equation. Indeed no numerical simulations of the full NLO BK equation have been performed yet, for technical reasons, but only simulations [859, 750, 860, 751, 867. of the BK equation with LO kernel and running coupling $\alpha_{s}$, with various prescriptions used to set the scale in the coupling. By contrast, it is non-trivial to go from fixed coupling to running coupling in the analytical studies, since it leads to a different class

\footnotetext{
${ }^{3}$ More precisely, in the QCD case, that asymptotic behavior in rapidity is reached from any initial condition compatible with perturbative QCD in the UV.
} 
of wave-front solutions, for which universality of the asymptotics is not fully established. The inclusion of other NLO corrections gives however no additional difficulty. Let us first discuss the effects of running coupling only.

A priori, the running of the coupling brings the additional scale $\Lambda_{Q C D}$ into the problem, which may spoil the geometric scaling property. Indeed, there is no interval where the solutions of the running coupling BK equation show exact geometric scaling, by contrast to fixed coupling solutions, but they satisfy an approximate geometric scaling in some range. Equivalently, the wave-front in the solutions is being slowly distorted during its propagation, instead of being uniformly translated as in the fixed coupling case.

Running coupling effects turn the asymptotic behavior of the saturation scale into $\log Q_{s}^{2}(Y) \propto \sqrt{Y}$, as found in early analytical studies [742, 755, 861, 863. More precisely its large $Y$ asymptotics writes

$$
\log \left(Q_{s}^{2}(Y) / \Lambda_{Q C D}^{2}\right)=b_{1 / 2} \sqrt{Y}+b_{1 / 6} Y^{1 / 6}+b_{0}+b_{-1 / 6} Y^{-1 / 6}+b_{-1 / 3} Y^{-1 / 3}+\mathcal{O}\left(Y^{-1 / 2}\right)
$$

where the first five terms are universal and known, whereas the following ones of order $Y^{-1 / 2}$ or less are sensitive to the initial conditions. The universality of the constant term $b_{0}$ in (5.60) implies that initial conditions effects such as the nuclear $A^{1 / 3}$ enhancement of $Q_{s}^{2}$ are washed-out at high rapidity when the coupling is running, as first predicted in [869]. Numerically, it has been found $[750,860,870$, that this effect happens at very high rapidity. Hence, the nuclear enhancement of $Q_{s}^{2}$, which is one of the motivations for doing nuclear DIS at the EIC, should still be present in the kinematical range accessible at the EIC. Remarkably, the evolution of the saturation scale in the running coupling case is such that very good fits of DIS data can be performed with solutions of the running coupling BK equation [85, 820], by contrast to the fixed coupling case, without the inclusion of other NLO effects.

Other NLO effects: Apart from the contributions re-summed into the running of the coupling, there are large NLO corrections to the BK kernel, related to the large NLO corrections to the BFKL kernel [722, 723].

In a conformal gauge field theory, terms of arbitrary $\mathrm{N}^{n} \mathrm{LO}$ order from the kernel would contribute at each order of the expansion (5.59). By contrast, the running of the coupling is dynamically quenching the effect on the solutions of higher order terms in the kernel. NLO contributions start to appear at order $Y^{0}$ in (5.60), NNLO contributions at order $Y^{-1 / 2}$ and so on. Moreover, the coefficient $b_{-1 / 6}$ has been found to be NLO-independent [868]. Apart from the running of the coupling, NLO contributions thus affect mostly the normalization of $Q_{s}^{2}(Y)$ at large $Y$, via $b_{0}$, and only mildly the asymptotic $Y$-evolution of $Q_{s}^{2}(Y)$, via $b_{-1 / 3} Y^{-1 / 3}$ and further subleading terms. That property is indeed seen in numerical simulations with running coupling and a subset of other NLO contributions included [867]. That result shed some light on the spectacular success of the running coupling LO BK equation to describe DIS data. There is a degeneracy in (5.60) between the contribution of $\Lambda_{Q C D}$ and $b_{0}$ to $Q_{s}^{2}(Y)$. Hence, treating $\Lambda_{Q C D}$ as a free fit parameter as in Refs. [85, 820] allows one to fit the bulk of NLO effects, without actually simulating the BK evolution with NLO kernel.

Several prescriptions [833, 834] have been proposed to split NLO corrections into contributions to the running coupling or to the kernel. Hence, BK equations with running

\footnotetext{
${ }^{4}$ The calculation of $b_{0}, b_{-1 / 6}$ and $b_{-1 / 3}$ has been performed recently in 868 .
} 
coupling and LO kernel obtained following different prescriptions differ formally by terms of order NLO and beyond in the kernel. In numerical simulations of such running coupling LO BK equations [751], solutions with different prescriptions differ at large $Y$ mostly by a constant rescaling of $Q_{s}^{2}(Y)$, in agreement with our previous discussion.

The problems brought by the impact-parameter dependence: Implicitly, we have discussed so far only results from studies of impact parameter independent solutions of the BK equation. The BK equation preserves unitarity at fixed impact parameter. However, its impact parameter dependent solutions violate unitarity since they violate the Froissart bound [871] on the cross-section [872, 867], due to the unphysical possibility of gluon emission at arbitrarily long range in the transverse plane. The running of the coupling reveals another problem: there is a reappearance of the diffusion into the infrared [867], which was thought to be cured by gluon saturation, from studies of impact parameter independent solutions of the BK equation. Hence, the impact parameter dependent solutions of the BK solutions are very sensitive to strongly coupled infrared physics, which is not yet implemented in the formalism. This is the most challenging open theoretical problem with regard to gluon saturation. Therefore, it is not yet clear to what extent the results about impact parameter independent solutions presented in the previous sections are reliable for realistic proton or nuclear targets. 


\subsection{Diffractive DIS $\left(\mathbf{F}_{2}^{D}, \mathbf{F}_{L}^{D}\right.$, charm contribution)}

\section{Diffraction in $\mathrm{e}+\mathrm{p}$ and $\mathrm{e}+\mathrm{A}$ collisions}

\section{Cyrille Marquet}

A non-negligible fraction of the events in DIS are diffractive, meaning that the hadronic target, of mass $M$, escapes the collision intact. As a colorless object has been exchanged in the t-channel, there is rapidity gap void of particles in the final state, between the outgoing target and the diffractive final state $X$, made up of all the other particles in the event. On top of $x$ and $Q^{2}$, two additional kinematic invariants are needed to characterize diffraction in DIS: the momentum transfer $t<0$ at the hadronic vertex, and the mass $M_{X}$ of the diffractive final state. In practice, the variable $M_{X}$ is sometimes traded for $\beta$ and the variable $x$ is traded for $x_{\mathbb{P}}$-these are defined as

$$
\beta=\frac{Q^{2}}{Q^{2}+M_{X}^{2}-t} ; x_{\mathbb{P}}=\frac{x}{\beta}=\frac{Q^{2}+M_{X}^{2}-t}{Q^{2}+W^{2}-M^{2}} .
$$

Small values of $\beta$ refer to events with diffractive masses much bigger than the photon virtuality, while values of $\beta$ close to unity refer to the opposite situation. $x_{\mathbb{P}}$ is useful because it characterizes the size of the rapidity gap $\Delta \eta \simeq \ln \left(1 / x_{\mathbb{P}}\right)$.

There are events in which the hadronic target, instead of staying intact, may dissociate into a low-mass excited state $\mathrm{Y}$, while still leaving a rapidity gap in the final state. These events are also classified as diffractive, they occur only if the mass $M_{Y}$ of the excited state is close enough to the initial mass $M$. Coherent diffraction is employed when the target scatters elastically $(\mathrm{ep} \rightarrow \mathrm{eXp}$ ), while incoherent diffraction refers to the more general case $\mathrm{ep} \rightarrow \mathrm{eXY}$ which is a sum of coherent diffraction $(\mathrm{Y}=\mathrm{p})$ and target-dissociative diffraction $(Y \neq p)$. The former dominates at low $|t|$ and the latter at large $|t|$.

While in the leading-twist approximation of QCD there is a collinear factorization theorem to compute diffractive structure functions in DIS at large $Q^{2}$, the description of hard diffraction in this framework is not as natural as for inclusive events. This is reflected in the fact that standard parton distribution functions (pdfs) are of no help to compute $F_{2}^{D}$, and one has to introduce a different set of parton distributions called diffractive pdfs (dpdfs). Therefore in the collinear factorization framework, the description of the parton content of the proton depends on whether or not the final state is diffractive. While this is successful - and should be since collinear factorization is a good approximation of QCD at large $Q^{2}$ - conceptually it is not so satisfactory as one would like to be able to describe any process with a single proton wave function.

No further conceptual advances are expected within the leading-twist approximation of QCD. There are some technical improvements that can be made, for instance it is nowadays practically impossible to extract dpdfs without assuming what is called Regge factorization: $\operatorname{dpdf}\left(x_{\mathbb{P}}, t, \beta, Q^{2}\right)=f\left(x_{\mathbb{P}}, t\right) g\left(\beta, Q^{2}\right)$. This is not satisfactory, since such a factorization is not a property of QCD. However, there is little doubt that if one could bypass this practical problem - perhaps with a larger data sample in all four directions: $Q^{2}, \beta, x_{\mathbb{P}}$ and $t$ - this approach would succeed at large $Q^{2}$.

But in fact, the purpose of an electron-ion collider is not to check whether DGLAP evolution will work at large $Q^{2}$, the goal is rather to explore what we don't know as well: the non-linear regime of QCD where collinear factorization breaks down. To be more specific, we are interested in the regime $Q^{2}<5 \mathrm{GeV}^{2}$ and $x$ as small as possible. Interestingly enough, studying the non-linear saturation regime will be easier with diffractive than with inclusive 
measurements. This is so because at small $x$, diffractive processes are mostly sensitive to quantum fluctuations in the proton wave function that have a virtuality of order $Q_{s}^{2}$, instead of $Q^{2}$. As a result, power corrections (not the generic $\Lambda_{Q C D}^{2} / Q^{2}$ corrections, but rather the sub-class of them of order $Q_{s}^{2} / Q^{2}$ important at small $x$ ) are expected to come into play starting from a higher value of $Q^{2}$ in diffractive DIS, compared to inclusive DIS. In fact, there is already a hint that this is happening at HERA: collinear factorization starts to fail below about $2 \mathrm{GeV}^{2}$ in the case of $F_{2}$, while already below about $8 \mathrm{GeV}^{2}$ in the case of $F_{2}^{D}$.

The QCD description of diffractive DIS in the small- $x$ limit turns out to be much more insightful than that of the large- $Q^{2}$ limit. It is so because at small $x$, DDIS can be expressed in the Good-Walker picture (which was originally imagined for soft diffraction in hadronhadron collisions), with the benefit that, thanks to the point-like nature of the photon, the modeling part of the Good-Walker approach can be replaced by actual QCD computations. This remarkable realization of the Good-Walker picture in small- $x$ DIS is more commonly referred to as the dipole picture: dipoles are eigenstates of high-energy scattering in QCD, and it is known how to expand the photon wave function onto the dipole basis. At the end in this approach, the parton content of the proton - both in the linear and non-linear regimes - is parametrized through the dipole cross section. As a result, diffractive structure functions also feature geometric scaling [873. Another important fact is that at small $x$, diffraction can be entirely predicted, once the dipole cross section has been constrained with inclusive data.

In spite of the fact that this approach has been able to successfully predict $F_{2}^{D}$ at small $x$, there is still important conceptual progress to be made. For instance, the transverse impact parameter dependence of the dipole scattering amplitude is very poorly constrained. Indeed, one has been able to describe $F_{2}$ and correctly predict $F_{2}^{D}$ with two kinds of impact parameter dependences, neither of which is fully satisfactory. In a first class of dipole models, the impact parameter profile of the proton is independent of energy, yielding a dipole cross section bounded from above. In the other class of models, the black-disk regime of maximal scattering strength spreads too quickly in the transverse plane with increasing dipole size $r$, leading to a dipole cross section which diverges for large $r$. It is quite clear that the LHeC is needed to help us understand better this issue.

Finally, let us say a few important words on ep $\rightarrow \mathrm{eXY}$ diffractive events. In past experiments, events with $Y \neq p$ have mostly been regarded as background, and model-dependent subtractions have been applied to data, yielding large normalization uncertainties. Within the kinematic reach of HERA, it has been observed that the ratio $d \sigma^{e p \rightarrow e X Y} / d \sigma^{e p \rightarrow e X p}$ is a constant independent of all kinematic variables other than $M_{Y}$ and $t$ (that ratio increases with $M_{Y}$ and $|t|$ ). Here we would like to emphazise that proton-dissociative events are also intrinsically interesting. For instance, at small $x$ the cross section difference $d \sigma^{e p \rightarrow e X Y}-d \sigma^{e p \rightarrow e X p}$ is $1 / N_{c}^{2}$ suppressed, meaning that if it were measured accurately, it would give access to details of the QCD dynamics which are untestable otherwise. The EIC provides such an opportunity.

After many fixed target experiments, it took a collider to discover diffractive events in $\mathrm{e}+\mathrm{p}$. Since no e+A collider has ever been built, diffraction in e+A has simply never been measured. That such a deficiency exists in our knowledge of nuclear structure is compelling enough to build the EIC. Everything we would learn about DDIS off nuclei at the EIC will be new, in any kinematical domain, implying a huge discovery potential. Nevertheless, we have expectations of what diffraction off nuclei should look like, based on our current understanding of QCD. For instance, the theory of nuclear shadowing allows the 
constuction of nuclear DPDFs for large $Q^{2}$ physics, while within the Color Glass Condensate framework, nuclear diffractive structure functions can be predicted at small $x$. Depending on these kinematics, different patterns of nuclear shadowing or antishadowing as a function of $\beta$ and $x_{\mathbb{P}}$ are expected. This is just one example out of many that should be checked with an e+A collider. Since the current predictions rely on rather simple models for impact parameter dependence, they need to be confronted to data, in order to, in return, improve our understanding.

Finally, there is one aspect of diffraction which is specific to nuclei that one should mention. The structure of incoherent diffraction $\mathrm{eA} \rightarrow \mathrm{eXY}$ is more complex than with a proton target, and also can teach us a lot more. In the case of a target nucleus, we expect the following qualitative changes in the $t$ dependence. First, the low- $|t|$ regime in which the nucleus scatters elastically will be dominant up to a smaller value of $|t|$ (to about $|t|=0.05 \mathrm{GeV}^{2}$ ) compared to the proton case, reflecting the larger size of the nucleus. Then, the nucleus-dissociative regime will comprise two parts: an intermediate regime in momentum transfer up to about $0.7 \mathrm{GeV}^{2}$ where the nucleus will predominantly break up into its constituents nucleons, and a large- $-|t|$ regime where the nucleons inside the nucleus will also break up, implying pion production in the $Y$ system for instance. These are only qualitative expectations, it is crucial to study this aspect of diffraction quantitatively in order to complete our understanding of the structure of nuclei.

\section{Expectations for e+A from the CGC}

\section{Cyrille Marquet}

In this work, hard diffraction in electron-nucleus $(\mathrm{e}+\mathrm{A})$ collisions is considered within the IPsat model, 600] corresponding to the classical limit of the Color Glass Condensate approach. This effective theory of QCD at high partonic density is the most natural framework to describe the saturation phenomenon, and therefore to study e+A scattering at high energies, in particular diffractive observables. Here we shall focus on the nuclear diffractive structure function $F_{2, A}^{D}$.

Let us recall the kinematics of diffractive DIS: $\gamma^{*} A \rightarrow X A$. With a momentum transfer $t \leq 0$, the proton/nucleus gets out of the $\gamma^{*}-A$ collision intact, and is separated by a rapidity gap from the other final-state particles whose invariant mass we denote $M_{X}$. The photon virtuality is denoted $Q^{2}$, and the $\gamma^{*}-A$ total energy $W$. It is convenient to introduce the following variables: $x=Q^{2} /\left(Q^{2}+W^{2}\right), \beta=Q^{2} /\left(Q^{2}+M_{X}^{2}\right)$ and $x_{\mathbb{P}}=x / \beta$. The size of the rapidity gap is $\ln \left(1 / x_{\mathbb{P}}\right)$.

The diffractive structure function is expressed as a function of $\beta, x_{\mathbb{P}}, Q^{2}$, and $t$, and we will only consider the $t$-integrated structure function $F_{2}^{D, 3}$. While at large values of $x_{\mathbb{P}}$ and $Q^{2}$, the leading-twist collinear factorization is appropriate to describe hard diffraction off protons, this is not the case at small $x_{\mathbb{P}}$ or off nuclei, as higher twists are enhanced by $\sim\left(A / x_{\mathbb{P}}\right)^{0.3}$. In this situation, the dipole picture is better suited to address the problem. It naturally incorporates the description of both inclusive and diffractive events into a common theoretical framework: [781, 874, 875] the same dipole-nucleus scattering amplitudes, which can be computed treating the nucleus as a CGC, enter in the formulation of the inclusive and diffractive cross-sections.

Diffractive structure functions in the dipole picture: In our approach, $F_{2}^{D}=F_{T}^{q \bar{q}}+$ $F_{L}^{q \bar{q}}+F_{T}^{q \bar{q} g}$ where the different pieces correspond to transversely (T) or longitudinally (L) 
polarized photons dissociating into a $q \bar{q}$ or $q \bar{q} g$ final state. For instance, the $q \bar{q}$ contributions are

$$
\begin{aligned}
& x_{\mathbb{P}} F_{T}^{q \bar{q}}\left(\beta, x_{\mathbb{P}}, Q^{2}\right)=\frac{N_{c} Q^{4}}{8 \pi^{3} \beta} \sum_{f} e_{f}^{2} \int_{0}^{1} d z \Theta\left(\kappa_{f}^{2}\right) z(1-z)\left[f_{T}(z) \varepsilon_{f}^{2}(z) I_{1}\left(\kappa_{f}, \epsilon_{f}\right)+m_{f}^{2} I_{0}\left(\kappa_{f}, \epsilon_{f}\right)\right], \\
& x_{\mathbb{P}} F_{L}^{q \bar{q}}\left(\beta, x_{\mathbb{P}}, Q^{2}\right)=\frac{N_{c} Q^{6}}{8 \pi^{3} \beta} \sum_{f} e_{f}^{2} \int_{0}^{1} d z \Theta\left(\kappa_{f}^{2}\right) z(1-z) f_{L}(z) I_{0}\left(\kappa_{f}, \epsilon_{f}\right)
\end{aligned}
$$

with

$\varepsilon_{f}^{2}(z)=z(1-z) Q^{2}+m_{f}^{2}, \quad \kappa_{f}^{2}(z)=z(1-z) M_{X}^{2}-m_{f}^{2}, \quad f_{T}(z)=z^{2}+(1-z)^{2}, \quad f_{L}(z)=4 z^{2}(1-z)^{2}$.

The $x_{\mathbb{P}}$ dependence comes in the functions $I_{\lambda}$ from $N_{A}\left(r, b, x_{\mathbb{P}}\right)$, the $q \bar{q}$ dipole-nucleus scattering amplitude:

$$
I_{\lambda}(\kappa, \epsilon)=\int d^{2} b\left[\int_{0}^{\infty} r d r J_{\lambda}(\kappa r) K_{\lambda}(\epsilon r) N_{A}\left(r, b, x_{\mathbb{P}}\right)\right]^{2}
$$

where $J_{\lambda}$ and $K_{\lambda}$ are Bessel functions. In equation (5.64), the integration variables $r$ and $b$ are the $q \bar{q}$-dipole transverse size and its impact parameter.

In principle, it is justified to neglect final states containing gluons, because these are suppressed by extra powers of $\alpha_{s}$. However, for small values of $\beta$ or large values of $Q^{2}$, the $q \bar{q}$ pair will emit soft or collinear gluons whose emissions are accomponied by large logarithms $\ln (1 / \beta)$ or $\ln \left(Q^{2}\right)$ which compensate the factors of $\alpha_{s}$. In those situations, multiple gluon emissions should be re-summed; in practice, including the $q \bar{q} g$ final state is enough to describe the HERA data. In both the small $-\beta$ and large $-Q^{2}$ limits, this can be done within the dipole picture. An implementation of the $q \bar{q} g$ contribution $F_{T}^{q \bar{q} g}$ that correctly reproduces both limits was recently proposed [876], while at large $\beta$ and small $Q^{2}$, the $q \bar{q}$ contributions in equation (5.62) dominate. The formulae that we shall use can be found in ref. [876].

The dipole-nucleus scattering amplitude: We shall use the IPsat parametrization to describe the dipole-nucleus scattering amplitude:

$$
N_{A}(r, b, x)=1-e^{-r^{2} F(r, x) \sum_{i=1}^{A} T_{p}\left(b-b_{i}\right)}, \quad F\left(x, r^{2}\right)=\frac{\pi^{2}}{2 N_{c}} \alpha_{s}\left(\mu_{0}^{2}+\frac{C}{r^{2}}\right) x g\left(x, \mu_{0}^{2}+\frac{C}{r^{2}}\right) .
$$

This is a model of a nucleus whose nucleons interact independently. Indeed, $N_{A}$ is obtained from $A$ dipole-nucleon amplitudes $N_{p}=1-\exp \left[-r^{2} F(r, x) T_{p}(b)\right]$ assuming that the probability $1-N_{A}$ for the dipole not to interact with the nucleus is the product of the probabilities $1-N_{p}$ for the dipole not to interact with the nucleons. This assumption is not consistent with the CGC quantum evolution, which sums up nonlinear interactions between the nucleons. However, the classical limit of the dipole-CGC scattering amplitude can be thought of an initial condition (5.65). Note that in the small $r$ limit, one has $N_{A}=\sum_{i} N_{p}$, and there is no leading twist shadowing.

In (5.65), $T_{p}(b) \propto \exp \left[-b^{2} /\left(2 B_{G}\right)\right]$ is the impact parameter profile function in the proton with $\int d^{2} b T_{p}(b)=1$, and $F$ is proportional to the DGLAP evolved gluon distribution. The parameters $\mu_{0}, C$, and $B_{\mathrm{G}}$ (as well as two other parameters characterising the initial condition for the DGLAP evolution) are fit to reproduce the HERA data on the inclusive proton 

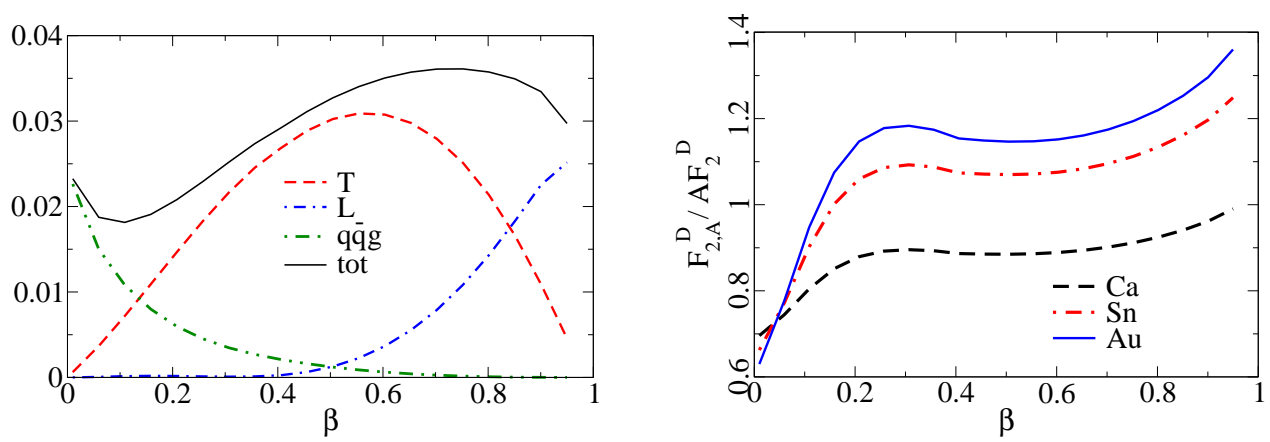

Figure 5.32. Left plot: $\beta$-dependence of the different contributions to the proton diffractive structure function $F_{2, p}^{D}$. Right plot: the ratio $F_{2, A}^{D} /\left(A F_{2, p}^{D}\right)$ as a function of $\beta$ for $\mathrm{Ca}$, Sn and Au nuclei. In both cases, results are for the "non breakup" case, and at $Q^{2}=5 \mathrm{GeV}^{2}$ and $x_{\mathbb{P}}=0.001$.

structure function $F_{2}$. The diffractive proton structure function $F_{2}^{D}$ is well reproduced [825] after adjusting $\alpha_{s}=0.14$ in the $q \bar{q} g$ component. Vector-meson production at HERA is also well described. 823

We introduced in (5.65) the coordinates of the individual nucleons $\left\{b_{i}\right\}$, they are distributed according to the Woods-Saxon distribution $T_{A}\left(b_{i}\right)$, which means that to compute an observable, one has to perform the following average

$$
\langle\mathcal{O}\rangle_{N} \equiv \int\left(\prod_{i=1}^{A} d^{2} b_{i} T_{A}\left(b_{i}\right)\right) \mathcal{O}\left(\left\{b_{i}\right\}\right)
$$

The Woods-Saxon parameters are measured from the electrical charge distribution, no additional parameters are introduced. The resulting dipole cross sections give a good agreement [826] with the small- $x$ NMC data on the nuclear structure function $F_{2, A}$. We will use this parametrization of $N_{A}$ to predict the nuclear diffractive structure function $F_{2, A}^{D}$.

Note that performing the average at the level of the amplitude (5.66), meaning calculating $\left\langle N_{A}\right\rangle_{N}^{2}$ in (5.62), imposes that the nucleus is intact in the final state. By contrast, when performing the average at the level of the cross-section, meaning calculating $\left\langle N_{A}^{2}\right\rangle_{N}$ in (5.62), one allows the nucleus to break up into individual nucleons, which will typically happen when the momentum transfer is bigger than the inverse nuclear radius. In what follows, we shall refer to those possibilities as "non breakup" and "breakup" cases.

Nuclear enhancement and suppression of $F_{2}^{D}$ : In fig. 5.32, the $\beta$ dependence of the diffractive structure function is displayed for $Q^{2}=5 \mathrm{GeV}^{2}$ and $x_{\mathbb{P}}=0.001$. In the left plot, the hierarchy of the different contributions is analysed in the case of $F_{2, p}^{D}$. The dominant contribution is: the $q \bar{q} g$ component for values of $\beta<0.1$, the longitudinally polarized $q \bar{q}$ component for values of $\beta>0.9$, and the transversely polarized $q \bar{q}$ component for intermediate values. In the case of $F_{2, A}^{D}$, this separation is still true but the $q \bar{q}$ and $q \bar{q} g$ components behave differently as a function of $A$. The $q \bar{q}$ components are enhanced compared to $A$ times the proton diffractive structure functions while the $q \bar{q} g$ component, on the contrary, is suppressed for nuclei compared to the proton (the $Q^{2}$ and $x_{\mathbb{P}}$ dependence of these effects will be discussed shortly).

This leads to a nuclear suppression of the diffractive structure function in the small $\beta$ region, and to an enhancement at large $\beta$. This is illustrated by the right plot of fig. 5.32 , where the ratio $F_{2, A}^{D} /\left(A F_{2, p}^{D}\right)$ is shown as a function of $\beta$ for different nuclei (for the "non 

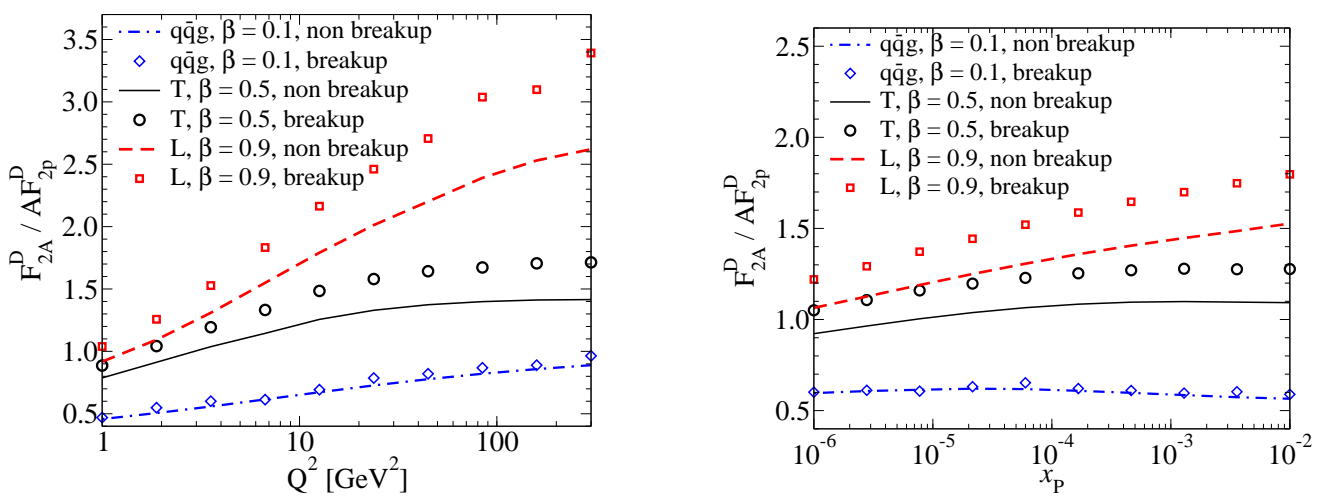

Figure 5.33. The ratios $F_{2, A}^{D, x} /\left(A F_{2, p}^{D, x}\right)$ of the different components $(x=q \bar{q} g, q \bar{q} T, q \bar{q} L)$ of the diffractive structure function for both "breakup" and "non breakup" cases. Left plot: as a function of $Q^{2}$ for $x_{\mathbb{P}}=0.001$. Right plot: as a function of $x_{\mathbb{P}}$ for $Q^{2}=5 \mathrm{GeV}^{2}$. In both cases, results are for $\mathrm{Au}$ nuclei and the different components are evaluated where they are dominant: at $\beta=0.1$ for $q \bar{q} g$, $\beta=0.5$ for $q \bar{q} T$ and $\beta=0.9$ for $q \bar{q} L$.

breakup" case). The net result of the different contributions is that $F_{2, A}^{D} / A$, for a large $\beta$ range down to 0.1 , is close to $F_{2, p}^{D}$, and is increasing with $A$.

In fig. [5.33, for the Au nucleus case, the ratios $F_{2, A}^{D} /\left(A F_{2, p}^{D}\right)$ of individual contributions are analyzed (for values of $\beta$ at which they are dominant). Comparisons between the "breakup" and "non breakup" cases are made, as functions of $Q^{2}$ (left plot) and $x_{\mathbb{P}}$ (right plot). For the $q \bar{q} g$ component, the nuclear suppression is almost constant (the suppression goes away slowly with $Q^{2}$ ). For the $q \bar{q}$ components, the enhancement becomes bigger with increasing $Q^{2}$ and $x_{\mathbb{P}}$. The result for the total diffractive cross-section in e+A scattering is that it decreases more slowly with increasing $Q^{2}$ or $x_{\mathbb{P}}$ compared to the e+p case. Finally, cross sections in the "non breakup" case are about $15 \%$ lower than in the "breakup" case.

Comparing with other approaches, we obtain similar features. We notice one interesting difference with the results obtained using diffractive parton distributions modified by leading twist shadowing [812]: even at large $\beta$, it is found that $F_{2, A}^{D} / A$ is suppressed compared to $F_{2, p}^{D}$ as a function of $Q^{2}$. This could be tested with measurements at a future EIC where diffraction will be an important part of a rich program. A typical nuclear enhancement of diffraction, for a $\mathrm{Au}$ nucleus, is a factor of $\sim 1.2$. Combining this with the typical nuclear suppression in the inclusive case $(\sim 0.8$, see 826$])$, we expect the fraction of diffractive events to be increased by a factor of $\sim 1.5$ compared to the proton, meaning 25 to $35 \%$ at the EIC.

\section{Expectations for diffraction e+A DIS from LT shadowing}

\section{Vadim Guzey and Mark Strikman}

The leading twist theory of nuclear shadowing (see section 5.2) that uses the connection between nuclear shadowing and diffraction [791] and allows one to predict nuclear parton distributions (PDFs) at small $x$ [803, 805, 806, 807] can also be used to predict nuclear diffractive PDFs and diffractive structure functions [812]. At small $x$ and in the nuclear target rest frame, the virtual photon interacts coherently with all nucleons of the nuclear target and the $\gamma^{*} A \rightarrow X A$ scattering amplitude is given by the sum of the multiple scattering contributions presented in Fig. 5.34. Graphs $a, b$, and $c$ correspond to the coherent 
interaction with one, two, and three nucleons of the nuclear target, respectively: graph $a$ is the impulse approximation; graphs $b$ and $c$ contribute to the shadowing correction. Note that the interactions with four and more nucleons (at the amplitude level) are not shown, but they are implied. The application of the Abramovsky-Gribov-Kancheli (AGK) cutting rules 804] allows one to relate these diagrams to the corresponding diagrams for the total cross section in $\gamma^{*} A$ scattering.
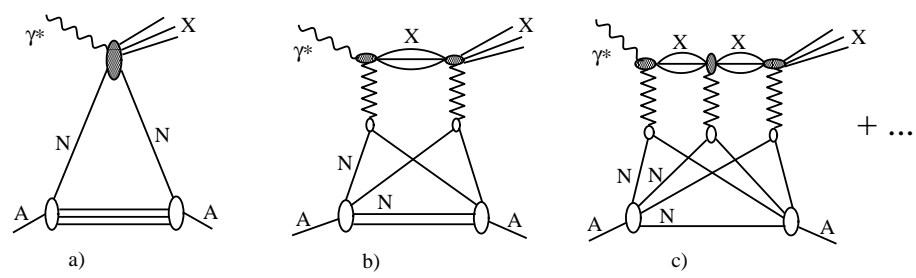

Figure 5.34. The multiple scattering series for the $\gamma^{*} A \rightarrow X A$ diffractive scattering amplitude. Graph $a$ is the impulse approximation; graphs $b$ and $c$ correspond to the interaction with two and three nucleons of the nuclear target, and contribute to the shadowing correction.

Combining the Glauber-Gribov multiple scattering formalism for the $\gamma^{*} A \rightarrow X A$ scattering amplitude with the QCD factorization theorem [808, one can derive the nuclear diffractive parton distribution of flavor $j$ [807, 812]:

$$
\begin{aligned}
\beta f_{j / A}^{D(3)}\left(\beta, Q_{0}^{2}, x_{\mathbb{P}}\right) & =4 \pi A^{2} \beta f_{j / N}^{D(4)}\left(\beta, Q_{0}^{2}, x_{\mathbb{P}}, t_{\mathrm{min}}\right) \int d^{2} b \\
& \times\left|\int_{-\infty}^{\infty} d z e^{i x_{\mathbb{P}} m_{N} z} e^{-\frac{A}{2}(1-i \eta) \sigma_{\mathrm{soft}}^{j}\left(x, Q_{0}^{2}\right) \int_{z}^{\infty} d z^{\prime} \rho_{A}\left(b, z^{\prime}\right)} \rho_{A}(b, z)\right|^{2}
\end{aligned}
$$

where the notation is the same as in eqs. (5.32) and (5.33).

While at the level of the interaction with two nucleons (graphs $a$ and $b$ in fig. 5.34) our predictions are model-independent, the contribution of the interaction with $N \geq 3$ nucleons requires additional model-dependent considerations since these interactions probe the details of the diffractive dynamics beyond what is encoded in the elementary diffractive distribution $f_{j}^{D(4)}$, as discussed in Section 5.2 . Viewing the hard probe (virtual photon) as a coherent superposition of the configurations that interact with the target nucleons with very different strengths (from align-jet configurations to point-like configurations) and which are present in the virtual photon with the probability $P(\sigma)$, one immediately sees from fig. 5.34 that diffractive scattering probes all moments of the cross section (color) fluctuations of the virtual photon, $\left\langle\sigma^{n}\right\rangle \equiv \int d \sigma P(\sigma) \sigma^{n}$, up to the order $n=2 A$. One should note that coherent diffraction probes these fluctuations differently from inclusive scattering. For instance, while the shadowing correction to the deuteron's usual parton distributions is proportional to $\left\langle\sigma^{2}\right\rangle$ (i.e., it is unambiguously expressed in terms of the corresponding diffractive PDFs, see eq. 5.32 in section 5.2 , the shadowing correction to the deuteron's diffractive PDFs is proportional to $\left\langle\sigma^{3}\right\rangle$ (interference of graphs $a$ and $b$ in fig. 5.34). (Note that the square of graph $b$ in fig. 5.34 proportional to $\left\langle\sigma^{4}\right\rangle$ also contributes, but its contribution is numerically very small.) As the cross section fluctuations of the virtual photon ( $\left\langle\sigma^{n}\right\rangle$ moments) are rather weakly constrained by the present data, predictions of the leading twist theory of nuclear shadowing contain unavoidable theoretical uncertainty associated with modeling of $\left\langle\sigma^{n}\right\rangle$ with $n \geq 3$. Precise measurements of the $t$ dependence of nuclear shadowing in $e D$ diffraction at an EIC will dramatically reduce this uncertainty by determining exactly these moments. 
Equation (5.67) determines nuclear diffractive PDFs at a certain initial scale $Q_{0}^{2}$ $\left(Q_{0}^{2}=4 \mathrm{GeV}^{2}\right.$ in our case). As a consequence of QCD factorization [808, the subsequent $Q^{2}$ evolution is given by the DGLAP evolution equations (at fixed $x_{\mathbb{P}}$ and $t$ ). As another consequence of the QCD factorization, the same nuclear diffractive PDFs $f_{j / A}^{D(3)}$ enter the perturbative QCD description of many processes and observables: the diffractive structure function $F_{2 A}^{D(3)}$, the longitudinal diffractive structure function $F_{L A}^{D(3)}$, the charm structure functions $F_{2 A}^{D(3)(c)}$ and $F_{L A}^{D(3)(c)}$, and diffractive
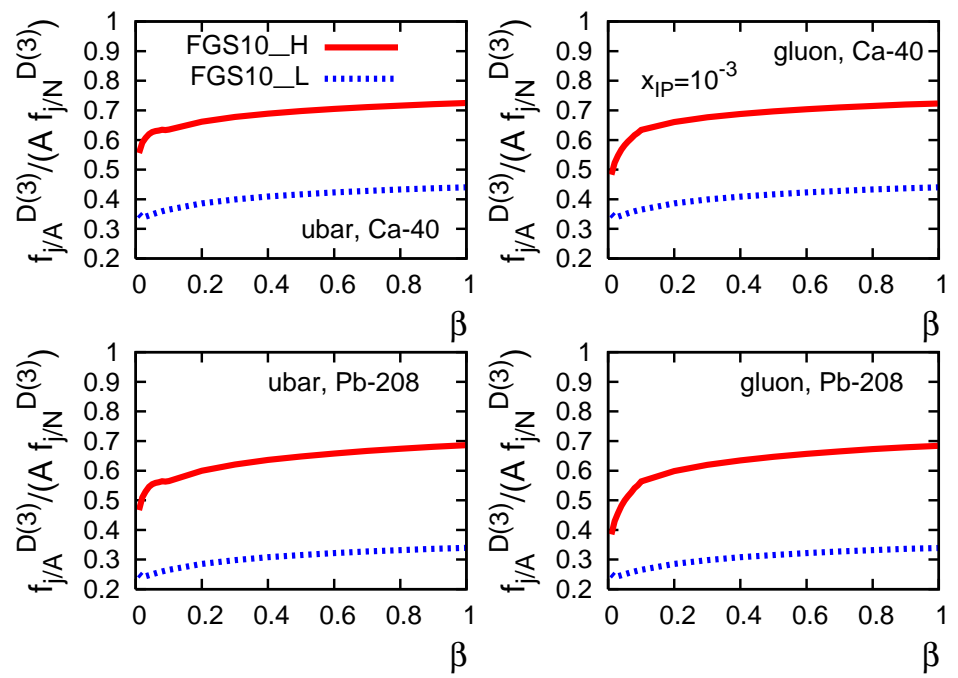

Figure 5.35. The leading twist theory of nuclear shadowing predictions for the ratio of nuclear to nucleon gluon and $\bar{u}$ quark diffractive PDFs, $f_{j / A}^{D(3)} /\left(A f_{j / N}^{D(3)}\right)$, as a function of $\beta$ at $x_{\mathbb{P}}=10^{-3}$ and $Q_{0}^{2}=4 \mathrm{GeV}^{2}$. The two sets of curves (labeled FGS10_H and FGS10_L) correspond to the two extreme scenarios of nuclear shadowing. electroproduction of jets and heavy flavors.

As an example of our predictions for nuclear diffractive PDFs, in fig. 5.35 we present the ratio of the nuclear $\left({ }^{40} \mathrm{Ca}\right.$ and $\left.{ }^{208} \mathrm{~Pb}\right)$ to nucleon diffractive PDFs, $f_{j / A}^{D(3)} /\left(A f_{j / N}^{D(3)}\right)$, as a function of $\beta$ at fixed $x_{\mathbb{P}}=10^{-3}$ and $Q_{0}^{2}=4 \mathrm{GeV}^{2}$. The left column of panels corresponds to the $\bar{u}$-quark distribution; the right column corresponds to the gluon distribution. The two sets of curves (labeled FGS10_H and FGS10_L) correspond to the two scenarios for the effective cross section $\sigma_{\text {soft }}^{j}$, which also determines shadowing effects as discussed in Section 5.2. As one can see from the comparison of fig. 5.35 to our predictions for the usual nuclear PDFs presented in fig. 5.19, nuclear diffractive PDFs are much more sensitive to the effect of the color fluctuations (the spread between the solid and dotted curves is much larger for $f_{j / A}^{D(3)} /\left(A f_{j / N}^{D(3)}\right)$ than for $\left.f_{j / A}\left(x, Q_{0}^{2}\right) /\left[A f_{j / N}\left(x, Q_{0}^{2}\right)\right]\right)$.

\begin{tabular}{|c|c|c|}
\hline$A /$ model & $F_{2 A, \text { incoh }}^{D(3)} / F_{2 A}^{D(3)}, x_{\mathbb{P}}=10^{-3}$ & $F_{2 A, \text { incoh }}^{D(3)} / F_{2 A}^{D(3)}, x_{\mathbb{P}}=10^{-2}$ \\
\hline${ }^{40} \mathrm{Ca}$, FGS10_H & 0.35 & 0.33 \\
${ }^{40} \mathrm{Ca}$, FGS10_L & 0.43 & 0.38 \\
${ }^{208} \mathrm{~Pb}$, FGS10_H & 0.12 & 0.11 \\
${ }^{208} \mathrm{~Pb}$, FGS10_L & 0.20 & 0.16 \\
\hline
\end{tabular}

Table 5.3. The leading twist theory of nuclear shadowing predictions for the ratio of the nuclear structure functions measured in incoherent and coherent diffraction in $e A$ DIS, $F_{2 A \text {, incoh }}^{D(3)} / F_{2 A}^{D(3)}$, at $x_{\mathbb{P}}=10^{-3}$ and $10^{-2}$ and $Q_{0}^{2}=4 \mathrm{GeV}^{2}$. The ratio is approximately $\beta$-independent.

The simplest observable to measure at an EIC is the diffractive structure function $F_{2 A}^{D(3)}$. Our predictions for $F_{2 A}^{D(3)} /\left(A F_{2 N}^{D(3)}\right)$ for $Q^{2} \sim$ few $\mathrm{GeV}^{2}$ are similar in shape and close in 
the absolute value for ${ }^{40} \mathrm{Ca}$ and model FGS10_H to the corresponding predictions made in the framework of the color dipole model, where the main contribution originates from the aligned-jet configurations [825]. (Note that at the level of the interaction with two nucleons, the expressions for the shadowing correction in our leading twist approach and in the dipole formalism are essentially the same and are unambiguously expressed in terms of $\gamma^{*}$-nucleon diffraction.) Hence, it appears that the $x_{\mathbb{P}}$ and $\beta$ dependence of coherent inclusive diffraction in $e A$ DIS at $Q^{2} \sim Q_{0}^{2}$ may not give unambiguous information on the onset of the non-linear regime of parton dynamics; to distinguish between the nonsaturation and saturation regimes one will need to study the $Q^{2}$ dependence of various diffractive observables.

In addition to inclusive coherent diffraction that we have discussed above, the leading twist theory of nuclear shadowing makes predictions for incoherent diffraction (with nuclear break-up into its constituents) in eA DIS, see [807] for details. An example of our predictions for the ratio of the nuclear structure functions measured in incoherent and coherent diffraction in $e A$ DIS at $x_{\mathbb{P}}=10^{-3}$ and $x_{\mathbb{P}}=10^{-2}$ and $Q_{0}^{2}=4 \mathrm{GeV}^{2}$ is presented in table 5.3. To a good accuracy, the ratio is approximately $\beta$-independent. 


\section{$5.5 k_{T}$-dependent gluons: SIDIS and jets}

\section{Dijet and Dihadron production at EIC}

Fabio Dominguez, Cyrille Marquet, Bowen Xiao and Feng Yuan

Dijet production at an EIC: The operator definition of the Weizsäcker-Williams (WW) gluon distribution can be written as follows [267, 283]:

$$
x G^{(1)}\left(x, k_{\perp}\right)=2 \int \frac{d \xi^{-} d \xi_{\perp}}{(2 \pi)^{3} P^{+}} e^{i x P^{+} \xi^{--i k_{\perp}} \cdot \xi_{\perp}}\left\langle P\left|\operatorname{Tr}\left[F^{+i}\left(\xi^{-}, \xi_{\perp}\right) \mathcal{U}^{[+] \dagger} F^{+i}(0) \mathcal{U}^{[+]}\right]\right| P\right\rangle
$$

where the gauge link $\mathcal{U}_{\xi}^{[+]}=U^{n}[0,+\infty ; 0] U^{n}\left[+\infty, \xi^{-} ; \xi_{\perp}\right]$ represents final state interactions with $U^{n}$ being the light-like Wilson line in covariant gauge. By choosing the light-cone gauge with certain boundary conditions for the gauge potential $\left(A_{\perp}\left(\zeta^{-}=\infty\right)=0\right.$ for the specific case above), we can drop out the gauge link contribution in equation (5.68) and find that this gluon distribution has the number density interpretation. Then, it can be calculated from the wave functions or the WW field of the nucleus target [729, 731, 877]. At small- $x$ for a large nucleus, it was found

$$
x G^{(1)}\left(x, k_{\perp}\right)=\frac{S_{\perp}}{\pi^{2} \alpha_{s}} \frac{N_{c}^{2}-1}{N_{c}} \int \frac{d^{2} r_{\perp}}{(2 \pi)^{2}} \frac{e^{-i k_{\perp} \cdot r_{\perp}}}{r_{\perp}^{2}}\left(1-e^{-\frac{r_{\perp}^{2} Q_{s}^{2}}{4}}\right),
$$

where $N_{c}=3$ is the number of colors, $S_{\perp}$ is the transverse area of the target nucleus, and $Q_{s}^{2}=\frac{g^{2} N_{c}}{4 \pi} \ln \frac{1}{r_{\perp}^{2} \lambda^{2}} \int d x^{-} \mu^{2}\left(x^{-}\right)$is the gluon saturation scale with $\mu^{2}$ the color charge density in a large nuclei.

The second gluon distribution, the Fourier transform of the dipole cross section, is defined in the fundamental representation

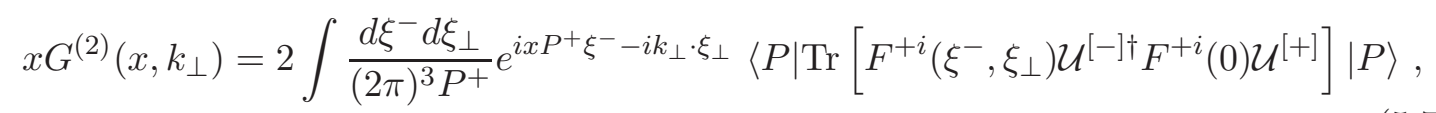

where the gauge link $\mathcal{U}_{\xi}^{[-]}=U^{n}[0,-\infty ; 0] U^{n}\left[-\infty, \xi^{-} ; \xi_{\perp}\right]$ stands for initial state interactions. It has been shown in ref. [283] that the Weizsäcker-Williams gluon distribution can be directly probed in the dijet production processes in DIS while the second gluon distribution enters in the total and semi-inclusive DIS cross sections. The quark-antiquark dijet cross section in DIS can be calculated in both the CGC formalism and the TMD approach. In the CGC formalism, the photon splits into a quark-antiquark pair which subsequently undergoes multiple interactions with the nucleus (see figure 5.36 left).

After averaging over the photon's polarization and summing over the quark and antiquark colors and helicities in the splitting functions $\psi_{\alpha \beta}^{T, L \lambda}\left(p^{+}, z, r\right)$, we obtain,

$$
\begin{aligned}
\frac{d \sigma^{\gamma_{T, L}^{*} A \rightarrow q \bar{q} X}}{d^{3} k_{1} d^{3} k_{2}}= & N_{c} \alpha_{e m} e_{q}^{2} \delta\left(p^{+}-k_{1}^{+}-k_{2}^{+}\right) \int \frac{\mathrm{d}^{2} x_{1}}{(2 \pi)^{2}} \frac{\mathrm{d}^{2} x_{1}^{\prime}}{(2 \pi)^{2}} \frac{\mathrm{d}^{2} x_{2}}{(2 \pi)^{2}} \frac{\mathrm{d}^{2} x_{2}^{\prime}}{(2 \pi)^{2}} \\
& \times e^{-i k_{1 \perp} \cdot\left(x_{1}-x_{1}^{\prime}\right)} e^{-i k_{2 \perp} \cdot\left(x_{2}-x_{2}^{\prime}\right)} \sum_{\lambda \alpha \beta} \psi_{\alpha \beta}^{T, L \lambda}\left(x_{1}-x_{2}\right) \psi_{\alpha \beta}^{T, L \lambda *}\left(x_{1}^{\prime}-x_{2}^{\prime}\right) \\
& \times\left[1+S_{x_{g}}^{(4)}\left(x_{1}, x_{2} ; x_{2}^{\prime}, x_{1}^{\prime}\right)-S_{x_{g}}^{(2)}\left(x_{1}, x_{2}\right)-S_{x_{g}}^{(2)}\left(x_{2}^{\prime}, x_{1}^{\prime}\right)\right]
\end{aligned}
$$

where $k_{1}$ and $k_{2}$ are momenta for the final state quark and antiquark, respectively. We further define $\vec{P}_{\perp}=\vec{k}_{1 \perp}-\vec{k}_{2 \perp}$ and $\vec{q}_{\perp}=\vec{k}_{1 \perp}+\vec{k}_{2 \perp}$. All transverse momenta are defined 

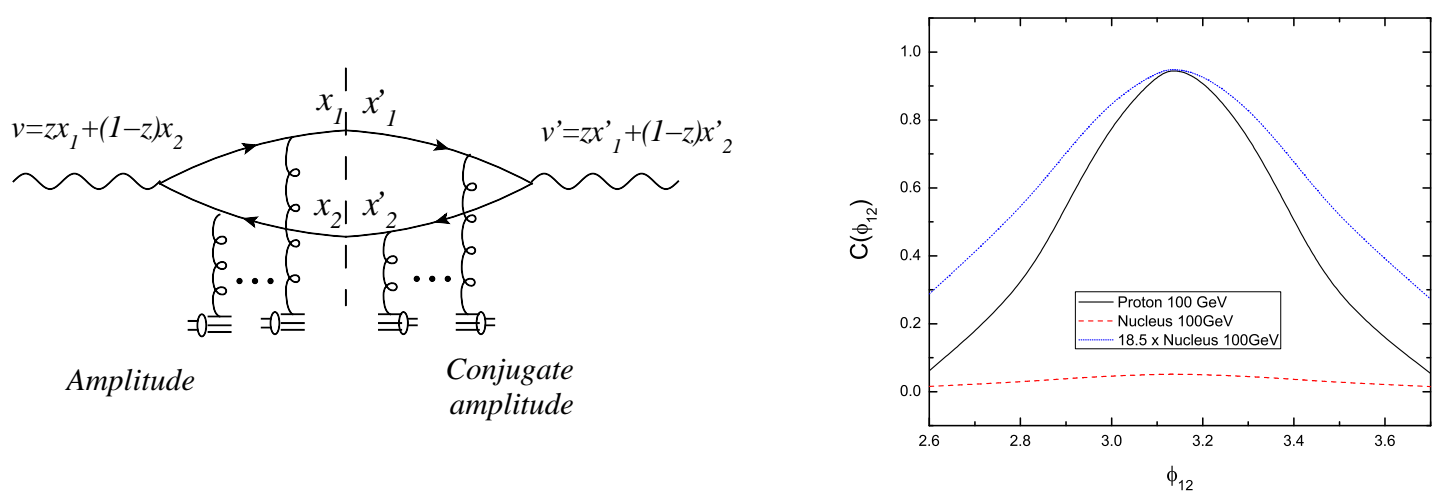

Figure 5.36. Left: Typical diagrams contributing to the cross section in the DIS at small- $x$ limit. Right: EIC dihadron correlation function

in the center of mass frame of the virtual photon and the nucleus target. The two- and four-point functions are defined as

$S_{x_{g}}^{(2)}\left(x_{1}, x_{2}\right)=\frac{1}{N_{c}}\left\langle\operatorname{Tr} U\left(x_{1}\right) U^{\dagger}\left(x_{2}\right)\right\rangle_{x_{g}}, S_{x_{g}}^{(4)}\left(x_{1}, x_{2} ; x_{2}^{\prime}, x_{1}^{\prime}\right)=\frac{1}{N_{c}}\left\langle\operatorname{Tr} U\left(x_{1}\right) U^{\dagger}\left(x_{1}^{\prime}\right) U\left(x_{2}^{\prime}\right) U^{\dagger}\left(x_{2}\right)\right\rangle_{x_{g}}$.

The notation $\langle\ldots\rangle_{x_{g}}$ is used for the CGC average of the color charges over the nuclear wave function where $x_{g}$ is the smallest fraction of longitudinal momentum probed, and is determined by the kinematics.

In order to simplify the above result and obtain a factorized expression, we take the correlation limit of equation (5.71). For convenience, we introduce the transverse coordinate variables: $u=x_{1}-x_{2}$ and $v=z x_{1}+(1-z) x_{2}$, and similarly for the primed coordinates. The respective conjugate momenta are $\tilde{P}_{\perp}=(1-z) k_{1 \perp}-z k_{2 \perp} \approx P_{\perp}$ and $q_{\perp}$, and therefore the correlation limit $\left(\tilde{P}_{\perp} \gg q_{\perp}\right)$ can be taken by assuming $u$ and $u^{\prime}$ are small and then expanding the integrand with respect to these two variables before performing the Fourier transform. Therefore, we can obtain the following expression which agrees perfectly with the TMD approach:

$$
\frac{d \sigma_{\mathrm{TMD}}^{\gamma_{T, L}^{*} A \rightarrow q \bar{q}+X}}{d \mathcal{P} . \mathcal{S} .}=\delta\left(x_{\gamma^{*}}-1\right) x_{g} G^{(1)}\left(x_{g}, q_{\perp}\right) H_{\gamma_{T, L}^{*} g \rightarrow q \bar{q}},
$$

where $x_{g}$ is the momentum fraction carried by the gluon and is determined by the kinematics, $x_{\gamma^{*}}=z_{q}+z_{\bar{q}}$ with $z_{q}=z$ and $z_{\bar{q}}=1-z$ being the momentum fractions of the virtual photon carried by the quark and antiquark, respectively. The phase space factor is defined as $d \mathcal{P} . \mathcal{S}$. $=d y_{1} d y_{2} d^{2} P_{\perp} d^{2} q_{\perp}$, and $y_{1}$ and $y_{2}$ are rapidities of the two outgoing particles in the lab frame. The leading order hard partonic cross section reads

$$
H_{\gamma_{T}^{*} g \rightarrow q \bar{q}}=\alpha_{s} \alpha_{e m} e_{q}^{2} \frac{\hat{s}^{2}+Q^{4}}{\left(\hat{s}+Q^{2}\right)^{4}}\left(\frac{\hat{u}}{\hat{t}}+\frac{\hat{t}}{\hat{u}}\right), \quad H_{\gamma_{L}^{*} g \rightarrow q \bar{q}}=\alpha_{s} \alpha_{e m} e_{q}^{2} \frac{8 \hat{s} Q^{2}}{\left(\hat{s}+Q^{2}\right)^{4}},
$$

with the usually defined partonic Mandelstam variables $\hat{s}=P_{\perp}^{2} /(z(1-z)), \hat{t}=-\left(P_{\perp}^{2}+\right.$ $\left.\epsilon_{f}^{2}\right) /(1-z)$, and $\hat{u}=-\left(P_{\perp}^{2}+\epsilon_{f}^{2}\right) / z$ with $\epsilon_{f}^{2}=z(1-z) Q^{2}$.

di-hadron correlations in DIS: By including the $k_{t}$ dependent fragmentation functions as proposed in ref. [332], one can compute the di-hadron production cross section and the 
correlation function $C\left(\phi_{12}\right)$ which is defined as follows

$$
C\left(\phi_{12}\right)=\frac{1}{\frac{d \sigma_{\text {tot SIDIS }}^{\gamma^{*} A h_{1} X}}{d z_{h 1}}} \frac{d \sigma_{\text {tot }}^{\gamma^{*} A \rightarrow h_{1} h_{2}+X}}{d z_{h 1} d z_{h 2} d \phi_{12}},
$$

where $z_{h 1}$ and $z_{h 2}$ are the longitudinal momentum fractions of two produced hadrons w.r.t. the photon momentum. $p_{1 \perp}$ and $p_{2 \perp}$ are the transverse momenta of these two back-toback hadrons and $\phi_{12}$ is the azimuthal angle between them. Thus, it is straightforward to numerically evaluate the correlation function and plot it in figure 5.36 right, where we fix $z_{h 1}=z_{h 2}=0.3, Q^{2}=4.0 \mathrm{GeV}^{2}, \sqrt{s}=100 \mathrm{GeV} . p_{1 \perp}$ and $p_{2 \perp}$ are integrated in the range $[2,3] \mathrm{GeV}$ and $[1,2] \mathrm{GeV}$, respectively. For the gluon distribution in gold nuclei, we have used a parametrization inspired by $G B W$ model. From figure 5.36, one sees the suppression of the away-side peak in nuclei due to gluon saturation.

Conclusion: First of all, we would like to compare the dijet production process in DIS to the inclusive and semi-inclusive DIS. As shown above, we derive that the dijet production cross section in DIS is proportional to the WW gluon distribution in the correlation limit. On the other hand, it is well-known that inclusive and semi-inclusive DIS involves the dipole cross section instead [878, which can be related to the second gluon distribution. This might look confusing at first sight, so let us take a closer look at equation (5.71). If one integrates over one of the outgoing momenta, say $k_{1}$, one can easily see that the corresponding coordinates in the amplitude and conjugate amplitude are identified $\left(x_{1}=x_{1}^{\prime}\right)$ and, therefore, the four-point function $S_{x_{g}}^{(4)}\left(x_{1}, x_{2} ; x_{2}^{\prime}, x_{1}^{\prime}\right)$ collapses to a two-point function $S_{x_{g}}^{(2)}\left(x_{2}, x_{2}^{\prime}\right)$. As a result, the SIDIS and inclusive DIS cross section only depend on two-point functions, thus they only involve the dipole gluon distribution.

Now we can see the unique feature of the dijet production process in DIS. By keeping the momenta of the quark and antiquark unintegrated, we can keep the full color structure of the four-point function which eventually leads to the WW gluon distribution in the correlation limit. Therefore, measuring the dijet production cross sections or dihadron correlations in DIS at future experimental facilities like EIC would give us a first direct and unique opportunity to probe and understand the Weizsäcker-Williams gluon distribution. Last but not least, by measuring the SIDIS and inclusive DIS cross section at EIC, one can also probe and constrain the dipole gluon distribution.

Acknowledgments: We thank A. Accardi, M. Diehl, L. McLerran, A. Mueller, J.-W. Qiu, A. Stasto and R. Venugopalan for stimulating discussion.

\section{Heavy quark production in $e+A$ collisions}

Victor P. Gonçalves

In this contribution we calculate the cross section of heavy quark production using the dipole approach and a nuclear saturation model based on the physics of the Color Glass Condensate (CGC) (For more details and references see Ref. [879]). The main input of our calculation is the dipole-nucleus cross section, $\sigma_{d A}(x, r)$, which is determined by the QCD dynamics at small $x$. In the eikonal approximation it is given by twice the impact-parameter $b$ integral of $\mathcal{N}^{A}(x, r, b)$, the forward dipole-target scattering amplitude for a dipole with size $r$ which encodes all the information about the hadronic scattering, and thus about the 
nonlinear and quantum effects in the hadron wave function. In our calculations we will assume as before that the forward dipole-nucleus amplitude is given by

$$
\mathcal{N}^{A}(x, r, b)=1-\exp \left[-\frac{1}{2} \sigma_{d p}\left(x, r^{2}\right) T_{A}(b)\right],
$$

where $\sigma_{d p}$ is the dipole-proton cross section and $T_{A}(b)$ is the nuclear profile function, which is obtained from a 3-parameter Fermi distribution for the nuclear density normalized to $A$. It is important to emphasize that this model describes the current experimental data on the nuclear structure function as well as includes the impact parameter dependence in the dipole nucleus cross section. For the dipole-proton cross section we will use the b-CGC model.

To estimate the magnitude of the saturation effects in heavy quark production, let us compare the CGC predictions with those associated to linear QCD dynamics. As a model for the linear regime we consider the leading logarithmic approximation for the dipole-target cross section, where $\sigma_{d A}$ is directly related to the nuclear gluon distribution $x g_{A}$ as follows

$$
\sigma_{d A}\left(x, r^{2}\right)=\frac{\pi^{2}}{3} r^{2} \alpha_{s} x g_{A}\left(x, 10 / r^{2}\right) .
$$

The use of this cross section in the formulae given below will produce results which we denote CT, from color transparency. In this limit we are disregarding multiple scatterings of the dipole with the nuclei and are assuming that the dipole interacts incoherently with the target. In what follows we consider two different models for the nuclear gluon distribution. In the first one we disregard the nuclear effects and assume that $x g_{A}\left(x, Q^{2}\right)=A \cdot x g_{N}\left(x, Q^{2}\right)$, with $x g_{N}$ being the gluon distribution in the proton and given by the GRV98 parameterization. We will refer to this model as CT. In the second model we take into account the nuclear effects in the nuclear gluon distribution as described by the EKS98 parameterization. We will call this model CT + Shad. In our calculations the charm quark mass is $m_{c}=1.5 \mathrm{GeV}$ and the bottom quark mass is $m_{b}=4.5 \mathrm{GeV}$.

Heavy quark production in the color dipole approach: Heavy quark production is usually estimated using the collinear factorization approach, where all partons involved are assumed to be on mass shell, carrying only longitudinal momenta, and their transverse momenta are neglected in the QCD matrix elements. On the other hand, in the large energy (small- $x$ ) limit, we have that the characteristic scale $\mu$ of the hard subprocess of parton scattering is much less than $\sqrt{s}$, but greater than the $\Lambda_{Q C D}$ parameter. In this limit, the effects of the finite transverse momenta of the incoming partons become important, and the factorization must be generalized, implying that the cross sections are now $k_{\perp}$-factorized into an off-shell partonic cross section and a $k_{\perp}$-unintegrated parton density function $\mathcal{F}\left(x, k_{\perp}\right)$, characterizing the $k_{\perp}$-factorization approach. Recently, an alternative approach to calculating the heavy quark production at high energies was proposed, considering the quasi-multi-Regge-kinematics (QMRK) framework. It is based on an effective theory implemented with the non-Abelian gauge-invariant action. The heavy quark production can also be calculated using the color dipole approach. This formalism can be obtained from the $k_{\perp}$-factorization approach after the Fourier transformation from the space of quark transverse momenta into the space of transverse coordinates. It is important to emphasize that this equivalence is only valid in the leading logarithmic approximation, being violated if the exact gluon kinematics is considered. A detailed discussion of the equivalence or 

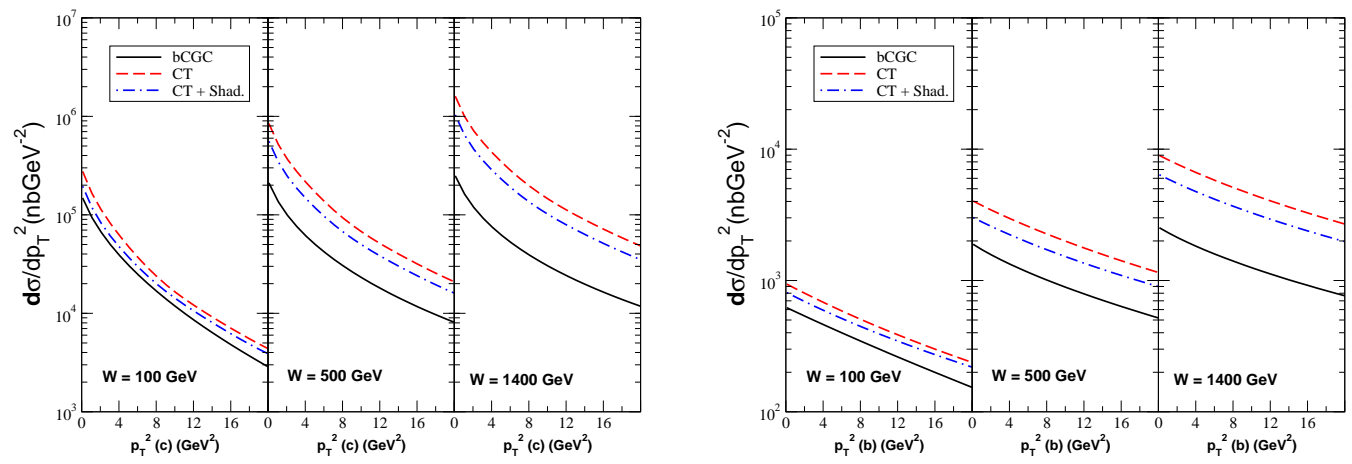

Figure 5.37. Transverse momentum charm spectrum (left) and bottom spectrum (right) for $Q^{2}=2$ $\mathrm{GeV}^{2}$ and different energies.

not between the dipole and the QMRK approaches still is an open question. The main advantage to use the color dipole formalism, is that it gives a simple unified picture of inclusive and diffractive processes and the saturation effects can be easily implemented in this approach.

In the color dipole approach, the heavy quark production cross section is given by

$$
\begin{aligned}
\frac{d \sigma\left(\gamma^{*} A \rightarrow Q X\right)}{d^{2} p_{Q}^{\perp}} & =\frac{6 e_{Q}^{2} \alpha_{e m}}{(2 \pi)^{2}} \int d \alpha\left\{\left[m_{Q}^{2}+4 Q^{2} \alpha^{2}(1-\alpha)^{2}\right]\left[\frac{I_{0}}{p_{Q}^{\perp 2}+\epsilon^{2}}-\frac{I_{2}}{4 \epsilon}\right]\right. \\
& \left.+\left[\alpha^{2}+(1-\alpha)^{2}\right]\left[\frac{p_{Q}^{\perp} \epsilon I_{1}}{p_{Q}^{\perp}+\epsilon^{2}}-\frac{I_{0}}{2}+\frac{\epsilon I_{2}}{4}\right]\right\}
\end{aligned}
$$

with

$$
I_{\lambda}=\int d r r J_{\lambda}\left(p_{Q}^{\perp} r\right) K_{\lambda}(\epsilon r) \sigma_{d A}(r) ; I_{2}=\int d r r^{2} J_{0}\left(p_{Q}^{\perp} r\right) K_{1}(\epsilon r) \sigma_{d A}(r)
$$

with $\lambda=0,1$, and $J_{0,1}$ and $K_{0,1}$ are Bessel functions, and $\epsilon^{2}=\alpha(1-\alpha) Q^{2}+m^{2}$.

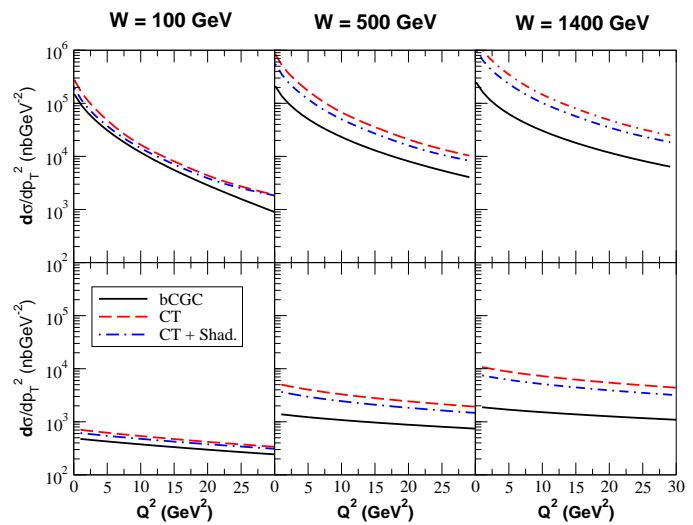

Figure 5.38. Dependence on the photon virtuality at $p_{T}^{2}=4 \mathrm{GeV}^{2}$.

Results: In Fig. 5.37 we show the transverse momentum spectrum of charm quarks. The main purpose of this figure is to show that the predictions of the linear physics (CT + 
Shad) differ from the total (i.e. bCGC) by a factor which increases with the energy $W$ and goes from $1.5(W=100 \mathrm{GeV})$ to $4(W=1400 \mathrm{GeV})$. Moreover, this difference persists for a wide momentum window. At very large $p_{T}$ we enter the deep linear regime and expect that the two curves coincide.

In Fig. 5.37 we show the transverse momentum spectrum of bottom quarks. As expected, we observe the same features of the charm distribution, except that now the nonlinear effects are weaker. Nevertheless they are still noticeable. In Fig. 5.38 we show the $Q^{2}$ dependence of the $p_{T}$ distribution at a fixed value $p_{T}=4 \mathrm{GeV}^{2}$ for different energies. The upper and lower panels show the charm and bottom distributions respectively. Here again, we observe a remarkable strength and persistence up to large virtualities of the differences between CT + Shad and bCGC.

Acknowledgments: The author thanks M. S. Kugeratski and F.S. Navarra for collaboration. 


\title{
5.6 b-dependent gluons: exclusive VM, DVCS
}

\section{Gluon Density in e+A : KLN, CGC, DGLAP Glauber, or Neither?}

\author{
William A. Horowitz
}

Perturbative quantum chromodynamics (pQCD) predicts a nontrivial expansion in the size of the nuclear wavefunction at small $x$ as the perturbative power law tails of the gluon distribution near the edge of the nucleus become important compared to the exponential dropoff due to confinement effects [871, 742, 766]. Similarly, in order to not violate unitarity, the enormous growth in the gluon parton distribution function as $x$ becomes small found via naïve application of DGLAP evolution (see [3] and references therein) must be tamed by perturbatively-calculable saturation effects [742, 766]. However it is not yet clear from a theoretical standpoint at what values of $x$ these nontrivial changes in the dominant dynamics occur [766]. Additionally a quantitative theoretical understanding of experimental heavy ion data requires a quantitative understanding of the initial geometry of a heavy ion collision. Certainly observables such as the azimuthal anisotropy of particles [880, 881, 882] are correlated with the anisotropy of the initial geometry; surprisingly the event-by-event fluctuations in the initial geometry also strongly affect these observables [883, 884]. In particular the viscosity to entropy ratio $(\eta / s)$ of the quark-gluon plasma (QGP) found by comparing hydrodynamics simulations to heavy ion collision data is directly related to the eccentricity of the initial thermal quark-gluon plasma distribution that is evolved hydrodynamically. Currently the uncertainty in the initial thermal distribution due to the uncertainty in the importance of saturation effects in the initial nuclear profiles is large enough that it is not clear whether the physics of the QGP is better described by leading order weakly-coupled perturbative quantum chromodynamics (LO pQCD) or by LO strongly-coupled anti-deSitter/conformal field theory (AdS/CFT) methods [881]. An experimental measurement of the spatial gluon distribution in a highly boosted nucleus, and hence the relevant physics in this kinematic range, would thus be a very interesting and important contribution to our understanding of QCD.

Exclusive vector meson production (EVMP) in e + A collisions has been proposed as a channel for just such a measurement [618, 885, 827]. In this section we will focus on the production of heavy vector mesons, in particular $J / \Psi$ mesons. To leading order, EVMP of a $J / \Psi$ meson occurs in an e + A collision when a photon emitted by the electron splits into a $c-\bar{c}$ pair which communicates with the gluon density in the highly boosted nucleus via a two gluon exchange and subsequently forms a $J / \Psi$ meson and nothing else (we will be interested here in coherent EVMP, in which case the nucleus remains intact); see figure 5.39 for a visualization of the process. It is precisely this two gluon exchange which yields a diffractive measurement of the gluon density in a nucleus.

Previous work 885] explored how modest changes in the Woods-Saxon distribution [886] of a nucleus might manifest themselves as changes in the diffractive peaks in EVMP if one assumes that the spatial distribution of gluons in a nucleus is proportional to the Glauber thickness function found from the Woods-Saxon distribution. That these modest changes do result in a visually obvious modification of the diffraction pattern motivated our further study, in which we consider whether two very different physical pictures of the gluon distribution in a highly boosted nucleus can be experimentally distinguished via EVMP: in particular we wish to compare the diffraction patterns that emerge when the gluon distribution 1) has normalization dictated by DGLAP evolution and spatial distribution 
given by the Glauber thickness function and 2) is given by the KLN parameterization (see [887, 888] and references therein) of the Color Glass Condensate (CGC) (see, e.g., [766, 889] for a review). We choose to investigate these two ansätze of the gluon distribution in nuclei as they have been the dominant models used in heavy ion physics calculations to estimate the uncertainty in the viscosity to entropy ratio of the QGP produced at RHIC due to the uncertainty of the currently poorly constrained initial conditions in heavy ion collisions [880, 881].

It is worth taking a moment to comment on some common - yet confusing - terminology in the EVMP field. As mentioned above, to leading order the coherent production of a vector meson in an e + A collision involves a two-gluon exchange between the $q-\bar{q}$ pair and the nucleus. If one assumes that all two-gluon exchanges occur independently, then one may exponentiate the single two-gluon exchange result. Making this independence assumption is often referred to in the EVMP field as using "saturation" physics because the cross section is unitarized via the exponentiation process. However this "saturation" does not refer to unitarizing the gluon distribution functions themselves. For instance in the "IP-Sat" 600] and "b-Sat" 823 models, where "Sat" is short for saturation, the $x$ evolution of the gluon PDF is effected through the use of the DGLAP equations. On the other hand, the "b-CGC" model [823] incorporates both the exponentiation of the two-gluon exchange and the CGC physics of the saturation of the gluon PDF. We note that, in principle, small- $x$ evolution effects and exponentiation effects in the dipole cross section should become appreciable simultaneously [737]. In order to (hopefully) make the presentation more clear, and to simplify some of the numerics, we will not exponentiate the two-gluon exchange; we will present results using only the leading order two-gluon exchange in which the gluon PDF is given either via DGLAP evolution or from the CGC. Any subsequent reference to "saturation" in this paper will refer to the saturation of the gluon distribution function alone.

Formalism: Following [600, 885, the diffractive production of a vector meson from a photon scattering off a target is

$$
\frac{d \sigma}{d t}=\frac{1}{16 \pi}\left|\int d^{2} \boldsymbol{r} \int \frac{d z}{4 \pi} \int d^{2} \boldsymbol{b}\langle V \mid \gamma\rangle_{T} e^{i \boldsymbol{b} \cdot \boldsymbol{\Delta}} \frac{d \sigma_{q \bar{q}}}{d^{2} \boldsymbol{b}}\right|^{2}
$$

where $\langle V \mid \gamma\rangle_{T}$ is the overlap of the vector meson wavefunction and the transversely polarized virtual photon wavefunction - the contribution from the longitudinally polarized photon is zero as we are interested in $Q^{2}=0$ photoproduction - and we used the photon-meson overlap and Gauss-LC model for the $J / \Psi$ wavefunction from [600] 5 , and $\boldsymbol{\Delta}^{2}=-t . d \sigma_{q \bar{q}} / d^{2} \boldsymbol{b}$ is the differential cross section for the interaction of the dipole with the target; its form depends on the physics assumptions we make for the nuclear gluon distribution, as we discuss in detail below.

DGLAP Evolution in $x$, Glauber Distribution of Gluons in $b$ : If we assume that the two gluon exchange from the dipole to the nucleus occurs within an individual nucleon then

$$
\frac{d \sigma_{q \bar{q}}}{d^{2} \boldsymbol{b}}=\frac{\pi^{2}}{N_{c}} r^{2} \alpha_{s}\left(\mu^{2}\right) x g\left(x, \mu^{2}\right) T(b),
$$

\footnotetext{
${ }^{5}$ Note that the normalization of the $J / \Psi$ wavefunction in 600 is erroneously reported as a factor of 100 smaller than the correct value; one can readily see this by comparing with the normalization condition defined in 600] and with the results reported in [823]. It is surprising that this error was not noted in 823 , in which the results found in [823] are explicitly compared to those in [600].
} 


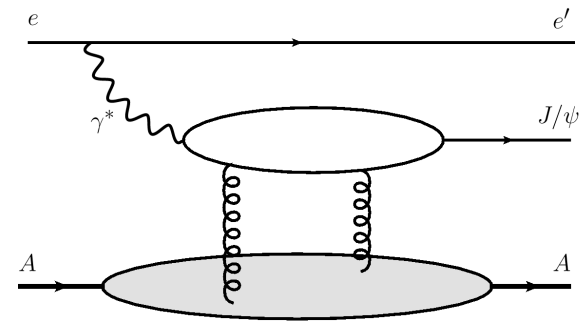

Figure 5.39. Leading order Feynman diagram for the exclusive vector meson production of a $J / \Psi$ meson.

where $r$ is the size of the dipole, $\mu=\sqrt{ }\left(\mu_{0}+C / r^{2}\right)$ is the relevant momentum scale for the dipole, $x g$ is the gluon distribution function, and

$$
T(b)=\frac{1}{2 \pi B_{G}} e^{-b^{2} / 2 B_{G}}
$$

is the assumed spatial distribution of gluons in a nucleon. We use the MSTW parameterization of the gluon PDF [22]. As described in [821], $\mu_{0}$ and $C$ are free parameters; as in [821, 600, 827, we take $\mu_{0}=1 \mathrm{GeV}^{2}$ and $C=4$. From HERA data [588] the measured slope of $d \sigma / d t$ yields $B_{G} \approx 4.25 \mathrm{GeV}^{-2}$ [600]. Then

$$
\frac{d \sigma^{D G L A P}}{d t}=4 \pi \sigma_{p}^{2} e^{-B_{G} t}\left|\int d b J_{0}(b \sqrt{t}) T_{A}(b)\right|^{2},
$$

where $J_{0}$ is the usual Bessel function, $T_{A}(b) \equiv \int d z \rho_{A}\left(\sqrt{b^{2}+z^{2}}\right)$, with $\int d^{2} \boldsymbol{b} T_{A}(b)=A$, is the usual thickness function, and $\rho_{A}$ is the density of the nucleus (here taken as the Woods-Saxon distribution of ${ }^{197} \mathrm{Au}$ with the usual $R=6.38 \mathrm{fm}$ and $a=0.535 \mathrm{fm}$ [890]) and

$$
\sigma_{p} \equiv \frac{1}{4 \pi} \int d^{2} \boldsymbol{r} \int d z\langle V \mid \gamma\rangle_{T} \frac{\pi^{2}}{N_{c}} r^{2} \alpha_{s}\left(\mu^{2}\right) x g\left(x, \mu^{2}\right)
$$

CGC Distribution of Gluons in $x$ and $b$ : Alternatively we may view the nucleus as a whole and that the gluon distribution is found from the CGC. In this case

$$
\frac{d \sigma_{q \bar{q}}}{d^{2} \boldsymbol{b}}=\frac{\pi^{2}}{N_{C}} r^{2} \alpha_{s}\left(\mu^{2}\right) x g_{A}\left(\mu^{2}, Q_{s}^{2}\right),
$$

where $x g_{A}$ is the integrated gluon distribution function related to the unintegrated gluon distribution (UGD) $\phi_{A}$ by

$$
x g_{A}\left(\mu^{2}, Q_{s}^{2}\right)=\int d^{2} \boldsymbol{k} \phi_{A}\left(k^{2}, Q_{s}^{2}\right)=\pi \int_{0}^{k_{\max }^{2}=\mu^{2}} d k^{2} \phi_{A}\left(k^{2}, Q_{s}^{2}\right)
$$

The $x$ and $b$ dependence of the two-gluon exchange dipole scattering formula, Eq. 5.84, comes in implicitly through the $x$ and $b$ dependence of $Q_{s}^{2}$ [888,

$$
Q_{s}^{2} \equiv \frac{2 \pi^{2}}{C_{F}} \alpha_{s}\left(Q_{s}^{2}\right) x g\left(x, Q_{s}^{2}\right) T_{A}(b),
$$


where $C_{F} \equiv\left(N_{c}^{2}-1\right) / 2 N_{c}$.

In principle one determines the UGD via the JIMWLK evolution equations or, in the large- $N_{c}$ limit, the BK evolution equations (see [766, 889] and references therein). However, instead of solving the full evolution equations many heavy ion physics calculations use instead the KLN prescription of the CGC (see, e.g., [887, 888]), which attempts to capture the main feature of CGC physics; in particular, the KLN UGD becomes saturated at momenta on the scale of the saturation scale $Q_{s}$. Because of its widespread use in heavy ion physics calculations and in order to simplify our own calculations we, too, will use the KLN UGD,

$$
\phi_{A}^{K L N}\left(k, Q_{s}^{2}\right)=\frac{\kappa C_{F} Q_{s}^{2}}{2 \pi^{3} \alpha_{s}\left(Q_{s}^{2}\right)} \begin{cases}\left(Q_{s}^{2}+\Lambda^{2}\right)^{-1}, & k^{2} \leq Q_{s}^{2} \\ \left(k^{2}+\Lambda^{2}\right)^{-1}, & k^{2}>Q_{s}^{2},\end{cases}
$$

where $\kappa$ is an $O(1)$ parameter meant to represent higher order corrections to the UGD, and $\Lambda=0.2 \mathrm{GeV}[888$.

In principle $\kappa$ is set by comparing to known experimental observables such as the measured multiplicity at midrapidity at RHIC [891, 892, 893, or LHC [894, 895] or to the diffractive cross sections for protons measured at HERA [588]. However we found that the results from the leading order multiplicity formula [887] are linearly dependent on the cutoff taken for $\alpha_{s}, \alpha_{s}^{\max }$. The KLN UGD itself, though, is not nearly as sensitive to $\alpha_{s}^{\max }$, so the multiplicity prescription does not provide a robust way of setting $\kappa$. We note in passing that the centrality dependence of the particles produced via the leading order CGC multiplicity formula using the KLN UGD's also depends on $\alpha_{s}^{\max }$. Perhaps the use of the next-to-leading order results in the UGD [896] and/or the production formula [897] will mitigate this dependence enough to make reasonable comparisons of CGC multiplicity to current data. Currently, though, there does not appear to be any quantitative estimate of the size of the dependence of the predicted CGC multiplicity as a function of centrality on $\alpha_{s}^{\max }$. $\kappa$ also cannot be set by comparing to the proton diffractive cross section as the currently available data does not probe regions of $x$ small enough such that $Q_{s}^{2}$ is a perturbative scale (at least when using the LO MSTW PDFs). In our calculations we will set $\kappa=1$.

It is important to contrast the interaction of the dipole in the KLN CGC approach taken here, in which the $q-\bar{q}$ pair interacts with the entire nucleus, and the Glauber approach, in which the pair interacts with individual nucleons. By interacting with individual nucleons the diffractive cross section for the DGLAP Glauber model picks up an extra exponential suppression in $t$ proportional to the square of the width of the nucleon, $B_{G}$.

Results: The saturation physics of the CGC has resulted in a wider and flatter gluon distribution than that from the Glauber treatment; the DGLAP growth of the small- $x$ gluon distribution - tamed by the saturation physics of the KLN CGC - leads to a significant enhancement in the cross section at $x=10^{-5}$ compared to that found using the KLN CGC gluon distribution. It is worth noting that the KLN prescription for the CGC satisfies the black disk limit.

We attempt to quantify the changes in both the nuclear gluonic width and density as a function of $x$ and note that even out to extremely small values of $x \sim 10^{-13}, b_{1 / 2}$ from the KLN CGC continues to rise sublinearly with $\log (s)$; thus the implementation of the KLN CGC used here, with the MSTW gluon PDF, satisfies the Froissart bound [871]. Intriguingly this sublinear (as opposed to linear) growth in radius as a function of $\log s$ is a surprise compared to other CGC parameterizations [872]. Note the enormous growth of 
the dipole cross section as $x$ decreases for the LO DGLAP-evolved gluonic density. This unitarity-violating enhancement is clearly reduced tremendously with the saturation physics of the KLN CGC.

The drastically faster increase in the gluon density from the DGLAP evolved PDF results in a cross section that increases much faster as a function of $x$ than for the KLN CGC case. As was shown in 827$]$ the incoherent cross section, in which the nucleus breaks up, begins to dominate the total diffractive cross section by $t \sim 0.02 \mathrm{GeV}^{-2}$. It is likely that the $t$ dependence of the incoherent EVMP of the two models will be different, although we do not provide a quantitative estimate here: the decrease in cross section as a function of $t$ for the DGLAP Glauber model will be enhanced by $\exp \left(-B_{G} t\right)$ due to the assumption that the heavy quark dipole interacts with individual nucleons. And in the case of coherent scattering one can discern a stronger $t$ dependence in the DGLAP Glauber results due precisely to the extra $\exp \left(-B_{G} t\right)$ factor that results from treating the nucleus as a collection of individual nucleons. More importantly, the much larger gluon density yields a particularly noticeable difference at $t=0$, where possible nuclear breakup effects are negligible.Even with the very large PDF uncertainties as $x$ decreases, there is a clear increase in the coherent diffractive cross section for the DGLAP Glauber dipole compared to the KLN CGC dipole.

Conclusions and Discussion: An enormous wealth of information on the gluonic structure of highly relativistic nuclei can be found using exclusive vector meson production. In particular we investigated the experimental signatures of the coherent scattering of a $c \bar{c}$ dipole onto a nucleus that results in an intact nucleus and a $J / \Psi$ meson in e + A collisions at eRHIC energies. We found that the diffractive cross section will readily experimentally differentiate between the two common initial highly boosted nucleus prescriptions used in heavy ion physics phenomenology: 1) the gluon density is found using DGLAP evolution and its spatial distribution is assumed to be proportional to the at-rest Glauber nuclear thickness function and 2) the gluon density and distribution is given by the KLN parameterization of the CGC. In particular there is the exciting possibility of literally watching a nucleus grow with center of mass energy as the positions in $t$ of the minima and maxima in the diffractive cross section for the saturation physics calculation depend quite strongly on $\log (x)$. On the other hand the DGLAP Glauber model yields a nucleus of constant size as a function of $x$; the positions in $t$ of the diffractive minima and maxima do not change as a function of $x$. At the same time one is determining the width of a nucleus in $\mathrm{e}+\mathrm{A}$ collisions, one will also measure the $x$ dependence of the normalization of $d \sigma / d t$. Due to the explosion of small- $x$ gluons the DGLAP Glauber approach yields a normalization that rapidly increases as a function of $x$; additionally the $t$ dependence of the DGLAP Glauber $d \sigma / d t$ is also quite strong as it is proportional to $\exp \left(-B_{G} t\right)$ due to the assumption that the $q-\bar{q}$ dipole interacts with individual nucleons. Conversely the KLN CGC dipole description does not have a strong $x$ dependence in its normalization due to its inclusion of saturation effects; similarly, the interaction of the dipole with the whole nuclear gluonic wavefunction yields a weaker $t$ dependence than is displayed by the DGLAP Glauber results.

It is clear that, at the very least, the striking difference between the $x$ dependence of the peaks and minima from the DGLAP Glauber model and the KLN CGC model are robust: these differences will persist should we use even more sophisticated models of these two physical pictures; the $x$ dependence of the peaks and minima will persist should we attempt to approximate multiple scattering within the nucleus by exponentiating the dipole cross

\footnotetext{
${ }^{6}$ fig. 8 in 885 also shows that the incoherent process quickly dominates the coherent one as a function of $t$, although we note that there was an error in the calculation of the figure and that the curves plotted do not correspond to the equations in the text of the paper.
} 
section, should we use a less approximate CGC calculation such as is found in [896], or should we examine the results from other vector mesons such as the $\phi$ or $\rho$. We regrettably leave the quantification of the diffractive cross section for these more sophisticated physical models and additional vector mesons for future work. Exponentiating the two-gluon exchange cross section will reduce the enormous growth in the diffractive cross section in the DGLAP Glauber picture compared to the CGC case; we suspect this reduction will not be too large, although we also leave the quantification of this reduction to future work.

Acknowledgments: The author wishes to thank E. Aschenauer, M. Diehl, Y. Kovchegov, H. Kowalski, T. Lappi, C. Marquet, T. Ullrich, and R. Venugopalan for invaluable discussions and the INT for its hospitality and support. The author wishes to especially thank Y. Kovchegov for reading and commenting on the manuscript.

\section{Coherent vs incoherent diffraction}

\section{Tuomas Lappi and Cyrille Marquet}

The purpose of this section is to investigate incoherent diffraction in a simpler context than with inclusive diffraction $\gamma^{*} A \rightarrow X Y$, mainly using diffractive vector meson production $\gamma^{*} A \rightarrow V Y$, where the diffractive final state $\mathrm{X}$ consists of a vector meson and nothing else, $A$ stands for the target nucleus and $Y$ for the final state it may dissociate into. At high energies, the $q \bar{q}$ dipole that the virtual photon has fluctuated into, scatters off the gluonic field of the nucleus before recombining into the vector meson. While this scattering involves a color-singlet exchange, leaving a rapidity gap in the final state, the nucleus can still interact elastically ( $Y=A$, this is called coherent diffraction) or inelastically (i.e. break up, called incoherent diffraction). In this process, the momentum transfer $t$ can be determined from the meson regardless of the fate of the target, and elastic and inelastic interactions of the target can be experimentally distinguished.

Kinematically, a low invariant mass of the system $Y$ corresponds to a large rapidity gap in the final state between that system and the vector meson, and implies that the longitudinal momentum of the meson is close to that of the incoming photon. In this case, the eikonal approximation can be assumed to compute the dipole-nucleus scattering. At small values of $x=\left(Q^{2}+M_{V}^{2}\right) /\left(Q^{2}+W^{2}\right)$ where $Q^{2}$ is the photon virtuality, $M_{V}$ the vector meson mass, and $W$ the energy of the $\gamma^{*}-A$ collision, a target proton can also be considered. Indeed in that case, since partons with an energy fraction as small as $x$ are probed in the target wave function, the dipole will scatter off large gluon densities generated by the QCD evolution.

In $\mathrm{e}+\mathrm{p}$ collisions, the cross-section is maximal at minimum momentum transfer with exclusive production (or coherent diffraction) dominating. As the transfer of momentum gets larger, the role of incoherent diffraction increases and eventually it becomes dominant, typically for momenta larger that the inverse target size; the elastic contribution decreases exponentially while the inelastic contribution decreases only as a power law. It is known that saturation models describe well the exclusive cross section [600, 898, 823, 899], while the BFKL Pomeron exchange approach works well for the target-dissociation cross-section [900, 901. In the section on proton breakup, we show that, within the Color Glass Condensate (CGC) picture of the small-x part of the hadronic wave function, both coherent and incoherent diffraction can be described in the same framework. We also explicitly calculate both contributions to the diffractive vector meson production cross-section using the 
McLerran-Venugopalan (MV) model for the CGC wave function, and discuss phenomenological consequences in the context of a future electron-ion collider [902].

Diffractive dissociation is an aspect of diffraction that changes qualitatively with nuclear targets. Indeed, the structure of incoherent diffraction $\mathrm{eA} \rightarrow \mathrm{eXY}$ is more complex than with a proton target, and also can teach us a lot more. In the case of a target nucleus, we expect the following qualitative changes in the $t$ dependence. First, the low- $|t|$ regime in which the nucleus scatters elastically will be dominant up to a smaller value of $|t|$ (to about $|t|=0.05 \mathrm{GeV}^{2}$ ) compared to the proton case, reflecting the bigger size of the nucleus. Then, the nucleus-dissociative regime will be made of two parts: an intermediate regime in momentum transfer up to about $0.7 \mathrm{GeV}^{2}$ where the nucleus will predominantly break up into its constituents nucleons, and a large- $-|t|$ regime where the nucleons inside the nucleus will also break up, implying pion production in the $Y$ system for instance. These are only qualitative expectations, it is crucial to study this aspect of diffraction quantitatively in order to complete our understanding of the structure of nuclei. The transition from the coherent to the intermediate regime is studied in the nuclear breakup section, following Ref. [827.

Proton breakup: In diffractive vector meson production, the relevant quantity is (the photon is a right mover, the CGC a left mover, and the gauge is $\mathcal{A}^{+}=0$ ):

$$
T_{\mathbf{x y}}\left[\mathcal{A}^{-}\right]=1-\frac{1}{N_{c}} \operatorname{Tr}\left(U_{\mathbf{y}}^{\dagger} U_{\mathbf{x}}\right), \quad \text { with } U_{\mathbf{x}}\left[\mathcal{A}^{-}\right]=\mathcal{P} \exp \left(i g_{S} \int d z^{+} T^{c} \mathcal{A}_{c}^{-}\left(z^{+}, \mathbf{x}\right)\right) .
$$

In terms of this object, the differential cross sections for a transversely $(\mathrm{T}$ ) or longitudinally (L) polarized photon are given by (with $t=-q_{\perp}^{2}$ the momentum transfer squared)

$$
\frac{d \sigma_{T, L}}{d t}=\frac{1}{4 \pi}\left\langle\left|\int d z d^{2} x d^{2} y e^{i q_{\perp} \cdot(z \mathbf{x}+(1-z) \mathbf{y})} \Psi_{T, L}(z, \mathbf{x}-\mathbf{y}) T_{\mathbf{x y}}\right|^{2}\right\rangle_{x},
$$

where $2 \Psi_{T}=\Psi_{V \mid \gamma}^{++}+\Psi_{V \mid \gamma}^{--}$and $\Psi_{L}=\Psi_{V \mid \gamma}^{00}$ with

$$
\Psi_{V \mid \gamma}^{\lambda^{\prime} \lambda}(z, \mathbf{r})=\sum_{h \bar{h}}\left[\phi_{\lambda^{\prime}}^{h \bar{h}}(z, \mathbf{r})\right]^{*} \phi_{\lambda}^{h \bar{h}}(z, \mathbf{r})
$$

the overlap between the photon and meson wave functions. $\lambda$ and $h$ denote polarizations and helicities while $z$ is the longitudinal momentum fraction of the photon carried by the quark and $\mathbf{x}$ and $\mathbf{y}$ are the quark and antiquark positions in the transverse plane.

The target average $\langle.\rangle_{x}$ is done with the CGC wave function squared $\left|\Phi_{x}\left[\mathcal{A}^{-}\right]\right|^{2}$ :

$$
\langle f\rangle_{x}=\int D A^{-}\left|\Phi_{x}\left[A^{-}\right]\right|^{2} f\left[A^{-}\right] .
$$

If one had imposed elastic scattering on the target side to describe the exclusive process $\gamma^{*} A \rightarrow V A$, the CGC average would be at the level of the amplitude, and the two-point function $\left\langle T_{\mathbf{x y}}\right\rangle_{x}$ inside the $|.|^{2}$ in (5.89), recovering the formula often used with dipole models.

Instead, when also including the target-dissociative part, the diffractive cross section involves the 4-point correlator $\left\langle T_{\mathbf{x y}} T_{\mathbf{u v}}\right\rangle_{x}$. In order to compute it, we must specify more about the CGC wave function. We shall use the McLerran-Venugopalan (MV) model [729, 903, 730, which is a Gaussian distribution for the color charges which generate the field $\mathcal{A}$ :

$$
\left|\Phi_{x}\left[A^{-}\right]\right|^{2}=\exp \left(-\int d^{2} x d^{2} y d z^{+} \frac{\rho_{c}\left(z^{+}, \mathbf{x}\right) \rho_{c}\left(z^{+}, \mathbf{y}\right)}{2 \mu^{2}\left(z^{+}\right)}\right),
$$


where the color charge $\rho_{c}$ and the field $\mathcal{A}_{c}^{-}$obey the Yang-Mills equation $-\nabla^{2} \mathcal{A}_{c}^{-}\left(z^{+}, \mathbf{x}\right)=$ $g_{S} \rho_{c}\left(z^{+}, \mathbf{x}\right)$. The variance of the distribution is the transverse color charge density squared along the projectile's path $\mu^{2}\left(z^{+}\right)$, with

$$
\left\langle\rho_{c}\left(z^{+}, \mathbf{x}\right) \rho_{d}\left(z^{\prime+}, \mathbf{y}\right)\right\rangle=\delta_{c d} \delta\left(z^{+}-z^{\prime+}\right) \delta^{(2)}(\mathbf{x}-\mathbf{y}) \mu^{2}\left(z^{+}\right) .
$$

The only parameter is the saturation momentum $Q_{s}$, with $Q_{s}^{2}$ proportional to the integrated color density squared. Note that there is no $x$ dependence in the MV model, it should be considered as an initial condition to the small- $x$ evolution.

The MV distribution is a Gaussian distribution, therefore one can compute any target average by expanding the Wilson lines in powers of $g_{S} \mathcal{A}_{c}^{-}$(see (5.88)), and then use Wick's theorem [904, 905]. The results for the 4-point function $\left\langle T_{\mathbf{x y}} T_{\mathbf{u v}}\right\rangle$ are given in [906]. We note that, in the large $-N_{c}$ limit, one has $\left\langle T_{\mathbf{x y}} T_{\mathbf{u v}}\right\rangle=\left\langle T_{\mathbf{x y}}\right\rangle\left\langle T_{\mathbf{u v}}\right\rangle$, which means that at small- $x$, the target-dissociative part of the diffractive cross-section in suppressed at large $N_{c}$, compared to the exclusive part.

The numerical results presented below are obtained with the $x$ evolution of the saturation scale modeled as in [83]: $Q_{s}(x)=\left(x_{0} / x\right)^{\lambda / 2} \mathrm{GeV}$, with $\lambda=0.277$ and $x_{0}=4.110^{-5}$ for the case of a target proton. The collinear logarithm of $Q_{s}$ is neglected, which corresponds to exact geometric scaling [752, 907, 873]: $F(x, \mathbf{r})=F\left[\mathbf{r}^{2} Q_{s}^{2}(x)\right]$. As an illustration, the resulting cross-section for diffractive $\mathrm{J} / \Psi$ production is displayed in Fig. 5.40, and separated into its coherent and incoherent contributions. The light-cone Gaussian J/ $\Psi$ wave function [908, 909] has been used in (5.90). At small values of $|t|$ where coherent diffraction dominates, our results are in agreement with HERA data 620 (one can get a better agreement with more realistic saturation models [600, 898, 823, 899], but this is not our point). Our model indicates that for $|t|>0.7 \mathrm{GeV}^{2}$ or so (this value slightly decreases when $Q^{2}$ increases), incoherent diffraction starts to dominate. This may be the reason why the data on exclusive production stop: there is too much proton-dissociative 'background'. We observe that this part of the cross-section decreases as a power law with $|t|$, rather than exponentially as the exclusive part does.

The model discussed in this work is well adapted to describe the low- and large- $-|t|$ regimes in the case of scattering off a nucleus, but not the intermediate

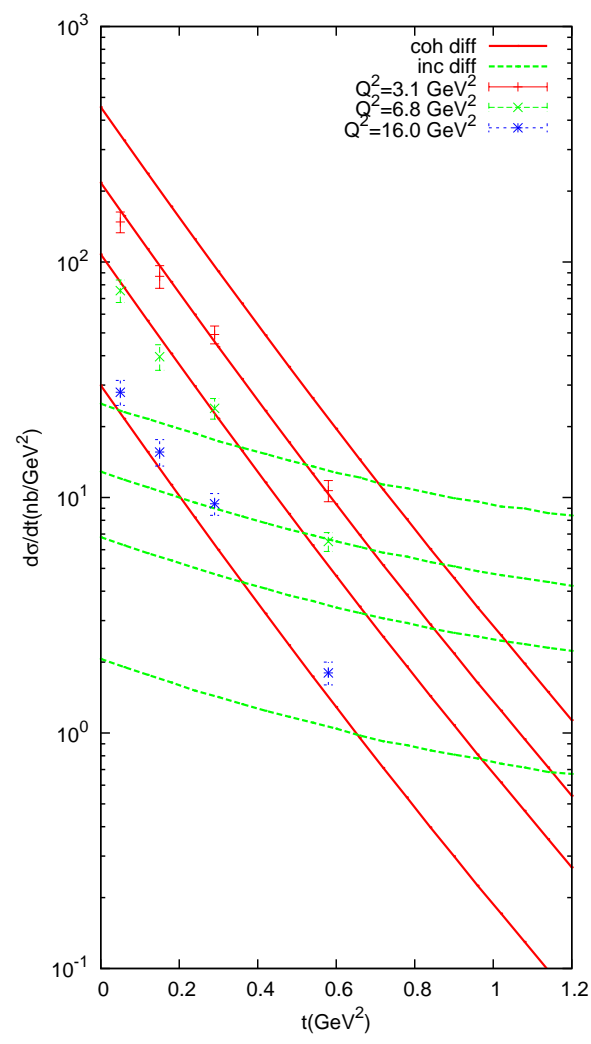

Figure 5.40. Diffractive $J / \Psi$ production in DIS at HERA for $W=90 \mathrm{GeV}$ and different $Q^{2}$ values. regime since the constituent nucleons are absent from the description. This problem has been addressed in a complementary setup in the case of inclusive diffraction off nuclei [826, 825], and the coherent diffraction regime was found to be dominant up to about $|t|=0.05 \mathrm{GeV}^{2}$. The vector meson production case will be addressed next. While in the proton case, both exclusive and diffractive processes can be measured, it is likely that at a future electron-ion collider, the exclusive cross section cannot 
be extracted: when the momentum transfer is small enough for the nucleus to stay intact, then it will escape too close to the beam to be detectable. Therefore the diffractive physics program will rely on our understanding of incoherent diffraction.

Nuclear breakup into its constituent nucleons: To simplify our calculation, we will here use a factorized impact parameter profile for the dipole cross section in a proton

$$
\frac{\mathrm{d} \sigma_{\mathrm{dip}}^{\mathrm{p}}}{\mathrm{d}^{2} \mathbf{b}_{T}}\left(\mathbf{b}_{T}, \mathbf{r}_{T}, x\right)=2\left(1-S_{p}\left(\mathbf{r}_{T}, \mathbf{b}_{T}, x\right)\right)=2 T_{p}\left(\mathbf{b}_{T}\right) \mathcal{N}(r, x)
$$

where $T_{p}$ is a Gaussian profile $T_{p}\left(\mathbf{b}_{T}\right)=\exp \left(-b^{2} / 2 B_{p}\right)$. In the following we shall consider two dipole cross section parametrizations, the IIM model [84, 910, 876], for which we take take $B_{p}=5.59 \mathrm{GeV}^{-2}$, and a factorized approximation of the IPsat parametrization 600, 823], for which $B_{p}=4.0 \mathrm{GeV}^{2}$. See [827] for a discussion of the origin of these values in different fits.

To extend the dipole cross section from protons to nuclei, we will take the independent scattering approximation that is usually used in Glauber theory and write the $S$-matrix as

$$
S_{A}\left(\mathbf{r}_{T}, \mathbf{b}_{T}, x\right)=\prod_{i=1}^{A} S_{p}\left(\mathbf{r}_{T}, \mathbf{b}_{T}-\mathbf{b}_{T i}, x\right) .
$$

Here $\mathbf{b}_{T i}$ are the nucleon coordinates. This independent scattering assumption is natural in IPsat-like parametrizations or the MV-model, where $r=\left|\mathbf{r}_{T}\right|, S\left(\mathbf{r}_{T}\right) \sim$ $e^{-r^{2} Q_{\mathrm{s}}{ }^{2} / 4}$ with a saturation scale $Q_{\mathrm{s}}{ }^{2}$ proportional to the nuclear thickness $T_{A}(b)$. High energy evolution, however, introduces an anomalous dimension that leads, in the nuclear case, to what could be called leading twist shadowing. With an anomalous dimension $S \sim e^{-\left(Q_{\mathrm{s}} r\right)^{2 \gamma}}$ with $\gamma \neq 1$, a proportionality $Q_{\mathrm{s}}{ }^{2} \sim T_{A}(b)$ is not equivalent to Eq. (5.95). A solution to this problem (see also the more detailed discussion in 825]) would require a realistic impact parameter dependent solution to the BK [770, 741, 747] equation which is not yet available. We point the reader to Ref. [872], for example, for a discussion of the difficulties. These are related to the long distance Coulomb tails that, physically, are regulated at the confinement length scale that is not enforced in

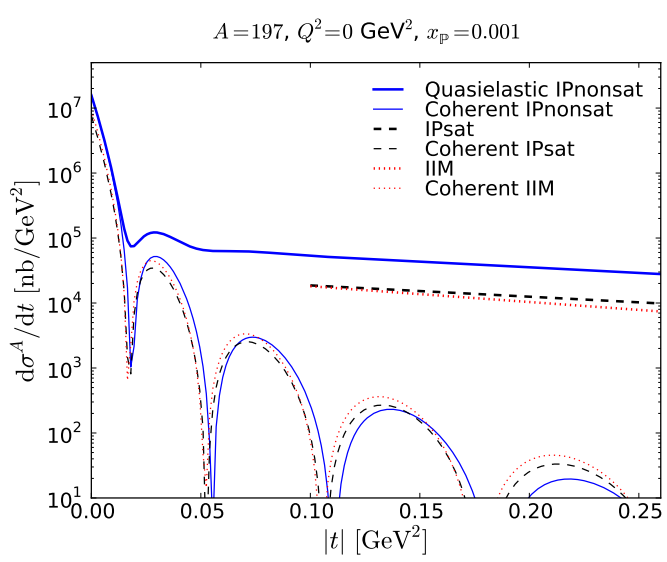

Figure 5.41. The quasielastic and coherent diffractive $J / \Psi$ cross sections in gold nuclei at $Q^{2}=0$ and $x_{\mathbb{P}}=0.001$. The IPsat and IIM parametrizations are shown. We also show the result for the linearized "IPnonsat" version (used e.g. in Ref. 911) where the incoherent cross section is explicitly $A$ times that of the proton. Our approximation (5.97) is not valid for small $|t|$ and has been left out of the plot. a first principles weak coupling calculation.

The average over the positions of the nucleon in the nucleus was given in eq. (5.66). The expectation valuedefined there is equivalent to the average over nucleon configurations in a Monte Carlo Glauber calculation. We are assuming that the positions $\mathbf{b}_{T i}$ are independent, i.e. neglecting nuclear correlations that would be a subject of interest in their own right (see e.g. [912]). The coherent cross section is obtained by averaging the amplitude before 
squaring it, $\left|\langle\mathcal{A}\rangle_{\mathrm{N}}\right|^{2}$, and the incoherent one is the variance $\left\langle|\mathcal{A}|^{2}\right\rangle_{\mathrm{N}}-\left|\langle\mathcal{A}\rangle_{\mathrm{N}}\right|^{2}$ that measures the fluctuations of the gluon density inside the nucleus. Because $\langle\mathcal{A}\rangle_{\mathrm{N}}$ is a very smooth function of $\mathbf{b}_{T}$, its Fourier transform vanishes rapidly for $\Delta \gtrsim 1 / R_{A}$. Therefore, at large $\Delta$, the quasielastic cross section is almost purely incoherent.

The cross section for quasielastic vector meson production is now expressed in terms of the dipole scattering amplitude as

$$
\begin{aligned}
\frac{\mathrm{d} \sigma^{\gamma^{*} A \rightarrow V A^{*}}}{\mathrm{~d} t}=\frac{R_{g}^{2}\left(1+\beta^{2}\right)}{16 \pi} \int \frac{\mathrm{d} z}{4 \pi} \frac{\mathrm{d} z^{\prime}}{4 \pi} \mathrm{d}^{2} \mathbf{r}_{T} \mathrm{~d}^{2} \mathbf{r}_{T}^{\prime} & \\
& \quad \times\left[\Psi_{V}^{*} \Psi\right](r, z, Q)\left[\Psi_{V}^{*} \Psi\right]\left(r^{\prime}, z^{\prime}, Q\right)\left\langle\left|\mathcal{A}_{q \bar{q}}\right|^{2}\left(x_{\mathbb{P}}, r, r^{\prime}, \boldsymbol{\Delta}_{T}\right)\right\rangle_{\mathrm{N}},
\end{aligned}
$$

where we have applied corrections for the skewedness, $R_{g}$, and the real part of the scattering amplitude (see e.g. 824]) We now average the square of the dipole scattering amplitude over the nucleon coordinates, using the assumptions of Eqs. (5.95) and (5.94) and taking the large $A$ limit. We are additionally assuming that $T_{A}$ is a smooth function on the distance scale defined by $B_{p}$. Averaging the square of the amplitude gives the total quasi-elastic contribution.

Note that Eqs. (5.95) and (5.94) have enabled us to write the leading contributions as proportional to the (Gaussian) proton impact parameter profile, which can then be Fourier-transformed analytically. Giving up either of these approximations would force us to numerically Fourier-transform the "lumpy" b-dependence corresponding to a fixed configuration of the nucleon positions. Keeping only the terms that contribute at large $|t| \gg 1 / R_{A}^{2}$ leaves us with the expression

$$
\begin{aligned}
\left|\mathcal{A}_{q \bar{q}}\right|^{2}\left(x_{\mathbb{P}}, r, r^{\prime}, \boldsymbol{\Delta}_{T}\right)= & 16 \pi^{2} B_{p}^{2} A \int \mathrm{d}^{2} \mathbf{b}_{T} \\
& \times e^{-B_{p} \boldsymbol{\Delta}_{T}^{2}} e^{-2 \pi B_{p}(A-1) T_{A}(b)\left[\mathcal{N}(r)+\mathcal{N}\left(r^{\prime}\right)\right]} \mathcal{N}(r) \mathcal{N}\left(r^{\prime}\right) T_{A}(b) .
\end{aligned}
$$

Equation (5.97) has a very clear interpretation. The squared amplitude is proportional to $A$ times the squared amplitude for scattering off a proton, corresponding to the dipole scattering independently off the nucleons in a nucleus. This sum of independent scatterings is then multiplied by a nuclear attenuation factor which accounts for the requirement that the dipole must not scatter inelastically off the other $A-1$ nucleons in the target (otherwise the interaction would not be diffractive). Note that factor $4 \pi B_{p} \mathcal{N}\left(r, x_{\mathbb{P}}\right)=\sigma_{p}\left(r, x_{\mathbb{P}}\right)$ is the proton-dipole cross section for a dipole of size $r$. Thus this attenuation corresponds to the probability of a dipole with a cross section which is the average of dipoles with $r$ and $r^{\prime}$ to pass though the nucleus. A similar expression can be found in Ref. [913] for example.

The coherent cross section in our approximation is given by

$$
\frac{\mathrm{d} \sigma^{\gamma^{*} A \rightarrow V A}}{\mathrm{~d} t}=\frac{R_{g}^{2}\left(1+\beta^{2}\right)}{16 \pi}\left|\left\langle\mathcal{A}\left(x_{\mathbb{P}}, Q^{2}, \boldsymbol{\Delta}_{T}\right)\right\rangle_{\mathrm{N}}\right|^{2},
$$

where in the large $A$ and smooth nucleus limit the amplitude is

$$
\left\langle\mathcal{A}\left(x_{\mathbb{P}}, Q^{2}, \boldsymbol{\Delta}_{T}\right)\right\rangle_{\mathrm{N}}=\int \frac{\mathrm{d} z}{4 \pi} \mathrm{d}^{2} \mathbf{r}_{T} \mathrm{~d}^{2} \mathbf{b}_{T} e^{-i \mathbf{b}_{T} \cdot \boldsymbol{\Delta}_{T}}\left[\Psi_{V}^{*} \Psi\right]\left(r, Q^{2}\right) 2\left[1-e^{-2 \pi B_{p} A T_{A}(b) \mathcal{N}\left(r, x_{\mathbb{P}}\right)}\right] .
$$

Figure 5.41 summarizes the $t$-dependence of the quasielastic and coherent cross sections. Also shown is the approximation used in [911] where nonlinear effects are left out. The most 
striking result is the large suppression by a factor of $\sim 3$ of the incoherent cross section due to nonlinear effects. The incoherent and coherent curves cross saround $|t| \approx 0.05 \mathrm{GeV}^{2}$, as anticipated. With a very good detection of the nuclear breakup events, the first, even the second, diffractive dips in the coherent cross section could be measurable at the EIC, providing detailed information about the average spatial distribution of gluons inside the nucleus. For understanding the initial conditions of ultra-relativistic heavy-ion collisions what has turned out to be equally important are the fluctuations in the gluon density, which are directly measured by the incoherent part of the spectrum.

\section{Electroproduction of $J / \Psi$}

\section{Boris Z. Kopeliovich}

Proton target: The diffractive electro-production of charmonia and the charmoniumnucleon elastic scattering are closely related. The amplitudes of diffractive electro-production of a charmonium and elastic charmonium-proton scattering in the dipole approach have the form,

$$
\begin{aligned}
\mathcal{M}_{\gamma^{*} p}\left(s, Q^{2}\right) & =\sum_{\mu, \bar{\mu}} \int_{0}^{1} d \alpha \int d^{2} r_{T} \Phi_{\Psi}^{*(\mu, \bar{\mu})}\left(\alpha, \vec{r}_{T}\right) \sigma_{q \bar{q}}\left(r_{T}, s\right) \Phi_{\gamma^{*}}^{(\mu, \bar{\mu})}\left(\alpha, \vec{r}_{T}, Q^{2}\right) \\
\mathcal{M}_{\Psi p}(s) & =\sum_{\mu, \bar{\mu}} \int_{0}^{1} d \alpha \int d^{2} r_{T} \Phi_{\Psi}^{*(\mu, \bar{\mu})}\left(\alpha, \vec{r}_{T}\right) \sigma_{q \bar{q}}\left(r_{T}, s\right) \Phi_{\Psi}^{(\mu, \bar{\mu})}\left(\alpha, \vec{r}_{T}\right)
\end{aligned}
$$

Here, $\mu$ and $\bar{\mu}$ are the spin indices of the $c$ and $\bar{c}$ quarks, $Q^{2}$ is the photon virtuality, $\Phi_{\gamma^{*}}\left(\alpha, r_{T}, Q^{2}\right)$ is the light-cone distribution function of the photon for a $c \bar{c}$ fluctuation of separation $r_{T}$ and relative fraction $\alpha$ of the photon light-cone momentum carried by $c$ or $\bar{c}$. Correspondingly, $\Phi_{\Psi}\left(\alpha, \vec{r}_{T}\right)$ is the light-cone wave function of $J / \Psi$, or $\Psi^{\prime}$, or $\chi$.

The wave functions of charmonia are calculated in 914 solving the Schrödinger equation with four realistic potentials, which are labelled as COR [915], BT [916, LOG [917], and POW [918]. Then one should make a Lorentz boost from the charmonium rest frame to the infinite momentum frame, and to switch from 3-dimensional coordinates to the light-cone variables, $p_{T}$ and $\alpha$, which are the $c$-quark transverse and fractional longitudinal momenta respectively. This was done in 914] using the popular prescription 919].

The important ingredient of the calculations performed in [914] (compare with 920]) is the Melosh spin rotation [921] which relates the 2-dimensional spinors $\chi_{c}$ and $\chi_{\bar{c}}$, describing $c$ and $\bar{c}$ in the infinite momentum frame, to the spinors $\bar{\chi}_{c}$ and $\bar{\chi}_{\bar{c}}$ in the rest frame:

$$
\bar{\chi}_{\mathbf{c}}=\widehat{\mathbf{R}}\left(\alpha, \tilde{\mathbf{p}}_{\mathbf{T}}\right) \chi_{\mathbf{c}}, \quad \bar{\chi}_{\overline{\mathbf{c}}}=\widehat{\mathbf{R}}\left(\mathbf{1}-\alpha,-\tilde{\mathbf{p}}_{\mathbf{T}}\right) \chi_{\overline{\mathbf{c}}},
$$

where the matrix $R\left(\alpha, \vec{p}_{T}\right)$ has the form:

$$
\widehat{R}\left(\alpha, \vec{p}_{T}\right)=\frac{m_{c}+\alpha M-i[\vec{\sigma} \times \vec{n}] \vec{p}_{T}}{\sqrt{\left(m_{c}+\alpha M\right)^{2}+p_{T}^{2}}} .
$$

Since the $c \bar{c}$ pair is in $S$-wave, the spatial and spin dependences in the wave function factorize, and one arrives at the following light cone wave function of the $c \bar{c}$ in the infinite momentum frame

$$
\Phi_{\psi}^{(\mu, \bar{\mu})}\left(\alpha, \vec{p}_{T}\right)=U^{(\mu, \bar{\mu})}\left(\alpha, \vec{p}_{T}\right) \cdot \Phi_{\psi}\left(\alpha, \vec{p}_{T}\right)
$$


where

$$
U^{(\mu, \bar{\mu})}\left(\alpha, \vec{p}_{T}\right)=\chi_{c}^{\mu \dagger} \widehat{R}^{\dagger}\left(\alpha, \vec{p}_{T}\right) \vec{\sigma} \cdot \vec{e}_{\psi} \sigma_{y} \widehat{R}^{*}\left(1-\alpha,-\vec{p}_{T}\right) \sigma_{y}^{-1} \widetilde{\chi}_{\bar{c}}^{\bar{\mu}}
$$

Now we can determine the light-cone wave function in the mixed longitudinal momentum - transverse coordinate representation:

$$
\Phi_{\psi}^{(\mu, \bar{\mu})}\left(\alpha, \vec{r}_{T}\right)=\frac{1}{2 \pi} \int d^{2} p_{T} e^{-i \vec{p}_{T} \vec{r}_{T}} \Phi_{\psi}^{(\mu, \bar{\mu})}\left(\alpha, \vec{p}_{T}\right) .
$$

With this wave function and with the standard distribution functions of the photon one can calculate the amplitudes in (5.100)-(5.101) and predict the cross section of $J / \Psi$ photoproduction on a proton. The results for the energy dependence are compared with HERA data (see references in [914]) in Fig. 5.42, The calculation was performed in 914 with two parametrizations of the dipole cross section labelled as GBW [788] and KST [795].

We see that only BP and LOG potentials describe the data well, which, however, are not sensitive to the choice of the phenomenological dipole cross section. The $Q^{2}$ dependence of the cross section is

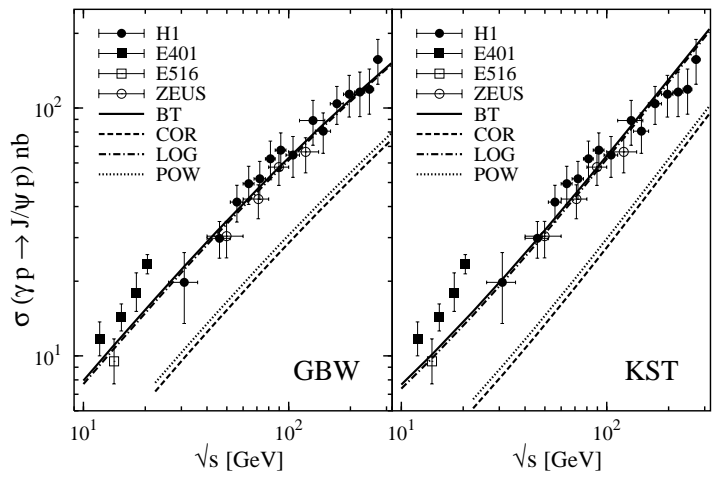

Figure 5.42. Integrated cross section for elastic photoproduction $\gamma p \rightarrow J / \Psi p$ with real photons $\left(Q^{2}=0\right)$ as a function of the energy calculated with GBW and KST dipole cross sections and for four potentials to generate $J / \Psi$ wave functions. compared to HERA data (see references in 914]) in Fig. 5.43 (left) for the LOG and BT potentials. It turns out that the effects of Melosh spin rotation have a gross impact on the cross section of elastic photoproduction $\gamma p \rightarrow J / \Psi(\psi) p$. It increases the photoproduction cross section by about $50 \%$. These effects have even more dramatic impact on the $\psi^{\prime}$, increasing the photoproduction cross section by a factor of 2-3 and eliminating the large discrepancy with data observed previously [920.

Eventually, we are in a position to predict the charmonium-proton total cross section, which is impossible to extract directly from photoproduction data, either on protons, or nuclear targets. Indeed, neither vector dominance [922, nor Glauber model [923] can be used for data analysis. We believe that the only way is to predict the charmonium cross section within a model, which successfully describe data on photoproduction in a parameter free way. Our predictions for the energy dependent charmonium-proton total cross section are depicted in Fig. 5.43 (right) for $J / \Psi$ and $\Psi^{\prime}$.

Nuclear targets: Charmonium photoproduction on nuclei is controlled by two length scales.

$$
l_{c}=\frac{2 \nu}{M_{c \bar{c}}^{2}+Q^{2}} \approx \frac{2 \nu}{M_{J / \Psi}^{2}+Q^{2}} ; l_{f}=\frac{2 \nu}{M_{\Psi^{\prime}}^{2}-M_{J / \Psi}^{2}},
$$

The first one is called coherence length can be interpreted as the lifetime of a $\bar{c} c$ fluctuation in the projectile photon in the nuclear rest frame. When $l_{c}$ is short compared to the mean nucleon spacing, one can treat $\bar{c} c$ production as instantaneous, with following propagation of the $\bar{c} c$ dipole through the nucleus. In the opposite limit of $l_{c} \gg R_{A}$ the $\bar{c} c$ dipole propagates and attenuates through the whole nucleus. The second scale $l_{f}$ is the formation length, 

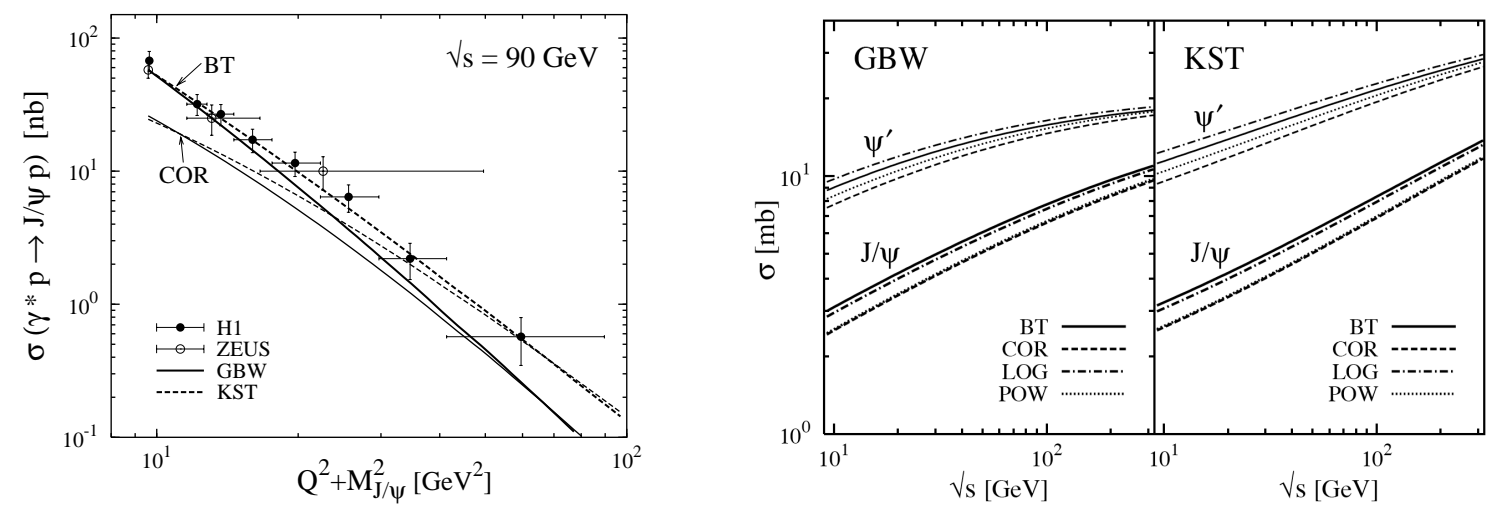

Figure 5.43. Left: Integrated cross section for elastic photo production as a function of the photon virtuality $Q^{2}+M_{J / \Psi}$ at energy $\sqrt{s}=90 \mathrm{GeV}$. Right: Total $J / \Psi-p$ (thick curves) and $\Psi^{\prime}-p$ (thin curves) cross sections with the GBW and KST parameterizations for the dipole cross section.

which characterizes the formation of the charmonium wave function. Indeed, the produced $\bar{c} c$ dipole has a certain size and interaction cross section, but does not have any certain mass. It might be the $J / \Psi$, or its radial excitation. To disentangle between them, takes time according to the uncertainty principle.

The cross section of charmonium photo-production on nuclei is easiest to write in the limit of long $l_{c} \gg R_{A}$. In this case, the size of the $\bar{c} c$ dipoles "frozen" by Lorentz time dilation for propagation of the dipole through the nucleus. The cross sections of incoherent (the nucleus break up to fragments) and coherent (the nucleus remains intact) production have the form [923, 924,

$$
\begin{array}{r}
\sigma_{i n c}^{\gamma_{T, L}^{*} A}\left(s, Q^{2}\right)=\int d^{2} b T_{A}(b)\left|\left\langle\Psi\left|\sigma_{\bar{c} c}\left(r_{T}, s\right) \exp \left[-\frac{1}{2} \sigma_{\bar{c} c}\left(r_{T}, s\right) T_{A}(b)\right]\right| \Psi_{c \bar{c}}^{T, L}\right\rangle\right|^{2} \\
\sigma_{c o h}^{\gamma_{T, L}^{*} A}\left(s, Q^{2}\right)=\int d^{2} b\left|\left\langle\Psi\left|1-\exp \left[-\frac{1}{2} \sigma_{\bar{c} c}\left(r_{T}, s\right) T_{A}(b)\right]\right| \Psi_{c \bar{c}}^{T, L}\right\rangle\right|^{2}
\end{array}
$$

where $\Psi_{\bar{c} c}^{T, L}$ are the photon wave functions given by Eq. (5.27); $\Psi\left(\vec{r}_{T}, \alpha\right)$ is the charmonium light-cone wave function calculated in the previous section. These expressions are significantly different from the Glauber model [925] and effectively include the Gribov corrections in all orders.

We define the nuclear ratios for coherent and incoherent reactions as,

$$
R_{\Psi}^{c o h}\left(s, Q^{2}\right)=\frac{\sigma_{c o h}^{\gamma^{*} A}\left(s, Q^{2}\right)}{A \sigma^{\gamma^{*} N}\left(s, Q^{2}\right)}, \quad R_{\Psi}^{i n c}\left(s, Q^{2}\right)=\frac{\sigma_{i n c}^{\gamma^{*} A}\left(s, Q^{2}\right)}{A \sigma^{\gamma^{*} N}\left(s, Q^{2}\right)} .
$$

These ratios, calculated with Eqs. (5.108)-(5.109) for real photoproduction of $J / \Psi$ and $\Psi^{\prime}$, are depicted as a function of energy in Fig. 5.44, For coherent production, the cross section rises with $A$ nearly as $A^{4 / 3}$, so the ratio may reach a large magnitude.

One can also predict the dependence on the momentum transfer $\vec{k}_{T}$ for the charmonium electro-production on nuclei. In the case of incoherent production, this dependence is the same as for production on free nucleons. However, in coherent production, the nuclear form factor comes into play and one has

$$
\frac{d \sigma_{c o h}^{\gamma_{T, L}^{*} A}\left(s, Q^{2}\right)}{d^{2} k_{T}}=\left|\int d^{2} b e^{i \vec{k}_{T} \cdot \vec{b}}\left\langle\Psi\left|1-\exp \left[-\frac{1}{2} \sigma_{\bar{q} q}\left(r_{T}, s\right) T_{A}(b)\right]\right| \Psi_{c \bar{c}}^{T, L}\right\rangle\right|^{2} .
$$



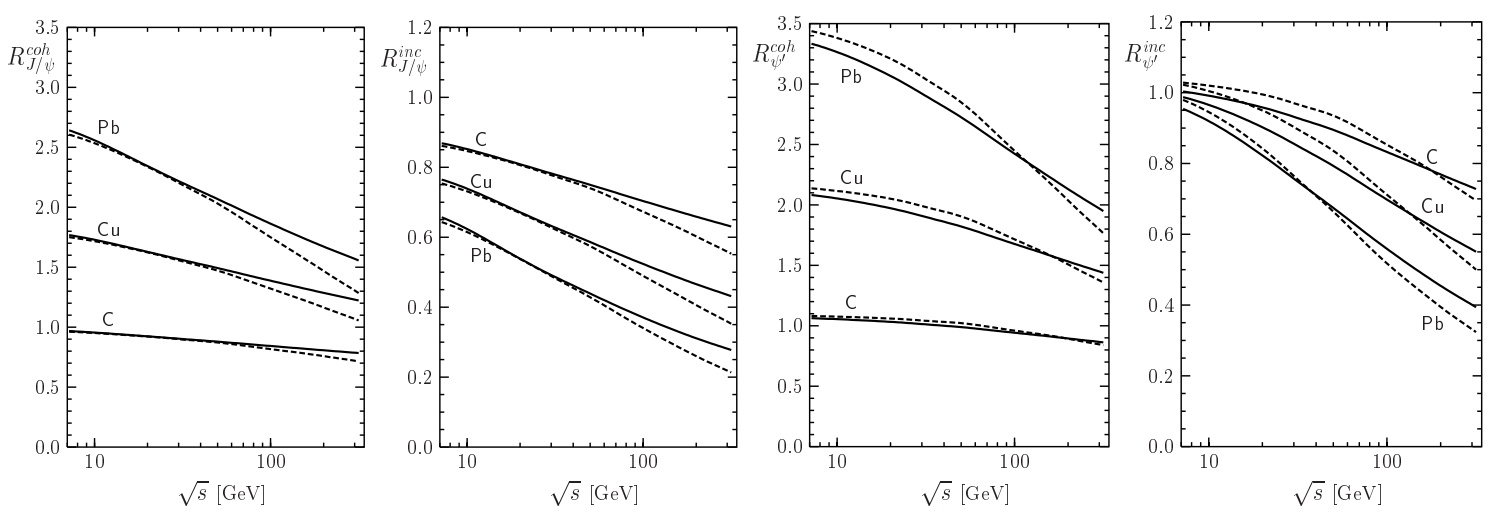

Figure 5.44. Ratios $R_{J / \Psi}^{c o h}, R_{J / \Psi}^{i n c}, R_{\Psi^{\prime}}^{c o h}$ and $R_{\Psi^{\prime}}^{c o h}$ for coherent and incoherent production on carbon, copper and Pbas function of $\sqrt{s}$ and at $Q^{2}=0$. The solid and dashed curves refer to the GBW and KST parameterizations respectively.

We introduce the ratios the sum of $T$ and $L$ components of Eq. (5.111) to the cross section at $Q^{2}=0$ and $k_{T}=0$,

$$
\mathcal{R}\left(s, Q^{2}, k_{T}\right)=\frac{d \sigma_{c o h}^{\gamma^{*} A}\left(s, Q^{2}\right)}{d^{2} k_{T}} /\left.\frac{d \sigma_{c o h}^{\gamma^{*} A}\left(s, Q^{2}=0\right)}{d^{2} k_{T}}\right|_{k_{T}=0}
$$

This ratio is plotted in Fig. 5.45 as a function of $k_{T}$ at $s=4000 \mathrm{GeV}^{2}$ for different virtualities of the photon. We see that the $k_{T}$ dependences are rather similar for $J / \Psi$ and $\Psi^{\prime}$. The shape of the distribution is determined mainly by the nuclear geometry (and not by the size of the (small) charmonium). The calculated curves show the familiar diffraction pattern known from elastic scattering on nuclei.
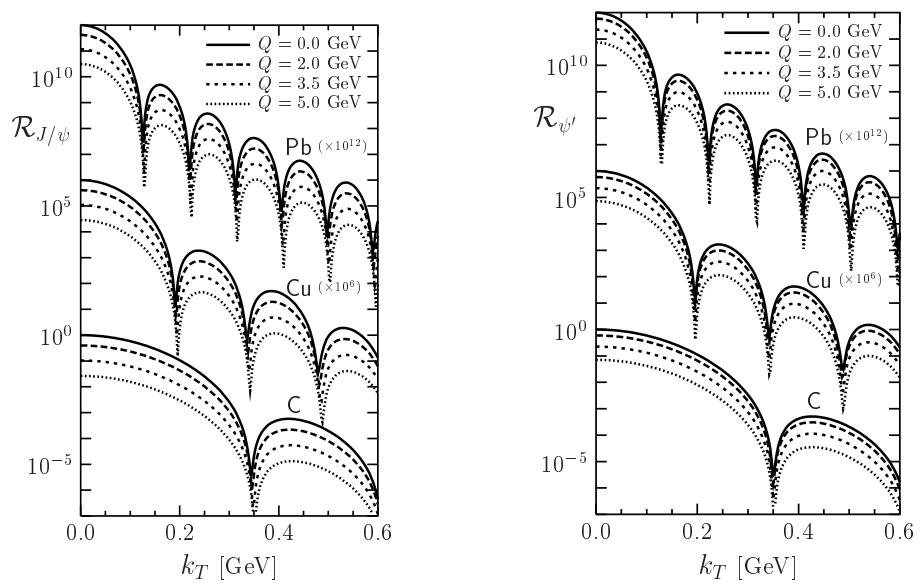

Figure 5.45. Ratios $\mathcal{R}_{J / \Psi}$ and $\mathcal{R}_{\Psi^{\prime}}$ as functions of $k_{T}$ at $s=4000 \mathrm{GeV}^{2}$ for different values of $Q$. All curves are calculated with the GBW parameterization of the dipole cross section.

It is interesting that the effects of gluon shadowing, calculated in [924], do not affect much the shape and position of the minima in $k_{T}$ dependence of the coherent cross section. However, the cross section integrated over $k_{T}$ may be significantly affected by gluon shadowing. To see the magnitude of gluon shadowing, we introduce the ratio of the cross sections calculated with and without gluon shadowing,

$$
S_{g}\left(s, Q^{2}\right)=\frac{\sigma_{g}^{\gamma^{*} A}\left(s, Q^{2}\right)}{\sigma^{\gamma^{*} A}\left(s, Q^{2}\right)} .
$$

for incoherent and coherent exclusive charmonium electroproduction. The predicted effects 
of gluon shadowing are depicted in Fig. 5.46,

We only plot ratios for $J / \Psi$ production, because ratios for $\Psi^{\prime}$ are practically the same. All curves are calculated with the GBW parameterization of the dipole cross section. We see that the onset of gluon shadowing happens at a c.m. energy of a few tens of GeV. This is controlled by the longitudinal nuclear form factor

$$
F_{A}\left(q_{c}^{g}, b\right)=\frac{1}{T_{A}(b)} \int_{-\infty}^{\infty} d z \rho_{A}(b, z) e^{i q_{c} z}
$$

where the longitudinal momentum transfer $q_{c}^{g}=1 / l_{c}^{g}$. For the onset of gluon shadowing, $q_{c}^{g} R_{A} \gg 1$, one can keep only the double scattering shadowing correction,

$$
S_{g} \approx 1-\frac{1}{4} \sigma_{e f f} \int d^{2} b T_{A}^{2}(b) F_{A}^{2}\left(q_{c}^{g}, b\right)
$$

where $\sigma_{\text {eff }}$ is the effective cross section which depends on the dynamics of interaction of the $\bar{q} q g$ fluctuation with a nucleon.

It was found in 787. that the coherence length for gluon shadowing is rather short, $l_{c}^{g} \approx\left(10 x m_{N}\right)^{-1}$, where Bjorken $x$ in our case should be an effective one, $x=\left(Q^{2}+\right.$ $\left.M_{\Psi}^{2}\right) / 2 m_{N} \nu$. The onset of shadowing according to (5.114) and (5.115) should be expected at $q_{c}^{2} \sim 3 /\left(R_{A}^{c h}\right)^{2}$ corresponding to $s_{g} \sim$ $10 m_{N} R_{A}^{c h}\left(Q^{2}+M_{\Psi}^{2}\right) / \sqrt{3}$, Figure 5.46. Ratios $S_{g}\left(s, Q^{2}\right)$, defined in (5.113), of cross sections calwhere $\left(R_{A}^{c h}\right)^{2}$ is the mean culated with and without gluon shadowing for incoherent and coherent square of the nuclear $J / \Psi$ production..

charge radius. This esti-

mate is in a good agreement with Fig. 5.46. Remarkably, the onset of shadowing is delayed with rising nuclear radii and $Q^{2}$. This follows directly from Eq. (5.115) and the fact that the formfactor is a steeper falling function of $R_{A}$ for heavy than for light nuclei, provided that $q_{c}^{G} R_{A} \gg 1$.

At medium energies, the effects of finite coherence length, $l_{c} \sim R_{A}$ become important. They increase the incoherent and suppress coherent cross sections of charmonium electroproduction. One can find the details of the corresponding calculations in [924].

\section{Exclusive processes in $e+A$ collisions}

\section{Victor P. Gonçalves}

Exclusive processes in deep inelastic scattering (DIS) have appeared as key reactions to trigger the generic mechanism of diffractive scattering. In particular, diffractive vector meson production and deeply virtual Compton scattering (DVCS) have been extensively 
studied at HERA and provide a valuable probe of the QCD dynamics at high energies. The cross sections for exclusive processes in DIS are proportional to the square of the scattering amplitude, which makes them strongly sensitive to the underlying QCD dynamics.

In this contribution, we present our estimate for the coherent and incoherent cross sections for exclusive $\rho, J / \Psi$, and $\phi$ production as well as for nuclear DVCS, making use of the numerical solution of the Balitsky-Kovchegov equation including running coupling corrections in order to estimate the contribution of the saturation physics to exclusive processes (For more details and references see Refs. [926, 927]).

Exclusive production: In the color dipole approach, exclusive production $\gamma^{*} A \rightarrow E Y$ $(E=\rho, \phi, J / \Psi$ or $\gamma)$ in electron-nucleus interactions at high energies $\left(l_{c} \gg R_{A}\right)$ is given by

$$
\sigma^{c o h}\left(\gamma^{*} A \rightarrow E A\right)=\int d^{2} b\left\langle\mathcal{N}^{A}(x, r, b)\right\rangle^{2}
$$

where

$$
\langle\mathcal{N}\rangle=\int d^{2} r \int d z \Psi_{E}^{*}(r, z) \mathcal{N}^{A}(x, r, b) \Psi_{\gamma^{*}}\left(r, z, Q^{2}\right)
$$

and $\mathcal{N}^{A}(x, r, b)$, defined in eq. (5.76), is the forward dipole-target scattering amplitude for a dipole with size $r$ and impact parameter $b$. We will assume that $\sigma_{d p}$ in eq. (5.76) is given by the bCGC saturation model or the solution of the running coupling BK equation.

On the other hand, if the nucleus scatters inelastically, i.e. breaks up $(Y=X)$, the process is called incoherent production. In this case, one sums over all final states of the target nucleus, except those that contain particle production. The $t$ slope is the same as in the case of a nucleon target. Therefore we have

$$
\sigma^{i n c}\left(\gamma^{*} A \rightarrow E X\right)=\frac{|\mathcal{I} m \mathcal{A}(s, t=0)|^{2}}{16 \pi B_{E}}
$$

where at high energies $\left(l_{c} \gg R_{A}\right)$ :

$$
|\mathcal{I} m \mathcal{A}|^{2}=\int d^{2} b T_{A}(b)\left|\Psi_{E}^{*}(r, z) \sigma_{d p} \exp \left[-\frac{1}{2} \sigma_{d p} T_{A}(b)\right] \Psi_{\gamma^{*}}\left(r, z, Q^{2}\right)\right|^{2}
$$

and $\sigma_{d p}$ is the dipole-proton cross section. In the incoherent case, the $q \bar{q}$ pair attenuates with a constant absorption cross section, as in the Glauber model, except that the whole exponential is averaged rather than just the cross section in the exponent. The coherent and incoherent cross sections depend differently on $t$. At small- $t\left(-t R_{A}^{2} / 3 \ll 1\right)$ coherent production dominates, with the signature being a sharp forward diffraction peak. On the other hand, incoherent production will dominate at large- $t\left(-t R_{A}^{2} / 3 \gg 1\right)$, with the $t$ dependence being to a good accuracy the same as in the production off free nucleons.

In Eqs. (5.117) and (5.119) the functions $\Psi^{\gamma}(z, r)$ and $\Psi^{E}(z, r)$ are the light-cone wavefunctions of the photon and the exclusive final state, respectively. The variable $r$ defines the relative transverse separation of the pair (dipole) and $z(1-z)$ is the longitudinal momentum fraction of the quark (antiquark). In the dipole formalism, the light-cone wavefunctions $\Psi(z, r)$ in the mixed representation $(r, z)$ are obtained through a two dimensional Fourier transform of the momentum space light-cone wavefunctions $\Psi(z, k)$. The photon wavefunctions are well known in the literature. For the meson wavefunction, we considered the Gauss-LC model. In the DVCS case, as one has a real photon in the final state, only 

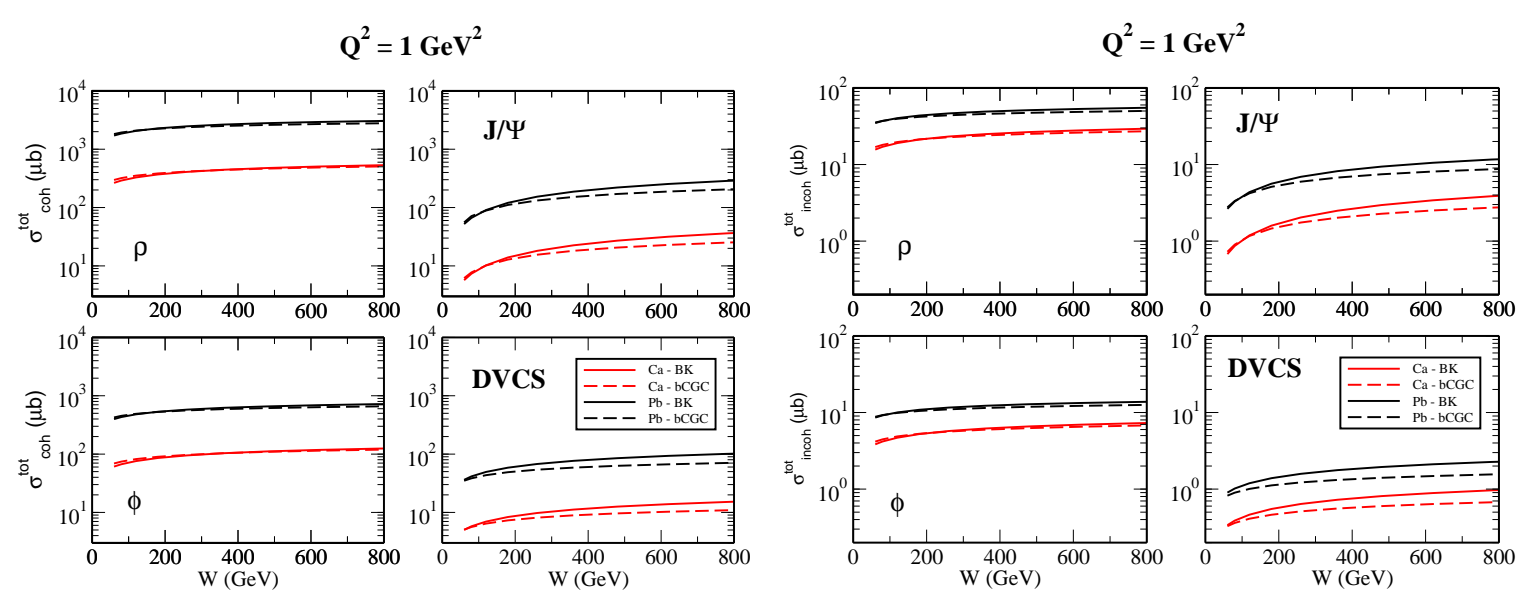

Figure 5.47. Energy dependence of the coherent (left) and incoherent (right) cross sections for different final states and $Q^{2}=1 \mathrm{GeV}^{2}$.

the transversely polarized overlap function contributes to the cross section. Summed over the quark helicities, for a given quark flavour $f$, it is given by,

$$
\left(\Psi_{\gamma}^{*} \Psi\right)_{T}^{f}=\frac{N_{c} \alpha_{\mathrm{em}} e_{f}^{2}}{2 \pi^{2}}\left\{\left[z^{2}+\bar{z}^{2}\right] \varepsilon_{1} K_{1}\left(\varepsilon_{1} r\right) \varepsilon_{2} K_{1}\left(\varepsilon_{2} r\right)+m_{f}^{2} K_{0}\left(\varepsilon_{1} r\right) K_{0}\left(\varepsilon_{2} r\right)\right\}
$$

where we have defined the quantities $\varepsilon_{1,2}^{2}=z \bar{z} Q_{1,2}^{2}+m_{f}^{2}$ and $\bar{z}=(1-z)$. Accordingly, the photon virtualities are $Q_{1}^{2}=Q^{2}$ (incoming virtual photon) and $Q_{2}^{2}=0$ (outgoing real photon).

Results: In Fig. 5.47 left, we show the coherent production cross section as a function of the photon-target c.m.s energy, $W$, for a fixed photon virtuality $Q^{2}=1 \mathrm{GeV}^{2}$. Fig. 5.47 right is the exact analogue for the corresponding incoherent cross sections. Each one of the panels shows the results obtained for one specific final state. In each figure, the two upper (lower) curves show the results for a $\mathrm{Pb}(\mathrm{Ca})$ target. In all figures, the dashed (solid) lines are obtained with the bCGC (rcBK) dipole-proton cross section. At low $W$, the bCGC and $\mathrm{rcBK}$ production cross sections are indistinguishable from one another because the dipole cross sections tend to coincide. These latter have been tuned to fit DIS data, which are taken in this kinematical region. Another expected feature is the observed decrease of the cross sections with increasing vector meson masses, which comes from the wave functions. Differences are expected to appear at higher energies, where we enter the lower $x$ (extrapolation) region. In all cases we see that the results obtained with the rcBK cross section are larger than those obtained with the bCGC one. This is related to the fact that the numerical solutions of the BK equation tend to reach the unitarity limit later. Due to this fact, the results obtained with the rcBK dipole cross section grow faster with energy than those obtained with the bCGC one. Another feature is that the differences between $\mathrm{bCGC}$ and rcBK are larger for heavier vector mesons. Comparing the results shown in Fig. 5.47 we verify the dominance of the coherent production with a small contribution coming from incoherent processes.

Acknowledgments: The author thank E.R. Cazaroto, F. Carvalho, M. S. Kugeratski, M.V.T. Machado, and F.S. Navarra by collaboration. 


\section{Constraining the $\rho$ wavefunction}

\section{Jeffrey R. Forshaw and Ruben Sandapen}

In the dipole model [735, 928], the imaginary part of the amplitude for diffractive $\rho$ production is written as 899

$$
\Im \mathrm{m} \mathcal{A}_{\lambda}\left(s, t ; Q^{2}\right)=\sum_{h, \bar{h}} \int \mathrm{d}^{2} \mathbf{r d} z \Psi_{h, \bar{h}}^{\gamma^{*}, \lambda}\left(r, z ; Q^{2}\right) \Psi_{h, \bar{h}}^{\rho, \lambda}(r, z)^{*} e^{-i z \mathbf{r} . \boldsymbol{\Delta}} \mathcal{N}(x, \mathbf{r}, \boldsymbol{\Delta})
$$

where $t=-|\boldsymbol{\Delta}|^{2}$. In a standard notation [929, 899, 898], $\Psi_{h, \bar{h}}^{\gamma^{*}, \lambda}$ and $\Psi_{h, \bar{h}}^{\rho, \lambda}$ are the light-cone wavefunctions of the photon and the $\rho$ meson respectively while $\mathcal{N}(x, \mathbf{r}, \boldsymbol{\Delta})$ is the imaginary part of the dipole-proton elastic scattering amplitude. The energy dependence of the latter is via the dimensionless variable $x$, taken here to be $x=\left(Q^{2}+4 m_{f}^{2}\right) /\left(Q^{2}+s\right)$ where $m_{f}$ is a phenomenological light quark mass 7 Setting $t=0$ in equation (5.121), we obtain the forward amplitude used in reference [929]:

$$
\left.\Im \mathrm{m} \mathcal{A}_{\lambda}\left(s, t ; Q^{2}\right)\right|_{t=0}=s \sum_{h, \bar{h}} \int \mathrm{d}^{2} \mathbf{r} \mathrm{d} z \Psi_{h, \bar{h}}^{\gamma, \lambda}\left(r, z ; Q^{2}\right) \hat{\sigma}(x, r) \Psi_{h, \bar{h}}^{\rho, \lambda}(r, z)^{*}
$$

where we have used the optical theorem to introduce the dipole cross-section $\hat{\sigma}(x, r)=$ $\mathcal{N}(x, r, \mathbf{0}) / s$. Note that since the momentum transfer $\boldsymbol{\Delta}$ is Fourier conjugate to the impact parameter $\mathbf{b}$, the dipole cross-section at a given energy is simply the $b$-integrated dipoleproton scattering amplitude:

$$
\hat{\sigma}(x, r)=\frac{1}{s} \int \mathrm{d}^{2} \mathbf{b} \mathcal{N}(x, r, \mathbf{b}) .
$$

This dipole cross-section can be extracted from the $F_{2}$ data since

$$
F_{2}\left(x, Q^{2}\right) \propto \int \mathrm{d}^{2} \mathbf{r} \mathrm{d} z\left|\Psi_{\gamma^{*}}\left(r, z ; Q^{2}\right)\right|^{2} \hat{\sigma}(x, r)
$$

and the photon's light-cone wavefunctions are known in QED, at least for large $Q^{2}$. The $F_{2}$-constrained dipole cross-section can then be used to predict the imaginary part of the forward amplitude for diffractive $\rho$ production and thus the forward differential cross-section,

$$
\left.\frac{d \sigma_{\lambda}}{d t}\right|_{t=0}=\frac{1}{16 \pi}\left(\Im \mathrm{m} \mathcal{A}_{\lambda}(s, 0)\right)^{2}\left(1+\beta_{\lambda}^{2}\right)
$$

where $\beta_{\lambda}$ is the ratio of real to imaginary parts of the amplitude and is computed as in reference [929]. The $t$-dependence can be assumed to be the exponential dependence as suggested by experiment 930]:

$$
\frac{d \sigma_{\lambda}}{d t}=\left.\frac{d \sigma_{\lambda}}{d t}\right|_{t=0} \times \exp (-B|t|), \quad B=N\left(14.0\left(\frac{1 \mathrm{GeV}^{2}}{Q^{2}+M_{\rho}^{2}}\right)^{0.2}+1\right)
$$

with $N=0.55 \mathrm{GeV}^{-2}$. After integrating over $t$, we can compute the total cross-section $\sigma=\sigma_{L}+\epsilon \sigma_{T}$ which is measured at HERA 8

\footnotetext{
${ }^{7}$ We shall take $m_{f}=0.14 \mathrm{GeV}$, the value used when extracting the dipole cross section from $F_{2}$ data.

${ }^{8}$ To compare with the HERA data, we take $\epsilon=0.98$.
} 
Presently, several dipole models [931, 824, 910, 821, 823] are able to fit the current HERA $F_{2}$ data and there is evidence that the data prefer those incorporating some form of saturation [932]. We can use the $F_{2}$-constrained dipole cross-section in order to extract the $\rho$ light-cone wavefunction using the current precise HERA data [930, 680]. This has recently been performed in reference [929] using the Regge-inspired FSSat dipole model [931] and we shall report the results of this work here. In addition, we repeat the analysis using two alternative models [910, 824, 823] both based on the original Colour Glass Condensate (CGC) model 84]. They differ from the original CGC model by including the contribution of charm quarks when fitting to the $F_{2}$ data. Furthermore in one of them [910, 824], the anomalous dimension $\gamma_{s}$ is treated as an additional free parameter instead of being fixed to its LO BFKL value of 0.63 . We shall refer to these models as CGC[0.74] and CGC [0.63] models where the number in the square brackets stands for the fitted and fixed value of the anomalous dimension respectively. For both models, we use the set of fitted parameters given in reference [824]. All three models, i.e FSSat, CGC[0.63] and CGC[0.74] account for saturation although in a $b$ - (or equivalently $t$-) independent way. Indeed, at a given energy, the dipole cross-section is equal to the forward dipole-proton amplitude or to the $b$-integrated dipole proton amplitude given by equation (5.123). Finally, all three dipole models we consider here give a good description of the diffractive structure function data [876, 933].

Boosted Gaussian predictions

\begin{tabular}{|c|c|}
\hline Dipole model & $\chi^{2} /$ data point \\
\hline FSSat & $310 / 75$ \\
\hline CGC $[0.74]$ & $262 / 75$ \\
\hline CGC $[0.63]$ & $401 / 75$ \\
\hline
\end{tabular}

BG fits

\begin{tabular}{|c|c|}
\hline Model & $\chi^{2} /$ d.o.f \\
\hline FSSat [929] & $82 / 72$ \\
\hline CGC[0.74] & $64 / 72$ \\
\hline CGC[0.63] & $83 / 72$ \\
\hline
\end{tabular}

\section{Improved fits}

\begin{tabular}{|c|c|}
\hline Model & $\chi^{2} /$ d.o.f \\
\hline FSSat [929] & $68 / 70$ \\
\hline CGC[0.63] & $67 / 70$ \\
\hline
\end{tabular}

Table 5.4. Left: Predictions of the $\chi^{2} /$ data point using the BG wavefunction. Center: $\chi^{2} /$ d.o.f obtained when fitting $R_{\lambda}$ and $b_{\lambda}$ to the leptonic decay width and HERA data. Right: $\chi^{2} /$ d.o.f obtained when fitting $b_{\lambda}, R_{\lambda} c_{T}, d_{T}$ the leptonic decay width and HERA data.

Fitting the HERA data: Previous work [898, 899, 824] has shown that a reasonable assumption for the scalar part of the light-cone wavefunction for the $\rho$ is of the form

$$
\begin{aligned}
\phi_{\lambda}^{\mathrm{BG}}(r, z)= & \mathcal{N}_{\lambda} 4[z(1-z)]^{b_{\lambda}} \sqrt{2 \pi R_{\lambda}^{2}} \exp \left(\frac{m_{f}^{2} R_{\lambda}^{2}}{2}\right) \exp \left(-\frac{m_{f}^{2} R_{\lambda}^{2}}{8[z(1-z)]^{b_{\lambda}}}\right) \\
& \times \exp \left(-\frac{2[z(1-z)]^{b_{\lambda}} r^{2}}{R_{\lambda}^{2}}\right)
\end{aligned}
$$

and is referred to as the 'Boosted Gaussian' (BG). This wavefunction is a simplified version of that proposed originally by Nemchik, Nikolaev, Predazzi and Zakharov [934]. In the original BG wavefunction, $b_{\lambda}=1$ while the parameters $R_{\lambda}$ and $\mathcal{N}_{\lambda}$ are fixed by the leptonic decay width constraint and the wavefunction normalization conditions [929]. However, when the BG wavefunction is used in conjunction with either the FSSat model or any of the CGC models, none of them is able to give a good quantitative agreement with the current HERA $\rho$-production data. This is illustrated by the large $\chi^{2}$ values in table 5.4, the situation is 


\section{Best fit parameters}

\begin{tabular}{|c|c|c|c|c|c|c|}
\hline & $R_{L}^{2}$ & $R_{T}^{2}$ & $b_{L}$ & $b_{T}$ & $c_{T}$ & $d_{T}$ \\
\hline FSSat [929] & 26.76 & 27.52 & 0.5665 & 0.7468 & 0.3317 & 1.310 \\
\hline CGC[0.63] & 27.31 & 31.92 & 0.5522 & 0.7289 & 1.6927 & 2.1457 \\
\hline CGC[0.74] & 26.67 & 21.30 & 0.5697 & 0.7929 & 0 & 0 \\
\hline
\end{tabular}

Table 5.5. Best fit parameters for each dipole model.

considerably improved by fitting $R_{\lambda}$ and $b_{\lambda}$ to the leptonic decay width and HERA data (we fit to the same data set and with the same cuts as in reference [929]).

For the FSSat and CGC[0.63] models, we can further improve the quality of the fit by allowing for additional end-point enhancement in the transverse wave-function, i.e. using a scalar wave-function of the form

$$
\phi_{T}(r, z)=\phi_{T}^{\mathrm{BG}}(r, z) \times\left[1+c_{T} \xi^{2}+d_{T} \xi^{4}\right]
$$

where $\xi=2 z-1$. The results are shown in table 5.4 .
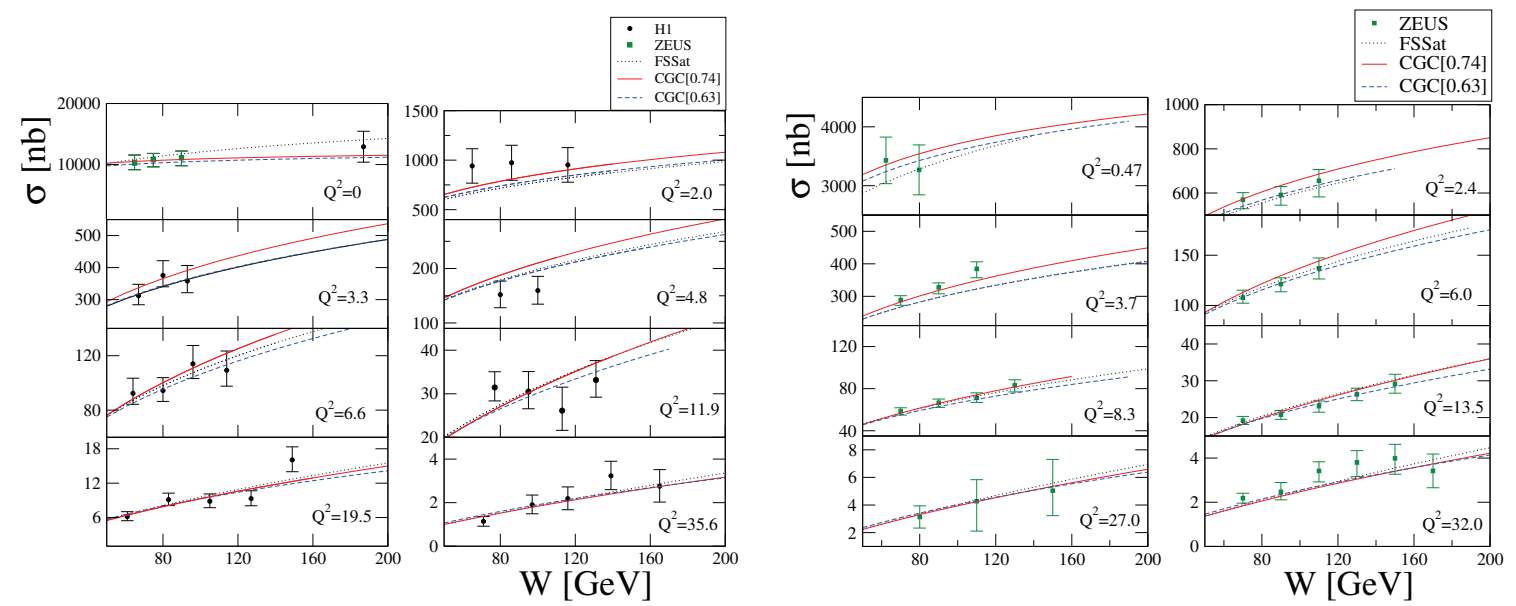

Figure 5.48. Best fits to the HERA (left) and ZEUS (right) total cross-section data. CGC[0.74]: solid; FSSat: dotted; CGC[0.63]: dashed.

The best fits obtained with each dipole model are compared to the HERA data in figures 5.48 and 5.49. The corresponding fitted parameters are given in table 5.5. Note that we achieve a lower $\chi^{2} /$ d.o.f $=0.89$ with CGC[0.74] than with CGC[0.63] and FSSat for which we obtain $\chi^{2} /$ d.o.f $=0.96$ and $\chi^{2} /$ d.o.f $=0.97$ respectively. Compared to the FSSat and $\mathrm{CGC}[0.63]$ fits, note that no additional enhancement in the transverse wavefunction is required in the CGC[0.74] fit. Nevertheless the extracted wavefunction still exhibits enhancement compared to the old BG wavefunction. The extracted light-cone wavefunctions are shown in figure 5.50 left.

Distribution Amplitudes: The leading twist-2 Distribution Amplitude (DA) reads [929]:

$$
\varphi(z, \mu) \sim\left(1-\mathrm{e}^{-\mu^{2} / \Delta(z)^{2}}\right) \mathrm{e}^{-m_{f}^{2} / \Delta(z)^{2}}[z(1-z)]^{b_{L}},
$$



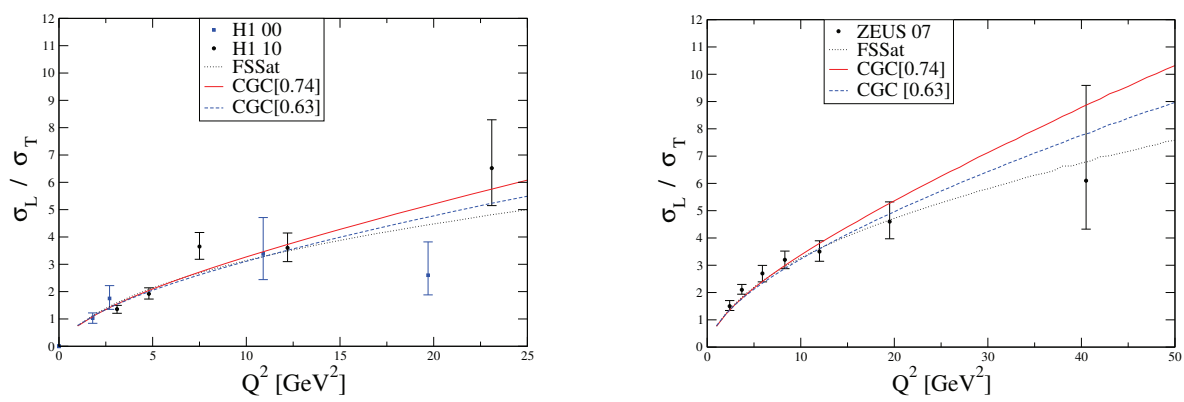

Figure 5.49. Best fits to the $\sigma_{L} / \sigma_{T}$ data. The $\mathrm{H} 1$ data are at $W=75 \mathrm{GeV}$ while the ZEUS data are at $W=90 \mathrm{GeV}$. CGC[0.74]: solid; FSSat: dotted; CGC[0.63]: dashed.
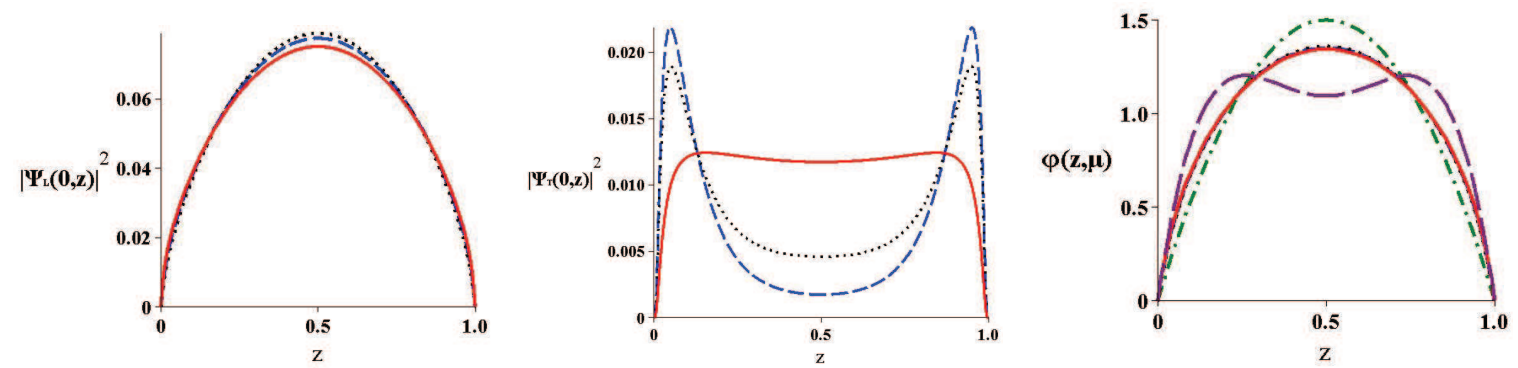

Figure 5.50. Left and center: The longitudinal and transverse light-cone wavefunctions squared at $r=0$. (CGC[0.74]: solid; FSSat: dotted; CGC[0.63]: dashed.) Right: The extracted leading twist-2 DAs at $\mu=1 \mathrm{GeV}$ compared to the DA of reference [935] also at $1 \mathrm{GeV}$ (long-dashed) and the asymptotic DA (dot-dashed).

where $\Delta(z)^{2}=8[z(1-z)]^{b_{L}} / R_{L}^{2}$. This leading twist DA is only sensitive to the longitudinal wavefunction and, as illustrated in figure 5.50 right, we expect little variation in the predictions using the different dipole models. To compare with existing theoretical predictions for the DA, we compute moments:

$$
\left\langle\xi^{n}\right\rangle_{\mu}=\int_{0}^{1} \mathrm{~d} z \xi^{n} \varphi(z, \mu)
$$

where by convention 929] $\int_{0}^{1} \mathrm{~d} z \varphi(z, \mu)=1$. In reference [929], we noted that our DA is very slowly varying with $\mu$ for $\mu>1 \mathrm{GeV}$, i.e our parameterization neglects the perturbatively known $\mu$-dependence of the DA. This statement remains true if we use the CGC $[0.63]$ or CGC[0.74] instead of the FSSat model.

Our results are compared with the existing predictions in table 5.6. The moments obtained with our best fit, i.e with the CGC[0.74] model, are very similar to those obtained with FSSat model or the CGC[0.63]. In all cases, the results are in very good agreement with expectations based on QCD sum rules and the lattice. Finally, in figure 5.50 right, we compare our DAs with that predicted by Ball and Braun [935, at a scale $\mu=1 \mathrm{GeV}$. The agreement is reasonable given that in reference [935, the expansion in Gegenbauer polynomials is truncated at low order, which is presumably responsible for the local minimum at $z=1 / 2$. Certainly, all 4 distributions are broader than the asymptotic prediction $\sim 6 z(1-z)$.

Conclusions: We have used the current HERA data on diffractive $\rho$ production to extract 
Moments of the leading twist DA at the scale $\mu$

\begin{tabular}{|c|c|c|c|c|c|c|c|}
\hline Reference & Approach & Scale $\mu$ & $\left\langle\xi^{2}\right\rangle_{\mu}$ & $\left\langle\xi^{4}\right\rangle_{\mu}$ & $\left\langle\xi^{6}\right\rangle_{\mu}$ & $\left\langle\xi^{8}\right\rangle_{\mu}$ & $\left\langle\xi^{10}\right\rangle_{\mu}$ \\
\hline (This paper) & CGC[0.74] fit & $\sim 1 \mathrm{GeV}$ & 0.227 & 0.105 & 0.062 & 0.041 & 0.029 \\
\hline (This paper) & CGC[0.63] fit & $\sim 1 \mathrm{GeV}$ & 0.229 & 0.107 & 0.063 & 0.042 & 0.030 \\
\hline$[929$ & FSSat fit & $\sim 1 \mathrm{GeV}$ & 0.227 & 0.105 & 0.062 & 0.041 & 0.029 \\
\hline (This paper) & Old BG prediction & $\sim 1 \mathrm{GeV}$ & 0.181 & 0.071 & 0.036 & 0.021 & 0.014 \\
\hline$[936$ & GenSR & $1 \mathrm{GeV}$ & $0.227(7)$ & $0.095(5)$ & $0.051(4)$ & $0.030(2)$ & $0.020(5)$ \\
\hline$[937$ & SR & $1 \mathrm{GeV}$ & 0.26 & 0.15 & & & \\
\hline$[935]$ & SR & $1 \mathrm{GeV}$ & $0.26(4)$ & & & & \\
\hline$[938$ & SR & $1 \mathrm{GeV}$ & 0.254 & & & & \\
\hline$[939$ & SR & $1 \mathrm{GeV}$ & $0.23 \pm_{0.02}^{0.03}$ & $0.11 \pm_{0.02}^{0.03}$ & & & \\
\hline$[940$ & Lattice & $2 \mathrm{GeV}$ & $0.24(4)$ & & & & \\
\hline & $6 z(1-z)$ & $\infty$ & 0.2 & 0.086 & 0.048 & 0.030 & 0.021 \\
\hline
\end{tabular}

Table 5.6. Our extracted values for $\left\langle\xi^{n}\right\rangle_{\mu}$, compared to predictions based on the QCD sum rules (SR), Generalised QCD Sum Rules (GenSR) or lattice QCD.

information on the $\rho$ light-cone wavefunction. We find that the corresponding leading twist-2 DA is broader than the asymptotic shape and agrees very well with the expectations of QCD sum rules and the lattice. We also find that the data prefer a transverse wavefunction with end-point enhancement although the degree of such an enhancement is model-dependent.

Acknowledgments: We thank H. Kowalski and C. Marquet for useful discussions. R.S. also thanks the organisers for their invitation and for making this workshop most enjoyable. 


\title{
5.7 Nuclear effects across the $x-Q^{2}$ plane: quarks and gluons
}

\author{
Introduction \\ Rodolfo Sassot, Marco Stratmann, Pia Zurita
}

In spite of the remarkable phenomenological success of QCD as the theory of strong interactions, a detailed understanding of the role of quark and gluon degrees of freedom in nuclear matter is still lacking and poses great challenges for the theory. Ever since the discovery that quark and gluons in bound nucleons exhibit momentum distributions different from those measured in free or loosely bound nucleons [941, the precise determination of nuclear parton distribution functions (nPDF) has attracted growing attention, driving both increasingly accurate and comprehensive nuclear structure functions measurements [942] and a more refined theoretical understanding of the underlying physics.

The precise knowledge of nPDFs is not only required for a deeper understanding of the mechanisms associated with nuclear binding from a QCD improved parton model perspective, but is also a crucial input for the theoretical interpretation and analyses of a wide variety of ongoing and future high energy physics experiments, such as, for instance, heavy ion collisions at BNL-RHIC [943], proton-nucleus collisions to be performed at the CERN-LHC [944], or neutrino-nucleus interactions in long baseline neutrino experiments 945. Consequently, the kinematic range and the accuracy at which nPDFs are known has evolved into a key issue in many areas of hadronic and particle physics.

The standard description of DIS processes off nuclear targets is customarily done in terms of the hard scale $Q$ set by the virtuality of the exchanged photon and a scaling variable $x_{A} \equiv Q^{2} /\left(2 p_{A} \cdot q\right)$, analogous to the Bjorken variable used in DIS off nucleons. Here, $p_{A}$ is the target nucleus momentum, and, consequently, $x_{A}$ is kinematically restricted to $0<x_{A}<1$, just like the standard Bjorken variable. Alternatively, one can define another scaling variable $x_{B} \equiv A x_{A}$, where $A$ is the number of the nucleons in the nucleus. Under the assumption that the nucleus momentum $p_{A}$ is evenly distributed between the nucleons $p_{N}=p_{A} / A$, this variable resembles the Bjorken variable corresponding to the scattering off free nucleons, $x_{B} \equiv Q^{2} /\left(2 p_{N} \cdot q\right)$. However, in the context of nuclear scattering, it spans the interval $0<x_{B}<A$ by definition, reflecting the fact that a parton may in principle carry more than the average nucleon momentum.

In a naive picture, parton distributions in a nucleus are simply given by the incoherent sum of the parton distributions in the $Z$ protons and $(A-Z)$ neutrons that constitute the nucleus. In that case, the ratios between the structure functions or cross sections of two iso-scalar nuclei (with the same proportion of protons and neutrons, such as carbon and deuteron) should be just proportional to the ratio of their respective number of nucleons (or to unity if we normalize the structure functions by the number of nucleons $A$ ).

If we take into account Fermi motion effects, one would expect that in the larger nuclei, the cross section extends up to larger $x_{B}$, so the rates should typically grow to larger than unity at high $x_{B}$. What the EMC experiment found was that in addition to this motion effect, there was a significant and quite unexpected drop in the rates between approximately $x_{B} \approx 0.3$ and $x_{B} \approx 0.7$. In fig, 5.51, we show a precise measurement that illustrates both effects. which was recently performed at JLab 946] Later on, it was found that the situation was even worse for the naive picture outlined above, because at lower $x_{B}$ values, the rates showed non-trivial patterns of suppression and enhancement. These effects are called shadowing and anti-shadowing, respectively. The phenomenon has been measured at 

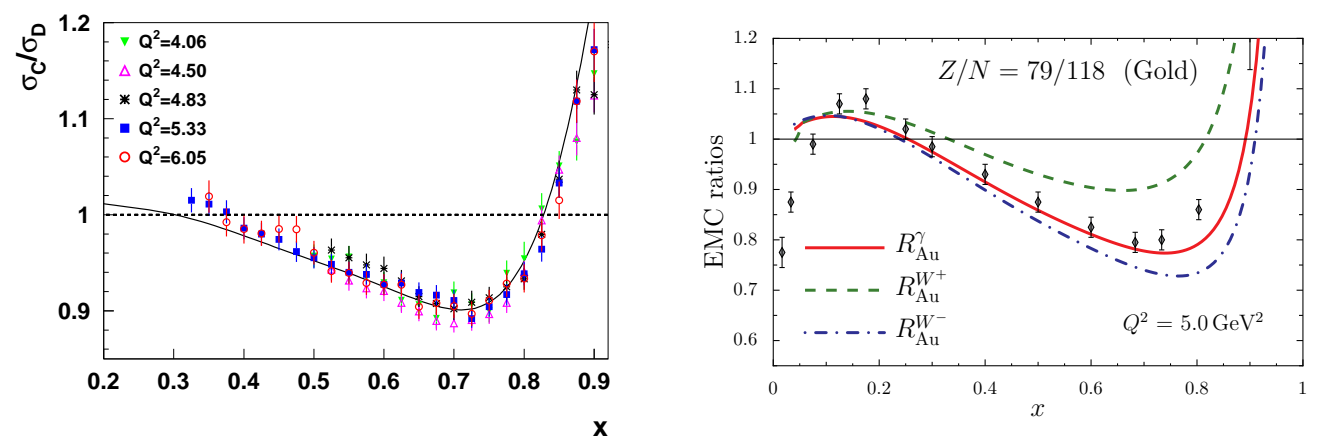

Figure 5.51. Left: EMC effect and Fermi motion as measured at JLab 946]. Right: The experimental results of Ref. [947] are illustrated for a gold target. The solid line is the result from Ref. 948] for the usual EMC effect and the dashed and dashed-dotted lines are respectively the EMC effects in the $F_{2}^{W^{+}}$and $F_{2}^{W^{-}}$structure functions. In both panels, $x \equiv x_{B}$ as defined in the text.

different $Q^{2}$ and persists at higher $Q^{2}$ but with a dependence specific for each $x_{B}$ region.

After more than 30 years of experimental and theoretical studies, a standard picture of nuclear modifications of structure functions and parton densities has not yet emerged. This is a clear target for detailed studies at the EIC, which have a large potential to qualitatively improve the current situation.

\section{The EMC effect at an EIC}

\section{Ian C. Cloët}

The EMC effect has an immediate parton model interpretation, which is that the valence quarks in nuclei carry a smaller momentum fraction than the valence quarks in a free nucleon. There have been numerous attempts to explain the EMC effect, for example nuclear structure 949, nuclear pion enhancement [950], dynamical rescaling and inter-nucleon color conductivity [951, 952, 953], point like configurations [954] and the medium modifications to the bound nucleons [955, 956, 957, 958. However, after more than a quarter of a century since the original EMC experiment, there is still no universally accepted explanation of the EMC effect. Therefore, it appears likely that to gain a deeper insight into the origins of the EMC effect we require new experimental information that is not accessed in traditional DIS.

An electron ion collier (EIC) provides excellent opportunities to access different aspects of the EMC effect, which are not as accessible with traditional fixed target experiments. A standout example is $W$-production via the DIS processes

$$
\begin{aligned}
& \ell^{-}+A \longrightarrow W^{-}+\nu_{\ell}+A \longrightarrow \nu_{\ell}+X \\
& \ell^{+}+A \longrightarrow W^{+}+\bar{\nu}_{\ell}+A \longrightarrow \bar{\nu}_{\ell}+X .
\end{aligned}
$$

The extraction of the target structure functions from these reactions is possible at an EIC because of the unique ability to reconstruct the final state and therefore avoid the need to directly determine the outgoing momentum of the neutrino or anti-neutrino. The parton model expressions for the $F_{2}$ structure functions that characterize these processes are [959]

$$
\begin{aligned}
& F_{2 A}^{W^{+}}(x)=\bar{u}_{A}(x)+d_{A}(x)+s_{A}(x)+\bar{c}_{A}(x), \\
& F_{2 A}^{W^{-}}(x)=u_{A}(x)+\bar{d}_{A}(x)+\bar{s}_{A}(x)+c_{A}(x),
\end{aligned}
$$


where $u_{A}(x), \bar{u}_{A}, \ldots$ are the various quark distributions of the target. In the valence quark region these $W^{ \pm}$structure functions are completely dominated by quark distributions of a single flavour, and hence a measurement of these structure functions provides direct access to the flavour decomposition of the nuclear parton distributions functions in this region. The flavour dependence of the EMC effect can then be determined, which will provide extremely important new information on the nature of this important phenomena.

The EMC effect ratio can be defined as

$$
R^{i}=\frac{F_{2 A}^{i}}{Z F_{2 p}^{i}+N F_{2 n}^{i}}, \quad \text { where } \quad i \in \gamma, W^{ \pm}
$$

and $F_{2 p}^{i}, F_{2 n}^{i}, F_{2 A}^{i}$ are respectively the proton, neutron and nuclear structure functions. The atomic number of the nucleus is labelled by $Z, N$ is the neutron number. Using the nuclear quark distribution results from Ref. [948, we can construct the usual EMC effect associated with the exchange of a virtual photon and also the EMC effect in the $W^{ \pm}$ structure functions. These results are illustrate in Fig. 5.51 for an Au nucleus.

Therefore, measurements of $F_{2 A}^{W^{ \pm}}(x)$ for various nuclei, for example $\mathrm{C}, \mathrm{Fe}, \mathrm{Au}$ and $\mathrm{Pb}$ would provide important new information on the flavour dependence of the EMC effect, which in ref. 948, is predicted to be large for nuclei like $\mathrm{Pb}$ and $\mathrm{Au}$. It is also claimed that a significant part of the $\mathrm{NuTeV}$ anomaly may also be explained by this isovector EMC effect 948. Therefore, these measurements present an excellent opportunity for an EIC and will undoubtedly help us understand the origins of the EMC effect, which is essential if we are to ever have a QCD based description of nuclei.

\section{Nuclear gluons}

\section{Hans J. Pirner}

Historically, the very accurate NMC measurements of DIS on Tin and Carbon nuclei has allowed one to extract the gluon distribution from the scaling violation in $F_{2}(A)$. This has been done by Gousset and myself [960] for the first time. That analysis shows an enhancement of $10 \%$ i.e. antishadowing for $x \approx 0.1$ and the same amount of shadowing, namely also $10 \%$ at $x \approx 0.01$. A high experimental accuracy is demanded, therefore only a trend could be established. The asymptotic calculation of heavy charmonium production on nuclei is often proposed as another method to extract the nuclear gluon distribution based on the gluon-gluon fusion process. As shown in various papers by Kopeliovich this production is more complicated, especially for $J / \Psi$, because of initial and final state effects. Measurements of the gluon distribution would give an experimental window on the importance of gluonic effects in nuclear binding. Very little is known about the role of gauge fields in nuclei.

To gain insight on gluons in bound nucleons system, we have studied an abelian QED model [961] where the nucleon is replaced by an atom and the nucleus by a molecule, i.e., we have analysed the structure function of the photon in the $H_{2}$-molecule and compared it with the structure function in the $H$-atom. The electron orbits of the hydrogen atoms in the molecule are polarized and modified by the electron exchange interaction leading to a suppression of photons at small $x$. At the momentum corresponding to the relative distance of the two protons, a small antishadowing peak is visible [961]. In analogy, gluon antishadowing in the region $x=0.1$ may indicate the distance $\Delta r \approx 2 \mathrm{fm}$ between the centers of the nucleons which act as color sources of common gluon fields between nucleons. 
A covalent binding of quarks may manifest itself as a density dependent lack of long range gluons at $x<0.1$ similarily to the deformation of the photon cloud in the hydrogen molecule. In addition, in non-Abelian QCD, one expects at small $x$ that the gluons from different nucleons overlap and merge. Both of these effects have also an interpretation in the nuclear rest frame in terms of the absorption of various partonic components in the wave function of the photon.

During the last ten years, the available data have been used to extract nuclear parton distributions and evolve them to high $Q^{2}$, as reviewed below. In a careful analysis one has to respect the large errors of the starting distribution at low $Q^{2}$ for the nuclear gluon distributions and also the larger $x$ region has to be included correctly - at least the fact that the nuclear gluon distribution 962 is more strongly affected by Fermi-motion of the nucleons than the quark distribution, since it has a stronger decrease at large $x$. Enhancement of the nuclear gluon distribution sets in already at $x=0.5$ which may be of importance for charmonium production at JLab 962 .

\section{Global fits of nuclear PDFs: current status}

\section{Rodolfo Sassot, Marco Stratmann, Pia Zurita}

From the point of view of perturbative QCD (pQCD), the extraction of nPDF can be performed in close analogy to what is routinely done for free nucleons: they are considered as non-perturbative inputs, to be inferred from data, whose relation to the measured observables and their energy scale dependence can be computed order by order in perturbation theory. Although one cannot discard potentially larger higher-twist or power corrections than in the case of free nucleons, or non-linear nuclear recombination effects, standard QCD factorization and universality of nPDFs are found to hold to a very good approximation in the kinematical range covered by present experiments.

At variance with PDFs for free nucleons, which, driven by the demand for increasingly precise predictions of the standard model, obtained an impressive degree of accuracy and refinement, extractions of nPDFs are done at a considerably lower level of sophistication. Not only the number, variety, kinematical coverage, and precision of nuclear data are much more limited, but the precise parameterization of nPDFs is also much more involved as it depends not only on the energy scale $Q$ and the parton's momentum fraction $x$, but also on the size of the nucleus characterized by the atomic number $A$. In the following, we present a brief summary of the current status of nPDFs and outline limitations in the analyses imposed by the data available so far.

Thanks to its variable beam energy, the possibility to run with different nuclei, and the envisioned large luminosities, an EIC will add invaluable novel information on nPDFs from studies of the inclusive structure functions $F_{2, L}$. It will extend the kinematic range toward lower values of $x$ as well as higher scales of $Q$, allowing precise determination of the gluon distribution from scaling violations of $F_{2}$, permit the flavour separation of the quark sea and the study the onset of non-linear saturation effects at small $x$ (see Section 5.3), which eventually spoil the factorized pQCD approach.

Status of Nuclear Parton Densities. From the point of view of pQCD and a factorized approach, the description of nuclear DIS can be viewed as follows. In a DIS processes off a nuclear target, we also have a hard momentum scale $Q$ that allows one to factorize the measured cross section into a point-like partonic cross section and non-perturbative parton 

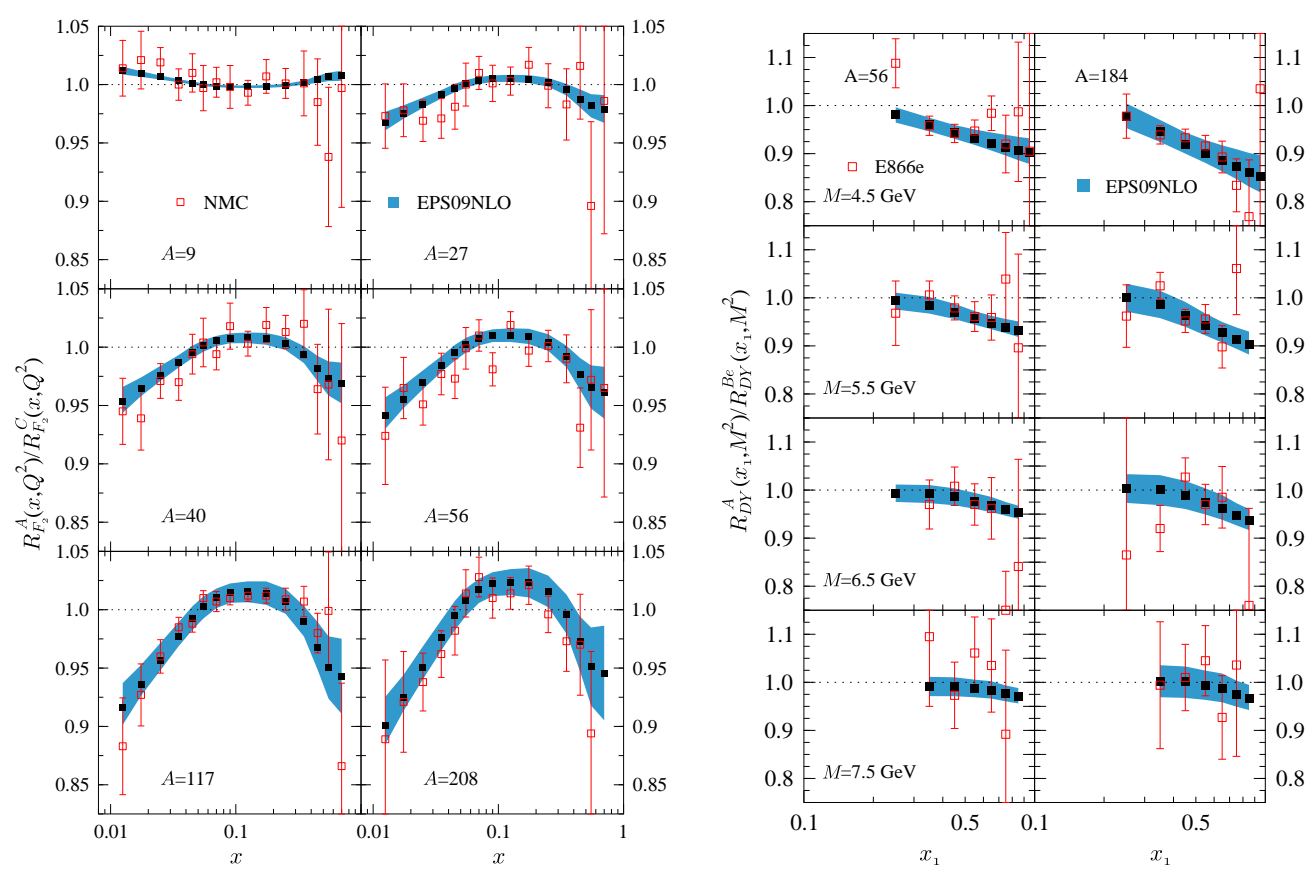

Figure 5.52. Quality of the fit to nuclear DIS and Drell-Yan data; taken from Ref. [40.

densities, characteristic of partons seen in that nucleus. These "effective" parton densities factorize and encode all the non-perturbative information, including the details about the nuclear structure, and every mechanism, interaction, or effect we can imagine. Since the hard partonic cross sections are just the same as those appearing in the factorization for free nucleons, the nuclear parton densities will evolve with scale in the same way as ordinary parton densities. For similar reasons, the approach could be extended to higher orders. What is clearly not obvious within this line of reasoning is why, or how, one could split the non-perturbative effective nuclear parton density into a piece containing only the effects related to quarks and gluons belonging to single nucleon from those related to the nucleons bound in the nuclei. No field theoretical tool gives us a precise prescription of how to achieve this. It is important to keep in mind that even in lepton-nucleon scattering standard PDFs are not just naive probability densities; they are non-trivial, though perfectly well defined, objects which depend on the choice of factorization scheme and contain other ingredients such as gauge links.

What can be done, of course, is to follow a program of global QCD analyses completely analogous to the one carried out for PDFs, i.e., to extract the nPDFs and their $A$ dependence from data. In doing so one can explore if the basic properties of factorization and universality still hold in a nuclear environment. The first QCD extractions of nPDFs defined in this way were done at the end of the 90's by two pioneering groups who performed leading order (LO) analyses of nuclear DIS data (EKS98, HKM01) [963, 828, 964.

When introducing nPDFs, the usual approach was to propose a very simple relation between the parton distribution of a proton bound in the nucleus, $f_{i}^{A}$, and those for free protons $f_{i}$,

$$
f_{i}^{A}\left(x_{B}, Q_{0}^{2}\right)=R_{i}\left(x_{B}, Q_{0}^{2}, A, Z\right) f_{i}\left(x_{B}, Q_{0}^{2}\right),
$$

in terms of a multiplicative nuclear correction factor $R_{i}\left(x_{B}, Q^{2}, A, Z\right)$, specific to a given 
nucleus $(A, Z)$, parton flavor $i$, and initial energy scale $Q_{0}^{2}$. Such a description is convenient since the ratio $R_{i}\left(x_{B}, Q^{2}, A, Z\right)$ compares directly the parton densities with and without nuclear effects, and is closely related to the most common nuclear DIS observables, which are the ratios between the nuclear and deuterium structure functions. In Ref. [39] the alternative to relate nPDFs to standard PDFs by means of a convolution was introduced. The convolution approach implements straightforwardly effects related to rescalings or shifts in the parton's momentum fraction due to interactions with the nuclear medium. In addition, convolution integrals are the most natural language for parton dynamics beyond the LO and allow for the straightforward application of the Mellin transform techniques, convenient for a numerical fast and accurate computation of the scale dependence of PDFs and relevant cross section estimates.

Following the developments for standard PDFs, nPDFs analyses subsequently incorporated various improvements such as a consistent next-to-leading order (NLO) framework (nDS) 39], a thorough uncertainty analysis (HKN04 LO) [965, and periodical updates of the different sets in order to incorporate new data (EKPS07 LO) [966, up to NLO accuracy (HKN07 NLO, EPS09 NLO) [38, 40]. In the latest sets [40, 36] particular attention has been paid to the possible impact of $\mathrm{d}+\mathrm{Au}$ collision data from RHIC and neutrino DIS data on the global fits. A typical comparison to nuclear DIS and Drell-Yan data is shown in Fig. 5.52 ,

It is worth noticing that the inclusion of $d+A u$ data in nPDF fits, although neglecting any nuclear modifications in the hadronization process, leads to significantly larger gluon shadowing and antishadowing, as has been pointed out in [40]. The same data, however, can be described with much more moderate nuclear gluon PDFs, but including medium modified nFFs [967], see Section [5.10.

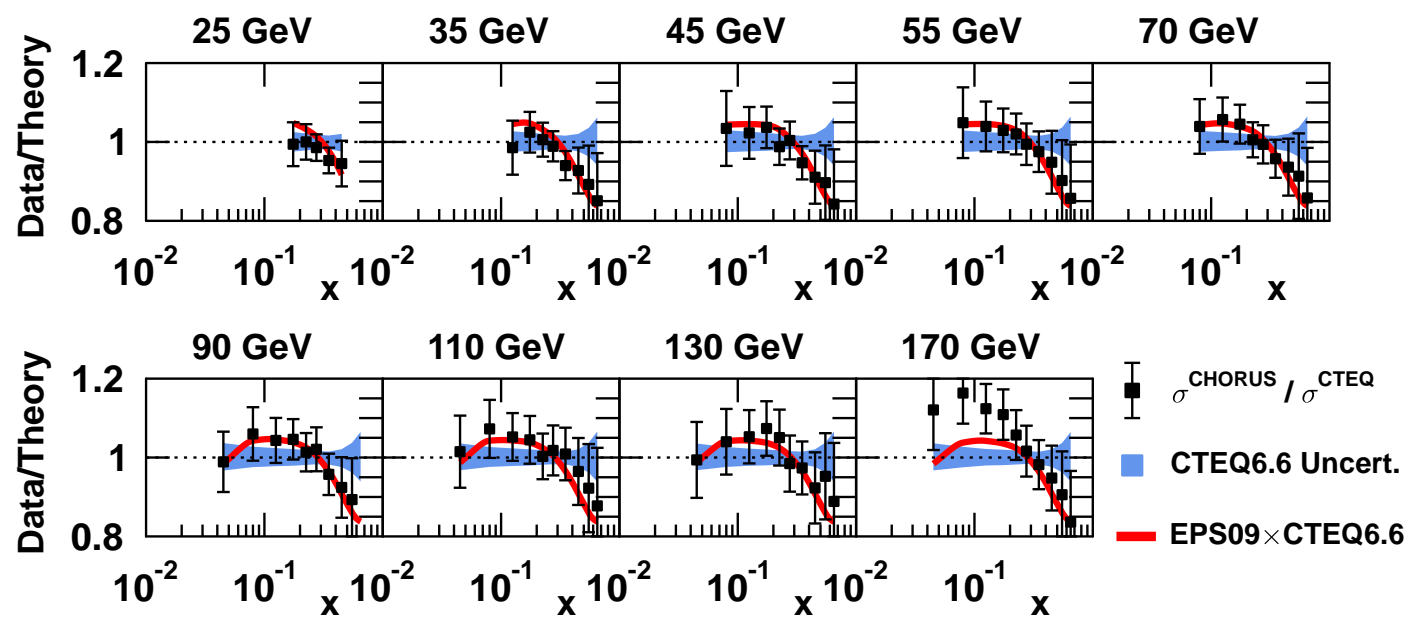

Figure 5.53. Comparison to neutrino data; taken from Ref. 968.

Regarding the impact of neutrino data, Schienbein et al. 36 claim that within their analysis it is not possible to reproduce simultaneously the trend of the data coming from electromagnetic nuclear DIS and some observables derived from neutrino DIS measurements. Of course, these conclusions are reached under rather stringent assumptions such as a very specific parameterization for nuclear effects and those implicit in the derivation of the neutrino DIS rates to deuteron, which have not been actually measured yet. On the other hand, using the EPS09 analysis and neutrino DIS data, Paukkunen and Salgado 968. find 


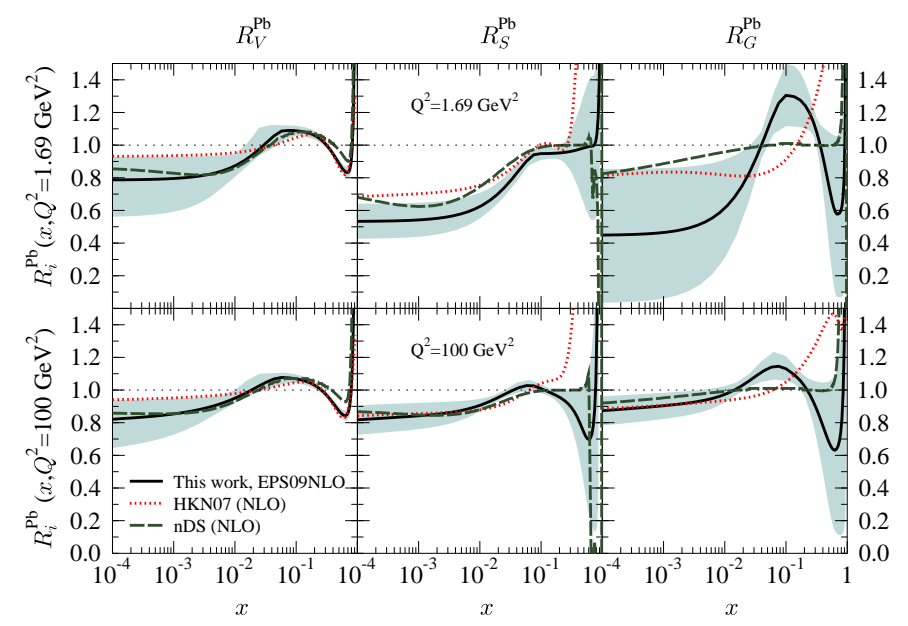

Figure 5.54. Comparison between different sets of nPDFs from 969].

no traces of such tension, besides some energy dependent fluctuations in the NuTeV data. A typical comparison to neutrino data is given in Fig. 5.53 .

Different recent extractions of nPDFs are shown in Fig. 5.54. A general shortcoming of all present fits is that independent nuclear modification factors can be determined only for gluons, valence, and sea quarks without distinguishing different quark flavors. Also, present fixed-target data do not constrain nPDFs below about $x_{B} \simeq 0.01$, and the curves shown at smaller values of $x_{B}$ are mere extrapolations. Uncertainties on $\mathrm{nPDF}$ s are large, in particular for the nuclear gluon distribution. There is clearly a need for more precise data covering also the small $x_{B}$ region.

Conclusions. In the last few years, our knowledge of the way that both parton densities and fragmentation probabilities are modified in a nuclear environment have improved significantly. Different studies performed so far have clearly demonstrated that pQCD factorization and universality are extremely good approximations within the precision and kinematic range of the available data. Although the uncertainties and differences between different QCD global analysis are still large, the availability of more data for different processes, and their subsequent inclusion in the analyses will certainly help to reduce them further. Ultimately, the EIC will be required for precise quantitative studies and to explore the small $x_{B}$ regime where novel non-linear recombination and saturation phenomena are expected. A preliminary study of the capabilities of the EIC in these respects has been presented in Section 5.3. the EIC has the potential to determine gluon and quark nPDFs to a precision comparable to the nucleon PDFs down to $x \sim 10^{-3}$, and indeed to detect saturation effects as a deviation from DGLAP linear evolution.

\section{HKN nuclear parton distribution functions}

\section{Shunzo Kumano}

The Hirai, Kumano and Nagai (HKN) nuclear PDFs [965, 38] are determined by a global analysis of world data on charged-lepton DIS and Drell-Yan processes with nuclear targets. Since the PDFs of the nucleon are relatively well determined, it is appropriate to 
parametrize the nPDFs at the initial $Q_{0}^{2}=1 \mathrm{GeV}^{2}$ using Eq. (5.134) and

$$
R_{i}\left(x_{B}, Q_{0}^{2}, A, Z\right)=1+\left(1-\frac{1}{A^{\alpha}}\right) \frac{a_{i}+b_{i} x+c_{i} x^{2}+d_{i} x^{3}}{(1-x)^{\beta_{i}}},
$$

The determined $u_{v}, \bar{q}$, and $g$ nPDFs from the HKN07 analysis [38] are shown for the calcium nucleus in Fig. 5.55 at $Q^{2}=1 \mathrm{GeV}^{2}$. LO and NLO results are shown with uncertainty bands, showing that $\mathrm{nPDF}$ s are determined more accurately at NLO. We obtain $\chi_{\min }^{2}$ /d.o.f. $=1.35$ and 1.21 for the LO and NLO fits, respectively.

The valence-quark modifications are well determined because of accurate measurements on the $F_{2}$ ratios at medium $x$. The small- $x$ region is fixed by the baryon-number and charge conservations together with the modifications in the medium- and large- $x$ regions. The antiquark modifications are also determined well at small $x$ due to measurements on $F_{2}$ shadowing, and they are also fixed at $x \sim 0.1$ because of Fermilab Drell-Yan measurements. However, the region at $x>0.2$ is not determined at all. The E906/SeaQuest collaboration is currently measuring this medium- $x$ region, and there is also a possibility to measure this region with an experiment at J-PARC. In the near future, the uncertainty bands should be significantly reduced for the antiquark.

The gluon distribution has the largest uncertainties since it contributes to the $F_{2}$ and Drell-Yan ratios only as higher-order effects, and the $Q^{2}$ dependence of $F_{2}^{A} / F_{2}^{A^{\prime}}$ is not measured accurately on nuclear targets, which makes it difficult to pin down the gluon modifications measured by scaling violations of $F_{2}$. The small- $x$ nPDFs are dominated by huge gluon distributions, so that it is essential to determine them accurately for new discoveries by high-energy heavyion experiments. Therefore, it is important to measure the $Q^{2}$ dependence of $F_{2}^{A} / F_{2}^{A^{\prime}}$ at EIC for determining nuclear gluon distributions.

In HKN07, the nPDFs are also investigated for the deuteron. In obtaining the "nucleonic" PDFs, deuteron data are used after crude nuclear corrections. Since the current PDFs could possibly contain nuclear effects, appropriate nuclear corrections should be applied in future for excluding such effects. Our codes for calculating the nPDFs and their uncertainties are available at the web site [970]. The technical details are explained in Refs. [965, 38] and within the subroutine.
Figure 5.55. Determined nuclear modifications in $\mathrm{Ca} 38$.

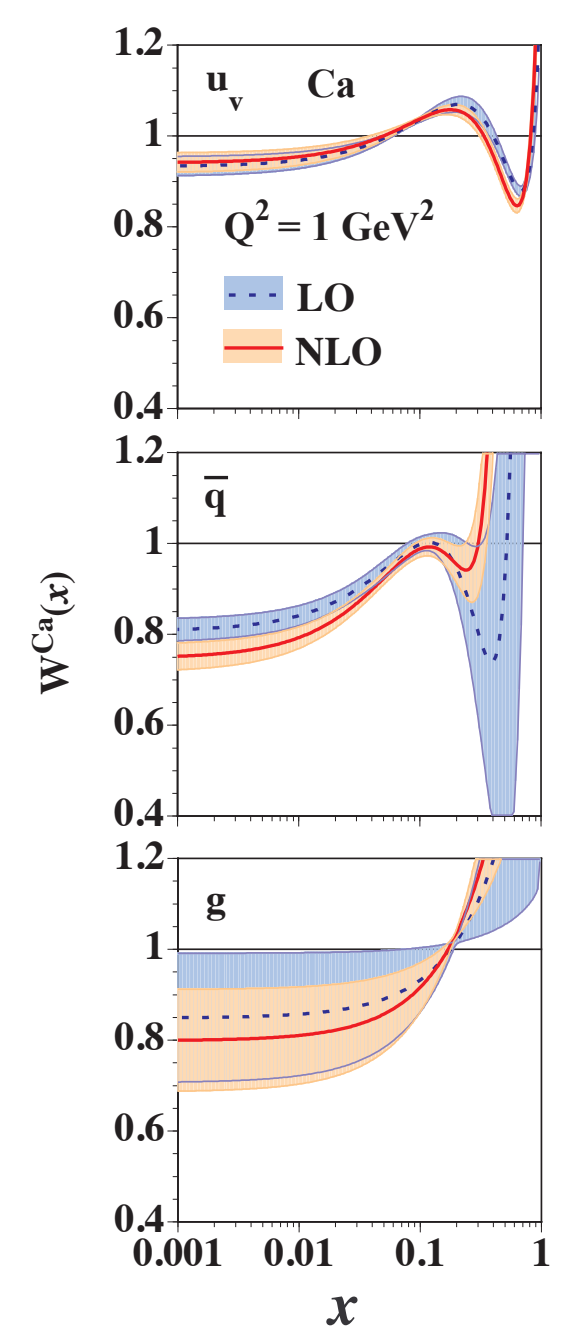




\title{
5.8 Color transparency
}

\section{Color transparency phenomena}

\author{
B. Z. Kopeliovich
}

The nuclear medium is more transparent for colorless hadronic wave packets than predicted by the Glauber model. One can treat this phenomenon either in the hadronic basis as a result of Gribov's inelastic corrections [791, or in QCD as a result of color screening [780], an effect called color transparency (CT). Although the two approaches are complementary, the latter interpretation is more intuitive and straightforward. Indeed, a point-like colorless object cannot interact with external color fields, therefore its cross section vanishes as $\sigma(r) \propto r^{2}$ when $r \rightarrow 0$ [780. When a colorless wave packet propagates through a nucleus, the fluctuations with small size have an enhanced survival probability which leads to a non-exponential attenuation $\propto 1 / L$ [780, where $L$ is the path length in nuclear matter.

Diffractive electro-production of vector mesons off nuclei is affected by shadowing and absorption which are different phenomena. Final state absorption of the produced meson exists even in the classical probabilistic approach which relates nuclear suppression to the survival probability $W(z, b)$ of the vector meson produced at the point with longitudinal coordinate $z$ and impact parameter $\vec{b}$,

$$
W(z, b)=\exp \left[-\sigma_{i n}^{V N} \int_{z}^{\infty} d z^{\prime} \rho_{A}\left(b, z^{\prime}\right)\right]
$$

where $\rho_{A}(b, z)$ is the nuclear density and $\sigma_{i n}^{V N}$ is the inelastic $V N$ cross section. Shadowing, is also known to cause nuclear suppression. In contrast to final state absorption, it is a pure quantum-mechanical effect which results from destructive interference of the amplitudes for which the interaction takes place on different bound nucleons. It can be interpreted as a competition between the different nucleons participating in the reaction: since the total probability cannot exceed one, each participating nucleon diminishes the chances of others to contribute to the process. The interplay between absorption and shadowing is controlled by the two time scales introduced for the case of charmonium in eq. (5.107). They are defined similarly for other hadrons.

In the low-energy limit of short $l_{c}<l_{f} \ll R_{A}$ (shorter than the mean nucleon spacing $\sim 2 \mathrm{fm}$ ) only final state absorption matters. The ratio of the quasielastic $\gamma^{*} A \rightarrow V X$ and $\gamma^{*} N \rightarrow V X$ cross sections reads,

$$
\left.R_{i n c}\right|_{l_{c}, l_{f} \ll R_{A}} \equiv \frac{\sigma_{V}^{\gamma^{*} A}}{A \sigma_{V}^{\gamma^{*} N}}=\frac{1}{A} \int d^{2} b \int_{-\infty}^{\infty} d z \rho_{A}(b, z) \exp \left[-\sigma_{i n}^{V N} \int_{z}^{\infty} d z^{\prime} \rho_{A}\left(b, z^{\prime}\right)\right]
$$

In the limit of long $l_{c} \gg R_{A}$, it takes a different form; in the Glauber approximation,

$$
\left.R_{i n c}\right|_{l_{c} \gg R_{A}}=\int d^{2} b T_{A}(b) \exp \left[-\sigma_{i n}^{V N} T_{A}(b)\right]
$$

One can see that the $V$ meson attenuates along the whole nucleus thickness in Eq. (5.138), but only along roughly half of that length in Eq. (5.137). The exact expression beyond VDM which interpolates between the two regimes (5.137) and (5.138) can be found in 925 . 

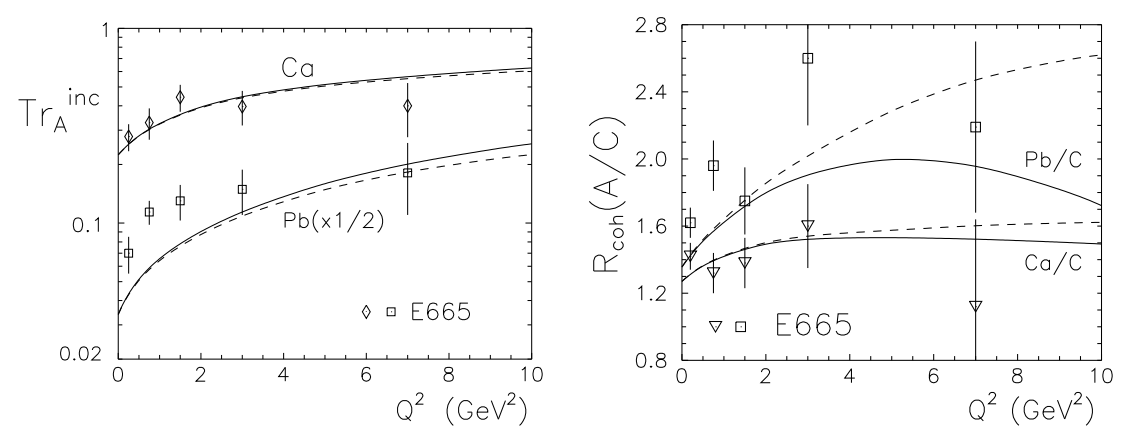

Figure 5.56. Comparison of the dipolea pproach with E665 data 971 for nuclear effect in electroproduction of $\rho$-mesons. Left panel: $Q^{2}$ - dependence of nuclear transparency for lead and calcium. Solid and dashed curves show the results of using the Green function approach and the "frozen" approximation respectively. Right panel: $Q^{2}$-dependence of the total cross section ratio $R_{c o h}(A / C)=12 \sigma_{c o h} / A \sigma_{c o h}$.

The effects of color transparency lead to deviation from this expression. These effects, which can be understood as Gribov inelastic corrections lead to equation (5.108), which should be used to study the effects of color transparency.

Light-cone distribution functions for the photons and vector mesons. In what follows, we rely on the dipole description and need to know the distribution functions for the photon and vector mesons. To be self-consistent, we should use the same light-cone potential for describing both. In equation (5.30) for the Green function, we chose the real part of the potential of the $\bar{q} q$ dipole as in Refs. [795, 913]. Solving Eq. (5.30) for the Green function with this potential and assuming similar spin structures for the vector mesons and photons, one can obtain an explicit formula for the vector meson light-cone wave function [913], depending on a "width" and a "quark mass" phenomenological parameters that were fitted to data in [934.

Cross section on a proton. Now we are in a position to calculate the forward electroproduction diffractive amplitudes, which have the following form, The forward scattering amplitude $\left.\mathcal{M}_{\gamma^{*} N \rightarrow V N}^{T, L}\left(s, Q^{2}\right)\right|_{t=0}$ can be extracted from eq. (5.100) discussed previously. These amplitudes are normalized as $\left|\mathcal{M}^{T, L}\right|^{2}=16 \pi d \sigma_{N}^{T, L} /\left.d t\right|_{t=0}$. In what follows we calculate the cross sections $\sigma=\sigma^{T}+\epsilon \sigma^{L}$ assuming that the photon polarization is $\epsilon=1$.

For HERA data, the normalization of the cross section and its energy and $Q^{2}$ dependence are remarkably well reproduced, see [913. This is important, since the absolute normalization is usually much more difficult to reproduce than nuclear effects, which we switch to in the nest section.

As a cross-check for the choice of the $\rho^{0}$ wave function, we also calculated the total $\rho^{0}$ nucleon cross section, which is usually expected to be roughly similar to the pion-nucleon cross section $\sigma_{\text {tot }}^{\pi N} \sim 25 \mathrm{mb}$. For the dipole cross section, we adopt the KST parameterization [795], which has been used above, and is designed to describe low- $Q^{2}$ data. Then, at $\nu=100 \mathrm{GeV}$, we obtain $\sigma_{\text {tot }}^{\rho N}=27 \mathrm{mb}$ which is quite a reasonable number.

Diffractive electroproduction on nuclei. In the high energy regime of $l_{c} \gg R_{A}$ one can rely on Eq. (5.111) for incoherent electroproduction of $\rho$-mesons (with different quark mass and meson wave function). As a manifestation of color transparency, the nuclear ratio, also called nuclear transparency, $\operatorname{Tr}_{A}^{i n c} \equiv R_{\text {inc }}$ defined in (5.110), was predicted in 972 ] to rise as 

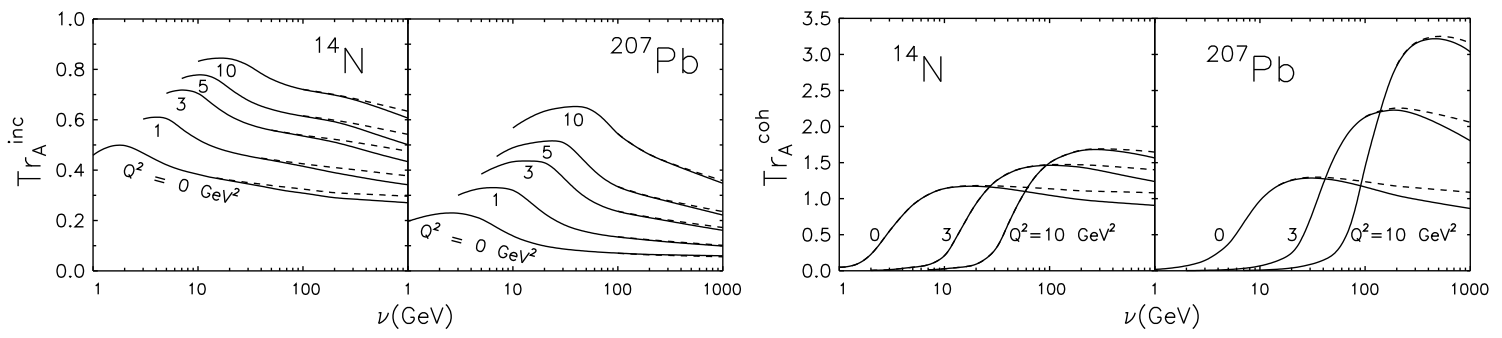

Figure 5.57. Nuclear transparency for incoherent and coherent electroproduction of $\rho^{0}$ on nitrogen and lead as function of energy. Solid and dashed curves correspond to calculations with and without gluon shadowing, respectively. Left two panels: Incoherent production at $Q^{2}=0,1,3,5,10 \mathrm{GeV}^{2}$. Right two panels: Coherent production at $Q^{2}=0,3,10 \mathrm{GeV}^{2}$.

function of $Q^{2}$. Indeed, the mean size of the $\bar{q} q$ component of the virtual photon decreases qith $Q^{2}$, so the nucleus becomes more transparent. The results of the E665 experiment at Fermilab 971 depicted in Fig. 5.56 are in a good accord with the predicted behavior. The calculations performed in the "frozen" approximation $\left(l_{c} \gg R_{A}\right)$ are presented with dashed curves. The more realistic results including finiteness of $l_{c}$ and $l_{f}$ are plotted by solid curves. While the "frozen" approximation is rather accurate for incoherent production, the deviation from its expectation for coherent process at the energy of the E665 experiment is significant.

The predicted energy dependence of the nuclear ratios in incoherent and coherent $\rho$ production on nitrogen and lead are depicted in Fig. 5.57. As was expected, the nucleus becomes more opaque with energy for incoherent production. This happens because when the hadronic fluctuations of the virtual photon live longer, they propagate through the whole nucleus and attenuate more. On the other hand, in incoherent production the phase shifts between the amplitudes of $\rho$ production on different nucleons must me very small in order the nucleus remained intact. This is why the nuclear ratio depicted in the bottom part of Fig. 5.57 is so suppressed at low energies.

At high energies, such as at an EIC, gluon shadowing causes an additional nuclear suppression of $\rho$ production. This correction is calculated as was described in Sect. 5.2 and the final results are plotted in Fig. 5.57 by solid curves. As was expected, the effect of gluon shadowing is not significant.

\section{From color transparency to color opacity}

\section{Mark Strikman}

Color transparency (CT) phenomena play several roles. They probe both the high energy dynamics of the strong interaction and the minimal small size components of the hadrons. In the case when some of the produced particles have energies smaller than $10 \mathrm{GeV}$ in the nucleus rest frame, these processes could be also used to study the space-time evolution of small wave packets - a question relevant for interpretation of heavy-ion collisions. They also provide an important link to the hard QCD black disk regime - the regime of strong absorption for the processes which at lower energies exhibit the CT regime, and determine the kinematics where factorization can be applied to generalized parton distribution studies.

The basic feature of $\mathrm{CT}$ is the suppression of the interaction of small size color singlet 
configurations: for a dipole of transverse size $d$, perturbative QCD gives

$$
\sigma\left(d, x_{N}\right)=\frac{\pi^{2}}{3} \alpha_{s}\left(Q_{e f f}^{2}\right) d^{2}\left[x_{N} G_{N}\left(x_{N}, Q_{e f f}^{2}\right)+2 / 3 x_{N} S_{N}\left(x_{N}, Q_{e f f}^{2}\right)\right],
$$

where $Q_{\text {eff }}^{2} \propto 1 / d^{2}, x_{N}=Q_{\text {eff }}^{2} / W^{2}$, and the second term is due to the contribution of quark exchanges which is important for intermediate energies [973. There are two critical requirements for CT phenomena: squeezing, the selection of small size configurations, and freezing, the selection of high enough energies to allow the squeezed configuration to live long enough.

At high energies, one can select CT processes by selecting special final states: for example, the diffraction of a pion into two high $p_{t}$ jets, or a small initial state $\gamma_{L}^{*}$ such as in the exclusive production of mesons. QCD factorization theorems [572, 973] were proven for these processes based on the CT property of QCD. The space time picture of these processes in the nucleus rest frame is as follows: long before the target, the projectile pion or virtual photon fluctuates into a $q \bar{q}$ configuration with transverse separation $d$, which elastically scatters off the target with an amplitude which for $t=0$ is given by Eq. (5.139) (up to small corrections due to different off shellness of the $q \bar{q}$ pair in the initial and final states), followed by the transformation of the pair into two jets or a vector meson. With a slight simplification, the amplitude for dijet diffractive production can be written as

$$
A(\pi N \rightarrow 2 \text { jets }+N)\left(z, p_{t}, t=0\right) \propto \int d^{2} d \psi_{\pi}^{q \bar{q}}(d, z) \sigma_{q \bar{q}-N(A)}(d, s) e^{i p_{t} d},
$$

where $z$ is the light-cone fraction of the pion momentum carried by a quark, and $\psi_{\pi}^{q \bar{q}}(z, d) \propto$ $z(1-z)_{d \rightarrow 0}$ is the quark-antiquark Fock component of the meson light cone wave function. The presence of the plane-wave factor in the final state leads to an expectation of an earlier onset of scaling than in the case of the vector meson production, where the vector meson wave function appears instead. CT was observed in the pion diffraction into two jets 974, confirming predictions in 975 . The HERA data on exclusive vector meson production are also well described.

\section{Investigations at an EIC}

Studies at an EIC will require investigations of different exclusive meson production channels as a function of $x, Q^{2}$. In the $\mathrm{CT}$ limit and $-t \geq 0.1 \mathrm{GeV}^{2}$, where coherence effects are negligible, one expects

$$
\sigma_{\gamma_{L}^{*} A \rightarrow " m e s o n "}^{i n c o h} A^{*}(t)=Z \sigma_{\gamma_{L}^{*} p \rightarrow " m e s o n " N}(t)+N \sigma_{\gamma_{L}^{*} n \rightarrow " m e s o n " N}(t)
$$

In EIC kinematics, the coherence length is $\gg 2 R_{A}$ so deviations from the CT prediction could be due to leading twist effects - leading twist shadowing, and higher twist effects of multiple interactions of the $q \bar{q}$ pair with the target nucleus. There are two distinctive regimes: $x \geq 0.03$ where nuclear PDFs are practically linear in $\mathrm{A}$, and $x \leq 0.01$ where a significant LT shadowing of nPDFs is expected (see discussion in section 5.2).

The $x \geq 0.03$ region. Multiple interactions should reduce the cross section. At an EIC, it would be possible to perform a scan as a function of $Q^{2}$. For low $Q^{2}$ and especially for $\sigma_{T}$, one expects a hadron-like regime in which absorption is strong and $\sigma_{\gamma_{L}^{*} A \rightarrow \text { coh }}^{i n e s o n " A^{*}}(t) \propto A^{1 / 3}$. With an increase of $Q^{2}$, one expects a transition from soft dynamics with Gribov-Glauber type screening to the CT regime without significant LT gluon shadowing. In the case of $J / \psi$ 
production, one expects the $\mathrm{CT}$ regime already at low $Q^{2}$ while for the light mesons, the onset of CT can be much slower as essential transverse sizes of the $q \bar{q}$ pair decrease rather slowly with $Q^{2}$ as manifested in the slow convergence of the t-slope of $\rho$-meson production to the t-slope of $J / \psi$ production with increasing $Q^{2}$ [976].

The $x \leq 0.01$ region. In this regime, one expects large shadowing due to the LT mechanisms both for the incoherent and coherent contribution in which case [977], perturbative color opacity is given by

$$
\frac{d \sigma_{\gamma_{L} A \rightarrow V A}}{d t} / \frac{d \sigma_{\gamma_{L} N \rightarrow V N}}{d t}=G_{A}^{2}\left(x, Q_{e f f}^{2}\right) / G_{N}^{2}\left(x, Q_{e f f}^{2}\right) \cdot F_{A}^{2}(t)
$$

where $F_{A}(t)$ is the nucleus form factor. Typical results for the expected suppression effect are given in Fig. 5.58, Note here that effective $Q^{2}$, which enters in Eq 5.142 , is much smaller than $Q^{2}$ in the electro-production of light vector mesons. For example, in the case of the $\rho$ meson, $Q_{\text {eff }}^{2} \sim 3 \mathrm{GeV}^{2}$ for $Q^{2} \sim 10 \mathrm{GeV}^{2}$. For $J / \psi$ photo-production, $Q_{\text {eff }}^{2} \sim 3 \div 4 \mathrm{GeV}^{2}$ and grows slowly with $Q^{2}$ 978. Hence, for the top EIC energies, one expects a reduction in the coherent $J / \psi$ photo/electro production of at least a factor of two. Numerically, the LT shadowing mechanism leads to a larger screening effect for the interaction of the small dipoles than the HT dipole eikonal models (cf. [976]).

The incoherent cross section, $\sigma_{\text {incoh }}$, is shadowed somewhat more strongly than the coherent cross section, $\sigma_{c o h}$. The effect grows with the increasing strength of the elementary interaction. As a result, the ratio $B_{\gamma^{*} N \rightarrow "}^{-1}{ }^{\prime \prime} N \cdot \sigma_{i n c o h} / \sigma_{c o h}$ of incoherent and coherent cross sections integrated over $t$ and divided by the slope of the elementary cross section is expected to decrease slowly with decreasing $x$ at fixed $Q^{2}$ (cf. Fig.43 in [979]). For example, for $B=4 \mathrm{GeV}^{-2}, R \equiv \sigma_{\text {incoh }} / \sigma_{\text {coh }}$ changes from $R \approx 0.3$ in the impulse approximation limit to $R \approx 0.18$ in the regime of strong absorption (strength of dipole interaction of the order $\left.\sigma_{\text {tot }}(\pi N)\right)$. Simultaneous measurements of coherent and incoherent diffraction will allow the testing of the underlying dynamics in greater detail.

Note that it will be feasible to measure the coherent cross section at $t \sim 0$ due to the very steep $t$ dependence of coherent peak and the ability to kill most of the incoherent diffraction experimentally. At the same time, measurements of the $t$ dependence of coherent diffraction beyond the first minimum are unlikely (except for the lightest nuclei like ${ }^{4} \mathrm{He}$ ) due to the dominance of processes of the nuclear excitations for $-t \geq-t_{1}$. (Measurements of very soft photons at rather large opening angles are required [980].) Note that the cross section of inelastic diffraction with production of hadrons in the nucleus fragmentation region is comparable to that of quasi-elastic diffraction. Studies of the t-dependence of the meson production and/or hadron production in the nucleus fragmentation regionare required to separate these two processes.

Testing the onset of the black disk regime. The study of vector meson production provides a fine probe to test the onset of high density color opacity regime where the LT approximation breaks down - the black disk regime in which interactions of small dipoles with heavy nuclei become completely absorptive. In this limit, one can derive a model independent prediction for the cross section of the vector meson production [981]:

$$
\frac{d \sigma^{\gamma_{T}^{*}+A \rightarrow V+A}}{d t}=\frac{M_{V}^{2}}{Q^{2}} \frac{d \sigma^{\gamma_{L}^{*}+A \rightarrow V+A}}{d t}=\frac{\left(2 \pi R_{A}^{2}\right)^{2}}{16 \pi} \frac{3 \Gamma_{V} M_{V}^{3}}{\alpha\left(M_{V}^{2}+Q^{2}\right)^{2}} \frac{4\left|J_{1}\left(\sqrt{-t} R_{A}\right)\right|^{2}}{-t R_{A}^{2}}
$$

where $\Gamma_{V}$ is the electronic decay width $V \rightarrow e^{+} e^{-}, \alpha$ is the fine-structure constant. Eq. (5.143) corresponds to a drastically different result: a factor of $Q^{4}$ slower $Q^{2}$ dependence of the cross section than the LT result. 

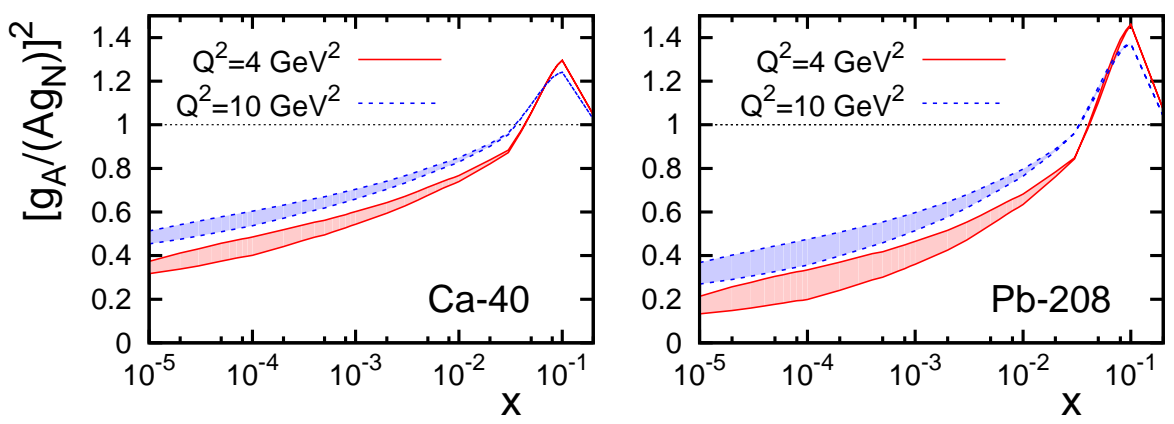

Figure 5.58. Leading twist shadowing effect for coherent vector meson production off $\mathrm{Ca}$ and $\mathrm{Pb}$. Bands reflect the range of predictions given by the FGS10_L and FGS10_H parametrizations of the gluon LT shadowing.

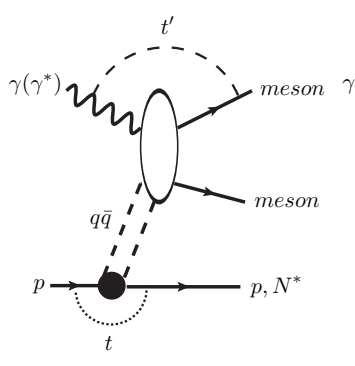

(a)

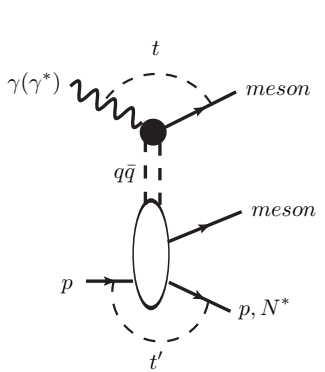

(b)

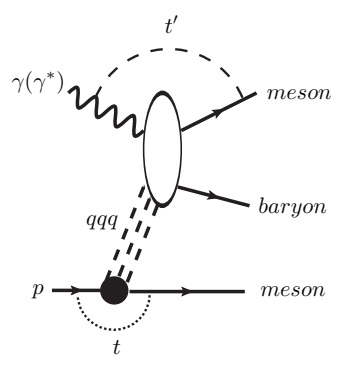

(c)

Figure 5.59. Examples of $2 \rightarrow 3$ processes probing (a) nucleon GPDs and large angle $\gamma^{*}(q \bar{q}) \rightarrow$ $\pi \pi(\pi \rho)$ scattering; (b) photon GPDs and large angle nucleon $(q \bar{q}) \rightarrow \pi N$ scattering, (c) nucleon $\rightarrow$ meson GPD and large angle $\gamma^{*}(q q q) \rightarrow \rightarrow \pi N$ scattering.

Other directions of studies. Recently, a number of novel processes were suggested to check the interplay between $\mathrm{CT}$ and color opacity phenomena as well as to use CT to understand the dynamics of various elementary processes.

1. It was demonstrated that it is possible to trace small dipoles through the center of the nucleus by selecting large $t \mathrm{VM}$ production with rapidity gap $\gamma^{*} A \rightarrow V+$ gap $+Y$ for $x_{g}=-t /\left(-t+M_{Y}^{2}\right)[982$.

2. It was suggested that amplitudes of high energy $2 \rightarrow 3$ branching processes: $a+b \rightarrow$ $c+d+e$, where $t=\left(p_{b}-p_{e}\right)^{2}$ is small, $t^{\prime}=\left(p_{a}-p_{c}\right)^{2}, s^{\prime}=\left(p_{c}+p_{e} d\right)^{2}$ are large, and $t^{\prime} / s^{\prime}=$ const can be written in a factorized form as a convolution of different nucleon quark GPDs and hard $2 \rightarrow 2$ amplitudes [983. Several examples of such processes are depicted in Fig. 5.59, In the case of the ep collider one would be able to study both the nucleon GPDs and GPDs of the real (virtual photon). Also, it will be possible to study large angle $\gamma\left(\gamma^{*}\right)+(q \bar{q}) \rightarrow$ meson $_{1}+$ meson $_{2}$ and $\gamma\left(\gamma^{*}\right)+(q q q) \rightarrow$ meson + baryon reactions.

3. Embedding these processes in nuclei, for example by studying the process $\gamma+A \rightarrow$ $\pi^{+} \pi^{+} A^{*}$, will make it possible to determine at what $p_{T}$ of the pions CT sets in and hence determine minimal $p_{T}$ for which these processes could be used to study various quark GPDs. The nuclear transparency for these processes is very sensitive 
to the size of the meson $\bar{q}$ configurations 984 . Hence it may be possible to determine the characteristic transverse size of the $q \bar{q}$ dipole involved in the hard process using Eq. (5.139). Also, by studying the transparency as a function of $s$ for fixed $s^{\prime}, t$ and $t^{\prime}$, one could measure in great detail the rate and the pattern of the space time evolution of small $q \bar{q}$ wave packets. 


\title{
5.9 Nuclear GPDs and TMDs
}

\subsubsection{Nuclear quark and gluon GPDs}

\author{
Vadim Guzey, Mark Strikman
}

Generalized parton distributions (GPDs) parameterize the response of hadronic targets (nucleon, nucleus) when probed by hard probes in exclusive reactions. The QCD factorization theorems state that GPDs are universal distributions that can be accessed in a wide range of hard exclusive processes: deeply virtual Compton scattering (DVCS) 624], electroproduction of mesons by longitudinal virtual photons [572], time-like Compton scattering, etc. GPDs are fundamental and rigorously-defined quantities that encode information on: (i) the distributions and correlations of partons in hadrons that is much richer than that contained in usual diagonal parton distributions and elastic form factors (in a certain sense, GPDs provide three-dimensional parton imaging), (ii) parton total angular momentum (thus, GPDs are believed to help resolve the so-called proton spin crisis), etc. For the detailed discussion of GPDs, see section 3.1 on "Imaging QCD Matter"

While what has been said above holds true for any hadronic target, nuclear GPDs are also interesting in their own right:

(i) Nuclear GPDs give access to both proton and neutron GPDs [985, 986, 987, 988, 989, Incoherent reactions (with nuclear break-up) can be used to study quasi-free neutron GPDs 990].

(ii) Traditional nuclear effects - off-diagonal EMC effect 991, 992, nuclear shadowing and antishadowing [993, 994, 807] — have been predicted to be more prominent than in the diagonal case.

(iii) Nuclear GPDs may be a good tool to study not well-established/controversial and novel nuclear effects such as the medium modifications of bound nucleon GPDs 992 , 995] and presence of non-nucleonic degrees of freedom 996].

Medium $x_{B}>0.05$

The cleanest way to study GPDs is deeply virtual Compton scattering (DVCS), $\gamma^{*}+A \rightarrow$ $\gamma+A^{\prime}$. Nuclear DVCS is more complex and versatile than that with the free proton because the nuclear target, $A$, can have various spins (the number of GPDs increases with the spin of the target) and many different final states, $A^{\prime}$, can be produced $\left(A^{\prime}=A, A^{*}, A+\pi, A-1+N\right.$, etc.). In the situation when the final nuclear state cannot be detected, one can sum over all final states $A^{\prime}$ assuming their completeness and obtain for the nuclear DVCS cross section [988]:

$$
\sigma_{\mathrm{DVCS}}=A(A-1) \sigma_{\mathrm{DVCS}}^{\mathrm{coh}}+A \sigma_{\mathrm{DVCS}}^{N} \cdot
$$

In this expression, the first term is the coherent-dominated contribution (without nuclear break-up or excitation) which is proportional to the nuclear form factor squared, $F_{A}^{2}$, and significant only at the small momentum transfer $t$. The second term is the incoherent contribution whose $t$ dependence is governed by that of the nucleon GPDs; this term dominates at large $t$.

Similarly to Eq. (5.144), the expressions interpolating between the coherent and incoherent regimes can also be derived for the interference between DVCS and Bethe-Heitler 
(BH) amplitudes and $\mathrm{BH}$ cross section. For instance, the coherent-dominated contribution to the interference between DVCS and BH amplitudes scales as $Z(A-1)$ and that to the $\mathrm{BH}$ cross section scales as $Z(Z-1)$ ( $Z$ is the nuclear charge). Therefore, one immediately and model-independently predicts the enhancement of the ratio of the nuclear to free proton DVCS beam-spin asymmetries at small $t, A_{\mathrm{LU}}^{A} / A_{\mathrm{LU}}^{p} \sim(A-1) /(Z-1)$ [987, 988]. At large $t$, the cross section is dominated by the incoherent contribution, no nuclear enhancement is expected, and $A_{\mathrm{LU}}^{A} / A_{\mathrm{LU}}^{p} \sim 1$ (in fact, the neutron contribution somewhat suppresses the ratio and makes $A_{\mathrm{LU}}^{A} / A_{\mathrm{LU}}^{p}<1$ [989]). While the HERMES analysis of nuclear DVCS with ${ }^{4} \mathrm{He},{ }^{14} \mathrm{~N},{ }^{20} \mathrm{Ne},{ }^{84} \mathrm{Kr}$, and ${ }^{132} \mathrm{Xe}$ targets supports that $A_{\mathrm{LU}}^{A} / A_{\mathrm{LU}}^{p} \sim 1$ at large $t$ and $A$-independent at all $t$, it finds that at small $t, A_{\mathrm{LU}}^{A} / A_{\mathrm{LU}}^{p}=0.91 \pm 0.19[672$.

Quark nuclear GPDs in the kinematic region of the off-diagonal EMC effect, $0.1<$ $x_{B}<0.3$, will be constrained with high precision by the analysis CLAS data on DVCS on ${ }^{4} \mathrm{He}$ [997]. The experiment measured purely coherent nuclear DVCS (the recoiled nucleus was detected using the BoNuS spectator tagger) and also DVCS on a quasi-free proton. The latter will probe possible nuclear medium modifications of the bound proton quark GPDs 995. Gluon GPDs in nuclei can be accessed best in hard exclusive production of heavy vector mesons. For instance, coherent $J / \psi$ production for $x_{B}>0.1$ can be used to learn about the off-diagonal EMC effect in the gluon channel. The incoherent production of $J / \psi$ can be used to probe medium modifications of the gluon GPD of the bound nucleon.

The EIC will be the only other accelerator beside JLab $12 \mathrm{GeV}$ to study GPDs in $e+A$ collisions, and will contribute considerably to their knowledge. In particular, it will access sea quark and gluon distributions, which are hard to measure at $6 \mathrm{GeV}$ due to the limited $x$ and $Q^{2}$ range, and open dedicated channels like $J / \Psi$ diffreactive production.

\section{Small $x_{B}<0.05$ : leading twist shadowing and exclusive diffraction}

The EIC will open the way to experimental measurements of nuclear GPDs at small $x_{B}$, where nuclear shadowing is known to occur for PDFs. The leading twist theory of nuclear shadowing (see section 5.2) allows one also to predict the impact parameter dependence of nuclear PDFs [807, 803, 805, 806]. The resulting impact parameter dependent nuclear PDFs, $f_{j / A}\left(x, Q^{2}, b\right)$ are the corresponding nuclear generalized parton distributions (GPDs) in the $\xi \rightarrow 0$ limit and in impact parameter space [994], $f_{j / A}\left(x, Q^{2}, b\right)=H_{A}^{j}\left(x, \xi=0, b, Q^{2}\right)$, where the latter GPD depends in general on two light-cone fractions $x$ and $\xi$; $\xi$ is fixed by the external kinematics, $\xi=x_{B} /\left(2-x_{B}\right)$, where $x_{B}$ is the standard Bjorken $x$. The number of GPDs depends on the spin of the target; we shall consider only spinless targets characterized by one twist-two chirally-even GPD $H^{j}$ ( $j$ is the parton flavor).

Using the predictions of the leading twist theory of nuclear shadowing for the impact parameter dependence of nuclear PDFs (Eq. (5.33) ) and the connection of these to GPDs, one can obtain the nuclear GPD $H_{A}^{j}$ at small $x$ in the $\xi=0$ limit. The final result for the GPDs in the momentum space is

$$
\begin{aligned}
H_{A}^{j}(x, \xi & \left.=0, t, Q^{2}\right)=A F_{A}(t) f_{j / N}\left(x, Q^{2}\right) \\
& -\frac{A(A-1)}{2} 16 \pi \Re e\left\{\frac{(1-i \eta)^{2}}{1+\eta^{2}} \int d^{2} b e^{i \vec{\Delta} \perp \cdot \vec{b}} \int_{\infty}^{\infty} d z_{1} \int_{z_{1}}^{\infty} d z_{2} \int_{x}^{0.1} d x_{\mathbb{P}} \rho_{A}\left(b, z_{1}\right)\right. \\
& \left.\times \rho_{A}\left(b, z_{2}\right) e^{i m_{N} x_{\mathbb{P}}\left(z_{1}-z_{2}\right)} e^{-\frac{A}{2}(1-i \eta) \sigma_{\mathrm{soft}}^{j}\left(x, Q^{2}\right) \int_{z_{1}}^{z_{2}} d z^{\prime} \rho_{A}\left(b, z^{\prime}\right)} \frac{1}{x_{\mathbb{P}}} f_{j}^{D(4)}\left(\beta, Q^{2}, x_{\mathbb{P}}, t_{\mathrm{min}}\right)\right\},
\end{aligned}
$$



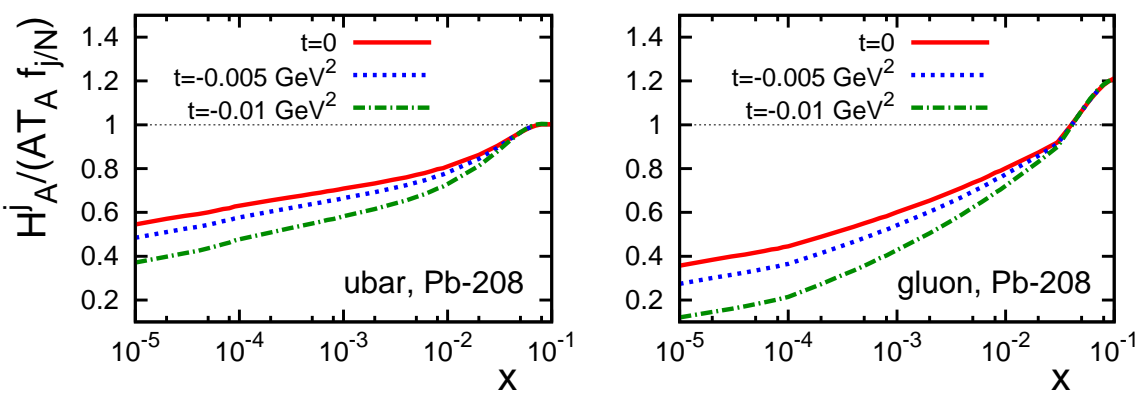

Figure 5.60. The ratio of the gluon and $\bar{u}$-quark $H_{A}^{j}(x, \xi=0, t) /\left[A F_{A}(t) f_{j / N}\left(x, Q^{2}\right)\right]$ for ${ }^{208} \mathrm{~Pb}$ as a function of $x$ for different values of $t$. All curves correspond to $Q^{2}=4 \mathrm{GeV}^{2}$ and model FGS10_H.

where the notation is the same as in eqs. (5.32) and (5.33).

Fig. 5.60 presents our predictions for the ratio $H_{A}^{j}\left(x, \xi=0, t, Q^{2}\right) /\left[A F_{A}(t) f_{j / N}\left(x, Q^{2}\right)\right]$ for ${ }^{208} \mathrm{~Pb}$ as a function of $x$ for different values of $t$. The left panel corresponds to the ratio of the $\bar{u}$-quark distributions; the right panel corresponds to the gluon distributions. All curves correspond to $Q^{2}=4 \mathrm{GeV}^{2}$ and model FGS10_H (see details in section [5.2). Since the $t$ dependence of the shadowing correction to $H_{A}^{j}(x, \xi=0, t)$ (second term in Eq. (5.145) ) is somewhat slower than that of the impulse approximation (the first term), the effect of nuclear shadowing increases as $|t|$ is increased, as expected.

Experimental observables measured in hard exclusive processes such as, e.g., $\gamma^{*}+A \rightarrow$ $\gamma(J / \Psi, \rho, \ldots)+A$, probe the GPD $H_{A}^{j}\left(x, \xi, t, Q^{2}\right)$ integrated over the entire region of the light-cone variable $x, 0 \leq x \leq 1$. However, at high energies (small $\xi$ or $x_{B}$ ), the situation simplifies: the predominantly imaginary $\gamma^{*}+A \rightarrow \gamma(J / \Psi, \rho, \ldots)+A$ scattering amplitudes are expressed solely in terms of the GPDs at the $x=\xi$ cross-over line, $H_{A}^{j}\left(\xi, \xi, t, Q^{2}\right)$ (to the leading order in the strong coupling constant $\alpha_{s}$ ). In addition, it was shown in [592] that at high energies and in the leading logarithmic approximation (LLA), GPDs at an input scale $Q_{0}^{2} \sim$ few $\mathrm{GeV}^{2}$ can be approximated well by the usual parton distributions, i.e., it is safe to neglect the effect of the skewness $\xi$. Therefore, for instance, for the imaginary part of the coherent nuclear deeply virtual Compton scattering (DVCS) amplitude $\left(\gamma^{*}+A \rightarrow \gamma+A\right)$, we have at the leading order in $\alpha_{s}$ :

$$
\begin{aligned}
\Im m \mathcal{A}_{\mathrm{DVCS}}\left(\xi, t, Q^{2}\right) & =-\pi \sum_{q} e_{q}^{2}\left[H_{A}^{q}\left(\xi, \xi, t, Q^{2}\right)+H_{A}^{\bar{q}}\left(\xi, \xi, t, Q^{2}\right)\right] \\
& \approx-\pi \sum_{q} e_{q}^{2}\left[H_{A}^{q}\left(\xi, \xi=0, t, Q^{2}\right)+H_{A}^{\bar{q}}\left(\xi, \xi=0, t, Q^{2}\right)\right]
\end{aligned}
$$

where $e_{q}$ are the quark charges; $H_{A}^{q}\left(\xi, \xi=0, t, Q^{2}\right)$ are given by Eq. (5.145).

The cleanest way to access GPDs is via DVCS. At the photon level, the $\gamma^{*}+A \rightarrow \gamma+A$ cross section reads, (see, e.g., 625]):

$$
\frac{d \sigma_{\mathrm{DVCS}}}{d t}=\frac{\pi \alpha_{\mathrm{em}}^{2} x^{2}\left(1-\xi^{2}\right)}{Q^{4} \sqrt{1+\epsilon^{2}}}\left|\Im m \mathcal{A}_{\mathrm{DVCS}}\left(\xi, t, Q^{2}\right)\right|^{2},
$$

where $\alpha_{\text {em }}$ is the fine-structure constant; $\epsilon^{2}=4 x^{2} m_{N}^{2} / Q^{2} ; \Im m \mathcal{A}_{\text {DVCS }}$ is given by Eq. (5.146).

The DVCS process interferes and competes with the purely electromagnetic BetheHeitler $(\mathrm{BH})$ process. The $\mathrm{BH}$ cross section at the photon level can be written in the 

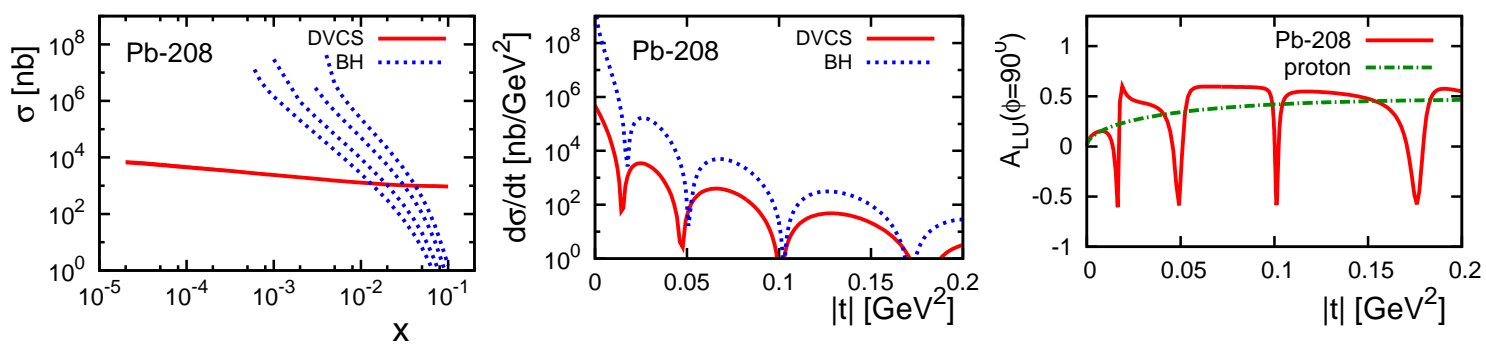

Figure 5.61. The DVCS (solid curves) and Bethe-Heitler (dot-dashed curves) cross sections for ${ }^{208} \mathrm{~Pb}$ at $Q^{2}=4 \mathrm{GeV}^{2}$. Left panel: $t$-integrated cross sections vs. $x$. The four $\mathrm{BH}$ curves correspond, from left to right, to $\sqrt{s}=(32,44,66,90) \mathrm{GeV}$. Middle panel: differential cross sections vs. $|t|$ at fixed $x=5 \times 10^{-3}$; the BH curve corresponds to $\sqrt{s}=32 \mathrm{GeV}$. Right panel: beam-spin asymmetry $A_{L U}$.

following form 625:

$$
\frac{d \sigma_{\mathrm{BH}}}{d t}=\frac{\pi \alpha_{\mathrm{em}}^{2}}{4 Q^{2} t(1+\epsilon)^{5 / 2}\left(1-y-y^{2} / 2\right)} \int_{0}^{2 \pi} \frac{d \phi}{2 \pi} \frac{1}{\mathcal{P}_{1}(\phi) \mathcal{P}_{2}(\phi)}\left|\mathcal{A}_{\mathrm{BH}}\left(\xi, t, Q^{2}, \phi\right)\right|^{2},
$$

where $y=\left(q \cdot P_{A}\right) /\left(k \cdot P_{A}\right)=Q^{2} /(x s)$ ( $k$ is the incoming lepton momentum, $q$ is the momentum of the virtual photon, $P_{A}$ is the momentum of the incoming nucleus, $s$ is the total invariant energy squared); $\phi$ is the angle between the lepton and hadron scattering planes; $\mathcal{P}_{1}(\phi)$ and $\mathcal{P}_{2}(\phi)$ are proportional to the lepton propagators; $\left|\mathcal{A}_{\mathrm{BH}}\left(\xi, t, Q^{2}\right)\right|^{2}$ is the $\mathrm{BH}$ amplitude squared. The expressions for $\mathcal{P}_{1,2}(\phi)$ and $\left|\mathcal{A}_{\mathrm{BH}}\left(\xi, t, Q^{2}\right)\right|^{2}$ can be found in [625]. Note that $\left|\mathcal{A}_{\mathrm{BH}}\left(\xi, t, Q^{2}\right)\right|^{2}$ is proportional to the nuclear electric form factor squared $\left(\left|F_{A}(t)\right|^{2}\right)$ and the nucleus charge squared $\left(Z^{2}\right)$.

Integrating the differential cross sections in Eqs. (5.147) and (5.148) over $t$, one obtains the corresponding $t$-integrated cross section $\sigma_{\mathrm{DVCS}(\mathrm{BH})}$ between $t_{\min } \approx-x^{2} m_{N}^{2}$ and $t_{\max }=$ $-1 \mathrm{GeV}^{2}$ :

In fig, 5.61 we present our predictions for a ${ }^{208} \mathrm{~Pb}$ target: in the left plot, the DVCS and $\mathrm{BH}$ cross sections at $Q^{2}=4 \mathrm{GeV}^{2}$, in the middle plot the differential cross sections as a function of $|t|$ at fixed $x=5 \times 10^{-3}$, and in the right plot the $A_{L U}$ asymmetry.

In the considered kinematics, the $t$-integrated $\mathrm{BH}$ cross section is much larger than the DVCS cross section for $x<10^{-2}$ due to the dramatic enhancement of the BH cross section at small $t \approx t_{\text {min }}$ by the factor $1 / t$, see Eq. (5.148). Therefore, in order to extract a small DVCS signal on the background of the dominant BH contribution for such $x$, one needs to consider the observable differential in $t$. The $t$ dependence of the DVCS and BH differential cross sections has the characteristic shape of the nuclear form factor squared, with distinct minima and maxima. However, the minima of the DVCS cross section are slightly shifted towards smaller $t$ : this is the effect of the leading twist nuclear shadowing in quark nuclear GPDs. The small shift of the minima toward smaller $t$ can be interpreted as an increase of the transverse size of the distributions of quarks in nuclei. One can enhance the effect by using lighter nuclei (e.g., ${ }^{4} \mathrm{He}$ and ${ }^{12} \mathrm{C}$ ) or by considering observables sensitive to the interference between the BH and DVCS amplitudes. For instance, the DVCS beam-spin asymmetry at $A_{\mathrm{LU}}\left(\phi=90^{\circ}\right)$, dramatically oscillates as a function of $|t|$ [994, and the sole reason for these oscillations is the leading twist nuclear shadowing.

Another possibility to study nuclear shadowing in DVCS is offered by processes with nuclear break-up. In this case, the nuclear modification (suppression due to shadowing) of the DVCS break-up cross section (as compared to the impulse approximation) is as large - or even bigger - as that for the coherent case. At the same time, in the impulse approximation, 
the relative contribution of the DVCS and BH cross sections is enhanced by $A / Z$ compared to the ep case. It allows one to observe the DVCS signal on the large BH background down to much smaller $x$ than in the $e p$ case, see the discussion in section 3.1 .

The leading twist theory of nuclear shadowing allows one also to make predictions for certain observables in exclusive electroproduction of heavy vector mesons $(J / \psi, \Upsilon)$ with nuclear targets which probe the nuclear gluon distribution, with a pattern similar to that discussed for small- $x$ nuclear DVCS [807. See the discussion by M.Strikman in Section 5.8.

\subsubsection{Nuclear TMDs}

Jian-Hua Gao, Zuo-tang Liang, Xin-Nian Wang, Jian Zhou

Transverse momentum dependent distributions (TMDs) were discussed extensively for nucleons earlier in this report. TMDs play an important role in studying final/initial state multiple re-scattering effects in nuclei. Indeed, the leading power nuclear effect comes from the gauge link appearing in the nuclear TMDs, in which the re-scattering effect is encoded.

The extraction of the TMDs from high energy scattering data relies on TMD factorization theorems, established in the $e^{+} e^{-}$annihilation process [241] and semi-inclusive deepinelastic (SIDIS) lepton-nucleon scattering [256]. It is not so clear whether TMD factorization still holds in SIDIS off a large nucleus target. In our recent work [998, we simply assume that it does. Correspondingly, one can introduce leading power unpolarized nuclear TMDs. For simplicity, we restrict our discussion to the light cone gauge, $A^{+}=0$ [263], where

$$
f_{q}^{A}\left(x, \vec{k}_{\perp}\right)=\int \frac{d y^{-}}{2 \pi} \frac{d^{2} y_{\perp}}{(2 \pi)^{2}} e^{i x p^{+} y^{-}-i \vec{k}_{\perp} \cdot \vec{y}_{\perp}}\left\langle A\left|\bar{\psi}\left(0, \overrightarrow{0}_{\perp}\right) \frac{\gamma^{+}}{2} \mathcal{L}_{\perp}(0, y) \psi\left(y^{-}, \vec{y}_{\perp}\right)\right| A\right\rangle
$$

and the transverse gauge link is $\mathcal{L}_{\perp} \equiv P \exp \left[-i g \int_{\overrightarrow{0}_{\perp}}^{\vec{y}_{\perp}} d \vec{\xi}_{\perp} \cdot \vec{A}_{\perp}\left(\infty, \vec{\xi}_{\perp}\right)\right]$. This gauge link is not only crucial to ensure the gauge invariance of the TMD parton distribution functions, but also leads to physical consequences such as single-spin asymmetries in SIDIS and the Drell-Yan process in $e+p$ collisions [251, 261, 262]. For DIS off a nucleus target, it should also contain information on the quark transverse momentum broadening due to multiple scattering inside the nucleus [998].

In the study of either cold or hot nuclear matter, parton transverse momentum broadening plays a crucial role in unraveling the medium properties. One important parameter that controls parton energy loss is the parton transport parameter $\hat{q}$, i.e., the transverse momentum broadening squared per unit of propagation length [999]. Therefore, the calculation and measurement of the jet transport parameter is an important step toward understanding the intrinsic properties of the QCD medium. Much effort has been devoted to the study of transverse momentum broadening in high energy collisions within different approaches [999, 1000, 1001, 1002, 1003, 1004, 1005, 1006].

In this contribution, we start from the matrix element definition of the nuclear TMD and identify the gauge link as the main source of leading nuclear effects. The broadened distribution has a Gaussian form, as found in earlier studies [1005], and suppresses the azimuthal asymmetry in SIDIS off nuclear targets. This in turns gives direct experimental access to the cold nuclear matter transport coefficient $\hat{q}$, and offers a way to determine the relative magnitude of the intrinsic transverse momentum in various nucleon TMDs.

Nuclear TMDs and nucleon TMDs. The effect of final state interactions that lead to transverse momentum broadening can be encoded in the gauge link. In fact, the nuclear 
dependent part of the quark TMD can be isolated from the gauge link so that the nuclear TMD can be expressed as a convolution of the Gaussian broadening and the nucleon TMD. Assuming a weakly bound nucleon, neglecting the correlation between different nucleons, and keeping only the matrix elements with nuclear enhancement one obtains the nuclear TMD,

$$
f_{q}^{A}\left(x, \vec{k}_{\perp}\right)=\frac{A}{\pi \Delta_{2 F}} \int d^{2} \ell_{\perp} e^{-\left(\vec{k}_{\perp}-\vec{\ell}_{\perp}\right)^{2} / \Delta_{2 F}} f_{q}^{N}\left(x, \vec{\ell}_{\perp}\right),
$$

as a convolution of the nucleon TMD and a Gaussian with a width $\Delta_{2 F}$ given by the total transverse momentum broadening squared,

$$
\Delta_{2 F}=\frac{1}{A f_{q}^{N}(x)} \int d^{2} k_{\perp} k_{\perp}^{2}\left[f_{q}^{A}\left(x, \vec{k}_{\perp}\right)-f_{q}^{N}\left(x, \vec{k}_{\perp}\right)\right]=\int d \xi_{N}^{-} \hat{q}_{F}\left(\xi_{N}\right) .
$$

where the quark transport parameter $\hat{q}_{F}\left(\xi_{N}\right)$ is defined as

$$
\hat{q}_{F}\left(\xi_{N}\right)=-\frac{g^{2}}{2 N_{c}} \rho_{N}^{A}\left(\xi_{N}\right) \int \frac{d \xi^{-}}{2 p^{+}}\left\langle N\left|F_{+\sigma}(0) F_{+}^{\sigma}\left(\xi^{-}\right)\right| N\right\rangle=\frac{2 \pi^{2} \alpha_{s}}{N_{c}} \rho_{N}^{A}\left(\xi_{N}\right)\left[x f_{N}^{g}(x)\right]_{x=0},
$$

with $\rho_{N}^{A}\left(\xi_{N}\right)$ is the spatial nucleon density inside the nucleus and $f_{g}^{N}(x)$ is the gluon distribution function in a nucleon. Eq. (5.150) is our main result.

Nuclear dependence of azimuthal asymmetry in SIDIS. One can generalize the above approach to the nuclear modification of higher twist TMD parton distributions. The case of twist-3 and twist-4 TMDs [243, 247, 1007, which account for the $\cos \phi$ and $\cos 2 \phi$ azimuthal asymmetries in SIDIS, has been recently investigated in Ref. [1008, 1009]. Here we review the nuclear dependent $\cos \phi$ azimuthal asymmetry in the two kinematic regions: at small transverse momentum $P_{h \perp} \sim \Lambda_{Q C D}$ and intermediate transverse momentum $\Lambda_{Q C D} \ll P_{h \perp} \ll Q$, where $Q$ is the virtual photon momentum. The central ingredient of the treatment in Ref. 1008 is the relation between the nucleon twist-3 TMDs and nuclear ones. If we look at jet production in SIDIS, the azimuthal asymmetry is solely determined by one twist-3 TMD distribution $f^{\perp}\left(x, k_{\perp}\right)$. The ratio of the asymmetry between SIDIS off nucleons and nuclei is,

$$
\frac{\langle\cos \phi\rangle_{e A}}{\langle\cos \phi\rangle_{e N}}=\frac{f_{\perp}^{A}\left(x, k_{\perp}\right) / f^{A}\left(x, k_{\perp}\right)}{f_{\perp}^{N}\left(x, k_{\perp}\right) / f^{N}\left(x, k_{\perp}\right)}
$$

The ratio depends on how the twist-3 TMD distributions $f_{\perp}^{A}$ is enhanced/suppressed due to the stronger final state interaction taking place inside a nucleus. Following the same approach applied to the twist-2 TMD distribution, we relate the function $f_{\perp}^{A}$ to $f_{\perp}^{N}$,

$$
f_{\perp}^{A}\left(x, k_{\perp}\right) \approx \frac{A}{\pi \Delta_{2 F}} \int d^{2} \ell_{\perp} \frac{\left(\vec{k}_{\perp} \cdot \vec{\ell}_{\perp}\right)}{\vec{k}_{\perp}^{2}} e^{-\left(\vec{k}_{\perp}-\vec{\ell}_{\perp}\right)^{2} / \Delta_{2 F}} f_{\perp}^{N}\left(x, \ell_{\perp}\right)
$$

Given the TMDs $f^{N}\left(x, k_{\perp}\right)$ and $f_{\perp}^{N}\left(x, k_{\perp}\right)$, one will be able to calculate the ratio (5.154). To illustrate the nuclear dependence of the asymmetry qualitatively, we consider an ansatz of the Gaussian distributions in $k_{\perp}$ for both TMDs,

$$
f^{N}\left(x, k_{\perp}\right)=\frac{1}{\pi \alpha} f_{q}^{N}(x) e^{-k_{\perp}^{2} / \alpha}, \quad f_{\perp}^{N}\left(x, k_{\perp}\right)=\frac{1}{\pi \beta} f_{q \perp}^{N}(x) e^{-k_{\perp}^{2} / \beta} .
$$

As shown in Fig. 5.62, the azimuthal asymmetry is suppressed in $e+A$ SIDIS as compared to that in $e+N$ SIDIS. Note also that the suppression pattern as a function of $k_{\perp}$ is sensitive to the relative magnitude of the intrinsic transverse momentum in the nucleon TMDs. 

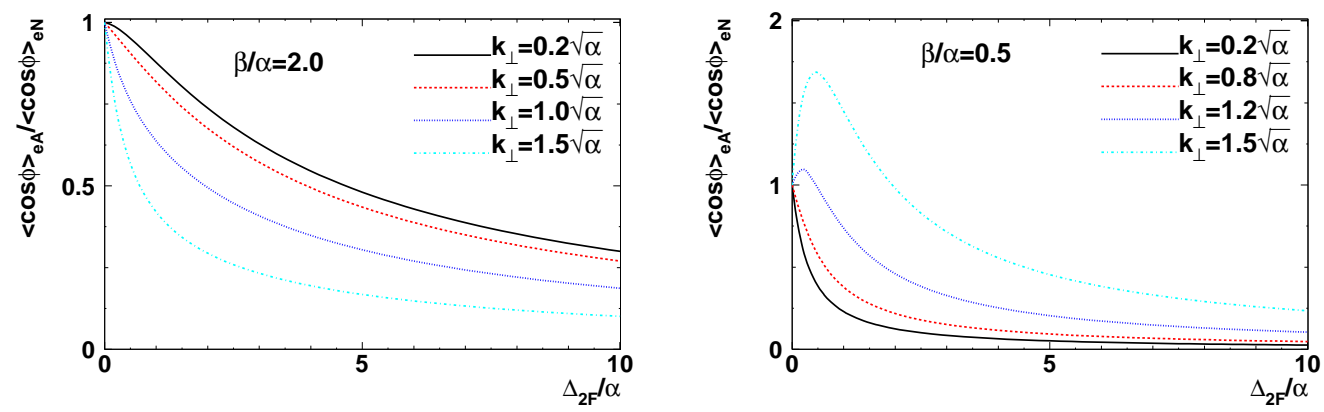

Figure 5.62. Ratio $\frac{\langle\cos \phi\rangle_{e A}}{\langle\cos \phi\rangle_{e N}}$ as a function of $\Delta_{2 F}$ for different $k_{\perp}$ and the relative width $\beta / \alpha$.

Now let us discuss the asymmetry at intermediate transverse momentum. The fact that TMDs are perturbatively calculable when $p_{\perp} \gg \Lambda_{Q C D}$ or $k_{\perp} \gg \Lambda_{Q C D}$ allows us to reduce the theoretical uncertainty, since the twist-3 TMDs are poorly known so far. In the parton model, the azimuthal asymmetry for hadron production in SIDIS can be expressed as a convolution of a few TMD distributions and TMD fragmentation functions [243, 247]. It turns out that fragmentation functions $H_{1}^{\perp}$ and $\tilde{H}$ are power suppressed compared to $\tilde{D}_{\perp}$ and $D$ at large $p_{\perp}$ [292, 296, 297]. Therefore, at intermediate transverse momentum, the leading power terms are proportional to $f_{1} \tilde{D}_{\perp}$ and $f_{\perp} D$. In the current fragmentation region, where $p_{\perp}$ is large, we make a collinear expansion around $p_{\perp}=q_{\perp}$ in terms of the power $k_{\perp} / q_{\perp}$ and keep the quadratic terms $k_{\perp}^{2} / q_{\perp}^{2}$ in order to extract the nuclear dependent contributions. After carrying out the integrals over $p_{\perp}$, we find the nuclear dependent azimuthal asymmetry is related to the term $D(z) \int \frac{k_{\perp}^{2}}{q_{\perp}^{2}} f_{1}\left(x, k_{\perp}\right) d^{2} k_{\perp}$. Therefore, the difference of the $\cos \phi_{h}$ azimuthal asymmetry is proportional to the transverse momentum broadening.

$$
\left\langle\cos \phi_{h}\right\rangle_{e A}-\left\langle\cos \phi_{h}\right\rangle_{e N} \propto \int \frac{k_{\perp}^{2}}{q_{\perp}^{2}}\left[f_{1}^{A}\left(x, k_{\perp}\right)-f_{1}^{N}\left(x, k_{\perp}\right)\right]=\frac{\Delta_{2 F}}{q_{\perp}^{2}}
$$

Conclusions. In summary, we can get a direct handle on the crucial transport parameter $\hat{q}$, which descrcibed the properties of the QCD medium, by measuring the nuclear dependent azimuthal asymmetry at intermediate transverse momentum. Conversely, the target nucleus can be used as a filter to study nucleon TMDs, e.g., to determine the relative magnitude of the intrinsic transverse momentum of $f^{N}$ and $f_{\perp}^{N}$.

Acknowledgments: J.Z. thanks A. Metz and M. Diehl for helpful discussion. 


\subsection{Current fragmentation}

\section{Introduction and the role of $e+A$ collisions}

Raphaël Dupré and Alberto Accardi

The fragmentation process, by which hard partons turn into hadrons, is only partly known due to its non perturbative nature. Fragmentation functions, which encode the probability that a parton fragments into a hadron, have been obtained by fitting experimental data covering large kinematic ranges and numerous hadron species, see Section 5.10 . However, knowledge about the dynamics of hadronization remains fragmentary: this process has been studied in a number of model calculations, but lacks a first-principles description in QCD. One possible scenario for the hadronization process is sketched in figure 5.63 as an example for DIS. At LO the virtual photon strikes a quark, which then propagates quasifreely emitting gluons; after a time called production time, the quark neutralizes its color and gluon emission stops. The quark becomes a pre-hadron, which will eventually form a hadron at the formation time. In fact, a color string connects the struck quark to its nucleon, and hadrons can be formed all along this string, but we focus our attention on the hadron that contains the struck parton. In nuclear DIS, the hadronization process happens at least in part in the target nucleus (cold nuclear matter). Thus the quark is subject to energy loss by medium-induced gluon brehmsstrahlung, and the prehadron (as well as the hadron) can have inelastic interactions with the surrounding nucleons, leading to attenuation and broadening of the produced particle spectra. The relative weight of one mechanism compared to the other is determined by the magnitude of the color neutralization time. For full reviews, see Refs. [1010, 1011, 1012]. Alternative scenarios are also feasible and final states in nuclear DIS (nDIS) can help untangle these from the scenario outlined here to provide genuine insight into the hadronization process.

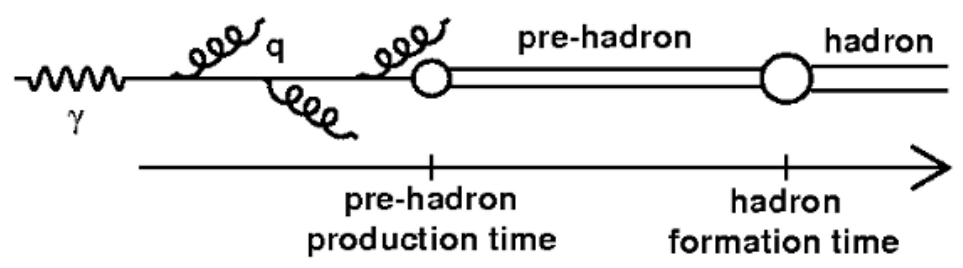

Figure 5.63. A model sketch of the hadronization process.

These nuclear effects are both an opportunity for a first principles study hadronization and nuclear properties as well as important benchmarks for reducing existing uncertainties in many nuclear measurements. For example, in neutrino experiments, nuclei are used to maximize the cross section and the kinematics are reconstructed from the hadronic final state. Therefore, a poor knowledge of hadron attenuation leads to a tangible systematic error. In heavy-ion collisions, hadrons are produced in hot and expanding nuclear matter, whose properties can be measured, among other methods, by the modifications of highenergy particle spectra compared to proton-proton and proton-nucleus collisions. It is clear that the details and the time scales of the hadronization process can profoundly modify the interpration of the data, see Fig. 5.2 .

The role of $e+A$ collisions. Nuclear deep inelatic scattering provides a known and stable 
cold nuclear medium and a low-multiplicity final state with strong experimental control on the kinematics of the hard scattering. This permits one to use nuclei as femtometer-scale detectors and study the time scales of the hadronization process and calibrate theoretical models for parton energy loss and prehadronic scattering, that can then be applied, for instance, to the study of the QGP, see Figure 5.2. Initial state parton energy loss can furthermore be studied in isolation from hadronization in Drell-Yan lepton pair production in $p+A$ collisions, where however it can be masked by nuclear modification of the target wave function such as antishadowing and the EMC effect. So, an interplay of nuclear DIS and nuclear Drell-Yan can help isolate hadronization effects on one hand, and on the other to clarify the differences in quark and anti-quark antishadowing. Perhaps more interestingly, the study of hadronization in nuclear DIS can give direct information about the gluon structure of the nuclei. For example, one can link energy loss and transverse momentum broadening to the gluon density [1013] or more directly to the saturation scale [801]. In models like GiBUU 1014, focussing on hadron absorption, access to the pre-hadron evolution and its color transparency evolution is possible. All these physical interpretation of the data are model dependent and based on very different assumptions about the relative importance of the interaction mechanisms, therefore they are fragile and need to be carefully validated and calibrated with precise data.

The typical observables used to explore hadronization in nuclear DIS are the multiplicity ratio and the transverse momentum broadening, in both cases they are comparison of deuterium with heavier nuclei. The multiplicity ratio, representing the production rate of a hadron $h$ in a nuclear target $A$ compared to Deuterium, is defined as

$$
R_{A}^{h}\left(Q^{2}, \nu, z_{h}, p_{T}^{2}\right)=\frac{N_{A}^{h}\left(Q^{2}, \nu, z_{h}, p_{T}^{2}\right) / N_{A}^{e}\left(Q^{2}, \nu\right)}{N_{D}^{h}\left(Q^{2}, \nu, z_{h}, p_{T}^{2}\right) / N_{D}^{e}\left(Q^{2}, \nu\right)}
$$

with $N_{t}^{e}$ and $N_{t}^{h}$ respectively the number of electrons and the number of semi-inclusive hadrons $h .1-R_{A}^{h}$ is the attenuation of hadron production in a nucleus of atomic mass $A$. This ratio minimizes the influence of nuclear PDF modifications, which have been shown to cancel to a large degree up to NLO. The hadron transverse momentum broadening, representing the increase of transverse momentum in a nuclear target A compared to Deuterium, is defined by $\Delta\left\langle p_{T}^{2}\right\rangle=\left\langle p_{T}^{2}\right\rangle_{A}-\left\langle p_{T}^{2}\right\rangle_{D}$, with $\left\langle p_{T}^{2}\right\rangle_{t}$ the average hadron transverse momentum measured in a nucleus. When integrated over a large kinematic range, these observables they are dominated by the geometry of the nuclei and do not discriminate well between the models. One needs to also consider more differential observables, including a multi-dimensional analysis of $R_{M}$ and $\Delta\left\langle p_{T}^{2}\right\rangle$, and hadron-hadron and photon-hadron correlations.

Another possibility is to use experimental settings in which we can isolate the involved processes. In the case of EIC, the high energy boost imparted to the struck quark in events with large $\nu$ can increase dramatically the production length, which leads to prehadron production far outside the nuclei and an experimental isolation of pure parton energy loss effects. Since the pre-hadron production time is expected to roughly be inversely proportional to the mass squared of the hadron, measuring attenuation and $p_{T}$-broadening of many meson and baryon species, together with the large $\nu$ leverage afforded by the EIC, will give another important handle in the exploration of the hadronization mechanism. New features will be availabe at the EIC, the high rate for heavy flavor production $(D$ and $B$ mesons) will allow the measurement of heavy quark energy loss. Finally, jet production, will open the possibility to study the dynamics of parton showers and the detailed transport properties of cold nuclear matter using specific jet observables. 
Overview of theoretical models. Three processes are typically included in theoretical descriptions of in-medium hadronization: quark energy loss, pre-hadron absorption and modified fragmentation functions. The models in the literature are usually based on one or two of those and neglect the others. In this section we will discuss a few examples to give an idea of the abundant existing literature; for a detailed review, including models specific to heavy-ion collision experiments, see Ref. [1010].

Pure quark energy loss models assume a very long production time and are typically used to describe hadron suppression in the hot nuclear matter produced in heavy-ion collision. In a few cases they have been applied to nDIS data as well [1015, 1016] permitting a common interpretation of hadron suppression in cold and hot nuclear matter. In these models, hadron suppression is due to the lower energy of the quark when it fragments, so that hadrons are produced in lower number and at lower energy. The differences in the models depend on the way calculations of medium-induced gluon radiation are performed, on the modeling of the medium, and on assumptions about its coupling to the hard parton.

Typically, parton energy loss is determined by the transport coefficient $\hat{q}$, which is defined as the transverse momentum square transfered to a quark after propagating through a length of nuclear matter and is a characteristic property of that matter. It is expected to be much larger in a Quark-Gluon Plasma than in the nucleus of a nDIS experiment, which is what is observed from the analysis of experimental data from RHIC and HERMES [1015, 1016, 1017, 1018. The $\hat{q}$ transport coefficient is directly related to the observed broadening of the $p_{T}$ distribution of hadrons in nDIS; it follows that the main challenge for these pure energy loss models is to make a coherent picture of both multiplicity ratios and hadron $p_{T}$ broadening. In particular, for some of the models, the $\hat{q}$ extracted from multiplicity ratios is larger by an order of magnitude than what one would estimate from the hadron transverse momentum broadening. This has led some authors [1019] to the conclusion that quark energy loss is not enough to explain the observed nuclear effects; nevertheless, the variation between theoretical models is still too big for a definitive statement.

The GiBUU model [1014] is an absorption model based on Boltzmann equation including only hadronic and pre-hadronic interactions, see Section 5.10, It assumes short productions times obtained from the Lund string model and neglects gluon brehmstralung from the partonic stage. It can describe very well well most of the hadron multiplicity ratios measured at HERMES and EMC using a linear growth of the pre-hadron cross section between production time and formation time. Other pure absorption models [1020, 1021, 1022] are also successful in describing hadron attenuation. However, the transverse momentum broadening remains a challenge for this kind of models; some progress within GiBUU has been presented during the meeting by Kai Gallmeister.

To resolve the problems of the previous "pure" models, Kopeliovich et al. [1019] describe hadronization including both quark energy loss and hadron absorption. In their model, the transverse momentum broadening is linked to quark energy loss and the multiplicity ratio suppression is explained by hadron absorption, therefore the two processes can be independently quantified. This model describes HERMES data to a large extent, and highlights the fact several processes are involved and need to be disentangled.

Recently, HERMES data have also been described by assuming factorization and universality to hold at the nuclear level not only for parton distributions but also for fragmentation functions, and a set of nuclear Fragmentation Functions have been fitted to experimental data using both $e+A$ interactions and $d+A u$ collisions at RHIC. In this case, no dynamical assumption is made of the physical mechanism for nuclear modifications of hadron production; this information is subsumed into the non-perturbative nuclear FFs-see Section 

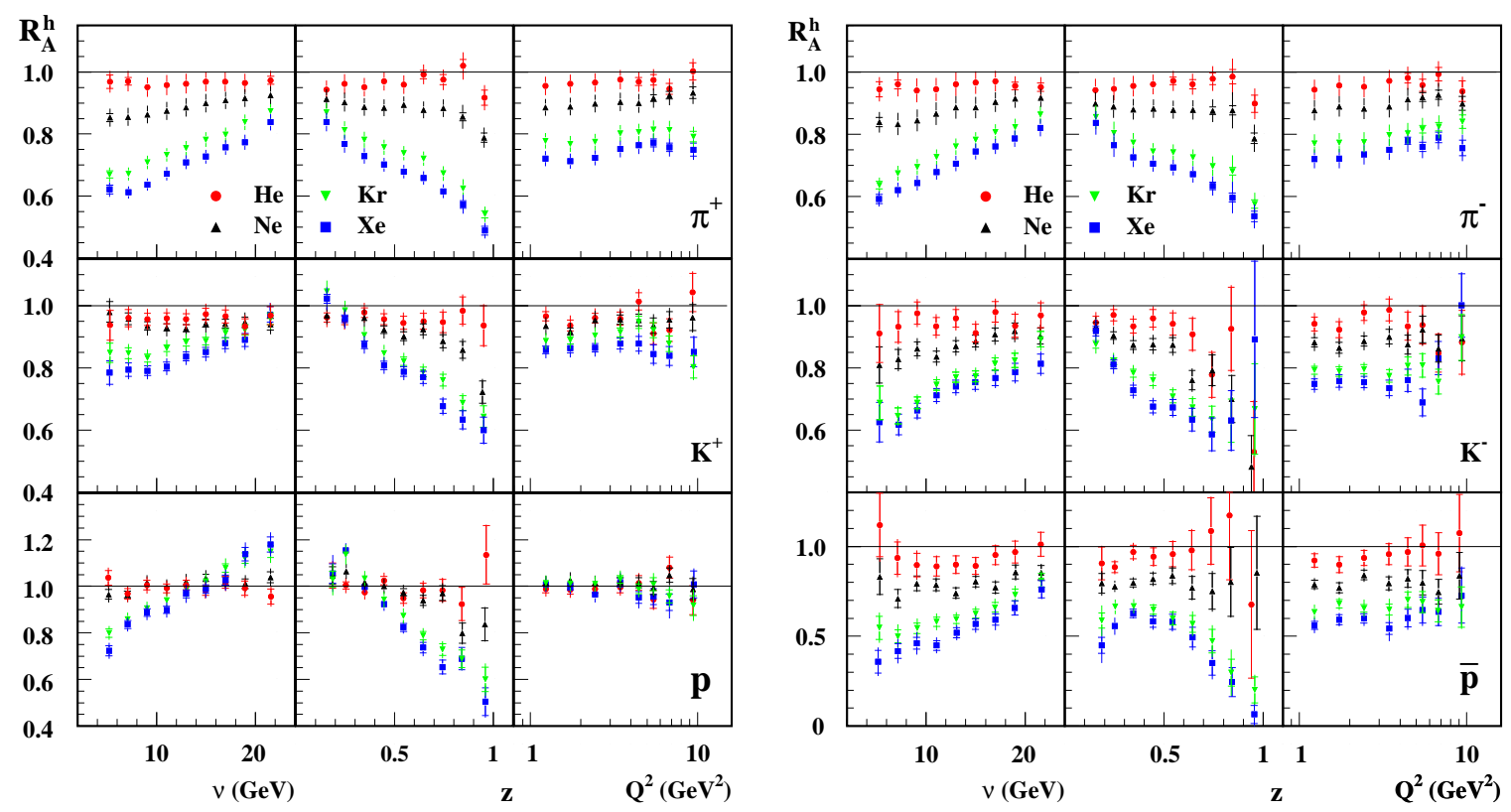

Figure 5.64. Multiplicity ratio of positively charged hadrons (left) and negatively charged hadrons (right) from the HERMES experiment [1029]

5.10.

A number of other models exist using different variants of the discussed mechanisms, and most of them are able to describe the existing data to a good extent: no consensus is reached yet on which mechanisms are dominant, and indeed this is the main motivation for future precise measurements of hadronization at Jefferson Lab 1023, which will be completed by the time EIC starts its operations, and will help settle some of the issues related to early time color dynamics and interaction in cold nuclear matter.

Previous mesurements and open questions. Unidentified charged hadron multiplicity ratios in nuclei were measured in numerous lepton facilities, the earlier results were by Osborne et al. [1024 at SLAC, Hand et al. [1025] and the E665 collaboration [1026] at FNAL, and the European Muon Collaboration [1027, 1028] at CERN. Those measurements revealed a general picture: hadron suppression is stronger at low $\nu$ and high $z$. On the opposite side, at low $z$, an increase of the number of hadron is observed.

In the more recent data from the HERMES collaboration [1029, 1030] several hadrons are studied individually (Fig. 5.64), and new observables such as transverse momentum broadening (figures 5.67 and 5.68) and two hadrons multiplicity ratios [1031] are measured. Because of their improved precision and the large number of hadron species, these data provide us today with a much more detailed picture, which leads to new questions. The behavior of the kaons, for example, is very interesting: $\mathrm{K}^{+}$are less suppressed than pions, but $\mathrm{K}^{-}$have the same behavior as pions (figure 5.64). This difference is not reproduced by existing models, showing that the relatively simple phenomenological models utilized so far cannot fully describe the data. Furthermore, the introduction by HERMES of precise and flavor dependent $\Delta p_{T}^{2}$ measurement [1030] has revealed another strange behavior: the $p_{T}$ broadening of $K^{+}$is larger than for the pion (Figure 5.67 right). This seems to indicate more interaction for kaons, and yet they are less suppressed. To solve this apparent incongruity, one may have to consider models based on different processes involved at different 
stages of hadronization, like in Reference [1019, reinforcing the indications coming from kaon suppression. Furthemore, no model is able to describe the $z$ dependence of the $p_{T}$ broadening, highlighting once again the need for a more detailed theoretical understanding of hadronization. Finally, proton observables are very different from anti-protons (figure 5.64), and no model is yet able to reproduce them correctly, although few attempts have been made [1014, 1032. At the low energies of HERMES, part of the problem may be due to protons coming from the target fragmentation region, which is interesting in its own right. The collider geometry and the large energy range of EIC will permit to experimentally separate clearly target and current fragmentation, allowing to address hadronization in either region. Indeed developing a consistent picture within a given model for both current and target fragmentation would be a great theoretical progress.

To complete the review of existing data, we should mention the preliminary results on pion and kaon production from the CLAS collaboration at Jefferson Lab, where electrons up to $5 \mathrm{GeV}$ scatter on fixed targets ranging from Carbon to Lead [1033, 1034].

\section{Studying hadronization at an EIC}

\section{Raphaël Dupré and Alberto Accardi}

The experimental study of the hadronization process using nDIS is well established; however the high energy available at the EIC creates novel opportunities. The main interest in going at higher energy is to ensure that hadron formation occurs outside of the nuclei, in order to isolate in-medium parton interactions and energy loss. Furthemore, an EIC will permit, for the first time in $e+A$ collisions, the study of hadronization of the open charm and eventually open bottom mesons. Recent results from RHIC [1035, 1036] are showing unexpected results for open charm and bottom suppression in $A+A$ collisions, and several contrasting explainations have already been suggested, with more detailed experiments planned at RHIC. However, due to the intricated interplay of the many variables in $A+A$ collisions and to the poorly known nature of the Quark-Gluon Plasma partons, the $e+A$ input seems necessary to confirm any interpretation. Also, the considerable energy leverage offered by an EIC is a chance to map precisely the $Q^{2}$ evolution of parton energy loss, and determine possible nuclear modifications of DGLAP evolution. The high luminosity will also facilitate the study of two particle correlations (such as hadron-hadron or photon-hadron) over a wide energy range, largely improving recent HERMES measurements, and complementing the low-energy measurements planned at CLAS. Finally, high energy permits access to jets, which give an opportunity to use new observables with improved sensitivity to quark energy loss and the medium modification of fragmentation functions, see Section 5.11. They also facilitate a detailed determination of the cold nuclear matter transport coefficients, which encode basic information on the non perturbative gluonic structure of the nuclei and can be calculated from first principles, e.g., in lattice QCD [1037].

To illustrate the possibilities offered by EIC, we show projections done using the PYTHIA Monte-Carlo generator to evaluate cross sections at $s=200$ or $1000 \mathrm{GeV}^{2}$, and $L=$ $200 \mathrm{fb}^{-1}$. We apply a series of cuts on the generated events to ensure the DIS nature of the interaction $\left(Q^{2}>1 \mathrm{GeV}^{2}\right.$ and $\left.W^{2}>4 \mathrm{GeV}^{2}\right)$, to limit radiative corrections $(y<0.85)$, to insure that we can detect the scattered electron $(y>0.1)$ and to limit di-parton production in the hard scattering of the virtual photon $\left(x_{B j}>0.1\right)$. Finally we assume an acceptance of $50 \%$ for pions, eta meson and kaons, and, an acceptance of $2 \%$ for heavy mesons. The acceptance is set low for heavy mesons to account for the small number of decay channels 

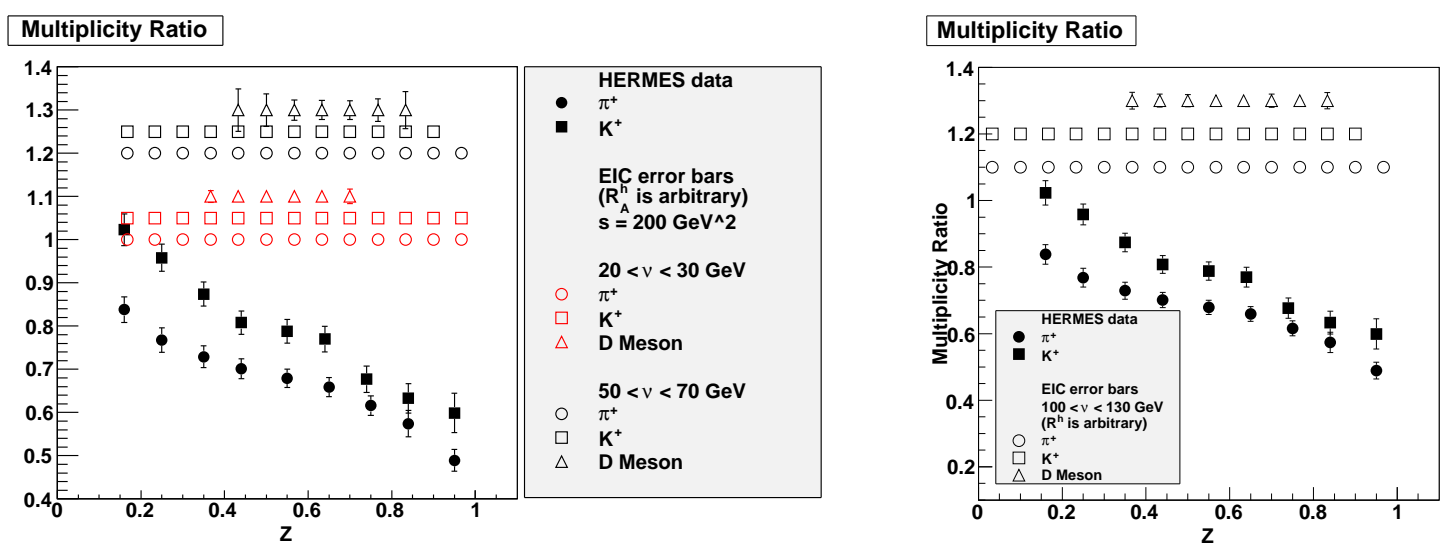

Figure 5.65. Multiplicity ratio in function of $\mathrm{z}$ for various $\nu$ bins. Full points are data from HERMES 1029, empty are projections for statistical errors at the EIC, at arbitrary vertical position. The left panel shows EIC measurements at $s=200 \mathrm{GeV}^{2}$, for 2 different $\nu$ cuts $(20<\nu<30 \mathrm{GeV}$ and $50<\nu<70 \mathrm{GeV}$ ); the right panel at $s=1000 \mathrm{GeV}^{2}$ with $100<\nu<130 \mathrm{GeV}$.

that can be effectively detected. EIC observables are plotted on arbitrary vertical scales, and include statistical errors only.

An EIC is the perfect tool for precise measurement of quark energy loss and transverse momentum broadening. One may object that at the higher EIC energies, because of the large $\nu \gtrsim 150 \mathrm{GeV}$, the relative effect on the quark momentum is too little to produce an appreciable hadron attenuation. This is true at least for the pions, as shown by EMC data. However, attenuation may in fact disappear at a yet higher value of $\nu$ for large $z$ or for heavier particles, because of reduced production times, or for large $Q^{2}$, because of a faster evolution in virtuality as discussed in Section 5.11. Anyway, because of the EIC kinematic flexibility, interesting multiplicity ratios can be measured. For example, Figure 5.65 shows projections for light and heavy flavors, which would shed light on the heavy quarks at RHIC, where they unexpectedly display a similar suppression compared to their light counterparts. It is also interesting to compare mesons of different mass but the same valence quark contents, such as $\pi^{0}$ vs. $\eta$, and $K^{0}$ vs $\Phi$. Figure 5.66 shows projections for the former case compared to calculations in a pure energy loss or pure prehadron absorption scenario. The sensitivity of such measurement to the hadronization time scales is obvious.

Changing observables, measurements of the hadron transverse momentum broadening permit getting around the small values of hadron attenuation at large energies. Indeed the $p_{T}$ broadening to first approximation is independent of $\nu$, and even very little effects can be experimentally observed; moreover, the induced transverse momentum has a theoretical interpretation in terms of transport coefficients. However, one should keep in mind that $\Delta\left\langle p_{T}^{2}\right\rangle$ of pions or other hadrons is not a direct measurement of $\hat{q}$, which is the parton transverse momentum broadening, and that it is essential to use dependences in $\nu$ and $z$ to make a model independent extraction of $\hat{q}$. One may also access $\hat{q}$ through nuclear modifications of hadron azimuthal asymmetries, see Section 5.9.2. The importance of this topic, especially in the scope of other EIC measurements, is enhanced by the connection between $\hat{q}$ and the saturation scale [801, enabling an independent large- $x$ measurement of the latter, complementary to the more traditional small- $x$ measurements discussed in Section 5.2. An EIC will not only allow one to make those measurements with pions but 


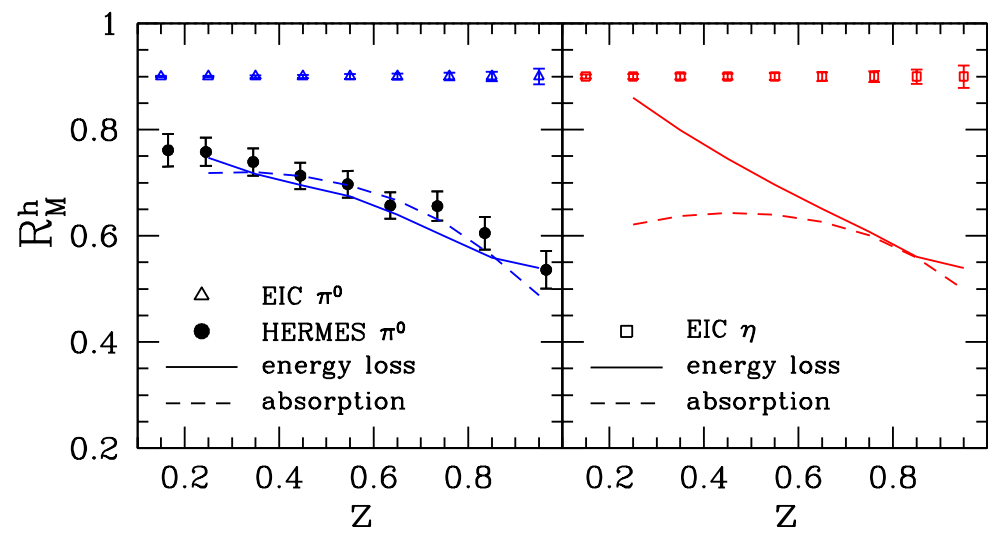

Figure 5.66. Multiplicity ratio for $\pi^{0}$ and $\eta$ mesons compared to pure energy loss and pure prehadron absorption computations.
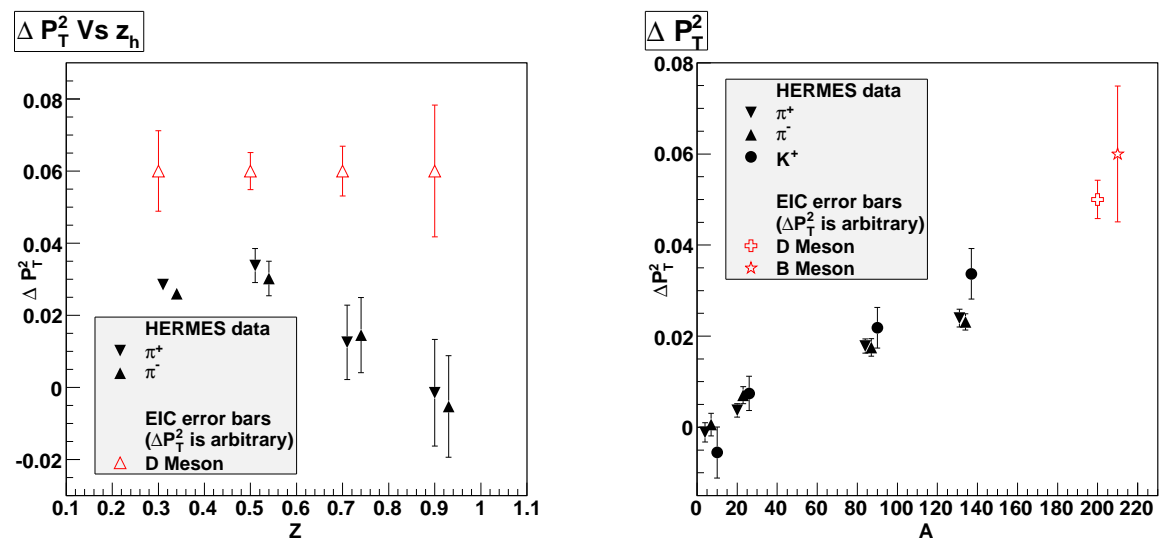

Figure 5.67. Transverse momentum broadening in function of z (left) and A (right), empty triangles and star are projections for EIC at $s=1000 \mathrm{GeV}^{2}$, full points are HERMES data.

also, and uniquely compared to previous e+A facilities, with heavy mesons (see figures 5.67).

The $Q^{2}$ evolution of hadron attenuation is not clearly understood: HERMES data indicate a small rise of the transverse momentum broadening, but the $Q^{2}$ coverage is not large enough to make a definite statement. An EIC can do a far better job as shown in figure 5.68 and provide a unique probe to detect any modification of the DGLAP evolution in nuclear medium.

The scaling of the hadronization times and the quark energy loss with the mass of quarks is an important question that can be used to reveal pQCD effects in parton energy loss and non perturbative effects in hadronization [1038, 1039. Many measurements to explore this at the EIC are possible, as the figures in this section illustrate.

To achieve the discussed measurement the key experimental requirement are good particle ID in general; for heavy flavors one needs in particular a very good vertex detector resolution, which needs to be of the order of few tens of micrometer, and high luminosity to reach a statistical precision allowing unambigous theoretical interpretations. Having a $\nu$ range covering low values for studies of hadronization and large values for studies of parton propagation and energy loss will require energies spanning $s=200-1000 \mathrm{GeV}^{2}$. The 

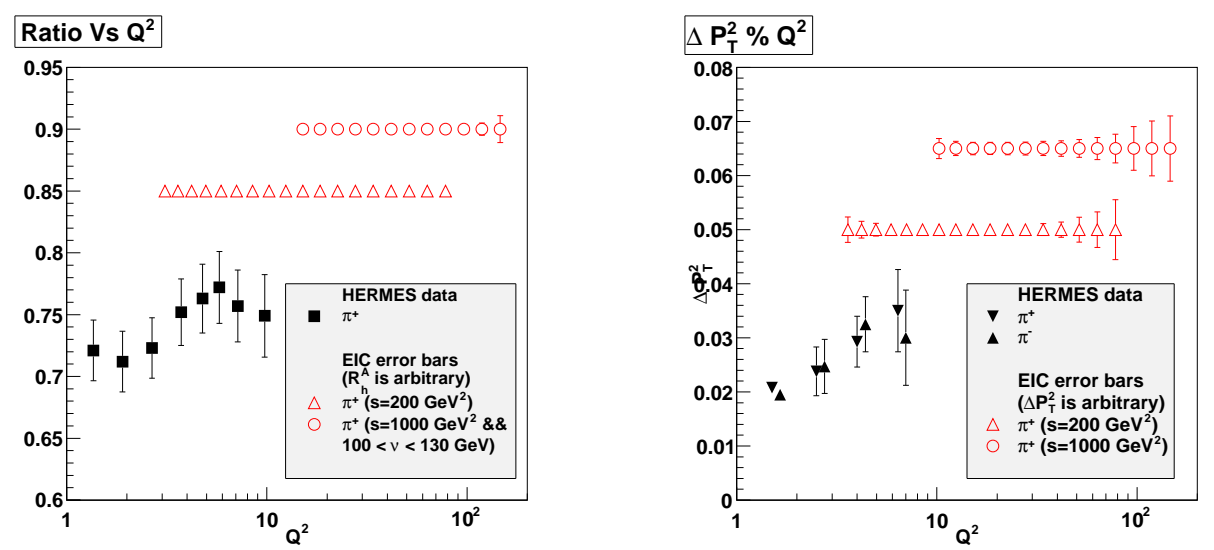

Figure 5.68. Multiplicity ratio (left) and transverse momentum broadening (right) in function of $Q^{2}$, empty markers are projections for EIC at $s=200 \mathrm{GeV}^{2}$ (triangles) and at $s=1000 \mathrm{GeV}^{2}$ (circles), full markers are HERMES data.

lowest required energy can be increased provided measurements of $y<0.1$ can be achieved for SIDIS observables.

Finally, the high energy of an EIC provides the chance, for the first time in $e+A$ collisions, to study hadronization through jet observables. Jets are a new and independent way to access transport coefficient $\hat{q}$ and confirm other measurements, to explore in detail the medium induced gluon radiation and transport properties of cold nuclear matter, and to study the conversion of the parton shower into hadrons, see Section 5.11.

\section{Hadronization in $e+A$ collisions within GiBUU}

\section{Kai Gallmeister and Ulrich Mosel}

The study of the interaction of hadrons, produced by elementary probes in a nucleus, with the surrounding nuclear medium can help to investigate important topics, such as color transparency and hadronization time scales. We investigate this by means of the semiclassical GiBUU transport code [1040, which not only allows for the absorption of newly formed hadrons, but also for elastic and inelastic scattering as well as for side feeding through coupled channel effects. A study of parton interactions in cold, ordinary nuclear matter of known properties is important to disentangle effects of the interaction of partons from those of the medium in which they move.

We summarize here the main features of our model, for details see [1014]. The model relies on a factorization of hadron production into the primary interaction process of the lepton with a nucleon, essentially taken to be the free one, followed by an interaction of the produced hadrons with nucleons. We have modeled the prehadronic interactions such that the description is applicable at all energy regimes and describes the transition from high to low energies correctly. For the first step, we use the PYTHIA model that has been proven to very successfully describe hadron production, also at the low values of $Q^{2}$ and $\nu$ treated in our studies. This model contains not only string fragmentation but also direct interaction processes such as diffraction and vector-meson dominance. In this first step, we take nuclear effects such as Fermi motion, Pauli blocking and nuclear shadowing into account 1041. The relevant production and formation times [1014] are obtained directly from PYTHIA [1042]. In the second step we introduce prehadronic interactions between 

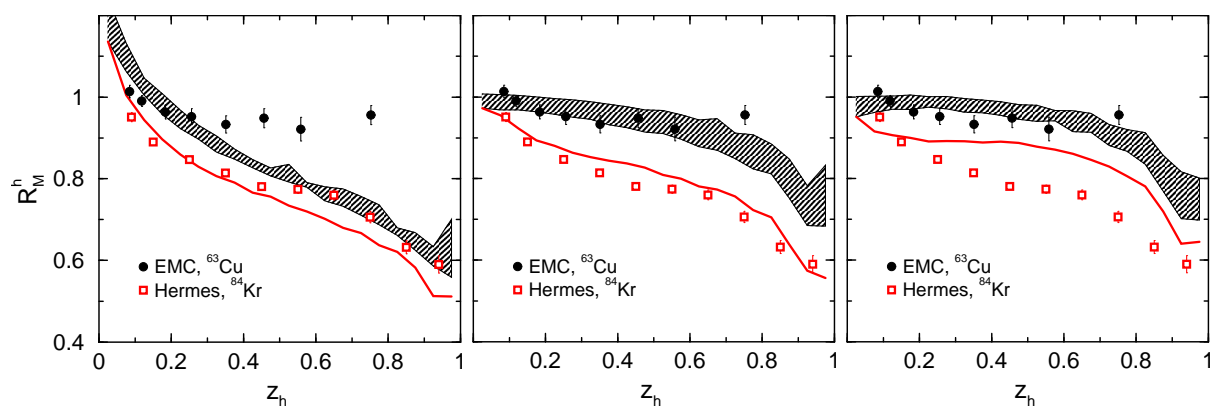

Figure 5.69. Nuclear modification factor for charged hadrons. Experimental data are shown for HERMES at $27 \mathrm{GeV}$ and for EMC at 100-280 GeV. The cross section scenarios are (from left to right): constant, linear and quadratic increase with time after production.

the production and the formation time and the full hadronic interactions after the hadron has been formed.

The actual time dependence of the prehadronic interactions presents an interesting problem in QCD. Dokshitzer et al. [1043] have pointed out that QCD and quantum mechanics lead to a time-dependence somewhere between linear and quadratic. We also note that a linear behavior has been used by Farrar et al. [1044] in their study of quasi-exclusive processes. In our calculations, we work with different time-dependence scenarios, among them a constant, lowered pre-hadronic cross section, a linearly rising one, and a quadratically rising one. In addition, we study a variant of the latter two, where the cross section for leading hadrons, i.e., hadrons that contain quarks of the original target nucleon, starts from a pedestal value $\sim 1 / Q^{2}$, thus taking into account possible effects of color transparency.

Fig. 5.69 shows a comparison of these various model assumptions to HERMES and EMC data on unidentified charged hadron attenuation. A good description of both data sets simultanousely is obtained only with a linear time dependence of the cross sections. Furthermore, a nearly perfect agreement is observed in HERMES data for pions, kaons, and protons, which

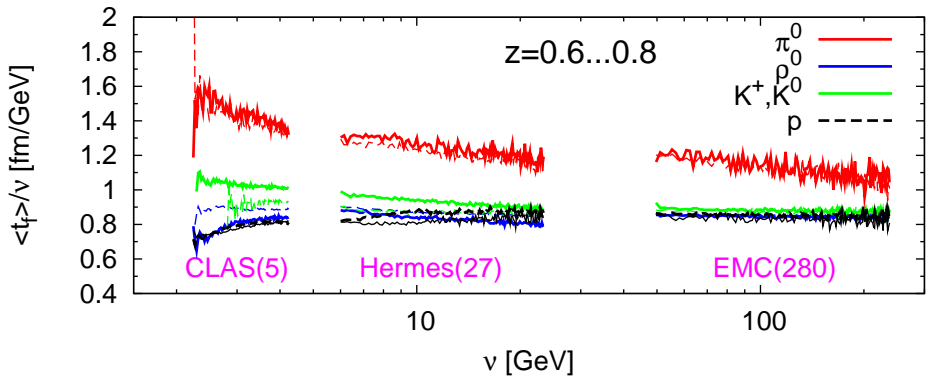

Figure 5.70. The average formation time of different particles divided by $\nu$ as a function of $\nu$ for several experimental setups.

a function of energy transfer $\nu$, relative energy $z_{h}=E_{h} / \nu$, momentum transfer $Q^{2}$ and the squared transverse momentum $p_{T}^{2}$ [1029]. The rise of $R_{M}$ with $\nu$ is mainly an acceptance effect, as we have shown in 1041, whereas the weaker rise of $R_{M}$ with $Q^{2}$ reflects the pedestal value $\sim 1 / Q^{2}$ of the pre-hadronic cross sections.

In Fig. 5.70 we show the average formation time for different particle species as a function of the boson energy $\nu$. One realizes a smooth transition from CLAS at $5 \mathrm{GeV}$ up to EMC at $280 \mathrm{GeV}$ for all particle species. One observes a somehow larger formation time for pions than for the heavier particles. Nevertheless, this effect, being somewhere on a $50 \%$ 
level, is much smaller than mass ratios would suggest: $m_{N} / m_{\pi} \sim 7$. Thus, recalling the basic boost relation, $t_{h}=\gamma_{h} \tau_{h}=\left(E_{h} / m_{h}\right) \tau_{h}$, the factor $\tau_{h}$ and the factor $m_{h}$ in the nominator/denominator cancel each other. We therefore conclude that, within our model, the formation time of a hadron in its rest frame is proportional to its mass, $\tau_{f} \propto m_{H}$, contrary to common assumptions of a constant formation time for all hadron species, which can also be obtained from uncertainty principle considerations [1038, 1010].
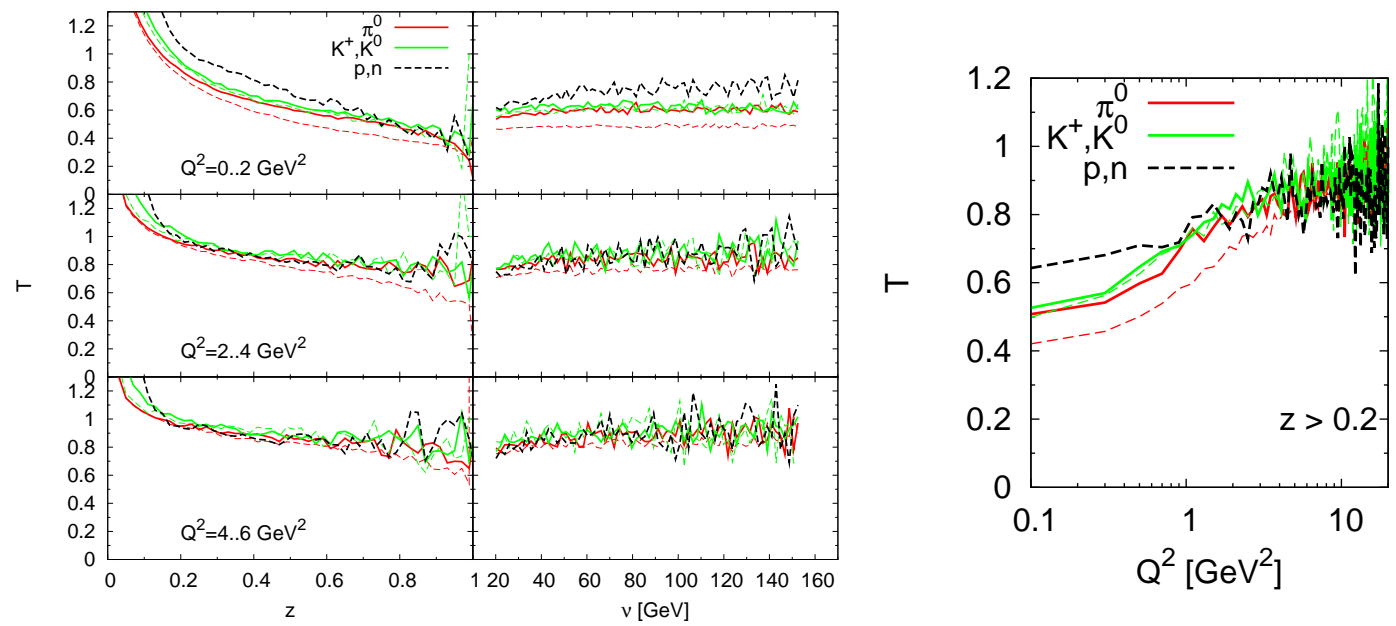

Figure 5.71. The hadron attenuation for different hadron species within several $Q^{2}$ bins as function of $z$ (left panel) and $\nu$ (for $z>0.2$, right panel) for a collider setup $(3+30) \mathrm{GeV}$.

Hadron Attenuation at an EIC: Strong $Q^{2}$ Dependence. One may now look at hadron attenuation at an EIC. Fig. 5.71] shows the expected attenuation for different hadron species within several $Q^{2}$ bins as functions of $\nu$ and $z$ for a very low energy collider setup $(3+30) \mathrm{GeV}$, which is close to former EMC conditions. One observes a large $Q^{2}$ dependence: while for low $Q^{2}$ values, the attenuation of all hadron species decreases to approx. 0.5 at $z \rightarrow 1$, the attenuation is only approx. 0.8 for $Q^{2}>4 \mathrm{GeV}^{2}$. This is also shown in Fig. 5.71, where the same attenuation is shown, but now as a function of $Q^{2}$ and integrated over all $\nu$ and $z>0.2$ values. It is worthwhile mentioning that there is nearly no $\nu$ dependence for all $Q^{2}$ bins visible in our calculations.

Hadron Attenuation at an EIC: $\pi^{0}$ vs. $\boldsymbol{\eta}$. As already shown in Fig. 5.71, some differences in the resulting attenuation ratio show up for different hadron species. In Section 5.10, it has been suggested that a comparison of $\eta$ and $\pi^{0}$ attenuation ratios will distinguish between energy-loss models and absorption models. In Fig. 5.72 we show our results for the attenuation of these two particle species. Both attenuation signals are close to each other, but show stronger absorption for $\pi^{0}$ than for $\eta$ mesons, in which case the discriminatory power would weaken. In Fig. 5.72 we also show the hadronic interaction cross section of pions and eta mesons with nucleons. For laboratory momenta larger than $2 \mathrm{GeV}$, these are nearly identical. Thus differences in the attenuation are due to formation time effects.

\section{A global fit of nuclear fragmentation functions}

\section{Rodolfo Sassot, Marco Stratmann, Pia Zurita}

Similarly to modifications of PDFs in nuclei, the production of hadrons in the finalstate is known to be affected when occurring in a nuclear environment. For example, 

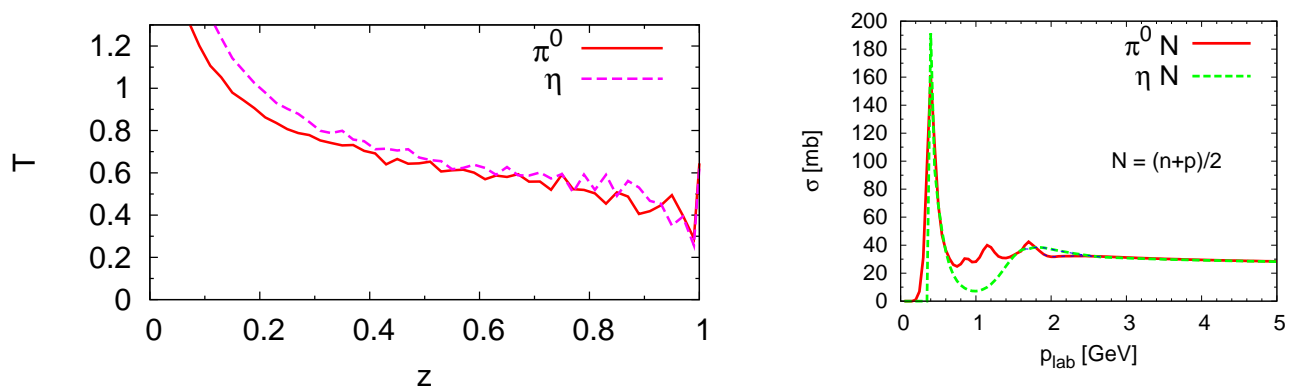

Figure 5.72. Left panel: The hadron attenuation for $\pi^{0}$ and $\eta$ mesons for a collider setup of $3+30$ GeV. Right panel: The hadronic interaction cross section of $\pi^{0}$ and $\eta$ mesons with nucleons at rest as a function of the meson momentum.

semi-inclusive deep-inelastic scattering (SIDIS) off large nuclear targets shows significant differences as compared to hadron production off light nuclei or proton targets, as reviewd in Section 5.10.

The past few years have seen a significant improvement in the pQCD description of hadron production processes, and, more specifically, in the precise determination of vacuum fragmentation functions (FFs), including estimates of their uncertainties [74]. FFs carry the details of the non-perturbative hadronization process, factorized from the hard scattering cross section in the same way as for PDFs. The most important result of these studies is that the standard pQCD framework not only reproduces data on electron-positron annihilation into hadrons, but it describes with remarkable precision also other processes like semiinclusive deep-inelastic scattering and hadron production in proton-proton collisions. It is then quite natural to ask if pQCD factorization can be also generalized to final-state nuclear effects, i.e., to introduce medium modified or nuclear fragmentation functions (nFFs), and to assess how good such an approximation works or to determine where and why it breaks down. From theoretical considerations alone, the answer is, however, not obvious since on the one hand, interactions with the nuclear medium may spoil the requirements of the factorization theorems, but, on the other hand, any estimates of possible factorization breaking effects are strongly model dependent.

Within the factorization ansatz, nFFs should contain (factorize) all the non-perturbative details related to hadronization in a nuclear environment, would be exchangeable from one process to another (universal), and would allow for QCD estimates at any given order in perturbation theory in a well defined and unified framework. These features can be explicitly tested using data from an increasing but still limited number of experiments that have performed precise measurements of hadron production off nuclear targets, for instance, in SIDIS by HERMES [1029] or in deuteron-gold collisions studied at RHIC [1045, 1046]. Both type of processes are compatible with a universal nuclear modification of the hadronization mechanism in the currently accessible kinematic regime. The inclusion of next-to-leading order QCD corrections and the possibility to use different observables have been proven to be crucial for an accurate parametrization of nFFs [967.

In addition to the primary goal of testing the factorization properties of nFFs and to constrain them from different data sets in a consistent theoretical framework (for further comparison with the different model estimates), a thorough analysis of $\mathrm{nFFs}$ also serves as a baseline for ongoing studies of hadron production processes in heavy-ion collisions performed at RHIC and the LHC [1047]. In the following, we present a brief summary 

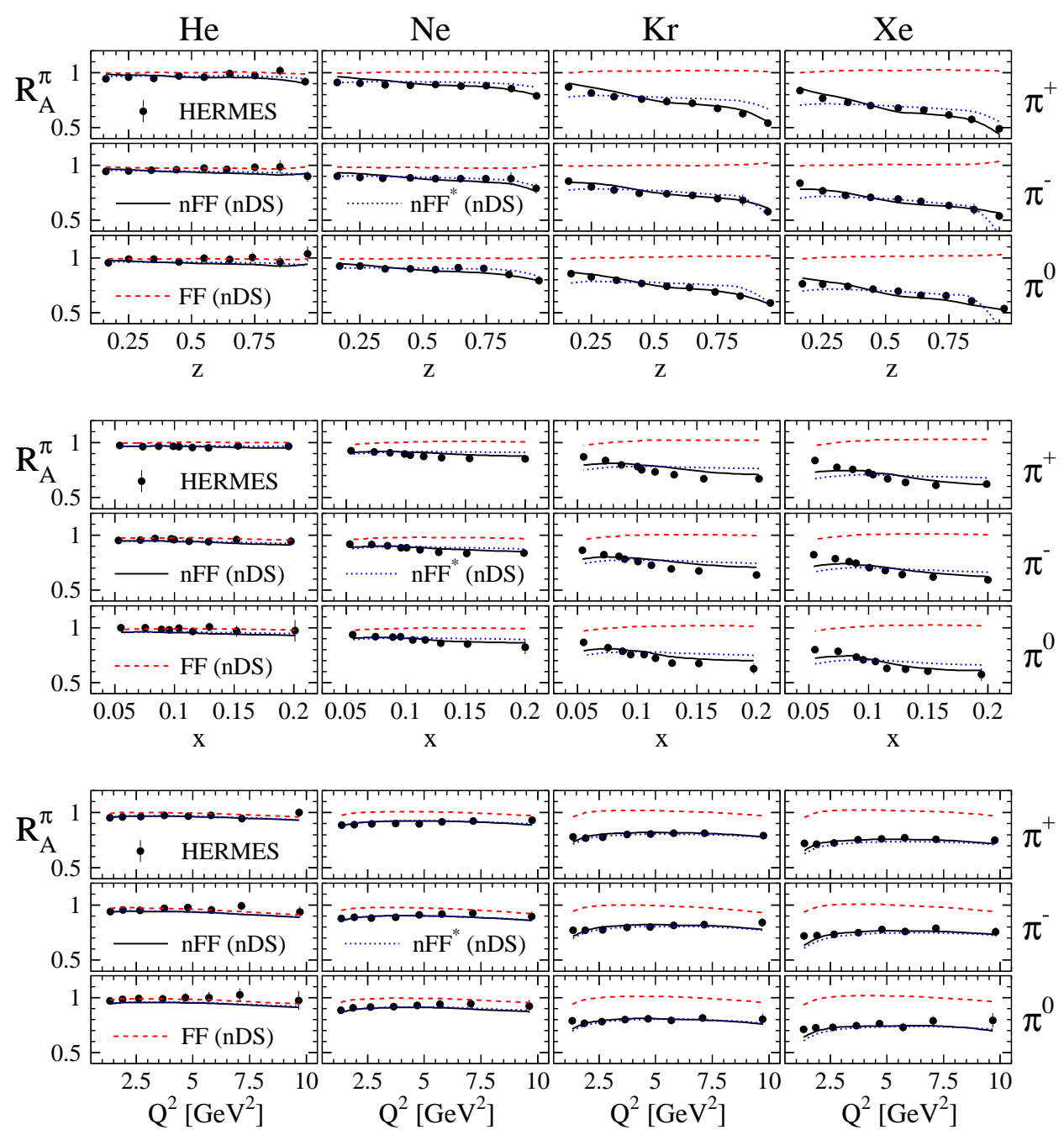

Figure 5.73. Quality of the nFF fit to nuclear SIDIS data from HERMES.

of the first global fit of nFFs and outline limitations in the analysis imposed by the data available so far.

Medium Modified Fragmentation Functions. Even though nuclear effects in the hadronization process have been known to be significant for quite some time, only recent experiments have become precise enough and selective from a kinematical point of view to allow for more detailed and quantitative studies. Specifically, the HERMES collaboration has performed a series of measurements of pion, kaon and proton attenuation on different nuclear targets as a function of the hadron momentum fraction $z$ and the photon virtuality $Q^{2}$, which both are used to characterize fragmentation functions, as well as the virtual photon energy $\nu$, that can be related to the nucleon momentum fraction $x$ carried by initial-state parton, see Fig. 5.64,

Single-inclusive identified hadron yields obtained in $d+A u$ collisions at mid-rapidity at BNL-RHIC, which show a characteristic nuclear suppression and enhancement pattern as a function of the hadron transverse momentum $p_{T}$, are another source of information on nuclear modification effects in the hadronization process. These measurements are often 


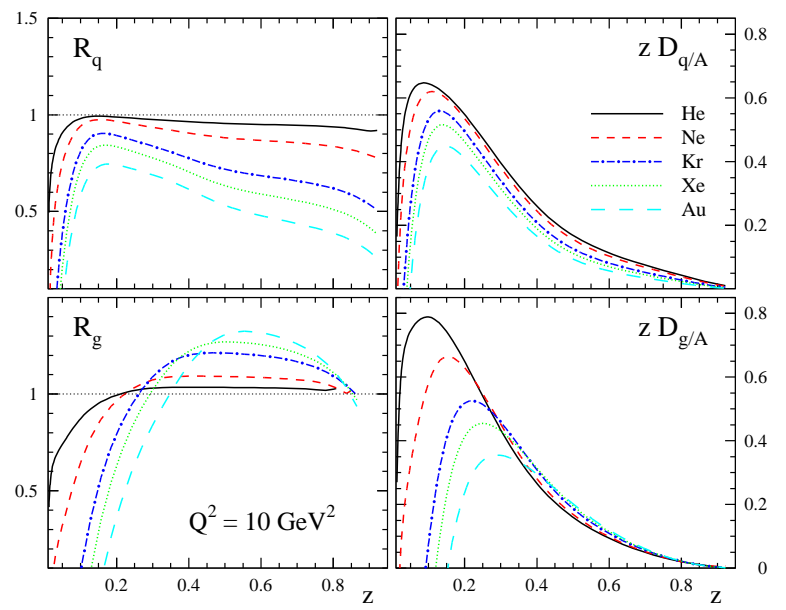

Figure 5.74. Comparison of medium modified and standard FFs.

seen as "control experiments" associated with the heavy-ion program at RHIC to explore the properties of nuclear matter under extreme conditions. However, in view of the evidence for strong medium induced effects in the fragmentation process found in SIDIS, $d+A u$ data are also of particular relevance for extracting $\mathrm{nFFs}$ and testing the assumed factorization and universality properties.

To perform global nFF fits, it was proposed in Ref. [967] to relate the medium modified fragmentations to the standard ones in a convolution approach with a very simple ansatz for the weight functions. The fits gives a very good description of the full kinematic dependence of the HERMES data as can be seen in Fig. 5.73 while an approach which ignores all finalstate nuclear effects clearly fails. The same set of $\mathrm{nFF}$ s that account for nuclear modification in SIDIS also reproduce the main features of the $d+A u$ data from RHIC. The peculiar $p_{T}$ dependence of the effects is found to come from an interplay between quark and gluon fragmentation as a function of $p_{T}$ in the hadron production cross section. It is interesting to notice that there seems to be no visible conflict between the standard $Q^{2}$ dependence assumed for the nFFs and the data. In this respect, there have been many interesting suggestions and model dependent calculations at the LO level, motivating the use of medium modified evolution equations. However, in the range of $Q^{2}$ covered by present SIDIS and $d+A u$ data, there is no evidence for any significant departure from standard time-like evolution equations [1048, 1049, 1050, 1051].

The pattern of medium induced modifications is rather different for quarks and for gluons, see Fig. 5.74. The dominant role of quark fragmentation in SIDIS leads to a suppression, i.e., $R_{q}^{\pi}<1$, increasing with nuclear size $A$ as dictated by the pattern of hadron attenuation found experimentally. The enhancement of hadrons observed in $d+A u$ collisions for $p_{T} \approx 10 \mathrm{GeV}$, along with the dominant role of gluon fragmentation at low values of $p_{T}$ explains that $R_{g}^{\pi}>1$ for $z \rightarrow 0.2$. Below $z \simeq 0.2$, where all the data used in the fit have very limited or no constraining power, both quark and gluon nFFs drop rapidly. For the time being, the behavior in this region could easily be an artifact of the currently assumed functional form for the parameterization. The extended $Q^{2}$ range of EIC will allow one to accurately test the factorization assumption for nFFs, which is at the basis of the presented approach to nuclear modfications of hadron production. 


\section{Heavy quarks and quarkonia in a nuclear environment}

\section{B. Z. Kopeliovich}

Time dependence of vacuum radiation. The color field of a quark originating from a hard reaction (DIS, high- $p_{T}, e^{+} e^{-}$, etc.) is stripped off, i.e., such a quark is lacking a color field up to transverse frequencies $q \lesssim Q$, and starts regenerating its field by radiating gluons, i.e., forming a jet. This can be described by means of an expansion of the initial "bare" quark over the Fock states containing a physical quark and different number of physical gluons with different momenta. Originally, this is a coherent wave packet equivalent to a single bare quark $|q\rangle$. However, different components have different invariant masses and they start gaining relative phase shifts as a function of time. As a result, the wave packet is losing coherence and gluons are radiated in accordance with their coherence times. The required time is to the jet energy, since the radiation time (or length) depends on the gluon energy and transverse momentum $k$ (relative to the jet axis),

$$
l_{c}=\frac{2 E}{M_{q g}^{2}-m_{q}^{2}}=\frac{2 E x(1-x)}{k^{2}+x^{2} m_{q}^{2}} .
$$

Here, $x$ is the fractional light-cone momentum of the radiated gluon; $m_{q}$ is the quark mass; $M_{q g}^{2}=m_{q}^{2} /(1-x)+k^{2} / x(1-x)$ is the invariant mass squared of the quark and radiated gluon.

One can trace how much energy is radiated over the path length $L$ by the gluons which have lost coherence during this time interval [1052, 1019, 1053, 1054, 1055,

$$
\Delta E(L)=E \int_{\Lambda^{2}}^{Q^{2}} d k^{2} \int_{0}^{1} d x x \frac{d n_{g}}{d x d k^{2}} \Theta\left(L-l_{c}\right),
$$

where $Q \sim p_{T}$ is the initial quark virtuality; the infra-red cutoff is fixed at $\Lambda=0.2 \mathrm{GeV}$. The radiation spectrum reads

$$
\frac{d n_{g}}{d x d k^{2}}=\frac{2 \alpha_{s}\left(k^{2}\right)}{3 \pi x} \frac{k^{2}\left[1+(1-x)^{2}\right]}{\left[k^{2}+x^{2} m_{q}^{2}\right]^{2}}
$$

where $\alpha_{s}\left(k^{2}\right)$ is the running QCD coupling, which is regularized at low scale by the substitution: $k^{2} \Rightarrow k^{2}+k_{0}^{2}$ with $k_{0}^{2}=0.5 \mathrm{GeV}^{2}$. In the case of heavy quark the $k$-distribution Eq. (5.160) peaks at $k^{2} \approx x^{2} m_{q}^{2}$, corresponding to the polar angle (in the small angle approximation) $\theta=k / x E=m_{q} / E$. This is known as the dead cone effect [1056, 1057].

The step function in Eq. (5.159) creates another dead cone [1055]: since the quark is lacking a gluon field, no gluon can be radiated unless its transverse momentum is sufficiently high, $k^{2}>2 E x(1-x) / L-x^{2} m_{q}^{2}$. This bound relaxes with the rise of $L$ until it reaches $k^{2} \sim x^{2} m_{q}^{2}$, characterizing the heavy quark dead cone at $L_{q}=E(1-x) / x m_{q}^{2}$. The radiation of such a "naked" quark has its own dead cone controlled by its virtuality $Q^{2} \gg m_{q}^{2}$, and is much wider than the one related to the quark mass. Therefore, there is no mass dependence of the radiation until the quark virtuality cools down to $Q^{2} \Rightarrow Q^{2}(L) \sim m_{q}^{2}$. At the early stage of hadronization, when $Q^{2}(L) \gg m_{q}^{2}$, all quarks radiate equally, and the results of [1057] for a reduced energy loss of heavy quarks should be applied with a precaution. The numerical results demonstrating this behavior are depicted in Fig. 5.75 . 
One can see that a substantial difference between the radiation of energy by charm and light quarks onsets at rather large distances, above $10 \mathrm{fm}$. However the $b$-quark radiation is suppressed already at a short distance, less than one fermi. Moreover, it completely regenerates the color field already at a distance of the order of $1 \mathrm{fm}$ and does not radiate any more. Of course, this $b$-quark still may have a medium induced radiation, which is very weak according to [1057]. Notice that the interference between vacuum and induced radiations is absent because they occur on different time scales.

Production and formation length. One should clearly distinguish between the production time scales for a colorless dipole (pre-hadron) and the final hadron. The former signals color neutralization, which stops the intensive energy loss caused by vacuum radiation following the hard process, while the latter is a much longer time taken by the dipole to gain the needed hadronic mass, i.e. to develop the hadron wave function. While the former is proportional to $1-z_{h}$ and contracts at large fractional momentum $z_{h}$ of the hadron, the latter keeps rising proportionally to $z_{h}$. These two time scales are frequently mixed up. The shortness of the production lengths at large $z_{h}$ is dictated by energy conservation. Indeed, a parton originating from a hard reaction intensively radiates, losing energy. This cannot last long, otherwise the parton energy will drop below the energy of the detected hadron. Only the creation of a colorless pre-hadron, which does not radiate gluons any more, can stop the dissipation of energy. Energy conservation thus imposes a restriction on the color neutralization time [1058], $l_{p} \leq \frac{E_{q}}{\langle d E / d z\rangle}\left(1-z_{h}\right)$, which must vanish at $z_{h} \rightarrow 1$. One should also distinguish between the mean hadronization time of a jet, whose energy is shared between many hadrons, and specific events containing a leading hadron with $z_{h} \rightarrow 1$. The production of such a hadron in a jet is a small probability fluctuation, usually associated with large rapidity gap events. The space-time development of such an unusual jet is different from the usual averaged jet. It is illustrated in Fig. 5.76. Notice that one should not mix up the production time with the time scale evaluated in [1059], Eq. (2), which is just the well known coherence time. This is not the time of duration of hadronization which we are interested in. If hadronization were lasting as long as the coherence time, energy conservation would be broken. Besides, a pre-hadron does not have any certain mass, since according to the uncertainty relation it takes time, called formation time, to resolve between the ground and excited states, which have certain masses. Therefore, one cannot evaluate the production time of a pre-hadron relying on the mass of the hadron.

Since the produced pre-hadron strongly attenuates in the nuclear medium, the position of the color neutralization point is crucial for the resulting nuclear suppression. Notice 


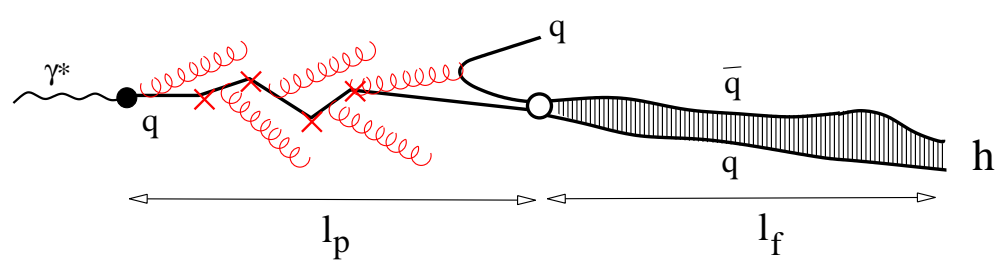

Figure 5.76. The two-step process of leading hadron production. On the production length $l_{p}$ the quark is hadronizing experiencing multiple interactions broadening its transverse momentum and inducing an extra energy loss. Eventually, the quark color is neutralized by picking up an antiquark. The produced color dipole (pre-hadron) is attenuating in the medium and developing the hadron wave function over the formation path length $l_{f}$.

that such a picture of space-time development of hadronization is classical. In quantum mechanics one cannot say with certainly whether the pre-hadron is produced inside or outside the medium: the inside-outside interference term is significant [1060].

Heavy flavored hadrons. The production length distribution calculated for light quarks [1052, 1019, 1054] should be similar to that for charm quarks, which have a similar vacuum radiation during the first several fermi. However, a bottom quark, according to Fig. 5.75, dissipates considerably less energy, moreover, its vacuum radiation ceases at the distance of about $1 \mathrm{fm}$, because the quark completely restores its color field. Of course, confinement does not allow a colored quark, even with a restored field, to propagate freely. It keeps losing energy via nonperturbative mechanisms 1054, like in the string (flux tube) model. Surprisingly, nonperturbative dynamics is more involved into hadronization of heavy compared with light quarks. However, one should remember that this is true only for jets which end up producing leading hadrons with $z_{h} \rightarrow 1$.

A high-energy heavy quark always escapes from the medium and produces an open flavor hadron with no suppression. Therefore, a break-up of a light-heavy dipole propagating in a medium should not lead to a suppression, unless the fractional momentum $z_{h}$ of the detected hadron is fixed at a large value. In such a case, break up of the dipole ignites continuation of vacuum energy loss, which slows down the quark to smaller values of $z_{h}$. This is why a quark should stop radiating at a distance $l \sim l_{p}$ and produce a colorless dipole, which then survives through the medium.

It is interesting that the produced heavy-light, $c-q$ or $b-q$ dipoles expand their sizes faster than a light $\bar{q} q$ dipole. This happens because of the very asymmetric sharing of the longitudinal momentum in such dipoles. Minimizing the energy denominator one gets the fractional momentum carried by the light quark, $\alpha \sim \frac{m_{q}}{m_{Q}}$, which indeed is very small, about 0.1 for charm and 0.03 for bottom. Then according to [1055, 1061, the dipole size is evolving with time as $r_{T}^{2}(t)=\frac{2 t}{\alpha(1-\alpha) E}+r_{0}^{2}$, where $r_{0}$ is the initial dipole separation: the $b-q$ dipole is expanding much faster than $\bar{q} q$.

Conclusions. The hadronization of charm and bottom quarks ends up at a short distance $l_{p}$ with production of a colorless dipole which is strongly absorbed by the medium. This may explain why both of them are strongly suppressed in $A+A$ collisions. Studies of light vs. heavy meson productions at the EIC will clearly be able to validate the discussed effects. 


\title{
5.11 Jets
}

\section{Jets, in-medium parton propagation and nuclear gluons}

\author{
Alberto Accardi, Matthew A. C. Lamont, Gregory Soyez
}

Preliminary results from the SLAC E665 fixed target experiment have demonstrated jet production in $e+A$ collisions at $s \approx 1000 \mathrm{GeV}^{2}$ [1062, 1063]. Thus, the start of the jet study programme should be feasible in a Phase-I EIC. This can be confirmed by further simulations, required to study the capabilities in a collider experiment as opposed to a fixed-target experiment like E665.

As will be discussed in detail in the next 2 contributions, the nuclear modification of $1+1$ jet production, i.e., 1 jet from current fragmentation and 1 from target fragmentation, is of great interest to study parton propagation through cold nuclear matter, in order to extract cold nuclear transport coefficients, and probing soft gluons in nuclei. In addition, the nucleus can be used as a femtometer-scale detector of the evolution of parton showers, allowing to test their perturbative descriptions (e.g., $k_{T}$-ordering $v s$. rapidity ordering) and Monte-Carlo implementations, which are used pervasively in all fields of high-energy physics to analyze experimental data.

The case of $2+1$ jets is also interesting. Indeed, the cross section for this prcess reads

$$
\frac{\mathrm{d}^{2} \sigma_{2+1}}{\mathrm{~d} x_{p} \mathrm{~d} Q^{2}}=A_{q}\left(x_{p}, Q^{2}\right) q_{A}\left(x_{p}, Q^{2}\right)+A_{g}\left(x_{p}, Q^{2}\right) g_{A}\left(x_{p}, Q^{2}\right)
$$

where the two terms correspond to the quark-initiated and gluon-initiated processes respectively, and the coefficients $A_{q}$ and $A_{g}$ are matrix elements that can be computed at given order in perturbation theory. Unlike the $1+1$ case which is dominated by quark initiated processes, the $2+1$ cross section is now also sensitive to nuclear gluons, and offers yet another way to measure them.

Since the outgoing jets have to travel in the medium, the coefficients $A_{q}$ and $A_{g}$ will be affected by in-medium propagation. We shall assume here that the measurements of $1+1$ jet cross-sections allow to control in medium quark jets, hence $A_{q}$. Then, by tagging or vetoing gluon jets in $2+1$ events one can study, respectively, gluon jets in-medium propagation and the nuclear gluon distributions. In Fig. 5.77, we show the expected kinematic reach of the gluon measurements for a phase-I and phase-II EIC, and for various cuts on the jet transverse momentum $p_{T}$. Details can be found in 1064. Detailed simulations are planned to study the feasibility and physics reach of these jet studies.

\section{Monte-Carlo for hard jets in e+A collisions}

\section{A. Majumder}

The production and modification of hard jets produced in lepton nucleus collisions is considered. The assumption of factorization of the hard scattering cross section from the structure functions and final fragmentation function allow one to compute the final medium modified fragmentation function in both cold nuclear matter and in a hot Quark-GluonPlasma (QGP) in an identical formalism. This allows for both a cross check of the basic energy loss formalism used in these reactions, and a comparative study of the partonic sub-structure of these different phases of QCD matter. Detailed descriptions are provided 


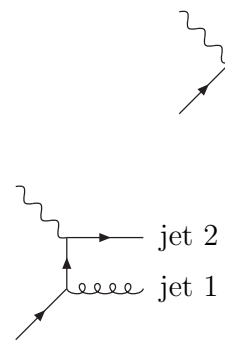

(b)
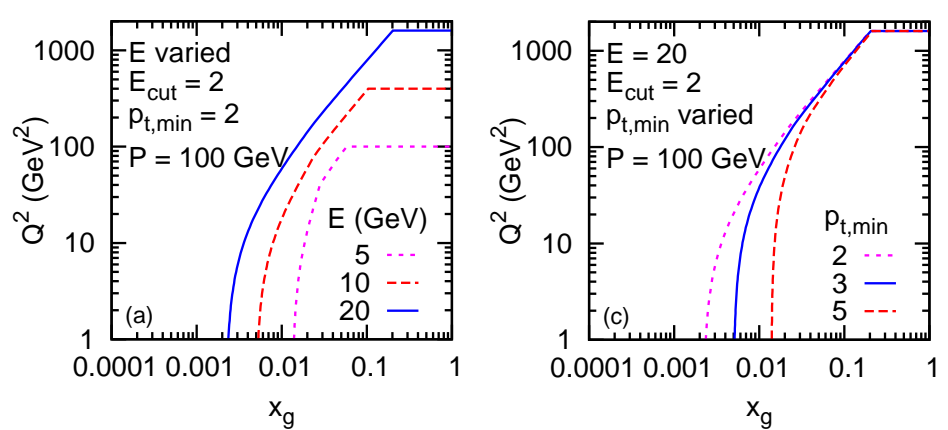

Figure 5.77. Left: Parton-level processes that contribute (a) to the $1+1$ and (b,c) $2+1$ jet crosssection. Middle and Center: Accessible kinematic range in $x_{p}$ and $Q^{2}$ for the $2+1$ jets scenario. The accessible region is plotted for different energies $E$ of the electron beam and hadron beam energy $E_{p}=100 \mathrm{GeV}$, corresponding to a phase-I and phase-II EIC, for different jet transverse momentum cuts $p_{T}>p_{T, \text { min }}$ at fixed jet energy cut $E_{c u t}$.

via a Monte-Carlo simulation of such calculations. We compare the results of analytical calculations in these two regimes and present preliminary Monte-Carlo simulations for jets produced in deep-inelastic collisions.

Introduction to in-medium DGLAP. Collision processes which involve a hard scale can be factorized into separate probabilities of hard and soft processes which are convoluted via a single dimensionless variable [716]. For example, for the case of single hadron inclusive production in deep-inelastic-scattering (DIS), the differential cross section may be expressed as,

$$
\frac{d \sigma}{d z}=\int d x G\left(x, Q^{2}\right) \otimes \frac{d \hat{\sigma}}{d Q^{2}} \otimes D\left(z, Q^{2}\right)
$$

where, $G\left(x, Q^{2}\right)$ represents the parton distribution function, $\frac{d \hat{\sigma}}{d Q^{2}}$ represents the electron quark scattering cross section via single photon exchange. $D\left(z, Q^{2}\right)$ represents the fragmentation function to produce a hadron with a momentum $z \nu$ from the fragmentation of the outgoing quark jet. The structure functions and fragmentation functions are defined and factorized from the hard cross sections at a given scale $\mu^{2}$ which, in this case, is chosen to be equal to the hard scale of the process $Q^{2}$. They only need to be measured at a single scale, and the change of these functions with scale is given by the DGLAP evolution equations [856]. For fragmentation functions, these equations read

$$
\frac{\partial D\left(z, Q^{2}\right)}{\partial \ln Q^{2}}=\frac{\alpha_{S}}{2 \pi} \int \frac{d y}{y} P(y) D\left(\frac{z}{y}, Q^{2}\right)
$$

where, $P(y)$ is the gluon splitting function and represents the probability for a quark to radiate a gluon and retain a fraction $y$ of its light cone momentum.

In the case of DIS on a large nucleus, one may simply include the entire effect of the medium by including a length dependent multiplicative factor to the gluon splitting function [1065], which accounts for the fact that the radiated gluon will scatter in the medium influencing its radiation amplitude, i.e., $P(y) \rightarrow P(y) K\left(y, q^{-}, L^{-}, Q^{2}\right)$. The medium dependent factor given as [1066],

$$
K\left(y, q^{-}, L^{-}, Q^{2}\right)=\int_{0}^{L^{-}} d \zeta^{-} \frac{\hat{q}}{Q^{2}}\left[2-2 \cos \left(\frac{Q^{2} \zeta^{-}}{2 p^{+} q^{-} y(1-y)}\right)\right]
$$



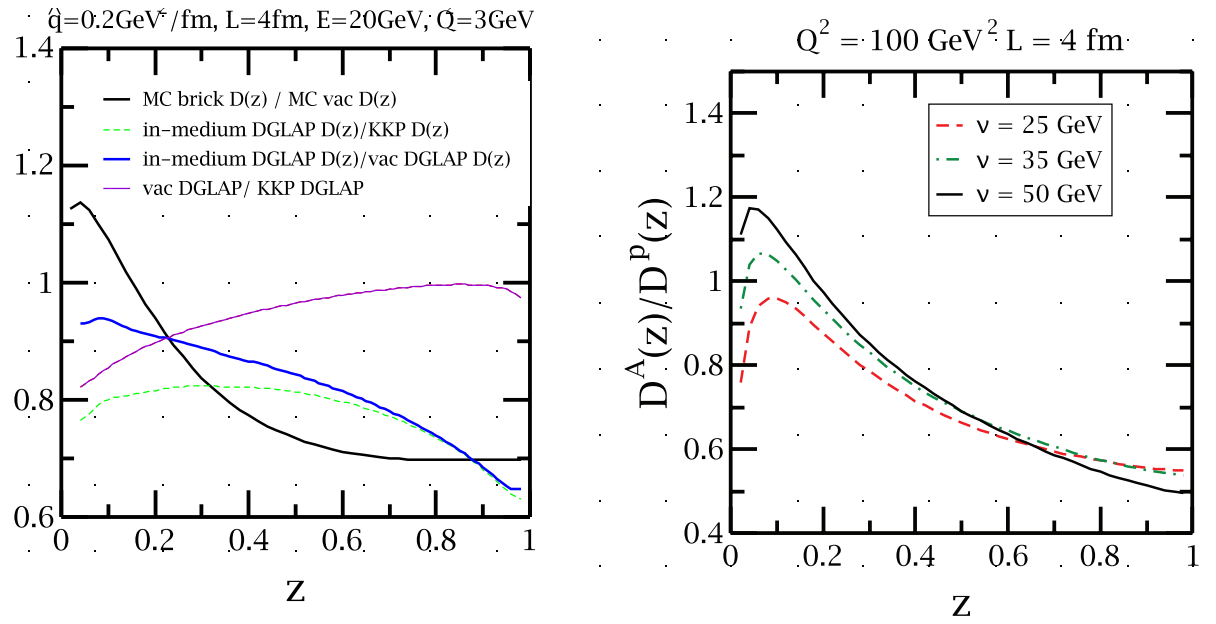

Figure 5.78. Left: A comparison of the results of an analytic DGLAP evolution calculation and a Monte-Carlo shower calculation for the same choice of input parameters. Right: Results of a set of Monte-Carlo simulations of a jet propagating through a $4 \mathrm{fm}$ brick.

In the equation above, $L^{-}$is the maximum possible length traversed in the medium in the course of one emission. In an evolution equation, the formation time of the final radiation is chosen to be larger than the maximum medium length. This restricts the length to be no larger than $q^{-} / Q_{\text {min }}^{2}$, where $Q_{\text {min }}$ is the minimum allowed virtuality on exit from the medium. In an analytic solution to the DGLAP equation, one requires an input fragmentation function. The most unambiguous input is to use the known vacuum fragmentation function at the scale $Q_{\min }^{2}$ where we have stipulated that the jet has emerged from the medium. This is then evolved in $Q^{2}$ up to the hard scale of the process using the medium modified evolution equation which includes the kernel of Eq. (5.164).

Results from such an in-medium DGLAP evolution are plotted in Fig. 5.78. The input distribution in vacuum is taken from KKP at an input scale of $\mu_{i n}^{2}=1 \mathrm{GeV}^{2}$ and evolved up to $Q^{2}$. Its ratio to the KKP fragmentation at the scale $Q^{2}$ is plotted as the green dashed line in Fig. 5.78, Note that our numerical implementation of the DGLAP equation is different from that of KKP and so for comparison, we plot the ratio of the vacuum evolved fragmentation function in our implementation versus that in the KKP where both calculations start from the same input distribution i.e. the KKP function at the scale $\mu_{i n}^{2}$, and are compared at the higher scale of $Q^{2}$. The ratio is plotted as the magenta curve in Fig. 5.78. While over the range of $z$ considered, the curve is close to unity, it may deviate by up to $20 \%$ at lower values of $z$.

The solid blue line in Fig. 5.78 represents the ratio of the medium modified fragmentation function to the vacuum fragmentation function, where both numerator and denominator are calculated using the same numerical routine (for the vacuum FF we simply use $\hat{q}=0$ ). This ratio can be approximately compared to the ratio of hadron yields in DIS experiments. It should be pointed out that in all the calculations reported in this article, the medium is assumed to be static and uniform with a fixed length. This fixed length is travelled by each jet. Realistic geometries will be considered in the future.

Monte-Carlo implementation. In any realistic calculation of jet modification in an extended medium a variety of approximations need to be made. For example, in the inmedium DGLAP evolution equations reported in the previous sections, we assumed that 
the entirety of the parton shower exits the medium and fragments in vacuum. This is obviously not the case. In reality, a large portion of the shower is trapped in the medium and does not undergo vacuum fragmentation. Such effects cannot be treated in a DGLAP setup where the input is the final vacuum fragmentation. Note that such effects may be included with a position dependent input fragmentation function. However, such input is always ambiguous and the computation of the evolution of a position, energy and obviously $z$-dependent fragmentation functions are prohibitively numerically intensive.

The obvious solution to this is to use a Monte-Carlo jet routine. Unlike analytic inmedium DGLAP calculations which evolve upwards, numerical Monte-Carlo routines evolve downwards in virtuality. As such, they are a more natural calculation which reconstructs the shower forwards in time. One starts with the original produced hard virtual parton and then constructs the Sudakov factor

$$
\Delta\left(Q^{2}, \mu^{2}\right)=\exp \left[-\frac{\alpha_{S}}{2 \pi} \int_{\mu^{2}}^{Q^{2}} \frac{d q^{2}}{q^{2}} \int d y P(y)\left\{1+K\left(y^{-}, q^{-}, L^{-}, q^{2}\right)\right\}\right],
$$

which yields the probability of no resolvable emission between $Q^{2}$ and $\mu^{2}$ and uses this to numerically estimate the probability of the initial parton being produced with a maximum virtuality of $\mu^{2}$. One then samples the splitting function to estimate the probability that the produced partons have fractions $y$ and $1-y$ of the parent parton. Unlike the case of the vacuum Sudakov factor, the equation above also contains in addition the medium dependent kernel $K$ defined in Eq. (5.164). This means that at each point, the shower may undergo either a vacuum split or a medium induced split. It also clearly demonstrates how the probability of splitting increases in the medium. At each point, we estimate the location of the parton based on its formation time, which may be easily obtained from its virtuality and its energy.

This showering routine is repeated to obtain partons with lower and lower virtuality. We terminate the shower when the virtuality of the parton reaches $\Lambda_{0}=1 \mathrm{GeV}$. If at this point the parton is found outside the medium, then it is convoluted with a vacuum fragmentation function. If it is found inside the medium then it is removed from the final shower. We point out again that the medium in all these calculations is not a real nucleus, but rather a static brick. Once the shower is calculated in the medium, it is then repeated in vacuum. Thus, both numerator and denominator of the ratio of fragmentation functions are calculated by an identical routine.

Using this implementation we may repeat our calculations in the HERMES-like systematics of Fig. 5.78, The results of the Monte-Carlo is represented by the solid black line. We should mention in passing that the fragmentation function used in the Monte-Carlo calculation is BKK while that in the DGLAP is KKP. We note that the ratio of fragmentation functions are rather similar. The Monte-Carlo results are for the most part below the DGLAP calculation. This is because of the mechanism by which we can systematically remove the partons which fragment in the medium, which can only be done in the MC calculation. The excess at lower $z$ is partially due to the use of a different fragmentation function and partially due to some of these partons having a long formation time.

Having tested the Monte-Carlo calculation in HERMES-like systematics $(E=20 \mathrm{GeV}$ and $\left.Q^{2}=3 \mathrm{GeV}^{2}\right)$, we apply the MC calculation to the EIC-like systematics $(E=25,35,50$ $\mathrm{GeV}$ and $Q^{2}=100 \mathrm{GeV}^{2}$ ). First off, we note that even with the larger energies there is a considerable amount of suppression. This is due to the larger $Q^{2}$ of the produced jet. Such jets tend to shower a lot and thus end up being considerably affected by the medium. This 
goes beyond what is known at HERMES that increasing the energy reduces the observed suppression. We also find a kind of universal suppression at large $z$ which is independent of energy. This kind of universal suppression was also noted in the DGLAP calculations performed for comparison with the HERMES data. In the earlier DGLAP calculations, the reason for the scaling was due to the vanishing of the real part of the evolution equation, leaving the same virtual corrections for different energies. It is difficult to state at this point if the scaling observed in the Monte-Carlo calculations is due to a similar reason, i.e., the vanishing of the real part of the equivalent DGLAP calculation.

If the results reported here are verified by a future EIC, this would represent an interesting observation: to find an almost $50 \%$ suppression in the large $z$ yield even for 50 $\mathrm{GeV}$ jets. Such high $Q^{2}$ jets should be describable using perturbation theory over a large part of their lifetime and would thus yield deep probes of the medium through which they propagate. This would allow for a much clearer understanding of the gluonic structure of nucleons inside nuclei. It would also greatly facilitate our understanding of how jets are modified in a dense extended environment, which would allow for more refined probes of matter produced in heavy-ion collisions.

\section{Jet evolution in hot and cold matter}

\section{Hans J. Pirner}

We will discuss jet propagation in hot matter first before addressing jet propagation in the "cold" matter of electron-nucleus collisions. A common interpretation of the large pion attenuation in $\mathrm{Au}+\mathrm{Au}$ collisions at RHIC is parton energy loss, where hadronization occurs outside of the hot zone and is not affected by the medium. There is no doubt that gluon radiation plays an important role for the energy loss and the parton evolution at RHIC and the LHC. The respective virtualities of partons are around $Q=20 \mathrm{GeV}$ and $Q=100 \mathrm{GeV}$. In our modeling of jet evolution [1067, 1068, the parton shower is treated together with the propagation of the parton in the medium which is more realistic because of the relevant time scales. A typical shower at RHIC lasts about $\tau_{\text {evo }}=2 \mathrm{fm}$. The non-perturbative part of hadronization involves the decay of the resonances at the pre-confinement scale $Q_{0}=1-2 \mathrm{GeV}$ into 3-4 pions. The lifetime of the plasma can be estimated at $\tau_{c}=3.3 \mathrm{fm}$. Comparing the two time estimates, we see that at the end of the evolution at RHIC, resonances interact with hadronic resonance matter. This process can be described by a hadronic theory with cross sections slightly larger than hadronic cross sections in vacuum. Because of these large cross sections, absorptive effects play a decisive role in the observed suppression of hadrons in RHIC experiments. We have advocated two scenarios. Scenario 1 uses the conservative radiative energy loss obtained from QCD and includes pre-hadron formation and resonance absorption. Scenario 2 neglects the resonance phase but tunes up the energy loss parameter to fit the data.

In more detail, our model [1067] works as follows: The parton produced in a hard process radiates successively to reduce its virtuality and become on mass-shell. This parton shower is modified by scattering in the medium. As both terms enter the same equation, one cannot separate scattering and radiation. This equation includes truly radiative energy loss, but without coherence. Quark fragmentation at RHIC and gluon fragmentation at the LHC should give the essential results. The indices on the fragmentation functions and the splitting functions can then be dropped and the formalism becomes simpler. For the in-medium fragmentation function $D^{m}\left(x, Q^{2}\right)$ we include into the DGLAP evolution the 
scattering term $S\left(x, Q^{2}\right)$.

$$
\frac{\partial D^{m}\left(x, Q^{2}\right)}{\partial \ln Q^{2}}=\frac{\alpha_{s}\left(Q^{2}\right)}{2 \pi} \int_{x}^{1} \frac{\mathrm{d} z}{z} P(z) D^{m}\left(\frac{x}{z}, Q^{2}\right)+S\left(x, Q^{2}\right)
$$

with

$$
S\left(x, Q^{2}\right) \simeq f \frac{n_{g} \sigma\left\langle q_{\perp}^{2}\right\rangle}{2 m_{s} Q^{2}}\left(D\left(x, Q^{2}\right)+x \frac{\partial D}{\partial x}\left(x, Q^{2}\right)\right) .
$$

The quantity appearing in the scattering term is the jet transport parameter $\hat{q} \simeq \bar{n} \bar{\sigma}\left\langle q_{\perp}^{2}\right\rangle$, which describes the mean acquired transverse momentum of the parton per unit length.

To allow a direct fit of experimental data with only parton energy loss, we introduce a possible enhancement factor $f$ in the scattering term. The scattering term is most relevant at small virtualities $Q \simeq Q_{0}$ and consequently we have used the scale $Q_{0}$ in $\alpha_{s}$ to arrive at an upper boundary for $\hat{q}$. More explicitly, these expressions give $\hat{q}=0.5 \mathrm{GeV}^{2} / \mathrm{fm}$ for a temperature of $T=0.3 \mathrm{GeV}$ for RHIC and $\hat{q}=5.2 \mathrm{GeV}^{2} / \mathrm{fm}$ for $T=0.5 \mathrm{GeV}$ corresponding to the LHC. As shown in ref. [1067] we can fit the RHIC data including prehadron absorption in the final state resonance gas. The prediction for LHC gives $R_{A A} \approx 0.4$. If we use an enhancement factor $f=8$ which is beyond any higher order QCD correction, the measurement of hadrons with high transverse momentum would be totally suppressed at the LHC.

Let us now discuss jets in cold matter resulting from DIS on nuclei. Electron scattering on a target at intermediate Bjorken $x$ can be treated along similar lines as the DGLAP evolution of the quark jet in the cold medium, whereas electron-nucleus scattering at low $x$, in principle necessitates the evolution of the quark and antiquark produced from photongluon fusion. It is not clear whether the cascades from the two reaction products behave independently when they propagate through the target. In the Ariadne model, two strings result from the quark and antiquark produced by photon-gluon fusion. The first string connects the antiquark with the quark which emitted the gluon. The second string combines the quark with the remnant di-quark of the proton. Due to the aligned jet configuration, one of the two strings only contains a few low momentum particles and perhaps may be neglected in the first approximation. The evolution equation outlined above can then be applied to jet propagation in cold matter, and applications to an EIC are planned. Scattering partners of the quark are nucleons and the quantity $\left\langle\sigma q_{\perp}^{2}>\right.$ can be derived from the dipole cross section on nucleons. The resulting transport parameter at HERMES energies is very small $\hat{q}=0.035 \mathrm{GeV}^{2} / \mathrm{fm}$ and has been tested in hadronic broadening of the produced hadrons [1069]. For a high energy machine with an electron-nucleon energy $E_{c m}=100 \mathrm{GeV}$ the transport parameter will be larger due to the increasing dipole cross section, we estimate that the transport parameter will increase to about $\hat{q}=0.1 \mathrm{GeV}^{2} / \mathrm{fm}$. So effects should well be observable, but smaller than at RHIC. 


\title{
5.12 Target fragmentation
}

\section{Fragmentation of nuclei - a critical tool for novel QCD phenomena}

\author{
Mark Strikman
}

The main focus of the discussions on quark propagation through the nucleus has been on current fragmentation processes, e.g., the suppression of the leading hadron spectrum, $p_{t}$ broadening and jet propagation in nuclear matter. So far, very little attention has been paid to nuclear fragmentation in DIS. To some extent, this is due to the lack of experimental data as such measurements are very challenging. However, while nuclear effects in the current fragmentation region decrease with increasing $Q^{2}$ at fixed $x$, the nuclear effects in the fragmentation region persist in this limit, and are likely to depend on $x$. They may help address a number of important questions:

- Are color tubes formed in propagation of quarks through nuclear media?

- How different are the propagations of gluons and quarks through the nuclear media?

- How different are the propagations of quark and dipole?

To visualize these questions, it is convenient to consider the process in the nuclear rest frame and distinguish three kinematic regions: (a) For $x \geq 0.1$, a quark is knocked out (or a gluon if we consider for example a leading di-jet or charm production in DIS), (b) for $0.1>x \geq 1 /\left(2 R_{A} m_{N}\right)$ the virtual photon converts to a $q \bar{q}$ pair inside a heavy nucleus, and (c) for $x<1 / 2 R_{A} m_{N}, \gamma^{*} \rightarrow q \bar{q}$ transition occurs predominantly before the target, see Fig. 5.79

In the case of of $x>0.1$ and large $Q^{2}$ corresponding to the knock out of a quark, a color triplet $q q$ system is left inside the nucleus and it is typically moving along the virtual photon momentum direction with a relatively small velocity. The knocked out quark fragments into partons/hadrons at longitudinal distances $y \geq 2 p_{q} / \Delta m^{2} \gg R_{A}$, where $\Delta m^{2} \sim 1 \mathrm{GeV}^{2}$ can be estimated based on the current DIS data [1014]. It is similar to that for color transparency processes. As a result, the leading hadron spectrum at large $Q^{2}$ approaches the universal limit given by pQCD. This pattern is consistent with the experimental data. Different to the naive expectations of the parton model, an A-dependent $p_{t}$ broadening in present in this limit. Naively the hadrons produced in the fragmentation of the quark are formed at distances given by $y \geq 2 p_{h} / \Delta m^{2}$, so that there should be a depletion in the spectrum at $p_{h}^{\text {crit }} \sim \Delta m^{2} R_{A} / 2$ followed by an enhancement at rapidities close to the nuclear rapidity (hadron pileup). Since for heavy nuclei $p_{h}^{\text {crit }} \sim 10 \div 20 \mathrm{GeV} / \mathrm{c}$, one would expect a strong deformation of the hadron spectrum with a large increase of multiplicity for $\left|y-y_{A}\right| \leq 2 \div 3$ for $A \sim 200$. In particular, it would be manifested in the strong break up of the heavy

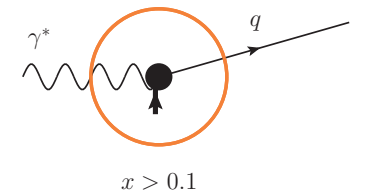

(a)

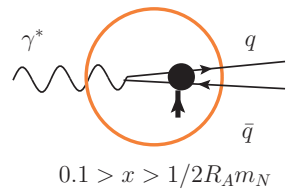

(b)

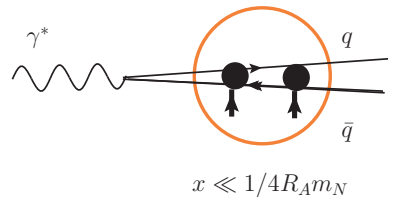

(c)

Figure 5.79. Space-time picture of DIS in the nucleus rest frame for different $x$ 

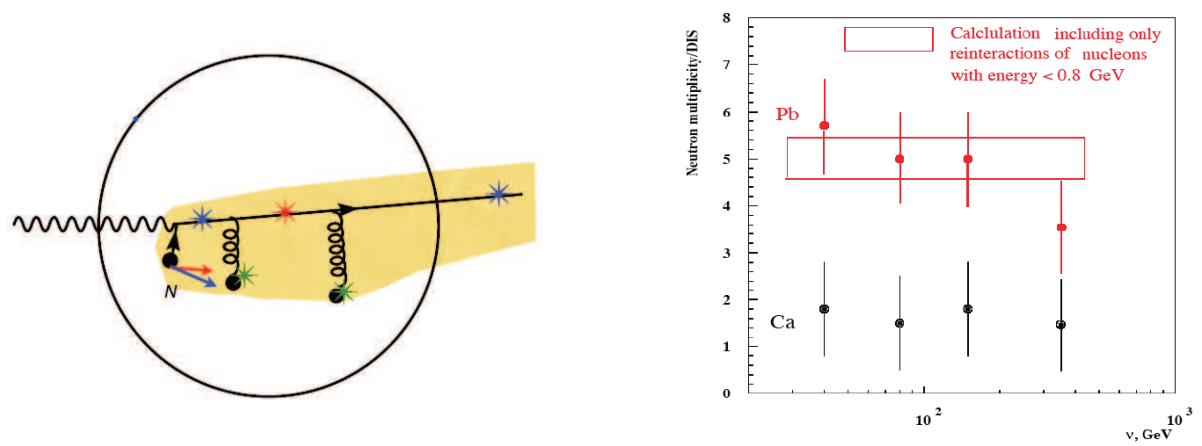

Figure 5.80. Left: Coulomb exchanges may lead to formation of extended spatial regions where color is not screened. Right: The E665 data [1070] for the soft neutron multiplicity compared with the calculation of [1071].

nuclei which is associated with emission of many soft neutrons. One should also expect an increase of the multiplicity of soft neutrons with an increase of $p_{t}$ of the leading hadron, since large $p_{t}$ selects events with extra Coulomb exchanges which are more likely for longer quark paths inside the nucleus and should result in a larger number of wounded nucleons. These may also lead to the creation of large unscreened color regions in the nucleus - see Fig 5.80. An open question is how these expectations could be affected by a high degree of coherence in the emission of the partons in pQCD. Such a coherence may lead to strong screening effects in the formation of the final state and in particular a reduction of $\Delta m^{2}$ away from the current fragmentation region. Also, if the color tube is very narrow, a chance that the tube intersects with other nucleons maybe significantly reduced.

For intermediate $x \sim 0.05$, the virtual photon also penetrates any point in the nucleus but it can hit either quark or antiquark, so in principle, by studying the properties of the leading hadron one can compare the structure of the final state interaction for the removal of quark and antiquark which maybe different, for example since $\bar{q}$ can belong to a color singlet $q \bar{q}$ cluster.

For small $x \leq 0.03$, the virtual photon predominantly transforms into a $q \bar{q}$ pair before the target nucleus. In the aligned jet model one would expect that the number of wounded nucleons would be given by $A \sigma(e N) / \sigma(e p)$ with the hadrons formed at the similar distances as in the large $x$ case. Hence naively one would expect that many nucleons will be wounded in a heavy nucleus, leading to a strong excitation of the nucleus which is known to be associated with multiple neutron emission, and emission of protons with momenta of $\geq 300$ $\mathrm{MeV} / \mathrm{c}$, see also Section 5.12 .

The process of neutron emission in DIS off $\mathrm{Pb}$ was studied by the E665 collaboration at FNAL for average $x \sim 0.05$ and $Q^{2} \sim$ few $\mathrm{GeV}^{2}[1070$. The results of the measurement are compared the theoretical calculation of [1071] in Fig. 5.80. Calculations using a Monte Carlo event generator tuned to reproduce the neutron emission in the proton-nucleus scattering reproduces both the neutron multiplicity and the neutron momentum distribution, provided only recoil nucleons with energy smaller than $1 \mathrm{GeV}$ are allowed to interact in the nucleus. Taken at face value, this suggests a very strong reduction of the final state interactions at large energies which is consistent with the trend of the E665 data to have a smaller neutron multiplicity for larger $\nu$.

At very small $x$ and moderate $Q^{2}$, one may reach the black disk regime. In this regime, the leading hadron spectrum is reduced and the pQCD factorization for the parton fragmen- 
tation breaks down in a gross way [981, see also Section 5.8, In this limit, the selection of events with enhanced activity in the nuclear fragmentation region should lead to reduction of the forward spectrum: this would provide a clear signal for a new regime, since no such correlation is possible in the leading-twist pQCD regime.

In summary, hadron production in the nuclear fragmentation region is very sensitive to the dynamics of space-time evolution of the triplet and octet color tubes as well as of color dipoles. This is one of the unexplored frontiers where the collider kinematics will allow a qualitative improvements in the data, and likely lead to the discovery of a series of new regularities. This may include a much higher degree of coherence in the fragmentation (hinted at by the E665 data) than suggested by the current models. Understanding of the fragmentation dynamics will be also of great help for understanding the dynamics in the nuclear fragmentation region in heavy ion collisions, where high density quark-gluon systems may be produced.

\section{In-medium hadronization and EMC effects in nuclear SIDIS}

\section{Ciofi degli Atti, L. P. Kaptari, B. Z. Kopeliovich, and C. B. Mezzetti}

The SIDIS process $A\left(e, e^{\prime}(A-1)\right) X$ in which, instead of the leading hadron, a nucleus $(A-1)$ in the ground or in low excitation states is detected in coincidence with the scattered electron, can provide new information about the mechanism of hadronization and the origin of the EMC effect [1072, 1073, 1074, 1075, 1076]. Two main advantages of the new SIDIS process over the classical SIDIS [1010] and inclusive $A\left(e, e^{\prime}\right) X$ scattering [1077] are worth mentioning here. Firstly, it can provide a new insight into the space-time development of hadronization at the early stage, which can be probed only by placing additional scattering centers at microscopic distances, i.e. by using nuclear targets. By detecting a jet produced on a nuclear target, one can get information about its time development, but in a rather indirect and complicated way, since cascading inside the nuclear medium essentially modifies the observables. Measuring the recoil nucleus supplies additional and cleaner information about the dynamics of hadronization; in particular, this process is free of the uncertainties caused by cascading, and the survival probability of the recoil nucleus is extremely sensitive to the multiparticle components of the jet [1072]. Secondly, a proper ratio of the cross sections on a nucleus $A$ taken at different values of the Bjorken scaling variable $x_{B j}$ provides information on the nucleon structure functions in the medium, $F_{2}^{N / A}$. Several experimental projects to investigate the new process at $12 \mathrm{GeV}$ have been proposed thanks to the development of proper recoil detectors [1078, and the experiment on Deuteron targets has already been performed [1079].

The basic ingredients of the theoretical calculation are the nuclear momentum distributions, the nucleon structure function $F_{2}^{N / A}$ in the medium, and the effective cross section of interaction between the hadronizing nucleon debris and the spectator nucleons. This last reads 1072 .

$$
\sigma_{e f f}\left(z, x_{B j}, Q^{2}\right) \equiv \sigma_{e f f}(z)=\sigma_{t o t}^{N N}+\sigma_{t o t}^{\pi N}\left[n_{M}(z)+n_{G}(z)\right]
$$

where $\sigma_{\text {tot }}^{N N}$ and $\sigma_{\text {tot }}^{\pi N}$ are the total nucleon-nucleon $(N N)$ and pion-nucleon $(\pi N)$ cross sections, and the $Q^{2}$ - and $x_{B j}$-dependent quantities $n_{M}(z)$ and $n_{G}(z)$ denote the pion multiplicities due to the breaking of the color string and to gluon radiation, respectively. Their explicit form directly follows from the hadronization mechanism proposed in Ref. [1080], leading to a satisfactory description of the grey track production in DIS off nuclei [1032]. 

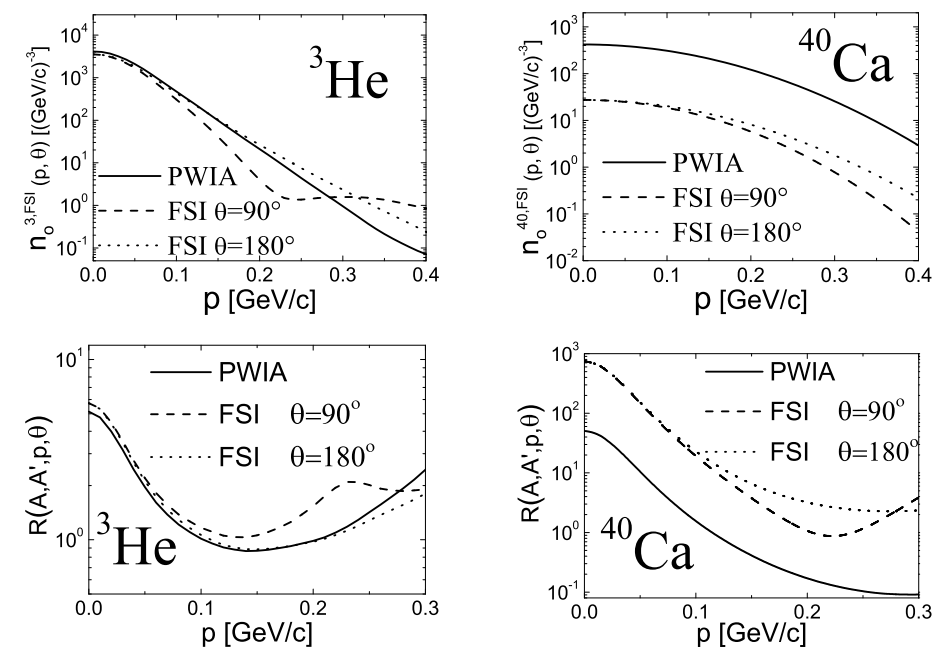

Figure 5.81. Top panels: the distorted momentum distributions $n_{0}$ with $\theta=\theta_{\widehat{\mathbf{P}_{A-1} \mathbf{q}}}$ and $p \equiv\left|\mathbf{P}_{A-1}\right|$ for ${ }^{3} \mathrm{He}$ and ${ }^{40} \mathrm{Ca}$. Bottom panels: The ratio $R\left(A, A^{\prime}\right)$ of Eq. 5.171 with $A=2$, and $A^{\prime}={ }^{3} \mathrm{He}$ or ${ }^{40} \mathrm{Ca}$.

The cross section of the $A\left(e, e^{\prime}(A-1)\right) X$ process [1072, 1074] schematically reads

$$
\frac{d \sigma^{A, F S I}}{d x_{B j} d Q^{2} d \mathbf{P}_{A-1}}=F_{2}^{N / A}\left(x_{A}, Q^{2}, k^{2}\right) \otimes n_{0}^{A, F S I}\left(\mathbf{P}_{A-1}\right)
$$

where $x_{A}=x_{B j} / z_{1}^{(A)}, z_{1}^{(A)}=\left(M_{A} k \cdot q\right) /\left(m_{N} P_{A} \cdot q\right), k$ is the four-momentum of the bound nucleon and $P_{A}$ of the target nucleus. In this equation, $n_{0}^{A, F S I}\left(\mathbf{P}_{A-1}\right)$ is the distorted momentum distribution of the bound nucleon after final state interaction (FSI) with the debris nucleon $\left(\mathbf{k}_{1}=-\mathbf{P}_{A-1}\right.$ in Plane Wave Impulse Approximation):

$$
n_{0}^{A, F S I}\left(\mathbf{P}_{A-1}\right)=\frac{1}{2 J_{A}+1} \sum_{\mathcal{M}_{A}, \mathcal{M}_{A-1}}\left|\int d \mathbf{r}_{1}^{\prime} e^{i \mathbf{P}_{A-1} \mathbf{r}_{1}^{\prime}}\left\langle\Psi_{J_{A-1}, \mathcal{M}_{A-1}}^{0}\left|S_{F S I}^{X N}\right| \Psi_{J_{A}, \mathcal{M}_{A}}^{0}\right\rangle\right|^{2}
$$

where $S_{F S I}^{X N}$ is the debris-nucleon eikonal scattering $S$-matrix which differs from the Glauber form because of the $z$ dependence of $\sigma_{\text {eff }}$ [1081. The results of some calculations are presented in what follows, using for Deuteron and ${ }^{3} \mathrm{He}$ realistic wave functions [1082] corresponding to the AV18 interaction [1083, and for heavy nuclei single particle mean field wave functions. A good agreement between our parameter-free calculation [1076] and the experimental data for $2 H\left(e, e^{\prime} p\right) X$ around $\theta \simeq 90^{\circ}$ is exhibited.

The distorted momentum distributions of ${ }^{3} \mathrm{He}$ and ${ }^{40} \mathrm{Ca}$ at kinematics more appropriate for an EIC are shown in Fig. 5.81, As already pointed out, the FSI is governed by the details of $\sigma_{e f f}$ and strongly affects the survival probability of $(A-1)$, as it can be seen by comparing the results for ${ }^{3} \mathrm{He}$ and ${ }^{40} \mathrm{Ca}$. Let us denote the cross section (5.169) by $\sigma^{A, F S I}$. Then, if our description is correct, the ratio of cross sections on different nuclei,

$$
R\left(A, A^{\prime}, \mathbf{P}_{A-1}\right)=\frac{\sigma^{A, \exp }\left(x_{B j}, Q^{2},\left|\mathbf{P}_{A-1}\right|, z_{1}^{(A)}, y_{A}\right)}{\sigma^{A^{\prime}, \exp }\left(x_{B j}, Q^{2},\left|\mathbf{P}_{A-1}\right|, z_{1}^{\left(A^{\prime}\right)}, y_{A^{\prime}}\right)} \rightarrow \frac{n_{0}^{(A, F S I)}\left(\mathbf{P}_{A-1}\right)}{n_{0}^{\left(A^{\prime}, F S I\right)}\left(\mathbf{P}_{A-1}\right)}
$$

should be governed only by the FSI, as shown in Fig. [5.81, 

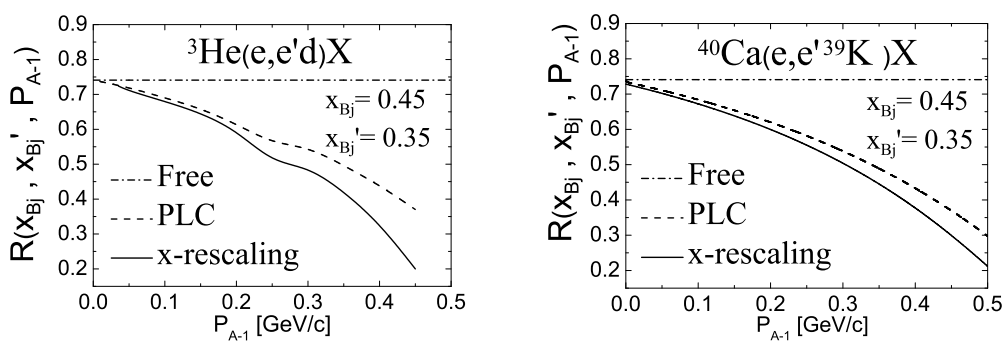

Figure 5.82. The ratio $R\left(x_{B j}, x_{B j}\right)$ of Eq. (5.172) for the process ${ }^{3} \mathrm{He}\left(e, e^{\prime} d\right) X$ and ${ }^{40} \mathrm{Ca}\left(e, e^{\prime 39} \mathrm{~K}\right) \mathrm{X}$ calculated with different nucleon structure functions: i) free structure function; ii) off mass-shell (xrescaling) structure function; iii) with suppression of point-like configurations (PLC) in the medium depending upon the nucleon virtuality 1084$]\left(P_{A-1} \equiv\left|\mathbf{P}_{A-1}\right|\right)$.

In order to tag bound nucleon structure functions, whose nuclear modification is one of the causes of the EMC effect, one has to get rid of the distorted nucleon momentum distributions and other nuclear structure effects. This can be achieved by considering the ratio of the cross sections on a nucleus $A$ measured at two different values of the Bjorken scaling variable, $x_{B j}$ and $x_{B j}^{\prime}$, leaving unchanged all other quantities in the two cross sections, i.e., the ratio

$$
R\left(x_{B j}, x_{B j}^{\prime},\left|\mathbf{P}_{A-1}\right|\right)=\frac{\sigma^{A, \exp }\left(x_{B j}, Q^{2},\left|\mathbf{P}_{A-1}\right|, z_{1}^{(A)}, y_{A}\right)}{\sigma^{A, \exp }\left(x_{B j}^{\prime}, Q^{2},\left|\mathbf{P}_{A-1}\right|, z_{1}^{(A)}, y_{A}\right)} \approx \frac{F_{2}^{N / A}\left(x_{A}, Q^{2}, k^{2}\right)}{F_{2}^{N / A}\left(x_{A}^{\prime}, Q^{2}, k^{2}\right)}
$$

which depends only upon the nucleon structure function $F_{2}^{N / A}$. Calculations of the ratio (5.172) have been performed [1076] using three different structure functions, namely, the free one, giving no EMC effect, and two medium dependent structure functions, yielding only a few percent difference in the inclusive cross section. It can be seen from Fig. 5.82 that the discrimination of different models of the medium dependence of $F_{2}^{N / A}\left(x_{A}, Q^{2}, k^{2}\right)$ can indeed be achieved, especially at large $P_{A-1} \equiv\left|\mathbf{P}_{A-1}\right|$.

In conclusion, from what shown here and in the original papers 1073, 1072, 1074, 1075, 1076 it appears that the SIDIS process $A\left(e, e^{\prime}(A-\right.$ 1)) $X$, with detection of a complex nucleus $(A-$ $1)$, would be extremely useful to clarify the origin of the EMC effect and to study the early stage of hadronization at short formation times. At EIC kinematics (large $Q^{2}$ and $W_{X}^{2}$ ), the

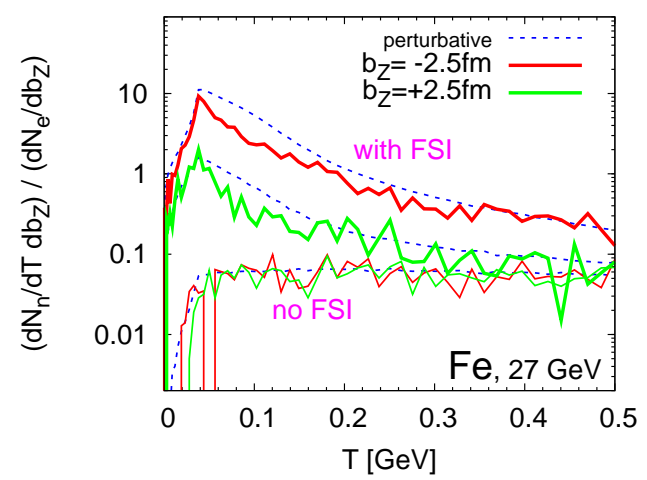
theoretical assumptions underlying Eqs.(5.168)-

Figure 5.83. The production cross section of neutrons with low momenta for different longitudinal production points, normalized to the corresponding number of events. (The calculations are preliminary.)

(5.170) are expected to be of higher validity than at lower energy. The problem remains as to whether experiments of the kind we are discussing, i.e. the detection of low-momentum light nuclei at specific angles, could be performed at an Electron Ion Collider. We have calculated the process ${ }^{3} \mathrm{He}\left(e, e^{\prime} d\right) X$ at various EIC kinematics and found that, e.g. at $Q^{2} \simeq 30 \mathrm{GeV}^{2}$ and $x_{B j} \simeq 0.7$, when the Deuteron is emitted at about $90^{0}$ in the target 
rest frame, this corresponds to about $1^{0}$ in the direction of the incident nucleus in the collider CM frame.

\section{Proving the microscopic origin of nuclear forces}

\section{Mark Strikman}

An important task for the EIC is to probe nuclear forces on the microscopic level using hard probes. Before describing some of the possible avenues for EIC research, it is worth summarising what is already known from the analyses of the experimental studies of the nuclear pdfs.

- The quark distributions at large $x$ are suppressed as compared to the naive expectations based on the picture of the nucleus built of nucleons with internal parton distributions coinciding with the free nucleon pdfs, the so called EMC effect - for a review see e.g. 942. However, the EMC effect modification of the nucleon pdfs remains small $-\leq 2 \%$ for $x \leq 0.5$ after one takes into account the Coulomb field contribution into the wave function of the heavy nuclei and uses the proper scaling variable $x_{A}=A Q^{2} / 2 q_{0} M_{A}$ for the comparison of the nuclear cross sections [1085]. The modification of the nucleon pdfs strongly grows with $x$ at $x>0.5$ reaching $\sim 10 \%$ at $x=0.6$.

- The $A$-dependence of the EMC effect at large $x$ indicates that the main contribution to the EMC effect is due to scattering off the short-range correlations (SRC) in nuclei.

- Experiments at JLab confirm approximate $A$-independence of the momentum distribution of nucleons in the short-range correlations, though the absolute probability is a factor of $\sim 5$ larger in heavy nuclei than in the deuteron, for a recent review see [1086.

- The measurements of the antiquark distributions in nuclei were performed using the Drell-Yan process. No enhancement of the $\bar{q}_{A} / \bar{q}_{N}$ ratio was observed for $x \sim 0.1$ where the models of nuclear forces with dynamic pion fields predicted $10 \div 20 \%$.

- Application of the baryon and momentum sum rules indicate that the valence quarks and gluons are enhanced in nuclei at $x \sim 0.1$ [1087, 828].

The region of $x \sim 0.1$ is especially interesting for the purposes of studying the QCD origin of the nuclear forces since it corresponds to the Ioffe distances $\sim 1 / 2 x m_{N} \sim 1 \mathrm{fm}$, characteristic of more medium and short-distance nuclear forces. The regularities listed above suggest that meson exchanges which lead to the enhancement of the sea quark distributions are less important than it is suggested in the meson models of the nuclear forces, while quark and gluon interchanges between nearby nucleons play a significant role. The inclusive measurements at the EIC will directly measure $V_{A} / V_{N}, G_{A} / G_{N}$ for $x \sim 0.1$.

A new tool which will be available at the EIC is exclusive hard processes for which the QCD factorization theorem has been proven for the processes $\gamma_{L}+T \rightarrow V M+T^{\prime}$ for the Bjorken limit and the mass of the final system $T^{\prime}$ being fixed [572]. We will focus on the processes with deuteron target since in this case it is easier to select scattering off the compact proton - neutron configurations and measure a complete final state. The rational here is that the structure of the SRC in nuclei is approximately the same while using a 
heavier target, say ${ }^{4} \mathrm{He}$ would increase the impulse approximation interaction rate by a factor of $\sim 3$ only (due to a higher probability of SRCs in ${ }^{4} \mathrm{He}$ ). However this apparent gain will be compensated to a large extent by the final state absorption/distortions and multistep processes significantly complicating the interpretation of the observations.

The first question one can address is whether the quark and gluon transverse distributions in bound nucleon are the same. The simplest possible processes are break up of the deuteron

$$
\gamma+{ }^{2} H \rightarrow J / \psi+p+n
$$

and

$$
\gamma_{L}^{*}+{ }^{2} H \rightarrow \rho^{-}+p+p .
$$

which probe gluon and quark transverse distributions.

The exclusivity of the process could be tested by measuring $p_{t}\left(N_{1}\right)+p_{t}\left(N_{2}\right)+p_{t}(V M)=$ 0 . To avoid an ambiguity which of the nucleons was interacting via the hard process $\gamma^{*}+N \rightarrow V M+N$, one needs to select transverse momenta of the vector meson $\geq 600 \div$ $700 \mathrm{MeV} / \mathrm{c}$ with momentum of the nucleon $N_{1}$ in approximately the opposite direction. For the spectator to belong to the SRC one needs to ensure that it has a large momentum in the deuteron rest frame $\geq 0.3 \mathrm{GeV} / \mathrm{c}$. It could be either mostly longitudinal or have a transverse component sufficiently deviating from the direction opposite to $p_{t}(V M)$.

The measurement involves studying the dependence of the ratio of the cross section of the reaction (5.173), (5.174) and elementary reaction

$$
R\left(p_{s} p\right)=\frac{\frac{d \sigma\left(\gamma^{*}+{ }^{2} H \rightarrow N N+V M\right)}{d W_{\gamma N} d Q^{2} d t, d p_{s p}}}{\frac{d \sigma\left(\gamma^{*}+N \rightarrow N+V M\right)}{d W_{\gamma N} d Q^{2} d t}}
$$

on the momentum transfer to the vector meson - $t \approx-p_{t}(V M)^{2}$ for fixed values of $p_{s p}$. Deviations of the t-dependence from a constant (which can be calculated in the two nucleon approximation) would signal the change of the size of the bound nucleon. The theoretical expectation is that such effects are proportional to the nucleon "off-shellness" so they should rapidly increase with increasing $p_{s p}$, roughly $\propto p_{s p}^{2}$ [1088, 1084]. It is worth noting that nucleon deformation along and transverse to the direction between the nucleons may differ (like in the case of polarization of the atoms in the molecules). Hence a nontrivial dependence of $R\left(p_{s} p\right)$ on the angle between $p_{s p}$ and $p_{t}(V M)$ is possible (such a dependence is absent if the deformation depends only on the virtuality). It would be possible to study the dependence of $R\left(p_{s} p\right)$ on $x$ (for photoproduction of $J / \psi$ on $m_{J / \psi}^{2} / W^{2}$ probing how the nucleon deformation depends on $x$ of the gluon in the bound nucleon wave function.

Another possible direction for studies is probing directly the pion exchange mechanism using exclusive hard processes - for example $\gamma+{ }^{2} H \rightarrow J / \psi+\pi^{-}+p p$ with transverse momenta of $J / \psi$ and $\pi^{-}$back to back and large deuteron rest frame momenta of both protons (to ensure that the process occurs off the SRC).

The discussed class of the reactions is well suited also for looking for non-nucleonic baryonic components in the SRCs (six quarks, $\Delta \Delta, \ldots$ ). For example one can study the process $\gamma+{ }^{2} H \rightarrow J / \psi+\Delta^{++}+\Delta^{-}$where transverse momenta of $J / \psi$ and one of $\Delta$ 's are back to back. The advantage of this reaction as compared to medium energy processes is the absence of a non-vacuum exchange in t-channel. 


\title{
Slow neutrons and final-state interaction length
}

\author{
Kai Gallmeister, Ulrich Mosel
}

With collider kinematics, it is very instructive to look at "slow" nucleons of energy less than $10 \mathrm{GeV}$, considered slow with respect to the (fast) target nucleon [1071, see also Section 5.12, Performing some exploratory simulations within the GiBUU framework (see Section 5.10) we are confronted with a lot of complications. In Fig. 5.83 we show some distributions of slow neutrons as a function of energy for different production points in the longitudinal axis, normalized to the corresponding number of scattered electrons. This result is to be considered as preliminary, since we learned that we need a more accurate treatment of Pauli-blocking and binding effects in the few $\mathrm{MeV}$ region. In addition, we need to take into account the production of slow nucleons via evaporation and fragmentation. This work is currently in progress by inclusion of a multi-fragmentation framework (SMM) [1089. and correcting for effects of the large energy gap between initial interaction and fragmenting nucleons.

It has been proposed by Ciofi degli Atti and coworkers in many papers (see Section 5.12) that the interaction cross section of the jet particles within a SIDIS event with the debris of the target nucleus shows interesting formation length dependencies. We see a large potential for our GiBUU model to study all these questions. 


\title{
5.13 Bose-Einstein correlations at an electron-ion collider
}

\author{
Gerald P. Gilfoyle
}

QCD directs the formation of hadrons from quarks and gluons in hard scattering. However, our understanding of this process is ad hoc; there is no full, QCD-based theory to explain hadronization and fragmentation. To probe these processes, we propose to take advantage of an iconic quantum mechanical effect, the symmetrization of the wave function required for bosons. Particles formed near one another will have overlapping wave functions and the interference of the wave functions produces correlations in the intensity and momentum dependence of the final particles. These Bose-Einstein Correlations (BEC) (or the Hanbury-Brown Twiss effect) are examples of intensity interferometry and can be used to study the space-time extent of the source of the particles and/or learn about the dynamics of their formation. They have been used to investigate hot nuclear matter, but there are only a few cases where $e+A$ interactions have been studied. That work revealed that BECs can be used to study the QCD string in hard scattering and our simulations show we will be able to make precise measurements of the BEC source size at an EIC.

Bose-Einstein Correlations arise when two identical bosons are detected and their joint wave function $\left|p_{1} p_{2}\right\rangle$ ( $p_{i}$ is the particle 4-momentum) must be symmetric under particle exchange. In other words, when the two bosons are detected from different points in spacetime, the observer cannot distinguish the origin of each particle so their amplitudes must add. This requirement gives rise to interference terms in the intensity that do not exist for non-identical particles. In fact, for identical fermions there would an anti-correlation between the particles. The BEC in energy-momentum space is related to the extent of the source in its spatial dimensions and the correlation function can be written as

$$
R\left(Q_{12}\right)=\frac{d N / d Q_{12}}{d N_{\text {ref }} / d Q_{12}}
$$

where $Q_{12}=\sqrt{-\left(p_{1}-p_{2}\right)^{2}}$ is the Lorentz-invariant momentum difference between the identical bosons and $N_{\text {ref }}$ is a reference spectrum constructed with no BECs. The correlation function is often parameterized as

$$
R\left(Q_{12}\right)=\alpha\left(1+\lambda \Omega\left(Q_{12} r_{12}\right)\right)\left(1+\beta Q_{12}\right) .
$$

In static models of particle sources, $\Omega\left(Q_{12} r_{12}\right)$ can be interpreted as the Fourier transform of the spatial distribution of the emission region of bosons with overlapping wave functions and is characterized by the size parameter $r_{12}$ of the source. It is typically treated as a Gaussian $\left(e^{-Q_{12}^{2} r_{12}^{2}}\right)$ or an exponential $\left(e^{-Q_{12} r_{12}}\right)$. The parameter $\lambda$ measures the coherence of the source, $\alpha$ is a normalization factor, and $\beta$ accounts for long range correlations.

Existing measurements. There is a long history of the study of BECs in particle and nuclear physics going back to 1960 when two-pion correlations were measured in $p \bar{p}$ collisions [1091. They have been used to study geometric properties in $e+p$ reactions [1090, the space-time extent of hot nuclear matter in $A u+A u$ collisions [1092, 1093, and the dynamical properties of hadrons extracted from $A u+A u$ collisions [1094. Figure 5.84 shows the two-pion correlation function from Ref. [1090] for $e+p$ reactions measured at the DESY collider for an electron momentum $p_{e}=27.6 \mathrm{GeV}$ and proton momenta $p_{p}=-820 \mathrm{GeV}$ and $p_{p}=-920 \mathrm{GeV}$. It shows several of the important features seen in many correlation functions. There is a clear correlation that is maximal at $Q_{12}=0$ and drops rapidly to unity 

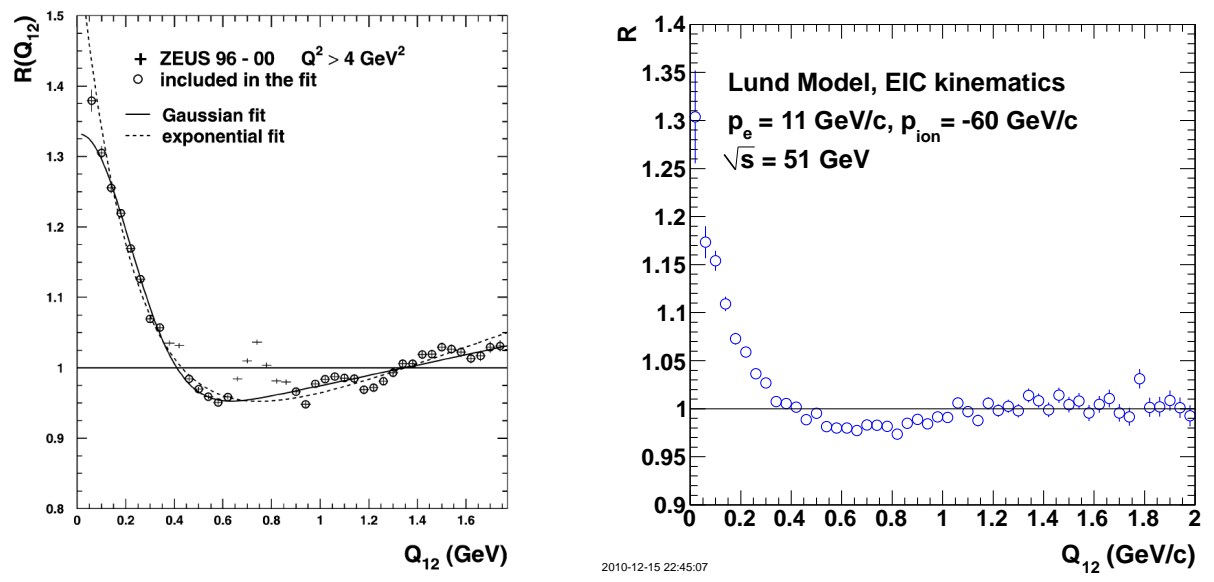

Figure 5.84. Left: the measured Bose-Einstein correlation function, $R\left(Q_{12}\right)$, together with Gaussian and exponential fits 1090. The error bars show the statistical uncertainties. The data points included in the fit are marked with the circles. The other points are excluded from the fit because the correlation is dominated by resonance effects. Right: Pythia simulation of $\pi^{+} \pi^{+}$Bose-Einstein correlations (BEC) at Electron-Ion Collider kinematics. The BEC parameters were taken from Ref. [1090. The Lund fragmentation model was used.

and below with increasing momentum difference. The height of the correlation function at $Q_{12}=0$ measures the coherence in the source. At moderate $Q_{12}$ the correlation drops below one, reflecting the usual practice of requiring the integral of the entire correlation function to go to one. There is a steady rise in $R$ at larger $Q_{12}$ due to long-range effects. Recall the denominator Eq. (5.175); It should be free of the correlations arising from Bose-Einstein statistics, but will not be free of all correlations: momentum conservation will push $R$ up at large $Q_{12}$. The width of the peak at $Q_{12}=0$ reflects the size of the source of the two bosons, i.e. large width in momentum space implies a small spatial source. The width of $R$ in Fig. 5.84 corresponds to $r_{12} \approx 0.9 \mathrm{fm}$ for an exponential fit and is largely independent of $Q^{2}$, the square of the four-momentum transfer.

BECs at an EIC. Measurements with the CLAS detector of a different type of correlations (i.e., two protons) have been performed on nuclear targets. Some of the results are shown in the left-hand panel of Fig. 5.85 [1095]. The figure shows the effects on the source size $r_{r m s}$ (extracted from the correlation) of the average pair momentum $\left(p=\left|\vec{p}_{1}+\vec{p}_{2}\right| / 2\right)$ and the nuclear size on the correlation function. At low average pair momentum $r_{r m s}$ increases for the heavier nuclei and approaches the nuclear size; implying the possible dominance of proton rescattering. The density of the source was extracted in Ref. 1095 and found to be about 2-3 times the nuclear density in helium. In the right-hand panel of Fig. 5.85 we show preliminary results on $\pi^{+} \pi^{+}$pairs on several nuclear targets [1099]. Below $Q_{12} \approx$ $0.15 \mathrm{GeV} / \mathrm{c}$ the correlations from all nuclei rise to a large positive correlation. Above $Q_{12} \approx 0.15 \mathrm{GeV} / \mathrm{c}$ the correlation functions overlap one another within the statistical uncertainty.

Measurement of Bose-Einstein correlations at an EIC will provide a new portal to studies of cold, high-density nuclear matter and the process of hadronization. The ground-state properties of nuclei are now well understood. Ab initio calculations of the nuclear ground state are successful for nuclei up to $A=8$ and higher [1100, 1101] and lattice QCD calculations continue to make progress toward a fundamental understanding of the nucleon 

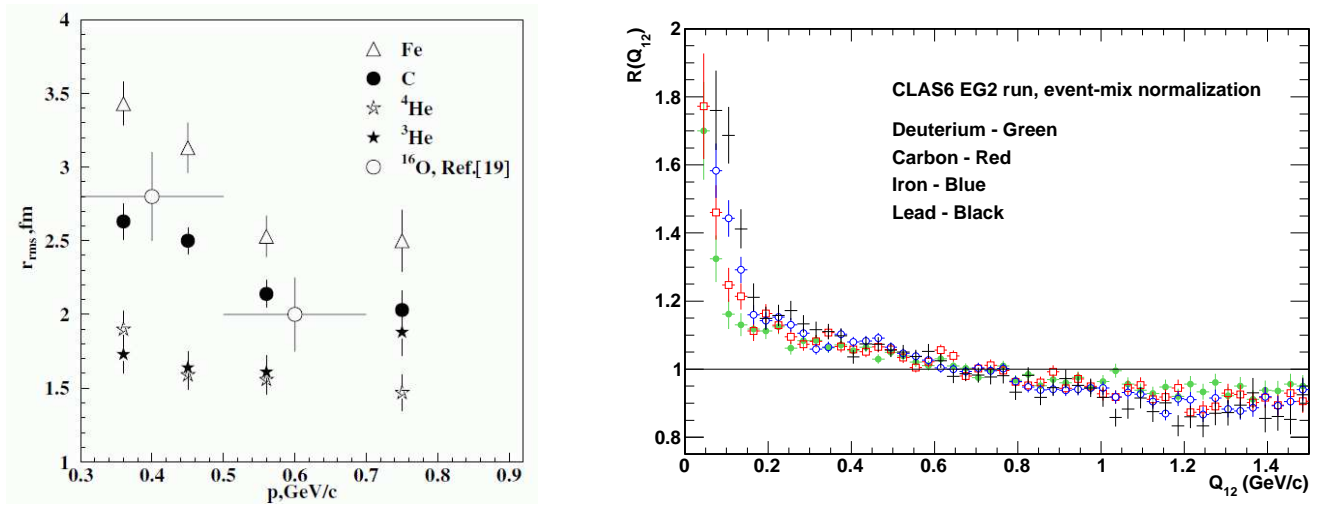

Figure 5.85. Left panel: The size parameter $r_{r m s}$ as a function of the mean pair momentum $p=$ $\left|\vec{p}_{1}+\vec{p}_{2}\right| / 2$ is shown for different nuclear targets [1095]. Data from Refs [1096, 1097, 1098, are shown which correspond to $e-{ }^{16} \mathrm{O}$ interactions at initial energy of $5 \mathrm{GeV}$ and $Q^{2}<0.1(\mathrm{GeV} / \mathrm{c})^{2}$ are shown for comparison. Right panel: Preliminary correlation functions for $\pi^{+} \pi^{+}$from the CLAS detector at Jefferson Lab [1099.

[102. However, the high-momentum components of the nuclear ground state are only now being revealed. These high-momentum nucleons are often paired with another, nearby neutron or proton forming regions of cold, dense nuclear matter. Short-range correlations have shown the importance of high-density components and the influence of the tensor force [1103, 1104]. The results of Ref. [1095] (left-hand panel of Fig. 5.85) demonstrated the use of correlations to extract density information. Measurements at an EIC could also help us to understand neutron stars 1105] and the EMC effect [1106].

Simulations. We have simulated Bose-Einstein correlations for $\pi^{+} \pi^{+}$pairs at the kinematics of an Electron-Ion Collider to investigate the feasibility of measuring BECs at an EIC. For our starting point we used the results for $\pi^{+} \pi^{+}$correlations from $e p$ reactions at DESY that are shown in Fig. 5.84 [1090]. That measurement covered the range $Q^{2}=4-8000(\mathrm{GeV} / \mathrm{c})^{2}$ and there was limited $Q^{2}$ dependence in the BEC parameters they extracted. It is reasonable to believe those parameters may also apply to the EIC kinematics. We chose the $\pi^{+} \pi^{+}$ channel because we expect them to be abundant and there is data from other experiments that enable us to make comparisons. We took advantage of several existing tools to perform the simulations. The Pythia program [81] was used to generate events with either Lund string model or independent fragmentation. The code also includes a feature to simulate Bose-Einstein correlations [1107, 1108]. The algorithm for the BECs starts with the usual fragmentation simulation and then pairs of identical particles (i.e. $\pi^{+} \pi^{+}$) are selected. For these pairs the relative 4-momentum $Q_{12}$ is modified according to the desired parameterization (see discussion of Eq. (5.176) above) with the constraint that the total 3-momentum of the pair remains the same in the center-of-mass (CM). The overall effect of applying the algorithm is to preserve momentum conservation, but reduce the energy. To compensate for the energy reduction, the CM momentum vectors are then rescaled.

As a consistency check, we compared the simulated correlation function $R$ for $\pi^{+} \pi^{+}$ pairs with the measurements from DESY shown in Fig. 5.84. The simulated correlation was weaker than the measured one, $R\left(Q_{12}=0\right)=1.2$ (simulated) versus $R\left(Q_{12}=0\right)=1.38$ (measured), and not as wide, but still experimentally significant. Since we are studying the possibility of observing BECs, the parameters from Ref. [1090] will provide a more 

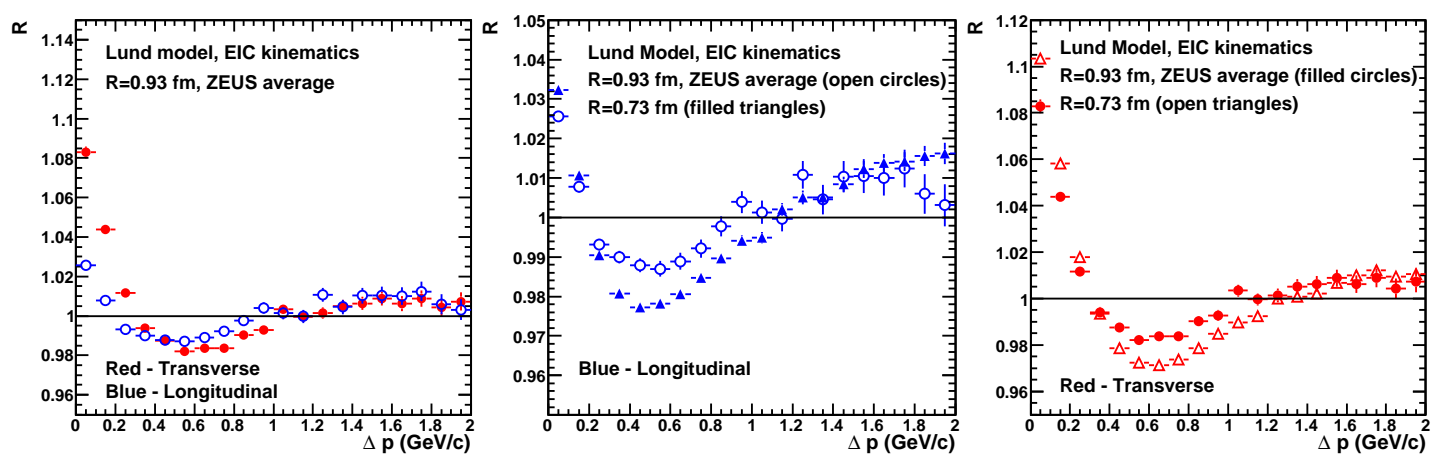

Figure 5.86. Longitudinal and transverse $(L T)$ correlation functions calculated with Pythia. The left-hand panel shows the correlations functions using the Ref. [1090] parameters. The other two panels show a comparison between those results and ones from a calculation with a smaller source size $r_{12}$.

conservative (and safer) test. We also simulated the BECs at the same kinematics as the preliminary results shown in the right-hand panel of Fig. 5.85 $\left(p_{e}=5 \mathrm{GeV}\right.$ and fixed target). Here we found the simulated correlation disappeared entirely. The multiplicity of the events generated by Pythia dropped significantly at these kinematics reflecting the limitations of the code at these lower energies.

At EIC kinematics $\left(p_{e}=11 \mathrm{GeV} / \mathrm{c}, p_{\text {ion }}=-60 \mathrm{GeV} / \mathrm{c}, \sqrt{s}=51 \mathrm{GeV}\right)$, we used the BEC parameters from ZEUS [1090]. Since the EIC will run at energies lower than at HERA, but above the current ones at Jefferson Lab, our estimates of the BECs are again conservative ones. Our simulation of $R$ at EIC kinematics is shown in the right panel of Fig. [5.84, There is, like in the Ref. [1090] data, a sizable correlation at $Q_{12}=0$, a decrease in $R$ with width $\approx 0.2 \mathrm{GeV} / \mathrm{c}$, a dip below unity (recall discussion of Fig. [5.84) and then the data approach one at high $Q_{12}$. The Lund model was used here for the fragmentation and a calculation using the independent fragmentation model in Pythia yielded similar results. This result shows we can expect sizable correlation functions at the EIC.

One of the possible effects we may see at an EIC is the stretching of the QCD color string at high $Q^{2}$ and/or changes in the string tension (recall Ref. [1109]). The fragmentation region may not be spherical as observed in Ref. [1109, but may have different sizes in the longitudinal and transverse directions. Such a difference was measured in Ref. [1090] where the longitudinal radius was $0.26 \pm 0.03 \mathrm{fm}$ bigger than the transverse one. To search for such an effect in our simulation requires a different approach to extracting $R$. We worked in the longitudinal Center-of-Mass System (LCMS), where the longitudinal components of the pair momentum add to zero and extracted the transverse and longitudinal 3-momentum differences $\Delta p$. Our initial results are shown in the left-hand panel of Fig. 5.86. The transverse (red, filled circles) and longitudinal (blue, open circles) produce the characteristic shapes seen above for $R$, but with significant quantitative differences between the two. The transverse correlation is about twice the longitudinal one at $Q_{12}=0$ and the widths are similar. The large difference between the correlation functions suggests this may be a useful tool for studying space-time properties of the emission source. To delve deeper into this question, we considered the sensitivity of the $L T$ distributions to changes in the size parameter in the BEC parameterization. The middle and right-hand panels in Fig. 5.86 show a comparison of the same $L T$ correlation functions shown in the left-hand panel with ones calculated with a smaller size parameter $\left(r_{12}=0.73 \mathrm{fm}\right.$ versus $r_{12}=0.93 \mathrm{fm}$ 
from Ref. [1090]). The smaller radius amplifies the shape of the correlation functions (the maximum at $Q_{12}=0$ increases and the dip at $Q_{12} \approx 0.6 \mathrm{GeV} / \mathrm{c}$ is deeper. We can clearly separate the two distributions within the Monte Carlo statistics shown here. We expect the statistical uncertainties for an EIC measurement to be better than the Monte Carlo statistical uncertainties shown here. The cross sections for these reactions (from Pythia) multiplied by the EIC luminosity suggest a production rate of $10^{5} \mathrm{~Hz}$. We also fitted the correlation functions with Eq. (5.176) and obtained uncertainties on the size parameter $r_{12}$ less than $0.15 \mathrm{fm}$ which is comparable to the precision of the results in Ref. [1090. Thus, we will be able to discriminate between different size parameters at least at the $0.2 \mathrm{fm}$ level. Conclusions. Bose-Einstein correlations will be an important tool at an Electron-Ion Collider for studying high-density nuclear matter, the dynamics of the QCD string in hard scattering, and to gain a deeper understanding of fragmentation and hadronization. Our simulations have shown us that we can expect large (20\%) effects in the correlation function at small $Q_{12}$. The longitudinal-transverse correlations are sensitive to the size parameter to a fraction of a $\mathrm{fm}$. Finally, the large $\pi^{+} \pi^{+}$BECs observed at JLab that are not reproduced in our simulations hold the promise of new physics to be uncovered with the EIC. 


\subsection{4 e+A Monte Carlo simulation tools}

\subsubsection{A Monte Carlo Generator for Diffractive Events in e+A Collisions}

Tobias Toll and Thomas Ullrich

While there is a rich set of Monte Carlo (MC) event generators for $e+p$ collisions available (e.g. PYTHIA6 [1110, HERWIG++ [1111, LEPTO [91], PEPSI [155, RAPGAP [1112, ARIADNE [1113, CASCADE [1114, SHERPA [1115]), the situation for eA collisions is less favorable. The exception is DPMJET [1116] which attempts to describe deep-inelastic $e \mathrm{~A}$ events but does not include the rich physics accessible via diffractive events.

In strong interactions, diffractive events can be interpreted as resulting from scattering via the exchange of a pomeron that carries the quantum numbers of the vacuum, as discussed in 5.4. It was a surprise to see that a large fraction (approximately 15\%) of all $e+p$ events at HERA were diffractive. Calculations predict this fraction to be even larger in $e+\mathrm{A}$ collisions at EIC where the large nuclei remain intact $\sim 25-30 \%$ of the time (e.g. [826, 825]). In fact diffractive events are considered the most sensitive means of studying saturation since the dipole scattering amplitude is proportional to the square of the gluon momentum distribution $x g\left(x, Q^{2}\right)$. Another fascinating aspect of the study of diffractive events at an EIC is that that it would allow us to measure the intensity and the spatial distribution of the strong field that binds the nucleus together 885.

For all the above measurements the most important process to study is the production of exclusive diffractive vector mesons, such as $J / \Psi, \phi$, and $\rho$ mesons, as well as Deeply Virtual Compton Scattering (DVCS) photons. These processes give very clean final states, consisting of the scattered electron and nucleus and one extra particle: a vector meson or a real photon. This is a process which is dominated by small momentum fractions $x<10^{-2}$. $J / \Psi$ production is particularly well suited for studies of the spatial gluon distribution inside nuclei due to its well known wave function, narrow decay width, and its large branching ratio for electromagnetic decays $J / \Psi \rightarrow e^{+}+e^{-}\left(\right.$or $\mu^{+}+\mu^{-}$).

The measurement of exclusive vector meson production in diffractive events will be one of the key measurements at an EIC. Therefore these processes has been the starting point in our efforts to realise a new multi-purpose MC generator.

The Dipole Model: The dipole model is an important tool in investigations of diffractive processes and for the purpose of applying it to $e+\mathrm{A}$ collisions, we needed an impact parameter dependent model as starting-point. Two known models fulfil this requirement: bSat (or IPSat) 823] and bCGC [823, 824]. They are the underlying building blocks used in the generator. In what follows, we will concentrate on the bSat model and not discuss the technical details of the generator but focus on how the dipole models are applied with emphasis on the extension to $e+\mathrm{A}$ collisions.

The parameters of the dipole models described below have been tuned to inclusive HERA data, and they describe a wide variety of HERA measurements exceptionally well [823, 824].

The Dipole Model in $e+p$ : The production of exclusive vector mesons and DVCS photons at small $x$ for $e p$ collisions, $e+p \rightarrow e^{\prime}+p^{\prime}+V / \gamma$, in the dipole model has been extensively studied [823, 824]. Here the virtual photon splits into a quark-antiquark dipole which interacts with the target diffractively via one or many two-gluon pomeron exchanges 

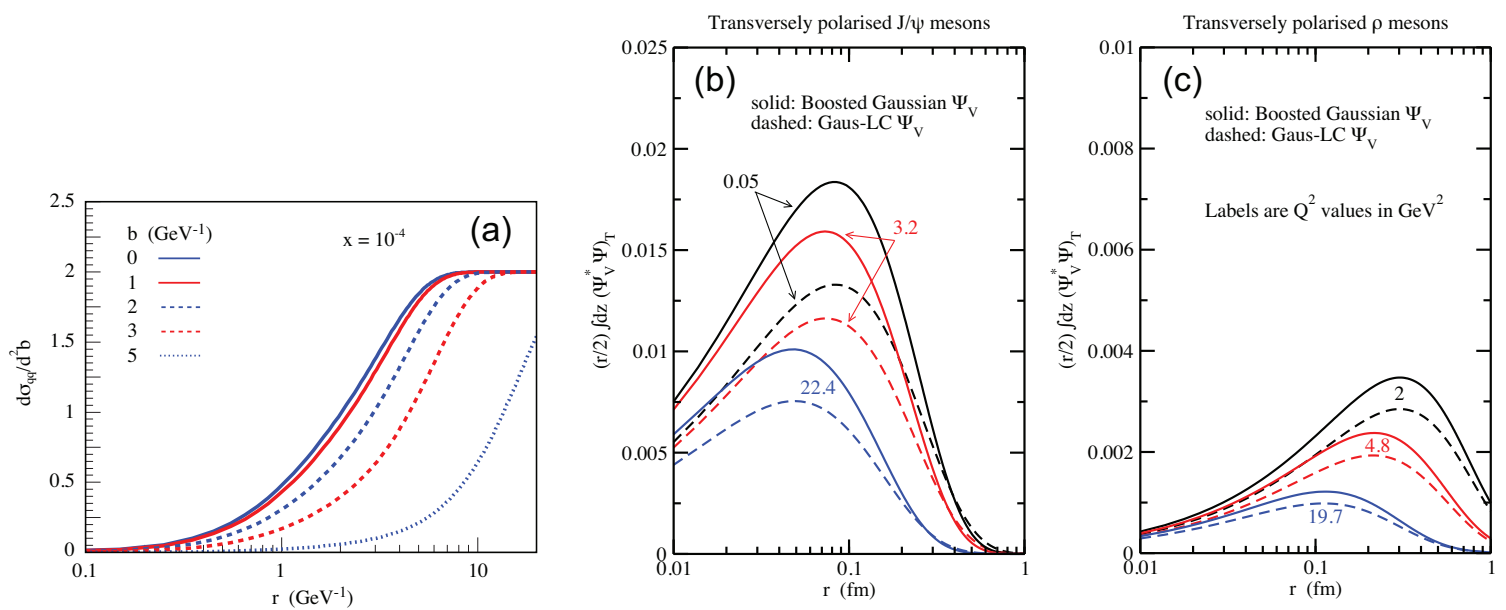

Figure 5.87. (a) shows the dipole cross-section for various impact parameters as a function of dipole size in the bSat model. (b) and (c) depict the wave overlap functions for $J / \Psi$ and $\rho$ mesons respectively as a function of $r$ for various $Q^{2}$ for transversely polarized photons (from 823 ).

(see Fig. 3.14). The amplitude for this process is

$$
\mathcal{A}_{T, L}^{\gamma^{*} p \rightarrow V p}(x, Q, \boldsymbol{\Delta})=i \int \mathrm{d}^{2} \mathbf{r} \int \frac{\mathrm{d} z}{4 \pi} \int \mathrm{d}^{2} \mathbf{b}\left(\Psi_{V}^{*} \Psi\right)(r, z) e^{-i \mathbf{b} \cdot \Delta} \frac{\mathrm{d} \sigma_{q \bar{q}}^{(p)}}{\mathrm{d}^{2} \mathbf{b}}(x, r, \mathbf{b})
$$

Here $T$ and $L$ represent the transverse and longitudinal polarizations of the virtual photon, $r$ is the size of the dipole, $z$ the energy fraction of the photon taken by the quark, $\Delta=\sqrt{-t}$ is the transverse part of the four-momentum difference of the outgoing and incoming proton, and $\mathbf{b}$ is the impact parameter of the dipole. The wave function of the produced vector meson or real photon is $\Psi_{V}$ while that of the incoming photon that splits into the dipole is $\Psi$.

In the bSat model the dipole cross-section in terms of the dipole scattering amplitude $\mathcal{N}^{(p)}(x, r, \mathbf{b})$ is

$$
\frac{\mathrm{d} \sigma_{q \bar{q}}^{(p)}}{\mathrm{d}^{2} \mathbf{b}} \equiv 2 \mathcal{N}^{(p)}(x, r, \mathbf{b})=2\left[1-\exp \left(-\frac{\pi^{2}}{2 N_{C}} r^{2} \alpha_{S}\left(\mu^{2}\right) x g\left(x, \mu^{2}\right) T(b)\right)\right],
$$

where $\mu^{2}=4 / r^{2}+\mu_{0}^{2}$ and $\mu_{0}^{2}$ is a cut-off scale in the DGLAP evolution of the gluons $g\left(x, \mu^{2}\right)$. The nucleon shape function $T^{(p)}(b)=1 /\left(2 \pi B_{G}\right) \exp \left(-b^{2} /\left(2 B_{G}\right)\right)$. The parameter $B_{G}$ is determined through fits to HERA data 823 . We use $B_{G}=4 \mathrm{GeV}^{-2}$. It should be noted that bSat is a model of multiple two-gluon exchanges and does not contain any gluon-gluon recombinations. It is however, by construction, a model that obeys unitarity, so in this respect it is a saturation model.

Figure 5.87(a) shows the dipole cross-section as a function of $r$ for different impact parameters. Figure 5.87(b) and (c) depict the wave overlap, $\left(\Psi_{V}^{*} \Psi\right)(r, z)$, for $J / \Psi$ (b) and $\rho$ mesons (c) 823] used in Eq. 5.177. It should be noted that the $J / \psi$ is not necessarily the best suited vector meson for probing saturation effects. Studying saturation implies probing large dipole radii $r \gtrsim 2 \mathrm{GeV}^{-1}(0.4 \mathrm{fm})$. However, the wave overlap with the $J / \Psi$ vanishes almost entirely for these dipole sizes. The lighter vector mesons $\rho$ and $\phi$ certainly appear more suited in this case. Unfortunately the wave functions of the lighter vector mesons are less well known than that of the $J / \Psi$ increasing the uncertainties in model-data 
comparisons. This can be overcome in the future by improving our knowledge of the light vector meson wave functions.

Phenomenological Corrections to the Cross-Section: In the derivation of the dipole amplitude (eq. (5.177)) only the real part of the $S$-matrix is taken into account, making the amplitude purely imaginary. The real part of the amplitude can be included by multiplying the cross-section by a factor $\left(1+\beta^{2}\right)$, where $\beta$ is the ratio of real to imaginary part of the amplitude. It is calculated using

$$
\beta=\tan \left(\lambda \frac{\pi}{2}\right), \quad \text { where } \lambda \equiv \frac{\partial \ln \left(\mathcal{A}_{T, L}^{\gamma * p \rightarrow V p}(x, Q, \Delta)\right)}{\partial \ln (1 / x)}
$$

Also, the two gluons interacting in each event do not carry the same momentum fraction $x$. In the leading $\ln (1 / x)$ limit, this skewedness effect disappears, but can still be accounted for by a factor $R_{g}(\lambda)$, where $R_{g}(\lambda)=2^{2 \lambda+3} \Gamma(\lambda+5 / 2) / \Gamma(\lambda+4) / \sqrt{\pi}$.

$R_{g}$ is multiplied to the gluon distribution $x g\left(x, \mu^{2}\right)$ and $\lambda$ is defined as the derivative of $\ln \left(x g\left(x, \mu^{2}\right)\right)$ with respect to $\ln (1 / x)$. It should be noted that while the correction of the real part of the amplitude is on firm theoretical footing, the skewedness correction should be viewed as a purely phenomenological correction. Also, the correction variable $\lambda$ is only well behaving for small values of $x<10^{-2}$. The combined magnitude of both corrections is $x$ dependent and is typically of the order of $10-60 \%$.

Extending the Dipole Model from $e+p$ to $e+\mathbf{A}$ : When going from $+e p$ to $e+\mathbf{A}$ scattering we will use the independent scattering approximation (see also eq. (5.95)),

$$
1-\mathcal{N}^{(A)}=\prod_{i=1}^{A}\left(1-\mathcal{N}^{(p)}\left(x, r,\left|\mathbf{b}-\mathbf{b}_{i}\right|\right)\right)
$$

where $\mathbf{b}_{i}$ is the position of each nucleon in the nucleus. Here, these positions are generated according to the Wood-Saxon potential. Combining equations (5.178) and (5.180) the bSat dipole cross-section for $e+\mathrm{A}$ becomes:

$$
\frac{\mathrm{d} \sigma_{q \bar{q}}^{(A)}}{\mathrm{d}^{2} \mathbf{b}}(x, r, \mathbf{b}, \Omega)=2\left[1-\exp \left(-\frac{\pi^{2}}{2 N_{C}} r^{2} \alpha_{S}\left(\mu^{2}\right) x g\left(x, \mu^{2}\right) \sum_{i=1}^{A} T^{(p)}\left(\mathbf{b}-\mathbf{b}_{i}\right)\right)\right]
$$

At small gluon momentum fractions, $x<10^{-2}$, the dipole interacts coherently with large volumes of the nucleus. Therefore the configuration of nucleons in the nucleus is not an observable. To obtain the total cross-section, these nucleon configurations have to be averaged over:

$$
\frac{\mathrm{d} \sigma_{\text {total }}}{\mathrm{d} t}=\frac{1}{16 \pi}\left\langle\left|\mathcal{A}\left(x, Q^{2}, t, \Omega\right)\right|^{2}\right\rangle_{\Omega}
$$

where $\Omega$ denotes nucleon configurations.

One defines two different kinds of diffractive events in $e \mathrm{~A}$ : coherent and incoherent. In incoherent diffractive processes the nucleus breaks up into two or more color neutral fragments, something not possible in diffractive $e p$. If the nucleus stays intact the diffractive processes are coherent. In the Good-Walker picture [1117] (also found in [885]) the incoherent cross-section is proportional to the variance of the amplitude with respect to the initial nucleon configurations $\Omega$ of the nucleus:

$$
\frac{\mathrm{d} \sigma_{\text {incoherent }}}{\mathrm{d} t}=\frac{1}{16 \pi}\left(\left\langle\left|\mathcal{A}\left(x, Q^{2}, t, \Omega\right)\right|^{2}\right\rangle_{\Omega}-\left|\left\langle\mathcal{A}\left(x, Q^{2}, t, \Omega\right)\right\rangle_{\Omega}\right|^{2}\right)
$$


where the first term on the R.H.S is the total cross-section and the second term is the coherent part of the cross-section.

The Generator: The Monte Carlo event generator is implemented in $\mathrm{C}++$ through a set of modular classes. A rich set of input parameters let the user select beam energy and species (A), wave function model, dipole model, kinematic range and the final state particle to study: $\rho, \pi, J / \Psi$, or $\gamma$ (DVCS). Internally, the variables $t, Q^{2}$, and $W^{2}$ are generated following a probability density function (pdf). From these three variables, the complete final state consisting of the scattered electron, the scattered proton or nucleus, and the produced vector meson or photon can be unambiguously calculated.

Generating Events for $e p$ : The variables are generated from a probability density function which for $e p$ is

$$
\operatorname{pdf}\left(Q^{2}, W^{2}, t\right)=\frac{\partial^{3} \sigma_{\text {tot }}}{\partial Q^{2} \partial W^{2} \partial t}=\frac{1}{16 \pi} \sum_{T, L} f_{T, L}^{\gamma^{*}}\left(Q^{2}, W^{2}\right)\left|\mathcal{A}_{T, L}^{\gamma^{*} p \rightarrow V p}\left(W^{2}, Q^{2}, t\right)\right|^{2}
$$

where $f_{T, L}^{\gamma^{*}}$ is the photon flux for transversely and longitudinally polarized photons. The user may also choose to include the corrections for the real part of the amplitude and/or the skewedness effect as described above.

Generating Events for $e \mathbf{A}$, the MC-Glauber Approach: For $e$ A the pdf is

$$
\frac{\partial^{3} \sigma_{\text {total }}}{\partial Q^{2} \partial W^{2} \partial t}\left(Q^{2}, W^{2}, t\right)=\frac{1}{16 \pi} \sum_{T, L} f_{T, L}^{\gamma^{*}}\left(Q^{2}, W^{2}\right)\left\langle\left|\mathcal{A}_{T, L}^{\gamma^{*} A \rightarrow V A}\left(Q^{2}, W^{2}, t, \Omega\right)\right|^{2}\right\rangle_{\Omega}
$$

Here the average of an observable $\mathcal{O}$ with respect to the initial nucleon configurations $\Omega$ is defined as $\langle\mathcal{O}\rangle_{\Omega} \equiv \frac{1}{C_{\max }} \sum_{j=1}^{C_{\max }} \mathcal{O}\left(\Omega_{j}\right)$, where a number of $C_{\max }$ configurations $\Omega_{j}$ are generated and summed over. This sum will converge to the true average for large $C_{\max }$. We call this way of performing the average the MC-Glauber approach. It should be noticed that this method of averaging the initial nucleon configurations is different than in previous publications, e.g. in [826] and 885].

For each event, the coherent part of the cross-section is calculated simultaneously with the total cross-section, by averaging the amplitude before squaring it. It is then decided probabilistically that the nucleus breaks up if

$$
\left(\frac{\partial^{3} \sigma_{\text {total }}}{\partial Q^{2} \partial W^{2} \partial t}-\frac{\partial^{3} \sigma_{\text {coherent }}}{\partial Q^{2} \partial W^{2} \partial t}\right) / \frac{\partial^{3} \sigma_{\text {total }}}{\partial Q^{2} \partial W^{2} \partial t}>R
$$

where $R$ is a random number from a uniform distribution on $[0-1]$. When this happens, the final state does not contain a scattered nucleus but rather the decay products resulting from the break-up of the nucleus.

Generating Events for $e \mathbf{A}$, the Optical Approach: A simpler and faster way of doing the average over the initial nucleon configurations is what we call the optical approach. Here the average is done implicitly in the dipole cross-section which becomes [826]

$$
\left\langle\frac{\mathrm{d} \sigma_{q \bar{q}}^{A}}{\mathrm{~d}^{2} \mathbf{b}}\right\rangle_{\Omega, \text { Optical }}=2\left[1-\left(1-\frac{T_{A}(\mathbf{b})}{2} \sigma_{q \bar{q}}(x, r)\right)^{A}\right] .
$$

For processing speed reasons we approximate the integrated dipole cross-section using the GBW model [819]:

$$
\sigma_{q \bar{q}}^{\mathrm{GBW}}(x, r)=\sigma_{0}\left(1-\exp \left(-\frac{r^{2} Q_{s}^{2}(x)}{4}\right)\right)
$$



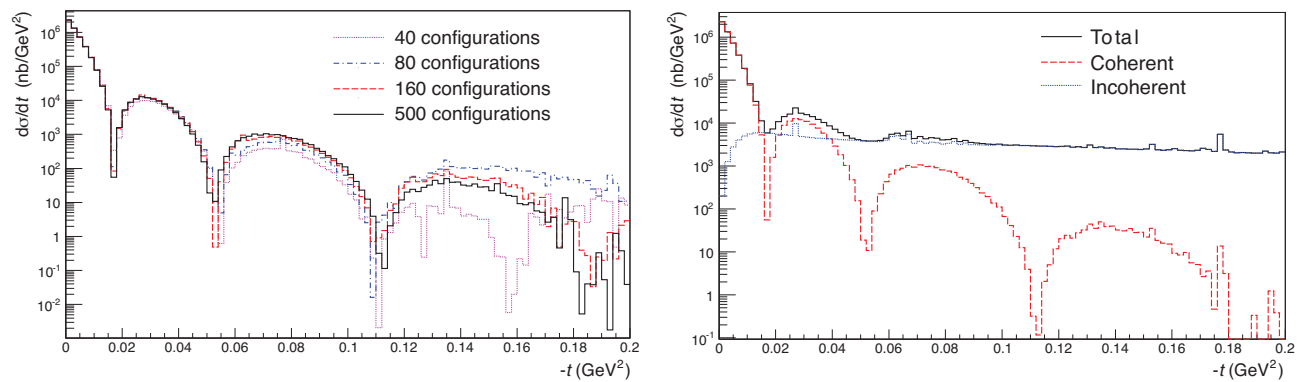

Figure 5.88. Left plot. The coherent part of the cross-section as a function of $|t|$ for electron-gold scattering at $Q^{2}=10^{-4} \mathrm{GeV}^{2}$ and $x_{p}=0.006$ averaged over 40, 80, 160 and 500 configurations respectively. Right plot. The total, coherent, and incoherent cross-sections as a function of $|t|$ for $e$ Au scattering at $Q^{2}=10^{-4} \mathrm{GeV}^{2}$ and $x_{p}=0.006$ averaged over 500 configurations.

where $Q_{s}^{2}(x)=\left(x_{0} / x\right)^{\lambda}$. Here, $\sigma_{0}=23.9 \mathrm{mb}, \lambda=0.287$ and $x_{0}=1.1 \cdot 10^{-4}\left[823\right.$. $T_{A}$ is the projection of the Woods-Saxon potential in the transverse plane. This approximation is valid for large nuclei. In the optical approach, only the coherent part of the cross-section can be calculated, since it gives the average of the amplitude, but not of the amplitude squared. It is implemented in the program as a fast alternative to the more accurate but CPU-time intensive MC-Glauber approach.

Results: In the following we only show results from the $e+\mathrm{A}$ part of the generator. In Figure 5.88, the coherent part of the crosssection for $e+\mathrm{A} \Rightarrow e^{\prime}+\mathrm{A}^{\prime}+J / \Psi$ is shown as a function of $|t|$, with $Q^{2}=10^{-4} \mathrm{GeV}^{2}$ and $x_{p}=0.006$. The nucleus used is gold with $\mathrm{A}=197$. The cross-section is calculated for different numbers of averaged nucleon configurations $C_{\max }$. The target is probed by the dipole at a scale $\Delta$ which means that at large $|t|$ the cross-section is much more sensitive to smaller variations in the positions of the nucleons than it is for small $|t|$. Therefore, for small $|t|$, the sum over configurations converges quickly, while for larger $|t|$, more configurations are needed for the sum to converge. As indicated in Fig. 5.88 approximately 100 configurations are needed to describe $e$ A scatterings up to $|t| \approx 0.2 \mathrm{GeV}^{2}$. In Figure 5.88 the total

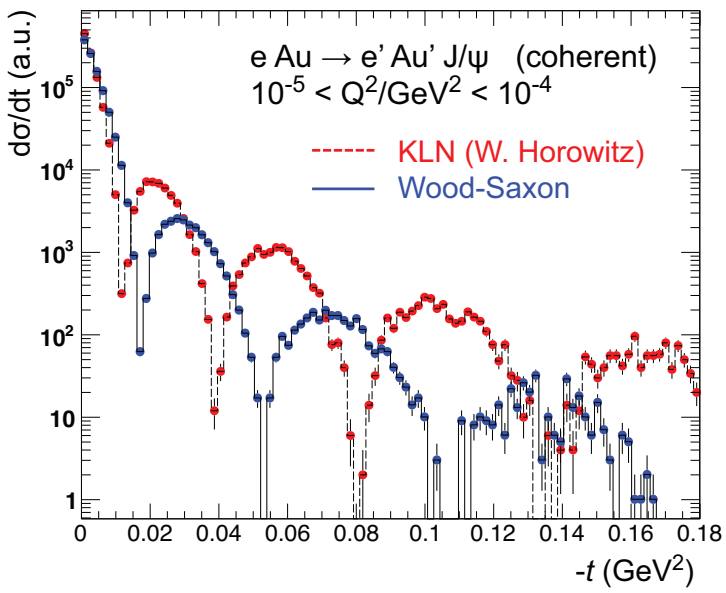
cross-section and the incoherent part of the cross-section are shown as averaged over 500 nuclear configurations. The $t$-slope of the incoherent cross-section is close to $6 \mathrm{GeV}^{-2}$. This is a bit steeper than is found in [827, where the impact parameter dependence was factorized out in the dipole cross-section and therefore the $t$-slope $=B_{G}=4 \mathrm{GeV}^{-2}$.

In order to measure the spatial distribution of gluons inside the nucleus, the coherent cross-section has to be well measured as a function of $t$. The inverse Fourier transformation 
of this will then give the transverse spatial dependence of the amplitude. To do this the position of the several coherent maxima in the $t$-distribution have to be measured accurately.

Experimentally, this requires the suppression of the large incoherent fraction, which is of course also of great interest in itself [825]. Coherent and incoherent processes can be separated by detecting the nuclear-breakup, i.e., detecting the nuclear fragments. While this is experimentally straight forward in fixed target experiments it is rather challenging at an EIC since the charged fragments are transported along the ion beam line. The most promising approach is the measurement of emitted neutrons via zero-degree-calorimeters, a technique used extensively at RHIC. Preliminary studies using de-excitation models (e.g. Gemini++ [1119] and SMM [1120]) and a realistic layout of an EIC interaction region showed that rejection factors of larger than $10^{5}$ can be achieved.

In Fig. 5.88, the nucleon configurations have been explicitly generated according to the Woods-Saxon configuration. Fig. 5.89 shows the same Woods-Saxon distribution in the optical approach compared with a KLN distribution motivated by the CGC as discussed in [1118. It can be seen that the difference when using different initial nucleon distributions within the nucleus is considerable and easily measurable by an EIC. It also demonstrates the flexibility of the generator in adapting different models at all stages of the generation process.

Summary and Outlook: A new event generator for the generation of diffractive events in $e p$ and $e \mathrm{~A}$ collisions has been implemented based on an impact parameter dependent dipole model. It describes the coherent and incoherent contributions to the cross-sections. In its current version it is limited to the production of exclusive vector mesons and DVCS photons. We intend to include more general diffractive processes in the same framework, $e+p / \mathrm{A} \rightarrow e^{\prime}+p^{\prime} / \mathrm{A}^{\prime}+X$ where $X$ is a general final state consisting of two or more hadrons. When completed it will be the first diffractive event generator for $e \mathrm{~A}$ collisions with a broad range of processes relevant for the physics of a future EIC.

Acknowledgments: The authors would like to thank the INT for their hospitality and support. Also many thanks to G. Beuf, M. Diehl, A. Dumitru, W. Horowitz, H. Kowalski, T. Lappi, and R. Venugopalan for many helpful discussions.

\subsubsection{Parton propagation and hadronization simulations: overview}

\section{Alberto Accardi}

The "Parton Propagation and Fragmentation" working group is currently working on several Monte Carlo simulations to address hadronization in the cold nuclear medium. More information, references and links are available on the PPF working group wiki [1121].

- PyQM. The "Pythia Quenching Model" is an energy-loss simulation based on Pythia, see Section 5.14.3. The partons created in the hard scattering are allowed to lose energy according to the Salgado-Wiedemann quenching weights, and then fed into the Lund string fragmentation Pythia module. The goal is to determine if the Lund string fragmentation leads to observable differences compared to using Fragmentation Functions to describe leading hadron attenuation (as implemented e.g. in PQM, see below), and to provide a simulation for a broader range of hadron flavors.

- Q-Pythia extension to DIS. Q-Pythia is an energy loss simulation by Armesto, Cunqueiro and Salgado based on medium-modified DGLAP evolution equations. Cur- 
rently, only energy loss in the QGP is implemented, and we are working on implementing energy loss in the cold nuclear target. Pursuing this simulation is likely to have a very big pay-off: it will allow to study jet nuclear modifications, the effects of medium modified DGLAP evolution on hadron observables, and compare this to the BDMPS energy loss formalism in the integrated PQM simulation, and the implementation of the Higher-Twist energy loss formalism. Comparison to simulations done with Q-Herwig, would also allow one to gauge the effects of cluster vs. Lund string hadronization.

- PQM. The "Parton Quenching Model" is a simulation by Dainese, Loizides and Paic, which uses Pythia as a parton level generator, and then applies the SalgadoWiedemann quenching weights to determine the parton energy loss before using Fragmentation Functions to determine single hadron attenuation. It has been integrated in Q-Pythia by C. Loizides.

- PyQM integration. It will be interesting to integrate PyQM in Q-Pythia, to provide a direct comparison between hadronization performed according to the Lund string model and using Fragmentation Functions.

- Higher-Twist energy loss. The Higher-Twist energy loss formalism has recently been extended to include a resummation of all higher-twist contributions, and inmplemented in a Monte-Carlo simulation, see Section 5.11,

- GiBUU. This is (among other things) a simulation of nuclear modifications of hadron production in DIS based on the Lund string model and BUU coupled-channel transport equation for the (pre)hadrons, and completely neglects energy loss, see Section 5.10. It has been extensively tested on HERMES and EMC data, and is ready to use at the EIC energy. It will be interesting to implement the few variations in the space-time prehadron production schemes available on the market and investigate possible observable differences. Inclusion of target fragmentation is currently in progress in the multi-fragmentation framework (SMM) [1089] and correcting for effects of the large energy gap between initial interaction and fragmenting nucleons.

\subsubsection{PyQM: a pure energy loss Monte-Carlo simulation}

\section{Raphaël Dupré and Alberto Accardi}

Pure quark energy loss models are widely used to describe jet quenching in relativistic heavy ion collisions (RHIC), however most of the calculations were never applied to the nDIS experiments, which are usually at lower energy, making any comparison difficult. EIC is the chance to have data of both processes at similar kinematic, in this context it is natural to develop PyQM, a pure energy-loss Monte-Carlo simulation for nDIS based on the Salgado-Wiedemann quenching weights formalism [1122] widely used to analyze RHIC data. This simulation will be utilized as a tool to evaluate the future EIC capabilities concerning quark energy loss measurement; since it also provides rate estimates and the kinematics of particles to detect, this information will be used to discuss the relevance and interest of various observables and the accelerator and detector requirements to access them.

The PyQM Monte-Carlo simulation is based on PYTHIA [81] for the DIS interaction and the fragmentation process, which is described by the Lund string model. Between the intial hard scattering and string fragmentation, we apply quark energy loss on the struck 
parton, using a nuclear density profile 1123 to estimate the quantity of matter the quark has to go through, and the Salgado-Wiedmann quenching weights [1124] to calculate the energy loss itself. To account for the geometry of the nuclei, we follow Ref. [1125], and pick randomly the interaction point according to the nuclear density distribution; the thickness of the nuclear matter seen by the parton is then given by $R=\frac{2 \bar{\omega}_{C}^{2}(\vec{b}, y)}{\int_{y}^{\infty} d z \hat{q}_{A}(\vec{b}, z)}$ with $y$ the position along the propagation direction with its origin at the interaction point, and $\vec{b}$ the transverse position of the $y$ axis relative to the center of the nucleus. The characteristic energy $\omega_{C}$, and the local transport coefficient $\hat{q}$ are given by

$$
\omega_{C}(\vec{b}, y)=\int_{y}^{\infty} d z(z-y) \hat{q}_{A}(\vec{b}, z) \quad \hat{q}_{A}(\vec{b}, y)=\frac{\hat{q}_{0}}{\rho_{0}} \rho_{A}(\vec{b}, y)
$$

Then the only free parameter for the quenching weights, and indeed for the whole simulation, is $\hat{q}_{0}$, the transport coefficient at the center of the nucleus. This is found to be $\hat{q}_{0}=0.6 \mathrm{GeV}^{2}$ $\mathrm{fm}^{-1}$ from a fit of the HERMES data [1029] (figure 5.90), in agreement with the analytic calculations of [1125, 1010]. A full description of the results of this simulation compared to HERMES would be beyond the scope of this presentation; here we note that its results are satisfactory for the multiplicity ratio, but require a seemingly too large $\hat{q}$ compared to HERMES data on pion $p_{T}$-broadening. We are currently working on an implementation of $p_{T}$-broadening in our simulation, which, puzzingly, appears instead to produce the right amount of integrated $p_{T}$ broadening as a function of $A$. This issue is directly linked to the quenching weight calculation and work is in progress to better understand it.

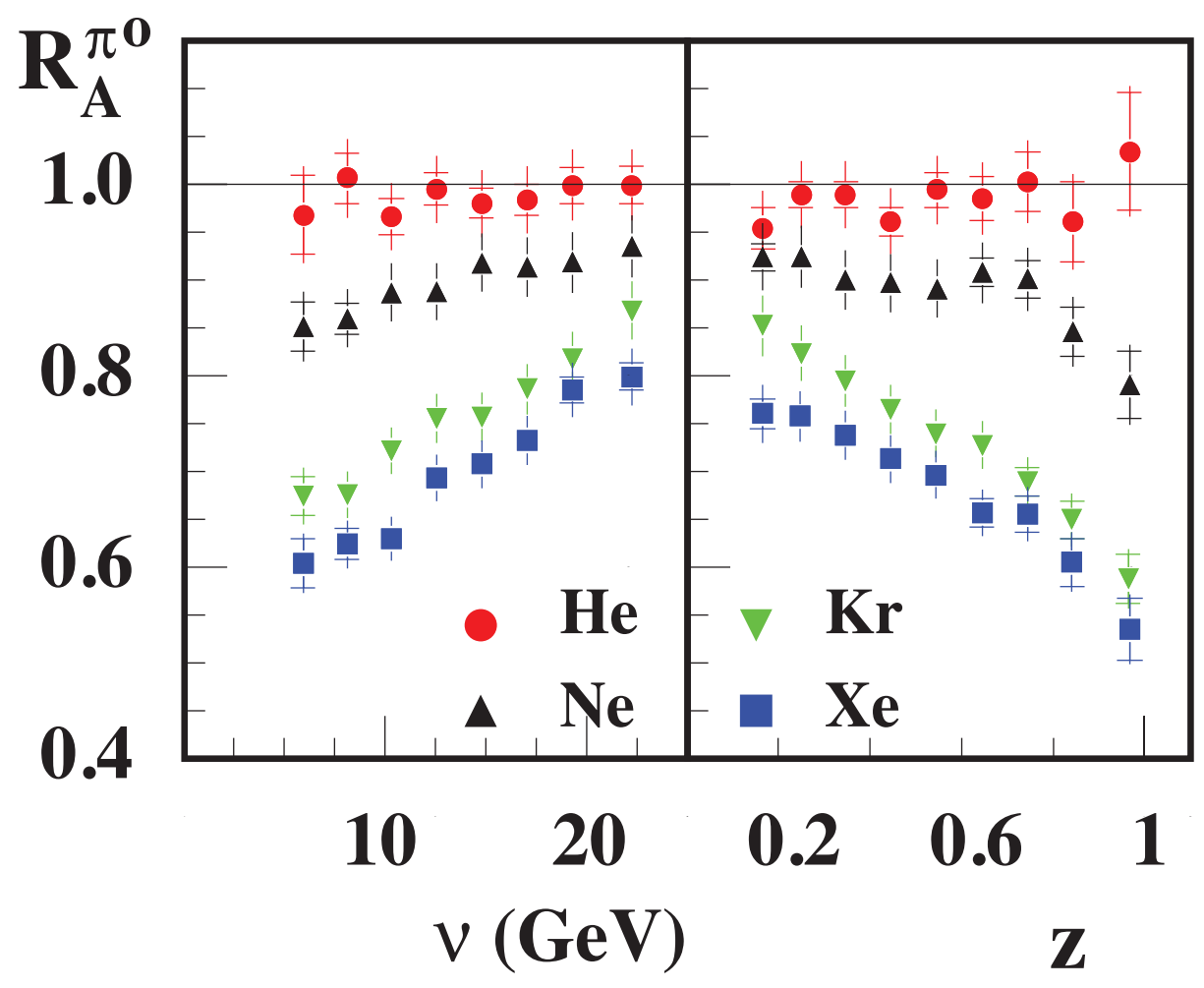

Figure 5.90. Multiplicity ratio of positive pions from HERMES[1029] (points) compared to the PyQM pure energy loss simulation (lines). 


\title{
5.15 Connections to other fields
}

\subsubsection{Gluon Tomography in Nuclei - The Heavy Ion Collision Initial State}

\author{
William A. Horowitz
}

The main purpose of colliding large nuclei is the creation and study of the quark-gluon plasma (QGP), the deconfined state of QCD matter at high temperatures $(T \sim 200 \mathrm{MeV})$ and low baryon chemical potential $(\mu \sim 0)$. Measuring the properties of the QGP is interesting as it is a known phase of the strong force, one of the four known forces in Nature. The QGP is fascinating from a theoretical standpoint as there exists the possibility of experimentally measuring the emergent many-body properties of the non-linear, nonAbelian QCD field theory. It was hoped that the collision of large, ultra-relativistic nuclei in a heavy-ion collision (HIC) might provide an experimental window with which to observe the properties of the QGP, and it appears that just such a novel phase of matter has been created at RHIC [1126, 1127, 1128, 1129, 1130].

But what are the properties of this QGP that has been created? Qualitatively: is the medium strongly or weakly coupled; what are its relevant degrees of freedom; does viscous relativistic hydrodynamics describe the bulk physics of the QGP; does either pQCD or the phenomenological string theory methods of the AdS/CFT correspondence or neither describe the physics of either the bulk medium or the high momentum probes of the medium? Quantitatively: what is the viscosity of the medium; what are the values of its transport coefficients? Is the QGP at RHIC the most perfect fluid created by mankind? The difficulty faced when trying to answer these questions is that a heavy-ion collision is an incredibly complex event. It is useful to think about a HIC, as currently best understood, as a series of separate stages: 1) $t=-\infty$, the time before overlap, when the nuclei are boosted to 200 GeV per nucleon at RHIC; 2) $t=0$, the actual collision of the nuclei and creation of large chromodynamic fields; 3 ) $0 \lesssim t \lesssim 1 \mathrm{fm} / \mathrm{c}$, the initial large chromodynamic fields rapidly thermalize; 4) $1 \lesssim t \lesssim 3 \mathrm{fm} / \mathrm{c}$, evolution as a QGP; 5) $3 \lesssim t \lesssim 4 \mathrm{fm} / \mathrm{c}$, hadronization; 6) 4 $\mathrm{fm} / \mathrm{c} \lesssim t \rightarrow \infty$, evolution as a hadron gas. A cartoon of a typical central heavy ion collision is shown in figure 5.91 .

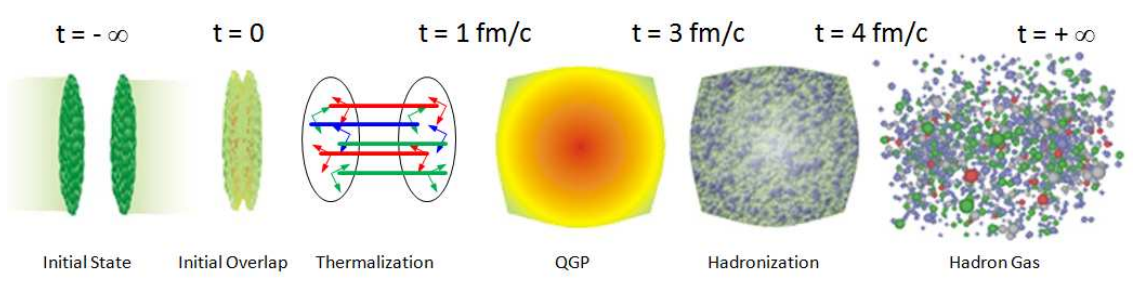

Figure 5.91. Cartoon of the stages of a heavy ion collision. Timescales are approximate.

As one can see, the system is in the QGP phase for only a brief period of its entire spacetime evolution! These times are important to understand not only because they are interesting in their own right-What are the non-linear evolution effects on the color charge density of highly boosted nuclei? How do very large chromodynamic fields thermalize so rapidly? How does hadronization occur? - but also because the interpretation of experimental observables associated with the QGP is sensitive to the details of the physics of the other stages of a HIC. Any new means of experimentally extending our understanding of these other stages would provide a qualitative leap forward in our understanding of the 


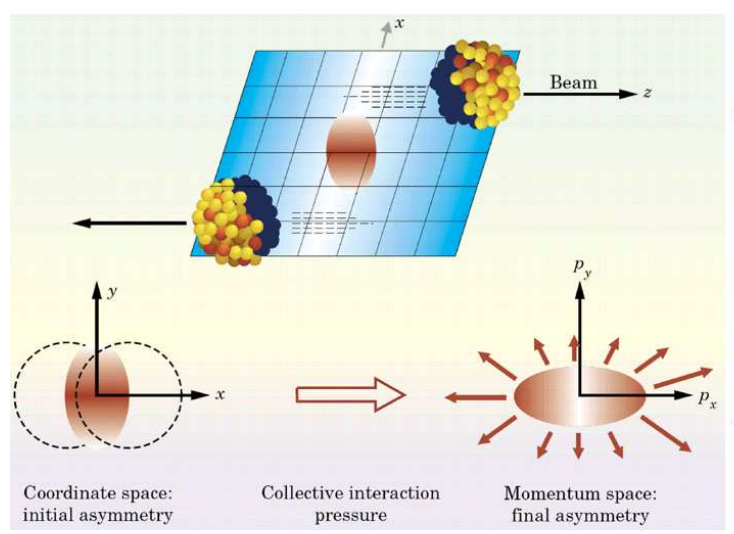

(a)
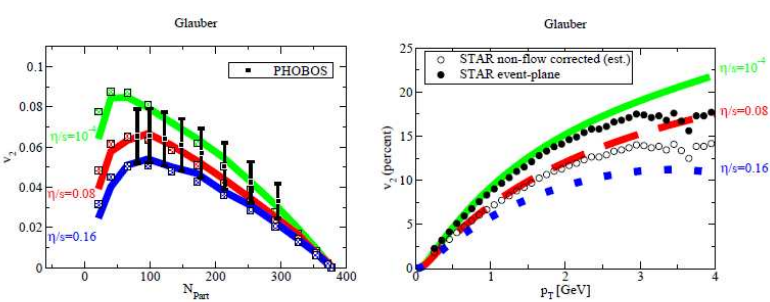

$\operatorname{cGc}$
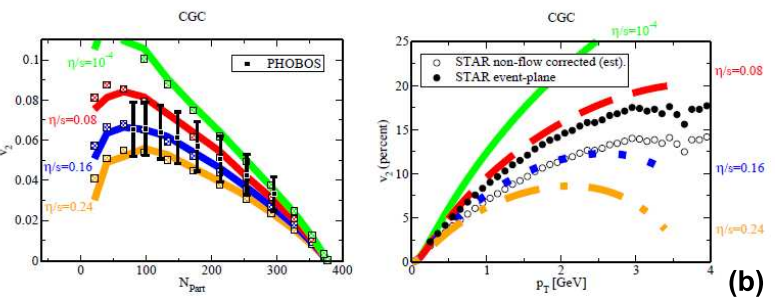

Figure 5.92. (a) Initial spatial anisotropy evolves into momentum anisotropy in non-central heavy ion collisions. Hydrodynamics aims to quantitatively model this process to gain information on the medium and its properties. (b) Comparison of data and theoretical predictions using viscous relativistic hydrodynamics for $v_{2}^{h}\left(N_{\text {part }}\right)$ (left) and $v_{2}^{h}\left(p_{T}\right)$ (right). Viscous hydrodynamics predictions use Glauber-like initial conditions (top) or a simplified implementation of color glass condensate (CGC) physics (bottom). Note the 100\% difference in extracted $\eta / s$ from the two naive geometry models. figures adapted from [1131, 881].

QGP created at RHIC. Of particular importance are the initial conditions of a heavy-ion collision, from $t=-\infty$ to $t \sim 1 \mathrm{fm} / \mathrm{c}$, from the time before the collision up through thermalization. An electron-ion collider that probes gluons at $x \sim 10^{-3}$ could provide precisely this qualitatively new physics understanding of the initial conditions.

The two most striking discoveries of the RHIC heavy-ion program so far are perfect fluidity and jet suppression. The naive interpretation of the measured flow of low momentum particles is that the QGP is a strongly coupled fluid whose properties are described by $\mathrm{AdS} / \mathrm{CFT}$; the naive interpretation of the measured jet suppression is that the QGP is a weakly coupled plasma whose properties are described by pQCD. These two interpretations are both mutually exclusive and highly dependent upon the initial conditions of HIC.

Hydrodynamics. The stunning success of ideal relativistic hydrodynamics at RHIC as compared to its failure in lower energy machines [1132, 1133, 880, led to the proclamation of the creation of a perfect fluid at RHIC [1134, 1135, 1136]. In HIC particle spectra are often conveniently reported as

$$
\frac{d N^{h}}{d p_{T}}\left(p_{T}, \phi, N_{\text {part }}\right)=\frac{d N^{h}}{d p_{T}}\left(p_{T}, N_{\text {part }}\right)\left(1+2 v_{2}^{h}\left(p_{T}, N_{\text {part }}\right) \cos 2 \phi+\ldots\right),
$$

where $\phi$ is the angle of the observed particle with respect to the semiminor axis of the overlap region; see figure 5.92 (a). As pictured in figure 5.92 (a) the $v_{2}^{h}$ develops from pressure gradients that build up as a result of the spatial anisotropy created in the initial overlap of the two nuclei.

The nearly ideal fluid flow as surmised from hydrodynamics is exciting because the extracted value of $\eta / s$, the shear viscosity to entropy ratio, is smaller than for any other known substance [1137. From a theoretical standpoint, this nearly ideal flow is a huge success for string phenomenology: the lower bound for $\eta / s$ in a strongly-coupled liquid as computed using the AdS/CFT correspondence is $1 / 4 \pi$, in natural units. This value of $1 / 4 \pi \simeq 0.1$ should be compared to the naive estimate from $\mathrm{pQCD}, \eta / s \sim 1$. Conservative 

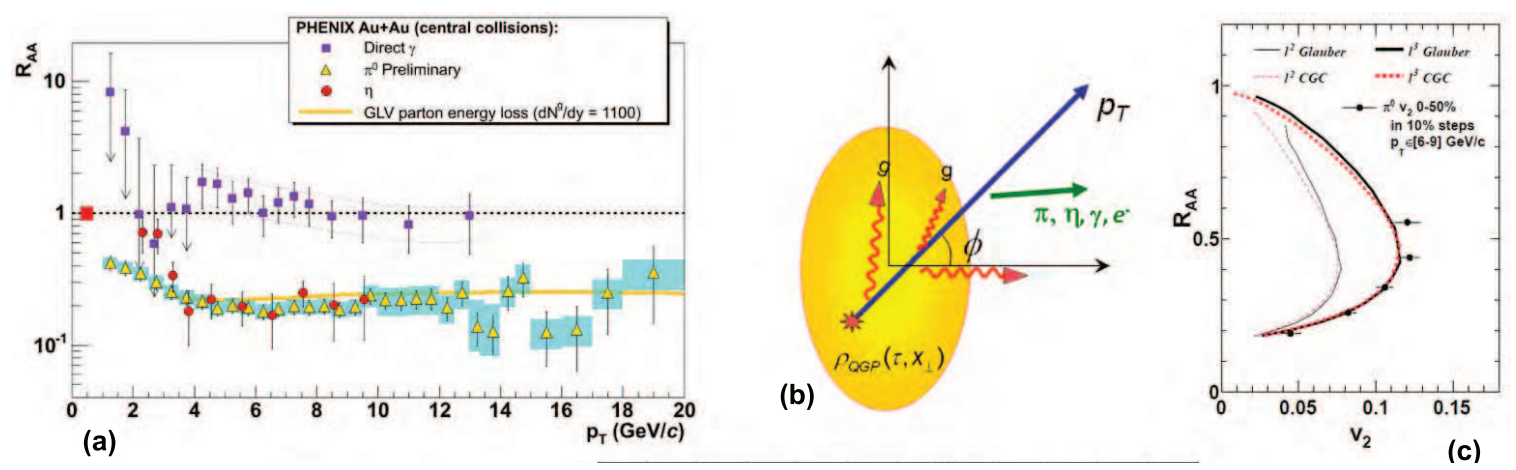

Figure 5.93. (a) A plot of the early success of pQCD energy loss calculations in describing $R_{A A}\left(p_{T}\right)$, Eq. 5.191 (b) Cartoon of the energy loss from a high- $p_{T}$ parton in the QGP medium. The longer the pathlength $L$ the greater the energy loss: the spatial anisotropy manifests as a suppression anisotropy, which is represented by $v_{2}^{h}$. (c) pQCD $\left(\Delta E \sim L^{2}\right)$ energy loss significantly underpredicts the anisotropy while AdS/CFT $\left(\Delta E \sim L^{3}\right)$ loss is consistent. The simultaneous description of $R_{A A}$ and $v_{2}$ seems to require both $L^{3}$ energy loss and a CGC-like initial state. figures adapted from 1140, 884.

estimates of the extracted value of $\eta / s$ from comparison between theoretical calculations and experimental data yield $\eta / s \sim 0.1-0.5$ [1137]. Hydrodynamics is a set of partial differential equations: initial conditions, for which hydrodynamics can tell us nothing, must be supplied. Figure 5.92 (b), in which a $2+1 D$ viscous hydrodynamics calculation is compared with data, shows the at least factor of 2 uncertainty in the extracted value of $\eta / s$ that arises from the poorly constrained mean value of the initial geometry. The uncertainty from fluctuations [1138], in which hot and cold spots appear in the initial conditions, might also be very large [1139. This very large range of $\eta / s$ means that one cannot definitively claim that the medium is better understood as strongly coupled and near the lower bound set by AdS/CFT or weakly coupled, with pQCD providing a good physical description.

High-pT Physics. Originally, high- $p_{T}$ particles were hoped to provide a tomographic probe of the QGP medium produced at RHIC. Jet tomography, then, would provide a means, independent of hydrodynamics, for determining many medium properties; most important, jet tomography could be a tool to investigate the initial geometry of the HIC. While early work showed great promise, see figure 5.93 (a), there are several observables for which the perturbative energy loss calculations do not provide a good description of the data (see, e.g., figure 5.93(c)). There is currently not much theoretical control over the inmedium energy loss experienced by high- $p_{T}$ partons: different assumptions about the best physics approximations have yielded very different energy loss calculations (see, e.g., [1141, [1011]), and all these calculations suffer from large, mostly unquantified uncertainties due to simplifying mathematical approximations [1142]. Nevertheless, qualitatively fascinating discoveries can be made from high- $p_{T}$ observables. In particular, one may compare the results of strong coupling calculations derived using the AdS/CFT correspondence to those derived using traditional pQCD methods; in this way, energy loss holds out the possibility of rigorously investigating, independent of hydrodynamics, whether RHIC creates a stronglycoupled perfect fluid or a weakly-coupled plasma.

High-energy particle spectra are often reported as normalized by the $p+p$ spectrum multiplied by $N_{\text {coll }}\left(N_{\text {part }}\right)$, where $N_{\text {coll }}\left(N_{\text {part }}\right)$ is the expected number of $p+p$-like hard 
collisions in an $A+A$ collision with a given number of participants:

$$
R_{A A}^{h}\left(p_{T}, \phi, N_{\text {part }}\right)=\frac{d N_{A A}^{h}}{d p_{T}}\left(p_{T}, \phi\right) / N_{\text {coll }}\left(N_{\text {part }}\right) \frac{d N_{p p}^{h}}{d p_{T}}\left(p_{T}\right),
$$

where $h$ is the measured hadron species and $\phi$ is the same angle as was defined in the discussion of hydrodynamics. This ratio is also often reported as a Fourier expansion, with $v_{2}^{h}$ again representing twice the first Fourier coefficient (the same $v_{2}^{h}$ as in hydrodynamics). However the physical understanding of the origin of the high- $p_{T} v_{2}^{h}$ is very different from the hydrodynamics physics which dominates the generation of the low- $p_{T} v_{2}^{h}$. For high$p_{T}$ observables, $v_{2}^{h}$ comes from high- $p_{T}$ partons traversing a medium asymmetric from the initial geometry: less energy loss occurs for partons traveling the short direction of the almond-shaped overlap region compared to those partons that travel the long direction. A cartoon of this physical picture is shown in figure 5.93 (b). The size of $v_{2}^{h}$ is then an entangled measure of the geometry of the medium and the pathlength dependence of the energy loss mechanism: perturbative elastic energy loss, which goes as $L^{1}$, produces less $v_{2}^{h}$ for a given geometry than perturbative inelastic energy loss, which goes as $L^{2}$, which produces less $v_{2}^{h}$ than strong-coupling energy loss, which, for light partons, goes as $L^{3}$ and as $\exp (-L)$ for heavy partons. $v_{2}^{h}$ is of particular interest because it was recently measured out to $p_{T} \sim 13 \mathrm{GeV}$, well beyond momentum scales where hadronization effects might be important. That the observed $v_{2}^{h}$ is significantly larger than that predicted by perturbative methods, shown in figure 5.93 (c), is perhaps the best high- $p_{T}$ experimental evidence that $\mathrm{AdS} / \mathrm{CFT}$, as opposed to $\mathrm{pQCD}$, is the best approximation to the relevant physics at RHIC.

As the theoretical prediction of high- $p_{T} v_{2}^{h}$ comes directly from the azimuthal anisotropy of the QGP medium, knowledge and constraint of the initial geometry is crucially important for a rigorous scientific conclusion to be made: the sharper the produced medium the larger the $v_{2}^{h}$, regardless of energy loss mechanism. As one can see from figure 5.93, there are reasonable initial conditions for which no known energy loss calculation describes the data. And just as in hydrodynamics, fluctuations may play an important, even outsized, role.

Measuring the Initial State. From the above discussion it is clear that knowledge of the initial conditions at RHIC is crucial for interpreting the experimental data. The density of the charged and neutral matter density of nuclei at rest is well understood from diffraction pattern experiments (see, e.g., [1143]). Knowledge of the rest frame density of protons and neutrons in nuclei has been used extensively in estimating the initial matter density created in HIC. Matter production in HIC, though, depends on the distribution of quarks and, especially, gluons in the nuclear wavefunction. Below some value of Bjorken $x$ that is not yet precisely known, non-Abelian, non-linear QCD evolution effects become important. The (mostly) gluonic initial state medium at midrapidity at RHIC consists of particles of $x \sim p_{T} / \sqrt{ } s \sim 10^{-3}$, which is at the order of magnitude for which small- $x$ physics likely becomes relevant. Unfortunately the theory of small- $x$ physics in $A+A$ collisions is very complicated, and current knowledge is incomplete. Additionally, the aforementioned theoretical calculations of $v_{2}^{h}$ are in fact most sensitive to the the quantitative shape of the edge of the initial nuclear overlap in HIC; it is just in this region that many of the theoretical tools developed for small- $x$ physics study break down. It turns out, though, that through careful measurements, diffraction patterns may be measured at an electronion collider using deeply-virtual Compton scattering and vector meson production. These diffraction patterns, in turn, may be inverted to constrain the initial gluon and quark densities of the highly boosted nuclei. Fortuitously, these experimental measurements give 
the most sensitive determination of these densities at the edge of the nucleus, the region of the overlap which hydrodynamics and energy loss calculations are most sensitive to.

\subsubsection{Constraining initial conditions in $\mathrm{A}+\mathrm{A}$ collisions}

\section{Adrian Dumitru}

Understanding small- $x$ gluon production in the initial state of relativistic A+A collisions constrains the amount of additional entropy produced via "final-state" interactions such as parton thermalization / QGP formation [1144] and its subsequent hydrodynamic expansion. If these processes provide a significant contribution, then that should presumably show in the centrality dependence of the multiplicity in the final state: final state interactions should be much more prevalent for a head-on collision of two large nuclei than for a grazing shot or $\mathrm{p}+\mathrm{A}$ or (minimum bias) $\mathrm{p}+\mathrm{p}$ collisions. It is therefore very important to test models for initial particle production over a broad range of centralities - perhaps down to the level of $\mathrm{p}+\mathrm{p}$ collisions - in order to constrain entropy production due to thermalization and viscous hydrodynamic expansion [1145].

To compute the number of small- $x$ gluons released from the wavefunctions of the colliding nuclei, one frequently employs the $k_{\perp}$-factorization formalism [742, 1146],

$$
\begin{aligned}
& \frac{d N}{d^{2} \mathbf{r}_{\perp} d y}=\mathcal{N} \frac{N_{c}}{N_{c}^{2}-1} \int \frac{d^{2} p_{\perp}}{p_{\perp}^{2}} \int^{p_{\perp}} d^{2} k_{\perp} \alpha_{s}\left(Q^{2}\right) \\
& \quad \times \phi_{A}\left(x_{1}, \frac{\left(\mathbf{p}_{\perp}+\mathbf{k}_{\perp}\right)^{2}}{4} ; \mathbf{r}_{\perp}\right) \phi_{B}\left(x_{2}, \frac{\left(\mathbf{p}_{\perp}-\mathbf{k}_{\perp}\right)^{2}}{4} ; \mathbf{r}_{\perp}\right)
\end{aligned}
$$

where $N_{c}=3$ is the number of colors, and $p_{\perp}, y$ are the transverse momentum and the rapidity of the produced gluons, respectively. $x_{1,2}=p_{\perp} \exp ( \pm y) / \sqrt{s_{N N}}$ denote the lightcone momentum fractions of the colliding gluon ladders, $\sqrt{s_{N N}}$ is the collision energy, and typically one chooses $Q^{2}=\max \left(\left(\mathbf{p}_{\perp}+\mathbf{k}_{\perp}\right)^{2},\left(\mathbf{p}_{\perp}-\mathbf{k}_{\perp}\right)^{2}\right) / 4$. The normalization factor $\mathcal{N}$ can be fixed from peripheral collisions, where final-state interactions should be suppressed. It effectively also absorbs NLO corrections and the contribution from sea (anti-)quarks. The unintegrated gluon distribution $\phi$ is related to the dipole scattering amplitude in the adjoint representation, $N_{G}$, through a Fourier transform [1147]:

$$
\phi\left(x, k_{\perp}^{2} ; \mathbf{r}_{\perp}\right)=\frac{C_{F}}{\alpha_{s}\left(k_{\perp}\right)(2 \pi)^{3}} \int d^{2} \mathbf{s}_{\perp} e^{-i \mathbf{k}_{\perp} \cdot \mathbf{s}_{\perp}} \nabla_{\mathbf{s}_{\perp}}^{2} N_{G}\left(x, s_{\perp} ; \mathbf{r}_{\perp}\right) .
$$

The multiplicity in heavy-ion collisions. Figure 5.94 (left) shows the centrality dependence of particle production for heavy-ion collisions at $200 \mathrm{GeV}$ and $2760 \mathrm{GeV}$, respectively, obtained by integrating eq. (5.192) over the transverse overlap of the colliding nuclei. The unintegrated gluon distributions are solutions of the local (impact parameter independent) Balitsky-Kovchegov (BK) equation with running-coupling corrections according to the Balitsky prescription [751. The impact parameter dependence is due entirely to the initial condition where it has been assumed that essentially $Q_{s}^{2}\left(x_{0} ; \mathbf{r}_{\perp}\right)=Q_{0}^{2} \sigma_{0} T_{A}\left(\mathbf{r}_{\perp}\right)$ increases in proportion to the thickness of the nucleus $\left(Q_{0}\right.$ and $\sigma_{0}$ denote constant scales; for details see ref. [896]). Neglecting the impact parameter dependence of the dipole scattering amplitude $N_{G}$ in a nucleon relies on the scale separation $R_{A} \gg R_{N} \gg Q_{s}^{-1}$ where $R_{A}$ is the size of the overlap region in the collision [1150]. 

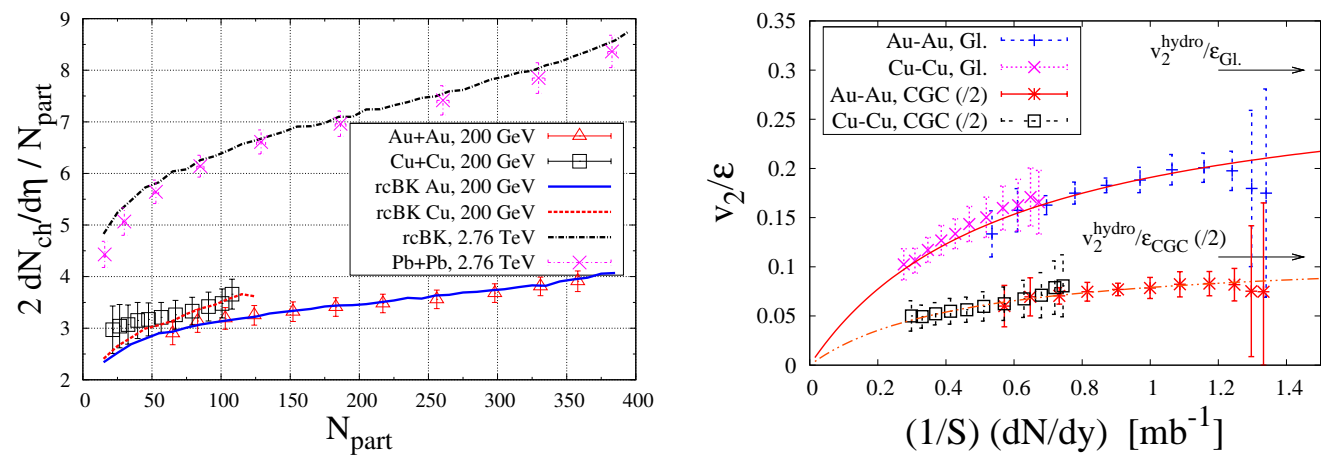

Figure 5.94. Left: Centrality dependence of the multiplicity at $200 \mathrm{GeV}$ and $2760 \mathrm{GeV}$, respectively, from $k_{\perp}$ factorization with running-coupling BK unintegrated gluon distributions (see [896] for details). PHOBOS data: 891] $(\mathrm{Au}+\mathrm{Au})$, 1148] $(\mathrm{Cu}+\mathrm{Cu})$; ALICE data from ref. [894. Right: $v_{2} / \varepsilon$ versus the transverse particle density [1149; $v_{2} / \varepsilon_{\mathrm{CGC}}$ has been scaled by $1 / 2$ for better visibility.

Apparently, the model calculation describes both the centrality and the energy dependence of particle production fairly well. If so, this constrains final-state entropy production and correlates the thermalization time and the shear viscosity to entropy density ratio: extremely rapid thermalization and/or $\eta / s \gtrsim 0.3$ would be excluded by stringent entropy production bounds [1145].

Several caveats remain. As already mentioned above, the absolute normalization of the gluon density at small $x$ (alternatively, the factor $\mathcal{N}$ in the $k_{\perp}$ factorization formula) can be fixed in practice only from very peripheral $\mathrm{A}+\mathrm{A}$ or $\mathrm{p}+\mathrm{p}$ collision 9 . For $\mathrm{p}+\mathrm{p}$ collisions, however, the impact parameter dependence of the dipole scattering amplitude over distance scales $\sim R_{N}$ can not be neglected, see for example ref. [1151].

Furthermore, it may be important to consider in more detail the structure of running coupling corrections to the $k_{\perp}$-factorization formula (5.192) [897] and the effect of a full NLO treatment of BK evolution. Indeed, if such corrections modify the centrality dependence of particle production in $\mathrm{A}+\mathrm{A}$ collisions then they will also affect entropy production constraints and thus the fundamental understanding of the thermalization processes and time scales as well as estimates of the shear viscosity of thermal QCD.

The eccentricity in heavy-ion collisions. Other quantities of relevance for the interpretation of heavy-ion collisions exhibit even greater sensitivity to the actual distribution of produced gluons in the transverse $\mathbf{r}_{\perp}$ plane than its integral $d N / d y$. A collision of two heavy ions at non-zero impact parameter, neglecting fluctuations of the local density of participant nucleons, leads to a momentum asymmetry called "elliptic flow", $v_{2} \sim\langle\cos 2 \phi\rangle$, as described in section 5.15.1. In the absence of any scales (such as the freeze-out temperature $T_{f}$, the phase transition temperature $T_{c}$, or a non-vanishing mean free path $\lambda$ ), hydrodynamics predicts that $v_{2}$ is proportional to the eccentricity $\varepsilon$ of the overlap area [1152, $\varepsilon=\left\langle y^{2}-x^{2}\right\rangle /\left\langle y^{2}+x^{2}\right\rangle$. The average is taken with respect to the distribution of produced gluons in the transverse $x-y$ plane. Clearly, $\varepsilon$ involves large cancellations of the contributions of gluons produced near the center $r_{\perp} \sim 0$ of the overlap zone and so is more sensitive to particle production in the periphery.

A simple geometry based initial condition assumes that by analogy to the Glauber model for soft particle production $d N / d y d^{2} r_{\perp} \sim \rho_{\text {part }}^{\text {ave }}\left(r_{\perp}\right) \equiv\left(\rho_{\text {part }}^{A}\left(r_{\perp}\right)+\rho_{\text {part }}^{B}\left(r_{\perp}\right)\right) / 2$, where $\rho_{\text {part }}^{i}$

\footnotetext{
${ }^{9}$ Small- $x$ partons do not contribute significantly to the momentum sum rule and a precise matching to the parton distributions at large $x$ and low $Q^{2}$ is lacking.
} 
is the density of participants of nucleus $i$ per unit transverse area. High-density QCD (the "Color-Glass Condensate") predicts a somewhat different distribution of gluons in the transverse plane, corresponding to a higher eccentricity $\varepsilon$. In particular, in the " $\mathrm{p}+\mathrm{A}$ limit" when one of the nuclei is very dense while the other is dilute, the number of produced particles is proportional only to the density of the dilute collision partner, whose partons add up linearly. Hence, in the reaction plane, $d N / d y d^{2} r_{\perp} \sim \min \left(Q_{s, A}^{2}, Q_{s, B}^{2}\right) \sim \min \left(\rho_{\text {part }}^{A}, \rho_{\text {part }}^{B}\right)$ drops more rapidly as one moves towards the edge of the overlap zone than $d N / d y d^{2} r_{\perp} \sim$ $\rho_{\text {part }}^{\text {ave }}$ 1153. Thus, a higher eccentricity is a generic effect due to a dense target or projectile. Specific numerical estimates rely on an accurate determination of the unintegrated gluon distribution, however. Ref. [896] finds that the energy dependence of $\varepsilon$ from RHIC to LHC is very weak.

Figure 5.94 (right) shows the elliptic flow $v_{2}$ measured in heavy-ion collisions at RHIC scaled by the eccentricity $\varepsilon$ of the overlap zone [1149]. As already mentioned above, in the absence of any scales such as a non-zero mean free path, $v_{2} / \varepsilon$ would be independent of the transverse density of particles. Indeed, if the $v_{2}$ data is scaled by the eccentricity obtained from a CGC model implementation then the required breaking of scale invariance is lower than for purely geometry based (Glauber-like) initial conditions. Actual solutions of viscous hydrodynamics (for $v_{2}$ ) appear to confirm this simple observation in that the slope of $v_{2} / \varepsilon$ versus transverse density is sensitive to the distribution of produced particles [1154].

More recent studies attempt to understand also the relation of higher moments of anisotropic flow $v_{n}$ to corresponding moments of the initial eccentricity $\varepsilon_{n}$ - such as the "triangularity" [1155, 1156, 1157, 883, 1158, which is non-zero because of fluctuations of the large- $x$ sources in the transverse impact parameter plane before the collision. A quantitative interpretation of the "response" $v_{n}$ of the Quark-Gluon Plasma medium to the initial geometry will also rely on a good understanding of particle production in high-energy QCD.

\subsubsection{Particle production at low- $x$ and gluon saturation: from $p+A$ to $e+A$}

\section{Kirill Tuchin}

In the beginning of the RHIC era, the $p(d)+A$ program was perceived as merely a useful baseline reference for the heavy-ion program. It very soon turned out that due to a wise choice of colliding energy, RHIC probes the transition region to a new QCD regime of gluon saturation. While the first hints of gluon saturation were observed in DIS experiments at HERA, it is fair to say that gluon saturation was discovered in $d A$ collisions at RHIC. At present, as we are heading toward the era of EIC, it is important to review what we have learned at RHIC and how it can be used to optimize the EIC program. The purpose of this section is to review phenomenological studies of gluon saturation at RHIC.

The reason why $p A$ and $e A$ high energy physics programs are closely related is provided by the Pomerantchuk theorem, which states that all high energy scattering processes are mediated by the exchange of a collective gluon state - known as a Pomeron - that has vacuum quantum numbers. For this reason, inclusive processes in both programs share many similarities in the low $x$ region. The main distinction arises from the difference in the characteristic scales of the projectile: in protons it is a soft scale $\Lambda$, while in virtual photons, it is the photon virtuality $Q^{2}$, which depends on the electron kinematics. A possibility to control the $Q^{2}$ is a great advantage of DIS. In particular, it allows one to study the total cross sections/structure functions. However, in practice, the requirement to keep $x$ low 
significantly restricts the range of $Q^{2}$ 's available for low $x$ studies.

The relation between $p A$ and $e A$ scattering at low- $x$ becomes particularly apparent in the framework of the dipole model [737]. In the dipole model, the cross section for $e A \rightarrow X$ or $p A \rightarrow X$ scattering, where $X$ is an arbitrary final state, can be represented as

$$
d \sigma_{p\left(\gamma^{*}\right)+A \rightarrow X}=\int d^{2} r_{\perp} \Phi^{p\left(\gamma^{*}\right)}\left(r_{\perp}\right) d \sigma_{\mathfrak{d}+A \rightarrow X}\left(r_{\perp}\right)
$$

where $\mathfrak{d}$ stands for color dipole (letter $d$ is reserved for deuteron) of size $r_{\perp}$ in the transverse plane. Eq. (5.194) is based on the separation of scales: the interaction length $\ell_{i} \sim R_{A}$ (in the target rest frame) is much smaller than the coherence length $\ell_{c}=\gamma / M_{N}$, where $\gamma \gg 1$ is the Lorentz factor and $M_{N}$ is the nucleon mass. $\Phi^{p\left(\gamma^{*}\right)}\left(r_{\perp}\right)$ is the light-cone "wave function" describing the Fourier decomposition of a projectile into dipoles; it can be calculated in QED (for $\gamma^{*}$ ), or modeled (for proton), see e.g. [1159, 1160]. The main theoretical concern in low $x p A / e A$ scattering is calculation of the dipole-nucleus cross section, which is universal for both processes. With this observation in mind, we are going to consider some of the $p A$ processes at RHIC that are of relevance for low- $x$ physics at EIC.

Inclusive hadron production: $p+A \rightarrow h+X$. The cornerstone for phenomenological applications of the Color Glass Condensate (CGC), which is the theory of gluon saturation, is the factorization theorem proved in [1147], where the cross section was derived that resums all leading logarithmic contributions $\alpha_{s} \ln (1 / x) \sim 1$ (LLA) for a heavy nucleus in the quasi-classical limit $\alpha_{s}^{2} A^{1 / 3} \sim 1$. A similar result was reported in [1161, 1162, 1163]. One does not expect that any of the hard perturbative QCD (hpQCD) factorizations apply in this case because higher twist interactions of valence quarks and gluons give contributions of order unity. Nevertheless, despite the fact that individual diagrams break factorization in covariant and light-cone gauges, the final re-summed expression can be cast in the $k_{T}$-factorized form. Unlike in hpQCD, the physical quantity that is factorized - the unintegrated gluon distribution $\varphi\left(x, Q^{2}\right)$ - can be calculated perturbatively owing to the existence of a hard scale $Q_{s} \gg \Lambda_{\mathrm{QCD}}$. Another surprising fact is that contrary to naive expectations, $\varphi\left(x, Q^{2}\right)$ is related not to the momentum space Fourier-image of the nucleus gluon-field correlation function $\left\langle A_{\perp}\left(0_{\perp}\right) \cdot A_{\perp}\left(x_{\perp}\right)\right\rangle$, but rather to the Fourier-image of $\nabla_{r}^{2} N\left(r_{\perp}, b_{\perp}, y\right)$, where $N\left(r_{\perp}, b_{\perp}, y\right)$ is the imaginary part of the forward elastic scattering amplitude of a color dipole of size $r_{\perp}$ at impact parameter $b_{\perp}$ and rapidity $y=\ln (1 / x)$ in the heavy nucleus. Although the inclusive gluon production in $p A$ collisions is the only known case were $k_{T}$-factorization holds, factorization of the multipoles in the transverse coordinate space is a general feature of the low- $x$ cross sections. It must be stressed that this multipole factorization does not imply hpQCD factorizations $\left(k_{T}\right.$ or collinear ones) and neither opposite is generally true.

The $k_{T}$-factorization formula derived in [1147] led to successful phenomenology of inclusive hadron production in $d A$ collisions at RHIC, where the suppression of hadrons at forward rapidities and Cronin enhancement at mid-rapidity were qualitatively predicted [1164, 1165] and then quantitatively described in the CGC framework [1166, 1167, 1168. The production of valence quarks in the forward direction gives an important contribution to inclusive hadron production at large- $x$ of the proton and was discussed in [1169, 1170, 1171].

By integrating the gluon spectrum over $p_{\perp}$, one arrives at the total hadron yield as a function of rapidity $y$. It is rather weakly dependent on the details of the gluon distributions. Therefore, a simple model suggested in [1172] is able to describe inclusive hadron yield with remarkable accuracy. 
Open charm (beauty) production: $\boldsymbol{p}+\boldsymbol{A} \rightarrow \boldsymbol{D}+\boldsymbol{X}$. The production of heavy quarks in $p A$ collisions at low- $x$ was calculated in [1173, 905, 1174]. One expects that the hpQCD factorization is applicable if the saturation momentum is much smaller than the quark mass $m$ [1175]. At RHIC, $Q_{s} \sim m$ for charm and bottom, hence factorization is broken in both cases. Indeed, analysis of [1176] indicates that semi-classical calculations of [905] disagree with $k_{T}$-factorization by about $10 \%$ at the $t$-channel gluon transverse momenta around $m$. hpQCD factorization is restored in the kinematic region where the operator product expansion is applicable, i.e. at transverse momenta much higher than the saturation momentum.

The phenomenology of open heavy quark production at RHIC was developed in [1177, where it was found that the production pattern of heavy quarks is qualitatively similar to that of light quarks and gluons, although the magnitude of nuclear effects (Cronin and suppression) slowly decrease with increasing quark mass. These qualitative features are in good agreement with preliminary data.

Inclusive production of $\boldsymbol{J} / \Psi: p+\boldsymbol{A} \rightarrow \boldsymbol{J} / \boldsymbol{\Psi}+\boldsymbol{X}$. In addition to the scales $\ell_{i}$ and $\ell_{c}$ mentioned earlier, the production of a charmonium state is characterized by another scale: formation length $\ell_{f}=\gamma / \Delta M$, where $\Delta M$ is its binding energy. The key theory observation is strong ordering of the scales at high energies: $\ell_{i} \ll \ell_{c} \ll \ell_{f}$ [977, 1178. Consequently, we can distinguish three stages of $J / \Psi$ production. (i) $g^{*} \rightarrow c \bar{c}$ described by the light-cone amplitude $\psi^{g}\left(k_{\perp}, z\right)$ often referred to as gluon's light-cone wave function, (ii) interaction of the gluon or the $c \bar{c}$ with the target depending on whether the splitting has occurred after or before the interaction, and (iii) formation of charmonium wave function. Unlike stages (i) and (ii), which can be described using perturbation theory owing to the weakness of the strong interaction at the $J / \Psi$-mass scale, stage (iii) is non-perturbative because $\Delta M \ll M$. This, however, does not preclude us from using perturbation theory for calculating the $J / \Psi$ production cross section, since the fragmentation process is independent of energy and atomic weight $\left(\ell_{f} \gg R_{A}\right)$. In other words, fragmentation happens in the vacuum long after any interaction with the target.

Thus, the problem of calculating the $J / \Psi$ production cross-section reduces to the calculation of the cross section of $\mathfrak{d}+A \rightarrow\left[c \bar{c}\left(1^{--}\right)\right]+X$ dipole-nucleus scattering. This calculation was done in [1179, 1180]. Note, that interaction depends on the quantum state of the $c \bar{c}$ pair, which must be in the $1^{--}$color singlet state. Therefore, only those higher twist contributions may be taken into account that lead to this quantum state, and which are also enhanced by $\alpha_{s}^{2} A^{1 / 3} \sim 1$. At the lowest order in $\alpha_{s}$, the projectile gluon in the proton wave-function has two interaction possibilities: (i) leading twist processes $g+g \rightarrow J / \Psi+g$, which is of order $\mathcal{O}\left(\alpha_{s}^{5} A^{1 / 3}\right)$ and (ii) higher twist process $g+g+g \rightarrow J / \psi$ (initial gluons come from different nucleons), which is of the order $\mathcal{O}\left(\alpha_{s}^{6} A^{2 / 3}\right)$. Since $\alpha_{s}^{2} A^{1 / 3} \sim 1$, the higher twist mechanism (ii) is parametrically enhanced. Notice, that this leading contribution explicitly breaks $k_{T}$-factorization as it is proportional to $x G\left(x_{1}\right)\left[x G\left(x_{2}\right)\right]^{2}$. Results reported in [1179, 1180] show strong coherence effects consistent with expectations of CGC theory.

Electromagnetic probes. The main advantage of electromagnetic probes, such as photons and dileptons, is that they are directly observable without an intermediate hadronization process, in contrast to quarks and gluons. Therefore, they are a cleaner probe of low$x$ nuclear matter. Their disadvantage is a low production rate due to the smallness of electromagnetic coupling. Prompt photon production in $p A$ collisions was considered in [1181 through the process $q A \rightarrow \gamma q X$. The production of di-leptons in a similar process $q A \rightarrow l^{+} l^{-} q X$ was addressed in [1182, 1183, 1184]. At higher energies, gluons become 
much more abundant than quarks in the central rapidity region which implies that photon (dilepton) production will go via the process $g^{*} A \rightarrow q \bar{q} X \gamma\left(l^{+} l^{-}\right)$. It is suppressed by $\alpha_{s}$ but enhanced by a positive power of energy. There have been no detailed phenomenological studies of electromagnetic probes in $p A$ collisions at RHIC.

Double inclusive hadron production and correlations. Azimuthal correlations are an important tool to investigate properties of QCD at low $x$. In [1185] it was proposed that azimuthal correlations of hadrons produced at large rapidity separation $(\Delta y \gg 1)$ may be depleted due to a quasi-classical nature of the saturated gluon fields. Unfortunately, accurate theoretical calculations in the region of large but finite $\Delta y$ are challenging as they must involve complicated NLO BFKL effects. Important progress has been made in the investigation of azimuthal correlations at smaller $\Delta y$.

It has been suggested that correlations at small $\Delta y$ in the forward direction can be effectively used to study gluon saturation [1186], where the forward direction corresponds to low- $x$ of the nucleus where saturation effects are strongest. Theory predicts that back-toback correlations are suppressed due to gluon saturation. Phenomenological models based on the CGC were suggested in [1186, 1187] and [1188] and rely on different approximations. An approach of [1186, 1187] is based on the dipole model [737] in which double inclusive gluon [1189], quark-anti-quark [1173, 905, 1174] and valence quark-gluon [1186] cross sections were calculated. Another approach [1188] is based on an approximate $k_{T}$-factorization and relies on calculating double-inclusive production based on NLO BFKL [1190, 1191].

Both models give a reasonable quantitative description of experimental data. However, in order to use azimuthal correlations to study low- $x$ physics in the most effective way, work remains to be done to reconcile the existing approaches and reduce model-dependencies in calculations. Measurements of forward azimuthal correlations in $e A$ will have a clear advantage over that in $p A$ due to much better theoretical control of the projectile current. Diffraction. One of the most sensitive probes of low- $x$ QCD is diffraction. This is because scattering in the high energy limit of QCD is mediated by the same collective gluon state (Pomeron) as the diffractive scattering. Saturation effects on diffractive processes in $p A$ collisions were investigated in [1159, 1192, 1193, 1160, 1194] where the main focus was on diffractive hadron production. (In [1195, 1196] this work was extended to DIS).

In diffraction on nuclear targets, it is important to distinguish two processes: coherent and incoherent diffraction, depending on the final state of the target. Coherent diffractive hadron production in $p A$ collisions is a process $p+A \rightarrow X+h+[L R G]+A$, where $[L R G]$ stands for Large Rapidity Gap. Coherent diffractive production exhibits a much stronger dependence on energy and atomic number than the corresponding inclusive process. Indeed, the diffractive amplitude is proportional to the square of the inelastic one. At asymptotically high energies, coherent diffractive events are expected to constitute up to a half of the total cross section, the other half being all inelastic processes. Therefore, coherent diffraction is a powerful tool for studying the low- $x$ dynamics of QCD.

In all phenomenological applications of the CGC formalism, one usually relies on the mean-field approximations in which only the lowest order Green's functions are relevant. Although corrections to the mean-field approximation, i.e. quantum fluctuations about the classical solution, are assumed to be small in $p A$ collisions at RHIC, their detailed phenomenological study is absent. An observable that is directly sensitive to quantum fluctuations is incoherent diffraction: $p+A \rightarrow X+h+[L R G]+A^{*}$, where $A^{*}$ denotes excited nucleus that subsequently decays into a system of colorless protons, neutrons and nuclei debris. Incoherent diffraction measures fluctuations of the nuclear color field. Calculations show that unlike the nuclear modification factor for coherent diffractive gluon production, 
the nuclear modification factor for incoherent diffraction is not expected to exhibit a significant rapidity and energy dependence [1194]. Therefore, the two diffractive processes can in principle be experimentally distinguished and yield unique information about low- $x$ QCD. Unfortunately, the study of diffraction in $p A$ collisions at RHIC is a virgin subject in part due to technical difficulties associated with measurements at very small forward angles.

Instead of a summary. Studying particle production in DIS at low $x$ has two main advantages: (i) one has much better theoretical understanding of the forward kinematic region owing to the weakness of the QED coupling and (ii) new kinematic regions open up for investigation depending on values of momentum scales $Q^{2}, k_{\perp}^{2}$ and $Q_{s}^{2}$, where $Q^{2}$ is photon virtuality, $Q_{s}^{2}$ is saturation scale and $k_{\perp}$ is transverse momentum of produced hadron.

\subsubsection{Small- $x$ dynamics in ultraperipheral heavy ion collisions at the LHC}

\section{Mark Strikman}

Experiments at HERA have demonstrated that reactions with quasi real photons provide an effective tool of probing pQCD which complements studies of DIS processes. In the near future it will be possible to extend these studies to ultra-high energy photon - nucleus collisions via the study of ultra-periperal collisions (UPCs) of heavy ions (protons and ions) at the LHC. The feasibility and the possible reach of these investigations was explored in a five year long study undertaken by the collaboration of theorists and experimentalists. The results of the study were published as a volume of Physics Reports [979. Due to the high energy of the colliding nuclei and very good acceptance of the CMS and ATLAS detectors at large rapidities, UPCs at the LHC allows to study a wide range of the processes sensitive to the small-x dynamics for $W_{\gamma N} \leq 1-2 \mathrm{TeV}$. This would extend the $x$ range probed at HERA down by at least by a factor of ten. A further advantage

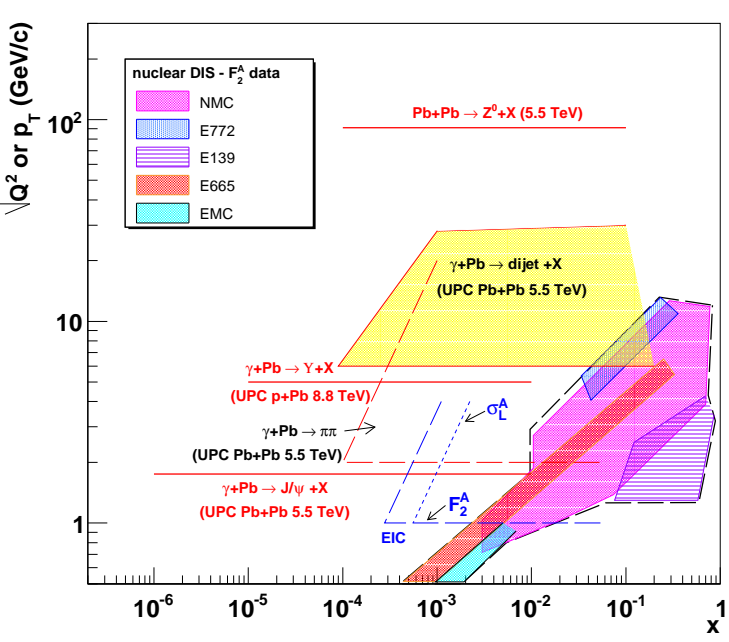

Figure 5.95. The kinematic range in which UPCs at the LHC can probe gluons in protons and nuclei in quarkonium, di-jet and di-hadron production. For comparison, the kinematic ranges for $J / \psi$ at RHIC, $F_{2}^{A}$ and $\sigma_{L}^{A}$ at eRHIC and $Z^{0}$ hadroproduction at the LHC are also shown. for the search for non-linear effects will be the use of the nuclear targets.

The kinematic range for which studies of several processes of interest will be feasible is presented in figure 5.95 (taken from [979]) as a function of $x$ and $Q$ which is the typical gluon virtuality which, as the transverse momentum of the jet or leading pion, sets the scale for dijet and $\pi \pi$ production respectively. The typical gluon virtuality scale for exclusive quarkonium photoproduction is shown for $J / \psi$ and $\Upsilon$. Below we list some of the directions of the planned studies. 
Dijet production. Dijet production in the discussed kinematic range is dominated by photon - gluon fusion. Estimates of the counting rates including cuts due to the acceptance of the CMS detector were performed in [1197]. It was found that measurements of the nuclear gluon pdfs will be feasible down to $x \sim 10^{-4}$ via study of several channels: dijet, charm, beauty jets, providing a number of cross checks. Use of the zero degree calorimeters (ZDCs) will also allow the separation of diffractive events and hence measure the nuclear gluon diffractive pdfs in the same kinematics. Hence, it will be possible to test a prediction of the leading twist theory of nuclear shadowing that the probability of the gluon induced coherent diffraction at large $p_{T}$ and small $x$ should be of the order $10-15 \%$ [812].

The cutoff $p_{t}($ jet $) \geq 6-8 \mathrm{GeV} / \mathrm{c}$ (necessary for selecting dijet production) reduces non-linear effects in dijet production. The parameter which governs non-linear effects is $R_{N L}=C_{F}^{2} \alpha_{s}(Q) x G_{T}\left(x, Q^{2}\right) / \pi r_{T}^{2} Q^{2}$, where $C_{F}^{2}$ is the Casimir operator, equal to $4 / 3$ for $q \bar{q}$ and 3 for $g g$, and $r_{T}$ is the transverse area of the target. For the smallest $x, p_{T}$ corner, $R_{N L}$ for the UPC processes $R_{N L}$ is about the same as for $F_{2 A}\left(x, Q^{2} \sim 2-4 G e V^{2}\right)$ for the lowest $x$ which could be reached at the EIC.

It will be also possible to reach larger $R_{N L}$ at smaller virtualities and $x \sim 10^{-4}$ using leading pion production in the central detectors $|y| \leq 2.4$ - see dashed area in figure 5.95, This is a kinematics similar to the production of two forward pions in $\mathrm{d}+\mathrm{Au}$ collisions at RHIC. Within the mechanism of fractional energy losses [981, 1198], one expects a strong suppression of the two pion yield as compared to the single pion yield which would allow one to perform clean tests of the onset of the black disk regime (BDR).

Another sensitive probe of the onset of BDR would be exclusive diffractive production of two jets in the process $\gamma+\mathrm{A} \rightarrow 2$ jets $+\mathrm{A}$. In the case of light quark jets, this process is strongly suppressed in the $\mathrm{pQCD}$ regime, while it is a dominant contribution to the diffraction mechanism in the BDR [981.

The interaction of small dipoles with nuclear media. In the leading twist approximation, the suppression of onium coherent production is given by the square of the ratio of the gluon densities in the nucleus and the proton gluon pdfs. It will be feasible to investigate the suppression of coherent $J / \psi, \Upsilon$ production in nucleus-nucleus collisions down to $x \sim m_{\text {onium }} / 2\left(E_{A} / A\right)$ corresponding to production at the central rapidities. At rapidities away from zero, photons of smaller energies dominate in the production of $J / \psi$, making it very difficult to probe smaller $\mathrm{x}$ for virtualities $\sim 3 \mathrm{GeV}^{2}$ characteristic for $J / \psi$ coherent photoproduction. However, the use of incoherent diffractive onium production appears to solve this problem as one can use production of soft neutrons to determine which of the nuclei emitted a photon and which was involved in the strong interaction [979]. As a result, there is a potential for probing $J / \psi$ production down to $x \sim 10^{-6}$, see figure 5.95.

A complementary method of tracking a small dipole through the nuclear media will be provided by the $J / \psi$ production in the $-t \geq$ few $\mathrm{GeV}^{2}$ process $\gamma+A \rightarrow J / \psi+$ rapidity gap $+\mathrm{Y}$ [982]. It is possible in this case to select the kinematics where $x_{g}$ of the gluon involved in the hard process is $x_{g} \geq 0.01$. In this case, scattering at central impact parameters dominates and one can probe the propagation of a small dipole through $\sim 10 \mathrm{fm}$ of the nuclear media up to $W_{\gamma N} \sim 1 \mathrm{TeV}$.

In conclusion, it appears that UPC studies to be performed at the LHC in the next few years will allow for the search of several signals of the onset of the BDR. However, it will not be possible to perform a precision scan of the range of moderate $Q^{2}$ sensitive to the transition between non-linear and linear regimes in the $x$ range to be covered by the EIC. Hence the UPC - LHC and EIC programs will nicely complement each other. 


\section{Chapter 6}

\section{Electroweak physics}

Convenors and chapter editors:

K. Kumar, Y. Li, W. Marciano 


\title{
6.1 Electroweak physics at the EIC
}

\author{
Krishna Kumar, Yingchuan Li, William J. Marciano
}

\subsubsection{Introduction}

The $S U(3)_{C} \times S U(2)_{L} \times U(1)_{Y}$ standard model of particle physics has been extremely successful in describing strong and electroweak interactions. Its unbroken gauge symmetry, $S U(3)_{C}$ or Quantum Chromodynamics (QCD), taken on its own, represents a "perfect theory" "with no arbitrary free parameter. Nevertheless, it beautifully encompasses all the basics of strong interactions: quark confinement, chiral symmetry breaking, asymptotic freedom, etc. The electroweak sector is potentially much more mutable. In addition to its, as yet, undiscovered Higgs scalar remnant of $S U(2)_{L} \times U(1)_{Y}$ symmetry breaking, it contains many arbitrary free masses, couplings and mixing angles. They are accommodated but not understood at a deep level. Questions such as: why parity violation, why 3 generations of quarks and leptons? etc suggest that simplifying principles must await future new discoveries. However, precision measurements and searches for rare phenomena still have important roles to play. They have the capability of indirectly probing scales of physics beyond collider facilities and expanding the horizons of electroweak physics.

The EIC is being proposed mainly for the study of strong interactions but also has a unique ability to measure parity violating structure functions involving $W^{ \pm}$and $Z$ boson mediated interactions. The high energy and luminosity combined with polarized electrons and protons as well as a variety of heavy ion targets will provide a wealth of data in an area never explored before.

Two EIC capabilities for electroweak measurements, outlined in table 6.1, are: 1) Precision measurements of the weak mixing angle over a broad range in $Q^{2}$ and 2) Searches for $e \rightarrow \tau$ flavor changing conversion. For the former, we show how parity violating, right-left, deep-inelastic polarized $e p$ and $e d$ asymmetries can be used to precisely determine the running $\sin ^{2} \theta_{W}(Q)$ as a function of $Q^{2}$. The comparison of those measurements with precision values obtained from other lower energy or Z-pole studies can be used to find hints of "new physics". Alternatively, the overall World average of $\sin ^{2} \theta_{W}$ can be compared with precisely determined quantities such as $\alpha_{E M}, G_{F}, m_{Z}$, and $m_{W}$ to test the SM at the quantum loop level and probe "new physics" effects. In the case of $e-\tau$ conversion, the $e p \rightarrow \tau X$ reaction is examined, including isolation cuts and $\tau$ identification. First estimates suggest that backgrounds are under control and the high luminosity goals of the EIC allow the search of reactions well beyond HERA sensitivities.

\begin{tabular}{|c|c|c|c|c|}
\hline Deliverables & Observables & What we learn & Phase I & Phase II \\
\hline \hline $\begin{array}{c}\text { Weak mixing } \\
\text { angle }\end{array}$ & $\begin{array}{c}\text { Parity violating } \\
\text { asymmetries in } \\
e p \text { - and } e d \text {-DIS }\end{array}$ & $\begin{array}{c}\text { physics behind EW } \\
\text { symmetry breaking } \\
\text { \& BSM physics }\end{array}$ & $\begin{array}{c}\text { good precision } \\
\text { over limited } \\
\text { range of scales }\end{array}$ & $\begin{array}{c}\text { high precision } \\
\text { over wide range } \\
\text { of scales }\end{array}$ \\
\hline e- $\tau$ conversion & ep $\rightarrow \tau, \mathrm{X}$ & $\begin{array}{c}\text { flavour violation } \\
\text { induced by BSM } \\
\text { physics }\end{array}$ & challenging & very promising \\
\hline
\end{tabular}

Table 6.1. Science Matrix for Electroweak physics at an EIC. 


\title{
6.2 The weak mixing angle via polarized electron scattering asymmetries
}

\author{
Krishna Kumar, Yingchuan Li, William J. Marciano, Seamus Riordan
}

\subsubsection{Introduction}

The nature of spontaneous gauge symmetry breaking implies that the masses and couplings of weak gauge bosons $W$ and $Z$ are related by natural lowest order relations $\sin ^{2} \theta_{W}^{0}=e_{0}^{2} / g_{0}^{2}=1-m_{W}^{0}{ }^{2} / m_{Z}^{0}{ }^{2}$. The weak mixing angle plays a central role in those correlations. In the context of the Standard Model (SM) as a complete stand alone theory, the renormalized weak mixing angle is related to the other precisely measured quantities

$$
\begin{aligned}
\alpha^{-1} & =137.03599959(40) \\
G_{\mu} & =1.1663788(7) \times 10^{-5} \mathrm{GeV}^{-2} \\
m_{Z} & =91.1871(21) \mathrm{GeV}
\end{aligned}
$$

via

$$
\sin ^{2} 2 \theta_{W}\left(m_{Z}\right)_{\overline{M S}}=\frac{4 \pi \alpha}{\sqrt{2} G_{\mu} m_{Z}^{2}\left[1-\Delta \hat{r}\left(m_{t}, m_{H}\right)\right]},
$$

where $\Delta \hat{r}$ denotes loop corrections that depend on the top quark and Higgs masses while the renormalized weak mixing angle is defined by modified minimal subtraction $\overline{M S}$ [1199, 1200]. The value of $\sin ^{2} 2 \theta_{W}\left(m_{Z}\right) \overline{M S}$ can be determined from parity violating asymmetries and other weak interaction measurements. A comparison of the parameters in equation 6.2 at a high level of precision was used in the past to constrain the top quark mass (before its discovery) and more recently, to provide an upper bound on the Higgs boson mass, the missing particle of the SM. After the Higgs mass is directly measured, equation 6.2 will be used to probe for "new physics" effects at the tree or loop level.

Incorporating $m_{W}=80.398(25) \mathrm{GeV}$ via

$$
\sin ^{2} \theta_{W}\left(m_{Z}\right)_{\overline{M S}}=\frac{\pi \alpha}{\sqrt{2} G_{\mu} m_{W}^{2}\left[1-\Delta r\left(m_{Z}\right)_{\overline{M S}}-0.0085 S-\mathcal{O}(1) m_{W}^{2} / m_{W^{*}}^{2}\right]},
$$

with $\Delta r\left(m_{Z}\right)_{\overline{M S}}=0.0696(2)$ representing loop corrections insensitive to $m_{t}$ and $m_{H}$, one has another handle on "new physics" parameters such as $S$ [1201, 1202, a measure of possible new heavy chiral doublets such as a 4 th generation, or $m_{W^{*}}$, the scale of possible Kaluza-Klein excitations.

The most precise determinations of $\sin ^{2} \theta_{W}$ come from two measurements at SLAC [1203] and CERN 1204]

$$
\begin{array}{ll}
\sin ^{2} \theta_{W}\left(m_{Z}\right)_{\overline{M S}}=0.23070(26) & (\mathrm{SLAC}) \\
\sin ^{2} \theta_{W}\left(m_{Z}\right)_{\overline{M S}}=0.23193(29) & (\mathrm{CERN}),
\end{array}
$$

with both extracting $\sin ^{2} \theta_{W}$ at the $\mathrm{Z}$ pole and carrying an error of roughly $0.1 \%$ level. Unfortunately, they disagree by about 3 sigma and therefore, individually provide completely different implications for the Higgs mass and possible "new physics". For example, the SLAC left-right asymmetry result weighs heavily in the leptonic Z pole average which indicates a Higgs mass of

$$
m_{H} \approx 50_{-23}^{+34} \mathrm{GeV}
$$




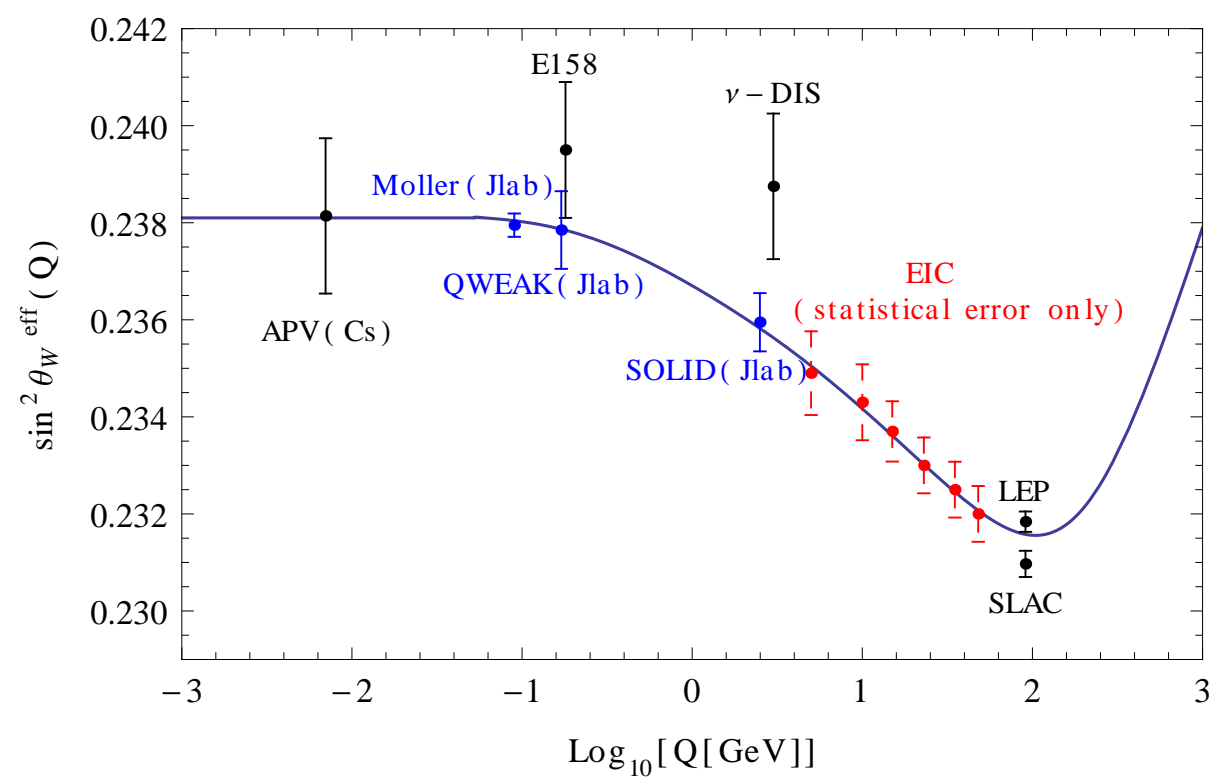

Figure 6.1. The past, currently running, and future experiments on extracting $\sin ^{2} \theta_{W}(Q)$.

with the center value significantly below the LEP II direct search limit [3]

$$
m_{H}>114 \mathrm{GeV} \text { (95\%C.L.). }
$$

On the other hand, the LEP $Z \rightarrow b \bar{b}$ forward-backward asymmetry weights heavily in the hadronic Z pole average which implies a rather heavy Higgs [1205]

$$
m_{H} \approx 480_{-230}^{+350} \mathrm{GeV} .
$$

The often quoted bound $m_{H}<150 \mathrm{GeV}$ results mainly from the Z-pole world average $\sin ^{2} \theta_{W}\left(m_{Z}\right) \overline{M S}=0.23125(16)$. Is the world average correct? We may have to wait and see what the LHC tells us.

In addition to experiments at the $\mathrm{Z}$ pole, several precision measurements of $\sin ^{2} \theta_{W}$ have been carried out at lower $Q^{2}$, including atomic parity violation [1206], polarized Moller scattering [1207], and deep-inelastic neutrino scattering [1208], but with uncertainties about an order of magnitude larger, i.e. $\mathcal{O}(1 \%)$. Together all such measurements play an important role in constraining "new physics" appendages to the SM, such as heavy $Z^{\prime}$ bosons of $\mathcal{O}(1$ $\mathrm{TeV}$ ) and are useful for demonstrating the running of $\sin ^{2} \theta_{W}(Q)$, due to $\gamma-Z$ loop mixing, at about the 6 sigma level.

It is highly desirable to have other experimental extractions of $\sin ^{2} \theta_{W}$ with a precision roughly comparable to $\mathrm{Z}$ pole measurements, given the $3 \sigma$ discrepancy between the two best values. Fortunately, several new measurements are in progress or planned at Jefferson lab, including $Q_{\text {weak }}$ using elastic ep scattering [1209] ( \pm 0.0008$)$, polarized Moller scattering [1210] $( \pm 0.00025)$, and SOLID using polarized ed-DIS $( \pm 0.0006)$, which aim to extract $\sin ^{2} \theta_{W}$ at low $Q^{2}$ in very high luminosity fixed target experiments. Their projected uncertainties are shown in figure 6.1.

Here, we focus on the feasibility of measuring $\sin ^{2} \theta_{W}$ at high $Q^{2}$ using an Electron-Ion Collider (EIC). Since the center of mass energy of the EIC is expected to be much higher than 
fixed target experiments, parity violating asymmetries are larger and, therefore, potentially more sensitive to weak interaction effects. In addition, the EIC enables one to extract $\sin ^{2} \theta_{W}(Q)$ and demonstrate its evolution over a wide range of $Q^{2}$. Those measurements will test the predicted running of $\sin ^{2} \theta_{W}\left(Q^{2}\right)$, improve the world average $\sin ^{2} \theta_{W}\left(m_{Z}\right)_{\overline{M S}}$, and test for "new physics" such as $Z^{\prime}$ bosons via comparison with Z-pole and low $Q^{2}$ results. We demonstrate those capabilities for an EIC with integrated luminosity of $200 \mathrm{fb}^{-1}, \sqrt{s} \approx 140$ $\mathrm{GeV}$ and electron (as well as perhaps hadron) polarization. A statistical determination of $\sin ^{2} \theta_{W}\left(Q^{2}\right)$ to about $\pm 0.25 \%$ is found for a range of $Q^{2}$ with overall precision roughly equal to the best Z-pole and proposed polarized $e^{-} e^{-}$measurements.

\subsubsection{Extracting $\sin ^{2} \theta_{W}$ from parity-violating right-left polarization asym- metries}

Various parity violating asymmetries in $e p$ - and $e d($ deuteron)-DIS can be obtained from ratios of differences and sums of cross-sections with opposite polarizations

$$
\begin{aligned}
& d \bar{\sigma}\left(P_{e}, P_{p, d}\right)-d \bar{\sigma}\left(-P_{e},-P_{p, d}\right) \propto \\
& \frac{1}{2} \Sigma_{i} f_{i}(x)\left\{\left(P_{e}+\tilde{f}_{i}(x) P_{p, d}\right)\left(d \sigma_{R R}^{i}-d \sigma_{L L}^{i}\right)\right. \\
& \left.+\left(P_{e}-\tilde{f}_{i}(x) P_{p, d}\right)\left(d \sigma_{R L}^{i}-d \sigma_{L R}^{i}\right)\right\}
\end{aligned}
$$

and

$$
\begin{aligned}
& d \bar{\sigma}\left(P_{e}, P_{p, d}\right)+d \bar{\sigma}\left(-P_{e},-P_{p, d}\right) \propto \\
& \frac{1}{2} \Sigma_{i} f_{i}(x)\left\{\left(1+\tilde{f}_{i}(x) P_{e} P_{p, d}\right)\left(d \sigma_{R R}^{i}+d \sigma_{L L}^{i}\right)\right. \\
& \left.+\left(1-\tilde{f}_{i}(x) P_{e} P_{p, d}\right)\left(d \sigma_{R L}^{i}+d \sigma_{L R}^{i}\right)\right\}
\end{aligned}
$$

where $P_{e}$ and $P_{p, d}$ are longitudinal polarizations of the electron and proton (deuteron) beams. The $\alpha$ and $\beta$ in $d \sigma_{\alpha \beta}^{i}(\alpha, \beta=R, L)$ label polarizations of the electron and quark of type $i$, respectively. The $f_{i}(x)$ is the unpolarized parton distribution function and

$$
\tilde{f}_{i}(x) \equiv \Delta f_{i}(x) / f_{i}(x)
$$

is the ratio of polarized and unpolarized parton distribution function. The quantity $\tilde{f}_{i}(x) P_{p, d}$ can be viewed as the effective quark longitudinal polarization in a polarized proton (deuteron).

The polarized electron-quark cross-sections are proportional to [1211]

$$
\begin{aligned}
d \sigma_{R R}^{i} & \propto\left(\frac{Q_{R e}^{\gamma} Q_{R i}^{\gamma}}{Q^{2}}+\frac{Q_{R e}^{Z} Q_{R i}^{Z}}{Q^{2}+M_{Z}^{2}}\right)^{2} \\
d \sigma_{L L}^{i} & \propto\left(\frac{Q_{L e}^{\gamma} Q_{L i}^{\gamma}}{Q^{2}}+\frac{Q_{L e}^{Z} Q_{L i}^{Z}}{Q^{2}+M_{Z}^{2}}\right)^{2} \\
d \sigma_{R L}^{i} & \propto\left(\frac{Q_{R e}^{\gamma} Q_{L i}^{\gamma}}{Q^{2}}+\frac{Q_{R e}^{Z} Q_{L i}^{Z}}{Q^{2}+M_{Z}^{2}}\right)^{2}(1-y)^{2} \\
d \sigma_{L R}^{i} & \propto\left(\frac{Q_{L e}^{\gamma} Q_{R i}^{\gamma}}{Q^{2}}+\frac{Q_{L e}^{Z} Q_{R i}^{Z}}{Q^{2}+M_{Z}^{2}}\right)^{2}(1-y)^{2} .
\end{aligned}
$$


Left-handed and right-handed couplings of electrons and quarks to the photon are the same

$$
Q_{L}^{\gamma}=Q_{R}^{\gamma} \equiv Q^{\gamma}
$$

while those to the $Z$ are different (giving rise to parity violation)

$$
\begin{aligned}
Q_{L}^{Z} & =\frac{e}{\sin \theta_{W} \cos \theta_{W}}\left(T_{3 L}-Q^{\gamma} \sin ^{2} \theta_{W}\right) \\
Q_{R}^{Z} & =\frac{e}{\sin \theta_{W} \cos \theta_{W}}\left(-Q^{\gamma} \sin ^{2} \theta_{W}\right)
\end{aligned}
$$

with

$$
\begin{aligned}
& Q_{u}^{\gamma}=\frac{2}{3}, Q_{d}^{\gamma}=-\frac{1}{3}, Q_{e}^{\gamma}=-1, \\
& T_{3 L}^{u}=\frac{1}{2}, T_{3 L}^{d}=T_{3 L}^{e}=-\frac{1}{2} .
\end{aligned}
$$

For an ep collider, there are two single-polarization parity violating right-left asymmetries

$$
\begin{aligned}
A_{e p}^{e} & \equiv \frac{d \bar{\sigma}\left(P_{e}, P_{p}=0\right)-d \bar{\sigma}\left(-P_{e}, P_{p}=0\right)}{d \bar{\sigma}\left(P_{e}, P_{p}=0\right)+d \bar{\sigma}\left(-P_{e}, P_{p}=0\right)} \\
& =P_{e} \frac{\Sigma_{i} f_{i}(x)\left[\left(d \sigma_{R R}^{i}-d \sigma_{L L}^{i}\right)+\left(d \sigma_{R L}^{i}-d \sigma_{L R}^{i}\right)\right]}{\Sigma_{i} f_{i}(x)\left[\left(d \sigma_{R R}^{i}+d \sigma_{L L}^{i}\right)+\left(d \sigma_{R L}^{i}+d \sigma_{L R}^{i}\right)\right]}
\end{aligned}
$$

and

$$
\begin{aligned}
A_{e p}^{p} & \equiv \frac{d \bar{\sigma}\left(P_{e}=0, P_{p}\right)-d \bar{\sigma}\left(P_{e}=0,-P_{p}\right)}{d \bar{\sigma}\left(P_{e}=0, P_{p}\right)+d \bar{\sigma}\left(P_{e}=0,-P_{p}\right)} \\
& =P_{p} \frac{\Sigma_{i} \Delta f_{i}(x)\left[\left(d \sigma_{R R}^{i}-d \sigma_{L L}^{i}\right)-\left(d \sigma_{R L}^{i}-d \sigma_{L R}^{i}\right)\right]}{\Sigma_{i} f_{i}(x)\left[\left(d \sigma_{R R}^{i}+d \sigma_{L L}^{i}\right)+\left(d \sigma_{R L}^{i}+d \sigma_{L R}^{i}\right)\right]}
\end{aligned}
$$

with electron and proton separately polarized.

These asymmetries are simplified for an ed collider since the deuteron is an iso-singlet. Restricting to the large $x$ region $(x>0.2)$, the anti-quark contributions can be neglected. To first approximation, the parton distributions of $u$ and $d$ quark are the same (up to charge symmetry violation effects) in the deuteron and can thus be factored out of the sum over quark flavors. They then cancel in the asymmetries

$$
\begin{aligned}
\left.A_{e d}^{e}\right|_{x>0.2} & \equiv \frac{d \bar{\sigma}\left(P_{e}, P_{d}=0\right)-d \bar{\sigma}\left(-P_{e}, P_{d}=0\right)}{d \bar{\sigma}\left(P_{e}, P_{d}=0\right)+d \bar{\sigma}\left(-P_{e}, P_{d}=0\right)} \\
& =P_{e} \frac{\Sigma_{i}\left[\left(d \sigma_{R R}^{i}-d \sigma_{L L}^{i}\right)+\left(d \sigma_{R L}^{i}-d \sigma_{L R}^{i}\right)\right]}{\Sigma_{i}\left[\left(d \sigma_{R R}^{i}+d \sigma_{L L}^{i}\right)+\left(d \sigma_{R L}^{i}+d \sigma_{L R}^{i}\right)\right]}
\end{aligned}
$$

and

$$
\begin{aligned}
\left.A_{e d}^{d}\right|_{x>0.2} & \equiv \frac{d \bar{\sigma}\left(P_{e}=0, P_{d}\right)-d \bar{\sigma}\left(P_{e}=0,-P_{d}\right)}{d \bar{\sigma}\left(P_{e}=0, P_{d}\right)+d \bar{\sigma}\left(P_{e}=0,-P_{d}\right)} \\
& =\tilde{f}^{D} P_{d} \frac{\Sigma_{i}\left[\left(d \sigma_{R R}^{i}-d \sigma_{L L}^{i}\right)-\left(d \sigma_{R L}^{i}-d \sigma_{L R}^{i}\right)\right]}{\sum_{i}\left[\left(d \sigma_{R R}^{i}+d \sigma_{L L}^{i}\right)+\left(d \sigma_{R L}^{i}+d \sigma_{L R}^{i}\right)\right]}
\end{aligned}
$$



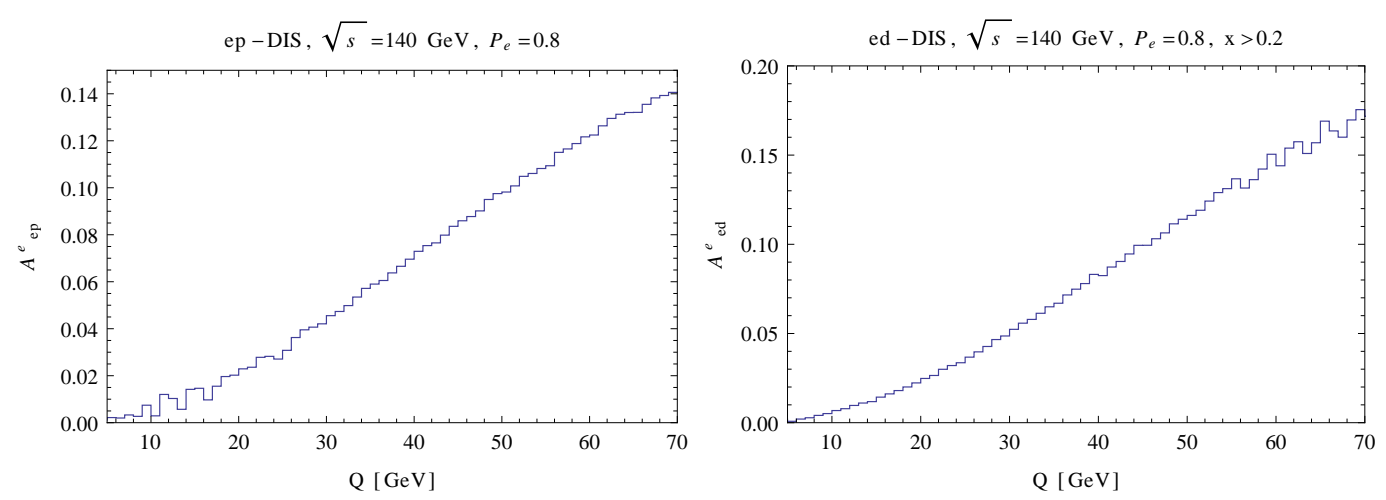

Figure 6.2. The right-left asymmetries $A_{e p}^{e}$ and $A_{e d}^{e}$ as functions of $Q$ for $e p$ - and $e d$ - DIS at $\sqrt{s}=140$ $\mathrm{GeV}$ with polarized electron $\left(P_{e}=0.8\right)$.

This leads to some simplification in the case of the single-polarization asymmetry $A_{e d}^{e}(x)$ for the $e d$ collider over $A_{e p}^{e}(x)$ for the $e p$ collider. Both $A_{e p}^{e}(x)$ and $A_{e d}^{e}(x)$ are proportional to electron polarization $P_{e}$ and thus carry smaller uncertainties than asymmetries $A_{e p}^{p}(x)$ and $A_{e d}^{d}(x)$ which are proportional to the hadron polarization $P_{p, d}$ which has a larger uncertainty. In fact, the single-polarization asymmetries $A_{e p}^{p}(x)$ and $A_{e d}^{d}(x)$ with a hadron beam polarized would hardly play any role for the purpose of measuring $\sin ^{2} \theta_{W}$ to high precision due to the large uncertainty in $P_{p, d}$ expected to be $\mathcal{O}( \pm 5 \%)$. Instead, hadron polarization (or quark polarization) may be precisely determined from the asymmetries.

The double-polarization asymmetries

$$
\begin{aligned}
& A_{e p, e d}^{e p, e d} \equiv \frac{d \bar{\sigma}\left(P_{e}, P_{p, d}\right)-d \bar{\sigma}\left(-P_{e},-P_{p, d}\right)}{d \bar{\sigma}\left(P_{e}, P_{p, d}\right)+d \bar{\sigma}\left(-P_{e},-P_{p, d}\right)} \\
= & \frac{\Sigma_{i} f_{i}(x)\left\{\left(P_{e}+\tilde{f}_{i}(x) P_{p, d}\right)\left(d \sigma_{R R}^{i}-d \sigma_{L L}^{i}\right)+\left(P_{e}-\tilde{f}_{i}(x) P_{p, d}\right)\left(d \sigma_{R L}^{i}-d \sigma_{L R}^{i}\right)\right\}}{\Sigma_{i} f_{i}(x)\left\{\left(1+\tilde{f}_{i}(x) P_{e} P_{p, d}\right)\left(d \sigma_{R R}^{i}+d \sigma_{L L}^{i}\right)+\left(1-\tilde{f}_{i}(x) P_{e} P_{p, d}\right)\left(d \sigma_{R L}^{i}+d \sigma_{L R}^{i}\right)\right\}}
\end{aligned}
$$

for both $e p$ and ed collider running depend on hadron polarization; however, there are circumstances for which the asymmetry can be simplified and carry a reduced uncertainty. First, the $d \sigma_{R L, L R}$ are proportional to $(1-y)^{2}$ and thus suppressed in the kinematic region $y \rightarrow 1$. Second, the double-polarization asymmetry can be further simplified for a ed collider at large $x$. As a result, the asymmetry

$$
\left.A_{e d}^{e d}\right|_{y \rightarrow 1, x>0.2} \approx P_{\text {eff. }} \frac{\sum_{i}\left(d \sigma_{R R}^{i}-d \sigma_{L L}^{i}\right)}{\sum_{i}\left(d \sigma_{R R}^{i}+d \sigma_{L L}^{i}\right)}
$$

is proportional to the effective polarization

$$
P_{\text {eff. }} \equiv \frac{P_{e}+\tilde{f}(x) P_{d}}{1+\tilde{f}(x) P_{e} P_{d}}
$$

which carries a reduced fractional uncertainty.

From the above discussion, it is clear that with regard to precision $\sin ^{2} \theta_{W}$ measurements, the most useful asymmetries are the two single-polarization asymmetries $A_{e p}^{e}$ and $A_{e d}^{e}$ with only the electron polarized for $e p$ and $e d$ collisions, respectively, and the double-polarization 

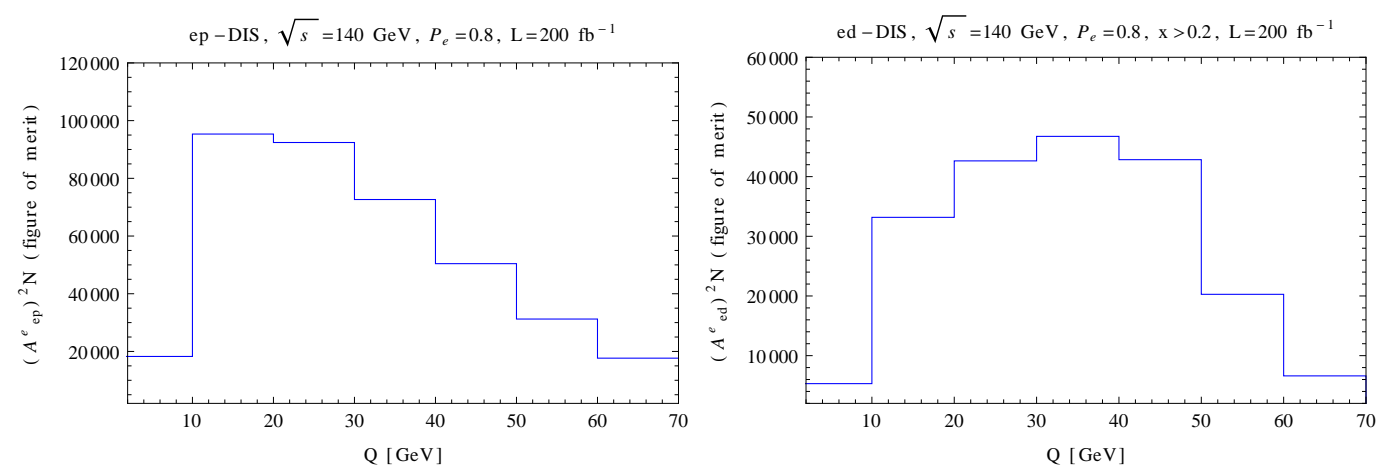

Figure 6.3. The figure of merit of measuring the asymmetries $A_{e p}^{e}$ and $A_{e d}^{e}$ at an ep and $e d$ collider with $\sqrt{s}=140 \mathrm{GeV}$ and polarized electron $\left(P_{e}=0.8\right)$, integrated luminosity of $200 \mathrm{fb}^{-1}$, for bin size of $10 \mathrm{GeV}$. A cut of $x>0.2$ is imposed for ed collisions.

asymmetry $A_{e d}^{e d}$ for the $e d$ collision, since they carry the smallest systematic polarization errors.

In general, the high energy EIC gains some advantage over experiments at low energy. For example, the error from higher $1 / Q^{2}$ twist effects should be negligible at high $Q$. In addition, since the uncertainty from parton distributions largely cancel in $A_{e d}^{e}$, the major source of systematic error comes from the polarization of electron beam $P_{e}$ which is expected to carry an uncertainty of roughly $\pm 0.5 \%$. This leads to an uncertainty of $\pm 0.5 \%$ in the single-polarization asymmetries $A_{e p, e d}^{e}$ and roughly $\pm 0.25 \%$ in $\sin ^{2} \theta_{W}$. One possible way to obtain some leverage on extracting $\sin ^{2} \theta_{W}$ with further reduced systematic error is to make use of the $y$ dependence to extract the term proportional to the vector coupling $g_{V}^{e} \propto 1-4 \sin ^{2} \theta_{W}$ of electrons to the $Z$ boson. It is well known that this coupling is very sensitive to $\sin ^{2} \theta_{W}$. An accuracy of $1 \%$ of the asymmetry proportional to this coupling determines $\sin ^{2} \theta_{W}$ at the $0.1 \%$ level. This may help with the systematic precision but unlikely with the statistical one since the latter would decrease in extracting various pieces from the $y$ dependence. To assess the statistic error in measuring $\sin ^{2} \theta_{W}$, we carry out a Monte Carlo simulation for polarized $e p$ - and $e d$ - DIS at $\sqrt{s}=140 \mathrm{GeV}$ as an example. We use the parton distribution functions of CTEQ6L [82]. We have included $u$ and $d$ quark and anti-quark contributions. For ed-DIS, a cut of $x>0.2$ is imposed to suppress the anti-quark contribution as needed to simplify the asymmetry in equation 6.17. We show the asymmetries $A_{e p}^{e}$ and $A_{e d}^{e}$ for $e p$ and $e d$ collider with polarized electron $\left(P_{e}=0.8\right)$ in figure 6.2. The asymmetries grow with $Q$ and reach $14 \%$ and $17 \%$ for $Q \approx 70 \mathrm{GeV}$, for ep and $e d$ collisions, respectively.

Based on these polarized cross-sections, one can further obtain the statistical figure of merit (F.O.M.) $A^{2} N /\left(1-A^{2}\right) \approx A^{2} N$ for measuring the asymmetry and the statistical errors for a given luminosity. In figure 6.3, we show the figure of merit for $e p$ and $e d$ collisions with integrated luminosity of $200 \mathrm{fb}^{-1}$ as function of $Q$, with bin size of $10 \mathrm{GeV}$.

The corresponding statistical errors, $\Delta A / A \approx\left(A^{2} N\right)^{-1 / 2}$, are shown in figure 6.4 for $e p$ and $e d$ colliders. For an ed collider, the energy of the deuteron beam is shared by the proton and neutron, thus effectively the CM energy for e-nucleon is reduced from $140 \mathrm{GeV}$ to roughly $100 \mathrm{GeV}$. For both ep and ed collider, with $10 \mathrm{GeV}$ bin, the statistical error is about $\pm 0.5 \%$ for $Q$ between 10 and $50 \mathrm{GeV}$. For $Q>50$ and $Q<10 \mathrm{GeV}$ region, the statistical error is significantly higher. However, a smaller error is achievable for $Q>50$ 

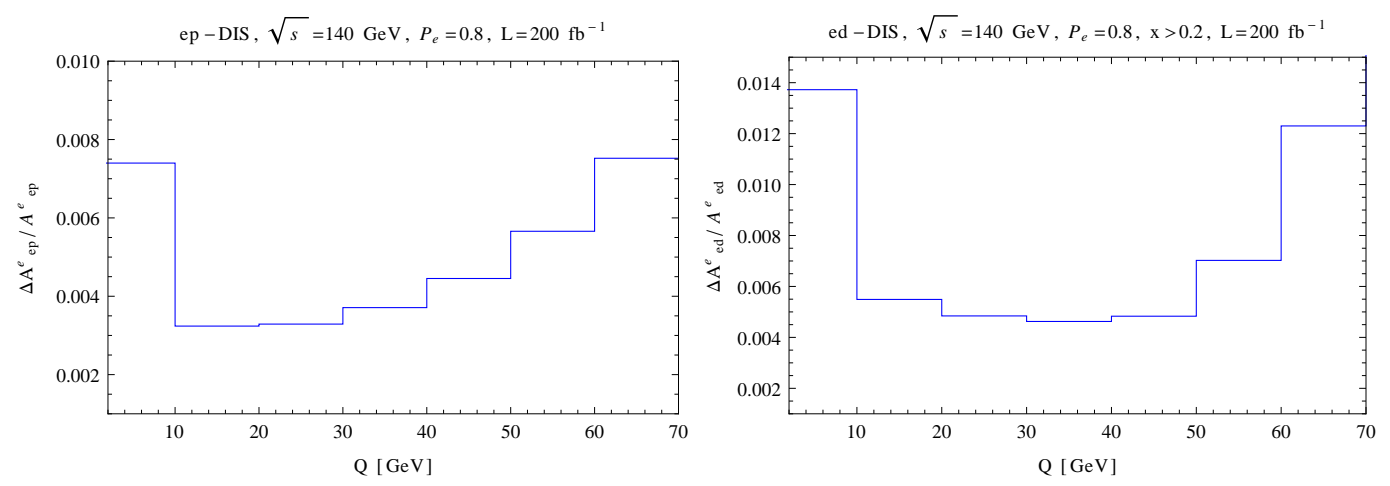

Figure 6.4. The statistical error expected for the asymmetries $A_{e p}^{e}$ and $A_{e d}^{e}$ for $e p$ and $e d$ collider at $\sqrt{s}=140 \mathrm{GeV}$ with polarized electron $\left(P_{e}=0.8\right)$, and with luminosity of $200 \mathrm{fb}^{-1}$, for bin size of $10 \mathrm{GeV}$. A cut of $x>0.2$ is imposed for $e d$ collider. The statistical error for $\sin ^{2} \theta_{W}(Q)$ is roughly $1 / 2$ the percentage error on $A_{e p}^{e}$ or $A_{e d}^{e}$.

$\mathrm{GeV}$ region if a larger bin is used. Overall, the error in extracting $\sin ^{2} \theta_{W}$ is roughly half of the error in the asymmetry. Therefore, the statistical error in extracting $\sin ^{2} \theta_{W}$ for most of the $Q$ region between a few $\mathrm{GeV}$ and Z-pole is below $\pm 0.25 \%$ level.

\subsubsection{Conclusions}

The advantage of measuring $\sin ^{2} \theta_{W}$ at a polarized EIC lies in its high $Q^{2}$, which enhances the parity violating asymmetry, reduces some of the uncertainty from higher twist effects, and most importantly enables one to extract $\sin ^{2} \theta_{W}$ over a wide range of $Q$ from a few $\mathrm{GeV}$ to $Q \approx m_{Z}$. We demonstrated the capability of measuring $\sin ^{2} \theta_{W}$ for an EIC with integrated luminosity of $200 \mathrm{fb}^{-1}, \sqrt{s} \approx 140 \mathrm{GeV}$ and electron (as well as perhaps hadron) polarization. A statistical determination of $\sin ^{2} \theta_{W}\left(Q^{2}\right)$ to about $\pm 0.25 \%$ is found for most of the region of $Q$ with overall precision roughly equal to the best Z-pole measurements. In figure 6.1, we have plotted values of $\sin ^{2} \theta_{W}(Q)$ obtained from past, ongoing and planned as well a possible EIC measurements. The running of $\sin ^{2} \theta_{W}(Q)$ is based on ref. [1212, 1213]. The error bar for EIC measurements only represents the statistical error based on figure 6.4. A combination of all the measurements of $\sin ^{2} \theta_{W}$ at various scales will play very important roles in revealing the physics behind EWSB and other "new physics".

\section{Acknowledgments}

We thank T. Han for providing the Fortran code HANLIB that is used in the Monte Carlo simulations. 


\subsection{Electron-to-Tau conversion}

Abhay Deshpande, Cyrus Faroughy, Matthew Gonderinger, Krishna Kumar, Swadhin Taneja

\subsubsection{Introduction and Motivation}

Every conservation law in the Standard Model (SM) is anticipated to have a symmetry associated with it. We have no knowledge of a symmetry that asserts Lepton Flavor Conservation in the Standard Model (SM) of particle physics and yet its (direct) violation has never been seen. Although discovery of neutrino oscillations [1214, 1215] indicates that charged Lepton Flavor Violation (LFV) processes such as $\mu \rightarrow e \gamma$ should be allowed (within the $\mathrm{SM})$, its rate is expected to be very small $\left(\mathrm{BR}(\mu \rightarrow e \gamma)<10^{-54}\right)$ due to the very small values of the neutrino masses. This level of sensitivity is beyond the reach of any present or planned experiment. However, many models of physics Beyond the SM (BSM) predict rates of charged lepton flavor violation significantly higher than those within the SM, some of them even within the reach of present or planned experiments. LFV hence becomes a very attractive process for experimental discovery of physics beyond the Standard Model.

Many searches for specific reactions which violate lepton flavor have been performed. The most sensitive include searches for $\mu+N \rightarrow e+N$ using low energy muons (from the SINDRUM II collaboration [1216]), the muon decay $\mu \rightarrow e \gamma$ (MEGA collaboration [1217, 1218]), and decays of kaons ([1219]). The limits from these processes, though extremely precise, are all sensitive to $e \leftrightarrow \mu$ transitions (abbreviated $\operatorname{LFV}(1,2)$ ) and not to $e \leftrightarrow \tau$ transitions $(\mathrm{LFV}(1,3))$. Also, each of these processes involve specific quark flavors: in some, only the 1st generation quarks participate; in others the same quark flavor must couple to the initial and final leptons, or strange quarks must participate. These stringent bounds are related to the opportunities for such searches afforded by specific experimental apparatuses. None of these searches involved the $\tau$ lepton either in the initial or in the final state. Since a general model with lepton flavor violation may involve a $\tau$ lepton and also initial and final state quarks of different flavors (not necessarily including strange quarks), the above measurements would be blind to such LFV mechanisms. Existing best limits on $e \leftrightarrow \tau$ conversion come from the BaBar Collaboration $(\tau \rightarrow e \gamma)$ [1220 and the BELLE Collaboration $(\tau \rightarrow 3 e)$ [1221]. These are notably worse than the limits on $e \leftrightarrow \mu$ by several orders of magnitude. LFV searches at proposed future experiments would further improve limits on $e \leftrightarrow \mu$ transitions.

The search for LFV involving $\tau$ leptons has been performed by the high energy lepton - hadron collider experiments H1 and ZEUS. The LFV process could proceed via exchange of a leptoquark (LQ), a color triplet boson - scalar or vector - with both lepton and baryon quantum numbers which appears naturally in many extensions of the SM such as GUTs, supersymmetry, compositeness, and technicolor (for a concise review of LFV in several such models, see [1222]). The most recent limits on the search for $e p \rightarrow \mu X$ and $e p \rightarrow \tau X$ were set by the H1 collaboration using HERA collisions at $320 \mathrm{GeV}$ center-of-mass energy and an integrated luminosity of $0.5 \mathrm{fb}^{-1}$. They did not find any evidence for lepton flavor violation [1223, 1224, and in turn they put limits on the mass and couplings of the leptoquarks in the Buchmüller-Rückl-Wyler (BRW) effective model [1225.

A high energy, high luminosity electron-proton/ion collider (EIC) is being considered by the US nuclear science community with a variable center-of-mass energy of $50 \rightarrow 160$ $\mathrm{GeV}$ and with 100 - 1000 times the accumulated luminosity of HERA over a comparable operation time, see sections 7.1 and 7.2. In a recent study [1226] it has been argued that a 
$90 \mathrm{GeV}$ center-of-mass e-p collider with $10 \mathrm{fb}^{-1}$ of integrated luminosity could set a limit on leptoquark coupling-over-mass ratios that would surpass the current best limits from HERA experiments. The study also shows that the proposed EIC could compete or surpass the updated leptoquark limits from $\tau \rightarrow e \gamma$ for a subset of quark flavor diagonal couplings. Lastly, the authors found that although $e \rightarrow \tau$ LFV is indeed severely suppressed, $e \rightarrow \tau$ transition could still exist within the reach of the EIC, under certain situations [1226]. The present study of search for leptoquarks at the EIC was motivated by these exciting possibilities.

For completeness, we studied leptoquark couplings with first, second and third generation leptons $(e \rightarrow e, \mu, \tau)$ in our simulations, although the main focus of this study was the $e \rightarrow \tau$ transition. We comment here on all three.

1. Leptoquark decays to first generation leptons lead to final states similar to those in SM deep inelastic scattering (DIS) neutral current (NC, ep $\rightarrow e X$ ) and charged current $(\mathrm{CC}, e p \rightarrow \nu X)$ interactions. These processes contribute as backgrounds by mimicking the final state signature of the signal events, and hence are indistinguishable. Other SM backgrounds arise from photo-production $\gamma p \rightarrow X$, lepton-pair production $(e p \rightarrow$ $\left.e l^{+} l^{-} X\right)$, and $\mathrm{W}$ production $(e p \rightarrow e W X)$. We simulate them and study the angular correlations of the final states and the missing momentum spectra in cases where neutrinos are involved in the final state. Possibilities of misidentification of events due to detector inefficiencies will be commented upon in section 6.3.7

2. Leptoquark decays with a $\mu$ in the final state give a back-to-back muon and hadronic system event characteristic in the transverse plane. Since muons typically deposit a very small fraction of their energy in a calorimeter, in real experiments, such events are characterized by a large missing calorimetric transverse momentum. Additionally, such muons are typically required to be isolated, well separated from the hadronic jets or tracks in such an event. Such selections strongly suppress the NC component of the SM backgrounds, which mainly arise from muon-pair production and muonic decays of $\mathrm{W}$ bosons. See details in [1223, 1224].

3. The $1 \rightarrow 3$ transition, $e p \rightarrow \tau X$, is the principle focus of this study; it is studied using three $\tau$ decay channels: electronic, muonic and hadronic. Electronic decays $\tau \rightarrow e \nu_{e} \nu_{\tau}$ have a topology similar to high $Q^{2} \mathrm{NC}$ events, except for missing transverse momentum due to the escaping neutrinos, which can be exploited to reduce this background. Muonic decays $\tau \rightarrow \mu \nu_{\mu} \nu_{\tau}$ result in similar final states as the electronic decay of $\tau$ and hence a similar criteria for their selection is used. Hadronic decays of $\tau$ lead to a high transverse momentum, narrow jet resulting in a signal topology of a di-jet event with no leptons. These events can be selected using various well known algorithms to identify and separate the $\tau$-jet from other hadronic jets in NC DIS and photoproduction events.

Many of the above mentioned strategies require detailed detector simulation of the response. This is not done in the present study. However, we studied the event topologies of the SM processes and the leptoquark events through simulations with beam energies and detector acceptance guidelines suggested on the INT website [131]. The differences in event topologies generated by $p_{T}^{\text {miss }}$ (the missing transverse momentum) and the angle $\phi$ (between the $\tau$-jet and the missing transverse momentum vector) present in SM and LQ events with final state neutrinos were studied. We ask in this study: are they different enough to be 
distinguishable from one another at the EIC energies, and for what range of leptoquark couplings and masses could the LFV LQ events be differentiated from a SM event at the EIC.

This report proceeds as follows. In section 6.3.2, the leptoquark framework is introduced and the findings of [1226] are summarized and updated to reflect recent developments regarding higher EIC integrated luminosities and the proposed reach of Super-B experimental searches for $\tau \rightarrow e \gamma$. Section 6.3.3 discusses the possibility of $e \rightarrow \tau$ searches at the EIC in the broader context of an effective operator framework. Concluding remarks for the theoretical analysis are presented in section 6.3.4. An experimental analysis begins with section 6.3.5 in which we present the SM process generation and its study for the above correlations. In section 6.3.6 we detail the MC generator study for the leptoquark and study some of its parameters (leptoquark mass dependence and the coupling strength dependence) on the observable missing $p_{T}$ and $\phi$ spectra. In section 6.3.7 we compare some of the selected spectra from SM and the leptoquark and show potentially how leptoquarks may be identified at a future EIC. We then conclude with a comment on the limitations of this study and a brief plan for the near future.

\subsubsection{Theory I: Leptoquark Framework}

We begin our study of $e \rightarrow \tau$ conversion at the EIC by assuming a leptoquark framework. Leptoquarks (abbreviated LQs) are particles coupling to leptons and quarks which arise in models such as Pati-Salam color-SU(4) and $S U(5)$ GUTs. Leptoquarks provide a useful framework for an initial analysis of $e \rightarrow \tau$ conversion because they allow for the conversion process to occur at tree level, as described further below, and so larger cross sections may be expected relative to other models which induce LFV through loop effects. Additionally, searches for leptoquark-induced $e \rightarrow \tau$ were performed at HERA, and so direct comparisons can be made between limits from HERA and potential limits from the EIC.

The class of particles which may be described as "leptoquarks" have a variety of properties: spin 0 or 1; fermion number $F=3 B+L=0$ or $\pm 2 ; S U(2)_{L}$ singlet, doublet, or triplet representations; and chiral couplings to $L$ - or $R$-handed leptons. We use the BuchmüllerRückl-Wyler (BRW) parameterization of LQs [1225]. In this parameterization, there are 14 different LQs encompassing all allowed combinations of the listed properties; their interactions with quarks and leptons are given by the renormalizable SM gauge-invariant Lagrangian in equation (6.22).

$$
\begin{aligned}
& \mathcal{L}_{L Q} \quad=\quad \mathcal{L}_{F=0}+\mathcal{L}_{|F|=2} \\
& \mathcal{L}_{F=0}=h_{1 / 2}^{L} \bar{u}_{R} \ell_{L} S_{1 / 2}^{L}+h_{1 / 2}^{R} \bar{q}_{L} \epsilon e_{R} S_{1 / 2}^{R}+\tilde{h}_{1 / 2}^{L} \bar{d}_{R} \ell_{L} \tilde{S}_{1 / 2}^{L} \\
& +h_{0}^{L} \bar{q}_{L} \gamma_{\mu} \ell_{L} V_{0}^{L \mu}+h_{0}^{R} \bar{d}_{R} \gamma_{\mu} e_{R} V_{0}^{R \mu}+\tilde{h}_{0}^{R} \bar{u}_{R} \gamma_{\mu} e_{R} \tilde{V}_{0}^{R \mu} \\
& +h_{1}^{L} \bar{q}_{L} \gamma_{\mu} \vec{\tau} \ell_{L} \vec{V}_{1}^{L \mu}+\text { h.c. } \\
& \mathcal{L}_{|F|=2}=g_{0}^{L} \bar{q}_{L}^{c} \epsilon \ell_{L} S_{0}^{L}+g_{0}^{R} \bar{u}_{R}^{c} e_{R} S_{0}^{R}+\tilde{g}_{0}^{R} \bar{d}_{R}^{c} e_{R} \tilde{S}_{0}^{R}+g_{1}^{L} \bar{q}_{L}^{c} \epsilon \vec{\tau} \ell_{L} \vec{S}_{1}^{L} \\
& +g_{1 / 2}^{L} \bar{d}_{R}^{c} \gamma_{\mu} \ell_{L} V_{1 / 2}^{L \mu}+g_{1 / 2}^{R} \bar{q}_{L}^{c} \gamma_{\mu} e_{R} V_{1 / 2}^{R \mu} \\
& +\tilde{g}_{1 / 2}^{L} \bar{u}_{R}^{c} \gamma_{\mu} \ell_{L} \tilde{V}_{1 / 2}^{L \mu}+\text { h.c. }
\end{aligned}
$$

In equation (6.22), $q_{L}$ and $\ell_{L}$ are the $S U(2)_{L}$ doublet quarks and leptons, $u_{R}, d_{R}, e_{R}$ are the $S U(2)_{L}$ singlet quarks and charged lepton, $\epsilon$ is the $S U(2)_{L}$ antisymmetric tensor $\left(\epsilon_{12}=-\epsilon_{21}=+1\right), \vec{\tau}=\left(\tau_{1}, \tau_{2}, \tau_{3}\right)$ are the Pauli matrices, and the charge conjugated fermion 
is defined as $\psi^{c} \equiv C \bar{\psi}^{T}=i \gamma_{2} \gamma_{0} \bar{\psi}^{T}$ in the Dirac basis for the $\gamma$ matrices. Color, $S U(2)_{L}$, and flavor (generation) indices have been suppressed. We follow the notation used in the recent literature where spin-0 leptoquarks are $S$ and spin-1 are $V$, the subscript indicates the $S U(2)_{L}$ quantum number (0 for a singlet, $1 / 2$ for a doublet, 1 for a triplet), the superscript $L, R$ indicates the chirality of the lepton coupling to the leptoquark, and a tilde $\left(^{(}\right)$is used to distinguish between leptoquarks which have different hypercharges but are otherwise identical. The dimensionless coupling constants $g$ and $h$ (which we assume to be real) carry the same lepton chirality and $S U(2)_{L}$ labels as their associated leptoquarks. Lepton flavor violating processes mediated by LQs arise if the couplings — which are matrices in flavor space - have non-zero off-diagonal elements.

The $e \rightarrow \tau$ conversion process mediated by LQs is shown at the partonic level in the Feynman diagrams in figure 6.5. For simplicity, the couplings $g$ and $h$ in equation (6.22) have been replaced by $\lambda_{i j}$ where the first index corresponds to the lepton generation and the second index the quark generation. The cross section for the deep inelastic scattering conversion process $e^{-}+p \rightarrow \tau^{-}+X$ mediated by a single leptoquark is calculated using the Feynman rules derived from the Lagrangian of equation (6.22) and convoluting the partonic subprocess with the appropriate parton distribution functions for the initial state quark or antiquark. In the high mass approximation, where the LQ mass is much larger than the center-of-mass energy and all fermion masses are neglected, the momentum dependence of the LQ propagator can be neglected, effectively shrinking the propagator to a four fermion contact interaction. The cross section is then given by [1227]

$$
\begin{aligned}
& \sigma_{F=0}=\sum_{\alpha, \beta} \frac{s}{32 \pi}\left[\frac{\lambda_{1 \alpha} \lambda_{3 \beta}}{M_{L Q}^{2}}\right]^{2}\left\{\int d x d y x \bar{q}_{\alpha}(x, x s) f(y)\right. \\
& \left.+\int d x d y x q_{\beta}(x,-u) g(y)\right\}, \\
& \sigma_{|F|=2}=\sum_{\alpha, \beta} \frac{s}{32 \pi}\left[\frac{\lambda_{1 \alpha} \lambda_{3 \beta}}{M_{L Q}^{2}}\right]^{2}\left\{\int d x d y x q_{\alpha}(x, x s) f(y)\right. \\
& \left.+\int d x d y x \bar{q}_{\beta}(x,-u) g(y)\right\} .
\end{aligned}
$$

The functions $f$ and $g$ are defined differently for scalar and vector leptoquarks:

$$
f(y)=\left\{\begin{array}{cc}
1 / 2 & (\text { scalar }) \\
2(1-y)^{2} & \text { (vector) }
\end{array}, g(y)=\left\{\begin{array}{cc}
(1-y)^{2} / 2 & \text { (scalar) } \\
2 & \text { (vector) }
\end{array}\right. \text {. }\right.
$$

The parton distribution functions for the quarks and antiquarks are $q\left(x, Q^{2}\right)$ and $\bar{q}\left(x, Q^{2}\right)$, respectively, evaluated at momentum fraction $x$ and energy scale $Q^{2}$. Also, $u=x s(y-1)$ and both $x$ and $y$ are integrated from 0 to 1 . As equation (6.23) shows, in the high mass approximation the unknown leptoquark couplings and masses appear in the cross section as the ratio $\lambda_{1 \alpha} \lambda_{3 \beta} / M_{L Q}^{2}$.

In the $e^{ \pm} p$ collisions at HERA, no $e \rightarrow \tau$ conversion events were observed. Limits on the LQ ratios $\lambda_{1 \alpha} \lambda_{3 \beta} / M_{L Q}^{2}$ were set by both the ZEUS [1228] and H1 [1224] collaborations. In our analysis, we determine how the EIC might improve on these limits set by ZEUS and $\mathrm{H} 1$ by answering the question, to what values of the ratios $\lambda_{1 \alpha} \lambda_{3 \beta} / M_{L Q}^{2}$ would the EIC be sensitive? As with the ZEUS and H1 analyses, we consider all combinations of the quark 

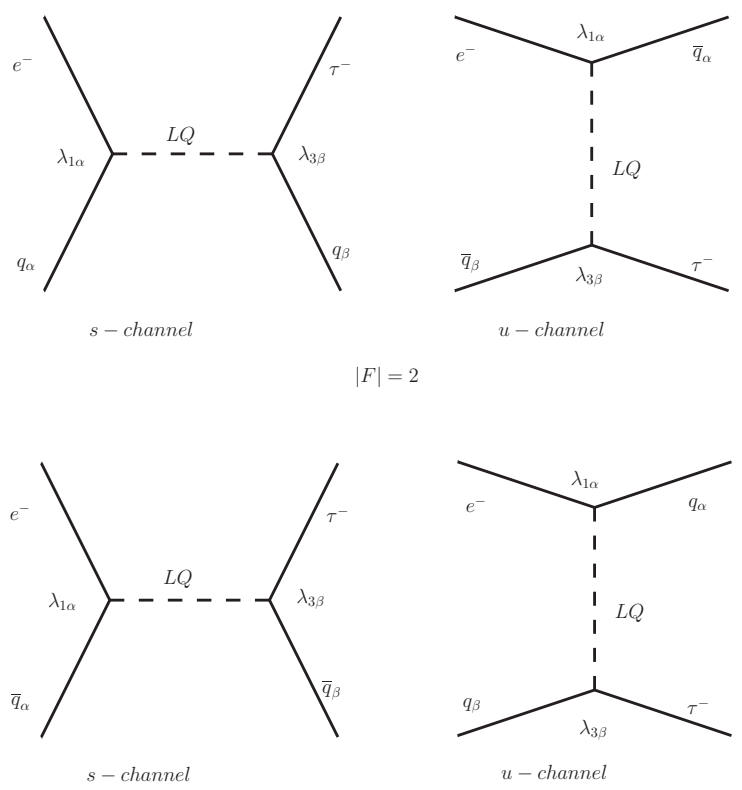

$F=0$

Figure 6.5. Feynman diagrams showing the leptoquark-mediated $e \rightarrow \tau$ conversion process. $\alpha$ and $\beta$ are the quark generation indices.

generations $\alpha$ and $\beta$ (excluding the top quark) for all 14 BRW leptoquarks. It is assumed that one of the BRW LQs dominates the cross section and the LQs in $S U(2)_{L}$ multiplets are degenerate in mass. Full results of this analysis can be found in [1226]; in this report, we summarize the results and discuss a few representative examples.

With $1000 \mathrm{fb}^{-1}$ of integrated luminosity (attainable within a reasonable length of time at a high luminosity machine such as the EIC), the EIC would in principle be sensitive to $e \rightarrow \tau$ conversion cross sections at a level of $0.001 \mathrm{fb} 1$ This would yield on the order of one $e \rightarrow \tau$ conversion events (not accounting for backgrounds, $\tau$ reconstruction efficiency, etc.). Using this number for the cross section, and assuming a center-of-mass energy $\sqrt{s}=90 \mathrm{GeV}$, the LQ ratios $\lambda_{1 \alpha} \lambda_{3 \beta} / M_{L Q}^{2}$ can be calculated from equation (6.23). Generally, for nearly all leptoquarks and combinations of quark generations $\alpha$ and $\beta$, the EIC could probe values of the ratios $\lambda_{1 \alpha} \lambda_{3 \beta} / M_{L Q}^{2}$ that are smaller than the HERA limits by a factor between 10 and 200. This is demonstrated for the LQ $S_{0}^{R}$ in figures 6.6 and 6.7 where the cross sections for the different quark generation combinations $(\alpha \beta)$ are plotted as a function of the number $z$, defined to be the LQ ratio $\lambda_{1 \alpha} \lambda_{3 \beta} / M_{L Q}^{2}$ scaled by the corresponding HERA limit. For example, the cross section for first generation initial and final state quarks (the red line in figure (6.6) is equal to $0.001 \mathrm{fb}$ at $z \simeq 0.05$. This means that the EIC could improve the HERA limit on the ratio $\lambda_{11} \lambda_{31} / M_{L Q}^{2}$ for the leptoquark $S_{0}^{R}$ by as much as a factor of 20; or, if such a leptoquark exists and has properties such that $\lambda_{11} \lambda_{31} / M_{L Q}^{2}$ is between 0.05 and 1 times the HERA limit, this LQ could induce a number of $e \rightarrow \tau$ conversion events sufficiently large enough to be observed at the EIC.

Also shown in figure 6.6 are the values of the LQ ratios $\lambda_{1 \alpha} \lambda_{3 \beta} / M_{L Q}^{2}$ (again scaled by the HERA limits) to which future Super-B experiments may be sensitive2; these are indicated

\footnotetext{
${ }^{1}$ Reference $[1226$ focused on a lower integrated luminosity and a larger cross section.

${ }^{2}$ Reference [1226] used only the current $\tau \rightarrow e \gamma$ limit.
} 


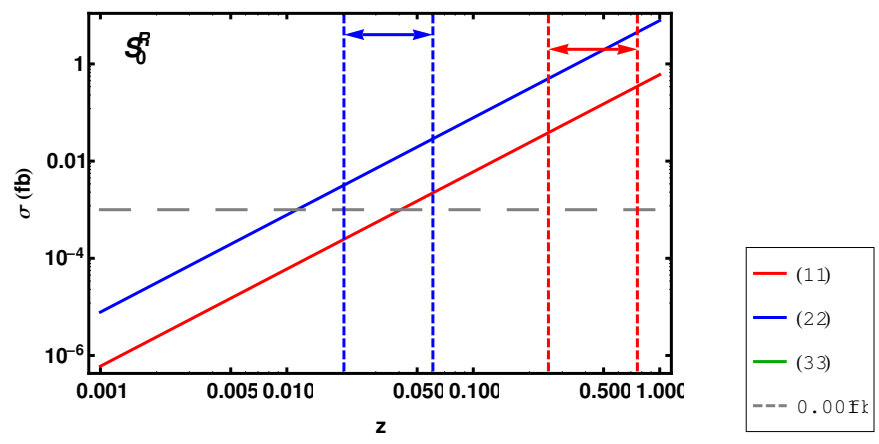

Figure 6.6. The $e \rightarrow \tau$ cross section for the leptoquark $S_{0}^{R}$ plotted as a function of $z$, defined to be the ratio $\lambda_{1 \alpha} \lambda_{3 \beta} / M_{L Q}^{2}$ scaled by the HERA limit. A cross section of $0.001 \mathrm{fb}$, corresponding to order 1 events with $1000 \mathrm{fb}^{-1}$ integrated luminosity, is indicated with a gray dashed line. The cross section is plotted for the different quark generation combinations, $(\alpha \beta)$. Shown here are the quark flavor-diagonal contributions with $\alpha=\beta$. The vertical dashed lines indicate the range of these ratios to which the Super-B experiments may be maximally sensitive from $\tau \rightarrow e \gamma$ searches.

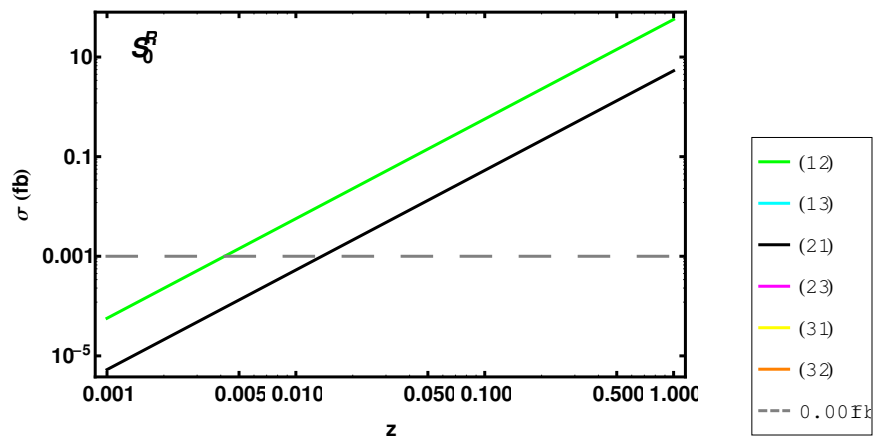

Figure 6.7. As for figure 6.6 but shown here are the quark flavor-off-diagonal contributions with $\alpha \neq \beta$. No $\tau \rightarrow e \gamma$ limits exist in this case.
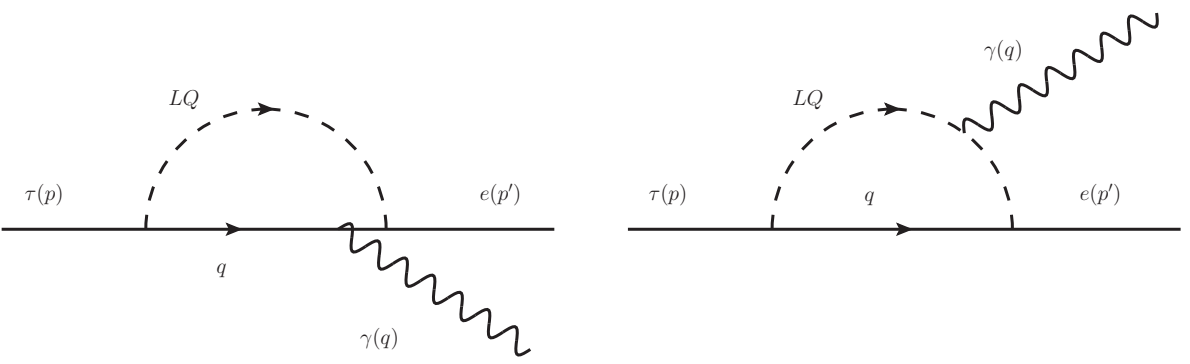

Figure 6.8. Feynman diagrams showing the leptoquark loops contributing to the $\tau \rightarrow e \gamma^{*}$ process. 
with vertical dashed lines in the figure. The scalar leptoquarks can contribute to the $\tau \rightarrow e \gamma$ decay through loop diagrams shown in figure $6.83^{3}$ Limits on the LQ ratios are derived as follows. The amplitude for the process $\tau \rightarrow e \gamma^{*}$ has the general form [1229]

$$
\begin{array}{r}
\mathcal{M}_{\tau \rightarrow e \gamma^{*}}=e \epsilon^{* \nu} \bar{u}_{e}\left(p^{\prime}\right)\left[\left(q^{2} \gamma_{\nu}-q_{\nu}(q \cdot \gamma)\right)\left(A_{1}^{L} P_{L}+A_{1}^{R} P_{R}\right)\right. \\
\left.+i m_{\tau} q^{\alpha} \sigma_{\nu \alpha}\left(A_{2}^{L} P_{L}+A_{2}^{R} P_{R}\right)\right] u_{\tau}(p),
\end{array}
$$

where the $A_{1}$ s and $A_{2}$ s are model-dependent factors. For a real photon, $q^{2}=0$, and so $|\mathcal{M}|^{2}$ depends only on the factors $A_{2}^{L, R}$. Then, the $\tau$ decay rate ratio is given by

$$
R(\tau \rightarrow e \gamma) \equiv \frac{\Gamma\left(\tau^{-} \rightarrow e^{-} \gamma\right)}{\Gamma\left(\tau^{-} \rightarrow e^{-} \bar{\nu}_{e} \nu_{\tau}\right)}=\frac{48 \pi^{3} \alpha_{E M}}{G_{\mu}^{2}}\left(\left|A_{2}^{L}\right|^{2}+\left|A_{2}^{R}\right|^{2}\right)
$$

Recent work by the BABAR collaboration [1220] has set a 90\% C.L. limit $\Gamma(\tau \rightarrow e \gamma) / \Gamma_{\text {total }} \leq$ $3.3 \times 10^{-8}$. The current consensus is that future Super-B experiments will be able to improve this limit by a single order of magnitude, and so we take $R \leq 1.85 \times 10^{-8}$. The coefficients $A_{2}^{L}$ and $A_{2}^{R}$ can be determined for each scalar leptoquark by computing the amplitude for the $\tau \rightarrow e \gamma$ loop diagrams and picking out terms proportional to the magnetic moment operator $q^{\alpha} \sigma_{\nu \alpha}$. When neglecting the lepton masses and expanding in powers of $m_{q}^{2} / M_{L Q}^{2}$, at zeroth order the $A_{2}$ s will depend on a sum over $\alpha$ of the ratios $\lambda_{1 \alpha} \lambda_{3 \alpha} / M_{L Q}^{2}$ (here, $\alpha=\beta$ since there is only one quark present in the loop). Thus, the experimental limit on $R$ determines a range of upper limits on the LQ ratios: the stronger upper limit is set by assuming all three quark generations contribute to the $A_{2}$ coefficients equally, while the weaker upper limit assumes only a single quark generation contributes to the $A_{2}$ coefficients. Both upper limits on $\lambda_{1 \alpha} \lambda_{3 \beta} / M_{L Q}^{2}$ (again scaled by the HERA limit) are indicated by vertical dashed lines for each quark generation in figure 6.6.

As figure 6.6 shows for the $S_{0}^{R} \mathrm{LQ}$, the EIC could potentially surpass upper limits on the LQ ratios derived from an improved Super-B factory $\tau \rightarrow e \gamma$ limit. For the other scalar leptoquarks, it is generally true that the EIC would be competitive with or surpass the future limits from Super-B factories. The EIC also has two additional advantages over $\tau \rightarrow e \gamma$ searches. First, $\tau \rightarrow e \gamma$ only constrains those leptoquark ratios $\lambda_{1 \alpha} \lambda_{3 \beta} / M_{L Q}^{2}$ for which $\alpha=\beta$, while the EIC can probe all combinations of quark generations. Second, it is possible for the LQ-induced $\tau \rightarrow e \gamma$ to be suppressed relative to $e \rightarrow \tau$ conversion: the first non-zero contribution to the $A_{2}$ coefficient may be proportional to $m_{q}^{2} / M_{L Q}^{2} \ll 1$ because of a cancellation of electric charges in the zeroth order term. Under these circumstances, the $\tau \rightarrow e \gamma$ yields relatively weak upper bounds on the LQ ratios. This occurs for the scalar leptoquark $\tilde{S}_{1 / 2}^{L}$.

Finally, we discuss the impact of $\operatorname{LFV}(1,2)$ searches on the leptoquark limits. As in the case of the effective operators below, a priori there is nothing in the BRW leptoquark parameterization that relates the LQ couplings to second generation leptons to LQ couplings to third generation leptons. Therefore, experimental limits on $\mu \rightarrow e$ conversion, $\mu \rightarrow e \gamma$, and $\mu \rightarrow 3 e$ do not necessarily affect the expected size of the cross sections expected for leptoquark-mediated $e \rightarrow \tau$ conversion at the EIC. Only by considering a specific model with an additional symmetry does a connection between LQ-induced $\operatorname{LFV}(1,2)$ and $\operatorname{LFV}(1,3)$ exist. An example is the $S U(5)$ GUT studied in [1230, 1231]. The leptoquark present in this model has the same spin and gauge group quantum numbers as the BRW leptoquark $\tilde{S}_{1 / 2}^{L}$.

\footnotetext{
${ }^{3}$ The contribution of the vector leptoquarks is less clear, for reasons explored in 1226, so we restrict our discussion to the scalar LQs.
} 

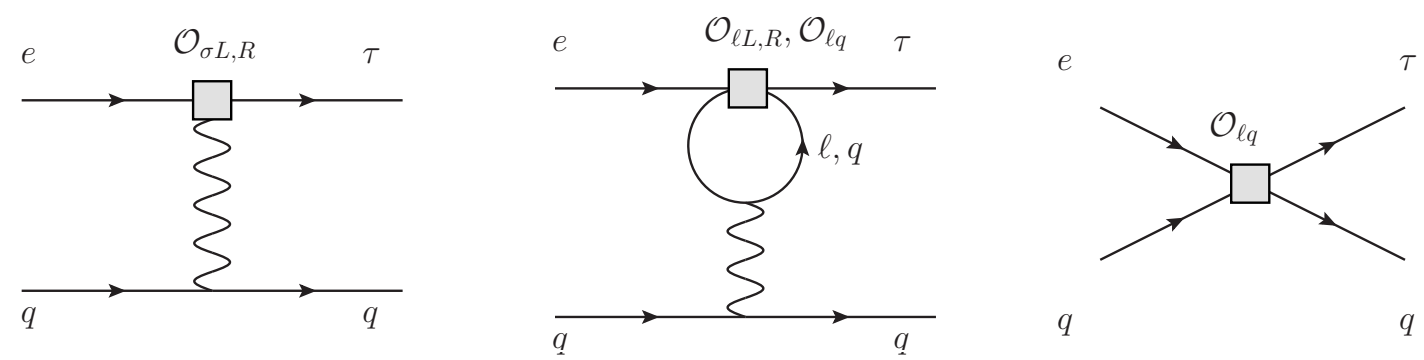

Figure 6.9. Feynman diagrams showing the contributions of the magnetic moment $\left(\mathcal{O}_{\sigma L, R}\right)$, four lepton $\left(\mathcal{O}_{\ell L, R}\right)$, and four fermion $\left(\mathcal{O}_{\ell q}\right)$ operators to the $e \rightarrow \tau$ conversion process.

As mentioned above, this particular LQ evades limits from $\tau \rightarrow e \gamma$ as well as $\mu \rightarrow e \gamma$ for the same reason. Additionally, the $S U(5)$ symmetry implies that the leptoquark couplings are proportional to the neutrino mixing angles and squared mass differences, and the stringent experimental bounds on $\mu \rightarrow e$ conversion further constrain the LQ couplings. Imposing all of these limits, the LQ couplings can still yield an $e \rightarrow \tau$ conversion cross section within reach of the EIC with $1000 \mathrm{fb}^{-1}$ integrated luminosity (for details, see [1231, 1226]). In particular, the $e^{-}+p \rightarrow \tau^{-}+X$ cross section is dominated by the partonic subprocess $e+d \rightarrow \tau+b$, implying that a $\tau$ plus a $b$-jet may be a unique experimental signatature of this particular $S U(5)$ GUT at the EIC.

\subsubsection{Theory II: Effective Operators}

We now examine the $e \rightarrow \tau$ conversion process at the EIC from the perspective of model-independent effective operators. A complete list of $S U(3)_{C} \times S U(2)_{L} \times U(1)_{Y}$ gaugeinvariant dimension- 5 and -6 operators built from the SM field content can be found in [1232] (for an updated list, see [1233]). There are three classes of operators which can contribute to $e \rightarrow \tau$ conversion and are of particular interest [1234, 1235]:

1. magnetic moment operators (written here after electroweak symmetry breaking)

$$
\mathcal{O}_{\sigma L}=i m_{j} \bar{\ell}_{L i} \sigma^{\mu \nu} \ell_{R j} F_{\mu \nu}+\text { h.c. ; }
$$

2. four lepton operators

$$
\mathcal{O}_{\ell L}=\bar{\ell}_{L i} \ell_{L j}^{C} \bar{\ell}_{L k}^{C} \ell_{L m}
$$

3. four fermion (two quark, two lepton) operators

$$
\mathcal{O}_{\ell q}=\bar{\ell}_{i} \Gamma_{\ell} \ell_{j} \bar{q} \Gamma_{q} q
$$

We use indices $i, j, k, l$ to indicate the lepton generations and suppress the gauge group indices; the superscript ${ }^{C}$ indicates charge conjugation. Note that analogous operators with right-handed fields can also be constructed. These operators can contribute to the deep inelastic electron-to-tau conversion process, as shown in figure 6.9.

The leptonic current for the photon exchange diagrams in figure 6.9 (left and middle) has a general parameterization similar to the $\tau \rightarrow e \gamma$ amplitude in equation (6.25):

$$
\begin{array}{r}
j^{\mu}=\bar{u}_{e}\left[\left(q^{2} \gamma^{\mu}-q^{\mu}(q \cdot \gamma)\right)\left(A_{1}^{L} P_{L}+A_{1}^{R} P_{R}\right)\right. \\
\left.+i m_{\tau} q_{\nu} \sigma^{\mu \nu}\left(A_{2}^{L} P_{L}+A_{2}^{R} P_{R}\right)\right] u_{\tau} .
\end{array}
$$




\begin{tabular}{|l|l|}
\hline$\tau \rightarrow 3 e$ & $\Gamma\left(\tau^{-} \rightarrow e^{-} e^{+} e^{-}\right) / \Gamma_{\text {total }}<3.6 \times 10^{-8}$ \\
\hline$\tau \rightarrow e \mu \mu$ & $\Gamma\left(\tau^{-} \rightarrow e^{-} \mu^{+} \mu^{-}\right) / \Gamma_{\text {total }}<3.7 \times 10^{-8}$ \\
\hline$\tau \rightarrow \mu e e$ & $\Gamma\left(\tau^{-} \rightarrow \mu^{-} e^{+} e^{-}\right) / \Gamma_{\text {total }}<2.7 \times 10^{-8}$ \\
\hline$\tau \rightarrow 3 \mu$ & $\Gamma\left(\tau^{-} \rightarrow \mu^{-} \mu^{+} \mu^{-}\right) / \Gamma_{\text {total }}<3.2 \times 10^{-8}$ \\
\hline$\tau \rightarrow e \gamma$ & $\Gamma\left(\tau^{-} \rightarrow e^{-} \gamma\right) / \Gamma_{\text {total }}<3.3 \times 10^{-8}$ \\
\hline$\tau \rightarrow \mu \gamma$ & $\Gamma\left(\tau^{-} \rightarrow \mu^{-} \gamma\right) / \Gamma_{\text {total }}<4.4 \times 10^{-8}$ \\
\hline
\end{tabular}

Table 6.2. All limits are taken from 3 and are at a 90\% C.L.

The two structures in this current are the charge radius term, $q^{2} \gamma^{\mu}-q^{\mu}(q \cdot \gamma)$, with $A_{1}^{L, R}$ coefficients, and the magnetic moment term, $i m_{\tau} q_{\nu} \sigma^{\mu \nu}$, with $A_{2}^{L, R}$ coefficients. The loop diagram (middle) in figure 6.9 which contains the four fermion and four lepton operators contributes to the charge radius term of the leptonic current, and so the Wilson coefficients of the operators $\mathcal{O}_{\ell L, R}$ and $\mathcal{O}_{\ell q}$ appear in the coefficients $A_{1}^{L, R}$. These contributions are loop-suppressed but receive potentially large logarithmic enhancements which go like $\ln \left(\Lambda_{L F V}^{2} / m^{2}\right)$ (where $m$ is the mass of the quark or lepton in the loop and $\Lambda_{L F V}$ is the scale at which new degrees of freedom that induce LFV are no longer integrated out of the theory) [1234]. The left photon exchange diagram in figure 6.9 containing the magnetic moment operator implies that the $A_{2}^{L, R}$ factors in the leptonic current depend on the Wilson coefficients of the $\mathcal{O}_{\sigma L, R}$ operators. These effective operators also are loop suppressed since they appear in the effective theory when heavy particles in loop diagrams (e.g., the leptoquarks in figure 6.8) are integrated out of the full theory.

The four fermion operator in figure 6.9 (right), similar to the Fermi theory for weak interactions, is a contact interaction that arises when a massive propagator is integrated out of the full theory at external momentum scales much smaller than the propagator's mass. This operator contributes to $e \rightarrow \tau$ conversion at tree level. Its cross section is expected to be larger than the cross sections from the other diagrams and operators discussed above (assuming the Wilson coefficients for all of the operators in equations (6.27)-(6.29) are all roughly the same order).

Limits on the magnetic moment and four lepton operator coefficients can be determined directly from relevant $\tau$ decay limits, some of which are listed in table 6.2. The smallness of the limits on these $\tau$ decays, in conjunction with loop suppression factors, ensures that the contributions of the $\mathcal{O}_{\sigma L, R}, \mathcal{O}_{\ell L, R}$ coefficients to the leptonic current in equation (6.30) are negligible. Therefore, as stated previously, it is expected that the greatest contributions to $e \rightarrow \tau$ conversion will come from the four fermion operators $\mathcal{O}_{\ell q}$, while photon exchange contributions will be negligibly small. Limits on the four fermion operators' coefficients can be determined from the limits on the leptoquark ratios $\lambda_{1 \alpha} \lambda_{3 \beta} / M_{L Q}^{2}$. The 14 leptoquarks in the BRW parameterization correspond to 7 of the four fermion operators listed in [1232], as shown in table 6.3 (though the correspondence is not one-to-one). Hence the leptoquark limits set by direct $e \rightarrow \tau$ searches at HERA as well as the rare process searches cited by the HERA analyses [1228], such as $\tau \rightarrow \pi e$ and decays of $B$ and $K$ mesons, allow limits to be set on the four fermion operator coefficients.

We conclude our discussion of the effective operators by noting that searches for $\mu \rightarrow e$ conversion, $\mu \rightarrow e \gamma$, and $\mu \rightarrow 3 e$ bound the coefficients of the operators in (6.27)-(6.29) which mix first and second generation leptons. However, a priori, limits on such $\operatorname{LFV}(1,2)$ 


\begin{tabular}{|l|l|l|}
\hline $\mathcal{O}_{\ell q}^{(1)}$ & $\bar{\ell}_{L} \gamma_{\mu} \ell_{L} \bar{q}_{L} \gamma^{\mu} q_{L}$ & $\left(S_{0}^{L}, \vec{S}_{1}^{L}\right) ;\left(V_{0}^{L}, \vec{V}_{1}^{L}\right)$ \\
\hline $\mathcal{O}_{\ell q}^{(3)}$ & $\bar{\ell}_{L} \gamma_{\mu} \tau^{a} \ell_{L} \bar{q}_{L} \gamma^{\mu} \tau^{a} q_{L}$ & $\left(S_{0}^{L}, \vec{S}_{1}^{L}\right) ;\left(V_{0}^{L}, \vec{V}_{1}^{L}\right)$ \\
\hline $\mathcal{O}_{e u}$ & $\bar{e}_{R} \gamma_{\mu} e_{R} \bar{u}_{R} \gamma^{\mu} u_{R}$ & $S_{0}^{R} ; \tilde{V}_{0}^{R}$ \\
\hline $\mathcal{O}_{e d}$ & $\bar{e}_{R} \gamma_{\mu} e_{R} \bar{d}_{R} \gamma^{\mu} d_{R}$ & $\tilde{S}_{0}^{R} ; V_{0}^{R}$ \\
\hline $\mathcal{O}_{\ell u}$ & $\bar{\ell}_{L} u_{R} \bar{u}_{R} \ell_{L}$ & $S_{1 / 2}^{L} ; \tilde{V}_{1 / 2}^{L}$ \\
\hline $\mathcal{O}_{\ell d}$ & $\bar{\ell}_{L} d_{R} \bar{d}_{R} \ell_{L}$ & $\tilde{S}_{1 / 2}^{L} ; V_{1 / 2}^{L}$ \\
\hline $\mathcal{O}_{q e}$ & $\bar{q}_{L} e_{R} \bar{e}_{R} q_{L}$ & $S_{1 / 2}^{R} ; V_{1 / 2}^{R}$ \\
\hline $\mathcal{O}_{q d e}$ & $\bar{\ell}_{L} e_{R} \bar{d}_{R} q_{L}$ & \\
\hline $\mathcal{O}_{\ell q}$ & $\bar{\ell}_{L} e_{R} \epsilon \bar{q}_{L} u_{R}$ & \\
\hline
\end{tabular}

Table 6.3. List of four fermion operators. For the operator names, we follow the notation of [1232]. In the middle column, we maintain the same notation as in equation (6.22). The right column lists the leptoquarks from which these operators are obtained upon integrating out the LQs. Some operators are a linear combination of different LQs which are enclosed in parentheses.

operators do not constrain the $\operatorname{LFV}(1,3)$ operators relevant for $e \rightarrow \tau$ conversion. Only by assuming the existence of an additional symmetry or a particular underlying model can the two sets of operators be related. One example of such an additional symmetry is the theory of minimal flavor violation $(\mathrm{MFV})$ in the lepton sector [1236. Under the assumptions of MFV, the breaking of the lepton flavor symmetry group $S U(3)_{L} \times S U(3)_{E}$ (for the left-handed doublets and the right-handed charged leptons) arises solely from the charged lepton and neutrino mass matrices 4 As a result, all higher-dimensional lepton flavor violating operators constructed from the lepton bilinears $\bar{\ell}_{L}^{i} \Gamma \ell_{L}^{j}, \bar{e}_{R}^{i} \Gamma \ell_{L}^{j}$, and $\bar{e}_{R}^{i} \Gamma e_{R}^{j}$ are suppressed by one or more powers of lepton masses and/or neutrino mixing parameters. This is true even of the four fermion type operators. Under the MFV hypothesis, the $e \rightarrow \tau$ conversion cross section is unobservably small; it is probable that any observation of $e \rightarrow \tau$ conversion at the EIC would therefore rule out the MFV hypothesis.

\subsubsection{Theory III: Conclusions and Future Work}

The theoretical analysis of the $e \rightarrow \tau$ DIS process presented in [1226] and section 6.3 .2 shows that leptoquarks provide a framework in which $e \rightarrow \tau$ conversion searches at the EIC are feasible. Present leptoquark limits are not prohibitive, and the EIC would be competitive with future Super-B experiments ( $\tau \rightarrow e \gamma$ searches) on similar time scales, for several reasons: the EIC would have high luminosity and be sensitive to small cross sections; the EIC like HERA can set limits for all combinations of quark generations while $\tau \rightarrow e \gamma$ is more limited in this region; and the EIC could probe leptoquarks which may evade $\tau \rightarrow e \gamma$ searches.

Limits from $\operatorname{LFV}(1,2)$ searches may or may not be relevant for leptoquarks. While the BRW framework implies no connection between $\operatorname{LFV}(1,2)$ limits and $\operatorname{LFV}(1,3)$ processes, in general it is presumed that leptoquarks will arise from physics at the high scale which does in fact constrain $\operatorname{LFV}(1,3)$ processes given the current stronger limits on $\operatorname{LFV}(1,2)$ processes. However, at least one model, the $S U(5)$ GUT discussed above, exists in which

\footnotetext{
${ }^{4}$ We limit our discussion here to the scenario of "minimal field content" described in [1236].
} 
limits from $\operatorname{LFV}(1,2)$ searches $(\mu \rightarrow e$ conversion, $\mu \rightarrow e \gamma), \tau \rightarrow e \gamma$ searches, and the neutrino sector can be implemented and still allow for observable $e \rightarrow \tau$ conversion cross sections at the EIC.

An estimation of $e \rightarrow \tau$ cross sections using model-independent effective operators and present limits on $L F V$ processes suggests that the best hope for observing $e \rightarrow \tau$ conversion at the EIC is with models which give rise to four fermion operators through tree level processes at low energies. Four lepton and magnetic moment operators are generally too suppressed to give rise to large enough $e \rightarrow \tau$ cross sections via photon exchange, especially when the relevant limits (e.g., $\tau \rightarrow 3 e, \tau \rightarrow e \gamma$ ) are imposed on the operator coefficients. Limits from additional $\operatorname{LFV}(1,2)$ searches like $\mu \rightarrow e$ conversion and $\mu \rightarrow e \gamma$ can be applied to the effective operator analysis if an additional symmetry such as MFV is imposed. MFV results in a suppression of all the LFV operators, including the four fermion operators, and hence negligibly small $e \rightarrow \tau$ cross sections.

There are several theoretical topics worthy of further attention for the $e \rightarrow \tau$ EIC search. First is the study of leptoquarks and $\operatorname{LFV}(1,3)$ flavor structure at LHC. While studies of LQ searches at the LHC have been performed in the past (as an example, see [1237]), such work has focused only on first generation fermions coupling to leptoquarks and has not considered LFV leptoquark final states. Further work is required to determine the extent to which the EIC and the LHC may provide complementary probes of the leptoquark flavor violating parameter space.

An additional topic which merits further study is a broader analysis of model-dependent $e \rightarrow \tau$ searches at the EIC. Non-leptoquark models or symmetries may give promising results for the $e \rightarrow \tau$ conversion process. For example, R-parity violating supersymmetry allows for tree level $e \rightarrow \tau$ conversion mediated by squarks; this suggests that large cross sections perhaps may be expected. Furthermore, depending on the models which give rise to the effective operators discussed above, there may be large log enhancements in the charge radius contribution to photon exchange $e \rightarrow \tau$ which could overcome the limits on the four lepton operators.

Finally, we observe that many experiments have over many years placed limits on a wide variety of flavor-violating processes. Many of these experiments constrain the leptoquark parameter space, as analyzed in [1238. Updated limits from experimental searches for other flavor-violating processes may exist and still need to be considered in analyzing the potential of the EIC (and LHC) to search for LQ-mediated LFV events. Such limits may also be relevant for non-LQ scenarios. Improved limits from ongoing and future experiments searching for $\operatorname{LFV}(1,2)$ processes also need to be included, depending on the context for the $e \rightarrow \tau$ analysis.

This concludes the discussion of the theoretical analysis of leptoquark-induced $e \rightarrow \tau$ conversion in deep-inelastic scattering at the EIC. The analysis so far has been optimistic and disregarded important experimental considerations that would impact a search for

$e \rightarrow \tau$ events. The next several sections address the questions of SM backgrounds and $\tau$ detection.

\subsubsection{Experiment I: Standard Model Backgrounds \& the Analysis Strat- egy}

In this section, we discuss the main SM processes that could mimic the $\operatorname{LFV}(1,3)$ signal at the EIC. In the SM, ep scattering is caused by the exchange of an electroweak gauge boson between the electron and a quark inside the proton. Photon exchange dominates when 
the momentum transfer $Q$ is low, but the amplitude of weak gauge bosons becomes more important as $\left|Q^{2}\right|$ approaches $M_{W^{ \pm}}^{2}$ and $M_{Z^{0}}^{2}$. Standard Model NC and CC DIS processes are shown in figure 6.10. The EIC acceptance from the beampipe $\left(0.1^{\circ}<\theta<179.9^{\circ}\right)$ restricts all EIC kinematics to $Q^{2}>0.01 \mathrm{GeV}^{2}[1239$. This cut was implemented in the SM simulations at low momentum transfer. However, we focused our SM background analysis on events with very high $Q^{2}$ since a cut of $Q^{2}>1000 \mathrm{GeV}^{2}$ was used in all simulations involving leptoquarks (given the range of LQ masses chosen, see table 6.6).
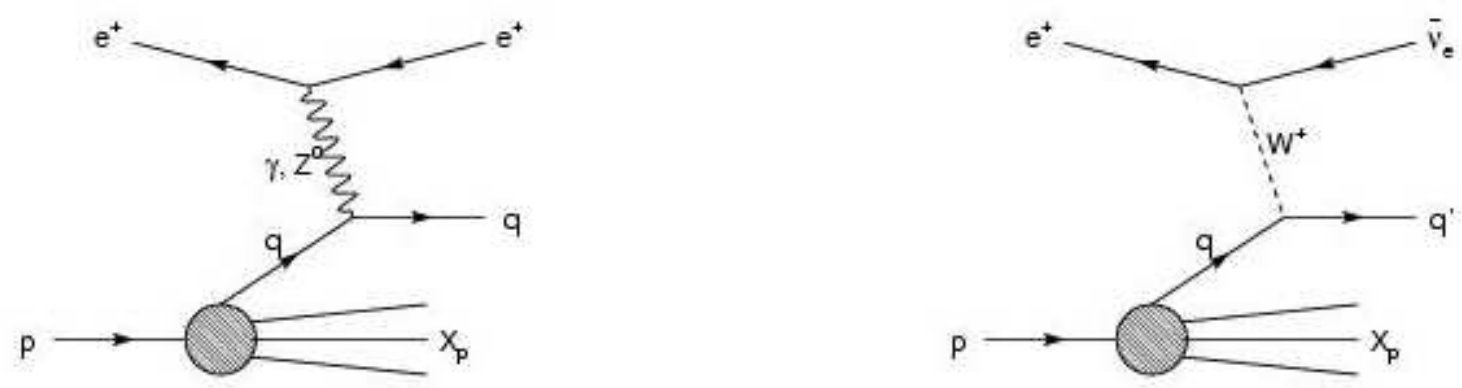

Figure 6.10. NC and CC DIS diagrams.

Ignoring rare processes, the final state $\tau$ in the $\operatorname{LFV}(1,3)$ event can decay in three different ways: electronic channel, muonic channel, and hadronic channel. Like previous searches done at HERA [1223, 1224, we consider five different SM events whose final states could be misidentified with a decaying $\tau$ : NC DIS, CC DIS, photoproduction, lepton-pair production, and real $\mathrm{W}$ boson production.

SM processes lead to final state particles that could be misidentified as our candidate $\tau$. In other words, they produce particles that leave tracks in the detector that look like the leptons or hadrons produced in a $\tau$ decay. However, the geometry of the SM events and the $\operatorname{LFV}(1,3)$ events do differ. Indeed, the identified $\tau$ lepton in an $e p \rightarrow \tau X$ conversion must be back-to-back in azimuth with the hadronic sector $X$. In addition, the angular distribution in the $\theta$ direction of the decay products of scalar LQs, vector LQs, and SM DIS background will differ because their corresponding cross sections have a different $y$ dependence 5 By inspecting equations (6.23) and (6.24) we can see that in the s-channel (see figure 6.5) vector LQs are distributed according to $d \sigma / d y \propto(1-y)^{2}$, whereas scalar LQs decay isotropically (flat $d \sigma / d y$ distribution) in their rest frame (and vice-versa in the u-channel). In contrast, NC DIS events have $d \sigma / d y \propto y^{-1 / 2}$, and this difference between LQ and SM $y$ spectra could be exploited to identify background DIS events [1240.

At the detector level, the events in all channels at the EIC must be accepted by a trigger for a large imbalance in the transverse energy flow. The energy flow summation runs over all energy deposits in the calorimeters and missing transverse momentum is associated to the neutrinos that escape the detector without any energy deposit. In this initial analysis, the missing transverse momentum $p_{T}^{\text {miss }}$ is defined as:

$$
p_{T}^{m i s s}=\sqrt{\left(\sum P_{x, i}\right)^{2}+\left(\sum P_{y, i}\right)^{2}}
$$

where $i$ runs over all final state particles in an event, excluding all neutrinos.

PYTHIA 6.4.23 is used to generate all SM events with the CTEQ 5L parametrization

\footnotetext{
${ }^{5}$ The Bjorken scattering variable is given by $y=Q^{2} / s_{e p} x=1 / 2(1-\cos \hat{\theta})$ where $\hat{\theta}$ is the decay polar angle of the lepton relative to the incident proton in the center-of-mass frame.
} 
of the parton distribution functions of the proton [1241]. Initial and final state radiation are included. No GEANT simulation of the EIC detector has been used. Two different energies are chosen for the ep MC simulations: $20 \times 325 \mathrm{GeV}$ with $\sqrt{s}=161.25 \mathrm{GeV}$ and $10 \times 250 \mathrm{GeV}$ with $\sqrt{s}=100.01 \mathrm{GeV}$. Although not directly relevant for the conclusions of this topological study, we allowed ourselves the possibility of gathering a total integrated luminosity of $1000 \mathrm{fb}^{-1}$ as suggested on this workshop's web page [1239].

\section{Standard Model Event Generation}

NC DIS: $(e p \rightarrow e X)$

NC DIS events are mediated by a photon or a $Z^{0}$ boson, and the final state includes an electron. The final state event topology of the tau electronic decay $\left(\tau \rightarrow e \nu_{e} \nu_{\tau}\right)$ is therefore very similar to that of high $Q^{2}$ NC DIS. By energy-momentum conservation the $\sum\left(E-P_{z}\right)$ distribution for NC DIS events is peaked at $2 E_{0}$, where $E_{0}$ is the electron beam energy (10 or $20 \mathrm{GeV}$ ). We can also select NC events by implementing an upper and lower cut to the quantity $\sum\left(E-P_{z}\right)$ measured. In contrast, the $\tau$ decay exhibits a large missing transverse momentum due to the neutrinos in the decay.

CC DIS: $(e p \rightarrow \nu X)$

CC DIS events are mediated by a $W^{ \pm}$boson and are characterized by high missing transverse momentum $p_{T}^{m i s s}$ and higher $Q^{2}$.

\section{Photoproduction: $(\gamma p \rightarrow X)$}

Events from photoproduction processes occur in the low $Q^{2}$ limit and may contribute to the final selection if a narrow hadronic jet fakes the tau signature or is misidentified as an electron. For $\gamma p$ events simulated with PYTHIA, the photon can be either direct (point-like) or resolved (VMD and GVMD/anomalous). A photon is assumed to be direct (point-like) when it can only interact in processes which explicitly contain the incoming photon [81], such as $f_{i} \gamma \rightarrow f_{i} g$. A photon is considered to be resolved when it interacts through its constituent quarks and gluons. Each photoproduction subprocess leads to a different event structure and has a cross section that depends strongly on the virtuality of the photon. For high virtualities (high $Q^{2}$ ), DIS events dominate, and the photon is very virtual $\left(\gamma^{*}\right)$. For very low $Q^{2}$, however, the photon can be treated as real and can have a partonic structure that can interact in different ways with the proton's quark (e.g., resolved photoproduction).

However, the LFV processes were simulated with a $Q^{2}>1000 \mathrm{GeV}^{2}$ cut and hence the SM photoproduction background will automatically be reduced to zero. As table 6.4 below suggests, most of the background that concerns us is therefore in the DIS region where the photon is very virtual.

\section{Lepton-pair Production: $\left(e p \rightarrow e l^{+} l^{-} X\right)$}

Lepton-pair production events contribute to the background because they may lead to high momentum leptons in the final state. An analysis of the event geometry is required to avoid misidentifying the three pencil-like tracks in the tau decay with the tracks left 


\begin{tabular}{|c|c|c|}
\hline Subprocess & \% of tot. events, $Q^{2}>0.01$ & \% of tot. events, $Q^{2}>1000$ \\
\hline VMD & 61.56 & 0 \\
\hline Direct & 11.28 & 0 \\
\hline Anomalous & 9.05 & 0 \\
\hline DIS $\left(\gamma^{*} q \rightarrow q\right)$ & 18.11 & 100 \\
\hline \hline
\end{tabular}

Table 6.4. Event statistics for photoproduction/DIS subprocesses simulated at $20 \times 325 \mathrm{GeV}$ with $Q^{2}>0.01 \mathrm{GeV}^{2}$ and $Q^{2}>1000 \mathrm{GeV}^{2}$.

by $l^{+}, l^{-}$and $X$ when the scattered electron is missed. The background samples include $e^{+} e^{-}, \tau^{+} \tau^{-}$and $\mu^{+} \mu^{-}$production. The simulation of these processes was not included in this analysis due to its very low cross section given the chosen EIC energy range. However, they can be included in the future using a better suited generator with improved efficiency compared to PYTHIA.

\section{W Production: $(e p \rightarrow e W X)$}

Real W boson production leads to final states with isolated leptons with high transverse momentum. The simulated $\mathrm{W}$ production samples include hadronic $\mathrm{W}$ decays (which can fake a tau decay) and leptonic $\left(l \bar{\nu}_{l}\right)$ decays that contribute to the missing transverse momentum and could potentially produce a non-LFV $\tau$. However, the cross section of this process $\left(2.449 \times 10^{-13} \mathrm{mb}\right.$ for $10 \times 250$ and $5.343 \times 10^{-11} \mathrm{mb}$ for $\left.20 \times 325\right)$ at EIC energies and luminosities is negligible.

\section{Results}

Figures 6.11 and 6.12 include all SM processes. Shown are the $p_{T}^{\text {miss }}$ and acoplanarity

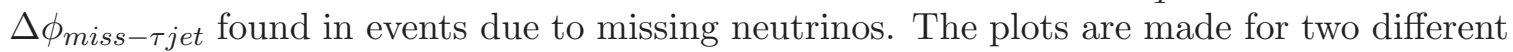
beam energy combinations (top and bottom). It is apparent that beam energies do not matter, the plots are very similar. Two different $Q^{2}$ conditions were studied: left and right, which isolate predominantly high and low $Q^{2}$ events, respectively. With no $Q^{2}$ cut, the event sample is dominated by low $Q^{2}$ photo-production background. If a cut of $Q^{2}>1000 \mathrm{GeV}^{2}$ is made the EW-physics (W) events become apparent. Figure 6.12 shows the acoplanarity $\Delta \phi_{\text {miss- }-\tau \text { jet }}$, the angle between the reconstructed $\tau$-jet direction and the missing momentum direction (presumably the neutrinos in the primary collision) for the two different energies and virtualities. The figures also reveal that the shapes of the curves are very similar for the two different center-of-mass energies.

The particles in table 6.3.5 are primarily produced from the decays of hadrons in the hadronic sector $X$; e.g., $\tau$ s can be produced from $D_{s}$ meson decay, but also include leptons from the processes mentioned above. The low $P_{T}$ suggests that these background particles can be partially avoided by restricting the kinematics phase space to high $Q^{2}$ and high transverse momentum. 


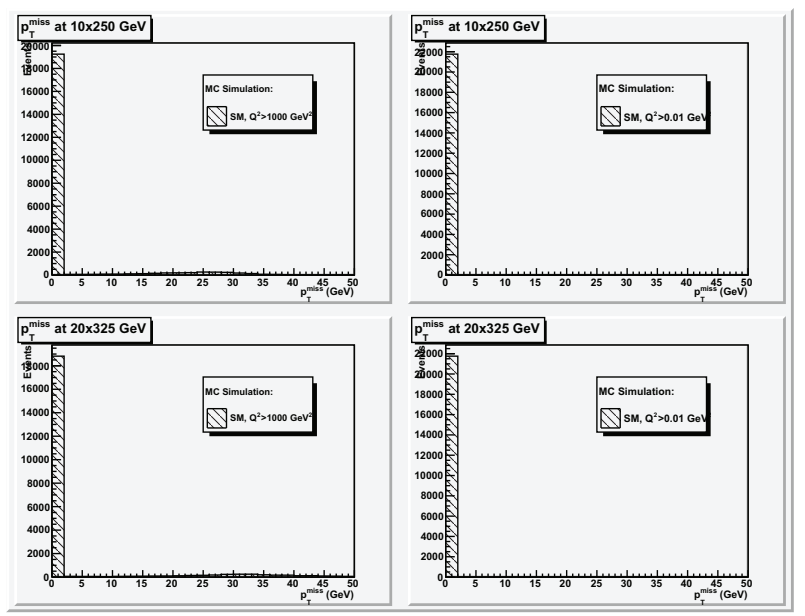

Figure 6.11. $p_{T}^{\text {miss }}$ at $10 \times 250$ and $20 \times 325 \mathrm{GeV}$ with low $Q^{2}$ and $Q^{2}>1000 \mathrm{GeV}^{2}$.

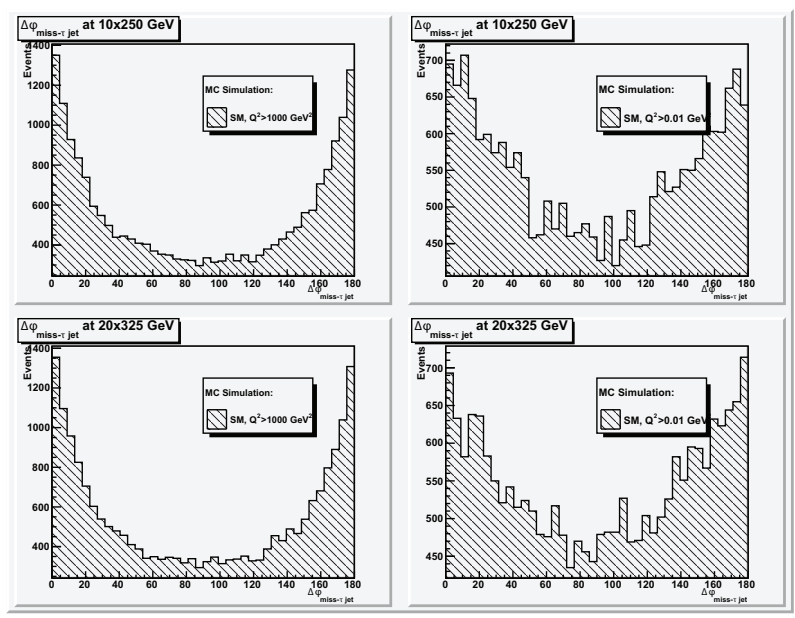

Figure 6.12. Acoplanarity $\Delta \phi_{\text {miss- }- \text { jet }}$ at $10 \times 250$ and $20 \times 325 \mathrm{GeV}$ with low $Q^{2}$ and $Q^{2}>1000$ $\mathrm{GeV}^{2}$.

\subsubsection{Experiment II: Leptoquark Simulation Study}

\section{Type of Leptoquark Studied: Parameter Space}

In this work we present the distribution of $p_{T}^{\text {miss }}$ generated from a LFV signal Monte Carlo sample of the leptoquark $\tilde{S}_{1 / 2}^{L}{ }_{6}^{6}$ The mass of the leptoquark is determined from the ratios $z \equiv \frac{\lambda_{i} \lambda_{j}}{M^{2}}$. The smallest value of the these ratios [1242] which the EIC will potentially probe are listed in table 6.6. The LFV signal Monte Carlo events were generated using a LQ generator called "LQGENEP" [1243]. LQGENEP is a LQ generator for electron/positronproton scattering which simulates processes involving LQ production and exchange using the BRW [1225] effective model. The generator is interfaced with the PYTHIA event generator. The value of $\lambda_{i}=\lambda_{j}=0.3$ is taken throughout this study. The values of $\lambda$ are correlated with $z$ through their relation to the mass of the leptoquarks, $M_{L Q}$.

\footnotetext{
${ }^{6}$ See section 6.3 .2 for a description of the notation. This leptoquark's interactions are given by the Lagrangian terms $\lambda \bar{d}_{R} \ell_{L} \tilde{S}_{1 / 2}^{L}+$ h.c. Also note that this leptoquark evades limits from $\tau \rightarrow e \gamma$ as explained in section 6.3 .2
} 


\begin{tabular}{|c|c|c|c|c|}
\hline Particle ID & $N_{1}$ & $N_{2}$ & $\mathbf{1 0 \times 2 5 0}$ & $\mathbf{2 0 \times 3 2 5}$ \\
\hline$\tau^{-}$ & 531 & 316 & $p_{T}<4, \theta_{p} \sim 2$ & $p_{T}<4, \theta_{p} \sim 2$ \\
\hline$\tau^{+}$ & 512 & 385 & $p_{T}<4, \theta_{p} \sim 1$ & $p_{T}<4, \theta_{p} \sim 1$ \\
\hline$\mu^{-}$ & 38771 & 27849 & $p_{T}<2, \theta_{p} \sim 4$ & $p_{T}<2, \theta_{p} \sim 4$ \\
\hline$\mu^{+}$ & 38691 & 27523 & $p_{T}<2, \theta_{p} \sim 4$ & $p_{T}<2, \theta_{p} \sim 4$ \\
\hline$\nu_{\tau}$ & 1043 & 701 & $p_{T}<1, \theta_{p} \sim 4$ & $p_{T}<1, \theta_{p} \sim 4$ \\
\hline$\nu_{\mu}$ & 37200 & 26170 & $p_{T}<2, \theta_{p} \sim 4$ & $p_{T}<2, \theta_{p} \sim 4$ \\
\hline$\nu_{e}$ & 38343 & 27255 & $p_{T}<2, \theta_{p} \sim 4$ & $p_{T}<2, \theta_{p} \sim 4$ \\
\hline$\overline{\nu_{\tau}}$ & 1043 & 701 & $p_{T}<1, \theta_{p} \sim 4$ & $p_{T}<1.5, \theta_{p} \sim 4$ \\
\hline$\overline{\nu_{\mu}}$ & 37280 & 26496 & $p_{T}<2, \theta_{p} \sim 4$ & $p_{T}<2, \theta_{p} \sim 4$ \\
\hline$\overline{\nu_{e}}$ & 38836 & 28004 & $p_{T}<2, \theta_{p} \sim 3$ & $p_{T}<2, \theta_{p} \sim 4$ \\
\hline \hline
\end{tabular}

Table 6.5. Statistics of selected SM background particles for 10 million $e^{-} p$ collisions generated with PYTHIA. $N_{1}$ and $N_{2}$ are the number of times the particle is produced out of the 10 million events at energies of $20 \times 325 \mathrm{GeV}$ and $10 \times 250 \mathrm{GeV}$ respectively. $\theta_{p}$ is the peak of the particle's polar angle distribution in degrees with a FWHM $\sim 11^{\circ}$ and $p_{T}$ is the transverse momentum in GeV. All $\phi$ distributions are flat.

\begin{tabular}{|c|c|c|}
\hline$\left(q_{i} q_{j}\right)$ & $z\left(\mathrm{TeV}^{-2}\right)$ & Mass $(\mathrm{GeV})$ \\
\hline 11 & 0.024 & 1936.5 \\
\hline 13 & 0.03 & 1732.0 \\
\hline 22 & 0.039 & 1519.1 \\
\hline 23 & 0.047 & 1383.8 \\
\hline 31 & 0.03 & 1732.0 \\
\hline 32 & 0.06 & 1224.7 \\
\hline 33 & 0.084 & 1035.1 \\
\hline \hline
\end{tabular}

Table 6.6. The initial and final quark flavors $\left(q_{i} q_{j}\right)$ in the subprocess $e q_{i} \rightarrow \tau q_{j}$, the ratio $z$ and the mass of the LQ for $\lambda_{i}=\lambda_{j}=0.3$.

\section{Leptoquark Event Characterization}

Electron-to-tau events were generated using the LFV generator LQGENEP for two EIC energies, namely, $10 \times 250 \mathrm{GeV}$ and $20 \times 325 \mathrm{GeV}$, in ep scattering. These events were restricted to sub-processes with a specific intermediary BRW LQ, $\tilde{S}_{1 / 2}^{L}$. The kinematic region was restricted to $Q^{2}>1000 \mathrm{GeV}^{2}$ and $y>0.1$.

\section{Electronic \& muonic $\tau$ decays}

The leptonic decays of the tau, $\tau \rightarrow e \bar{\nu}_{e} \nu_{\tau}, \mu \bar{\nu}_{\mu} \nu_{\tau}$, were studied. Background for these events is present from SM neutral current events in $e p$ DIS. The $p_{T}^{\text {miss }}$ distribution for $10 \times 250$ and 20x325 are shown in figure 6.3.6 (left) and (right), respectively. The plots shown are for electron final states. The muon final state plots are identical. The different panels indicate the $p_{T}^{\text {miss }}$ spectrum for each combination of quarks $i, j$ involved. The $p_{T}^{\text {miss }}$ spectrum is wider at higher center-of-mass energies, but otherwise the spectra are generally similar. 


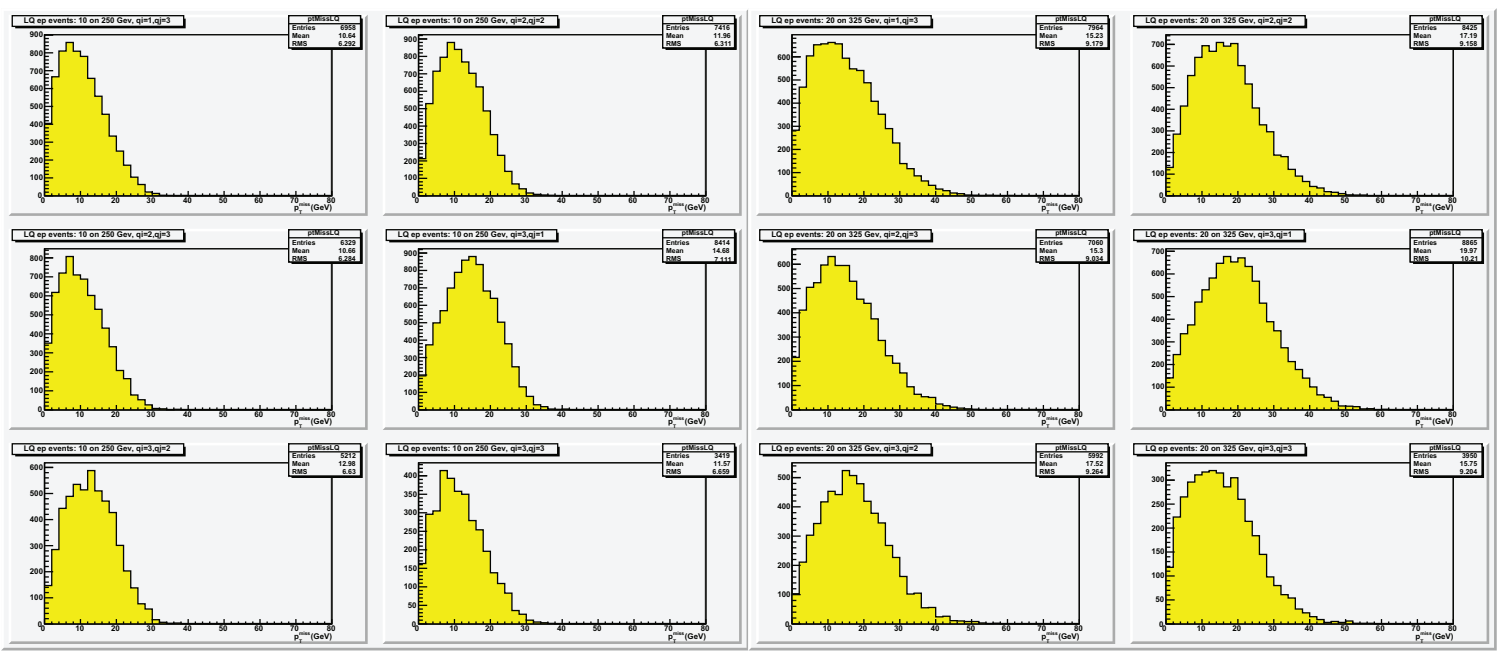

Figure 6.13. Missing transverse momentum in the electronic $\tau$ decay channel in the $e p$ scattering with $10 \times 250$ (left) and 20x325 (right) energies and the ratios, $z$, from table 6.6 for $\left(q_{i} q_{j}\right) \equiv 13,22,23,31,32$ and 33. The lepton-quark couplings are $\lambda_{i}=\lambda_{j}=0.3$.

\section{Hadronic $\tau$ decays}

The hadronic decay of high- $P_{T} \tau$ leptons results in a characteristic narrow "pencillike" jet with three pions. The event $e p \rightarrow \tau X$ would look like a a di-jet event with one narrow and one wide/high multiplicity jet. The jet associated with the $\tau$ decay is narrow. Thus one narrow and one wide jet in a di-jet event is a potential candidate for the signal. Various standard algorithms are used to identify such events [1224]. We did not simulate the detector response - this is a topic for a future detector study - but we studied the event characteristics and topology for such events.

The $p_{T}^{\text {miss }}$ distribution for 10x250 and 20x325 are shown in figure6.3.6 (left) and (right), respectively. Also plotted is the acoplanarity, $\Delta \phi_{\text {miss }-\tau \text { jet }}$, between the $\tau$-jet and the missing transverse momentum. $\Delta \phi_{m i s s-\tau j e t}$ for the EIC energies $10 \times 250$ and 20x325, shown in figure 6.3.6 (left) and (right) respectively. Figure 6.3.6 shows the same results as previous two figures but with an additional requirement of $\Delta \phi_{\text {miss-rjet }}$ below $20^{\circ}$. A small

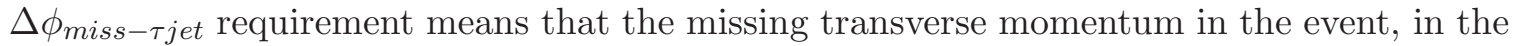
form of a $\tau$ neutrino, is aligned with the $\tau$ jet. These should be the events in which the $\tau$ decayed with neutrinos in the final state.

\subsubsection{Experiment III: Concluding Remarks}

We have studied the topological differences between events in the SM and a BRWleptoquark extension of the SM. Leptoquark searches in electron-hadron machines are sensitive to the ratio of the product of coupling constant to the square of the leptoquark mass. Motivated by recent theoretical expectations first presented in [1226] and summarized above in section 6.3.2, we have studied this for a range of leptoquark masses. While we studied the topologies of leptoquark-mediated transitions between the electron and all three generations of charged leptons, we limit our comments to the $\operatorname{LFV}(1,3)$ transition, for now. 


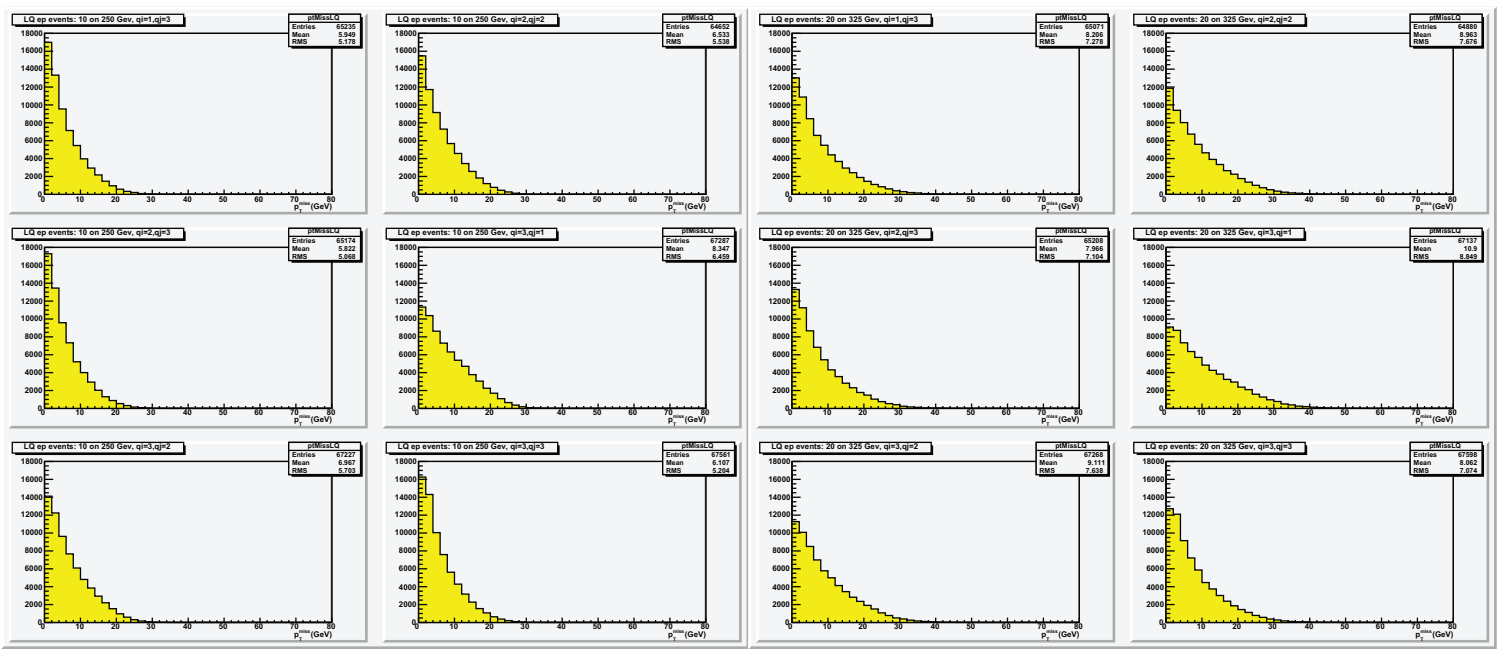

Figure 6.14. Missing transverse momentum in the hadronic $\tau$ decay channel in the ep scattering with 10x250 (left) and 20x325 (right) energies and the ratios, $z$, from table 6.6 for $\left(q_{i} q_{j}\right) \equiv 13,22,23,31,32$ and 33. The lepton-quark couplings are $\lambda_{i}=\lambda_{j}=0.3$.
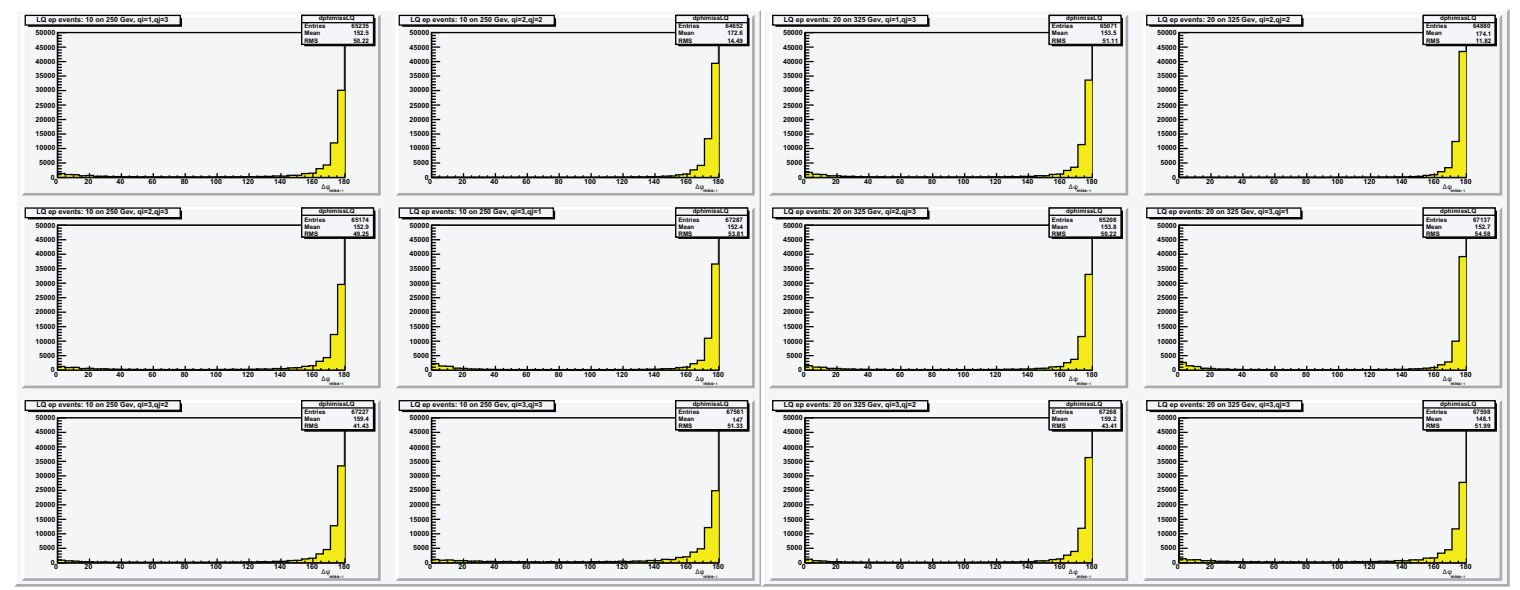

Figure 6.15. The acoplanarity, $\Delta \phi_{\text {miss }-\tau j e t}$, between the $\tau-j$ et and the missing transverse momentum in the hadronic $\tau$ decay channel in the ep scattering with 10x250 (left) and 20x325 (right) energies respectively and the ratios, $z$, from table 6.6 for $\left(q_{i} q_{j}\right) \equiv 13,22,23,31,32$ and 33.The leptonquark couplings are $\lambda_{i}=\lambda_{j}=0.3$. 


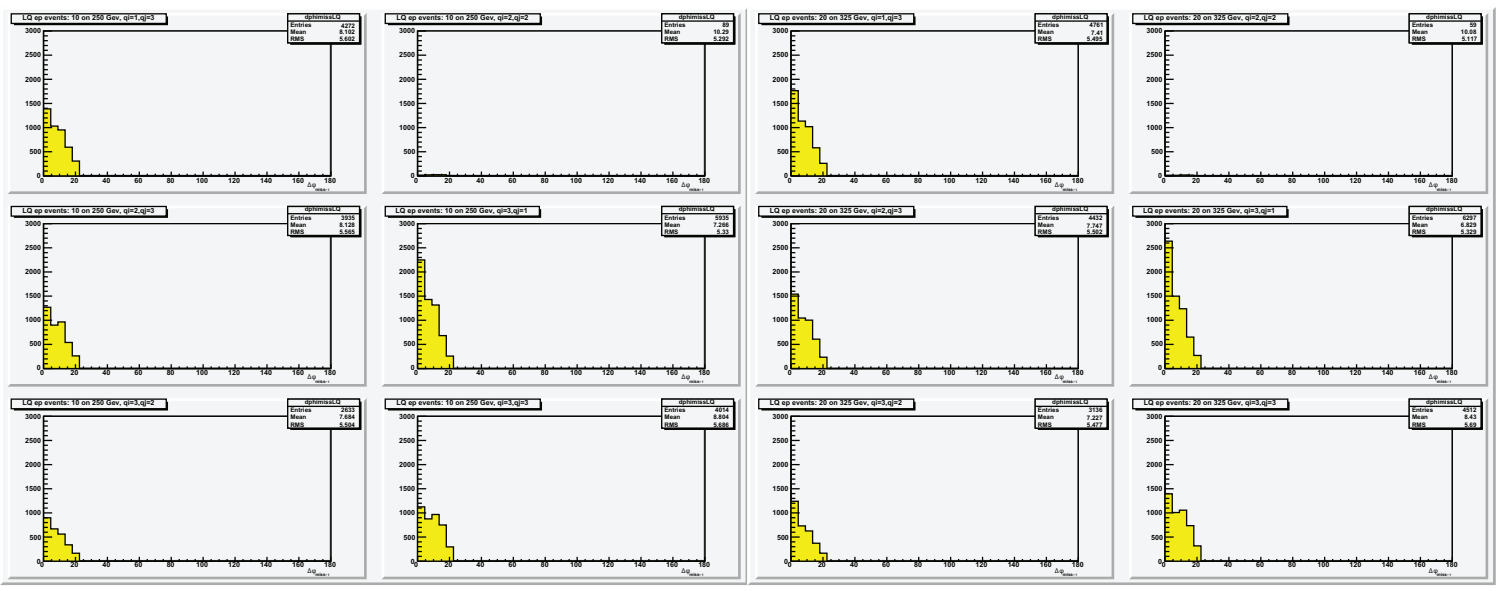

Figure 6.16. The acoplanarity, $\Delta \phi_{\text {miss }-\tau j e t}$, between the $\tau-j$ et and the missing transverse momentum in the hadronic $\tau$ decay channel in the ep scattering with 10x250 (left) and 20x325 (right) energies respectively and the ratios, $z$, from table 6.6 for $\left(q_{i} q_{j}\right) \equiv 13,22,23,31,32$ and 33 . The

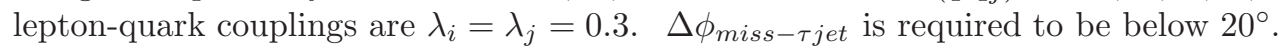

\section{Observations}

The topological features of a SM event that produces a $\tau$ lepton which decays and an event in which a $\tau$ is created as a decay of a leptoquark are distinct in two different variables routinely studied in colliders. They are: 1) $p_{T}^{\text {miss }}$ spectrum, transverse missing momentum in such an event, and 2) $\Delta \phi_{\text {miss- }}$ jet , defined as the transverse angle $\phi$ between the $\tau$ created in the event and the vector direction of missing momentum.

As shown in figure 6.17, the $p_{T}^{\text {miss }}$ spectrum is extremely narrow for SM events with final state leptonic or hadronic decays of the $\tau$ created in the collisions. This implies that the neutrinos released in the decay of the SM-produced $\tau$ are boosted in the direction of the $\tau$ and hardly any noticeable transverse momentum is lost. In contrast, the $\tau$ produced in a leptoquark decay tends to have a larger spread in the $p_{T}^{\text {miss }}$ spectrum. These general features of the $p_{T}^{\text {miss }}$ spectra do not depend on the center-of-mass of the collision: the top histograms in figure 6.17 correspond to $100 \mathrm{GeV}$ center-of-mass energy, while on the bottom they correspond to $160 \mathrm{GeV}$. Note that these plots are not normalized amongst themselves.

Figure 6.18 shows the acoplanarity plots, the angle between the $\tau$-jet in the event and the missing momentum vector reconstructed in the transverse plane using all other observable hadronic and leptonic activity (whether part of a jet or not). The left plots at each centerof-mass energy are unnormalized acoplanarity distributions showing that the SM events are distributed widely over the $\phi$ range, while the leptoquark-produced $\tau$ jets are narrowly peaked at $180^{\circ}$. If a proximity requirement cut of $20^{\circ}$ is made - meaning that the missing neutrinos were very close in $\phi$ angle with the direction of the generated $\tau$ - the distribution switches sides, indicating two categories of such events (the plots on the right of figure). Again, the top two histograms are for $100 \mathrm{GeV}$ center-of-mass energy and the bottom are for $160 \mathrm{GeV}$. They show no differences based on these center-of-mass energies. This feature by itself will be less deterministic of the event topology of the leptoquarks, but we expect (as was done in previous searches [1223]) the $p_{T}^{\text {miss }}$ spectrum and the acoplanarity distributions together will be utilized in a maximum likelihood or neural network analysis to search for excesses seen in future EIC events. 


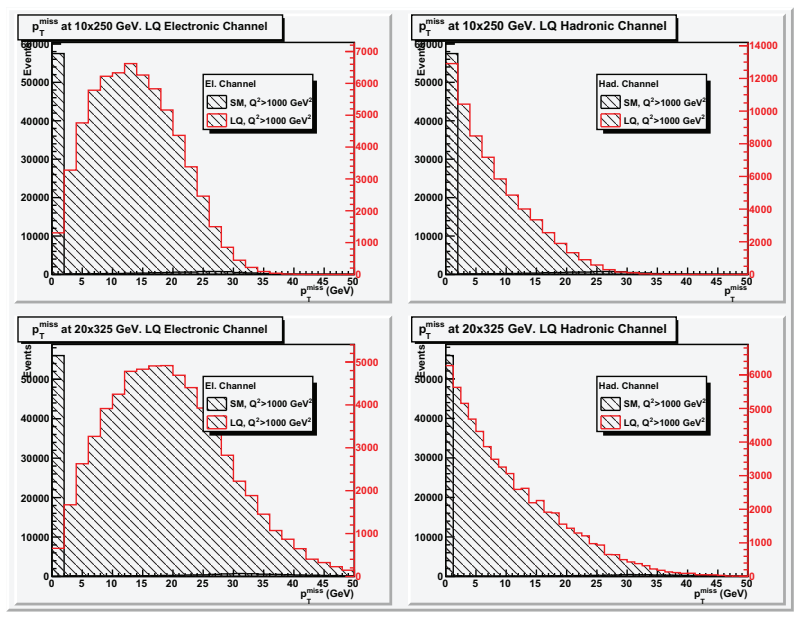

Figure 6.17. SM and LQ (both hadronic and electronic channels) $p_{T}^{\text {miss }}$ at $10 \times 250$ and $20 \times 325 \mathrm{GeV}$ with $Q^{2}>1000 \mathrm{GeV}^{2}$. These plots are not normalized amongst themselves.

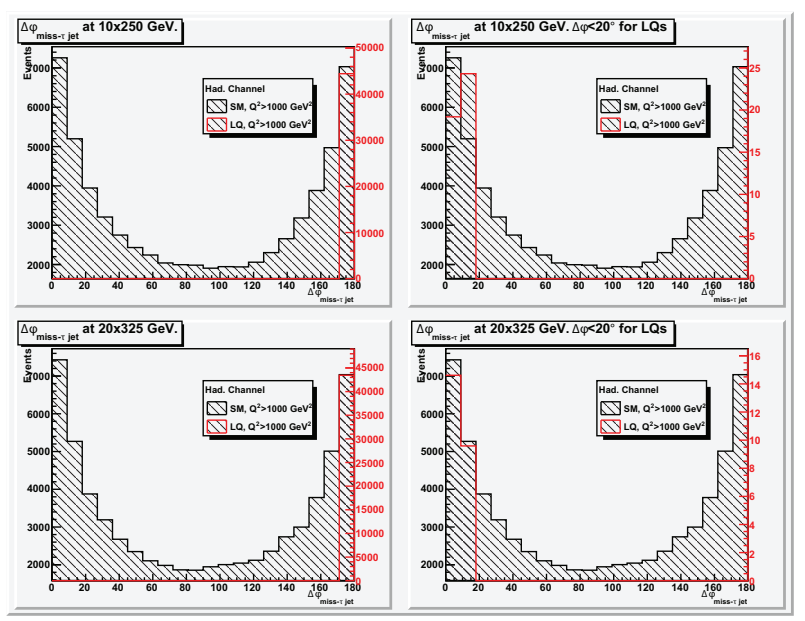

Figure 6.18. Acoplanarity $\Delta \phi_{\text {miss }, \tau j e t}$ at $10 \times 250$ and $20 \times 325 \mathrm{GeV}$ with $Q^{2}>1000 \mathrm{GeV}^{2}$. The two plots in the right side have a $\Delta \phi<20^{\circ}$ cut implemented on the LQ simulation. In this case, the LQ preserves the quark flavor: $\left(q_{i}=1 q_{j}=1\right)$. These plots are not normalized amongst themselves. 


\section{Outlook}

The study we performed is only a beginning. The estimates made in the theoretical motivation in section 6.3.2 and [1226] assume a $100 \%$ efficiency of final state leptonic and hadronic decay reconstruction of the $\tau$ created in the final state. The experimental publications [1223, 1224 indicate that their detailed simulation of the $\mathrm{H} 1$ detector resulted in a range of $7 \%$ to $15 \%$ in the reconstruction efficiency of the $\tau$ in those final states. BELLE and $\mathrm{BaBar}$ detectors have reported higher efficiencies reaching about $20 \%$. It is reasonable to assume that a future EIC detector may be able to achieve at least that. Assuming this we note that the luminosity requirements stated in section 6.3 .2 and [1226] for the EIC to probe $e \rightarrow \tau$ cross sections at the level stated are an underestimate by about 10-to- 5 times. This means at $90 \mathrm{GeV}$ center-of-mass, the stated $10 \mathrm{fb}^{-1}$ could be as high as 100 or as low as $50 \mathrm{fb}^{-1}$.

The studies we performed were based on HERA studies in which the collision energies were about $300 \mathrm{GeV}$ in the center-of-mass frame. The efficiencies of some cuts and selection criteria would certainly be better at those energies than at the EIC 100 and $160 \mathrm{GeV}$ centerof-mass energies, so the cut efficiencies may not transfer exactly as it has been assumed in these estimates. However, it is not unreasonable to assume that a similar but equally (if not more) efficient set of cuts and analysis techniques may be eventually found for this search at the future EIC.

The group now formed hopes to continue these studies with detailed detector simulation as it will become available in near future.

\section{Acknowledgments}

The authors would like to thank William Marciano and Yingchuan Li for useful discussions, and helpful suggestions. 


\section{Chapter 7}

\section{Experimental aspects}

Chapter editors:

E. C. Aschenauer, R. Ent 


\subsection{High-energy high-luminosity electron-ion collider eRHIC}

Vladimir N. Litvinenko, Joanne Beebe-Wang, Sergei Belomestnykh, Ilan Ben-Zvi, Michael M. Blaskiewicz, Rama Calaga, Xiangyun Chang, Alexei Fedotov, David Gassner, Harald Hahn, Lee Hammons, Yue Hao, Ping He, William Jackson, Animesh Jain, Elliott C. Johnson, Dmitry Kayran, Jörg Kewisch, Yun Luo, George Mahler, Gary McIntyre, Wuzheng Meng, Michiko Minty, Brett Parker, Alexander Pikin, Eduard Pozdeyev, Vadim Ptitsyn, Triveni Rao, Thomas Roser, Brian Sheehy, John Skaritka, Steven Tepikian, Yatming Than, Dejan Trbojevic, Evgeni Tsentalovich, Nicholaos Tsoupas, Joseph Tuozzolo, Gang Wang, Stephen Webb, Qiong Wu, Wencan Xu, Anatoly Zelenski

\subsubsection{Introduction}

In this paper, we describe a future electron-ion collider (EIC), based on the existing Relativistic Heavy Ion Collider (RHIC) hadron facility, with two intersecting superconducting rings, each $3.8 \mathrm{~km}$ in circumference [1244]. The replacement cost of the RHIC facility is about two billion US dollars, and the eRHIC will fully take advantage and utilize this investment. We plan adding a polarized 5-30 GeV electron beam to collide with variety of species in the existing RHIC accelerator complex, from polarized protons with a top energy of $325 \mathrm{GeV}$, to heavy fully-stripped ions with energies up to $130 \mathrm{GeV} / u$.

Brookhavens innovative design, (figure 1), is based on one of RHICs hadron rings and a multi-pass energy-recovery linac (ERL). Using the ERL as the electron accelerator assures high luminosity in the $10^{33}-10^{34} \mathrm{~cm}^{-2} \mathrm{sec}^{-1}$ range, and for the natural staging of eRHIC, with the ERL located inside the RHIC tunnel. eRHIC will provide electron-hadron collisions in up to three interaction regions. We detail eRHICs performance in subsection 7.1.2.

Since the first paper on eRHIC in 2000, its design has undergone several iterations. Initially, the main eRHIC option (the so-called ring-ring, RR, design) was based on an electron ring, with the linac-ring (LR) option as a backup. In 2004, we published the detailed "eRHIC 0th-Order Design Report" including a cost-estimate for the RR design [1245]. After detailed studies, we found that an LR eRHIC has about a 10-fold higher luminosity than the RR. Since 2007, the LR, with its natural staging strategy and full transparency for polarized electrons, became the main choice for eRHIC. In 2009, we completed technical studies of the design and dynamics for MeRHIC with 3-pass 4-GeV ERL. We learned much from this evaluation, completed a bottom-up cost estimate for this $\$ 350 \mathrm{M}$ machine, but then shelved the design.

In the same year, we turned again to considering the cost-effective, all-in-tunnel sixpass ERL for our design of the high-luminosity eRHIC (figure 7.1). In it, electrons from the polarized pre-injector will be accelerated to their top energy by passing six times through two SRF linacs. After colliding with the hadron beam in up to three detectors, the e-beam will be decelerated by the same linacs and dumped. The six-pass magnetic system with small-gap magnets [1246] will be installed from the start. We will stage the electron energy from $5 \mathrm{GeV}$ to $30 \mathrm{GeV}$ stepwise by increasing the lengths of the SRF linacs.

We considered several IR designs for eRHIC. The latest one, with a $10 \mathrm{mrad}$ crossing angle and $\beta^{*}=5 \mathrm{~cm}$, takes advantage of newly commissioned $\mathrm{Nb}_{3} S n$ quadrupoles [1247]. Subsection 7.1.3 details the eRHIC lattice and the IR layout.

The current eRHIC design focuses on electron-hadron collisions. If justified by the EIC physics, we will add a $30 \mathrm{GeV}$ polarized positron ring with full energy-injection from the 
(a)

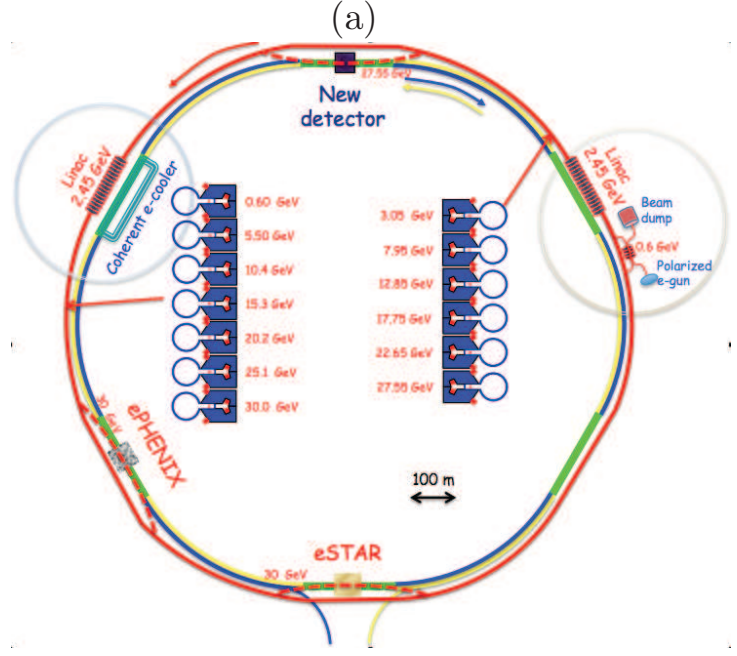

(b)

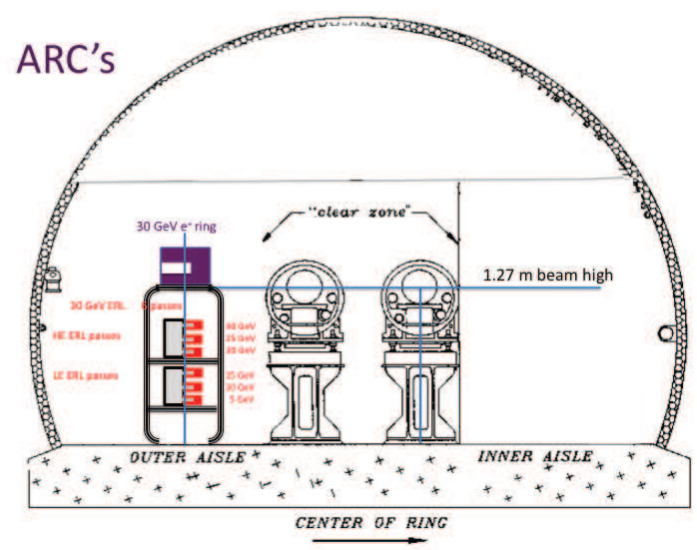

Figure 7.1. (a) Layout of the ERL-based, all-in-RHIC-tunnel, $30 \mathrm{GeV}$ x $325 \mathrm{GeV}$ high-energy high-luminosity eRHIC. (b) Location of eRHICs six recirculation arcs in the RHIC tunnel.

eRHIC ERL. This addition to the eRHIC facility provides for positron-hadron collisions, but at a significantly lower luminosity than those attainable in the electron-hadron mode.

As a novel high-luminosity EIC, eRHIC faces many technical challenges, such as generating $50 \mathrm{~mA}$ of polarized electron current. eRHIC also will employ coherent electron cooling (CeC) 1248] for the hadron beams. Staff at BNL, JLab, and MIT are pursuing vigorously an $\mathrm{R} \& \mathrm{D}$ program for resolving addressing these obstacles. In collaboration with Jlab, BNL plans experimentally to demonstrate $\mathrm{CeC}$ at the RHIC. We discuss the structure and the status of the eRHIC R\&D in subsection 7.1.4.

\subsubsection{Main eRHIC parameters}

eRHIC is designed to collide electron beams with energies from 5- to 30-GeV1 with hadrons, viz., either with heavy ions with energies from 50- to $130-\mathrm{GeV}$ per nucleon, or with polarized protons with energies between 100- and 325-GeV. Accordingly, eRHIC will cover the C.M. energy range from 44.7- to $197.5-\mathrm{GeV}$ for polarized e-p, and from 31.6- to $125-\mathrm{GeV}$ for electron heavy-ion-collisions.

Several physics and practical considerations influenced our choice of beam parameters for eRHIC. Some of these limitations, such as the intensity of the hadron beam, the space charge and beam-beam tune shift limits for hadrons, come from experimental observations at RHIC or other hadron colliders. Some of them, for example $\beta^{*}=5 \mathrm{~cm}$ for hadrons, are at the limits of current accelerator technology, while others are derived either from practical or cost considerations. For example, from considering the operational costs, we limit the electron beam's power loss for synchrotron radiation to about $7 \mathrm{MW}$, corresponding to a $50 \mathrm{~mA}$ beam current at $20 \mathrm{GeV}$. Above $20 \mathrm{GeV}$, the electron beam's current will decrease in inverse proportion to the fourth power of energy, and will be restricted to about $10 \mathrm{~mA}$ at an energy of $30 \mathrm{GeV}$. It means that the luminosity of eRHIC operating with $30 \mathrm{GeV}$ electrons will be a $1 / 5$ th of that with $20 \mathrm{GeV}$.

\footnotetext{
${ }^{1}$ There is no accelerator problem with using lower energy electron beams.. According to statements from EIC physicists, using electron energies below $5 \mathrm{GeV}$ would not contribute significantly to the physics goals.
} 
Since the ERL provides fresh electron bunches at every collision, the electron beam can be strongly abused, i.e., it can be heavily distorted during a collision. The only known effect that might cause a serious problem is the so-called kink instability. The ways of suppressing it within range of parameters accessible by eRHIC is well-understood [1249] and it no longer presents a problem.

We list below some of our assumed limits and parameters:

1. Bunch-intensity limits:

a. For protons: $210^{11}$

b. For $\mathrm{Au}$ ions: $1.210^{9}$

2. Electron-current limits:

a. Polarized current: $50 \mathrm{~mA}$

b. Un-polarized current: $250 \mathrm{~mA}$

3. Minimum $\beta^{*}=5 \mathrm{~cm}$ for all species

4. Space-charge tune shift for hadrons: $\leq 0.035$

5. Proton (ion) beam-beam parameter: $\leq 0.015$

6. Bunch length (with coherent electron-cooling):

a. Protons: $8.3 \mathrm{~cm}$ at energies below $250 \mathrm{GeV}, 4.9 \mathrm{~cm}$ at $325 \mathrm{GeV}$

b. Au ions: $8.3 \mathrm{~cm}$ in all energy ranges

7. Synchrotron radiation intensity limit is defined as that of a $50 \mathrm{~mA}$ beam at $20 \mathrm{GeV}$

8. Collision rep-rate $\leq 50 \mathrm{MHz}$.

The limitations on luminosity resulting from various considerations are involved. The main trend is that eRHIC's luminosity does not depend on the electron beam's energy (below $20 \mathrm{GeV}$ ) and reaches its maximum at the hadron beam's highest energy. We mentioned the exception for energies of electrons above $20 \mathrm{GeV}$. The top eRHIC performance for various species is shown in table 7.1 .

Table 7.2 lists the luminosity of a polarized electron-proton collision for a set of electronand proton-energies. Table 7.3 contains this information for a polarized electron beam colliding with $\mathrm{Au}$ ions, while tables 7.4 and 7.5 provide data for the case of unpolarized electrons.

An additional major parameter describing eRHIC's overall performance is its expected average luminosity. Since the plans for eRHIC are to use coherent electron cooling to control the parameters of hadron beam, its lifetime will be affected only by scattering on residual gas, and by burn-off in collisions with electrons. Hence, the hadron beam's luminosity lifetime could be as long as a few days, and, in the most likely scenario, the average delivered luminosity will be determine by the reliability of RHIC systems. Hence we anticipate that the average luminosity will be $\sim 70 \%$ of that listed in the tables.

\subsubsection{The eRHIC interaction region}

The current high-luminosity eRHIC IR design incorporates a $10 \mathrm{mrad}$ crab-crossing scheme; thus, hadrons traverse the detector at a $10 \mathrm{mrad}$ horizontal angle, while electrons go straight through. Figure 7.2 plots this scheme. The hadron beam is focused to $\beta^{*}=5 \mathrm{~cm}$ by a special triplet wherein the first magnet is a combined function magnet (1.6 $\mathrm{m}$ long with $2.23 \mathrm{~T}$ magnetic fields and a $-109 \mathrm{~T} / \mathrm{m}$ gradient). It has two functions; it focuses the hadron beam while bending it $4 \mathrm{mrad}$. Two other quadrupoles do not bend the hadron beam but serve only for focusing. Importantly, all three magnets provide zero magnetic fields along the electron beam's trajectory. Quadrupoles for this IR require very high gradients, and can be built only with modern superconducting technology [1247, 1250] 


\begin{tabular}{|c|c|c|c|c|c|}
\hline & e & $\mathrm{p}$ & ${ }^{2} \mathrm{He}^{3}$ & ${ }^{79} \mathrm{Au}{ }^{197}$ & ${ }^{92} \mathrm{U}^{238}$ \\
\hline Energy, GeV & $5-20$ & 325 & 215 & 130 & 130 \\
\hline $\mathrm{CM}$ energy, GeV & & $80-161$ & 131 & 102 & 102 \\
\hline $\begin{array}{l}\text { Number of bunches or distance be- } \\
\text { tween bunches }\end{array}$ & 74 nsec & 166 & 166 & 166 & 166 \\
\hline Bunch intensity (nucleons), $10^{11}$ & 0.24 & 2 & 3 & 3 & 3.15 \\
\hline Bunch charge, nC & 3.8 & 32 & 30 & 19 & 20 \\
\hline Beam current, $\mathrm{mA}$ & 50 & 420 & 390 & 250 & 260 \\
\hline $\begin{array}{l}\text { Normalized emittance of hadrons } \\
95 \%, \mathrm{~mm} \cdot \mathrm{mrad}\end{array}$ & & 1.2 & 1.2 & 1.2 & 1.2 \\
\hline $\begin{array}{l}\text { Normalized emittance of elec- } \\
\text { trons, rms, } m m \cdot \operatorname{mrad}\end{array}$ & & $5.8-23$ & $7-35$ & $12-57$ & $12-57$ \\
\hline Polarization, \% & 80 & 70 & 70 & none & none \\
\hline RMS bunch length, $\mathrm{cm}$ & 0.2 & 4.9 & 8.3 & 8.3 & 8.3 \\
\hline$\beta^{*}, \mathrm{~cm}$ & 5 & 5 & 5 & 5 & 5 \\
\hline $\begin{array}{l}\text { Luminosity per nucleon, } 10^{34} \\
\mathrm{~cm}^{-2} \mathrm{~s}^{-1}\end{array}$ & & 1.46 & 1.39 & 0.86 & 0.92 \\
\hline
\end{tabular}

Table 7.1. Projected eRHIC luminosity for various hadron beams at top energy.

\begin{tabular}{|c|c|c|c|c|}
\hline Plectrons & $100 \mathrm{GeV}$ & $130 \mathrm{GeV}$ & $250 \mathrm{GeV}$ & $325 \mathrm{GeV}$ \\
\hline $5 \mathrm{GeV}$ & $0.62 \cdot 10^{33}$ & $1.4 \cdot 10^{33}$ & $9.7 \cdot 10^{33}$ & $15 \cdot 10^{33}$ \\
\hline $10 \mathrm{GeV}$ & $0.62 \cdot 10^{33}$ & $1.4 \cdot 10^{33}$ & $9.7 \cdot 10^{33}$ & $15 \cdot 10^{33}$ \\
\hline $20 \mathrm{GeV}$ & $0.62 \cdot 10^{33}$ & $1.4 \cdot 10^{33}$ & $9.7 \cdot 10^{33}$ & $1.5 \cdot 10^{33}$ \\
\hline $30 \mathrm{GeV}$ & $0.12 \cdot 10^{33}$ & $0.3 \cdot 10^{33}$ & $1.9 \cdot 10^{33}$ & $3 \cdot 10^{33}$ \\
\hline
\end{tabular}

Table 7.2. Projected eRHIC luminosity (in $\mathrm{cm}^{-2} \mathrm{sec}^{-1}$ ) for polarized electron-proton collisions.

\begin{tabular}{|c|c|c|c|c|}
\hline Au ions & $50 \mathrm{GeV} / \mathrm{u}$ & $75 \mathrm{GeV} / \mathrm{u}$ & $100 \mathrm{GeV} / \mathrm{u}$ & $130 \mathrm{GeV} / \mathrm{u}$ \\
\hline $5 \mathrm{GeV}$ & $0.49 \cdot 10^{33}$ & $1.7 \cdot 10^{33}$ & $3.9 \cdot 10^{33}$ & $8.6 \cdot 10^{33}$ \\
\hline $10 \mathrm{GeV}$ & $0.49 \cdot 10^{33}$ & $1.7 \cdot 10^{33}$ & $3.9 \cdot 10^{33}$ & $8.610^{33}$ \\
\hline $20 \mathrm{GeV}$ & $0.49 \cdot 10^{33}$ & $1.710^{33}$ & $3.9 \cdot 10^{33}$ & $8.6 \cdot 10^{33}$ \\
\hline $30 \mathrm{GeV}$ & $0.1 \cdot 10^{33}$ & $0.34 \cdot 10^{33}$ & $0.8 \cdot 10^{33}$ & $1.7 \cdot 10^{33}$ \\
\hline
\end{tabular}

Table 7.3. Projected eRHIC luminosity (in $\mathrm{cm}^{-2} \mathrm{sec}^{-1}$ ) for polarized electrons and Au ions. 


\begin{tabular}{|c|c|c|c|c|}
\hline Plectrons & $100 \mathrm{GeV}$ & $130 \mathrm{GeV}$ & $250 \mathrm{GeV}$ & $325 \mathrm{GeV}$ \\
\hline $5 \mathrm{GeV}$ & $3.1 \cdot 10^{33}$ & $5 \cdot 10^{33}$ & $9.7 \cdot 10^{33}$ & $15 \cdot 10^{33}$ \\
\hline $10 \mathrm{GeV}$ & $3.1 \cdot 10^{33}$ & $5 \cdot 10^{33}$ & $9.7 \cdot 10^{33}$ & $15 \cdot 10^{33}$ \\
\hline $20 \mathrm{GeV}$ & $0.62 \cdot 10^{33}$ & $1.4 \cdot 10^{33}$ & $9.7 \cdot 10^{33}$ & $15 \cdot 10^{33}$ \\
\hline $30 \mathrm{GeV}$ & $0.12 \cdot 10^{33}$ & $0.3 \cdot 10^{33}$ & $1.9 \cdot 10^{33}$ & $3 \cdot 10^{33}$ \\
\hline
\end{tabular}

Table 7.4. Projected eRHIC luminosity $\left(\right.$ in $\left.\mathrm{cm}^{-2} \mathrm{sec}^{-1}\right)$ for polarized protons and unpolarized electrons.

\begin{tabular}{|c|c|c|c|c|}
\hline Au ions & $50 \mathrm{GeV} / \mathrm{u}$ & $75 \mathrm{GeV} / \mathrm{u}$ & $100 \mathrm{GeV} / \mathrm{u}$ & $130 \mathrm{GeV} / \mathrm{u}$ \\
\hline $5 \mathrm{GeV}$ & $2.5 \cdot 10^{33}$ & $8.3 \cdot 10^{33}$ & $11.4 \cdot 10^{33}$ & $18 \cdot 10^{33}$ \\
\hline $10 \mathrm{GeV}$ & $2.5 \cdot 10^{33}$ & $8.3 \cdot 10^{33}$ & $11.4 \cdot 10^{33}$ & $18 \cdot 10^{33}$ \\
\hline $20 \mathrm{GeV}$ & $0.49 \cdot 10^{33}$ & $1.710^{33}$ & $3.9 \cdot 10^{33}$ & $8.6 \cdot 10^{33}$ \\
\hline $30 \mathrm{GeV}$ & $0.1 \cdot 10^{33}$ & $0.34 \cdot 10^{33}$ & $0.8 \cdot 10^{33}$ & $1.7 \cdot 10^{33}$ \\
\hline
\end{tabular}

Table 7.5. Projected eRHIC luminosity (in $\mathrm{cm}^{-2} \mathrm{sec}^{-1}$ ) for unpolarized electrons and Au ions.

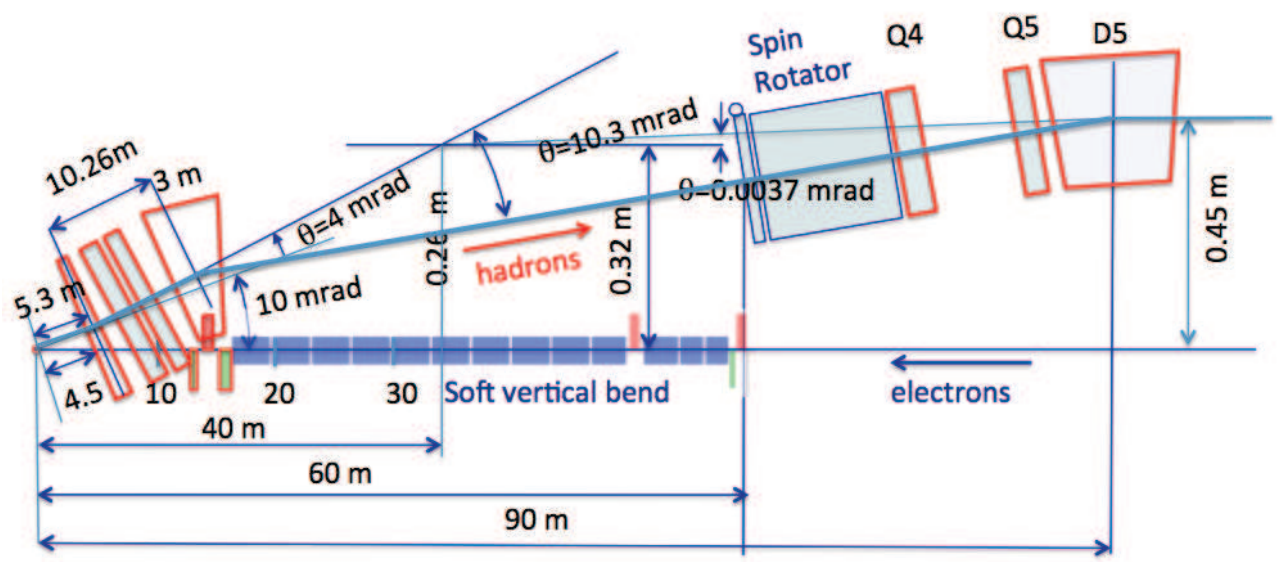

Figure 7.2. Layout of the right side of eRHIC IR from the IP to the RHIC arc. The spin rotator is the first element of existing RHIC lattice remaining in place in this IR design. 
This configuration guaranties the absence of harmful high-energy X-rays from synchrotron radiation. Furthermore, the electron beam is brought into the collision via a 130 meter long merging system (figure 7.3). The radiation from regular bending magnets would be absorbed. The last 60 meters of the merging system use only soft bends: downwards magnets have strength of $84 \mathrm{Gs}$ ( for $30 \mathrm{GeV}$ beam ) and the final part of the bend used only 24 Gs magnetic field. Only $1.9 \mathrm{~W}$ of soft radiation from the later magnets would propagate through the detector.

One important factor in the IR design with low $\beta^{*}=5 \mathrm{~cm}$ is that the chromatism of the hadron optics in the IR should be controlled, which is reflected in the maximum $\beta$-function of the final focusing quadrupoles. Figure 7.4 a shows the designed $\beta$ - and dispersion-functions for the hadron beam. The values of the $\beta$-function are kept under $2 \mathrm{~km}$, and the chromaticity held at the level typical for RHIC operations with $\beta^{*} \sim 1 \mathrm{~m}$. We are starting full-fledged tracking of hadron beams in RHIC, including characterizing beam-beam effects and all known nonlinearities of RHIC magnets: we do not anticipate any serious chromatic effects originating from our IR design.

(a)

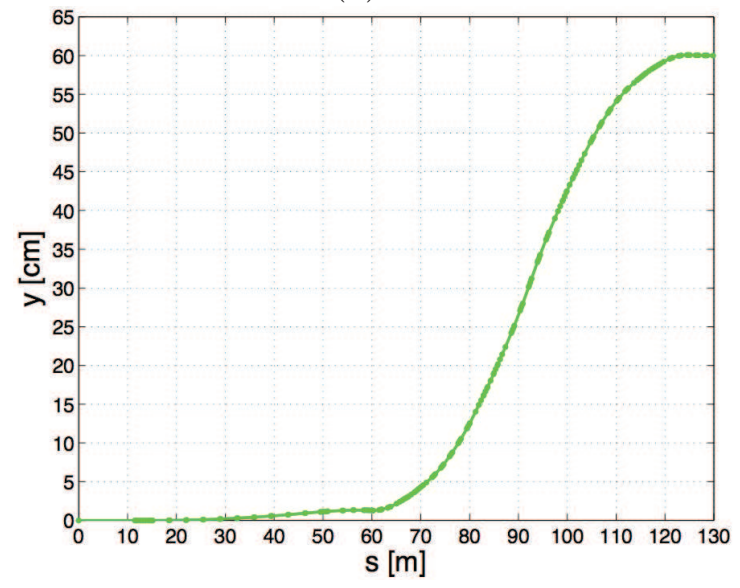

(b)

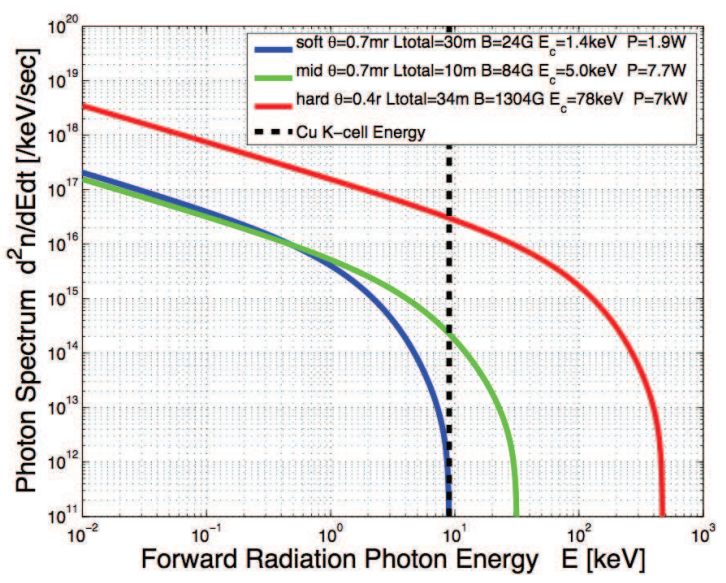

Figure 7.3. (a) Vertical trajectory of $30 \mathrm{GeV}$ electron beam merging over 130 meters into the IP. (b) Spectra of the radiation from various part of the merger. Only $1.9 \mathrm{~W}$ of soft X-ray radiation will propagate through the detector; the absorbers intercept the rest of it.

Furthermore, we introduced the bending field in the first quadrupole for the hadrons thereby to separate the hadrons from the neutrons. Physicists considering processes of interest for EIC science requested our installing this configuration. Since the electrons are used only once, the optics for them is much less constrained. Hence, it does not present any technical- or scientific-challenges, and so we omit its description here.

Finally, beam-beam effects play important roles in eRHIC's performance. While we will control these effects on the hadron beam, i.e., we will limit the total tune shift for hadrons to about 0.015 , the electron beam is used only once and it will be strongly disrupted during its single collision with the hadron beam. Consequently, the electrons are strongly focused by the hadron beam (pinch effects), and the e-beam emittance grows by about a factor of two (disruption) during the collision. These effects, illustrated in figure 7.5, do not represent a serious problem, but will be carefully studied and taken into account in designing the optics and the aperture .

More details on the lattice and IR design are given in reference [1251]. 
(a)

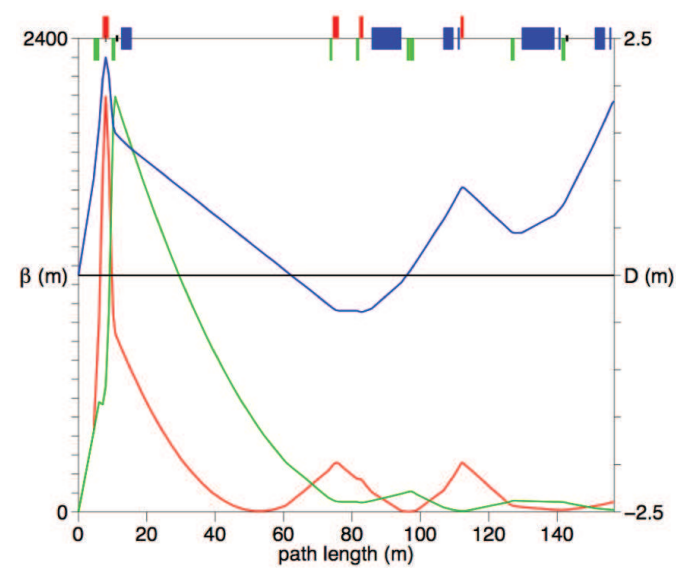

(b)

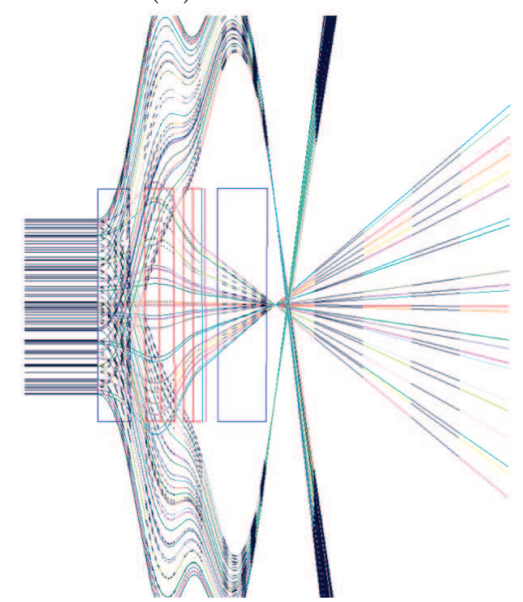

Figure 7.4. (a) Hadron beam's optics at the eRHIC IR. The $5 \mathrm{~cm} \beta^{*}$ is matched into the RHIC's arc lattice that starts about $60 \mathrm{~m}$ from the IR. (b) Tracking of hadrons with an energy deviation of $\pm 0.1 \%$ through the first four magnets at the IR.

(a)

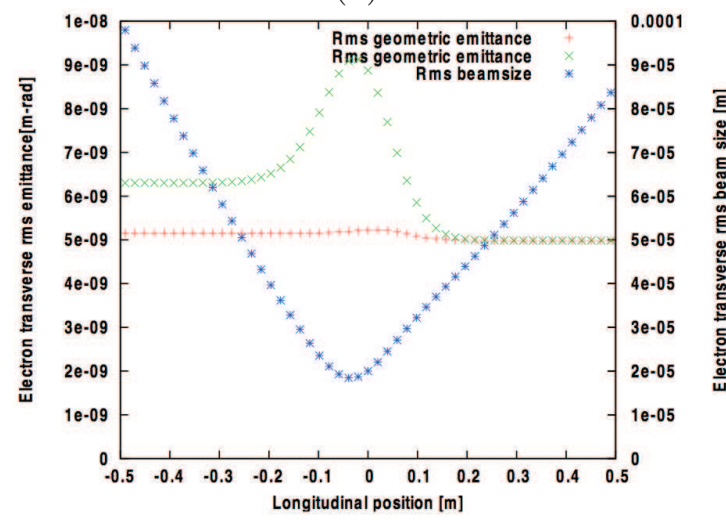

(b)

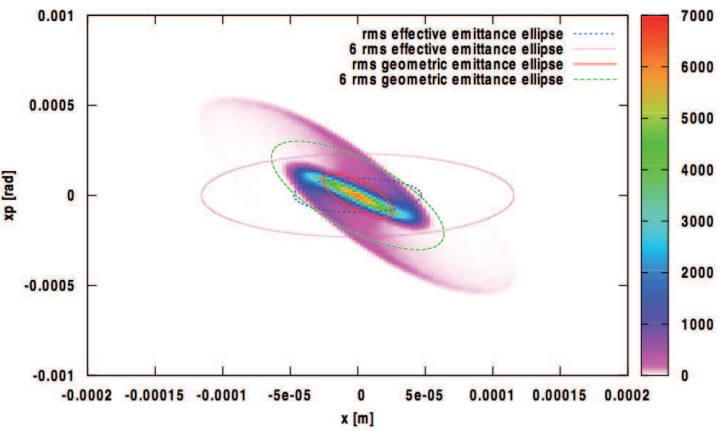

Figure 7.5. (a) The optimized e-beam envelope during collision with the hadron beam in eRHIC; (b) Distribution of electrons after colliding with the hadron beam in eRHIC.

\subsection{4 eRHIC R\&D}

The list of the needed accelerator R\&D on eRHIC is quite extensive, ranging from the $50 \mathrm{~mA}$ CW polarized source [1252, 1253, 1254] to Coherent Electron Cooling [1248]. It includes designing and testing multiple aspects of SRF ERL technology in BNL's R\&D ERL [1255].

Coherent Electron Cooling (figure (7.6) promises to cool both ion beams by an order of magnitude (both transversely and longitudinally) in under half an hour. Traditional stochastic or electron cooling techniques could not satisfy this demand. Being a novel unverified technique, $\mathrm{CeC}$ will be tested in a proof-of-principle experiment at RHIC in a collaboration between scientists from BNL, JLab, and TechX [1256].

Another important R\&D effort, supported by an LDRD grant, focuses on designing and prototyping small-gap magnets and vacuum chamber for cost-effective eRHIC arcs [1246]. In addition to their energy efficiency and inexpensiveness, small-gap magnets assure 
a very high gradient as room-temperature quadrupole magnets. Figure 7.7 shows two such prototypes; they were carefully tested and their fields were mapped using high-precision magnetic measurements. While the quality of their dipole field is close to satisfying our requirements, the quadrupole prototype was not manufactured to our specifications. We will continue this study, making new prototypes using various manufacturers and techniques.

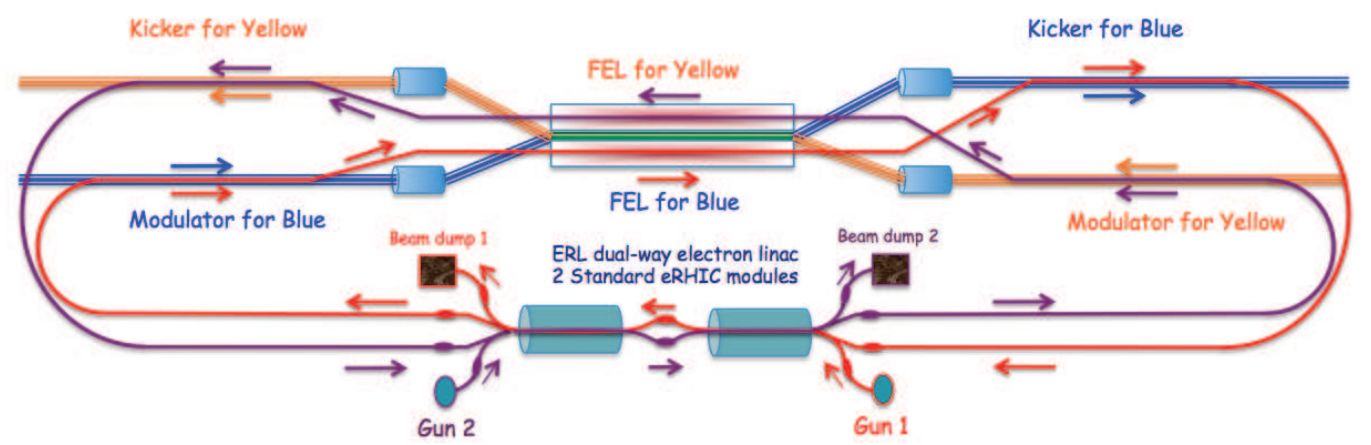

Figure 7.6. Possible layout of RHIC CeC system cooling for both the yellow and blue beams.

(a)

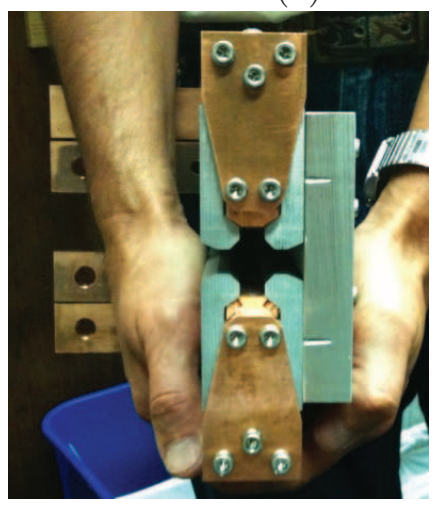

(b)

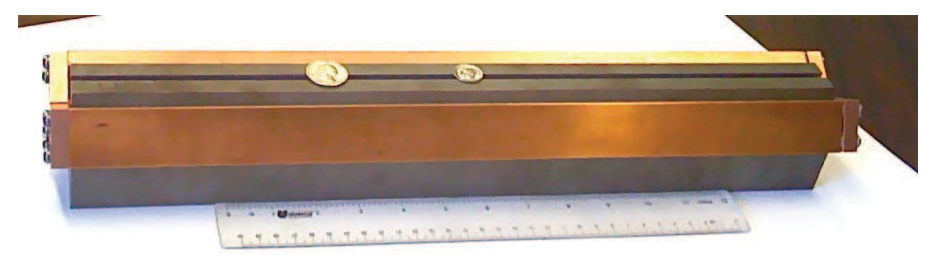

Figure 7.7. (a) A prototype of eRHIC quadrupole with $1 \mathrm{~cm}$ gap; (b) Assembled prototype of eRHIC dipole magnet with $5 \mathrm{~mm}$ gap.

Another part of our R\&D encompasses testing the RHIC in the various modes that will be required for eRHIC's operation.

\subsubsection{Conclusions and Acknowledgements}

We are making steady progress in designing the high-energy, high-luminosity electronion collider eRHIC and plan to continue our R\&D projects and studies of various effects and processes. So far, we have not encountered a problem in our proposal that we cannot resolve. Being an ERL-based collider, eRHIC offers a natural staging of the electron beam's energy from 5-6 to $30 \mathrm{GeV}$. During this year, we will complete our cost estimate of all eRHIC stages.

The authors would like to acknowledge contributions and advice from E.-C.Aschenauer, D. Bruhwiler, G. Bell, A. Cadwell, A. Deshpande, R. Ent, W. Gurin, A. Hutton, H. Kowalski, G. Krafft, M. Lamont, T. W. Ludlam, R. Milner, M. Poelker, R. Rimmer, B. Surrow, B. Schwartz, T. Ullrich, S. Vigdor, R. Venugopalan, and W. Vogelsang. 


\subsection{A Polarized Medium-Energy Electron-Ion Collider at JLab}

B. Terzić, Y. Zhang, S. Abeyratne, S. Ahmed, A. Bogacz, P. Chevtsov, Ya. Derbenev, B. Erdelyi, A. Hutton, A. Kondratenko, G. Krafft, R. Li, S. Manikonda, F. Marhauser, V. Morozov, P. N. Ostrumov, F. Pilat, R. Rimmer, T. Satogata, H. Sayed, M. Spata, M. Sullivan, H. Wang, B. Yunn

The conceptual design of MEIC, a polarized ring-ring electron-ion collider based on CEBAF, has been continuously optimized for best supporting the nuclear science program. MEIC covers a medium CM energy region up to $65 \mathrm{GeV}$ (for $6 \mathrm{~T}$ superconducting dipole magnets) and achieves a luminosity of above $10^{34} \mathrm{~cm}^{-2} \mathrm{~s}^{-1}$ for one high-luminosity and one full-acceptance detectors. The unique compact figure- 8 shaped collider rings, designed to accommodate 3 to $11 \mathrm{GeV}$ electrons and up to $96 \mathrm{GeV}$ protons or 48 (38) GeV/u for light (heavy) ions (128 GeV protons or $64(51) \mathrm{GeV} / \mathrm{u}$ light (heavy) ions for $8 \mathrm{~T}$ superconducting magnet), provide a great advantage for delivering and preserving high polarization of ion beams (including polarized deuterons) for collisions at multiple interaction points. The design is upgradable to accommodate $20 \mathrm{GeV}$ electrons and about $250 \mathrm{GeV}$ proton energies at a late stage, with luminosities up to $10^{35} \mathrm{~cm}^{-2} \mathrm{~s}^{-1}$. The present focus of the Jefferson Lab accelerator team is to develop a coherent machine design that integrates all of the design features that have been explored over recent years, based upon state-of-the-art performance criteria. Various collider components including ion linac and boosters, spin rotators, and interaction regions have been designed and integrated into a unified design. These advances will be discussed in detail.

\subsubsection{Introduction}

Over the last decade, Jefferson Lab has been developing a conceptual design of an electron-ion collider for future nuclear physics research. This facility, fully utilizing the 12 GeV upgraded CEBAF, will provide collisions between polarized electrons and polarized light ions or unpolarized light to heavy ions up to lead over a wide CM energy range at multiple interaction points (IPs). Requirements of the science programs drive the design efforts to focus on achieving ultra-high luminosity $\left(10^{34} \mathrm{~cm}^{-2} \mathrm{~s}^{-1}\right.$ or above) per detector, and high polarization (over 80\%) for both electron and light ion beams.

Our primary design focus at the present time is a Medium-energy Electron-Ion Collider (MEIC), with a CM energy up to $65 \mathrm{GeV}$, which covers electron energy up to $11 \mathrm{GeV}$, proton energy up to $96 \mathrm{GeV}$ and ion energy up to $48 \mathrm{GeV}$ per nucleon. It is considered as an optimal compromise between science, technology and project cost. We also maintain a well-defined upgrade capability to higher energies, ELIC, which can reach up to $20 \mathrm{GeV}$ electron energy, and $250 \mathrm{GeV}$ proton energy or $100 \mathrm{GeV} / \mathrm{u}$ heavy ion energies (typically, for heavy ions the proton number is about $40 \%$ of the atomic mass number). In both instances, high luminosity and high polarization remain the main design drivers.

The present MEIC design features a traditional ring-ring collider with a high luminosity at a level of $10^{34} \mathrm{~cm}^{-2} \mathrm{~s}^{-1}$ per detector, over up to three IPs, by taking full advantage of an electron beam from the upgraded $12 \mathrm{GeV}$ CEBAF recirculated SRF linac. As a design concept, the high luminosity of MEIC is attained by utilizing high bunch repetition rate, crab-crossing colliding electron and ion beams with short bunch length and small transverse 
emittances, and strong final focusing at collision points. Our choice of this luminosity concept was motivated by the remarkable success of two electron-positron colliders at KEK and SLAC B-factories, which had reached luminosities over $2 \times 10^{34} \mathrm{~cm}^{-2} \mathrm{~s}^{-1}$. In a way, Jefferson Lab is poised to replicate the same success in a collider involving hadron beams. The new concept requires the colliding ion beams of MEIC to be very different from all existing or previously operated hadron colliders in terms of bunch intensity (very small), bunch length (very short), transverse emittances (very small) and repetition frequency (very high), while, at the same time, it pushes the final focusing parameter $\beta^{*}$ to be much smaller than what has been achieved in hadron colliders. To support such a conceptual design, extensive R\&D programs have been established at Jefferson Lab, supplemented by several external collaborations.

As a design strategy, we are taking a conservative technical position by limiting many MEIC design parameters within or close to the present state-of-the-art in order to minimize technical uncertainty. This conservative technical design will form a baseline for future design optimization guided by the evolution of the science program, technology innovations and R\&D advances.

\subsubsection{Baseline Design}

The MEIC main parameters are summarized in table 7.2 .2 for a design point of $60 \mathrm{GeV}$ proton and $5 \mathrm{GeV}$ electron. Figure 7.8 presents luminosities as a function of CM energy for both proton and ions. In deriving this set of design parameters, we have imposed certain limits on several key machine or beam parameters in order to reduce technical risk and the accelerator $\mathrm{R} \& \mathrm{D}$ challenges and to improve robustness of the design. These limits, based on largely previous lepton and hadron collider experiences and state-of-art of accelerator technologies, are:

- Average current of the stored beams are up to $1 \mathrm{~A}$ for protons/ions and $3 \mathrm{~A}$ for electrons,

- Electron synchrotron radiation power density is less than $20 \mathrm{~kW} / \mathrm{m}$,

- Peak bending field of ion superconducting dipole is no larger than $6 \mathrm{~T}$,

- The maximum betatron value at the beam extension area near an IP is no larger than $2.5 \mathrm{~km}$,

- Frequency of accelerating RF cavity in the electron ring is less than $1 \mathrm{GHz}$.

Also, different nuclear programs usually require different detector acceptances and arrangement of interaction regions (IR). While such detector requirements are still in a formation stage, we have considered two different types of IR designs, one for a full-acceptance detector (with 0.5 to 179.5 degree solid angular acceptance before the ion final focusing magnets, and the apertures of the latter sufficient to allow particles with angles up to 0.5 degrees to go through the bore of the magnet for downstream detection), the other for a high-luminosity detector. The key difference of the IR designs is a space between the collision point and the location of the first final focusing quad, and values of these distances for the two detectors are $7 \mathrm{~m}$ and $4.5 \mathrm{~m}$ respectively for ion beams, while the space for electron beams can be as low as $3 \mathrm{~m}$ for both cases. The relatively short distance of $4.5 \mathrm{~m}$ enables a further reduction of the final focusing $\beta^{*}$ to $8 \mathrm{~mm}$, thus resulting in a more than a factor two increase of luminosity for that detector configuration as shown in table 7.2.2. 


\begin{tabular}{lccc} 
Quantity & Unit & $p^{-}$beam & $e$ beam \\
Beam energy & $\mathrm{GeV}$ & 60 & 5 \\
Collision frequency & $\mathrm{MHz}$ & \multicolumn{2}{c}{749} \\
Particles per bunch & $10^{10}$ & 0.416 & 2.5 \\
Beam current & $\mathrm{A}$ & 0.5 & 3 \\
Polarization & $\%$ & $>70$ & $\sim 80$ \\
Energy spread & $10^{-3}$ & 0.3 & 0.71 \\
RMS bunch length & $\mathrm{mm}$ & 10 & 7.5 \\
Horiz. emit. (norm.) & $\mu \mathrm{m}$ & 0.35 & 53.5 \\
Vertical emit. (norm.) & $\mu \mathrm{m}$ & 0.07 & 10.7 \\
Horizontal $\beta^{*}$ & $\mathrm{~cm}$ & $10(4)$ & $10(4)$ \\
Vertical $\beta^{*}$ & $\mathrm{~cm}$ & $2(0.8)$ & $2(0.8)$ \\
Vertical beam-beam tuneshift & & 0.015 & 0.03 \\
Laslett tuneshift & & 0.06 & $\mathrm{small}$ \\
Distance from IP to ${ }^{\text {st }}$ final focusing quad & $\mathrm{m}^{-2}$ & $7(4.5)$ & 3.5 \\
Luminosity per IP & $10^{33} \mathrm{~cm}^{-2} \mathrm{~s}^{-1}$ & $5.6(14.2)$
\end{tabular}

Table 7.6. MEIC design parameters for the full-acceptance detector. Values for the high-luminosity detector are given in parentheses.

The MEIC design calls for the construction of a green-field ion accelerator complex and two collider rings, one for electrons and the other for medium energy ions, as shown in figure 7.9. There are four crossing points of these figure- 8 collider rings which will accommodate three detectors, at least two of which are available for medium-energy collisions, and the other for low-energy collisions with ions stored in a large booster. As presently envisaged, the two collider rings of identical circumferences are vertically stacked and the ion beams are transported into the plane of the electron ring via a vertical chicane, where horizontal crab crossings were used to collide the two beams at the collision points.
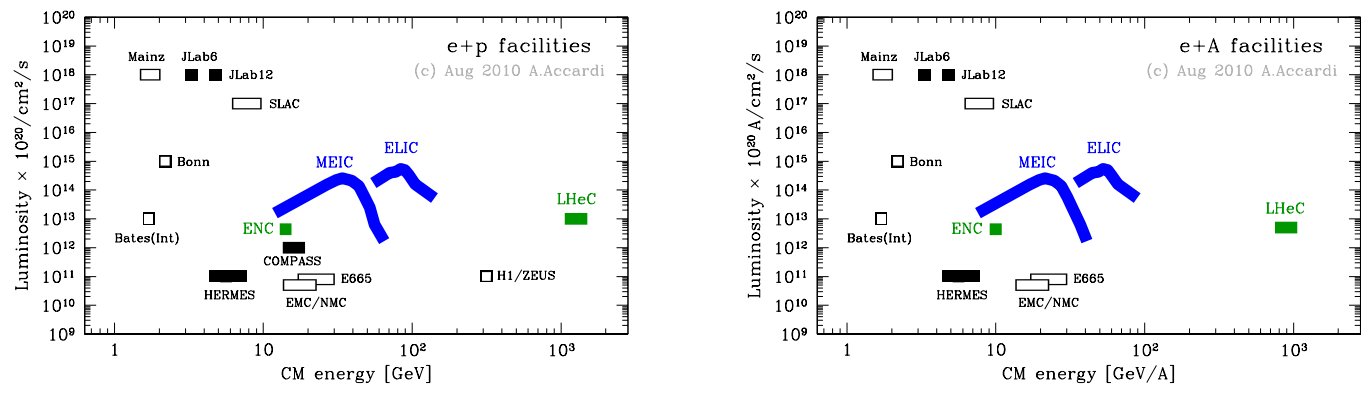

Figure 7.8. MEIC and its high-energy upgrade ELIC in CM energy-luminosity space.

The ion complex consists of ion sources, a $200 \mathrm{MeV}$ SRF linac, a $3 \mathrm{GeV}$ pre-booster and a large booster with energy up to $20 \mathrm{GeV}$. The ion beams are formed and accelerated in multiple stages in the low-energy ion complex, and are then filled into the collider ring for further acceleration to the colliding energy and stored for collision. A large figure- 8 ring, also drawn in Fig. 7.9 (in grey), accommodates high-energy ion beams in a future energy upgrade. In that case, the compact medium-energy collider ring will act as another large booster. On the electron side, a $12 \mathrm{GeV}$ upgraded CEBAF SRF linac will serve as a 
full-energy injector into the electron collider ring, which could also be operated in a top-off mode in order to maintain high beam current. It is possible to continue the fixed target program for the CEBAF whenever there is a need, since each filling of the electron ring is very short.

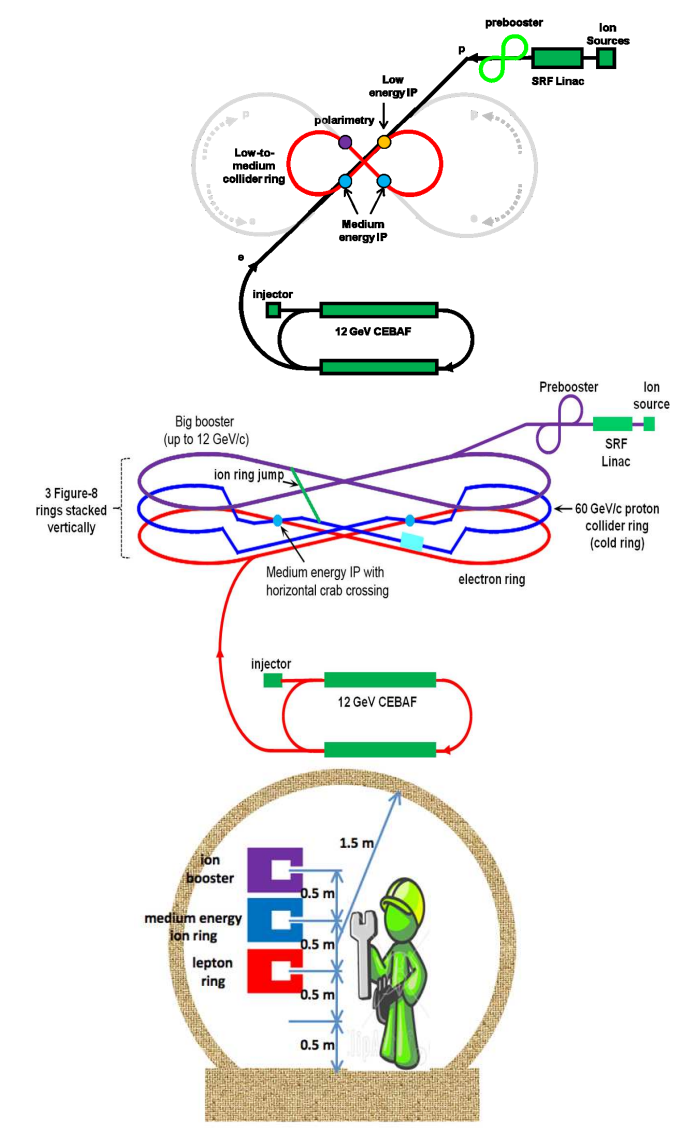

The MEICs figure- 8 shape in all rings is an optimal solution to preserve full polarization of light ion beams by avoiding spin resonances during acceleration in multiple booster and collider rings. It is also the only practical way to accelerate polarized deuterons and to arrange for longitudinal spin polarization at IP. The figure- 8 layout allows for energy independence of the spin tune, as well as the transverse polarization of deuterons.

An essential component of every version of Jefferson Lab electron-ion collider design is an electron cooling facility, which is required for reducing ion beam transverse emittance and along with strong RF bunching, shortening the bunch length to $1 \mathrm{~cm}$.

\subsubsection{Ion Complex}

Being primarily a lepton lab, Jefferson Lab does not have an ion complex at this time. This is usually considered a disadvantage to the Jefferson

Figure 7.9. Layout and side view of the MEIC and cross-section of tunnel.

Lab electron-ion collider design effort, since a new ion complex is usually more expensive than a new electron complex. However, a green-field ion complex provides an excellent opportunity for applying new concepts and accelerator technologies which have been developed, tested and perfected over the last half century. Therefore, the MEICs ion complex could, in principle, be built to be far superior to the existing or legacy hadron facilities, thereby offsetting the relatively high project cost.

The main design goal of MEIC ion complex is to create and accelerate polarized or un-polarized ion beams with appropriate time, spatial and phase space structure matching the electron beam in order to implement the new luminosity concept. It is important to note that, while, on the one hand, the MEIC design requires bunch length and transverse emittances order of magnitude smaller than that of the conventional ion beams, on the other hand, due to a high bunch repetition rate, the MEIC ion bunch intensity is unusually low (at $4 \times 10^{9}$ ), approximately 50 times smaller than RHIC ion beam, thereby drastically easing the process of forming such ion beams and intensity dependent collective beam instabilities.

The MEIC ion complex is shown in figure 7.9. Its layout also characterizes the scheme of 
ion beam acceleration and formation. The ions, coming out from the polarized or unpolarized sources, will be accelerated step-by-step to the colliding energy in the following major machine components: a $200 \mathrm{MeV}$ SRF linac, a $3 \mathrm{GeV}$ pre-booster, a $20 \mathrm{GeV}$ large booster and finally a medium-energy collider ring of 20 to $60 \mathrm{GeV}$. All rings are in figure- 8 shape for the benefit of ion polarization. We will present a brief description on each component in the rest of this section. The pre-booster is also an accumulator ring, accepting and stacking ions (0.5 to $1 \mathrm{~A}$ average current) from a SRF linac in a multi-turn injection with assistance of a conventional DC electron cooling (except the case of $\mathrm{H}^{-} / \mathrm{D}^{-}$for which a phase space paint technique will be used). The accumulated ion beam in the pre-booster will become a coasting beam and will be re-bunched later in the medium-energy collider ring in order to decouple the RF frequencies in the linac and collider rings, as well as to suppress space charge tune-shift at low-energy stage.

\section{Ion Sources}

The MEIC ion sources will rely on existing and mature technologies. We will have an Atomic Beam Polarized Ion Source (ABPIS) with Resonant Charge Exchange ionization for producing polarized light ions $\mathrm{H}^{+} / \mathrm{D}^{+}$and ${ }^{3} \mathrm{He}^{++}$. For unpolarized light to heavy ions, we will utilize Electron-Beam Ion Source (EBIS) which is current in operation at BNL. It is a realistic extrapolation, given future $R \& D$, that an ABPIS should be able to deliver $10 \mathrm{~mA}$ polarized $\mathrm{H}^{+} / \mathrm{D}^{+}$pulses at $5 \mathrm{~Hz}$ repetition frequency, over a $0.5 \mathrm{~ms}$ pulse length with a polarization better than $90 \%$. An EBIS, on the other, hand is expected to generate unpolarized ${ }^{208} \mathrm{~Pb}^{30+}$ pulses also at $5 \mathrm{~Hz}$ repetition rate and about $1.6 \mathrm{~mA}$ averaged current over a much shorter pulse length of 10 to $40 \mu \mathrm{s}$. Alternatively, an Electron Cyclotron Resonance Source (ECR) can generate heavy ion beams with similar averaged currents, but 10 to 50 times longer pulse lengths, resulting in a factor of 10 or more pulse charges. In all of these instances, the present ion source technologies should be able to meet the requirement of the MEIC.

\section{Ion Linac}

A technical design of an advanced SRF ion linac, originally developed at Argonne National Laboratory as a heavy-ion driver accelerator for Rare Isotope Beam Facility, has been adopted for the MEIC proposal. This 150-m-long linac, as shown in figure 7.10, is very effective in accelerating a wide variety of polarized and unpolarized ions from $\mathrm{H}(285 \mathrm{MeV})$ to ${ }^{208} \mathrm{~Pb}^{67+}(100 \mathrm{MeV} / \mathrm{u})$. Economic acceleration of lead ions up to $100 \mathrm{MeV} / \mathrm{u}$ requires a stripper with an optimal stripping energy of $13 \mathrm{MeV} / \mathrm{u}$. The stripping efficiency of ${ }^{208} \mathrm{~Pb}$ ion beam to the most abundant charge state $67+$ is $21 \%$.

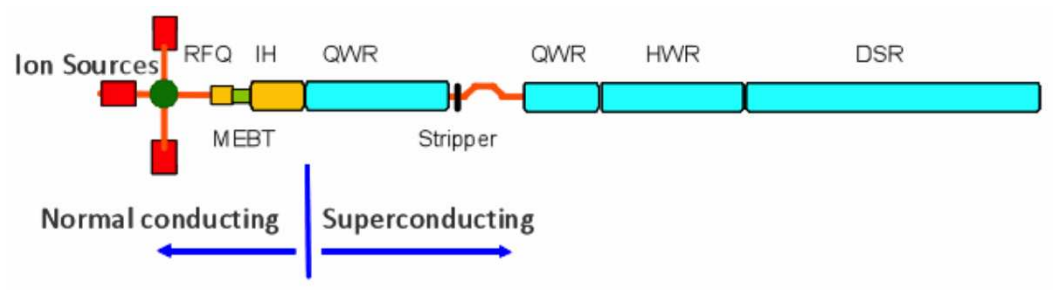

Figure 7.10. Schematic drawing of MEIC SRF linac conceptual design. 


\section{Pre-Booster/Accumulator Ring}

The pre-booster ring, as shown in figure [7.11, is an essential component of the ion accelerator complex, which accepts beam pulses of any ions from the ion linac and, after accumulation and/or acceleration, transfers the beam to the subsequent large booster for further acceleration. The exact mechanisms of pre-booster operation depend on the ion species, relying on either combined longitudinal and transverse paint technique for $\mathrm{H}^{-} / \mathrm{D}^{-}$or conventional DC electron cooling for lead or other heavy metals during multiturn injection from the SRF linac. One important design consideration of the pre-booster is sufficiently high transition gamma, such that the ions never cross the transition energy during acceleration in order to prevent associated particle loss. In addition, the betatron motion working point should be carefully chosen such that the tune footprint does not cross low-order resonances.

\begin{tabular}{|c|c|c|c|c|c|}
\hline Length & Crossing angle & Max. beam size & $\gamma$ for $3 \mathrm{GeV}$ particles & Transition $\gamma$ & Mom. compaction \\
\hline \hline $234 \mathrm{~m}$ & $75 \mathrm{deg}$ & $2.3 \mathrm{~cm}$ & 4.22 & 5 & 0.04 \\
\hline
\end{tabular}

Table 7.7. Parameters for the pre-booster ring.

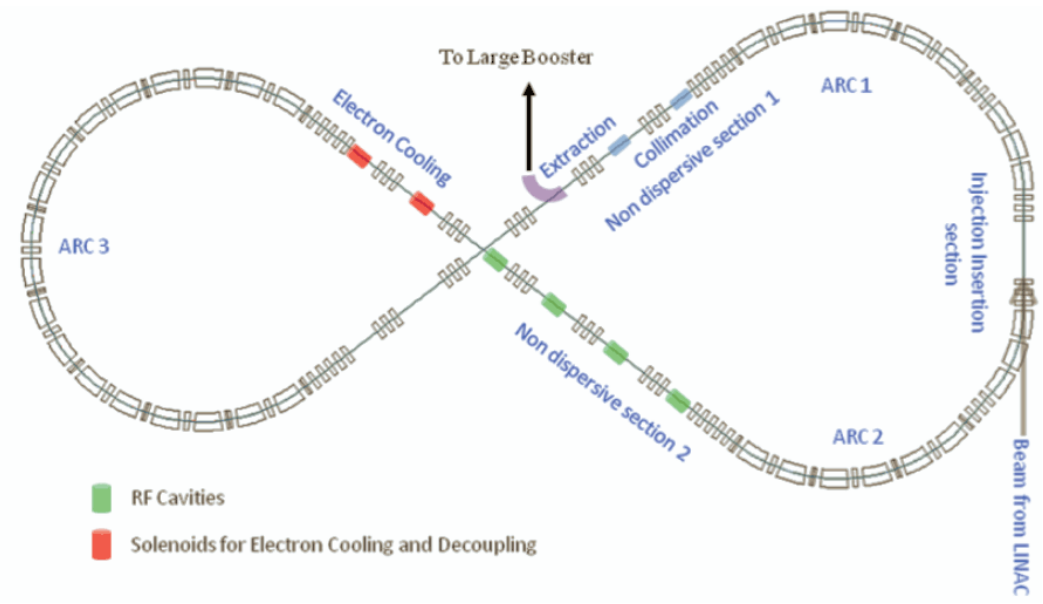

Figure 7.11. A figure-8 shaped pre-booster ring.

\section{Large Booster}

The MEIC large booster shares the same tunnel as the electron and ion collider ring. It accelerates protons from $3 \mathrm{GeV}$ to 12 to $20 \mathrm{GeV}$ before sending them to the medium-energy collider ring. The extraction energy will be determined in the further design optimization. The boosters can handle all ion species. However, the energy will be affected by the ratio of charge and mass of the ion species. In principle, higher extraction energy is preferred. The key design requirements are that the ring must be also a figure- 8 shape, its magnetic lattice should be built by warm magnets and a crossing of the transition energy must be avoided. It should be pointed out that the large booster will be also used as a low-energy collider ring for collision energies in the region of 5 to $20 \mathrm{GeV}$ per nucleon over only one detector. Special insertions such as an interaction region and an electron cooling facility must be inserted or added to meet such physics demand. 


\subsubsection{Collider Rings}

Figure 7.12 shows a scaled layout of the electron and ion collider rings. When designing the optics of the electron and ion collider rings, the following geometric constraints must be taken into account:

- Figure-8 shape, and four intersection points in two straights,

- Two short $(20 \mathrm{~m})$ straights in the middle of the two arcs for two Siberian snakes,

- A 60 m Universal Spin Rotator consisting of two solenoids and two sets of arc bending dipoles on each end of two electron arcs,

- Layouts of the interaction regions must match for both electron and ion rings,

- Footprints of the two collider rings must be very close so that they can be housed in one tunnel,

- The ion ring circumference must be equal to the big boosters length and be an integer multiple of the pre-boosters length for the purpose of RF matching.

The circumferences of the electron and ion rings are about $1340 \mathrm{~m}$. The circumferences can be modified by adjusting length of straights, crossing angle and arc radii. The figure- 8 crossing angle is $60^{\circ}$. The electron and ion rings IPs coincide. The maximum separation of the electron and ion beam lines in the design is less than $4 \mathrm{~m}$, which can be further reduced to be within the required limits once the optics design concept is finalized. The parameters of the electron ring and optics design are summarized in table 7.8 .

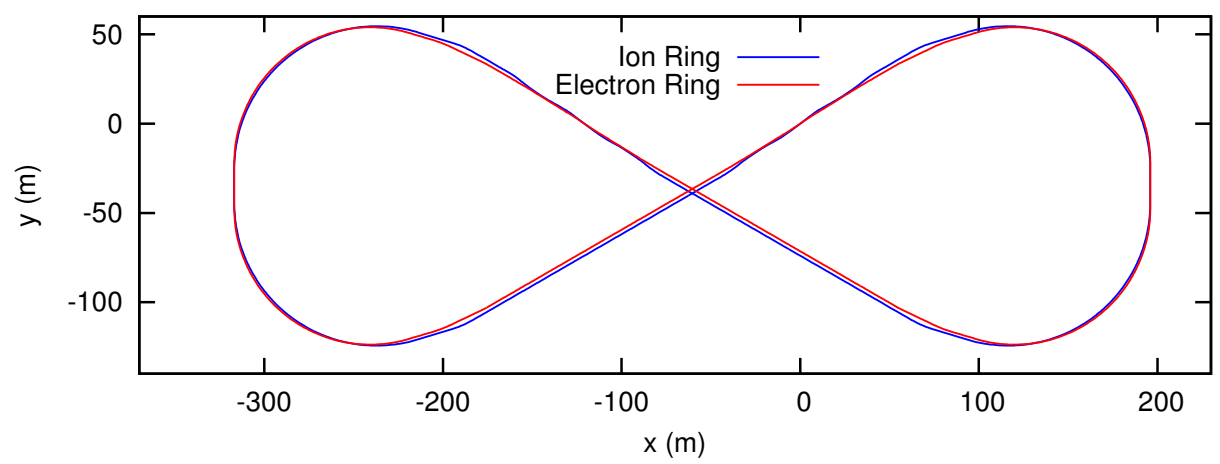

Figure 7.12. Layout of the electron and ion collider rings.

\begin{tabular}{|c|c|c|c|c|c|}
\hline Arc's net bend & Crossing angle & Arc length & Arc avg. radius & straight length & circumference \\
\hline \hline $240 \mathrm{deg}$ & $60 \mathrm{deg}$ & $405.75 \mathrm{~m}$ & $96.86 \mathrm{~m}$ & $264.46 \mathrm{~m}$ & $1340.41 \mathrm{~m}$ \\
\hline
\end{tabular}

Table 7.8. Parameters for the collider ring.

\subsubsection{Interaction Region}

\section{Detector}

The primary detector of the MEIC will be unique in its ability to provide almost full acceptance for the produced particles of electron-ion collisions. To accomplish this, a high 
level of integration with the interaction region of the accelerator is required. The central detector will be built around a solenoid barrel, providing tracking, particle identification, and calorimetry for all particles, and two end-caps focusing on the detection of electrons and hadrons respectively. See section 7.3 for further discussion.

\section{Crab Crossing}

With a $749 \mathrm{MHz}$ bunch repetition rate for both colliding beams of MEIC, the bunch spacing is about $40 \mathrm{~cm}$. A crab crossing of the colliding beams provides a simple way to separate quickly the two colliding beams near an IP to avoid undesired parasitic collisions. In the present MEIC IR design, the horizontal crabbing angle is $50 \mathrm{mrad}$. There are two ways currently under consideration to tilt the orientation of the electron or ion bunches in horizontal plane by a half crab crossing angle in order to restore head-on collisions. The first approach is placing crab cavities on each side of an IP. Such approach has been proved recently at KEK-B factory, which led to a record-high luminosity. It has been estimated that we need approximately 1.26 and 16.2 MV integrated transverse kicking voltage for 5 $\mathrm{GeV}$ electron and $60 \mathrm{GeV}$ protons respectively. While a KEK-type squashed crab cavity should be readily adopted for the MEIC electron ring, the ion ring needs a set of such KEK crab cavities to achieve the design goal. At Jefferson Lab, a new type of crab cavity, which is more compact and promises much higher field, has been recently conceptually designed using transverse electromagnetic field (TEM), as another candidate of crab cavities for the MEIC. An alternative approach is dispersive crabbing, in which tilting of a bunch is achieved through purposely leaking the horizontal dispersion in the normal accelerating RF cavities.

\section{Interaction Region Design}

As mentioned earlier, the two collider rings of MEIC are stacked vertically. In an early version of the IR design, the electron beam was vertically bent into a crab crossing, while the ion ring remains in a plane. Such design layout was abounded due to several problems: (i) bending electrons will generate excessive synchrotron radiation near an IP, which, consequently, interferes with the detector and degrades the background; (ii) the synchrotron radiation and quantum excitations in a beam extension area (betatron values are still very large) could enlarge the electron beam emittance by an order of magnitude; (iii) polarization decrease caused by vertical bend could be also very significant. The present MEIC IR design now requires the ions to undergo a vertical excursion to facilitate a horizontal crab crossing at an IP. For a 50 mrad crab crossing angle, the required bending field of the dipoles is quite modest for our ion energy range.

A typical magnet lattice layout of the MEIC interaction region is illustrated in figure 7.42 At a medium-energy region, the ion beams are modestly asymmetric in two transverse dimensions, as a result of balance of electron cooling and intra-beam scatterings. For instance, the emittance aspect ratio of a $60 \mathrm{GeV}$ proton bunch is about 5 . For such modestly flat beams, a final focusing quad doublet is a good choice.

For the MEIC IR design with a $2 \mathrm{~cm}$ or less $\beta^{*}$, chromatic aberration of the final focusing quads is one important issue that special attention must be paid. The chromaticity, defined as a ratio of betatron tune shift and momentum spread, could be as high as 110 per IR. A dedicated chromaticity compensation block, consisting of a set of sextupoles, will be inserted in the beam extension area on both sides of an IR to mitigate the problem. The initial studies indicate that, with proper values of these sextupoles, the chromaticity can be 
reduced dramatically to single digits. Particle tracking simulations for dynamic aperture are currently underway.

\subsubsection{Electron Cooling}

Cooling of the ion beam is essential to achieve high luminosity in MEIC. At low energy, DC electron cooling is employed to help stacking of the ions in the pre-booster. At the collider ring, we rely on a concept of staged cooling of bunched ion beams of medium energies. Electron cooling is first called in the injection energy for reduction of the area that the ion beam occupies in the $6 \mathrm{D}$ phase space. After ions are accelerated to the collision energy, electron cooling will be utilized again for conditioning the beam to the design values. And most importantly, electron cooling will be continued during the collision mode to suppress the intra-beam scattering induced beam heating and emittance growth. Shortening the bunch length (down to $1 \mathrm{~cm}$ or less) that results from electron-cooling of the ion beam captured in a high voltage SRF field, in particular, it is critical for the high luminosity in MEIC, since it facilitates two important advances: an extreme focusing of the colliding beams and implementation of crab crossing at the IPs for achieving the highest bunch collision rate (up to $1.5 \mathrm{GHz}$ ) and luminosity.

A schematic drawing of the MEIC ERL based electron cooler is shown in figure 7.13 , Two technologies, namely, energy recovery linac (ERL) and circulator ring, play critical roles to the success of this facility. A high-charge electron bunch from a photo-cathode is accelerated in a SRF ERL linac to required energy 10 to $50 \mathrm{MeV}$ and then sent to a specially designed circulator cooler ring, with optics matching the cooling channel for cooling of a proton or ion bunch. The photo-injector and SRF linac ensure a high quality of the injected cooling bunch. An individual bunch circulates a large number of revolutions (up to a few hundred) in the ring before its quality is degraded by intra- and inter-beam scatterings, after which it returns to the same SRF linac for energy recovery. The recovered energy will be used for accelerating a new electron bunch. This circulator ring reduces the average current from a photo-cathode by a factor equal to the number of recirculation. Therefore, it provides a near-perfect solution for two bottlenecks of the facility: the high current and high power of the cooling electron beam. For example, a $3 \mathrm{~A}, 50 \mathrm{MV}$ (e.g. $150 \mathrm{MW}$ of power) cooling beam can effectively be provided by $30 \mathrm{~mA}, 2 \mathrm{MV}$ (e.g $60 \mathrm{~kW}$ of active beam power) from the electron injector.

\subsubsection{R\&D}

For an advanced accelerator design like MEIC, there are many R\&D issues needed to be completed to solidify the design. We have identified a list of critical R\&D issues: electron cooling of the bunch ion beams at medium energy; crab crossing and crab cavity; polarization life time and spin tracking; beam-beam effects; non-linear collective beam effects and feedback systems; interaction region design and dynamic aperture, etc. The Jefferson Lab accelerator team and its collaborators are currently working on each of these issues. The details of the ongoing research are reported in a number of publications: overall design [1257, 1258, lattice design [1259, 1260, ion complex [1261, 1262], beam-beam simulations [1263, 1264, crab cavity [1265, electron cloud [1266], beam instabilities [1267], and others. For brevity, here we only highlight one topic from our R\&D list - beam-beam simulations.

Beam-beam interactions present a key limitation to collider performance, because they may lead to appreciable emittance growth of colliding beams and rapid reduction of lumi- 


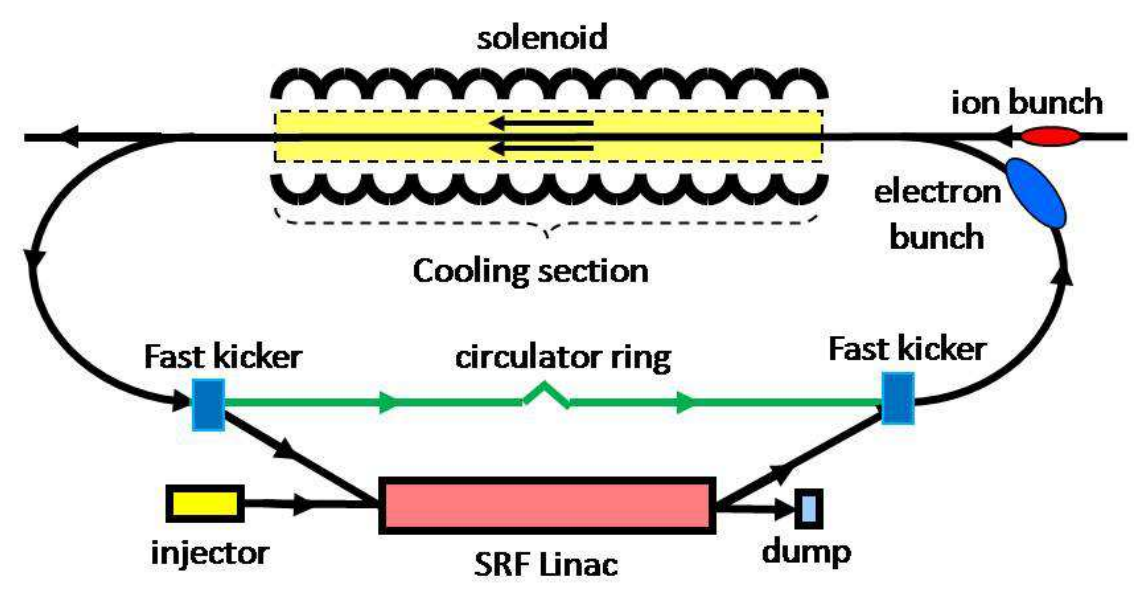

Figure 7.13. Schematic of electron cooling for the MEIC.

nosity. Such nonlinear collective beam effects can pose a significant design challenge when the machine parameters are pushed into a new regime. In order to lend credibility to the conceptual design, we use computer simulations to examine beam-beam instabilities, to optimize and explore limits of machine parameters.

A first phase of the beam-beam simulations of MEIC at Jefferson Lab, featuring a simplified model with linear transfer map, head-on collisions, and perfect chromaticity correction, has been already carried out for the current medium-energy configuration [1263, 1264, 1268] using the state-of-the-art beam collision code BeamBeam3D [1269]. These studies established that both designs were safely away from coherent beam-beam instabilities.

Furthermore, we use an evolutionary (genetic) algorithm [1270, 1271] to search for the optimal working point in the tune space, and demonstrated that such an approach is orders of magnitude more efficient than the simple tune scans [1272]. Figure 7.14 illustrates how the evolutionary algorithm successfully navigates the 4D betatron tune space (2 tunes for each beam) to find a (near-)optimal working point for which the luminosity exceeds the design luminosity by about $30 \%$.

\subsubsection{Summary}

The MEIC is the future of nuclear physics at Jefferson Lab. It is optimized to collide a wide variety of polarized light ions and unpolarized heavy ions with polarized electrons. It covers an energy range matched to the science program proposed by the Jefferson Lab nuclear physics community $\left(\sim 4200 \mathrm{GeV}^{2}\right)$, with luminosity exceeding $10^{34} \mathrm{~cm}^{-2} \mathrm{~s}^{-1}$. An upgrade path to higher energies $\left(250 \times 20 \mathrm{GeV}^{2}\right)$ has been developed and should provide luminosity of close to $10^{35} \mathrm{~cm}^{-2} \mathrm{~s}^{-1}$. The design is based on a figure- 8 ring for optimum polarization, and an ion beam with high repetition rate, small emittances and short bunch lengths.

We reported on the status of the design for the MEIC at Jefferson Lab. Our design is both mature, having addressed all the required aspects of the design in the various level of detail, and flexible, being able to accommodate revisions in design specifications and advances in accelerator $\mathrm{R} \& \mathrm{D}$. We have identified the critical accelerator $\mathrm{R} \& \mathrm{D}$ topics for the MEIC, and are presently working on them. 


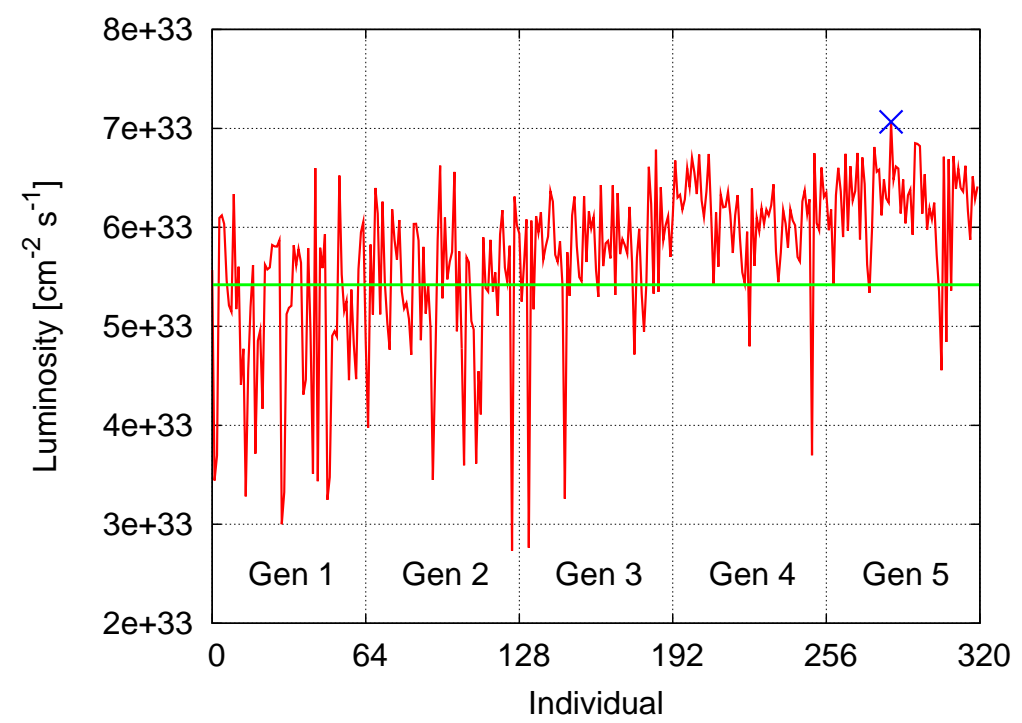

Figure 7.14. MEIC beam-beam simulation with Evolutionary algorithm. Green line represents the design luminosity. The simulation locates a (near-)optimal working point within only 320 simulations (blue $\mathrm{x}$ ).

\section{Acknowledgment}

We would like to thank members of the Jefferson Lab EIC nuclear science study group as well as the CEBAF user community for working with us on developing the MEIC design. In particular, we are grateful to Rolf Ent for coordinating the collaboration between the accelerator and nuclear physics groups, Alberto Accardi for generating Figure 7.14, Pawel Nadel-Turonski and Tanja Horn for their contributions on detector and interaction region design. 


\title{
7.3 Kinematics and detector designs for the different EIC machine designs
}

\author{
E.C. Aschenauer, R. Ent, T. Horn, P. Nadel-Turonski, H. Spiesberger
}

\subsubsection{Kinematics and Requirements for an EIC Detector}

The physics program of an EIC imposes several challenges on the design of a detector, and more globally, the extended interaction region, as it spans a wide range in center-of-mass energy, different combinations of both beam energy and particle species, and several different physics processes. The various physics processes encompass inclusive measurements $\left(e p / A \rightarrow e^{\prime}+X\right)$, which require detection of the scattered lepton and/or the full scattered hadronic debris with high precision; semi-inclusive processes $\left(e p / A \rightarrow e^{\prime}+h+X\right)$, which require detection in coincidence with the scattered lepton of at least one (current or target region) hadron; and exclusive processes $\left(e p / A \rightarrow e^{\prime}+N^{\prime} / A^{\prime}+\gamma / m\right)$, which require detection of all particles in the reaction. The following figures in this section demonstrate the differences in particle kinematics of some representative examples of these reaction types, as well as differing beam energy combinations. For these plots, the directions of the beams are defined as for HERA at DESY: the hadron beam is in the positive $\mathrm{z}$ direction $\left(0^{\circ}\right)$ and the lepton beam is in the negative z-direction $\left(180^{\circ}\right)$. The upper panel of fig. 7.15 illustrates that the lower $\mathrm{Q}^{2}$ is, the closer the momentum of the scattered lepton is to the original lepton beam energy. For all lepton-hadron beam energy combinations (indicated by the panel in each of the plots), the scattered lepton goes in the direction of the original lepton beam for low $\mathrm{Q}^{2}$ and more and more into a central detector acceptance for higher $\mathrm{Q}^{2}$. For a fixed hadron beam energy the lepton scattering angle becomes smaller at a fixed $\mathrm{Q}^{2}$ with increasing lepton energy.

Fig. 7.16 shows the $\mathrm{x}-\mathrm{Q}^{2}$ plane for two different center-of-mass energies. In general, the correlation between $x$ and $Q^{2}$ for a collider environment is weaker than for fixed target experiments. Nonetheless, it becomes stronger for small scattering angles or corresponding small inelasticity $y$, and momentum and scattering angle resolution for the scattered lepton become an issue, at HERA roughly at $y=0.1$. To circumvent this problem, HERA reconstructed the lepton kinematics from the hadronic final state using the Jacquet-Blondel method [1273, 1274], and has reached successful measurements down to $y$ of 0.005. The main reason why this hadronic method renders better resolution at low $y$ follows from the equation $y_{J B}=E-P_{z}^{h a d} / 2 E_{e}$, where $E-P_{z}^{\text {had }}$ is the sum over the energy minus the longitudinal momentum of all hadronic final-state particles and $E_{e}$ is the electron beam energy. This quantity has no degradation of resolution for $y<0.1$ as compared to the electron method, where $y_{e}=1-\left(1-\cos \theta_{e}\right) E_{e}^{\prime} / 2 E_{e}$. This is directly correlated to the relative resolutions for both quantities: $\Delta y_{J B} / y_{J B} \sim$ constant and $\Delta y_{e} / y_{e} \sim 1 / y_{e}$.

Typically, one can obtain for a given center-of-mass energy squared roughly a decade of $Q^{2}$ reach at fixed $x$ when using only an electron method to determine lepton kinematics, and roughly two decades when including the hadronic method. If only using the electron method, one can increase the range in accessible $Q^{2}$ by lowering the center-of-mass energy, as can be seen from comparing the two panels of fig. 7.16. This may become relevant for some semi-inclusive and exclusive processes. The advantages and disadvantages of this solution are discussed in the two machine-specific detector sections of this section.

In general, one would like to access as large a range in $Q^{2}$ at fixed $x$ as possible for a given beam energy combination, and reach as low $y_{J B}$ as possible. This requirement 

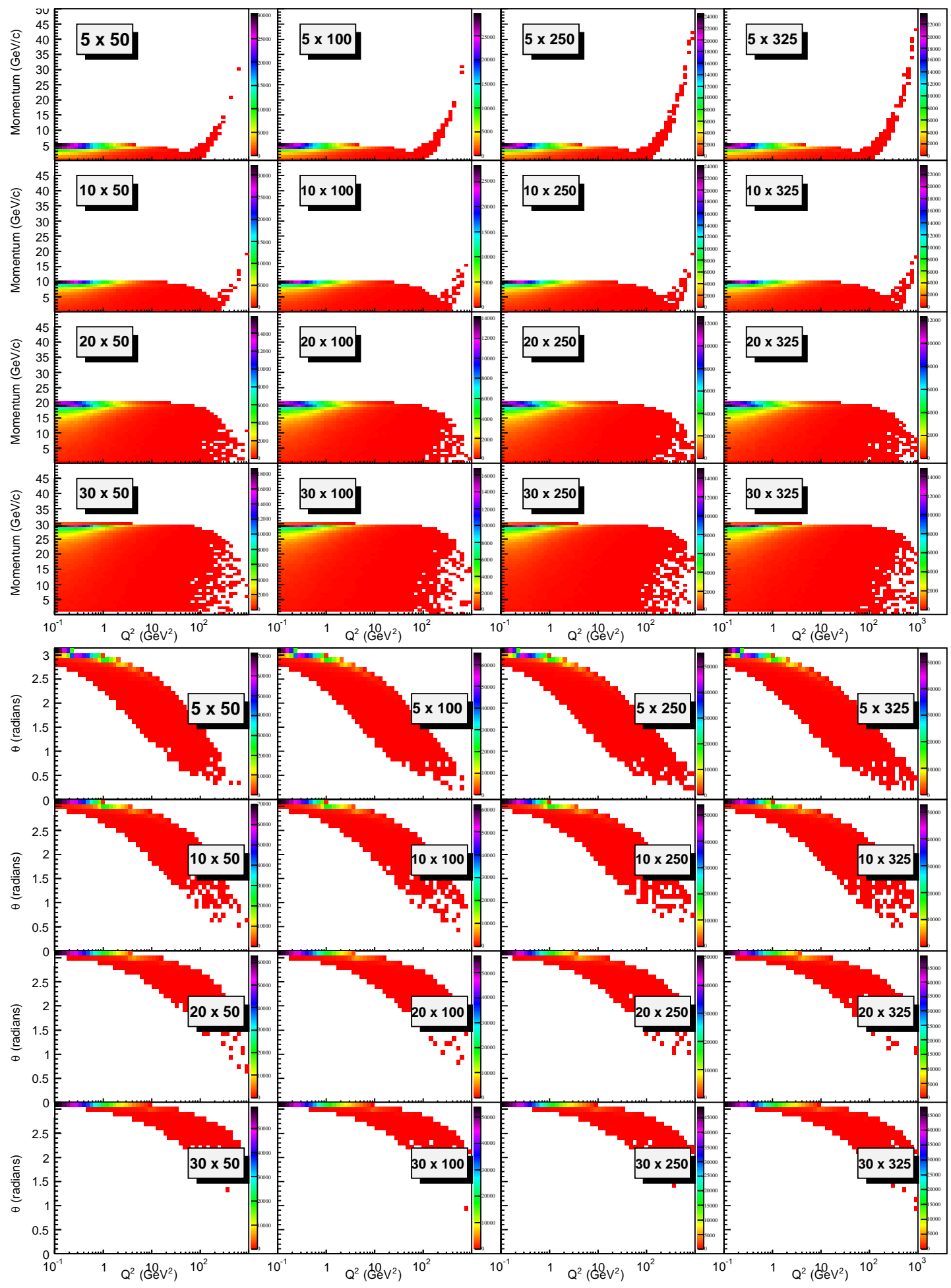

Figure 7.15. $\mathrm{Q}^{2}$ vs. momentum (upper panel) and $\mathrm{Q}^{2}$ vs. scattering angle (lower panel) of the scattered lepton in the laboratory frame. The following cuts have been applied in both figures: $\mathrm{Q}^{2}>0.1 \mathrm{GeV}^{2}, 0.01<\mathrm{y}<0.95$. The lepton-hadron beam energy combinations are indicated by the panel in each individual plots 
directly implies two important considerations for the detector design:

- good hadronic coverage in the forward direction

- low noise and/or good noise suppression algorithms in the hadronic calorimeter to allow for hadron detection down to $0.5 \mathrm{GeV}$. More detailed detector simulations are needed to confirm these requirements.
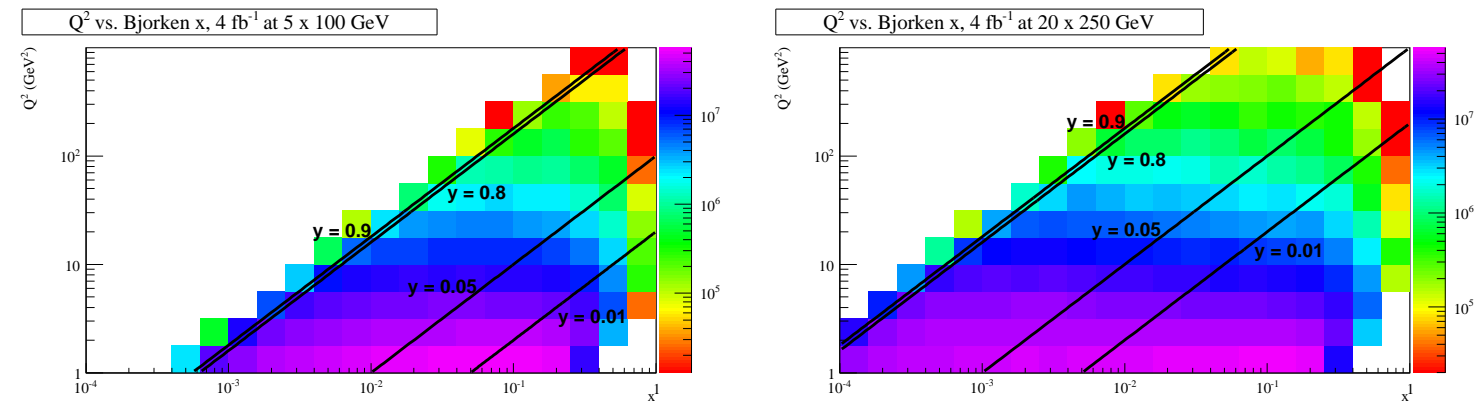

Figure 7.16. The $\mathrm{x}-\mathrm{Q}^{2}$ plane for center-of-mass energy $45 \mathrm{GeV}$ (left) and $140 \mathrm{GeV}$ (right). The black lines indicate different y-cuts placed on the scattered lepton kinematics.

It is important to point out that the reconstruction of the event kinematics from the hadronic final state is also important in suppressing events with radiation of a real or virtual photon from the incoming or outgoing lepton (radiative corrections); for details please see section 7.3 .2 ,

One should keep in mind that there are additional complications at low $y$ for the measurement of asymmetries and/or polarized cross sections, to for example extract the helicitydependent parton distributions. A depolarization factor, defined in [1275] as:

$$
D=\frac{y\left[\left(1+\gamma^{2} y / 2\right)(2-y)-2 y^{2} m_{e}^{2} / Q^{2}\right]}{y^{2}\left(1-2 m_{e}^{2} / Q^{2}\right)\left(1+\gamma^{2}\right)+2(1+R)\left(1-y-\gamma^{2} y^{2} / 4\right)}
$$

is needed to correct the measured helicity-dependent asymmetries $\left(A_{\|}\right)$. The depolarization factor corrects for the polarization transfer from the lepton to the virtual photon, and is small at low $y$. This reduces the effective polarized luminosity and increases the uncertainties of the measured polarized quantities at low y $\left(\delta A_{1}=\delta A_{\|} / D\right)$. Therefore, the $x-Q^{2}$-plane of precision polarized cross section measurements will be reduced as compared to unpolarized ones, for fixed center-of-mass energy.

Fig. 7.17 shows the momentum versus scattering angle distributions in the laboratory frame for pions originating from semi-inclusive reactions, for different lepton and proton beam energy combinations. For lower lepton energies, pions are scattered more in the forward (ion) direction. For fixed low lepton energy of $5 \mathrm{GeV}$, this pattern remains more or less constant as a function of proton energy. With increasing lepton beam energy, the hadrons increasingly populate the central region of the detector, and at the highest lepton energies, hadrons are even largely produced going backward (i.e. in the lepton beam direction). The kinematic distributions for kaons and protons, applying the same cuts as for pions, are essentially identical to those of the pions. The distributions for semi-inclusive events in electron nucleus collisions may be slightly altered due to nuclear modification effects, but the global features will remain. 


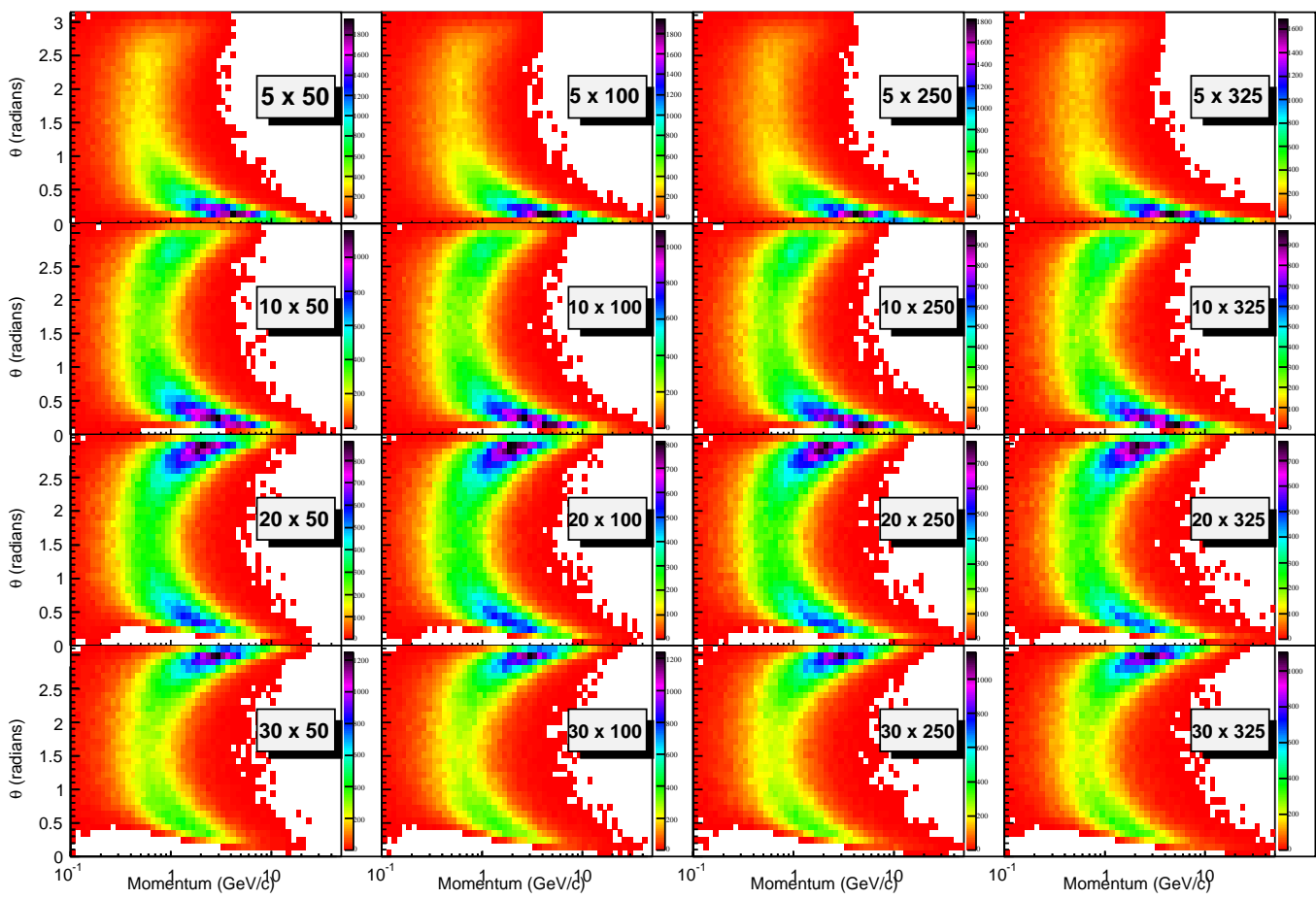

Figure 7.17. Momentum vs. scattering angle in the laboratory frames for pions from non-exclusive reactions. The following cuts have been applied: $\mathrm{Q}^{2}>1 \mathrm{GeV}^{2}, 0.01<\mathrm{y}<0.95$ and $0.1<\mathrm{z}<0.9$

Fig. 7.17 also indicates a shift of the momentum range of pions towards higher momenta in the central-angle region for higher lepton energy, to typical momenta of about $10 \mathrm{GeV} / \mathrm{c}$, which has implications for the required particle identification (PID). To be able to identify the different hadron types over a wide momentum and angular range an EIC detector needs to have detectors capable of good PID in the forward, central and backward direction. For the higher hadron momenta, typically in the forward ion direction and also in the backward direction for higher lepton beam energies, the most viable detector technology is a RingImaging Cherenkov (RICH) detector with dual-radiators. In the central detector region a combination of high resolution time-of-flight ( ToF) detectors (preferentially with timing resolutions $\delta \mathrm{t} \sim 10 \mathrm{ps}$ ), a DIRC, or a proximity focusing Aerogel RICH may be adequate detector technologies.

For certain kinematics, the hadrons (both charged and neutral) will be produced in the backward ion direction (see fig. 7.18) and need to be disentangled from the scattered leptons. The kinematic region in rapidity $\eta$, over which hadrons and photons need to be suppressed with respect to electrons, shifts to more negative rapidity with increasing centerof-mass energy. This can be cross-correlated with the angular and momentum patterns for scattered leptons of fig. 7.15. For the lower center-of-mass combination, electron, photon and charged hadron rates are roughly comparable at $1 \mathrm{GeV} / \mathrm{c}$ total momentum and $\eta=$ -3. For the higher center-of-mass energy, electron rates are a factor of 10-100 smaller than photon and charged hadron rates, and comparable again at a $10 \mathrm{GeV} / \mathrm{c}$ total momentum.

This adds another requirement to the detector: good electron identification. The kinematic region in rapidity $\eta$ over which hadrons and also photons need to be suppressed, typically by a factor of 10 - 100, shifts to more negative rapidity with increasing center- 
of-mass energy. Measuring the ratio of the lepton energy and momentum, $\mathrm{E}_{e}^{\prime} / \mathrm{p}_{e}^{\prime}$, typically gives a reduction factor of $\sim 100$ for hadrons. This requires the availability of both tracking detectors (to determine momentum) and electromagnetic calorimetry (to determine energy) over the same rapidity coverage. This availability also immediately suppresses the misidentification of photons in the lepton sample, by requiring that a track must point to the electromagnetic cluster. Of course, the availability of good tracking detectors over similar coverage as electromagnetic calorimetry similarly aids in $y$ resolution at low y from a lepton method only (see earlier), as the angular as well as the momentum resolution for trackers are much better than for electromagnetic calorimeters. The hadron suppression can be further improved by adding a Cherenkov detector to the electromagnetic calorimetry. Combining the electromagnetic calorimeter response and the response of Cherenkov detectors may especially help in the region of low-momentum scattered leptons, about 1 $\mathrm{GeV} / \mathrm{c}$. Other detector technologies, such as transition radiation detectors, may provide another factor 100 hadron suppression for lepton momenta greater than $4 \mathrm{GeV} / \mathrm{c}$.

An additional advantage of a collider detector over a fixed target experiment is the large coverage in transverse momentum. This is especially important for measurements linking the perturbative high-transverse momentum $p_{T}$ region to the region of small transverse momentum, $p_{T} \sim \Lambda_{Q C D}$, where single-spin asymmetries as functions of $p_{T}, x, Q^{2}, z$ and $\phi$ are the prime observable to extract TMDs - Transverse Momentum Dependent Parton Distributions (see chapter 2), like the Sivers function. Fig. 7.19 shows the coverage in hadron $\mathrm{p}_{T}$ measured with respect to the virtual photon vs. $z=E_{h} / \nu$ assuming an angular acceptance of a detector $0.5^{\circ}<\theta<179.5^{\circ}$. One can see that for all beam energy combinations a large range in transverse momentum is achievable. In general, such physics does not drive the most forward (or backward) detector requirements, leaving ample phase space in transverse momentum with respect to the virtual-photon direction - typically more central.

There is specific interest in detecting events with heavy quarks (charm or bottom). To measure the inclusive structure functions, $\mathrm{F}_{2}^{c}, \mathrm{~F}_{L}^{c}$, and $\mathrm{F}_{2}^{B}$ for heavy quarks, it is sufficient to tag the charm and the bottom quark content via the detection of additional leptons (electron, positron, muons) to the scattered lepton. The leptons from charmed mesons can be identified via a displaced vertex of the second lepton $(<\tau>\sim 150 \mu \mathrm{m})$. This can be achieved by integrating a high-resolution vertex detector into the detector design. For measurements of the charmed (bottom) fragmentation functions, or to study medium modifications of heavy quarks in the nuclear environment, at least one of the charmed (bottom) mesons must be completely reconstructed to have access to the kinematics of the parton. This requires, in addition to measuring the displaced vertex, good particle identification to reconstruct the meson via its hadronic decay products, e.g. $D_{0} \rightarrow K^{ \pm}+\pi^{\mp}$.

Fig. 7.20 (upper panel) shows the momentum versus scattering angle distributions for pions following from an exclusive reaction with a $\rho^{0}$ vector meson production $\left(Q^{2}>1.0\right.$ $\mathrm{GeV}^{2}$ ), in the laboratory frame and for different beam energy combinations. As in fig. 7.17, two familiar patterns arise. For increasing lepton beam energy, the pion distribution goes from being more peaked in the forward-angle direction to a distribution with both a peak in the forward and backward ion direction, and the momentum in the forwardion direction is slightly reduced. Most of the forward-ion direction pions in fig. 7.20 are correlated with lower- $Q^{2}$ processes, though possibly of less interest for these processes. If one would use a $Q^{2}>10 \mathrm{GeV}^{2}$ cutoff in these exclusive processes, only a peak in the backward-ion direction would remain and in that sense, lower lepton energies correspond to lower hadron momenta on average and reduced particle identification requirements. The distributions for kaons from exclusive $\phi$-mesons production as well as for muons/electrons 

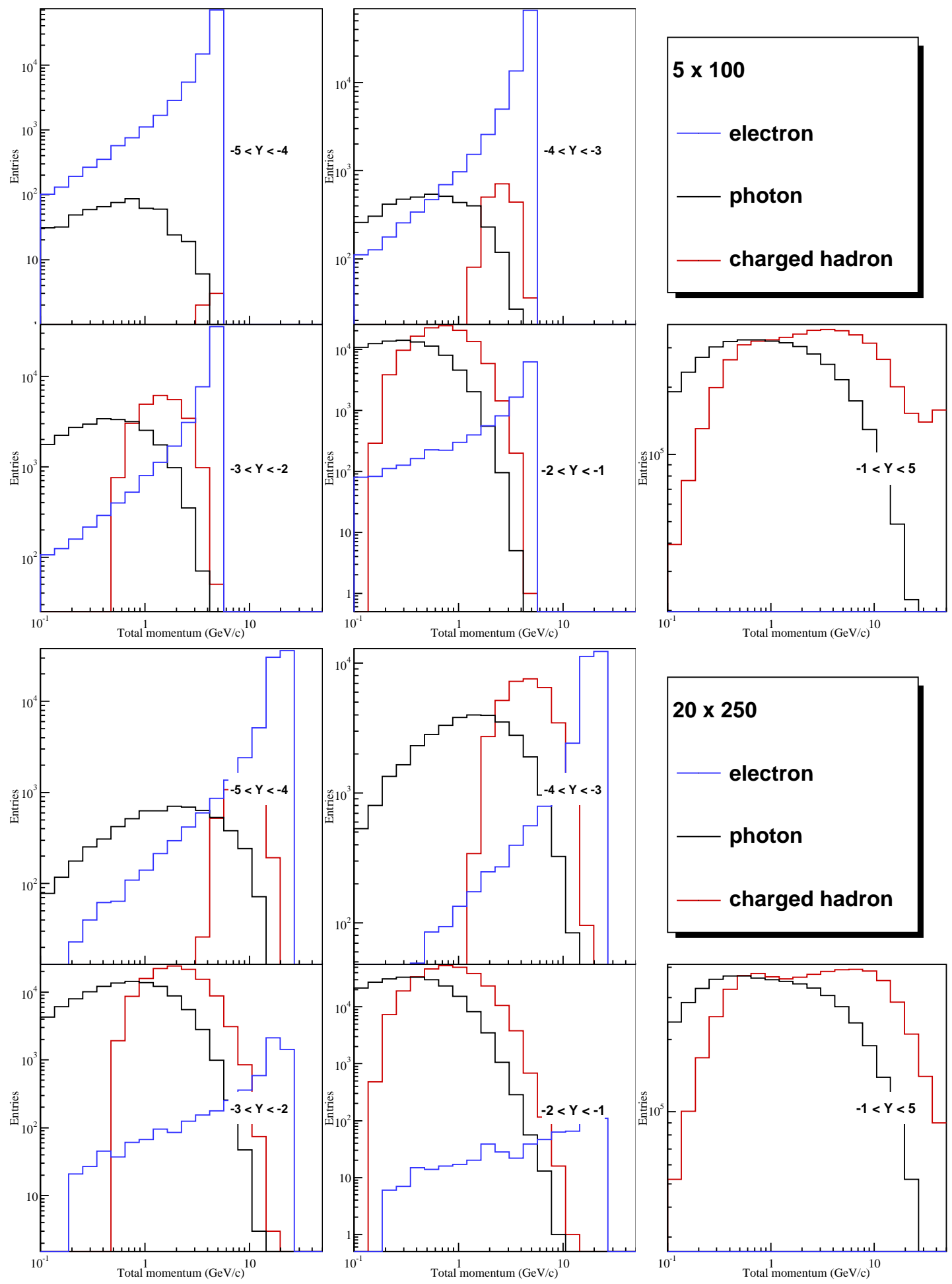

$20 \times 250$
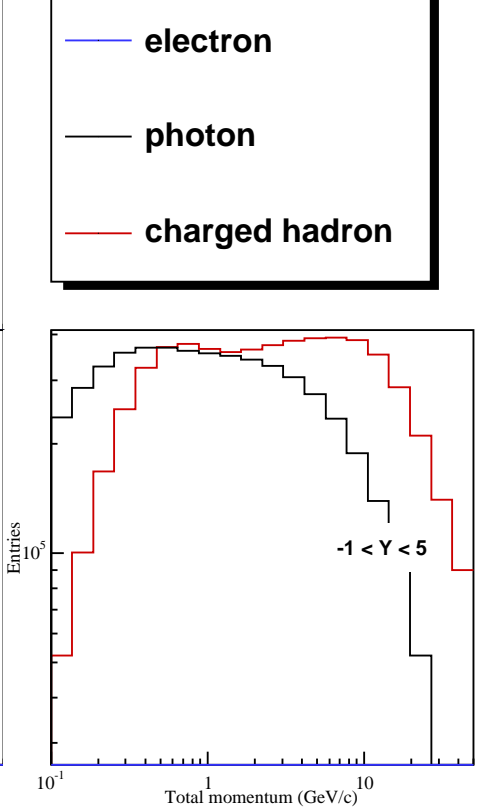

Figure 7.18. The number of photons and hadrons as well as the number of scattered leptons in a rapidity bin vs momentum having $5 \mathrm{GeV}$ leptons colliding with $100 \mathrm{GeV}$ protons and $20 \mathrm{GeV}$ leptons colliding with $250 \mathrm{GeV}$ protons. No kinematic cuts are applied. 


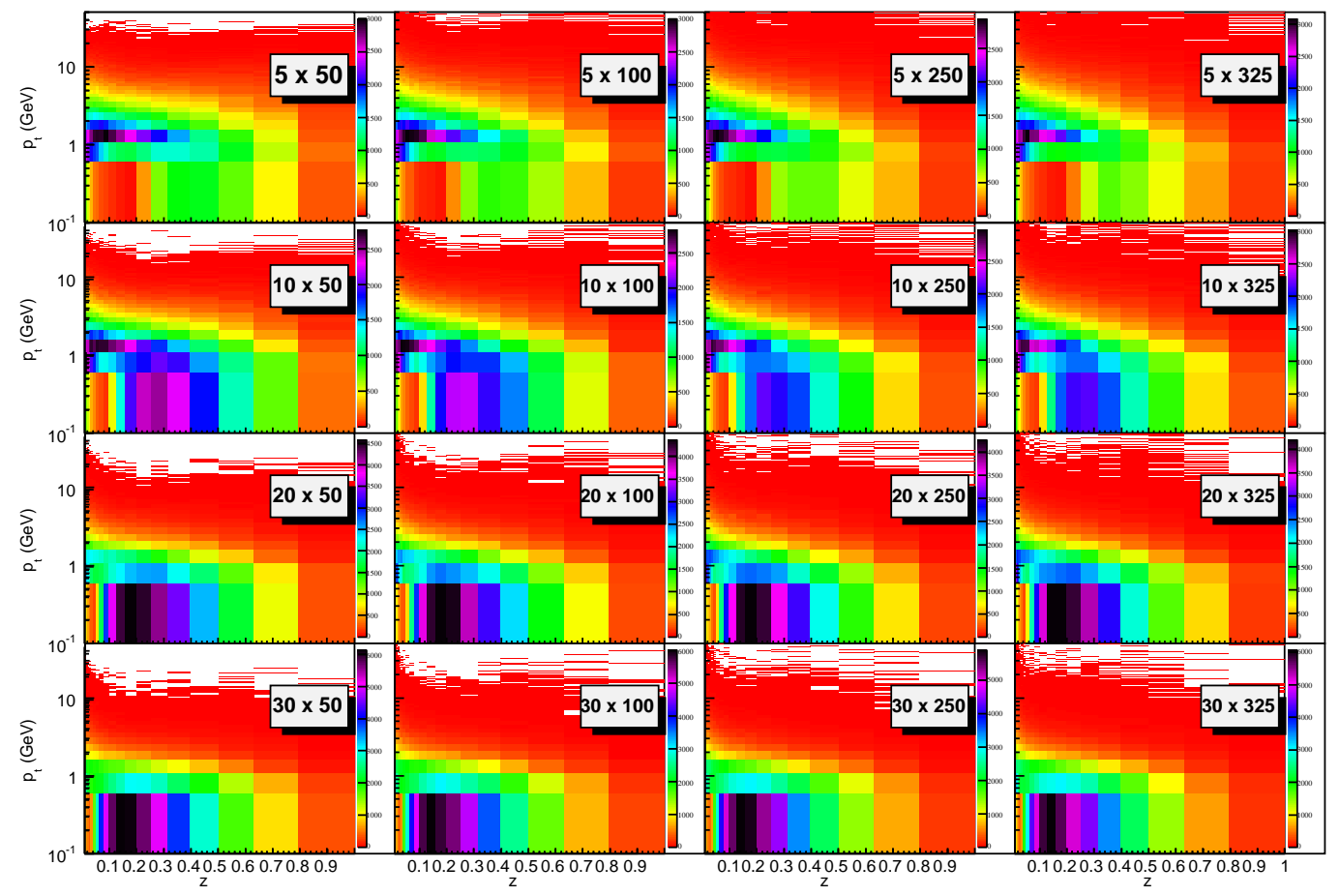

Figure 7.19. Transverse momentum vs. $\mathrm{z}$ for pions applying the following cuts $\mathrm{Q}^{2}>1 \mathrm{GeV}^{2}, 0.01<$ $\mathrm{y}<0.95,0.5^{\circ}<\theta<179.5^{\circ}$ and $\mathrm{p}>1 \mathrm{GeV}$. A momentum cut is applied to simulate the threshold of potential particle-identification-detectors.

from exclusive $J / \psi$ production look very similar, see lower panel of fig. 7.20. The most challenging constraints on the detector design for exclusive reactions compared to semiinclusive reactions is, however, not given by the hadrons originating from vector mesons, but from the detection of the exclusive hadronic state remaining.

As one specific example of an exclusive reaction, deeply virtual compton scattering (DVCS) was chosen, fig. 7.21 (top) shows the energy versus scattering angle distributions of photons in the laboratory frame, for different beam energy combinations. A cut of $\mathrm{Q}^{2}>$ $1 \mathrm{GeV}^{2}$ is assumed, although larger values of $\mathrm{Q}^{2}$ may be required. Lower lepton energies show a more symmetric distribution, and higher lepton energies are more backward-ion angle peaked. The distributions show relatively homogeneous distributions of the DVCS photons from forward to backward, with a small preference for the backward direction. The latter is true for all lepton-hadron beam energy combination.

Fig. 7.21 (bottom) correlates the distribution of the photon angle and the electron scattering angle in the laboratory frame, for different beam-energy combinations. With increasing lepton beam energy, the photon and scattered lepton tend towards the same detector hemisphere. Following fig. 7.21 (top), electromagnetic calorimetry is required over the entire rapidity range of the detector. Fig. 7.21 (bottom) illustrates that tracking and electromagnetic calorimetry capabilities covering similar rapidity range will greatly aid the separation of the photon and lepton, reducing a difficulty encountered by the ZEUS collaboration in their DVCS event reconstruction.

For exclusive reactions in general, with DVCS as the example above, it is extremely important to ensure that the remaining nucleon (or the nucleus) remains intact during the 

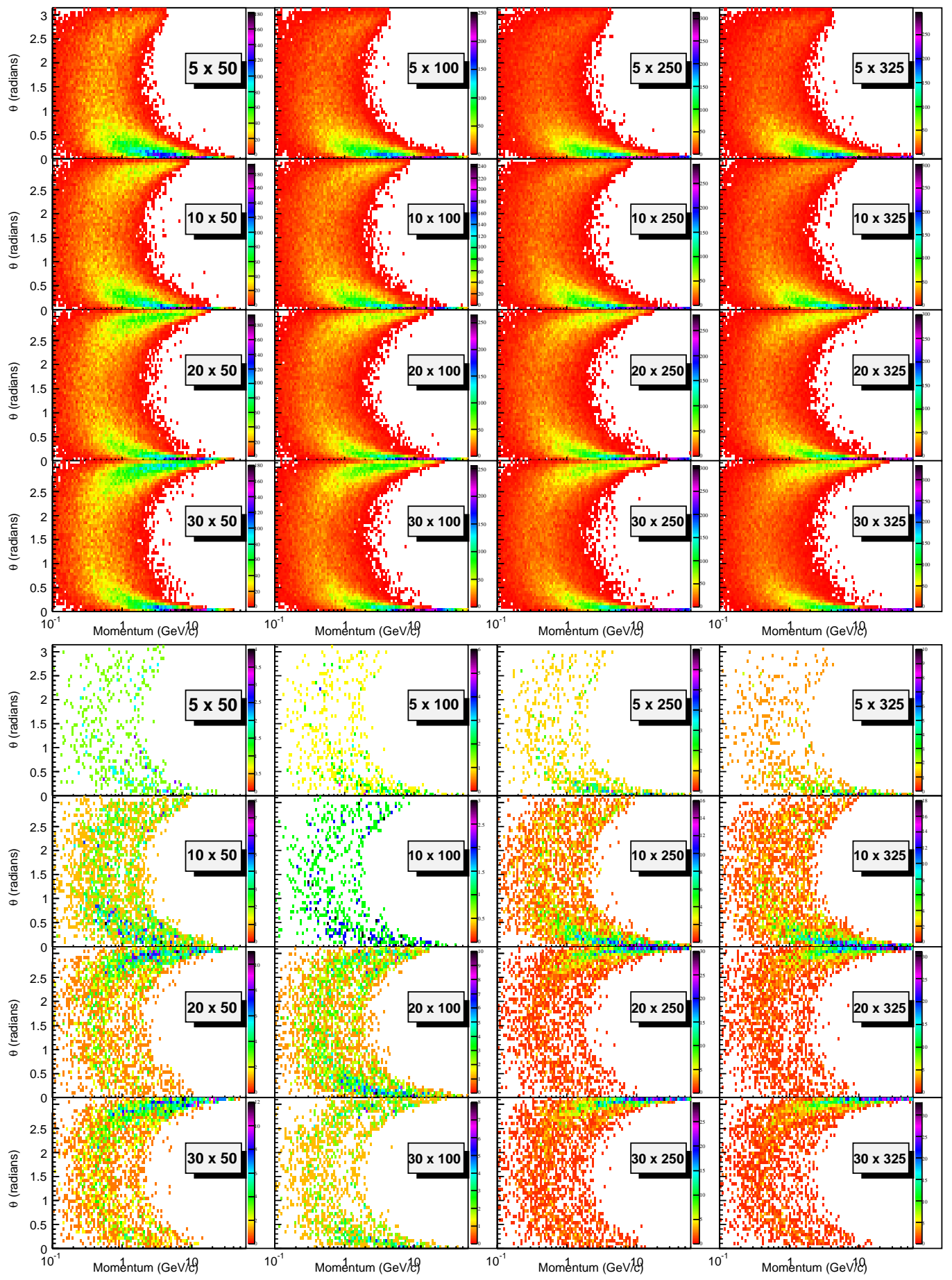

Figure 7.20. Upper Panel: Momentum vs. scattering angle in the laboratory frames for pions following from exclusive $\rho^{0}$ vector meson production. The following cuts are applied: $\mathrm{Q}^{2}>1.0$ $\mathrm{GeV}^{2}, 0.01<\mathrm{y}<0.95$.

Lower Panel: Momentum vs. scattering angle in the laboratory frames for muons following from exclusive $\mathrm{J} / \psi$ vector meson production. No cuts on $\mathrm{Q}^{2}$ have been applied as a hard scale for the process is given by the $\mathrm{J} / \psi$ mass. 

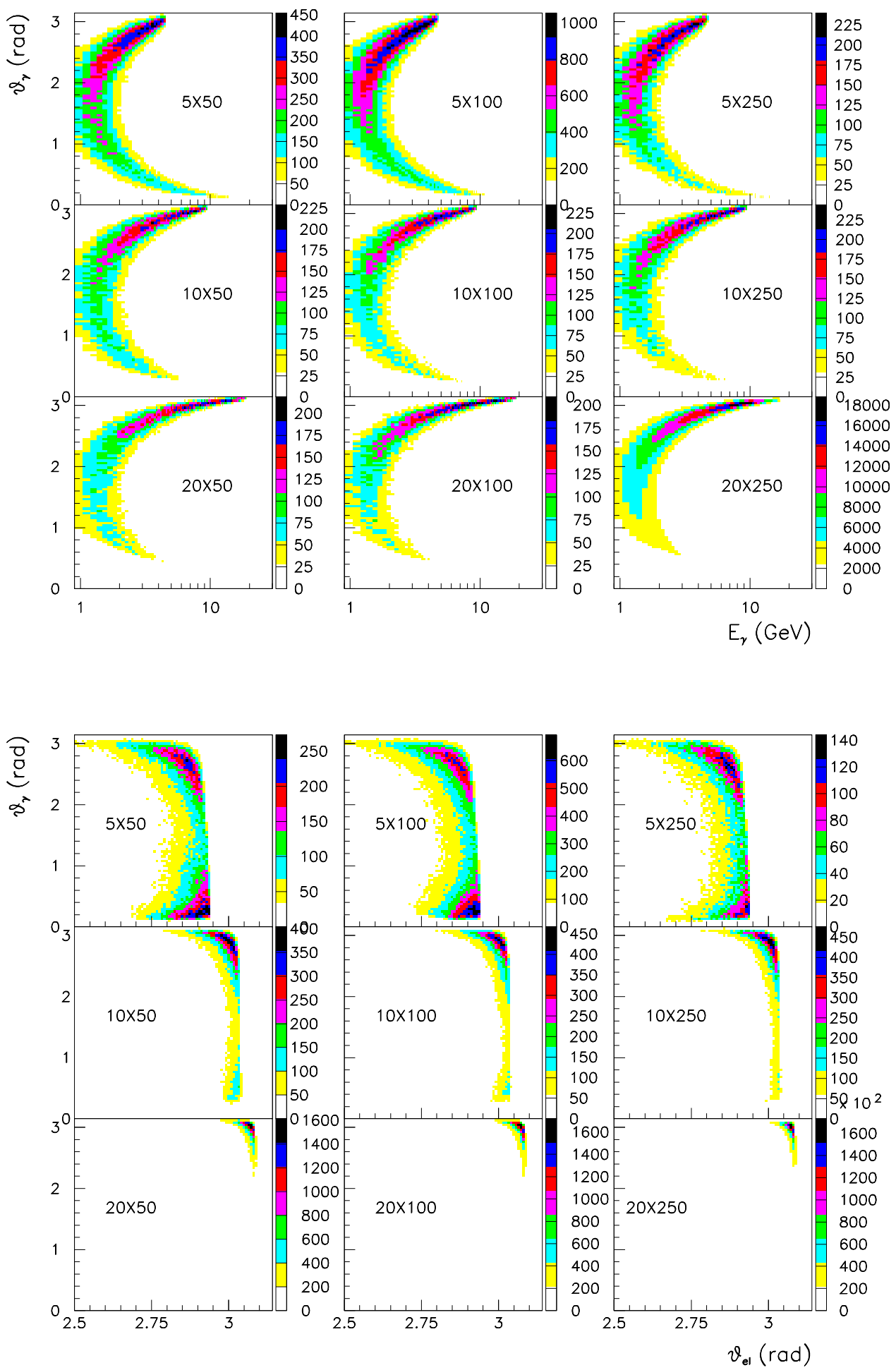

Figure 7.21. Upper panel: Energy vs. scattering angle in the laboratory frame for photons from DVCS. The following cuts have been applied: $\mathrm{Q}^{2}>1.0 \mathrm{GeV}^{2}, 0.01<\mathrm{y}<0.95$ and $\mathrm{E}_{\gamma}>1$. GeV. Lower Panel: The scattering angle in the laboratory frame of the photon vs. that of the scattered lepton for DVCS events. The following cuts have been applied: $\mathrm{Q}^{2}>1.0 \mathrm{GeV}^{2}, 0.01<\mathrm{y}<0.95$ and $\mathrm{E}_{\gamma}>1 . \mathrm{GeV}$ 
scattering process. Hence, one has to ensure exclusivity by measuring all products. Fig. 7.22 illustrates the kinematic requirements for the DVCS case, showing the scattered proton momentum versus its scattering angle for three different beam energy combinations. In general, for exclusive reactions one wishes to map the four-momentum transfer (or Mandelstam variable) $t$ to the hadronic system, and then obtain an image by a Fourier transform, at relatively low t of up to $1-2 \mathrm{GeV}$. The angle of the recoiling hadronic system is directly correlated with $t$ and the proton energy $E_{p}$, as $\sqrt{t} / E_{p}$. As can be seen in fig. 7.22, the proton scattering angle requirements indeed linearly (and inversely) scale with proton energy.

Even at a proton energy of $50 \mathrm{GeV}$, the proton scattering angles only range to about $1-2^{\circ}$. At proton energies of $250 \mathrm{GeV}$, this number is reduced to one fifth. In all cases, one obtains small to extremely small scattering angles, extending to or completely within the $0.5^{\circ}$ angular detector cutoff often used above. Because of this, the detection of these protons, or more general recoil baryons, is extremely dependent on the exact interaction region design and will therefore be discussed in more detail in the machine-dependent part of this chapter.

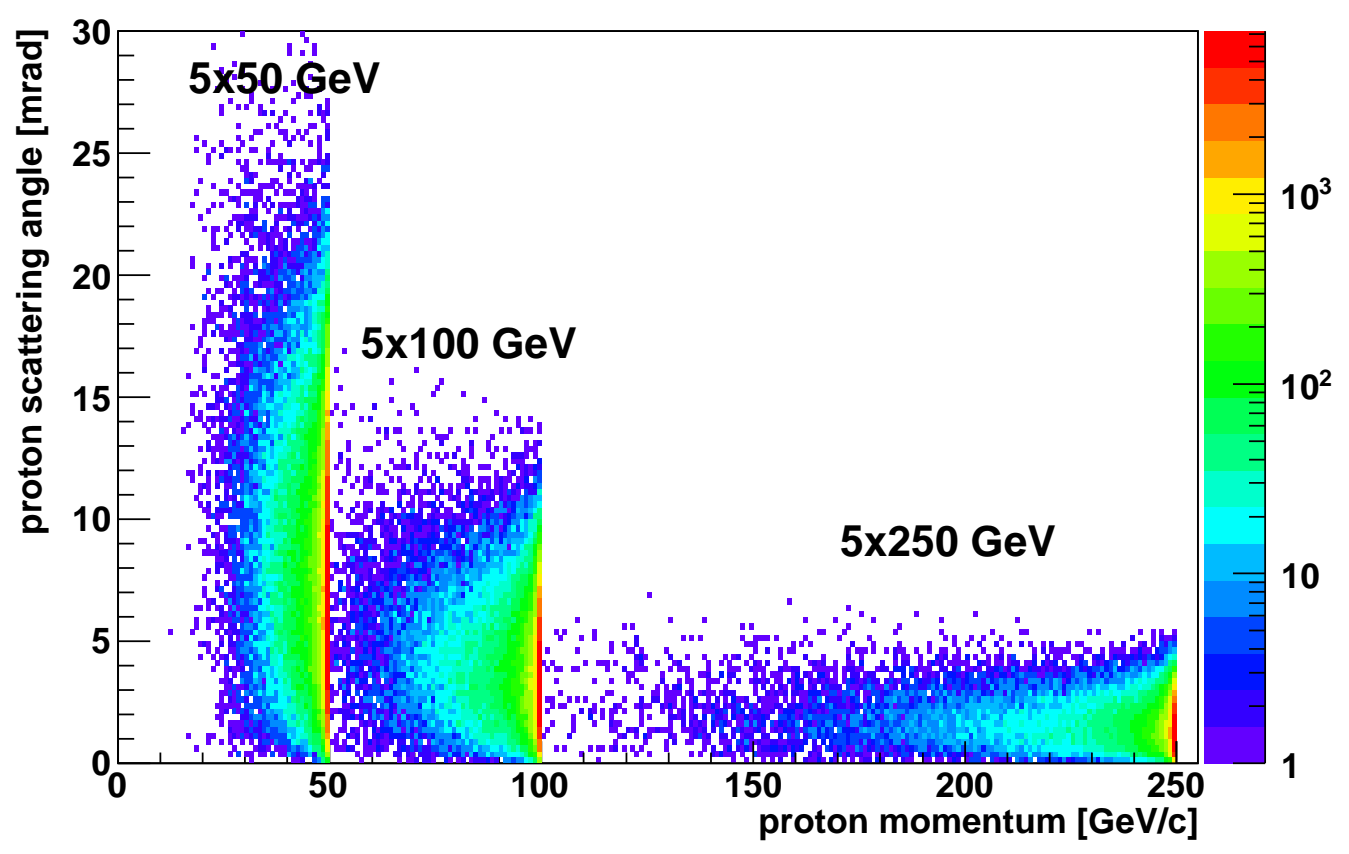

Figure 7.22. Scattered proton momentum vs. scattering angle in the laboratory frames for DVCS events with different beam energy combinations. The following cuts have been applied: $1 \mathrm{GeV}^{2}<$ $\mathrm{Q}^{2}<100 \mathrm{GeV}^{2}, 10^{-5}<\mathrm{x}<0.7$ and $0<\mathrm{t}<2 \mathrm{GeV}^{2}$.

Detection of the intact nucleus following an exclusive reaction in eA collisions is even more complicated. The binding energy in heavy nuclei is of the order $8 \mathrm{MeV}$ per nucleon. In general, the smallest measurable outgoing angle of heavy scattered or fragmented nuclei, $\theta_{\text {min }}$, is limited by the beam angular divergence and the requirement to have a $\sim 10 \sigma$ clearance of any detector element (often 'Roman pots') from the beam. For a beam divergence of say $0.1 \mathrm{mrad}$ and an ion beam of $100 \mathrm{GeV} / \mathrm{u}$, the transverse momentum required in the nuclear breakup to be beyond the so-called machine 'beam-stay-clear' area of $\sim 10 \sigma$ is 100 
$\mathrm{MeV}$, well beyond the $8 \mathrm{MeV}$ (or so) needed for a single nucleon. This would assume that the transverse momentum is equal to the excitation energy of the nucleus.

The diffractive slope at $\mathrm{t}=0$ depends on the size of the nucleus. Fig. 5.88 shows, for small $t \sim 1 / R_{A}^{2}$, a very steep t dependence, $\sim \exp \left(-t R_{A}^{2} / 3\right)$, and then several diffractive minima $\left(R_{A}=(1.12 \mathrm{fm}) A^{1 / 3}-(0.86 \mathrm{fm}) A^{-1 / 3}\right.$, for details see [1276] $)$. The incoherent background starts to dominate at $t$ values at which the coherent cross section has fallen to $1 / e$. These $t$ values can be estimated by $\exp \left(-|t| B_{0} A^{2 / 3}\right)=1 / A$, with $B_{0}=(1.12 f m)^{2 / 3}$. These values of $t$ are much smaller than the $t$ value corresponding to the first minimum in the coherent cross section and the $t$-values corresponding to the smallest measurable outgoing angle of scattered heavy nuclei. Therefore the strategy to ensure exclusive production on a nucleus is to veto nuclear breakup, by detecting the neutrons from incoherent events.

Another possibility can be to require a rapidity gap between the hadron beam and the produced jet, (vector) meson or real photon (where all events represent the sum of elastic and incoherent events). The left panel of fig. 7.23 shows the rapidity distribution of the most forward particle in deep-inelastic scattering (blue filled distribution) and diffractive events (unfilled histogram), respectively, for a $5 \mathrm{GeV}$ electron and a $100 \mathrm{GeV}$ proton beam energy combination. The $100 \mathrm{GeV}$ is here chosen to mimic the $100 \mathrm{GeV} / \mathrm{u}$ ion beam. The right panel of fig. 7.23 shows the efficiency and purity for diffractive events to DIS events (1:1) as function of rapidity, varying the lepton beam energy while keeping the hadron beam energy fixed. If one requires 4 units of rapidity between the hadron beam and a produced jet, vector meson or real photon, an efficiency of above $60 \%$ and a purity close to $100 \%$ for diffractive events would be obtained. A detector with wide rapidity coverage is essential for
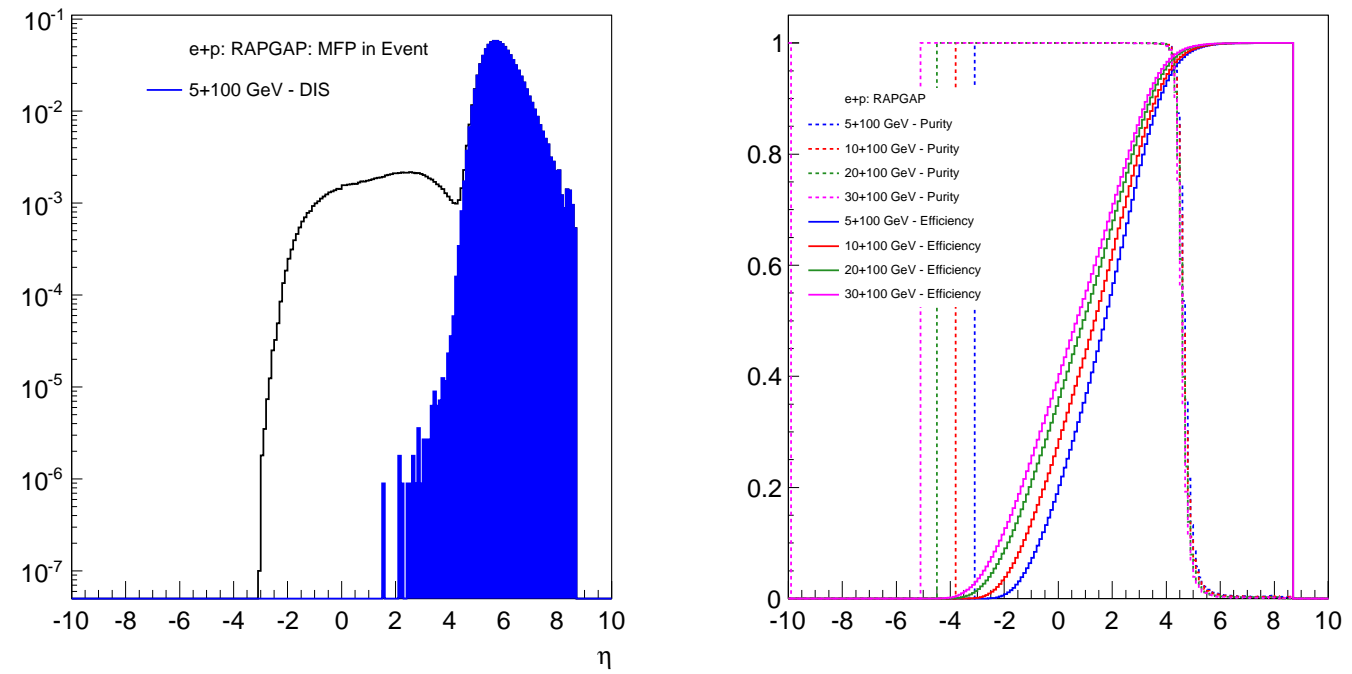

Figure 7.23. Left: Rapidity distribution of DIS and diffractive events for the most forward particle (MFP) in the event. Right: Efficiency and Purity for diffractive events with respect to DIS events $(1: 1)$ as a function of the detector rapidity coverage and the center-of-mass energy.

such events. 


\subsubsection{Radiative Corrections}

The radiation of real and virtual photons leads to large additional contributions to the observable cross section of electron scattering at high energies. Precision measurements of the nucleon structure require a good understanding of these radiative corrections. For neutral-current lepton nucleon scattering, a gauge-invariant classification into leptonic, hadronic and interference contributions can be obtained from Feynman diagrams. The Feynman diagrams for leptonic corrections are shown in fig. 7.24, Leptonic corrections dominate and strongly affect the experimental determination of kinematic variables.

Usually, the cross section is measured as a function of $Q^{2}$ and Bjorken-x, $x_{B}$, defined as

$$
Q^{2}=-\left(l-l^{\prime}\right)^{2}, \quad x_{B}=\frac{Q^{2}}{2 P \cdot\left(l-l^{\prime}\right)},
$$

where $l$ and $l^{\prime}$ denote the 4-momenta of the incoming and outgoing lepton, respectively, and $P$ is the $4-$ momentum of the incoming nucleon. The true values of these variables seen by the nucleon when a photon with 4 -momentum $k$ is radiated are, however, given by (see fig.)

$$
\tilde{Q}^{2}=-\left(l-l^{\prime}-k\right)^{2}, \quad \tilde{x}_{B}=\frac{\tilde{Q}^{2}}{2 P \cdot\left(l-l^{\prime}-k\right)} .
$$

If the photon momentum is large and balancing the transverse momentum of the scattered lepton, $\tilde{Q}^{2}$ can be shifted to small values, leading to an enhancement of the radiative corrections. This effect is similar to the radiative tail of a resonance.

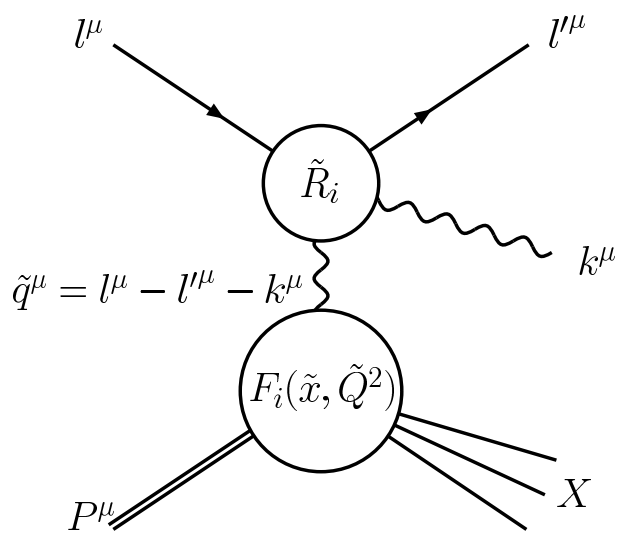

Kinematics of leptonic radiation.
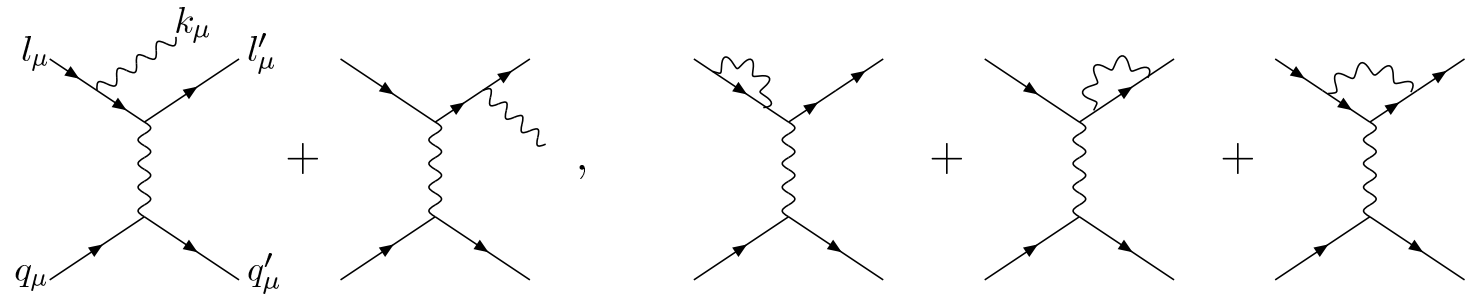

Figure 7.24. Feynman diagrams for leptonic radiation in lepton-quark scattering.

The effect of radiation of photons from the lepton can be described with the help of radiator functions $\tilde{R}_{i}\left(l, l^{\prime}, k\right)$. There is one $\tilde{R}_{i}$ for every structure function $F_{i}, i=2, L$. The radiator functions comprise both real radiation from the initial and the final state as well as the contribution from vertex and self-energy diagrams. Using $\tilde{x}_{B}$ and $\tilde{Q}^{2}$ from equation (7.3) to parametrize the integration over the phase space of emitted photons, one can express the observed structure functions as convolutions,

$$
F_{i}^{\text {obs }}\left(x_{B}, Q^{2}\right)=\int \mathrm{d} \tilde{x}_{B} \mathrm{~d} \tilde{Q}^{2} R_{i}\left(x_{B}, Q^{2}, \tilde{x}_{B}, \tilde{Q}^{2}\right) F_{i}^{\text {true }}\left(\tilde{x}_{B}, \tilde{Q}^{2}\right) .
$$


The integration limits are determined by the energy allowed for the radiated photon which, in the photon-nucleon center-of-mass frame, is given by

$$
E_{\gamma}^{\max }=\sqrt{\frac{1-x_{B}}{x_{B}} Q^{2}}
$$

Radiative corrections are, therefore, large at large $Q^{2}$ and small $x_{B}$. In contrast, at small $Q^{2}$ and large $x_{B}$, the phase space for photon emission is restricted and negative virtual corrections dominate.

From equation (7.4) it is obvious that the determination of the true structure functions $F_{i}^{\text {true }}\left(\tilde{x}_{B}, \tilde{Q}^{2}\right)$ requires unfolding, a procedure which is in general only possible in an iterative way and with reasonably chosen assumptions about the starting values. Moreover, the observed structure functions depend on the way in which the kinematic variables are measured. For example, if the momentum of the hadronic final state, $p_{X}$, could be measured, $\tilde{x}_{B}$ and $\tilde{Q}^{2}$ would be known. In practice this will be difficult to achieve; however, any information about the hadronic final state could contribute to a narrowing down of the phase space available for photon emission, thereby reducing the size of radiative corrections.

The radiator functions are dominated by peaks in the angular distribution for the collinear radiation of photons from the initial state (ISR) or from the final state (FSR). At high energies, it is a good approximation to assume that photon radiation can be described by a simple rescaling of the lepton momentum, $l \rightarrow z l$ for ISR and $l^{\prime} \rightarrow l^{\prime} / z$ for FSR. The radiator function in the collinear approximation takes the simple, universal form

$$
R_{\mathrm{coll}}=\frac{\alpha}{2 \pi} \log \frac{Q^{2}}{m_{e}^{2}}\left(\frac{1+z^{2}}{1-z}\right)_{+}
$$

so that the cross section is obtained from

$$
\mathrm{d} \sigma_{\text {ISR }}=\int \frac{\mathrm{d} z}{z} R_{\text {coll }}(z) \mathrm{d} \sigma_{\text {Born }}\left(l^{\mu} \rightarrow z l^{\mu}\right)
$$

(and similarly for FSR). The potentially large logarithm $\log Q^{2} / m_{e}^{2}$ may reach the order of $10 \%$ at large $Q^{2}$.

As an example, we show numerical results for electron proton scattering at two typical sets of beam energies: $E_{e}=5 \mathrm{GeV}$ with $E_{p}=50 \mathrm{GeV}$ (left panel of fig. 7.25) and $E_{e}=30$ $\mathrm{GeV}$ with $E_{p}=325 \mathrm{GeV}$ (right panel). The figures show the correction factor

$$
r_{c}(y)=\frac{\mathrm{d} \sigma /\left.\mathrm{d} y\right|_{O(\alpha)}}{\mathrm{d} \sigma /\left.\mathrm{d} y\right|_{B o r n}}-1
$$

where $y=Q^{2} / Q_{\max }^{2}, Q_{\max }^{2}=x_{B} S, S=2 l \cdot P$. The different curves correspond to different ranges of $x_{B}$ : at the lower center-of-mass energy (left panel of fig. 7.25, from the bottom up): $0.1<x_{B}<0.4,10^{-2}<x_{B}<10^{-1}$ and $10^{-3}<x_{B}<10^{-2}$; at the higher center-ofmass energy (right panel, again from the bottom up): $0.1<x_{B}<0.4,10^{-2}<x_{B}<10^{-1}$, $10^{-3}<x_{B}<10^{-2}, 10^{-4}<x_{B}<10^{-3}$, and $10^{-5}<x_{B}<10^{-4}$. The general features following from the preceding discussion are clearly visible: corrections are large at large $y$ and small $x_{B}$, while corrections become negative at large $x_{B}$ and small $y$.

Lacking a full Monte Carlo event simulation for scattering with heavy nuclei at present, we have studied the influence of a simple cut on the invariant mass of the hadronic final state. Imposing the condition $W_{\text {had }}>1.4 \mathrm{GeV}$ would remove the elastic tail and the contribution 

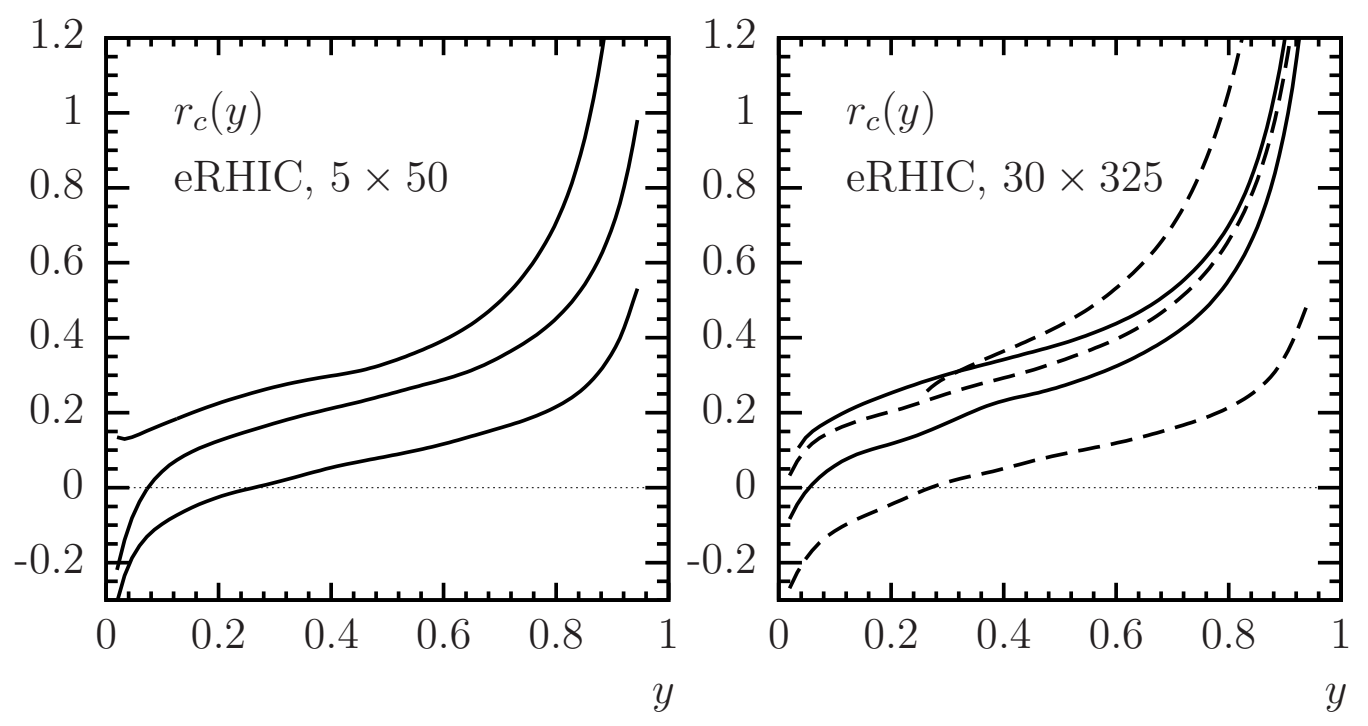

Figure 7.25. $y$-dependence of the leptonic radiative correction factor for electron proton scattering with different beam energies and in different $x_{B}$ ranges. Left: $E_{e}=5 \mathrm{GeV}, E_{p}=30 \mathrm{GeV}$ and the curves from the bottom up correspond to $0.1<x_{B}<0.4,10^{-2}<x_{B}<10^{-1}, 10^{-3}<x_{B}<10^{-2}$; Right: $E_{e}=30 \mathrm{GeV}, E_{p}=325 \mathrm{GeV}$ and $0.1<x_{B}<0.4,10^{-2}<x_{B}<10^{-1}, 10^{-3}<x_{B}<10^{-2}$, $10^{-4}<x_{B}<10^{-3}, 10^{-5}<x_{B}<10^{-4}$ (full and dashed lines alternating for better visibility).
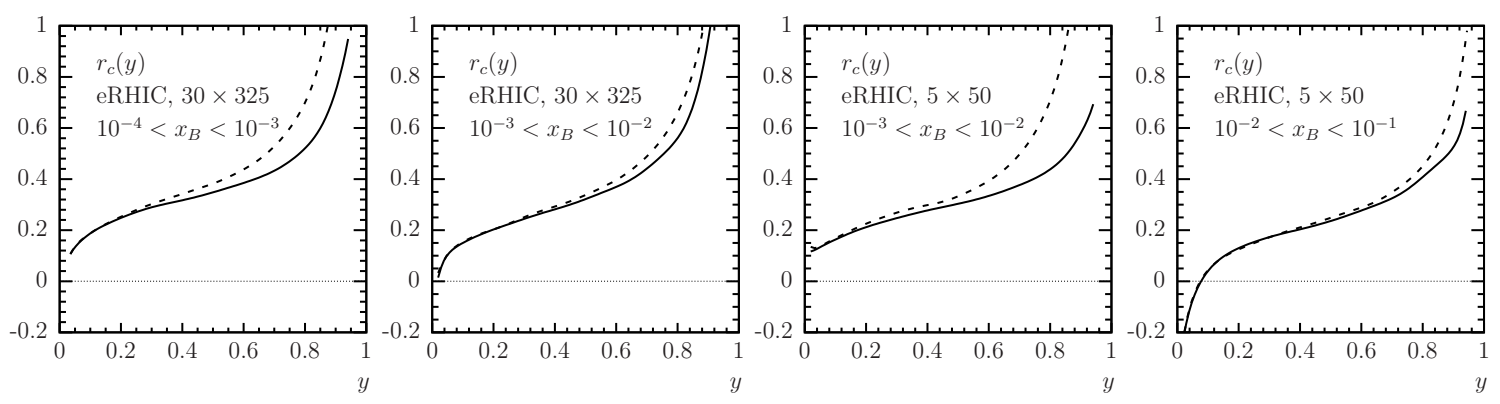

Figure 7.26. Influence of a cut on the mass of the hadronic final state on the leptonic radiative correction factor for a proton target in different $x_{B}$ ranges and beam energies as indicated in the figures. Dashed curves are without a cut, full curves are obtained after a cut of $W_{\text {had }}>1.4 \mathrm{GeV}$. 
from low-lying resonances. A similar effect can be achieved cutting on $E-p_{z}$ from the Jacquet-Blondel method. The effect of such a naive cut is shown in fig. 7.26. The reduction of the radiative corrections is considerable at largest $y$ and at small $x_{B}$, but probably not yet sufficient at larger values of $x_{B}$. From similar studies for electron-nucleus scattering at HERA [1277, 1278, 1274], one can expect to obtain a much stronger reduction of radiative corrections, if more refined prescriptions for the measurement of kinematic variables are found.
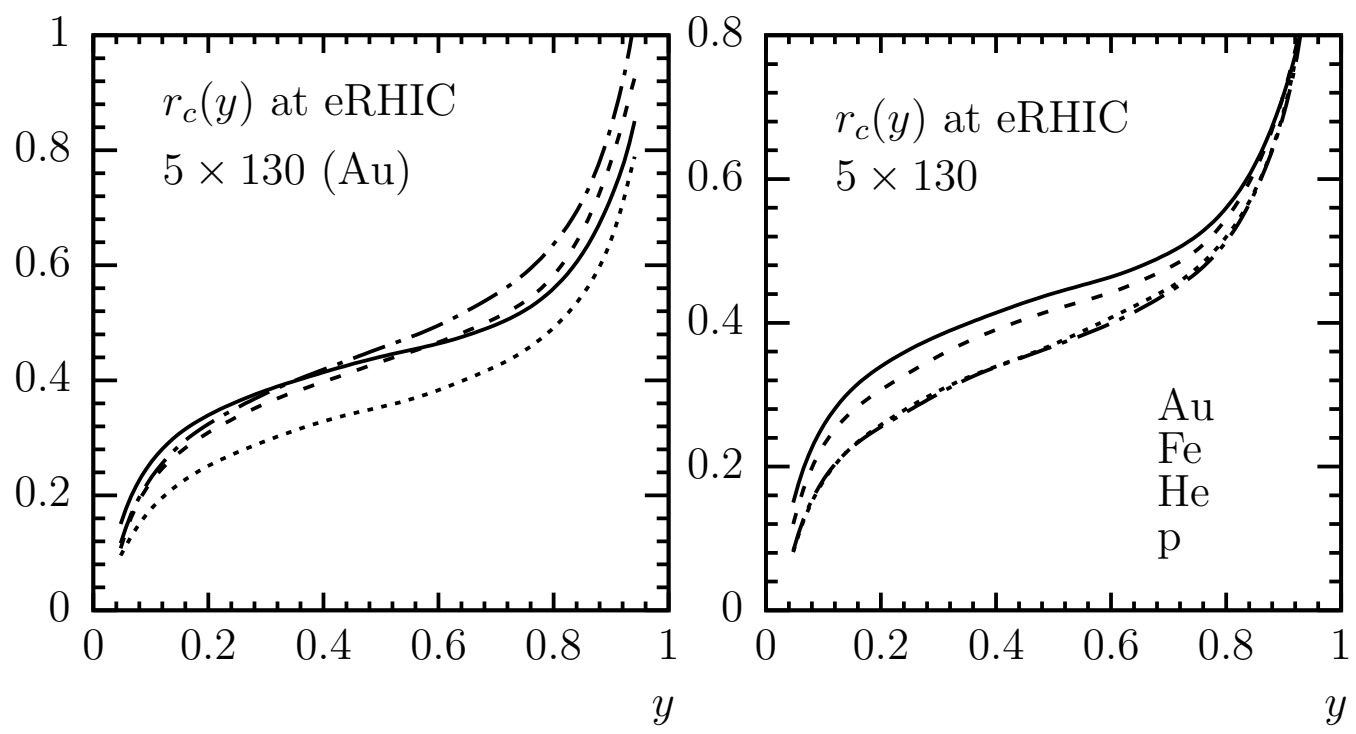

Figure 7.27. Left: Radiative corrections for electron scattering off a Au nucleus at $5 \times 130 \mathrm{GeV}^{2}$ beam energies, $10^{-3}<x_{B}<10^{-2}, Q^{2}>1 \mathrm{GeV}^{2}, W_{\text {had }}>1.4 \mathrm{GeV}$ with different models for nuclear PDFs: EPS09 (full curve), EPS08 (dash-dotted line), EKS98 (dashed line) and HKN (dotted line). Right: Radiative corrections for different nuclei with CTEQ61M PDFs modified by the EPS09 prescription. Beam energies and kinematic range as in the left figure. From the bottom up: proton, ${ }^{4} \mathrm{He},{ }^{56} \mathrm{Fe}$, ${ }^{197} \mathrm{Au}$.

Since the determination of the true structure functions requires an iterative unfolding procedure, it is important to show that the radiative corrections do not depend too strongly on the assumed input structure functions. In fig. 7.27 a we show the correction factor $r_{c}(y)$ as defined above for the case of electron scattering off an ${ }^{197} \mathrm{Au}$ nucleus, assuming different parameterizations of parton distribution functions corrected for nuclear effects, as available in the literature $[38,828,1279,40]$. Although differences at the level of $10 \%$ are visible, one can still observe a similar overall behavior of radiative corrections. Finally, in fig. 7.27b, we show results for scattering off different nuclei, again supporting the assumption that a common unfolding procedure would allow one to obtain the true structure functions.

Corrections due to the emission of photons from the hadrons, or quarks in the deep inelastic regime, require a careful separation into contributions which should be considered as a part of the hadron structure (leading to an electromagnetic contribution to scaling violations [1280]) and contributions which can, in principle, be related to the observation of direct photons radiated from quarks. The interference of radiation from the lepton and the quark is small [1277]. In certain phase space regions one may expect higher than one-photon corrections to be important. For example, soft-photon exponentiation will be necessary at small $y$ and large $x_{B}$. The procedure is well-known and straightforward. Finally, multi- 
photon radiation may become important at large $y$ and small $x_{B}$. In this case, the collinear approximation is sufficient to reach a precision at the level of one percent [1281].

\subsubsection{Detector Design for eRHIC}

The BNL design of an EIC allows for collisions at three interaction regions: one at IP-12 with a new dedicated EIC detector, and at IP-6 and IP-8 with the current RHIC detectors STAR and PHENIX. In the following, first the design considerations for a dedicated EIC detector are described and then the capabilities of PHENIX and STAR for ep / eA collisions are discussed.

\section{A dedicated EIC detector}

Combining all the requirements described in section 7.3.1 and in the physics chapters before, a schematic view of the emerging dedicated eRHIC detector is shown in fig. 7.28. As already discussed, it is important to have equal rapidity coverage for tracking and

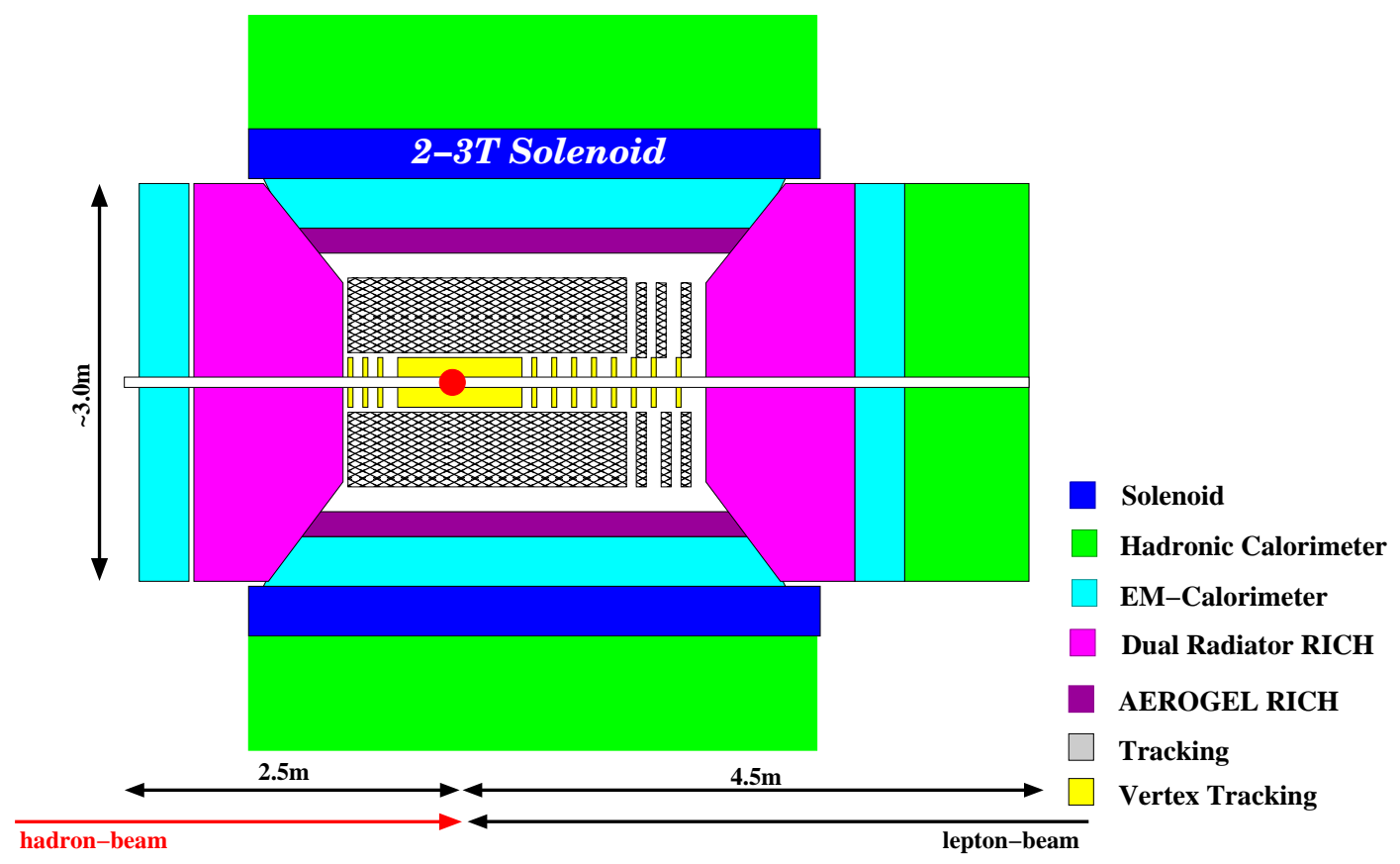

Figure 7.28. A schematic view of a dedicated EIC detector. Details of the GEANT-3 model can be found at https://wiki.bnl.gov/eic/index.php/Detector_Design.

electromagnetic calorimetry. This will provide good electron identification and give better momentum and angular resolutions at low inelasticity $y$ than with an electro-magnetic calorimeter alone.

The significant progress in the last decade in the development of Monolithic Active Pixel Sensors (MAPS), in which the active detector, analog signal shaping, and digital conversion take place in a single silicon chip (i.e. on a single substrate; see 1282 and references therein), provides a unique opportunity for a $\mu$-vertex detector for an eRHIC detector. These devices, built using CMOS technology, use an epitaxial layer as the active sensing element. Ionization deposited in the epitaxial layer is collected by $\mathrm{N}+$ wells embedded in 
the epitaxial layer. The "pixel" pitch is determined by the location of the $\mathrm{N}$ wells so there is no need for actual segmentation of the detector as is done with traditional hybrid pixel detectors. As a result, CMOS pixel detectors can be built with high segmentation, limited primarily by the space required for additional shaping and digital conversion elements. The key advantage of CMOS MAPS detectors is the reduced material required for the detector and the (on substrate) on-detector electronics. Such detectors have been fabricated and extensively tested (see e.g. [1283]) with thicknesses of about $50 \mu \mathrm{m}$, corresponding to $0.05 \%$ of a radiation length.

For tracking at larger radii, there are several possibilities which need to be investigated first through Monte Carlo studies for position resolution and material budget, and later through R\&D and building prototypes. The two most prominent options for the barrel tracker are a TPC and a cylindrical GEM-Tracker. For large radii, forward tracking GEMTrackers are the most likely option. The projected rates for a luminosity of $10^{34} \mathrm{~cm}^{-2} \mathrm{~s}^{-1}$ range, depending on the center-of-mass energy, between 300 and $600 \mathrm{kHz}$, with an average of 6 to 8 charged tracks per event. These numbers do not impose strong constraints on the technology for a tracker.

Due to the momentum range to be covered, the only solution for PID in the forward direction is a dual radiator $\mathrm{RICH}$, combining either Aerogel with a gas radiator like $\mathrm{C}_{4} \mathrm{~F}_{10}$ or $\mathrm{C}_{4} \mathrm{~F}_{8} \mathrm{O}$ if $\mathrm{C}_{4} \mathrm{~F}_{10}$ is no longer available, or combining the gas radiator with a liquid radiator like $\mathrm{C}_{6} \mathrm{~F}_{14}$.

In the barrel part of the detector several solutions are possible, as the momenta of the majority of the hadrons to be identified are between $0.5 \mathrm{GeV}$ and $5 \mathrm{GeV}$. The technologies available in this momentum range are high resolution ToF detectors ( $\mathrm{t} \sim 10 \mathrm{ps}$ ), a DIRC or a proximity focusing Aerogel RICH.

For the electromagnetic calorimetry in the forward and backward direction, a solution based on $\mathrm{PbWO}_{4}$ crystals would be optimal. The advantages of such a calorimeter would be a small Molière radius of $2 \mathrm{~cm}$ and a factor of two better energy resolution and higher radiation hardness than, for example, lead-glass. To increase the separation of photons and $\pi^{0} \mathrm{~s}$ to high momenta and to improve the matching of charged tracks to the electromagnetic cluster, it would be an advantage to add, in front of all calorimetry, a high resolution pre-shower. We follow for the barrel part of the detector the concept of very compact electromagnetic calorimetry (CEMCal). A key feature is to have at least one preshower layer with 1-2 radiation lengths of tungsten and silicon strip layers (possibly with two spatial projections) to allow separation of single photons from $\pi^{0}$ to up $p_{T} \approx 50 \mathrm{GeV}$, as well as enhanced electron-identification. A straw-man design could have silicon strips with $\Delta \eta=0.0005$ and $\Delta \phi=0.1$. The back section for full electromagnetic energy capture could be, for cost effectiveness and good uniformity, an accordion Lead-Scintillator Design, which would provide gain uniformity and the ability to calibrate the device. A tungsten- and silicon-strip-based pre-shower would also be a good solution for the forward and backward electromagnetic calorimetry.

To achieve the physics program as described in earlier sections, it is extremely important to integrate the detector design into the interaction region design of the collider. As already described, particularly challenging is the detection of forward-going scattered protons from exclusive reactions, as well as of decay neutrons from the breakup of heavy ions in nondiffractive reactions. Previous experience of electron colliders (SLAC, KEK B-factories) and HERA, an electron-proton collider, indicated difficulties with synchrotron radiation coming from bending the electron beam close to the interaction region (IR). The newest large improvements in luminosity at KEK in Japan, by introducing crab cavities, show 
that colliding heavy ions or protons with electrons could be obtained without bending the electrons close to the IR, but that it is possible to use the crossing angle between the two beams without losing luminosity. This is the path chosen in the eRHIC design: a 10 mrad crossing angle between the protons or heavy ions during collisions with electrons. This choice removes potential problems for the detector induced by synchrotron radiation. To obtain luminosities higher than $10^{34} \mathrm{~cm}^{-2} \mathrm{~s}^{-1}$, very strong focusing close to the IR is required to have the smallest beam sizes at the interaction point. A small beam size is only possible if the beam emittance is also very small. The focusing triplets are 4.5 meters away from the interaction point (IP). The strong focusing quadrupoles induce very large chromaticities. The current eRHIC design has its highest values of the amplitude betatron functions of the same size as the present operating conditions of the RHIC collider. In addition the design allows a correction of the first, second and third order chromaticities by using sextupoles at the triplets as well as 180 degrees away from the quadrupoles source (as shown in fig. 7.29).

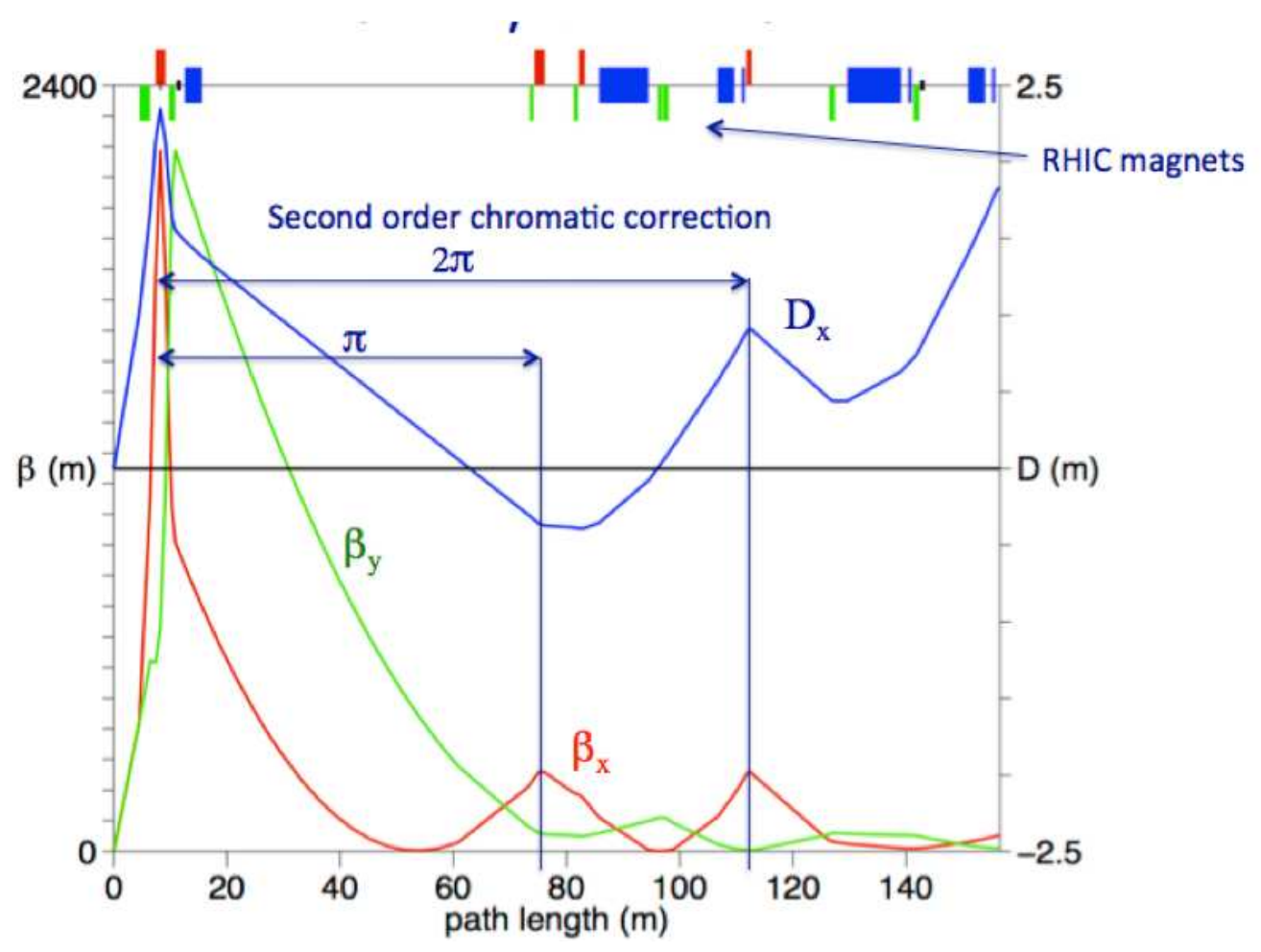

Figure 7.29. The Beta-function along the eRHIC hadron ring.

While the above accomplishes a small-emittance electron beam, the ions and protons need to be cooled by coherent electron cooling to have small emittance. The eRHIC interaction region design relies on the existence of small emittance beams with a longitudinal RMS of $5 \mathrm{~cm}$, resulting in $\beta^{*}=5 \mathrm{~cm}$. Strong focusing is obtained by three high-gradient quadrupole magnets using recent results from the LHC quadrupole magnet upgrade program (reaching gradients of $200 \mathrm{~T} / \mathrm{m}$ at $120 \mathrm{~mm}$ aperture). To ensure the previously described requirements from physics are met, four major requirements need to be fulfilled: high luminosity ( $>100$ times that of HERA), the ability to detect neutrons, measurement of the scattered proton from exclusive reactions (i.e. DVCS) and the detection of low-momentum 
protons $\left(\mathrm{p} \sim \mathrm{p}_{0} / 2.5\right)$ from heavy-ion breakup. The eRHIC IR design fulfills all these requirements: the first magnet in the high focusing quadrupole triplet is a combined function magnet producing a $4 \mathrm{mrad}$ bending angle of the ion/proton beam (see fig. 7.30). The 120 $\mathrm{mm}$ diameter aperture of the last quadrupole magnet allows detection of neutrons with a solid angle of $\pm 4 \mathrm{mrad}$, as well as the scattered proton from exclusive reactions, i.e. DVCS, up to a solid angle of $\sim 9 \mathrm{mrad}$. The electrons are transported to the interaction point through the heavy ion/proton triplets, seeing zero magnetic field as shown in fig. 7.30.
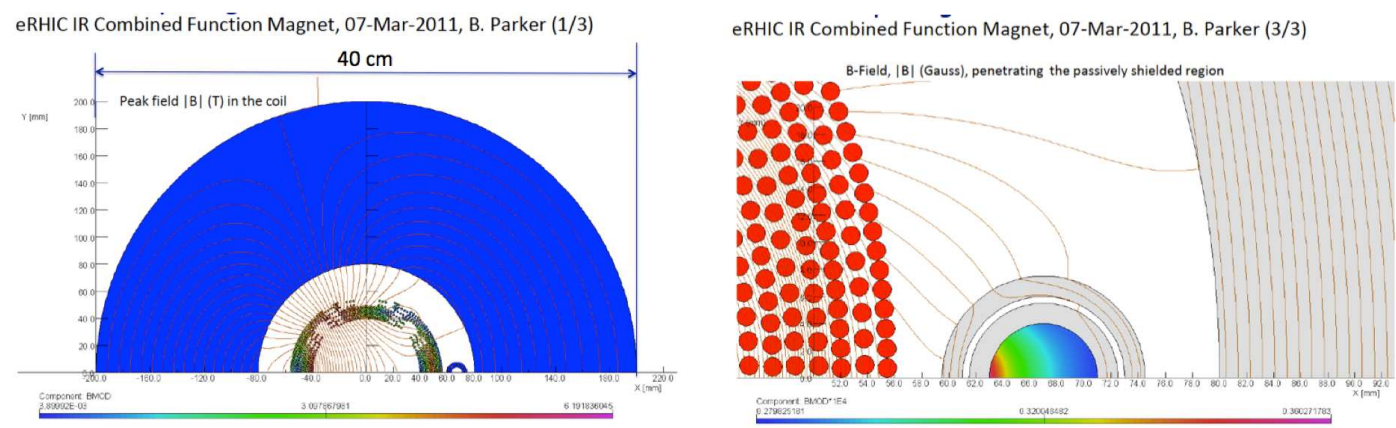

Figure 7.30. Combined-function magnet of the hadron beam high focusing quadrupole triplet.

Fig. 7.31 shows the current eRHIC interaction region design in the direction of the outgoing hadron beam. The other side of the IR is mirror symmetric for the incoming hadron beam. For the outgoing lepton beam we are currently investigating how to best integrate a low scattering-angle lepton tagger. Such a tagger is critical for any low $Q^{2}$ physics, like elastic $J / \psi$ production in eA collisions (see section 5.14).

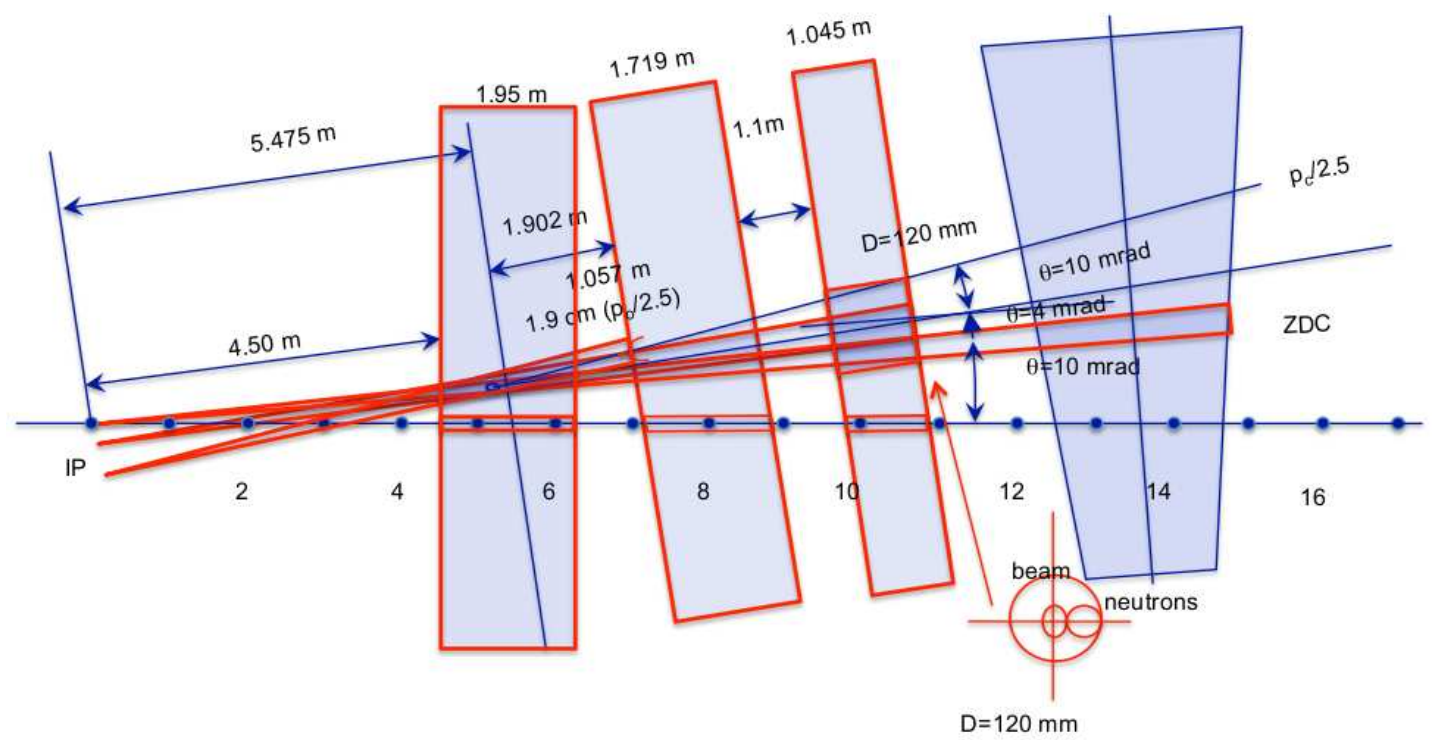

Figure 7.31. Schematic view of the eRHIC interaction region design in the direction of the outgoing hadron beam.

The scattered proton from DVCS events were tracked through this design and beam optics using HECTOR [1284]. The DVCS events have been generated with MILOU, a MC 
code dedicated to DVCS 673]. From fig. 7.32 it is clear that protons from DVCS events can be measured in 'Roman Pots' after the high-focusing quadrupole triplet with a high detection efficiency for hadron beam energies starting from $100 \mathrm{GeV}$ (as example are shown the results for $50 \mathrm{GeV}$ and $250 \mathrm{GeV}$ ). More studies are needed to determine the momentum and angular resolution that can be achieved depending on the 'Roman Pot' design.

As pointed out previously, equally challenging is the detection of the breakup neutrons from heavy ions to veto incoherent events. The nuclear breakup of Au nuclei depending on the excitation energy E* was simulated using the Monte Carlo generator GEMINI++ [1285] and SMM [1286]. The MC simulation showed that whenever the nucleus breaks up there will be at least one neutron emitted. At very low excitation energies there is the possibility that only a photon is emitted, while the nucleus remains intact. The possibility of detecting these photons still needs to be investigated. Fig. 7.33 shows the angular distribution of the breakup neutrons for three different excitation energies. The aperture of $120 \mathrm{~mm}$ diameter of the last quadrupole magnet allows detection of neutrons with a solid angle of $\pm 4 \mathrm{mrad}$, which is indicated by the simulations to be sufficient.

Fig. 7.34 shows the detection inefficiency for these neutrons for three different excitation energies as function of the maximal aperture of the last magnet. For apertures discussed for the IR design the inefficiencies are $10^{-2}$ or much lower for all excitation energies. This assumes a 100\%-efficient zero degree calorimeter (ZDC). The critical question is: to suppress incoherent events at high $t$ in eA collisions, can the detection inefficiencies be controlled on the $10^{-3}$ to the $10^{-4}$ level?

There are many detector, interaction region and machine parameters still to be worked out in detail, but one of the hardest questions for an EIC will be to estimate the limiting factors for the systematic uncertainties. Due to the high luminosity, many inclusive and semi-inclusive physics observables will be systematics-limited after a relatively short time of data taking, assuming a 50\% operations efficiency. This requires great care to be taken to consider the possible systematic limitations from the beginning and to integrate solutions to minimize them into the design. Only some of the possible limiting systematic effects that will need to be addressed with great care in the design are listed here. Their impact on key physics observables still needs to be studied.

- Absolute luminosity measurements between different beam energy combinations. This is extremely important for measurements like the structure function $\mathrm{F}_{L}$.

- Relative luminosity measurements between bunches with different bunch helicities, i.e. ,,++---+ and +- . Here it will be important to investigate whether Bremsstrahlung can be used for this measurement, as the Bremsstrahlung cross section has a term that is dependent on polarization.

- The measurements of the absolute hadron and electron beam polarization. To date the best precision in the measurement of lepton beam polarization at high energies in a collider was obtained during HERA-I running with 1.6\% 1287. At RHIC the best hadron polarization measurement achieved to date is $\sim 5 \%$ [1288, 1289, 1290] for a polarized proton beam. For high energy polarized ${ }^{3} \mathrm{He}$ beams, R\&D is needed to determine how to measure an absolute polarization. 

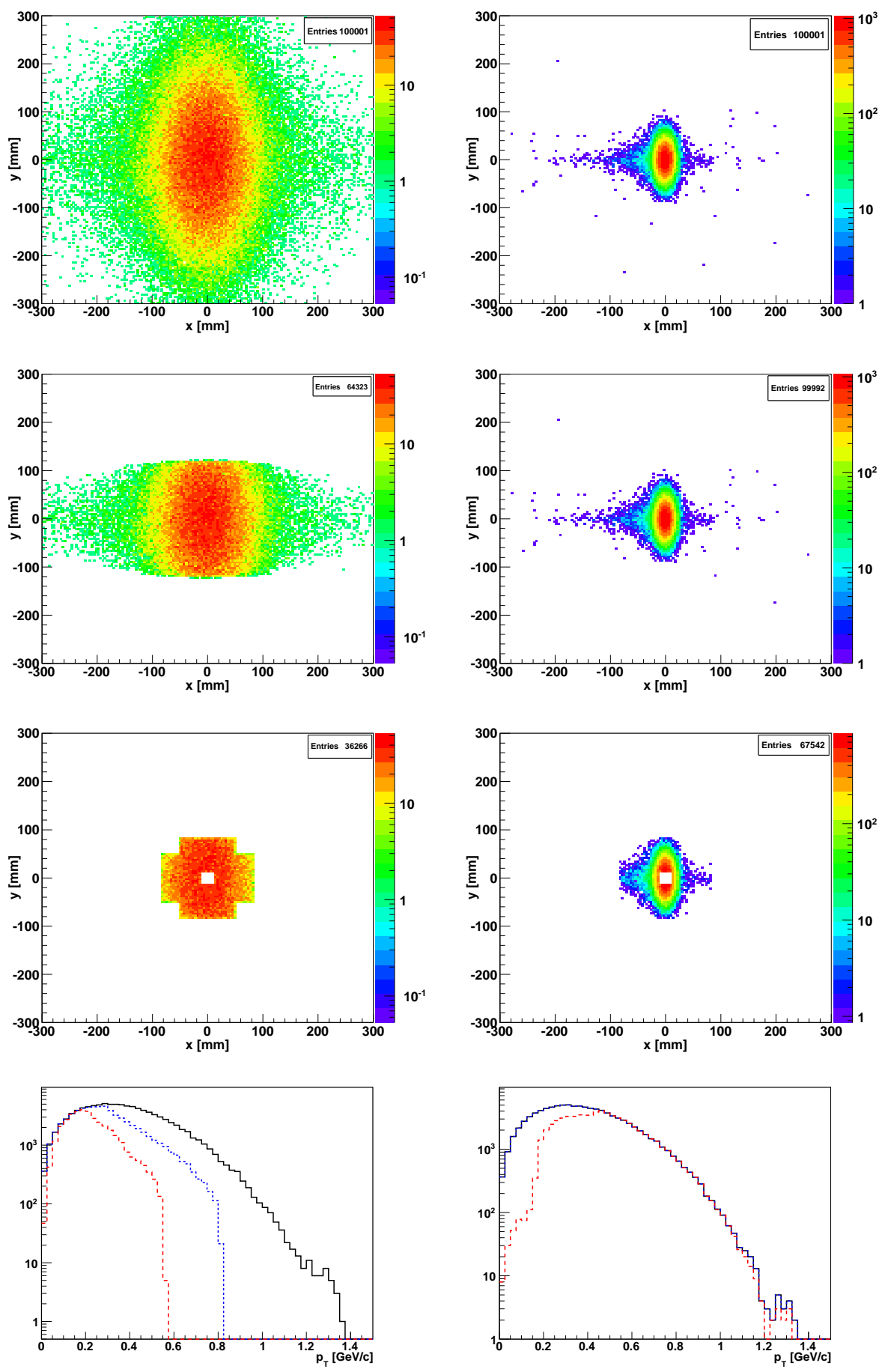

Figure 7.32. Row-1: Spatial distribution of the scattered protons from DVCS events at $20 \mathrm{~m}$ from the IP for 2 different beam energy combinations. Row-2: As Row-1, applying the aperture limitations due to the magnets. Row-3: As Row-2, applying the limitations due to the $10 \sigma$ beam clearance and the acceptance of 'Roman Pots' as currently used by pp2pp at STAR. Row-4: Comparison of the $p_{T}$ spectrum of generated protons (black), those accepted by the quadrupole aperture (blue) and those detected in the 'Roman Pots' (red). 

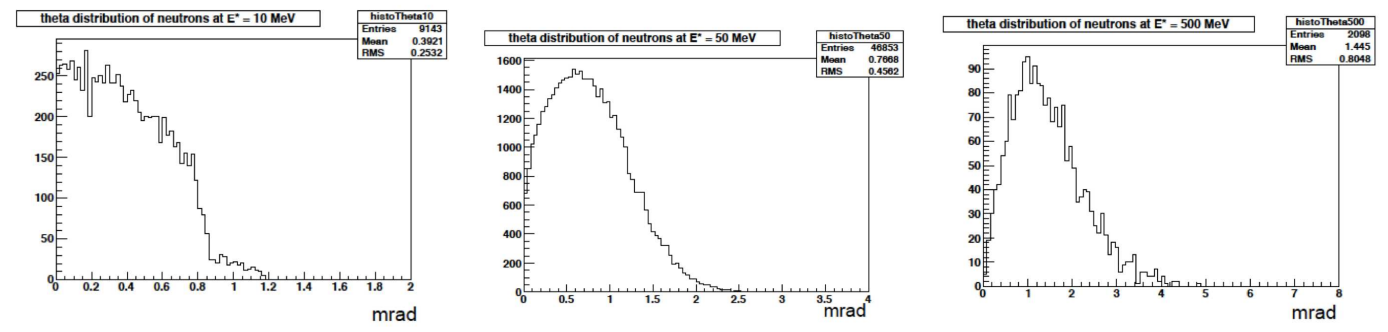

Figure 7.33. The angular distribution of neutrons from the breakup of a Au nucleus depending on the excitation energy.
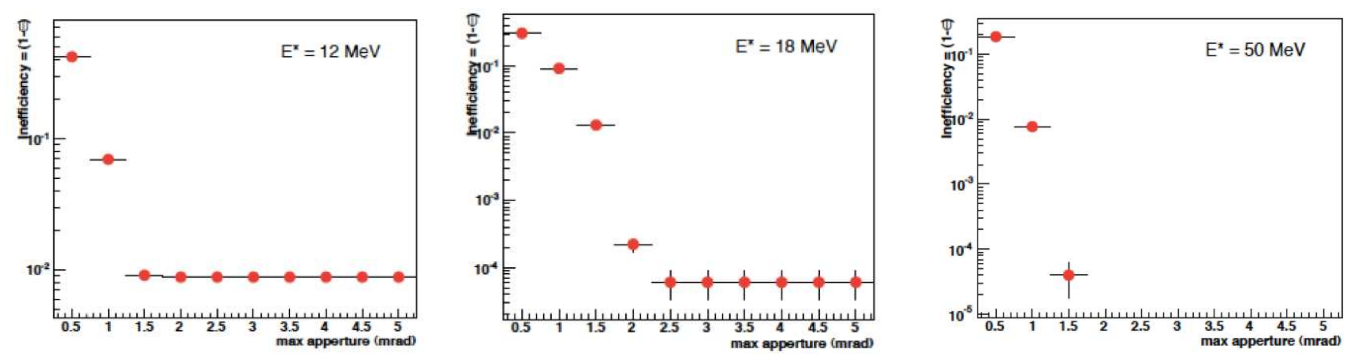

Figure 7.34. The inefficiency to detect the neutrons from the breakup of a Au-nucleus as function of the maximal aperture of the last magnet for different excitation energies.

\section{ePHENIX}

PHENIX is one of the two large dedicated RHIC detectors, located at IP-8. The PHENIX detector consists of two muon spectrometer arms and two central arms sitting in a 1 tesla solenoid. Over the years the detector has been upgraded to the configuration shown in fig. 7.35. Fig. 7.35 and the upper plot of fig. 7.36 show clearly that PHENIX
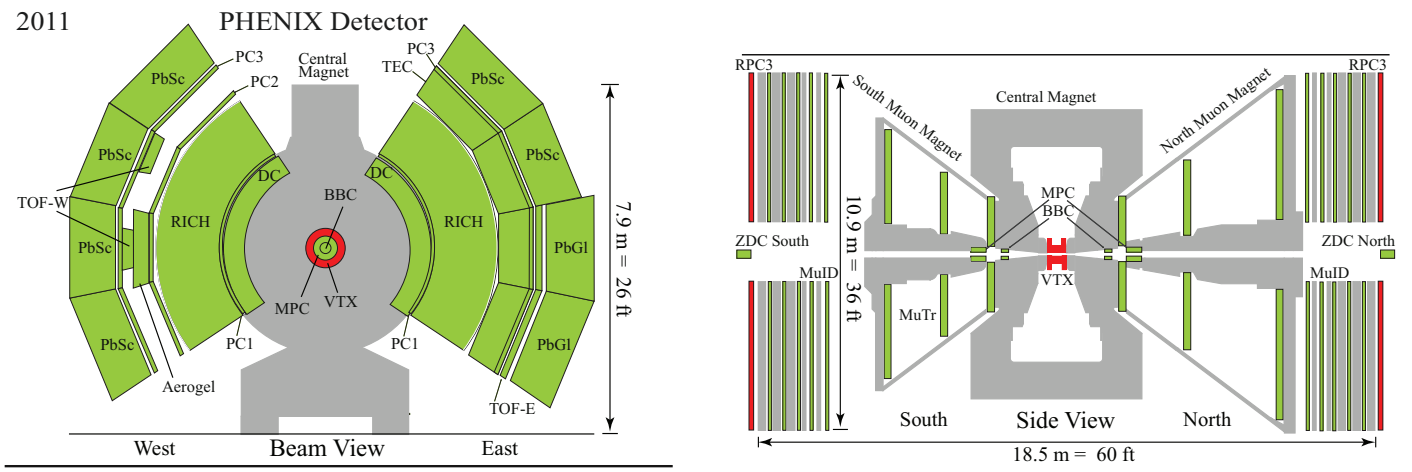

Figure 7.35. A schematic view of the current (2011) configuration of the PHENIX detector.

in its current configuration has only a very small acceptance $(|\eta|<0.35)$ for the scattered lepton. This makes the current PHENIX detector basically not usable for DIS physics.

For the RHIC decadal plan covering the period 2010 - 2020, PHENIX has proposed a major upgrade of the current detector [1291]. The decadal plan outlines an exciting program 

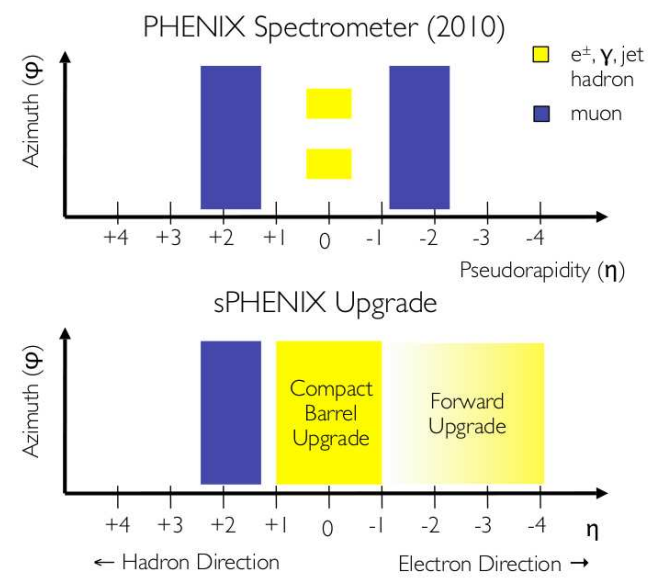

Figure 7.36. Rapidity coverage of the current PHENIX detector compared to the strawman new PHENIX detector. The central barrel detector covers $|\eta|<1.0$; the forward detector has tracking coverage for $-4<\eta<-1$, with full EMCal and HCAL coverage for $-4.0<\eta<-2.0-(-1.5)$ with the exact range dependent on the final design configuration

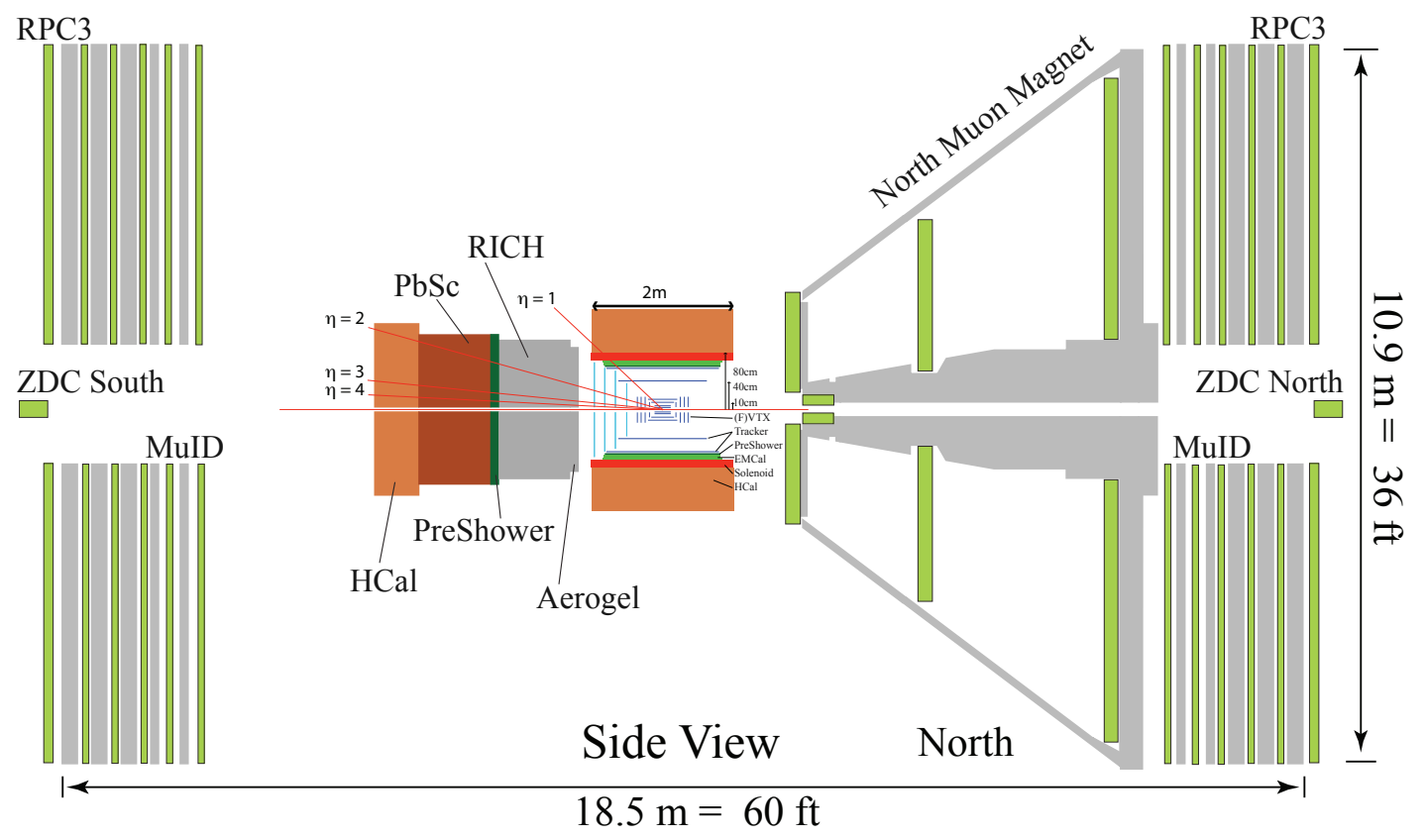

Figure 7.37. Schematic drawing of the new PHENIX detector. 
in heavy ion and spin physics in polarized pp collisions, focused on an investigation of the interplay between perturbative and nonperturbative physics in QCD and on the relative importance of strong and weak coupling. The physics aims have been translated into an extensive set of required physics observables to answer the key scientific questions, leading to the design of the new PHENIX detector. The upgrade plan involves replacing the PHENIX central magnet with a new compact solenoid. The limited aperture provided by the outer central arm detectors would be replaced with a compact EMCal and a Hadronic Calorimeter covering two units in pseudorapidity and full azimuth, complemented by the existing VTX and FVTX inner silicon tracking. Two additional tracking layers would be added. We highlight that the large acceptance and excellent detector capability is combined with high rate and bandwidth. The limited forward coverage of the current PHENIX detector does not allow one to adequately address the questions driving the nucleon structure and cold nuclear matter community, nor does it provide any capabilities for $\mathrm{e}+\mathrm{p}$ or $\mathrm{e}+\mathrm{A}$ collisions. Hence, an upgrade is being considered where one muon arm would be replaced by a new largeacceptance forward spectrometer with excellent PID for hadrons, electrons, and photons and full jet reconstruction capability. The modified detector layout is shown schematically in fig. 7.37. The increase in overall acceptance is shown in the lower part of fig. 7.36. The new compact barrel component at midrapidity is designed for excellent jet reconstruction and PID for photons, electrons, and $\pi^{0}$ in $\mathrm{p}+\mathrm{p}$, proton-nucleus, through central nucleusnucleus collisions. The forward upgrade design is driven by nucleon structure physics and cold nuclear matter physics. Such a forward spectrometer added to PHENIX would not only allow measurements of the single spin asymmetry at forward rapidity to test the QCD prediction that the Sivers function in Drell-Yan and SIDIS is opposite, but would also allow the unique possibility to detect the scattered lepton in $\mathrm{e}+\mathrm{p} / \mathrm{e}+\mathrm{A}$ collisions in the era of an eRHIC to virtualities $\mathrm{Q}^{2}>0.1 \mathrm{GeV}^{2}$. To realize these physics goals it is necessary to upgrade significantly the current PHENIX detector to a detector with high acceptance at forward rapidity $1<\eta<4.0$.

The strawman design for the central barrel has already been described. The forward detectors of the strawman design consist of a RICH, a preshower, an EMCal, an HCal, and additional tracking detectors to provide good momentum definition of the particles going forward. This combination of detectors is motivated by both Drell-Yan and e+p/e+A physics to emphasize the detection of electrons with high efficiency and purity.

It must be stressed again that the PHENIX detector upgrades as discussed above are driven by $\mathrm{p}+\mathrm{p}, \mathrm{p}+\mathrm{A}, \mathrm{A}+\mathrm{A}$ physics. But, comparing the requirements for the physics program at an EIC as described in section 7.3.1, it becomes clear that this detector upgrade also provides opportunities to carry out an $\mathrm{e}+\mathrm{p}$ and $\mathrm{e}+\mathrm{A}$ physics program, referred to as ePHENIX. The upgraded PHENIX is well suited for

- Inclusive $\mathrm{e}+\mathrm{p}$ physics to measure polarized and unpolarized structure functions.

- Inclusive e+A physics to measure unpolarized structure functions and derive nuclear parton distribution functions (nPDFs).

- $\mathrm{e}+\mathrm{p} / \mathrm{e}+\mathrm{A}$ physics involving charm and bottom

- Elastic diffractive physics, i.e. elastic vector meson production and deeply virtual Compton scattering. These measurements require the addition of 'Roman pot' detectors. 
nearly independent of the center-of-mass energy and lepton and hadron beam combination. Unfortunately due to the limited PID capabilities of the ePHENIX design, most of the SIDIS physics program for an EIC will not be possible.

There are still several open question on the detailed performance of the upgraded PHENIX detector in ep / eA collisions, which need to be studied in the next months. Some examples are given below. Of course, some of these concerns can easily be solved by addressing them by design changes.

- How can ePHENIX be integrated in the current IR design of eRHIC?

- What does the current material budget do to the momentum and angular resolution of the scattered lepton?

- Does the current compact solenoid provide enough bending power to achieve sufficient momentum resolution for the scattered lepton at low $\mathrm{Q}^{2}$ ?

- How can a luminosity measurement for ep/eA collisions be integrated in the design?

- Are the currently planned electromagnetic calorimeter designs suited in energy resolution to separate leptons from hadrons via $E / p$, and to get the required resolution for the DVCS photon?

\section{eSTAR}

STAR is the other of the two large dedicated RHIC detectors, located at IP-6. Fig. 7.38 shows STAR in the configuration anticipated in 2014.

The unique strength of STAR (solenoidal tracker at RHIC) [1292] is its large, uniform acceptance capable of measuring and identifying a substantial fraction of the particles produced in heavy ion collisions. The heart of STAR is its main tracking device: a TPC, covering full azimuthal angle and \pm 1.5 units of pseudo-rapidity. A dE/dx resolution of $8 \%$ can be achieved by requiring the tracks of charged particles to have at least 20 out of a maximum of 45 hits in the TPC. Detailed descriptions of the TPC and its electronics system have been presented in [1293, 1294]. The TPC sits in a 0.5 tesla solenoid, surrounded by electromagnetic calorimetry (EMC Barrel, EMC End Cap, FMS) covering $-1<\eta<4$, muon identification (MTD) covering $-1<\eta<1$ and a high-resolution time of flight system (MRPC ToF Barrel) covering $-1<\eta<1$. The tracking in STAR will be further improved by 2014 by adding a forward GEM tracker (FGT) covering $1<\eta<2$ and a high-resolution silicon detector (HFT) covering $-1<\eta<1$. The HFT gives the possibility to separate events with charmed mesons from those with beauty mesons through the detection of the displaced vertex for charmed mesons. Identification in the lepton sector will be enhanced with the Muon Telescope Detector (MTD), which will tag muons for $-1<\eta<1$. This will enable dilepton studies in the $\mu-\mu$ and $e-\mu$ channels, with a focus on separating the Upsilon states and constraining charm backgrounds to the thermal continuum in intermediate mass dileptons. Another unique feature of STAR is the 'Roman Pots' around the main detector; their main focus is to detect protons from elastic diffractive events in pp collisions.

In addition to large coverage in tracking and electromagnetic calorimetry, STAR has good particle identification capabilities. For stable charged hadrons, the TPC provides $\pi / \mathrm{K}(\pi+\mathrm{K} / \mathrm{p})$ identification to $\mathrm{p}_{T} \sim 0.7(1.1) \mathrm{GeV} / \mathrm{c}$ by the measurement of ionization energy loss $(\mathrm{dE} / \mathrm{dx})$. The STAR PID capability is further enhanced by the TOF system with a time resolution of $<100 \mathrm{ps}$, which is able to identify $\pi / \mathrm{K}(\pi+\mathrm{K} / \mathrm{p})$ to $\mathrm{p}_{T} \sim 1.6(3.0)$ 


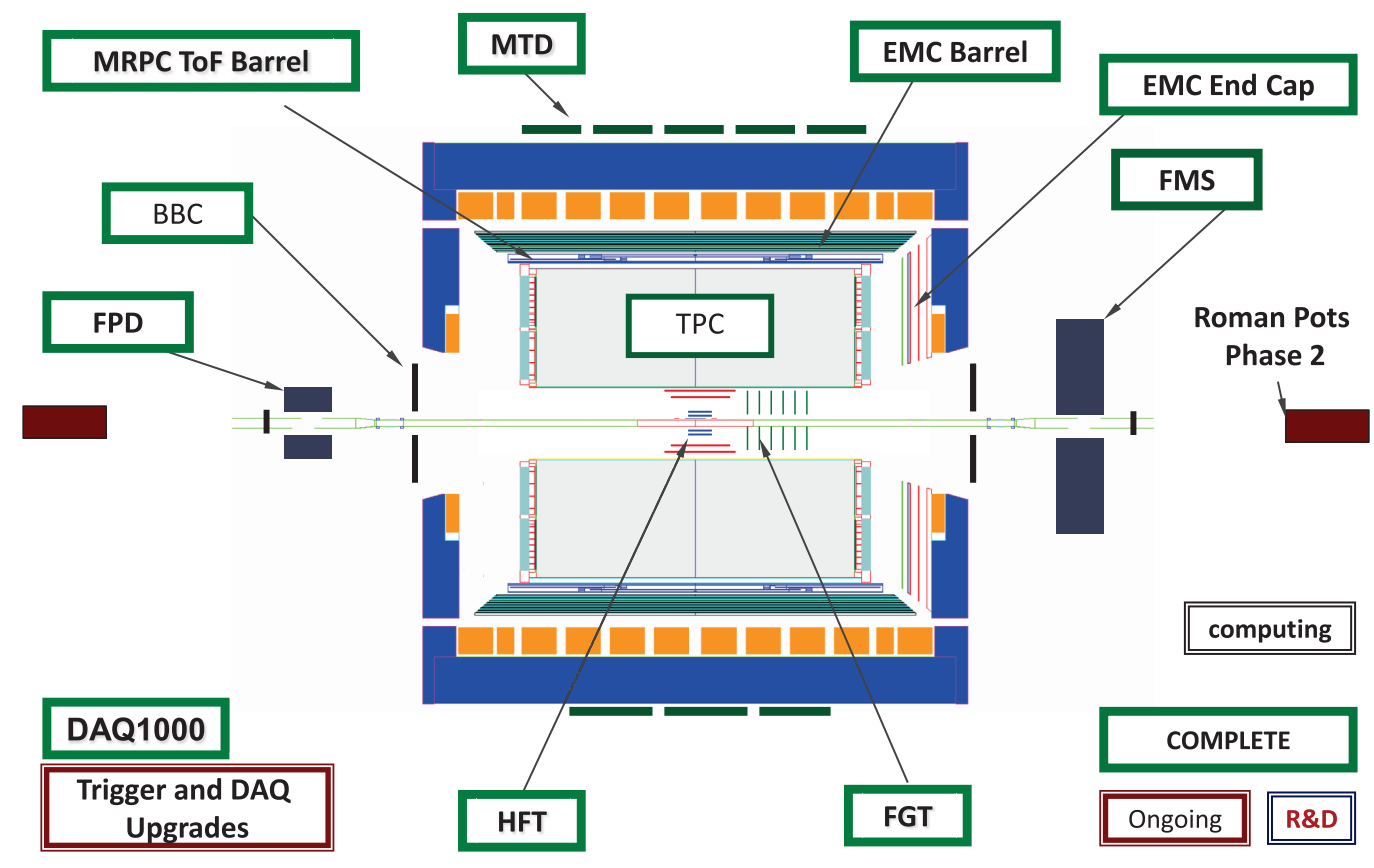

Figure 7.38. Schematic drawing of the STAR detector in 2014.
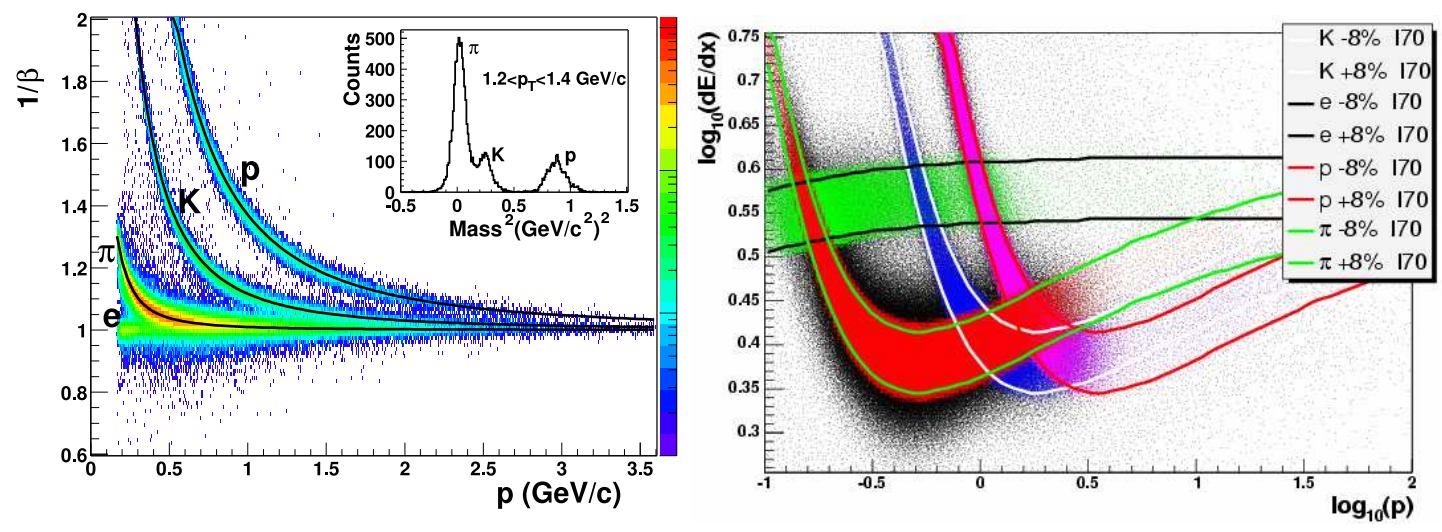

Figure 7.39. Left: $1 / \beta$ vs. momentum for $\pi^{ \pm}, K^{ \pm}$, and $(p \bar{p})$ from $200 \mathrm{GeV} \mathrm{d}+\mathrm{Au}$ collisions. Separation between pions and kaons (kaons and protons) is achieved up to $\mathrm{p}_{T} \sim 1.6(3.0) \mathrm{GeV} / \mathrm{c}$. The insert shows $m^{2}=p^{2}\left(1 / \beta^{2}-1\right)$ for $1.2<p_{T}<1.4 \mathrm{GeV} /$ c. Right: Distribution of $\log _{10}(d E / d x)$ as a function of $\log _{10}(p)$ for electrons, pions, kaons and (anti-)protons. The units of $d E / d x$ and momentum $(p)$ are $\mathrm{keV} / \mathrm{cm}$ and $\mathrm{GeV} / \mathrm{c}$, respectively. The color bands denote the $\pm 1 \sigma d E / d x$ resolution. 
$\mathrm{GeV} / \mathrm{c}$, as demonstrated in the left panel of fig. 7.39. In addition, with the relativistic rise of $\mathrm{dE} / \mathrm{dx}$ from charged hadrons traversing the TPC at intermediate/high $\mathrm{p}_{T}(>3 \mathrm{GeV} / \mathrm{c})$ and diminished yields of electrons and kaons at this $\mathrm{p}_{T}$ range, pions and protons can be identified up to very high $\mathrm{p}_{T}(\sim 10 \mathrm{GeV} / \mathrm{c})$ in $\mathrm{p}+\mathrm{p}, \mathrm{p}+\mathrm{A}$ and $\mathrm{A}+\mathrm{A}$ collisions (see right panel fig. (7.39).

STAR has, like PHENIX, provided a decadal plan outlining the physics program for pp, dA and AA collisions in the next 10 years [1295]. Contrary to PHENIX, the STAR upgrade plans are much more moderate and focus on forward rapidity $(2<|\eta|<4)$. On the side of the STAR detector at which the FMS is situated, the plan is to improve charged particle tracking by adding more tracking planes to the FGT to cover rapidities $2.5<\eta<4$. To improve lepton/hadron and $\gamma / \pi^{0}$ discrimination, as well as baryon/meson separation, a RICH detector and a preshower detector will be added in front of the FMS. The addition of a hadronic calorimeter behind the FMS will further improve the lepton/hadron separation, as well as give the possibility of measuring the energy due to neutral particles in jet reconstruction. The motivation for this upgrade is, like in the case of the PHENIX forward upgrade, transverse spin physics in pp collisions (Sivers asymmetry in Drell Yan) and the study of cold nuclear matter, i.e. parton saturation at small $x$.

The upgrade in rapidity $-4<|\eta|<-1$ is driven solely towards improving the detection capabilities of STAR for the scattered lepton in ep/eA collisions during the era of eRHIC. Currently, proposals include the addition of tracking and electromagnetic calorimetry as well as an additional ToF for PID. For tracking, it is proposed to combine high-resolution with electron identification by for example integrating a Cherenkov detector in the tracking detector.

Combining all these upgrades in fig. 7.40 shows that STAR will have very good acceptance for both the scattered lepton and for the hadrons produced by the current jet at the first stage of eRHIC, with $5 \mathrm{GeV}$ electron beams colliding with proton beams with energies as high as $325 \mathrm{GeV}$. From these figures it is also obvious that the upgrade at negative rapidity is essential to provide good coverage for the scattered lepton below $\mathrm{Q}^{2}$ of $10 \mathrm{GeV}^{2}$.
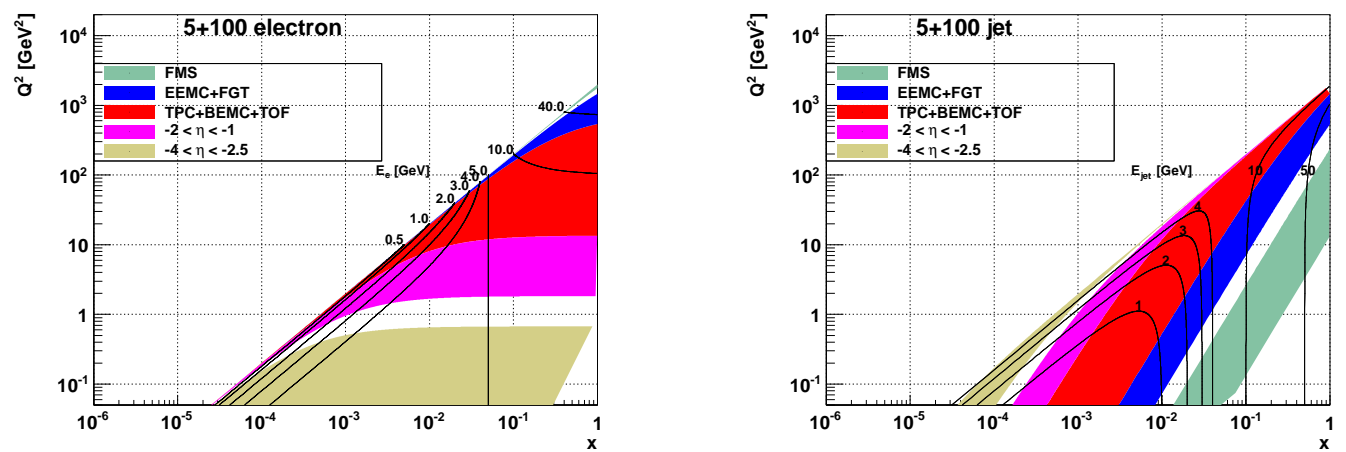

Figure 7.40. Kinematic coverage of the STAR detector in the $\left(\mathrm{x}, \mathrm{Q}^{2}\right)$ plane. Left: electron. Right: struck quark. The electron beam energy is $5 \mathrm{GeV}$, and the nucleus beam energy is $100 \mathrm{GeV} / \mathrm{u}$. Lines of constant laboratory energy of the electron and the struck quark are shown.

The list of questions which need to be answered is very similar to that listed in the ePHENIX section, and many further detailed simulations must be performed to understand in detail the performance of STAR for ep/eA collisions. However from the first studies it is 
clear that eSTAR will be able to make key measurements such as:

- Inclusive $\mathrm{e}+\mathrm{p}$ physics to measure polarized and unpolarized structure functions.

- Inclusive e+A physics to measure unpolarized structure functions and derive nuclear parton distribution functions (nPDFs).

- Elastic diffractive physics, i.e. elastic vector meson production and deeply virtual Compton scattering. Here the great advantage is that eSTAR already possesses 'Roman pot' detectors.

- The good particle ID capabilities also open the possibility of studying many of the semi-inclusive observables in ep/eA collisions, i.e. to do a flavour separation of the quark polarizations to understand both the helicity structure and the transverse spin structure (via Sivers and Collins functions) of the proton.

\subsubsection{Detector Design for MEIC/ELIC}

The Jefferson Lab design of an EIC is based on a novel figure-8 ring-ring design optimized for polarization preservation. The initial version of this EIC is termed the Medium-Energy EIC, or MEIC, which is upgradable to a higher-energy version termed Electron Ion Collider, or ELIC. The MEIC/ELIC will have minimal impact on continued operation of the Jefferson Lab (JLab) $12 \mathrm{GeV}$ fixed-target program.

The ring-ring design of the MEIC/ELIC allows simultaneous operation at high luminosity of multiple detectors located at different interaction points (IPs). Due to the nature of the figure-8, four IPs are foreseen with different functions. The MEIC detector/interaction region has concentrated on maximizing acceptance for deep exclusive processes and processes associated with very-forward going particles, which are the most challenging from the detector point of view. This section will describe the baseline full-acceptance detector in more detail, where it is understood that the various MEIC/ELIC interaction points can house detectors employing different technologies and having a slightly different physics focus.

Given that the detailed design of various subsystems does not have to be frozen for another decade or so, and dedicated pre-R\&D projects are only now under way, the focus of the JLab effort has been on formulating requirements, identifying and addressing critical design issues, and integrating the detector with the interaction region of the accelerator. A tentative detector configuration with estimates based on realistic projections has been adopted, however, to provide users with input for simulations.

\section{The Medium-energy EIC (MEIC)}

The current effort is geared towards the MEIC, for which the guiding principle has been based upon science motivation and design choices close to present state-of-the-art whenever possible. The exception to the latter is the ion beam properties, which have been established for electron-positron colliders but fundamentally depend on electron cooling for proton/ion beams. The fundamental choice for the MEIC design has been to assume short bunches, each carrying a small charge, and to achieve the requirements for the proton beam quality assume extrapolations from conventional electron cooling that have been successfully employed at Fermilab, albeit at modest proton energies. Extending this technology may be incremental, rather than transformational in nature. 
While ELIC would have a circumference of about $3 \mathrm{~km}$, and support proton energies up to $250 \mathrm{GeV}$ (as well as heavy ions up to $100 \mathrm{GeV} / \mathrm{A}$ ), and electrons post-accelerated up to about $20 \mathrm{GeV}$, the MEIC would be somewhat smaller than the $1.4 \mathrm{~km}$ of the CEBAF accelerator, from which it would inject electron or positron beams between 3 and $11 \mathrm{GeV}$. The maximum proton energy would be around $100 \mathrm{GeV}$ (or $40 \mathrm{GeV} / \mathrm{A}$ for heavy ions), but the often quoted design point for which performance parameters are being worked out in detail, is $60 \mathrm{GeV}$. The choice of a mid-range energy for these studies is primarily based on two considerations. On the accelerator side, a proton energy of $60 \mathrm{GeV}$ is a somewhat more conservative value for which one could anticipate the performance projections for the electron cooling to become valid at an early stage of operations. On the physics side, a range of measurements, for instance related to the 3D structure of the nucleon, place strong demands on the resolution in $t$ and the luminosity at modest values of proton energy, corresponding to $s \sim 2000 \mathrm{GeV}^{2}$.

To further illustrate the importance of a mid-range energy for detailed imaging studies through exclusive reactions, we come back to the kinematics associated with these processes, but for a cut in $Q^{2}>10 \mathrm{GeV}^{2}$, a likely must for the valid partonic interpretation of such studies. If one implies a $Q^{2}>10 \mathrm{GeV}^{2}$ cutoff in such exclusive processes, the kinematic patterns of earlier fig. 7.20 drastically change. The upper panels of fig. 7.41 shows how the momentum distribution of mesons associated with exclusive pseudoscalar meson production change with lepton and proton energy. Compared to fig. 7.20, the peak in the forward-ion direction has disappeared completely. Lower lepton energies also push towards lower hadron momenta in the central-angle region, and thus reduced particle identification requirements. The bottom panels of fig. 7.41 show one of the most challenging constraints on the detector and interaction region design for exclusive reactions from the need for detection of the exclusive hadronic state remaining in the exclusive process. The figures show the direct correlation between $t$ and proton energy, scaling like $1 / E_{p}$, and shows the remaining baryonic state goes very much in the forward-ion direction, but far less so (and with lower momenta) for lower proton energies, which are thus much easier to peel off from any beam-stay-clear area. Even more, assuming a fixed resolution in $t$, there are obvious benefits of lower proton energies for imaging. Of course, any high-energy ELIC would in turn greatly benefit from the experience gained from the construction and operation of the MEIC.

While maintaining a future upgrade path to the high-energy ELIC is important and always folded into the MEIC design, emphasis has been placed on ensuring that ELIC will not simply supersede the MEIC, but rather provide a complementary capability. The MEIC is thus designed to excel in the kinematic range that it will cover (i.e., on one hand having an overlap with JLab $12 \mathrm{GeV}$, and on the other with HERA data with $y<0.3$ ). Overlap in science goals is in part achieved by various accelerator features. Perhaps one of the most prominent is the figure- 8 shape, which could allow storage of polarized deuterium beams. By tagging the spectator proton in the small-angle ion spectrometer (discussed below), this will allow to carry out measurements on quasi-free (polarized) neutrons. A high luminosity over a broad kinematic range will make it possible to accumulate sufficient statistics for multiple beam energy settings. The capability to vary the beam energies is essential for some measurements (e.g., $F_{L}$ ), but also makes it possible to optimize the data taking by reducing reliance on data taken at extreme values of $y$, where the systematic uncertainties grow. This can be achieved by having a lepton beam energy that can be varied continuously, and a series of closely spaced discrete ion beam energies. In the MEIC, the latter can be accomplished by changing the number of stored ion bunches by one, and the bunch separation distance accordingly - a scheme facilitated by the high bunch repetition 

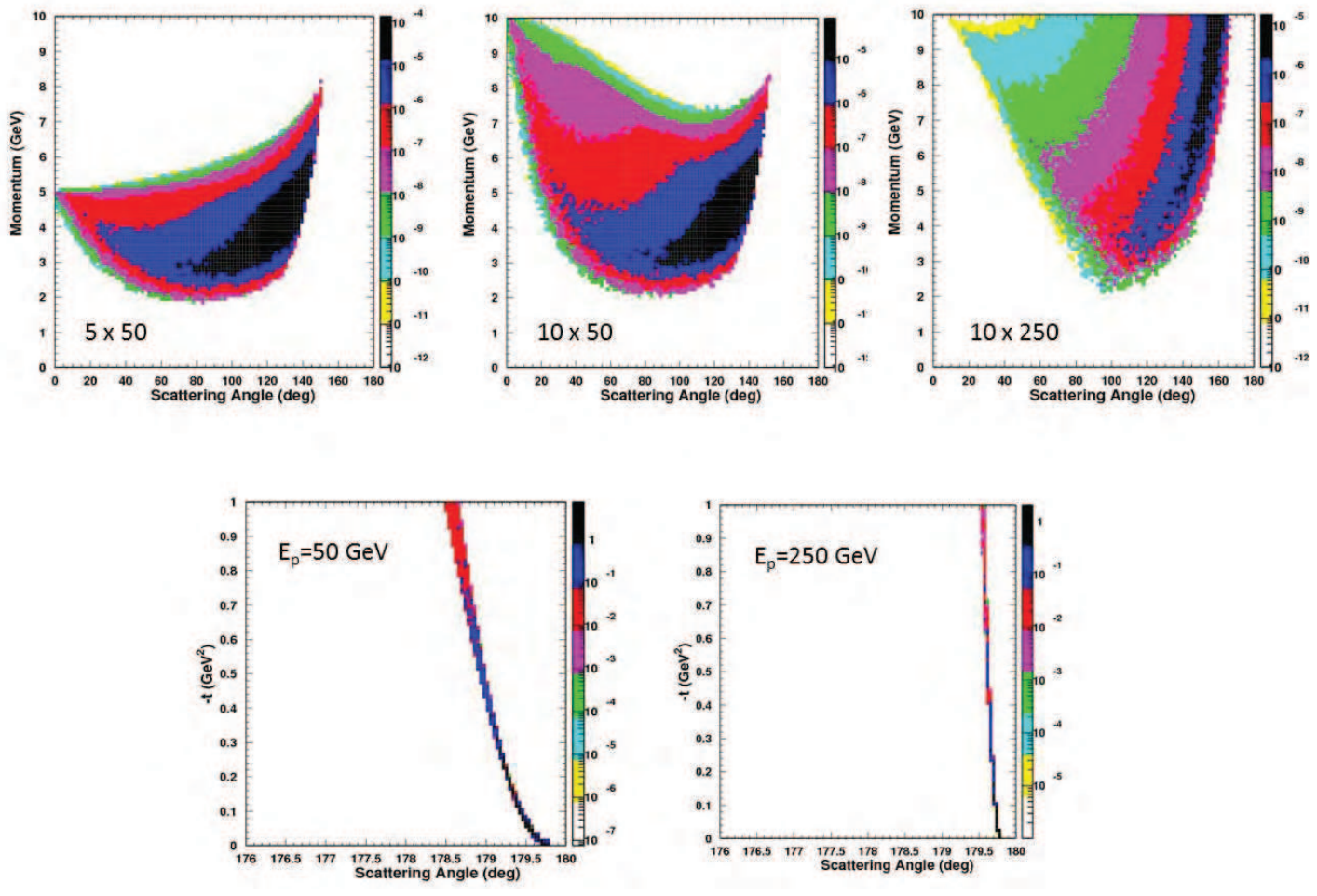

Figure 7.41. The momentum distribution of the exclusive hadronic final state as a function of the scattering angle for three different center of mass energies, $\sqrt{s}=31.6,44.7,100 \mathrm{GeV}$ (upper three panels), and the $t$ distribution as a function of scattering angle of the recoiling baryon in exclusive reactions for proton beam energies $E_{p}=50 \mathrm{GeV}$ and $250 \mathrm{GeV}$ (lower two panels). A cut of $Q^{2}>10$ $\mathrm{GeV}^{2}$ is applied to select the kinematic range of interest for exclusive processes. For lower center of mass energies, the momentum distribution tends towards more central scattering angles and covers lower momenta. The angle of the recoiling hadronic system is directly and inversely correlated with the proton energy. It thus decreases with increasing proton energy. For instance, as shown here, the baryon scattering angle ranges to about $1-2^{\circ}$ at a proton energy of $50 \mathrm{GeV}$ and is reduced to one fifth of that as the proton energy increases to $250 \mathrm{GeV}$. 
frequency. Independently varying beam energies also makes it possible to choose the most suitable lab kinematics at a certain value of $s$, potentially improving acceptance, resolution, and particle identification for the reaction of interest (see also fig. 17.41).

Having small, short ion bunches with a high bunch repetition frequency also facilitates the use of SRF crab crossing cavities, which were originally developed for KEKB to allow beams collide at an angle without significant loss of luminosity. In the context of an EIC, these were pioneered in the ELIC design, and the possibility of creating a significant crossing angle (at least $50 \mathrm{mrad}$ ) became early on a key feature of the small-angle detection for the MEIC (see section 7.3.4).

\section{Detector Placement and Backgrounds}

The figure-8 ring can support two IPs per straight section, one of which will be a "highluminosity" IP with the full crossing angle. In order to minimize backgrounds, the two high-luminosity IPs will be located close to where the ion beam exits the arc, and far away from the arc where the lepton beam exits. The latter helps to decrease synchrotron radiation (and the secondary neutron flux) at the IP, which is anyway already reduced due to the use of crab crossing (with the ion beam, not the electron beam, making the horizontal bend correction). The synchrotron background is reduced even further by lowering the strength of the last arc dipoles. The short distance between the ion arc and IP suppresses detector backgrounds from interactions of the beam with residual gas in the beam pipe by providing a smaller "target" with line-of-sight to the detector. A shorter section of the beamline is

also easier to bake and keep at at ultra-high vacuum. A comparison with HERA, also taking into account the lower $p-p$ (and $p-A$ ) cross section and lower hadron multiplicity at the $100 \mathrm{GeV}$, suggests that the hadronic background will be about an order of magnitude lower in the MEIC at comparable vacuum and ion beam current, leaving a lot of headroom to increase the latter. Due to the bends associated with the horizontal crossing, the secondary IPs on each straight section will not have a line-of-sight along the full straight section, but there this is less of an issue since they are intended to either have diagnostics equipment (e.g., polarimetry), or special detectors which are less sensitive to backgrounds or intended to operate at lower beam currents.

\section{Detector and Interaction Region Layout}

A global outline of the fully integrated MEIC detector and interaction region is given in fig. 7.42. We will in the subsequent subsections go in more detail over the central detector region, defined as the region of the detectors operating within the solenoid, the electron and ion endcaps, and the strategy to accomplish a full-acceptance detector. The latter has two ingredients, a relatively simple approach to incorporate low- $Q^{2}$ electron detection and a more challenging solution to measure forward and ultra-forward (in the ion direction) going hadronic or nuclear fragments. Here, we make critical use of various ingredients of the MEIC detector/interaction region design: i) the $50 \mathrm{mrad}$ crossing angle; ii) the range of proton energies; iii) a small 1-2 Tm dipole field to allow measurement down to $0.5^{\circ}$ before the ion final focusing magnets; iv) ion final focusing magnets with apertures sufficient for particles with angles up to at least $0.5^{\circ}$; and v) a large $20 \mathrm{Tm}$ dipole field much more downstream to peel off spectator particle and allow for very small-angle detection.

The strategy will be that various detector elements, amongst which zero degree calorimeters for neutron detection and various small-angle detectors, will be placed in the region 


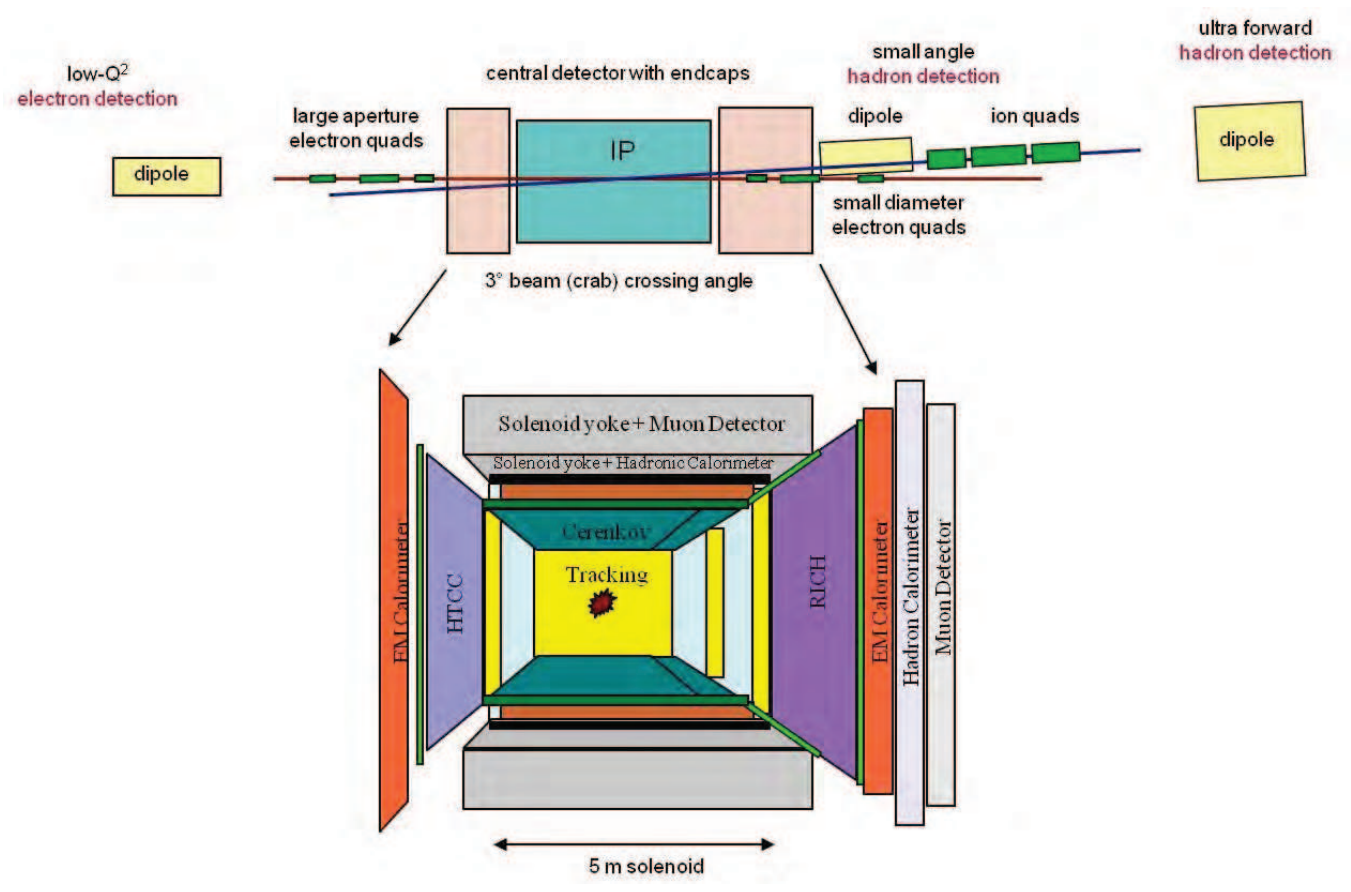

Figure 7.42. Interaction region and central detector layout, and its placement in the general integrated detector and interaction region. The central detector includes endcaps in both the electron and ion direction.

between the ion final focusing quads and the $20 \mathrm{Tm}$ dipole field, and also beyond this $20 \mathrm{Tm}$ dipole field. This then results in an essentially 100\% full acceptance detector. The electron beam traverses the center region of the solenoid, while the proton/ion beam traverses at the crab crossing angle. This choice minimizes any electron steering and synchrotron radiation. Note that the $50 \mathrm{mr}$ crab crossing angle also facilitates the small-diameter electron final focusing quads to be moved in to 3.5 meter distance of the interaction point. The lower electron beam energies and hence lower-field requirements for the electron beam allows the construction of relatively small-sized quadrupoles, much simplifying the electron optics design.

\section{Central Detector}

To fulfill the requirement of hermeticity, the central detector will be built around a solenoid magnet (with a length of about $5 \mathrm{~m}$ ). Due to the asymmetric beam energies, the interaction point (IP) will be slightly offset towards the electron side $(2 \mathrm{~m}+3 \mathrm{~m})$. This will allow more distance for the tracking of high-momentum hadrons produced at small angles, and a larger bore angle for efficient detection of the scattered beam leptons.

The characteristics of the solenoid are guided by the desire to optimize the tracking resolution, which at central angles scales like $\Delta p / p \sim \sigma p / B R^{2}$, where $\sigma$ is the position resolution, $p$ the particle momentum, $B$ the magnetic field, and $R$ the radius of the central tracker. At forward angles, however, the resolution depends on the scattering angle, but is independent of $R$ as the particle leaves the cylindrical central tracking system from the front side (see the left panel of fig. 7.43). The resolution will then deteriorate rapidly given the lack of transverse field along the central axis of a solenoid. This will later be remedied 
by adding a small dipole field, as high ion energies boost the outgoing hadrons to high momenta at forward angles and one wishes to optimize resolutions also in the forward-ion direction. To obtain a roughly better than $1 \%$ momentum resolution for central angles and particles in the $5-10 \mathrm{GeV} / \mathrm{c}$ momentum range, a field $B$ in the 2-4 $\mathrm{T}$ range seems highly desirable. This high field requirement suggests a magnet with a reasonably small diameter, preferably not larger than about $4 \mathrm{~m}$, putting radial space at a premium. Of course, a smaller diameter has the advantage of simplifying the magnet design, with the additional advantage of reducing detector cost (which scale with the radius for the barrel calorimeter and roughly as the radius squared for the endcaps). An alternate solution may be to increase the space for tracking in the central solenoid while reducing the required solenoid field, as illustrated in the right panel of fig. 7.43. Here, the resolution improvement for pions with $10 \mathrm{GeV} / \mathrm{c}$ momentum and a scattering angle of $90^{\circ}$ is shown as a function of the tracking length and solenoidal field. Thus, there is strong incentive to reduce the space requirements for particle identification detectors within the central solenoid as much as possible, to use available space for tracking, or reduce the solenoid diameter.
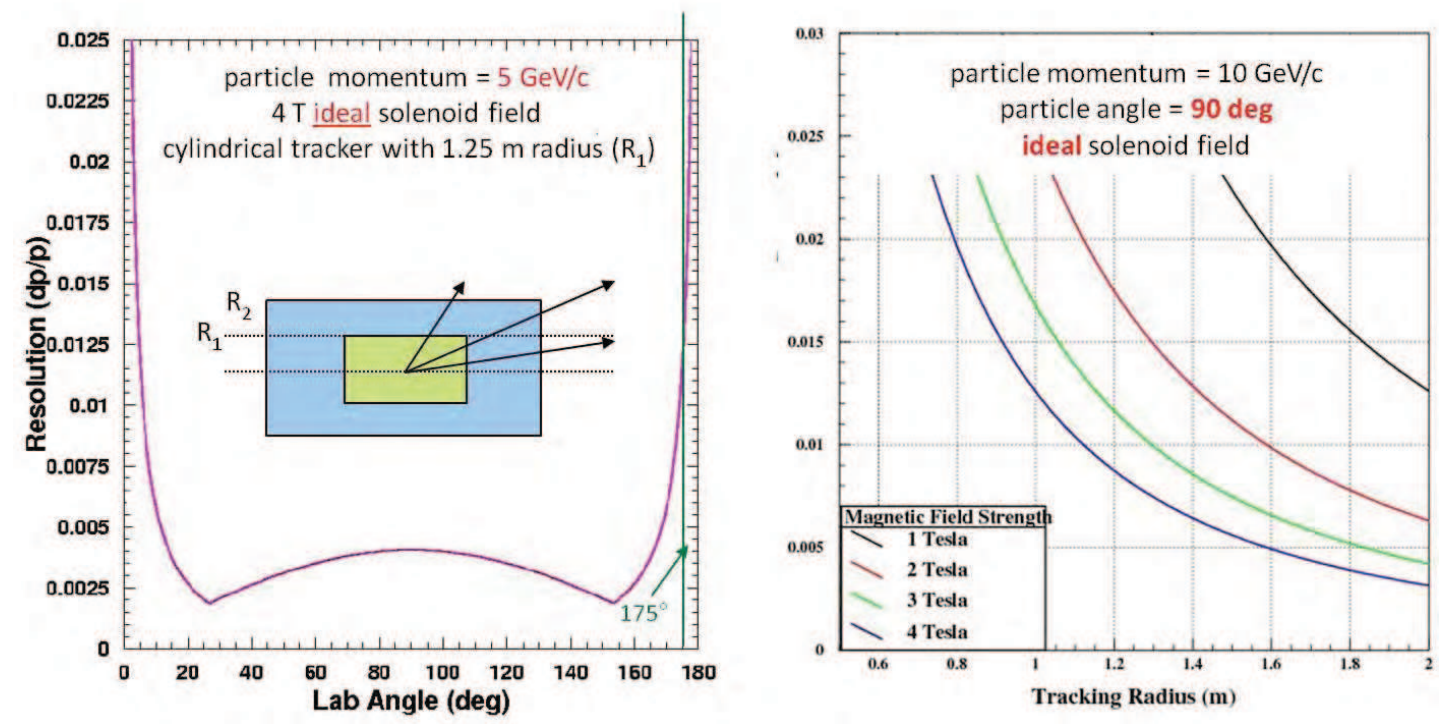

Figure 7.43. (left) The resolution as a function of lab angle for a particle (pion) momentum of 5 $\mathrm{GeV} / \mathrm{c}$ in a $4 \mathrm{~T}$ ideal solenoidal field and with a cylindrical tracker of radius $1.25 \mathrm{~m}$; (right) The resolution as function of solenoidal field strength and tracker radius for a particle (pion) momentum of $10 \mathrm{GeV} / \mathrm{c}$ and a scattering angle of $90^{\circ}$.

The central detector would contain a tracker, particle identification, and calorimetry. A three layer configuration of the central tracker was suggested at the JLab EIC detector workshop (June 4-5, 2010)2. The first layer would consist of a low-mass vertex tracker with sufficient resolution to separate primary and secondary vertices in charm production. The middle layer would be a Time-Projection Chamber (TPC) with GEM-based readout, and the outer layer would be a cylindrical GEM tracker. The position resolution of the TPC would be about $50 \mu \mathrm{m}$, which is a factor two improvement over the inner drift chambers of CLAS12. In conjunction with the outer GEM layer, it should provide adequate $(r, \theta, \phi)$ in-

${ }^{2}$ http://conferences.jlab.org/eic2010 
formation. Ongoing R\&D for vertex and micropattern detectors (including GEMs), suggest that such a high-performance tracker could be built for the EIC detector. Nevertheless, a radius of at least $1 \mathrm{~m}$ would be required.

Particle identification in the central detector is the most open design question. At low momenta, $\mathrm{dE} / \mathrm{dx}$ (in the TPC) or TOF can be helpful. With precise timing, the momentum range of the latter could be extended somewhat (although this would require a comparable uncertainty on the track length determination in order to get a good $t_{0}$ ). The most challenging requirement is, however, for a radially compact detector providing $\pi / K$ identification over a sufficiently wide momentum range. Taking up $8 \mathrm{~cm}$ of radial space, a BaBar-type DIRC could satisfy this condition, providing $3 \sigma \pi / K$ separation up to $4 \mathrm{GeV} / \mathrm{c}, e / \pi$ separation close to $1 \mathrm{GeV} / \mathrm{c}$, and p/K separation up to $7 \mathrm{GeV} / \mathrm{c}$. An aerogel barrel RICH could provide almost comparable performance. Neither is sufficient for the exclusive (GPD) or semi-inclusive (TMD) programs. The current baseline design thus includes a Low-Threshold Cherenkov Counter (LTCC) with $C_{4} F_{10}$ or $C_{4} F_{8} O$ gas in addition to the DIRC. This would provide $e / \pi$ separation between 1 and $3 \mathrm{GeV} / \mathrm{c}$, and $\pi / K$ separation from 4 to $9 \mathrm{GeV} / \mathrm{c}$, but at a price of $50-70 \mathrm{~cm}$ of radial space. Adding $C_{4} F_{10}$ to a barrel RICH would increase the radius by at least $80-90 \mathrm{~cm}$, although a RICH could extend the momentum coverage to $14 \mathrm{GeV} / \mathrm{c}$. Ultimately the allocation of radial space to PID and tracking is a matter of priorities, and with multiple detectors one could easily imagine that these would offer complementary capabilities. On the other hand, if one could improve the $\theta_{c}$ resolution for a DIRC by about a factor of two, its $3 \sigma \pi / K$ separation could be extended to about $6 \mathrm{GeV} / \mathrm{c}$, with the upper limits for the other particle species shifting accordingly, eliminating the need for the gas Cherenkov. Given the size of the EIC detector, an all-crystal electromagnetic calorimeter would be financially expensive and only needed in critical regions. Tungsten powder / scintillating fiber or other technologies may provide a more affordable alternative for the barrel without an excessive loss of resolution. If needed, the return yoke of the solenoid magnet can be used as part of a hadronic calorimeter, and as an absorber for muon detection (along the lines of CMS).

\section{Detector Endcaps}

The electron side endcap would face requirements quite similar to those of CLAS12, and it is natural to adopt a similar design. Due to the offset of the IP, lower particle momenta, and simpler small-angle detection (see section 7.3.4), the electron side is not nearly as crowded as the ion one. For lepton detection at small polar angles $(\theta)$, the main priority of the tracking would be to provide good $\theta$ resolution, as this directly impacts the reconstruction of the event kinematics. The inner part of the endcap tracker should thus be an extension of the vertex tracker, using semiconductor detectors. At larger angles, the requirements are not as demanding and the choice of technology is not as crucial. It could include planar GEMs or even cheaper drift chambers with a small cell size. Given the generous space constraints, a final tracking region could be added outside of the solenoid itself to improve tracking performance. Lepton identification will also use an electromagnetic calorimeter and a High-Threshold Cherenkov Counter (HTCC) with $C F_{4}$ gas or equivalent. The light can be collected by mirrors, producing a cost-effective readout. In this endcap region, hadron identification will be partially provided by a TOF detector, for which the endcap is more suitable than the barrel due to the longer flight path. The $\pi / K$ identification range, again in the electron endcap region, could be extended through the use of a LowTheshold Cherenkov Counter (LTCC) with $C_{4} F_{8} O$ gas or equivalent, possibly operating 
slightly above atmospheric pressure to lower the pion detection threshold. Of course, to push $\pi / K$ identification to larger momenta, $\sim 10 \mathrm{GeV} / \mathrm{c}$, a RICH detector may need to be considered, but there does not seem to be a compelling need in this electron endcap region for the MEIC. Given the space available on the electron side, there is no strong requirement for a compact electromagnetic calorimeter. Since the momentum resolution from tracking deteriorates at small angles, where also the rates go up, the ideal configuration would involve an inner circle of high-resolution, radiation-hard crystals, and a more budget-friendly outer part. Both could be covered by the same pre-shower calorimeter.

The ion side endcap would have to deal with hadrons with a wide range of momenta, some approaching that of the ion beam. The forward tracking would thus greatly benefit from good position resolution (e.g., planar GEMs), at least on par with the $50 \mu \mathrm{m}$ of the TPC. The smallest angles can be covered by semiconductor detectors as on the electron side. Of course, a good position resolution will also put significant demands on the detector alignment and field knowledge. The most important feature of the forward tracker, however, is related to the ion beam crossing angle with respect to the electron beam. In addition to being a key component of the small-angle detection, this turns the tracking resolution into a $2 \mathrm{D}$ problem. Whereas the momentum resolution in a solenoidal field deteriorates rapidly at small angles with respect to the axis, the hadron scattering angle is essentially defined with respect to the ion beam line. Given that the proton/ion beam traverses the solenoid at a 50 $\mathrm{mr}$ (crab crossing) angle, so already encounters some transverse magnetic field component, hadrons scattered away from the electron beam will end up in a part of the detector with better momentum resolution than those scattered towards the electron beam. Taking the $2 \mathrm{D}$ character of the problem into account, and the significant $50 \mathrm{mr}$ beam crossing angle, the spot of poor resolution will be moved into the periphery covering and only a small range in the azimuthal angle $\phi$ will be affected. For most processes, all particle tracks will remain in the zone of good resolution. In contrast, if the crossing angle is small, all particle tracks at very forward angles will suffer from poor momentum resolution, as shown in the right panel of fig. 7.43 ,

To identify particles of various species over the full momentum range, one would ideally want to use several radiators. A typical combination could include aerogel (perhaps with more than one index of refraction), $C_{4} F_{10}$ or equivalent gas, and $C F_{4}$. This would make some kind of RICH detector an attractive option, in particular if the endcap radius was not too large. Still, there are several possible approaches which eventually will need to be studied in detail. One could, for instance, imagine a dual radiator gas RICH combined with a disk DIRC (as in PANDA), with the latter providing $\pi / K$ identification up to about $4 \mathrm{GeV} / \mathrm{c}$. Having the longest flight path from the IP, the ion endcap is also where one could achieve the best results with high-resolution TOF (perhaps even integrated with the readout of the RICH). Regardless of technical solution, the total thickness of the stack of PID detectors is assumed not to exceed $1.5 \mathrm{~m}$. Calorimetry in the ion endcap will include both electromagnetic and hadronic parts. The main focus of the former will be to study various reaction products rather than the scattered lepton. However, the same resolution arguments apply as for the electron endcap, and a solution with an inner high-resolution circle, and a more cost-effective outer part makes sense here as well. The magnetic enclosure of the endcap can, as in the case of the return yoke of the central detector, be integrated with a hadronic calorimeter, and serve as an absorber for muon detection. 


\section{Small-angle Detection}

The design for the full-acceptance detector envisions small-angle detection on both sides of the central detector. The naming convention used here will be that the "ion side" or "ion endcap" refers to the side of the outgoing ion and incoming electron beam. The "electron side" refers to the other one.

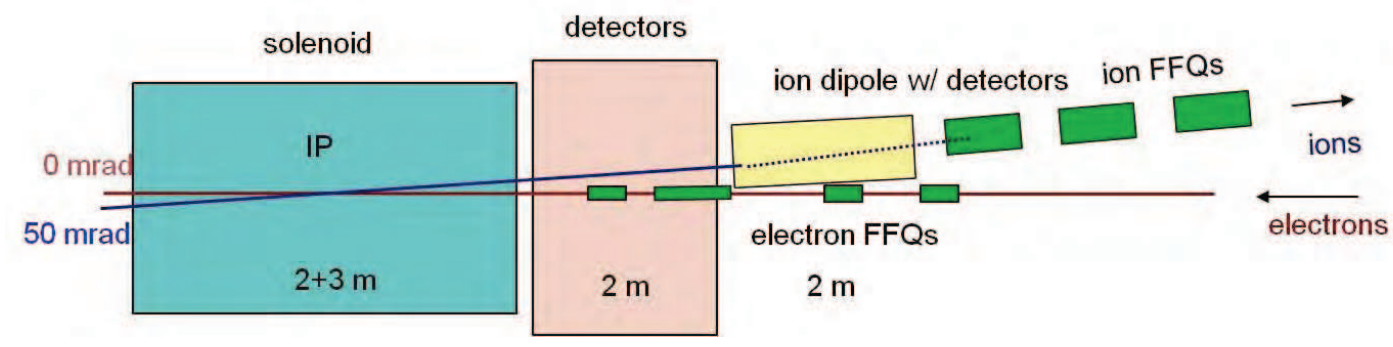

Figure 7.44. Forward ion detection with $50 \mathrm{mrad}$ crossing angle for the full-acceptance detector. Note that the distance to the final focusing quadrupoles are located $7 \mathrm{~m}$ from the IP.

On the ion side, the detection will be performed in three stages as illustrated in fig. 7.44. The first stage is the endcap (discussed in section 7.3.4), which will cover all angles down to the acceptance of the forward spectrometer. This in turn has two stages, one upstream of the ion Final Focus Quadrupoles (FFQs), covering down to $0.5^{\circ}$, and one downstream covering up to at least $0.5^{\circ}$. The former will use a 1-2 Tm dipole to augment the solenoid in the range where the resolution is poor. The magnet will be about $1 \mathrm{~m}$ long and cover the distance to the electron beam (corresponding to the horizontal crossing angle of $50 \mathrm{mrad}$ ), and about twice that in the other directions, for a total acceptance of $150 \mathrm{mrad}$ in the horizontal and $200 \mathrm{mrad}$ in the vertical plane. An important feature of the magnet design is to ensure that the electron beam line stays field free. The dipole will have trackers at the entrance and exit, and a calorimeter covering the ring-shaped area in front of the first ion FFQ. For neutrons, the primary goal of this calorimeter is to have good angular resolution. This intermediate stage is essential for providing good coverage and resolution in $-t$, and to investigate target fragmentation. The former is of particular importance for the study of exclusive processes, essential for the 3D imaging of the nucleon, requiring detection of the recoil baryon. Since $t \sim \theta_{p}^{2} E_{p}^{2}$, the $t$-resolution depends on the angular resolution that can be achieved. With a $50 \mathrm{GeV}$ proton beam, a $-t$ of $1 \mathrm{GeV}^{2}$ corresponds to about 27 mrad (see fig. (7.41). With an angular resolution of $1 \mathrm{mrad}$, the intermediate detection stage would be able to cover $-t$ up to $2 \mathrm{GeV}^{2}$ with a resolution of about $40-50 \mathrm{MeV}^{2}$ a value that would scale with angular resolution of the inner silicon forward tracker. Recoil baryons with larger values of $-t$ would be detected in the endcap. At higher ion beam energies the $t$-acceptance of the dipole increases, but the resolution deteriorates rapidly (due to the $E_{p}^{2}$ factor). Going to lower ion energies, the opposite is true.

The last stage is the ultra-forward detection that is crucial for the tagging of spectator protons in deuterium, as well as other recoil baryons/nuclei. The design is heavily integrated with the accelerator (see fig. 7.45), using two key features. One is, again, the horizontal crossing angle for the ion beam, which needs to be "corrected" some distance downstream of the interaction point (IP). For a $50 \mathrm{mrad}$ crossing angle, this corresponds to a bend of close to $100 \mathrm{mrad}$, and the required $20 \mathrm{Tm}$ dipole(s) can also serve as a dedicated forward spectrometer, using the long drift space beyond for detection of both charged and neutral 


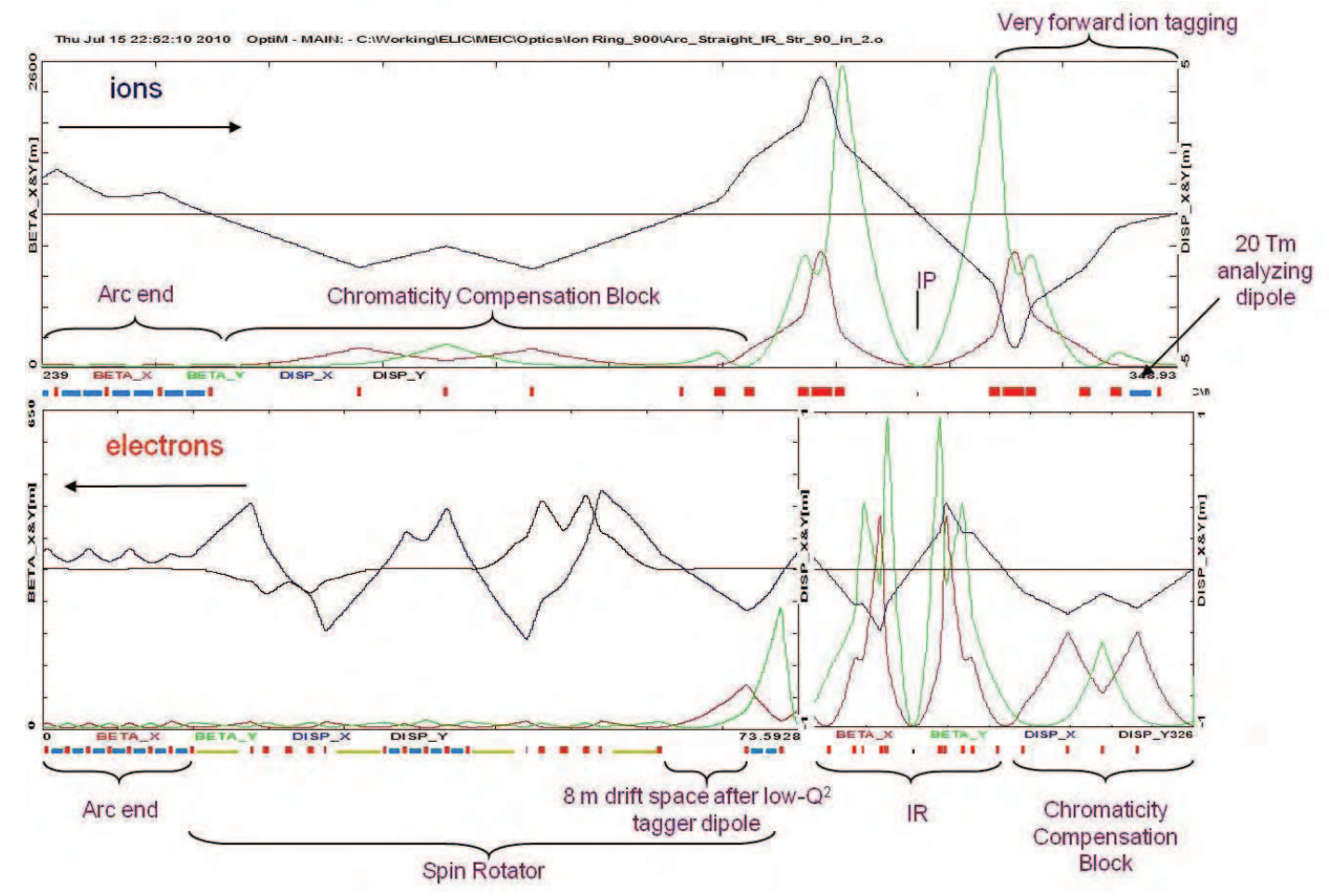

Figure 7.45. The integration of particle detection in the accelerator.

particles. The other feature is a beam optics requiring low quadrupole gradients, allowing large aperture magnets. In the current design, the maximum quad gradient is less than 65 $\mathrm{T} / \mathrm{m}$. With a $10 \mathrm{~cm}$ aperture, this creates a $6.5 \mathrm{~T}$ peak field (simply the product of the aperture radius and gradient), which the magnet design should be able to support if larger peak fields were acceptable, the apertures would increase accordingly. The gradients are further arranged so that they drop off faster than the distance from the IP to that specific location, allowing the apertures to become correspondingly larger, and thereby making sure that no bottlenecks are created. This defines a geometrical acceptance through the ion final-focusing quads (FFQs) of $10 \mathrm{mrad}$, or well beyond $0.5^{\circ}$, on each side of the beam (20 $\mathrm{mrad}$ in total). To focus the $250 \mathrm{GeV}$ beams in ELIC, the maximum quadrupole gradients would have to be 2.5 times larger than for the $100 \mathrm{GeV}$ of the MEIC, and the apertures reduced accordingly.

The acceptance for charged particles depends on both the polar and azimuthal angles (since quads focus in one plane and defocus in the other), as well as their momentum. This can be optimized by placing a dipole spectrometer relatively close to the FFQs. To give a numerical example, a $100 \mathrm{mrad}$ bend to a deuterium beam would equate to a $200 \mathrm{mrad}$ bend for a spectator proton. Over a drift space of $10 \mathrm{~m}$ (a relatively modest distance), the spectator proton would acquire a transverse separation of $1 \mathrm{~m}$ from the main beam. For heavy nuclei $(\mathrm{A} / \mathrm{Z}=2.5)$ with a negligible scattering angle at close to the beam momentum, this would increase to $1.5 \mathrm{~m}$, while fragments with other $\mathrm{A} / \mathrm{Z}$ ratios would be lined up in between (in particular, $\mathrm{N}=\mathrm{Z}$ would be at $25 \mathrm{~cm}$, while neutron rich fragments would be deflected to the other side). Ions scattered at zero degrees and having $98 \%$ of the beam momentum would be $2 \mathrm{~cm}$ from the beam after $10 \mathrm{~m}$ of drift. Due to the large deflection 
in a well known field (including the few preceding elements), the momentum resolution of the spectrometer would be excellent. Since no position measurements would be possible within the beam-stay-clear area, the angular resolution would depend on the knowledge of the optics between the IP and detection point. The reconstruction of the angle would be aided by the scattered particles quickly exiting the beam-stay-clear area after having passed the spectrometer, with multi-point tracking to be applied in the drift region. Nevertheless, some some low-momentum particles scattered at large angles will not make it all the way to the dipole spectrometer. To detect these particles, some ad hoc detectors ("Roman pots") may be placed along the way, although an interesting idea currently under investigation is to have a small-diameter compensating solenoid between the FFQs and the $20 \mathrm{Tm}$ dipole. In addition to its benefits for the accelerator, such a magnet could help in tracking charged particles that do not reach the final spectrometer dipole.

The low- $Q^{2}$ tagger on the electron side will complement the electron detection in the central detector and electron side endcap. Since the electron quad gradients required for 11 $\mathrm{GeV}$ beams are very small compared with what is needed for $100 \mathrm{GeV}$ protons, one can make the apertures very large without being constrained by peak fields (the different apertures on the incoming and outgoing sides do not affect the optics). The optimal transition point from the calorimeter to tagger coverage will ultimately be determined by physics simulations. The quads would be followed by a dipole spectrometer with sufficient drift space $(8 \mathrm{~m}$ in the current layout) to detect leptons with a significant fraction of the beam energy.

\section{Beam Helicity Reversals}

The electron and ion beam polarimetry has been given a special "interaction region" in the MEIC/ELIC design, in part due to the often large amount of space needed for Compton polarimetry. With the anticipated work in systematic understanding of Compton polarimetry in both JLab Halls A and C, and further plans to cross-calibrate this with atomic beam Moller polarimetry for a future demanding parity-violating Moller experiment, electron beam polarization determination through Compton polarimetry may well achieve sub- $0.5 \%$ uncertainties. Ion beam polarimetry remains more complicated, although efforts to reduce uncertainties are underway and possibilities are studied in elastic and inelastic electron-proton scattering experiments in situ.

The MEIC design will need both fast electron spin helicity reversal or flip for double-spin experiments and a program of deep-inelastic parity-violating experiments, and fast ion-spin flip for single-nucleon spin asymmetry experiments. The latter can also be an alternate method for double-spin experiments. The MEIC design, with its $750 \mathrm{MHz}$ bunch trains, does not assume bunch-to-bunch spin flips, but also does not need it. A helicity-reversal frequency of $0.1 \mathrm{~Hz}$ will be at about the level needed for experiments.

For double-spin experiments, it is to first order equivalent to perform fast helicity reversals of electron or ions. The choice is a question of detailed precision, as shown later. For single-spin asymmetry experiments, these techniques are however totally different, and can not replace each other. Single-electron spin asymmetry (flipping electrons only) is mostly useful for parity violation experiments, while single-nucleon spin asymmetry (flipping ions) is mostly useful for nucleon transverse-spin and other TMD experiments. Both type of experiments are routinely performed at JLab, and both will become an important part of the EIC science program.

The rate of the required helicity flips is closely related to the systematic understanding of the precision. Typically, although already very difficult, one can control the systematic 
uncertainties between two helicity states to about $1 \%$. To further reduce this asymmetry, to a level of $10^{-8}$ for the case of typical parity-violating experiments at JLab, or to a level of $10^{-5}$ for transverse-spin experiments, one has to provide a suppression faction of $10^{-6}$ (for electron spin flip) or $10^{-3}$ (for ion spin flip) by fast spin flip techniques. The suppression factor by such fast helicity reversal is proportional to $1 / \sqrt{N}$, where $N$ is the number of pairs of spin flip. If we assume a typical single-nucleon spin asymmetry experiment of 3 months of continuous running (assuming one can keep control of the systematic uncertainties between the two helicity states at the $1 \%$ level for the full period), one needs to accumulate $10^{6}$ pairs to reach a suppression of 1000 , or about 8 flips per minute. This is the root of the present $0.1 \mathrm{~Hz}$ beam helicity reversal assumption mentioned above. 


\section{Acknowledgments}

Major support for this report and the INT program were provided by Brookhaven Science Associates, LLC under U.S. DOE Contract No. DE-AC02-98CH10886, by the Institute for Nuclear Theory at the University of Washington under U.S. DOE Contract No. DEFG02-00ER41132, and by Jefferson Science Associates, LLC under U.S. DOE Contract No. DE-AC05-06OR23177.

The United States Government retains a non-exclusive, paid-up, irrevocable, world-wide license to publish or reproduce the published form of this manuscript, or allow others to do so, for United States Government purposes.

We acknowledge additional support from ANPCyt, CONICET, and UBACyT (Argentina), CNPq and FAPERGS (Brazil), FESR of the Université de Moncton (Canada), Fondecyt (Chile), Conicyt-DFG (Chile and Germany), the Ministry of Science and Education (Croatia), the European Union, the Academy of Finland, ANR (France), BMBF, DAAD, DFG, GSI, and the Helmholtz Association (Germany), MIUR and RAS (Italy), the Ministry of Science and Higher Education (Poland), STFC (UK), DOE, the Lightner-Sams Foundation, NSF, and the PSC-CUNY Research Award Program (USA). 


\section{References}

[1] D. Gross and F. Wilczek, Phys. Rev. D8, 3633 (1973).

[2] D. Gross and F. Wilczek, Phys. Rev. D9, 980 (1974).

[3] Particle Data Group, K. Nakamura et al., J. Phys. G 37, 075021 (2010).

[4] S. Moch, J. Vermaseren, and A. Vogt, Nucl. Phys. B688, 101 (2004).

[5] A. Vogt, S. Moch, and J. Vermaseren, Nucl. Phys. B691, 129 (2004).

[6] W. van Neerven and E. Zijlstra, Phys. Lett. B272, 127 (1991).

[7] E. B. Zijlstra and W. L. van Neerven, Nucl. Phys. B383, 525 (1992).

[8] E. B. Zijlstra and W. L. van Neerven, Phys. Lett. B297, 377 (1992).

[9] S. Moch and J. Vermaseren, Nucl. Phys. B573, 853 (2000).

[10] J. Vermaseren, A. Vogt, and S. Moch, Nucl. Phys. B724, 3 (2005).

[11] S. Moch, J. A. M. Vermaseren, and A. Vogt, Nucl. Phys. B813, 220 (2009).

[12] R. Mertig and W. van Neerven, Z. Phys. C70, 637 (1996).

[13] W. Vogelsang, Nucl. Phys. B475, 47 (1996).

[14] A. Vogt, S. Moch, M. Rogal, and J. Vermaseren, Nucl. Phys. Proc. Suppl. 183, 155 (2008).

[15] E. B. Zijlstra and W. L. van Neerven, Nucl. Phys. B417, 61 (1994).

[16] A. Daleo, D. de Florian, and R. Sassot, Phys. Rev. D71, 034013 (2005).

[17] B. Kniehl, G. Kramer, and M. Maniatis, Nucl. Phys. B711, 345 (2005).

[18] A. Daleo and R. Sassot, Phys. Rev. D73, 054014 (2006).

[19] S. Alekhin, J. Blumlein, S. Klein, and S. Moch, Phys. Rev. D81, 014032 (2010).

[20] H1 and ZEUS Collaboration, F. D. Aaron et al., JHEP 01, 109 (2010).

[21] P. Jimenez-Delgado and E. Reya, Phys. Rev. D79, 074023 (2009).

[22] A. Martin, W. Stirling, R. Thorne, and G. Watt, Eur. Phys. J. C63, 189 (2009).

[23] J. Blumlein and H. Bottcher, Nucl. Phys. B841, 205 (2010).

[24] M. Gluck, E. Reya, M. Stratmann, and W. Vogelsang, Phys. Rev. D63, 094005 (2001).

[25] D. de Florian, R. Sassot, M. Stratmann, and W. Vogelsang, Phys. Rev. Lett. 101, 072001 (2008).

[26] D. de Florian, R. Sassot, M. Stratmann, and W. Vogelsang, Phys. Rev. D80, 034030 (2009).

[27] M. Hirai and S. Kumano, Nucl. Phys. B813, 106 (2009).

[28] E. Leader, A. V. Sidorov, and D. B. Stamenov, Phys. Rev. D82, 114018 (2010). 
[29] T. Alexopoulos et al., Electron deuteron scattering with HERA, a letter of intent for an experimental programme with the H1 detector, DESY-03-194, 2003.

[30] H1 Collaboration, F. D. Aaron et al., Eur. Phys. J. C71, 1579 (2011).

[31] S. A. Larin and J. A. M. Vermaseren, Phys. Lett. B259, 345 (1991).

[32] P. Baikov, K. Chetyrkin, and J. Kuhn, Phys. Rev. Lett. 104, 132004 (2010).

[33] H1 and ZEUS Collaboration, H1prelim-10-044, ZEUS-prel-10-008.

[34] H1 and ZEUS Collaboration, H1prelim-10-141, ZEUS-prel-10-017.

[35] H1 and ZEUS Collaboration, H1prelim-10-142, ZEUS-prel-10-018.

[36] I. Schienbein et al., Phys. Rev. D80, 094004 (2009).

[37] K. Kovarik et al., Phys. Rev. Lett. 106, 122301 (2011).

[38] M. Hirai, S. Kumano, and T. H. Nagai, Phys. Rev. C76, 065207 (2007).

[39] D. de Florian and R. Sassot, Phys. Rev. D69, 074028 (2004).

[40] K. J. Eskola, H. Paukkunen, and C. A. Salgado, JHEP 04, 065 (2009).

[41] I. Schienbein et al., Phys. Rev. D77, 054013 (2008).

[42] NuTeV Collaboration, M. Tzanov et al., Phys. Rev. D74, 012008 (2006).

[43] P. M. Nadolsky et al., Phys. Rev. D78, 013004 (2008).

[44] F. Olness et al., Eur. Phys. J. C40, 145 (2005).

[45] H.-L. Lai et al., JHEP 0704, 089 (2007).

[46] H.-L. Lai et al., Phys. Rev. D82, 074024 (2010).

[47] R. D. Ball et al., Nucl. Phys. B838, 136 (2010).

[48] CCFR/NuTeV Collaboration, U.-K. Yang et al., Phys. Rev. Lett. 86, 2742 (2001).

[49] BCDMS Collaboration, A. C. Benvenuti et al., Phys. Lett. B223, 485 (1989).

[50] New Muon Collaboration, P. Amaudruz et al., Phys. Lett. B295, 159 (1992).

[51] D0 Collaboration, V. Abazov et al., Phys. Rev. D77, 011106 (2008).

[52] D0 Collaboration, V. Abazov et al., Phys. Rev. Lett. 101, 211801 (2008).

[53] NNPDF Collaboration, R. D. Ball et al., Nucl. Phys. B849, 112 (2011).

[54] A. Accardi et al., Phys. Rev. D81, 034016 (2010).

[55] J. Pumplin et al., Phys. Rev. D80, 014019 (2009).

[56] S. J. Brodsky, P. Hoyer, C. Peterson, and N. Sakai, Phys. Lett. B93, 451 (1980).

[57] S. J. Brodsky, C. Peterson, and N. Sakai, Phys. Rev. D23, 2745 (1981).

[58] E. Kuraev, L. Lipatov, and V. S. Fadin, Sov. Phys. JETP 44, 443 (1976).

[59] E. Kuraev, L. Lipatov, and V. S. Fadin, Sov. Phys. JETP 45, 199 (1977).

[60] I. I. Balitsky and L. N. Lipatov, Sov. J. Nucl. Phys. 28, 822 (1978).

[61] C. Boros, F. M. Steffens, J. T. Londergan, and A. W. Thomas, Phys. Lett. B468, 161 (1999).

[62] R. D. Ball, D. A. Harris, and K. S. McFarland, hep-ph/0009223.

[63] S. Kretzer et al., Phys. Rev. D64, 033003 (2001).

[64] A. D. Martin, R. G. Roberts, W. J. Stirling, and R. S. Thorne, Eur. Phys. J. C23, 73 (2002). 
[65] C. Boros, J. T. Londergan, and A. W. Thomas, Phys. Rev. D59, 074021 (1999).

[66] C. Boros, J. T. Londergan, and A. W. Thomas, Phys. Rev. Lett. 81, 4075 (1998).

[67] NA51 Collaboration, A. Baldit et al., Phys. Lett. B332, 244 (1994).

[68] NuSOnG Collaboration, T. Adams et al., Int. J. Mod. Phys. A25, 909 (2010).

[69] A. D. Martin, R. G. Roberts, W. J. Stirling, and R. S. Thorne, Eur. Phys. J. C39, 155 (2005).

[70] M. Roth and S. Weinzierl, Phys. Lett. B590, 190 (2004).

[71] M. Gluck, P. Jimenez-Delgado, and E. Reya, Phys. Rev. Lett. 95, 022002 (2005).

[72] R. D. Ball et al., Nucl. Phys. B849, 296 (2011).

[73] HERMES Collaboration, A. Airapetian et al., Phys. Lett. B666, 446 (2008).

[74] D. de Florian, R. Sassot, and M. Stratmann, Phys. Rev. D75, 114010 (2007).

[75] S. Moch and A. Vogt, Phys. Lett. B659, 290 (2008).

[76] G. Altarelli, R. K. Ellis, G. Martinelli, and S.-Y. Pi, Nucl. Phys. B160, 301 (1979).

[77] W. Furmanski and R. Petronzio, Z. Phys. C11, 293 (1982).

[78] P. Nason and B. R. Webber, Nucl. Phys. B421, 473 (1994).

[79] D. de Florian, M. Stratmann, and W. Vogelsang, Phys. Rev. D57, 5811 (1998).

[80] M. Stratmann and W. Vogelsang, Phys. Rev. D64, 114007 (2001).

[81] T. Sjostrand, S. Mrenna, and P. Z. Skands, JHEP 05, 026 (2006).

[82] J. Pumplin et al., JHEP 07, 012 (2002).

[83] K. J. Golec-Biernat and M. Wusthoff, Phys. Rev. D59, 014017 (1998).

[84] E. Iancu, K. Itakura, and S. Munier, Phys. Lett. B590, 199 (2004).

[85] J. L. Albacete, N. Armesto, J. G. Milhano, and C. A. Salgado, Phys. Rev. D80, 034031 (2009).

[86] New Muon Collaboration, M. Arneodo et al., Nucl. Phys. B483, 3 (1997).

[87] ZEUS Collaboration, S. Chekanov et al., Phys. Lett. B682, 8 (2009).

[88] G. A. Schuler and H. Spiesberger, in proceedings of the workshop on Physics at HERA, vol. 3, 1419 (1992).

[89] A. Arbuzov et al., Comp. Phys. Commun. 94, 128 (1996).

[90] A. Kwiatkowski, H. Spiesberger, and H. J. Mohring, Comp. Phys. Commun. 69, 155 (1992).

[91] G. Ingelman, A. Edin, and J. Rathsman, Comp. Phys. Commun. 101, 108 (1997).

[92] H1 Collaboration, F. D. Aaron et al., Eur. Phys. J. C65, 89 (2010).

[93] H1 Collaboration, F. D. Aaron et al., Eur. Phys. J. C71, 1509 (2011).

[94] ZEUS Collaboration, S. Chekanov et al., Eur. Phys. J. C65, 65 (2010).

[95] ZEUS Collaboration, H. Abramowicz et al., Eur. Phys. J. C69, 347 (2010).

[96] E. Witten, Nucl. Phys. B104, 445 (1976).

[97] J. Babcock, D. W. Sivers, and S. Wolfram, Phys. Rev. D18, 162 (1978).

[98] V. A. Novikov, M. A. Shifman, A. I. Vainshtein, and V. I. Zakharov, Nucl. Phys. B136, 125 (1978). 
[99] J. P. Leveille and T. J. Weiler, Nucl. Phys. B147, 147 (1979).

[100] E. Laenen, S. Riemersma, J. Smith, and W. L. van Neerven, Nucl. Phys. B392, 162 (1993).

[101] S. Riemersma, J. Smith, and W. L. van Neerven, Phys. Lett. B347, 143 (1995).

[102] M. Buza et al., Nucl. Phys. B472, 611 (1996).

[103] E. B. Zijlstra and W. L. van Neerven, Phys. Lett. B273, 476 (1991).

[104] I. Bierenbaum, J. Blumlein, and S. Klein, Nucl. Phys. B780, 40 (2007).

[105] I. Bierenbaum, J. Blumlein, and S. Klein, Phys. Lett. B648, 195 (2007).

[106] I. Bierenbaum, J. Blumlein, S. Klein, and C. Schneider, Nucl. Phys. B803, 1 (2008).

[107] I. Bierenbaum, J. Blumlein, and S. Klein, Nucl. Phys. B820, 417 (2009).

[108] J. Blumlein, A. De Freitas, W. L. van Neerven, and S. Klein, Nucl. Phys. B755, 272 (2006).

[109] I. Bierenbaum, J. Blumlein, and S. Klein, PoS DIS2010, 148 (2010).

[110] J. Ablinger et al., (2011).

[111] M. Gluck, S. Kretzer, and E. Reya, Phys. Lett. B380, 171 (1996).

[112] M. Buza and W. L. van Neerven, Nucl. Phys. B500, 301 (1997).

[113] J. Blumlein, A. Hasselhuhn, P. Kovacikova, and S. Moch, arXiv:1104.3449.

[114] M. Gluck, E. Reya, and M. Stratmann, Nucl. Phys. B422, 37 (1994).

[115] I. Bierenbaum, J. Blumlein, and S. Klein, Phys. Lett. B672, 401 (2009).

[116] M. Buza, Y. Matiounine, J. Smith, and W. L. van Neerven, Eur. Phys. J. C1, 301 (1998).

[117] J. C. Collins, Phys. Rev. D58, 094002 (1998).

[118] J. Blumlein and W. L. van Neerven, Phys. Lett. B450, 417 (1999).

[119] J. Blumlein, Mod. Phys. Lett. A25, 2621 (2010).

[120] Z. J. Ajaltouni et al., prepared for HERA and the LHC: 4th Workshop on the Implications of HERA for LHC Physics, Geneva, 2008.

[121] E. Laenen and S.-O. Moch, Phys. Rev. D59, 034027 (1999).

[122] S. Alekhin and S. Moch, Phys. Lett. B672, 166 (2009).

[123] N. A. L. Presti, H. Kawamura, S. Moch, and A. Vogt, PoS DIS2010, 163 (2010).

[124] S. Alekhin and S. Moch, Phys. Lett. B699, 345 (2011).

[125] K. Lipka, private communication.

[126] J. C. Collins and W.-K. Tung, Nucl. Phys. B278, 934 (1986).

[127] M. A. G. Aivazis, J. C. Collins, F. I. Olness, and W.-K. Tung, Phys. Rev. D50, 3102 (1994).

[128] B. W. Harris, J. Smith, and R. Vogt, Nucl. Phys. B461, 181 (1996).

[129] J. Pumplin, H. L. Lai, and W. K. Tung, Phys. Rev. D75, 054029 (2007).

[130] J. Pumplin, Phys. Rev. D73, 114015 (2006).

[131] Projections of the EIC parameters can be found at http://wiki.bnl.gov/eic

[132] N. Y. Ivanov, arXiv:1010.5424.

[133] COMPASS Collaboration, M. G. Alekseev et al., Phys. Lett. B680, 217 (2009).

[134] COMPASS Collaboration, M. G. Alekseev et al., Phys. Lett. B693, 227 (2010). 
[135] M. J. Savage and J. Walden, Phys. Rev. D55, 5376 (1997).

[136] S.-L. Zhu, G. Sacco, and M. J. Ramsey-Musolf, Phys. Rev. D66, 034021 (2002).

[137] S. Collins et al., PoS LATTICE2010, 134 (2010).

[138] L. Del Debbio, A. Guffanti, and A. Piccione, JHEP 11, 060 (2009).

[139] J. Rojo et al., PoS DIS2010, 244 (2010).

[140] D. de Florian and W. Vogelsang, Phys. Rev. D81, 094020 (2010).

[141] S. J. Brodsky, M. Burkardt, and I. Schmidt, Nucl. Phys. B441, 197 (1995).

[142] H. Avakian, S. J. Brodsky, A. Deur, and F. Yuan, Phys. Rev. Lett. 99, 082001 (2007).

[143] R. L. Jaffe and A. Manohar, Nucl. Phys. B337, 509 (1990).

[144] S. Bashinsky and R. L. Jaffe, Nucl. Phys. B536, 303 (1998).

[145] R. J. Crewther, Phys. Lett. B397, 137 (1997).

[146] D. de Florian, S. Frixione, A. Signer, and W. Vogelsang, Nucl. Phys. B539, 455 (1999).

[147] B. Jager, M. Stratmann, and W. Vogelsang, Phys. Rev. D70, 034010 (2004).

[148] B. Jager, A. Schafer, M. Stratmann, and W. Vogelsang, Phys. Rev. D67, 054005 (2003).

[149] D. de Florian, Phys. Rev. D67, 054004 (2003).

[150] E. Mirkes and C. Ziegler, Nucl. Phys. B429, 93 (1994).

[151] J. Feltesse, F. Kunne, and E. Mirkes, Phys. Lett. B388, 832 (1996).

[152] A. D. Watson, Z. Phys. C12, 123 (1982).

[153] I. Bojak and M. Stratmann, Phys. Lett. B433, 411 (1998).

[154] I. Bojak and M. Stratmann, Nucl. Phys. B540, 345 (1999).

[155] L. Mankiewicz, A. Schafer, and M. Veltri, Comp. Phys. Commun. 71, 305 (1992).

[156] J. Bartels, B. I. Ermolaev, and M. G. Ryskin, Z. Phys. C70, 273 (1996).

[157] J. Bartels, B. I. Ermolaev, and M. G. Ryskin, Z. Phys. C72, 627 (1996).

[158] B. I. Ermolaev, M. Greco, and S. I. Troyan, Phys. Lett. B622, 93 (2005).

[159] B. I. Ermolaev, M. Greco, and S. I. Troyan, Phys. Lett. B579, 321 (2004).

[160] G. T. Bodwin and J.-W. Qiu, Phys. Rev. D41, 2755 (1990).

[161] W. Vogelsang, Z. Phys. C50, 275 (1991).

[162] S. A. Larin, F. V. Tkachov, and J. A. M. Vermaseren, Phys. Rev. Lett. 66, 862 (1991).

[163] J. Blumlein and H. Bottcher, Nucl. Phys. B636, 225 (2002).

[164] S. L. Adler, Phys. Rev. D10, 3714 (1974).

[165] P. A. Baikov, K. G. Chetyrkin, and J. H. Kuhn, Phys. Rev. Lett. 101, 012002 (2008).

[166] V. M. Braun, G. P. Korchemsky, and D. Mueller, Prog. Part. Nucl. Phys. 51, 311 (2003).

[167] G. Grunberg, Phys. Lett. B95, 70 (1980).

[168] A. Deur, V. Burkert, J.-P. Chen, and W. Korsch, Phys. Lett. B650, 244 (2007).

[169] D. Diakonov et al., Nucl. Phys. B480, 341 (1996).

[170] D. Diakonov et al., Phys. Rev. D56, 4069 (1997).

[171] M. Wakamatsu and T. Kubota, Phys. Rev. D60, 034020 (1999). 
[172] M. Wakamatsu, Phys. Rev. D67, 034005 (2003).

[173] K. S. Kumar and P. A. Souder, Prog. Part. Nucl. Phys. 45, S333 (2000).

[174] H1 and ZEUS Collaboration, Z. Zhang, J. Phys. Conf. Ser. 110, 042034 (2008).

[175] C. Diaconu et al., Ann. Rev. Nucl. Part. Sci. 60, 101 (2010).

[176] M. Tzanov, AIP Conf. Proc. 1222, 243 (2010).

[177] C. Nash, Nucl. Phys. B31, 419 (1971).

[178] D. A. Dicus, Phys. Rev. D5, 1367 (1972).

[179] D. Wray, Nuovo Cim. A9, 463 (1972).

[180] E. Derman, Phys. Rev. D7, 2755 (1973).

[181] M. A. Ahmed and G. G. Ross, Nucl. Phys. B111, 441 (1976).

[182] A. S. Joshipura and P. Roy, Ann. Phys. 104, 460 (1977).

[183] J. Kaur, Nucl. Phys. B128, 219 (1977).

[184] J. A. Bartelski, Acta Phys. Polon. B10, 923 (1979).

[185] D. Hochberg, Nucl. Phys. B256, 1 (1985).

[186] B. Lampe, Phys. Lett. B227, 469 (1989).

[187] W. Vogelsang and A. Weber, Nucl. Phys. B362, 3 (1991).

[188] V. Ravishankar, Nucl. Phys. B374, 309 (1992).

[189] P. Mathews and V. Ravindran, Phys. Lett. B278, 175 (1992).

[190] P. Mathews and V. Ravindran, Int. J. Mod. Phys. A7, 6371 (1992).

[191] X.-D. Ji, MIT-CTP-2141, 1992 (unpublished).

[192] D. de Florian and R. Sassot, Phys. Rev. D51, 6052 (1995).

[193] M. Stratmann, A. Weber, and W. Vogelsang, Phys. Rev. D53, 138 (1996).

[194] J. Blumlein and N. Kochelev, Phys. Lett. B381, 296 (1996).

[195] J. Blumlein and N. Kochelev, Nucl. Phys. B498, 285 (1997).

[196] M. Anselmino, P. Gambino, and J. Kalinowski, Z. Phys. C64, 267 (1994).

[197] M. Anselmino, P. Gambino, and J. Kalinowski, Phys. Rev. D55, 5841 (1997).

[198] J. G. Contreras, A. de Roeck, and M. Maul, hep-ph/9711418.

[199] S. Forte, M. L. Mangano, and G. Ridolfi, Nucl. Phys. B602, 585 (2001).

[200] W. Vogelsang, Phys. Rev. D54, 2023 (1996).

[201] W. K. Tung et al., JHEP 02, 053 (2007).

[202] P. Souder et al., JLab experiment E12-09-012 (SoLID).

[203] T. Gottschalk, Phys. Rev. D23, 56 (1981).

[204] M. Gluck, S. Kretzer, and E. Reya, Phys. Lett. B398, 381 (1997).

[205] S. Kretzer and M. Stratmann, Eur. Phys. J. C10, 107 (1999).

[206] R. M. Barnett, Phys. Rev. Lett. 36, 1163 (1976).

[207] ZEUS Collaboration, S. Chekanov et al., Phys. Rev. D76, 072011 (2007).

[208] M. Klasen, Rev. Mod. Phys. 74, 1221 (2002). 
[209] M. Klein and R. Yoshida, Prog. Part. Nucl. Phys. 61, 343 (2008).

[210] R. Nisius, Phys. Rept. 332, 165 (2000).

[211] M. Gluck, E. Reya, and A. Vogt, Phys. Rev. D46, 1973 (1992).

[212] G. Kramer and H. Spiesberger, Eur. Phys. J. C38, 309 (2004).

[213] B. A. Kniehl, G. Kramer, I. Schienbein, and H. Spiesberger, Phys. Rev. D71, 014018 (2005).

[214] B. A. Kniehl, G. Kramer, I. Schienbein, and H. Spiesberger, Eur. Phys. J. C62, 365 (2009).

[215] G. Kramer and H. Spiesberger, Eur. Phys. J. C22, 289 (2001).

[216] G. Kramer and H. Spiesberger, Eur. Phys. J. C28, 495 (2003).

[217] B. A. Kniehl, G. Kramer, I. Schienbein, and H. Spiesberger, Eur. Phys. J. C41, 199 (2005).

[218] P. Aurenche, M. Fontannaz, and J. P. Guillet, Eur. Phys. J. C44, 395 (2005).

[219] T. Kneesch, B. A. Kniehl, G. Kramer, and I. Schienbein, Nucl. Phys. B799, 34 (2008).

[220] Belle Collaboration, R. Seuster et al., Phys. Rev. D73, 032002 (2006).

[221] CLEO Collaboration, M. Artuso et al., Phys. Rev. D70, 112001 (2004).

[222] ALEPH Collaboration, R. Barate et al., Eur. Phys. J. C16, 597 (2000).

[223] OPAL Collaboration, G. Alexander et al., Z. Phys. C72, 1 (1996).

[224] OPAL Collaboration, K. Ackerstaff et al., Eur. Phys. J. C1, 439 (1998).

[225] D. de Florian and S. Frixione, Phys. Lett. B457, 236 (1999).

[226] M. Stratmann and W. Vogelsang, Phys. Lett. B386, 370 (1996).

[227] G. F. Sterman and S. Weinberg, Phys. Rev. Lett. 39, 1436 (1977).

[228] M. Furman, Nucl. Phys. B197, 413 (1982).

[229] F. Aversa, P. Chiappetta, M. Greco, and J. P. Guillet, Nucl. Phys. B327, 105 (1989).

[230] F. Aversa, M. Greco, P. Chiappetta, and J. P. Guillet, Z. Phys. C46, 253 (1990).

[231] F. Aversa et al., Z. Phys. C49, 459 (1991).

[232] F. Aversa, M. Greco, P. Chiappetta, and J. P. Guillet, Phys. Rev. Lett. 65, 401 (1990).

[233] J. P. Guillet, Z. Phys. C51, 587 (1991).

[234] B. Jager, Phys. Rev. D78, 034017 (2008).

[235] M. Stratmann and W. Vogelsang, Z. Phys. C74, 641 (1997).

[236] B. Jager, M. Stratmann, and W. Vogelsang, Eur. Phys. J. C44, 533 (2005).

[237] COMPASS Collaboration, E. S. Ageev et al., Phys. Lett. B633, 25 (2006).

[238] B. Jager, M. Stratmann, and W. Vogelsang, Phys. Rev. D68, 114018 (2003).

[239] J. M. Butterworth, N. Goodman, M. Stratmann, and W. Vogelsang, hep-ph/9711250.

[240] J. C. Collins and D. E. Soper, Nucl. Phys. B193, 381 (1981).

[241] J. C. Collins and D. E. Soper, Nucl. Phys. B194, 445 (1982).

[242] A. Kotzinian, Nucl. Phys. B441, 234 (1995).

[243] P. J. Mulders and R. D. Tangerman, Nucl. Phys. B461, 197 (1996).

[244] D. Boer and P. J. Mulders, Phys. Rev. D57, 5780 (1998).

[245] R. L. Jaffe, hep-ph/9602236. 
[246] K. Goeke, A. Metz, and M. Schlegel, Phys. Lett. B618, 90 (2005).

[247] A. Bacchetta et al., JHEP 02, 093 (2007).

[248] M. Anselmino et al., Phys. Rev. D73, 014020 (2006).

[249] J. C. Collins, Nucl. Phys. B396, 161 (1993).

[250] M. Anselmino et al., Phys. Rev. D75, 054032 (2007).

[251] D. W. Sivers, Phys.Rev. D41, 83 (1990).

[252] H. Avakian et al., Phys. Rev. D77, 014023 (2008).

[253] A. Bacchetta, U. D’Alesio, M. Diehl, and C. A. Miller, Phys. Rev. D70, 117504 (2004).

[254] M. Diehl and S. Sapeta, Eur. Phys. J. C41, 515 (2005).

[255] M. Anselmino et al., Phys. Rev. D83, 114019 (2011).

[256] X.-d. Ji, J.-p. Ma, and F. Yuan, Phys. Rev. D71, 034005 (2005).

[257] X.-d. Ji, J.-P. Ma, and F. Yuan, Phys. Lett. B597, 299 (2004).

[258] J. C. Collins and A. Metz, Phys. Rev. Lett. 93, 252001 (2004).

[259] J. Collins, PoS LC2008, 028 (2008).

[260] S. M. Aybat and T. C. Rogers, Phys. Rev. D83, 114042 (2011).

[261] S. J. Brodsky, D. S. Hwang, and I. Schmidt, Phys. Lett. B530, 99 (2002).

[262] J. C. Collins, Phys. Lett. B536, 43 (2002).

[263] A. V. Belitsky, X. Ji, and F. Yuan, Nucl. Phys. B656, 165 (2003).

[264] D. Boer, P. J. Mulders, and F. Pijlman, Nucl. Phys. B667, 201 (2003).

[265] C. J. Bomhof, P. J. Mulders, and F. Pijlman, Phys. Lett. B596, 277 (2004).

[266] A. Bacchetta, C. J. Bomhof, P. J. Mulders, and F. Pijlman, Phys. Rev. D72, 034030 (2005).

[267] C. J. Bomhof, P. J. Mulders, and F. Pijlman, Eur. Phys. J. C47, 147 (2006).

[268] C. J. Bomhof and P. J. Mulders, Nucl. Phys. B795, 409 (2008).

[269] J.-W. Qiu, W. Vogelsang, and F. Yuan, Phys. Lett. B650, 373 (2007).

[270] J. Collins and J.-W. Qiu, Phys. Rev. D75, 114014 (2007).

[271] J. Collins, arXiv:0708.4410.

[272] T. C. Rogers and P. J. Mulders, Phys. Rev. D81, 094006 (2010).

[273] J. C. Collins, D. E. Soper, and G. F. Sterman, Nucl. Phys. B250, 199 (1985).

[274] X.-D. Ji, Phys. Rev. Lett. 78, 610 (1997).

[275] P. Hoodbhoy, X.-D. Ji, and W. Lu, Phys. Rev. D59, 014013 (1999).

[276] S. J. Brodsky and S. D. Drell, Phys. Rev. D22, 2236 (1980).

[277] M. Burkardt and G. Schnell, Phys. Rev. D74, 013002 (2006).

[278] X.-d. Ji, J.-P. Ma, and F. Yuan, Nucl. Phys. B652, 383 (2003).

[279] P. Hagler, B. U. Musch, J. W. Negele, and A. Schafer, Europhys. Lett. 88, 61001 (2009).

[280] B. U. Musch, P. Hagler, J. W. Negele, and A. Schafer, Phys. Rev. D83, 094507 (2011).

[281] P. J. Mulders and J. Rodrigues, Phys. Rev. D63, 094021 (2001).

[282] S. Meissner, A. Metz, and K. Goeke, Phys. Rev. D76, 034002 (2007). 
[283] F. Dominguez, B.-W. Xiao, and F. Yuan, Phys. Rev. Lett. 106, 022301 (2011).

[284] D. Boer, S. J. Brodsky, P. J. Mulders, and C. Pisano, Phys. Rev. Lett. 106, 132001 (2011).

[285] J. P. Ma and Q. Wang, Eur. Phys. J. C37, 293 (2004).

[286] A. V. Efremov and O. V. Teryaev, Sov. J. Nucl. Phys. 36, 140 (1982).

[287] A. V. Efremov and O. V. Teryaev, Phys. Lett. B150, 383 (1985).

[288] J.-w. Qiu and G. F. Sterman, Phys. Rev. Lett. 67, 2264 (1991).

[289] J.-w. Qiu and G. F. Sterman, Phys. Rev. D59, 014004 (1999).

[290] J. Zhou, F. Yuan, and Z.-T. Liang, Phys. Rev. D79, 114022 (2009).

[291] W. Vogelsang and F. Yuan, Phys. Rev. D79, 094010 (2009).

[292] X. Ji, J.-W. Qiu, W. Vogelsang, and F. Yuan, Phys. Rev. Lett. 97, 082002 (2006).

[293] X. Ji, J.-w. Qiu, W. Vogelsang, and F. Yuan, Phys. Rev. D73, 094017 (2006).

[294] X. Ji, J.-W. Qiu, W. Vogelsang, and F. Yuan, Phys. Lett. B638, 178 (2006).

[295] Y. Koike, W. Vogelsang, and F. Yuan, Phys. Lett. B659, 878 (2008).

[296] A. Bacchetta, D. Boer, M. Diehl, and P. J. Mulders, JHEP 08, 023 (2008).

[297] F. Yuan and J. Zhou, Phys. Rev. Lett. 103, 052001 (2009).

[298] B. Pasquini and F. Yuan, Phys. Rev. D81, 114013 (2010).

[299] M. Burkardt, Phys. Rev. D66, 114005 (2002).

[300] M. Burkardt and D. S. Hwang, Phys. Rev. D69, 074032 (2004).

[301] M. Burkardt, arXiv:0709.2966.

[302] R. D. Klem et al., Phys. Rev. Lett. 36, 929 (1976).

[303] J. Antille et al., Phys. Lett. B94, 523 (1980).

[304] E581 Collaboration, D. L. Adams et al., Phys. Lett. B261, 197 (1991).

[305] E581 Collaboration, D. L. Adams et al., Z. Phys. C56, 181 (1992).

[306] FNAL-E704 Collaboration, D. L. Adams et al., Phys. Lett. B264, 462 (1991).

[307] FNAL E704 Collaboration, D. L. Adams et al., Phys. Rev. D53, 4747 (1996).

[308] FNAL E704 Collaboration, A. Bravar et al., Phys. Rev. Lett. 77, 2626 (1996).

[309] STAR Collaboration, J. Adams et al., Phys. Rev. Lett. 92, 171801 (2004).

[310] G. L. Kane, J. Pumplin, and W. Repko, Phys. Rev. Lett. 41, 1689 (1978).

[311] BRAHMS Collaboration, I. Arsene et al., Phys. Rev. Lett. 101, 042001 (2008).

[312] BRAHMS Collaboration, J. H. Lee and F. Videbaek, arXiv:0908.4551.

[313] PHENIX Collaboration, A. Adare et al., Phys. Rev. D82, 112008 (2010).

[314] PHENIX Collaboration, S. S. Adler et al., Phys. Rev. Lett. 95, 202001 (2005).

[315] PHENIX Collaboration, V. Dharmawardane, PoS DIS2010, 222 (2010).

[316] STAR Collaboration, B. I. Abelev et al., Phys. Rev. Lett. 99, 142003 (2007).

[317] STAR Collaboration, B. I. Abelev et al., Phys. Rev. Lett. 101, 222001 (2008).

[318] STAR Collaboration, L. K. Eun, J. Phys. Conf. Ser. 230, 012041 (2010).

[319] U. D'Alesio and F. Murgia, Prog. Part. Nucl. Phys. 61, 394 (2008). 
[320] COMPASS Collaboration, E. S. Ageev et al., Nucl. Phys. B765, 31 (2007).

[321] COMPASS Collaboration, M. Alekseev et al., Phys. Lett. B673, 127 (2009).

[322] COMPASS Collaboration, M. G. Alekseev et al., Phys. Lett. B692, 240 (2010).

[323] COMPASS Collaboration, G. Pesaro, J. Phys. Conf. Ser. 295, 012058 (2011).

[324] HERMES Collaboration, A. Airapetian et al., Phys. Rev. Lett. 94, 012002 (2005).

[325] HERMES Collaboration, A. Airapetian et al., Phys. Rev. Lett. 103, 152002 (2009).

[326] X. Qian, 2010, PhD Thesis, Duke University.

[327] M. Anselmino et al., J. Phys. Conf. Ser. 295, 012062 (2011).

[328] A. V. Efremov et al., Phys. Lett. B612, 233 (2005).

[329] M. Anselmino et al., Phys. Rev. D72, 094007 (2005).

[330] W. Vogelsang and F. Yuan, Phys. Rev. D72, 054028 (2005).

[331] J. C. Collins et al., Phys. Rev. D73, 014021 (2006).

[332] M. Anselmino et al., Eur. Phys. J. A39, 89 (2009).

[333] A. Drago, Phys. Rev. D71, 057501 (2005).

[334] P. V. Pobylitsa, hep-ph/0301236.

[335] S. Kretzer, Phys. Rev. D62, 054001 (2000).

[336] M. Hirai, S. Kumano, T. H. Nagai, and K. Sudoh, Phys. Rev. D75, 094009 (2007).

[337] A. Bacchetta, F. Conti, and M. Radici, Phys. Rev. D78, 074010 (2008).

[338] L. P. Gamberg, G. R. Goldstein, and M. Schlegel, Phys. Rev. D77, 094016 (2008).

[339] A. Courtoy, S. Scopetta, and V. Vento, Phys. Rev. D79, 074001 (2009).

[340] H. Gao et al., Eur. Phys. J. Plus 126, 2 (2011).

[341] The CLAS12 programme is available at http://www.jlab.org/Hall-B/clas12.

[342] The COMPASS-II proposal, CERN-SPSC-2010-014, May 17, 2010, is available at http://cdsweb.cern.ch/record/1265628.

[343] M. Anselmino et al., Eur. Phys. J. A47, 35 (2011).

[344] Available at http://spin.riken.bnl.gov/rsc/write-up/dy-final.pdf.

[345] NA10 Collaboration, S. Falciano et al., Z. Phys. C31, 513 (1986).

[346] NA10 Collaboration, M. Guanziroli et al., Z. Phys. C37, 545 (1988).

[347] E. Anassontzis et al., Phys. Rev. D38, 1377 (1988).

[348] FNAL-E866/NuSea Collaboration, L. Y. Zhu et al., Phys. Rev. Lett. 99, 082301 (2007).

[349] FNAL E866/NuSea Collaboration, L. Y. Zhu et al., Phys. Rev. Lett. 102, 182001 (2009).

[350] C. S. Lam and W.-K. Tung, Phys. Rev. D18, 2447 (1978).

[351] Z.-B. Kang and J.-W. Qiu, Phys.Rev. D81, 054020 (2010).

[352] H. Eguchi, Y. Koike, and K. Tanaka, Nucl. Phys. B763, 198 (2007).

[353] H. Eguchi, Y. Koike, and K. Tanaka, Nucl. Phys. B752, 1 (2006).

[354] H. Beppu, Y. Koike, K. Tanaka, and S. Yoshida, Phys. Rev. D82, 054005 (2010).

[355] C. Kouvaris, J.-W. Qiu, W. Vogelsang, and F. Yuan, Phys. Rev. D74, 114013 (2006). 
[356] Z.-B. Kang and J.-W. Qiu, Phys. Rev. D78, 034005 (2008).

[357] Z.-B. Kang, J.-W. Qiu, W. Vogelsang, and F. Yuan, Phys. Rev. D78, 114013 (2008).

[358] Y. Koike and K. Tanaka, Phys. Lett. B646, 232 (2007).

[359] Y. Koike and K. Tanaka, arXiv:0907.2797.

[360] Y. Koike and S. Yoshida, J. Phys. Conf. Ser. 295, 012083 (2011).

[361] K. Kanazawa and Y. Koike, Phys. Rev. D82, 034009 (2010).

[362] Y. Koike and T. Tomita, Phys. Lett. B675, 181 (2009).

[363] Z.-B. Kang, F. Yuan, and J. Zhou, Phys. Lett. B691, 243 (2010).

[364] D. Boer, P. J. Mulders, and O. V. Teryaev, Phys. Rev. D57, 3057 (1998).

[365] K. A. Oganesian, H. R. Avakian, N. Bianchi, and P. Di Nezza, Eur. Phys. J. C5, 681 (1998).

[366] V. Barone, A. Prokudin, and B.-Q. Ma, Phys. Rev. D78, 045022 (2008).

[367] V. Barone, S. Melis, and A. Prokudin, Phys. Rev. D81, 114026 (2010).

[368] E. L. Berger, J.-W. Qiu, and R. A. Rodriguez-Pedraza, Phys. Rev. D76, 074006 (2007).

[369] D. Boer, Nucl. Phys. B806, 23 (2009).

[370] Z. Lu and I. Schmidt, Phys. Rev. D81, 034023 (2010).

[371] M. Anselmino, M. Boglione, A. Prokudin, and C. Turk, Eur. Phys. J. A31, 373 (2007).

[372] Y. Koike, J. Nagashima, and W. Vogelsang, Nucl. Phys. B744, 59 (2006).

[373] A. M. Kotzinian and P. J. Mulders, Phys. Lett. B406, 373 (1997).

[374] D. Boer, L. Gamberg, B. U. Musch, A. Prokudin, "Bessel-weighted asymmetries in SIDIS", in preparation.

[375] D. Boer and W. Vogelsang, Phys. Rev. D69, 094025 (2004).

[376] M. Diehl, in preparation.

[377] M. Anselmino et al., Phys. Rev. D70, 074025 (2004).

[378] D. Boer, P. J. Mulders, and C. Pisano, Phys. Rev. D80, 094017 (2009).

[379] D. Boer, P. J. Mulders, and C. Pisano, Phys. Lett. B660, 360 (2008).

[380] J. C. Collins, D. E. Soper, and G. F. Sterman, in: Perturbative Quantum Chromodynamics, Ed. by A. H. Mueller (World Scientific, 1989).

[381] C. J. Bomhof and P. J. Mulders, JHEP 02, 029 (2007).

[382] A. Bacchetta, L. P. Gamberg, G. R. Goldstein, and A. Mukherjee, Phys. Lett. B659, 234 (2008).

[383] J. C. Collins, Acta Phys. Polon. B34, 3103 (2003).

[384] J. Collins, Foundations of Perturbative QCD (Cambridge University Press, 2011).

[385] F. Landry, R. Brock, P. M. Nadolsky, and C. P. Yuan, Phys. Rev. D67, 073016 (2003).

[386] R. Meng, F. I. Olness, and D. E. Soper, Phys. Rev. D54, 1919 (1996).

[387] P. M. Nadolsky, D. R. Stump, and C. P. Yuan, Phys. Rev. D61, 014003 (2000).

[388] D. Boer, Nucl. Phys. B603, 195 (2001).

[389] J.-w. Qiu and X.-f. Zhang, Phys. Rev. Lett. 86, 2724 (2001).

[390] Z.-B. Kang and J.-W. Qiu, Phys. Rev. D79, 016003 (2009). 
[391] V. M. Braun, A. N. Manashov, and B. Pirnay, Phys. Rev. D80, 114002 (2009).

[392] Z.-B. Kang, Phys. Rev. D83, 036006 (2011).

[393] A. Idilbi, X.-d. Ji, J.-P. Ma, and F. Yuan, Phys. Rev. D70, 074021 (2004).

[394] D. Boer, Z.-B. Kang, W. Vogelsang, and F. Yuan, Phys. Rev. Lett. 105, 202001 (2010).

[395] A. Metz, Phys. Lett. B549, 139 (2002).

[396] A. Afanasev and C. E. Carlson, hep-ph/0308163.

[397] A. Metz and M. Schlegel, Eur. Phys. J. A22, 489 (2004).

[398] A. Metz and M. Schlegel, Annalen Phys. 13, 699 (2004).

[399] E. Witten, Nucl. Phys. B160, 57 (1979).

[400] A. V. Efremov, K. Goeke, and P. V. Pobylitsa, Phys. Lett. B488, 182 (2000).

[401] S. J. Brodsky and S. Gardner, Phys. Lett. B643, 22 (2006).

[402] M. Anselmino, U. D'Alesio, S. Melis, and F. Murgia, Phys. Rev. D74, 094011 (2006).

[403] R. Jakob, P. J. Mulders, and J. Rodrigues, Nucl. Phys. A626, 937 (1997).

[404] L. P. Gamberg, G. R. Goldstein, and K. A. Oganessyan, Phys. Rev. D67, 071504 (2003).

[405] J. She, J. Zhu, and B.-Q. Ma, Phys. Rev. D79, 054008 (2009).

[406] Z. Lu and I. Schmidt, Phys. Rev. D82, 094005 (2010).

[407] S. J. Brodsky, D. S. Hwang, B.-Q. Ma, and I. Schmidt, Nucl. Phys. B593, 311 (2001).

[408] I. C. Cloet, W. Bentz, and A. W. Thomas, Phys. Lett. B659, 214 (2008).

[409] B. Pasquini, S. Cazzaniga, and S. Boffi, Phys. Rev. D78, 034025 (2008).

[410] B. Pasquini, S. Boffi, and P. Schweitzer, Mod. Phys. Lett. A24, 2903 (2009).

[411] G. A. Miller, Phys. Rev. C76, 065209 (2007).

[412] V. Y. Petrov and M. V. Polyakov, hep-ph/0307077.

[413] C. Lorce, Phys. Rev. D79, 074027 (2009).

[414] B. Pasquini and C. Lorce, arXiv:1008.0945.

[415] C. Lorce and B. Pasquini, PoS LC2010, 057 (2010).

[416] M. Wakamatsu, Phys. Rev. D79, 094028 (2009).

[417] H. Avakian, A. Efremov, P. Schweitzer, and F. Yuan, Phys. Rev. D81, 074035 (2010).

[418] R. L. Jaffe and X.-D. Ji, Nucl. Phys. B375, 527 (1992).

[419] H. Avakian, A. V. Efremov, P. Schweitzer, and F. Yuan, Phys. Rev. D78, 114024 (2008).

[420] P. Schweitzer, T. Teckentrup, and A. Metz, Phys. Rev. D81, 094019 (2010).

[421] A. V. Efremov, P. Schweitzer, O. V. Teryaev, and P. Zavada, Phys. Rev. D80, 014021 (2009).

[422] A. V. Efremov, P. Schweitzer, O. V. Teryaev, and P. Zavada, PoS DIS2010, 253 (2010).

[423] A. Efremov, P. Schweitzer, O. Teryaev, and P. Zavada, Phys. Rev. D83, 054025 (2011).

[424] C. Bourrely, J. Soffer, and F. Buccella, Mod. Phys. Lett. A21, 143 (2006).

[425] C. Bourrely, F. Buccella, and J. Soffer, Phys. Rev. D83, 074008 (2011).

[426] U. D'Alesio, E. Leader, and F. Murgia, Phys. Rev. D81, 036010 (2010).

[427] R. D. Tangerman and P. J. Mulders, hep-ph/9408305. 
[428] T. Teckentrup, A. Metz, and P. Schweitzer, Mod. Phys. Lett. A24, 2950 (2009).

[429] G. R. Goldstein and L. Gamberg, hep-ph/0209085.

[430] A. Bacchetta, A. Schaefer, and J.-J. Yang, Phys. Lett. B578, 109 (2004).

[431] Z. Lu and B.-Q. Ma, Nucl. Phys. A741, 200 (2004).

[432] K. Goeke, S. Meissner, A. Metz, and M. Schlegel, Phys. Lett. B637, 241 (2006).

[433] Z. Lu and I. Schmidt, Phys. Rev. D75, 073008 (2007).

[434] J. R. Ellis, D. S. Hwang, and A. Kotzinian, Phys. Rev. D80, 074033 (2009).

[435] F. Yuan, Phys. Lett. B575, 45 (2003).

[436] A. Courtoy, S. Scopetta, and V. Vento, Phys. Rev. D80, 074032 (2009).

[437] A. Courtoy, F. Fratini, S. Scopetta, and V. Vento, Phys. Rev. D78, 034002 (2008).

[438] I. O. Cherednikov, U. D’Alesio, N. I. Kochelev, and F. Murgia, Phys. Lett. B642, 39 (2006).

[439] P. Hoyer and M. Jarvinen, JHEP 10, 080 (2005).

[440] S. J. Brodsky, B. Pasquini, B.-W. Xiao, and F. Yuan, Phys. Lett. B687, 327 (2010).

[441] P. V. Pobylitsa, hep-ph/0212027.

[442] L. Gamberg and M. Schlegel, Mod. Phys. Lett. A24, 2960 (2009).

[443] L. Gamberg and M. Schlegel, Phys. Lett. B685, 95 (2010).

[444] M. Burkardt, Nucl. Phys. A735, 185 (2004).

[445] S. Meissner, A. Metz, and M. Schlegel, JHEP 08, 056 (2009).

[446] S. Meissner, A. Metz, M. Schlegel, and K. Goeke, JHEP 08, 038 (2008).

[447] M. Burkardt, Phys. Rev. D69, 091501 (2004).

[448] M. Burkardt and B. Hannafious, Phys. Lett. B658, 130 (2008).

[449] B. Zhang, Z. Lu, B.-Q. Ma, and I. Schmidt, Phys. Rev. D78, 034035 (2008).

[450] J. C. Collins, S. F. Heppelmann, and G. A. Ladinsky, Nucl. Phys. B420, 565 (1994).

[451] A. Bianconi, S. Boffi, R. Jakob, and M. Radici, Phys. Rev. D62, 034008 (2000).

[452] J. P. Ralston and D. E. Soper, Nucl. Phys. B152, 109 (1979).

[453] A. Hayashigaki, Y. Kanazawa, and Y. Koike, Phys. Rev. D56, 7350 (1997).

[454] S. Kumano and M. Miyama, Phys. Rev. D56, 2504 (1997).

[455] W. Vogelsang, Phys. Rev. D57, 1886 (1998).

[456] QCDSF Collaboration, M. Gockeler et al., Phys. Rev. Lett. 98, 222001 (2007).

[457] M. Wakamatsu, Phys. Lett. B653, 398 (2007).

[458] H.-X. He, Int. J. Mod. Phys. A18, 1289 (2003).

[459] B. Pasquini, M. Pincetti, and S. Boffi, Phys. Rev. D76, 034020 (2007).

[460] L. P. Gamberg and G. R. Goldstein, Phys. Rev. Lett. 87, 242001 (2001).

[461] V. Barone and P. G. Ratcliffe, River Edge, USA: World Scientific (2003).

[462] HERMES Collaboration, A. Airapetian et al., Phys. Lett. B693, 11 (2010).

[463] X. Artru, J. Czyzewski, and H. Yabuki, Z. Phys. C73, 527 (1997).

[464] A. Bacchetta, R. Kundu, A. Metz, and P. J. Mulders, Phys. Lett. B506, 155 (2001). 
[465] A. Bacchetta, R. Kundu, A. Metz, and P. J. Mulders, Phys. Rev. D65, 094021 (2002).

[466] L. P. Gamberg, G. R. Goldstein, and K. A. Oganessyan, Phys. Rev. D68, 051501 (2003).

[467] A. Bacchetta, A. Metz, and J.-J. Yang, Phys. Lett. B574, 225 (2003).

[468] D. Amrath, A. Bacchetta, and A. Metz, Phys. Rev. D71, 114018 (2005).

[469] X. Artru, arXiv:1001.1061.

[470] D. Boer, R. Jakob, and P. J. Mulders, Nucl. Phys. B504, 345 (1997).

[471] BELLE Collaboration, R. Seidl et al., Phys. Rev. D78, 032011 (2008).

[472] BELLE Collaboration, K. Abe et al., Phys. Rev. Lett. 96, 232002 (2006).

[473] Schäfer, A. and Teryaev, O. V., Phys. Rev. D 61, 077903 (2000).

[474] M. Anselmino et al., Nucl. Phys. Proc. Suppl. 191, 98 (2009).

[475] K. Konishi, A. Ukawa, and G. Veneziano, Phys. Lett. B78, 243 (1978).

[476] R. L. Jaffe, X.-m. Jin, and J. Tang, Phys. Rev. Lett. 80, 1166 (1998).

[477] X. Artru and J. C. Collins, Z. Phys. C69, 277 (1996).

[478] A. Bacchetta and M. Radici, Phys. Rev. D69, 074026 (2004).

[479] A. Bacchetta and M. Radici, Phys. Rev. D67, 094002 (2003).

[480] A. Bacchetta and M. Radici, Phys. Rev. D74, 114007 (2006).

[481] HERMES Collaboration, A. Airapetian et al., JHEP 06, 017 (2008).

[482] A. Bacchetta, F. A. Ceccopieri, A. Mukherjee, and M. Radici, Phys. Rev. D79, 034029 (2009).

[483] A. Efremov, L. Mankiewicz, and N. Tornqvist, Phys. Lett. B284, 394 (1992).

[484] M. Stratmann and W. Vogelsang, Phys. Lett. B295, 277 (1992).

[485] D. Boer, R. Jakob, and M. Radici, Phys. Rev. D67, 094003 (2003).

[486] J. She, Y. Huang, V. Barone, and B.-Q. Ma, Phys. Rev. D77, 014035 (2008).

[487] COMPASS Collaboration, H. Wollny, arXiv:0907.0961.

[488] BELLE Collaboration, A. Vossen et al., arXiv:1104.2425.

[489] D. Boer, Phys. Rev. D60, 014012 (1999).

[490] D. Boer and W. Vogelsang, Phys. Rev. D74, 014004 (2006).

[491] J. C. Collins and D. E. Soper, Phys. Rev. D16, 2219 (1977).

[492] J. C. Collins, Phys. Rev. Lett. 42, 291 (1979).

[493] E. Mirkes and J. Ohnemus, Phys. Rev. D51, 4891 (1995).

[494] J. S. Conway et al., Phys. Rev. D39, 92 (1989).

[495] Z. Lu and B.-Q. Ma, Phys. Rev. D70, 094044 (2004).

[496] M. Diehl and P. Hagler, Eur. Phys. J. C44, 87 (2005).

[497] M. Burkardt, Phys. Rev. D72, 094020 (2005).

[498] R. N. Cahn, Phys. Lett. B78, 269 (1978).

[499] R. N. Cahn, Phys. Rev. D40, 3107 (1989).

[500] CLAS Collaboration, M. Osipenko et al., Phys. Rev. D80, 032004 (2009). 
[501] HERMES Collaboration, F. Giordano and R. Lamb, AIP Conf. Proc. 1149, 423 (2009).

[502] COMPASS Collaboration, A. Bressan, arXiv:0907.5511.

[503] B. Zhang, Z. Lu, B.-Q. Ma, and I. Schmidt, Phys. Rev. D77, 054011 (2008).

[504] V. Barone, S. Melis, and A. Prokudin, Phys. Rev. D82, 114025 (2010).

[505] COMPASS Collaboration, A. Kotzinian, arXiv:0705.2402.

[506] L. L. Pappalardo, Nuovo Cim. B125N1, 51 (2010).

[507] A. Bacchetta, M. Boglione, A. Henneman, and P. J. Mulders, Phys. Rev. Lett. 85, 712 (2000).

[508] A. Metz, P. Schweitzer, and T. Teckentrup, Phys. Lett. B680, 141 (2009).

[509] S. Wandzura and F. Wilczek, Phys. Lett. B72, 195 (1977).

[510] J. Balla, M. V. Polyakov, and C. Weiss, Nucl. Phys. B510, 327 (1998).

[511] B. Dressler and M. V. Polyakov, Phys. Rev. D61, 097501 (2000).

[512] M. Gockeler et al., Phys. Rev. D63, 074506 (2001).

[513] M. Gockeler et al., Phys. Rev. D72, 054507 (2005).

[514] A. Kotzinian, B. Parsamyan, and A. Prokudin, Phys. Rev. D73, 114017 (2006).

[515] R. L. Jaffe and X.-D. Ji, Phys. Rev. D43, 724 (1991).

[516] A. Harindranath and W.-M. Zhang, Phys. Lett. B408, 347 (1997).

[517] A. Accardi, A. Bacchetta, W. Melnitchouk, and M. Schlegel, JHEP 11, 093 (2009).

[518] R. L. Jaffe, Comm. Nucl. Part. Phys. 19, 239 (1990).

[519] HERMES Collaboration, A. Airapetian et al., Phys. Rev. Lett. 84, 4047 (2000).

[520] HERMES Collaboration, A. Airapetian et al., Phys. Rev. D64, 097101 (2001).

[521] HERMES Collaboration, A. Airapetian et al., Phys. Lett. B562, 182 (2003).

[522] CLAS Collaboration, H. Avakian et al., Phys. Rev. D69, 112004 (2004).

[523] HERMES Collaboration, A. Airapetian et al., Phys. Lett. B622, 14 (2005).

[524] HERMES Collaboration, A. Airapetian et al., Phys. Lett. B648, 164 (2007).

[525] W. Gohn, H. Avakian, K. Joo, and M. Ungaro, AIP Conf. Proc. 1149, 461 (2009).

[526] COMPASS Collaboration, I. A. Savin, PoS DIS2010, 246 (2010).

[527] European Muon Collaboration, J. J. Aubert et al., Phys. Lett. B130, 118 (1983).

[528] European Muon Collaboration, M. Arneodo et al., Z. Phys. C34, 277 (1987).

[529] H. Mkrtchyan et al., Phys. Lett. B665, 20 (2008).

[530] COMPASS Collaboration, W. Kafer, arXiv:0808.0114.

[531] E665 Collaboration, M. R. Adams et al., Phys. Rev. D48, 5057 (1993).

[532] ZEUS Collaboration, J. Breitweg et al., Phys. Lett. B481, 199 (2000).

[533] ZEUS Collaboration, S. Chekanov et al., Phys. Lett. B551, 226 (2003).

[534] ZEUS Collaboration, S. Chekanov et al., Eur. Phys. J. C51, 289 (2007).

[535] K. A. Oganessian, H. R. Avakian, N. Bianchi, and A. M. Kotzinian, hep-ph/9808368.

[536] A. M. Kotzinian, K. A. Oganesian, H. R. Avakian, and E. De Sanctis, hep-ph/9908466. 
[537] M. Boglione and P. J. Mulders, Phys. Lett. B478, 114 (2000).

[538] B.-Q. Ma, I. Schmidt, and J.-J. Yang, Phys. Rev. D63, 037501 (2001).

[539] M. Wakamatsu, Phys. Lett. B509, 59 (2001).

[540] A. V. Efremov, K. Goeke, and P. Schweitzer, Nucl. Phys. A711, 84 (2002).

[541] B.-Q. Ma, I. Schmidt, and J.-J. Yang, Phys. Rev. D66, 094001 (2002).

[542] A. V. Efremov, K. Goeke, and P. Schweitzer, Czech. J. Phys. 55, A189 (2005).

[543] M. Anselmino and F. Murgia, Phys. Lett. B483, 74 (2000).

[544] E. De Sanctis, W. D. Nowak, and K. A. Oganesian, Phys. Lett. B483, 69 (2000).

[545] A. V. Efremov, K. Goeke, M. V. Polyakov, and D. Urbano, Phys. Lett. B478, 94 (2000).

[546] K. A. Oganessian, N. Bianchi, E. De Sanctis, and W. D. Nowak, Nucl. Phys. A689, 784 (2001).

[547] A. V. Efremov, K. Goeke, and P. Schweitzer, Phys. Lett. B522, 37 (2001).

[548] B.-Q. Ma, I. Schmidt, and J.-J. Yang, Phys. Rev. D65, 034010 (2002).

[549] A. Efremov, K. Goeke, and P. Schweitzer, Eur. Phys. J. C24, 407 (2002).

[550] A. Efremov, K. Goeke, and P. Schweitzer, Phys. Rev. D67, 114014 (2003).

[551] A. V. Efremov, K. Goeke, and P. Schweitzer, Phys. Lett. B568, 63 (2003).

[552] A. V. Efremov, K. Goeke, and P. Schweitzer, Eur. Phys. J. C32, 337 (2003).

[553] F. Yuan, Phys. Lett. B589, 28 (2004).

[554] P. Schweitzer and A. Bacchetta, Nucl. Phys. A732, 106 (2004).

[555] L. P. Gamberg, D. S. Hwang, A. Metz, and M. Schlegel, Phys. Lett. B639, 508 (2006).

[556] A. Bacchetta, P. J. Mulders, and F. Pijlman, Phys. Lett. B595, 309 (2004).

[557] M. Radici, R. Jakob, and A. Bianconi, Phys. Rev. D65, 074031 (2002).

[558] F. A. Ceccopieri, M. Radici, and A. Bacchetta, Phys. Lett. B650, 81 (2007).

[559] M. Burkardt, arXiv:0810.3589.

[560] Y. Koike and K. Tanaka, Phys. Rev. D51, 6125 (1995).

[561] Y. Koike and N. Nishiyama, Phys. Rev. D55, 3068 (1997).

[562] A. V. Belitsky, Phys. Lett. B405, 312 (1997).

[563] A. V. Belitsky and D. Mueller, Nucl. Phys. B503, 279 (1997).

[564] A. Ali, V. M. Braun, and G. Hiller, Phys. Lett. B266, 117 (1991).

[565] I. I. Balitsky, V. M. Braun, Y. Koike, and K. Tanaka, Phys. Rev. Lett. 77, 3078 (1996).

[566] J. Kodaira and K. Tanaka, Prog. Theor. Phys. 101, 191 (1999).

[567] D. Mueller et al., Fortsch. Phys. 42, 101 (1994).

[568] X.-D. Ji, Phys. Rev. D55, 7114 (1997).

[569] A. Radyushkin, Phys. Lett. B380, 417 (1996).

[570] A. Radyushkin, Phys. Lett. B385, 333 (1996).

[571] A. Radyushkin, Phys. Rev. D56, 5524 (1997).

[572] J. C. Collins, L. Frankfurt, and M. Strikman, Phys. Rev. D56, 2982 (1997). 
[573] S. J. Brodsky and G. Lepage, in: Perturbative Quantum Chromodynamics, Ed. by A. H. Mueller (World Scientific, 1989).

[574] K. Goeke, M. V. Polyakov, and M. Vanderhaeghen, Prog. Part. Nucl. Phys. 47, 401 (2001).

[575] M. Diehl, Phys. Rept. 388, 41 (2003), Habilitation thesis.

[576] A. Belitsky and A. Radyushkin, Phys. Rept. 418, 1 (2005).

[577] S. Boffi and B. Pasquini, Riv. Nuovo Cim. 30, 387 (2007).

[578] A. Radyushkin, Phys. Rev. D58, 114008 (1998).

[579] M. Burkardt, Phys. Rev. D62, 071503 (2000).

[580] M. Burkardt, Int. J. Mod. Phys. A18, 173 (2003).

[581] M. Guidal, M. Polyakov, A. Radyushkin, and M. Vanderhaeghen, Phys. Rev. D72, 054013 (2005).

[582] M. Diehl, T. Feldmann, R. Jakob, and P. Kroll, Eur. Phys. J. C39, 1 (2005).

[583] M. Diehl, T. Feldmann, R. Jakob, and P. Kroll, Eur. Phys. J. C8, 409 (1999).

[584] Hall A Collaboration, D. Hamilton et al., Phys. Rev. Lett. 94, 242001 (2005).

[585] A. Radyushkin, Phys. Rev. D59, 014030 (1999).

[586] L. Mankiewicz, G. Piller, and T. Weigl, Eur. Phys. J. C5, 119 (1998).

[587] M. V. Polyakov and C. Weiss, Phys. Rev. D60, 114017 (1999).

[588] ZEUS Collaboration, S. Chekanov et al., Eur. Phys. J. C24, 345 (2002).

[589] H1 Collaboration, A. Aktas et al., Eur. Phys. J. C46, 585 (2006).

[590] M. E. Binkley et al., Phys. Rev. Lett. 48, 73 (1982).

[591] L. Frankfurt, M. Strikman, and C. Weiss, Phys. Rev. D83, 054012 (2011).

[592] L. Frankfurt, A. Freund, V. Guzey, and M. Strikman, Phys. Lett. B418, 345 (1998).

[593] A. Martin et al., Eur. Phys. J. C63, 57 (2009).

[594] M. Strikman and C. Weiss, Phys. Rev. D69, 054012 (2004).

[595] L. Frankfurt, M. Strikman, and C. Weiss, Phys. Rev. D69, 114010 (2004).

[596] L. Frankfurt, M. Strikman, and C. Weiss, Ann. Rev. Nucl. Part. Sci. 55, 403 (2005).

[597] M. Strikman and C. Weiss, Phys. Rev. D80, 114029 (2009).

[598] L. Frankfurt, M. Strikman, D. Treleani, and C. Weiss, Phys. Rev. Lett. 101, 202003 (2008).

[599] L. Frankfurt, A. Radyushkin, and M. Strikman, Phys. Rev. D55, 98 (1997).

[600] H. Kowalski and D. Teaney, Phys. Rev. D68, 114005 (2003).

[601] T. Rogers, V. Guzey, M. Strikman, and X. Zu, Phys. Rev. D69, 074011 (2004).

[602] M. Burkardt, Phys. Lett. B582, 151 (2004).

[603] M. Diehl, 2010, talk in this program on 8 Nov. 2010, http://www.int.washington.edu/talks/WorkShops/int_10_3

[604] LHPC Collaboration, J. Bratt et al., Phys. Rev. D82, 094502 (2010).

[605] S. Goloskokov and P. Kroll, Eur. Phys. J. C59, 809 (2009).

[606] D. Mueller, 2010, talk in this program on 8 Nov. 2010, http://www.int.washington.edu/talks/WorkShops/int_10_3 
[607] G. A. Miller, Phys. Rev. C80, 045210 (2009).

[608] G. A. Miller, Phys. Rev. Lett. 99, 112001 (2007).

[609] G. A. Miller, Ann. Rev. Nucl. Part. Sci. 60, 1 (2010).

[610] D. E. Soper, Phys. Rev. D15, 1141 (1977).

[611] M. Diehl, Eur. Phys. J. C25, 223 (2002).

[612] G. A. Miller and J. Arrington, Phys. Rev. C78, 032201 (2008).

[613] J. A. Rinehimer and G. A. Miller, Phys. Rev. C80, 025206 (2009).

[614] S. Venkat, J. Arrington, G. A. Miller, and X. Zhan, Phys. Rev. C83, 015203 (2011).

[615] P. Blunden, W. Melnitchouk, and J. Tjon, Phys. Rev. C72, 034612 (2005).

[616] J. Arrington, W. Melnitchouk, and J. Tjon, Phys. Rev. C76, 035205 (2007).

[617] A. Puckett et al., Phys. Rev. Lett. 104, 242301 (2010).

[618] S. Munier, A. M. Stasto, and A. H. Mueller, Nucl. Phys. B603, 427 (2001).

[619] M. Diehl and W. Kugler, Phys. Lett. B660, 202 (2008).

[620] ZEUS Collaboration, S. Chekanov et al., Nucl. Phys. B695, 3 (2004).

[621] M. Diehl, 2010, talk in this program on 1 Nov. 2010, http://www.int.washington.edu/talks/WorkShops/int_10_3.

[622] M. Burkardt, A. Miller, and W.-D. Nowak, Rept. Prog. Phys. 73, 016201 (2010).

[623] J. P. Ralston and B. Pire, Phys. Rev. D66, 111501 (2002).

[624] J. C. Collins and A. Freund, Phys. Rev. D59, 074009 (1999).

[625] A. V. Belitsky, D. Mueller, and A. Kirchner, Nucl. Phys. B629, 323 (2002).

[626] O. Teryaev, hep-ph/0510031.

[627] K. Kumericki, D. Mueller, and K. Passek-Kumericki, Nucl. Phys. B794, 244 (2008).

[628] M. Diehl and D. Ivanov, Eur. Phys. J. C52, 919 (2007).

[629] K. Kumericki, D. Mueller, and K. Passek-Kumericki, Eur. Phys. J. C58, 193 (2008).

[630] D. Mueller and A. Schafer, Nucl. Phys. B739, 1 (2006).

[631] M. Polyakov and A. Shuvaev, hep-ph/0207153.

[632] M. Polyakov, Phys. Lett. B659, 542 (2008).

[633] K. M. Semenov-Tian-Shansky, Eur. Phys. J. A36, 303 (2008).

[634] M. V. Polyakov and K. M. Semenov-Tian-Shansky, Eur. Phys. J. A40, 181 (2009).

[635] D. Mueller, Phys. Lett. B634, 227 (2006).

[636] K. Kumericki, D. Mueller, K. Passek-Kumericki, and A. Schafer, Phys. Lett. B648, 186 (2007).

[637] M. V. Polyakov, Nucl. Phys. B555, 231 (1999).

[638] M. Diehl, T. Gousset, B. Pire, and J. P. Ralston, Phys. Lett. B411, 193 (1997).

[639] A. V. Belitsky and D. Mueller, Phys. Lett. B513, 349 (2001).

[640] D. Ivanov, L. Szymanowski, and G. Krasnikov, JETP Lett. 80, 226 (2004).

[641] M. Diehl and W. Kugler, Eur. Phys. J. C52, 933 (2007). 
[642] ZEUS Collaboration, S. Chekanov et al., Phys. Lett. B573, 46 (2003).

[643] H1 Collaboration, A. Aktas et al., Eur. Phys. J. C44, 1 (2005).

[644] H1 Collaboration, F. Aaron et al., Phys. Lett. B659, 796 (2008).

[645] ZEUS Collaboration, S. Chekanov et al., JHEP 0905, 108 (2009).

[646] K. Kumericki and D. Mueller, Nucl. Phys. B841, 1 (2010).

[647] L. Frankfurt, A. Freund, and M. Strikman, Phys. Rev. D58, 114001 (1998).

[648] H1 Collaboration, F. Aaron et al., Phys. Lett. B681, 391 (2009).

[649] S. Alekhin, Phys. Rev. D68, 014002 (2003).

[650] A. V. Belitsky and D. Mueller, Phys. Rev. D79, 014017 (2009).

[651] M. Penttinen, M. V. Polyakov, and K. Goeke, Phys. Rev. D62, 014024 (2000).

[652] K. Goeke et al., Phys. Rev. D75, 094021 (2007).

[653] F. Yuan, Phys. Rev. D69, 051501 (2004).

[654] K. Kumericki and D. Mueller, 1008.2762.

[655] A. Belitsky and D. Mueller, Phys. Rev. D82, 074010 (2010).

[656] HERMES Collaboration, A. Airapetian et al., JHEP 0911, 083 (2009).

[657] CLAS Collaboration, F. Girod et al., Phys. Rev. Lett. 100, 162002 (2008).

[658] Hall A Collaboration, C. Munoz Camacho et al., Phys. Rev. Lett. 97, 262002 (2006).

[659] S. Goloskokov and P. Kroll, Eur. Phys. J. C53, 367 (2008).

[660] J. Kelly, Phys. Rev. C70, 068202 (2004).

[661] HERMES Collaboration, A. Airapetian et al., JHEP 0806, 066 (2008).

[662] CLAS Collaboration, S. Chen et al., Phys. Rev. Lett. 97, 072002 (2006).

[663] HERMES Collaboration, A. Airapetian et al., JHEP 1006, 019 (2010).

[664] M. Guidal, Phys. Lett. B689, 156 (2010).

[665] Hall A Collaboration, M. Mazouz et al., Phys. Rev. Lett. 99, 242501 (2007).

[666] M. Guidal, Eur. Phys. J. A37, 319 (2008).

[667] M. Guidal and H. Moutarde, Eur. Phys. J. A42, 71 (2009).

[668] H. Moutarde, Phys. Rev. D79, 094021 (2009).

[669] N. Kivel and L. Mankiewicz, Eur. Phys. J. C21, 621 (2001).

[670] S. Forte, L. Garrido, J. I. Latorre, and A. Piccione, JHEP 0205, 062 (2002).

[671] HERMES Collaboration, A. Airapetian et al., Phys. Rev. D75, 011103 (2007).

[672] HERMES Collaboration, A. Airapetian et al., Phys. Rev. C81, 035202 (2010).

[673] E. Perez, L. Schoeffel, and L. Favart, hep-ph/0411389.

[674] R. Géraud, H. Moutarde, and F. F. Sabatié, Prophet: Platform for representing the organization of partons inside hadrons and experimental tomographies (in preparation), 2011.

[675] P. Guichon and M. Vanderhaeghen, Analytic ee $\gamma$ cross section, private communication, 2010.

[676] M. Vanttinen and L. Mankiewicz, Phys. Lett. B440, 157 (1998).

[677] D. Ivanov, A. Schafer, L. Szymanowski, and G. Krasnikov, Eur. Phys. J. C34, 297 (2004). 
[678] A. Radyushkin, Phys. Lett. B449, 81 (1999).

[679] S. Goloskokov and P. Kroll, Eur. Phys. J. C42, 281 (2005).

[680] H1 Collaboration, F. D. Aaron et al., JHEP 05, 032 (2010).

[681] R. D. Ball et al., Nucl. Phys. B809, 1 (2009).

[682] C. Weiss and V. Guzey, private communication, 2008.

[683] Jefferson Lab $F_{\pi}$ Collaboration, H. Blok et al., Phys. Rev. C78, 045202 (2008).

[684] G. R. Goldstein, J. G. Hernandez, and S. Liuti, arXiv:1012.3776.

[685] S. Ahmad, G. R. Goldstein, and S. Liuti, Phys. Rev. D79, 054014 (2009).

[686] G. R. Goldstein, J. Gonzalez Hernandez, and S. Liuti, work in progress, 2010.

[687] S. Liuti and G. R. Goldstein, arXiv:1009.1334.

[688] G. R. Goldstein and S. Liuti, Phys. Rev. D80, 071501 (2009).

[689] G. R. Goldstein and S. Liuti, arXiv:1006.0213.

[690] S. Ahmad, H. Honkanen, S. Liuti, and S. K. Taneja, Eur. Phys. J. C63, 407 (2009).

[691] P. Hagler, Phys. Lett. B594, 164 (2004).

[692] G. Goldstein and J. Owens, Phys. Rev. D7, 865 (1973).

[693] S. Goloskokov and P. Kroll, Eur. Phys. J. C65, 137 (2010).

[694] M. Diehl, Eur. Phys. J. C19, 485 (2001).

[695] M. Diehl, T. Gousset, and B. Pire, Phys. Rev. D59, 034023 (1999).

[696] J. C. Collins and M. Diehl, Phys. Rev. D61, 114015 (2000).

[697] D. Ivanov, B. Pire, L. Szymanowski, and O. Teryaev, Phys. Lett. B550, 65 (2002).

[698] R. Enberg, B. Pire, and L. Szymanowski, Eur. Phys. J. C47, 87 (2006).

[699] M. Beiyad et al., Phys. Lett. B688, 154 (2010).

[700] M. Beiyad et al., arXiv:0911.2611.

[701] M. Wakamatsu, Eur. Phys. J. A44, 297 (2010).

[702] A. W. Thomas, Phys. Rev. Lett. 101, 102003 (2008).

[703] CMS Collaboration, S. Chatrchyan et al., arXiv:1107.0330.

[704] CDF Collaboration, F. Abe et al., Phys. Rev. Lett. 79, 584 (1997).

[705] M. Diehl and A. Schafer, Phys. Lett. B698, 389 (2011).

[706] I. Cherednikov and N. Stefanis, Nucl. Phys. B802, 146 (2008).

[707] C. W. Bernard et al., Phys. Rev. D64, 054506 (2001).

[708] LHPC Collaboration, P. Hagler et al., Phys. Rev. D77, 094502 (2008).

[709] B. Pasquini, S. Boffi, A. Efremov, and P. Schweitzer, arXiv:0912.1761.

[710] H. Dorn, Fortsch. Phys. 34, 11 (1986).

[711] L. Maiani, G. Martinelli, and C. T. Sachrajda, Nucl. Phys. B368, 281 (1992).

[712] G. Martinelli and C. T. Sachrajda, Phys. Lett. B354, 423 (1995).

[713] B. U. Musch, PoS LATTICE2010, 151 (2010).

[714] J. J. Dudek et al., Phys. Rev. D82, 034508 (2010). 
[715] SciDAC Collaboration, LHPC Collaboration, UKQCD Collaboration, R. G. Edwards and B. Joo, Nucl. Phys. Proc. Suppl. 140, 832 (2005).

[716] J. C. Collins, D. E. Soper, and G. F. Sterman, Nucl. Phys. B261, 104 (1985).

[717] J. C. Collins, D. E. Soper, and G. F. Sterman, Nucl. Phys. B308, 833 (1988).

[718] S. Moch, J. A. M. Vermaseren, and A. Vogt, Phys. Lett. B606, 123 (2005).

[719] J. Collins and H. Jung, hep-ph/0508280.

[720] F. Caola, S. Forte, and J. Rojo, Phys. Lett. B686, 127 (2010).

[721] NNPDF Collaboration, R. D. Ball et al., Nucl. Phys. B823, 195 (2009).

[722] V. S. Fadin and L. N. Lipatov, Phys. Lett. B429, 127 (1998).

[723] M. Ciafaloni and G. Camici, Phys. Lett. B430, 349 (1998).

[724] M. Ciafaloni, D. Colferai, G. P. Salam, and A. M. Stasto, Phys. Rev. D68, 114003 (2003).

[725] M. Ciafaloni, D. Colferai, and a. A. M. S. G. P. Salam, Phys. Lett. B587, 87 (2004).

[726] M. Ciafaloni, D. Colferai, G. Salam, and A. Stasto, JHEP 0708, 046 (2007).

[727] G. Altarelli, R. D. Ball, and S. Forte, Nucl. Phys. B742, 1 (2006).

[728] G. Altarelli, R. D. Ball, and S. Forte, Nucl. Phys. B674, 459 (2003).

[729] L. D. McLerran and R. Venugopalan, Phys. Rev. D49, 2233 (1994).

[730] L. D. McLerran and R. Venugopalan, Phys. Rev. D50, 2225 (1994).

[731] L. D. McLerran and R. Venugopalan, Phys. Rev. D49, 3352 (1994).

[732] Y. V. Kovchegov, Phys. Rev. D54, 5463 (1996).

[733] J. Jalilian-Marian, A. Kovner, L. D. McLerran, and H. Weigert, Phys. Rev. D55, 5414 (1997).

[734] G. P. Lepage and S. J. Brodsky, Phys. Rev. D22, 2157 (1980).

[735] N. N. Nikolaev and B. G. Zakharov, Z. Phys. C49, 607 (1991).

[736] Y. V. Kovchegov and L. D. McLerran, Phys. Rev. D60, 054025 (1999).

[737] A. H. Mueller, Nucl. Phys. B335, 115 (1990).

[738] Y. V. Kovchegov, Phys. Rev. D55, 5445 (1997).

[739] L. D. Landau and E. M. Lifshitz, Quantum mechanics, non-relativistic theory, Vol. 3 (Butterworth-Heinemann, 2003).

[740] I. Balitsky, Nucl. Phys. B463, 99 (1996).

[741] Y. V. Kovchegov, Phys. Rev. D60, 034008 (1999).

[742] L. V. Gribov, E. M. Levin, and M. G. Ryskin, Phys. Rept. 100, 1 (1983).

[743] A. H. Mueller and J. w. Qiu, Nucl. Phys. B268, 427 (1986).

[744] J. Jalilian-Marian, A. Kovner, A. Leonidov, and H. Weigert, Phys. Rev. D59, 014014 (1998).

[745] E. Iancu, A. Leonidov, and L. D. McLerran, Nucl. Phys. A692, 583 (2001).

[746] I. Balitsky, hep-ph/9706411.

[747] Y. V. Kovchegov, Phys. Rev. D61, 074018 (2000).

[748] M. Braun, Eur. Phys. J. C16, 337 (2000).

[749] K. Golec-Biernat, L. Motyka, and A. M. Stasto, Phys. Rev. D65, 074037 (2002). 
[750] K. Rummukainen and H. Weigert, Nucl. Phys. A739, 183 (2004).

[751] J. L. Albacete and Y. V. Kovchegov, Phys. Rev. D75, 125021 (2007).

[752] A. M. Stasto, K. Golec-Biernat, and J. Kwiecinski, Phys. Rev. Lett. 86, 596 (2001).

[753] A. Freund, K. Rummukainen, H. Weigert, and A. Schäfer, Phys. Rev. Lett. 90, 222002 (2003).

[754] E. Levin and K. Tuchin, Nucl. Phys. B573, 833 (2000).

[755] E. Iancu, K. Itakura, and L. McLerran, Nucl. Phys. A708, 327 (2002).

[756] J. Jalilian-Marian, A. Kovner, A. Leonidov, and H. Weigert, Nucl. Phys. B504, 415 (1997).

[757] J. Jalilian-Marian, A. Kovner, A. Leonidov, and H. Weigert, Phys. Rev. D59, 014014 (1999).

[758] J. Jalilian-Marian, A. Kovner, A. Leonidov, and H. Weigert, Phys. Rev. D59, 034007 (1999).

[759] J. Jalilian-Marian, A. Kovner, and H. Weigert, Phys. Rev. D59, 014015 (1999).

[760] A. Kovner, J. G. Milhano, and H. Weigert, Phys. Rev. D62, 114005 (2000).

[761] H. Weigert, Nucl. Phys. A703, 823 (2002).

[762] E. Iancu, A. Leonidov, and L. D. McLerran, Phys. Lett. B510, 133 (2001).

[763] E. Ferreiro, E. Iancu, A. Leonidov, and L. McLerran, Nucl. Phys. A703, 489 (2002).

[764] F. Gelis and R. Venugopalan, Nucl. Phys. A776, 135 (2006).

[765] F. Gelis and R. Venugopalan, Nucl. Phys. A779, 177 (2006).

[766] E. Iancu, A. Leonidov, and L. McLerran, hep-ph/0202270.

[767] E. Iancu and R. Venugopalan, Quark Gluon Plasma 3 (World Scientific, 2004), hep$\mathrm{ph} / 0303204$.

[768] Y. V. Kovchegov, J. Kuokkanen, K. Rummukainen, and H. Weigert, Nucl. Phys. A823, 47 (2009).

[769] J.-P. Blaizot, E. Iancu, and H. Weigert, Nucl. Phys. A713, 441 (2003).

[770] I. Balitsky, Nucl. Phys. B463, 99 (1996).

[771] S. Jeon and R. Venugopalan, Phys. Rev. D70, 105012 (2004).

[772] S. Jeon and R. Venugopalan, Phys. Rev. D71, 125003 (2005).

[773] Y. Hatta, E. Iancu, K. Itakura, and L. McLerran, Nucl. Phys. A760, 172 (2005).

[774] Y. V. Kovchegov, L. Szymanowski, and S. Wallon, Phys. Lett. B586, 267 (2004).

[775] L. D. McLerran and R. Venugopalan, Phys. Rev. D59, 094002 (1999).

[776] R. Venugopalan, Acta Phys. Polon. B30, 3731 (1999).

[777] F. Gelis, T. Lappi, and R. Venugopalan, Phys. Rev. D78, 054019 (2008).

[778] F. Gelis, T. Lappi, and R. Venugopalan, Phys. Rev. D78, 054020 (2008).

[779] F. Gelis, T. Lappi, and R. Venugopalan, Phys. Rev. D79, 094017 (2009).

[780] B. Z. Kopeliovich, L. I. Lapidus, and A. B. Zamolodchikov, JETP Lett. 33, 595 (1981).

[781] N. Nikolaev and B. G. Zakharov, Z. Phys. C53, 331 (1992).

[782] J. Kogut and D. Soper, Phys. Rev. D1, 2901 (1970).

[783] J. Bjorken, J. Kogut, and D. Soper, Phys. Rev. D3, 1382 (1971).

[784] S. Brodsky and A. Mueller, Phys. Lett. B206, 685 (1988). 
[785] J. B. K. J. M. Bjorken, Phys. Rev. D8, 1341 (1973).

[786] J. Kopeliovich and B. Povht, Z. Phys. A356, 467 (1997).

[787] B. Kopeliovich, J. Raufeisen, and A. Tarasov, Phys. Lett. B440, 151 (1998).

[788] K. Golec-Biernat and M. Wüsthoff, Phys. Rev. D59, 014017 (1999).

[789] New Muon Collaboration, M. Arneodo et al., Nucl. Phys. B481, 3 (1996).

[790] New Muon Collaboration, P. Amaudruz et al., Nucl. Phys. B441, 3 (1995).

[791] V. Gribov, Sov. Phys. JETP 29, 483 (1969).

[792] A. B. Kaidalov, Phys. Rept 50, 157 (1979).

[793] Y. M. Kazarinov, B. Z. Kopeliovich, L. I. Lapidus, and I. K. Potashnikova, Sov. Phys. JETP 43, 598 (1976).

[794] B. Z. Kopeliovich, A. V. Tarasov, and A. Schäfer, Phys. Rev. C59, 1609 (1999).

[795] B. Kopeliovich, A. Schäfer, and A. Tarasov, Phys. Rev. D62, 054022 (2000).

[796] A. DiGiacomo and H. Panagopoulos, Phys. Lett. B285, 133 (1992).

[797] T. Schäfer and E. V. Shuryak, Rev. Mod. Phys. 70, 323 (1998).

[798] B. Z. Kopeliovich, I. K. Potashnikova, B. Povh, and I. Schmidt, Phys. Rev. D76, 094020 (2007).

[799] B. Z. Kopeliovich, J. Nemchik, A. Schäfer, and A. V. Tarasov, Phys. Rev. C65, 035201 (2002).

[800] J. L. Albacete et al., Phys. Rev. D71, 014003 (2005).

[801] B. Z. Kopeliovich, I. K. Potashnikova, and I. Schmidt, Phys. Rev. C81, 035204 (2010).

[802] B. Z. Kopeliovich, E. Levin, I. K. Potashnikova, and I. Schmidt, Phys. Rev. C79, 064906 (2009).

[803] L. Frankfurt and M. Strikman, Eur. Phys. J. A5, 293 (1999).

[804] V. A. Abramovsky, V. N. Gribov, and O. V. Kancheli, Sov. J. Nucl. Phys. 18, 308 (1974).

[805] L. Frankfurt, V. Guzey, and M. Strikman, Phys. Rev. D71, 054001 (2005).

[806] V. Guzey and M. Strikman, Phys. Lett. B687, 167 (2010).

[807] L. Frankfurt, V. Guzey, and M. Strikman, to be submitted to Phys. Rep.

[808] J. C. Collins, Phys. Rev. D61, 019902 (2000).

[809] H1 Collaboration, A. Aktas et al., Eur. Phys. J. C48, 715 (2006).

[810] A. Donnachie and P. V. Landshoff, Phys. Lett. B296, 227 (1992).

[811] N. Armesto, A. B. Kaidalov, C. A. Salgado, and K. Tywoniuk, Eur. Phys. J. C68, 447 (2010).

[812] L. Frankfurt, V. Guzey, and M. Strikman, Phys. Lett. B586, 41 (2004).

[813] H. Abramowicz, L. Frankfurt, and M. Strikman, Surveys High Energ. Phys. 11, 51 (1997).

[814] A. I. Shoshi, F. D. Steffen, and H. J. Pirner, Nucl. Phys. A709, 131 (2002).

[815] A. I. Shoshi, F. D. Steffen, H. G. Dosch, and H. J. Pirner, Phys. Rev. D66, 094019 (2002).

[816] J. Nian and H. J. Pirner, Nucl. Phys. A833, 119 (2010).

[817] H. J. Pirner and F. Yuan, Phys. Rev. D66, 034020 (2002). 
[818] J. Bartels, K. Golec-Biernat, and L. Motyka, Phys. Rev. D81, 054017 (2010).

[819] K. J. Golec-Biernat and M. Wusthoff, Phys. Rev. D60, 114023 (1999).

[820] J. L. Albacete et al., arXiv:1012.4408.

[821] J. Bartels, K. J. Golec-Biernat, and H. Kowalski, Phys. Rev. D66, 014001 (2002).

[822] A. P. Bukhvostov, G. V. Frolov, L. N. Lipatov, and E. A. Kuraev, Nucl. Phys. B258, 601 (1985).

[823] H. Kowalski, L. Motyka, and G. Watt, Phys. Rev. D74, 074016 (2006).

[824] G. Watt and H. Kowalski, Phys. Rev. D78, 014016 (2008).

[825] H. Kowalski, T. Lappi, C. Marquet, and R. Venugopalan, Phys. Rev. C78, 045201 (2008).

[826] H. Kowalski, T. Lappi, and R. Venugopalan, Phys. Rev. Lett. 100, 022303 (2008).

[827] T. Lappi and H. Mantysaari, Phys. Rev. C83, 065202 (2011).

[828] K. J. Eskola, V. J. Kolhinen, and C. A. Salgado, Eur. Phys. J. C9, 61 (1999).

[829] S. Forte, Acta Phys. Polon. B41, 2859 (2010).

[830] G. Altarelli, R. D. Ball, and S. Forte, Nucl. Phys. B799, 199 (2008).

[831] F. Caola, S. Forte, and J. Rojo, Nucl. Phys. A854, 32 (2011).

[832] E. R. Cazaroto, F. Carvalho, V. P. Goncalves, and F. S. Navarra, Phys. Lett. B669, 331 (2008).

[833] I. Balitskyi, Phys. Rev. D75, 014001 (2005).

[834] Y. V. Kovchegov and H. Weigert, Nucl. Phys. A784, 188 (2007).

[835] I. Balitsky and G. A. Chirilli, Phys. Rev. D77, 014019 (2008).

[836] V. S. Fadin, E. A. Kuraev, and L. N. Lipatov, Phys. Lett. B60, 50 (1975).

[837] I. Balitsky and G. A. Chirilli, Nucl. Phys. B822, 45 (2009).

[838] I. Balitsky and G. A. Chirilli, Phys. Rev. D79, 031502 (2009).

[839] I. Balitsky and G. A. Chirilli, Phys. Rev. D83, 031502 (2011).

[840] I. Balitsky, hep-ph/0101042.

[841] A. H. Mueller and J.-w. Qiu, Nucl. Phys. B268, 427 (1986).

[842] A. H. Mueller, Nuclear Physics B335, 115 (1990).

[843] L. Cornalba, M. S. Costa, and J. Penedones, JHEP 03, 133 (2010).

[844] J. Bartels and A. Kyrieleis, Phys. Rev. D70, 114003 (2004).

[845] J. Bartels, D. Colferai, S. Gieseke, and A. Kyrieleis, Phys. Rev. D66, 094017 (2002).

[846] J. Bartels, S. Gieseke, and A. Kyrieleis, Phys. Rev. D65, 014006 (2002).

[847] I. Balitsky and G. A. Chirilli, Phys. Lett. B687, 204 (2010).

[848] G. A. Chirilli, L. Szymanowski, and S. Wallon, Phys. Rev. D83, 014020 (2011).

[849] S. J. Brodsky, G. P. Lepage, and P. B. Mackenzie, Phys. Rev. D28, 228 (1983).

[850] E. Gardi, J. Kuokkanen, K. Rummukainen, and H. Weigert, Nucl. Phys. A784, 282 (2007).

[851] Y. V. Kovchegov and H. Weigert, Nucl. Phys. A789, 260 (2007).

[852] J. L. Albacete, Phys. Rev. Lett. 99, 262301 (2007). 
[853] M. A. Braun, Phys. Lett. B348, 190 (1995).

[854] E. Levin, Nucl. Phys. B453, 303 (1995).

[855] V. Gribov and L. Lipatov, Sov. J. Nucl. Phys. 15, 438 (1972).

[856] G. Altarelli and G. Parisi, Nucl. Phys. B126, 298 (1977).

[857] Y. L. Dokshitzer, Sov. Phys. JETP 46, 641 (1977).

[858] L. Lipatov, Sov. J. Nucl. Phys. 23, 338 (1976).

[859] K. J. Golec-Biernat, L. Motyka, and A. M. Stasto, Phys. Rev. D65, 074037 (2002).

[860] J. L. Albacete et al., Phys. Rev. D71, 014003 (2005).

[861] A. H. Mueller and D. N. Triantafyllopoulos, Nucl. Phys. B640, 331 (2002).

[862] S. Munier and R. B. Peschanski, Phys. Rev. Lett. 91, 232001 (2003).

[863] S. Munier and R. B. Peschanski, Phys. Rev. D69, 034008 (2004).

[864] S. Munier and R. B. Peschanski, Phys. Rev. D70, 077503 (2004).

[865] M. Bramson, Mem. Am. Math. Soc. 44, 285 (1983).

[866] U. Ebert and W. van Saarloos, Physica D146, 1 (2000).

[867] J. Berger and A. Stasto, Phys. Rev. D83, 034015 (2011).

[868] G. Beuf, arXiv:1008.0498.

[869] A. H. Mueller, Nucl. Phys. A724, 223 (2003).

[870] K. Dusling, F. Gelis, T. Lappi, and R. Venugopalan, Nucl. Phys. A836, 159 (2010).

[871] M. Froissart, Phys. Rev. 123, 1053 (1961).

[872] K. J. Golec-Biernat and A. M. Stasto, Nucl. Phys. B668, 345 (2003).

[873] C. Marquet and L. Schoeffel, Phys. Lett. B639, 471 (2006).

[874] A. Bialas and R. B. Peschanski, Phys. Lett. B378, 302 (1996).

[875] A. Bialas and R. B. Peschanski, Phys. Lett. B387, 405 (1996).

[876] C. Marquet, Phys. Rev. D76, 094017 (2007).

[877] Y. V. Kovchegov and A. H. Mueller, Nucl. Phys. B529, 451 (1998).

[878] C. Marquet, B.-W. Xiao, and F. Yuan, Phys. Lett. B682, 207 (2009).

[879] V. P. Goncalves, M. S. Kugeratski, and F. S. Navarra, Phys. Rev. C81, 065209 (2010).

[880] T. Hirano et al., Phys. Lett. B636, 299 (2006).

[881] M. Luzum and P. Romatschke, Phys. Rev. C79, 039903 (2009).

[882] J. Jia and R. Wei, Phys. Rev. C82, 024902 (2010).

[883] B. Schenke, S. Jeon, and C. Gale, Phys. Rev. Lett. 106, 042301 (2011).

[884] J. Jia, W. A. Horowitz, and J. Liao, arXiv:1101.0290.

[885] A. Caldwell and H. Kowalski, Phys. Rev. C81, 025203 (2010).

[886] R. D. Woods and D. S. Saxon, Phys. Rev. 95, 577 (1954).

[887] T. Hirano and Y. Nara, Nucl. Phys. A743, 305 (2004).

[888] A. Kuhlman, U. W. Heinz, and Y. V. Kovchegov, Phys. Lett. B638, 171 (2006).

[889] J. Jalilian-Marian and Y. V. Kovchegov, Prog. Part. Nucl. Phys. 56, 104 (2006). 
[890] B. Hahn, D. G. Ravenhall, and R. Hofstadter, Phys. Rev. 101, 1131 (1956).

[891] PHOBOS Collaboration, B. B. Back et al., Phys. Rev. C65, 061901 (2002).

[892] PHENIX Collaboration, S. S. Adler et al., Phys. Rev. C71, 034908 (2005).

[893] PHOBOS Collaboration, B. Alver et al., Phys. Rev. C80, 011901 (2009).

[894] The ALICE, B. Abelev et al., Phys. Rev. Lett. 105, 252301 (2010).

[895] ALICE Collaboration, K. Aamodt et al., Phys. Rev. Lett. 106, 032301 (2011).

[896] J. L. Albacete and A. Dumitru, arXiv:1011.5161.

[897] W. A. Horowitz and Y. V. Kovchegov, Nucl. Phys. A849, 72 (2011).

[898] J. R. Forshaw, R. Sandapen, and G. Shaw, Phys. Rev. D69, 094013 (2004).

[899] C. Marquet, R. B. Peschanski, and G. Soyez, Phys. Rev. D76, 034011 (2007).

[900] R. Enberg, J. R. Forshaw, L. Motyka, and G. Poludniowski, JHEP 09, 008 (2003).

[901] G. G. Poludniowski, R. Enberg, J. R. Forshaw, and L. Motyka, JHEP 12, 002 (2003).

[902] A. Deshpande, R. Milner, R. Venugopalan, and W. Vogelsang, Ann. Rev. Nucl. Part. Sci. 55, 165 (2005).

[903] L. D. McLerran and R. Venugopalan, Phys.Rev. D49, 3352 (1994).

[904] H. Fujii, Nucl. Phys. A709, 236 (2002).

[905] J. P. Blaizot, F. Gelis, and R. Venugopalan, Nucl. Phys. A743, 57 (2004).

[906] F. Dominguez, C. Marquet, and B. Wu, Nucl. Phys. A823, 99 (2009).

[907] C. Marquet, R. B. Peschanski, and G. Soyez, Nucl. Phys. A756, 399 (2005).

[908] H. G. Dosch, T. Gousset, G. Kulzinger, and H. J. Pirner, Phys. Rev. D55, 2602 (1997).

[909] G. Kulzinger, H. G. Dosch, and H. J. Pirner, Eur. Phys. J. C7, 73 (1999).

[910] G. Soyez, Phys. Lett. B655, 32 (2007).

[911] A. Caldwell and H. Kowalski, arXiv:0909.1254.

[912] M. Alvioli, H. J. Drescher, and M. Strikman, Phys. Lett. B680, 225 (2009).

[913] B. Z. Kopeliovich, J. Nemchik, A. Schafer, and A. V. Tarasov, Phys. Rev. C65, 035201 (2002).

[914] J. Hufner, Y. P. Ivanov, B. Z. Kopeliovich, and A. V. Tarasov, Phys. Rev. D62, 094022 (2000).

[915] E. Eichten et al., Phys. Rev. D21, 203 (1980).

[916] W. Buchmuller and S. H. H. Tye, Phys. Rev. D24, 132 (1981).

[917] C. Quigg and J. L. Rosner, Phys. Lett. B71, 153 (1977).

[918] A. Martin, Phys. Lett. B93, 338 (1980).

[919] M. V. Terent'ev, Sov. J. Nucl. Phys. 24, 106 (1976).

[920] P. Hoyer and S. Peigné, Phys. Rev. D61, 031501(R) (2000).

[921] H. J. Melosh, Phys. Rev. D9, 1095 (1974).

[922] J. Hufner and B. Z. Kopeliovich, Phys. Lett. B426, 154 (1998).

[923] B. Z. Kopeliovich and B. G. Zakharov, Phys. Rev. D44, 3466 (1991). 
[924] Y. P. Ivanov, B. Z. Kopeliovich, A. V. Tarasov, and J. Hufner, Phys. Rev. C66, 024903 (2002).

[925] J. Hufner, B. Kopeliovich, and J. Nemchik, Phys. Lett. B383, 362 (1996).

[926] V. P. Goncalves, M. S. Kugeratski, M. V. T. Machado, and F. S. Navarra, Phys. Rev. C80, $025202(2009)$.

[927] E. R. Cazaroto et al., Phys. Lett. B696, 473 (2011).

[928] A. H. Mueller and B. Patel, Nucl. Phys. B425, 471 (1994).

[929] J. R. Forshaw and R. Sandapen, JHEP 11, 037 (2010).

[930] ZEUS Collaboration, S. Chekanov et al., PMC Phys. A1, 6 (2007).

[931] J. R. Forshaw and G. Shaw, JHEP 12, 052 (2004).

[932] L. Motyka, K. Golec-Biernat, and G. Watt, arXiv:0809.4191.

[933] J. R. Forshaw, R. Sandapen, and G. Shaw, JHEP 11, 025 (2006).

[934] J. Nemchik, N. N. Nikolaev, E. Predazzi, and B. G. Zakharov, Z. Phys. C75, 71 (1997).

[935] P. Ball and V. M. Braun, Phys. Rev. D54, 2182 (1996).

[936] A. P. Bakulev and S. V. Mikhailov, Phys. Lett. B436, 351 (1998).

[937] V. L. Chernyak and A. R. Zhitnitsky, Phys. Rept. 112, 173 (1984).

[938] P. Ball, V. M. Braun, and A. Lenz, JHEP 08, 090 (2007).

[939] P. Ball and R. Zwicky, Phys. Rev. D71, 014015 (2005).

[940] RBC Collaboration, P. A. Boyle et al., PoS LATTICE2008, 165 (2008).

[941] European Muon Collaboration, J. J. Aubert et al., Phys. Lett. B123, 275 (1983).

[942] M. Arneodo, Phys. Rept. 240, 301 (1994).

[943] PHENIX Collaboration, S. S. Adler et al., Phys. Rev. Lett. 91, 172301 (2003).

[944] A. Accardi et al., hep-ph/0308248.

[945] E. A. Paschos and J. Y. Yu, Phys. Rev. D65, 033002 (2002).

[946] J. Seely et al., Phys. Rev. Lett. 103, 202301 (2009).

[947] J. Gomez et al., Phys. Rev. D49, 4348 (1994).

[948] I. C. Cloet, W. Bentz, and A. W. Thomas, Phys. Rev. Lett. 102, 252301 (2009).

[949] R. P. Bickerstaff and A. W. Thomas, J. Phys. G15, 1523 (1989).

[950] E. L. Berger and F. Coester, Phys. Rev. D32, 1071 (1985).

[951] F. E. Close, R. G. Roberts, and G. G. Ross, Phys. Lett. B129, 346 (1983).

[952] O. Nachtmann and H. Pirner, Z.Phys. C21, 277 (1984).

[953] H. J. Pirner and J. P. Vary, arXiv:1008.4962.

[954] L. L. Frankfurt and M. I. Strikman, Nucl. Phys. B250, 143 (1985).

[955] G. A. Miller and J. R. Smith, Phys. Rev. C65, 015211 (2002).

[956] J. R. Smith and G. A. Miller, Phys. Rev. C65, 055206 (2002).

[957] I. C. Cloet, W. Bentz, and A. W. Thomas, Phys. Rev. Lett. 95, 052302 (2005).

[958] I. C. Cloet, W. Bentz, and A. W. Thomas, Phys. Lett. B642, 210 (2006). 
[959] Particle Data Group, C. Amsler et al., Phys. Lett. B667, 1 (2008).

[960] T. Gousset and H. J. Pirner, Phys. Lett. B375, 349 (1996).

[961] M. Kaluza, A. G. Schneider-Neureither, and H.-J. Pirner, Phys.Rev. C48, 877 (1993).

[962] H. Merabet, J. Mathiot, J. Dolejsi, and H. Pirner, Phys.Lett. B307, 177 (1993).

[963] K. J. Eskola, V. J. Kolhinen, and P. V. Ruuskanen, Nucl. Phys. B535, 351 (1998).

[964] M. Hirai, S. Kumano, and M. Miyama, Phys. Rev. D64, 034003 (2001).

[965] M. Hirai, S. Kumano, and T. H. Nagai, Phys. Rev. C70, 044905 (2004).

[966] K. J. Eskola, V. J. Kolhinen, H. Paukkunen, and C. A. Salgado, JHEP 05, 002 (2007).

[967] R. Sassot, M. Stratmann, and P. Zurita, Phys. Rev. D81, 054001 (2010).

[968] H. Paukkunen and C. A. Salgado, PoS ICHEP2010, 166 (2010).

[969] H. Paukkunen, C. A. Salgado, and K. J. Eskola, PoS ICHEP2010, 349 (2010).

[970] The HKN-NPDF code is available at http://research.kek.jp/people/kumanos/nuclp.html

[971] E665 Collaboration, M. Adams et al., Phys.Rev.Lett. 74, 1525 (1995).

[972] B. Z. Kopeliovich, J. Nemchick, N. N. Nikolaev, and B. G. Zakharov, Phys. Lett. B309, 179 (1993).

[973] L. Frankfurt, G. A. Miller, and M. Strikman, Phys. Rev. D65, 094015 (2002).

[974] E791 Collaboration, E. Aitala et al., Phys.Rev.Lett. 86, 4773 (2001).

[975] L. Frankfurt, G. Miller, and M. Strikman, Phys.Lett. B304, 1 (1993).

[976] L. Frankfurt, W. Koepf, and M. Strikman, Phys. Rev. D54, 3194 (1996).

[977] S. J. Brodsky et al., Phys. Rev. D50, 3134 (1994).

[978] L. Frankfurt, W. Koepf, and M. Strikman, Phys. Rev. D57, 512 (1998).

[979] A. J. Baltz et al., Phys. Rept. 458, 1 (2008).

[980] S. White and M. Strikman, arXiv:1003.2196.

[981] L. Frankfurt, V. Guzey, M. McDermott, and M. Strikman, Phys. Rev. Lett. 87, 192301 (2001).

[982] L. Frankfurt, M. Strikman, and M. Zhalov, Phys. Rev. Lett. 102, 232001 (2009).

[983] S. Kumano, M. Strikman, and K. Sudoh, Phys. Rev. D80, 074003 (2009).

[984] S. Kumano and M. Strikman, Phys. Lett. B683, 259 (2010).

[985] E. R. Berger, F. Cano, M. Diehl, and B. Pire, Phys. Rev. Lett. 87, 142302 (2001).

[986] F. Cano and B. Pire, Eur. Phys. J. A19, 423 (2004).

[987] A. Kirchner and D. Mueller, Eur. Phys. J. C32, 347 (2003).

[988] V. Guzey and M. Strikman, Phys. Rev. C68, 015204 (2003).

[989] V. Guzey, Phys. Rev. C78, 025211 (2008).

[990] Hall A Collaboration, M. Mazouz et al., Phys. Rev. Lett. 99, 242501 (2007).

[991] S. Scopetta, Phys. Rev. C70, 015205 (2004).

[992] S. Liuti and S. K. Taneja, Phys. Rev. C72, 032201 (2005).

[993] A. Freund and M. Strikman, Eur. Phys. J. C 33, 53 (2004). 
[994] K. Goeke, V. Guzey, and M. Siddikov, Phys. Rev. C79, 035210 (2009).

[995] V. Guzey, A. W. Thomas, and K. Tsushima, Phys. Lett. B673, 9 (2009).

[996] V. Guzey and M. Siddikov, J. Phys. G32, 251 (2006).

[997] H. Egiyan et al., 2008, Jefferson Lab Exp. E08-024.

[998] Z.-t. Liang, X.-N. Wang, and J. Zhou, Phys. Rev. D77, 125010 (2008).

[999] R. Baier et al., Nucl. Phys. B484, 265 (1997).

[1000] G. T. Bodwin, S. J. Brodsky, and G. Lepage, Phys. Rev. D39, 3287 (1989).

[1001] M. Luo, J.-w. Qiu, and G. F. Sterman, Phys. Lett. B279, 377 (1992).

[1002] X.-f. Guo, Phys. Rev. D58, 114033 (1998).

[1003] U. A. Wiedemann, Nucl. Phys. B588, 303 (2000).

[1004] R. J. Fries, Phys. Rev. D68, 074013 (2003).

[1005] A. Majumder and B. Muller, Phys. Rev. C77, 054903 (2008).

[1006] F. D'Eramo, H. Liu, and K. Rajagopal, arXiv:1006.1367.

[1007] Z.-t. Liang and X.-N. Wang, Phys. Rev. D75, 094002 (2007).

[1008] J.-H. Gao, Z.-t. Liang, and X.-N. Wang, Phys. Rev. C81, 065211 (2010).

[1009] Y.-k. Song, J.-h. Gao, Z.-t. Liang, and X.-N. Wang, Phys. Rev. D83, 054010 (2011).

[1010] A. Accardi et al., Riv. Nuovo Cim. 32, 439 (2010).

[1011] A. Majumder and M. Van Leeuwen, Prog. Part. Nucl. PHYS66, 41 (2011).

[1012] S. Albino, Rev. Mod. Phys. 82, 2489 (2010).

[1013] F. Arleo, JHEP 11, 044 (2002).

[1014] K. Gallmeister and U. Mosel, Nucl. Phys. A801, 68 (2008).

[1015] E. Wang and X.-N. Wang, Phys. Rev. Lett. 89, 162301 (2002).

[1016] A. Accardi, Eur. Phys. J. C49, 347 (2007).

[1017] PHENIX Collaboration, A. Adare et al., Phys. Rev. C77, 064907 (2008).

[1018] S. A. Bass et al., Phys. Rev. C79, 024901 (2009).

[1019] B. Z. Kopeliovich, J. Nemchik, E. Predazzi, and A. Hayashigaki, Nucl. Phys. A740, 211 (2004).

[1020] A. Accardi, V. Muccifora, and H.-J. Pirner, Nucl. Phys. A720, 131 (2003).

[1021] A. Accardi, D. Grunewald, V. Muccifora, and H. J. Pirner, Nucl. Phys. A761, 67 (2005).

[1022] A. Accardi, D. Grunewald, V. Muccifora, and H. J. Pirner, hep-ph/0508036.

[1023] K. Hafidi et al., Jefferson Lab experiment E12-06-117, http://www.jlab.org/exp_prog/proposals/06/PR12-06-117.pdf.

[1024] L. S. Osborne et al., Phys. Rev. Lett. 40, 1624 (1978).

[1025] L. Hand et al., Z. Phys. C1, 139 (1979).

[1026] E665 Collaboration, M. R. Adams et al., Z. Phys. C65, 225 (1995).

[1027] European Muon Collaboration, A. Arvidson et al., Nucl. Phys. B246, 381 (1984).

[1028] European Muon Collaboration, J. Ashman et al., Z. Phys. C52, 1 (1991). 
[1029] HERMES Collaboration, A. Airapetian et al., Nucl. Phys. B780, 1 (2007).

[1030] HERMES Collaboration, A. Airapetian et al., Phys. Lett. B684, 114 (2010).

[1031] HERMES Collaboration, A. Airapetian et al., Phys. Rev. Lett. 96, 162301 (2006).

[1032] C. Ciofi degli Atti and B. Z. Kopeliovich, Phys. Lett. B606, 281 (2005).

[1033] CLAS Collaboration, K. Hicks and A. Daniel, Proceedings of DIS2009, Madrid, Spain, April 2009, http://dx.doi.org/10.3360/dis.2009.85.

[1034] W. K. Brooks and H. Hakobyan, Nucl. Phys. A830, 361c (2009).

[1035] PHENIX Collaboration, A. Adare et al., Phys. Rev. Lett. 98, 172301 (2007).

[1036] STAR Collaboration, B. I. Abelev et al., Phys. Rev. Lett. 98, 192301 (2007).

[1037] A. Majumder, (2010), See http://www.int.washington.edu/talks/WorkShops/int_10_3.

[1038] I. Vitev, J. Phys. G35, 104011 (2008).

[1039] W. A. Horowitz and M. Gyulassy, J. Phys. G35, 104152 (2008).

[1040] GiBUU homepage: http://gibuu.physik.uni-giessen.de/GiBUU/

[1041] T. Falter, Nuclear reactions of high energy protons, photons and leptons, PhD thesis, JustusLiebig-Universität Gießen, 2004, available at http://theorie.physik.uni-giessen.de.

[1042] K. Gallmeister and T. Falter, Phys. Lett. B630, 40 (2005).

[1043] Y. L. Dokshitzer, V. A. Khoze, A. H. Mueller, and S. Troian, (1991).

[1044] G. Farrar, H. Liu, L. Frankfurt, and M. Strikman, Phys. Rev. Lett. 61, 686 (1988).

[1045] PHENIX Collaboration, S. S. Adler et al., Phys. Rev. Lett. 98, 172302 (2007).

[1046] STAR Collaboration, J. Adams et al., Phys. Lett. B637, 161 (2006).

[1047] N. Armesto, arXiv:0903.1330.

[1048] X.-f. Guo and X.-N. Wang, Phys. Rev. Lett. 85, 3591 (2000).

[1049] A. Majumder, E. Wang, and X.-N. Wang, Phys. Rev. C73, 044901 (2006).

[1050] N. Armesto, L. Cunqueiro, C. A. Salgado, and W.-C. Xiang, JHEP 02, 048 (2008).

[1051] S. Albino, B. A. Kniehl, and R. Perez-Ramos, Nucl. Phys. B819, 306 (2009).

[1052] B. Kopeliovich, J. Nemchik, and E. Predazzi, nucl-th/9607036.

[1053] B. Kopeliovich, J. Nemchik, and I. Schmidt, Nucl. Phys. A782, 224 (2007).

[1054] B. Kopeliovich, H.-J. Pirner, I. Potashnikova, and I. Schmidt, Phys. Lett. B662, 117 (2008).

[1055] B. Z. Kopeliovich, I. K. Potashnikova, and I. Schmidt, Phys. Rev. C82, 037901 (2010).

[1056] Y. L. Dokshitzer, V. A. Khoze, and S. Troian, J. Phys. G G17, 1481 (1991).

[1057] Y. L. Dokshitzer and D. Kharzeev, Phys. Lett. B519, 199 (2001).

[1058] B. Kopeliovich and F. Niedermayer, Sov. J. Nucl. Phys. 42, 504 (1985).

[1059] C. Markert, R. Bellwied, and I. Vitev, Phys. Lett. B669, 92 (2008).

[1060] B. Kopeliovich et al., Phys. Rev. C78, 055204 (2008).

[1061] B. Z. Kopeliovich and J. Nemchik, PoS CERP2010, 025 (2010).

[1062] E665 Collaboration, C. W. Salgado, FERMILAB-CONF-93-345-E (1993).

[1063] E665 Collaboration, H. L. Melanson, FERMILAB-CONF-93-165-E (1993). 
[1064] G. Soyez, (2009), See https://eic.jlab.org/wiki/images/b/b0/Soyez.jet_note.pdf.

[1065] A. Majumder, arXiv:0901.4516.

[1066] A. Majumder, arXiv:0912.2987.

[1067] S. Domdey, B. Z. Kopeliovich, and H. J. Pirner, Nucl. Phys. A856, 134 (2011).

[1068] S. Domdey et al., Nucl. Phys. A808, 178 (2008).

[1069] S. Domdey, D. Grunewald, B. Z. Kopeliovich, and H. J. Pirner, Nucl. Phys. A825, 200 (2009).

[1070] E665 Collaboration, M. Adams et al., Phys. Rev. Lett. 74, 5198 (1995).

[1071] M. Strikman, M. G. Tverskoii, and M. B. Zhalov, Phys. Lett. B459, 37 (1999).

[1072] C. Ciofi degli Atti and B. Z. Kopeliovich, Eur. Phys. J. A17, 133 (2003).

[1073] C. Ciofi degli Atti, L. P. Kaptari, and S. Scopetta, Eur. Phys. J. A5, 191 (1999).

[1074] C. Ciofi degli Atti, L. P. Kaptari, and B. Z. Kopeliovich, Eur. Phys. J. A19, 145 (2004).

[1075] V. Palli et al., Phys. Rev. C80, 054610 (2009).

[1076] C. Ciofi degli Atti and L. P. Kaptari, Phys. Rev. C83, 044602 (2011).

[1077] P. Norton, Rept. Prog. Phys. 66, 1253 (2003).

[1078] See, e.g., Nuclear Exclusive and Semi-inclusive Physics with a New CLAS12 Low Energy Recoil Detector, LOI to the PAC Jlab, K. Hafidi et al.

[1079] CLAS Collaboration, A. V. Klimenko et al., Phys. Rev. C73, 035212 (2006).

[1080] B. Kopeliovich, J. Nemchik, E. Predazzi, and A. Hayashigaki, Eur. Phys. J. A19S1, 111 (2004).

[1081] R. J. Glauber, Lectures in Theoretical Physics (Interscience Publishers, 1959).

[1082] A. Kievsky, S. Rosati, and M. Viviani, Phys. Rev. Lett. 82, 3759 (1999).

[1083] R. B. Wiringa, V. G. J. Stoks, and R. Schiavilla, Phys. Rev. C51, 38 (1995).

[1084] C. Ciofi degli Atti, L. L. Frankfurt, L. P. Kaptari, and M. I. Strikman, Phys. Rev. C76, 055206 (2007).

[1085] L. Frankfurt and M. Strikman, Phys.Rev. C82, 065203 (2010).

[1086] J. Arrington, D. Higinbotham, G. Rosner, and M. Sargsian, (2011).

[1087] L. Frankfurt, M. Strikman, and S. Liuti, Phys.Rev.Lett. 65, 1725 (1990).

[1088] L. L. Frankfurt and M. I. Strikman, Phys. Rept. 160, 235 (1988).

[1089] J. Bondorf et al., Phys. Rept. 257, 133 (1995).

[1090] ZEUS Collaboration, S. Chekanov et al., Phys. Lett. B583, 231 (2004).

[1091] G. Goldhaber, S. Goldhaber, W.-Y. Lee, and A. Pais, Phys. Rev. 120, 300 (1960).

[1092] PHENIX Collaboration, S. S. Adler et al., Phys. Rev. Lett. 93, 152302 (2004).

[1093] CMS Collaboration, V. Khachatryan et al., Phys. Rev. Lett. 105, 032001 (2010).

[1094] T. Csorgo, R. Vertesi, and J. Sziklai, Phys. Rev. Lett. 105, 182301 (2010).

[1095] CLAS Collaboration, A. V. Stavinsky et al., Phys. Rev. Lett. 93, 192301 (2004).

[1096] P. Degtyarenko et al., Z. Phys. A335, 231 (1990).

[1097] P. Degtyarenko et al., Z. Phys. A350, 263 (1994). 
[1098] P. Degtyarenko et al., Z. Phys. A357, 419 (1997).

[1099] H. Hakobyan, 2010, private communication.

[1100] E. A. McCutchan et al., Phys. Rev. Lett. 103, 192501 (2009).

[1101] S. C. Pieper and R. B. Wiringa, Ann. Rev. Nucl. Part. Sci. 51, 53 (2001).

[1102] K. Jansen, PoS LATTICE2008, 010 (2008).

[1103] CLAS Collaboration, K. S. Egiyan et al., Phys. Rev. Lett. 96, 082501 (2006).

[1104] CLAS Collaboration, H. Baghdasaryan et al., Phys. Rev. Lett. 105, 222501 (2010).

[1105] L. Frankfurt and M. Strikman, AIP Conf. Proc. 1056, 241 (2008).

[1106] M. M. Sargsian et al., J. Phys. G29, R1 (2003).

[1107] L. Lonnblad and T. Sjostrand, Phys. Lett. B351, 293 (1995).

[1108] L. Lonnblad and T. Sjostrand, Eur. Phys. J. C2, 165 (1998).

[1109] B. Andersson and W. Hofmann, Phys. Lett. B169, 364 (1986).

[1110] T. Sjostrand et al., Comput. Phys. Commun. 135, 238 (2001).

[1111] M. Bahr et al., Eur. Phys. J. C58, 639 (2008).

[1112] H. Jung, Comput.Phys.Commun. 86, 147 (1995).

[1113] L. Lonnblad, Comput.Phys.Commun. 71, 15 (1992).

[1114] H. Jung, Comput. Phys. Commun. 143, 100 (2002).

[1115] T. Gleisberg et al., JHEP 02, 007 (2009).

[1116] S. Roesler, R. Engel, and J. Ranft, hep-ph/0012252.

[1117] M. Good and W. Walker, Phys.Rev. 120, 1857 (1960).

[1118] W. Horowitz, 2011, these proceedings.

[1119] R. J. Charity, 2011, private communication.

[1120] A. Botvina, 2011, private communication.

[1121] The EIC "Parton Propagation and Fragmentation" working group wiki: https://eic.jlab.org/wiki/index.php/EA_ppf.

[1122] C. A. Salgado and U. A. Wiedemann, Phys. Rev. Lett. 89, 092303 (2002).

[1123] H. De Vries, C. W. De Jager, and C. De Vries, Atom. Data Nucl. Data Tabl. 36, 495 (1987).

[1124] C. A. Salgado and U. A. Wiedemann, Phys. Rev. D68, 014008 (2003).

[1125] A. Accardi, Phys. Rev. C76, 034902 (2007).

[1126] PHENIX Collaboration, K. Adcox et al., Nucl. Phys. A757, 184 (2005).

[1127] STAR Collaboration, J. Adams et al., Nucl. Phys. A757, 102 (2005).

[1128] PHOBOS Collaboration, B. B. Back et al., Nucl. Phys. A757, 28 (2005).

[1129] BRAHMS Collaboration, I. Arsene et al., Nucl. Phys. A757, 1 (2005).

[1130] M. Gyulassy and L. McLerran, Nucl. Phys. A750, 30 (2005).

[1131] T. Ludlam and L. McLerran, Phys. Today 56N10, 48 (2003).

[1132] P. F. Kolb, P. Huovinen, U. W. Heinz, and H. Heiselberg, Phys. Lett. B500, 232 (2001).

[1133] NA49 Collaboration, C. Alt et al., Phys. Rev. C68, 034903 (2003). 
[1134] M. Riordan and W. A. Zajc, Sci. Am. 294N5, 24 (2006).

[1135] BNL press release, RHIC scientists serve up "perfect" liquid, 2005, http://www.bnl.gov/bnlweb/pubaf/pr/pr_display . asp?prid=05-38

[1136] B. Jacak and P. Steinberg, Phys. Today 63N5, 39 (2010).

[1137] J. I. Kapusta, arXiv:0809.3746.

[1138] J. Takahashi et al., Phys. Rev. Lett. 103, 242301 (2009).

[1139] M. Gyulassy, D. H. Rischke, and B. Zhang, Nucl. Phys. A613, 397 (1997).

[1140] PHENIX Collaboration, Y. Akiba, Nucl. Phys. A774, 403 (2006).

[1141] W. A. Horowitz and M. Gyulassy, Phys. Lett. B666, 320 (2008).

[1142] W. A. Horowitz and B. A. Cole, Phys. Rev. C81, 024909 (2010).

[1143] G. S. Blanpied et al., Phys. Rev. Lett. 39, 1447 (1977).

[1144] R. Baier, A. H. Mueller, D. Schiff, and D. T. Son, Phys. Lett. B539, 46 (2002).

[1145] A. Dumitru, E. Molnar, and Y.Nara, Phys. Rev. C76, 024910 (2007).

[1146] D. Kharzeev and E. Levin, Phys. Lett. B523, 79 (2001).

[1147] Y. V. Kovchegov and K. Tuchin, Phys. Rev. D65, 074026 (2002).

[1148] PHOBOS Collaboration, G. Roland et al., Nucl. Phys. A774, 113 (2006).

[1149] H. J. Drescher, A. Dumitru, C. Gombeaud, and J. Y. Ollitrault, Phys. Rev. C76, 024905 (2007).

[1150] A. Dumitru, Nucl. Phys. A854, 71 (2011).

[1151] P. Tribedy and R. Venugopalan, Nucl. Phys. A850, 136 (2011).

[1152] J. Y. Ollitrault, Phys. Rev. D46, 229 (1992).

[1153] A. Adil et al., Phys. Rev. C74, 044905 (2006).

[1154] U. W. Heinz, J. S. Morel, , and H. Song, Phys. Rev. C80, 061901 (2009).

[1155] B. Alver and G. Roland, Phys. Rev. C81, 054905 (2010).

[1156] B. H. Alver, C. Gombeaud, M. Luzum, and J. Y. Ollitrault, Phys. Rev. C82, 034913 (2010).

[1157] G.-Y. Qin, H. Petersen, S. A. Bass, and B. Muller, Phys. Rev. C82, 064903 (2010).

[1158] D. Teaney and L. Yan, arXiv:1010.1876.

[1159] Y. V. Kovchegov, Phys. Rev. D68, 039901 (2001).

[1160] Y. Li and K. Tuchin, Phys. Rev. C78, 024905 (2008).

[1161] M. A. Braun, Phys. Lett. B483, 105 (2000).

[1162] J. P. Blaizot, F. Gelis, and R. Venugopalan, Nucl. Phys. A743, 13 (2004).

[1163] M. A. Braun, Eur. Phys. J. C70, 73 (2010).

[1164] D. Kharzeev, Y. V. Kovchegov, and K. Tuchin, Phys. Rev. D68, 094013 (2003).

[1165] R. Baier, A. Kovner, and U. A. Wiedemann, Phys. Rev. D68, 054009 (2003).

[1166] D. Kharzeev, Y. V. Kovchegov, and K. Tuchin, Phys. Lett. B599, 23 (2004).

[1167] A. Dumitru, A. Hayashigaki, and J. Jalilian-Marian, Nucl. Phys. A770, 57 (2006).

[1168] J. L. Albacete and C. Marquet, Phys. Lett. B687, 174 (2010). 
[1169] A. Dumitru and J. Jalilian-Marian, Phys. Rev. Lett. 89, 022301 (2002).

[1170] F. Gelis and A. Peshier, Nucl. Phys. A697, 879 (2002).

[1171] F. Gelis and J. Jalilian-Marian, Phys. Rev. D67, 074019 (2003).

[1172] D. Kharzeev, E. Levin, and M. Nard, Nucl. Phys. A743, 329 (2004).

[1173] K. Tuchin, Phys. Lett. B593, 66 (2004).

[1174] Y. V. Kovchegov and K. Tuchin, Phys. Rev. D74, 054014 (2006).

[1175] D. Kharzeev and K. Tuchin, Nucl. Phys. A735, 248 (2004).

[1176] F. G. H. Fujii and R. Venugopalan, Phys. Rev. Lett. 95, 162002 (2005).

[1177] K. Tuchin, Nucl. Phys. A798, 61 (2008).

[1178] J. Hufner, B. Kopeliovich, and A. B. Zamolodchikov, Z. Phys. A357, 113 (1997).

[1179] D. Kharzeev and K. Tuchin, Nucl. Phys. A770, 40 (2006).

[1180] D. Kharzeev, E. Levin, M. Nardi, and K. Tuchin, Nucl. Phys. A826, 230 (2009).

[1181] J. Jalilian-Marian, Nucl. Phys. A753, 307 (2005).

[1182] F. Gelis and J. Jalilian-Marian, Phys. Rev. D66, 094014 (2002).

[1183] R. Baier, A. H. Mueller, and D. Schiff, Nucl. Phys. A741, 358 (2004).

[1184] J. Jalilian-Marian, Nucl. Phys. A739, 319 (2004).

[1185] D. Kharzeev, E. Levin, and L. McLerran, Nucl. Phys. A748, 627 (2005).

[1186] C. Marquet, Nucl. Phys. A796, 41 (2007).

[1187] J. L. Albacete and C. Marquet, Phys. Rev. Lett. 105, 162301 (2010).

[1188] K. Tuchin, Nucl. Phys. A846, 83 (2010).

[1189] J. Jalilian-Marian and Y. V. Kovchegov, Phys. Rev. D71, 079901 (2005).

[1190] V. S. Fadin, M. I. Kotsky, and L. N. Lipatov, hep-ph/9704267.

[1191] A. Leonidov and D. Ostrovsky, Phys. Rev. D62, 094009 (2000).

[1192] A. Kovner and U. A. Wiedemann, Phys. Rev. D64, 114002 (2001).

[1193] Y. Li and K. Tuchin, Phys. Rev. D77, 114012 (2008).

[1194] K. Tuchin, Phys. Rev. C79, 055206 (2009).

[1195] K. J. Golec-Biernat and C. Marquet, Phys. Rev. D71, 114005 (2005).

[1196] K. Tuchin and D. Wu, Phys. Rev. D83, 054027 (2011).

[1197] M. Strikman, R. Vogt, and S. N. White, Phys. Rev. Lett. 96, 082001 (2006).

[1198] L. Frankfurt and M. Strikman, Phys. Lett. B645, 412 (2007).

[1199] W. J. Marciano, Phys.Rev. D20, 274 (1979).

[1200] W. Marciano and A. Sirlin, Phys.Rev.Lett. 46, 163 (1981).

[1201] M. E. Peskin and T. Takeuchi, Phys.Rev. D46, 381 (1992).

[1202] W. J. Marciano and J. L. Rosner, Phys.Rev.Lett. 65, 2963 (1990).

[1203] SLD Collaboration, K. Abe et al., Phys.Rev.Lett. 84, 5945 (2000).

[1204] The LEP Collaborations, LEP Electroweak Working Group and the SLD Heavy Flavour and Electroweak Working Groups, A Combination of Preliminary Electroweak Measurements and Constraints on the Standard Model, 2000, Report No. CERN-EP/2000-016. 
[1205] W. Marciano, AIP Conf.Proc. 870, 236 (2006).

[1206] C. Wood et al., Science 275, 1759 (1997).

[1207] SLAC E158 Collaboration, P. Anthony et al., Phys.Rev.Lett. 95, 081601 (2005).

[1208] NuTeV Collaboration, G. Zeller et al., Phys.Rev.Lett. 88, 091802 (2002).

[1209] Qweak Collaboration, M. T. Gericke, AIP Conf.Proc. 1149, 237 (2009).

[1210] J. Benesch et al., 2008, http://www.jlab.org/ armd/moller-proposal.pdf.

[1211] R. N. Cahn and F. J. Gilman, Phys.Rev. D17, 1313 (1978).

[1212] A. Czarnecki and W. J. Marciano, Phys.Rev. D53, 1066 (1996).

[1213] A. Ferroglia, G. Ossola, and A. Sirlin, Eur. Phys. J. C34, 165 (2004).

[1214] SNO Collaboration, Q. Ahmad et al., Phys. Rev. Lett. 89, 011301 (2002).

[1215] Super-Kamiokande Collaboration, Y. Fukuda et al., Phys. Rev. Lett. 81, 1562 (1998).

[1216] SINDRUM II Collaboration, W. H. Bertl et al., Eur. Phys. J. C47, 337 (2006).

[1217] MEGA Collaboration, M. Brooks et al., Phys. Rev. Lett. 83, 1521 (1999).

[1218] MEGA Collaboration, M. Ahmed et al., Phys.Rev. D65, 112002 (2002).

[1219] P. Paradisi, Prepared for IFAE 2006 (in Italian), Pavia, Italy , 229 (2006).

[1220] BABAR Collaboration, B. Aubert et al., Phys. Rev. Lett. 104, 021802 (2010).

[1221] Belle Collaboration, Y. Miyazaki et al., Phys. Lett. B660, 154 (2008).

[1222] C. H. Albright and M.-C. Chen, Phys. Rev. D77, 113010 (2008).

[1223] H1 Collaboration, F. D. Aaron et al., Phys. Lett. B701, 20 (2011).

[1224] H1 Collaboration, A. Aktas et al., Eur. Phys. J. C52, 833 (2007).

[1225] W. Buchmuller, R. Ruckl, and D. Wyler, Phys.Lett. B191, 442 (1987).

[1226] M. Gonderinger and M. J. Ramsey-Musolf, JHEP 1011, 045 (2010).

[1227] ZEUS Collaboration, S. Chekanov et al., Phys.Rev. D65, 092004 (2002).

[1228] ZEUS Collaboration, S. Chekanov et al., Eur. Phys. J. C44, 463 (2005).

[1229] M. Ramsey-Musolf and S. Su, Phys. Rept. 456, 1 (2008).

[1230] I. Dorsner and P. Fileviez Perez, Nucl. Phys. B723, 53 (2005).

[1231] P. Fileviez Perez, T. Han, T. Li, and M. J. Ramsey-Musolf, Nucl. Phys. B819, 139 (2009).

[1232] W. Buchmuller and D. Wyler, Nucl. Phys. B268, 621 (1986).

[1233] B. Grzadkowski, M. Iskrzynski, M. Misiak, and J. Rosiek, JHEP 1010, 085 (2010).

[1234] M. Raidal and A. Santamaria, Phys. Lett. B421, 250 (1998).

[1235] V. Cirigliano, A. Kurylov, M. Ramsey-Musolf, and P. Vogel, Phys. Rev. Lett. 93, 231802 (2004).

[1236] V. Cirigliano, B. Grinstein, G. Isidori, and M. B. Wise, Nucl. Phys. B728, 121 (2005).

[1237] A. Belyaev, C. Leroy, R. Mehdiyev, and A. Pukhov, JHEP 0509, 005 (2005).

[1238] S. Davidson, D. C. Bailey, and B. A. Campbell, Z.Phys. C61, 613 (1994).

[1239] EIC Wiki, BNL website: https://wiki.bnl.gov/eic/index.php/Kinematics.

[1240] H1 Collaboration, C. Adloff et al., Eur. Phys. J. C11, 447 (1999). 
[1241] CTEQ Collaboration, H. Lai et al., Eur. Phys. J. C12, 375 (2000).

[1242] M. Gonderinger, 2010, private communication.

[1243] L. Bellagamba, Comp. Phys. Commun. 141, 83 (2001).

[1244] RHIC: http://www.bnl.gov/rhic.

[1245] M. Farkhondeh and V. Ptitsyn, editors, eRHIC - Oth Order Design Report, 2004.

[1246] Y. Hao et al., p. TUPEB040 (2010).

[1247] S. Caspi et al., p. MOPEB059 (2010).

[1248] V. N. Litvinenko and Y. S. Derbenev, Phys.Rev.Lett. 102, 114801 (2009).

[1249] Y. Hao, V. Litvinenko, and V. Ptitsyn, p. TUPEB041 (2010).

[1250] P. Wanderer, IEEE Trans.Appl.Supercond. 19, 1208 (2010).

[1251] D. Trbojevic et al., p. MOPEA028 (2010).

[1252] E. Tsentalovich, AIP Conf.Proc. 980, 79 (2008).

[1253] V. Litvinenko, (2011), C-AD/AP/417 Note.

[1254] X. Chang, Polarized Gatling gun design, http://faculty.cua.edu/hornt/EICC_CUA_2010/ Chang_Gatling_gun_design.ppt .

[1255] D. Kayran, Proc. XXIV Linear Accelerator Conf. (LINAC08) (2008).

[1256] DOE NP offices support this R\&D project starting in FY 2011.

[1257] Y. Zhang et al., Proc. of PAC 2011 (2011).

[1258] CASA 2011, http://casa.jlab.org/meic/meic.shtml.

[1259] A. Bogacz, JLAB-TN-11-007 (2011).

[1260] H. Sayed, PhD thesis, Old Dominion University (2011).

[1261] B. Erdelyi et al., Proc. of PAC 2011 (2011).

[1262] S. Abeyratne, B. Erdelyi, and S. Manikonda, Proc. of PAC 2011 (2011).

[1263] B. Terzić and Y. Zhang, Proc. of IPAC 2010 (2010).

[1264] B. Terzić and Y. Zhang, ICFA Newsletter 52, 144 (2010).

[1265] S. Ahmed, Y. Derbenev, G. Krafft, and Y. Zhang, Proc. of IPAC 2011 (2011).

[1266] S. Ahmed, G. Krafft, and B. Yunn, Proc. of IPAC 2011 (2011).

[1267] S. Ahmed, G. Krafft, and B. Yunn, Proc. of IPAC 2011 (2011).

[1268] B. Terzić, M. Kramer, and C. Jarvis, Proc. of IPAC 2011 (2011).

[1269] J. Qiang, M. Furman, and R. Ryne, Phys. Rev. ST: Acell. Beams 5, 104402 (2002).

[1270] E. Zitzler, M. Laumanns, and L. Thiele, Proc. of EUROGEN2011 (2001).

[1271] I. Bazarov and C. Sinclair, Phys. Rev. ST: Acell. Beams 8, 034202 (2005).

[1272] M. Kramer, C. Jarvis, and B. Terzić, JLAB-TN-11-007 (2010).

[1273] F. Jacquet and A. Blondel, DESY 79/48 (1979), in Proceedings of the Study of an ep facility for Europe, U. Amaldi (ed.).

[1274] U. Bassler and G. Bernardi, NIM A361, 197 (1995).

[1275] Spin Muon Collaboration, B. Adeva and other, Phys. Rev. D 62, 079902 (2000). 
[1276] A. Caldwell and H. Kowalski, Phys. Rev. C 81, 025203 (2010).

[1277] H. Spiesberger et al., Contribution to Workshop on Physics at HERA, Hamburg, Germany, 1991.

[1278] I. Akushevich and H. Spiesberger, Contribution to Workshop on Future Physics at HERA, Hamburg, Germany, 1995/96.

[1279] K. J. Eskola, H. Paukkunen, and C. A. Salgado, JHEP 07, 102 (2008).

[1280] H. Spiesberger, Phys. Rev. D52, 4936 (1995).

[1281] J. Kripfganz, H. Mohring, and H. Spiesberger, Z. Phys. C49, 501 (1991).

[1282] G. Gaycken et al., NIM A560, 44 (2006).

[1283] C. Hu-Guo et al., JINST 4, P04012 (2009).

[1284] J. de Favereau, X. Rouby, and K. Piotrzkowski, JINST 2, P09005 (2007).

[1285] R. J. Charity, Phys. Rev. C82, 014610 (2010).

[1286] A. Botvina, G. Chaudhuri, S. Das Gupta, and I. Mishustin, Phys. Lett. B668, 414 (2008).

[1287] M. Beckmann et al., NIM A479, 334 (2002).

[1288] A. Zelenski et al., in Proceedings of the 18th International Symposium on High-Energy Spin Physics, Charlottesville, 2008, AIP Conf. Proc. No 1149, p. 847.

[1289] A. Zelenski et al., NIM A536, 248 (2005).

[1290] Y. Makdisi et al., Proceedings of PAC07, p. 4671.

[1291] PHENIX Collaboration, The PHENIX Experiment at RHIC - Decadal Plan 2011 - 2020 , http://wWw.phenix.bnl.gov/phenix/WWW/docs/decadal/2010/ phenix_decadal10_full_refs.pdf.

[1292] K. H. Ackermann et al., NIM A499, 624 (2003).

[1293] M. Anderson et al., NIM A499, 659 (2003).

[1294] M. Anderson et al., NIM A499, 679 (2003).

[1295] STAR Collaboration, Decadal plan, http://www.bnl.gov/npp/docs/STAR_Decadal_Plan_Final [1].pdf. 


\section{List of Authors}

Abeyratne, S., 448

Accardi, Alberto, 240, 280, 354, 358, 370, 394, 395

Ahmed, S., 448

Aidala, Christine, 95

Alekhin, Sergey, 38

Anselmino, Mauro, 84

Aschenauer, Elke, 31, 34, 43,53, 95, 177, 459

Avakian, Harut, 138,149

Bacchetta, Alessandro, 95, 138, 149

Bartels, Jochen, 275

BC, Hikmat, 183

Beebe-Wang, Joanne, 440

Belomestnykh, Sergei, 440

Ben-Zvi, Ilan, 440

Beuf, Guillaume, 292

Blümlein, Johannes, 38

Blaskiewicz, Michael M., 440

Boer, Daniël, 10, 95, 120]

Bogacz, A., 448

Brodsky, Stanley J., 120

Burkardt, Matthias, 154, 183

Burton, Thomas, 95

Calaga, Rama, 440

Chang, Xiangyun, 440

Cherednikov, Igor O., 125

Chevtsov, P., 448

Chirilli, Giovanni A., 286

Ciofi degli Atti, Claudio, 378

Cloët, Ian C., 333

Cooper-Sarkar, Amanda, 22

Debbe, Ramiro, 34

Derbenev, Ya., 448

Deshpande, Abhay, 61, 418

Diehl, Markus, 1 [120, 170, 177.

Dominguez, Fabio, 304

Dumitru, Adrian, 401

Dupré, Raphaël, 354, 358, 395

Ent, Rolf, 459

Erdelyi, B., 448

Faroughy, Cyrus, 418
Fazio, Salvatore, 177, 197

Fedotov, Alexei, 440

Forshaw, Jeffrey R., 327

Géraud, Rémi, 205

Gallmeister, Kai, 361, 383

Gamberg, Leonard, 95

Gao, Jian-Hua, 351

Gassner, David, 440

Gelis, François, 261

Gilfoyle, Gerald P., 384

Goldstein, Gary, 219

Golec-Biernat, Krzysztof, 275

Gonçalves, Victor P., 283, 306, 324

Gonderinger, Matthew, 418

Guzey, Vadim, 154, 270, 280, 300, 347

Guzzi, Marco, 27, 44

Hägler, Philipp, 227

Hahn, Harald, 440

Hammons, Lee, 440

Hao, Yue, 440

Hasch, Delia, 95

He, Ping, 440

Horn, Tanja, 212, 459

Horowitz, William A., 310, 397

Huang, Min, 95

Hutton, A., 448

Jäger, Barbara, 81

Jackson, William, 440

Jain, Animesh, 440

Johnson, Elliott C., 440

Kang, Zhong-Bo, 95, 125

Kaptari, Leonid P., 378

Kayran, Dmitry, 440

Kewisch, Jörg, 440

Koike, Yuji, 95

Kondratenko, A., 448

Kopeliovich, Boris Z., 265, 320, 340, 367, 378

Kovchegov, Yuri V., 253,289

Krafft, G., 448

Kroll, Peter, 208

Kumano, Shunzo, 338

Kumar, Krishna, 61, 410, 411,418 
Kumerički, Krešimir, 185

Lamont, Matthew, 240, 370,

Lappi, Tuomas, 277, 315

Lautenschlager, Tobias, 185]

Li, R., 448

Li, Yingchuan, 410, 411

Liang, Zuo-tang, 351

Litvinenko, Vladimir N., 440

Liuti, Simonetta, 219

Luo, Yun, 440

Müller, Dieter, 185

Mahler, George, 440

Majumder, Abhijit, 370

Manikonda, S., 448

Marciano, William J., 410, 411

Marhauser, F., 448

Marquet, Cyrille, 240, 253, 295, 297, 304, 315

McIntyre, Gary, 440

Meškauskas, Mantas, 185

Meng, Wuzheng, 440

Metz, Andreas, 84, 138, 149

Mezzetti, Chiara Benedetta, 378

Miller, Gerald A., 174]

Milner, Richard, 1]

Minty, Michiko, 440

Moch, Sven-Olaf, 19, 38

Morozov, V, 448

Mosel, Ulrich, 361, 383

Motyka, Leszek, 275]

Moutarde, Hervé, 205

Mulders, Piet J., 120, 125

Musch, Bernhard, 95, 227

Nadel-Turonski, Pawel, 459

Nadolsky, Pavel, 27, 44

Olness, Fredrick, 27, 44

Ostrumov, P. N., 448

Parker, Brett, 440

Pasquini, Barbara, 125

Passek-Kumerički, Kornelija, 185

Pikin, Alexander, 440

Pilat, F., 448

Pire, Bernard, 222

Pirner, Hans J., 272, 334, 374

Pisano, Cristian, 120

Pozdeyev, Eduard, 440

Prokudin, Alexei, 95

Ptitsyn, Vadim, 440

Qian, Xin, 95

Qiu, Jian-Wei, 125
Radici, Marco, 138

Radyushkin, Anatoly, 156

Rao, Triveni, 440

Rimmer, R., 448

Ringer, Felix, 61

Riordan, Seamus, 61411

Rogers, Ted, 125

Rojo, Juan, 280

Roser, Thomas, 440

Sabatié, Franck, 154, 205

Sandapen, Ruben, 327

Sassot, Rodolfo, 46, 53, 332, 335,363

Satogata, T., 448

Sayed, H., 448

Schäfer, Andreas, 185,227

Schnell, Gunar, 95

Schweitzer, Peter, 84, 125, 149

Sheehy, Brian, 440

Skaritka, John, 440

Soyez, Gregory, 370

Spata, M., 448

Spiesberger, Hubert, 77, 779, 459

Staśto, Anna M., 250 251

Stefanis, Nicolaos G., 125

Stratmann, Marco, 14, 31, 34, 43, 46, 53, 75, 77. $81,332,335,363$

Strikman, Mark, 270, 300, 342, 347, 376, 381, 407

Sullivan, M., 448

Szymanowski, Lech, 222

Tanaka, Kazuhiro, 95

Taneja, Swadhin, 61, 418

Tepikian, Steven, 440

Terzić, Balša, 448

Than, Yatming, 440

Toll, Tobias, 389

Trbojevic, Dejan, 440

Tsentalovich, Evgeni, 440

Tsoupas, Nicholaos, 440

Tuchin, Kirill, 403

Tuozzolo, Joseph, 440

Ullrich, Thomas, 389

Venugopalan, Raju, 1

Vogelsang, Werner, 10 61

Vossen, Anselm, 95

Wallon, Samuel, 222

Wang, Gang, 440

Wang, H., 448

Wang, Xin-Nian, 351

Webb, Stephen, 440

Weiss, Christian, 166 
Wu, Qiong, 440

Xiao, Bo-Wen, 120, 304

$\mathrm{Xu}$, Wencan, 440

Yuan, Feng, 120, 304

Yunn, B., 448

Zelenski, Anatoly, 440

Zhang, Yuhong, 448

Zhou, Jian, 351

Zurita, Pia, 332, 335, 363 